\title{
LATE HOLOCENE VEGETATION HISTORY OF GREVENA PROVINCE, NORTHWESTERN GREECE
}

\author{
Volume I - Text \\ by \\ Pamela I. Chester \\ A thesis \\ submitted to the Victoria University of Wellington \\ in fulfilment of the \\ requirements for the degree of \\ Doctor of Philosophy \\ in Geology
}

Victoria University of Wellington,

New Zealand.

1998 


\begin{abstract}
Palynological investigations aimed at reconstructing the vegetation history of the Grevena Province, northwestern Greece were conducted in association with an archaeological research project. Fossil pollen, spores, microscopic charcoal particles, and sediment stratigraphies of radiocarbon-dated sediment cores from three sites in pine, beech, and oak woodland zones provide evidence of climatic changes, land use, and erosion during the last 3500 years. Identification of pollen and spores was aided by an extensive reference collection of local species and construction of a pollen key modelled on that of Faegri \& Iversen (1989).

Extant vegetation units are characterized by their contemporary pollen assemblages. Surface samples collected along an elevational transect show that a fairly direct relationship exists between the major vegetation zones and pollen deposition: pine, beech, and oak pollen predominate in their respective zones. Over-representation of pine pollen is notable.

The sequence from Gomara site in the pine wood-pasture zone at $1750 \mathrm{~m}$ asl covers the time span c. $1340 \mathrm{BC}$ to $700 \mathrm{AD}$. A local open pine wood was gradually replaced by beech after c. $890 \mathrm{BC}$, perhaps through reduced disturbance and/or increased precipitation. A herbaceous pollen spike at c. $80 \mathrm{BC}$ resulted from deposition of volcanic ash. Pine wood replaced beech at c. $330 \mathrm{AD}$. Two periods of accelerated erosion coincide with the pine wood phases and with anthropogenic burning and grazing. These periods are separated by a period of abandonment when the climate was probably wetter.

The sequence from Anelia site in the beech wood zone at $1440 \mathrm{~m}$ asl spans c. 15601989 AD. The site was surrounded by beech wood for the duration of the sequence. Regional and local burning of vegetation is indicated by an abundance of microscopic charcoal particles from c. 1560 until c. $1730 \mathrm{AD}$, when it ceased. Periods of erosion occurred during this period. After c. $1730 \mathrm{AD}$ a homogeneous peat formed on the wetland, suggesting a period of greater landscape stability. From pollen evidence, a variety of land-use practices such as cereal cultivation, grazing, coppicing, and lumbering were carried out in the vicinity of the site especially before $c .1730 \mathrm{AD}$, but these diminished after $1920 \mathrm{AD}$.

The lower part of the sequence from Kellia site in the oak wood-steppe zone at $580 \mathrm{~m}$ asl is insecurely dated, but the upper part spans c. 1230-1989 AD. The lowlands were covered with deciduous/semi-evergreen oak woods for the duration of the sequence. Since $c .1230 \mathrm{AD}$ land close to the site was intensively cultivated with a variety of cereals. Burning occurred frequently throughout the period. The ratio of deciduous to semi-evergreen oak pollen is correlated with temperature and indicates a decline from c. 1230-1680 AD, after which temperature increased, a pattern similar to that of the Little Ice Age.
\end{abstract}



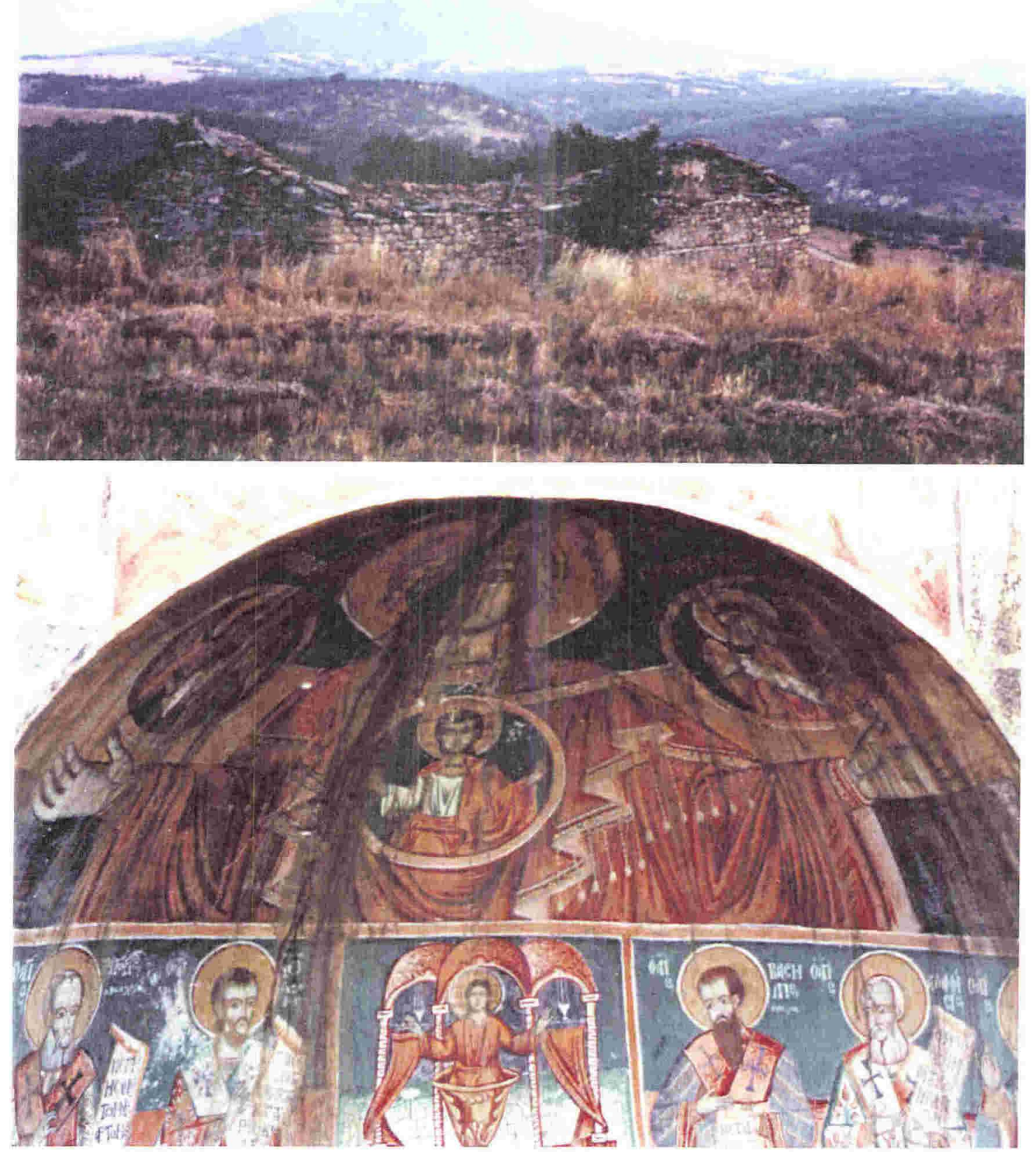

Frontispiece The cultural landscape of Grevena Province looking NE towards the Vourinos Mountains on the eastern border of Grevena, from a prominent hill about $1 \frac{1}{4} \mathrm{~km} \mathrm{NW}$ of the modern village of Itea. In the foreground is the stubble of a wheat field. Beyond is an 18th century church dedicated to Aghia Panaghia. The middle ground is the steppe oak wood pasture of Grevena plains. Recent erosion on the sides of gullies can be seen here, even though they appear to be well vegetated.

Frescoes in the church are shown in the enlargement. It stands on a registered archaeological site, Grevena Project $108\left(21^{\circ} 36.18^{\prime} \mathrm{E}, 40^{\circ} 04.29^{\prime} \mathrm{N}, 640 \mathrm{~m}\right.$ asl $)$ that covers more than $10,000 \mathrm{~m}^{2}$. Archaeological evidence dating to Early Iron Age, Archaic, Classical, Hellenistic, Roman, Early Medieval and Ottoman periods has been recorded. 
This thesis is dedicated to my father

\section{Hugh Chester}

$1913-1990$ 


\section{Acknowledgements}

Working on the Grevena Project gave me the opportunity to work on an interdisciplinary archaeological project with a dedicated group of scholars, experts in their respective fields of research. I wish to acknowledge my heartfelt gratitude to every member of the Grevena Project for their assistance with my research.

I am greatly indebted to many people who assisted me with my research. I wish to thank the director of the Grevena Project Dr Nancy Wilkie, Professor of Classics, Carleton College, Minnesota, and my initial supervisor and geologist of the Grevena Project Dr Mary Savina, Professor of Geology, Carleton College, for their assistance in many varied ways, their patience and friendship for the duration of my research. I am also indebted to Victoria University Geology Department supervisors: Drs Brad Pillans and James Shulmeister.

I was greatly assisted in the field by Drs Oliver Rackham, Cambridge University, plant ecologist for the Grevena Project and Ian Raine, palynologist, [N.Z.] Institute of Geological \& Nuclear Sciences (IGNS) who assisted with the overall approach to fieldwork and with collecting data. Others who assisted with the collection of field data were members of the Grevena Project: Dr Nancy Wilke, Dr Stanley Aschenbrenner, Dr Mary Savina, Richard Doyle, Mira Beaglehole; Maria Vaiopiulou, representative of the Greek Archaeological Service; Penelope Adamakopoulos; and staff of the Greek Forest Service, Grevena. The Greek Forest Service also allowed use of unpublished vegetation maps.

I would like to thank Peter Tijsen, head gardener, Wellington Botanic Gardens who gave permission to collect pollen from plants in the gardens, and Dr Patrick Brownsey, Curator of the Wellington National Museum herbarium and Dr Barry Sneddon, Senior lecturer, Biological Sciences, Victoria University, who gave permission to collect pollen from herbaria. Thanks to IGNS and staff who supplied facilities, chemicals and equipment for preparation of most of the pollen reference collection and to Drs Margaret Atherden, Tony Stevenson and Brian Huntley who donated reference slides. Thanks also to Greer Laboratories Inc., North Carolina, who donated Lycopodium spores; Ajax Chemicals, Auckland who donated silicone oil; and to Dr Linda Shane who supplied detailed instructions on making a spike.

I would like to thank several people of Victoria University who cheerfully helped in many varied ways while I carried out my research: staff of the workshop (manufacture of the piston corer); staff of Biological Sciences, Peter Watson (use of microscope), Tanya Clough (photographic processing), Philipa Spackman and Margaret Crimp (any and every problem to do with finding equipment); Brett Robertson (photographic processing); Dr Rodney Grapes (use of carbon analyser); Dr Margaret Harper (identification of diatoms); Chemistry Department (lending equipment); and reference librarians (acquiring interloan references).

Financial assistance was provided for fieldwork by grants from the Royal Society Hutton Memorial Fund, N.Z.; Victoria University Internal Grants Committee; Carleton College; the National Science Foundation, U.S.A.; and members of the Grevena Project. Victoria University and the N.Z. Lottery Board provided funding for radiocarbon dating. Funding was also provided by a Victoria University Postgraduate Scholarship, a Federation of University Women Postgraduate Fellowship, Mrs I.A. Chester and Dr Ian Raine.

I wish to thank my mother and siblings who tolerated my many absences from family celebrations, and my nieces and nephews who maintained an encouraging interest in my research. I am in greatest debt to Dr Ian Raine, my partner in life and mentor, who guided me through this project, assisted in the field and draughted the figures. 


\section{Contents}

VOLUME I - Text

\section{Chapter 1. Introduction}

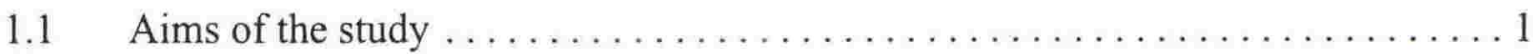

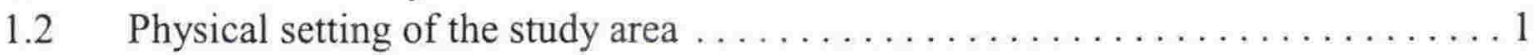

1.2 .1 Location . . . . . . . . . . . . . . . . . . . . . 3

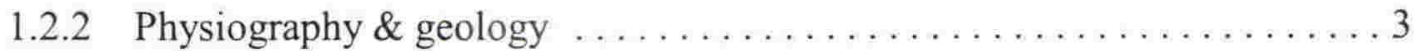

1.2 .3 Climate . . . . . . . . . . . . . . . . . . . . . . . . . . 7

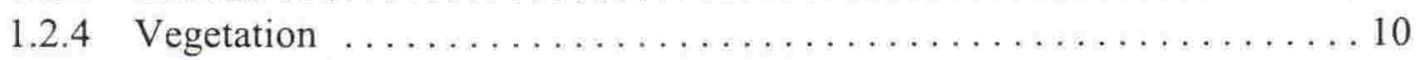

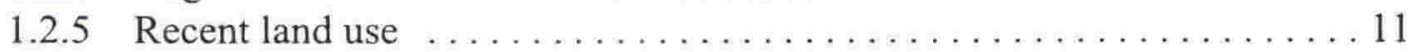

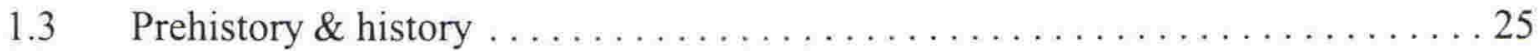

1.4 Summary of late Holocene pollen studies on mainland Greece $\ldots \ldots \ldots \ldots .28$

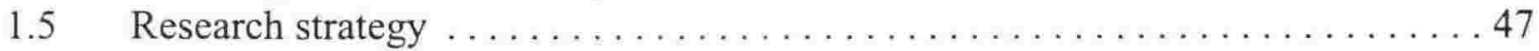

\section{Chapter 2. Field and laboratory techniques}

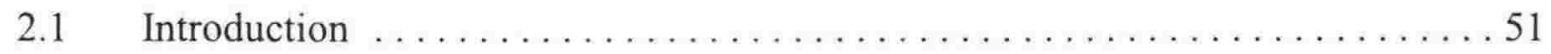

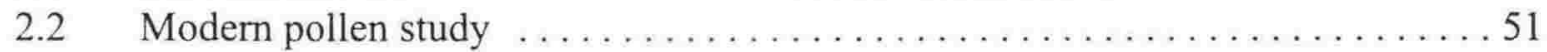

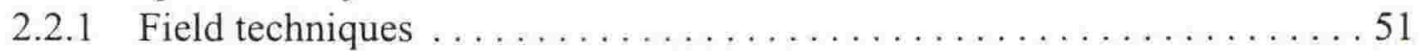

2.2.2 Laboratory preparation of surface pollen samples . . . . . . . . . 53

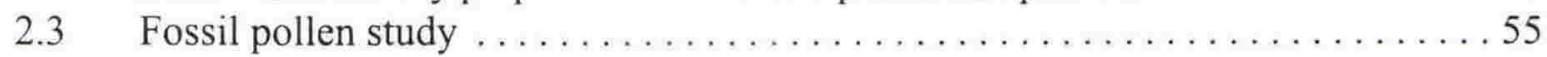

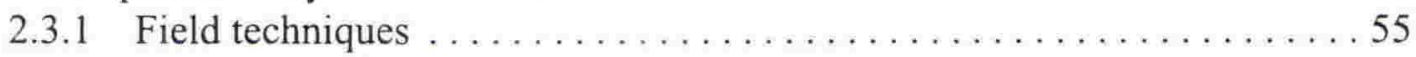

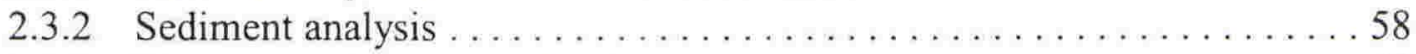

2.3.3 Plant macrofossil identification ..................... 62

2.3.4 Miscellaneous micro- and macrofossils . . . . . . . . . . . . . 62

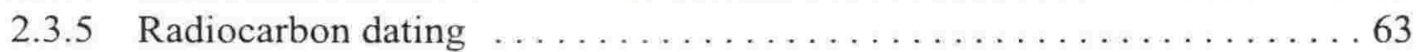

2.3.6 Laboratory preparation of fossil pollen samples . . . . . . . . . 66

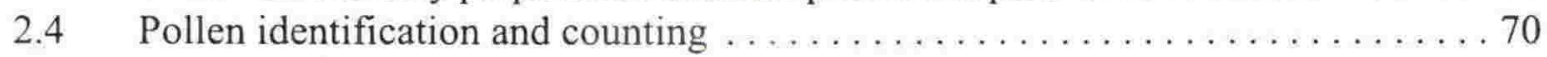

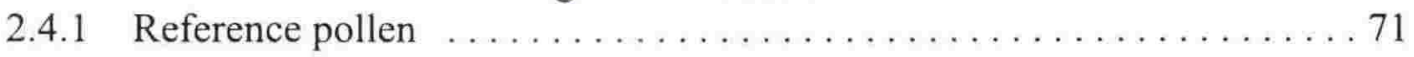

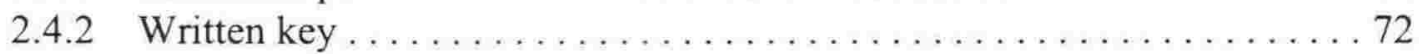

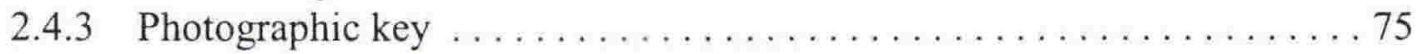

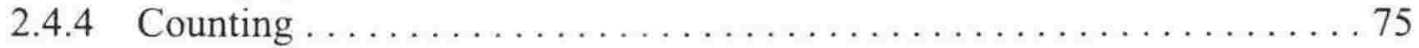

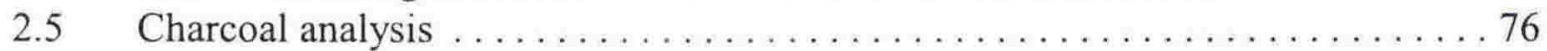

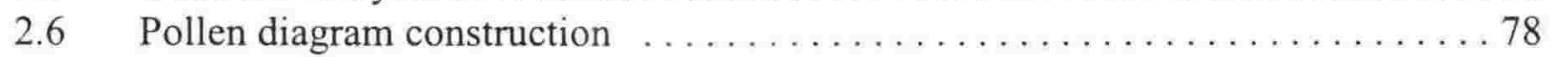

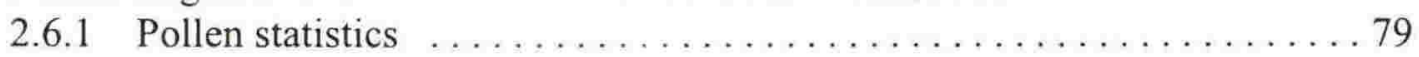

2.6 .2 Pollen taxon groupings $\ldots \ldots \ldots \ldots \ldots \ldots \ldots \ldots \ldots \ldots \ldots \ldots \ldots \ldots \ldots \ldots \ldots \ldots$

Chapter 3. Modern pollen deposition study - current vegetation \& pollen data

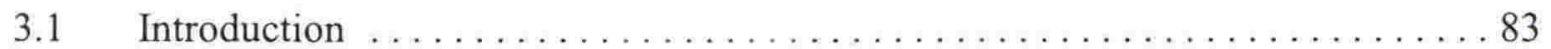

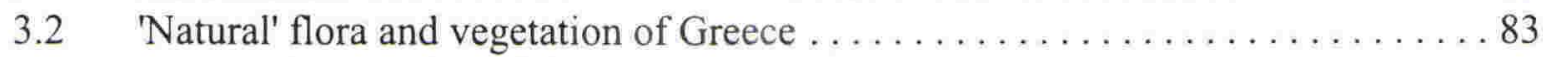

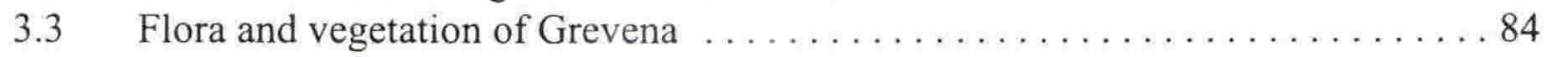

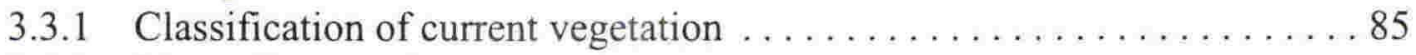

3.3 .2 'Natural' vegetation of Grevena . . . . . . . . . . . . . . 101 


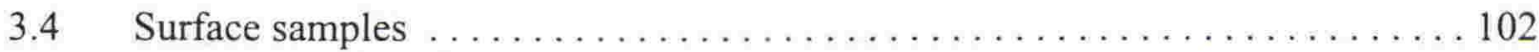

3.4.1 Sample collection sites . . . . . . . . . . . . . . . . . 102

3.4.2 Sample material and pollen preservation ................ 102

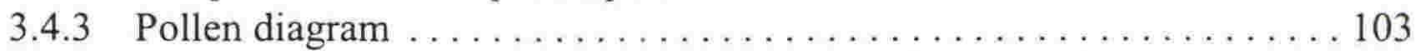

3.4.4 Relative pollen dispersal and representation of various plant taxa . . . 104

3.4.5 Characterisation of the vegetation zones in the pollen spectra . . . . . 112

3.4 .6 Charcoal . . . . . . . . . . . . . . . . . . . . . . . . . 116

3.5 Discussion . . . . . . . . . . . . . . . . . . . 117

3.5.1 Previous surface sample study in Grevena . . . . . . . . . . . . 117

3.5.2 Arboreal pollen to herbaceous pollen ratio $\ldots \ldots \ldots \ldots \ldots \ldots \ldots \ldots$

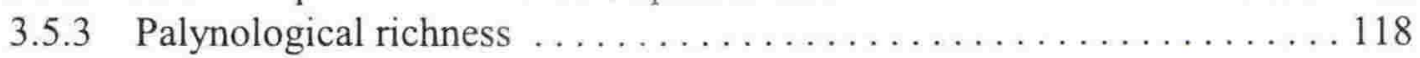

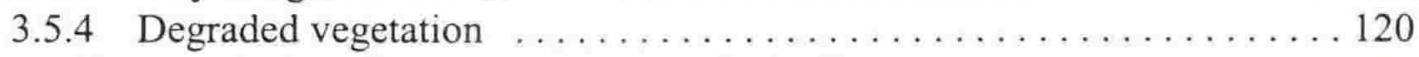

3.6 Implications for late Holocene pollen studies in Grevena . . . . . . . . . 120

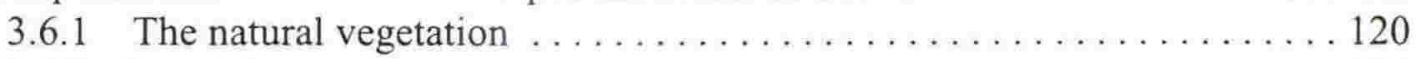

3.6 .2 Land clearance ... . . . . . . . . . . . . . . . . . . . . . 124

3.6 .3 Pastoralism . . . . . . . . . . . . . . . . . . . . . . . . 125

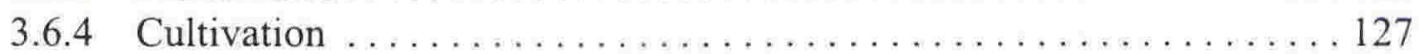

\section{Chapter 4. Gomara}

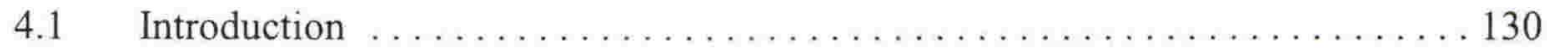

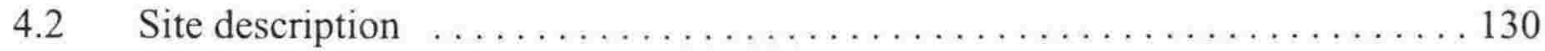

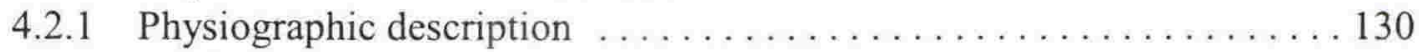

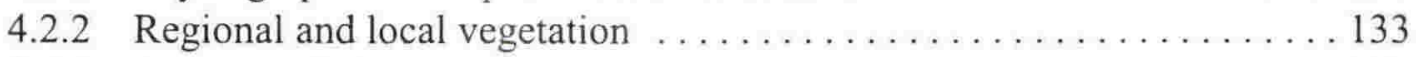

4.2 .3 Geology of site . . . . . . . . . . . . . . . . . . . . 133

$4.3 \quad$ Sediment analysis . . . . . . . . . . . . . . . . . . . . . . . 134

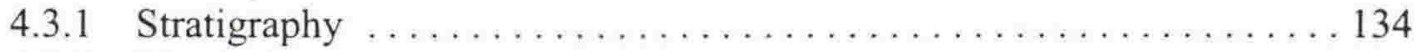

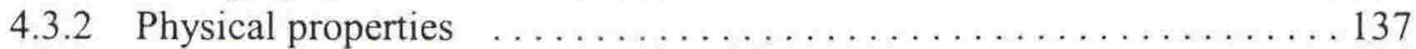

4.4 Chronostratigraphy . . . . . . . . . . . . . . . . . . . . . . 140

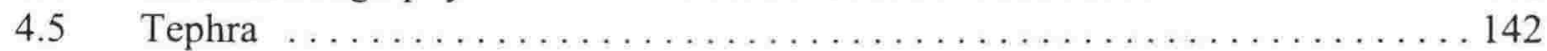

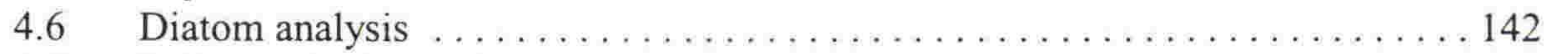

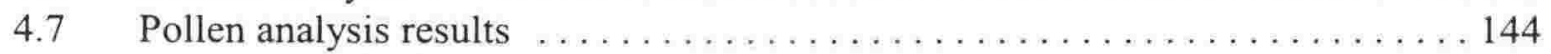

4.7 .1 Introduction . . . . . . . . . . . . . . . . . . . . . 144

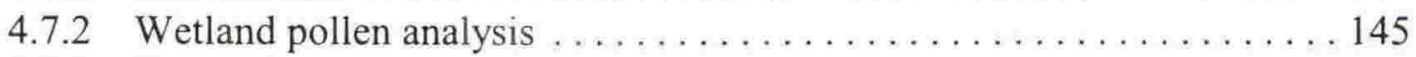

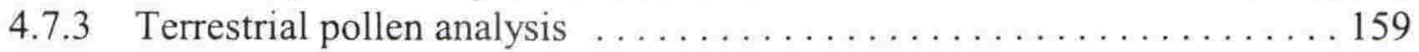

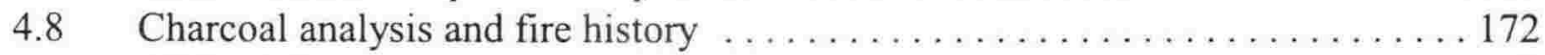

4.8.1 Microscopic charcoal analysis . . . . . . . . . . . . . . . . 172

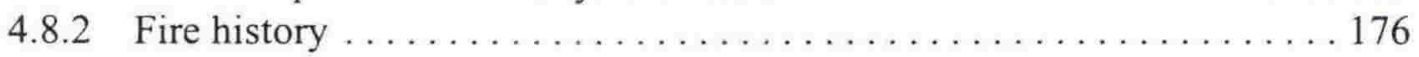

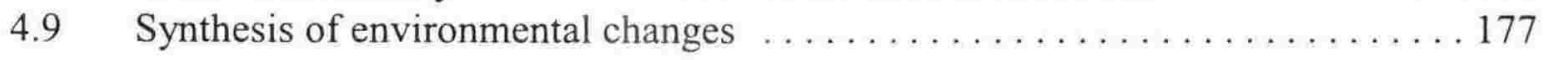

\section{Chapter 5. Anelia}

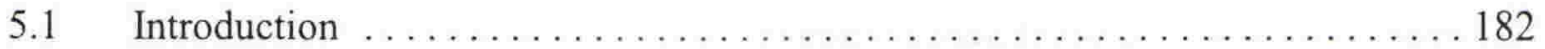

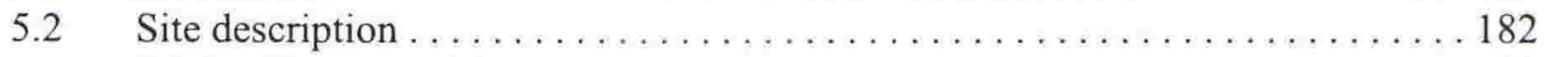

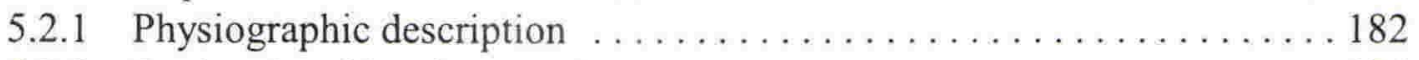

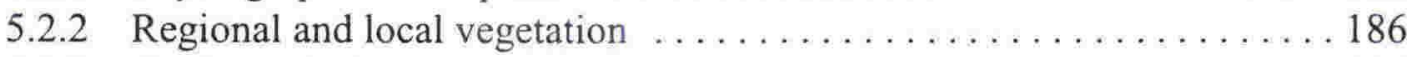

5.2 .3 Geology of site . . . . . . . . . . . . . . . . . . 186 
$5.3 \quad$ Sediment analysis . . . . . . . . . . . . . . . . . . . . . . . . . 187

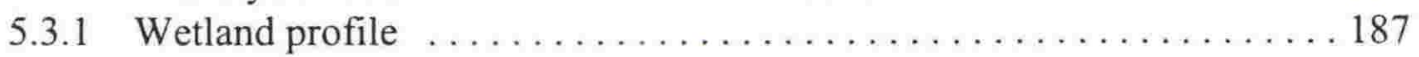

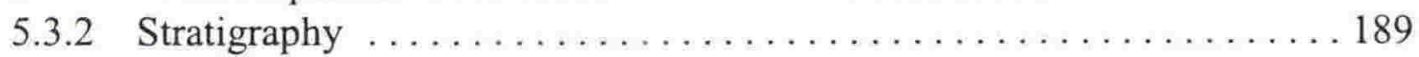

5.3 .3 Physical properties . . . . . . . . . . . . . . . . . . 193

5.4 Chronostratigraphy . . . . . . . . . . . . . . . . . . . . . . . . 195

5.4 .1 Radiocarbon dating . . . . . . . . . . . . . . . . . . . 195

5.4 .2 Age/depth plot . . . . . . . . . . . . . . . . . . . . 197

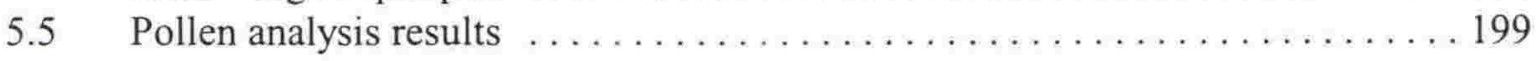

5.5 .1 Introduction . . . . . . . . . . . . . . . . . . . . 199

5.5 .2 Wetland pollen analysis . . . . . . . . . . . . . . . . 200

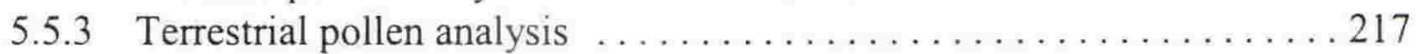

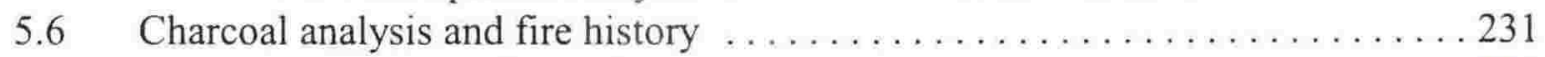

5.6 .1 Microscopic charcoal analysis . . . . . . . . . . . . . . 231

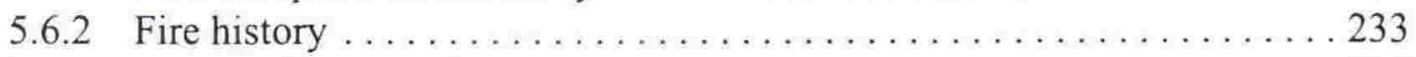

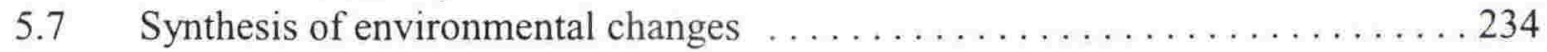

\section{Chapter 6. Kellia}

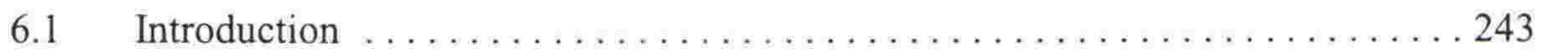

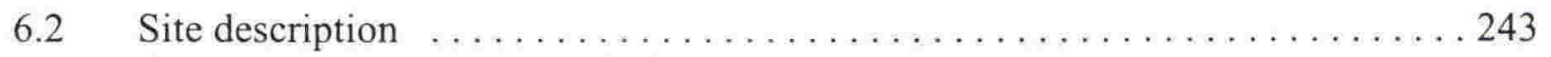

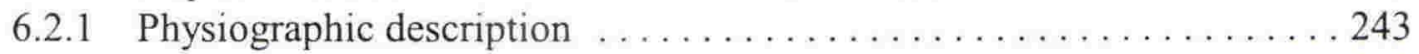

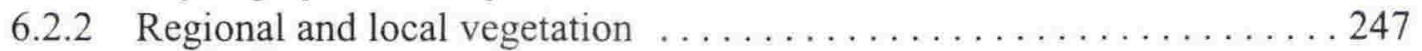

6.2 .3 Geology of site . . . . . . . . . . . . . . . . . . . . . . . 247

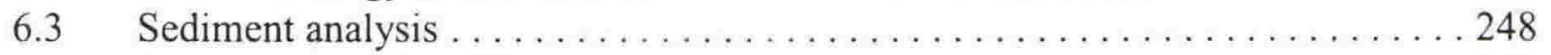

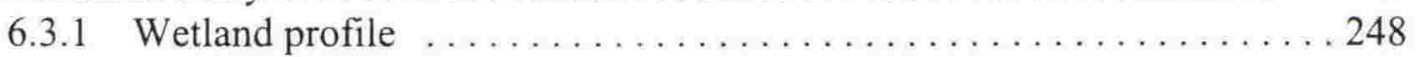

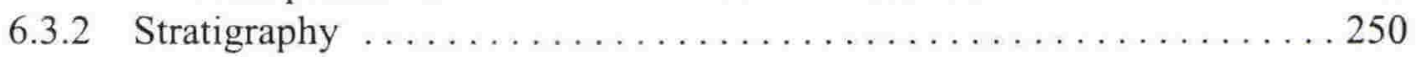

6.3 .3 Physical properties . . . . . . . . . . . . . . . . . . . 254

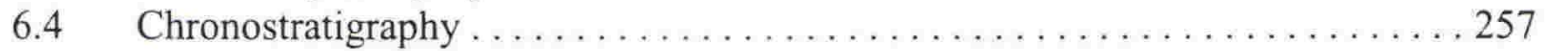

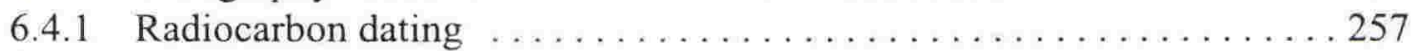

6.4 .2 Age/depth plot . . . . . . . . . . . . . . . . . . . . . . . 259

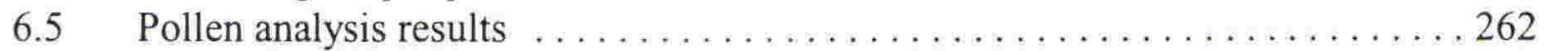

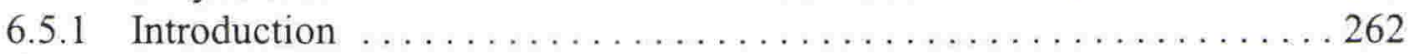

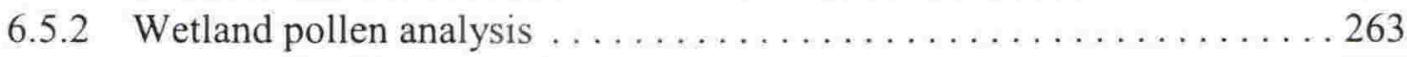

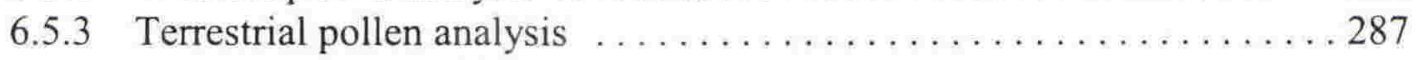

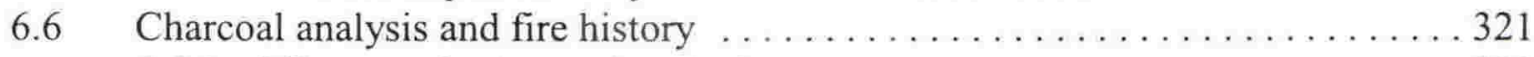

6.6 .1 Microscopic charcoal analysis . . . . . . . . . . . . . . . 321

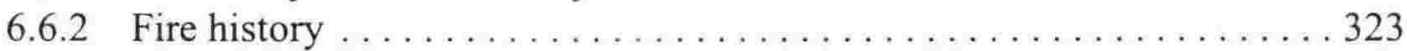

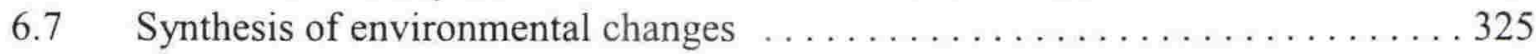

\section{Chapter 7. Discussion and conclusions}

7.1 Vegetation and interpretation of fossil pollen sequences . . . . . . . . . 347

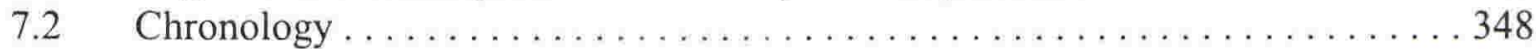

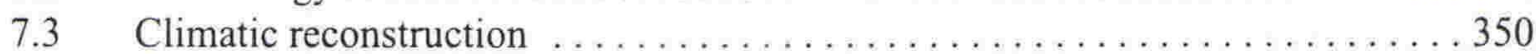

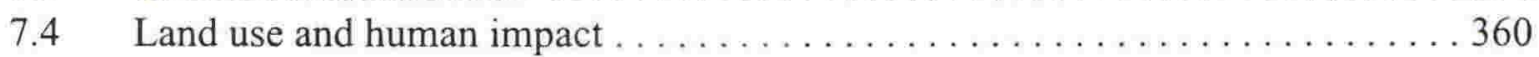

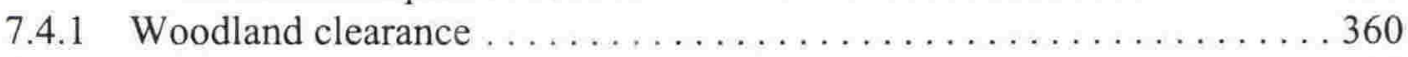

7.4 .2 Erosion . . . . . . . . . . . . . . . . . . . . . . . 364

7.4 .3 Pastoralism . . . . . . . . . . . . . . . . . . . . . . 365

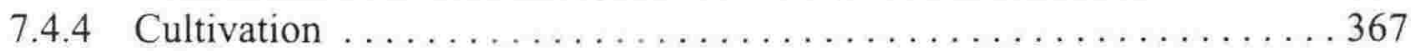

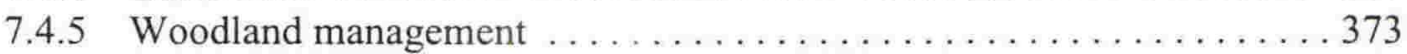




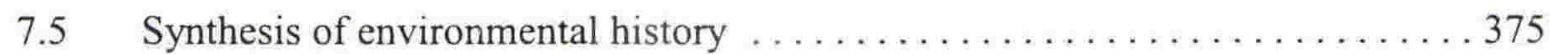

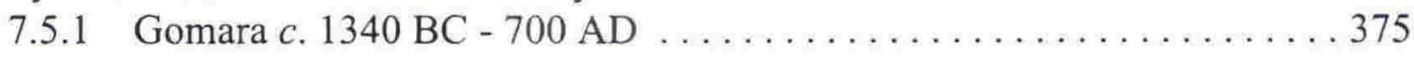

7.5.2 Anelia c. $1560-1989$ AD . . . . . . . . . . . . . . . . . 385

7.5.3 Kellia c. $1230-1989$ AD ............................ 387

References

\section{VOLUME II - Appendices}

I Herbarium specimens collected in Grevena Province $\ldots \ldots \ldots \ldots \ldots \ldots \ldots$ 1-10

II Laboratory preparation techniques for pollen and charcoal analysis . . . . 1-12 II.1 Laboratory preparation of moss polsters and litter, soil and wetland

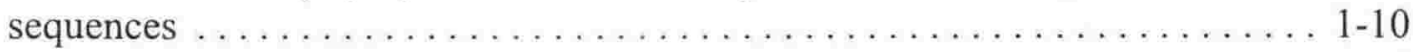

II.1.1 Laboratory preparation of moss polsters and litter samples . . . 2 2-4

II.1.2 Laboratory preparation of soil samples ... . . . . . . . 4-6

II.1.3 Laboratory preparation of wetland samples . . . . . . . . . 6-8

II.1.4 Lycopodium spike preparation . . . . . . . . . . . . . 8-9

II.1.5 Laboratory procedure for diatom extraction . . . . . . . . 9-10

II.2 Laboratory preparation of pollen reference material . . . . . . . . 10-11

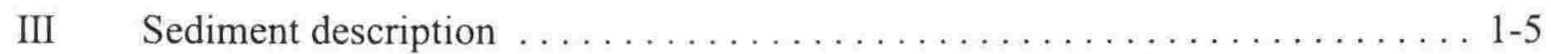

III.1 Troels-Smith characterization of unconsolidated sediments . . . . . . 1-5

III.2 Comparison of grain size class scales of inorganic sediments . . . . . . . . 5

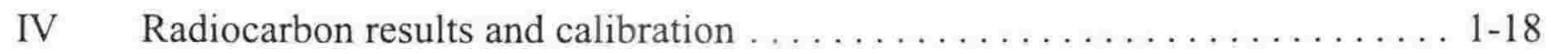

$\mathrm{V} \quad$ Modern pollen and spore reference collection $\ldots \ldots \ldots \ldots \ldots \ldots \ldots \ldots \ldots \ldots$

VI Pollen key . . . . . . . . . . . . . . . . . . . . . . . . . . .

VI.1 Key . . . . . . . . . . . . . . . . . . . . . . . . . . .

VI.2 Pollen types $\ldots \ldots \ldots \ldots \ldots \ldots \ldots \ldots \ldots \ldots \ldots \ldots \ldots \ldots \ldots \ldots \ldots$

VII Species lists for reconnaissance vegetation surveys $\ldots \ldots \ldots \ldots \ldots \ldots \ldots .4$

VIII Modern vegetation composition at pollen surface sample collection sites ... 1-33

IX Surface samples - Pollen and charcoal counts $\ldots \ldots \ldots \ldots \ldots \ldots \ldots \ldots \ldots$

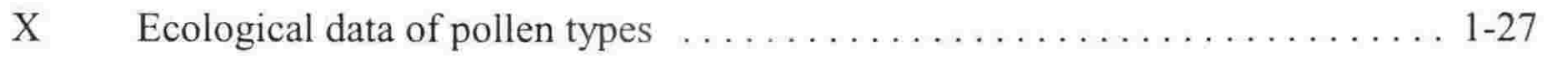

XI Relative dispersal and representation $\ldots \ldots \ldots \ldots \ldots \ldots \ldots \ldots \ldots \ldots \ldots \ldots \ldots \ldots .13$

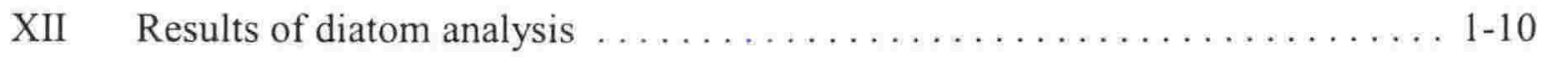

XIII Sediment sequences - Pollen and charcoal counts $\ldots \ldots \ldots \ldots \ldots \ldots \ldots$ 1-60

XIV Stratigraphy of exploratory boreholes in Anelia \& Kellia wetlands ........ 1-7 


\section{Figures}

\section{Chapter 1}

1.1 Location of Grevena Province; places referred to in the text $\ldots \ldots \ldots \ldots \ldots .2$

1.2 Late Holocene chronologies . . . . . . . . . . . . . . . . . . . . . . . 5

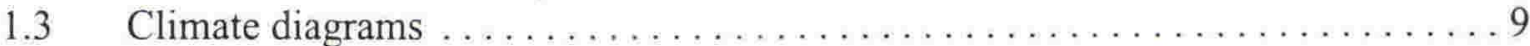

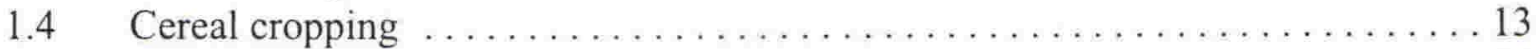

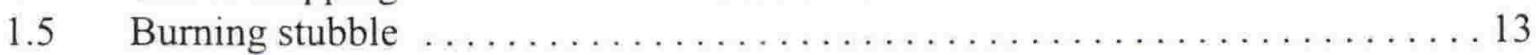

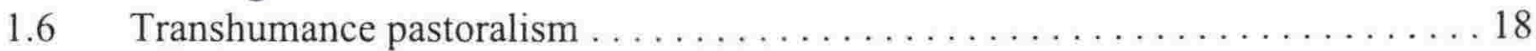

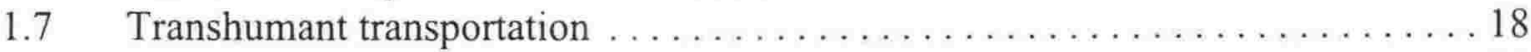

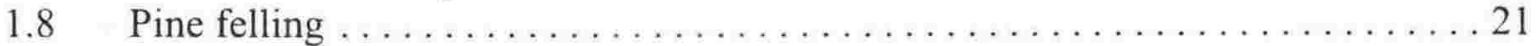

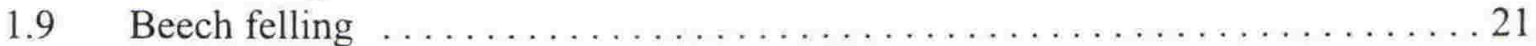

1.10 Pollarded oaks . . . . . . . . . . . . . . . . . . . . . . . . 23

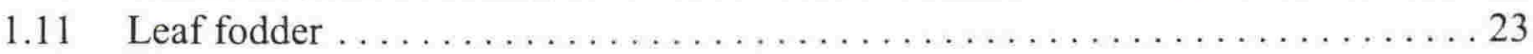

1.12 Late Holocene pollen sites on mainland Greece . . . . . . . . . . . . . . 29

\section{Chapter 3}

3.1 Diagrammatic section across Grevena, showing present-day distribution of

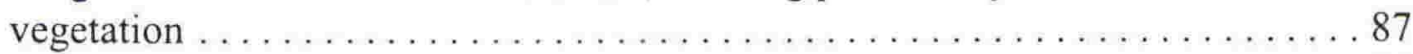

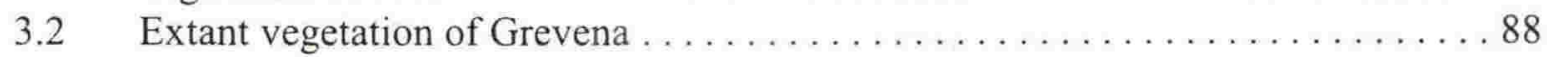

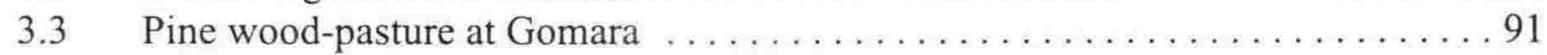

3.4 A huge Pinus leucodermis tree at Avgo . . . . . . . . . . . . . . 91

3.5 Beech woods around the wetland coring site of Anelia $\ldots \ldots \ldots \ldots \ldots \ldots 92$

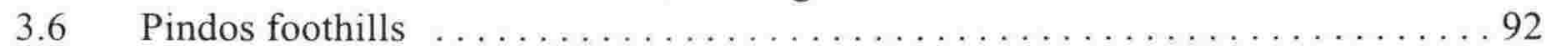

3.7 Mixed wood on limestone above Zakas . . . . . . . . . . . . . . . . . . . 94

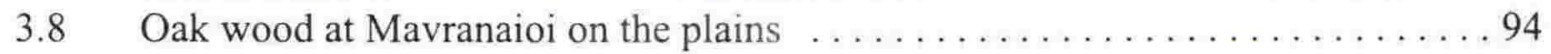

3.9 Pollen diagram - surface samples $\ldots \ldots \ldots \ldots \ldots \ldots \ldots \ldots \ldots \ldots \ldots$ pocket

\section{Chapter 4}

$4.1 \quad$ View of Gomara site from Smixi-Distraton road $\ldots \ldots \ldots \ldots \ldots \ldots \ldots \ldots 131$

4.2 Location of Gomara wetland in relation to local topography $\ldots \ldots \ldots \ldots \ldots 132$

4.3 Troels-Smith classification of Gomara core sediments . . . . . . . . . . . . 135

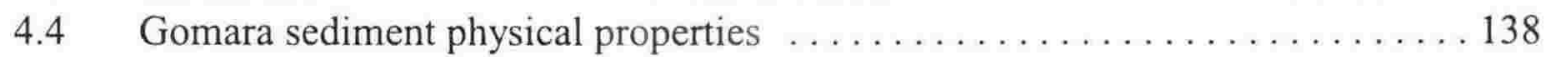

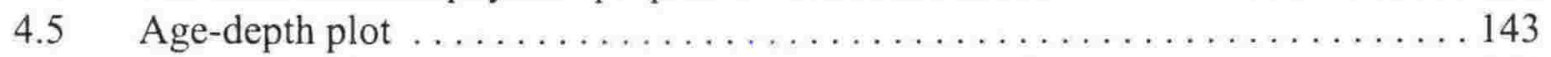

4.6 Gomara - relative frequency pollen diagram, wetland taxa . . . . . . . . . . . 146

4.7 Gomara - influx pollen diagram, wetland taxa $\ldots \ldots \ldots \ldots \ldots \ldots \ldots \ldots \ldots$

4.8 Gomara - summary relative frequency pollen diagram, terrestrial taxa $\ldots \ldots 160$

4.9 Gomara - resolved relative frequency pollen diagram, terrestrial taxa ..... pocket

4.10 Gomara - influx pollen diagram, selected terrestrial taxa . . . . . . . . . . 162

4.11 Gomara - charcoal particle percentage diagram $\ldots \ldots \ldots \ldots \ldots \ldots \ldots \ldots$

4.12 Gomara - charcoal particle influx diagram $\ldots \ldots \ldots \ldots \ldots \ldots \ldots \ldots \ldots \ldots$ 


\section{Chapter 5}

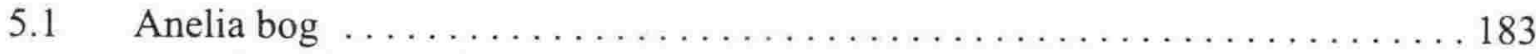

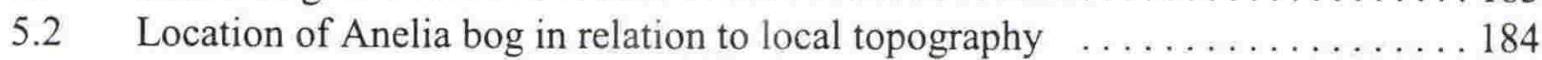

5.3 Anelia mire with location of exploratory borings and core site . . . . . . . . 185

5.4 Longitudinal and transverse sections through Anelia bog . . . . . . . . . . . 188

5.5 Troels-Smith classification of Anelia core sediments . . . . . . . . . . . . . . 190

5.6 Anelia sediment physical properties . . . . . . . . . . . . . . . . . . . . . . . 194

$5.7 \quad$ Age-depth plot . . . . . . . . . . . . . . . . . . . . . . . . . . . 198

5.8 Anelia - relative frequency pollen diagram, wetland taxa . . . . . . . . 201

5.9 Anelia - concentration pollen diagram, wetland taxa . . . . . . . . . . . . 202

5.10 Anelia - summary relative frequency pollen diagram, terrestrial taxa $\ldots \ldots 218$

5.11 Anelia - resolved relative frequency pollen diagram, terrestrial taxa ..... pocket

5.12 Anelia - concentration pollen diagram, selected terrestrial taxa . . . . . . . 219

5.13 Anelia - charcoal particle percentage diagram $\ldots \ldots \ldots \ldots \ldots \ldots \ldots 232$

\section{Chapter 6}

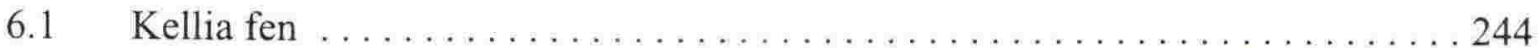

6.2 Location of Kellia fen in relation to local topography . . . . . . . . . . 245

6.3 Kellia fen, showing location of pollen core site ans exploratory borings . . . . 246

6.4 Longitudinal and transverse sections through Kellia fen . . . . . . . . . . . 249

6.5 Troels-Smith classification of Kellia core sediments . . . . . . . . . . 251

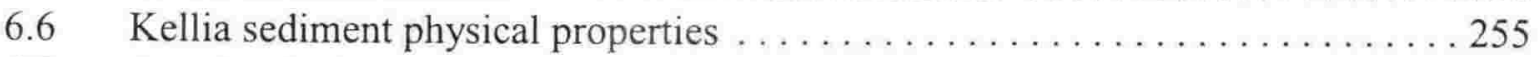

6.7 Age-depth plot . . . . . . . . . . . . . . . . . . . . . . . . 260

6.8 Cumulative Pinus pollen deposition plotted against depth $\ldots \ldots \ldots \ldots \ldots .261$

$6.9 \quad$ Kellia - relative frequency pollen diagram, wetland taxa . . . . . . . . . . . 264

$6.10 \mathrm{Kellia}$ - concentration pollen diagram, wetland taxa ............... 265

$6.11 \mathrm{Kellia}$ - summary relative frequency pollen diagram, terrestrial taxa . . . . . 288

6.12 Kellia - resolved relative frequency pollen diagram, terrestrial taxa ..... pocket

6.13 Kellia - concentration pollen diagram, selected terrestrial taxa . . . . . . . . 289

6.14 Kellia - charcoal particle percentage diagram . . . . . . . . . . . . . . . 322

\section{Chapter 7}

7.1 Relative abundance of the three Quercus pollen types $\ldots \ldots \ldots \ldots \ldots . \ldots 358$ 


\section{Tables}

\section{Chapter 3}

3.1 Range of pollen relative percentages by vegetation zone $\ldots \ldots \ldots \ldots \ldots \ldots$

\section{Chapter 4}

$4.1 \quad$ Radiocarbon dating results - Gomara $\ldots \ldots \ldots \ldots \ldots \ldots \ldots \ldots \ldots \ldots \ldots \ldots \ldots \ldots$

4.2 Major environmental changes in Gomara wetland and its catchment,

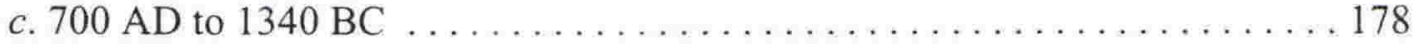

\section{Chapter 5}

5.1 Radiocarbon dating results - Anelia 196

5.2 Major environmental changes in Anelia wetland and the surrounding region, c. 1989-1560 AD

\section{Chapter 6}

6.1 Radiocarbon dating results - Kellia 258

6.2 Major environmental changes in Kellia wetland and the surrounding region, c. 1989-1230 $\mathrm{AD}$ (and ? c. 600-2000 BC)

\section{Chapter 7}

7.1 Major environmental changes in Grevena 376 


\section{Chapter 1. Introduction}

\subsection{Aims of the study}

Palynological techniques are used to determine the chronosequence of vegetation during the last 3500 years in Grevena Province $(\Gamma \rho \in \beta \in \nu \alpha$ No $\mu \circ \sigma)$, western Greek Macedonia, northwest Greece (Figure 1.1). This study was undertaken in association with the Grevena Project, an interdisciplinary archaeological research project run from Carleton College, United States of America, under the auspices of Dr Gallis of the IE' Ephoreia of Prehistoric and Classical Archaeology in Larisa, Greece, and the American School of Classical Studies in Athens. The principal aim of the project is to reconstruct the environmental and cultural history of Grevena Province since human settlement began there. Little was known of the area archaeologically before the project began.

The major aim of this thesis is to describe the natural environmental background, vegetation, and climatic conditions contemporary with the archaeological cultures found to have occupied Grevena since the Late Bronze Age, as determined by archaeologists of the Grevena Project conducting an initial all-period regional survey. Past human cultures cannot be properly understood without knowing what natural environmental conditions existed while they were extant and what natural resources were available. How humans behaved and interacted within particular natural environmental conditions and exploited natural resources is of major interest to archaeologists.

Another aim is to provide information on anthropogenic vegetation changes and the consequent landscape changes from land-use practices of agriculture, pastoralism (grazing by sheep and goats and fodder collection), cutting of wood for fuel and industrial purposes, and building settlements. This research is directed towards distinguishing natural from human-induced changes in vegetation, assessing the effects of land use (mostly agriculture and pastoralism) on the environment, and establishing a chronology of hillslope erosion and deposition.

By assessing the intensity of the impact of land use at different times and at different localities some idea of variation in population size and distribution is gained.

\subsection{Physical setting of the study area}

Grevena Province, formed in 1951 (Wilkie 1987), occupies a small (75 x $45 \mathrm{~km}$ ) inland basin in remote mountainous northwestern Greece, close to the borders of Albania and Macedonia of former Yugoslavia. The capital of Grevena Province is Grevena, hereafter referred to as Grevena city (the province as Grevena) (Figure 1.1). Transliteration of Greek place names varies because English letters do not correspond exactly to Greek sounds, but an 


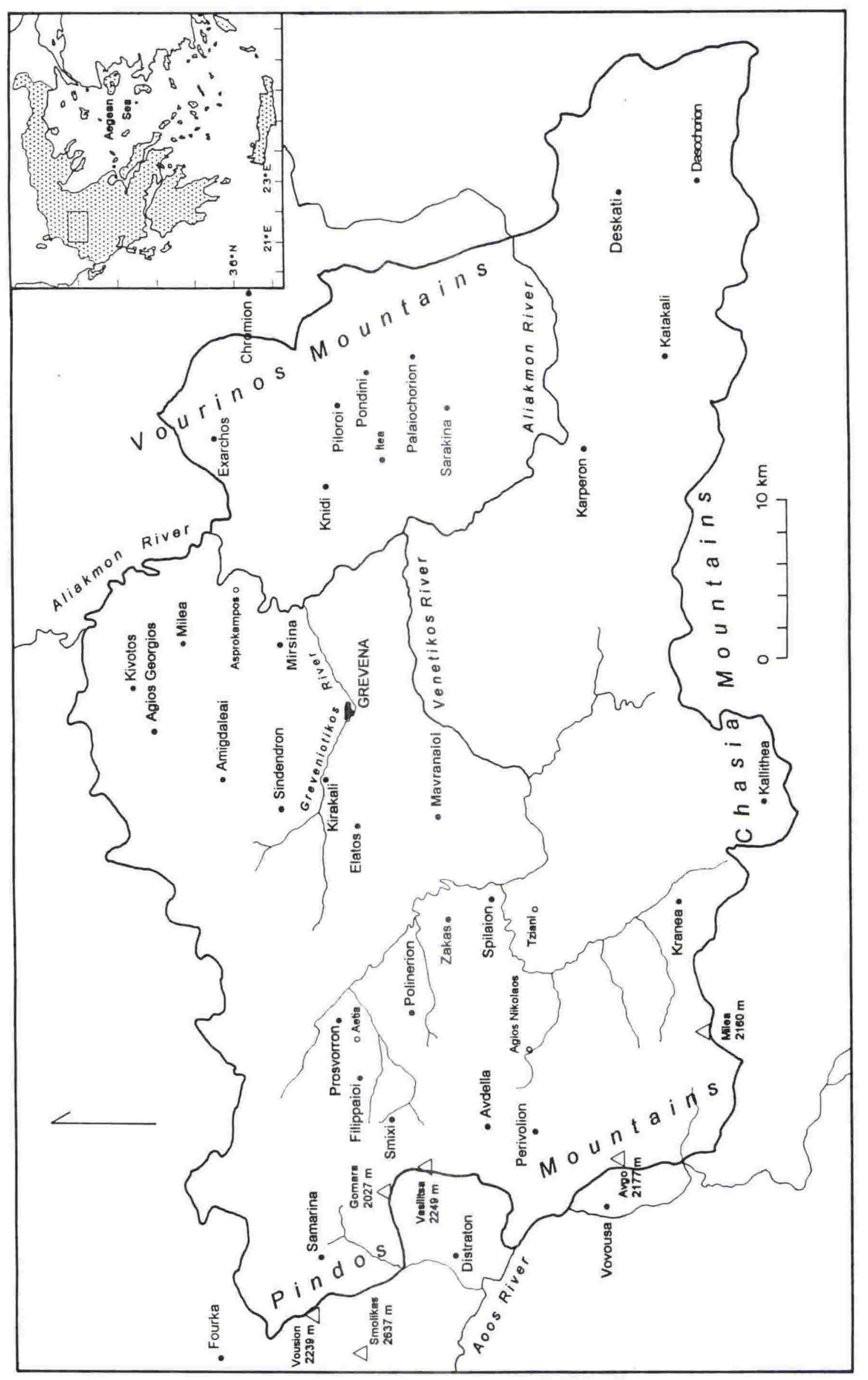

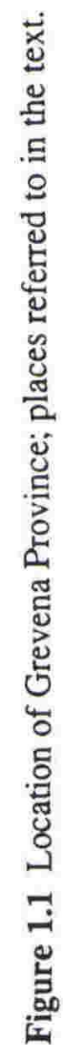


attempt has been made to follow the system used in Collins Gem Greek Dictionary (Hionides 1987), except where a difference from the usage of previous authors would create confusion.

\subsubsection{Location}

Currently Greece is divided into 51 provinces (nomoi) and 13 regions (Parker 1995:14). Grevena forms the south-west corner of the Greek region called Macedonia. Greek Macedonia is part of a larger area also called Macedonia, well known for its prominence in European history during the reigns of Philip II and his son Alexander the Great, which includes parts of former Yugoslavia and Bulgaria. Greek Macedonia in north-central mainland Greece borders the Aegean Sea which lies to the south and east.

Grevena borders the better-known Greek regions of Epirus to the west and Thessaly to the south. The provinces of Kastoria (NW) and Kozani (NE) border it to the north, Ioanninon to the west, Larisa to the east, and Trikala to the south.

\subsubsection{Physiography and geology}

Grevena landscape resembles Central European landscapes of mountains and plains. The province is bordered by mountains on three sides; in the west the Pindos massif reaches elevations of $2250 \mathrm{~m}$ asl, in the east the Vourinos Mountains reach elevations of $1850 \mathrm{~m}$ asl, and in the south the lesser hills of the Chasia Mountains reach $1482 \mathrm{~m}$. The Pindos and Vourinos ranges run almost parallel from NNW to SSE. Smolikas (2637 m), the highest peak in the Pindos range, is just across the northwestern border of Grevena, while many peaks in Grevena are over $2000 \mathrm{~m}$. The lower peaks of the Vourinos are generally closer to $1500 \mathrm{~m}$. The northern border of the province is not well defined topographically, although part of it is formed by the middle reaches of the Aliakmon River, the main drainage of Grevena. The central plains of the basin undulate between 400 and $700 \mathrm{~m}$ asl (Figure 1.1).

The province is drained by the Aliakmon and its major tributary, the Venetikos, which have incised up to $200 \mathrm{~m}$ (Savina 1993).

Thus Grevena can be divided into five major topographic regions: Pindos mountains, Pindos foothills, central plains or lowlands, flood plains, and the slopes of the Vourinos Mountains. At higher elevations towards the mountains to the east and west the slopes are steeper and more dissected, with more ravines and gullies than the central lowlands. The generally gently rolling hills of the lowlands cover almost half the total area of the province.

These topographic regions reflect the bedrock structure, which is well documented by the maps of the Greek national geological survey (Institute for Geology and Subsurface Research 1959, 1960, 1972; Institute for Geological and Mining Research 1979; Institute of Geology and Mineral Exploration 1980, 1987a, b). The Pindos and Vourinos mountain ranges 
are primarily Mesozoic ophiolites and early Cenozoic flysch (the Pindos Flysch). The Pindos include the full ophiolitic suite of ultramafic rocks, pillow basalts, chert, limestone, and clastic marine sediments, while the Vourinos Mountains include both ultramafic rocks and limestone. Structure is complex within the Pindos range, where many different lithologies are juxtaposed over short distances (Savina 1989). The central lowlands are underlain mainly by marine Oligocene to Miocene sediments of the Mesohellenic Trench. These range from interbedded siltstones and sandstones to thick conglomerate units, the last mainly basal. Near the Aliakmon River, older Tertiary sediments are covered unconformably by Plio-Pleistocene deposits, dominantly thick gravels derived from the Pindos and Vourinos, but also including thinly bedded clays and silts (probably lake deposits) and thicker sands and silts (possibly wind-blown) (Savina 1989). In the southeast the margins of the Deskati and Dasochorion basins are markedly different, being underlain by Paleozoic igneous and metamorphic rocks (the Pre-Alpine basement). These basins as well as the Karperon basin, also in the southeast, are filled with mainly fine-grained lacustrine sediments (marls, clays, and sandstones) of Pliocene to early Pleistocene age.

West of the Aliakmon, the Cenozoic sedimentary rocks dip to the northeast gently to steeply. South of the Venetikos and east of the Aliakmon the orientation of the Cenozoic rocks is more variable.

Quaternary deposits have been described by Savina (1993), and Doyle (1990). Holocene aggradation terraces formed over the last 10,000 years occur along many reaches of the Aliakmon and Venetikos rivers and also along many tributaries. Most catchments have at least one Holocene aggradation terrace that is younger than about 2300 years, e.g. broad fill terraces, generally less than $3 \mathrm{~m}$ above river bed. In the Leipsokouki catchment near Sindendron there are four terraces below $10 \mathrm{~m}$ above the modern stream, all of which formed during the last 2500 years (Doyle 1990).

Colluvial deposits ranging in age from Late Pleistocene to Late Holocene are distributed widely. In many parts of the Pindos range above $1000 \mathrm{~m}$ elevation a mantle of thick colluvium covers most hillslopes on which soils are well-developed. In the Vourinos broad colluvial fans mantle toeslopes. These also have well-developed soils. Elsewhere colluvium occurs either as thin sheets on hillslopes, especially on sandstone dip slopes, or as the filling of bedrock depressions. Most catchments appear to have several ages of Holocene fill. One or more periods of alluviation/colluviation occurred in most catchments during the last 2300 years (Figure 1.2).

The dominant orders and suborders of soils of the Grevena region are classified as mountain soils with various moisture and temperature regimes on steep slopes and variable relief and elevation; the soils vary greatly within short distances (Orvedal 1990:18 \& 19). 


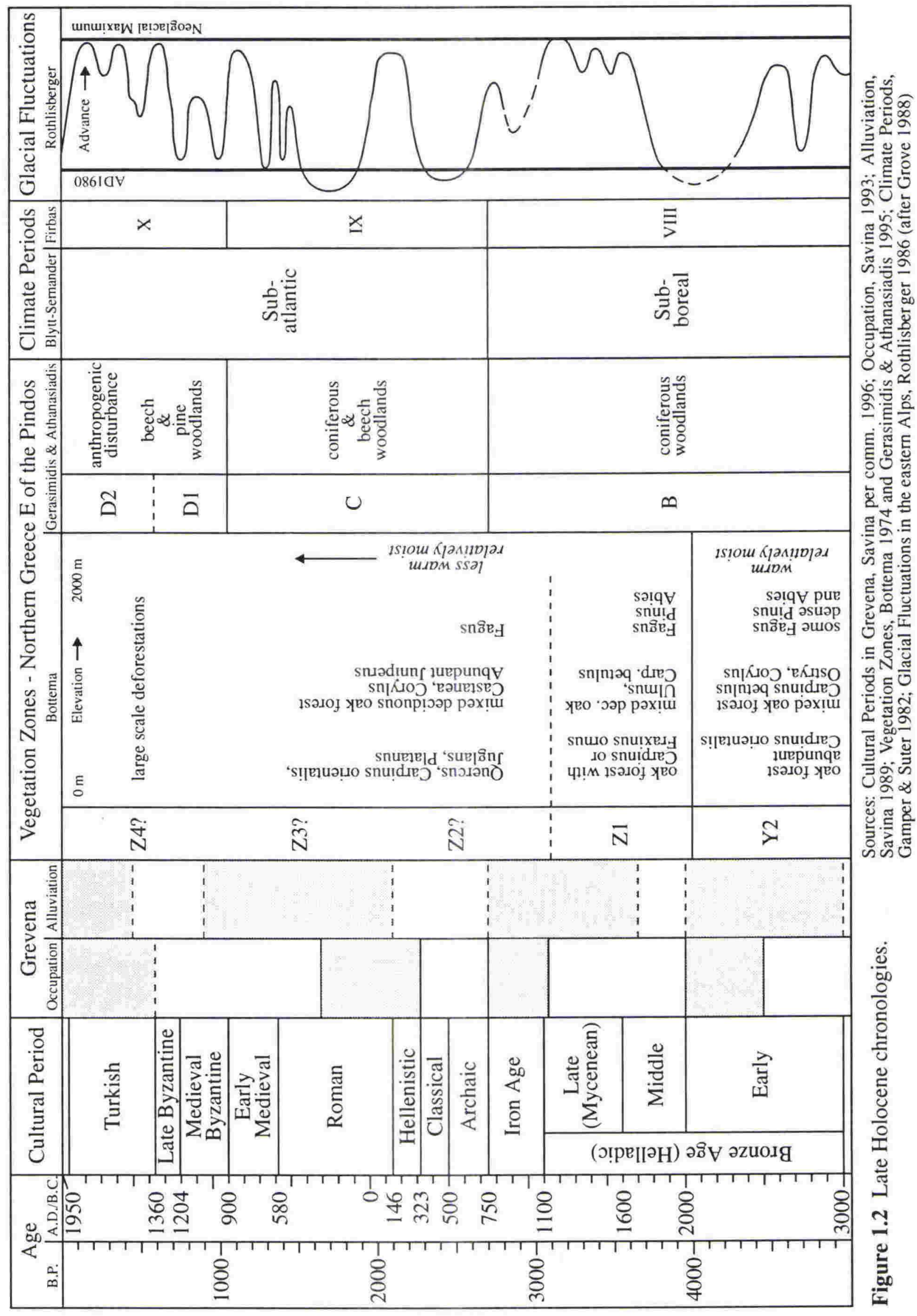


During a detailed study of cultivated soils in every village in Grevena, Oikonomou et al. (1988) analysed 1,072 surface soils and 1,040 subsoils.

Entisols occurred on recent alluvial deposits along rivers. In low hilly regions and on old plains, where the parent materials have a small to moderate (or no) content of free calcium carbonate, the soils formed are chiefly Alfisols ("red earth", mostly moderately heavy). On parent material where calcium carbonate is abundant (marl, usually) and there is little erosion, the soils are Mollisols ("blackearths", heavy), and of lesser extent Vertisols (the typically very heavy "black earths"). Vertisols are found chiefly on old plains and to a very small extent on low hilly areas. Where marls occur at shallow depths below the soil surface and/or on the surface due to severe erosion, the soils are Inceptisols ("white earths" or "grey", heavy soils) and Entisols ("white earths"). On many low hilly areas and old plains where the parent material is mixed (e.g. sandstones, conglomerate, gravel, sand, clay, marly sandstones) the soils are Inceptisols (moderately heavy and usually gravelly). They are usually dark brown and/or yellow soils. In the foothills of the Vourinos Mountains, where the parent material is chiefly serpentinites or peridotites, the soils are Inceptisols ("chocolate" or "red", heavy). Where the soils have formed from acid rocks (e.g. gneisses, mica schists, sandstones) the soils are Inceptisols ("yellow" to "red", light to moderately heavy) and also Alfisols ("red earths", moderately heavy).

In the semi-mountainous regions where relief varies greatly and erosion is more severe than in the low hilly regions, the steeper slopes and the general composition of the alluvium results in the soils of the oldest of the river terraces being friable and much less calcareous (sandstones, conglomerates, gravels, sands, and, on the fertile land, clays and marls). Relatively friable also are the compositions of the parent material in those areas where the provenance of the soils is from micaceous schist, gneiss, and sandstone. Generally the soils are gravelly, leached, usually moderately heavy, and with lower "active" depths.

Almost all the surface soils and subsoils vary from heavy to moderately heavy. The heavy soils are formed mostly on low hilly regions and old plains. Sixty-six percent of the soils ranged from slightly acid to slightly alkaline, and $22 \%$ were found to be very acid to exceedingly acid. Twenty-two percent of cultivated land in Grevena was too acid $(\mathrm{pH} \leq 5.5)$ for cultivation of wheat and barley (two crops most sensitive to acid) and corn, sunflower, soybeans, tobacco, fruit trees, vine, and garden crops (alfalfa and sugar beets require an even higher $\mathrm{pH}$ ) and required lime. The acid soils occur on the lowlands.

A characteristic diagnostic property of almost all the soils during the dry season of the year is cracking. Cracks may be small to very large (e.g. in the Vertisols). The clay content is largely composed of montmorillonites, which are swelling clays. These cracks contribute to large losses of water (and also fertilizer), which together and with the presence 
of gravel in the soils make them even drier, especially on steep slopes.

Currently Grevena appears to be an area of low seismicity with few active faults (Savina 1993), although an earthquake in 1995 destroyed many buildings, including the hotel we stayed in during our 1989 field season (Wilkie, pers. comm.). Faults with probable Holocene movement bound the Karperon basin on the north, and there are deformed lake deposits near Katakali on the south, indicating that deformation took place after sediment deposition. They trend W-E to WSW-ENE and may still be actively subsiding (Savina 1993:61). Other faults with apparent Quaternary movement in the Sarakina-Palaiochorion area and southwest of Grevena trend ENE-WSW (Savina 1993:61). Grevena is slowly rising at a rate of about $40-45 \mathrm{~cm} / 10^{3}$ year (Savina 1993).

\subsubsection{Climate}

Grevena has a Mediterranean climate characterised by winter rainfall and a long dry summer season (Trewartha 1990:8-9). However, inland Grevena, $140 \mathrm{~km}$ from the coast and at $40^{\circ} \mathrm{N}$ latitude, is near the northern limit of this climatic zone and is influenced by continental European climate, so winter temperatures are colder (snow falls at even the lowest elevations) and summer rainfall is generally greater than for more southerly or coastal Mediterranean climates (Walter et al. 1975:5; Sakellariou 1988:19).

In winter the weather system is controlled by the anticyclonic Eurasiatic high-pressure axis, the subtropical Azores high, and the frontal depressions of low pressure over the warm Mediterranean Sea (Biel 1944:5; Furlan 1977:216; Martyn 1992:127). There are regional highs over the northern Balkans and Serbia, and a centre of particularly low pressure occurs over the Adriatic. In winter the eastern Mediterranean becomes a principal region of cyclonic activity (Furlan 1977:192).

In summer the high pressure over the Balkans weakens, the Azores high moves westward, and the low-pressure centres over the Mediterranean are less pronounced. The main feature of the summer pressure pattern is a persistent heat low over Asia Minor. The transition to winter is abrupt and much more pronounced than the transition to summer conditions in April. In September east-bound disturbances start as the west-east gradient over the Mediterranean weakens, and high pressure is established over the cooling northern Balkans (Biel 1944:6-7). Depressions originate mainly over the central Adriatic and pass eastward, particularly in autumn. Though fairly weak, these lows are persistent (Martyn 1992:127).

The mountainous topography of northern Greece produces very different climates at different elevations (Walter et al. 1975:29, map 9), and there is a noticeable climatic difference between high- and lowlands in Grevena. Unfortunately climatic data for this part 
of Greece are scanty and mostly from the lowlands: climatic data have been collected from two stations within Grevena Province, one near Grevena city and one at Kranea in the southwest, over the last 20 years, but only limited records are available. Doyle (1990) analysed data from the Grevena station for the period 1978-1987, and this information can be compared with longer-period records from two lowland stations outside the province: Bitola (Bitolj, Monastir) in the mountainous region of Yugoslavian Macedonia, and Trikala on the plains of northern Thessaly (Biel 1944); and one station at high elevation, Pertouli in the Pindos to the south of Grevena between Trikala and Ioanninon (Athanasiadis 1975). For these stations climate diagrams (Walter et al. 1975) are shown in Figure 1.3. The typical Mediterranean climate pattern of winter rainfall and summer drought is exhibited at Trikala, while at Bitola the summer drought season is less pronounced. Because of its elevation, Pertouli displays the Mediterranean winter rainfall regime to a very marked degree, and this pattern can be expected to occur at higher elevations in the Grevena Pindos also.

Biel (1944) and Espenshade (1990) provide upper-level wind data from two Balkan stations, Drama in eastern Macedonia and Bitola. The net drift at $3 \mathrm{~km}$ above the surface is consistent: directions are west to northwest in winter, west-southwest to west-northwest in spring, west to north in summer, and west-southwest to north-northwest in autumn. January high-level winds reach 3-4 on the Beaufort scale, but in July the strength is very weak, Beaufort 0-3.

Local wind direction and velocity at the ground surface may vary greatly due to the great differences in relief and the ruggedness of the mountains. Except at high elevations, surface winds in Grevena are usually light, but two characteristic southeast European winds which probably do occur are the dry summer northerly wind (Etesian), which starts its steady drift around mid May and lasts until mid September, although breaks particularly in July are not unusual; and a cold, gusty winter northerly similar to the Vardarac of the Vardar Valley to the northeast (Biel 1944).

Annual precipitation is high in the Pindos and may reach $2000 \mathrm{~mm}$ (Martyn 1992:131). Convective clouds in the mountains are responsible for increased precipitation there during the summer. In winter most precipitation falls as snow and continues to lie till early summer. Above $1000 \mathrm{~m}$ snow may lie from mid-December to early March, while above 2000 m continuous snow may lie till mid-May (Moody \& Rackham pers. comm.; Polunin 1988:22). There is a high probability for the first snowfall to occur by midSeptember (Furlan 1977: 210). Last snowfall is about late March in the lowlands, but April or even May in the highlands. 

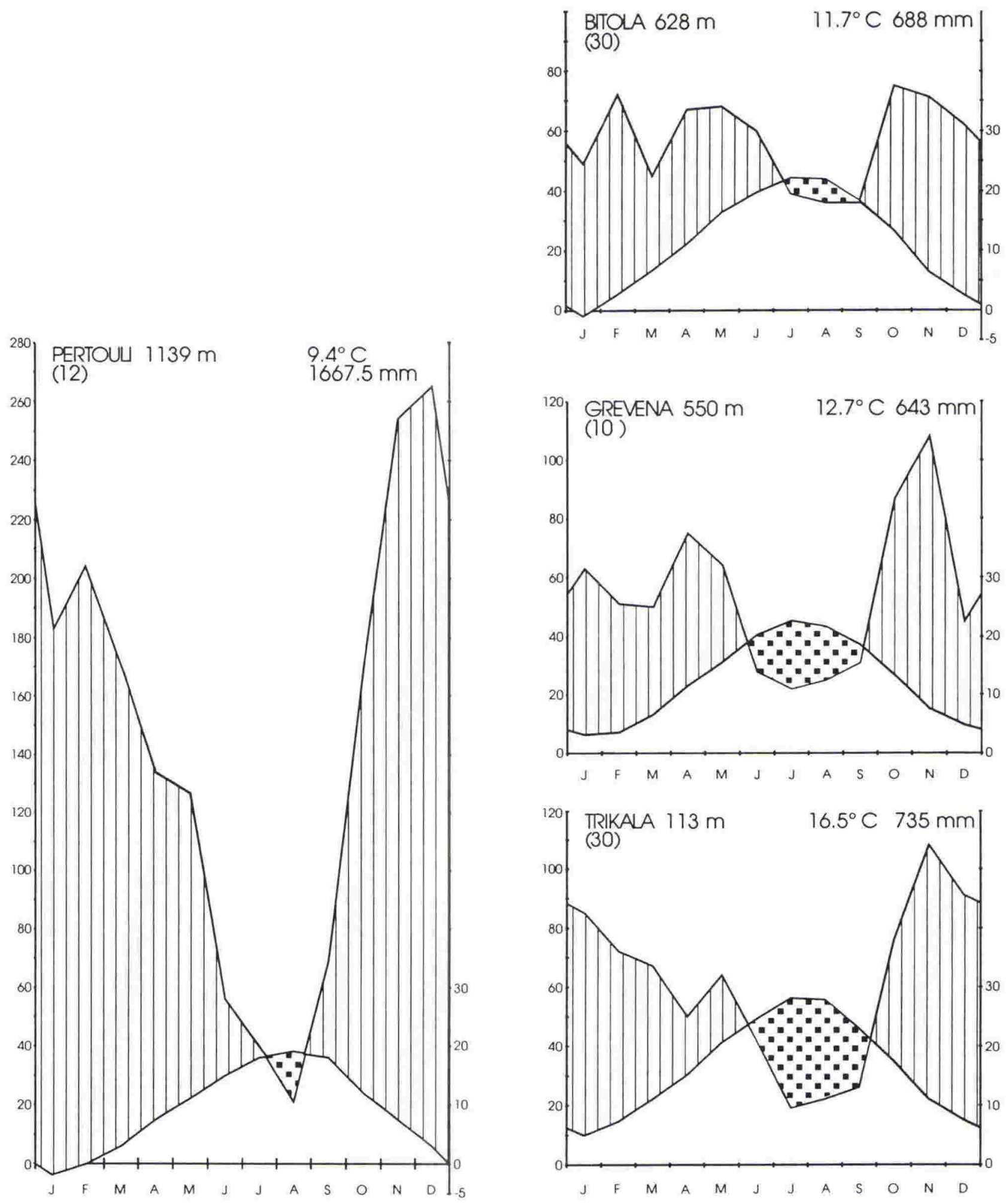

Figure 1.3 Climate diagrams for four Balkan stations

The form of the diagrams follows the convention established by Walter et al (1975). The abscissa represents the months from January to December, the warm season being in the centre of the diagram. Following the name of the station in the top left-hand corner is the height above sea level in metres; below this is the number of years of records. In the top right-hand comer is the mean annual temperature $\left({ }^{\circ} \mathrm{C}\right)$, then mean annual precipitation $(\mathrm{mm})$. The curves represent the mean monthly temperatures (scale on right, in ${ }^{\circ} \mathrm{C}$ ), and mean monthly precipitation (scale on left, in $\mathrm{mm}$ ). The dotted field indicates the relatively droughty season, and that with vertical lines the relatively humid season. As frost data is unavailable for some stations, this normal feature of Walter-diagrams is not shown. 
The Pindos act as an effective barrier to the predominantly westerly rain-bearing depressions (Furlan 1977:191) and cause a pronounced rain shadow. Mean annual rainfall thus decreases from about $750-1000 \mathrm{~mm}$ in the foothills to under $500 \mathrm{~mm}$ in the east of the province. Grevena weather station received an average of $643 \mathrm{~mm}$ of rainfall annually (Doyle 1990). Maximum rainfall months were in autumn, November being the wettest month with $108 \mathrm{~mm}$ average rainfall. Minimum rainfalls occurred in summer, with July at $22 \mathrm{~mm}$ the driest. The variability of annual precipitation is likely to be about $15-20 \%$ (Espenshade 1990:12-15).

The mean annual temperature at the Grevena weather station during the 1978-1987 period was $12.7^{\circ} \mathrm{C}$ (Doyle 1990). January was the coldest month at $3.2^{\circ} \mathrm{C}$, and July the warmest at $22.7^{\circ} \mathrm{C}$. Doyle (1990) estimated an annual average of 40 days of ground frost. These figures appear to be typical of the mountain basins of inland Macedonia ( $c f$. Furlan 1977; Martyn 1992). In winter there is commonly a temperature inversion: in January valleys and basins are $2-3^{\circ} \mathrm{C}$ colder than mountain slopes and peaks at the same height; the temperatures in basins occur on peaks 400-600 m higher (Furlan 1977:196). In summer the effect is less pronounced, and environmental lapse rates are higher. In general, the environmental lapse rate is $5.7^{\circ} \mathrm{C}$ per $1000 \mathrm{~m}$ (Savina 1993:60), but the highlands probably have a lower annual temperature range than is recorded in Grevena city, due to the cooling effects of prolonged snow lie and greater cloudiness.

Cloudiness is greatest in December and January and may be as high as $70-80 \%$ (Biel 1944). In summer to the east of the Pindos there is little cloudiness (probably $<20 \%$ ), which contributes to strong insolation. Doyle (1990) calculated mean monthly potential evaporation and soil moisture balance for the Grevena station, using the Thornthwaite \& Mather 1957 formula. Low rainfall and high monthly potential evaporation in summer (with a peak of $110 \mathrm{~mm}$ in July) cause a high summer soil-moisture deficit. Cooler temperatures and high rainfall in autumn result in recharging of soil moisture in September and early October, and soil-moisture surplus occurs throughout late autumn, winter, and spring. Minimum monthly potential evaporation is a mere $8 \mathrm{~mm}$ in January, and there is a net moisture surplus of $25 \mathrm{~mm}$ annually.

\subsubsection{Vegetation}

The landscape of Grevena is intensively used and little if any unmodified vegetation remains. The current vegetation of Grevena is described in detail in Chapter 3. Only the main features are mentioned here.

There are two conspicuous features of the vegetation in Grevena; it varies according to elevation and moisture. The highest elevations are occupied by alpine meadows and pine 
woods, the intermediate elevations by a mosaic of pine woods, beech woods, and mixed woods, and the drier rolling plains by deciduous oak woods and agricultural land. The west-to-east moisture gradient results in the western Pindos being much more moist and more vegetated than the eastern Vourinos Mountains. Woodland covers over half the entire land area.

Grevena differs greatly from southern Greece in both wild and cultivated plants. The province is very rich in flowering plants, but these tend not to be plants associated with Mediterranean climates characteristic of Greece, and even many 'sub-Mediterranean' plants associated with the fringes of the Mediterranean zone are rare or absent, winter conditions in Grevena being too harsh (Moody \& Rackham, in prep.). In fact, Grevena flora has a Central European character, with a number of species not found elsewhere in Greece. Some are characteristic of the steppes of the northern Balkans, Hungary, and south Russia, and many species are at or near their southern limit.

Plant growing season is limited by cold winters and dry summers (Moody \& Rackham 1988). The growth of most plants stops both in winter, as in Central Europe, and in summer, as in south Greece. The growing season is short - in most years only April, May and June. Even in mid May some of the more arid steppes begin to dry up (Moody \& Rackham 1988). For some wild plants, there is a second growing season in autumn (Moody \& Rackham, in prep.).

\subsubsection{Recent land use}

The entire landscape of Grevena is a resource base, there are no unproductive wastelands. The current land-use activities that have most impact on the landscape are agriculture, pastoralism, tree felling, burning, and settlements. These activities selectively favour some plant species over others, e.g. deciduous oaks invade abandoned land readily. They are resistant to browsing and have probably been encouraged at the expense of other trees.

Major landscape change has occurred this century due to the mechanization of agriculture, which has resulted in a reduction in cultivation in the Pindos Mountains and foothills, with a subsequent expansion of woodland and pasture. In the plains there has been a huge expansion of cultivation at the expense of oak wood and grassland. Today everything too steep to plough mechanically is pasture or woodland. 


\subsubsection{Agriculture}

In Grevena arable land represents only $14 \%$ of total surface area (Sakellariou 1988:24). The current agricultural economy in Grevena is based mostly on a few cereal cash crops and is confined mostly to the central plains. The most important crop is wheat (Triticum), then barley (Hordeum), and to a lesser extent rye (Secale cereale) (Figure 1.4). Rye is usually planted in fields with poor fertility or on the edges of wheat fields (Aschenbrenner 1988). A small amount of maize (Zea mays) is grown.

Vines (Vitis) are grown mostly for household use only. Other minor crops include tobacco (Nicotiana tabacum); alfalfa (Medicago sativa subsp. sativa); sunflowers (Helianthus annuus); soybeans (Glycine max); chickpeas (Cicer arietinum); melons (Cucumis melo); nut trees, e.g. almond (Prunus dulcis) and walnut (Juglans regia); and garden crops such as sugar beets (Beta vulgaris) and various brassicas (Brassiceae). Walnut is the most important cultivated tree. Its cultivation, however, is somewhat precarious, as it may be damaged by frost. Almond is common and often planted as hedges (Moody \& Rackham, in prep.). Sunflowers and tobacco are very recent introductions. Tobacco is grown widely but requires irrigation in the northeast of the province. Irrigation is also used to grow grass for fodder hay.

Modern mechanized wheat/barley cultivation involves once-a-year ploughing in autumn when the soils are moist and soft, followed by seeding. Seeds remain in the soil over the winter and germinate in the warmth of spring. The growing season lasts only a few months in spring and summer, and harvest is July-August. Between harvest and late September-early October stubble covers the fields prior to being burnt after the first autumn rain, just before ploughing (Figure 1.5). Agricultural soils lay bare during autumn and winter (Aschenbrenner, in prep.).

By the 1960s mechanized agricultural practices had replaced manual practices. In the plains great new fields were levelled with bulldozers from about 1973 to 1985 , obliterating most of the traditional small fields (Aschenbrenner, in prep.; Moody \& Rackham, in prep.). Today, the foothills still retain most of the old pattern of small fields, lanes, hedges, and isolated trees in fields. In some places these survive in use, but on steeper land they have been turned into pasture or woodland. In the mountains almost all previously cultivated land has become pasture or woodland.

Before mechanization manual subsistence agriculture was practised. Numerous relatively steep slopes were cultivated, including many areas above $1000 \mathrm{~m}$ in the Pindos with very thin soils. Some of these fields were ploughed in autumn, others in early spring. Pre-mechanized agriculture required the use of draught animals, which required fodder (crops and woodland). Both rye and wheat are known to have been much more extensively 


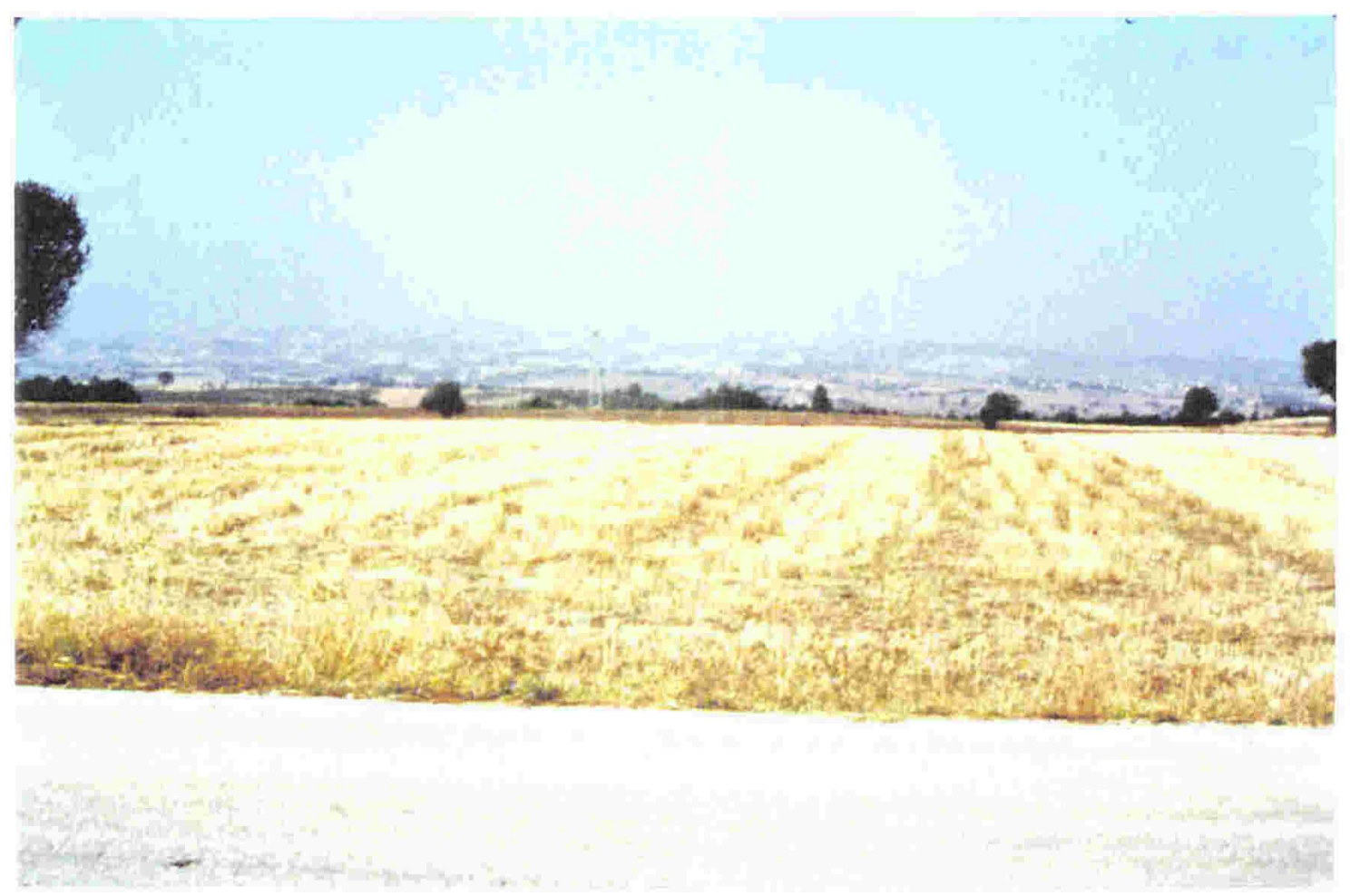

Figure 1.4 Cereal cropping. A field of barley stubble near Asprokampos on the central plains.

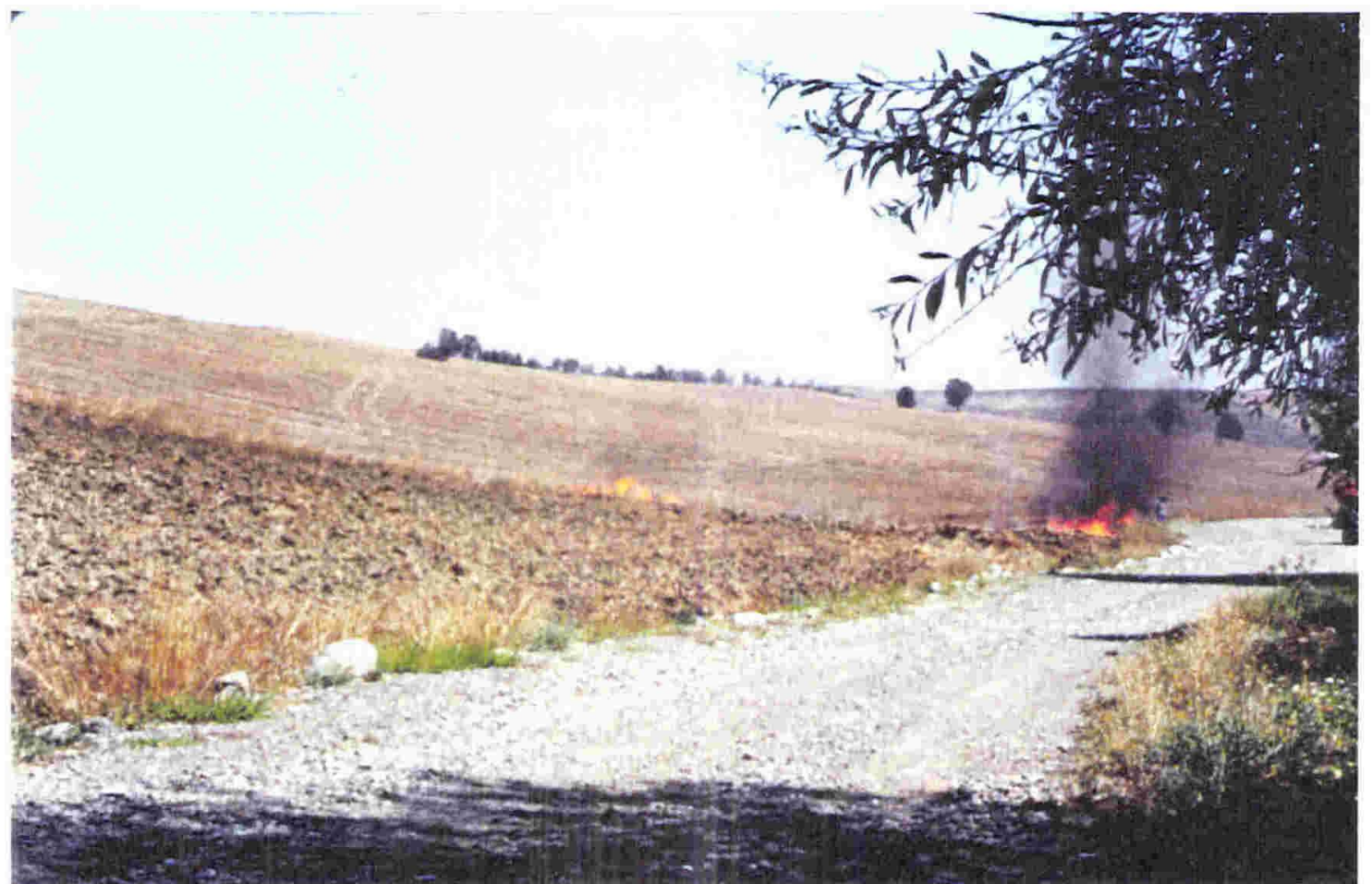

Figure 1.5 Burning stubble. Wheat stubble being set alight adjacent to the pollen analysis site at Kellia near Grevena city. Stubble burning was introduced after mechanized agriculture was introduced mainly to prevent machinery getting clogged. 
grown in historic times, in the foothills and even in the mountains (Leake 1835). Vines (Vitis) were extensively grown, e.g. around Spilaion.

For this study it is appropriate to look at pre-1940 agricultural systems, that is, before tractor-powered cultivation was employed and fertilizers were used. Prior to 1940, with the exception of the introduction of the iron mould-board plough in 1917, it is thought that there were few changes in the agricultural system for several centuries ( $c f$. the archaeological remains at Nea Nikomedeia dated c. 5600 BC (van Zeist \& Bottema 1971; Rodden 1972; Theocharis 1973:43)). Most of the following data come from enquiries of informants (Aschenbrenner $1988 \&$ in prep.).

The pre-mechanized agricultural economy was fundamentally a peasant subsistence type, for most households had insufficient land holdings to produce any significant surplus. Agriculture and pastoralism were integrated systems, even the transhumant communities of the Pindos Mountains practised a small amount of agriculture. In general each household grew a diversity of crops for human consumption (wheat; barley, probably mainly for livestock; maize; rye; beans; chickpeas; vegetables; fruits; nuts; and vine for table grapes, wine, and tsipouro). As well, a household grew crops for fodder (barley, oats, and several legume varieties, esp. vetch, pea hay, and alfalfa). Household livestock included oxen, cows, horses, mules, donkeys, pigs, chickens, and either goats or sheep or both. Animals served for draught and transport as well as providing food, e.g. meat, milk, cheese, and fat (Aschenbrenner 1988). A minimum complement of household livestock would include: one or two oxen, one donkey or horse or mule, one to two pigs, ten chickens, and 15-20 goats/sheep (Aschenbrenner, in prep.). Some households had a secondary occupation of commercial transport and kept mules (since they take less specialized feed). The size of a household's goat/sheep flock depended on personnel to herd them, available grazing areas, and cooperative arrangements with others for herding. In Milea and in Agios Georgios many also kept a cow, with which to produce calves and thus oxen, or animals to sell. Asia Minor refugees tended more often to keep cows, even for milk to drink, while indigenous Greeks tended to keep small herds of sheep/goats (Aschenbrenner 1988).

The cereal crops of wheat, barley, oats, and rye were rotated with either maize or a legume. The choice of rotation crop was based on considerations such as total cultivated area of a household, its need for bread and other cereal-based food, the needs of its draught animals, and the quality of its cultivatable land. Some households had difficulty meeting these needs and at the same time following the above rotation system. Two modes of compromise used were: either maize and rye were used instead of wheat, or the main group of cereal crops were rotated among themselves; but the same cereal crops were never planted in the same field in successive years. The latter was recognized as less desirable 
but was forced on people by poverty (Aschenbrenner 1988). Rotation was sometimes organized on a village-wide basis, e.g. at Agios Georgios, so that vast tracts of fields would be sown in cereals and another large tract in legumes. This allowed animals to more freely graze over cereal stubble without risk of their getting into a still unharvested legume field (Aschenbrenner 1988).

Fallowing was seldom practised because most households had so little land. When practice regularly it was by the few with large holdings, and then only fields of poor fertility were fallowed. Deliberate soil-fertility improvement other than crop rotation and fallowing was achieved by adding manure. This was done in two ways, either by grazing live stock (in herds or staked individually) in the stubble fields or by collecting manure from stables, yards, or sheep/goat pens and distributing it over the fields. This was done to fields with the poorest fertility. Reports vary as to how extensively or regularly this was done (Aschenbrenner 1988).

The introduction of the iron mould-board plough, which turned soil over, meant a dramatic increase in production. Prior to this the wooden, usually iron-tipped, scratch or ard plough was used. Ploughing of the predominantly clay-rich soils of the plains would have been difficult if not impossible. Ploughing of the higher-altitude soils would have been easier. By far the most common draught animal for ploughing was the ox, but a few used the horse. After 1923, refugee villages used water buffalo. Ploughing with draught animals tended to follow contours of the land. Hand-reaping left only short stubble, and the straw was taken to the threshing floor as part of grain sheaves. Only occasionally did a farmer prepare for ploughing by raking the corn stalk stubble into a heap to burn (Aschenbrenner 1988).

Grevena has a range of weeds of cereals. Weed seeds were discarded when crop seeds were selected. During the growing season a farmer would do as much weeding as time permitted, and many farmers said they could not do as much as they thought was necessary. Some weeds were fed to livestock (Aschenbrenner 1988).

Irrigable areas were scarce and small and hence devoted mainly to vegetable gardens. These plots were close to springs and streams. At Spilaion there are numerous springs, all with associated irrigated plots. These were up to $7 \mathrm{~km}$ from the village (Aschenbrenner 1988).

Average yields were $100 \mathrm{~kg} / \mathrm{str}\left(1\right.$ stremma $\left.=1000 \mathrm{~m}^{2}\right)$ for wheat with a ratio of seed to yield 1:3, but not infrequently yields only equalled the seed planted (Aschenbrenner 1987). At minimum a household needed to have about $32 \mathrm{str}$ allocated to production in the following manner: wheat 15 str; rye 2 str; maize 4 str; legumes/yoke oxen 6 str; oats/ horse or mule 2 str; vegetable garden 1 str; vineyard 2 str (Aschenbrenner 1988). In addition to 
the 32 str cultivated in various crops, a subsistence household required access to pasture and woodland, as a source of winter fodder and fuel (Aschenbrenner 1988).

A typical arrangement of fields would be a zone near the settlement in which each household had its vineyard. These fields often had fruit and nut trees surrounding them (Aschenbrenner, in prep.). Beyond this were grain fields with scattered large oak or pear trees. They were edged by a row of fruit or nut trees or a strip of scrub oak. The best fields were on stream terraces, where irrigated vegetable gardens were located. Most fields were within an hour's distance by foot from the settlement (Aschenbrenner 1988).

At Sindendron on the plains, where an unusually large amount of erosion has occurred, fields were most often cultivated ridge tops and segments of the gentler slopes. A few springs permitted irrigated gardens (Aschenbrenner 1988). At Spilaion in the Pindos foothills, where relief is greater, cultivated land included valley-bottom terraces (some irrigated), gentler slopes west of the village, and numerous isolated patches set on southfacing slopes in pine woods. Numerous springs were used for small irrigated gardens and bean fields. The distance to both grain fields and gardens was up to $2 \frac{1}{2}$ hours by foot. Nut and fruit trees, e.g. cherry and apple, were often planted on steeper slopes. Spilaion devoted such a relatively great proportion of its cultivated land to vineyards that wine could be exported to cover the deficiency in grain production. Many families had a second, fullyequipped house in which they lived permanently near distant cultivations.

During the twentieth century prior to mechanization, there was episodic widespread land clearance either to expand fields or open new fields. This type of abrupt widespread clearance may provide a model close to earlier land clearance, rather than the gradual pattern often suggested. In 1908 in the Lipsokouki Valley an area of oak wood and pasture changed hands and was subsequently quickly converted into cultivation fields. In 1924 when ethnic Turkish families returned to Turkey and Greek refugees came to Grevena from rurkey, land was allocated to the refugees, who set about fully exploiting and expanding their newly gained property. At Agios Georgios uncultivated areas were cleared by state work teams and added to the redistributed land (Aschenbrenner, in prep.).

\subsubsection{Pastoralism and agro-pastoralism}

The pastoral economy in Grevena is based on the herding of sheep and goats. Koster (1987) identified eight different zones of pastoral exploitation based on degree of dependence on transhumance or 'village pastoralism', ethnicity, vegetation types, and stocking rates. The two extremes in the system are the "Vlachohoria" in the Pindos, which are strictly transhumant, practice virtually no agriculture, use pastures seasonally, have moderate to high stocking rates and are ethnically Vlach; and the lowland agricultural areas, 
which are grazed by year-round 'village pastoralists', who practice intensive cereal cultivation, have high stocking rates, and are ethnically Kupatshar and/or Pondi. The following data on pastoralism and agro-pastoralism are taken from Koster (1987).

Almost the entire area is accessible to sheep and goats, and flocks are usually shepherded. Grazing restrictions are sometimes put in place by various authorities who administer public land, e.g. the parish council and the Forest Service. However, restrictions are not always adhered to. In parts of the Pindos National Park grazing is prohibited, but evidence of grazing is as great there as in areas where grazing is permitted. In much of the Pindos the Forest Service tries to confine grazing to designated areas by building huts and digging waterholes. A deterrent for grazing some areas of the Pindos is the presence of bears and wolves, which are legally protected; sheep and goats are rounded up into protective corrals at night, and large dogs are kept to protect the flocks.

The Grevena region is well known for the practice of transhumance, long-distance seasonal flock movement of small stock to and from winter and summer pastures (Figure 1.6). The mountain villages of Samarina, Perivolion, Avdella, and Smixi in the Pindos are some of the largest transhumance villages in Greece and are famous for their large flocks of sheep. They are also among the highest villages in Greece. The pastures around these villages are used exclusively in summer, May-October. However, in 1986 flock figures, though incomplete, suggested that less than $50 \%$ of the sheep in Grevena belonged to longdistance transhumant flocks as opposed to local forms of village pastoralism.

Village pastoralists are herders who are socially oriented toward a village throughout the year. They tend to have smaller flocks than the transhumant. In many of the more agricultural villages of Grevena, numbers of goats and sheep seem to be declining; a decline in village flocks by $20 \%$ of the total regional herd has been noted. In 1929 agricultural census figures for Grevena show a total of 140,646 small stock; in 1961, 184, 725; in 1971 there was little change; but in 1986 there was a decline of over 40,000, which brought the number of stock close to the 1929 figure again. This decline is almost entirely in village pastoralists and may be a response to a decline in available grazing land due to an increase in cultivated land, but there are also areas where both grazing and cultivation are declining. There is, however, an increase in villages in the south and east of the province.

In contrast, in the Vlachohoria small-stock numbers have stabilized over the past two decades: although there are fewer flocks, they are larger. In the highlands the average working flock today is about 425 animals, and there are about 200 families practising transhumance from the four Vlach villages. There were an estimated 420 families in 1967. Shepherds tend to return to the same milking and folding areas. Often two distinct grazing areas are used. The best pasture is reserved for milking ewes, while non-milking animals 


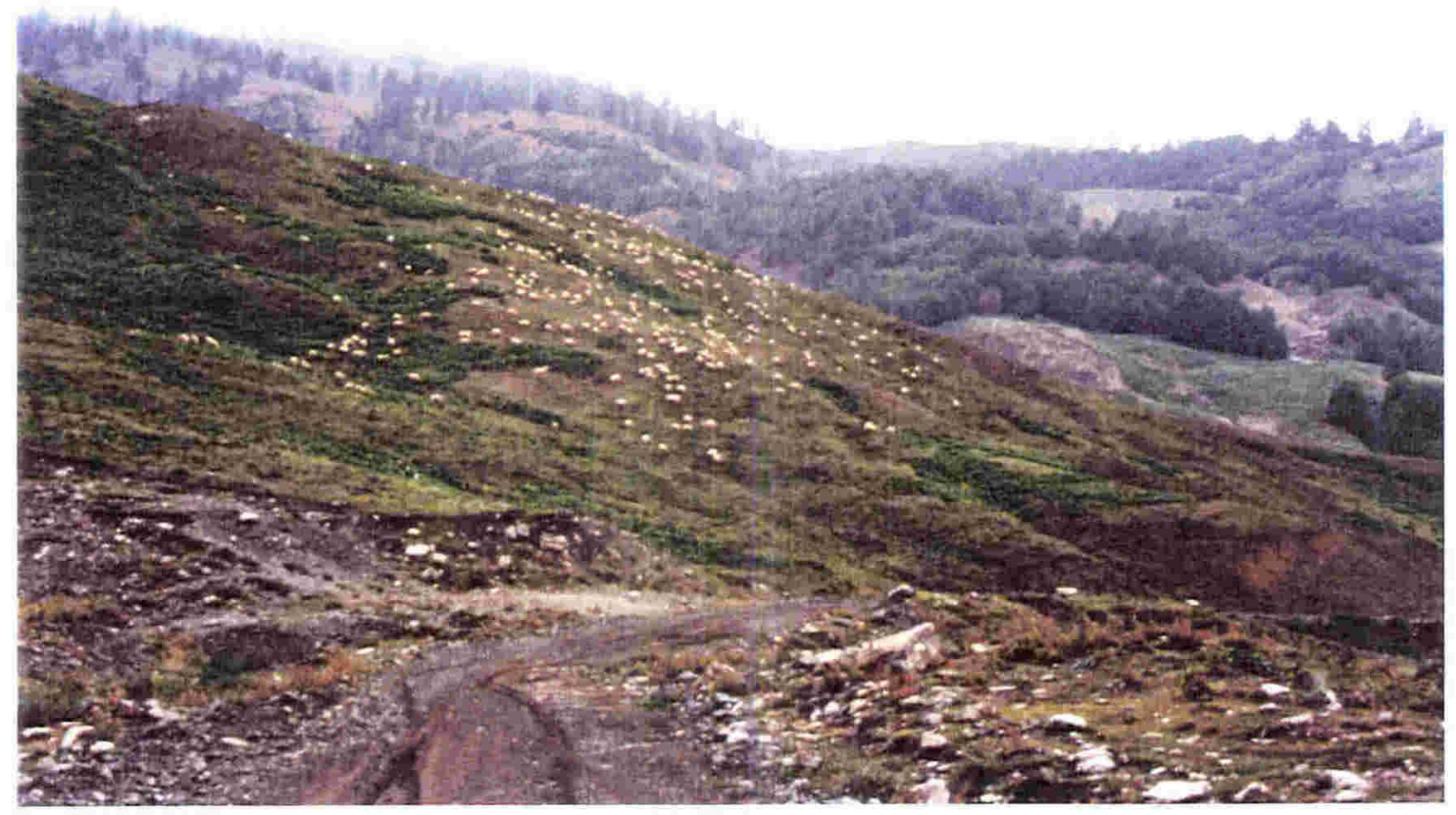

Figure 1.6 Transhumance pastoralism. A flock of sheep and goats grazing in the Pindos near Perivolion.

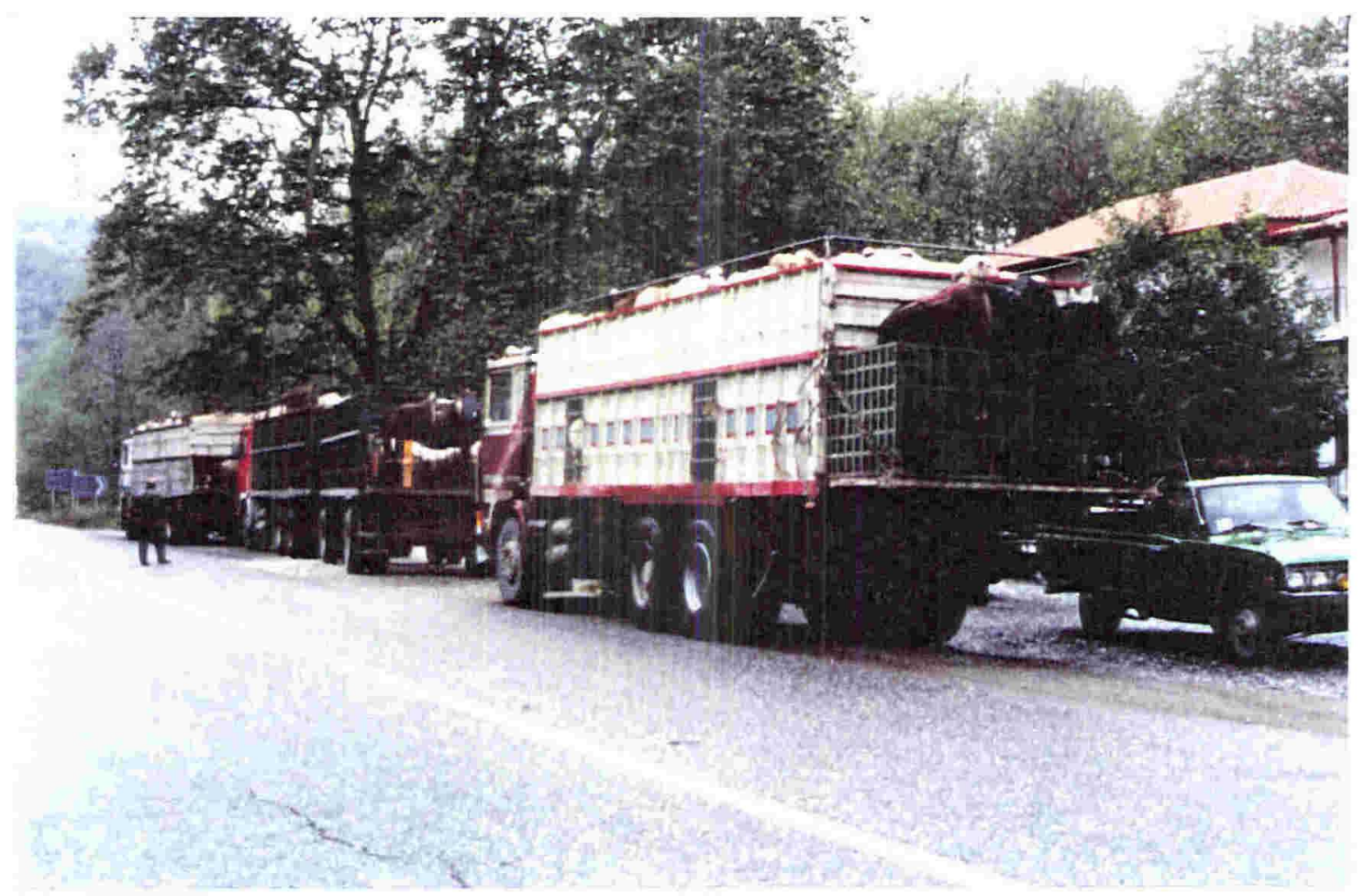

Figure 1.7 Transhumant transportation. Flocks being transported by truck, the usual mode of transport these days, from summer pastures in the Pindos to winter coastal pastures outside Grevena, probably in either Thessaly or Central Macedonia. 
graze among snowfields just below peaks, sometimes above 2400 meters. Flock territories are apparently not closely defended and are entirely informal, so that herders can adjust flock distribution to pasture condition. If pasture is poor, as noted for 1987 due to an unusually hot dry summer, flocks may leave the summer pastures early. Most herders keep some pack animals, as they graze their flocks up to as much as 7 hours travelling distance from their village.

The majority of transhumant flocks in the mountains are sheep, although there are also large goat flocks, and a small proportion of goats are kept with most sheep flocks. There are also 1800 head of cattle in the Vlachohoria. Before mechanized agriculture was introduced and more pasture existed in the lowlands, flocks would be transported on foot between summer and winter pastures, taking circuitous routes and grazing on stubble or natural pasture during the journey. Today they are usually trucked (Figure 1.7).

At lower altitudes in northern Pindos pastoralism is based primarily on seasonal summer pasture and mostly transhumance, but more villagers and flocks over-winter in the villages than in the Vlachohoria. Like the Vlachohoria there is a preference for sheep (except at Prosvorron, where there are large goat flocks). Currently there is very little agriculture (mostly irrigable), but subsistence cereal agriculture was practiced in the past. There are high stocking rates in this zone. Oak trees grow in this area and are shredded (see below) to provide either fresh forage or winter fodder. The same structures are built in this area as in the Vlachohoria, with the addition of well constructed winter folds and storage facilities for winter fodder. Ethnically this area is Kupatshar.

On the Pindos foothills there is a shift to a dominance of year-round village pastoralism. Most villages have transhumant flocks, but the majority of flocks over-winter in the village. Subsistence agriculture is integrated with herding. Both sheep and goats are herded, and stocking rates are low to moderate but were higher in the past. This area has the same structures as the area above. Ethnically this area is Kupatshar, but has some Pondi.

In the central agricultural villages where cereal cultivation is dominant, village pastoralism is year-round with no transhumance. Herding is an integral part of the production system. Although there has been a decline in stock numbers, stocking rates are high to moderate, with a preference for sheep. This area has undergone great changes since the introduction of mechanized agriculture. With the formation of large fields, which are planted continuously in cereals, much previously available grazing land has disappeared. In mid-summer herds graze on cereal stubble until it is burnt ready for ploughing. In winter they are taken into folds and fed alfalfa, straw, grain and, oak leaves. During the two periods between winter folding and cereal-stubble grazing, flocks are now forced to graze 
on reduced areas of pasture. Ethnically this area is Pondi today, but in the recent past was Valakhades and Kupatshar.

North of the central villages, in deeply eroded hill country, village pastoralism is dominant, but there is some transhumance. Herding is well integrated with cultivation, which involves about $1 / 3$ of the land surface. Stocking rates are moderate to low, with equal numbers of sheep and goats, except at Sindendron and Kirakali, where goats are dominant. The same structures that are associated with rear-round village pastoralism are found here. Ethnically this area is Valakhades, Kupatshar, and Pondi.

Village pastoralism is practised on the slopes of the Vourinos Mountains. Cereal cultivation is widespread. Stocking rates are some of the lowest in the province, with usually roughly equal sheep and goats, except in Palaiochorion and Chromion, where there are more goats, and Exarchos, Piloroi, and Pondini, where there are more sheep. A current practice is to graze in the Vourinos until winter and then descend to winter folds, where fodder of alfalfa, oak leaves, straw, and barley is supplied. The ethnicity of this area is unknown.

East of the central agricultural area along the Aliakmon River much of the surface area is thickly forested with deciduous oaks, and agriculture is limited. Village pastoralism is practiced, and there are few transhumants. Stocking rates are usually low to moderate with strong preference for goats. Winter folds are usual. Ethnicity is unknown.

The southern border of the province, except the Pindos, provides winter pasturage for many transhumant flocks belonging to the province. There are also many local village pastoral flocks. Large grazing areas are available, as well as stubble from well-developed agriculture. Stocking rates are moderate to high, with a dominance of sheep.

\subsubsection{Woodland management}

Grevena has very extensive woodlands for the size of its population. All woodlands have a history of management, which includes tree-felling, coppicing, pollarding, shredding and grazing. Pines are felled but not usually coppiced, pollarded, or shredded, while beech and oak and less dominant woodland trees may be felled, coppiced, pollarded, or less often shredded. Trees in woods are usually coppiced; free-standing oaks in pastures or fields are pollarded.

Timber production was well established 180 years ago (Leake 1835). In the past the main export of high-altitude villages such as Smixi and Samarina was milled pine planks (Figure 1.8). Beech and oak are also felled for timber - many local houses and outbuildings contain beams of oak. Today planks are produced for local use and for export. A recently established industry is that of particleboard manufacture (Figure 1.9). Wood 


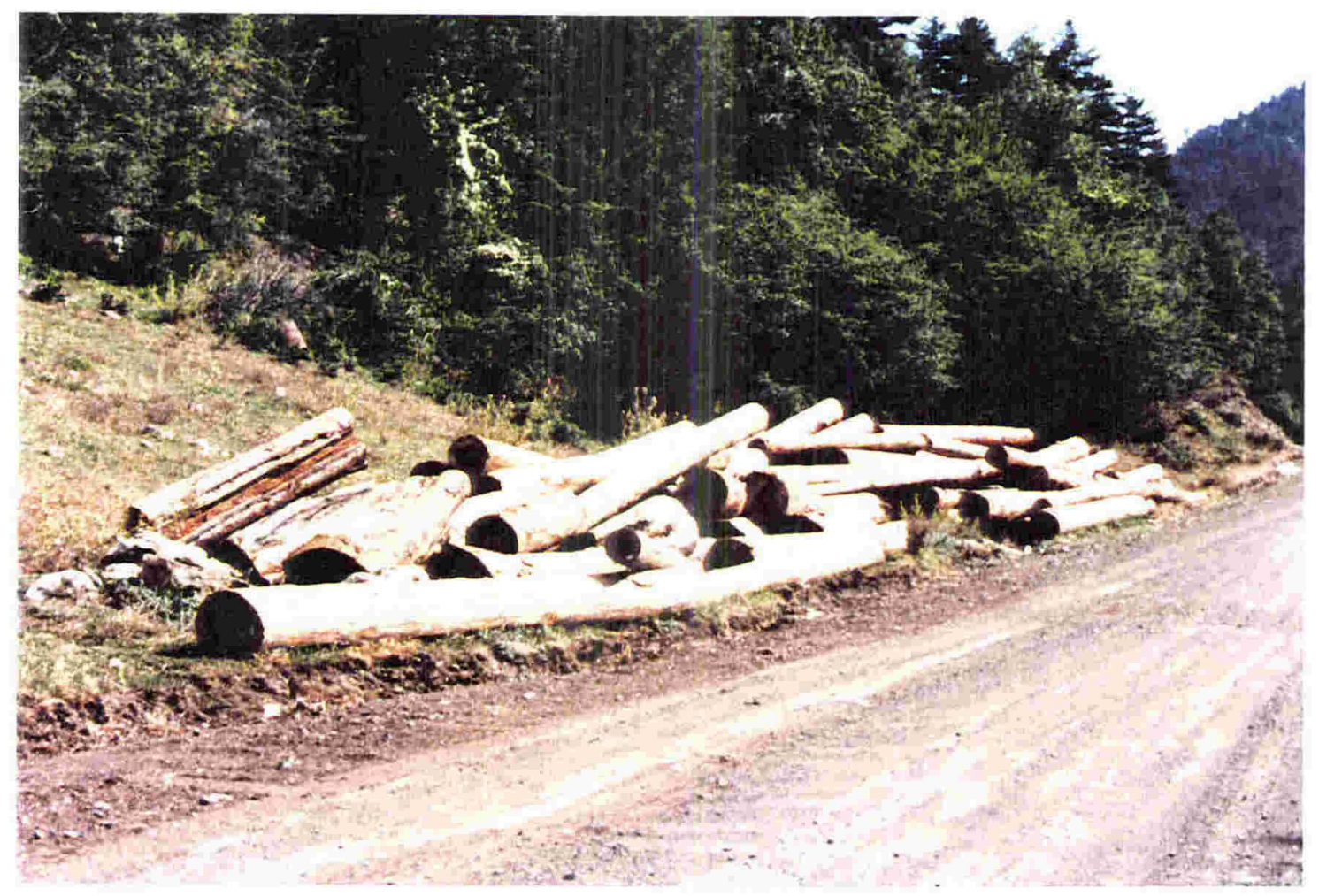

Figure 1.8 Pine felling. Pine logs on the side of the road in the Pindos Mountains between Zakas and Perivolion.

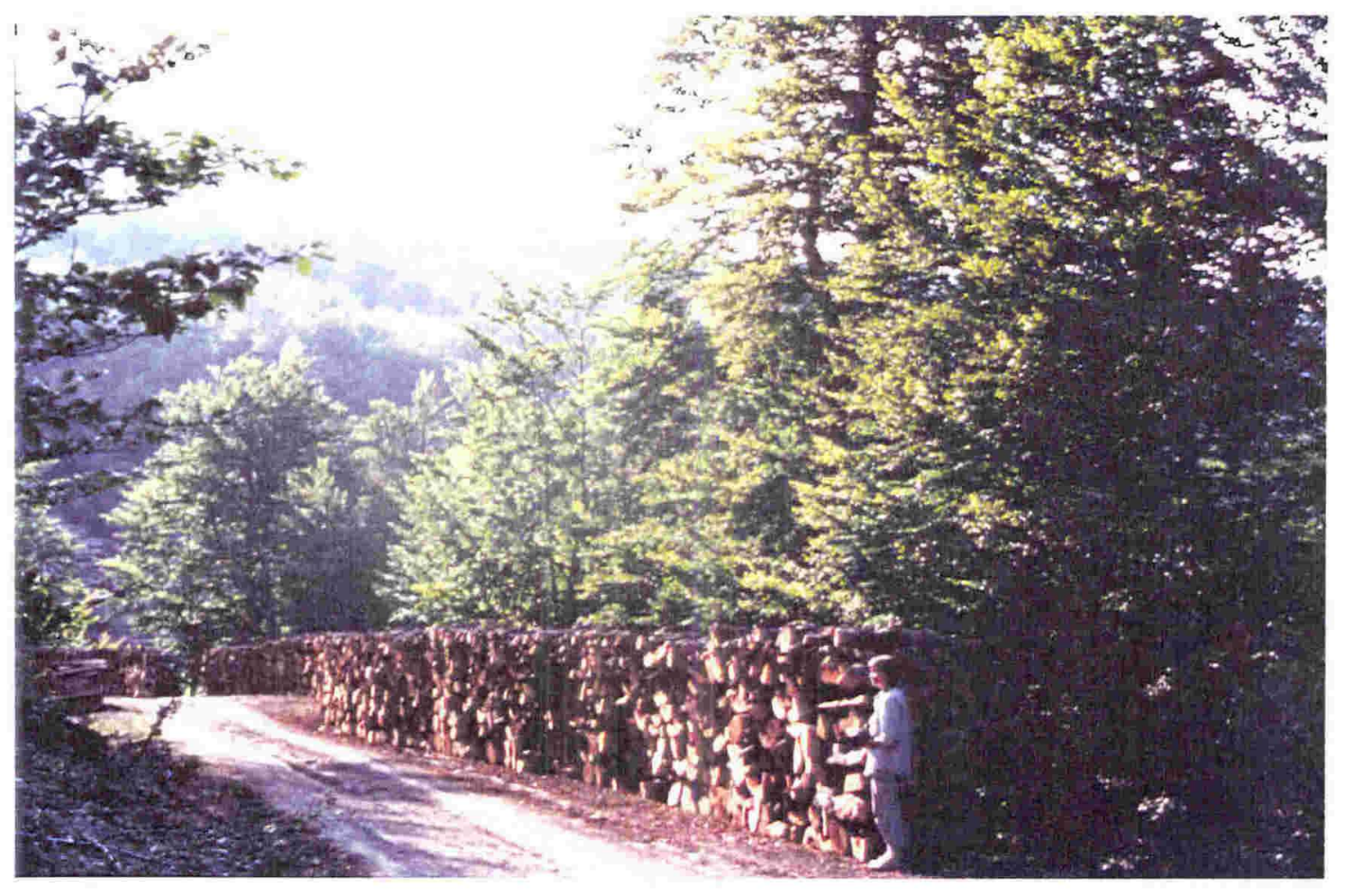

Figure 1.9 Beech felling. Beech billets for firewood or particleboard manufacture stacked beside the road between Avdella and Smixi in the Pindos. 
cutting has been nominally controlled by law since the late 1930 's, but this law was not enforced until 1955; now there is more careful control (Aschenbrenner, in prep.). Chainsaws appeared in 1965, and widespread cutting of larger trees commenced in the late 1970 's.

Coppicing, pollarding, and shredding are all ways of increasing the number of stems per tree. In coppice-woods, the majority of trees are cut down every so many years then allowed to sprout again from the stump, while scattered timber trees are allowed to grow longer to produce beams and planks. Pollards are cut like coppice stools but at $4 \mathrm{~m}$ above ground, while shredding is the removal of side branches so that successive crops of stems are produced (Figure 1.10). Stems are used mostly for winter fodder and fuel.

Coppicing has been widespread for many centuries, e.g. Tilia (lime) stools in the Aetia gorge measure $2 \frac{1}{2} \mathrm{~m}$ diameter, and at Anelia annual rings indicate that a beech stool was about 300 years old when last cut about 20 years ago. The practice still continues but on a diminished scale (Moody \& Rackham, in prep.). The coppicing methods used in Grevena are unusual in that the interval between cutting is unusually long (between 30 and 75 years), woods have no fixed boundaries but adjoin grassland or fields with no intervening earthwork, timber trees are sometimes left in groups (not scattered among the underwood), and animals are sometimes allowed to browse the young shoots for a time. Coppice is used for many purposes but usually for fuel.

Pollarding and shredding are both commonly used to provide leaf fodder. Leaf fodder production is a major industry (Figure 1.11). All oak species are so treated, but perhaps most frequently Quercus brachyphylla. Pollarding has been practiced for many centuries: in Aetia the stump of an oak was found that was first pollarded in $1556 \mathrm{AD}$ (from annual tree-ring count). Pollarding and shredding has occurred at very irregular intervals, from 4 to 75 years, with no apparent pattern. Almost all oaks have been cut or pollarded within the last 50 years (Moody \& Rackham, in prep.).

\subsubsection{Burning}

The natural vegetation of Grevena, except bracken (Pteridium) and juniper (Juniperus), is not very flammable. Many large pine trees have large lightning-strike scars but have not burnt. Steppe burns occasionally and this could limit trees by setting back juniper, which would protect young trees from browsing.

Since mechanized cultivation was introduced cereal stubble has been burnt (Figure 1.5). Although controlled by regulations these fires often spread into oak wood or steppe. The fires are lit when much of the vegetation is dormant, during summer drought, but plants 


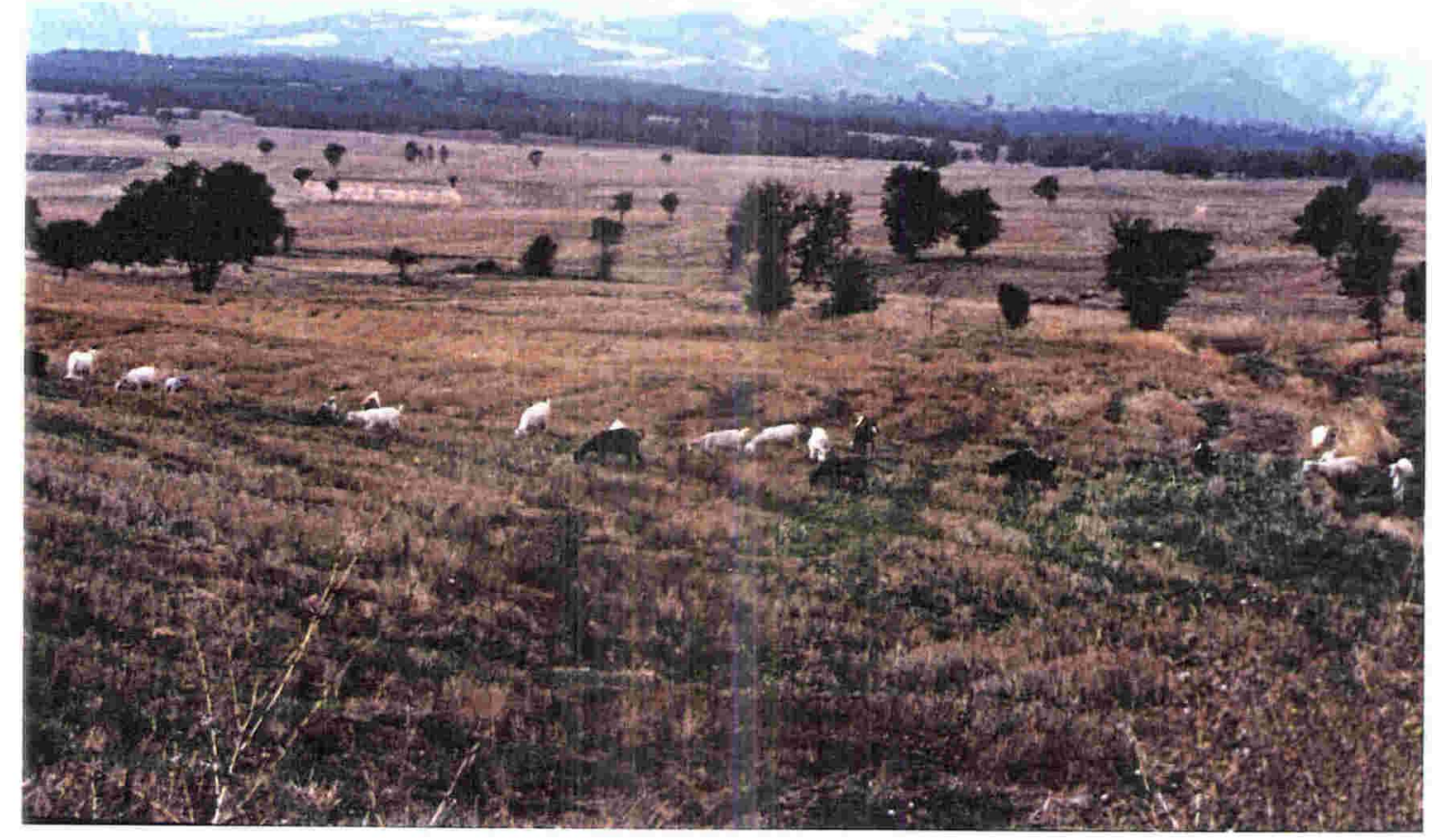

Figure 1.10 Pollarded oaks. Pollarded oaks, scattered in pasture near Mavranaioi, are cut about $4 \mathrm{~m}$ above ground level so that livestock cannot reach regrowth.

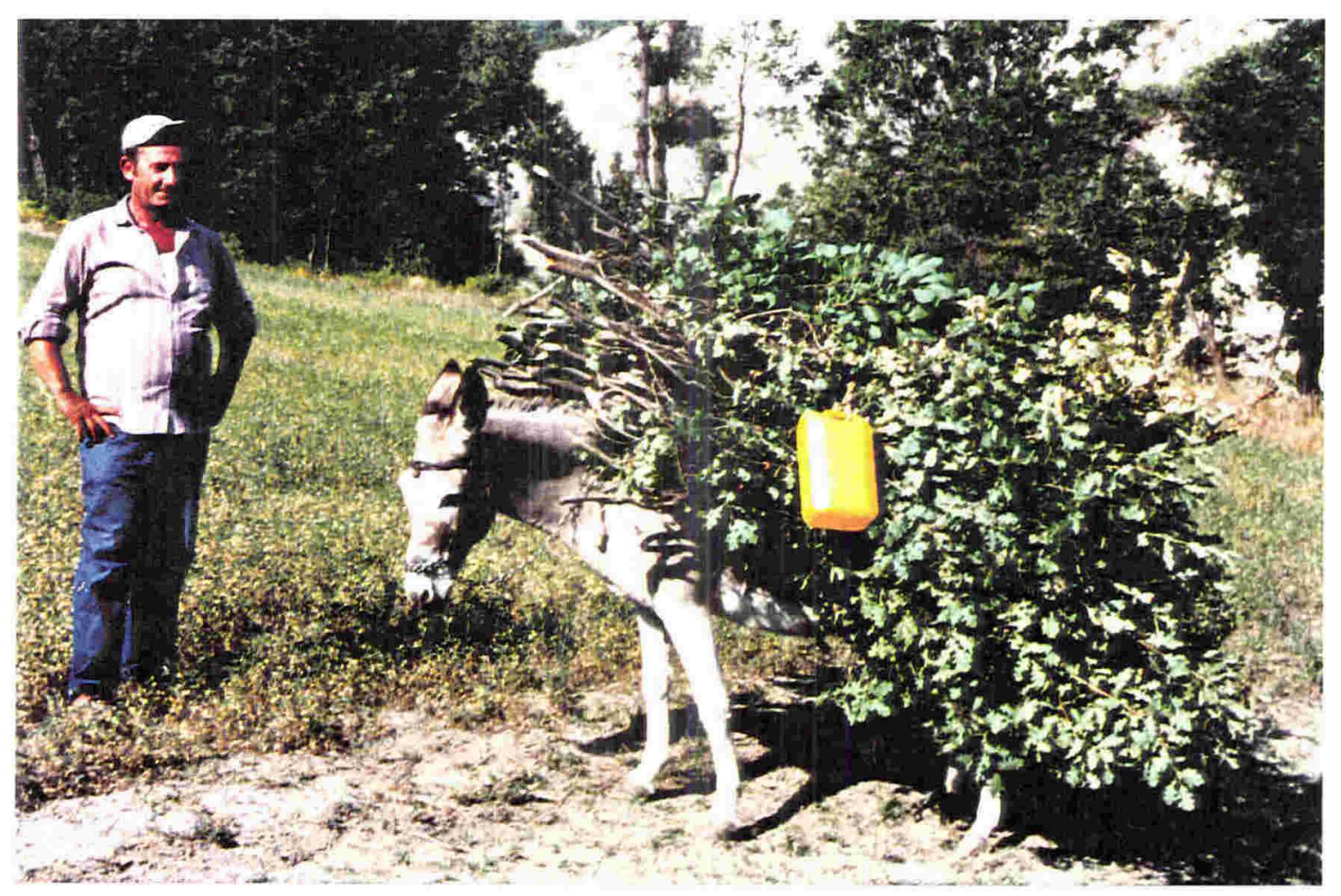

Figure 1.11 Leaf fodder. Oak leaves provide much of the winter food supply for stabled animals. 
that have an autumn growing season would be expected to be set back. Fire frequency has thus increased on the plain, but this does not seem to have changed the vegetation much, apart from the disappearance of the fire-sensitive juniper.

Burning natural communities to increase pasture is mentioned in the literature (Turrill 1929:210). I have not seen this done, but patches of Pteridium growing in pasture in the Pindos is indicative of this.

\subsubsection{Habitations and communication systems}

Today Grevena is one of the most sparsely populated areas of Greece. Grevena city, as the capital of the province, has become the commercial and manufacturing as well as administrative centre: its population in 1971 was 35,385 (Stevenson 1977:462). There are now approximately 100 modern villages and two cities in the province. Most of the population outside Grevena city is concentrated in the plains and rolling country to the west of the Aliakmon. Those engaged in agriculture tend to reside in villages rather than hamlets or isolated houses. There are relatively few villages in the mountains - in the Pindos because of the extreme winter conditions villages are occupied only during the summer. The relatively dry climate in the Vourinos limits agriculture and pastoralism.

Before World War II the structure of relations between settlements, their relative size, and their locations were different. Before the war Grevena city settlement was merely one of several small market towns hosting weekly markets and annual animal fairs: the relatively prosperous farming in the plains today has developed over the last 40 years. Farmers lived more often in hamlets and isolated houses than in villages as they do today. The principal structures associated with pre-mechanized farming have been described above.

The economy of the province was based on commerce in animal and timber products rather than those of local agriculture. Traditionally people in the Grevena region made their living by trade, especially in wool, the raising of sheep and goats, and cutting timber (Wilkie 1987). Settlements in the plains conducted their trade mostly on a local basis. Pindos villages, which were involved in long-distance trade, appear to have been more prosperous than those in the lowlands, and there were more and larger villages in the foothills and mountains than in the plains (Clark, M. 1988; Moody \& Rackham, in prep.).

There has been considerable instability of settlement in the lowlands over the last few hundred years. Settlements have formed and been abandoned. In recent times many villages have coalesced to form one new village closer to main roads. In the eighteenth and nineteenth century frequently the reverse was true as villages tried to avoid Turkish tax collectors. 
There is relatively easy access into Grevena basin, allowing much movement through this area. In historical times that movement has taken the form of trade, transhumant herding, migration, brigandage, and war (Clark, M. 1988). Many of the trading routes were along the edges of rivers and streams. The former trading passes over the Pindos have been retained as roadways, e.g. from Samarina to Fourka (the most northern pass), Smixi to Distraton (past Vasilitsa), through Avdella and Perivolion to Vovousa, and in the south from Kranea to Metsovon (Koster 1987:25). In 1912, Wace and Thompson (1972) traced the transhumant route from the coastal plains through Dasochorion to the Pindos Mountains. There are also several easy passes through the Chasia Mountains, at Velemishti as well as at Kutsophilani (Koster 1987:25).

The Greek Military Staff map of Grevena in 1929 shows cobbled roads in the lowlands (Clark, M. 1988). These radiated from larger villages such as Agios Georgios and Kivotos. The cobbled roads radiating out from Agios Georgios, Kivotos, and Amigdaleai also connected those villages. Roads also ran from Grevena to Kirakali and to Elatos and connected certain villages such as Mirsina and Asprokampos. There was a main road between Kalambaka and Kozani.

\subsection{Prehistory \& history}

Most of the following information is taken from the Grevena Project surface survey (Savina 1995; Wilkie 1995). The prehistoric cultural period represented by the largest number of archaeological sites is the Hellenistic period, next is the Early Iron Age. In historic times the Roman and Ottoman Periods are represented by many more sites than the Byzantine Period. Figure 1.2 indicates the periods of densest occupation based on abundance of archaeological sites.

Archaeological sites containing Late Bronze Age sherds are not numerous, less than 10. Some of the sites are typical citadels and capable of being fortified and controlling large areas of agricultural land.

The Early Iron Age seems to have been a period in which occupation of the region increased dramatically. Over 30 sites have been identified as definitely containing Early Iron Age material, and another 20 as possibly containing Early Iron Age material. Early Iron Age sites extend from the lowlands to elevations near $1000 \mathrm{~m}$ asl. Often these sites are on commanding promontories; some are probably quite large. At a number of sites archaeological material is common in the colluvium on the steep slopes (Wilkie 1988).

After the Iron Age there seems to have been a reduction in occupation. Only a few sites belonging to the Archaic and Classical Periods have been found: less than 40 from both periods. Both periods are represented mostly in the lowlands by settlement sites. 
These are mostly in the central plains and in the southeast of the province. There are a few fortified sites in the Pindos foothills.

In the Hellenistic Period the number and size (up to 10 hectares) of sites increases greatly: over 50 sites are definitely identified as representing this period, from the form of architecture and numerous sherds. Many Hellenistic sites are fortifications in the Pindos foothills, close to the upper elevation limit of permanent, year-round occupation. These sites are generally at elevations of approximately $1000 \mathrm{~m}$ (but not higher). There are also many sites of this period in the central part of the province - a number of quite large sites are scattered more or less evenly over the lowlands. Pottery was made locally. Loomweights are found for the first time, and in abundance. Sheep or goat bone and wheat/barley seeds have been found in association with Hellenistic sites. Parts of Grevena with few Hellenistic sites include areas above $1000 \mathrm{~m}$ in the Pindos and much of the eastern side of the province.

Archaeological sites dating to the Roman Period are more widespread than those of earlier periods. Romans occupied many places that have little record of Pre-Roman settlement, e.g. the northern part of Grevena, the Ventzia (between the Aliakmon River and Vourinos Mountains), and the south-central part of the province. Over 150 sites have been identified as belonging to this period, but the period spans a much longer time interval than the previous periods. There are more sites belonging to Early and Middle Roman times than to Late Roman times. North and northwest Grevena seem to have been virtually abandoned during Late Roman times, leaving only a few fortified outposts in the northwest and west. Most sites over $800 \mathrm{~m}$ in elevation were abandoned in the Late Roman Period. Roman settlements tend to be grouped, but do not exhibit a systematic hierarchial pattern. Road-building was introduced during this period. There was a substantial local ceramic industry, and wine was made locally (Savina 1995).

The Medieval and Byzantine Periods are represented by 80 to 90 sites. During the Early Medieval Period there are more than 40 certain and about 15 possible sites in Grevena, the same order of magnitude as the number of Late Roman sites. Slavic pottery has been recognized at only 3 sites. The areas that Late Roman sites occupied, that is, central Grevena, the Ventzia, and south-central Grevena, continued to be occupied, the Karperon basin was abandoned, but there was renewed settlement in the DeskatiDasochorion basin, and in the north and northwest. There are only a few Early Medieval sites at $1000 \mathrm{~m}$ or higher. Early Medieval sites tend to be widely spaced. About five Early Medieval sites were fortifiable, but at only one is it clear that the fortifications date from the Early Medieval Period or the following Medieval Byzantine Period. Some of the church crypts and mosaics in Grevena may date to the Early Medieval, or may be even 
older. A local ceramic industry existed, but production was reduced. The economy of Early Medieval times may have been based on agriculture and pastoralism: most of the lower elevation sites are in places suitable for agriculture. They are near a water source but not on main thoroughfares. The occupation of sites in the Pindos Mountains at about $1000 \mathrm{~m}$ elevation resembles the distribution of modern transhumant villages.

Compared to the number of Late Roman and Early Medieval sites, there are relatively few Medieval Byzantine sites in Grevena. There are 12 sites with definite Medieval Byzantine material. Of the five sites with many sherds of the period, all are in the central part of the province or the Ventzia. The two major sites in the drier Ventzia are associated with high-discharge springs. There are no fortified sites of this period in Grevena.

The Late Byzantine is represented by only 3 confirmed sites. The oldest standing church in Grevena (Agios Nikolaos near Perivolion, in the Pindos) was built in 1303 AD.

Grevena came under Ottoman control in about 1360. In contrast to the lack of settlement during the Byzantine Period, there are about 90 archaeologically recognizable Ottoman Period settlements distributed throughout the province, including central and south-central Grevena, the Ventzia, the Karperon basin, Dasochorion-Deskati basins, and the Pindos foothills. There are relatively few sites in the north. Some villages were highly specialized, e.g. Filippaioi was a community of technicians, including gunsmiths; Tziani had jewellery makers; and Ventzia, around Knidi, had charcoal making. Many villages had one or more grain storehouses. The agricultural areas were divided into large estates. Transhumant pastoralism was practised in the Pindos mountains and foothills. An extensive road and bridge network was built during the Ottoman Period. Lodges were built along these roads. There are also mill sites on several rivers.

Many Christian churches in Grevena date to the Ottoman period. A church at Spilaion, built in $1633 \mathrm{AD}$, is well known because it was attached to a famous monastery. It was decorated with frescoes between 1640 and 1658 AD (Kokkini 1976:48, in Savina 1995). According to local tradition, the monastery was very wealthy and had extensive estates.

At the end of Turkish rule, the former economic and cultural ties Grevena had with the continental hinterland weakened, and ties with the Greek peninsula and the Mediterranean strengthened. Turkish immigrants were transported back to Turkey and Greek refugee families living in Turkey were resettled in Grevena.

Between 1941 and 1950 Grevena suffered the effects of two wars, World War II and the Greek Civil War. Villages in Grevena, particularly in the Pindos, were bombed, and many people left the province. Fewer people have returned to the Pindos than the lowland 
villages and many of the houses in the Pindos today are used as only summer holiday homes. Today Grevena belongs to the parliamentary republic of Greece.

\subsection{Summary of late Holocene pollen studies on mainland Greece}

No late-Holocene pollen sequences have been studied previously in Grevena, but a number of studies have been undertaken elsewhere in mainland Greece, in areas with contrasting ecological conditions, current vegetation, and land-use history. In pollen diagrams that span the last few thousand years human activity appears to be a major factor influencing changes in the vegetation. The following account summarises the evidence presented by various researchers. Sites are presented more or less in chronological order of first examination. Figure 1.12 shows the location and elevation of the pollen-analysed sites; the site numbers are also given in the text below.

Van der Hammen et al. (1965) published the first late-Holocene pollen diagram from mainland Greece, from the Tenaghi Philippon marsh [4] on the Plain of Drama. Three pollen samples taken from above a level radiocarbon dated at 7850 $\pm 50 \mathrm{BP}$ (Wijmstra 1969) showed a dense forest dominated by Quercus and Pinus, but Tilia, Ulmus, Carpinus, and Alnus also occurred.

Wijmstra (1969) published a more detailed diagram from Tenaghi Philippon marsh. The base of the uppermost zone is placed where Quercus begins to rise after an ArtemisiaChenopodiaceae zone, just below a radiocarbon date of $7850 \pm 50 \mathrm{BP}$. By extrapolation the upper two subzones (Z4-Z5) are assigned to the last 5000 years. They are characterised by an open Quercus ilex/coccifera-Pistacia-Juniperus forest with Corylus and Ostrya. The forest became increasingly open, with the lower subzone being characterised by an increase in Fagus and Ericaceae, while the upper subzone is defined by increasing values of Pistacia and non-arboreal pollen and the presence of Rhus and Arbutus unedo.

Greig \& Turner (1974) and Turner \& Greig (1975) recored the Tenaghi Philippon marsh and analysed two overlapping cores from the most recently formed peat. A total of six radiocarbon dates were obtained, the oldest being $7556 \pm 85 \mathrm{BP}$ and the most recent $2867 \pm 60 \mathrm{BP}$ at $75 \mathrm{~cm}$ depth. Both pollen diagrams showed similar changes in pollen frequencies, and a local zonation scheme was applied to the diagrams. Six pollenassemblage subzones were recognized spanning the Early Neolithic to Classical Periods. At the base of the sequence, between 6500 and $2500 \mathrm{BC}$, is a Quercus-Tilia pollen assemblage; this changed to a Quercus-Tilia-Fagus-Ericaceae assemblage between 2500 and 1900 BC. Evidence of human disturbance begins in the Quercus-Olea-herbaceous zone between 1900-1360 BC, when there was a decline in Ulmus and Tilia. Dates for the 3 zones above this are extrapolated; these change from a zone with increased weedy species 


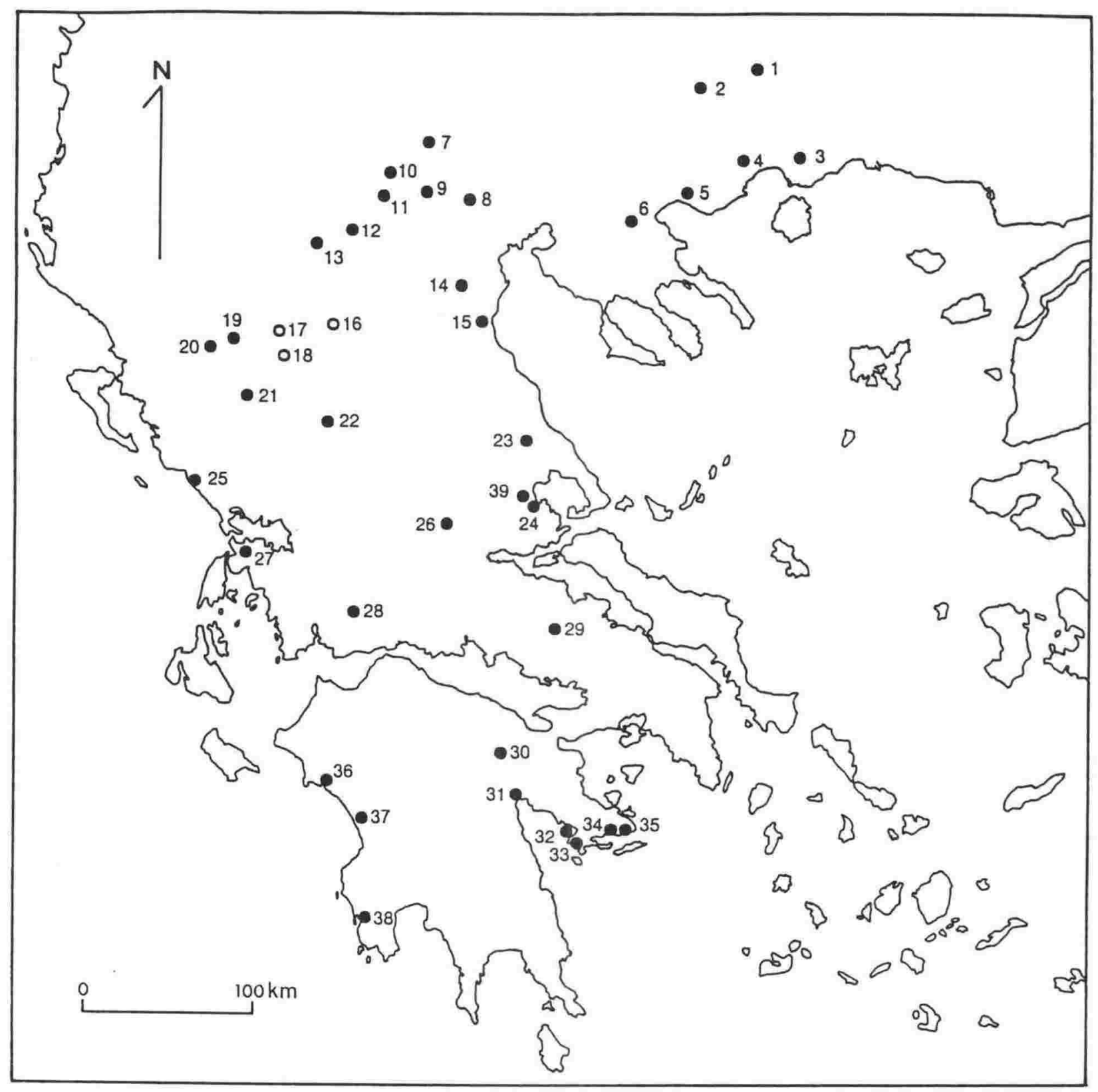

Figure 1.12 Location of Holocene pollen analysis sites, with altitudes (where known).

1 Rhodopi $1520 \mathrm{~m}$ (Athanasiadis et al 1993); 2 Lailias $1420 \mathrm{~m}$ (Gerasimidis 1985); 3 Gravouna c.0 m (Turner \& Greig 1975); 4 Tenaghi Philippon $40 \mathrm{~m}$ (Van der Hammen et al 1965; Wijmstra 1969; Greig \& Turner 1974; Turner \& Greig 1975); 5 Strimon c.0 m (Atherden 1989); 6 Volvi c. $100 \mathrm{~m}$ (Bottema 1981, 1982); 7 Voras $1640 \mathrm{~m}$ (Athanasiadis \& Gerasimidis 1986); 8 Giannitsa c. $10 \mathrm{~m}$ (Bottema 1974, 1975); 9 Edessa $350 \mathrm{~m}$ (Bottema 1974, 1975); 10 Paikon 1080 m (Athanasiadis \& Gerasimidis 1987); 11 Vegoritis 570 m (Bottema 1981, 1982; Bottema \& Woldring 1990); 12 Khimaditis $c .560 \mathrm{~m}$ (Bottema 1974, 1975); 13 Kastoria $650 \mathrm{~m}$ (Bottema 1974, 1975); 14 Pieria - Kokkini Brysi 1420 m \& Flampoura 1650 m (Gerasimidis 1985); 15 Litochoro $25 \mathrm{~m}$ (Athanasiadis 1975); 16 Kellia $600 \mathrm{~m}$ (this study); 17 Gomara $1750 \mathrm{~m}$ (this study); 18 Anelia $1440 \mathrm{~m}$ (this study); 19 Rezina 1800 m (Willis 1989, 1992b, c, 1994); 20 Gramousti 285 m (Willis 1989, 1992a, c, 1994); 21 Ioannina $470 \mathrm{~m}$ (Bottema 1967, 1974, 1975); 22 Pertouli $1275 \mathrm{~m}$ (Athanasiadis 1975); 23 Viviis c.50 m (Bottema 1979); 24 Halos c.0 m (Bottema 1988, 1990); 25 Korone 10 m (Yasuda 1985); 26 Xinias 500 m (Bottema 1978, 1979); 27 Voukaria c.0 m (Wright 1972); 28 Trikhonis $20 \mathrm{~m}$ (Bottema 1980, 1981, 1982, 1990; Bottema \& Woldring 1990); 29 Kopais 95 m (Greig \& Turner 1974; Turner \& Greig 1975; Allen 1986, 1990); 30 Kleonai (Nemea) (Atherden et al 1993); 31 Lerna (Jahns 1990, 1993); 32 Kiladha Bay -10.2 m (Bottema 1990); 33 Port Kheli c.0 m (Sheehan 1979); 34 Thermisia c.0 m (Sheehan 1979); 35 Saktouri c.0 m (Sheehan 1979); 36 Lake Agoulinitsa $c .0 \mathrm{~m}$ (Gennett 1982); 37 Kaiafa $c .0 \mathrm{~m}$ (Wright 1972); 38 Osmanaga -0.20 m (Wright 1972) \& Navarino No.3 c.5 m (Kraft et al 1980; Gennett 1982); 39 Zileria 1.5 m (Reinders \& Bottema 1983; Bottema 1994). 
(Quercus-herbaceous) to one from 1000-550 BC characterised by small amounts of Olea (Quercus-Olea-herbaceous) to one of increased weedy species again. It is interesting that oak forest appears to remain dominant (although it decreases slightly) throughout the diagram when there is archaeological evidence indicating that this area was occupied in the Neolithic and later times (Renfrew, C. 1971). Unfortunately the authors were not able to distinguish between evergreen and deciduous oak pollen or to separate Cereales from other Gramineae due to poor pollen preservation. Fagus appears with a coinciding increase in Ericaceae between levels dated to $4193 \pm 120$ and $3722 \pm 100$ BP. An increase in Fagus is also characteristic of Wijmstra's subzones Z4 and Z5.

Bottema (1967) published a preliminary diagram from Ioanninon [21], west of the Pindos Mountains. Four pollen zones were distinguished, but only the uppermost is relevant to this study, having a radiocarbon date near its base of $10200 \pm 90$ BP. Quercus (deciduous) played an important part in the forest cover of this zone, and in the second half Ostrya/Carpinus orientalis pollen type appears and becomes quite abundant. Quercus ilex appears and steadily increases soon afterwards. Bottema provides further details in a comprehensive pollen-analytical study of sites in northern Greece published in 1974 (see below).

Bottema $(1974,1975)$ analysed wetland sediment sequences from four basins in western Macedonia; Edessa [9], Khimaditis [12] (cores I, III \& IV), Kastoria [13], and Giannitsa [8]; as well as re-examining the Ioanninon sequence (cores I \& II) (Bottema 1967). Bottema established a series of zones based on characteristic tree pollen assemblages, reflecting the sequence of climatic changes and intensity of anthropogenic disturbance. The upper part of subzone $\mathrm{Y} 2$ and zone $\mathrm{Z}$ cover the late Holocene for the area northeast of the Pindos (Figure 1.2). The vegetation development in the Ioanninon area, farther south and to the west of the Pindos, differs in some aspects from the more northern area east of the Pindos. Subzone Y2 of the northeast area and zone Y (Bottema 1975, fig.3) of the southwest are contemporaneous and are both characterized by a sudden increase of Carpinus orientalis/Ostrya. During zone Y (subzone Y2) changes took place in the deciduous mixed oak forest. North of the Pindos some of the constituents of the mixed oak forest such as Corylus, Tilia, and Ulmus decreased in numbers, but Quercus does not decrease considerably. At higher elevations the situation did not change noticeably, as conifers maintained their position. Fagus was present in low numbers. The end of zone $\mathrm{Y}$ is difficult to date. At the northern sites radiocarbon dates of $4080 \pm 55 \mathrm{BP}$ (at Kastoria), $5260 \pm 65$ BP (at Edessa), and $3995 \pm 60$ BP (at Khimaditis I) were obtained. In the south, Ioanninon II gave a radiocarbon date of $4535 \pm 40 \mathrm{BP}$. 
Zone $\mathrm{Z}$ is divided into subzones in the northern area, based on rather local pollen assemblages, but not in the south. The main characteristic of subzone $\mathrm{Zl}$ is the expansion of Fagus in the belt of the Pinus and Abies forests. The deciduous forest below the conifer belt expanded upwards, while Abies and Pinus moved to higher elevations. More to the interior, north of the Pindos this was less pronounced, particularly at higher elevations, where coniferous forest gave way gradually to Quercus forest and Fagus. Coincident with the increase of Fagus was an expansion of Ericaceae in the mountains in both the north and south areas. In the south conifers were not affected, but they played only a restricted role. At medium elevations, in the upper Quercus (deciduous) belt, Corylus and Tilia decreased in abundance.

In the north the $\mathrm{Zl} / \mathrm{Z} 2$ boundary is based on the increase of Juglans, Platanus and Castanea and is dated at $3135 \pm 70 \mathrm{BP}$ in the Khimaditis III sequence and $3280 \pm 55 \mathrm{BP}$ in Edessa. Juglans and Castanea appeared for the first time, although in the south Juglans and Castanea did not appear until quite recently. Together with these trees, pollen of Vitis, cereal-type, Xanthium, Centaurea solstitialis-type, Plantago, and Humulus/Cannabis was found. The subzone Z2/Z3 boundary at Kastoria is dated $1095 \pm 60 \mathrm{BP}$. The subzones Z3 and $\mathrm{Z} 4$ are based on the intensity of human disturbance, Z4 showing much greater disturbance. Farming decreased during Z3, as indicated by the lower abundances of weeds and cereal-type at Khimaditis I and Edessa.

Wright (1972) published pollen data from three sites on the west coast, Osmanaga Lagoon [38], Kaiafa Lagoon [37] and Lake Voulkaria [27]. Two overlapping cores from Osmanaga Lagoon were analysed, with the base of the longer core being radiocarbon dated to $3940 \pm 270 \mathrm{BP}$. All changes in this sequence are attributed to human activities. The two pollen sequences were divided into 3 zones. The lowest zone is dominated by Pinus, Quercus, and Erica-type. Pinus disappeared during the Middle Bronze Age or early in the Late Bronze Age, perhaps due to woodcutting. The middle zone is dominated by two peaks of Olea separated by a maximum of Quercus (probably evergreen); Pistacia pollen becomes common in this zone. A radiocarbon date of $3220 \pm 120 \mathrm{BP}$ at the beginning of the Olea rise and another of $2820 \pm 100 \mathrm{BP}$ at maximum frequency places widespread olive cultivation at 1100-700 BC. In the top zone Quercus is reduced and declines steadily. Another peak in Olea occurs at the base of this zone in one of the sequences and may have occurred during the first millennium after Christ. A later study from nearby was carried out by Kraft et al. (1980) - see Navarino Bay, below.

Two basal radiocarbon dates are provided by Wright (1972) for the pollen sequence from Kaiafa Lagoon, $1680 \pm 100$ and $1650 \pm 100 \mathrm{BP}$. No zonation is provided, but there is a change in the dominant tree type midway up the sequence from Quercus at the base to 
Pinus at the top. Olea and Pistacia are present throughout the sequence at low levels while Phillyrea comes and goes. The increase in Pinus may be due to pine plantations.

No pollen diagram was provided by Wright (1972) from Lake Voulkaria, but it is described as having high pollen values of Quercus (deciduous) and Gramineae, with rare Olea in the lower half of the $8 \mathrm{~m}$ core. The upper half is marked by a rise in Olea pollen, followed by a decrease. Pollen of maquis plants such as Quercus (evergreen), Pistacia and Phillyrea are more abundant than below while Quercus (deciduous) remains about the same, but Pinus declines. Juglans (walnut), Castanea (chestnut), and Platanus (plane tree) are continuously represented, whereas below they are absent. A basal radiocarbon date gives $10900 \pm 300 \mathrm{BP}$ and one just below the Olea rise is $3040 \pm 120 \mathrm{BP}$.

Two pollen diagrams were published for a sediment core taken from Lake Kopais [29] in 1968 (Greig \& Turner 1974; Turner \& Greig 1975). This core was zoned in an independent, local scheme. One radiocarbon date of $5205 \pm 120 \mathrm{BP}$ dates a zone boundary at about $225 \mathrm{~cm}$, where Quercus and total tree pollen frequencies decrease markedly and do not recover. The zone immediately above this date is characterised by low Quercus and high Olea pollen with herbaceous pollen. Tilia and Ulmus also disappear during this zone. In the zone above Olea disappears but then appears again in the next zone. Erosion may have increased during the periods represented by the two zones with Olea present. Forest clearance and grazing are thought to have begun before the level of the radiocarbon date.

A second long sediment core (core KA) was pollen analysed from Kopais Basin and published by Allen $(1986,1990)$. The upper part of the core has a radiocarbon date of $9900 \pm 110 \mathrm{BP}$ and is also dated by correlation with a nearby exposure with a basal date of $9970 \pm 120 \mathrm{BP}$ and two more recent dates of $4620 \pm 150 \mathrm{BP}$ and $3480 \pm 150 \mathrm{BP}$. The pollen stratigraphy for core KA was found to closely resemble that previously found in the Kopais Basin. The postglacial record is dominated by Quercus until this declines after about 5000 BP. At a core depth of $3.9 \mathrm{~m}$ Quercus and Gramineae concentrations begin to decline, while Ostrya/Carpinus increases and Ericaceae appears. This level may correspond, assuming constant sedimentation rates, to the level in the Greig and Turner (1974) pollen sequence where a marked drop in Quercus pollen is noted. There are only 6 samples above this level, and the age of the top of the core is uncertain but may be about 2500 years BP. Tilia and Fagus are present in small amounts in these samples. In the top 3 pollen samples, above $3.2 \mathrm{~m}$, Olea increases while Quercus continues declining. The source of the Olea pollen may be wild plants rather than cultivated, but the relatively high percentage suggests the possibility of cultivation. There is a coincident inwash of eroded topsoil from the catchment, identified by mineral magnetic analysis at 2 and $4 \mathrm{~m}$. The onset of indications of human activity are thought to begin with the decline in Quercus. 
Turner \& Greig (1975) analysed lake sediments from Gravouna [3], farther east from Tenaghi Philippon on the Plain of Drama. Two major pollen-assemblage zones were recognized, a Quercus zone at the base and a Pinus-Gramineae-herbaceous zone at the top, with a transitional zone. The lowest zone resembles the top of their Tenaghi Philippon diagram, with Quercus the major pollen type. At the top of the sequence Quercus frequency drops significantly and the pollen assemblage is dominated by grasses and other herbs indicative of a non-forested landscape, and cereal-type pollen increases. The rather long transitional zone indicates that the disappearance of the oak forest took place fairly slowly, in at least two stages, the oak at first being replaced by Corylus and later by grass. No radiocarbon dates were obtained for this core, but the major deforestation may belong to the Medieval Period. The forest did not recover.

Athanasiadis (1975) published a pollen diagram from the Pindos Mountains, Pertouli [22], and another from near the east coast, Litochoro [15]. The sediment core from Pertouli was radiocarbon-dated at three levels, to $1290 \pm 110,1899 \pm 80$, and $1699 \pm 80 \mathrm{BP}$ (the lowest date is the youngest and is probably not reliable). The pollen diagram was divided into zones that correspond to those of Bottema (1974; Figure 1.2). Part of zone Y2 is represented and zone $\mathrm{Z}$ is divided into two subzones, $\mathrm{Z} 1-3$ and $\mathrm{Z4}$. Zone $\mathrm{Y} 2$ is divided into two phases, $\mathrm{a}$ and $\mathrm{b}$, which differ in their dominant arboreal pollen: there is a shift from an Abies-Quercus dominance to Quercus-Corylus dominance in phase b. Phases a and b are similar in other arboreal types represented, which include Tilia, Ulmus, OstryaCarpinus orientalis-type, Carpinus betulus, and Corylus in relatively high frequencies. Pinus, Fraxinus, Alnus, Acer, and Salix are present in small numbers, while Castanea and Juglans are present in phase b in very small numbers. High frequencies of non-arboreal pollen are characteristic of both subzones, mainly Pteridium and cereal-type, but also including Compositae, Cruciferae, Rumex-Oxyria, Polygonum, and Leguminosae. Subzone Z1-Z3 is characterised by dominant Abies, Quercus, and Corylus and an increase in the frequency of arboreal to non-arboreal pollen. Fagus, Ulmus, Alnus, and Acer increase slightly; Carpinus betulus and Ostrya-Carpinus orientalis-type decline slightly but are still quite abundant; while other arboreal pollen types remain about the same. Pteridium remains the dominant non-arboreal type, other herbs such as Cruciferae and Compositae increase slightly, while the frequency of cereal-type pollen declines but is still significant. Subzone Z4 is divided into three phases (d-f). The base of phase $d$ is radiocarbon-dated to $1890 \pm 80$ BP. This phase is remarkable for its dramatic increases in Fagus and Pteridium and decline in Corylus. Ostrya-Carpinus orientalis-type increases, although Abies and Quercus are the dominant arboreal types. Herbaceous pollen of Cruciferae and Compositae increases. The boundary between phases $\mathrm{d}$ and e is radiocarbon dated to $1690 \pm 80 \mathrm{BP}$. 
Phase e is characterised by its richness in non-arboreal pollen, mostly Compositae and Plantago. Other herbaceous pollen types that increase include cereal-type, Liliaceae, Leguminosae, and Sanguisorba-Poterium-type. Pteridium declines dramaticaliy. Quercus is the dominant arboreal type, but there is a significant increase in Castanea and an increase in Alnus. Phase $\mathrm{f}$ is characterised by increases in Abies and Juniperus. Quercus declines along with Ostrya-Carpinus orientalis-type. Herbaceous types decline and Pteridium becomes the non-arboreal dominant again.

Three radiocarbon dates are provided by Athanasiadis (1975) for the core from Litochoro: from lowest to highest $2400 \pm 110$ (base of core), $800 \pm 100$, and $940 \pm 100 \mathrm{BP}$. This diagram has also been divided into zones Y2 and Z1 to Z4 according to the Bottema (1974) scheme (Figure 1.2). In zone Y2 arboreal pollen is much more abundant than nonarboreal pollen: Pinus, Quercus, and Alnus all have high frequencies, and Abies, OstryaCarpinus orientalis-type, Salix, and Corylus are relatively abundant. Vitis has high frequency and Pteridium is also well represented and is the most abundant non-arboreal pollen. A variety of herbs is represented in small amounts, including Compositae and Chenopodiaceae. In subzone $\mathrm{Zl}$ arboreal pollen continues to be much more abundant than non-arboreal pollen, with Pinus, Alnus, and Quercus dominant. Pinus increases in frequency, Alnus remains about the same, while Quercus declines. The frequencies of Abies, Ostrya-Carpinus orientalis-type, and Salix remain about the same, while Corylus increases and Vitis declines somewhat; herbaceous pollen is relatively unimportant. The importance of non-arboreal pollen increases in subzone Z2, dominated by Pteridium. A variety of other non-arboreal types is also well represented. Salix dramatically increases during this subzone. Alnus also increases, but much less dramatically, as does Vitis, which increases from the middle of this subzone; Juglans forms a continuous curve, and Castanea appears in very low values. Other arboreal types either remain at the same frequency or decline slowly, e.g. Quercus. Alnus becomes the dominant arboreal type in subzone Z3, while all other arboreal types decline except Salix, which increases slightly; Juglans and Castanea disappear. Pteridium almost disappears, and only aquatic non-arboreal pollen appears in significant numbers. The base of subzone $\mathrm{Z} 4$ is radiocarbon-dated to $940 \pm 100 \mathrm{BP}$. This subzone is divided into three phases, e to g. Phase e is characterised by the increased importance of non-arboreal pollen, represented mostly by Cyperaceae and Lythrum. Pinus and Alnus are the two most important arboreal types: Pinus increases in importance towards the centre of this phase then declines, while Alnus does the reverse. Phase $\mathrm{f}$ is characterised by another major increase in Salix and a corresponding increase in Alnus. Other arboreal types decline except Juglans and Castanea, which appear in small numbers, and Vitis increases towards the end of the phase. Non-arboreal pollen is less 
important than in the phase below. In phase g non-arboreal pollen increases dramatically, particularly Chenopodiaceae and Compositae. Pinus also becomes much more abundant, while Alnus declines; other arboreal pollen continues to decline or disappears, except Salix.

A pollen diagram from former Lake Xinias [26] was first published in 1978 (Bottema). The uppermost zone, X, characterised by high arboreal values, had a basal radiocarbon date of $10680 \pm 90 \mathrm{BP}$. There are only 5 pollen samples above this date and so the diagram lacks the detail required for late-Holocene studies. A second pollen diagram was published in 1979: in this the zone above the radiocarbon date is shown in more detail and is divided into four subzones (Bottema 1979). Here subzone Z3 appears to correlate with zone Y2 of the Bottema 1974 zonation (Figure 1.2). At Xinias Z3 begins with an increase in Carpinus orientalis/Ostrya and Corylus. Arboreal pollen percentages and significant values of Poterium-type point to rather open forest. The climate responsible for this vegetation would have had dry and hot summers. Pistacia and possibly Poterium point to winters with a Mediterranean character, at least not extremely cold. Quercus cerris-type declines while Abies, Pinus, and Fraxinus excelsior increase and Fagus is present in small amounts. Plantago lanceolata appears. Subzone Z4 has high values of Quercus cerristype, whereas several other arboreal pollen types including Pistacia, Fraxinus ornus, Ulmus, Tilia, and Fraxinus excelsior quite suddenly decrease or disappear. Ericaceae and Centaurea solstitialis-type appear. Subzone Z5 is characterised by relatively high Fagus values, and Ericaceae increases correspondingly. Abies as well as Quercus coccifera-type also increase. Olea becomes quite abundant. The appearance of Juglans and Castanea in this zone is dated to $3000 \mathrm{BP}$ by comparison with their Holocene appearance in pollen diagrams from Edessa and Khimaditis.

A description of a pollen diagram from a sediment core taken from Lake Viviis [23] is given in this same publication, but unfortunately there is no pollen diagram (Bottema 1979). There are no radiocarbon dates, but the base of zone 2 is thought to date to about $6500 \mathrm{BP}$ because of an increase in Carpinus orientalis/Ostrya (by correlation with zone Y2 in Bottema 1974). Ericaceae appearing at this time is rather earlier than at Xinias. Zone 2 also has abundant Quercus (deciduous), Corylus, and Carpinus betulus, while Pinus has low values. Zone 3 is characterised by a brief increase of Pinus, which is not seen at Xinias. Zone 4 shows a constant decline of arboreal pollen values. Pinus and Quercus cerris-type decrease considerably and Ostrya-type, Ulmus, Tilia, Corylus, and Carpinus betulus disappear. Abies and Fagus are still present in low numbers. Castanea, Platanus, and Olea appear in the uppermost spectrum. Pteridium appears in this zone. Only a low percentage of cereal-type pollen occurs in one spectrum near the top of the core. It is 
thought that the uppermost part of the sequence is missing and that the top of the core may be a few thousand years old, perhaps about 4000 BP based on the absence of Juglans.

Sheehan (1979; also Whitehead \& Sheehan 1981) analysed coastal deposits from three lagoons in the Argolid Peninsula, Lake Thermisia [34], Lake Saktouri [35] and Port Kheli [33]. Chronology of the three pollen diagrams is based on a radiocarbon date of $2280 \pm 80$ BP near the base of the core from Lake Saktouri and a basal date of $4375 \pm 90$ BP from Lake Thermisia. The pollen sequence from the three sites was divided into 7 zones. In zone 1 (4500-4200 BP) Pinus, Quercus (evergreen), and Quercus (deciduous) are dominant. A variety of herbs is represented, particularly Liguliflorae, Umbelliferae, Plantago, and Cistaceae. Zone 2 (4200-4000 BP) has extremely low pollen concentrations, but Pinus is dominant. Zone 3 (4000-3900 BP) is characterised by its low ratio of arboreal pollen to non-arboreal pollen. A wide diversity of herbaceous pollen is represented, but mostly Compositae. The pollen concentrations of zone 4 (3900-2700 BP) are too low to make a reliable interpretation. In zone 5 (2700-2000 BP) Olea and Pistacia become important. Pinus, Quercus, Abies, and Poterium increase, as well as Ericaceae pollen towards the top of this zone. In zone 6 (2000-1200 BP) Olea pollen is dominant, and Pinus, Quercus, Pistacia, and Phillyrea are also very abundant, with relatively abundant Poterium and Ericaceae. Zone 7 (1200 BP-present) shows a rapid decline in Olea pollen, but this reverses at the end of this zone. Pinus, Quercus, and Pistacia remain dominant while Phillyrea declines. Herbaceous pollen increases, particularly Liguliflorae. Lake Thermisia spans all 7 of these zones while the Lake Saktouri core spans only zones 5, 6, and 7, and Port Kheli sediments probably represent only zones 6 and 7 .

The coring of Lake Trikhonis [28] was principally for palaeomagnetic investigation (Readman et al. 1976). The first published pollen diagram from this core is not very detailed, but it does show a layer of volcanic material (Bottema 1980). The pollen spectra of this volcanic ash layer are distinctive because the percentages of Quercus cerris-type (deciduous oak) increase dramatically, while at the same time Quercus coccifera-type shows much lower values. Bottema interprets this as evidence of regeneration and assumes that the volcanic ash comes from the Santorini eruption dated at $3370 \pm 100 \mathrm{BP}$ and $3527 \pm 44$ BP (Opdyke et al. 1972), based on comparison of palynological data of this core with others from Greece. However, this is not in agreement with the findings of Creer et al. (1981), who associate the ash with the Pompeii eruption of Soma-Vesuvius in 79 AD (1870 BP), based on comparison of archaeomagnetic records of radiocarbon dated sediment cores taken from the same lake. Bottema (1981) published a further note on the relative dating of this core by palynological correlation plus two other cores collected from Lakes Volvi and Vegoritis on the same expedition, also principally for the purposes of 
palaeomagnetic investigation. The palynological sequences of these lakes were correlated with radiocarbon-dated pollen sequences from nearby sites. The pollen types Ostrya/Carpinus orientalis, Fagus, Juglans, Platanus, and Zea mays were of particular importance, as well as the arboreal/non-arboreal ratio.

The basal sediments of the pollen core analysed from Lake Trikhonis are assumed to be younger than $6500 \mathrm{BP}$, since the increase in Carpinus orientalis/Ostrya characteristic of zone Y2 of Bottema's 1974 scheme has already occurred (Figure 1.2). The level of 300 $320 \mathrm{~cm}$ is dated at $3100-3300 \mathrm{BP}$ according to the curves of Juglans and Platanus. The upper part of the core is dated younger than $1700 \mathrm{AD}$ at $110 \mathrm{~cm}$ since pollen of Zea mays was identified at this level (Bottema 1981). The lowest date is in close accord with a radiocarbon date from about the same depth in another core taken from this lake, but the two upper dates are much younger than the radiocarbon dates from similar levels in another core (Creer et al. 1981).

A pollen diagram prepared from this core was published later (Bottema 1982, 1990; Bottema \& Woldring 1990). The diagram is divided into five zones. Zone I demonstrates lower arboreal pollen values than the following zones. Quercus (mostly deciduous) and Carpinus orientalis/Ostrya are co-dominant. An upper subzone is based on higher values of Artemisia, Plantago lanceolata-type, and Centaurea solstitialis-type. In zone II Juglans appears and Platanus begins a continuous curve, increasing to the top of the diagram. By comparison with other Greek diagrams the beginning of this zone is dated to about 3500 BP. Zone II includes the layer of volcanic ash discussed above. During zone II the percentages of Quercus cerris-type increase and those of Quercus coccifera-type decrease dramatically. Artemisia values decrease. In zone III Quercus cerris decreases and Quercus coccifera-type increases, a response perhaps to renewed human use of the landscape. Zone IV shows another increase in Quercus cerris-type, and in zone V there is a steady decline of Quercus cerris-type while Quercus coccifera-type increases.

At Lake Volvi [6], the oldest part of the core is argued to be younger than $4000 \mathrm{BP}$, based on correlation with Tenaghi Philippon, as Fagus is already present in the lowest spectrum (Bottema 1981). Further, presence of Juglans at that level dates the sediment as even younger than $3000 \mathrm{BP}$. High Olea values found at 480-600 cm, together with high cereal-type percentages, may point to the end of the Classical Period and the beginning of the Roman Period at about 2200 BP. The decrease in arboreal pollen and the increase of cereal-type pollen at about $350 \mathrm{~cm}$ may represent the end of the Byzantine Period and the beginning of the Turkish occupation. An increase in arboreal pollen at about $190 \mathrm{~cm}$ may correspond to the return of Christian refugees to the lowlands about $570 \mathrm{BP}$. The level at $70 \mathrm{~cm}$ must be younger than about 250 years, from the presence of pollen of Zea mays. A 
radiocarbon date of another core taken from the lake of $1743 \pm 55 \mathrm{BP}$ at $384-416 \mathrm{~cm}$ compares well with the palynological correlations, but another radiocarbon date of $844 \pm 50 \mathrm{BP}$ at $124-159 \mathrm{~cm}$ is a few hundred years older (Creer et al. 1981). The pollen diagram (Bottema 1982) is divided into five pollen zones. Zone A is defined by relatively low arboreal pollen values, but Olea is very well represented. Vitis, Juglans, and Platanus are present. Some herbaceous pollen types represented in relatively important frequencies include Artemisia, Plantago, Humulus/Cannabis, cereal-type, Sanguisorba minor/Poterium, Rumex acetosa-type, Rumex hydrolapathum-type, and spores of Pteridium. Zone B is characterised by an increase of arboreal pollen, especially Quercus (deciduous), Carpinus orientalis/Ostrya, Pinus, and Fagus (inferred to come from higher elevations), but Quercus coccifera-type decreases. Vitis and Juglans are less important. Zone $\mathrm{C}$ is characterised by an increase in herbaceous pollen, especially Gramineae. Zone D shows similar characteristics to zone $\mathrm{B}$. Zone $\mathrm{E}$ is characterised by a pronounced decrease in arboreal pollen, especially in Quercus cerris-type.

At Lake Vegoritis [11], the lowest sample was dated younger than $6500 \mathrm{BP}$, as Ostrya/Carpinus orientalis is already present. Values of Tilia and Ulmus suggest that the lowest part is not much younger than $6000 \mathrm{BP}$. From $410-440 \mathrm{~cm}$ the percentages of Fagus increase, so this level is placed at about 4000 BP. Juglans and Platanus appear between 300 and $340 \mathrm{~cm}$. This level is dated to $3100-3300$ BP. At $30 \mathrm{~cm}$ Zea mays was identified, so a date of less than $250 \mathrm{BP}$ is inferred. These dates are somewhat younger than dates obtained from radiocarbon dating of a different core from the same lake, but they agree with the magnetic age-depth curve (Bottema 1981). A pollen diagram published the following year (Bottema 1982; Bottema \& Woldring 1990) is divided into pollen zones Y and Z. Zone $\mathrm{Y}$ is subdivided into subzones $\mathrm{Y} 1$ and $\mathrm{Y} 2$. During the time of subzone $\mathrm{Y} 1$ a forest of Pinus and Abies grew on the higher ground, while a deciduous forest dominated by Quercus (deciduous) and an admixture of Carpinus orientalis and/or Ostrya carpinifolia, Ulmus, Tilia, Corylus, and Carpinus betulus grew at lower altitudes. Low herbaceous pollen percentages suggest a closed canopy. Very low values of Fagus are recorded, and there is an absence of anthropogenic indicators. In subzone Y2 pollen of cereal-type, Chenopodiaceae, Artemisia, Sanguisorba minor/Poterium, and Polygonum aviculare-type appear. Zone $\mathrm{Z}$ is characterised by a sharp decline of coniferous pollen types, the appearance or increase of Juglans, Platanus, Vitis, Plantago lanceolata-type, cereal-type, Gramineae, Rumex acetosa-type, Polygonum aviculare-type, and higher values of Pteridium spores. Zone $\mathrm{Z}$ is subdivided into subzones based on the fluctuating arboreal/non-arboreal pollen ratio: in subzone $\mathrm{Z} 1$ it is low; in $\mathrm{Z} 2$ it increases due to increases in Pinus and Abies; in Z3 it is low again. 
Kraft et al. (1980) presented a palaeogeomorphic study of Navarino Bay on the southwestern Peloponnese coast, with pollen analysis of a core, Navarino No.3 [35], by Gennett. The upper part of the core is dated by two radiocarbon samples reported as 6983 and 4280 years BP. By 6963 BP a coastal Pinus forest probably dominated the area, but between 6963 and 4280 BP sand began to be deposited at the coring site, and pollen becomes very sparse, so no pollen spectra above that dated by interpolation as $6700 \mathrm{BP}$ are shown on the pollen diagram. Pollen types identified in higher levels are reported to have included Pinus, Ostrya-Carpinus, Quercus, Juniperus, Ulmus, Alnus, Olea, Chenopodiaceae, Compositae, and Caryophyllaceae, so the coastal Pinus forest phase is thought to have continued to $4000 \mathrm{BP}$. There is no pollen evidence after this time.

Gennett later (1982) published a note without pollen diagrams on the Navarino Bay sequence and an additional site, Lake Agoulinitsa [36]. She reported that the pollen record at Lake Agoulinitsa begins shortly after 4850 BP. Initially (at $190 \mathrm{~cm}$ ) Pinus and maquis plants such as Quercus and Erica are represented equally well. Pine percentages increase until, at the $100 \mathrm{~cm}$ level, almost no maquis taxa are found. Above this level, Quercus and Erica are again important, but there are substantial amounts of herbaceous pollen, especially that of Gramineae and Compositae.

Yasuda (1985) published a pollen diagram from the analysis of sediments from Korone moor in coastal Epirus [25]. A basal radiocarbon date of $6360 \pm 30$ BP suggests that the moor was formed about 6500 years BP; another date from about $4 \mathrm{~m}$ is $4500 \pm 35 \mathrm{BP}$. Charcoal abundance was calculated as well as pollen percentages. Unfortunately only Quercus pollen percentages are shown against the charcoal curve, but there is a clear correspondence between peaks of charcoal abundance and low frequency of Quercus pollen. Between 6000 and 4500 years BP charcoal is very abundant but fluctuating. Quercus abundance fluctuates in correspondence to the charcoal, and the sedimentation rate is rapid during this period. Evidence for cereal cultivation begins at the beginning of this period also. Pollen of Abelia[?], Pinus, Rhododendron[?], and Ericaceae is reported as having high values during this time. (The pollen types reported as Abelia (Caprifoliaceae) and Rhododendron (Ericaceae) are thought to be spurious. Abelia may be a misspelling of Abies, and Rhododendron might be another member of the Ericaceae family misnamenamed.) After 4500 years BP charcoal abundance is very low, and there is a corresponding increase in Quercus. Olea and Pistacia also increase, but a decrease is reported in cerealtype, Ericaceae, Rhododendron[?], and Abelia[?]. After about 1500 years BP charcoal increases again and Quercus type pollen decreases, while cereal-type pollen and Olea increase. 
An alluvial sediment core from near the archaeological site of Halos [24] has been pollen-analysed (Bottema 1988, 1990). The pollen diagram has been divided into three pollen zones, I-III, and radiocarbon dated at 4 levels. The radiocarbon date of $6520 \pm 100 \mathrm{BP}$ dates the base of zone II, which corresponds to zone Y2 of Bottema's 1974 zonation scheme (Figure 1.2). It is characterised by high arboreal pollen percentages, including Quercus cerris-type and Pinus, with a conspicuous increase in Carpinus orientalis and /or Ostrya carpinifolia. The boundary with the next zone, III, is drawn where arboreal pollen percentages decrease, about 2900 BP. Zone III shows low arboreal pollen values and high herbaceous frequencies, dominantly Chenopodiaceae, Plantago-types, Liguliflorae, Polygonum aviculare-type, and Sparganium-type.

Two sediment cores from the Strimon delta [5] (Strimon 1, from Tuzla Marsh at the eastern end of the delta; and Strimon 3, from the north side of a lagoon in the central part of the delta) have been pollen-analysed by Atherden (1989), but only preliminary results are available. Neither core has been radiocarbon-dated. The presence of Platanus, Castanea, and Juglans at the base of the Strimon 1 core suggests that this level post-dates 3200 BP. In the Strimon 1 pollen diagram there are two main sections of good pollen preservation, $90-210 \mathrm{~cm}$ and $260-310 \mathrm{~cm}$. In the lower zone Pinus and Quercus have relatively low abundance along with other trees. High values for Olea and the presence of cereal-type suggest agricultural land nearby. The upper zone has higher Pinus and Quercus (especially deciduous oak), lower Olea, and less cereal-type pollen, suggesting a decrease in agricultural activity. Higher values for Chenopodiaceae are probably from saltmarsh plants becoming established in the local area as the water became shallower. Ericaceae is represented throughout the diagram. Again in the Strimon 3 core there are two sections where pollen preservation is relatively good: from $2.7-2.0 \mathrm{~m}$ and $1.4-0.7 \mathrm{~m}$. The first of these zones is dominated by pollen of grasses and sedges, probably derived mainly from local marsh plants, but agricultural activity in the vicinity of the site is suggested by the records for Olea and Vitis pollen and also for many weed species. Cereal pollen is represented by only one record. A wide range of tree species are represented, but all are at low frequencies: Pinus has the highest value, while Quercus (both deciduous and evergreen) is present in small numbers; other trees include Alnus, Carpinus, Corylus, Abies, Salix and Platanus. Pollen of maquis species is also present. Towards the top of this zone Chenopodiaceae increases until in the upper zone it is dominant. Olea is still present, although at slightly lower values, but Vitis and cereals have disappeared. Pollen frequencies of tree and shrub species are similar to that for the earlier zone and the maquis elements are also fairly stable throughout the diagram. This may be interpreted as woodland and maquis vegetation at some distance from the site. 
Willis $(1989,1992 \mathrm{a}, \mathrm{b} \& \mathrm{c}, 1994)$ published data from two pollen studies close to Grevena, but on the western side of the Pindos Mountains in Epirus. These sites, Gramousti Lake [20] and Rezina Marsh [19], are only $20 \mathrm{~km}$ apart but differ greatly in elevation, being at 285 and $1800 \mathrm{~m}$ respectively. Sediment chemistry and mineral magnetic analyses were undertaken on the cores, which were dated by AMS radiocarbon dating of individual macrofossils. Within the section of interest of the core from Gramousti there is one accepted radiocarbon date of $1930 \pm 180 \mathrm{BP}$. This section has been divided into two pollen-assemblage zones, GL5 and GL6. The rate of sedimentation is constant throughout these two zones. The date of the base of zone GL5, $5200 \mathrm{BP}$, is extrapolated from a lower radiocarbon date of $6330 \pm 180 \mathrm{BP}$. The base of this zone is delimited by a sharp increase in the percentage of Quercus pollen (exceptionally high influx levels of Quercus are recorded) and a large decrease in the frequency of Gramineae and all other non-arboreal taxa. Pinus is present as are Carpinus orientalis/Ostrya carpinifolia, Corylus, Abies, and Phillyrea but at lower values, and Alnus is present at an even lesser extent. Organic content of the sediment increases along with a decrease in sediment influx, indicating a stable phase in the sedimentary history of the basin. Zone GL6 (4320-1200 BP) extends to the top of the core. It is characterised by a reduction in Quercus and an increase in Gramineae. Pinus remains at about the same frequency until it declines near the top of the sequence. Carpinus orientalis/Ostrya carpinifolia, Corylus, Abies, and Phillyrea are present throughout the zone at relatively high frequencies, but Olea has lower frequency and is confined to the top of the zone. Non-arboreal taxa such as Ranunculaceae, Crucifereae, Plantago type, and Centaurea occur in low frequencies. Sediment chemistry and mineral magnetic analysis suggest that erosion occurred between about 4300 and $3500 \mathrm{BP}$ and suddenly decreased about 3500-3000 BP before resuming between about 2500 and $2000 \mathrm{BP}$.

The upper part of the Rezina Marsh sequence has been divided into four pollen assemblage zones, RM5-8, and dated by interpolation between four radiocarbon dates, $6320 \pm 130,4440 \pm 150,3830 \pm 60$ and $2990 \pm 80 \mathrm{BP}$. The base of zone RM5 about $6160 \mathrm{BP}$, is characterised by a large increase in non-arboreal pollen and a reduction in the pollen frequency of most arboreal taxa, although Pinus, Quercus, Abies, and Carpinus orientalis/Ostrya are all well represented. Corylus, Ulmus, and Acer occur in smaller numbers. Carpinus betulus increases towards the top of the zone. Gramineae is the dominant herbaceous pollen, and only small percentages of other herbaceous pollen types occur: Chenopodiaceae, Umbelliferae, Poterium sanguisorba, Rubiaceae, and Caryophyllaceae. The base of zone RM6 (4250-1900 BP) is characterised by a further increase in Gramineae, as well as in Rumex and other herbaceous taxa. A further decline 
in arboreal taxa (excluding Pinus) occurs: only Quercus, Carpinus orientalis/Ostrya carpinifolia, Abies, Corylus, and Phillyrea show a continuous presence in this zone. The frequency of Pinus, however, increases towards the middle of the zone and then declines. RM7 (1900-1170 BP) is a narrow zone characterised by a large and sudden increase in the percentage of Pinus and a rapid decline in Gramineae. In RM8 (1170-100 BP) there is a decrease in the percentage of Pinus and an increase in Gramineae. All other arboreal taxa maintain a low presence, while Abies declines further. At the very top of the zone Quercus increases along with Plantago, Umbelliferae, Caryophyllaceae, Rumex, Compositae Tubuliflorae, and Compositae Liguliflorae. Before about 4500 BP sedimentation was slow, but after this time accumulation of sediment increased dramatically with a large increase in the concentration of magnesium and calcium, suggesting that erosion of the surrounding terrain was occurring. Erosion declined after about $3500 \mathrm{BP}$, but further erosion took place about 2000 BP.

Pinus is continuously higher at Rezina than at Gramousti, peaking at about $4000 \mathrm{BP}$ and 2000 BP. Abies decreases at both sites from 4500 BP, but, except at about 2000 BP, it is almost continuously higher at Rezina than at Gramousti. Quercus values are continuously higher at Gramousti than at Rezina. Carpinus orientalis/Ostrya is higher at Rezina except between about 3500 and 1500 BP. Corylus, Ulmus, Tilia, Phillyrea, and Salix show about the same abundance at each site throughout the sequence. Gramineae is more abundant at Rezina until about $2500 \mathrm{BP}$, when after a slight fluctuation it becomes more abundant at Gramousti at about $2000 \mathrm{BP}$.

A pollen diagram of sediment core OK $85 / 11$ collected from Kiladha Bay [32] was published by Bottema in 1990. The base of the sediments is dated to about $6700 \mathrm{BP}$, based on sea-level-change data and radiocarbon dates of nearby cores. The pollen diagram is divided into two zones, I (thought to correspond to the later Neolithic and Bronze Age) and II. Zone I is characterised by low arboreal pollen frequencies (although Quercus cerristype, including deciduous and semi-evergreen oaks, is quite abundant), low conifer and mediterranean xerophytes; and a large variety of herbaceous pollen types with relatively high values. There is a wide range of anthropogenic indicators, including Plantago lanceolata, Vitis, and very high values for Cerealia (higher than Gramineae). High concentrations of cereal-type pollen may be due to summer-deposited dust containing cereal chaff or by in-wash from streams. These two factors may also be responsible for the very high percentages of Chenopodiaceae and Liguliflorae pollen types. Zone II is characterised by high arboreal pollen percentages and is dominated by Pinus, while Mediterranean xerophytic species also show high values. This zone is divided into two subzones. Zone IIa begins perhaps about $2700 \mathrm{BP}$ and may correspond to the Iron Age. It is characterised 
by increasing arboreal pollen values. Cereal-type pollen is still present with high values. Subzone Ilb has high arboreal pollen values and is dominated by Pinus, Olea, and Quercus coccifera-type, with deciduous oak also well represented, and pollen from other deciduous trees such as Corylus, Tilia, and Ulmus is present. Platanus becomes more common, and Ericaceae becomes important. An abundance of small charcoal particles was observed in the sediment of zone I, but these decrease in zone II. Sedimentation rates are calculated to be higher during zone I than in subzone IIa and may be even higher in subzone IIb.

A pollen diagram has been drawn from a sediment core $5.57 \mathrm{~m}$ long from former Lake Lerna [31] on the Argive Plain (Jahns 1990, 1993). Chronology of the sequence is based on 9 radiocarbon dates (there are two inversions). The pollen-bearing sequence begins at $6800 \mathrm{BP}$ and is divided into 4 zones (I-IV). Zone I (6800-6300 BP) is characterised by large amounts of pollen of deciduous oak and other deciduous trees such as Corylus, Fraxinus ornus, and Carpinus orientalis/Ostrya. Some evergreen taxa such as Phillyrea, Pistacia, Quercus coccifera/ilex, and Juniperus as well as non-arboreal pollen types display relatively high values. This zone may have been influenced by human impact. Zone II (6300-4800 BP) is characterised by a decrease of Pistacia and Phillyrea values and an increase in Quercus pubescens type. This dense deciduous oak woods lasted until the beginning of the Bronze Age. During this zone Erica shows a continuous curve, and single grains of Myrtus, Castanea, and Platanus occur for the first time. Zone III (4800-1100 BP) is characterised by a decrease in Quercus pubescens values and an increase of Pinus and Carpinus orientalis/Ostrya. Zone III is divided into two subzones, a and b. Subzone b differs from a by showing a decrease in Carpinus orientalis/Ostrya and an increase of Olea. From zone IIlb Juglans shows an almost continuous curve. The zone border between zones III and IV is marked by a decrease in Olea. During zone IV Pinus is dominant and Quercus pubescens declines continuously. Juniperus and Quercus coccifera/ilex show relatively high values. During zones III and IV strong human influence is indicated by the spread of maquis, phrygana, and pine. After about $2700 \mathrm{BP}$ there is evidence of extensive olive farming.

Atherden et al. (1993) published a pollen diagram from Kleonai [30] in the Nemea valley of the northern Peloponnese, in which pollen preservation is patchy and only two zones, $4(57-87 \mathrm{~cm})$ and $5(0-10 \mathrm{~cm})$, corresponding to the Bronze and Roman Periods, contained countable quantities of pollen. Radiocarbon dates at $61-64 \mathrm{~cm}, 79-83 \mathrm{~cm}$, and $95-99 \mathrm{~cm}$ giving ages of $3345 \pm 70 \mathrm{BP}, 3820 \pm 50 \mathrm{BP}$, and $4770 \pm 70 \mathrm{BP}$ respectively, provide good age control of the pollen-bearing Bronze Age sediments. The age of the top of the core was extrapolated to $1820 \mathrm{BP}$. During zone 4 there is an expansion of maquis elements, including Quercus coccifera-type, Phillyrea, Pistacia, and Leguminosae. 
Records for Pinus and Abies increase slightly. Deciduous trees include Quercus pubescenstype, Tilia, Ostrya, Castanea, Juglans, and Platanus. There are significant records for Olea. Gramineae is very abundant, and weed species are well represented. In zone 5 there is a decrease of deciduous trees and an increase of Pinus and maquis shrubs, including Quercus coccifera-type and Juniperus. Olea and Cereales are recorded. Weed species and Gramineae are present. The authors note that similarities exist between zone 4 in this diagram and zone III from the former Lake Lerna: the higher ratio of pine to deciduous oaks, the records for Juglans and Olea, and the importance of maquis elements. The apparent increased human impact on the landscape during the Bronze Age fits well with other pollen records from the Peloponnese (Wright 1972; Sheehan 1979; Whitehead \& Sheehan 1981; Bottema 1990; Jahns 1990, 1993).

Five pollen-analytical investigations by Athanasiadis and Gerasimidis have been undertaken in mountainous areas in northern Greece; Lailias [2] (Gerasimidis 1985); Flampouro and Kokkini Brysi at Pieria [14] (Gerasimidis 1985); Voras [7] (Athanasiadis \& Gerasimidis 1986); Paikon [10] (Athanasiadis \& Gerasimidis 1987); and Rhodopi [1] (Athanasiadis et al. 1993). The results of these studies have been published in either Greek or German, but Gerasimidis \& Athanasiadis (1995) have published a review in English with pollen diagrams. They found that overall trends in the woodland history at these sites were comparable, but there were also substantial differences that they attributed to the effects of human influence. An early phase (A), corresponding to the Atlantic period, is characterised by thermophilous mixed deciduous woodlands with Quercus or Tilia as the main components (Figure 1.2). Phase B represents the Subboreal period (Firbas zone VIII) and is characterised by coniferous woodlands dominated by Abies and Pinus (Figure 1.2). At this time Fagus gradually expanded except at Paikon where, at the end of the phase, Fagus was reduced and Quercus expanded. At Rhodopi, severe destruction of the upland woodlands, evidently by people, affected the pattern of vegetation development and favoured Betula which, as a pioneer species, spreads in areas cleared of woodland. The relatively strong representation of Quercus probably reflects oak growing in low-lying areas, which appear to have been unaffected by human activity.

Phase C relates to the earlier Subatlantic (Firbas zone IX) and is characterised by conifer and beech woodlands. Fagus competed with Pinus and Abies, as well as with Picea in Rhodopi. Beech woodlands dominated especially at Voras. Quercus representation remained steady while the other deciduous trees continued to be reduced and became extinct in most areas by the end of this phase. A feature of the phase is reduction in the ratio of arboreal pollen to non-arboreal pollen, especially at the top. In the Lailias profile arboreal pollen values fall at the beginning of this phase, while at Paikon arboreal pollen 
representation is low throughout. This suggests that woodland clearance, especially in the uplands, was quite severe. At Voras, Paikon, and Lailias Juglans pollen is recorded at the beginning of the phase and, at the first two sites, Castanea is also recorded. These two trees are normally regarded as associated with human manipulation of vegetation (Athanasiadis 1975; Bottema 1974, 1980), so the presence of their pollen is a further indication of intensive and systematic human disturbance. The record of Juglans at the base of the Rhodopi profile is probably due to the existence of a natural population in this area. It is assumed that people were largely responsible for its spread from centres of natural populations such as that at Rhodopi (Athanasiadis et al. 1993; Turrill 1929).

Phase D relates to the later Subatlantic (Firbas zone X). The woodland vegetation of the mountains in northern Greece looked more or less similar to that of the present day. Fagus is of greater importance than Quercus, and Pinus is the main conifer except at Rhodopi, where Picea is also important. Abies has very low representation at the base of this phase and subsequently almost disappears after a sharp decline in arboreal pollen which is recorded in all profiles. The division into subphases D1 and D2 marks the latter development. Clearance of woodland has not necessarily taken place at the same time in the various mountain ranges, but that recorded at the D1/D2 boundary probably coincides with the Turkish occupation of Greece in the 14-15th century.

More recently a review of the prehistoric environment of Greece has been published by Bottema (1994), in which he also includes new palynological data from Zileria [39]. The sediments of the small lake of Mikro Zileria were deposited very rapidly. A pollen diagram is shown of selected characteristic pollen types identified in a sediment core. The base of the core has been radiocarbon dated to $1440 \pm 90$ BP. From 500 to 1500 AD there are four periods dominated by deciduous oak pollen, separated by periods of grass pollen dominance. A large variety of tree pollen (other than oak) maintains constant low percentages and represents trees growing at a distance. The periods (200 years on average) alternating between oak and grass dominance are thought to represent cycles of oak clearance and regeneration. Bottema suggests that the presence of high values for Sanguisorba minor-type during the clearance phases indicates that no intensive cultivation of crops took place, and sheep grazing prevented regeneration of oaks. Above these clearance cycles the environment of Zileria appears to have changed; the sediment changes from gyttja to clay and oak pollen almost vanishes. The plain was changed into a steppe that lacked protective cover. There is some regeneration of trees, including oaks, in the uppermost $20 \mathrm{~cm}$.

In his review Bottema (1994) points out that many of the palynological studies undertaken in Greece, all during the last 30 years, have been undertaken in conjunction with 
archaeological projects and were focused towards reconstructing the botanical environment of prehistoric people and to explain past climate. He suggests that the field of research should be oriented towards the relation between the natural evolution of vegetation and human impact on plant regimes. A summary of Bottema's review is given below.

Around $6500 \mathrm{BP}$, the Greek landscape changed. The cause of this change is not known, but it may have been human disturbance. Universally on the Greek mainland Ostrya carpinifolia (hop hornbeam) and Carpinus orientalis (eastern hornbeam) expanded in the deciduous tree belt (zone Y2, in Figure 1.2). Other changes in the pollen record and related vegetation had a regional character (due mostly to variations in elevation). In northern Greece in both the mountains and lowlands we have evidence of a further expansion of the coniferous forest, and Fagus (beech) appears for the first time, probably in the coniferous belt. On the Epirus (west) side of the Pindos Mountains and in Thessaly, the quantities of beech and needle-leaved tree pollen remained unchanged. Increased forest canopy cover is inferred from the disappearance of light-demanding types such as Sanguisorba minor (burnet). At Halos, on the coast, there is no change. Kopais, in Boetia, which has a much more pronounced Mediterranean climate today, displayed comparable differences during this period.

There is clear proof of human impact upon vegetation from about $4000 \mathrm{BP}$ (zone Z1, in Figure 1.2). At this time a series of herb-pollen types appears; these include primary human disturbance indicators such as cereal-type pollen and secondary indicator types such as the weeds Plantago and Polygonum. Besides pollen of plants that are the direct result of farming, secondary changes in the vegetation triggered by human activities can be seen. A sudden increase in Fagus in the northern mountain belt and the Epirotic part of the Pindos Mountains may be the result of vegetation disturbance by humans. At higher elevations in Thessaly Pinus increased and replaced Juniperus. At this time some leaching of the soil may have taken place through increased precipitation, as concluded from the appearance of Ericaceae.

At about 3200 BP (zone Z1/Z2 boundary, in Figure 1.2) a suite of trees exploited by humans appears - Juglans regia (walnut), Castanea sativa (sweet chestnut), Fraxinus ornus (manna ash), and Platanus orientalis (plane tree). Coincident with this is an increase or appearance of primary and secondary types indicative of farming. In the stratigraphic record this palynological event directly follows a Santorini volcanic event that can be identified by tephra. Furthermore, there are indications of a change in sedimentation. Climatic conditions were cooler, moister, or even cloudier between about 3200 and $1500 \mathrm{BP}$. 


\subsection{Research strategy}

Previous pollen-analytical studies in Greece are summarised in the preceding section. Some generalities that can be extracted include the following:

- Many studies were directed towards a vegetation history of the area in terms of glacial/interglacial climatic cycles and not primarily directed towards a study of the impact of humans upon natural vegetation. In general, zones were identified based on characteristic tree-pollen spectra, reflecting the sequence of climatic changes through the Late Glacial and Holocene. Each of these zones may cover two or three millennia.

- Many sequences are not continuous to the present day.

- The abundance of microscopic charcoal, giving a fire history, was calculated for only one site.

- Some of the cores have no absolute dating, and none has sufficient absolute dating to be sure of chronology.

- Most are too coarse-grained to show changes in the vegetation that may have been caused by humans, and sample intervals are too large for a detailed study of human impact on the vegetation.

It appears from the pollen studies undertaken so far in Greece that very local variation occurs. This is not surprising, since there is such a great variation in altitude and topography over short distances. Turner \& Greig (1975:203) found a difference of over three thousand years in the time at which the major part of the oak forest was destroyed in the Kopais and Tenaghi Philippon regions of Greece. In general, however, although there is evidence of domesticated plants at Franchthi in northeastern Peloponnese as early as c. 8000 years BP (Hansen 1985:177), extensive forest clearance and plant cultivation is not recorded in pollen diagrams until about 3500 years ago, when major reduction in tree pollen occurred, along with expansion of agricultural weeds and increase or appearance of such cultivated types as Juglans (walnut), Castanea (chestnut), Olea (olive), and Vitis (grape).

These observations encourage one to expect that a local vegetation-history investigation, undertaken in conjunction with archaeological, geomorphic, and humanecological studies, may make a contribution to understanding the wider picture, particularly if close attention is paid to obtaining a record of the past few thousand years, to radiocarbon dating, and to identification of pollen-analytical indicators of human activity.

The primary objective of this thesis, then, is to reconstruct the vegetation history of Grevena since the Late Bronze Age and to seek possible causes and mechanisms of any vegetation changes found. Subsurface samples of unconsolidated sediments from soils and wetland sequences were collected for analysis. Fossil pollen, spores, and microscopic 
charcoal were the principal items identified, but the composition of the sediments was also analysed. Identification of pollen and spores was aided by an extensive reference collection of local species and construction of a pollen key modelled on that of Faegri \& Iversen (1989). An absolute chronology was provided by radiocarbon dating of organic detritus within the sediments.

An attempt is made to shed light on the history of agriculture and pastoralism, and the intensity of land use through time. The recognition of anthropogenic activities and their palynological signal is based on knowledge of present-day and historically documented conditions, especially old cultivation techniques. By backwards extrapolation the recent pollen deposition of such landscapes can serve as a guide to the vegetational interpretation of pollen diagrams.

Wetland sites are the usual focus of pollen-analytical studies because pollen is usually preserved best in water-logged situations. Preservation of pollen in dryland sites is less common than in wetland sites, but pollen is preserved at dryland sites if some factor, such as aridity or high acidity, exists in the microenvironment that inhibits microbiological decay (Dimbleby 1985:1; Andersen 1986; Chester 1991; Matthews 1994). Some soils in Grevena are both arid and high in acidity (Oikonomou et al. 1988). To obtain direct evidence of the local vegetation contemporary with particular archaeological cultures, ten samples were collected from horizons in soil sequences where evidence of human occupation occurred (Andersen 1986). These were dry localities, and it was hoped that information could be gained (that would be masked in a regional pollen study) on the environmental background of the particular archaeological cultures and their land-use practices, such as cultivation of food plants for humans and draught animals, and animal husbandry (Dimbleby 1985). Unfortunately, no palynomorphs were found in these samples.

Four freshwater wetlands were selected from the three major topographic regions of Grevena; the high Pindos Mountains, low Pindos Mountains, and the central plains or lowlands, for the collection of palynological samples for the study of regional vegetation history. The distribution of these sampling sites, although to a large degree limited by the distribution of suitable wetlands, was selected to assist with distinguishing between natural and human-induced changes in the vegetation so that, in particular, the history of settlement patterns and the development of agriculture and pastoralism in Grevena could be deduced The four sites differ in elevation, physiography, and vegetation. Land-use practices at each site are quite different today and probably they were also in the past. The highest wetland site, Gomara, is in an area that is sparsely populated today and may have been so in the past, and it would thus be expected to reveal a history of vegetation little disturbed by human activity. It is also at an elevation where the vegetation would be sensitive to 
relatively small climate changes. The second highest site, Anelia, although also sparsely populated, showed much evidence of woodland management over the past few hundred years. Only one of the two lowland sites was pollen-analysed - Kellia. This site is on the densely populated plains of Grevena and is surrounded by cultivated fields, which would contribute eroded sediment to this fen. The fen is within the boundaries of a designated archaeological site. A core collected from the other lowland site, Mirsina, was not pollenanalysed, as a radiocarbon date from the base of this sequence gave a radiocarbon age of less than 100 years. The diversity of the remaining sites, however, made it possible for the relationship between vegetation history, land use, and climate change to be investigated in different ecological zones.

Close sampling of the core sequences was undertaken so that the time period between each sample was reduced. Both concentration and influx data were obtained to assist with interpretation of the significance of fluctuations in relative pollen abundance. Concentration data give the absolute number of pollen grains per unit of sediment, rather than relative percentages, dependent on the frequency of other pollen taxa. Pollen influx (grains per unit area per year) was calculated for the highland site where sedimentation accumulation was relatively steady and where slow successional changes may have occurred.

Surface pollen samples were collected from representative plant communities (including both little disturbed and much disturbed "anthropogenic" communities) in conjunction with local vegetation surveys, to study the relationship of the modern vegetation to the contemporary pollen deposition, and as modern analogues for comparison with fossil spectra. The ecological tolerance of individual extant plant species and communities (particularly of anemophilous taxa) determined from surveys of the current vegetation, was used to interpret climatic change. In addition, the flora on each wetland was recorded so that a correlation could be made between the deposit-forming plant communities and sediment type and the wetland trophic state.

To obtain a history of the environment of the wetlands, sediments were characterised by the Troels-Smith classification system, estimating moisture content, bulk density, organic content, carbonate content, and non-carbonate inorganic content. Plant macrofossils and gastropods encountered during sediment description were noted and also give evidence of local conditions. Diatoms identified in sediments from the highland site assisted in palaeoecological interpretation of that wetland. Ostracods were sought in the lowland sediments but none was found.

Close determination of palynomorphs was undertaken, as many anthropogenic indicator species are morphologically similar to natural species, e.g. wild species of 
Leguminosae and those grown for fodder, cereal-pollen types and wild Gramineae species. Pollen counts, where possible, were made large enough so that weeds associated with human disturbance were represented despite low relative abundance, e.g. especially at the highland site where anemophilous Pinus is over-represented. Even early cereals were self-pollinated and the legumes are insect-pollinated, so the pollen of neither of these groups of domesticated plants is well-represented in the pollen rain (Wright 1985:189). However, plant communities associated with agriculture can often be identified by indicator species such as the weeds of cultivation or ruderals, for example Agrostemma githago, Centaurea cyanus, Plantago lanceolata, or Rumex acetosa, although many of these also have low representation.

A reduction in canopy cover, interpreted from a reduction in the arboreal to nonarboreal pollen ratio, may result from either climatic factors or land-use practices such as wood cutting, pastoralism, and fodder production. An often used method of effecting deforestation is fire. To assist with establishing the causes of deforestation, a fire history of Grevena was established by obtaining the fire history of each pollen core site from counts of microscopic charcoal particles. Also pyrophytic plants may have a palynological record, e.g. those that sprout readily after a fire (Pteridium) and those that are fire sensitive (Juniperus).

The chronology of slope erosion and valley alluviation in response to natural (e.g. climatic, tectonic) and human factors (primarily agriculture and pastoralism) was also investigated through sediment analyses. The organic content of the wetland sediments was determined so that changes in the ratio of inorganic content to organic content could be seen. Special attention was paid to the timing of increases in sedimentation and possible correlation with changes in vegetation seen through pollen analysis, e.g. reduction of forest or changes in grazing and agriculture. Rates of sediment accumulation were also calculated, as increased accumulation may indicate soil erosion.

Finally, results of the present study were compared with other results of the Grevena Project, such as changing population density estimated from the number of archaeological sites found by surface survey, investigations of lowland soil stratigraphy and landscape stability, and vegetation history and archaeological results farther afield. 


\section{Chapter 2. Field and laboratory techniques}

\subsection{Introduction}

In general, the techniques used in this study follow usual Quaternary palynological and palaeoecological techniques as outlined in the standard palynology and palaeoecological textbooks (Wodehouse 1935; Brown 1960; Kummel \& Raup 1965; Erdtman 1969; Faegri \& Iversen 1975,1989; Moore \& Webb 1978; Birks \& Birks 1980; Birks \& Gordon 1985; Dimbleby 1985; Berglund 1986a; Moore et al. 1991).

\subsection{Modern pollen study}

Interpretation of fossil pollen sequences is generally made via reconstruction of the original plant communities. Unfortunately, the ratio of the abundance of pollen of plants represented in the pollen diagram to abundance in the original vegetation is never unity, due to differences in relative pollen production, transport, and preservation (Prentice 1986a). In addition, a landscape such as that of Grevena with a long cultural history has been subject to many human activities, such as arable and pastoral farming, timber cutting, building settlements, and communication systems, which have altered the natural plant communities and created new complex communities.

\subsubsection{Field techniques}

To assist with the reconstruction of past plant communities from fossil pollen assemblages, the modern vegetation of Grevena was studied by compiling detailed modern vegetation maps and species lists, and by undertaking vegetation surveys in conjunction with surface pollen sampling. These studies were undertaken to formulate modern analogues of plant communities that might be preserved in the wetland sequences, and to gain an understanding of the ecological demands of species extant in Grevena, for example, species with well-defined narrow ecological tolerance that can be used as indicator species. Surface pollen samples were collected so that the pollen spectra representative of plant communities could be compared with fossil assemblages and to assist with compensating for the variability in pollen representation in fossil pollen spectra. The contemporary pollen rain was studied by comparing vegetation surveys with pollen representation in surface pollen samples. The major extant vegetation zones are classified according to land-use, physiognomy, and floral composition and follow Moody \& Rackham (in prep.).

It was important to obtain a modern vegetation map of Grevena so that a research strategy could be followed that covered all the major vegetation zones. Moreover, since lack of time in the field precluded extensive vegetation surveys and numerous surface pollen 
sample collections, obtaining a modern vegetation map of Grevena was necessary to assist with interpretation of the modern pollen rain in the study area. An unpublished 1:50 000 scale vegetation map of Grevena, hand-coloured by the Greek Forest Service and held in their Grevena office, was copied (Karasavvidis, unpub.). In addition, a detailed vegetation map (at 1:20000 scale) of the wetland fossil pollen sampling site of Anelia was also hand-copied from hand-coloured maps. Unfortunately no 1:20 000-scale vegetation maps were available for the other wetland sites.

I also obtained of an unpublished vegetation survey from the NE Pindos Mountains (Adamakopoulos 1989). The species list from that survey and the species list compiled by a member of the Grevena Project have been of great assistance in gaining an overall understanding of the vegetation in Grevena (Rackham 1991).

The modern vegetation of Grevena was studied by surveying a variety of sites by both qualitative and quantitative methods. In the site surveys, herbarium specimens were prepared from plants that could not be identified in the field, a total of 363 being prepared (Appendix I). Identifications were made by Dr O. Rackham, Cambridge University.

Reconnaissance surveys (qualitative surveys where a species list only was made) were undertaken at six sites. These surveys were focused mostly towards plant communities of disturbed areas; rather than plant communities of the main vegetation zones of the province. Pollen of species of these disturbed areas was expected to be mostly zoophilous and thus grossly under-represented on a regional scale, but grossly over-represented on a very local scale. Therefore no surface pollen sample was collected. However, the presence of species of disturbed areas taken collectively in the pollen diagram may be important in the interpretation of anthropogenic disturbance of the vegetation, so it was important to know something of their abundance and habitats.

Qualitative surveys were also undertaken at three sites of special interest where surface pollen samples were collected. A composite moss polster was collected from around a mountain lake. This lake has the potential of providing a long pollen sequence dating to before human disturbance. Unfortunately, this site is located in the Pindos National Park and the permit required by Greek authorities for coring in the lake was not granted until the field season had ended. In any case this site provided a useful surface sample from the Pindos Mountains. Several large pine trees had been cut down, leaving annual rings exposed in the remaining stumps. The number of annual rings of three trees were counted, providing a standard from which the age of other pine trees could be estimated and additional ecological data of this area could be interpreted. In the Pindos foothills, a composite moss polster was collected from the edge of an mixed wood in a limestone gorge. The type of mixed wood (described in Chapter 3) at this site is rare in Grevena and is thought to be a relic of preglacial 
forests. On the plains, surface soil was collected randomly over an entire $100 \times 100 \mathrm{~m}$ barley field which had been harvested of its crop a few weeks prior, and weeds were beginning to grow (Lewis \& Ogden 1965:615; Dimbleby 1985; Wright 1985; Hall 1989). A vegetation survey was made of the field to gain some information about weeds of cultivation.

Quantitative vegetation data and surface pollen samples were collected at seven sites representing the major vegetation zones from a transect across Grevena (Wright et al. 1967; Moore \& Chapman 1986; Jahns 1993). These sites also represent the major physiographic regions of Grevena and span the altitudinal range of the province, from mountains to lowlands. At sites where the vegetation comprised mostly herbaceous plants, quantitative vegetation surveys were undertaken of $2 \times 2 \mathrm{~m}$ plots; where the vegetation being surveyed included many woody plants, quadrats of usually $30 \times 30 \mathrm{~m}$ (under tree canopies) or larger were surveyed (Andersen 1973; Birks 1977). Relative abundance of species was estimated by making a visual assessment of percent cover (Andersen 1970, 1973; Birks 1973, 1977; Greig-Smith 1983; Moore \& Chapman 1986). Where more than one vegetational layer was present, each stratum was considered separately for estimating the relative cover values. Taxa absent from the plot but present nearby in a stand were also recorded.

Pollen surface samples from these sites composed of the green parts of composite moss polsters (except for one litter sample (Lewis \& Ogden 1965:615)), were collected randomly at ground level near the centre of the surveyed area. Neither the period of accumulation nor the extent of preservation of accumulated pollen is known precisely in these types of sample (Faegri \& Iversen 1964:35; Bradshaw 1981; Boyd 1986) (Benninghoff (1960) suggested less than 10 years, while Bradshaw (1981:50) suggests perhaps only 5 years if only the green moss stems and leaves are sampled), but, to a greater or lesser degree, these samples simulate the collection of pollen by a bog or lake surface and can be used to study the relationship between pollen frequency and vegetation, and the relative representation of pollen taxa (Carroll 1943; Potter \& Rowley 1960; Andersen 1967; Wright et al. 1967; Markgraf 1980; Bradshaw 1981; Prentice 1986a; Fall 1992).

Surface pollen samples were also collected and both quantitative and non-quantitative vegetation surveys undertaken, in the same manner as described above, on the wetland and in the vicinity of the coring sites.

\subsubsection{Laboratory preparation of surface pollen samples}

Extraction of pollen and charcoal particles from the surface samples was carried out in the laboratory of the Research School of Earth Sciences, Geology Department, Victoria University of Wellington. Care was taken to ensure that samples were not contaminated with pollen circulating in the laboratory air. 
The surface pollen samples are composed of a variety of materials, including wet undecomposed surface peat, moist moss polsters from wetland surfaces, wet and dry mud from wetlands, dry ground-surface moss polsters, dry woodland litter, and surface soil from an agricultural field (Lewis \& Ogden 1965:615; Wright 1985:185). Laboratory preparation techniques to extract the pollen, spores, and charcoal were chosen so that unwanted minerals and organic debris were removed, and the pollen, spores, and charcoal were concentrated.

All moss polster and litter samples were prepared for analysis in the same way. Seven steps were used; hot potassium hydroxide solution $(\mathrm{KOH})$ treatment, wet sieving, cold dilute hydrochloric acid $(\mathrm{HCl})$ treatment, cold hydrofluoric acid ( $\mathrm{HF})$ treatment, acetolysis, cold $\mathrm{KOH}$ and distilled water wash, and filtration, before being dehydrated in tertiary butyl alcohol (TBA) so that evaporation would be complete when mounting in silicone oil. Appendix II details the procedure followed.

The soil surface sample was prepared in the same manner as the subsurface soil samples (those abandoned after processing since they were devoid of pollen), the main difference from the moss and litter samples being steps to disperse the soil and cause more effective removal of siliceous matter and other minerals. Extra steps undertaken were an initial step to disaggregate the sample, a stronger HF treatment (hot rather than cold), and the inclusion of a concentrated $\mathrm{HCl}$ treatment following the $\mathrm{HF}$ treatment. Appendix II describes the procedure followed.

Wetland surface samples were prepared when the subsurface wetland samples were prepared (see below).

The concentrated pollen and spores were not stained. Silicone oil was used as the mounting medium, so that the orientation of the grains could be changed during examination, especially for critical identification such as for the Gramineae. Silicone oil has many favourable features for viewing palynomorphs microscopically. These features include a low refractive index (1.40 at 2000 centistokes viscosity), availability in a wide range of viscosities, non-volatility, and chemical purity and stability. Moreover, pollen grains mounted in it do not swell as they do in glycerine and glycerine jelly (Andersen 1960, 1978; Whitehead 1961). Mounting techniques are described in Appendix II.

Identification of palynomorphs followed the procedures outlined below. Palynomorphs were counted along evenly spaced traverses of the microscope slide until at least 400 grains had been counted; in one exceptional case many slides were counted before a total of only 200 grains was reached, due to sparse pollen. Identification and calculation of abundance of charcoal particles followed the procedures outlined below for the subsurface samples. 


\subsection{Fossil pollen study}

The three pollen-analysed wetlands were found by examination of air photographs, 1:50 000 topographic maps, reconnaissance by car, and revisiting wetlands where preliminary palynological investigation had been done the previous year by members of the Grevena Project.

\subsubsection{Field techniques}

The main aim of fieldwork was to collect uncontaminated samples of the deposits for pollen analysis, to define as exactly as possible the conditions under which the samples were taken, to determine sediment types of the deposits, and to explain the origin of the stratigraphy. Additional aims, described in other sections, were to describe the present vegetation of the site, make a floral list, survey the plant communities of the surrounding area, and note the geology and geomorphology of the surrounding area.

\subsubsection{Sampling equipment}

Four different types of samplers were used to explore the stratigraphy of the wetland sites and collect cores for pollen analysis, depending upon the texture (organic and minerogenic content), water content, and consolidation of the sediment: stationary piston corer; Russian corer; Hiller corer; and soil auger (Wright et al. 1965:494-509; Aaby \& Digerfeldt 1986:182-7; Faegri \& Iversen 1989:60-5; Moore et al. 1991:31-4).

The stationary piston corer is end-filling and superior to side-filling corers, such as the Russian and Hiller corers, for the collection of uncontaminated cores for pollen analysis. With the stationary piston corer an undisturbed continuous core can be extracted. The outside surface of the core is smeared along the whole length of the jacket, but this thin smear can be easily removed in the laboratory. The continuous core allows study of the entire sediment and pollen sequence. Unfortunately, although it works well in homogeneous fine grained sediments, this type of corer is unsuitable for very fibrous, very compact or very fluid deposits.

For the collection of pollen cores in Grevena, a modified Livingstone stationary piston corer was machined in Victoria University workshop using specifications of Walker (1964). The major modification is in the mechanism that holds the piston in place while the sampler is inserted to the required depth ready for driving. Retractable pins at the bottom of the piston have been replaced by a long shaft mounted above the piston, which is locked into position with lugs until sampling begins, then released by rotating the piston rod. The principles of construction and operation are described in detail in Walker (1964), Deevey (1965:521-529), Wright et al. (1965:498-506), Aaby \& Digerfeldt (1986:184-7). This particular corer had a 
$100 \mathrm{~cm}$ long chamber, with an internal diameter of $47 \mathrm{~mm}$. The internal diameter of the removable cutting shoe at the open end of the chamber was $45 \mathrm{~mm}$.

The Russian corer had a chamber with D-shaped section $5 \mathrm{~cm}$ in diameter and $48 \mathrm{~cm}$ long. The Hiller corer had a cylindrical chamber of $3 \mathrm{~cm}$ diameter and a length of $50 \mathrm{~cm}$ with a cone shaped bottom and was used for reconnaissance sampling only. The operation of these samplers is described in detail in Aaby \& Digerfeldt (1986:182-1984), Faegri \& Iversen (1989:60-63), and Moore et al. (1991:31-3). The soil auger had a $5 \mathrm{~cm}$ diameter and $20 \mathrm{~cm}$ long chamber and was used only where sediments were too stiff to penetrate with the other samplers (Wright et al. 1965:508-9).

\subsubsection{Wetland profiles}

Before the most suitable location for extracting cores for pollen analysis can be chosen, something of the origin and history of the wetland must be known, so that the general shape can be deduced (Faegri \& Iversen 1975:98). Usually the deepest deposits of the wetland are sampled, where least erosional loss has occurred and the most complete section through the deposit occurs. At the Gomara site it was apparent that the wetland filled a small basin, and chances of erosional loss were small (Faegri \& Iversen 1989:57), so a core was taken close to the centre of the basin, after only a couple of exploratory cores had been checked for stratigraphic integrity (Faegri \& Iversen 1965:491; 1989:57).

At Anelia and Kellia the origin and history, and thus the shape, of the wetland was not immediately obvious from surface evidence. Therefore, the profiles of these two wetlands were more fully investigated, so that stratigraphic cross sections, or profiles, could be drawn. First, a transect through the longest axis of the wetland was investigated, then a transect at $90^{\circ}$ to it through the centre of the wetland. The Anelia wetland was probed at $5 \mathrm{~m}$ intervals, along the two intersecting transects, to find the base of the wetland, then exploratory cores were extracted with the Hiller corer at $10 \mathrm{~m}$ intervals, to examine the stratigraphy. At Kellia, the Hiller corer and soil auger were used to extract cores for examination at mostly 10 or $20 \mathrm{~m}$ intervals. The composition and texture of the sediments were described by observation and feel; the colour by using a standard Munsell soil colour chart (Oyama \& Takehara 1967). A Brunton pocket transit was used for levelling. From these data the shape of the wetland could be determined and the best position for the extraction of cores for pollen analysis selected.

\subsubsection{Extraction of cores for pollen analysis}

Sampling of the wetlands was done either from the sides of pits dug into the wetland, or by use of one of the hand-operated samplers described above: stationary piston and Russian corers or a soil auger. Successive retrieval of material was necessary to extract deposits to the 
base of each wetland sequence. Sampling alternated between two adjacent holes, an overlap being maintained between successive samples.

The preferred method of collecting pollen samples in peat deposits is by cutting vertical pillars, or monoliths, from exposed profiles (Faegri \& Iversen 1989:58-60). This method avoids the sediment distortion, compression, and contamination associated with use of coring devices. The upper peaty material of the sequences at Anelia and Kellia was collected in this manner. A large hole was first dug to expose a face, then an approximately $15 \mathrm{~cm}$ square monolith was cut with a sharp knife from the top of the sequence to the bottom to reduce the risk of contamination (Faegri \& Iversen 1975:87). At Anelia one continuous monolith was cut down to $110 \mathrm{~cm}$, while at Kellia four overlapping monoliths from two contiguous columns were cut to a depth of $215 \mathrm{~cm}$. The monoliths were wrapped tightly in polythene sheet and sealed with plastic tape for removal to the field laboratory.

Where possible, deposits beneath the peaty material were collected with the stationary piston corer, the preferred corer for collection of pollen samples. At the Kellia site, the piston corer was used to extract a core from 2-3 m. Below this the deposits were very stiff, so the soil auger was used to retrieve the remainder of the core. At Anelia the Russian corer was used alternately with the piston corer (in alternate holes), down to $2.40 \mathrm{~m}$. The lowest $10 \mathrm{~cm}$ were retrieved with the soil auger. Corers were cleaned between each sampling with clean water and paper towelling.

The piston corer was used to extract the wetland sequences from Gomara and Mirsina. The cores were extruded from the piston corer in the field into longitudinally split $48 \mathrm{~mm}$ internal diameter rigid plastic tubing, which was lined with a strip of polythene sheet. The polythene sheet was sealed immediately with plastic tape (to avoid moisture loss), and wooden plugs were placed between sections of uncut core and at the ends of the tubes. The plastic tubes were then sealed and labelled with the site name, indication of upper end of the core, initial depth, final depth, and length of core. The cores were then ready for packing into boxes for shipment to New Zealand.

All the deposits collected for pollen analysis (except those extracted with the piston corer) were cut and packaged for shipment in the field laboratory, to ensure stratigraphic integrity of the samples would be maintained. To reduce contamination of the pollen samples, $2 \mathrm{~cm}$ slices were cut away from the outside of monoliths, and smaller amounts were cut away from the outside of samples collected with coring devices. Profile samples (about $30 \mathrm{~cm}$ long, $10 \mathrm{~cm}$ wide, and $2 \mathrm{~cm}$ thick) were first cut from the face of monoliths, then the monoliths were cut horizontally into $1 \mathrm{~cm}$ thick slices. The remainder of the samples for shipment were also cut into $1 \mathrm{~cm}$ slices. Care was taken not to cross-contaminate samples. The knife was cleaned between each cut with paper towelling, which was discarded after each cleaning. These 
samples were all packaged in zip-lock plastic bags and placed in square plastic containers with airtight lids to avoid moisture loss. The samples were shipped to New Zealand.

\subsubsection{Sediment analysis}

The aim of the sediment analysis was to obtain information that would elucidate the origin of the sediments, the environment in which they were deposited, and events occurring in the catchment area. Information provided by the character of the deposits is integral to the study - it both provides supplementary evidence and aids in the interpretation of the past environment derived from pollen analysis. For example, plants forming a deposit will probably have produced their own pollen, whereas sediments originating from eroded material will bring pollen and spores from the parent material. Further, the physical and chemical characteristics of the deposits influence the state of preservation of palynomorphs.

Stratigraphic analysis and sediment analysis are two facets of physical description of sediments of a wetland site. Stratigraphic evidence is based on the stratigraphy of the entire swamp/basin in which the pollen core was collected. As described above, transects bored across two of the wetland sites to obtain a stratigraphic cross section (profile) assisted with interpretation of the stratigraphic history of these basins and thus the sequences of the pollenanalysis cores.

Detailed field descriptions and laboratory analyses were undertaken so that the sediments could be described following the Troels-Smith classification system for unconsolidated sediments (Troels-Smith 1955). In the field at the time of core collection details of the component elements, physical properties, humification, and colour (with a Munsell colour chart) were recorded (Oyama \& Takehara 1967). In the laboratory, field descriptions were checked and refined during subsampling; and again after laboratory analyses had been done. Laboratory analyses included testing the degree of humicity of organic matter with an alkali solubility test and measuring the proportions of the major components moisture, organic matter, carbonate, and non-carbonate inorganic matter using a Leco carbon analyser (Davis 1976:141; Nelson \& Sommers 1982).

In addition, the flora of the wetland was recorded at the time when the sediment samples were collected so that a correlation could be made among the deposit-forming plant communities, sediment type, and wetland trophic state.

\subsubsection{Sediment description}

The sediments are described in detail so that the maximum amount of environmental information can be extracted. Because of the predominantly organic character of the sediments, the Troels-Smith classification system for unconsolidated sediments is used 
(Troels-Smith 1955). This is not an exact characterization, but a semi-quantitative estimation based on observation. An advantage of this classification system is that it is purely descriptive and does not require a knowledge of the origin of the sediments prior to description, although origin of the sediments can be deduced from these descriptions. Other advantages include the possibility of describing admixtures of elements, and producing standard formulas to describe the sediments. A detailed description of the terms used in this classification system can be found in Appendix III.

Troels-Smith suggests using a standard colour chart to describe the exact colour of the sediments. The Munsell system, Standard Soil Colour Chart was used (Oyama \& Takehara 1967).

Sediments were first described in the field by the scheme outlined above. During subsampling in the pollen laboratory descriptions were checked and additions made, mostly of macrofossils, e.g. gastropods and seeds. Additional observations were made on degree of humification during chemical processing of pollen samples. Every pollen sample was treated with alkali in which humic acids (products of decomposition) are soluble: $10 \%$ potassium hydroxide $(\mathrm{KOH})$ solution was added to the samples, which were placed in a boiling waterbath for ten minutes. The colour of the extract after boiling was compared to those on the Munsell chart: highly humified samples produce a brown or blackish brown extract, while those little humified produce straw-coloured or colourless extracts. Description of the sediments is also based on other laboratory analyses described below.

For each wetland site a stratigraphic column has been drawn, with symbols indicating the major component elements and the proportions in which they occurred, the accessory elements, the degree of humicity of the organic matter (unless detritus), and the degree of definition of boundaries between strata (limes) (the columns are included in the pollen diagrams in simplified form.) To the side of each column is a formula also indicating the relative proportions of the major component elements and the degree of humicity of the organic matter (humositas). The fresh colour of the sediments, as noted in the field according to the Munsell chart, is also noted as well as structural properties of the sediments (structura). Additional columns have been drawn showing the physical properties of nigror, stratificatio, elasticitas, and siccitas.

\subsubsection{Moisture content}

Water content varies widely according to depositional environment. General decrease in water content with depth in sediment cores is due to compaction, but vertical variation in the water content also depends on other factors, such as the rate of sedimentation, the particle 
size and composition of the deposits, and the degree of bioturbation (Bengtsson \& Enell 1986:427).

Moisture loss from the time of collection to the time of analysis was avoided as much as possible by wrapping samples in plastic and placing them in airtight containers. They were stored at $5^{\circ} \mathrm{C}$ once they reached New Zealand.

The moisture content was measured by calculating weight loss between the original state of the sediment and after oven-drying. This was accomplished in the following manner. One cc of wet sample (in its natural state) was subsampled with a $1 \mathrm{cc}$ disposable syringe with the needle cut off. The sample was immediately placed in a tared $50 \mathrm{ml}$ centrifuge tube with an airtight cap and weighed on a Mettler PE 360 single pan electronic balance with precision to $0.001 \mathrm{~g}$. The sample was then dried in an oven at $50^{\circ} \mathrm{C}$ for 24 hours, placed in a desiccator to cool, then weighed again. The loss in weight represents the moisture content. The moisture content was calculated as a percentage of the wet weight ('natural state' of the sediment):

$$
\text { Moisture content }=\frac{(\text { wet weight }- \text { dry weight) } \times 100}{\text { wet weight }} \%
$$

\subsubsection{Bulk density}

The bulk density (dry density) is often measured to indicate the degree of decomposition of peat materials. As decomposition proceeds, the size of organic particles decreases, resulting in smaller pores and higher bulk density (Boelter 1969:607; Keys 1982:120). It also reflects the proportions of organic matter to inorganic matter in mixed sediments. Organic matter has densities of less than $0.4 \mathrm{~g} / \mathrm{cc}$. As organic matter decreases the value of this ratio increases. Many peats (composed almost entirely of organic matter) have bulk densities of $0.5 \mathrm{~g} / \mathrm{cc}$ or less, while the average density of mineral soils is about $1.25 \mathrm{~g} / \mathrm{cc}$.

The term bulk density (dry density) refers to the weight of oven-dry material held per unit volume of material in its natural state (Klemetti \& Keys 1982). This should be contrasted with the bulk density (wet density) based on the wet-sample weight (Bengtsson \& Enell 1986:427).

The bulk density was calculated on an oven-dry weight-wet bulk volume basis, using the measurements detailed above, as follows:

$$
\text { Bulk density }=\frac{\text { weight of oven-dried sample }}{\text { volume of sample in 'natural state' }} \mathrm{g} / \mathrm{cc}
$$




\subsubsection{Organic content}

To determine the organic content of the sediments, the elemental carbon content was determined by using a Leco RC-412 multiphase carbon analyser (Dean 1974:247; Nelson \& Sommers 1982). This was achieved by drying about $5-10 \mathrm{cc}$ of wet sample at $100^{\circ} \mathrm{C}$. The dried sample was ground with a mortar and pestle to a fine powder. A small amount (usually much less than a gram) of the powder was placed in a 'boat' and weighed immediately prior to placing it in the analyser furnace. The furnace chamber was heated to $550^{\circ} \mathrm{C}$ for 6 minutes while oxygen was added (Ball 1964; Davis 1976). On ignition of organic matter, carbon dioxide was evolved and measured by infra-red analysis. This machine expresses results in the form of elemental carbon as a proportion of the dry weight of the original sample (percent dry weight).

Inspection of the elemental carbon values obtained from the Leco carbon analyser indicated that a $47 \%$ elemental carbon value correlated to $100 \%$ organic matter in the sediment, suggesting that a conversion factor of 2.13 should be used to convert the instrument values to organic-matter content (as a proportion of dry weight). This conversion factor is the same as that found by Dean (1974:244) when he compared loss on ignition (at $550^{\circ} \mathrm{C}$ ) values with those of organic carbon determined chromatographically.

Values were corrected to a wet-weight basis using the moisture contents previously determined. As a check, duplicate samples from the Gomara core were measured. Degree of reproducibility was good, the difference between samples usually being less than 1 percent.

\subsubsection{Carbonate content}

Carbonate content was also determined by measuring evolved gas in the Leco RC-412 multiphase carbon analyser (Dean 1974:247). This was achieved by leaving the sample in the furnace after the organic carbon had been burnt, increasing the temperature of the furnace from $550^{\circ}$ to $900^{\circ} \mathrm{C}$, and holding it at that temperature for 3 minutes (Nelson \& Sommers 1982).

The analyser expresses the results in terms of elemental carbon as a percentage of dry weight of the original sample. Assuming the carbonate to be all in the form of calcium carbonate $\left(\mathrm{CaCO}_{3}\right)$, the percentage of carbonate on a dry weight basis is obtained by multiplying by the molecular weight ratio 100/12 (8.33). Values were corrected to a wetweight basis using the moisture contents previously determined.

Duplicate samples from the Gomara core were put through the analyser, and good reproducibility was again demonstrated.

Samples were also tested during pollen preparation with dilute hydrochloric acid $(\mathrm{HCl})$, and the presence/absence of resultant effervescence of carbon dioxide was recorded. 


\subsubsection{Non-carbonate inorganic content}

Inorganic matter was calculated by subtracting the sum of the three measured components above (organic matter, carbonate, moisture) from the total, and expressed as a percentage.

$$
\text { Inorganic matter }=\frac{100 \times(\text { total } w t-(\text { organic matter } w t+\text { carbonate } w t+\text { moisture } w t))}{\text { wet weight }} \%
$$

\subsubsection{Tephra analysis}

The wetland sediment sequences were examined for the presence of chronological markers such as abrupt stratigraphic changes, e.g. tephras. In the Gomara sequence a band of fine-grained sediment between 108.2 and $107.2 \mathrm{~cm}$ below ground surface was examined by Mr John Carter, Geology Department, Victoria University of Wellington, for the presence of glass shards. An attempt was then made by Mr John Paterson, Victoria University of Wellington, to identify the volcanic eruption from which the tephra had derived by determining the chemical composition of the glass shards.

\subsubsection{Plant-macrofossil identification}

Plant macrofossils are direct evidence that the particular plant was present nearby. Plant macrofossils are complementary to pollen data for reconstructing local plant communities, as they are not generally dispersed far from their source. Resources did not permit a detailed macrofossil analysis, however: only obvious, larger, fruits encounted during sediment description were identified.

\subsubsection{Miscellaneous micro- and macrofossils}

Other fossils likely to be preserved in the sediments besides pollen and spores, such as diatoms, ostracods, and gastropods were searched for where appropriate.

Diatoms, unicellular algae with silica tests, are useful as palaeoecological indicators, as they have distinct ecological tolerances determined by such conditions as water depth, $\mathrm{pH}$ of water, and nutrient level. Several samples from both upland mires were tested for the presence of diatoms. No diatoms were found in the samples from the Anelia site, but abundant diatoms were found at the Gomara site. Ten samples of $0.5 \mathrm{gm}$ were analysed from the Gomara core. The diatoms were identified and counted by Dr Margaret Harper, Geology Department, Victoria University of Wellington. The extraction technique is detailed in Appendix II.

Ostracods, minute crustaceans with calcified shells, are best preserved under calcareous or saline conditions. It has been demonstrated that they are useful as ecological indicators 
(Delorme 1969; Delorme et al. 1977). The sediment of the lower parts of the Kellia sequence appeared to be suited to the preservation of ostracods. Two samples at 3.91-3.92 $\mathrm{m}$ and 3.35$3.36 \mathrm{~m}$ were analysed by a staff member of the Geology Department, Victoria University of Wellington, Stephen Eager, but no ostracods were found.

Molluscs are reliable indicators of local habitat conditions near the site of deposition, but not of regional conditions, as they can occupy locally favourable microhabitats in generally unfavourable conditions. Freshwater molluscs thrive best in alkaline situations where there is a readily available supply of calcium carbonate for their shells. Their shells are also preserved best in such conditions (Birks \& Birks 1980:121). Molluscs are useful indicators of the destruction of woodland and the creation of grassland habitats (Birks \& Birks 1980:127). Gastropods were noted in the lower sediments of the Kellia sequence but not specifically identified.

\subsubsection{Radiocarbon dating}

The establishment of accurate age control within the wetland sediment cores is important for temporal and spatial correlation of events and for analysis of rates of change. Chronologies are based on a series of dates obtained from radiocarbon dating. A total of 13 radiocarbon dates are provided: 5 for Kellia, 3 for Anelia, and 5 for Gomara. (Two dates were also obtained from the Mirsina sequence.) All material dated was taken from within the core sediments used for pollen analysis. Initially, two samples were dated from each core, one near the bottom and one at intermediate depth, to determine the age range of the sediments. After identification and counting of palynomorphs further dates were obtained to date more precisely particular events seen in the pollen record.

The first set of 8 samples were sent to two different radiocarbon-dating laboratories. Seven samples were submitted to the University of Waikato radiocarbon-dating laboratory, Hamilton, where the liquid-scintillation technique is used (Hogg 1989), and one, which contained only a small amount of carbon, to the Rafter laboratory, Institute of Geological and Nuclear Sciences, Lower Hutt, where accelerator mass spectrometry is used (Lowe \& Judd 1987; Wallace et al. 1987). Subsequently 7 samples were submitted to the Rafter radiocarbondating laboratory, for dating using accelerator mass spectrometry. The radiocarbon ages of these samples were compatible with those from the laboratory using the liquid-scintillation technique. 


\subsubsection{Pretreatments}

All pretreatments carried out were done by the dating-laboratory technicians. Pretreatments usually carried out at the Rafter laboratory for peats, soils, charcoals, leaf litter, and degraded woods consist of leaching with dilute hot $\left(80^{\circ} \mathrm{C}\right), 2 \mathrm{~N}$ hydrochloric acid for 15 30 minutes to remove carbonates; rinsing with distilled water to a neutral $\mathrm{pH}$; treatment with dilute alkali solution ( $0.1 \mathrm{M}$ sodium hydroxide and sodium pyrophosphate) for 15-30 minutes at $80-90^{\circ} \mathrm{C}$ to extract humic acids; and subsequent washing with distilled water to a neutral $\mathrm{pH}$. Finally, the insoluble fraction from the alkali treatment, humin, is acidified to remove carbon dioxide absorbed during the alkali treatment; rinsed with distilled water to a neutral pH; and oven dried (Goh \& Molloy 1972). The acid and alkali treatments are repeated if necessary. The samples are centrifuged or filtered and oven-dried between each step.

Two samples from lake sediment and two peat samples from a bog, dated at the University of Waikato dating laboratory, received no pretreatment: Gomara $81-87 \mathrm{~cm}$; Gomara $186-190 \mathrm{~cm}$; Anelia $81-83 \mathrm{~cm}$, and Anelia $240-245 \mathrm{~cm}$. Two organic samples from the Kellia calcareous fen, $166-168 \mathrm{~cm}$ and $296-307 \mathrm{~cm}$, were pretreated with acid washes $(0.5 \mathrm{M} \mathrm{HCl}$ at $80^{\circ} \mathrm{C}$ ) for 1 hour, then washed thoroughly with distilled water. These were also dated at the University of Waikato.

Sediment samples dated in the Rafter laboratory contained less organic material than those dated in the University of Waikato dating laboratory so required careful pretreatment. Where possible plant material was removed from the sediments for dating, then treated consecutively with hot solutions of acid, alkali, and acid, as described above. The samples treated in this manner were: Anelia 124-125 cm, Gomara $156-157 \mathrm{~cm}$, and Kellia $71-72 \mathrm{~cm}$. The remaining samples had little visible plant material, so they were ground and then treated consecutively with hot solutions of acid, alkali, and acid. Thus the humin fraction (acid and alkali insoluble) of the entire sediment was extracted and dated (Goh \& Molloy 1972). Samples treated in this manner were: Kellia $315-316 \mathrm{~cm}$, Kellia 394-395 cm, Gomara 52$53 \mathrm{~cm}$, and Gomara $111-112 \mathrm{~cm}$.

It is assumed in this study that all the samples dated in this method formed in isotopic $\left({ }^{14} \mathrm{C}\right)$ equilibrium with atmospheric $\mathrm{CO}_{2}$, so no allowance has been made for reservoir effect. Although the samples from Gomara are from lake sediments, the lake was very small and probably shallow, so the reservoir effect is probably absent. Also the contribution of submerged plants, which if present would result in a radiocarbon age too old, was probably minimal.

Many samples dated could have contained organic matter washed in from the surrounding catchment, some of which could have been older than the contemporary vegetation. This kind of contamination would be most likely in the inorganic samples from 
the Kellia site, less likely in samples from Gomara, and least likely in the peat samples of Anelia.

The samples from Anelia and Kellia, which are spring-fed wetlands, may have been subject to ground water with either an elevated or depleted carbon content.

\subsubsection{Calibration}

The dating of these samples was done over the course of some years, and the results of the radiocarbon ages were reported differently. Furthermore, atmospheric radiocarbon is modulated by the geomagnetic field, causing long-term variations and cosmic ray intensity causing short-term variations (Stuiver 1971, 1980; Webb \& Webb 1988; Aitken 1990). To standardize the results, correct for secular variations, and convert the radiocarbon years to calendar years, all the conventional radiocarbon ages that had been determined and reported by the radiocarbon-dating laboratories were calibrated. The conventional radiocarbon ages based on the half-life of 5568 years were normalized to $\delta{ }^{13} \mathrm{C}=-25$ per mil in the PDB-scale and related to the international dating standard.

The computerized calibration program of Stuiver and Reimer (1993), CALIB 3.0.3c (revised version 1995), available on the Internet at the University of Washington ftp site, was used. CALIB runs on any IBM compatible computer with MS-DOS and at least $480 \mathrm{kB}$ available memory.

CALIB converts the radiocarbon age to calibrated calendar years. It yields single or multiple calibrated ("Cal") $\mathrm{AD} / \mathrm{BC}(\mathrm{BP})$ ages that are compatible with a certain ${ }^{14} \mathrm{C}$ age, the range(s) of calibrated ages that correspond(s) to the standard deviation in the ${ }^{14} \mathrm{C}$ age (and calibration curve), and the probability that a certain calibrated age is the actual sample age. An option to calibrate selected radiocarbon ages by a simple intercept with a linear interpolation of the data points is also available. That option was not chosen. The conventional radiocarbon ages and calibration results are presented in text and graphs, edited output from the program, in Appendix IV.

Calibration is based on the Seattle-Belfast bidecadal calibration curve derived from the radiocarbon age of tree rings of known age (Pearson \& Stuiver 1993; Stuiver \& Pearson 1993). The ${ }^{14} \mathrm{C}$ age errors are small because most bidecadal data points were obtained by averaging two Seattle decadal ${ }^{14} \mathrm{C}$ ages and one Belfast bidecadal ${ }^{14} \mathrm{C}$ age. It is assumed that the radiocarbon age of these trees from the northern hemisphere would be very similar to the radiocarbon ages of coeval samples from Grevena, and that there would be no systematic difference. 
The radiocarbon-age error reported by the Rafter radiocarbon laboratory comprises statistical errors in sample and standard determinations, as well as a system error component of 0.6 , so a laboratory error multiplier $(\mathrm{K})$ of 1.0 was chosen. On the other hand, the reported radiocarbon age error margin from the University of Waikato of \pm 1 standard deviation was based on counting statistics alone, so a laboratory error multiplier $(\mathrm{K})$ of 1.6 was chosen to increase the standard deviation for samples dated in that laboratory (Stuiver \& Pearson 1986:807).

To smooth the calibration curve and minimize multiple calibrated age ranges for samples spanning more than 30 years, a weighted moving average of the data set was used. Samples with an estimated calendar-year span of 30 years or less were calibrated without any smoothing.

Two radiocarbon dates, in addition to those reported above, were obtained on samples from the Mirsina wetland. Samples were sent to the two dating laboratories used for this study. The upper sample, $94.5-100 \mathrm{~cm}$, was sent to the University of Waikato radiocarbon dating laboratory and the basal sample, $328-331 \mathrm{~cm}$, to the Rafter radiocarbon dating laboratory. Pretreatment of the basal sample was carried out in the Rafter laboratory and consisted of extracting plant material by wet sieving, then washing the plant material in hot water and acid. A radiocarbon age of less than 100 years was obtained. The upper sample received no pretreatment and also gave a 'modern' radiocarbon age. No further analyses were undertaken on this sequence.

Radiocarbon ages are expressed as ${ }^{14} \mathrm{C}$ years before $1950 \mathrm{AD}$ (years BP) and calendar ages are expressed as years $\mathrm{Cal} \mathrm{BC}$ or $\mathrm{AD}$.

\subsubsection{Laboratory preparation of fossil pollen samples}

Sediments for fossil pollen analysis were processed so that the actual numbers of palynomorphs in a sample (concentration) could be calculated as well as the relative frequencies. This required precise subsampling and special procedures and care during extraction procedures and counting of grains on microscope slides. To avoid volume or biochemical changes between field collection and subsampling, wetland samples were stored in a cool store at $5^{\circ} \mathrm{C}$ in the Biological Sciences Department, Victoria University of Wellington. 


\subsubsection{Subsampling}

High-precision measurements of wetland sediment samples were taken so that calculations could be made of pollen concentrations, the number of grains per unit weight or volume of wet (fresh) sediment, could be calculated (grains/cc or grains/gm) (Birks \& Birks 1980:206). Samples were subsampled volumetrically and weighed.

Subsampling was done in the palynology laboratory of the Geology Department, Victoria University, where an air filtration system had been fitted.

Two different methods were used to determine palynomorph concentration, a direct method (aliquot method), which estimates the actual number of palynomorphs in a sample, and an indirect method, which relies on the addition of a known concentration of exotic marker grain or tracer (Benninghoff 1962; Davis 1965, 1966; Matthews 1969; Bonny 1972). Both methods require precise measurement of a volume or weight of sample.

The cores collected with the piston corer were shipped to New Zealand intact, so after removing the surface smear of disturbed sediment, transversely with a sharp knife, the length of the core, lithology, and stratigraphy were checked against field notes. These cores were then sliced into equal units in the laboratory at Victoria University just prior to subsampling. Because the cores were collected with the removable cutting shoe attached to the bottom end of the piston corer and the internal diameter of the chamber is greater than the internal diameter of the removable shoe, depths within core segments had to be adjusted to compensate for the reduction in length of the sediment. Slices of $1 \mathrm{~cm}$ 'true' length were cut.

Samples were chosen for analysis from these slices and those cut in the field laboratory. Generally samples were taken at $5 \mathrm{~cm}$ intervals. The selection of samples was based on a combination of uniform and structural subsampling principles (Bengtsson \& Enell 1986:424). Initially, a uniform $5 \mathrm{~cm}$ interval was chosen, but where changes in sediments occurred within that interval samples were taken at closer intervals, so that all sediment types are represented. This resulted in all samples from Kellia being either $5 \mathrm{~cm}$ or less apart except between 2.95 and $3.05 \mathrm{~m}$ where an interval of $10 \mathrm{~cm}$ exists because the sample at $3.00 \mathrm{~m}$ was used for radiocarbon dating. The Anelia sampling interval is $5 \mathrm{~cm}$ or less, except in the upper $1.00 \mathrm{~m}$, where radiocarbon dates showed that deposition may have been extremely rapid, so the sampling interval was increased to $10 \mathrm{~cm}$. The sampling interval for the Gomara site is $5 \mathrm{~cm}$ or less, but samples above $25 \mathrm{~cm}$, except the surface sample, were not analysed since the top $25 \mathrm{~cm}$ of the lake had been trampled and was dry and cracked. This resulted in 82 samples from Kellia, 39 from Anelia, and 36 from Gomara: a total of 157.

All wetland fossil pollen samples were volumetrically measured and weighed before laboratory processing. Two subsamples of $1 \mathrm{cc}$ each were taken from the centre of the slices chosen for analysis, one for pollen analysis, and one for sediment analysis (see above). 
Subsamples were taken from the wet slices with a disposable $1 \mathrm{cc}$ syringe with the needle cut off. The samples were immediately extruded into weighed $50 \mathrm{ml}$ polypropylene centrifuge tube with airtight screw top lids. The centrifuge tubes plus the samples were then weighed to $0.001 \mathrm{~g}$ precision using a Mettler AK160 scale. The difference between the wet weights of the duplicate pairs averaged $1 \%$ of the mean weight of each pair for the Gomara and Anelia cores and only $0.5 \%$ for the Kellia core. The samples remained in these centrifuge tubes in a cool store at $5^{\circ} \mathrm{C}$ to discourage biochemical activity, until extraction or sediment analyses were carried out.

The subsampling with the $1 \mathrm{cc}$ disposable syringe was relatively easy where the sediment was not very fibrous, but it was much more difficult and time-consuming where the sediments were fibrous, e.g. the top one metre of Anelia and almost the entire Gomara sequence.

\subsubsection{Palynomorph concentration calculation}

Pollen concentrations reflect more closely the actual plant populations in the past than proportional pollen frequencies. They give information on the absolute abundance of each pollen taxon that is not dependent upon fluctuations in other taxa.

\subsection{Aliquot method}

The direct method or aliquot method, described by Davis $(1965,1966)$ is based on methods described by Erdtman (1943). This method was used for samples from the Gomara site where palynomorphs were abundant.

The precisely measured volume of wet ('fresh') sample was processed in the laboratory to extract palynomorphs as described below, and the residue remaining after the extraction process was suspended in a known volume of tertiary butyl alcohol (TBA). Aliquots of known volume were withdrawn from this suspension and mounted on a microscope slide in silicone oil. This is described in detail in Appendix II.

The number of pollen grains in the original suspension is derived from the number of grains found on the slide. A significant error could be introduced in this method by using aliquots of small volume. This error is reduced by diluting the sample with a volatile liquid to a size where aliquots can be measured more accurately (Davis 1965:674).

All pollen grains should be counted on the microscope slide. This means that assumptions must be made initially concerning the approximate pollen density so that a reasonable dilution and aliquot volume can be estimated. Initially a few test slides were processed, then estimates were made from counted slides before processing subsequent samples. 


\subsection{Exotic marker grain (tracer) method}

The indirect method, exotic pollen or tracer method, described first by Benninghoff (1962) and subsequently tested and described more fully by Matthews (1969), Pennington \& Bonny (1970), and Bonny (1972), compares the ratio of the number of native fossil pollen grains to the known number of exotic marker or tracer pollen grains (or other appropriate particles) added to a known volume of sediment prior to preparation. During analysis the tracers are counted with the native pollen of the sample. All subsequent abundance determinations can then be carried out in relation to counts of the tracer grains.

$$
\text { Fossil pollen concentration }=\frac{\text { fossil pollen counted }}{\text { exotic pollen counted }} \times \frac{\text { exotic pollen added }}{\text { sample size }}
$$

This method was used for all three wetland sites.

The first requirement for this technique is a suspension of known concentration of an exotic marker grain (or other marker particle) of an appropriate size, which disperses easily in a suspension medium and does not agglomerate into clumps, is easily recognized to the observer, and does not occur naturally in the pollen sample. Lycopodium spores were chosen as the exotic marker because they do not occur in the flora of Grevena and are easily recognized and of a suitable size. A spike of Lycopodium spores with a concentration of 82900 Lycopodium grains $/ \mathrm{ml}$ was prepared. Briefly the preparation consisted of acetolysis to rid the spores of waxes, oils, and protoplasm; staining; suspending the spores in a known volume of suitable liquid; then calibrating the concentration of the spores in the suspension. Calibration was achieved by mounting microscope slides with a known volume of the suspension, then counting all the spores on the slide. Stockmarr (1973) found that this method of calibration gave almost identical results to calibrating with a Coulter counter (Stockmarr 1973:89). Appendix II details the preparation procedures.

Aliquots of known volume of the Lycopodium suspension were added to samples before extraction was begun, so any fossil grains lost during processing would be accompanied by an equivalent loss in the exotic grains, and the overall proportion of fossil grains to exotic grains would remain unchanged. A tally was kept of the number of Lycopodium spores encountered during counting of microscopic slides made from a prepared pollen sample. The pollen concentration of any taxon or of total pollen in a given sample could then be determined as above.

The number of marker grains to be added to a sample depends upon the expected pollen density in the fossil sample. As pollen density varies widely, often according to sediment type, several samples from different sediment types were mounted and counted, so that the approximate number of Lycopodium spores to be added could be estimated. The ideal goal, 
according to Maher (1981) is two exotic grains for each fossil native grain, although Berglund \& Ralska-Jasiewiczowa (1986:456) have found that the relationship of counted fossil pollen /counted exotic grains should be about 1:1. Lycopodium spores were added at an estimated ratio of 2 exotic grains for each fossil grain.

\subsubsection{Extraction}

Special attention was paid to prevent contamination of these samples because of the paucity of palynomorphs in some sediments and the abundance of aerial pollen in New Zealand that belongs to the same types as those occurring in Grevena.

Extraction techniques usually vary according to the characteristics (e.g. lithology, compaction, age, degree of weathering) of the material from which the palynomorphs are being extracted. Although the characteristics of the sediments from the Grevena wetlands varied from organic to mineral, only one set of extraction procedures was followed, to reduce differences in proportions of species between samples.

A known volume (and weight) of sediment, in this case $1 \mathrm{cc}$, of sample in its original state was placed in a $50 \mathrm{ml}$ centrifuge tube, then a known volume of the exotic marker (Lycopodium spores) was added to the tube. The extraction procedures that followed were standard methods outlined above (Appendix II).

\subsection{Pollen identification and counting}

In modern pollen analysis it has become clear that palaeoecological interpretations are much enhanced by a detailed determination of pollen types. Frequently it becomes impossible to key out the fossil types to the species level, but identification of the pollen grains to taxa of the lowest possible rank is highly desirable, so that as many pollen taxa as possible are ecologically meaningful.

Although there are pollen keys of western-European Quaternary pollen floras such as those of Faegri \& Iversen (1950, 1964, 1975, 1989) (NW European pollen flora), Beug (1961a, b) (pollen flora of middle Europe), Erdtman et al. (1961, 1963) (Scandinavian pollen flora), Moore \& Webb (1978) (British pollen flora), Belmonte et al. (1986) (Iberian Peninsula pollen flora), Valdes et al. 1987 (pollen flora of the Andalucía Occidental), and Moore et al. (1991) (mostly British pollen flora), none exists for the Balkan Peninsula. Keys treating special taxonomic or other groups are available, such as Cerceau (1959) - Umbelliferae, Chanda (1962) - Caryophyllaceae, Praglowski (1962) - Swedish trees, Punt (1976-91), Andersen (1979) - Gramineae, and Ubera et al. (1988) - Plantago. These are also mainly of western European species. 
Palynomorph identification was aided by use of a modern pollen and spore reference collection and both a written key and a photographic key of the pollen and spore flora of Grevena.

\subsubsection{Reference pollen}

A reference collection of pollen and spores representative of pollen and spore taxa likely to be found in late-Holocene palynological samples from Grevena was made to assist with identification of fossil grains. This permitted greater precision in the identification of fossil grains than could have been made with only keys or published photographs, for direct comparisons could be made.

A species list of the recent and extant flora in Grevena was compiled from the following publications: Pouqueville (1826), Leake (1835), Turrill (1929), Tutin et al. (1964-80), Wace \& Thompson (1972), Sfikas (1978), Polunin \& Huxley (1987), Polunin (1988), Huxley \& Taylor (1989), Strid (1989), Strid \& Tan (1991), and the following unpublished literature from Grevena Project members: Moody \& Rackham (1988), Chester (1989a), Chester (1989b), Chester (1989c), Rackham (1991), Moody \& Rackham (in prep.), and an unpublished Greek Forest Service report: Adamakopoulos (1989). The species list includes 143 families and almost 800 genera. Specimens for the reference collection were based on this species list.

Throughout this study taxonomic names of vascular European species follow Tutin et al. (1964-80). In some exceptional cases, for example where taxa have been described more recently than the publication of the above volumes, taxonomic names follow those used in Strid (1989) and Strid \& Tan (1991). Taxonomic names of non-vascular species follow Watson (1968).

The pollen and spore reference collection was prepared from 1517 plant specimens, both dried herbarium specimens and fresh living plants, and lacks few important taxa from the modern Grevena flora. Anthers and sporangia were collected from two herbaria holding world-wide collections; the Natural History Unit, Museum of New Zealand, Wellington (WELT) and the Biological Sciences Department, Victoria University of Wellington (WELTU). Anthers taken from these collections rendered well preserved pollen suitable for comparative identification purposes. Six hundred and thirty-nine herbarium sheets were sampled from WELT and 689 from WELTU.

Living plant material was collected in Grevena. Herbarium specimens were prepared from the plant material (often whole plants) according to Fosberg (1960). Although fieldwork in Grevena was undertaken from the middle to the end of summer, a large number of plants, especially herbs, were in flower. Weeds, particularly those growing in cultivated fields, disturbed areas, and woodland clearings, were sought, as these are particularly important in 
interpreting changes in pollen diagrams. Weeds of cultivation are particularly important indicator plants due to the difficulty of distinguishing cereal pollen from that of other grass genera and the low numbers of cereal pollen grains found in pollen diagrams (Faegri \& Iversen 1989:127). One hundred and thirty seven plants collected in Grevena had flowers from which pollen was extracted for the reference collection. It was subsequently fumigated and added to the Southern European herbarium collection of the Victoria University of Wellington Biological Sciences Department. A catalogue of the pollen reference slides made from these specimens was deposited with the herbarium specimens.

Living plants were also collected from various gardens in the Wellington area, mainly the Wellington City Council Botanic Garden, but also private and public gardens in Lower Hutt and my own garden in Johnsonville. These were also prepared as herbarium specimens and deposited in the Biological Sciences Department herbarium.

The collection of pollen followed the recommendations of Traverse (1965). The laboratory preparation of the reference collection was undertaken in the Palynology Section of the Institute of Geological and Nuclear Sciences, Lower Hutt. Pollen and spores were extracted from plant material, concentrated, and mounted on microscope slides. Duplicate microscope slides were made. One set of slides of the reference collection is held in the Geology Department, Victoria University, and the other is held in the Palynology Section of the Institute of Geological and Nuclear Sciences, where it is incorporated in their modern pollen reference collection. A detailed description of the laboratory procedures appears in Appendix II.

In addition to the reference material described above, prepared slides (296) were obtained by exchange with Dr Margaret Atherden, College of Rippon \& York, York, England, and Dr Tony Stevenson, Newcastle University, Newcastle-on-Tyne, England.

The reference collection is listed in Appendix V. The following data are included: reference collection registration number (VUWG etc. number), taxonomic data (family, genus, species), herbarium collection number (where applicable), and location where the plant grew.

\subsubsection{Written key}

In the written key pollen and spores of extant plants (those occurring naturally and in cultivation) in Grevena, are described at the lowest possible morphological rank with a light microscope (Appendix VI). Descriptions of the grains were collected from many sources, the main literary sources being the major morphological works of Erdtman (1943, 1952, 1957, 1965, 1969), Ikuse (1956), Erdtman et al. (1961), Erdtman et al. (1963), Erdtman \& Sorsa (1971), Faegri \& Iversen (1975, 1989), Belmonte et al. (1986), Ferrarini et al. (1986), Moore et al. (1991), Nilsson et al. (1977), various articles in The Northwest European Pollen Flora, 
volumes 1-6, edited by Punt (1976-1991, in the key these are referred to by "NW" followed by the volume number), Roure (1985), Valdes et al. (1987), and smaller works in the journals of Grana palynologica, Grana, and Review of paleobotany and palynology. In addition, to assist with the description of pollen and spores reference was made to the comprehensive pollen and spore reference collection of local species from plants collected in Grevena and from herbarium specimens. Although this key was made to cover extant species in northwestern Greece, it is likely that it would cover most of the common pollen and spores encountered in Quaternary deposits in the Central Balkans. A full list of species included in each pollen type is given at the end of this key. Unfortunately the morphology of many species still remains unexamined, but the probable morphology of these species can be based on plant taxonomy, allowing them to be placed in the most likely pollen type. These species are listed at the end of each species list and can be identified by the '?' that precedes them. Nomenclature follows Flora Europaea (Tutin et al. 1964-80).

The purpose of this key is to supply a practical means of identifying pollen and spores without specialist pollen-morphological knowledge. The key is intended for use with a light microscope, mostly with a X40 objective and occasionally an oil immersion X100 objective. The terms nexine and sexine are used to describe the strata of the exine because the sculptured sexine is easily distinguished from the unsculptured nexine when viewed with a light microscope, while the ektekine and endexine layers are difficult to distinguish with a light microscope (Faegri \& Iversen 1989:225). The sexine is considered to be made up of (from outermost to innermost layer) sculpture element (sexine 3), tectum (sexine 2), and columella (sexine 1). The nexine is made up of the footlayer (nexine 1) and the endexine (nexine 2) (Moore et al. 1991:64).

Special attention has been paid to closely identifying pollen and spores of particular interest to the archaeologist, e.g. cultivated plants, weeds of cultivation, and plants indicating disturbance of the natural vegetation, especially indicator species. Unfortunately, among the major cereals only Secale cereale (rye) and Zea mays (maize) are specifically identifiable. Triticum (wheat), Hordeum (barley), and Avena (oats) can be distinguished only to the level of types, including several closely related species, not all of which are or have been cultivated. Other important cultivated species such as Vicia faba (broad bean) and Pisum sativum (pea) can be identified, but Isatis tinctoria (woad) and Camelina sativa (gold-of-pleasure), and most other crucifers and legumes cannot be specifically identified. The same pertains to many important weeds and ruderal species.

The key has been modelled on the key in Faegri \& Iversen (1989), and a glossary to most terms used can be found in that text. The principle of the terminology is to use terms in a strictly descriptive sense, for example the polar axis is defined as the "unique axis of 
maximum symmetry" and not in terms of ontogeny (Faegri \& Iversen 1989:219). Some important terms describing aperture shape are anulus, margo, costae, vestibulum, and fastigium.

The definition of these terms follows Moore et al. (1991:71):

anulus - a sudden thickening or thinning of the sexine around an ectoporus, e.g. Plantago lanceolata;

margo - a sudden thickening or thinning of the sexine around an ectocolpus, e.g. Euphorbia; costae - thickening or thinning of the nexine around an endoaperture or below the edge of an ectoaperture, e.g. Polygonum aviculare;

vestibulum - the cavity formed at the edge of the pore in porate grains when the sexine and nexine separate, e.g. Betula;

fastigium (geniculus in van Benthem et al. 1984:87) - the cavity formed around pores in colporate grains, e.g. Quercus.

In principle the key is binary, but more than two entries at the same level have been used where taxa are very similar. Aperture type, number, shape, and arrangement of apertures, is the major entry in the key. Exine structure and sculpture, as defined by Faegri \& Iversen (1989:227-9), are considered next. Because these features vary and are sometimes difficult to discern, some pollen taxa occur in more than one place in the key. After the above features have been considered, measurements given should be considered and used cautiously. During the course of researching the morphology of the pollen and spores included in this key many widely differing measurements were found in the literature for the same pollen and spore taxa. Also different chemical treatments will affect the size of pollen and spores variously (Reitsma 1969). Thus, no particular attention has been paid to the mounting mediums used, and the measurements should be considered approximate. Measurements do, however, give a rough guide to whether the grains are large or small. Occasionally size is used as a distinguishing feature, as for example in Quercus, where it has been used prudently. Particular distinguishing features have been italicized.

Spores are placed here in the aperture classes originally devised to separate angiosperm pollen grains, for example pteridophyte spores with one groove are included under monocolpate instead of being in a class on their own, that is, monolete.

The right-hand column gives the ultimate breakdown to taxa. A genus name alone refers to species of that genus only; similarly a species name signifies only that species belongs to the pollen taxon. The addition of the term 'type', abbreviated "t.", implies a pollen morphological type common to several families, genera, or species, as the case may be. 


\subsubsection{Photographic key}

Photographs of pollen and spores were taken using a Carl Zeiss Oberkochen mark III photomicroscope with X40 and X100 oil-immersion objectives; X1.25, 1.6, 2, and 3.2 tube magnification; and Ilford PanF 50ASA and TMX 100 ASA films.

The photographs were mounted on cards with full latin name, common name, and pollen type; morphological details were added with major references to descriptions of the pollen type; and ecological data, including life form and habitat and method of pollination.

\subsubsection{Counting}

Palynomorphs were identified with a Zeiss Standard LAB 06 microscope, with tube factor X1.25 and phase-contrast capability. Eye pieces with magnification of X10 were used. The objectives used for counting palynomorphs were Plan X40 (non-phase and phase), and an Achromat X100 oil-immersion objective when higher magnification and resolution was required. For regular identifications a total magnification of X500 was used. A $35 \mathrm{~mm}$ SLR Olympus $2 \mathrm{~N}$ camera with automatic exposure was fitted to the microscope so that those grains not identified immediately could be photographed and identified at a later date. Palynomorphs were photographed on Fujicolor Super HGII, 100 and 400 ASA film. A comparison microscope was used to view reference material.

Counting was carried out along contiguous transects of the microscope slides. The scale on an eye-piece micrometer was used as a gauge for advancing the stage. At the completion of each traverse, the stage was advanced a distance equal to the length of the scale. This method of advancement is more accurate than using the scale on the microscope stage as a gauge. All grains that lay within the scale or overlapped the upper edge were counted, those that overlapped the lower edge were not counted. In this way all grains on the microscope slide were counted once.

Within the Gomara sequence, where pollen was less abundant, at least 250 pollen grains representing trees and shrubs were counted, but where pollen was more abundant many more were counted, to maintain a diversity in the count despite the dominance of Pinus (Rull 1987:472). In the samples from Anelia and Kellia the pollen content was much lower than in the Gomara sequence, and the dominating effect of Pinus was lower. At Anelia, at least 200 grains of trees and shrubs were counted. In the samples from Kellia pollen and spores were counted until about 200 grains of woody plants had been counted (Barkley 1934; Rull 1987).

In the case of the Gomara samples where the aliquot method for calculating pollen concentrations was used, as well as the exotic marker grain method, the entire slide was always counted, but in the samples from Anelia and Kellia, where the exotic marker grain 
method only was used, sometimes only part of a slide was counted, if pollen of woody plants was abundant. In these cases, counting transects were spaced evenly over the slide, to avoid a statistically inaccurate sample due to an uneven distribution of pollen under the cover slip (Brookes \& Thomas 1968).

Many of the Pinus grains were broken at the junction between the body and bladder, so each body or bladder of a Pinus grain was counted as $1 / 3$ of a grain.

\subsection{Charcoal analysis}

An understanding of fire history is important in evaluating interactions between vegetation, climate, and human disturbances. Analyses were undertaken to assist with reconstructing the intensity, size, and distance of fires from the site of charcoal deposition. Generally, large heavy particles and those with a high ratio of volume to surface area tend to move shorter distances (Patterson et al. 1987:5).

To obtain the fire history of each pollen-core site microscopic charcoal fragment numbers, individual fragment areas, and size classes were determined for each pollen sample using a semiautomatic computerized image-analysing system. Relative abundances of charcoal particles in surface samples were also quantified to assist with interpretation of the core sequences.

Microscopic analyses were performed on the same slides as were scanned for pollen. Care was taken not to break charcoal particles during sample preparation by using a vortex stirrer instead of a stirring rod wherever possible (Clark, R. 1984:564). All samples were sieved through a $260 \mu \mathrm{m}$ mesh sieve before microscope slide preparation: residues retained on the sieve were later inspected by eye to determine relative abundance of large charcoal particles.

The charcoal-scanning equipment consisted of a high-resolution JVC colour video camera mounted on a Zeiss Photomicroscope II with a X16 objective. The camera was interfaced to an IBM-compatible PC and monitor with 486DX processor and $16 \mathrm{Mb}$ of RAM, using an Oculus-TCX video digitizing board (Raine et al. 1996). The commercial imageanalysis software package Optimas 5.1 was used to access the active image in the digitizing board. Optimas delimits images by colour and brightness; thresholds were set with a 3-colour mode. Charcoal was identified based on optical density (opacity), following interactive adjustments of microscope light intensity, camera sensitivity, and software parameters. Once a set of values had been optimised for charcoal particle discrimination, these were used for all further measurements. The digital image dimensions were calibrated into Optimas by measuring a microscope micrometer slide: for the X16 objective and microscope/camera optical arrangement the imaged area was 245 x $181 \mu \mathrm{m}$. Operating information was displayed 
on the PC monitor. A second monitor attached to the digitizing board displayed the microscope image. The microscope mechanical stage was positioned by hand.

Under control of an Optimas macro program the system outlined individual particles of interest and performed an initial classification based on average grey value, tagging as "charcoal" those with density and maximum dimension greater than a predetermined threshold (minimum particle size for consideration by Optimas was set at $5 \mu \mathrm{m}$ ). The resulting classified image was then presented on the second monitor for observer approval.

Some workers have found that charcoal concentrations calculated using an automated scanner correlate well with charcoal concentrations determined using standard visual techniques: although visual estimates are usually slightly higher, they display the same variations and lead to the same interpretation. MacDonald et al. (1991) have attributed this result to small particles being missed by the imaging system, and to light 'bleeding' at the edges of charcoal particles. Horn et al. (1992:8) attributed their higher visual counts to the image analyser missing out-of-focus particles. These workers used a more completely automated system than the system used here, with a mechanical stage positioned by the computer system, but with a set focus. They suggested that improvements to the method could be made by specialised extraction techniques to remove optically dense non-charcoal particles such as mineral grains or darkly stained pollen or plant material. They also suggested using an automatic focus control and thinner-than-normal slides specifically prepared for charcoal scanning, as well as the development of artificial intelligence techniques (which, however, would require more sophisticated hardware and software).

The preparation of special slides and development and availability of elaborate software and hardware were beyond the scope and resources of the present study. The difficulties mentioned above, and also the problem of overlapping particles in dense or clumped slide preparations, were overcome by using a semi-automatic technique, interactively modifying the data being recorded while following the guidelines of Griffin \& Goldberg (1979) and R.L. Clark (1984:571-2) for charcoal particle identification. After the image-analysis system completed its initial classification and displayed the result, discrimination of charcoal particles was checked for each frame by viewing the frame area on the monitor and microscope. Particles classed as charcoal, but which through visual examination could be correctly identified as mineral grains, could quickly be reclassified using the computer "mouse" as a pointer. In cases of charcoal-particle overlap, or partly out-of-focus particles being incorrectly outlined, the outline of the charcoal could be traced by hand, again with the mouse. Frames could also be repeated to record particles at different focus levels.

Once an acceptable classification had been obtained (and in most cases the macro produced an entirely acceptable result), a computer keystroke initiated export of particle area, 
maximum linear dimension, etc. of the individual charcoal particles to a linked Excel spreadsheet.

To enable abundances of measured charcoal particles to be related to pollen abundances, each pollen-counted microscope slide from the wetland sequences was scanned for charcoal using 100 fields each $245 \times 181 \mu \mathrm{m}$ evenly spaced over the area of the cover slip. (In the case of surface-sample pollen slides, charcoal was recorded in fields spaced $1 \mathrm{~mm}$ apart along pollen-counted traverses.) The raw measurement data were tabulated in size classes based on the area of individual particles following a geometric progression: $<25,25-50,50-100,100-$ 200, 200-400, and $>400 \mu \mathrm{m}^{2}$ (Patterson et al. 1987:18). The frequency of the size classes is expressed as a percentage of the pollen sum. Charcoal influx is also calculated for the Gomara site only.

\subsection{Pollen-diagram construction}

To aid interpretation of the plant taxa represented in the surface and wetland samples, the pollen-analytical data are expressed in a series of graphs or pollen diagrams. The pollen diagrams were produced on an "IBM compatible" personal computer, and output on a laser printer with a PostScript interpreter. Diagrams were plotted using psimpoll 2.23 (Bennett 1994). Files include data on: sediment stratigraphy (using the Troels-Smith notation), radiocarbon dates, local pollen assemblage zones, pollen and spore counts, charcoal abundance, rarefaction analysis, and rate-of-change (for the Gomara wetland site only).

Pollen diagrams of wetland sequences are presented by depth and, in the case of the surface pollen study, sample-site name. Pollen diagrams of the wetland sequences are separated into two types: local (wetland) taxa and extra-local and regional (terrestrial) taxa (Janssen 1973). Wetland pollen diagrams include a summary curve showing the ratio of mire to aquatic taxa, and the number of wetland types identified and total number of wetland taxa counted.

Terrestrial pollen diagrams include summary curves showing the ratio of woody plants to herbaceous plants, and of taxa grouped according to habitat preference. The number of pollen grains counted of trees and shrubs, the total number of all terrestrial pollen and spores, and the total of all pollen and spores counted in the sequence (wetland and terrestrial) is given. The number of terrestrial pollen types (not normalized for unequal pollen sums) is also included for each spectrum. 


\subsubsection{Pollen statistics}

In the case of the surface samples, the pollen-analytical data are expressed in relative frequencies only. However, by obtaining absolute data from the wetland sequences, both relative and absolute frequencies of pollen abundance could be calculated, so the changing abundance of plant taxa are illustrated as both relative proportions and absolute concentration (by volume). Pollen influx rates were calculated for the Gomara site only, where a relatively uniform rate of sedimentation has been assumed.

\subsubsection{Relative frequency pollen calculations}

The main pollen diagram for each site is a percentage diagram. In these diagrams the relative proportions of the pollen taxa are expressed as percentages of a pollen sum. Diagrams of this type are the most readily produced, as they do not require knowledge of pollen concentrations or sedimentation rates and in fact smooth out variations in these factors. A further advantage of using percentages is that all components within the pollen sum are less than $100 \%$, whereas many large numbers of certain taxa may have to be plotted on concentration or influx diagrams. The principal and important disadvantage is that each pollen type influences all other types within the sum.

Pollen taxa included in the pollen sum vary according to the question being asked. The pollen sum of the surface pollen study and wetland sequences of terrestrial (extra-local and regional) pollen types includes all identified terrestrial pollen and spore types and excludes obligate aquatic plants and swamp, bog, and fen species (Janssen 1973:36). A pollen sum including only wetland pollen types is used for the wetland (local) pollen diagrams.

\subsubsection{Pollen influx calculations}

The pollen-influx diagram is the most meaningful one in an ecological context, as it allows variations in abundance of the various taxa to be assessed entirely independently, but it can only be calculated where the rate of sedimentation can be deduced (Davis \& Deevey 1964; Maher 1972).

Pollen influx (also termed pollen-accumulation rate or pollen-deposition rate), the net number of grains accumulated per unit area of sediment surface per unit time, was calculated for only one site, Gomara, where the rate of sedimentation of the deposit could be calculated with reasonable confidence.

Pollen influx $=$ Pollen concentration $x$ sediment accumulation rate (grains $/ \mathrm{cm}^{2} /$ year), where the sediment-matrix accumulation rate is the net thickness of sediment accumulated per unit time after compaction and diagenesis ( $\mathrm{cm} /$ year). 


\subsubsection{Absolute pollen concentration calculations}

Pollen concentration diagrams also have the advantage that each pollen taxon can be evaluated separately but are more difficult to interpret than pollen-influx diagrams, for concentrations vary with non-vegetation factors such as changing sediment type and variations in deposition rate. Concentration diagrams were drawn for the two wetland sites of Anelia and Kellia, where the rate of sedimentation is very variable.

\subsubsection{Zonation}

The sequences of pollen spectra have been divided into local pollen-assemblage zones (Hedberg 1976). Zones are based mostly on relative frequency pollen and spore data and are formed to simplify discussion and interpretation of the pollen diagram and facilitate a discussion of the vegetational events and comparison with other pollen sequences. The zone boundaries are placed where change in the pollen spectra is most marked so that the enclosed units are relatively homogeneous. They can be juxtaposed with the climate periods of Blytt and Sernander (considered as chronozones) (de Beaulieu 1982) and cultural periods.

Numerical zonation by the optimal divisive method, using information content (Bennett 1994) was used to assist with the determination of the number of zones to be used and the placement of zone boundaries. The broken-stick model was used to assess the significance of zones based on the structure in the data set, by using a model of random distribution of zones within the sequences as a comparison. Simulation modelling was used to assess the reliability of zone-boundary locations, given the errors of the original data (Bennett 1996). A threshold value of $5 \%$ was used (Bennett 1996), but this was reduced to $2 \%$ for the diagrams of terrestrial pollen types from the Gomara and Anelia sites, where the pollen of Pinus is greatly over-represented, and the remaining subset data were recalculated to proportions of the sum of types included in the subset. Final selection of the best number of zones to be used and the placement of the zonation boundaries was determined by visual inspection and by consideration of influx and concentration data and rate of vegetation change (see below).

\subsubsection{Palynological richness}

The number of taxa recorded per spectrum is of interest, for an opening of a forest canopy above a site of deposition will permit the entry of more light, thus allowing lightdemanding plants an opportunity to grow and also permitting entry of a wider variety of pollen taxa, for more of the canopy and regional component pollen types enter the sedimentary environment. Increasing numbers of taxa may therefore indicate canopy opening and may lead to interpretative conclusions about forest catastrophe or human impact. 
Palynological richness (diversity) is estimated by rarefaction analysis. Rarefaction analysis standardises the sum on which palynological richness is determined and provides minimum variance unbiased estimates of the expected number of taxa for the standardised sum in each sample (Birks \& Line 1992; Bennett 1994). Estimates of palynological richness are shown with $95 \%$ confidence intervals.

\subsubsection{Rate-of-change}

Rates of vegetation change were calculated for the Gomara wetland site only. The dissimilarity coefficient of Chi-squared coefficient 2 was used on unsmoothed pollen and spore relative frequency data and calibrated radiocarbon ages (Bennett 1994; Bennett \& Humphry 1995).

\subsubsection{Pollen taxon groupings}

Vegetation communities can best be reconstructed by grouping together taxa with similar known ecological preferences. Groupings are based mostly on results of vegetation surveys and surface pollen analyses in Grevena (see Chapter 3) and data from Turrill (1929) and Ellenberg (1988). The main terrestrial groups are: alpine shrubs, alpine herbs, coniferous and deciduous canopy trees, woodland understorey trees and shrubs, woodland herbs, riverine trees, evergreen woodland trees, plants of open dry rocky habitats, herbs of open ground, and cultivars. Wetland taxa have been divided into two main vegetation-community types: aquatic (open water) and emergent taxa.

A number of problems are connected with arranging ecological groups from pollen types:

1) Each pollen type must be assigned to one of the basic groups on the basis of the present ecological amplitude of the species. But the ecological amplitudes may have been different in the past. The climate may have fluctuated in the time span considered here, but the flora was probably not too different from that of today. It is therefore assumed that competition among the various species was similar to that found today, and ecological groups have been so constructed.

2) The flora of some plant communities is discrete, but there is overlap between others, for example the floras of steppe and woodland edge have many species or higher taxa in common with weeds of cultivation (Moody \& Rackham, in prep.).

3) Many pollen types cannot be identified to species level. Pollen types may thus comprise species with contrasting ecological requirements and consequently may be placed in more than one ecological group. 
4) An interpretation of pollen assemblages in terms of plant communities largely depends on knowing the source of the pollen grains, i.e. whether or not a pollen assemblage comes from plants that could have occurred together in the area.

5) It is difficult to determine the present ecological amplitude of a species. Comprehensive surveys over the whole range of plant communities in Grevena have not been completed. Thus characteristic and differential species have been determined on the basis of a small part of the vegetation. Comparison of the fossil assemblages with recent plant communities was aided by the work of Dr O. Rackham (Moody \& Rackham, in prep.). 


\section{Chapter 3. Modern pollen deposition study - vegetation \& pollen data}

\subsection{Introduction}

This chapter discusses the flora and vegetation of Grevena and the relationship between the extant vegetation and pollen deposition. First the flora and vegetation of Greece are presented to provide a wider context for Grevena, then the flora and vegetation of Grevena are discussed. The relative dispersal and representation of pollen and spores found in surface samples from the major extant vegetation types in Grevena is presented. Abundance of microscopic charcoal particles recorded in these surface samples is also presented.

\section{2 'Natural' flora and vegetation of Greece}

It is ninety years since a detailed comprehensive flora of Greece was published (Halácsy 1900-08, in Strid 1989). Unfortunately, the forthcoming Flora Hellenica has not yet been published. Flora Europaea (Tutin et al. 1964-80) represents a milestone in European floristics; it is, however, very brief and Greece is probably the country with least coverage. The two volumes of Strid (1989) and Strid and Tan (1991) provide better coverage of the mountain flora. They describe all vascular plants commonly occurring above an altitude of $1800 \mathrm{~m}$ within the borders of present-day Greece, with an emphasis on the mountains in northern Greece. In addition, taxa that only occasionally reach this altitude or are commonly found in open tree-less habitats above $1500 \mathrm{~m}$ have also been included but are more briefly treated. The rules were interpreted with some flexibility because the treeline, although often lying around $1800 \mathrm{~m}$, varies greatly as a consequence of anthropogenic disturbance, e.g. logging and fire. The aim of their study was to exclude typical forest taxa and to include all taxa of open subalpine and alpine habitats, thus excluding many taxa extant in Grevena and also many taxa of importance to palynological studies in general and the present study in particular. Strid and Tan (1991) found that $1 / 3$ of the taxa they recorded had not been recorded for Greece in Flora Europaea or were recorded under a different name and/or with a different taxonomic rank.

Today about $85 \%$ of the ground surface of Greece is bare of trees. This $85 \%$ is covered with scrubland (maquis), fields, and pasturage, as well as land completely barren and eroded (Sfikas 1978). Most of the forests in Greece are found at intermediate altitudes, on the flanks of the larger mountain ranges in the north in Thrace, Macedonia, and Epirus and in central Greece at high elevations. These woodlands are rich in species. The greater part of Greek forests consists of conifers, with deciduous trees such as Fagus (beech), Castanea (chestnut), and Quercus (oak). Other species grow rather sporadically and scattered in the forests, on scrubland or in fields, and are rarely found in extensive or homogenous stands (Sfikas 1978). 
Most of the trees found in Greece are common to all of Europe. However, there are also some Asian species, e.g. Fagus moesiaca, which does not exist in other European countries (Sfikas 1978:10). Several coniferous trees commonly occurring in Greece, and indeed Grevena, are endemics to the Balkan area, for example Abies cephalonica, Pinus heldreichii, and Pinus leucodermis. Also several trees and shrubs which, although they originated in other countries, have become very common in certain regions and have acclimatised themselves so well that they grow like native species, e.g. Ailanthus altissima (tree of heaven) from Asia and Robinia pseudacacia (false acacia) from America.

The most common Greek forests can be roughly divided into three categories, according to the predominant species:

1) Coniferous forests - coniferous highland forests throughout Greece, made up of different species of Pinus (pine) and Abies (fir);

2) Deciduous forests - deciduous highland forests in the north, consisting of Fagus (beeches), either by themselves or mixed in with other deciduous trees;

3) Evergreen forest - Mediterranean forests on the lower mountain slopes where Pinus halepensis (aleppo pine) is by far the most common, often mingling with other needled conifers; Quercus coccifera (kerm-oak or holly oak); Quercus ilex (holm-oak); Fraxinus (ash); and various shrub species (Sfikas 1978:9-10).

\subsection{Flora and vegetation of Grevena}

In contrast to the bareness of much of Greece (Rackham 1982), Grevena province is covered by large tracts of woodland. Of the 200 tree and large shrub species (belonging to about 85 different genera) growing in Greece today, according to Sfikas (1978), about 149 of these species (and 63 genera belonging to 69 pollen taxa) are probably extant in Grevena (Tutin et al. 1964-80; Moody \& Rackham 1988; Polunin 1988; Adamakopoulos 1989; Strid 1989; Rackham 1991; Strid \& Tan 1991; Appendices I, VII \& VIII; Moody \& Rackham, in prep.). Ninety seven additional tree and large shrub species, 39 trees (including 7 that are not native, but planted), and 58 shrubs have been identified as probably also extant in Grevena. In addition, 83 dwarf shrubs and woody climbers have been identified, making a total of 329 woody plants. About 118 of these woody species have been observed by Grevena Project members, and an additional 8 woody species were recorded by Adamakopoulos (1989) during her survey of the NE Pindos. 
The flora of Grevena is very rich in herbaceous species also. Factors contributing to this rich flora include:

1) the proximity of other floras, notably the central European, Anatolian, and Pontic floras from which migration has taken place;

2) an isolated mountain range, which has resulted in the fragmentation, isolation, and migration of species;

3) an old flora containing many Tertiary species that have survived the Quaternary glaciations; and

4) the profound influence of humans in destroying and changing the natural plant cover, creating new habitats, and introducing species from outside the Balkan Peninsula (Turrill 1929).

Plant communities of Grevena are varied and complex. With decreasing elevation they range from alpine vegetation of mountain peaks, mountain pine woods and beech and fir woods of Central European aspect, deciduous oak woods in the foothills, to lowland Mediterranean-type woods containing some evergreen species such as Quercus coccifera and Phillyrea media, and dry steppe reminiscent of the Hungarian and Russian steppe. A unique local climate coupled with the wide differences in bedrock formation, altitude, and aspect, as well as the varied origin of species, make for great diversity in plant communities. In consequence of these many unique factors the vegetation of Grevena has many local variants and exceptions.

As yet there is no published study of the vegetation of the province per se. For this study, much ecological information on the vegetation of Grevena is taken from studies made of the Balkan Peninsula, especially the phytogeographical study of Turrill (1929) and the study of Polunin (1988).

\subsubsection{Classification of extant vegetation}

The systematic classification of Mediterranean vegetation began in France with the Braun-Blanquet hierarchical classification based on floristics (Whittaker 1962). This is a formalized system with Latin names analogous to those used for naming plant species, genera, and families. The system became fashionable among European phytosociologists and has been extended to many countries, including Greece. The extant vegetation of Grevena is the result of centuries of interaction of wild plants and animals with anthropogenic activities including woodcutting, grazing, burning, cultivation, and building settlements and communication systems, so this type of formal classification is not suited to its description. Recently Moody \& Rackham (in prep.) classified the extant vegetation of Grevena using a less formal system that is more pragmatic and more suited to the extant vegetation of Grevena and 
to this study. They divide the vegetation into woodland, steppe, and riverine vegetation. Woodland is the dominant vegetation type in Grevena and covers about half the province. The woodlands are divided into pine and beech woods of the Pindos Mountains, mixed woods on limestone outcrops at the upper altitudes of the Pindos foothills, deciduous oak woods of the Pindos foothills and lowland plains, and pseudo-maquis woodland in the southeast.

Distinct vegetation zones coincide with five geographic sub-regions of Grevena:

1) Pindos mountains,

2) Pindos foothills,

3) lowland plains,

4) flood plains,

5) and eastern mountains.

Generally in the Pindos Mountains over $1000 \mathrm{~m}$ woodland is dominated by Pinus (pine), less commonly Fagus (beech). Pasture also occurs, but very little of the past cultivation now remains. In the Pindos foothills oak wood now usually predominates over cultivation, although there are abundant remains of former small fields. On the lowland plains cultivation predominates over woodland except in the valleys, but where woodland occurs it is usually deciduous oak. The flood plains have a band of riverine woodland of Salix (willow), Tamarix (tamarack), and Platanus (plane) and other riverine vegetation, as well as some cultivation. The eastern mountains are less well vegetated than the Pindos Mountains - where vegetation exists it is mostly dry shrubbery (Figure 3.1).

Plant communities within these zones are described below according to physiognomy, land use, and floral composition. These descriptions are based on fieldwork carried out during summer field seasons between 1987 and 1989, mostly by Dr O. Rackham. The author spent one field season (1989) with Dr Rackham as well as conducting her own botanical fieldwork. An unpublished Greek Forest Service 1:50 000 map provides abundant detail on the extant vegetation of Grevena (Karasavvidis, unpub.), which, although based on dominant trees, can be interpreted in terms of the Moody \& Rackham classification (Figure 3.2).

\subsubsection{Pindos Mountains}

The highest elevations are occupied by alpine meadows and pastures. (These were not studied but are adequately covered in Polunin (1988).) Below the treeline, about 1800$2100 \mathrm{~m}$, and above $800 \mathrm{~m}$ are mountain pine woodlands. Within the pine woods at intermediate elevations is a mosaic of pine-beech-fir woods. 


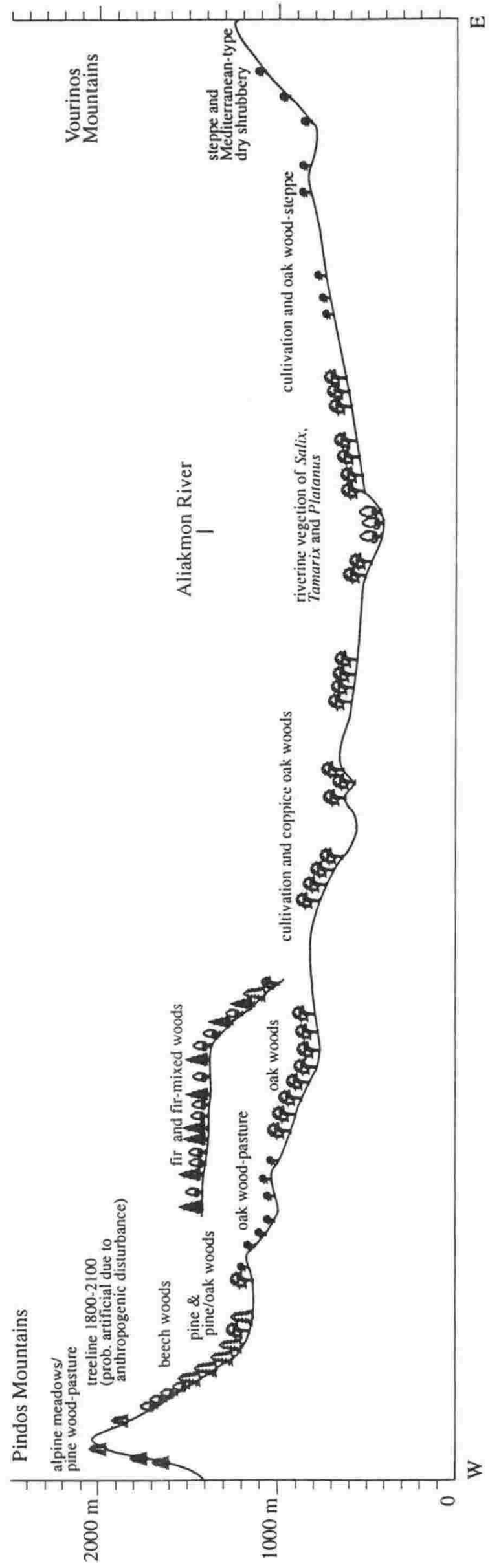

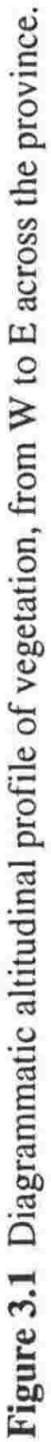




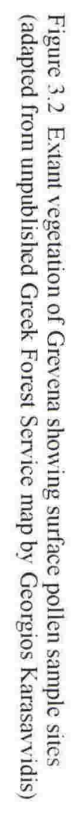
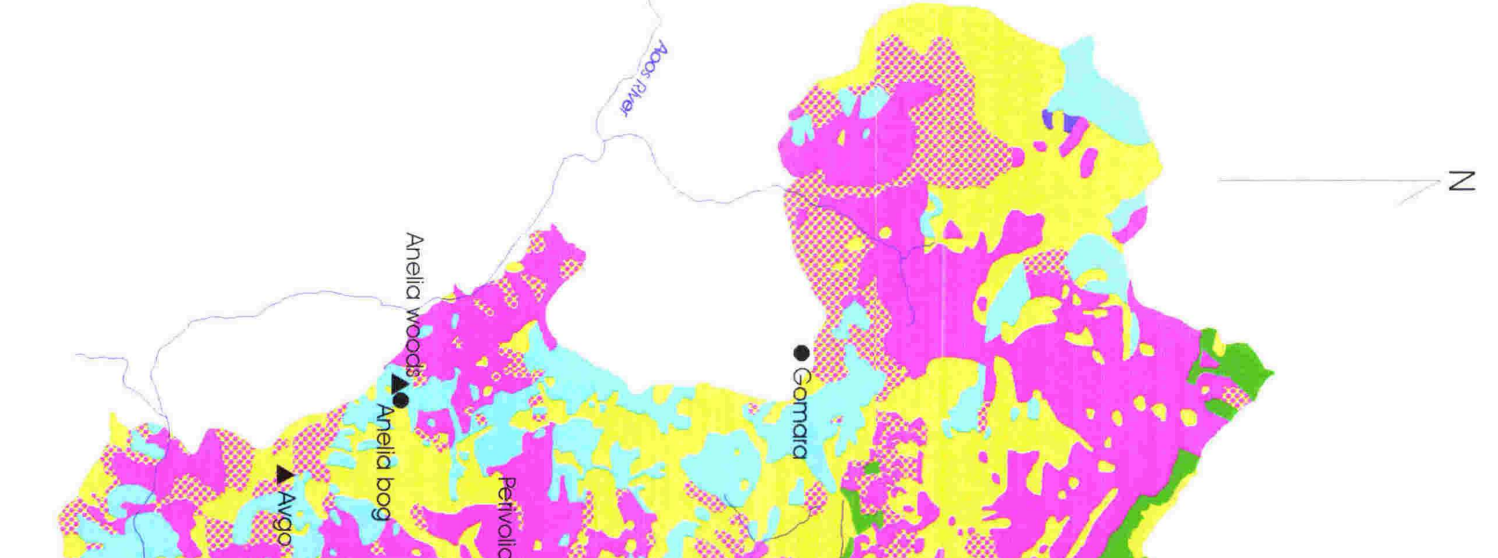

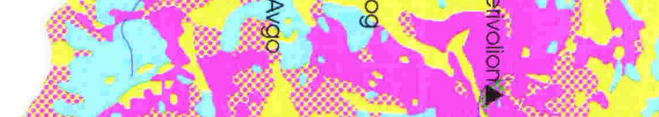

\%

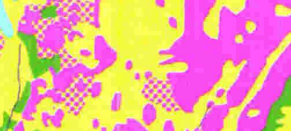

1.

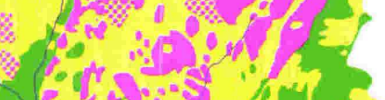

, 0.0

\% 2

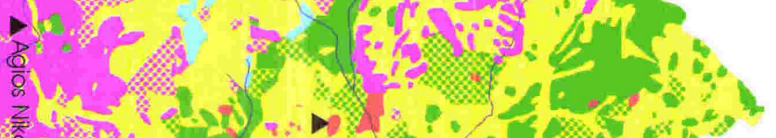

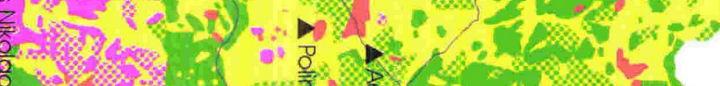

$\frac{0}{8}-A$.

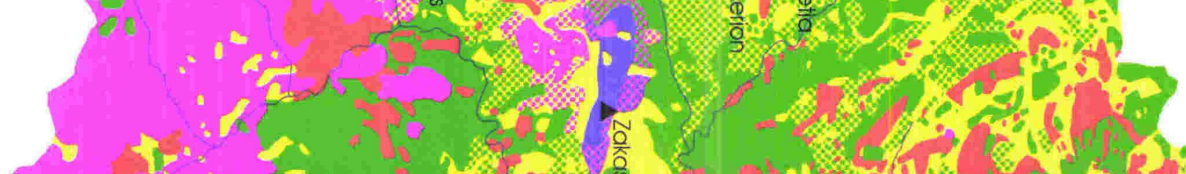

$+1 ; \div$

$2, \frac{1}{2}$

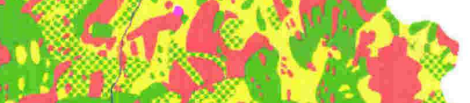
$\therefore y+2=0$

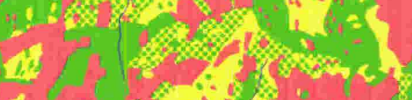

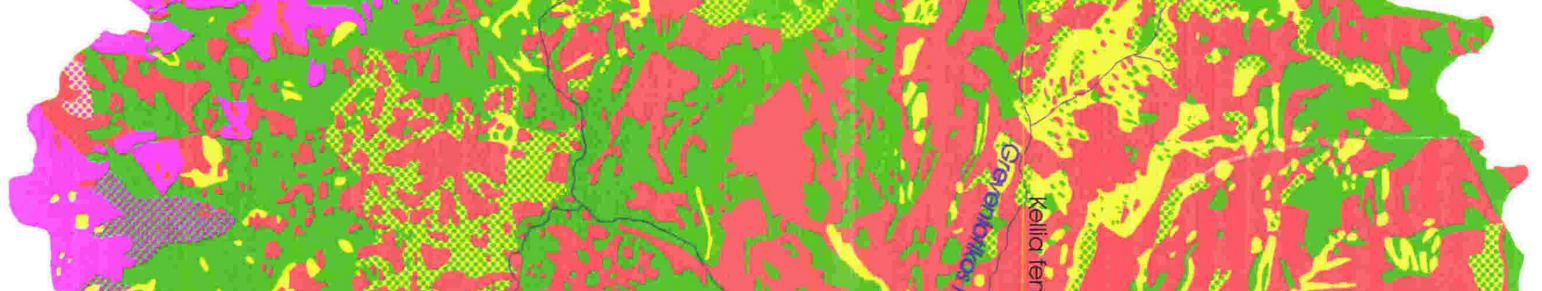

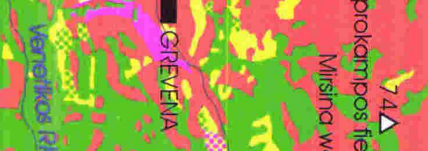

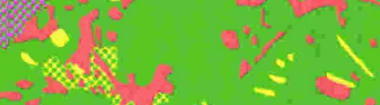

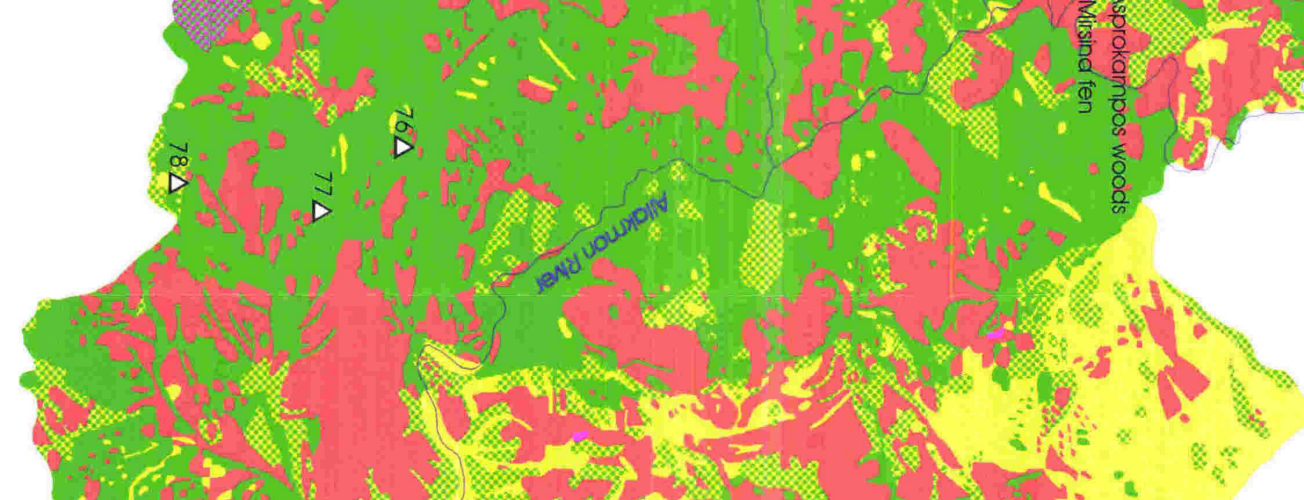

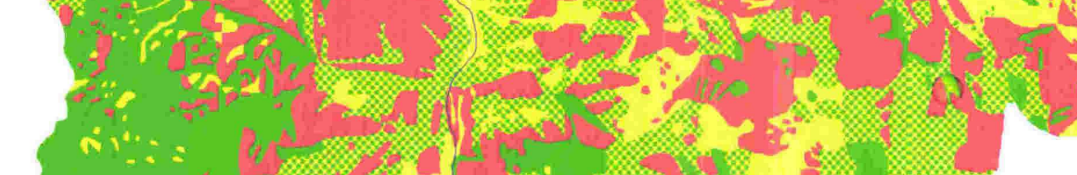

.

s.

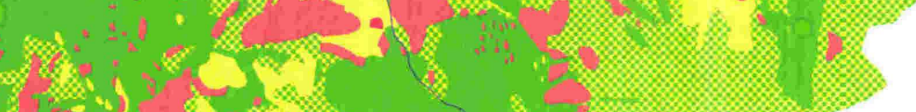
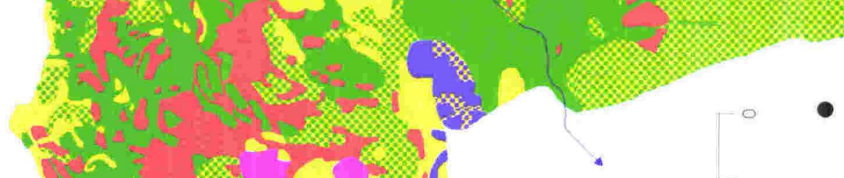

$\triangleright$

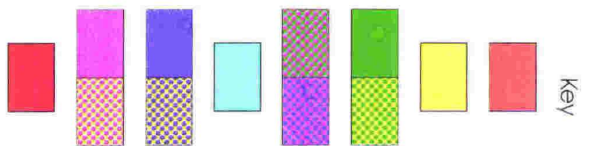

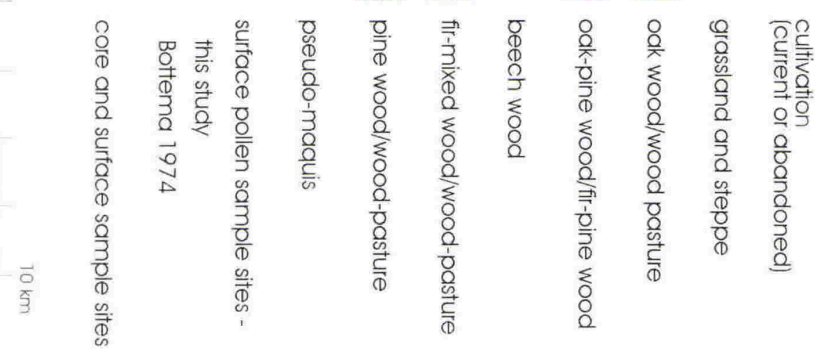


The growth of trees within these woods is episodic: any one area has trees of two or three different ages (estimated from tree-ring data), rather than a range of ages. This may reflect the history of grazing and to some degree woodcutting, although a contributory effect from weather cycles cannot be ruled out.

In general, in the last 200 years pine has invaded former pasture land. Beech, much of which 200 years ago seems to have been reduced to bush size through grazing, has increased at the expense of pine and of pasture (Leake 1835). Fir has probably declined (Turrill 1929), and in many areas is represented by only scattered giant trees. There has been a slight encroachment on the mountain zone by oak wood from lower altitudes. Much of the younger pine and beech is of an age to have started growing during and after the second World War. There are also many young trees of both the 1930s and 1950s. There is evidence that some of the beeches have been shredded and coppiced in the past (Moody \& Rackham 1988).

Fire is not a major ecological factor here. The woods, even the pine woods, do not burn easily; their leaf-litter is compact and soon rots. Evidence of frequent lightning strikes without subsequent conflagration is abundant. Juniper, which fire kills, is abundant almost everywhere (Moody \& Rackham 1988).

\section{Mountain pine woods and wood-pastures}

Of the three pine species that grow in the pine zone of the Pindos, Pinus nigra is a local variant of a species fairly widespread in Central and sub-Mediterranean Europe. The other two pines, $P$. leucodermis and $P$. heldreichii, are Balkan endemics. The ecological differences between these three are not great. $P$. leucodermis tends to grow at the highest altitudes, with $P$. heldreichii intermediate.

Pines are not very palatable and are not used as fodder. P. leucodermis can survive being grazed to a bush but retains a gnarled, twiggy base if it later grows into a tree. Pines are killed by felling. They will pollard, but this is rarely done in Grevena. These pines have shallow-spreading roots.

There are two types of vegetation structure resulting from two different land uses, pine woods and pine wood-pastures. In pine woods, trees grow close together and often all three pine species grow mixed together, e.g. between Zakas to Agios Nikolaos (Appendix VII, i). They are usually young (up to 150 years) and commonly two or three generations of pines grow in any one place. Juniper grows as an understorey shrub in pine woods and acts as a nurse plant to seedling pines. Pines are felled for timber.

In pine wood-pasture the pines are scattered and set in grassland or bracken (Pteridium aquilinum); species are not mixed together but grow according to elevation preferences. This type of vegetation occurs around the wetland core site of Gomara and at Avgo (Appendices 
VII, ii \& VIII) (Figure 3.3). Occasionally pines are pollarded (e.g. at Samarina). They often carry the pine variant of Viscum album, the most widespread European mistletoe. The shapes of pines in this vegetation structure indicate that they have always been free-standing - at least since they were very young - and are not the remains of a continuous wood.

Some pines are of great age and huge size. Ancient pines are commonly 30-40 m high and $1 \frac{1}{2}-21 / 2 \mathrm{~m}$ in diameter (Figure 3.4). Tree-ring counts show that growth-rate varies according to altitude: thus a big $P$. nigra at $1200 \mathrm{~m}$ below Smixi is no more than 195 years old while a similar-size tree at $1400 \mathrm{~m}$ above Smixi is about 420; pines on the pass to Distraton $(1750 \mathrm{~m})$ are 470-550 years old; P. leucodermis at Avgo (1760 m), up to $1.4 \mathrm{~m}$ diameter, are 630-700. About half the ancient pines have been struck by lightning, some of them many times. This can burn a great scar down one side of the tree without killing it.

There is a tendency for beech to invade pine areas. There are frequent beech coppices among the giant pines in areas that must have once been pine wood-pasture. A few pollard oaks extend well into the pine zone. These are either along watercourses or associated with settlements (e.g. beside the church of Agios Nikolaos). In the latter situation especially, this may indicate that pine has lost ground to oak wood-pasture, but below Kranea, in the southwest, there is some evidence of pine moving down to invade oak wood and oriental hornbeam (Carpinus orientalis) wood.

Grassland of the pine wood-pastures is dominated by species of Festuca and other perennial grasses. Among the grasses is a rich flora of mainly perennial herbs, such as Arabis subflava, Doronicum orientale, Geranium macrostylum, Valeriana tuberosa, and Vicia lathyroides.

\section{Mountain beech and fir woods}

The European beech, Fagus sylvatica, and the endemic fir, Abies cephalonica, form woods in the Pindos between 600 and $1600 \mathrm{~m}$. Although most of the beech woods in Grevena are pure Fagus sylvatica, as around the wetland core site of Anelia (Appendix VIII) (Figure 3.5), Fagus moesiaca occurs with F. sylvatica in the southwest of the province in Pindos National Park. In Grevena Fagus is close to its southern limit and Abies cephalonica to its northern limit.

Beech and fir overlap with the pine zone and tend to replace pine in the more westerly mountains. This is probably due to the higher rainfall to the west. On a local scale, beech and fir tend not to mix with pine. They grow in the damper areas. Beech sometimes, but not often, mixes with a few other trees, notably Sorbus aucuparia.

Fir is much less abundant than beech, often occurring only as scattered individuals of great age among the beeches. Between Samarina and Fourka an Abies tree, the oldest of four 

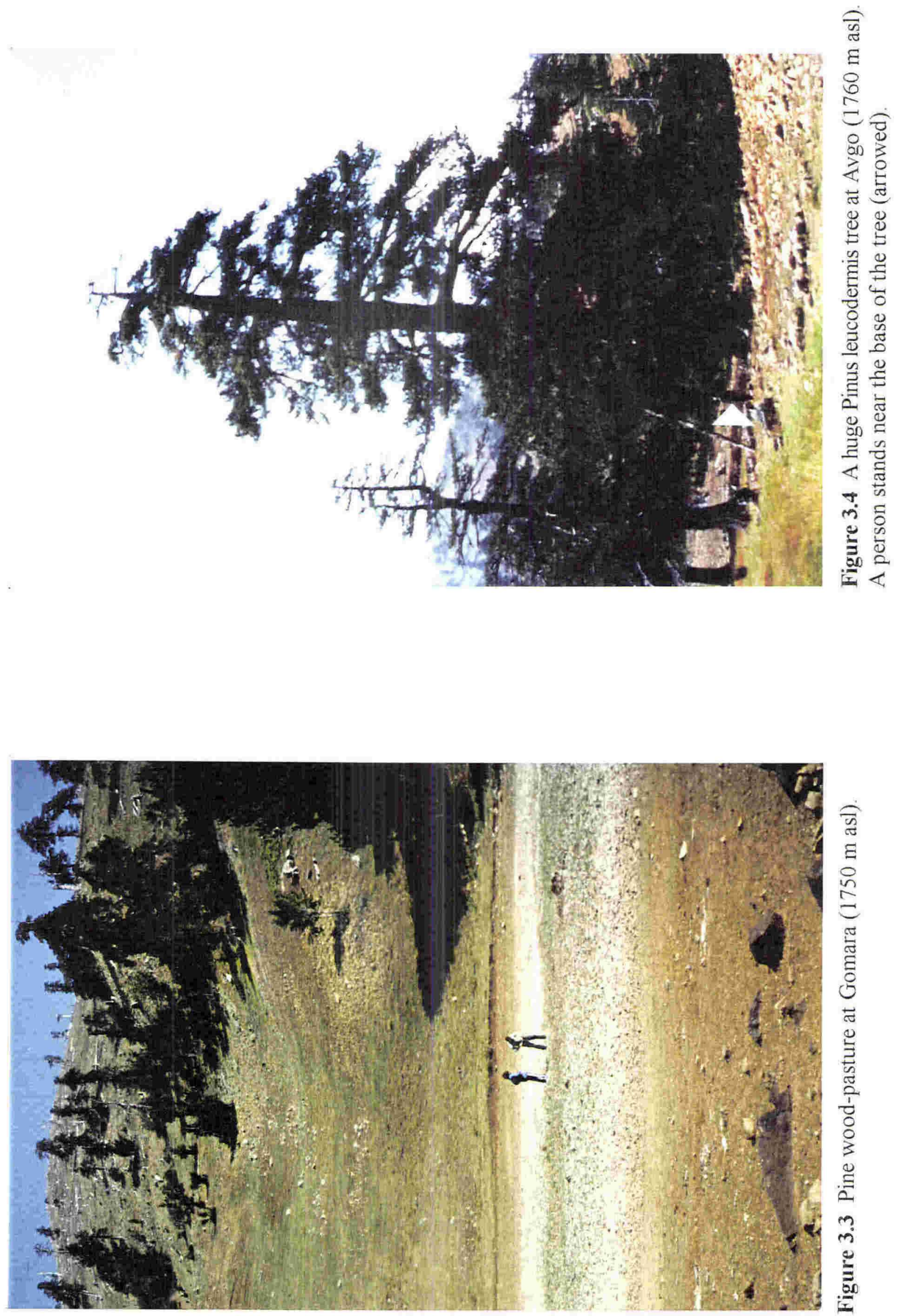


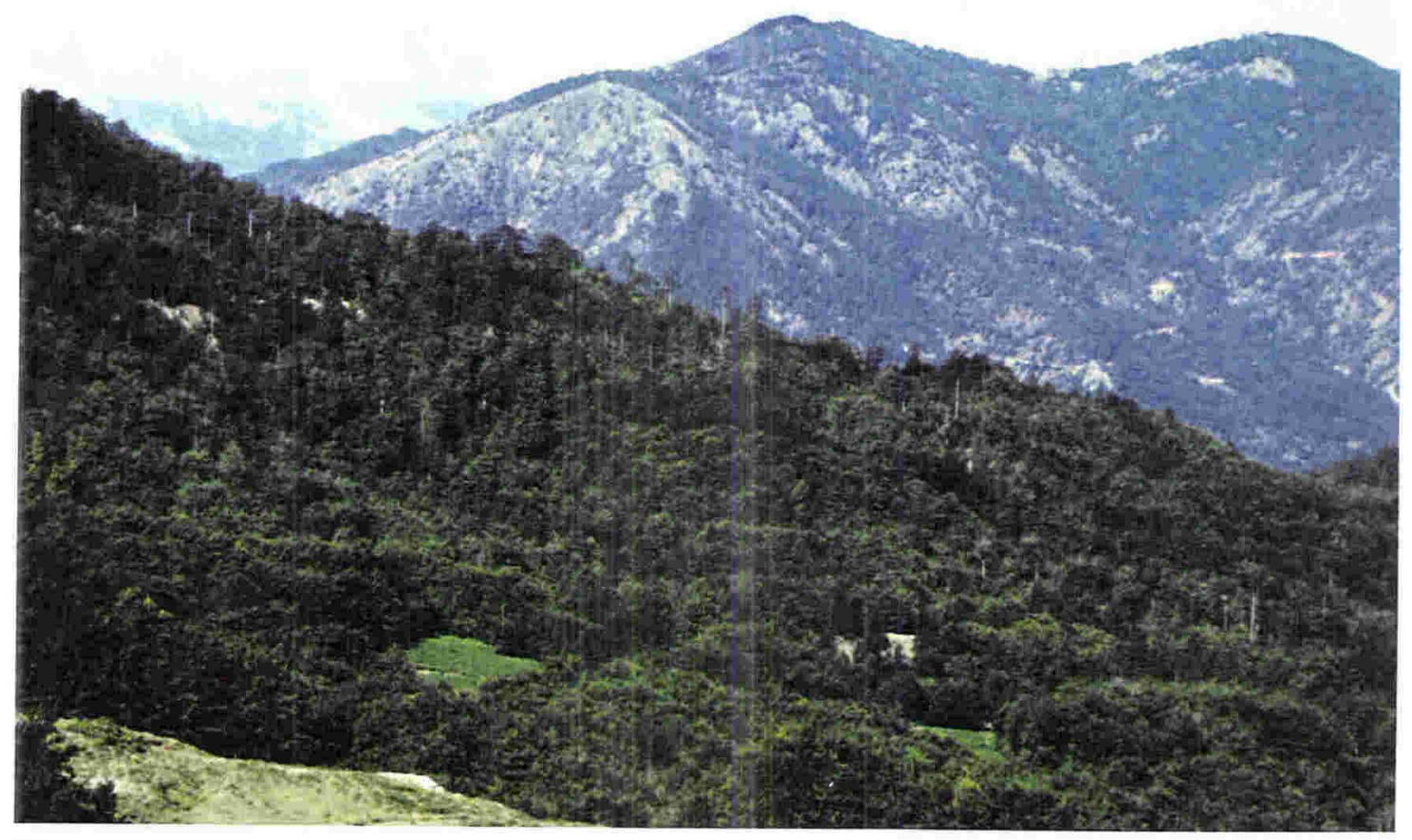

Figure 3.5 Beech woods around the wetland coring site of Anelia (1440 m asl). Pine grows above the beech.

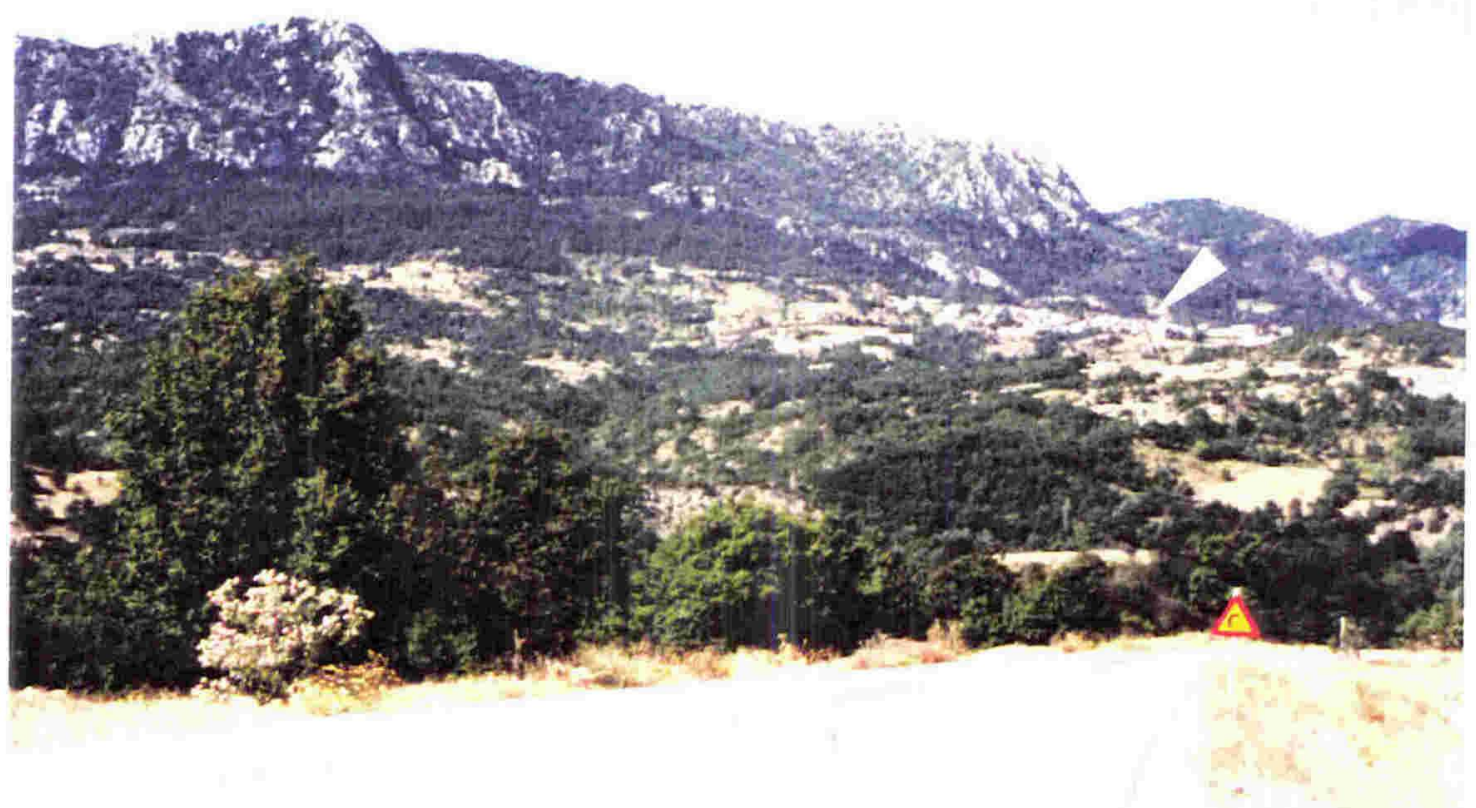

Figure 3.6 Pindos foothills. Limestone ridge behind the village of Zakas (on the righthand side of the photograph, arrowed). Deciduous oak wood-pasture covers the lower slopes, with mixed wood above. 
generations, was estimated to be $35 \mathrm{~m}$ in height and $1 \mathrm{~m}$ in diameter; the second generation was about 300 years old.

Both trees occasionally form wood-pastures, but less often than pines or oaks. A common pattern is for beech woods to occur in small valleys while the rest of the landscape is grassland with or without scattered pines.

Both Fagus sylvatica and Abies cephalonica can survive long periods of browsing when young. They do not burn. Beech sprouts when felled, but fir is killed. Both can be pollarded, but this is rarely seen in Grevena. Beech can be very shallow-rooted.

Beech woods are generally of coppice type, felled every 40-75 years, but many have not been cut for centuries. Beeches with stems up to $1.5 \mathrm{~m}$ in diameter and at least 300 years old are locally common, e.g. around the Anelia coring site. Coppice stools are up to $3 \mathrm{~m}$ across, and are presumably many centuries old. Some stools were last coppiced 250-320 years ago. The growth-rate of beech is very variable from decade to decade, due either to weather or to damage: many old beeches have lost their tops.

Buxus sempervirens (box) is common in mountain beech woods. It replaces juniper as an understorey shrub, perhaps on more calcareous soils. Beech-fir woods are rich in flowering plants, for example Helleborus cyclophyllus (hellebore), Primula vulgaris (primrose - a west European plant which does not like drought), Cardamine bulbifera, Scilla bifolia, Crocus biflorus, Erica herbacea, Lilium martagon, Primula veris, and P. elatior, a coppice plant (cf. Appendix VII, iii, where plants were noted in a disturbed area in beech wood near the wetland core site of Anelia). Lichen are prolific on trees in the beech woods, indicating an unpolluted atmosphere.

Castanea sativa (chestnut) woods, which are widespread but local in the wetter mountains of Greece, occur just north and west of Grevena (Moody \& Rackham, in prep.).

\subsubsection{Pindos foothills}

The slopes are steeper and more dissected here than anywhere else in the province, with many ravines and gullies. There are large areas of continuous deciduous oak wood on these slopes and two small patches of mixed woods (Figures $3.6 \& 3.7$ ).

\section{Deciduous oak woods}

Grevena is one of the most oak-dominated regions in Europe (Moody \& Rackham, in prep.). At least seven species (perhaps up to 12, Appendix VI.2) of deciduous (includes deciduous or semi-evergreen) oaks are extant: Quercus brachyphylla, Q. cerris, Q. frainetto, $Q$. pedunculiflora, $Q$. pubescens, $Q$. trojana, and $Q$. virgiliana (Moody \& Rackham, in press). Only Quercus trojana (deciduous or semi-evergreen) stands out as a clearly defined species; 


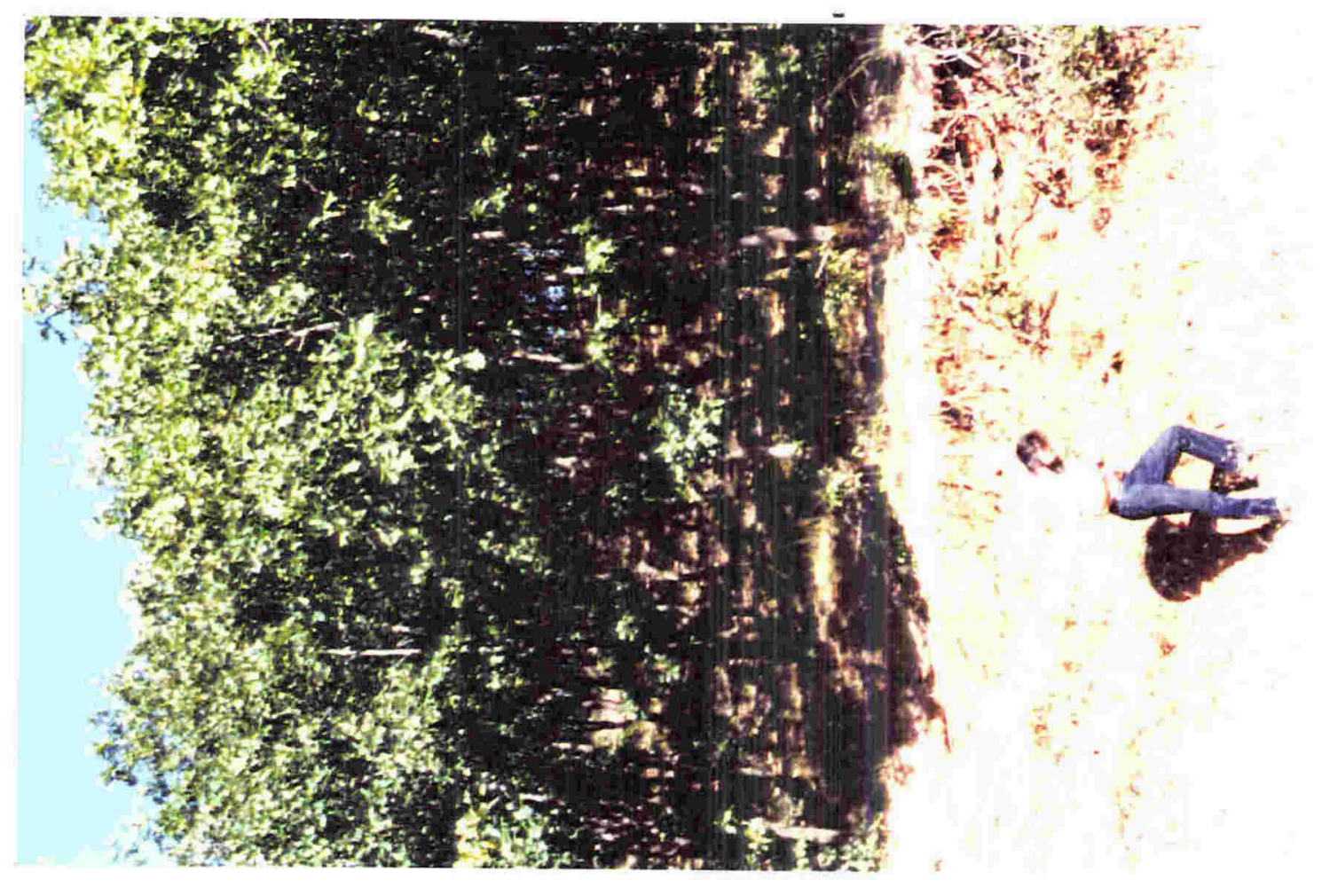

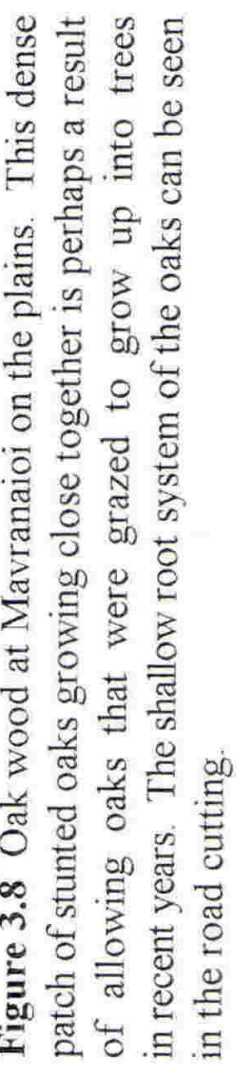


the others appear to hybridize and form a continuum in which some individuals are more or less typical of one or other species, but many are intermediate. Any one area is usually a mixture of at least three species. There is little ecological difference among the oaks except that Q. trojana frequents warmer and drier sites.

The oak woods are usually pure stands. Only occasionally other trees such as Fraxinus ornus (manna-ash) and Sorbus torminalis (service) occur in them. Juniperus (juniper) sometimes forms an understorey, this is less common in the foothills than in the plains.

Availability of moisture appears to be a factor in the spacing of the oak trees. Oaks in the foothills grow closer together than those on the drier plains. Much oak wood is interspersed with areas of grassland, usually of a very dry type. The growth of oaks has been episodic: in any one area there are usually two or three distinct ages of pollards or other big oaks. (The age of one giant oak was estimated at 700-1000 years old from annual rings.)

The structure of oak woods in the foothills varies from wood-pasture (scattered big oaks in grassland) to coppice-woods and sometimes to 'oak-pasture' (tracts of deciduous oak grazed by sheep and goats to less than $2 \mathrm{~m}$ high, sometimes with upstanding pollard trees as well). Oak wood-pasture is more common in the foothills than in the plains and usually occurs at the highest altitudes of the oak woods, with coppice-woods at a lower altitude. 'Oak-pasture' occurs mostly in the north.

The grassland between the trees of the oak wood-pasture includes many steppe species. The dominant grasses are perennials, species of Festuca and Poa timoleontis. Perennial herbs include Acinos alpinus subsp. alpinus, Erysimum linariifolium (a Balkan endemic), Euphorbia myrsinites; legumes such as Astragalus monspessulanus, Lathyrus digitatus, Trifolium physodes; and bulbs such as Muscari neglectum. Annuals include Cerastium brachypetalum subsp. pindigenum (endemic), Evax pygmaea, Lamium bifidum subspecies balcanicum (Balkan endemic), and Scleranthus uncinatus (cf. species noted near the surface sample site at Polinerion listed in Appendix VII, iv).

In their shady uncut state the ground vegetation of oak woods is rather sparse; it includes Aremonia agrimonoides, Buglossoides purpurocaerulea, Cephalanthera damasonium (an orchid), Cyclamen repandum, Fragaria vesca, Scilla autumnalis, and Stachys alopecuros (cf. Mirsina oak-wood quadrat, Appendix VIII). Vegetation is richer at the edges of woods, characteristic species being Dictamnus albus, Lathyrus laxiflorus, L. nissolia, and Melittis melissophyllum (Rackham \& Moody, in prep.).

Oaks produce acorns in the later part of each coppicing cycle, except Quercus frainetto, which takes at least 50 years. Pollards often carry the oak mistletoe Loranthus europaeus. After felling, coppice-woods develop a varied and characteristic flora of herbaceous plants distinct from (though overlapping with) the floras of steppe, woodland edges, and cultivation. 
They include perennials that are present (but suppressed) before felling such as Fragaria sp., Lathyrus laxiflorus, Melittis melissophyllum, Symphytum ottomanum; steppe plants (perhaps present in patches of steppe) such as Arenaria conferta (an endemic), Bellis sylvestris, Polygala nicaeensis, Trifolium repens; seed plants (growing from seed buried since last felling) Euphorbia amygdaloides, possibly Cerastium brachypetalum subsp. tenoreanum, Lathyrus aphaca, Ranunculus cf. constantinopolitanus, Veronica chamaedrys, and Vicia grandiflora; plants with a dispersal mechanism that allows them to move around the wood from one felled area to another, e.g. Cardamine cf. hirsuta, Senecio squalidus; and another plant of unknown origin Geranium asphodeloides (Moody \& Rackham, in prep.).

Near Polinerion at over $1000 \mathrm{~m}$, numerous pockets of deep soil were cultivated about 50 years ago, but probably not for long (Appendix VIII, Polinerion plot a). These areas are still very distinct from the adjacent undisturbed steppe. They are dominated by the annual grass Taeniatherum caput-medusae and other annual grasses, e.g. Bromus species and Cynosurus elegans, annual herbs such as Galium setaceum, and weeds such as Convolvulus arvensis and Polygonum aviculare (Moody \& Rackham, in prep.).

Oaks are resistant to grazing; they are quite palatable to livestock but can live even if grazed to less than a metre high. They are not killed by felling but sprout from the stump. They do not burn easily, but their litter can be ignited by adjacent stubble or grass fires, and young oaks are killed to the ground but sprout (Moody \& Rackham, in prep.). Oaks (except $Q$. frainetto) easily invade abandoned farmland and have undoubtedly increased at the expense of pasture: early last century there were probably wider areas of grazed oaks than there are now (Leake 1835).

\section{Mixed woods}

Two small limestone outcrops, one near Aetia and another above Spilaion and Zakas, have small areas of woodland growing on them that include a much greater number of species than any of the other woods in Grevena. Most of these species are rare or do not occur anywhere else in the province. These woods have been supposed to be relics of preglacial forests (Moody \& Rackham, in prep.). The woodland in the Aetia gorge, at $1000 \mathrm{~m}$, is very distinctive, with the common trees of Grevena missing apart from occasional oaks (Appendix VIII). Trees growing in the wood include a mixture of north European, central European, Balkan, and sub-Mediterranean species, including a surprising number of thermophilous trees for such a high altitude. This wood is partly inaccessible, due to the steep sides of the gorge, but where it is accessible there are scattered pollards, including such seldom-pollarded trees as Acer monspessulanum, Prunus mahaleb, and Tilia tomentosa, and intervening trees are coppiced and sometimes browsed. 
The mixed wood above Spilaion and Zakas includes most of the trees of the mixed wood near Aetia but differs from it in that it is dominated by Abies cephalonica and includes the two Mediterranean trees Phillyrea media and Pistacia terebinthus (terebinth) (Figure 3.7). This wood is browsed but has a protective understorey of unpalatable Buxus (box). With the protection of box, palatable species such as Phillyrea and hornbeam can grow into trees. This mixed wood continues on to flysch and ophiolite on the damp north side of Mount Orliakas. Here Tilia rubra (lime), Ulmus glabra (elm), Populus tremula (aspen), and Prunus avium (cherry) grow amongst the lower edge of the mountain beech and fir woods (Moody \& Rackham, in prep.).

Another mixed wood occurs on the southwestern boundary of the province between Kranea and Milea on ophiolite and gabbro cliffs. This wood differs from the two above on limestone in that there is not such a range of species, and the more common Fagus and Abies are usually dominant. Carpinus and Ostrya (hop-hornbeam) are frequent in this wood. Rare trees include Aesculus (horse-chestnut), Tilia tomentosa (silver lime), and Acer obtusatum (Moody \& Rackham, in prep.).

\subsubsection{Lowland plains}

The deciduous oak woods of the plains are discussed separately because their physiognomy, use, and structure differ from those of the oak woods of the foothills. Today cultivation dominates over oak woods on the lowland plains.

\section{Deciduous oak woods}

The plains are more arid than the foothills, and the effects of moisture deficit are greater. The plains are too arid to support continuous oak woodland as in much of the foothills. Intervals between trees covered with very dry grassland (steppe) are much larger than those of the foothills; the shadier north-facing slopes are covered with more trees than the southfacing slopes or the ridgetops (where not cultivated). Oaks have shallow roots that extend under the grassland and take moisture from it, creating a mosaic of oaks and steppe (Figure 3.8).

Moisture also appears to limit the growth of oak. Oaks in woods are extremely slowgrowing and often dead at the top. They can take 70 years to reach $15 \mathrm{~cm}$ in diameter, but free-standing oaks between fields in better-watered places can put on annual rings of $5 \mathrm{~mm}$ or more (Moody \& Rackham, in prep.). The present structure of patches of dense, stunted oak wood and patches of steppe may be to some extent artificial. It may be a result of allowing oaks that were grazed in the past to grow up into trees in recent years and compete with each 
other for moisture. The natural state would probably be a thin scatter of bigger oaks, with widely spreading root systems, within grassland (Figure 3.8).

Juniper, Juniperus oxycedrus, grows mostly as an understorey shrub in the lowland oak woods. In some areas it is more abundant than in others, forming a continuous understorey. It is not very shade-tolerant, and its dead remains (which persist for many decades) are evidence that the woods were once more open. In many areas juniper is completely absent, even in well-lit situations. This probably results from a history of burning the steppe or the leaf-litter of the woods: juniper is much more sensitive to fire than oak. The presence of juniper may also be related to the history of pasturage or of erosion (Moody \& Rackham 1988). The rare mistletoe Arceuthobium oxycedri grows on this juniper.

In very dry places wild pear, Pyrus amygdaliformis, is scattered among oaks (Moody \& Rackham, in prep.). Steppe in the plains may have a small component of undershrubs that belong to the Mediterranean garrigue, e.g. Teucrium polium and Cistus incanus. There is a great variety of herbaceous plants in plains steppe. Some are deep-rooted perennials like Galium verum and Dictamnus albus, or annuals such as Xeranthemum annuum. The steppe contains many endemics, including Achillea grandifolia, the Centaurea species, Iris reichenbachii, Onobrychis ebenoides, Stachys scardica, and Cerastium brachypetalum subspecies pindigenum. Many of the steppe species have affinities with Eastern Europe, and especially the Hungarian plain. These include the perennial pink Dianthus pontederae subspecies giganteiformis, Inula ensifolia, and Veronica chamaedrys subspecies vindobonensis (additional species are listed in Appendix VII, v \& vi).

Grasses of the steppe are perennials of an arid kind, a mixture of Mediterranean species such as Hyparrhenia hirta, Dichanthium ischaemum, and Chrysopogon gryllus and of Central European steppe grasses, e.g. Phleum phleoides, Koeleria splendens, and members of Festuca. There are also annual grasses such as Cynosurus echinatus and Aegilops and Bromus species.

\section{Hornbeam woods}

On the eastern side of the plains and on the less arid bases of the eastern mountains are hornbeam woods. Hornbeam (Carpinus orientalis) grows in damper places and has fewer patches of steppe than the plains oak wood. Near Mirsina hornbeam grows on the damper NW-facing slopes, while oak woods and shrubbery grow on the drier SW-facing slopes (Moody \& Rackham, in prep.). These woods are an alternative to oak wood and intergrade with it. They are not as pure as oak woods, having Fraxinus ornus (manna-ash) and Acer monspessulanum (maple) frequently occurring in them. Hornbeam is commonly grazed in the manner of oak-pasture. It is also coppiced, but not pollarded or shredded, for leaf fodder. 


\section{Mixed wood}

Eight km SE of Grevena on the Venetikos River is a mixed wood of an unusual type. It is dominated by deciduous oaks (at least 4 species occur here) with much hombeam. There are also many other trees, some Balkan and sub-Mediterranean (e.g. Acer opalus, Cornus mas, Fraxinus ornus, Tilia rubra), and some from central and northern European (Acer campestre, Corylus avellana, Salix caprea, Sorbus torminalis). A row of Tilia tomentosa overhangs the river. The rich flora of this small wood includes the rare Carex depauperata, $C$. depressa subsp. transsilvanica, Lathyrus laxiflorus, Primula elatior, and P. vulgaris. The flora is very much richer than oak woods apparently growing on similar soil (Moody \& Rackham, in prep.). This wood with its richer-than-usual flora may resemble more closely a natural vegetation than the more common pure stands.

\section{Shiblyak}

A Balkan-type of shrubbery, shiblyak, of long-lived deciduous plants, often deep-rooted, forms brushwood plant communities in dry places, particularly in the plains (Moody \& Rackham, in prep.). Although oak wood may develop from this shiblyak, it also forms a narrow permanent zone between woodland and steppe. It is more extensive on south-facing slopes and especially on eroding slopes too unstable for trees to become established, and without enough soil for grassland. Cotinus coggygria is characteristic of the latter situation; it appears to be adapted to having its roots exposed by erosion. On stable sites, shrub thickets often include Prunus spinosa, Colutea arborescens, and Paliurus spina-christi. Juniperus oxycedrus is a very abundant shrub in permanent shiblyak.

\section{Weeds and ruderals}

Weeds of arable land and ruderals of the Balkans have been defined by Turrill (1929). The following families are the most important, on the basis of the number of species contributing to the weed and ruderal floras: Compositae, Leguminosae, Cruciferae, Umbelliferae, and Gramineae. Unfortunately some of the weeds and ruderals, such as the ruderal grass Cynodon dactylon and the weed Cyperus rotundus, cannot be distinguished palynologically from other species that are not weeds or ruderals.

Extant weeds of cereals include Mediterranean, Balkan, and general European species such as: Consolida regalis subsp. paniculata, Dasypyrum villosum, Nigella arvensis subsp. arvensis, Orlaya kochii, Vicia pannonica subsp. striata, Buglossoides arvensis subsp. sibthorpiana, Agrostemma githago, Centaurea cyanus, Asperula arvensis, Brassica nigra, Erysimum cheiranthoides, Galium tricornutum, and Ranunculus arvensis (Moody \& Rackham, in prep.). 


\section{Hedges}

There is no tradition of planted hedges in Grevena, except for rows of almonds (Prunus dulcis). A few hedges occur around villages, defining small irregular fields. They are usually comprised of a mixture of species and usually include a greater variety of trees than woods; Ulmus (elms) and Fraxinus ornus being common. Sometimes they consist of only undershrubs such as bramble. Often they consist of shrubs with oaks at intervals. A common plant of hedges is Sambucus ebulus (danewort). Hedges may have been more common in the past before mechanized agriculture was introduced.

\subsubsection{Flood plains}

Most of the river terraces are cultivated, but a variety of wild vegetation survives in unstable areas. The flood plains of the Aliakmon, Venetikos, and Pramoritza and their bigger tributaries support woods of Alnus glutinosa (alder) and the willows Salix elaeagnos, S. alba, and S. amplexicaulis. The Balkan Tamarix parviflora (tamarisk) forms thickets in places with the Central European Myricaria germanica. Smaller woods of Platanus (plane) grow on the flood plains of the Aliakmon and Venetikos and their bigger tributaries, reaching $970 \mathrm{~m}$ above Kranea, high for a Mediterranean tree (Moody \& Rackham, in prep.). River gravels often have a rich flora, including endemics such as Silene haussknechtii, Dianthus gracilis subsp. haussknechtianus), and plants washed down from higher altitudes (Euphorbia myrsinites).

Along smaller watercourses are patches of suckering Populus alba (poplar) and Ulmus minor (elm). Springs often feed little clayey fens with thickets of Salix (willow) and Prunus cerasifera (cherry-plum), patches of bramble (Rubus species), and herbaceous plants that are often of widespread European species such as Equisetum telmateia, Lythrum salicaria, Anchusa arvensis, Scirpus holoschoenus, and Teucrium scordium (cf. Kellia, Appendix VIII).

\subsubsection{Eastern mountains}

Vegetation is usually very sparse on these extremely dry mountains and there are few trees. Exceptions to this occur in the south, where small patches of Abies, Pinus, and Fagus grow. Buxus sempervirens (box) forms permanent shrubbery in places in these mountains, especially on the dry serpentinite.

\section{Pseudo-maquis}

In the southeast corner of Grevena on a patch of limestone with red soils is a brushwood community of mixed evergreen and deciduous trees, pseudo-maquis. This corresponds to the pseudomacchie of Turrill (1929). Pseudo-maquis is related to the 'true' maquis of coastal and southern Greece, but unlike the 'true' maquis, which has several dominants, pseudo-maquis 
is usually dominated by only one species, such as Quercus coccifera, and can withstand harsher climatic conditions than 'true' maquis.

The stand in Grevena includes the Mediterranean evergreens of Quercus coccifera (prickly oak) and Phillyrea media; deciduous Mediterranean species Acer sempervirens, Crataegus heldreichii (hawthorn), and Pistacia terebinthus (terebinth); Cornus mas and Paliurus spina-christi; and the deciduous temperate trees Quercus brachyphylla, Q. trojana, and Carpinus orientalis (hornbeam).

The stand forms a kind of oak-pasture structure, in which most of the trees are browsed into shrubs, with occasional pollards of deciduous oak and maple. Some of the pollards are 200-300 years old. Although sheep and goats still browse in winter, some prickly oaks (evergreen oaks) are growing into trees, often through the protection of juniper bushes.

\subsection{2 'Natural' vegetation of Grevena}

The extant vegetation of Grevena resembles to some extent the 'natural' undisturbed vegetation cover that would grow in similar climatic conditions, especially in the dominant tree flora and vegetation. The major vegetation zones of pine at the highest montane altitudes, Fagus/Abies at lower montane altitudes and oak woods in the foothills and lowlands are probably close to major altitudinal zones that would exist in undisturbed vegetation. The tree line, however, would probably be higher, as it has probably been depressed by grazing.

The vegetation of the foothills and lowland plains is limited by rainfall. Coppicing has increased the number of tree stems per area, so increased competition between trees results in a slow growth rate. In wood-pasture the number of trees per area has been reduced, resulting in a relatively fast growth rate. The natural undisturbed state would thus be one of fewer trees in the coppice woods and more trees in wood-pasture.

Small patches of mixed woods growing in the less disturbed areas of today, scattered amongst almost pure stands in disturbed areas, suggest that an undisturbed vegetation would be more varied in taxa than is common today. In the foothills and lowlands oaks would be dominant, but there would be a wider range of trees, especially lime and hornbeam. The common two-layer structure of much of the woodland today, with ground and canopy cover only and little or no understorey, is probably a result of grazing. Undisturbed woodlands would have more luxuriant understorey. In the foothills trees would be more continuous, but the lowlands would be more savannah-like. Shiblyak would be abundant, especially on unstable land. 


\subsection{Surface samples}

The results of a study investigating the relationship between the extant vegetation of Grevena and pollen deposition are given below. Due to the time constraints of the field season (and the impossibility of quantifying many relevant variables - Lichti-Federovich \& Ritchie 1965), which resulted in the small sample size of the present study, a rigorous statistical analysis (e.g. Davis 1963; Webb et al. 1981; Bradshaw \& Webb 1985; Prentice 1986a) was not attempted, and a semiquantitative approach is adopted instead. A comparison is made between the actual distribution of the vegetation units described above, the relative abundance of plant taxa within these units, and relative frequencies of contemporary pollen spectra. Microscopic charcoal abundance and dispersal is also reported. Counts of all pollen and spore types and charcoal particles are listed in Appendix IX.

\subsubsection{Sample collection sites}

The selection and description of surface-sample sites is based on the Moody and Rackham classification. The aim was to sample the range of major vegetation communities described by Moody and Rackham (in prep.) along a west-to-east transect beginning in the Pindos Mountains and traversing the Pindos foothills and the lowland plains. Unfortunately, time precluded sampling the slopes of the Vourinos Mountains in the east. The locations of the 14 surface samples of the present study (and those of Bottema 1974, see below) are shown in Figure 3.2. Site descriptions and species lists, with numerical ratings where quantitative surveys were undertaken, are given in Appendix VIII.

The geographic area represented by the pollen data (pollen source area) varies, but where duplicate samples were collected from the same plant community they are approximately the same (Andersen 1970; Jacobson \& Bradshaw 1981; Prentice 1986a). The two mountain sites in the pine wood-pasture plant community, Avgo and Gomara, are both small closed-drainage lakes (basin size less than $200 \mathrm{~m}$ diameter with no inlet streams) in open environments. The beech-wood sites at Anelia are approximately $30 \mathrm{~m}$ apart; one in the woods and the other on a small bog $(90 \times 50 \mathrm{~m})$ surrounded by the beech wood. Samples from the two mixed woods growing on limestone in the Pindos foothills, Zakas and Aetia, were both collected under the canopy of the woods. In the lowlands, two samples were collected from open environments surrounded by cultivated fields (although one site is a fen, Kellia, and the other a cultivated field, Asprokampos). Samples collected from the oak woods, Asprokampos and Mirsina, in the lowlands were both collected under the canopy of open woods. 


\subsubsection{Sample material and pollen preservation}

Several different types of sample material were used; peat from the surface of wetlands, moss polsters, soil, and litter. In general preservation of palynomorphs was good in all samples. Preservation was best in samples from the wetlands, where it was excellent. Palynomorphs extracted from moss polsters collected from damp locations were well preserved, except that in some samples pollen of Fagus had been attacked by fungus (e.g. samples from Anelia woods and Agios Nikolaos). Pollen and spores from dry samples were less well preserved, being broken and crumpled (but the exine is normal - Cushing 1967), or in the worst cases degraded (thinning of exine) (Delcourt \& Delcourt 1980). The latter case applies to samples from Asprokampos woods and Polinerion, where the maximum percentage frequency of indeterminate grains of $9 \%$ was recorded. The dry-litter sample collected from Mirsina woods contained poorly preserved, degraded palynomorphs, while the palynomorphs extracted from the surface soil at Asprokampos suffered little degradation, perhaps because of a shorter period of accumulation.

In some cases poor preservation prevented identification of grains, but the composition of the pollen flora was probably not altered greatly, judging from the small amount of corrosion seen (Havinga 1984).

\subsubsection{Pollen diagram}

Pollen and spore relative frequencies are presented in Figure 3.9 (in two parts - in pocket). The pollen sum on which the relative frequencies are based includes all identified terrestrial pollen and spore taxa and excludes marsh and aquatic taxa. Relative frequencies of $0.2 \%$ or less are indicated with a dot.

Pollen spectra have been arranged according to landform and vegetation zones (more or less from highest elevation to lowest) following the west-east sampling transect and the above description of extant vegetation.

Pollen and spore types are arranged according to habitats drawing on data from Turrill (1929) and other sources (Appendix X). Many pollen types contain species that grow in a variety of habitats, and assignment to a particular habitat presents some difficulties. In general in such cases assignment is based on consideration of the habitat where plants of that pollen type were seen growing and/or the type of site where the surface sample was collected. A separate group for steppe plants is not included, as plants growing in steppe are not exclusive to that community; they all occur in other communities.

Two plant groups associated with anthropogenic activities (anthropogenic indicators) have been added: cultivars and herbaceous plants of open ground. The latter group includes herbaceous plants that are light-demanding and are therefore increased by human activities 
(and natural catastrophes), which remove forest cover. This group includes many weeds and ruderals (Bottema 1982). Many of these plants may also grow in natural grasslands. In this study the terms 'plant communities', 'weeds', and 'ruderals' follow the definitions of Art (1993).

Pollen types within habitat groups are arranged alphabetically except for the dominant tree types of Pinus (Diploxylon), Fagus, Quercus frainetto type, and Quercus trojana type, which are placed at the beginning of the 'coniferous and deciduous canopy trees' group.

Two summary diagrams are shown in part 2 of the diagram: one showing the ratio of terrestrial woody (trees and shrubs) to herbaceous plants; and the other, summary curves of each habitat group. A curve of palynological richness (rarefaction analysis) follows (Birks \& Line 1992; Bennett 1994). The number of terrestrial pollen types (not normalized for unequal pollen sums) in each spectrum is shown next. Then the number of pollen grains of trees and shrubs, the total number of all terrestrial pollen and spores, and the total of all pollen and spores counted is given.

Charcoal abundance is expressed as frequency of particles of charcoal, in size classes based on the projected particle area, relative to the terrestrial pollen sum (Patterson et al. 1987).

\subsubsection{Relative pollen dispersal and representation of various plant taxa}

Reconstruction of past vegetation from fossil pollen spectra can be considerably aided with information regarding the dispersal potential of different species. This assists with interpreting the source area of represented taxa and thus the distribution of the original vegetation (Oldfield 1970). Difficulties in interpretation arise because a well dispersed pollen type might be abundant in a pollen diagram, but this does not necessarily imply that the parent species was growing close to the sample site. On the other hand, abundance of a poorly dispersed pollen type indicates that the parent plant was growing in the vicinity. Hamilton (1972) introduced a three-class classification of pollen types in terms of dispersal power (termed 'relative export' by Flenley $(1973: 137)$ ). Pollen types that occur in all or almost all surface samples wherever collected are designated of high dispersal power. Those that occur in samples from adjacent areas are of medium dispersal power, and pollen types occurring only in samples from vegetation in which parent plants are growing are of low dispersal power. This classification assists with the distinction of the pollen source area and thus with reconstructing the actual distribution of the vegetation from fossil assemblages. The concepts of local and regional pollen deposition as defined by Janssen (1973) are adhered to in this study.

Relative dispersal and representation of pollen and spore types whose source plants were noted in the areas surveyed and/or whose pollen was present in the surface pollen samples are 
summarized in Appendix XI. The term 'representation' refers to the relationship between the registration of a taxon in the pollen count and in the actual vegetation (Faegri \& Iversen 1989:3). It differs to a large extent due to differences in pollen productivity, dispersal, deposition, and preservation (and the various treatments that analysts apply and their ability to identify pollen and spores (Bottema 1982)) (Havinga 1964, 1967, 1984; Tauber 1965; 1967; Erdtman 1969; Webb et al. 1978; Prentice 1985). Known pollination mechanisms have been included in Appendix XI as an indication of pollen production, that is, anemophilous plants generally produce much more pollen than plants with other pollination mechanisms (Knuth 1906:7; Erdtman 1969).

A discussion of the relative dispersal and representation of various taxa follows. This discussion is based on surface samples and so does not take differential preservation in sediments into account. The spectra from the surface sample of the wetland core sites provides the best modern analogue for that wetland sequence (although they may contain pollen that would not have been preserved). It is not easy to assess the dispersal abilities of pollen types with low frequencies. Accordingly, the dispersal abilities of common pollen types produced by common species are evaluated mostly. The failure of many pollen types to appear in the spectra is attributed to low pollen production, poor dispersal, or differential decomposition.

Pinus The long-distance dispersal capability and enormous production of Pinus pollen is well known (Moore et al. 1991; Faegri \& Iversen 1989). Results of this study are consistent with these characteristics. Even pollen spectra of the lowland plains sites record pollen relative frequencies that are quite high (13.5 to 19.8\%). An exception is the cereal field site at Asprokampos, where large relative frequencies of local weeds suppress the pine relative frequency to $3.5 \%$.

Abies Abies is strongly under-represented in the pollen record of the site where it is growing. At Zakas Abies cephalonica forms $60 \%$ of the canopy, but it is represented by less than $15 \%$ in the pollen diagram. Thus pollen production appears to be small, but dispersal is good as small relative frequencies are recorded in the pollen record at most sites, even though it is known to grow at only two locations in Grevena, at Zakas, and in the southeast (Figure $3.2)$.

Fagus Relative dispersal and representation of Fagus pollen are similar to that of Abies; it is under-represented at sites where it grows but is dispersed long distances at small relative frequencies. Fagus pollen is recorded at all sites, but the sample collected beneath a beech wood canopy, where pollen deposition is expected to be primarily local (Anderson 1970, 1974), recorded only $29.4 \%$ relative frequency. The bog beside the wood recorded $23.5 \%$. 
Quercus Quercus has been divided into three pollen types: Quercus frainetto type includes deciduous species only, Quercus trojana type includes mostly species that are semievergreen or deciduous (one species, $Q$. cerris, is deciduous), and $Q$. coccifera type includes evergreen species (van Benthem et al. 1984).

Habit and pollen morphology of the species that belong to Quercus frainetto type and Quercus trojana type are very similar, so pollen production and dispersal are probably also very similar. They have high relative dispersal power and are recorded at almost all sites. The species belonging to these two pollen types occur in a number of different vegetation communities, and relative pollen representation differs according to plant community. At Perivolion, where oaks ( $Q$. frainetto and $Q$. trojana types) represent $1 / 3$ of the tree canopy and pine the remaining $2 / 3$, only $5.7 \%$ oak pollen is recorded. At Polinerion in oak wood-pasture, where oak represents only $5 \%$ of the vegetation, $26.5 \%$ is recorded in the pollen spectrum. In the steppe/oak woods at Asprokampos, where the canopy is made up entirely of oaks (although this is only $50 \%$ cover), oaks record $40 \%$ pollen relative frequency. Representation may be affected by coppicing and pollarding.

The habit and pollen morphology of the two evergreen species, Q. coccifera and $Q$. ilex, included in the Quercus coccifera pollen type, are different from the species included in the above types. Quercus coccifera type is represented in almost every pollen sample but does not grow at any of the sampling sites. Only $Q$. coccifera is known to grow in Grevena and then only in a small stand in the southeast corner. Pollen representation is greatest on the western side of the province. Pollen in these spectra are presumed to be transported from woods on the western (windward) side of the Pindos Mountains (Moody \& Rackham, in prep.). Bottema (1974) also found Q. coccifera is over-represented.

Carpinus type and Ostrya type These two types are combined because the same three species occur in both pollen types, although in different proportions; Carpinus type is represented mostly by Carpinus betulus, while Ostrya type is represented mostly by Ostrya carpinifolia and Carpinus orientalis (Bottema 1974:23). Both types have good dispersal as they appear in almost all spectra. However, production may not be very high, as the relative frequencies of pollen recorded at sites where species belonging to these types occur are about the same as at sites where no plants are growing. At the sampling site of Mirsina woods, Carpinus sp. forms $15 \%$ of the sub-canopy layer under an oak canopy, but pollen of Carpinus type records only $1.9 \%$ and Ostrya type $1 \%$. Species which belong to the two types occur as canopy trees in the mixed deciduous woods at Zakas and Aetia, but only $0.5 \%$ and $0.7 \%$ of Carpinus type are recorded at the two sites respectively, while Ostrya type records $5.8 \%$ and $3 \%$ respectively. These relative pollen frequencies are no higher than at sites where species of this types are not recorded, e.g. at Anelia bog Ostrya type is $4.8 \%$ and at Asprokampos 
woods $4.5 \%$. The situation is the same for Carpinus type, but the relative frequencies are lower. Bottema (1974) also found that low shrubs of Carpinus orientalis are poorly represented.

Corylus Corylus also has good dispersal, but pollen production is not very high. Corylus pollen is represented at almost all sampling sites yet is recorded growing only at Aetia and near Mirsina fen. The surface sample from Aetia was collected beneath a Corylus canopy tree, and even here the pollen relative frequency is only $5.9 \%$. Pollen relative frequencies recorded where no Corylus is growing reach $2 \%$ at Asprokampos woods.

Tilia cordata type Tilia species grow as canopy trees in the two mixed deciduous woods at Zakas and Aetia. Relative pollen frequencies of $0.02 \%$ were recorded at each of these sites. Pollen was not recorded at any other sites. Pollen relative dispersal is low, so Tilia is under-represented (it is entomophilous).

Castanea sativa Castanea sativa is represented by very low frequencies in several spectra. No trees of this species were seen in Grevena, but dense woods exist just to the north and west (Bottema 1974; Polunin 1988; Moody \& Rackham, in prep.).

Platanus orientalis Platanus orientalis is self-propagating in Grevena but is probably also planted. It does not grow at any of the sampling sites, but it forms part of the riverine vegetation. Pollen was identified in most of the samples at very low relative frequencies (usually less than $2 \%$ ), suggesting that dispersal is medium. Perhaps production is not very high.

Juglans regia Juglans regia is the most cultivated tree in Grevena. No trees were recorded close to any of the sampling sites, but pollen grains were recorded at several sites at very low relative frequencies. Dispersal is probably high, but perhaps production of pollen is not very high.

Fraxinus ornus Although Fraxinus ornus trees were recorded at only three sampling sites, it is probably quite widespread in the extant vegetation of Grevena, albeit scattered and sparse. Pollen was recorded at several sites, thus relative dispersal of pollen is good. Relative representation is recorded as "over" in the summary table (Appendix XI) because pollen was identified at sites where no plants had been recorded. However, when relative frequencies of pollen abundance are compared to the relative abundance of its occurrence in the vegetation at the sampling site, it does not appear to be over-represented. Bottema (1974) found Fraxinus ornus to be well-represented.

Salix triandra Salix triandra pollen was identified in samples from Anelia bog, Kellia fen, Asprokampos field, Mirsina woods, and Mirsina fen. Salix species grow at the Kellia and Asprokampos sites. The Mirsina sites are close to the Aliakmon River, and Salix species, although not Salix triandra, have been identified in the flood plain vegetation of Grevena. 
Salix may have been growing in the vicinity of Anelia bog. No estimates were made of the relative cover of Salix at the sampling sites. At the two fen sites relative pollen frequencies of $11.9 \%$ and $14.4 \%$ are recorded. The available data suggest that Salix triandra has low relative dispersal but is locally well-represented.

Juniperus Juniper is widespread in Grevena. It is represented in all the surface sample spectra except that of Avgo in the Pindos Mountains. Relative frequencies are greatest in the foothill sites but are never greater than $16 \%$.

It is impossible to determine the relative dispersal of juniper pollen, as plants are so widespread in Grevena. The relative representation of juniper pollen appears rather erratic, probably because it grows in such a variety of plant communities and is affected by the relative frequencies of other pollen types in the same way as the deciduous oaks. However, it is probably under-represented. It is interesting to note that the digestion of juniper pollen by Collembola has been recorded (Scott \& Stojanovich 1963).

Buxus sempervirens Buxus is also widespread in Grevena. Relative dispersal is poor; it is only represented in the pollen spectra where it occurs at the sampling site. Relative representation is probably good. Bottema (1974) found that Buxus has rather good pollen production but poor dispersal.

Cotinus coggygria This shrub is widespread in the plains as part of the shiblyak community. No pollen was found. Bottema (1974:42) obtained a similar result.

Polygonum aviculare type Polygonum aviculare type includes well known ruderals and weeds of cultivation. Species belonging to this type were recorded at the sites of Polinerion, Kellia fen, Gomara bog, and Asprokampos field. At Gomara bog Polygonum sp. (probably $P$. aviculare) covered over $40 \%$ of the surface of the plot in the driest zone of the bog. At Asprokampos, Polygonum aviculare was the dominant weed growing in the cereal field.

Polygonum aviculare type pollen occurs in the pollen spectra of all the sampling sites except Anelia woods and Aetia. Relative pollen frequency is always less than $1 \%$ except at Kellia fen, where $2.2 \%$ relative pollen frequency was recorded, and Gomara bog and Asprokampos cereal field. At the latter two sites, $3.1 \%$ and $26 \%$ respectively were recorded. It is difficult to compare the relative importance of Polygonum aviculare type at these two sites because of the effect of the over-representation of pine pollen suppressing the relative frequencies of other pollen types. The effect would be much greater at the mountain site of Gomara, where $51.3 \%$ relative frequency is recorded for pine, than at the lowland site of Asprokampos, where only $3.5 \%$ is recorded.

Although this type is autogamous and entomophilous implying low pollen production and dispersal, it is well represented, and its dispersal capability is greater than expected. This can be explained by its system of autogamy. Self-fertilization is achieved by pollen falling 
from the anthers onto the stigma (Knuth 1909:348). During this process some pollen would undoubtedly drop to the ground (and perhaps later be caught up in wind currents) while other pollen may be dispersed by wind currents when falling.

Plantago lanceolata type Plants belonging to this pollen type were recorded at the sites of Anelia bog, Polinerion, and Asprokampos field. Plantago lanceolata type is represented in all the pollen spectra, except at Avgo. Relative pollen frequency is usually less than $1 \%$ at sites where it was not recorded in the vegetation. At sample sites where plants were recorded, usually between 2 and $4 \%$ cover, relative pollen frequency is between 2.3 and $2.5 \%$.

The relative dispersal of this pollen type is medium. It is considered to be overrepresented because it is present in the pollen spectra for sites where it is not growing locally. At sites where it is growing it appears to be well-represented.

Chenopodiaceae The only sampling site where species belonging to this pollen type were recorded was Asprokampos field. Pollen of this type is recorded in spectra from all sites except Aetia. Relative pollen frequencies are less than 1\%, except at the sites of Anelia bog, Kellia fen, Asprokampos fields, and Asprokampos woods, where relative frequencies range from $1.2 \%$ to $2.55 \%$. This pollen type has high relative dispersal and is over-represented.

Rumex acetosa group Plants belonging to this pollen group were not observed at any of the sampling sites. Pollen belonging to the group is represented in the pollen spectra of most of the open sampling sites. Relative pollen frequencies are less than $1 \%$, except at Anelia bog, Kellia fen, and Asprokampos field. This pollen type has medium relative dispersal and is over-represented.

Compositae A number of Compositae pollen types are represented in the pollen spectra. They are all represented by extremely low relative frequencies (usually less than $0.5 \%$ ), even when they are abundant in the local vegetation. This is expected, for they are mostly entomophilous. The highest relative pollen frequencies occur in the spectrum of the Kellia fen site, where these weeds are abundant, but even here, where Cirsium is recorded as "frequent" on the fen and recorded with $20 \%$ cover in one of the plots, relative pollen frequency reaches only $8.6 \%$. With the exception of Artemisia, Compositae pollen types are extremely under-represented and dispersal is low.

Artemisia, on the other hand, is recorded in several pollen spectra (relative frequencies less than $0.7 \%$ ), although not recorded in the vegetation at the sampling sites. It is known to be anemophilous and well dispersed.

Pteridium Pteridium grows in the woods and around the bog at the Anelia sites and as understorey at Agios Nikolaos (30\% cover), Perivolion (10\% cover), and Zakas ( $15 \%$ cover). Pteridium spores occur in only five pollen-diagram spectra: at the four wetland sites and at 
Zakas. Relative frequency is less than $1 \%$ except in the Gomara spectra, where it is $1.3 \%$. Pteridium has medium relative dispersal and is under-represented.

Dactylis type Species belonging to this pollen type grow in the wetland sites of Avgo, Anelia ("+"), and Kellia and in vegetation surrounding the bog at Anelia (up to $10 \%$ cover in one plot) and Mirsina fen (up to $15 \%$ cover in one of the plots). It also grows in woodlands and grasslands: Perivolion (15\% ground cover), Polinerion (up to $5 \%$ ground cover in one of the plots), Zakas ( $0.5 \%$ ground cover), and Asprokampos woods and Mirsina woods ( $15 \%$ ground cover).

Pollen is recorded in all pollen spectra except that of Agios Nikolaos. Relative pollen frequencies are less than $1 \%$ at Avgo, Anelia woods, Polinerion, Zakas, and Aetia, but at Gomara 6.4\%, Anelia bog 5.4\%, Perivolion 1\%, Kellia fen 2.8\%, Asprokampos field $15.6 \%$, Asprokampos woods 4.5\%, Mirsina woods 5.6\%, and Mirsina fen 5\% is recorded.

Relative representation of this pollen type is difficult to determine because pollen may be contributed by either the local vegetation of the wetland and/or the regional vegetation. Relative representation of this pollen type is lower than expected. This may be due to grazing preventing flowering.

Festuca type Species contributing to the Festuca type grow at the sites of Avgo, Agios Nikolaos ( $30 \%$ ground cover), Polinerion (dominant ground cover), Zakas, in steppe close to Kellia fen, Asprokampos woods, Mirsina woods ( $5 \%$ ground cover), and Mirsina fen ( $15 \%$ ground cover in plot d).

This pollen type comprises wild grasses of grassland. Festuca type occurs in every pollen spectrum except that of Perivolion. Relative pollen frequencies are lowest at the wetland sites of Anelia bog, Kellia fen, and Mirsina fen, where they range from 0.9 to $1.4 \%$. They are also low in the Pindos Mountains, but this may be partly due to the overrepresentation of pine. The highest relative frequencies of $23 \%$ are recorded at Polinerion in an oak wood-pasture.

Pollen of this type is probably severely affected by grazing, e.g. the relative pollen frequencies at the sites of Avgo and Gomara in pine wood-pasture are only $3.3 \%$ and $2 \%$ respectively, while that at the site of Anelia woods under the canopy of beech wood is $3 \%$. Pollen of this type is probably mostly contributed from regional sources.

Avena This pollen type includes pollen from both domesticated cereals and wild species. Domesticated cereals (oats) belonging to Avena are known to have been grown in Grevena before 1940 but are not known to be cultivated now. Plants producing Avena pollen are recorded in the vegetation of only one site, the Asprokampos cereal field. The species is probably the wild Avena sterilis. Relative pollen frequencies are less than $0.5 \%$ in three of the 
four sites where it is recorded. In the spectrum from the cereal field at Asprokampos, the relative frequency increases to $3.1 \%$.

The pollen record appears to be one of wild species of Avena (domesticated cereals producing pollen of this type are autogamous, and domesticated oats is pseudocleistogamous). The pollen source at Asprokampos is probably wild plants, such as Avena sterilis, behaving as weeds of cereals (perhaps growing from seed in contaminated seedstocks), and/or cultivation relics (Thurston 1982).

Hordeum type This pollen type includes both domesticated cereals (barley and einkorn) and wild grasses. Only one grain of this pollen type was identified (from Kellia fen). No pollen was identified in the sample from the cereal field at Asprokampos, which had been planted in barley the previous season.

Plants with pollen that possibly belongs to this pollen type occurred at only two other sampling sites; Polinerion and Asprokampos woods. No pollen was identified in either of the samples from these sites. Domesticated barley is cleistogamous (Knuth 1906-9).

Secale cereale This pollen type includes pollen of only the cereal Secale cereale (rye). No vegetation at the sampling sites included plants of Secale cereale, but it is currently planted as a crop on a small scale. Pollen of this type is recorded in several spectra in very low relative frequencies, $0.6 \%$ and less, even at the sites surrounded by cultivated fields.

Triticum type This pollen type includes only domesticated cereal species. Plants that produce Triticum-type pollen were identified at two sampling sites; Asprokampos field and Mirsina fen. Pollen is recorded in several spectra with relative frequencies less than $0.6 \%$. In three pollen spectra relative frequencies are higher; Kellia fen, Asprokampos field, and Asprokampos woods, reaching $4.4 \%$ in the Asprokampos field sample. Wheat may have been planted in the field prior to the year it was sampled. These relative frequencies are extremely low considering the amount of wheat cultivated in Grevena, especially in the lowland plains.

Zea mays Zea mays was not recorded in the vegetation at any of the sampling sites, but pollen was identified in four samples; one grain only being identified in the sample from Gomara. Relative frequencies of $0.8 \%, 2.1 \%$, and $0.2 \%$ were recorded at the lowland sites of Kellia fen, Asprokampos field, and Mirsina woods respectively. These relative frequencies are very low in comparison with the amount of Zea mays cultivated in Grevena.

Carex flacca type and Carex hallerana type Carex species were identified in the vegetation of several sampling sites. Carex hallerana type includes species that grow in marshes as well as some that prefer dry habitats, while Carex flacca type includes mostly species that prefer damp sites. In agreement with this distribution, Carex hallerana type pollen is recorded in many pollen spectra, both wetland and dryland, while Carex flacca type pollen is recorded in the spectra of samples from only wetland sites. 
Cyperus longus type Species that produce Cyperus longus type pollen grow on the fens at Kellia and Mirsina. This pollen type is represented in the pollen record for these two fens as well as for the Gomara wetland site and the Anelia woods site. Pollen representation for the last site may be due to small numbers of plants growing on the bog $30 \mathrm{~m}$ away and not to long-distance dispersal; it is present in upper levels of the Anelia core. It is the most abundant (by far) marsh pollen type represented in the Kellia fen spectra.

Cyperus michelianus This species was not identified in the vegetation at any of the sampling sites, but some of the Cyperus species that grow on the fens at Kellia and Mirsina may be $C$. michelianus. Pollen from this species is recorded at the wetland sites of Avgo, Gomara, Anelia bog, Kellia fen, and Mirsina fen. It was also recorded in the spectra from Asprokampos field (at very low frequency).

Glyceria type At Gomara Glyceria species cover $20 \%$ of the ground in the driest sampling plot. Pollen is recorded at the four wetland sites, including Gomara, where it is most abundant. It was not recorded in the vegetation at the other wetland sites and may occur in the pollen spectra as a result of long-distance dispersal, but it is more likely that it actually grew at the wetland sites since some of the species belonging to this type are marsh plants.

Phragmites australis Pollen of this species is recorded in spectra of samples from all the wetland sites as well as from the Asprokampos field site. Mirsina fen is the only sampling site where Phragmites australis grows close to the sampled area. Here it completely covers the fen, but pollen relative frequency is not much greater than that of Cyperus michelianus. Carex hallerana and Cyperus longus also have high relative frequencies.

Sparganium erectum Kellia fen is the only sampling site where Sparganium erectum was seen growing. Pollen of this marsh plant was identified in samples from Kellia fen, Mirsina woods, and Mirsina fen. At Kellia this pollen type recorded quite high relative frequency. At the other two sites relative frequency is low.

Sparganium angustifolium Few pollen grains of aquatic plants are recorded; relative frequencies are less than $1 \%$ with the exception of Sparganium angustifolium, which reaches $1.7 \%$ in the spectrum of Kellia fen.

\subsubsection{Characterisation of the vegetation zones in the pollen spectra}

Inspection of Figure 3.9 shows that the pollen composition of each vegetation zone as classified by Moody \& Rackham (in prep.) is distinctive. The ranges of relative frequencies of dominant tree species for each zone are given in Table 3.1. The Pindos Mountains are characterised by large relative pollen frequencies of Pinus and Fagus and small relative frequencies of Quercus pollen (Perivolion an exception with very low relative frequencies of Fagus). The Pindos foothills are characterised by lower relative frequencies of Pinus pollen 
and higher relative frequencies of Quercus and Juniperus pollen than in the mountains, very small relative frequencies of Fagus pollen, and high relative frequencies of Festuca type pollen. The lowlands are characterized by low relative frequencies of Pinus pollen, almost no Fagus pollen, high relative frequencies of Quercus, high relative frequencies of herbs of open ground and cultivated cereals.

Table 3.1

Range of pollen relative percentages by vegetation zone

$\begin{array}{llll} & \text { Pindos Mountains } & \text { Pindos foothills } & \text { Lowland Plains } \\ & & & \\ \text { Pinus (Diploxylon) } & 30.3-84.8 \% & 18.5-26.5 \% & 3.5-19.8 \% \\ \text { Fagus } & 0.5-29.4 \% & 0.05-2.3 \% & 0.6-1.4 \% \\ \text { Quercus frainetto type } & 0.3-8.8 \% & 14.6-21.9 \% & 1.2-19.8 \% \\ \text { Quercus trojana type } & 0.0-0.5 \% & 0.7-18.8 \% & 3.1-39.5 \% \\ \text { Abies } & 0.0-0.6 \% & 0.2-14.6 \% & 0.2-4.2 \% \\ \text { Ostrya type } & 0.8-4.8 \% & 1.7 \%-5.8 \% & 0.4-4.5 \% \\ \text { Juniperus } & 0.0-6.6 \% & 12.3-16 \% & 0.4-5.1 \% \\ \text { Festuca } & 0.0-4 \% & 8.7-32.1 \% & 0.9-11.2 \% \\ \text { Herbs of open ground } & & & \\ \text { (excluding Festuca } \text { type) } & 2.3-21.5 \% & 4.7-7.7 \% & 14.6-64 \% \\ \text { Cereales } & & & \\ \text { (excluding Avena) } & 0.0-0.5 \% & 0.0-0.2 \% & 0.6-6.9 \%\end{array}$

The relative pollen frequencies of vegetation communities within these zones are discussed below.

\subsubsection{Pindos Mountains}

Pine wood-pasture, Pinus leucodermis The pine wood-pasture vegetation community is characterised by the combination of very high relative frequencies of pine pollen $(50-70 \%)$, with reasonably high relative frequencies of Fagus (3.9\%) and also of herbs of open ground (14.6-23.4\%). Herbs that are particularly well represented are Dactylis type and Festuca type: $3.4 \%$ at Avgo and $6.5 \%$ at Gomara (both pollen types combined). The Plantago pollen types and Compositae (both Tubuliflorae and Liguliflorae) are abundant at the Gomara site.

Only the pine woods and pine/oak woods communities record such high relative pollen frequencies of pine. Pine wood-pasture is distinguished from those vegetation communities by its higher relative frequency of Fagus and the occurrence of herbs of open ground. 
Beech woods The beech wood community is distinguished from all other plant communities by its high relative frequency of Fagus pollen $(29.4 \%)$, which is much higher than in any other community.

Pine woods, Pinus nigra The pine woods community is characterised by high relative frequencies of pine pollen (64\%). The spectrum for this community is very similar to that of the pine/oak woods, and indeed the relative frequency of Quercus is slightly higher in the pine woods spectrum than in the pine/oak woods spectrum. This is probably a result of the smallscale mosaic nature of the plant communities in this particular area of the pine woods. A distinguishing feature between these two communities might be the greater relative frequency of juniper ( $6.6 \%$, in this spectrum) in the pine woods.

Pine/oak woods As discussed above, the pollen spectrum of this community is very similar to that of the pine woods. In an ideal situation, one would expect a greater relative frequency of oak to be the distinguishing feature between the two communities. The relative frequency of pine in the spectrum from this community is $84.8 \%$, while Quercus is $5.7 \%$.

\subsubsection{Pindos foothills}

Oak wood-pasture The pollen spectrum of this community has a much reduced relative frequency of pine pollen (18.5\%) compared to the plant communities of the Pindos Mountains. Oak relative frequency ( $Q$. frainetto $21.9 \%$ and $Q$. trojana $4.6 \%$ ) is greater than those of the Pindos Mountain communities. Juniperus (16\%) and Festuca type (23.1\%) pollen are also more abundant.

Mixed woods The two mixed woods are characterised by their diversity of tree pollen types. However, only Pinus and Quercus (deciduous and semi-evergreen) record higher than $20 \%$ relative frequency. Apart from Abies, which is the dominant canopy tree at Zakas and is represented by $14.6 \%$ relative frequency, Corylus and Ostrya record the next highest relative frequencies of not more than $5.9 \%$. The relative frequency of the remainder of the trees is very low, less than $1 \%$.

\subsubsection{Lowland plains}

Cultivated fields The two sites of Kellia fen and Asprokampos field are surrounded by cultivated fields of mostly cereals. The pollen spectra from these sites are distinct from those of the other lowland sites in their high relative frequencies of herbs of open ground and lower Quercus frequencies. Herbs of open ground reach $36.3 \%$ for Kellia and $70 \%$ for Asprokampos field. At Asprokampos Polygonum aviculare type is particularly well represented at $26.7 \%$. Other herbs of open ground that exhibit higher relative frequencies at these sites than at other sites are Rumex acetosa group (1.7 and 1.9\%), Chenopodiaceae (2.1 
and $2.5 \%$ ), Plantago lanceolata type (2.3\% at both sites), Solidago type (3 and $1.9 \%$ ), and Compositae (Tubuliflorae - Kellia 13\% and Asprokampos 6.5\% and Liguliflorae - Kellia $1.8 \%$ and Asprokampos 5\%), particulariy Cirsium type and Centaurea solstitialis type.

Pollen types that include domesticated cereals are highest in these two spectra and reach relative frequencies of 3.5\% for Kellia and 6.9\% for Asprokampos field (Avena is excluded as it is not now cultivated in Grevena, although it was in the recent past). The main contributing pollen type is Triticum.

Oak woods At the two sites of Asprokampos woods and Mirsina woods the surface samples were collected from beneath the canopy of stands of oak. The spectra of these two sites differ from the other lowland sites mostly in their higher relative frequencies of Quercus pollen types. The relative frequencies of Quercus frainetto type reach $19.8 \%$ for Asprokampos woods, while at Mirsina woods only 1.2\% is recorded. Quercus trojana type reaches $13.6 \%$ for Asprokampos woods and 39.5\% for Mirsina woods.

The relative frequencies of herbs of open ground are lower at these sites than for the cultivated fields, being $26.7 \%$ for Asprokampos woods and $25.2 \%$ for Mirsina woods. Pollen types that include domesticated cereals are not as well represented in the spectra from these two sites as those from the cultivated fields, aggregating only $2.4 \%$ and $1.2 \%$.

Steppe/oak woods The Mirsina fen site is only about $40 \mathrm{~m}$ from the Mirsina woods sampling site. The relative frequencies of Quercus types, Quercus frainetto type (16.6\%), and Quercus trojana type (3.6\%) are intermediate between those of the cultivated fields and the oak woods of the lowlands.

The relative frequencies of herbs of open ground are lower than at the sites surrounded by cultivated fields, but higher than the two oak wood sites, being $32.4 \%$ for Mirsina fen (this higher frequency is probably due to the steppe surrounding the fen). The $0.6 \%$ relative frequency of pollen types that include domesticated cereals is lower than for all the other lowland sites.

\subsubsection{Wetlands}

Pollen and spore types of wetland plants are seldom recorded outside the wetland sites, except those that include species that grow also on dry land, e.g. Carex hallerana type, Filipendula, Mentha type; or grow in other damp habitats, e.g Chrysosplenium alternifolium, Gratiola officinalis, Lychnis flos-cuculi, Salix fragilis type; or have good dispersal, e.g. Phragmites australis, Sparganium erectum, Typha angustifolia type. At dryland sites all these record less than 1\%, except for Phragmites australis (2.7\% for Asprokampos field) and Carex hallerana, which is recorded at several sites and probably represents plants growing on dry land. 
Lakes The only aquatic pollen types represented for the Avgo lake site are Hydrocotyle vulgaris $(0.3 \%)$ and Myriophyllum spicatum $(0.3 \%)$. At this site the two Carex pollen types record the highest relative frequencies of wetland types (Carex fiacca type 13.1\% and Carex hallerana type $3.6 \%$ ). All other wetland types reach only $0.3 \%$.

The site at Gomara is an ephemeral lake. Representation of aquatic pollen types is also low for this site: Hydrocotyle vulgaris $(0.2 \%)$ and Ranunculus aquatilis group $(0.2 \%)$. This site is distinguished from the lake at Avgo by a much greater representation of wetland pollen types: Carex hallerana (6.5\%), Cyperus michelianus (8.2\%), Glyceria type (4.9\%), and Phragmites australis (2.9\%).

Bog The bog community of Anelia is characterized by records of the following pollen types: Carex flacca type (1.2\%); Carex hallerana type (3\%); Cyperus michelianus (3.6\%); Glyceria (0.6\%); Phragmites australis (1.8\%); Mentha type (1.8\%), and Parnassia palustris $(3 \%)$.

Fens The two fen communities of Kellia and Mirsina differ from the bog community in having higher relative frequencies of pollen of Cyperus longus type (Kellia $116.4 \%$ and Mirsina 11.4\%) and Cyperus michelianus (27\%,21.9\%), and by the records of Equisetum $(5.8 \%, 2.2 \%)$ and Sparganium erectum $(16.6 \%, 0.3 \%)$. (Sparganium erectum grows very abundantly on Kellia fen.) Other wetland pollen types which are recorded for both bog and fen communities include Carex flacca type $(22.6 \%, 2.8 \%)$, Carex hallerana type $(2.6 \%$, $14.4 \%)$, Glyceria (2.8\% for both fens), and Phragmites $(1.2 \%, 27.4 \%)$. The last forms a canopy over the fen at Mirsina. Pollen of Mentha type is recorded in both fen spectra $(2.3 \%$, $2.5 \%)$, as well as Salix fragilis type $(1.2 \%, 1.7 \%)$.

\subsubsection{Charcoal}

Relative frequencies of charcoal particles of different size classes found in surface pollen samples provide a basis for interpretation of relative frequencies of charcoal particles in the wetland sequences. The wild vegetation of Grevena is not very flammable and, as something is known of the current anthropogenic fire regime, charcoal abundance and size of particles can be correlated with distance from the source of charcoal.

The sampling site at Avgo is within a national park, where lighting of fires is prohibited, and few if any fires are intentionally lit in the foothills, but the lowland sites are in a region where the stubble of cereals is burnt annually.

The relative frequencies of various size classes of charcoal particles counted in the surface samples show distinct patterns. The site at Avgo provides a good reference for an area where anthropogenic fires do not occur. Very low relative frequencies of all size classes are recorded here. Indeed, all mountain sampling sites record very low frequencies for all size 
classes of particles. (The high frequency for charcoal less than $25 \mu \mathrm{m}^{2}$ at the Anelia bog site is unreliable, due to small sample size, and must be disregarded.) Gomara records higher frequencies than the other mountain sites. This may be due to occasional burning to encourage summer pasture growth; no tradition of controlled burning to improve pasture is known to be practised in Grevena, although it is common in other parts of Greece (Forbes \& Koster 1976).

Sites in the foothills record slightly higher relative frequencies than the mountain sites, but only in the size classes containing charcoal particles smaller than $100 \mu \mathrm{m}^{2}$.

Relative frequencies recorded in the lowland vary greatly but are greater than those of the mountains or foothills. Fires are known to have occurred recently on or very near the sites of Kellia fen, Asprokampos field, and Mirsina fen. Charcoal of all size classes is abundant from these sites, especially from the two wetlands, where charcoal may have been washed in. Fires are not known to have occurred recently at the two lowland woodland sites, which show a marked contrast to the above three sites in having much lower charcoal particle frequencies in all size classes.

It can be concluded that charcoal particles of less than $25 \mu \mathrm{m}^{2}$ occur everywhere and must be transported long distances, while those of larger than $400 \mu \mathrm{m}^{2}$ are extremely local.

\subsection{Discussion}

\subsubsection{Previous surface-sample study in Grevena}

As part of a geographically wider study, Bottema (1974) analysed five surface pollen samples (numbers 74-78) from a north-south transect through the steppe/oak woods in the lowland plains of Grevena (Figure 3.2). The pollen sum included all pollen and spores of ferns (a second sum was introduced if one or more types were thought to be over-represented). The relative frequencies of Pinus pollen type range between about 5 and $10 \%$, while Fagus records less than 5\% for the first pollen sum, but up to $10 \%$ for the second. Quercus roburtype records a wide range, with sample numbers 76-78 recording much higher relative frequencies (between about 30 and 70\%) than samples 74 and 75 (about 10 and 5\% respectively). Another prominent woody pollen type, Juniperus, generally fluctuates between about 5 and $10 \%$, but one spectrum of one sample, \#77, records about $12 \%$. Abies records very low relative frequencies but reaches about $3 \%$ in the second pollen sum. Carpinus orientalis/Ostrya pollen type (equivalent to the Ostrya type in the present study) records less than $2 \%$ in the first pollen sum, but reaches about $4 \%$ in the second. Gramineae records between about 5 and $12 \%$. Cerealia-type and Zea mays pollen types added together record between about 0 and $5 \%$ in the first pollen sum, but almost $10 \%$ in the second. Many herbaceous pollen types are recorded with very low relative frequencies. However, there are a few which are recorded more consistently and/or are more abundant. These pollen types 
include: Chenopodiaceae, Plantago lanceolata type, Sanguisorba minor-type, Umbelliferae, Cruciferae, Liguliflorae, Matricaria-type (equivalent to the Achillea type of the present study), Centaurea solstitialis-type, Other Tubuliflorae, Leguminosae, Rumex acetosa-type, and Polygonum aviculare-type. All these types include many weeds and ruderals. Sample site number 74 is in a cultivated area and records higher frequencies of these than the other sites.

These pollen relative frequencies, which can be thought of as characterizing the steppe/oak vegetation zone of the lowland plains, are very close to those resulting from the present study (Table 3.1).

\subsubsection{Arboreal pollen to herbaceous pollen ratio}

The summary diagram showing the ratio of pollen frequency of trees and shrubs to that of herbaceous plants in each spectrum shows that the frequency of pollen of woody species is higher in the mountain and foothill spectra than in the lowland spectra.

The mountain and foothill spectra derived from sites which have canopies that are more than $50 \%$ closed have higher ratios than those with open canopies. This also applies to the lowland spectra. The ratio is greatest at the three sites of Anelia woods (0.916:0.084), Agios Nikolaos (0.906:0.094), and Perivolion (0.950:0.041). The samples from these three sites were collected under the canopy of a beech wood, pine wood, and pine/oak wood respectively. Samples collected from two foothill sites under the canopy of mixed deciduous woods have lower ratios of 0.803:0.197 (Zakas) and 0.854:0.146 (Aetia). The spectra from two samples collected beneath the canopy of lowland oak woods have lower ratios of 0.657:0.343 (Asprokampos woods) and 0.728:0.272 (Mirsina woods).

Two samples collected from open sites in pine wood-pasture recorded ratios of 0.803:0.197 (Avgo) and 0.699:0.301 (Gomara). The open oak wood-pasture site at Polinerion recorded a ratio of 0.671:0.329. The lowest ratio by far is that recorded for the Asprokampos field site $(0.165: 0.835)$.

\subsubsection{Palynological richness}

Although the size of the pollen sum varies considerably, the diversity of vegetation at the sampling sites is reflected in the pollen spectra. Spectra with the greatest number of terrestrial pollen types are those from the two pine wood-pasture sites (with 53 and 84 pollen types), and the lowland plains sites (range from 40 to 66 types). These are open sites apart from the two oak wood sites of Asprokampos and Mirsina.

Rarefaction analysis was carried out to standardize diversity, taking into account the varied pollen sums. The curve of the expected number of terrestrial pollen and spore types shows the same pattern as the unstandardized diversity, except that the Anelia bog spectrum 
now falls into the group of greater palynological richness (30 or above). The Anelia bog spectrum had previously had the lowest taxon count and estimates were based on it so no confidence interval is shown for this sample. The expected number of taxa for the group of lower palynological richness is from 15 to 25 .

In general, the results show a trend towards greater diversity for more open sites, and probably reflect in part a greater pollen source area for these sites. The greatest palynological richness (based on the rarefaction analysis) is recorded in the spectra from the wetland sites. Although pollen of marsh and bog, and of aquatic plants has been excluded from the sums used, herbaceous plants growing on the dry margins of these wetlands would contribute to the pollen sum. The palynological richness of the spectra from Asprokampos field and Polinerion is lower than expected, when the openness of the sites and the larger potential source area are considered, but this can be explained. In each case the lower diversity is a result of human disturbance. Asprokampos field is surrounded by cultivated fields, and local vegetation is mostly herbaceous and is ploughed each year except perhaps a narrow strip at the edge of the field. At Polinerion heavy grazing has greatly reduced diversity.

The relatively open oak woods with incomplete canopies record intermediate palynological richness, while woods with closed canopies have low palynological richness. The sample collected from beneath the canopy of the beech woods at Anelia records only 26 pollen taxa in the pollen spectra (and remains the same after rarefaction analysis), while the mixed woods at Zakas and Aetia record 36 and 33 pollen taxa respectively (but drop to almost 25 after rarefaction analysis). The lowest diversity (17 pollen taxa in the spectra, and after rarefaction) is recorded for the pine/oak woods in the Pindos Mountains.

Bottema (1982:270) identified surface pollen from a short transect across the Pindos just south of the Grevena border (with levels of identification similar to those of the present study). He identified 51 pollen types in a cultivated area in a valley, 49 in a herb-rich meadow just below a fir forest with bracken and forest at some distance, and 20 in samples within both a fir/beech forest and a beech forest. (His data were not corrected for dissimilar pollen sums, but his pollen sums were large, ranging from 700 to 1800.) In the present study, pollen samples from more or less comparable sites in Grevena displayed similar diversity: 40-66 pollen types (unstandardized sums) from the lowland plains, 33 pollen types from herb-rich meadows with bracken and forest at some distance (Polinerion), 36 from inside an Abiesdominated mixed wood on the Pindos foothills (Zakas), and 26 from a beech wood (Anelia woods). 


\subsubsection{Degraded vegetation}

Special mention is made of two vegetation communities, pseudo-maquis and shiblyak, because of their potential importance in the interpretation of fossil sequences in terms of human disturbance. It has been recorded that both these communities can be created from natural communities and maintained either directly or indirectly by anthropogenic disturbance (Turrill 1929), although shiblyak may also form a permanent community in Grevena (Moody \& Rackham, in prep.). Thus palynological recognition of these communities might indicate anthropogenic disturbance of an area. Pseudo-maquis is confined to a small area in Grevena, but shiblyak is widespread in the plains. Unfortunately, no surface samples were collected from these communities, but something can be said about their relative pollen dispersal and representation based on data from my study and previous studies.

Palynologically, pseudo-maquis is difficult to distinguish. In Grevena pseudo-maquis would be represented by Quercus coccifera type only. Other characteristic species of this vegetation community such as Juniperus, Buxus, Pistacia, and Phillyrea either occur in other plant communities or their pollen representation is very poor (Bottema 1974).

In Grevena shiblyak is characterised by Cotinus coggygria, Prunus spinosa, Colutea arborescens, and Paliurus spina-christi. All of these species are very poorly represented in the pollen record. Other characteristic taxa listed by Turrill (1929:153) that might occur in Grevena are Quercus pubescens, Berberis vulgaris, Rhus coriaria, Prunus amygdalus, Viburnum lantana, and Cercis siliquastrum. The first of these occurs in other plant communities while the remainder have very poor pollen representation.

\subsection{Implications for late-Holocene pollen studies in Grevena}

Although the number of samples is small and preservation variable, the relative frequency spectra of these surface pollen samples reveal much about the relationship between extant vegetation of Grevena and pollen deposition that is applicable to the reconstruction of palaeovegetation and environment from fossil pollen spectra. The data mainly reflect differences between pollen production and dispersal properties between taxa.

\subsubsection{The natural vegetation}

The extant vegetation of Grevena is dominated by woodland, which covers over half the entire land area; grassland and cultivation cover the remainder. The landscape is intensively used and little if any unmodified vegetation remains. Today land use includes agriculture, pastoralism, removal of trees by cutting, and maintenance of trees by coppicing, pollarding, and shredding. In spite of this intensive land use, the composition and structure of the woodlands varies according to elevation and moisture and forms distinct vegetation zones 
dominated by only a few tree genera. The highest elevations are occupied by alpine meadows and Pinus woods, at intermediate elevations by a mosaic of Pinus woods, Fagus woods, and mixed coniferous/deciduous woods, while the lower slopes of the Pindos foothills are dominated by deciduous Quercus woods and the plains by a mosaic of deciduous Quercus woods and steppe, and agricultural land. The west-to-east moisture gradient results in the dry eastern Vourinos Mountains supporting few trees. The trees that dominate the landscape, Pinus, Fagus, and Quercus, are also dominant in the surface pollen spectra, and the major vegetation zones which these trees dominate are characterized by corresponding high pollen relative frequencies: $51-84 \%$ Pinus (Diploxylon) in pine wood, 23.5-29.4\% Fagus in beech woods, and $26.5-40.7 \%$ Quercus frainetto and Quercus trojana types combined in deciduous oak woods.

As has been found in many other surface pollen studies, the proportion of pollen recorded in the pollen spectra is different from that of corresponding plant taxa in vegetation. An obvious feature of the results of this study is the over-representation of pine pollen, which suppresses the relative frequencies of all other pollen types. No site contained less than $13.6 \%$ Pinus pollen (Asprokampos field excepted), even in the lowlands where the sampling sites are $25 \mathrm{~km}$ from the main source of pine pollen (although there is a small stand of pine near Grevena city, $7 \mathrm{~km}$ from the sample sites). This extreme over-representation of Pinus necessitates that a high pollen sum be counted in samples collected from pine vegetation zones so that other pollen taxa may attain reasonable representation, and should be taken into account when interpreting the fossil sequences.

By extrapolating back from the extant disturbed vegetation in Grevena to an hypothetical undisturbed vegetation, given the same climatic and edaphic conditions, it is apparent that the same dominant tree vegetation and flora would exist at approximately the same elevations, but there would be greater diversity of trees. Small pockets of less disturbed vegetation such as the mixed woods growing on flysch and ultrabasics at the end of the limestone ridge above Zakas and Spilaion or the mixed wood growing in the oak/steppe zone are indicative of two types of mixed woods of different composition and habitats that presumably would be much more extensive. A former mixed wood of damp habitats and at an altitude between the mountain beech woods and oak wood of the foothills might include such species as Tilia rubra (lime), Ulmus glabra (elm), Populus tremula (aspen), and Prunus avium (cherry). A mixed wood of drier habitat and lower altitude might comprise Carpinus orientalis, Acer opalus, Cornus mas, Fraxinus ornus, Tilia rubra, Acer campestre, Corylus avellana, Sorbus torminalis, and Tilia tomentosa and be widespread in the oak/steppe zone in the plains.

The relative dispersal and representation of pollen of these trees varies (as demonstrated by the two surface samples collected from beneath the canopy of the relic mixed woods 
currently growing on limestone), but they all have lower relative dispersal and representation than Pinus, Fagus, and Quercus. In general the taller anemophilous trees contribute most pollen to the pollen rain. Many trees of the mixed woods are entomophilous and contribute almost no pollen, e.g. Acer, Cornus, and Tilia and genera of Rosaceae. Fraxinus ornus is also primarily entomophilous. If pollen of these trees is found in the pollen record they must have grown close to the sampling site. Poor representation of pollen of these trees makes detection of greater diversity of trees in woodlands difficult. A decline in the pollen of these types as a whole would probably be indicative of anthropogenic disturbance of the forests. Corylus avellana and Carpinus betulus are anemophilous, but although dispersal is good little pollen is produced, although production may be variable. Corylus is known to produce varying amounts of pollen depending upon its structural position in woods, i.e. it produces little pollen as an understorey shrub but produces much as a canopy tree (Iversen 1941; Moore 1976). A few pollen grains of Corylus may thus arise from a distant or local source.

One of the biggest problems in interpreting pollen data is defining the size of the area that contributes to the pollen rain. It is often impossible to tell whether a small percentage comes from a few trees in the vicinity or from stands at a distance. In a basin such as Grevena with distinct belts of vegetation zones at different elevations, it is possible to measure pollen dispersal distances from source vegetation. Long-distance transport of some pollen types identified in the surface pollen samples is apparent. Surface samples of the lowlands that contain pollen of trees that grow in the mountains, such as Pinus and Fagus are evidence of long-distance transport as are samples collected in the mountains that contain pollen of trees that grow in the lowlands and foothills, e.g. Quercus. A relative frequency of as much as $13 \%$, in samples from the lowland oak woods of Grevena is not indicative of pine growing on or near the site at the time of deposition of the pollen. Similarly threshold values for Fagus and Quercus ( $Q$. frainetto and $Q$. trojana types combined) of $1 \%$ and $6.5 \%$ respectively are proposed for Grevena. Another pollen type that clearly displays long-distance dispersal is Quercus coccifera, whose appearance in samples collected in the Pindos Mountains must be a result of long-distance transport from the western side of the mountains, taking into account the prevailing westerly winds.

Fagus pollen is relatively large and heavy, so although it is anemophilous only a small proportion of released pollen is carried very far. Evidence of this comes from the two sampling sites Avgo and Gomara, where scattered beech trees grow about 55 meters from each site and show only 3.9\% maximum relative frequency. At other Pindos Mountain sites the relative frequency declines to $1.7 \%$ or less, similar to that recorded in lowland sites. Two sites were sampled in the beech wood at Anelia, one under the canopy of the beech wood and the other on the surface of a bog $(90 \times 50 \mathrm{~m})$ within the woods. Thus it is expected that the 
greatest proportion of pollen is derived from local and extra-local source areas (Tauber 1965; Andersen 1970). Relative frequency of Fagus pollen recorded at these two sites is somewhat similar, $29.4 \%$ and $23.5 \%$, the greater frequency recorded in the woods. So a relative frequency of $20-30 \%$ would probably indicate that beech trees were growing close to the sampling site and dominate within the first half kilometre (but not that beech was dominating in the whole region). As shown, Fagus pollen is deposited mostly on a local and extra-local basis, and a record of a beech wood in the sediments at Anelia bog would indicate a local wood.

Dispersal patterns of oak are difficult to determine because oak trees are so widespread in Grevena and because of the possibility of long-distance transport of Quercus pollen from the western (windward) side of the Pindos. Some of the Quercus pollen recorded in samples collected from the Pindos Mountains may be a result of vertical transport from lower elevations in Grevena, but it is impossible to distinguish between a pollen source area on the western side of the Pindos Mountains from one on the eastern side (cf. Markgraf 1980).

In the late Holocene a change in the climate to cooler, moister, or even cloudier conditions between about 3200 and $1500 \mathrm{BP}$ has been suggested, based on the expansion of Fagus (Chapter 1). The modern micro-climatic patterns of Grevena are evident in the contemporary distribution of vegetation belts that change according to elevation. A change in climatic conditions of sufficient magnitude would result in a corresponding shift in the vegetation belts. The most obvious record of these changes in the palynological record would be in changes in the curves of the pollen of the dominant trees of these belts. Trees most sensitive to climatic changes are those near the limits of their ecological amplitude, especially Fagus, which is close to its southern limit, and Abies cephalonica, which is close to its northern limit.

Wetter or drier conditions might also be reflected in the palynological record of the wetlands as changes in hydrological conditions. Changes in the relative abundances of aquatic pollen types can indicate changes in water levels. Aquatic plants also react quicker than forest trees to thermal climatic changes, as water temperature changes relatively quickly and seed dispersal of water plants is good (Iversen 1964). Data from this study show that pollen from marsh and aquatic plants is mostly dispersed locally.

A problem in pollen analysis has been the differentiation between aquatic grasses and those representing terrestrial grasslands. A pollen curve comprising grasses from both habitats would severely reduce the diagnostic value of terrestrial grasses. Although the sample size is small and caution must be exercised, this study shows a positive correlation between the Festuca pollen type with pasture, and the Glyceria type and Phragmites type with wetlands. Iversen (1964:426) also noted that this distinction was possible. The distinction between 
terrestrial and wetland grasses allows more certain interpretation of a landscape becoming more open.

It was not possible to distinguish morphologically between pollen of terrestrial and wetland sedge plants in the Carex hallerana type. Thus plants growing locally on the wetland and those potentially growing in the regional vegetation are combined in one pollen type. Most of the plants recorded in the vegetation survey were from wetland sites, and the surface sample sites that record the greatest relative frequencies of Carex hallerana type are both fens, so it is probable that most of the pollen contributed to this type is actually from wetland plants. Carex hallerana type should therefore be excluded from a terrestrial taxa pollen sum.

\subsubsection{Land clearance}

The first signs of anthropogenic impact on the vegetation in the Balkan region have been recognized as a reduction or change in woodland composition, accompanied by the expansion of open-ground herbaceous types typical of disturbed agricultural ground in southeast Europe, as well as the presence of cereals (in combination with supporting evidence necessary because of the difficulty in distinguishing wild from domesticated grasses) (Willis \& Bennett 1994). Archaeological evidence suggests that the size and extent of the human population of Grevena have fluctuated. The number of archaeological sites recorded from surface surveys increases dramatically in the Early Iron Age (3000 BE), and sites extend from the lowlands to elevations near $1000 \mathrm{~m}$ asl. After a decline in the number of sites in the Archaic and Classic Periods, the number in the Hellenistic Period increases and again extends to the elevations previously occupied in the Early Iron Age. Many Hellenistic Period sites are fortifications in the Pindos foothills, and there is evidence for pastoralism and agriculture (Chapter 1). A corresponding successive reduction and regeneration of the woodland trees would be expected as populations requiring large areas of cleared land for their settlements, communication systems, and agriculture cleared land that later became abandoned when the population size reduced.

Agricultural economies require land on which to grow their crops, while pastoralists require grassland to feed their animals - woodland is an obstacle. Woodland can be destroyed by cutting or by burning; perhaps both methods have been employed in Grevena. The extant wild vegetation of Grevena is not very flammable, except Juniperus and Pteridium aquilinum, although steppe burns occasionally. Juniperus is killed by fire, and its absence may indicate the presence of fires. Juniperus is widespread in Grevena today, occurring in many different vegetation communities, but pollen representation is not very high. On the other hand, Pteridium aquilinum is encouraged by fire, regrowing quickly from rhizomes after being burnt. It is under-represented in the pollen record, so a continuous record may indicate anthropogenic burning. 
A guide to the frequency/intensity of fires at a site may also be gained from analysis of microscopic charcoal particles. Charcoal particles of less than $25 \mu \mathrm{m}^{2}$ represent charcoal derived from a distant source, while particles of $400 \mu \mathrm{m}^{2}$ and greater represent charcoal from a very local source.

Reduction of the canopy allows more light to penetrate to the forest floor, thus allowing herbaceous plants that require light to grow. Thus the ratio of non-arboreal pollen (NAP) to arboreal pollen (AP) has been used as a guide to the density of forest cover. In this study, higher ratios were found in the mountain and foothill woods than in open vegetation. The ratio was also found to vary according to tree genera forming the canopy. The ratio is higher in the mountain and foothill pine and beech woods than in the lowland oak woods. The lowest ratio was recorded, as expected, in the most open vegetation in the cultivated area of the lowlands.

Results from the present study show some correlation between openness of sites and palynological richness interpreted from estimated numbers of pollen and spore taxa standardized to a common count size using rarefaction analysis (Birks \& Line 1992). The values of expected number of taxa were highest for most open sites, while woods with closed canopies have the lowest values. Woods with incomplete canopies have intermediate palynological richness. Intensive land use, such as the current grazing at Polinerion and the agriculture at Asprokampos, lowered palynological richness. Other researchers have found that intermediate levels of disturbance, either natural or anthropogenic, are important in maximizing richness at the landscape scale (Birks \& Line 1992). Changes in palynological richness are interpreted as reflecting predominantly the changing floristic richness of the vegetation types in the pollen source area. These changes may be the result of many and varied causes. A reduction, or small openings, in a canopy would permit entry of a wider variety of pollen taxa from regional sources to fall on the deposition site and thus increase palynological richness.

\subsubsection{Pastoralism}

Pasture is today most abundant in the Pindos Mountains, where some of the largest modern transhumant Vlach villages are located. The earliest evidence of occupation above $1000 \mathrm{~m}$ in the Pindos Mountains in Early Medieval times is from archaeological sites distributed in a pattern similar to that of modern transhumant villages, which suggests the existence of a pastoral economy. Transhumant pastoralism is known to have been practised in the Pindos Mountains and foothills in the Ottoman Period. Today the Pindos pine woodpastures are intensively grazed during summer. This intensive grazing has reduced the number of trees to below the natural carrying capacity by not allowing seedlings to grow, and a 
reduction in grazing would result in regeneration of many more trees. Such a change in the density of trees would be recorded in the Gomara wetland site, which registers regional pollen deposition. The current relatively high frequencies of Compositae and Plantago at this site would probably decrease.

Intensive grazing in the dry south-east corner of Grevena has led to the development of pseudo-maquis. Unfortunately the extent and location of a degraded vegetation of pseudomaquis would be difficult to determine from the pollen record. The only pollen type indicative of this vegetation with reasonable representation is Quercus coccifera, which is over-represented and is transported long-distances. Recognition of shiblyak, whether humaninduced or natural, would also be difficult to detect due to poor pollen representation and the lack of characteristic pollen types.

The production of leaf-fodder for animal sustenance during winter is part of the pastoral economy in Grevena today. Iversen (1941) correlated the use of leaf-fodder of elm with the decline of elm pollen in pollen diagrams from Denmark during the Neolithic Period, although more recently Dutch elm disease has been suggested as a possible cause for the elm decline (Perry \& Moore 1987). Leaf-fodder production practised in Grevena today plays a vitally important role in animal husbandry (Chapter 1). The tree most used is Quercus, but there is much evidence that both Quercus and Fagus were regularly coppiced in the past few centuries. Coppicing of oak is most common at lower altitudes in the Pindos foothills, while pollarding of oak is most common in the lowlands. Unfortunately, no surface samples were collected from the coppice oak woods of the foothills, but the two oak woods that were sampled in the lowlands had been coppiced. Pollen relative frequencies of these woods were high (33.4 and $40.7 \%$ ), but it is impossible to say if coppicing has depressed pollen deposition, as no data are available against which to compare these frequencies. Oaks produce flowers in the later part of each coppicing cycle (30-40 years), except Quercus frainetto, which takes at least 50 years. It is not known how long Fagus takes to regrow and flower after coppicing, but if a species is regularly prevented from flowering, e.g. because it is regularly harvested before reaching flowering age, it will not show in a pollen diagram, even if it is common in the area. 


\subsubsection{Cultivation}

It has been demonstrated that an increase in the pollen of Castanea sativa, Juglans regia, and Platanus orientalis is associated with anthropogenic disturbance of woodlands in Greece (Bottema 1974, 1980, 1982; Bottema \& Woldring 1990). These trees are thought to have either profited from human-induced changes in habitats or were planted in prehistoric times. Juglans regia and Platanus orientalis are anemophilous, but pollen production is not very high. Thus low relative percentages in the pollen record may be due to long-distance dispersal or to a few trees growing in the vicinity of the sampling site. Castanea sativa is entomophilous primarily, but from the results of this study it appears capable of long-distance dispersal also. Long-distance dispersal of Castanea pollen has been reported by others (Ruffaldi 1994). The sap of Fraxinus ornus was used to make syrup in the past, but it is not known if this tree was planted (Bottema \& Woldring 1990). The relative dispersal and representation of this tree is discussed above.

Agriculture has a long history in Grevena, for archaeological surface surveys undertaken by the Grevena Project have revealed wheat/barley seeds in association with Hellenistic sites, and ethnoarchaeological studies indicate that a variety of crops was grown prior to $1940-$ before mechanization of agriculture (Chapter 1). The domesticated cereals of wheat, barley, rye, oats, and maize were grown for human consumption and animal fodder. Other crops included beans, chickpeas, vegetables, fruits, nuts, vines (grapes), vetch, pea hay, and alfalfa. Apart from maize these crops are likely to have been grown in Grevena in prehistoric times also (Secale cereale only since Roman times) (van Zeist \& Bottema 1971; Dennell 1972; Renfrew, J. 1969, 1973a, b; Hansen \& Renfrew, J. 1978; Hansen 1978, 1988; Jones 1981).

The results of the present study indicate that the cereal pollen types of Hordeum, Triticum, Secale cereale, and Zea mays are severely under-represented. Hordeum is cleistogamous and autogamous. No pollen of the Hordeum pollen type was found in the sample collected from the cultivated field where barley had been grown that summer and only one other grain of this type was found. Triticum type is better represented than that of Hordeum: although it is autogamous some pollen is released for atmospheric dispersal. Secale cereale is cross-pollinated by wind (Faegri \& Iversen 1989:127). The severe underrepresentation and poor dispersal of the domesticated cereals of Hordeum, Secale cereale, and Triticum demonstrated in this study is consistent with results from other studies of surface samples from cultivated fields (Vuorela 1972, 1973; O'Connell 1986; Hall 1989). Hall (1989), in a study of surface grass samples spaced only $5 \mathrm{~m}$ apart, found that although values of between 9 and $23 \%$ cereal pollen were recorded in surface samples from beneath crops, only $1-2 \%$ ( $3 \%$ from moss polsters) were recorded at distances of more than $1.5 \mathrm{~m}$ from the edge of the crop. No assessment of the representation of domesticated Avena could be made from 
the current study, but Vuorela $(1972,1973)$ found that Avena is well represented in pollen diagrams, although its pollen-dispersal capacity is less than that of Secale but greater than that of Triticum and considerably greater than that of Hordeum. On the other hand, O'Connell (1986) found that Avena representation is exceptionally low. Vuorela $(1972,1973)$ suggested that most cereal pollen is dispersed during harvest-time and not during the flowering season. Zea mays is anemophilous, but Raynor et al. (1972) found from experimentation that dispersal of Zea mays pollen is very poor: the concentration of pollen remaining airborne at $60 \mathrm{~m}$ from the source in the downwind direction is about $1 \%$ of that at $1 \mathrm{~m}$. The poor dispersal (and under-representation) makes representation of cereal-type pollen in a time-series study unlikely unless the sampling site is close to the fields that were cultivated.

Prior to mechanization of agriculture, if oxen were kept for draught a large proportion (a minimum of just over 1/5 (Chapter 1)) of the productive arable land was required for growing legume crops for fodder. Legumes are entomophilous, and their pollen has very low probability of being preserved in detectable frequencies in wetland deposits. Also it is difficult to differentiate crop pollen types from wild types. Medicago sativa subsp. sativa (alfalfa) is still grown as a fodder crop today in Grevena. A few pollen grains of the Medicago sativa pollen type were identified, but from locations that suggest they were from the wild subspecies. Chickpeas are also grown as a fodder crop today, but no pollen was identified.

Although no grape vine plants were recorded in the vegetation survey and no pollen was identified, they are mentioned here because they are known to have been grown much more extensively than they are in the household gardens of today, and because pollen of vines has been recorded in pollen diagrams from Greece (Chapter 1). Wine was made locally during Roman times (Chapter 1). Ethnographic studies of pre-1940's agriculture indicate that the minimum area of land required for a subsistence household for the production of grape vines was $2000 \mathrm{~m}^{2}$ (Chapter 1) and that extensive vineyards near Spilaion produced a surplus that was exported. Vine pollen is probably under-represented and has low dispersal power. Vines were usually planted close to settlements, so a sampling site such as Kellia, which is close to a settlement, might record this production. Fruit and nut trees usually surrounded the vineyards, so they might also be recorded in sites close to settlements.

While the pollen of herbaceous cultivars is under-represented in the pollen record, several weeds associated with cultivated fields are better represented. The surface sample from the cereal field at Asprokampos contained the pollen types of Polygonum aviculare type, Plantago lanceolata type, Rumex acetosa group, and Chenopodiaceae, which all include weed species. All of these except the first are anemophilous, and all have medium to high relative dispersal and are well- to over-represented. Pollen of several Compositae species were also identified in this sample. These pollen types are entomophilous, dispersing their pollen 
locally. Species belonging to these pollen types are light-demanding, and an increase in their relative frequency would provide evidence of an opening vegetation. These pollen types are included in Bottema's (1982) and Bottema \& Woldring's (1990) lists of anthropogenic indicator species.

In conclusion, plant communities characterised in this study may not provide modern analogues of past pollen assemblages because of the recent changes in land-use patterns, especially the contraction of agriculture to the lowlands since mechanisation (Chapter 1). Thus the indicator approach may be more helpful for interpretation. Indicator pollen types, especially those identifiable to species level or those of species groups that have a clearly defined ecology, are particularly diagnostic. Pollen types of the major trees would be the best indicators of climatic change, especially Fagus, which is near its ecological limits. Indicator pollen types of disturbance of woodland, whether anthropogenic or natural, would be those of weeds, ruderals, and pioneers. Herbaceous pollen types of cultivars, as well as weeds and ruderals, especially those that can be identified to species and those that are not sensitive to climatic influence, e.g. cereals, weeds, Pteridium aquilinum, would be particularly important for the interpretation of anthropogenic disturbance. 


\section{Chapter 4. Gomara}

\subsection{Introduction}

This chapter includes a description of the site and sediments of the Gomara wetland coring site and then presents the results of pollen analysis of a sediment sequence $190 \mathrm{~cm}$ long. The wetland was first identified as a potentially suitable pollen-analysis site by Moody \& Rackham (1988). These authors named the site 'Limni', a Greek word meaning lake, suggesting that the wetland was filled with water in early summer when they discovered it. Preliminary palynological investigations suggested that the sediments of this wetland were suitable for a full palynological study; fossil pollen was very abundant, and preservation was excellent (Huber \& Rapp 1988).

\subsection{Site description}

Gomara wetland is situated in the Pindos Mountains (latitude $40^{\circ} 03^{\prime} \mathrm{N}$, longitude $21^{\circ} 04^{\prime} \mathrm{E}$ ) on the pass between Smixi and Distraton at an elevation of $1750 \mathrm{~m}$ asl (Figure 1.1). It is about $1 \mathrm{~km}$ west of the Grevena border in the province of Ioanninon and about $4.5 \mathrm{~km}$ west of the Vlach village of Smixi (Figures 3.2, $4.1 \& 4.2$ ). The pass was a trading route during the Ottoman Period, and currently there is a small commercial ski field on the southern side of the pass on the slopes of Vasilitsa mountain. The Gomara-Vasilitsa area has pastures that are leased by herders of the Grevena transhumant (Vlach) villages of Smixi, Samarina, and Avdella (Koster 1987).

\subsubsection{Physiographic description}

Today an ephemeral lake, Gomara wetland is about $10 \mathrm{~m}$ north of the road between Smixi and Distraton in subalpine meadows (Figures $4.1 \& 4.2$ ). It is perched on a bench that drops steeply down to the Dristis River drainage, a narrow steep valley to the south. High peaks rise to the north and south, but the site is open to the west and east. The peak of Gomara $(2126 \mathrm{~m})$ rises to the NNW about $2 \mathrm{~km}$ from the wetland coring site, while the lesser peak Tambouri $(2026 \mathrm{~m}$ ) rises almost due north of the site and only about $0.8 \mathrm{~km}$ from it. To the SSE the slightly more distant (about $2.5 \mathrm{~km}$ ) and higher peak of Vasilitsa $(2248 \mathrm{~m})$ rises steeply (Hellenic Army Geographic Service 1970). The name given to the wetland site in this thesis is taken from Gomara peak.

The wetland is rheotrophic, receiving water not only directly from the atmosphere in the form of rain and snow but also from run-off. There is a shallow (about $25 \mathrm{~cm}$ deep) intermittent overflow channel from the lake to the SSE (Figure 4.2). Water was noted in the wetland basin at the beginning of the field season in 1989 (mid summer), but three weeks later 


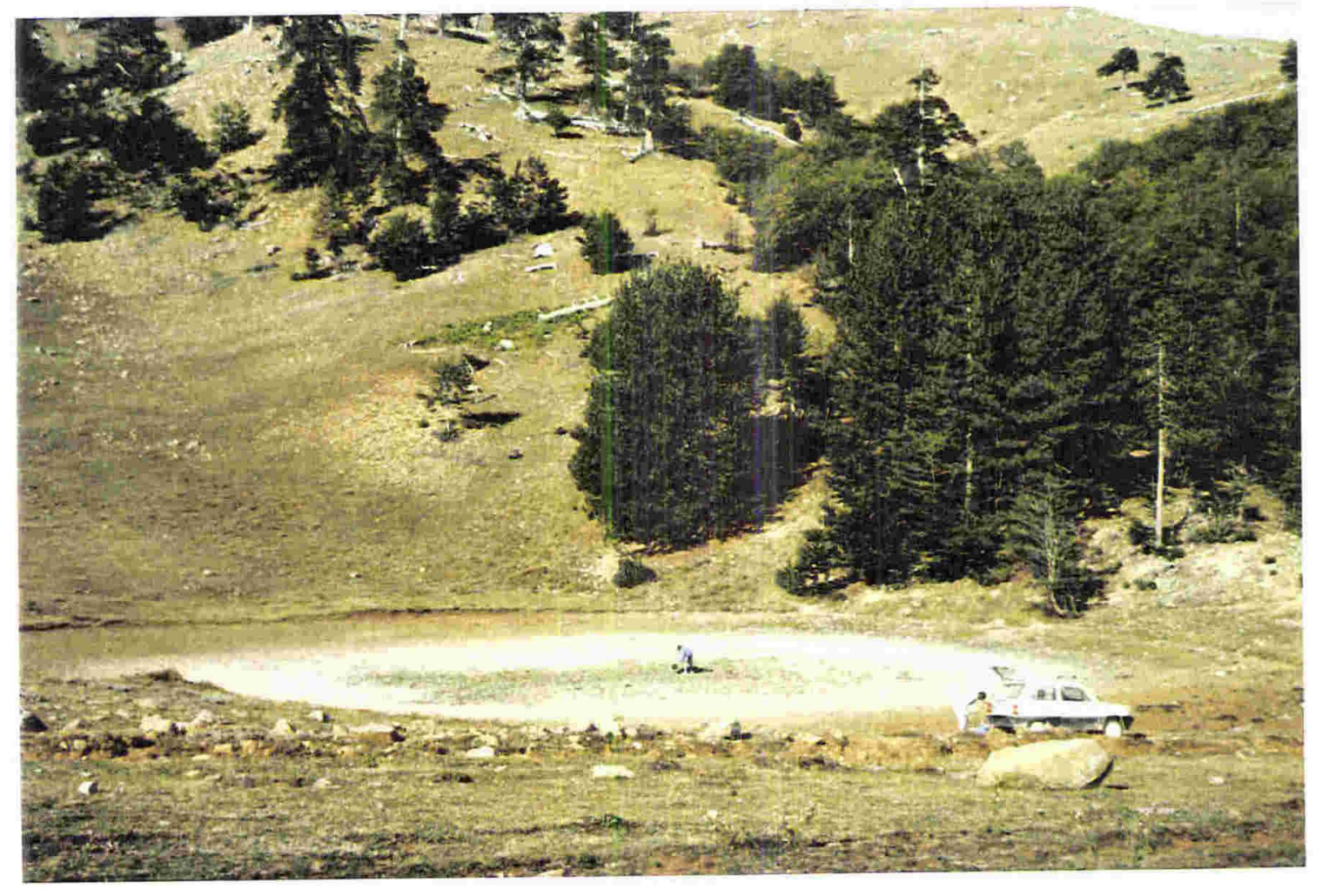

Figure 4.1 View of Gomara site from Smixi-Distraton road, looking NNE, across the dried lakebed. A small stand of beech trees can be seen on the right and scattered dead and living standing pine trees beyond. 


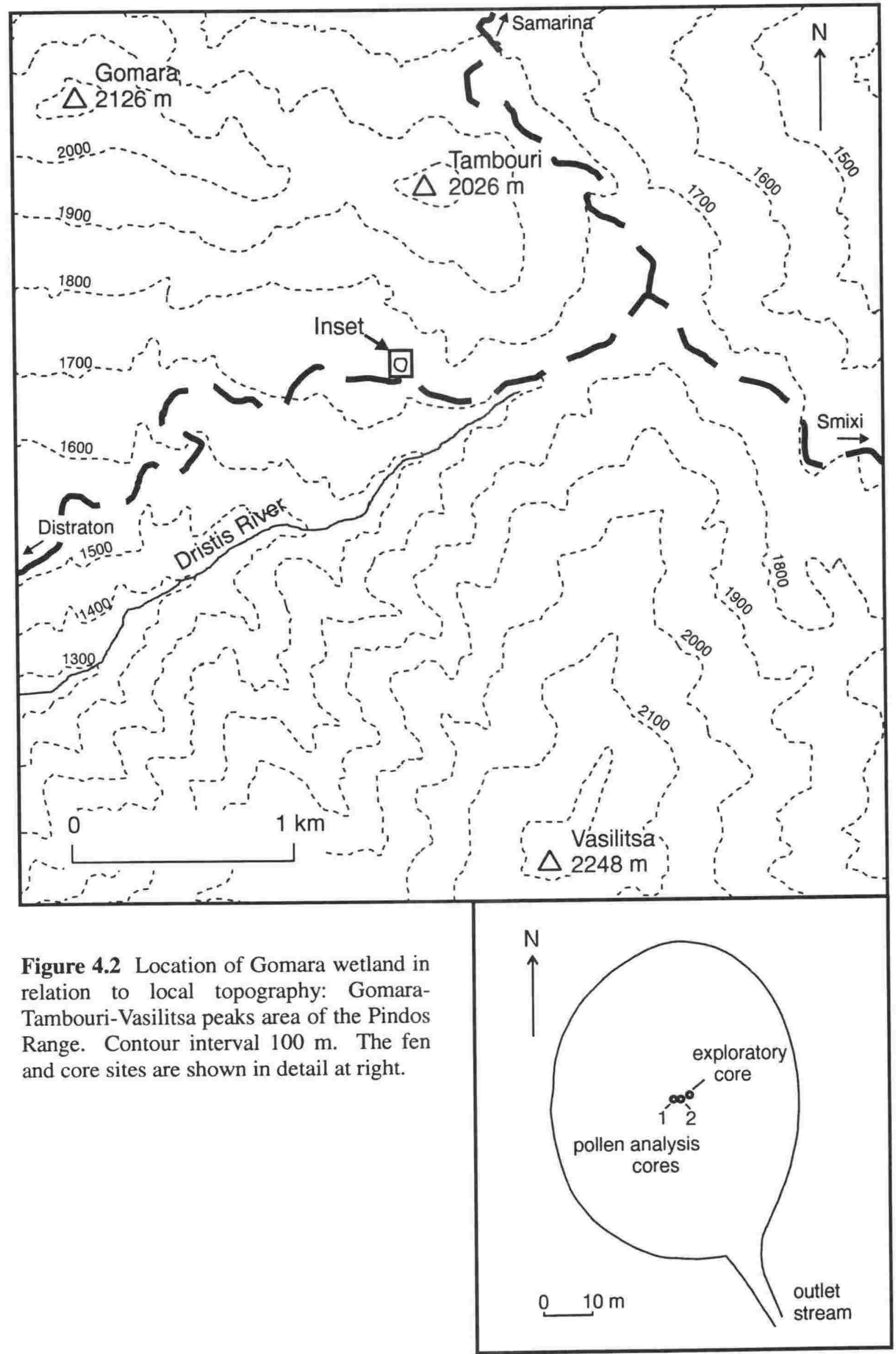


when coring was undertaken it was dry, and cracks had appeared on the surface of the lakebed (Figure 4.1). Presumably high water occurs in early summer following snow melt. The very strong westerly winds experienced while doing field work would act as a strong evaporative force.

The wetland is enclosed in a shallow almost circular basin, $65 \mathrm{~m}$ long and $50 \mathrm{~m}$ wide $\left(3250 \mathrm{~m}^{2}\right.$ in area). This is slightly smaller than the $5000 \mathrm{~m}^{2}(1 / 2 \mathrm{ha})$ ideal size suggested by Faegri \& Iversen $(1975: 86,1989: 57)$ for pollen analysis. The catchment of the wetland basin is approximately 75 ha with a SSW aspect and a mean slope of $19^{\circ}$ (estimated from contours) (Hellenic Army Geographic Service 1970).

\subsubsection{Regional and local vegetation}

The regional vegetation is the pine wood-pasture community described in Chapter 3 and is very similar to that surrounding the Avgo site, with the exception that it is grazed more intensively. The vegetation is very open, a characteristic of this vegetation type, with scattered trees, mostly Pinus leucodermis (the alpine pine), growing in grassland. About $30 \%$ of the ground is bare. In the vicinity of the lake pine trees are mostly about $20 \mathrm{~m}$ tall, and many are dead but still standing. A stand of Fagus sylvatica (beech) trees grows in a small valley close to the lake (Figure 4.1). They reach a maximum height of only $6 \mathrm{~m}$. Some grazing and coppicing of beech has occurred. Tree seedlings are grazed, thus prohibiting regeneration, and the slope below the lake has been logged recently.

The dried lakebed has a distinct seasonal fen vegetation (Appendix VIII). When fieldwork was undertaken vegetation on the lakebed was very low and sparse, and there was no hydrosere surrounding the perimeter of the lake.

\subsubsection{Geology of site}

Bedrock is mapped as the Pindos ophiolite ultramafics: peridotites and serpentinites (Institute for Geology and Subsurface Research 1960). These rocks weather easily and produce extensive screes (Strid 1989). Coarse well-draining colluvium with some large blocks surrounds the site. Soils in the catchment are brown, shallow, and stony. It has been speculated that "the coarse stone content and shallowness [of serpentinite soils] must result in a low water-holding capacity and a restricted soil depth for root penetration, and plants growing in these soils are likely to suffer from drought" (Proctor \& Woodell 1971:255).

The genesis of the lake basin is unknown, but impondment may have been the result of glacial activity, for example a kettlehole, glacial scour, or moraine-damming; local faulting; earthquake-induced slope failure; or human disturbance. Glacial activity is an unlikely cause since glacial activity in the Pindos Mountains during the last glaciation resulted in only 
relatively small glaciers forming on north and east facing slopes (Turrill 1929:71; Institute for Geology and Subsurface Research 1960; Savina 1993), and there are no obvious moraine features in the catchment. A more likely explanation is the coalescing of colluvial debris flows triggered by tectonic movement or a heavy rainstorm. Open vegetation cover, perhaps cleared by humans (Turrill 1929), may have predisposed the site to such an occurrence. Furthermore, landslides are a common feature of areas underlain by Pindos ophiolite (Institute for Geology and Subsurface Research 1960; Savina 1990).

The hardness of the sediment immediately below the lowest sample collected for pollen analysis suggests that $190 \mathrm{~cm}$ is about the full depth of the sedimentary basin. Rackham (pers. comm.) also managed to penetrate the sediments in the centre of the wetland to only $205 \mathrm{~cm}$ below surface.

\subsection{Sediment analysis}

The location of the coring sites is shown in Figure 4.2 and the results of the TroelsSmith (1955) classification and laboratory analyses of the physical properties of the pollenanalysed sediment sequence are reported below. Two borings $25 \mathrm{~cm}$ apart and overlapping $21 \mathrm{~cm}$ were made with a piston corer. In the diagrams and text, datum for depth (given in $\mathrm{cm}$ ) is the ground surface.

\subsubsection{Stratigraphy}

An illustrated description of the sediments of the pollen-analysed core is given in Figure 4.3. The Troels-Smith (1955) classification scheme using symbols and formulas is followed (Appendix III). Columns show the component elements, accessory elements, physical properties, and boundary definitions of the sediments. Component elements are shown in the first two columns in symbols and formulas. The proportion in which each element occurs is given as well as occurrence of accessory elements (in symbols only). The degree of definition of boundaries between strata (limes) is indicated where sediment composition changes, in symbols in the stratigraphic column and in numeric form to the right of the column (based on a 5-class scale). Notes on the structura of the sediments are given in the next column. Formulas (and text description) of the fresh colour of the sediments taken from standard Munsell colour charts (Oyama \& Takehara 1967) follow. Other physical properties, nigror, stratificatio, elasticitas, and siccitas are shown in symbolic form in columns on the right. (Since the whole sequence is water saturated, siccitas is 2 throughout.) Numbers indicating the degree of each of these properties (based on a 5-class scale) are shown to the right of the column. Colours are all fresh colours. 

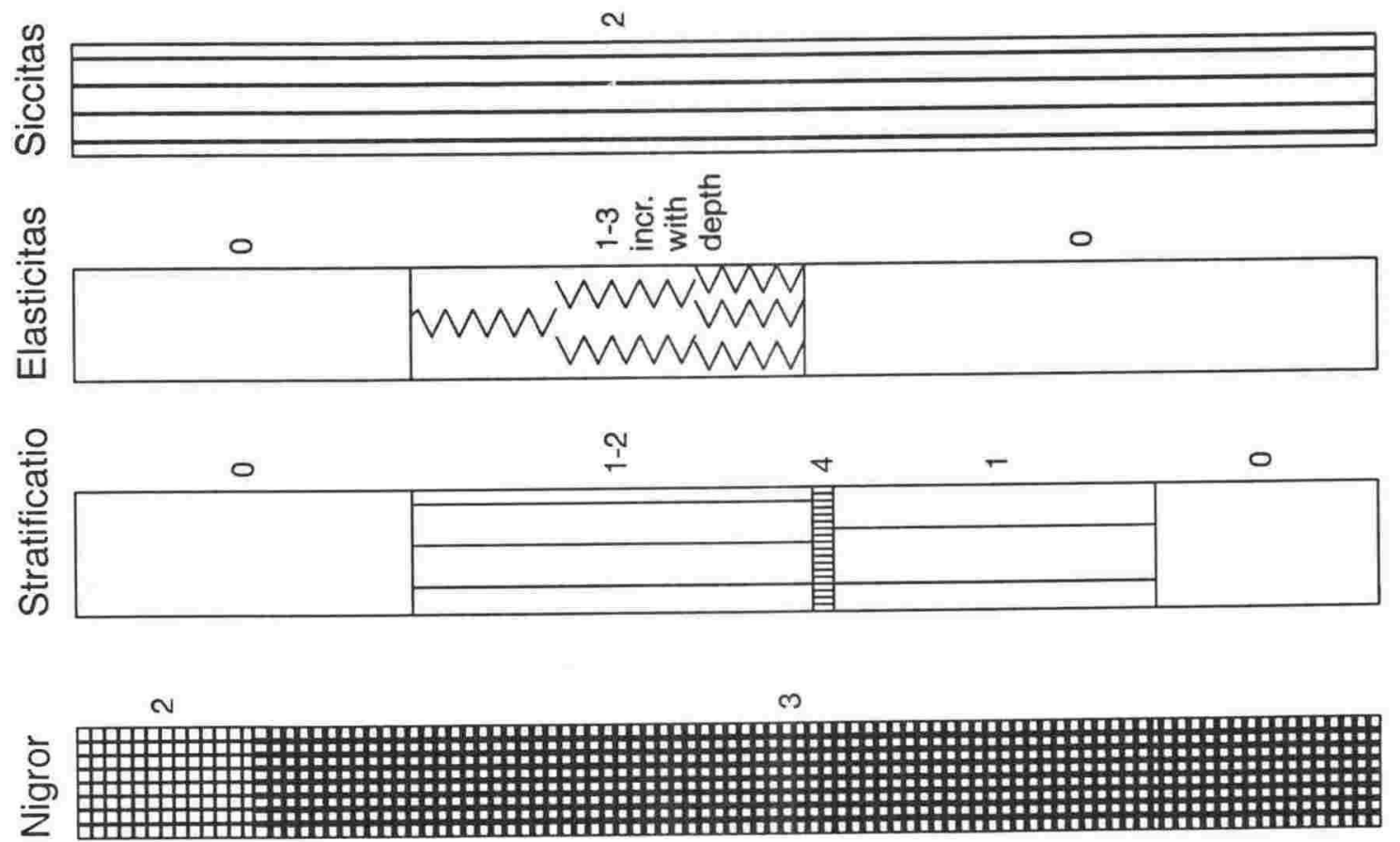

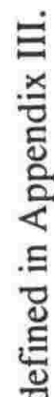

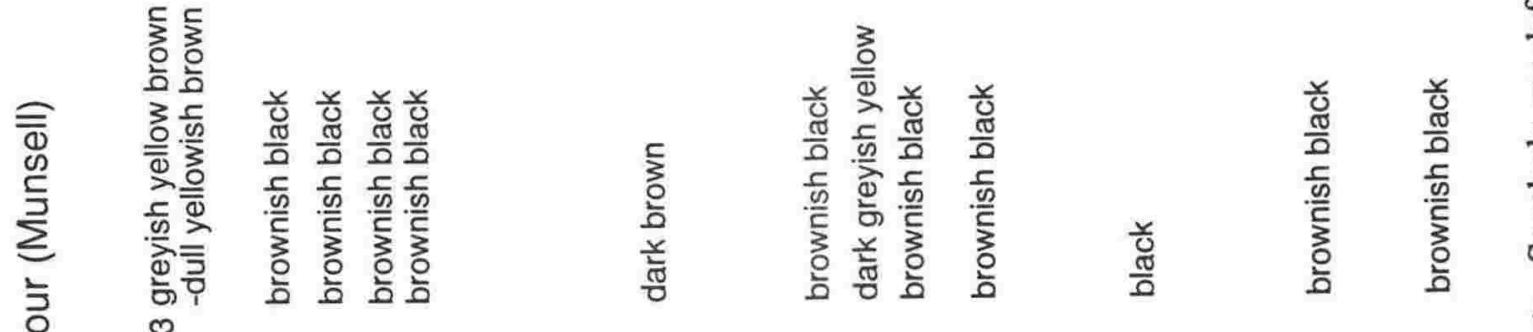

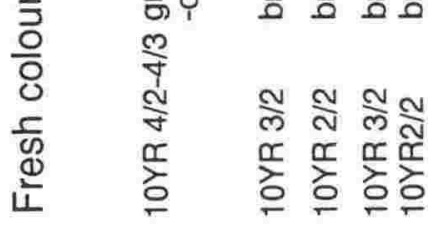

\begin{tabular}{|c|}
\hline 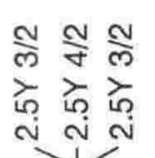 \\
\hline
\end{tabular}

$\begin{array}{lll}\pi & \frac{\pi}{\pi} \\ \frac{m}{\pi} & \frac{\pi}{2}\end{array}$

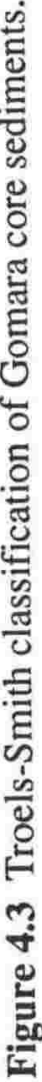

드

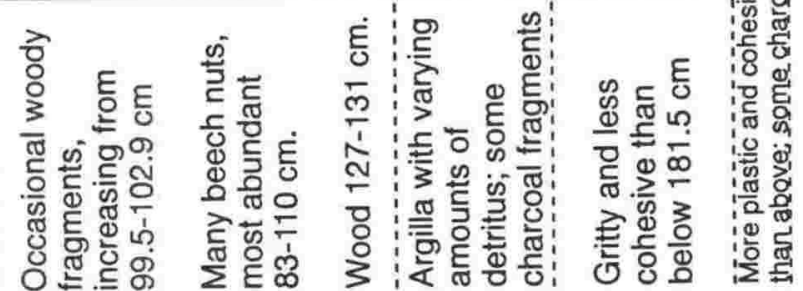

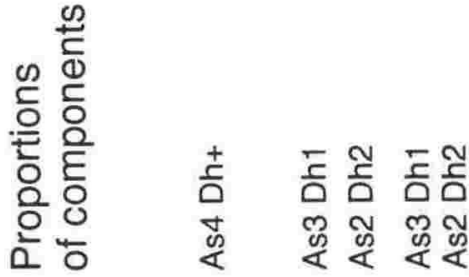

sәแ!า

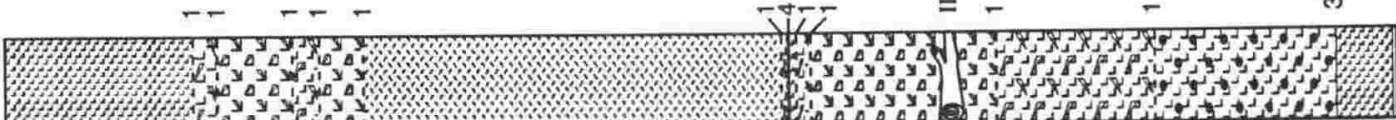

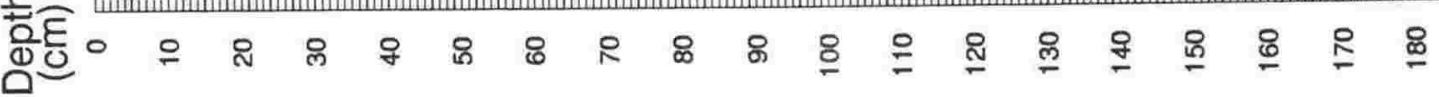


This sequence comprises minerogenic and organic deposits washed into the wetland from the catchment. No samples effervesced in $10 \% \mathrm{HCl}$, indicating the absence of calcium carbonate. This is consistent with a soil derived from the local bedrock of serpentinite (Spence 1957).

Macrofossils of superterranean fragments of herbaceous plants or parts of plants (Detritus herbosus) occur throughout the core, but from $83-157 \mathrm{~cm}$ macrofossils of superterranean woody parts of plants (Detritus lignosus) occurred. These are very abundant from $83-110 \mathrm{~cm}$, but they occur down to $157 \mathrm{~cm}$ decreasing in abundance with depth. Time constraints precluded a systematic numerical study of these macrofossils. Many of the fragments were twigs and pieces of wood that were not easily identifiable, but many were identifiable beech nuts, suggesting that beech grew in the vicinity of the wetland (Ryvarden 1971, 1975; Watts 1978; Glaser 1981; Wasylikowa 1986). The lowest beech nut was found at $155 \mathrm{~cm}$. A pine cone scale was noted at $107 \mathrm{~cm}$ and a piece of wood $4 \mathrm{~cm}$ thick was found from $127-131 \mathrm{~cm}$.

190.0-181.5 cm The basal sediments are composed of Argilla steatodes (mineral particles $<0.002 \mathrm{~mm}$ ) with only a trace of Detritus herbosus (organic detrital material derived from herbaceous plants). The sediments are brownish black (10YR 2/2), somewhat plastic and cohesive, and homogeneous (stratificatio 0 ). Small particles of charcoal were noted.

$181.5-157.0 \mathrm{~cm}$ The boundary is relatively distinct (limes 3 ) at $181.5 \mathrm{~cm}$ where the sediment becomes gritty with the addition of coarser-grained particles of Grana saburralia (mineral particles $0.6-2.0 \mathrm{~mm}$ ) (As3 Gs1 Dh+). This sediment is slightly drier, less cohesive, less plastic, and slightly lighter in colour (brownish black, $2.5 \mathrm{Y} 3 / 1$ ) than the unit below.

$157.0-135.5 \mathrm{~cm}$ Sediment composition changes gradually with an indistinct boundary (limes 1) at $157.0 \mathrm{~cm}$. Grana saburralia is replaced by Detritus herbosus, and D. lignosus (organic detrital material derived from ligneous plants) appears as a trace (As3 Dh1 Dl+). Charcoal particles were noted. The sediments are very dark in colour (black, 10YR 2/1), very slightly stratified (stratificatio 1), not elastic, and wetter than in the unit below.

$135.5-110.0 \mathrm{~cm}$ Again the sediment composition changes gradually at an indistinct boundary (limes 1) at $135.5 \mathrm{~cm}$, where the proportion of Detritus herbosus increases relative to Argilla steatodes (As2 Dh2 D1+). The colour of the sediments becomes less black (brownish black, 10YR 2/2), and the physical properties of stratificatio (1) and elasticitas (0) remain unchanged.

110.0-106.0 $\mathrm{cm}$ Above another indistinct boundary at $110.0 \mathrm{~cm}$, the preceding unit grades into a transitional unit below a thin but very distinct unit from $108.2-107.2 \mathrm{~cm}$. A sharp boundary (limes 4) occurs at $107.2 \mathrm{~cm}$, above which is another transitional unit of $0.8 \mathrm{~cm}$ thickness (up to $106.0 \mathrm{~cm}$ ). Between 108.2 and $107.2 \mathrm{~cm}$ a very distinct band of 
laminated dark greyish-yellow (2.5Y 4/2) pure Argilla steatodes (As4) occurs. On both sides of this band the narrow transitional bands of Argilla steatodes with Detritus herbosus (As 3 Dh1) are brownish black (2.5Y 3/2), the lower band differing from the upper by being weakly laminated. These units are not elastic (elasticitas 0 ).

106.0-49.0 cm Above these distinct units the sediment composition changes to one that is predominantly felted Detritus herbosus up to $49.0 \mathrm{~cm}$. At the base of this unit the sediments are composed of Detritus herbosus with some Argilla steatodes and a trace of Detritus lignosus (Dh3 As1 Dl+). The proportion of Detritus herbosus increases upwards; the sediment becomes pure Detritus herbosus ( $\mathrm{Dh} 4)$ at the top of the unit. This unit is dark brown (10YR 3/3), does not split easily into thin horizontal layers (stratificatio 1-2), but is somewhat elastic (elasticitas 1-3, increasing with depth).

49.0-25.7 cm An indistinct boundary (limes 1) occurs at about $49.0 \mathrm{~cm}$, where Argilla steatodes is mixed with Detritus herbosus. Above this boundary are several units comprised of a mixture of Detritus herbosus and Argilla steatodes in varying proportions. These units are alternating bands of firstly equal proportions of Argilla steatodes and Detritus herbosus (As2 Dh2) brownish black (10YR 2/2) in colour, then Detritus herbosus-rich Argilla steatodes (As3 Dh1) brownish black (10YR 3/2) in colour. These units are not laminated (stratificatio 0 ) or elastic (elasticitas 0$)$.

$25.7-0 \mathrm{~cm}$ The top $25.7 \mathrm{~cm}$ is highly disturbed by animal trampling and cracking (Figure $4.1 \& 4.3$ ). Cracking is a characteristic property of almost all Grevena soils during the annual dry season, due to swelling clays (Chapter 1). The deposit consists of Argilla steatodes with varying amounts of Detritus herbosus. A pebble at $c .24 \mathrm{~cm}$ may have been pushed in by animals trampling. This sedimentary unit is mottled with iron staining, but the general colour is greyish yellow brown to dull yellowish brown (10YR 4/2-4/3), which results in reduced nigror (2). These sediments are not laminated (stratificatio 0), or elastic (elasticitas 0$)$.

\subsubsection{Physical properties}

Organic matter, inorganic matter, moisture content, and dry density are plotted against depth and stratigraphy in Figure 4.4. The first three properties are expressed as a percentage of the original wet weight of the sample. Diatoms, although present, are thought to comprise only a minor proportion of total inorganic sediment. The curve of diatom concentration follows that of the inorganic matter (Appendix XII). Physical properties of the sediments suggest a division of the sequence into 8 sections $(\mathrm{A}-\mathrm{H})$, corresponding closely to the sediment types described above. The physical properties of the sediments are described below. 


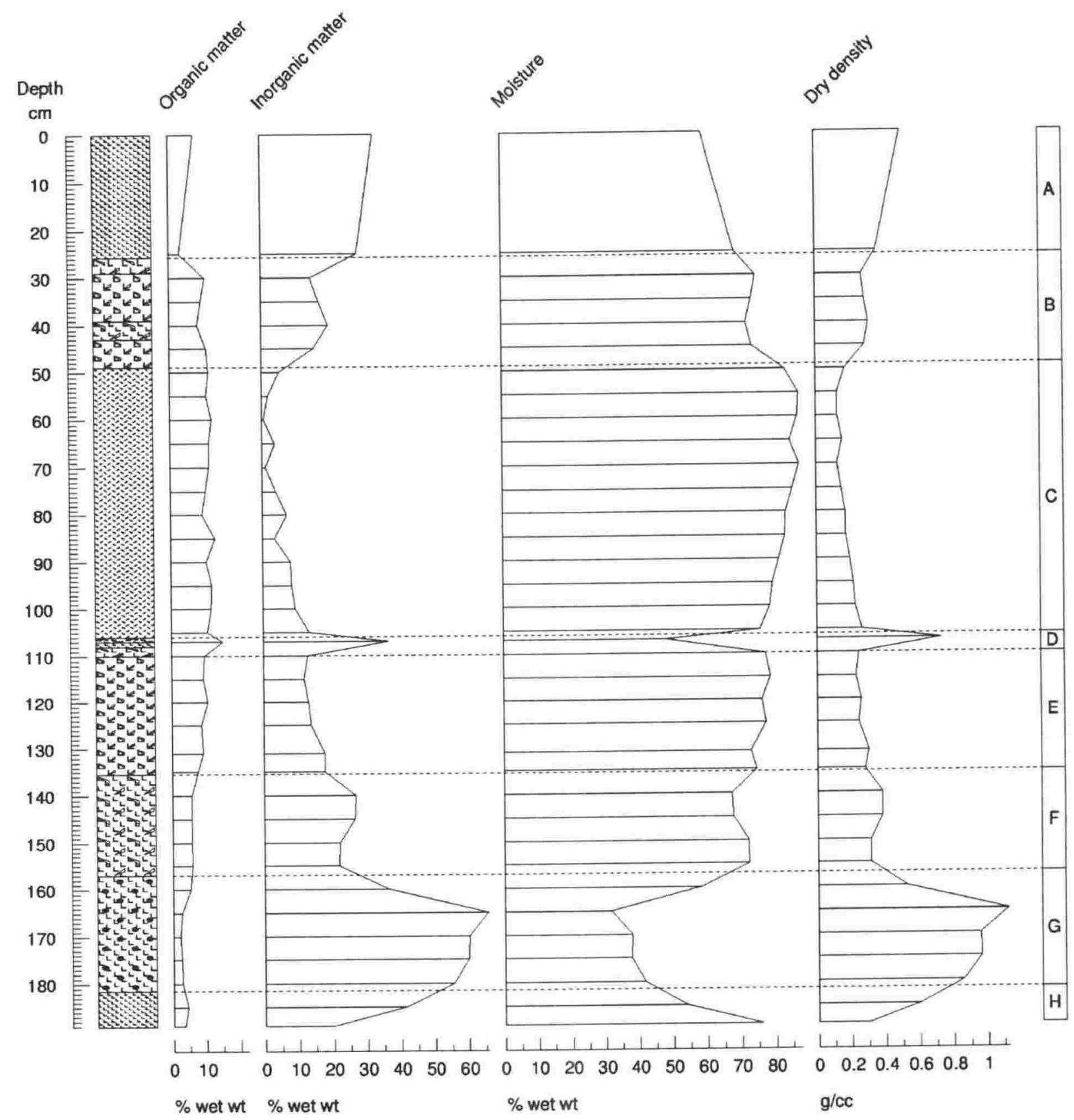

Figure 4.4 Gomara sediment physical properties: sediment type column (Troels-Smith classification 1955), organic matter, inorganic matter, moisture and bulk (dry) density. Sediment sections based on physical properties are indicated on the right. 
Section $\mathrm{H}, 190.0-181.5 \mathrm{~cm}$ The sediment in this section is moderately rich in organic matter $(3.5-4.4 \%)$, and moisture content is high (54.0-76.0\%). Dry density values of $0.31-0.60 \mathrm{~g} / \mathrm{cc}$ also suggests an organic sediment. Inorganic matter increases from 20.5 to $41.6 \%$ at the top of the section.

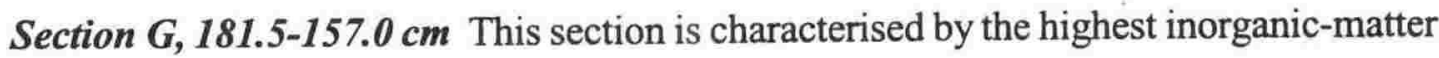
content of the sequence (peaking at $65.7 \%$ ), and correspondingly high dry density (peaking at $1.12 \mathrm{~g} / \mathrm{cc}$, which is close to the $1.25 \mathrm{~g} / \mathrm{cc}$ average dry density of a mineral soil). Moisture content is the lowest of the sequence, decreasing to $31.6 \%$, where inorganic matter content is greatest. Organic matter ranges from $2.2-2.8 \%$ and are the lowest of the sequence, with the exception of the uppermost sample, which increases to $5.3 \%$.

Section $F, 157.0-135.5 \mathrm{~cm}$ This section is characterised by higher organic content $(5.7-5.9 \%)$ than that of the section below, high moisture content (67.4-72.2\%), and much decreased but relatively high dry density $(0.32-0.38 \mathrm{~g} / \mathrm{cc})$. Inorganic content is only $21.9-26.8 \%$.

Section E, 135.5-110.0 cm Organic matter and moisture content are higher (and increase slightly upwards) in this section (maximum values of $10.7 \%$ and $78.7 \%$, respectively) than in that below, while inorganic matter and dry density are lower and decline slightly upwards (minimum values $11.8 \%$ and $0.23 \mathrm{~g} / \mathrm{cc}$, respectively).

Section $D, 110.0-106.0 \mathrm{~cm}$ This section is represented by only one sample. The physical properties of this sample are very different from those in the adjacent sections: organic and inorganic matter and dry density all increase $(15.3 \%, 36.6 \%$ and $0.73 \mathrm{~g} / \mathrm{cc}$, respectively), while moisture content decreases (48.1\%).

Section C, 106.0-49.0 cm Organic matter content is very high (9.4-13.2\%), recording maxima of the sequence, with the exception of section $\mathrm{D}$. Moisture content is correspondingly very high, reaching a maximum of $87.5 \%$ and in general recording the maxima of the sequence. Inorganic matter content is the lowest of the sequence: values are lower in the upper half of the section (minimum of $0.5 \%$ at $60.0 \mathrm{~cm}$ ). Dry density is also the lowest of the sequence and generally decreases upwards ranging from $0.13-0.27 \mathrm{~g} / \mathrm{cc}$.

Section $\boldsymbol{B}, 49.0-25.7 \mathrm{~cm}$ This section is characterised by an increase in inorganic content, which reaches $19.5 \%$ at $40.0 \mathrm{~cm}$. Dry density also increases (peaking at $0.32 \mathrm{~g} / \mathrm{cc}$ at $40.0 \mathrm{~cm}$ ), while moisture content declines to a minimum of $72.1 \%$, again at $40.0 \mathrm{~cm}$. Organic content declines slightly (8.4-11.0\%).

Section A, 25.7-0 cm Two samples were analysed in this section; a surface sample and one at $25.0 \mathrm{~cm}$. The physical properties of these samples differ from those below by having lower organic matter (7.6 and 3.2\%), higher inorganic matter (33.0 and 28.0\%), lower moisture content (59.4 and 69.0\%), and higher dry density ( 0.51 and $0.36 \mathrm{~g} / \mathrm{cc}$ ). 


\subsection{Chronostratigraphy}

The chronology of the Gomara sequence is based on radiocarbon ages of organic matter contained within the sediments. Samples for radiocarbon dating were selected to provide ages for various depositional events inferred from lithologic characteristics so are not evenly spaced.

The results of five critically positioned radiocarbon-dated samples are presented in Table 4.1. Conventional radiocarbon ages are shown with an analytical error of one standard deviation. These ages have been calibrated by tree-rings (Appendix IV).

Calibrated radiocarbon years before present (1950) and sidereal years are plotted against depth and stratigraphy (Figure 4.5). In Figure 4.5 the calibrated calendar date, if only one date results from calibration, or the median of the calibrated calendar dates, if several result from calibration, are joined by straight-line segments, indicating mean rate of sediment deposition. (This assumes a constant rate of sedimentation.) The line between the highest two radiocarbon ages has been extended to the next change in sediment composition. The solid line above this sediment change has been drawn with the same slope as that at $156.5-111.5 \mathrm{~cm}$ because of the similarity in the sediment compositions in these two intervals. Above $25.0 \mathrm{~cm}$ organic sediment is absent and oxidation has occurred, so the line is dashed.

Most of these sediments were apparently continuously deposited under gradually changing conditions and processes, so mean sediment-accumulation rates between dated samples were based on a linear age/depth function (Figure 4.5). A major change in the mean sediment-accumulation rate can be seen near the centre of the sequence, where a corresponding change in sediment composition occurs. However, mean sedimentaccumulation rates either side of this major change are very similar. In the lower part of the sequence the two mean rates of sediment accumulation are 0.069 and $0.058 \mathrm{~cm} / \mathrm{yr}$, while sediments above accumulated more than twice as rapidly at 0.152 and $0.145 \mathrm{~cm} / \mathrm{yr}$. This rapid accumulation rate is attributed to the coarseness of incompletely decomposed organic matter. The lower, more slowly accumulating sediment comprises mostly fine-grained mineral particles. These rates are close to the optimal rate of $1 \mathrm{~mm} /$ year, and the normal accumulation rate of $0.5 \mathrm{~mm} /$ year suggested for pollen-analysis sites in lakes (Berglund 1986b:119).

These mean accumulation rates do not measure possible rapid sediment accumulation episodes of short duration. One obvious change in sediment type which is likely to represent a rapid depositional event is that at $108.2-107.2 \mathrm{~cm}$. Unfortunately, this sediment has not been bracketed by radiocarbon ages. However, it is interpreted as a tephra (see below) and considered to be deposited 'instantaneously'. 


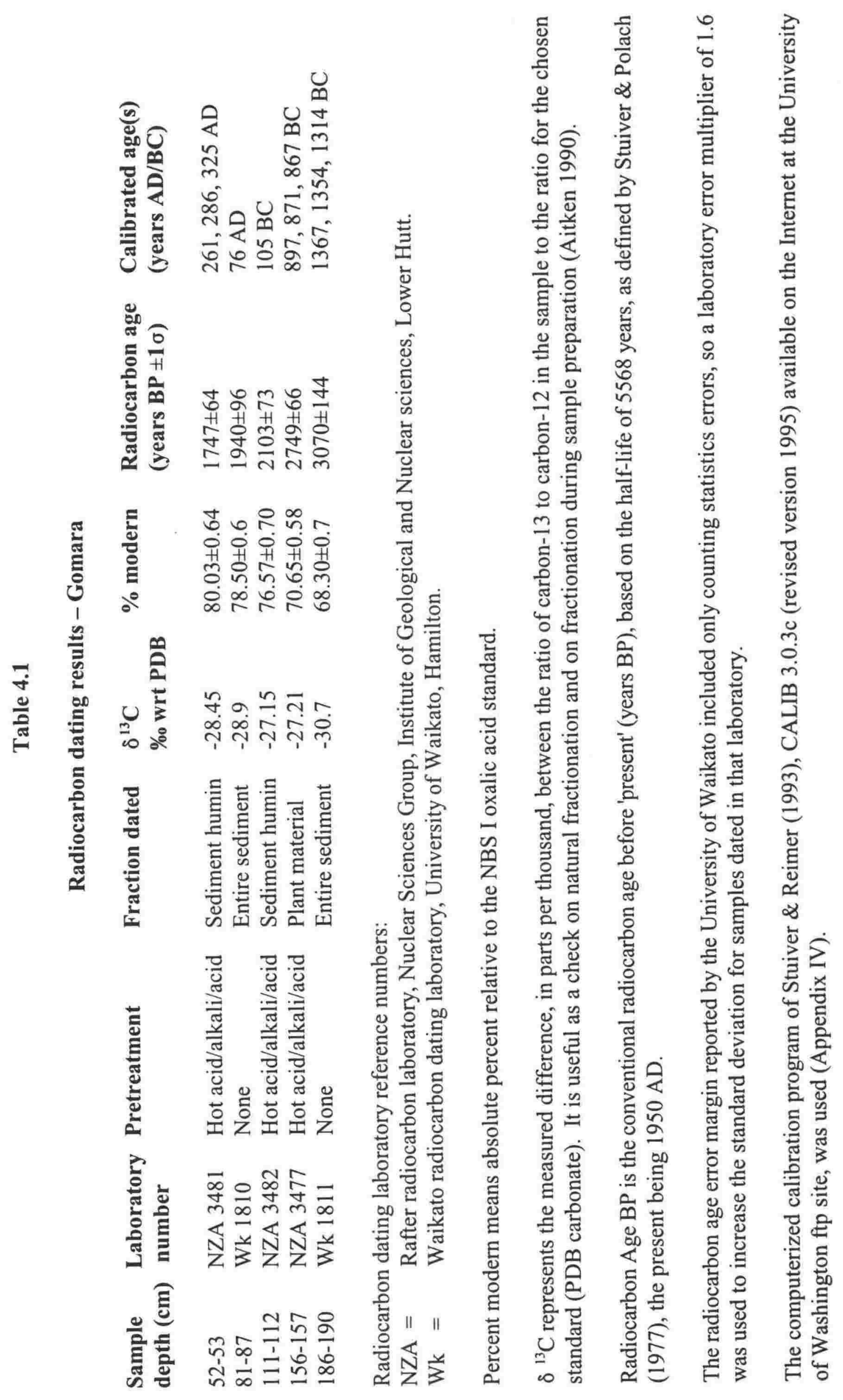


Pollen samples of one centimetre thickness from below $107.0 \mathrm{~cm}$ span a time interval of about 14.5-15.2 years, while those above span about 5.8-6.9 years. The intervals between each sample in the lower part of the sequence represent about 58-61 years, while those in the upper part represent about 23-28 years. These time resolutions fall within the range of 20-50 years for a sample of $1 \mathrm{~cm}$ thickness recommended for pollen analysis reference sites of 100 $300 \mathrm{~m}$ diameter in open mountainous areas (Berglund 1986b:114).

The calibrated calendar dates and the rates of sediment accumulation are considered to be a reasonable approximation of the 'true' time span represented by the sediments in this sequence, so a timescale has been derived for this sequence using the age/depth function of Figure 4.5.

\subsection{Tephra}

On visual examination of the sediment sequence it was suspected that the layer at $108.2-107.2 \mathrm{~cm}$ was a tephra because of the absence of plant material. On microscopic examination small glass shards were seen, so it is almost certainly a volcanic ash (J. Carter, pers. comm.). Unfortunately, microprobe analysis proved difficult because of alkaline etching of the glass shards, so identification of a particular eruption based on chemical composition of the shards could not be determined (J. Paterson, pers. comm.). Thus the tephra could not be used as a chronological marker.

The small size of the glass shards suggests that it has been transported a long distance. A radiocarbon date just below the tephra $(111-112 \mathrm{~cm})$ of $105 \mathrm{BC}$ suggests that the tephra is from an Italian volcanic eruption at Etna, Monte Albano, or Vulcano (Simkin et al. 1968).

\subsection{Diatom analysis}

Only eleven samples were analysed, so these are much more widely spaced than samples for sediment and pollen analyses, but all the major sediment types and pollen-assemblage zones are sampled. Samples $1 \mathrm{~cm}$ thick were analysed at 1, 10, 35, 56, 84, 106, 119, 139, 154, 172 , and $189 \mathrm{~cm}$, and at least 200 frustules per sample were counted. The results of the diatom identifications made by Dr M. Harper are presented in Appendix XII in relative frequency graphs, where diatoms have been grouped according to their various ecological tolerances (van Dam et al. 1994). Separate graphs have been drawn of $\mathrm{pH}$ tolerance, moisture requirements, habitat, trophic levels, oxygen requirements, and organic nitrogen uptake metabolism. A summary of the results, according to the local wetland pollen assemblage zones is also presented in Appendix XII. 


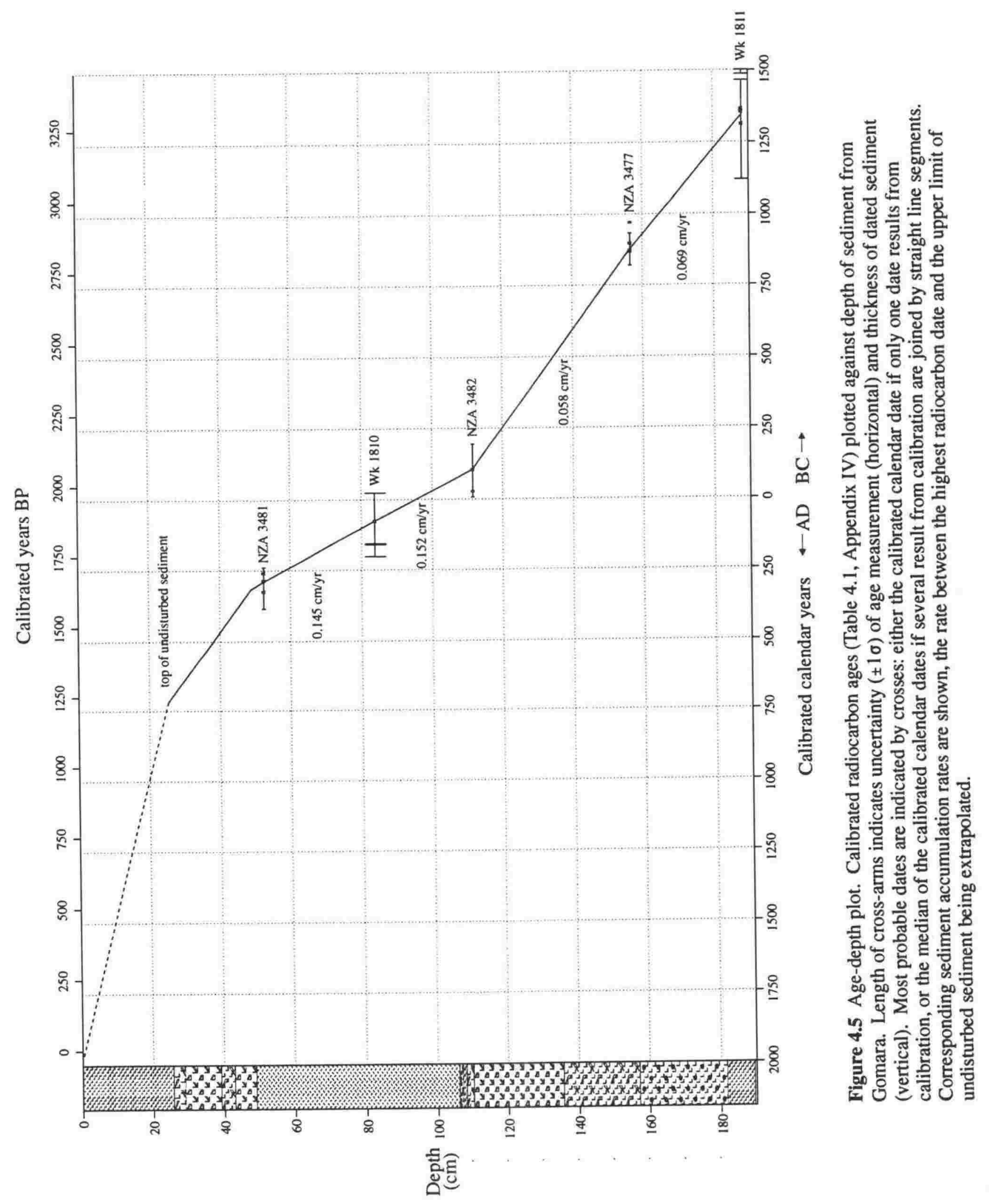




\subsection{Pollen analysis results}

\subsubsection{Introduction}

Relative frequency as well as concentration data are used to interpret the vegetation history of the Gomara area. Concentration data are converted to influx rates, using the sediment deposition rates calculated from the radiocarbon chronology. Both the aliquot and exotic marker grain (tracer) methods of calculating palynomorph concentrations were used, and pollen diagrams using the results from these two methods show a high degree of correlation. Separate diagrams are drawn for local (wetland) taxa, extra-local and regional (terrestrial) taxa, and microscopic charcoal data. Data used to calculate pollen and spore concentrations, all pollen and spore types counted, and charcoal abundances are tabulated in Appendix XIII.

\subsubsection{Pollen preservation/pollen abundance}

Palynomorphs are very abundant and preservation excellent. The principal mode of deterioration of grains is breakage, and this is usually confined to gymnosperm pollen with bladders that are easily separated from the body of the pollen grain. The ratio of broken pine grains to the total number of pine grains counted is highest at $140-55 \mathrm{~cm}$. An index of pollen preservation is given by the abundance of indeterminable pollen (not included in the pollen sum because they could be either wetland or terrestrial taxa). Indeterminate grains are usually less than $4 \%$, exceeding $5 \%$ in only 2 spectra. Unidentified monolete spores are rare, being recorded at $0.1 \%$ in 3 spectra. Unidentified trilete spores are even rarer, being recorded in one spectrum at $0.2 \%$. Unknown grains are rare, exceeding $1 \%$ in only one spectrum.

In general, concentration values were highest in inorganic sediments of low accumulation rate and lowest in organic sediments of high accumulation rate. The highest concentration of total terrestrial pollen and spores is in samples at $180-160 \mathrm{~cm}\left(222-433 \times 10^{3}\right.$ grains $\left./ \mathrm{cm}^{3}\right)$. The two basal samples $(189$ and $185 \mathrm{~cm}$ ) and those from $155-107 \mathrm{~cm}$, at $25 \mathrm{~cm}$, and the surface display the next highest concentrations. Concentrations are lowest from $105-30 \mathrm{~cm}$ (very low at $70-55 \mathrm{~cm}, 12-45 \times 10^{3}$ grains $/ \mathrm{cm}^{3}$ ).

The total terrestrial influx curve differs somewhat from this pattern (Figure 4.10). Samples at $180-160 \mathrm{~cm}$ have some of the highest values, but the two samples at 110 and $107 \mathrm{~cm}$ have equally high influx of $25-30 \times 10^{3}$ grains $/ \mathrm{cm}^{2} /$ year, and samples at $105-75 \mathrm{~cm}$ have the next highest influx of 10-12 x $10^{3}$ grains $/ \mathrm{cm}^{2} /$ year, while those at $155-115 \mathrm{~cm}$ have lower influx $\left(5-10 \times 10^{3}\right.$ grains $/ \mathrm{cm}^{2} /$ year $)$. The depth interval recording lowest influx is 70 $0 \mathrm{~cm}\left(2-7 \times 10^{3}\right.$ grains $/ \mathrm{cm}^{2} /$ year $)$.

The concentration curve of total wetland taxa is similar to that of total terrestrial taxa, displaying highest values near the base of the sequence in inorganic sediments at $185-160 \mathrm{~cm}$ 
$\left(92-181 \times 10^{3}\right.$ grains $\left./ \mathrm{cm}^{3}\right)$. But the basal sample and those at $155-107 \mathrm{~cm}$ are lower, while those at $107 \mathrm{~cm}$, at $50 \mathrm{~cm}$ and at $45 \mathrm{~cm}$ are higher; concentrations are similarly high at $25 \mathrm{~cm}$ and at the surface sample. Lowest concentrations are displayed from $105-55 \mathrm{~cm}$, with very low concentrations at $85-70 \mathrm{~cm}\left(5.5-8.5 \times 10^{3}\right.$ grains $\left./ \mathrm{cm}^{3}\right)$.

Total wetland taxa influx also displays high values at the base of the sequence and peaks at $107 \mathrm{~cm}$, at $50 \mathrm{~cm}$, and at $45 \mathrm{~cm}$, with lower values in the more organic deposits from 155 $110 \mathrm{~cm}$ and $105-55 \mathrm{~cm}$ (Figure 4.7). The influx curve differs from the concentration curve in displaying low influx from $40 \mathrm{~cm}$ to the surface.

\subsubsection{Wetland pollen analysis}

\subsubsection{Pollen diagram construction}

Pollen and spore relative frequencies are presented graphically in Figure 4.6 and influx data in Figure 4.7. Pollen and spore types are arranged according to the degree of wetness of habitat of plant communities from which they derive. Wetland plant communities are divided into the two broad habitat groups of emergent and open water (aquatic macrophytes). Emergent plants are divided into the habitat groups of wet-soil and sedge and reed swamp, while the aquatic macrophytes are divided into the habitat groups of floating-leaved and submerged macrophytes. Floating-leaved macrophytes are those that have leaves that float on the surface of the water at least for part of the time, while the submerged macrophytes are totally submerged or only flowers emerge above the surface of the water. The pollen sum on which the relative frequencies are based includes all wetland pollen and spore taxa. Relative frequencies of $0.2 \%$ or less are indicated with a dot. Only diagrams showing relative frequency and influx, using data obtained from the exotic spike method, are shown.

Pollen spectra are arranged according to $\mathrm{cm}$ depth below sediment surface. The column next to the depth column shows the sidereal timescale estimated from the radiocarbon chronology. The column to the right shows the major sediment types in the sequence according to the Troels-Smith classification (1955). The final column before the curves of pollen and spores indicates the conventional radiocarbon ages of sediment samples.

On the right of the resolved diagram, a summary diagram shows the ratio of emergent types to aquatic types. A curve of palynological richness (rarefaction analysis) follows (Birks \& Line 1992; Bennett 1994); rarefaction analysis is based on the smallest total count of the wetland pollen and spores, 48 at $110 \mathrm{~cm}$. The number of wetland types (not normalized for unequal pollen sums) in each spectrum is shown next. Rates of vegetation change were calculated using the dissimilarity coefficient of Chi-squared coefficient 2 on unsmoothed pollen frequencies of the 11 most abundant pollen and spore types (that is, those that were $\geq 5 \%$ of total wetland pollen and spores) and calibrated radiocarbon ages (Bennett \& Humphry 


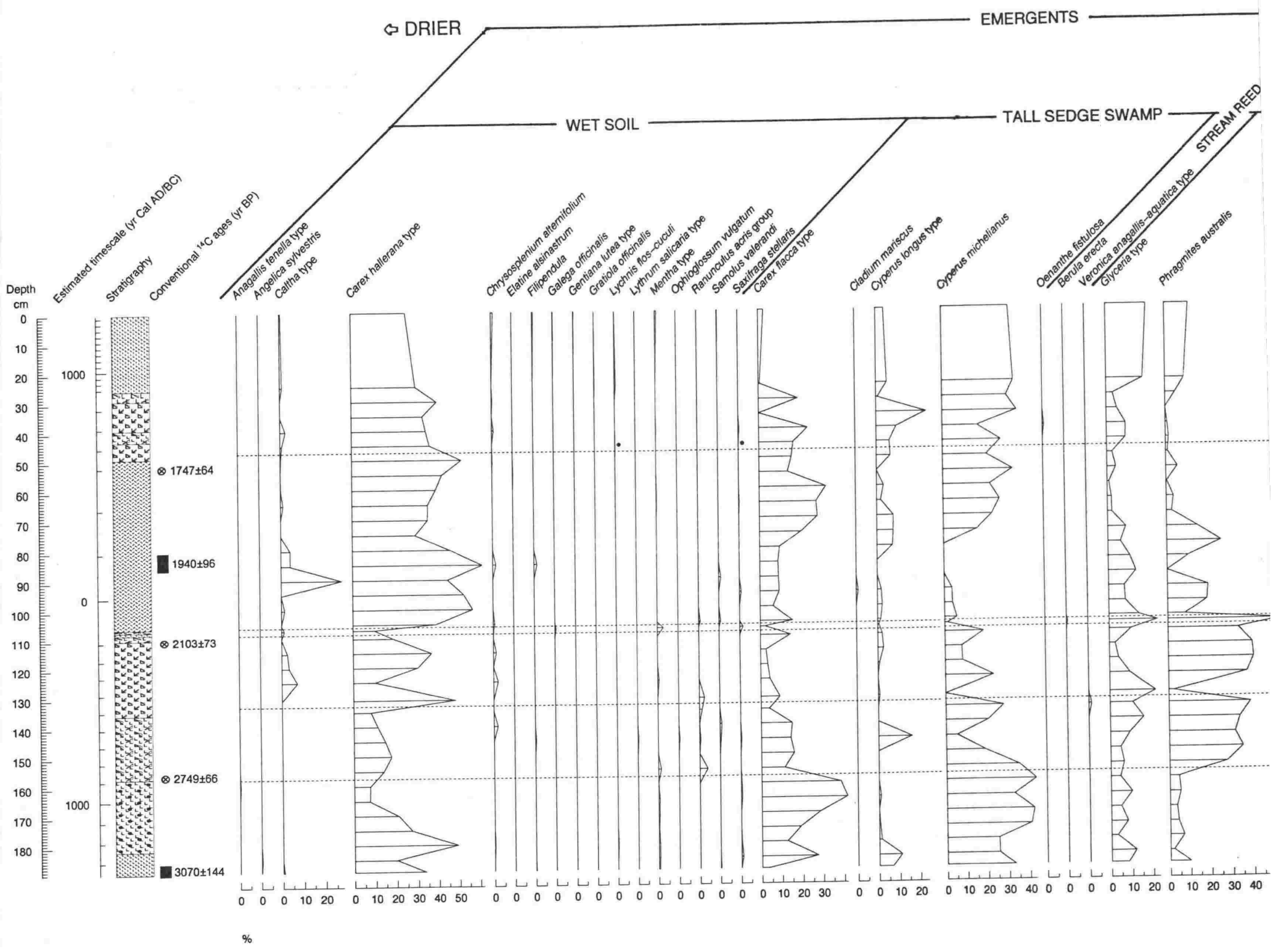

Figure 4.6 Gomara - relative frequency pollen diagram, wetland taxa (pollen sum is total wetland taxa) 


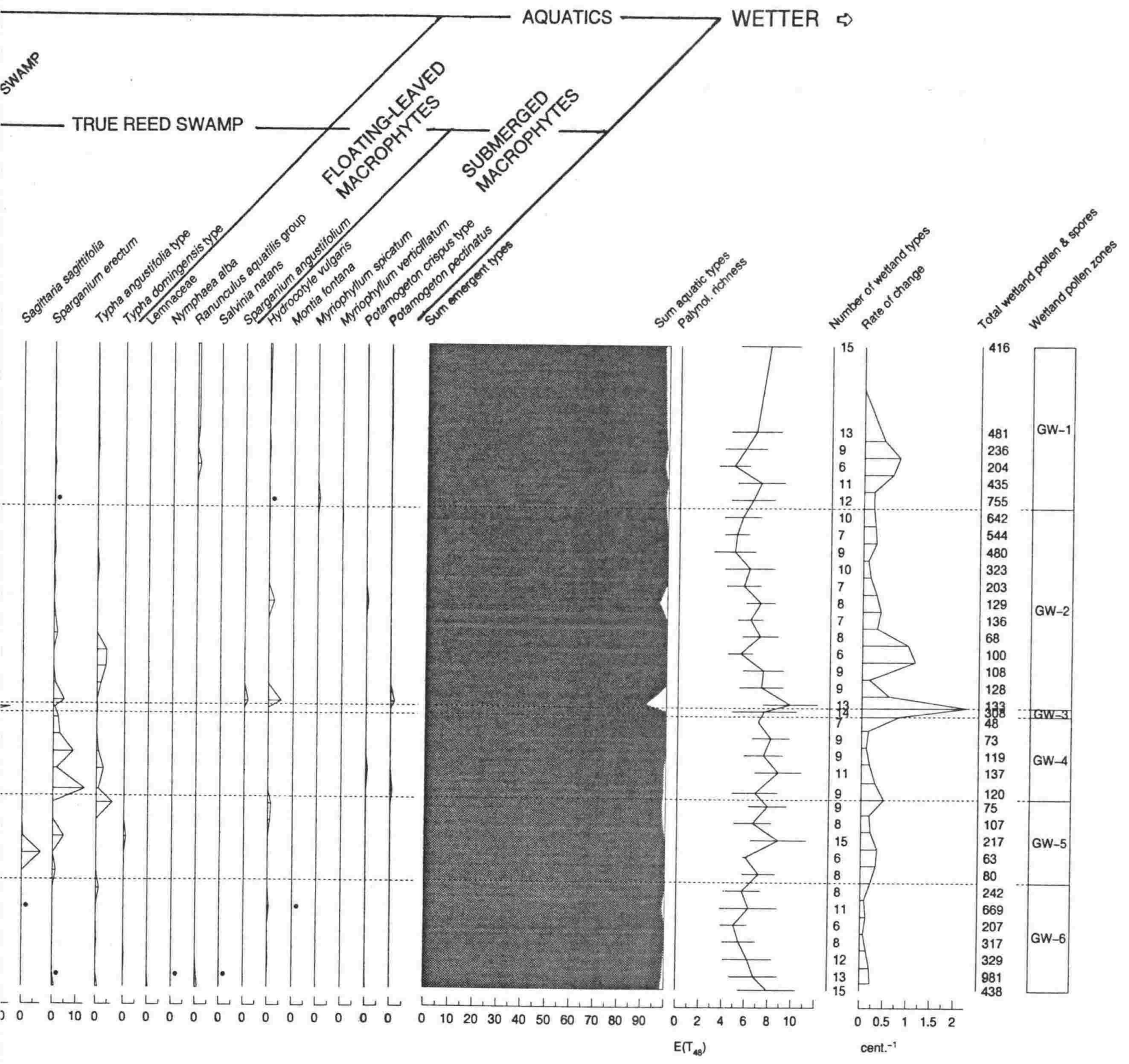

Figure 4.6 


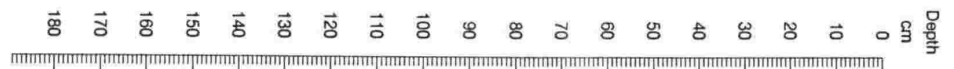
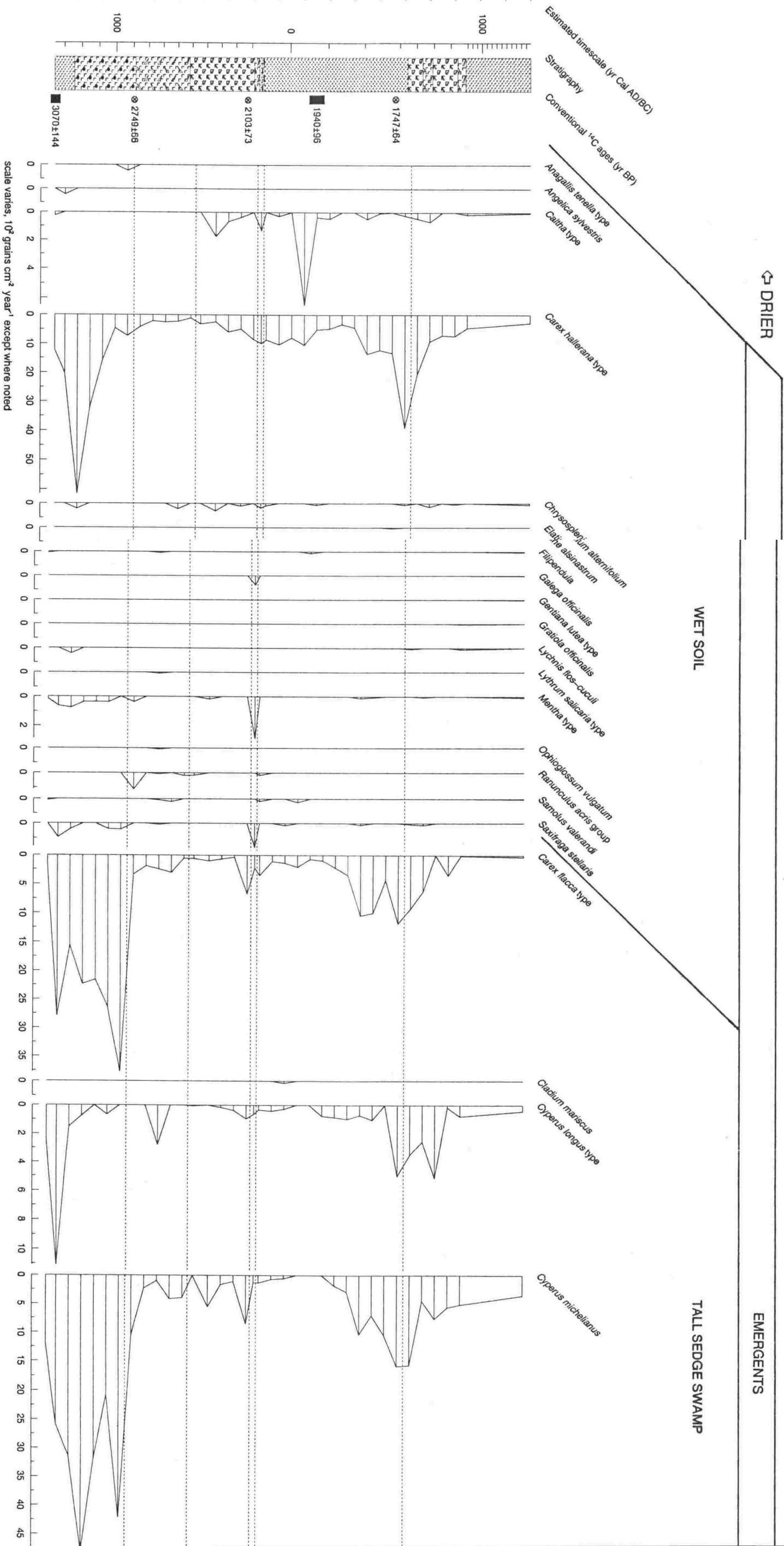


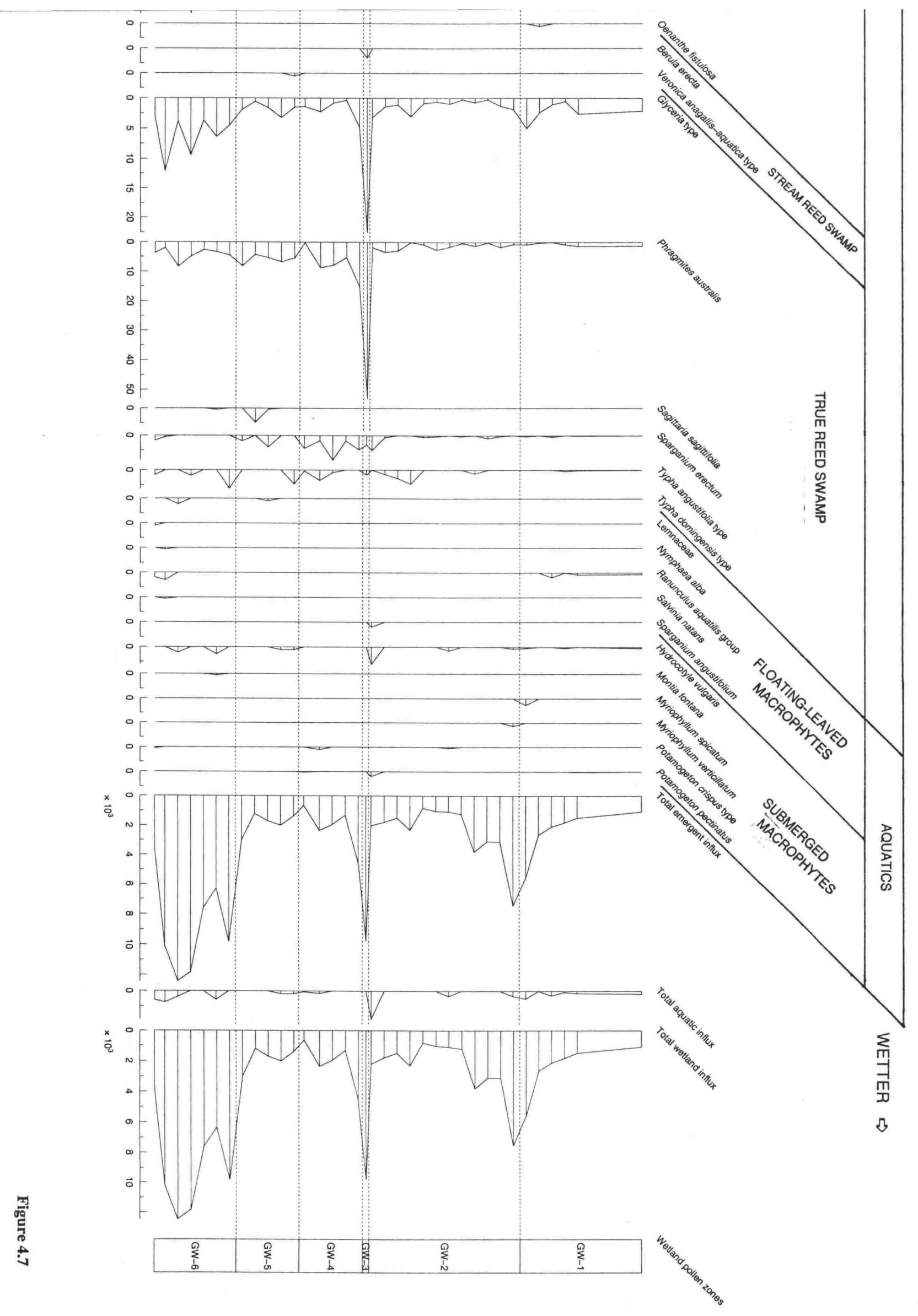


1995). The types included in the calculations are Caltha type, Carex flacca type, Carex hallerana type, Cyperus longus type, Cyperus michelianus, Glyceria type, Phragmites australis, Sparganium erectum, Typha angustifolia type, Hydrocotyle vulgaris, and Sagittaria sagittifolia. Lastly, the total of all wetland pollen and spores counted is given.

\subsubsection{Pollen record of wetland plant communities}

Wetland plant communities are floristically and ecologically more uniform throughout the Balkan Peninsula than any other group of plant communities, and division into Mediterranean and Central European domains is less clearly indicated than for other communities. Relatively few Balkan Peninsula types and endemics occur in them, and the species found are mostly such as are distributed in similar habitats throughout a large part of Europe. Wetland plant communities discussed below are based mostly on information from four sources: Turrill (1929); Haslam et al. (1975); Ellenberg (1988); and the fieldwork carried out for this research (Chapter 3).

Although a high proportion of wetland pollen and spore types can be identified to species level, some types present problems because they include plants that grow in dryland habitats, for example Bromus species that grow in dry habitats have pollen morphology similar to that of Glyceria maxima (Appendix VI.2). Also the comparative pollen morphology of a few wetland plants is unknown, e.g. Phalaris arundinacea, a grass that grows in reed and sedge swamps.

Wetland plant communities are often under-represented by pollen and spores and are often represented by only a few species (Chapter 3). A maximum of only 15 pollen and spore types were identified in any spectrum from Gomara; average is 10. Estimates of palynological richness by rarefaction analysis are low (5-9 expected types).

Emergent communities The wetland pollen and spore sequence is dominated by types that derive from emergent plant communities of swamp and fen habitats which commonly form marginal belts around lakes, according to depth of water (Figures $4.6 \& 4.7$ ). These plant communities usually grow in water to a maximum depth of $2 \mathrm{~m}$ (Spence 1982). Pollen and spores of types belonging to sedge- and reed-swamp communities are most numerous. Sedge swamps have many species in common with reed swamps, and they both belong to the same order, Phragmitetalia (Ellenberg 1988). Many of the species included in the Carex flacca type (Appendix VI.2) have been placed by Ellenberg (1988) in the tall-sedge swamp alliance (Magnocaricion). Those included are: Carex appropinquata, C. distans, C. disticha, C. elata, C. paniculata, C. riparia, C. rostrata, and C. vulpina. The species Cyperus longus and Oenanthe fistulosa are also included. Cladium mariscus often grows in a mixed sedge and reed swamp. Berula erecta and Veronica anagallis-aquatica belong to the stream-reed swamp 
alliance (Sparganio-Glycerion). Species belonging to the true-reed swamps (Phragmition alliance) are: Phragmites australis, Glyceria maxima (Glyceria type), Sagittaria sagittifolia, Sparganium erectum, and Typha angustifolia and T. latifolia (comprising Typha angustifolia type) (Ellenberg 1988).

Most of the species included in the types placed in the habitat group of 'wet soil' (Figures $4.6 \& 4.7$ ) grow in wet marshy ground and are included in the orders Molinietalia, of moist meadow and stream-bank communities, and Cyperetalia fusci, of dwarf plant communities that are inundated in winter but dry out in summer (Ellenberg 1988). Included are the species of Anagallis tenella type, Angelica sylvestris, Elatine alsinastrum, Gratiola officinalis, Lychnis flos-cuculi, Lythrum salicaria type, and Ophioglossum vulgatum. Caltha palustris, included in the Caltha type, is also placed in the order Molinietalia, while the other species in this type, Myosurus minimus and Aquilegia vulgaris, are placed in the wet-soil plant communities of the order Agrostietalia stoloniferae, which form pioneer swards of flooded and damp places, and the class Querco-Fagetea of broadleaved woods and scrub on more fertile soils, respectively. Saxifraga stellaris belongs to the wet-soil community of the order Montio-Cardaminetalia of spring communities. Chrysosplenium alternifolium is included in the wet-soil order of Fagetalia, alliance Alno-Ulmion of alder and noble broadleaved woods of flood plains (Ellenberg 1988).

Some pollen and spore types in the 'wet soil' habitat group include a mixture of species from wetland and dryland communities. One of the two species comprising Filipendula (Appendix VI.2) belongs to the order Molinietalia, while the other grows in dry grassland and belongs to the dryland plant communities of the class Festuco-Brometea. Gentiana lutea type includes one species included in order Molinietalia, one that grows mainly on soils of average dampness (but absent from both wet ground and places which may dry out), and one in order Brometalia erecti (communities of more or less arid swards). Mentha type includes some species that belong to emergent wetland communities as well as some species that grow in dryland communities. Ranunculus acris type includes several species; they grow in wetmeadow communities mostly (Ellenberg 1988).

Species in the Carex hallerana type are not included in Ellenberg's (1988) classification scheme. They have a wide range of habitats, which include damp soils at high altitude. Samolus valerandi is not included in Ellenberg's (1988) classification, but Turrill (1929) identifies it as part of the Balkan marsh community. Galega officinalis grows in damp meadows and by rivers (Polunin 1988).

Aquatic communities A few pollen and spores of aquatic types occur sporadically throughout the sequence, suggesting the possibility of an open-water habitat, at least sporadically (Figures $4.6 \& 4.7$ ). All free-floating aquatics are warmth-requiring, preferring 
waters that remain ice-free and require water that is protected from wind; these factors may partly account for the lack of pollen and spores identified from these communities (Ellenberg 1988). Floating-leaved communities usually grow on the lakeward side of reed swamps in water depths to a maximum of $2.5 \mathrm{~m}$ (Spence 1982), and submerged communities grow in the innermost belt in deeper water, usually at a depth of about $3 \mathrm{~m}$ (although they may grow in water depths up to $15 \mathrm{~m}$ ) (Spence 1982).

Types that include species of floating-leaved communities are: Lemnaceae, Nymphaea alba, Ranunculus aquatilis group, Salvinia natans, and Sparganium angustifolium. Lemna minor is the only species of the Lemnaceae type which occurs at altitudes higher than $1000 \mathrm{~m}$, so it is probably the species represented here. It belongs to the free-floating still-water communities of waters more or less rich in nutrients, order Lemnetalia (Ellenberg 1988). Salvinia natans also belongs to the order Lemnetalia (Ellenberg 1988). Nymphaea alba belongs to rooted waterplant communities (order Potamogetonetalia). Most species of the Ranunculus aquatilis group (Ranunculus aquatilis, R. peltatus, and $R$. trichophyllus) are also placed in the order Potamogetonetalia. Sparganium angustifolium belongs to the shorehairgrass shallow-water swards and related communities, order Littorelletalia (Ellenberg 1988).

Types that include species that may belong to submerged communities are: Hydrocotyle vulgaris, Montia fontana, Myriophyllum spicatum, Myriophyllum verticillatum, Potamogeton crispus type, and Potamogeton pectinatus. Hydrocotyle vulgaris and Montia fontana may be submerged or emergent (Haslam et al. 1975). Species included in the Potamogeton crispus type belong to rooted waterplant communities (order Potamogetonetalia) and are capable of living in either deep or shallow water, either submerged or floating, and subterrestrially in dried-up situations. Myriophyllum spicatum, Myriophyllum verticillatum, and Potamogeton pectinatus are exclusively submerged. They also belong to the order Potamogetonetalia (Ellenberg 1988).

\subsubsection{Wetland (local) pollen assemblage zones}

Wetland pollen diagrams are divided into six local pollen assemblage zones GW-1 to GW-6, based mostly on relative frequency data. These are defined below.

Zone $G W-6,190.0-157.5 \mathrm{~cm}$ This basal zone is characterised by high relative frequencies of Carex flacca type (12.5-41.6\%, with the exception of the lowest spectrum at $3.7 \%$ ), Carex hallerana type (19.9-49.2\%, except in the upper two spectra at about $7.0 \%$ ), and Cyperus michelianus (25.2-43.0\%). Glyceria type (3.0-11.8\%) and Phragmites australis (1.8$10.0 \%$ ) are represented at moderate frequencies, while Cyperus longus type occurs at moderate frequencies at the base of the zone (6.6 and 10.9\%) then declines sharply (zero at the top). 
Several other emergent types occur at very low frequencies, but only Typha angustifolia type exceeds $1.0 \%$, and this is in the uppermost spectrum only. Aquatic types are very poorly represented, occurring in only four spectra, and total relative frequency exceeds $1.0 \%$ in only one spectrum. Individual aquatic types (Hydrocotyle vulgaris, Lemnaceae, Montia fontana, Nymphaea alba, Potamogeton crispus type, Ranunculus aquatilis group, and Salvinia natans) all occur at less than $1.0 \%$.

Zone $G W-5,157.5-133.0 \mathrm{~cm}$ The boundary with the preceding zone is marked by a sudden decline in Carex flacca type (11.3-15.9\%, then declines further in the uppermost spectrum to $4.0 \%$ ) and a sudden increase in Phragmites australis, which is the dominant type (27.5-38.7\%). Carex hallerana type, Cyperus michelianus, and Glyceria type are also important throughout this zone. Carex hallerana type rises steadily to a maximum of $17.5 \%$ and then declines steadily to $8.0 \%$. The curve of Cyperus michelianus has the converse pattern; $35.0 \%$ at the bottom of the zone, $5.5 \%$ in the middle, then $28.0 \%$ at the top. Glyceria type increases from the bottom of the zone to the top, $4.8-15.9 \%$.

A few other emergent types record significant relative frequencies. Ranunculus acris group appears sporadically, recording a maximum of $3.8 \%$ at the bottom of the zone. Sparganium erectum also appears sporadically, reaching a maximum of $4.6 \%$ at $145 \mathrm{~cm}$. Sagittaria sagittifolia appears at $150 \mathrm{~cm}$ with $7.9 \%$, drops to $0.5 \%$ in the spectrum above, then disappears. Several types occur only once: Chrysosplenium alternifolium at $140 \mathrm{~cm}(1.9 \%)$, Cyperus longus type at $145 \mathrm{~cm}(16.1 \%)$, Mentha type at the bottom of the zone (1.3\%), and Veronica anagallis-aquatica type at the top of the zone (1.3\%).

The only aquatic type that exceeds $1.0 \%$ is Hydrocotyle vulgaris. It appears at $140 \mathrm{~cm}$ at $0.9 \%$, then increases to $1.3 \%$ in the spectrum above at the top of the zone.

Zone $G W-4,133.0-108.5 \mathrm{~cm}$ The boundary with the preceding zone is marked by a dramatic increase in the relative frequency of Carex hallerana type and an increase in Sparganium erectum. The zone is characterised by high relative frequencies in general of Phragmites australis (33.3-40.3\%, but the lowest spectrum records $2.5 \%$ ) and of Carex hallerana type (18.8-48.3\%, but one spectrum falls to $10.2 \%$ ), and fluctuating but moderate frequencies of Cyperus michelianus (zero in the lowest spectrum, otherwise $8.2-23.4 \%$ ), moderate frequencies of Glyceria type (declines from $21.7-2.7 \%$, then increases in the top spectrum to $10.4 \%$ ), a continuous curve of Sparganium erectum $(1.5-8.4 \%)$, the presence of Caltha type (occurs in 3 of 5 spectra in the middle of the zone, 2.7-7.3\%), and generally lower frequencies of Carex flacca type (2.7-9.2\%, but the top spectrum records $14.6 \%$ ) than those of the zone below.

Other emergent types that occur in significant abundance are: Chrysosplenium alternifolium (occurs in 2 spectra, 1.4 and 2.2\%), Cyperus longus (zero in one spectrum, but 
reaches a maximum of $2.7 \%$ ), Ranunculus acris group (occurs in the bottom spectrum only, $2.5 \%$ ), and Typha angustifolia type (occurs in the 3 lowest spectra, reaching a maximum of $2.9 \%$ ).

Aquatic types are again very poorly represented; all types record less than $1.0 \%$.

Zone $G W-3,108.5-106.0 \mathrm{~cm}$ This zone comprises one spectrum only, but it is quite distinct from the zone below. Phragmites australis (54.2\%) and Glyceria type (23.1\%) increase, while Carex flacca type (2.3\%), Carex hallerana type (10.1\%, but influx increases slightly), Cyperus longus (0.6\%), Cyperus michelianus (1.3\%), and Sparganium erectum $(0.6 \%)$ decline. Other emergent types that occur in significant abundance are Caltha type $(1.3 \%)$, Mentha type (2.9\%), and Saxifraga stellaris (1.6\%). No aquatic types occur.

Zone $G W-2,106.0-47.5 \mathrm{~cm}$ The boundary with the preceding zone is marked by an increase in relative frequency of Carex hallerana type, Carex flacca type, Cyperus michelianus, and a decrease in Phragmites australis and Glyceria type. The zone is characterised by the dominance of Carex hallerana type (29.5-61.8\%) and is more similar to zone GW-4 than to GW-3 but differs from it in having higher relative frequencies of Carex hallerana type and Carex flacca type and lower frequencies of Phragmites australis and Sparganium erectum.

The relative frequency curves suggest a division of this zone at $77.5 \mathrm{~cm}$, as indicated by numerical zonation based on relative frequency data. At this level a change occurs in the relative frequencies of the dominant emergent types of Carex hallerana type, Carex flacca type, Cyperus michelianus, Glyceria type, and Phragmites australis, and frequencies remain reasonably consistent above this change, while below this level frequencies fluctuate.

Curves of the concentration and influx diagrams do not display such a convincing division. No significant change occurs in Carex hallerana type until the uppermost spectrum of the zone, where concentration and influx increase (a slight increase is shown below this, not a decrease as shown in the relative frequency diagram). Carex flacca type and Cyperus michelianus begin to increase in concentration and influx but not until $65 \mathrm{~cm}$, while there is no significant change in Phragmites australis and Glyceria type. Rate of change is higher between several spectra in the lower part of the zone (that is, at $102.5 \mathrm{~cm}$ and at 92.5 and $87.5 \mathrm{~cm}$, where it is very high) than at $77.5 \mathrm{~cm}$, where it is moderately low.

On the relative frequency diagram Carex flacca type increases in general from the base of the zone upwards (ranging from 6.3 to $32.3 \%$ ). Cyperus michelianus is more abundant from $75 \mathrm{~cm}$; below this level it fluctuates from $0-6.0 \%$, while above it fluctuates from $16.3-33.6 \%$. Glyceria type displays a declining trend from about $14.3 \%$ to around $1.0-2.0 \%$. The curve of Phragmites australis also displays a declining trend from about $19.0 \%$ to less than $1.0 \%$. Cyperus longus type fluctuates (0-7.9\%), being most abundant from the middle 
of the zone upwards. Caltha type fluctuates $(0-28.0 \%)$, and highest frequencies are from $90-80 \mathrm{~cm}$. Sparganium erectum occurs at highest frequency $(4.5 \%)$ at the base of the zone and then declines to occur sporadically at $1.5 \%$ or less. Typha angustifolia type occurs most often towards the base of the zone ( $4.0 \%$ maximum). Other less abundant emergent types include Chrysosplenium alternifolium, which occurs infrequently and always at less than $2.0 \%$. Samolus valerandi occurs twice but at $1.0 \%$ and less. Filipendula occurs only at $85 \mathrm{~cm}$, at $1.5 \%$.

Aquatic types appear sporadically and always at very low relative frequencies. Those exceeding $1.0 \%$ are Hydrocotyle vulgaris, which occurs three times (5.3\% at the base of the zone, $2.3 \%$ in the middle, and $0.2 \%$ at the top), and Potamogeton pectinatus and Sparganium angustifolium, which occur at the base of the zone only (1.5\% each).

Zone $G W-1,47.5-0 \mathrm{~cm}$ The boundary with the preceding zone is marked by a decline in Carex hallerana type and an increase in Glyceria type. The zone is characterized by declining Carex hallerana type. Carex hallerana type and Cyperus michelianus are co-dominant (25.5-40.3\% and 17.0-34.3\%, respectively). Carex flacca type fluctuates from $16.6-23.7 \%$ in the lowest spectra, to zero, then to $19.1 \%$, before declining to 0.6 and $2.6 \%$ in the two top spectra. The curve of Cyperus longus type is the reverse of that of Carex flacca type reaching a maximum of $24.0 \%$ when Carex flacca type declines to zero. Cyperus longus type then drops to $0.8 \%$ before increasing to 5.2 and $3.8 \%$ in the two top spectra. Glyceria type is about $9.0 \%$ in the two lowest spectra, then declines to $3.0 \%$ before increasing dramatically in the two top spectra (17.3 and 19.2\%). Phragmites australis has very low values at the base of the zone, declining to zero, but then increasing again to reach a maximum of $11.5 \%$ at the top of the sequence. Fluctuations in relative frequencies are reflected in high rates of change, but increases in relative frequencies at the top of the zone are not supported by influx data, particularly for Cyperus michelianus, Glyceria type, and Phragmites australis. Total influx steadily declines upwards. Other emergent types recording $1.0 \%$ or more are Caltha type $(2.5 \%$ at $40 \mathrm{~cm}$ and $1.0 \%$ at $25 \mathrm{~cm})$ and Chrysosplenium alternifolium $(1.0 \%$ in the surface spectrum).

Aquatic types are poorly represented; only Ranunculus aquatilis group exceeds $1.0 \%$, occurring three times with relative frequencies ranging from 0.8 to $1.5 \%$.

\subsubsection{Sedimentary and local vegetation history}

The combination of evidence from deposited sediments and the wetland pollen sequence indicates a hydroseral succession from a permanently wet environment (small lake) to a seasonally wet fen as the deposition basin filled in from c. $1340 \mathrm{BC}$ to $700 \mathrm{AD}$. (Dates are interpolated from the radiocarbon chronology.) The sediments were continuously wet and 
there is good pollen preservation throughout the sequence until c. $700 \mathrm{AD}$ when intermittent drying of the lake bed and oxidation of surface deposits began. Currently the fen is too wet for forest trees yet sufficiently dry in summer to prevent effective accumulation of peat. Diatom results (Appendix XII) are somewhat difficult to interpret due to the small number of samples analysed, but in general they are in agreement with those of pollen analysis.

The permanent water was shallow, probably fluctuating between 1 and $2 \mathrm{~m}$ depth. Water condition throughout the sequence was fresh, slightly alkaline, and rich in oxygen. The wetland has never been nutrient-poor. Trophic level is highest at the base of the sequence (eutrophic), and organic nitrogen compounds are most abundant at the base and top. In the centre of the sequence the trophic level drops to meso-eutrophic, and organic nitrogen levels are low. A marginal swamp vegetation of sedges and reeds, with a Carex swamp community on the landward side of the reed swamp, surrounded the lake. Only occasional submerged and floating-leaved aquatic macrophytes from a deeper water zone inside the Phragmites australis belt were identified.

Broadly, the sediments represent two episodes of soil erosion, indicated by sediments with a high proportion of inorganic matter, separated by a period of reducing soil erosion and increasing soil stability from $c .80 \mathrm{BC}$ to $330 \mathrm{AD}$ when sediments were highly organic. Proportions of inorganic to organic matter contained in the sediments do not correspond to influx of wetland pollen, so it is proposed that organic matter as well as inorganic matter in the lake sediments is derived from soils on the catchment rather than from production within the lake (Pennington \& Lishman 1971). This is also consistent with Walker's (1970) claim that Phragmites australis swamps do not leave any visible organic remains. Only one abrupt stratigraphical change occurs, that at $107.2 \mathrm{~cm}$, where a catastrophic event is represented - a volcanic ash fall. All other changes in lithology are indistinct and represent slower environmental changes.

The sedimentary history of the basin is closely linked to the local wetland vegetation history, so major sedimentary units defined above coincide with the established wetland pollen zones. These zones are used, for convenience, in the discussion below.

Zone GW-6, 190.0-157.5 cm, c. 1340-890 BC At the base of the sequence the dominant plant community is a tall-sedge-swamp community. A wet-soil community has developed and a small reed swamp exists. Both floating-leaved and submerged communities of open water are represented by a few pollen and spore types. In the upper half of the zone, the wet-soil community decreases while the sedge-swamp community increases.

Development of a wetland plant community from the base of the sequence is suggested by low initial influx of pollen and spores, comparatively high palynological richness ( 8 expected types) at the base of the zone indicative of a colonising wetland plant community, 
and the occurrence of a greater proportion of diatoms that live on silt rather than on plants. Two sedimentary sections were described above that correspond to this zone. The lower section at the base of the sequence between 190.0 and $181.5 \mathrm{~cm}$ comprises clay with detritus, an indication of open water rather than fen or bog conditions at the time of sedimentation. Further evidence of open-water conditions are the dominance of diatoms that live on silt or in shallow water rather than on plants, and the presence of plant species that grow in shallow open-water conditions, e.g. Lemnaceae, Nymphaea alba, Potamogeton crispus, P. pectinatus, Ranunculus aquatilis, and Salvinia natans. Marginal fen and sedge communities establish around the edge of the lake, with a reed community on the lakeside in deeper water.

Small particles of charcoal incorporated within the sediment of this lowest section suggest that burning of vegetation may have contributed to the onset of erosion.

At the base of the next sediment section at $181.5 \mathrm{~cm}(c .1250 \mathrm{BC})$ sediments become gritty with the addition of coarser-grain particles, which would require higher transport energy, perhaps increased storminess (Bormann et al. 1974). The proportion of inorganic matter in the sediments reaches the highest levels for the sequence, and the average sedimentaccumulation rate in this zone is higher than that in other highly inorganic sediments in the sequence. Also, the colour of the sediment is lighter, perhaps as a result of less litter being humified. This could result from fewer trees in the catchment producing litter, and perhaps from grazing destroying the litter horizon. Once bare soil is exposed to alternating rain and drought, oxidation of organic matter is accelerated. The soil structure has been modified, losing its aggregation properties and becoming incohesive (Butzer 1974).

Diatom evidence also points to increasing erosion, with increasing abundance of alkaliphilous diatoms and increasing levels of nutrients. Alkalinity of the lake sediments, which increases in the second section of this zone, is consistent with soil derived from local bedrock. Eutrophication also corresponds to elevated levels in the amounts of soluble nutrients being washed into lake basins (Oldfield 1977). High nutrient level and correspondingly depressed oxygen content, as shown in the diatom record in this zone to increase in the second section of the zone, may thus be an expression of erosion of nutrient-rich soils (van Dam et al. 1994).

There is parallel evidence from the pollen record for high nutrient levels. Several types indicative of high nutrient levels were identified, e.g. Lemnaceae, Nymphaea alba, Lychnis flos-cuculi, and Glyceria, which indicate eutrophic conditions; and Ranunculus aquatilis group and Sagittaria sagittifolia, which indicate meso- to eutrophic conditions (Haslam et al. 1975; Ellenberg 1988). Marginal plant communities dominated by Phragmites australis and Carex species indicate mesotrophic nutrient levels in the lake (Ellenberg 1988). 
Lower water depth or greater seasonal fluctuation in water depth is indicated in the second section of this zone by the high relative frequency of diatoms that live in ephemeral water, which increases while those that live in permanent water falls. The only aquatic plant represented at this time, Hydrocotyle vulgaris, can also live as an emergent.

Zone $G W-5,157.5-133.0 \mathrm{~cm}, \mathrm{c.} 890-480 \mathrm{BC}$ The lower boundary of this zone coincides with a change in the sediment composition to one that suggests less intense erosion; coarsegrain mineral particles disappear, and organic detritus from both herbaceous and ligneous plants occurs. This suggests the accumulation of humus under an expanding woody vegetation in the catchment. The colour of the sediments is darker than that in the zone below. Less intense erosion is also suggested by a much reduced influx of inorganic matter, and a decline in nutrient level of the lake as shown by diatoms. Also, the average sedimentaccumulation rate of this and the following zone is lower than that of zone GW-6.

A steadier water level and an increase in water depth is suggested by an increase in diatoms that live in permanent water and a decline in those that live in ephemeral water. Pollen evidence also supports this; pollen of the shallower-water plant communities of wetsoil and sedge swamp declines, while that of the reed swamp increases and becomes dominant. Phragmites australis is the dominant species of the reed swamp. At Gomara Phragmites australis is probably close to its ecological limit, considering current climatic conditions. (Chapter 1 - no precise climatic data are available for the Gomara site). Studies show that vegetative growth of Phragmites australis only begins when the temperature has risen to about $8-10^{\circ}$ C. In trial tanks without water Phragmites australis was killed in January at temperatures of $-8.7^{\circ} \mathrm{C}$ and in March could only tolerate $-0.8^{\circ} \mathrm{C}$ (Dykyjová et al. 1971 in Ellenberg 1988). This raises doubts about the identification of the grains assigned to this pollen type based on their small size (Appendix VI), for they may belong to another species of Gramineae, the grain size of which has been reduced due to harsh growing conditions. However, the dense stand of Phragmites australis described in Chapter 3 and Appendix VIII on Mirsina fen in the lowlands of Grevena survives through winter minimum temperatures of $3.2^{\circ} \mathrm{C}$ and 40 days of ground frost and snow. The Gomara wetland site is at a much higher altitude than the Mirsina fen, but in winter there is commonly a temperature inversion: in January valleys and basins are $2-3^{\circ} \mathrm{C}$ colder than mountain slopes and peaks at the same altitude. Occurrence of pollen of other reed-swamp plants, such as Sagittaria sagittifolia, Sparganium erectum, and Typha species, supports the identification of Phragmites, and the morphology of the pollen in the Gomara sequence compares well with reference pollen collected from Mirsina fen (Appendix V). No macrofossil evidence of Phragmites australis was noted, but it is thought that Phragmites australis swamps do not leave any visible organic remains (Walker 1970). Phragmites australis usually dominates at an average water depth of 
1.2 to $2 \mathrm{~m}$, although its ecological amplitude is great and it can tolerate short periods of dry ground (Ellenberg 1988).

The floating-leaved aquatic community is not represented, and only Hydrocotyle vulgaris, which may be emergent, represents the submerged aquatic community.

Macroscopic charcoal particles were observed again, suggesting that burning of vegetation was taking place in the vicinity.

Zone $G W-4,133.0-108.5 \mathrm{~cm}, c .480-80 \mathrm{BC}$ The sediments of this zone indicate a further decline in the intensity of erosion taking place in the catchment. The proportion of organic detritus to clay increases and the occurrence of macrofossils such as beech nuts indicates the presence of beech trees in the catchment. Supporting evidence for reduced erosion is the lower relative abundance of alkaliphilous diatoms and the lower nutrient status indicated by the diatom record.

The reed-swamp community remains the dominant community, but the wet-soil community has expanded, Caltha type giving support to Carex hallerana type. The tall-sedge swamp has declined but is still a prominent community. The aquatic communities are again poorly represented, with no record of the floating-leaved community and extremely low representation of the submerged community.

An increase in marginal lake vegetation is suggested by an increase in the influx of Phragmites australis pollen as well as that of Carex hallerana type. An increase in the relative abundance of epiphytic/epilithic diatoms is also suggestive of an increase in plants growing in water. There is also an increase in diatoms that live in fairly permanent water and ephemeral water, while those that live in permanent water decline. This may indicate a lower water level and greater seasonal fluctuation. High palynological richness (about 8 expected types) also supports this.

The short severe decline in Phragmites australis at the base of the zone may be a result of anthropogenic activity, such as cutting the grass for fodder. Bottema (1974:66) noticed inhabitants at Lake Khimadhitis performing such a task.

Zone $\mathbf{G W}-3,108.5-106.0 \mathrm{~cm}, \mathrm{c.} 80 \mathrm{BC}$ The sediment at $108.2-107.2 \mathrm{~cm}$ is interpreted as a tephra. It represents primary deposition, as indicated by the absence of detrital materials from surrounding soils, and has a very distinct upper boundary. Deposition would have been almost instantaneous. Thus the pollen assemblage is representative only of species flowering at the time the tephra was deposited.

Zone $G W-2,106.0-47.5 \mathrm{~cm}, c .80 \mathrm{BC}-330 \mathrm{AD}$ This is a period of steady water level, probably of greatest depth reached, as shown by the abundance of diatoms living in permanent water and an increase in pollen of aquatic macrophytes. Declining palynological richness (8-5 expected types) suggest that the water level rose at the base of the zone, then remained at a 
high level with little seasonal fluctuation. The fine-grained tephra deposit may have contributed to an increased permanence of depth by reducing the permeability of the lake bottom.

The sediments become increasingly organic until, near the top of this zone at $60 \mathrm{~cm}$, they are composed almost entirely of organic matter, and deposition of minerogenic material has almost ceased. This results in a decline in the trophic status of the lake. The coarse nature of the detritus is indicative of several factors: detritus derived from forest canopy/forest soil; slow decomposition; and lack of torrential outflow from the lake. At $60 \mathrm{~cm}$ a closed forest canopy produced abundant organic matter, which covered the forest floor and left no mineral soil exposed for erosion.

Average rate of sediment accumulation is the highest for the entire sequence. This is attributed to the coarse nature of the organic detritus and the high moisture content.

The marginal swamp vegetation is reduced to a narrow band of mostly plants of a wetsoil habitat. The reed swamp is much reduced at the base of the zone and continues to decline, perhaps due to lowered trophic level, until the uppermost spectrum, where influx of Glyceria type increases slightly. The tall-sedge swamp increases in importance in the upper part of the zone, and the wet-soil community is important throughout the zone.

Pollen-influx data suggest that the marginal vegetation expanded prior to a visually detectable change in sediment composition at $49 \mathrm{~cm}$. This may be due to an increase in inwashed nutrients. Bormann et al. (1974) found that the first and immediate response of the ecosystem to deforestation is increased solubilization of nutrients; that is, more nutrients are dissolved in drainage water than was the case in the undisturbed ecosystem. They found that between 1.5 and 2.5 times the expected total annual uptake of nitrogen in the forested ecosystem was lost as dissolved nitrogen during the first 2 years of deforestation. Unfortunately diatom samples are too widely spaced around this transition to detect the onset of nutrient inwash, but an increase in organic nitrogen compounds is displayed by diatom data at $56 \mathrm{~cm}$, when the proportion of inorganic matter in the sediments begins to increase (Figure 4.4).

Zone $G W-1,47.5-0 \mathrm{~cm}$, c. 330-700 AD \& present Sediments of this zone are remarkably similar to the minerogenic sediments in the two zones immediately below the tephra and are interpreted as evidence of the onset of another period of more intense soil erosion, when the woodland canopy is open. Inorganic sedimentation begins again just below the pollen-zone boundary, and a change in sediment composition can be seen at $49 \mathrm{~cm}$. In this zone alkaliphilous diatoms and those tolerant of organic nitrogen increase. Trophic level increases, as indicated by diatom evidence and the presence of pollen types such as Lychnis flos-cuculi and Ranunculus aquatilis group and an increase in Glyceria type. Accumulation 
rate of the sediments is unknown, due to an absence of dated material, but it is assumed to be similar to that of zones GW-4 and GW-5, where sediments are of a similar particle size and composition.

Wet-soil and tall-sedge swamp communities are dominant but reduced. Some reed swamp still exists, but it is now probably dominated by Glyceria, which currently grows at Gomara. The floating-leaved community has very low representation, as has the submerged community, but the high abundance of diatoms that live in permanent water and low abundance of those that live in fairly permanent water and ephemeral water suggest that water level was high with little seasonal fluctuation.

At about $25 \mathrm{~cm}$ below surface the infill of the lake reached a threshold where seasonal lowering of water level through evaporation and outflow caused the lake to become ephemeral, with a dry bed in the summer.

\subsubsection{Terrestrial pollen analysis}

\subsubsection{Pollen diagram construction}

Pollen and spore relative frequencies are presented in Figures 4.8 and 4.9 (in pocket). Pollen and spore types are included in habitat groups defined in Chapter 2. The pollen sum on which the relative frequencies are based includes all identified terrestrial pollen and spore taxa and excludes wetland taxa. Relative frequencies of $0.2 \%$ or less are indicated with a dot.

Style of the pollen diagrams is similar to that of the wetland diagram (Figure 4.6). Figure 4.8, the summary diagram, shows the coniferous and deciduous canopy trees group in resolved form, then a summary column showing the ratio of terrestrial trees and shrubs to terrestrial herbaceous plants. This is followed by summary curves of each habitat group, then a curve of palynological richness derived from rarefaction analysis and based on the smallest total count of terrestrial pollen and spores, 292 at $70 \mathrm{~cm}$ (Birks \& Line 1992; Bennett 1994). This is followed by the number of terrestrial types in each spectrum (not normalized for unequal pollen sums). A curve of the rate of vegetation change is shown next. Rates of vegetation change were calculated using the dissimilarity coefficient of Chi-squared coefficient 2 on unsmoothed pollen frequencies of the 10 most abundant types (that is, those that were $\geq 5 \%$ of total terrestrial pollen and spores) and calibrated radiocarbon ages (Bennett 1994; Bennett \& Humphry 1995). The pollen types included in the calculations are: Pinus (Diploxylon), Fagus, Quercus frainetto type, Q. trojana type, Abies, Betula pendula, Ostrya type, Dactylis type, Festuca type, and Rumex acetosa group. Finally, the number of pollen grains of trees and shrubs, the total number of all terrestrial pollen and spores, and the total of all pollen and spores counted in this sequence (wetland and terrestrial) are given. 


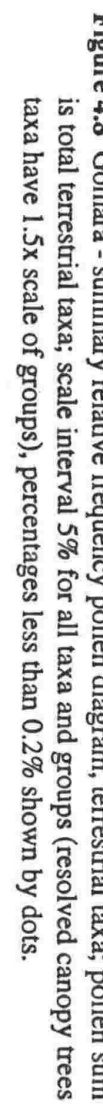

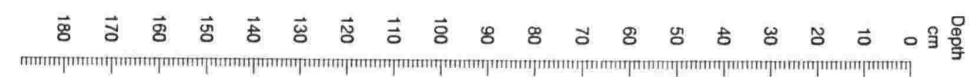

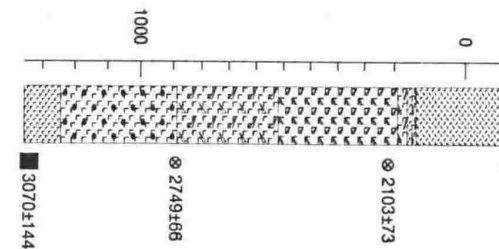

$\circ \quad \circ$

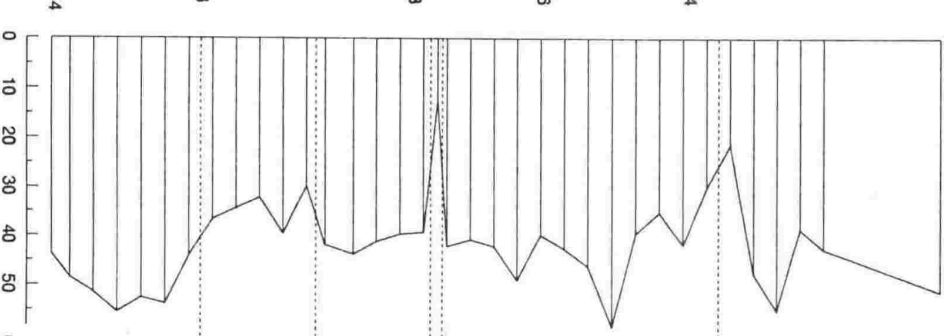

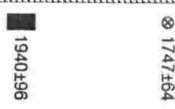

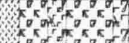

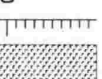

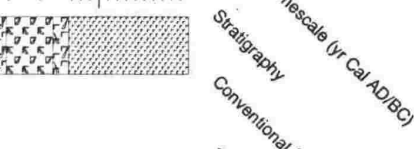

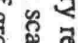

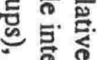

글

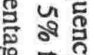

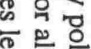

$\overrightarrow{\mathrm{g}}$

.

品

명

的

茞.

㱐

ب.

음

范苛
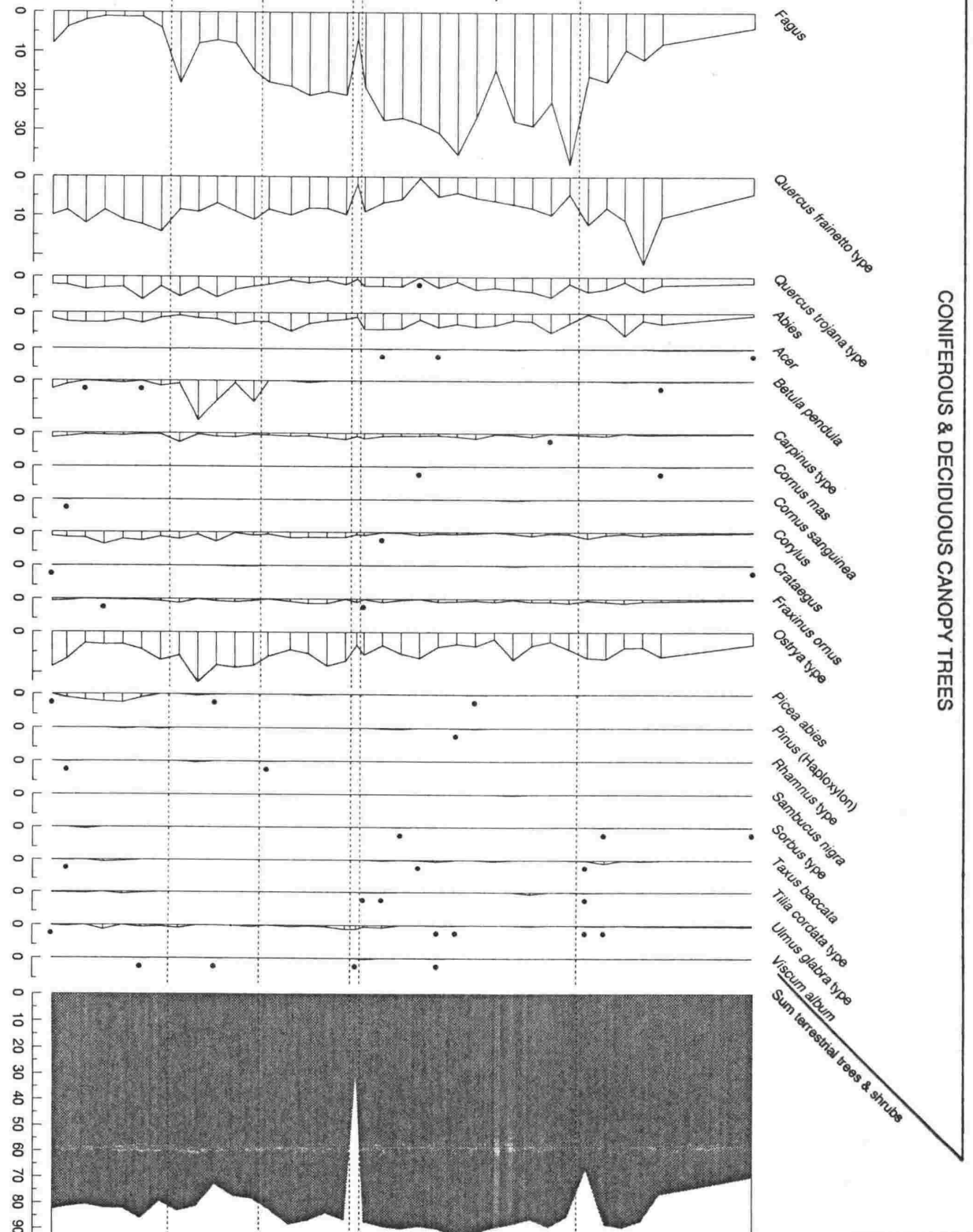


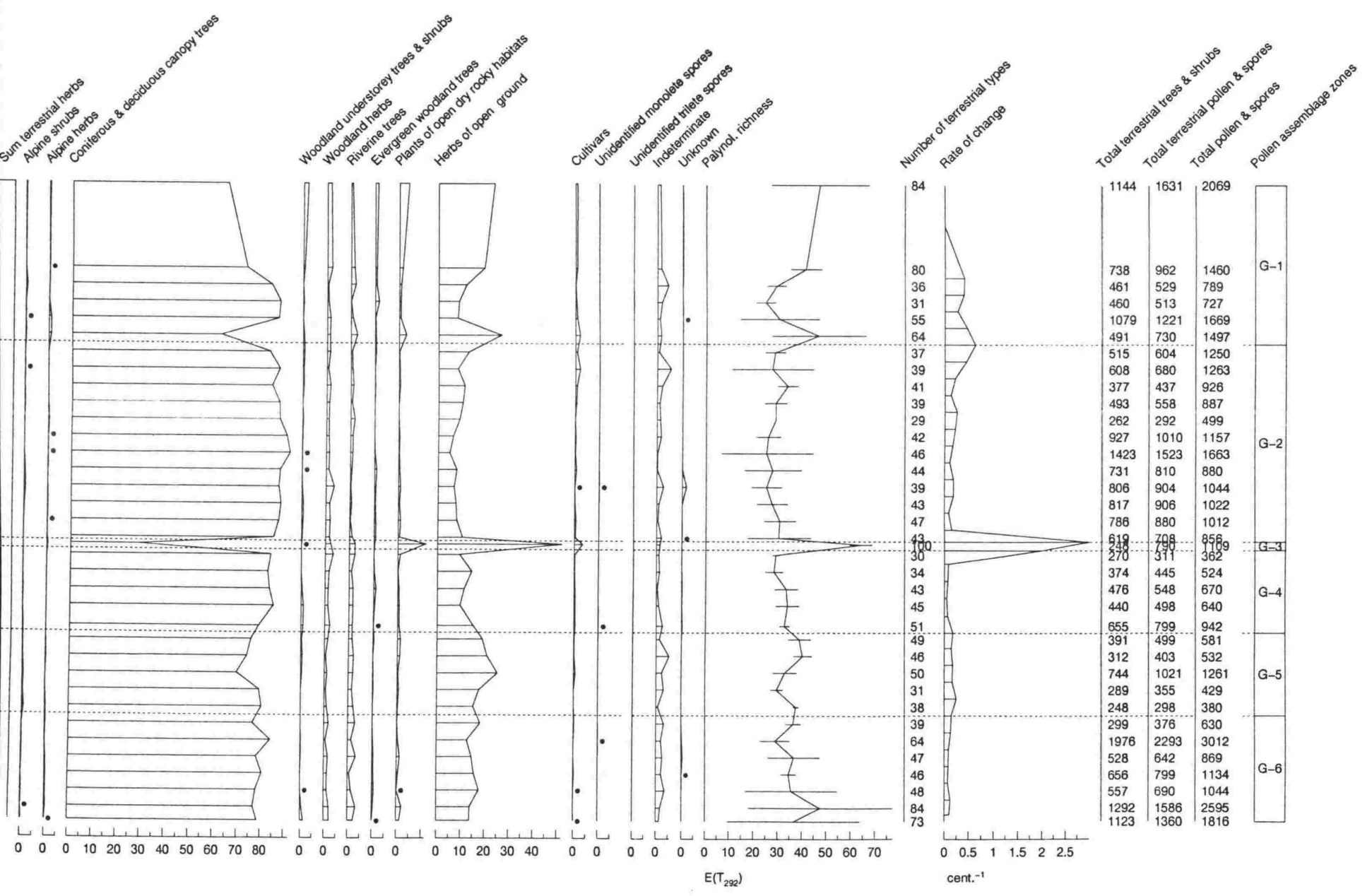

Figure 4.8 
The fully resolved diagram, Figure 4.9 (in pocket), displays all terrestrial pollen and spore taxa recognised in the sequence, in order of habitat groups. Within these groups they are arranged alphabetically except for the dominant tree types of Pinus (Diploxylon), Fagus, Quercus frainetto type, and Quercus trojana type, which are placed at the beginning of the 'coniferous and deciduous canopy trees' group.

Pollen and spore influx data are presented in Figure 4.10, in which are included only influx of pollen and spore types recorded in significant abundance; habitat groups; and total terrestrial herbs, total terrestrial trees and shrubs, and total terrestrial pollen and spores. The influx diagram takes a form similar to the relative frequency diagram.

\subsubsection{Terrestrial pollen assemblage zones}

Terrestrial pollen diagrams are divided into 6 local pollen assemblage zones, based mostly on relative frequency data; boundaries occur at the same level as those of the wetland sequence. They are prefixed by the site designation ' $G$ '. These zones are defined below. Influx data are discussed in the following section.

Zone $G-6,190.0-157.5 \mathrm{~cm}$ The basal zone is characterised by high relative frequencies of Pinus (Diploxylon) (43.7-55.4\%), the highest frequencies of the sequence of Corylus (1.0-3.0\%) and Picea abies (2.0\% maximum), and lowest of Fagus (1.1-4.0\%, with the exception of the basal spectrum, which reaches 7.9\%). Quercus frainetto type and Quercus trojana type occur at frequencies that are about average for the sequence $(8.5-14.1 \%$ and $2.0-5.8 \%$, respectively). Abies occurs at quite low frequencies (1.3-2.6\%), while Betula pendula records very low values (0.1-2.1\%). Other types of the 'coniferous and deciduous canopy trees' habitat group are very poorly represented, with the exception of Ostrya type $(2.8-8.5 \%)$.

Relative frequencies of the pollen types in the habitat groups of 'alpine shrubs' and 'alpine herbs' are extremely low $(0.3 \%$ maximum). The habitat group of 'woodland understorey trees and shrubs' is extremely poorly represented; no individual type exceeds $0.7 \%$. 'Woodland herbs' occur at about average frequencies for the sequence (0.6-2.2\%), while individual pollen types in this group are extremely poorly represented ( $0.9 \%$ maximum). The group 'riverine trees' records, in general, the maximum frequencies of the sequence, but reaches only $3.3 \%$. Alnus glutinosa is the most abundant pollen type of this group (0.5-1.9\%). The group 'evergreen woodland trees' records extremely low frequencies ( $0.7 \%$ maximum). Frequencies of the group 'plants of open dry rocky habitats' are slightly higher $(2.1 \%$ maximum). 


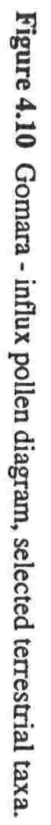

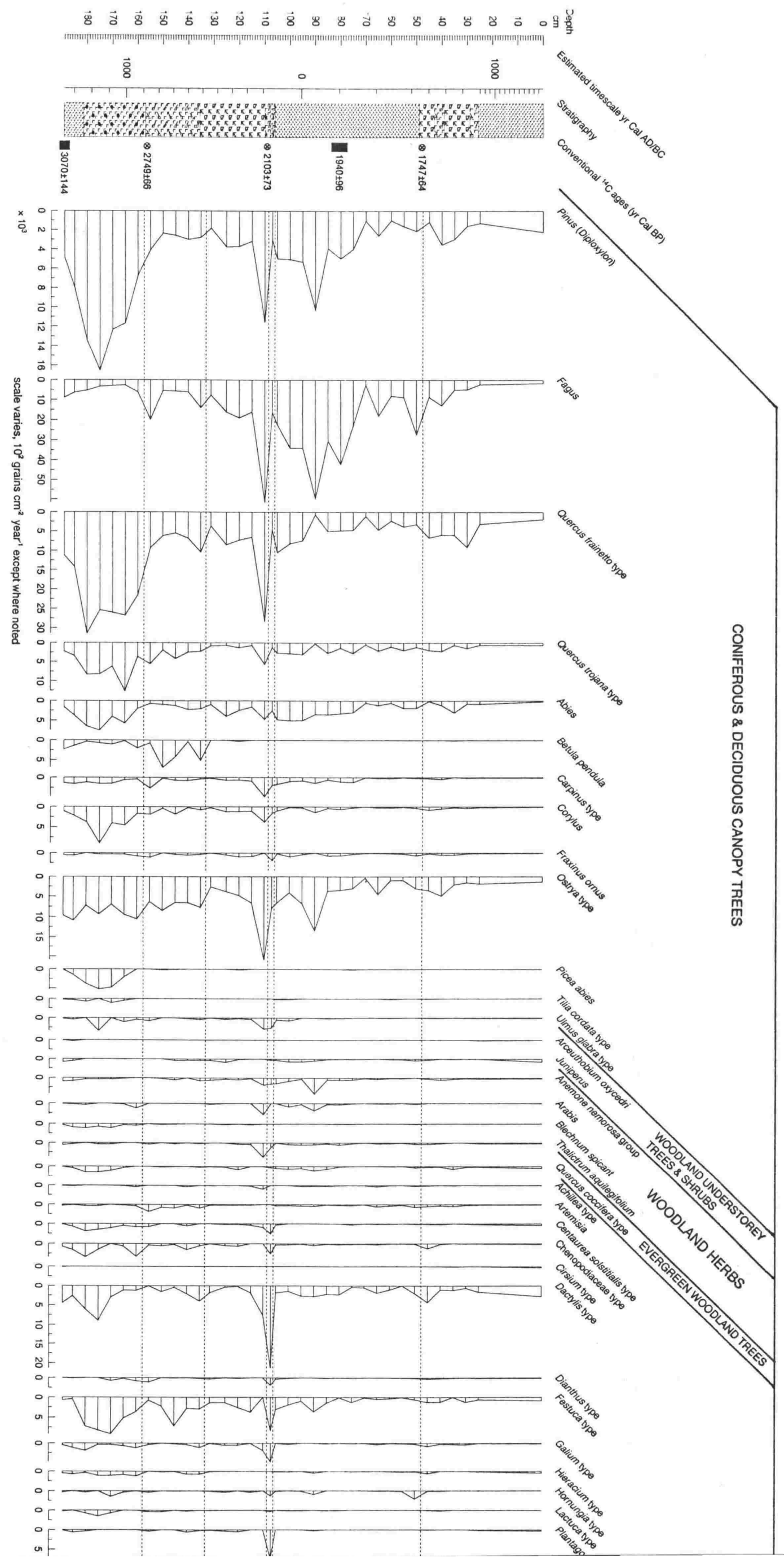




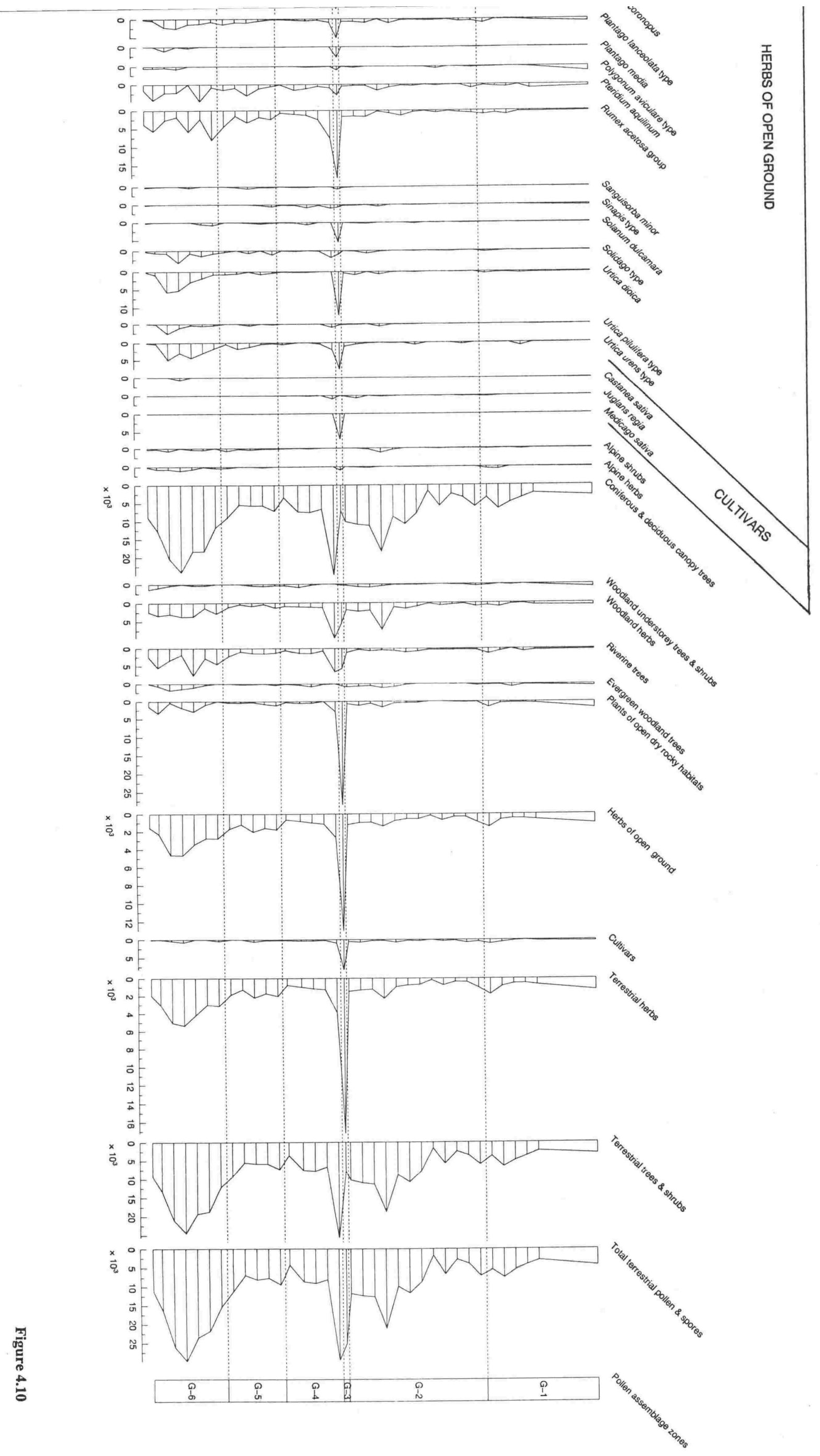


'Herbs of open ground' (12.6-18.1\%) is the second most abundant habitat group after 'coniferous and deciduous canopy trees'. Many of the types of this group are represented, the most important are: the two grasses, Dactylis type and Festuca type, Pteridium aquilinum, Rumex acetosa group, Urtica dioica, and U. urens type. Chenopodiaceae type, Plantago lanceolata type, and Solidago type are also important. Frequencies of Dactylis type are highest in the lower half of the zone (reaching 3.9\%), while Festuca type records low values in the two lowest spectra, then increases (2.4-3.9\%). Pteridium aquilinum reaches a maximum $(2.6 \%)$ in the second spectrum from the base of the zone, then declines, but reaches $2.1 \%$ in the spectrum second from the top. Frequencies of Rumex acetosa group are highest at the base and the top of the zone $(5.3 \%$ maximum). Urtica dioica and $U$. urens type both have continuous curves, but at $2.2 \%$ or less. Chenopodiaceae type, Plantago lanceolata type, and Solidago type occur mostly at less than $1.0 \%$.

Representation of the 'cultivars' group is very poor; no individual type exceeds $0.3 \%$.

Zone $G-5,157.5-133.0 \mathrm{~cm}$ The boundary with the preceding zone is marked by a decline in the relative frequencies of Pinus (Diploxylon) and Quercus frainetto type, and an increase in Fagus and Artemisia. The zone is characterised by low relative frequencies of Pinus (Diploxylon); increased frequencies of Fagus, Betula pendula, Ostrya type, Artemisia, Festuca type, and the habitat group of 'herbs of open ground'; and decreased frequencies of Quercus frainetto type, Corylus, Picea abies, and the habitat group of 'coniferous and deciduous canopy trees'.

The habitat groups of 'alpine shrubs' and 'alpine herbs' continue to be very poorly represented; no individual pollen type exceeds $0.7 \%$.

'Coniferous and deciduous canopy trees' decline, although some pollen types within this group increase. Pinus (Diploxylon) declines (29.7-39.5\%) to comparatively low frequencies for this sequence, while Fagus increases, ranging from $7.1 \%$ (only one spectrum) to $17.8 \%$. Quercus frainetto type declines slightly (6.8-9.0\%, with the exception of one spectrum of $14.8 \%$ ). Quercus trojana type and Abies fluctuate between the same frequencies as those of the preceding zone. In the second spectrum from the base of the zone Betula pendula increases dramatically to $10.1 \%$, declines to $5.2 \%$, then $0.5 \%$, and then increases to $5.4 \%$. Carpinus type increases very slightly (maximum of $2.3 \%$ at the base of the zone). Corylus declines slightly $(0.2-2.3 \%)$. Ostrya type increases slightly (12.4\% maximum). Picea abies almost disappears, occurring in only two spectra ( $0.3 \%$ maximum). Other pollen types in this habitat group either remain at very low relative frequencies or do not appear.

The habitat group of 'woodland understorey trees and shrubs' continues to be very poorly represented (maximum 0.5\%); only Juniperus and Buxus occur. Frequencies of 'woodland herbs' remain very low (less than $1.5 \%$ ), as do those of 'riverine trees' ( $2.0 \%$ maximum). 
Alnus glutinosa is again the most abundant type of the latter group, occurring at the same values as those of the preceding zone. 'Evergreen woodland trees' record a maximum of $0.4 \%$. Representation of 'plants of open dry rocky habitats' is also low (maximum 1.2\%).

'Herbs of open ground' is again the second most abundant habitat group, increasing to a maximum of $25.2 \%$. Most types of this group are represented. The most abundant types are: Artemisia, Chenopodiaceae type, Dactylis type, Festuca type, Plantago lanceolata type, Pteridium aquilinum, Rumex acetosa group, Solidago type, Urtica dioica, and U. urens type. Most of these types have frequencies of about the same value as those in the preceding zone, but a few have increased: Artemisia (1.0-2.0\%), Dactylis type and Festuca type (increase slightly to maximum frequencies of $4.2 \%$ and $8.9 \%$, respectively), and Pteridium aquilinum (increases to $3.7 \%$, in the centre of the zone).

'Cultivars' are again extremely poorly represented; no individual type exceeds $0.2 \%$.

Zone $G-4,133.0-108.5 \mathrm{~cm}$ The boundary with the preceding zone is marked by an increase in the relative frequencies of Pinus (Diploxylon), Fagus, and the habitat group of 'coniferous and deciduous canopy trees'; a decrease in Betula pendula; and a slight decline in Ostrya type and the habitat group of 'herbs of open ground'. This zone is characterised by increased relative frequencies, in general, of Pinus (Diploxylon); increased and increasing frequencies of Fagus and of the habitat group of 'coniferous and deciduous canopy trees'; and decreased frequencies of Betula pendula, Ostrya type and the habitat group of 'herbs of open ground'.

'Alpine shrubs' and 'alpine herbs' continue to be very poorly represented, no type exceeds $0.3 \%$.

'Coniferous and deciduous canopy trees' increase (maximum 85.3\%); Pinus (Diploxylon) increases (39.2-41.9\%), Fagus increases steadily towards the top of the zone (17.6-20.9\%), Abies increases slightly, while Quercus frainetto type and Carpinus type occur with frequencies similar to those in the zone below, and Quercus trojana type, Ostrya type, Corylus, Betula pendula, and Picea abies decline. The latter two almost disappear, occurring only once at $0.4 \%$ and $0.2 \%$, respectively.

'Woodland understorey trees and shrubs' are again extremely poorly represented, with only Juniperus exceeding $0.2 \%$, and then in only one spectrum and at $0.9 \%$. 'Woodland herbs' increase slightly (maximum 3.2\%), representation being mostly from Anemone nemorosa group (0.2-1.4\%) and Thalictrum aquilegifolium $(0.4 \%$ in two spectra and $1.3 \%$ in one spectrum). Frequencies of 'riverine trees' remain very low (1.4-2.3\%), as do those of 'evergreen woodland trees' $(0.9 \%$ maximum) and 'plants of open dry rocky habitats' $(1.0 \%$ maximum). 
Although frequencies of 'herbs of open ground' have declined (9.0-14.9\%), many types within this group continue to be represented but at lower frequencies. 'Cultivars' are again extremely poorly represented; no individual type exceeds $0.3 \%$.

Zone $G-3,108.5-106.0 \mathrm{~cm}$ This zone includes only one spectrum. The boundary with the preceding zone is marked by a dramatic decline in Pinus (Diploxylon), Fagus, and Quercus frainetto type, resulting in a reduction in the 'coniferous and deciduous canopy trees' habitat group. Herbaceous plants increase, with the exception of those that grow in woodlands. This results in an increase in the habitat groups of 'plants of open dry rocky habitats' and 'herbs of open ground'.

'Alpine shrubs' are not represented, but 'alpine herbs' are represented by three types, each at $0.1 \%$. 'Coniferous and deciduous canopy trees' drop to $28.4 \%$. Frequencies of the most important types in this group all decline dramatically; Pinus (Diploxylon) to $12.2 \%$, Fagus to $6.5 \%$, and Quercus frainetto type to $1.8 \%$. Other types in this group such as Quercus trojana type, Abies, Carpinus type, Corylus, and Ostrya type also decline, while Fraxinus ornus and Ulmus glabra type either increase very slightly or remain at very low frequencies.

'Woodland understorey trees and shrubs' are represented by only Juniperus $(0.1 \%)$. 'Woodland herbs' and 'riverine trees' decline slightly to $2.2 \%$ and $2.3 \%$, respectively. Representation of 'evergreen woodland trees' remains very low at $0.3 \%$.

The habitat groups of 'plants of open dry rocky habitats' and 'herbs of open ground' which include many herbaceous plants, both increase dramatically, the former to $11.4 \%$ and the latter to $51.8 \%$. Some types appear for the first time, while others increase. 'Cultivars' also increase, owing to an increase in Medicago sativa which in this situation is probably represented by a native wild subspecies.

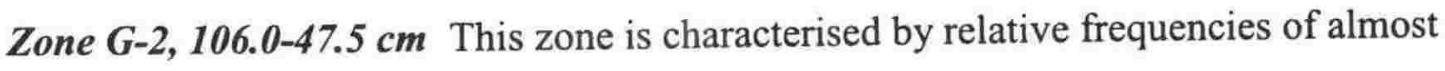
all types returning to the values at the top of zone G-4 and those of Pinus (Diploxylon) and Fagus increasing steadily to the centre of the zone and then declining slightly.

'Alpine shrubs' increase slightly to $0.6 \%$, but 'alpine herbs' reach a maximum of only $0.3 \%$.

The most abundant types in the group 'coniferous and deciduous canopy trees', Pinus (Diploxylon) and Fagus, both reach maximum relative frequencies of the sequence $(58.2 \%$ and $38.6 \%$, respectively). The relative frequencies of Quercus frainetto type fluctuate with high frequencies at the top and bottom of the zone, and lower frequencies in the centre, where, in general, minima of the sequence for this type occur (minimum $0.3 \%$ ). Quercus trojana type and Abies increase slightly to maxima of $5.1 \%$ and $4.9 \%$, respectively. Relative frequencies of Carpinus type remain extremely low (0.1-1.4\%), and those of Corylus ( $0.9 \%$ maximum) and Ostrya type (6.8\% maximum) decline slightly. 
Relative frequencies of 'woodland understorey trees and shrubs' remain low, with Juniperus continuing to be the most abundant type but with a maximum of only $0.7 \%$. 'Woodland herbs' increase slightly at the base of the zone but then decline again (0.6-3.4\%). Frequencies of 'riverine trees' (1.7\% maximum) and 'evergreen woodland trees' $(0.9 \%$ maximum) continue to be extremely low, and 'plants of open dry rocky habitats' return to the extremely low relative frequencies of zone G-4 ( $0.8 \%$ maximum).

'Herbs of open ground' record the lowest relative frequencies of the sequence (4.7-12.7\%). Types of this group that dominated in the lower zones continue to be dominant but at lower values.

'Cultivars' continue to record extremely low relative frequencies but increase slightly at the top of the zone, reaching a maximum of $1.9 \%$. This is due mostly to Juglans regia $(0.6 \%$ maximum at $100 \mathrm{~cm})$ and Secale cereale $(1.5 \%$ maximum at $55 \mathrm{~cm})$.

Zone $G-1,47.5-0 \mathrm{~cm}$ The boundary with the preceding zone is marked by an increase in the relative frequencies of Quercus frainetto type, Dactylis type, Festuca type, and the habitat group 'herbs of open ground'; and a decrease in Fagus and the habitat group of 'coniferous and deciduous canopy trees'. This uppermost zone is characterised by declining relative frequencies of Fagus, increasing relative frequencies of Quercus frainetto type (apart from the top two samples), and high relative frequencies of Dactylis type.

'Alpine shrubs' and 'alpine herbs' continue to be very poorly represented, as they have been throughout the sequence, with a maximum of $0.6 \%$ for both groups.

Relative frequency curves of the most abundant types in 'coniferous and deciduous canopy trees' form different patterns. The curve of Pinus (Diploxylon) increases rapidly from low values, then declines slightly (from a minimum of $21.4 \%$ to a maximum of $55.0 \%$, then to $51.3 \%$ in the surface spectrum). The curve of Quercus frainetto type is similar to that of Pinus (Diploxylon); bottom spectrum $12.2 \%$, peaks at $30 \mathrm{~cm}$ at $22.1 \%$, and surface spectrum $4.4 \%$ ). However, it peaks in a spectrum above the maximum of Pinus (Diploxylon). The curve of Fagus declines from a maximum of $17.4 \%$ in the spectrum second from the bottom to $3.9 \%$ in the surface spectrum. Other types in this group record frequencies very similar to those in the zone below.

'Woodland understorey trees and shrubs', 'woodland herbs', 'riverine trees', and 'evergreen woodland trees' all remain at very low frequencies, although Juniperus increases to $1.8 \%$ in the surface spectrum. 'Plants of open dry rocky habitats' increase and reach a maximum of $4.0 \%$ in the surface spectrum.

Relative frequencies of 'herbs of open ground' fluctuate, being highest at the base $(26.3 \%)$ and top $(23.4 \%)$ of the zone, with a minimum of $8.1 \%$ in the second spectrum from the base. Dactylis type follows a similar pattern, fluctuating from 1.4 to $7.9 \%$. Frequencies 
of Festuca type (3.0\% maximum), Pteridium aquilinum (3.3\% maximum), Rumex acetosa group (1.9\% maximum), Urtica dioica (1.0\% maximum), and U. urens ( $2.1 \%$ maximum) are higher than those in the zone below.

'Cultivars' continue to show the slightly elevated values of the top of the preceding zone ( $1.9 \%$ maximum). Pollen types contributing most pollen are again Juglans regia $(0.5 \%$ maximum at $45 \mathrm{~cm})$ and Secale cereale $(1.2 \%$ maximum at $45 \mathrm{~cm})$.

\subsubsection{Terrestrial vegetation history}

This sequence records the vegetation history of the Gomara area from c. $1340 \mathrm{BC}$ to $700 \mathrm{AD}$, at $25 \mathrm{~cm}$. (Dates are interpolated from the radiocarbon chronology.) The lake basin is a hollow within a small steeply sided drainage basin so it receives a substantial proportion of pollen from run-off from the catchment as well as directly from the atmosphere. Given the small size of the lake basin and catchment it is reasonable to assume that a large proportion of the pollen derives from extra-local plants, but a regional pollen source is also represented.

Pollen of the habitat group 'coniferous and deciduous canopy trees' are by far the most abundant throughout the sequence. Pinus (Diploxylon) is the most abundant pollen type in this habitat group, with Fagus and Quercus frainetto type next most abundant. The second most abundant group is 'herbs of open ground'. All the remaining habitat groups are poorly represented.

The terrestrial vegetation history of the Gomara area is discussed according to local pollen assemblage zones below.

Zone G-6, 190.0-157.5 cm, c. 1340-890 BC Pinus (Diploxylon) occurs at consistently very high relative frequencies; values are similar to those of the surface spectrum from this site. However, influx data suggest that initially influx is relatively low (but twice that of the surface spectrum), then increases three-fold before returning to values similar to those recorded initially. Both relative frequency and influx values of Fagus are very low and follow the same pattern of higher at the base and top of the zone, a pattern opposite to total influx. Relative frequencies of Quercus frainetto type (deciduous oaks), although in general slightly higher than in any other zone (with the exception of zone G-1), is only slightly higher than the maximum recorded in surface samples in the Pindos Mountains (Table 3.1), suggesting that the source of pollen may not be closer to the site than that of today, that is, the Pindos foothills (Chapter 3 ). The influx values of this pollen type are the highest for the sequence (with the exception of one spectrum at $110 \mathrm{~cm}$ ). Relative frequencies of Quercus trojana type (semievergreen oaks) and Abies are similar to those throughout the sequence, but influx values are the highest for the sequence. Influx of Abies is lower at the base and top of the zone. Relative frequencies of Ostrya type fluctuate, but in general are about average for the sequence, while 
influx values are the highest for the sequence (with the exception of two spectra - 110 and $90 \mathrm{~cm}$ ) and are of a consistent value. The highest relative frequencies and influx values of the sequence are recorded for Corylus and Picea abies; influx curves form a pattern similar to those of Pinus (Diploxylon) and Abies.

The habitat groups 'alpine shrubs' and 'alpine herbs' are very poorly represented in this zone, and indeed, throughout the sequence. In general plants from these habitats are poor pollen producers. 'Woodland understorey trees and shrubs' and 'woodland herbs' are poorly represented, suggesting an absence of a layered woodland structure or canopy cover. The relative frequencies and influx values of 'riverine trees' are so low that a presence in the vicinity of the site is very unlikely. Similarly, extremely low relative frequencies and influx values of the habitat groups of 'evergreen woodland trees' and 'plants of open rocky habitats' point to the absence of a Mediterranean-type vegetation. The relative frequency and influx values of 'herbs of open ground' are high and suggest an open-canopy vegetation.

Palynological richness values declining from very high values of 40 and 45 expected types at the base of the zone to high values of 30 and 35 at the top suggest that the canopy was very open at the base of the zone and then began to close. The number of terrestrial taxa is also high to very high, the maximum of 84 occurring in the spectrum second from the base, where palynological richness is highest. The ratio of terrestrial trees and shrubs to terrestrial herbs also suggests an open-canopy vegetation; terrestrial herbs represent about $20 \%$ of total terrestrial pollen throughout this zone.

The vegetation surrounding the Gomara site at this time was an open pine or mixed conifer-deciduous wood, but it was not as open as it is today. Two possibilities of the spatial distribution of woodland represented can be envisaged. Either an almost pure pine wood surrounds the site with a deciduous wood growing at a lower altitude or an open woodland of predominantly pine with scattered other conifers and deciduous species is widespread. Although no mixed coniferous-deciduous wood exists in Grevena today at high altitude on Pindos ophiolite ultramafics, a woodland that might resemble the vegetation represented by the pollen and spores of this zone occurs just across the Grevena border in the Pindos Mountains near Samarina at $1300 \mathrm{~m}$. Here Pinus nigra, Taxus baccata, Ostrya carpinifolia, Fagus sylvatica, Sorbus aucuparia, Acer obtusatum, Euonymus latifolius, and Fraxinus excelsior occur together (Polunin 1988). (A notable difference from this woodland is the absence of Fagus from the catchment.) In addition, the occurrence of such entomophilous (but sometimes anemophilous) pollen types as Tilia cordata type, Sorbus type, and Fraxinus ornus (this species may have been planted (Bottema \& Woldring 1990)) suggests the possibility of the latter reconstruction. The light demanding colonizer Corylus was probably growing in the catchment. 
Zone G-5, 157.5-133.0 cm, c. 890-480 BC In zone G-5 the relative frequencies of Pinus (Diploxylon) decline to low values for the sequence. Influx values also decline to very low values, suggesting a decline in the pollen source within the catchment. The relative froquencies and influx of Fogus increase indicating the sccurrence of Fagus in the catchment. Relative frequency and influx values of Betula pendula increase, while both values of Corylus decline and Picea abies almost disappears. Sorbus type, Taxus baccata, and Tilia cordata type disappear completely. The changes in pollen abundance of these types may represent changes taking place in the vegetation within the catchment or regionally, whereas that of Abies (in which relative frequencies remain about the same but influx declines) probably represents a change in the regional woodland. Trees represented by oak pollen types, Ostrya type, and Carpinus type probably occur at lower altitude. The relative frequencies of semievergreen oaks increase slightly, although influx values decrease, while both relative frequencies and influx values of deciduous oaks decline. The relative frequencies of Ostrya type and Carpinus type increase, but influx values decline slightly.

The habitat groups 'alpine shrubs' and 'alpine herbs' are again very poorly represented. 'Woodland understorey trees and shrubs' and 'woodland herbs' are represented by even fewer pollen grains. Similarly, the values of 'riverine trees', 'evergreen woodland trees', and 'plants of open dry rocky habitats' either remain unchanged or decline. Relative frequencies of 'herbs of open ground' increase due to a decrease in 'coniferous and deciduous canopy trees', while influx values decline due to a decline in total influx. However, values are relatively high and suggest an open-canopy vegetation. The most abundant herbs of this group are the same as previously, with the exception of an increase in Artemisia.

High palynological richness values of 30-40 expected types, and the declining ratio of terrestrial trees and shrubs to terrestrial herbs (terrestrial herbs comprising over $25 \%$ of total terrestrial pollen and spores in the centre of the zone) also suggest an open-canopy vegetation.

The pine wood has declined and the diversity of the extra-local/regional coniferousdeciduous woodland is reduced. Abies and Picea abies have almost disappeared. Fagus and Betula pendula have expanded. At $155 \mathrm{~cm}$ the abundance of beech pollen rises to exceed $15.0 \%$, and a beech nut found at the same depth supports the occurrence of beech trees in the catchment. Betula pendula, a pioneer, now plays a more significant role, perhaps as a result of increased exposure of mineral soils. The pioneering role is now played more strongly by Betula pendula than by Corylus. Betula pendula is here identified to the species level based on the current distribution of species of Betula (Tutin et al. 1964-80; Sfikas 1978) and size of pollen grains (Birks 1968; Prentice 1981; Roure 1985).

Zone G-4, 133.0-108.5 cm, c. 480-80 BC In zone G-4 the relative frequency and influx values of Pinus (Diploxylon), Fagus, and Abies increase, while those of Corylus, the 
deciduous oaks, and Carpinus type remain unchanged. Both values of the semi-evergreen oaks and Ostrya type decline in all spectra with the exception of the uppermost, in which an increase occurs in the influx value. A similar increase occurs in most types of the 'coniferous and deciduous canopy trees' group in this spectrum. Betula pendula has almost disappeared.

The habitat groups 'alpine shrubs' and 'alpine herbs' are again very poorly represented. 'Woodland understorey trees and shrubs' and 'woodland herbs' are represented by a slightly higher number of pollen grains. Again, the values of 'riverine trees', 'evergreen woodland trees' and, 'plants of open dry rocky habitats' either remain unchanged or decline. While the values of palynological richness ( 35 expected types at the base of the zone and 30 at the top) are still indicative of an open canopy, decreased frequency and influx values of 'herbs of open ground' and an increase in pollen influx of the habitat groups indicative of woodlands suggest that the canopy is closing. An increase in the ratio of the total terrestrial trees and shrubs to terrestrial herbs also suggests a slight closing of the canopy, terrestrial trees and shrubs representing $82.0-88.4 \%$ of total terrestrial pollen.

Pine woods have expanded and/or become denser as well as beech woods. Representation of Fagus pollen now reaches $20.0 \%$ relative frequency. The surface sample from Anelia bog, which is surrounded by beech wood, recorded only $23.5 \%$ (Chapter 3 ). Since beech pollen is heavy and usually does not travel far from its source, this high relative frequency value suggests that beech woods were growing extra-locally. In support of an extralocal beech wood is the steadily increasing abundance of beech nut macrofossils.

Abies has increased to a maximum of $4.6 \%$. Abies was found to be greatly underrepresented but well dispersed (Chapter 3), so this value may or may not represent an extralocal occurrence.

Zone G-3, 108.5-106 cm, c. $80 \mathrm{BC}$ Zone G-3 includes only one spectrum. The ratio of terrestrial trees and shrubs to terrestrial herbs declines dramatically; terrestrial herbs comprise about $70 \%$ of total terrestrial pollen and spores.

Sediment of this spectrum comprises a tephra, which was deposited very rapidly, that is, deposition took less than a full pollination season, and it therefore does not contain the full annual pollen and spore complement. Changes in palynomorph representation in this zone thus do not represent a change in vegetation.

There are no significant differences in palynomorph content of the sediments directly above and below the tephra. Mehringer et al. (1977) obtained the same result when pollen analysing sequences including known tephras.

Zone $G-2,106.0-47.5 \mathrm{~cm}$, c. $80 \mathrm{BC}-330 \mathrm{AD}$ In zone G-2 the pollen record of the vegetation history of the Gomara area is continuous with the top of zone G-4. Relative frequencies of Pinus (Diploxylon) and Fagus increase slightly and influx values of these 
pollen types are high until the upper part of the zone. The decline in influx values at the top of the zone may due to an increased sedimentation rate (which has not been completely compensated), or a decline in pollen in-wash. Relative frequency and influx values of the deciduous oaks, Carpinus type, Corylus, and Ostrya type decline slightly, while those of semievergreen oaks and Abies increase slightly.

The habitat groups 'alpine shrubs' and 'alpine herbs' are again very poorly represented, but increase slightly. 'Woodland understorey trees and shrubs' are very poorly represented. 'Woodland herbs' increase slightly at the base of the zone, but then decline. This fluctuation is interpreted as a response firstly to an increasing woodland canopy cover, then to a closed canopy cover of a Fagus woodland, which usually has few plants growing under its dense canopy. 'Riverine trees', 'evergreen woodland trees', and 'plants of open dry rocky habitats' all decline.

'Cultivars' increase towards the top of the zone due to a slight increase in the nut tree Juglans regia and the cereal Secale cereale, but pollen representation is very low and may result from wild plants or from long-distance dispersal from cultivated areas.

'Herbs of open ground' also declines and palynological richness declines (30 or less expected types), suggesting that canopy cover is complete. A high ratio of terrestrial trees and shrubs to terrestrial herbs (highest ratio of the sequence, peaking near the centre of the zone, where trees and shrubs represent more than $90 \%$ of total terrestrial pollen) is supporting evidence for complete canopy cover.

High relative frequencies of Fagus are indicative of a closed canopy beech wood surrounding the site. The relative frequencies of Fagus are higher than those recorded under the beech wood at Anelia (Chapter 3). Fagus woodland has now replaced the former pine wood or mixed coniferous-deciduous wood in the catchment. At the present time a small stand of beech grows in a small valley close to the coring site. Where beech and pines occur together in Grevena today, the former tends to expand at the expense of the latter. Beech has a much greater power of rejuvenation than pine, owing to its ability to develop dormant or adventitious buds, which form a dense brushwood of stumps and broken branches long before conifer seedlings can become established. A noticeable feature of Balkan vegetation today is that on non-calcareous rocks beech is usually dominated by the pines, fir, and spruce, where they occur together, and the latter retain their dominance unless they have been cleared by humans. There are, however, exceptions to this where beech forms a complete zone up to the tree limit (Turrill 1929).

Zone G-1, 47.5-0 cm, c. 330-700 AD and present In zone G-1 relative frequency and influx values of Pinus (Diploxylon), semi-evergreen oaks, Abies, Corylus, and Ostrya type continue at about the same values as at the top of zone G-2. The relative frequency and influx 
values of beech and Carpinus type decline while those of the deciduous oaks increase initially and then decline. Betula pendula reappears.

The habitat groups 'alpine shrubs' and 'alpine herbs' are again very poorly represented. A slight increase in 'woodland understorey trees and shrubs' towards the top of the zone is due to an increase in Juniperus, which grows as an understorey shrub in the open pine woods of today. Both frequency and influx values of 'woodland herbs' remain about the same as at the top of zone G-2. 'Riverine trees', 'evergreen woodland trees', and 'plants of open dry rocky habitats' all increase slightly: this is interpreted to result from a regional pollen source due to reduced extra-local arboreal pollen production. However, plants that grow in dry rocky habitats may have increased extra-locally due to surface soils becoming drier through a reduction in canopy cover and greater evaporation at ground surface.

Pollen of the 'cultivars' of Juglans regia and Secale cereale initially occur at the same abundance as that of the preceding zone and then decline. Juglans regia does not occur in the surface spectrum. Again the pollen source may be either wild plants or distant cultivated areas.

Palynological richness fluctuates from very high (45 expected types at the base of the zone) to low (minimum value of 25 ) near the centre and then to high values again (40 expected types at the top of the zone), suggesting an open, then more closed, then open canopy. The number of terrestrial taxa counted also fluctuates from high to low and then to high again. Rate of change is high, reflecting these fluctuations. The curve of the ratio of terrestrial trees and shrubs to terrestrial herbs follows this pattern also (at the base of the zone terrestrial trees and shrubs contribute about $65 \%$ of total terrestrial pollen, increase to $90 \%$, then decline to about $65 \%$ ), suggesting an open, then closed, then open canopy. This pattern is also followed by the habitat group 'herbs of open ground'. This is interpreted as a more open canopy of the catchment vegetation due to a reduction in the extra-local beech wood at c. 330 AD. Pine wood recovers somewhat, replacing beech wood, but then declines at c. $600 \mathrm{AD}$.

\subsection{Charcoal analysis and fire history}

\subsubsection{Microscopic charcoal analysis}

Results of microscopic charcoal particle analyses are presented as relative frequencies and influx in Figures 4.11 and 4.12. The local zonation scheme of the terrestrial pollen and spores is used for convenience of correlation. Curves show abundance of charcoal of different size classes and total abundance. The charcoal record shows continuous registration of charcoal particles in the total charcoal curves. High total charcoal abundance occurs in zones G-6, G-5, G-3, and G-1, while in zones G-4 and G-2 total charcoal abundance is very low. 


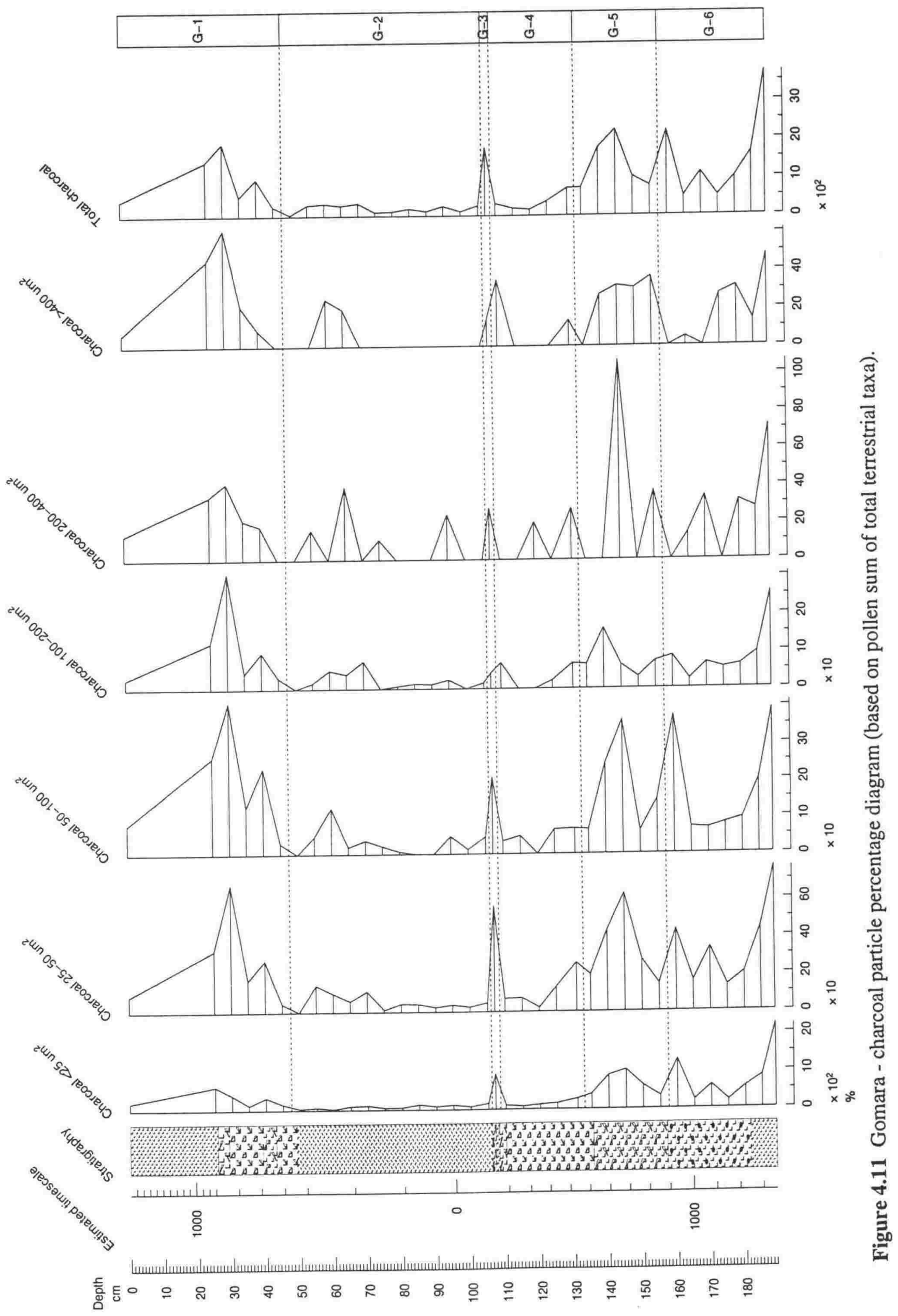

173 


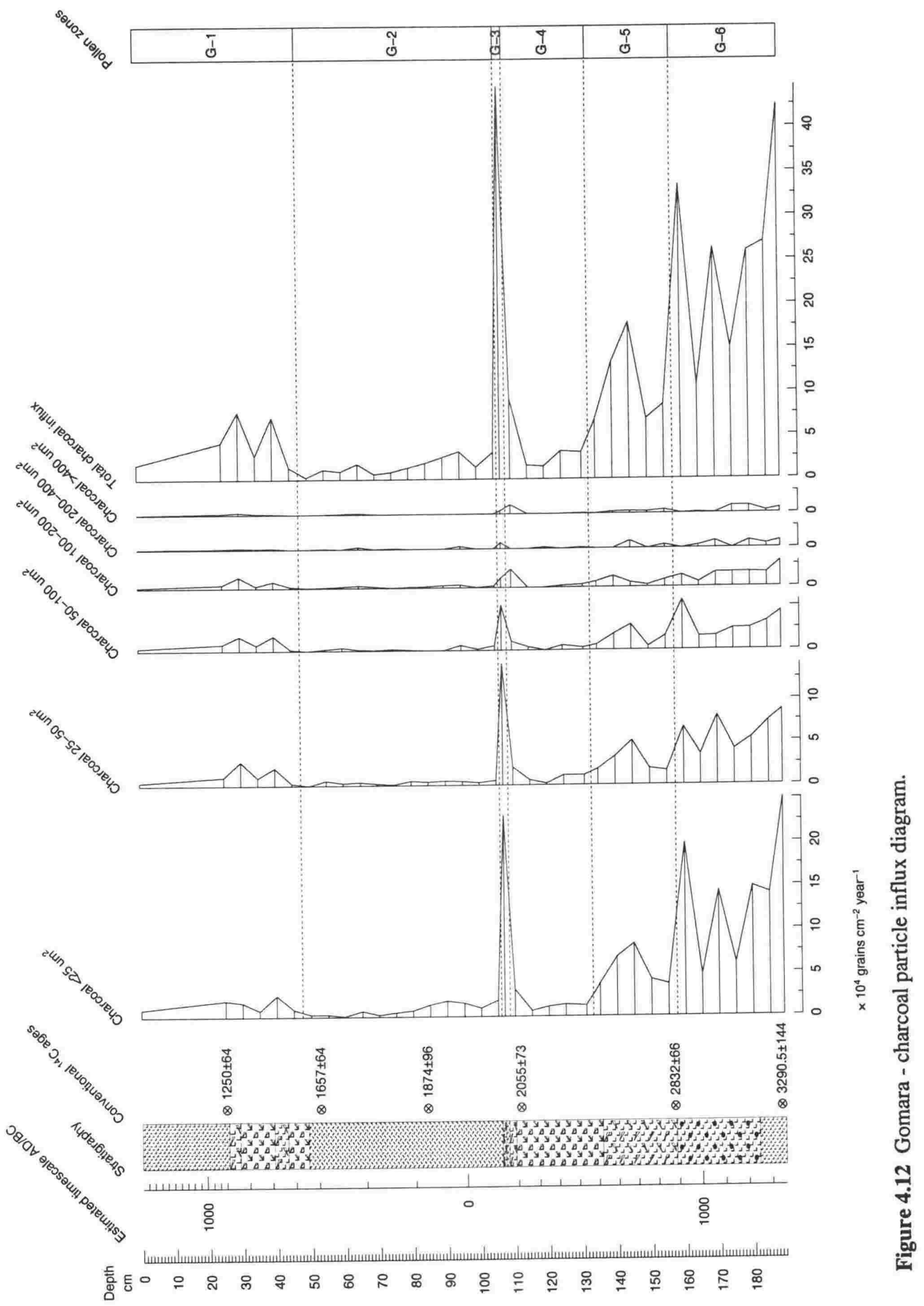


In zone G-6 relative frequencies of total charcoal fluctuate from the highest recorded of the sequence in the basal spectrum to about average in the centre of the zone, to one of the highest peaks of the sequence in the uppermost spectrum. Influx total charcoal data also show very high charcoal levels and confirms the very high peaks at the base and top of the zone, suggesting that fires were more intense/frequent in the region at that time. Charcoal particles are much more abundant in the smaller than in the larger size classes, and the peaks in the total charcoal curve are reflected in the three smallest size classes. Charcoal particles of the four smallest size classes, that is up to $200 \mathrm{um}^{2}$, are represented in all spectra. In the two largest size classes, no charcoal particles are recorded at $175 \mathrm{~cm}$ in the $200-400 \mathrm{um}^{2}$ size class and at $170 \mathrm{~cm}$ none is recorded in the $>400 \mathrm{um}^{2}$ size class. At $160 \mathrm{~cm}$ no particles were recorded for either of these classes. Charcoal particles in the size class $>400 \mathrm{um}^{2}$ are frequent in the lower half of this zone, suggesting that fires occurred in the catchment.

Zone G-5 exhibits high charcoal content. The total relative frequency curve shows abundances similar to those of the basal zone, with a peak in the centre of the zone (at $145 \mathrm{~cm}$ and $140 \mathrm{~cm}$ ), while influx data suggest that abundances were lower than those in the basal zone. The total influx curve also displays the peak in the centre of the zone. Charcoal particles of the smaller size classes are again much more abundant than those of the larger sizes and occur continuously, whereas those of the larger sizes occur sporadically. In the size class $200-400 \mathrm{um}^{2}$ no particles were recorded at 150,140 , and $135 \mathrm{~cm}$. In the largest size class, that of $>400 \mathrm{um}^{2}$, no particles were recorded at $135 \mathrm{~cm}$. The peak recorded by the total charcoal curve in the centre of the zone is reflected only in the three smallest size classes $\left(<100 \mathrm{um}^{2}\right)$ in the relative frequency and influx curves, although a peak is shown at $140 \mathrm{~cm}$ in the $100-200 \mathrm{um}^{2}$ size class and one at $145 \mathrm{~cm}$ in the $200-400 \mathrm{um}^{2}$ size class. Charcoal particles in the size class $>400 \mathrm{um}^{2}$ are frequent, with the exception of the uppermost spectrum, suggesting that fires occurred in the catchment (Chapter 3).

Zone G-4 exhibits low charcoal content. The total relative frequency and influx curves show much reduced charcoal content. Particle abundance of all size classes has declined, with the exception of a peak in the uppermost spectrum (of equal abundance to values in the lower two zones in the largest size class). Occurrence is sporadic in all size classes apart from the two smallest, i.e. smaller than $50 \mathrm{um}^{2}$.

Zone G-3 exhibits very high total charcoal content (in the influx diagram as high as that in the basal spectrum of the sequence) in the one spectrum that comprises this zone, and charcoal particles of all size classes are recorded. The smaller size classes are the most abundant. 
Zone G-2 exhibits very low charcoal content. Total charcoal content is extremely low and all size classes occur sporadically, apart from the two smallest size classes (particles $<50 u^{2}$ ).

In zone G-1 charcoal abundance increases in all size classes. Two peaks occur, at $40 \mathrm{~cm}$ and $30 \mathrm{~cm}$, when fires in the region were probably more intense or more frequent. These peaks are recorded in all size classes, with the exception of the peak at $40 \mathrm{~cm}$ in the largest two size classes $\left(200-400 \mathrm{um}^{2}\right.$ and $\left.>400 \mathrm{um}^{2}\right)$. The upper peak is the larger of the two and reaches relative frequencies similar to those in the basal spectrum of the sequence, especially in the larger size class particles. However, the influx curve is much lower. The surface spectrum shows greatly reduced charcoal abundance.

\subsubsection{Fire history}

There is continuous accumulation of microscopic charcoal particles throughout the sediment sequence. Visual inspection of residues retained by the $260 \mu \mathrm{m}$ mesh sieve during pollen-sample preparation, prior to mounting the processed samples onto microscope slides, showed that optically visible charcoal occurred in only those samples where the largest-sized particles were observed through the microscope.

Interpretation of charcoal data is complicated because erosion after a fire and the redeposition of sediment within the lake basin could produce smoothed peaks or even anomalous peaks. Also, the magnitude of a charcoal peak may be obscured if a sample spanning several years contains only a few years of high influx that are then averaged over the total number of years spanned by the sample. Thus the frequency of individual fires cannot be interpreted from the microscopic charcoal data, but periods when fires occurred more frequently or more intensely can be distinguished.

Two phases of more frequent/intense fires are evident; from c. 1340 to $480 \mathrm{BC}$, and from c. 500 to $700 \mathrm{AD}$ at the top of the undisturbed sediments. In the former phase the charcoal record shows continuously high values of charcoal influx of particles in the four lower size classes and almost continuous influx in the two largest size classes. During this period fire was widespread and of major ecological importance in the region of the site, and perhaps also in the catchment. Optically visible charcoal particles in the sediments from $190.0-181.5 \mathrm{~cm}$ and $157.0-133.0 \mathrm{~cm}$ provide supporting evidence for the importance of fires during this phase. In the later phase, total charcoal abundance is lower, but again the four smaller and the two largest size classes are continuously represented are also continuously represented. Separating these two phases is a period of about 900 years when fires were more distant and/or sporadic and were of little ecological significance. Today fire is not a major ecological factor around the Gomara site. 
Pollen evidence also suggests a high frequency of fires in the Gomara region in the lower part of the sequence. Between 190.0 and $157.5 \mathrm{~cm}$, the pollen assemblage zone (G-6) where charcoal is most abundant, the curve of total influx of pollen and spores is the mirror image of that of total charcoal influx. This is interpreted as resulting from the depletion of the pollen source through the vegetation being burnt. Total influx of pollen and spores is also low in the following pollen assemblage zone, where charcoal is also abundant. The influx of pollen of the conifers Pinus, Picea, and Abies, which are slow to recover from burning because they become established by seed reproduction only, declines during the phase of frequent fires in the lower part of the sequence. Influx of pollen of the early successional genera of Betula pendula, Corylus, Pteridium, and Gramineae, which can sprout from underground root collars following destruction of above-ground biomass after burning, is highest in that part of the sequence.

\subsection{Synthesis of environmental changes}

A summary of the major environmental changes in the wetland and its catchment from c. $1340 \mathrm{BC}$ to $700 \mathrm{AD}$ is given in Table 4.2.

There is close correspondence between changes in the composition of the sediment and changes in the pollen profiles of both wetland and terrestrial pollen and spore types. This close correspondence is explained by these three elements responding to environmental changes in the catchment. Thus the boundaries of the wetland and terrestrial pollen zones are synchronous. Further, this synchronism implies that many of the pollen and spores in the sediments derive from the catchment: the terrestrial pollen record reflects primarily the extralocal rather than regional vegetation.

The Gomara wetland is continuously wet and comprised a small lake 1-2 $\mathrm{m}$ deep from c. $1340 \mathrm{BC}$ to $700 \mathrm{AD}$. After this period seasonal drying of the lake bed occurred. The water of the lake is fresh, slightly alkaline, and oxygen-rich. The highland regional vegetation is pine wood throughout the sequence.

The genesis of the wetland, radiocarbon dated at $c .1340 \mathrm{BC}$, is coincident with soil erosion in the catchment as evidenced by the high proportion of inorganic matter of the wetland sediment, high trophic level (eutrophic), and high organic nitrogen content of the lake water. At c. $1250 \mathrm{BC}$ erosion intensifies, as indicated by coarser sediment particles, increased alkaliphilous diatoms, and increased nutrient levels of the lake. 


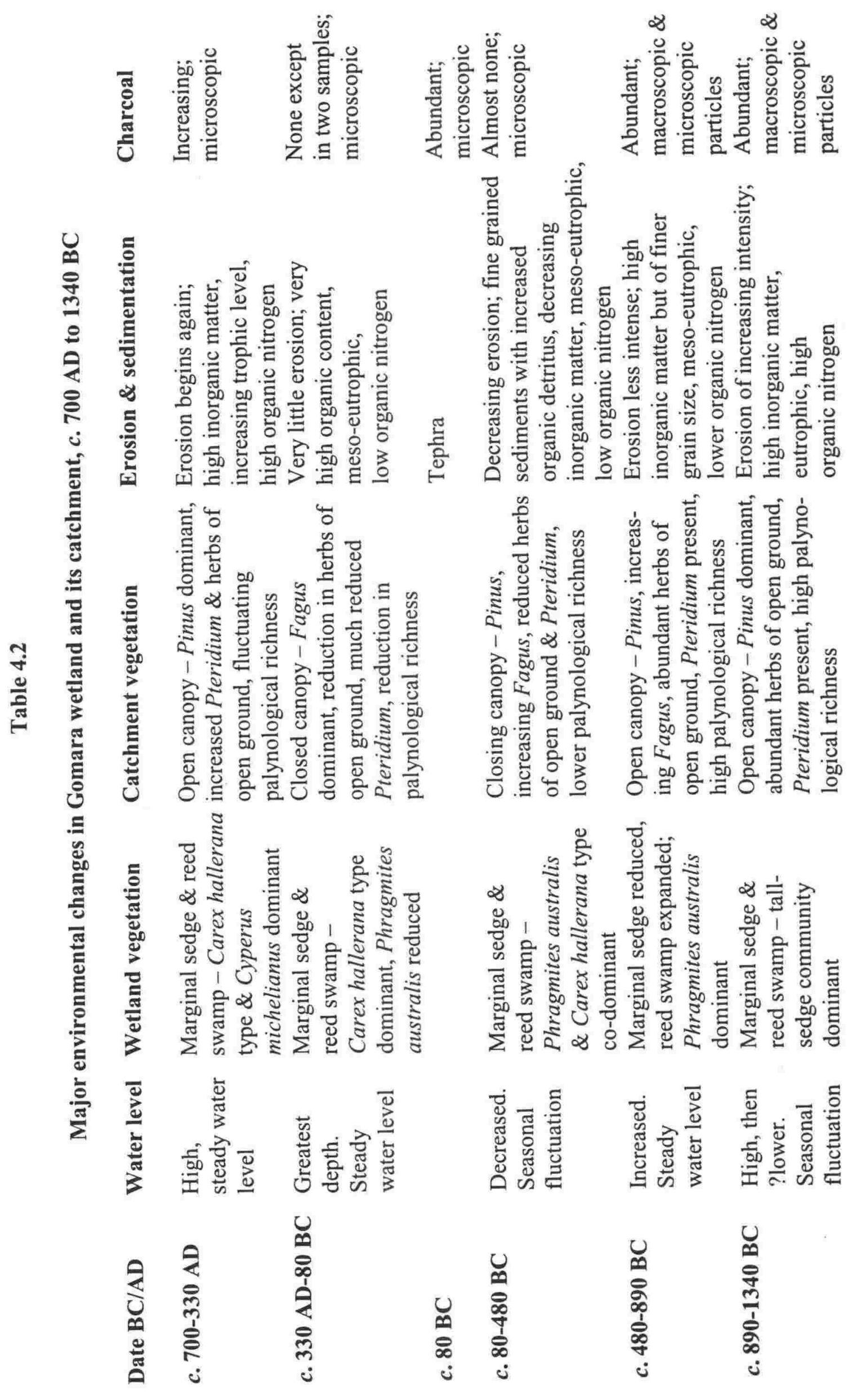


Between c. 1340 and $890 \mathrm{BC}$ a marginal sedge and reed swamp vegetation surrounds the lake with the tall-sedge-swamp community dominant. The water level fluctuates seasonally and average water level may decline. The catchment vegetation is either an open canopy pine or mixed conifer-deciduous wood, although not as open as today. Pine wood occurs over a wide area, either pure or mixed with other conifers and deciduous trees. Abies, Picea abies, Corylus, and Betula pendula are present either extra-locally and/or regionally. 'Herbs of open ground' are abundant especially terrestrial grasses, Rumex species, Urtica species, and Pteridium aquilinum. Species belonging to the pollen types Chenopodiaceae type, Plantago lanceolata type, and Solidago type are also important. A mixed oak wood including Ostrya and Carpinus occurs at lower elevation. Burning of the catchment and regional vegetation is suggested by the occurrence of abundant macroscopic and microscopic charcoal particles, lower influx of coniferous pollen (Pinus, Abies, and Picea abies) where charcoal is most abundant, and, perhaps, the presence or greater abundance of pollen of earlysuccessional genera such as Betula pendula, Corylus, and Gramineae and spores of Pteridium aquilinum.

Between c. 890 and $480 \mathrm{BC}$ less intense soil erosion is suggested by finer grain size of inorganic matter, a reduced average sedimentation-accumulation rate, a decline in trophic level to meso-eutrophic, and lower organic-nitrogen content of the lake. Water depth increases and there is less seasonal fluctuation. In the mixed marginal sedge and reed vegetation Phragmites australis becomes more important. Vegetation of the catchment changes to an open canopy mixed pine-beech wood. The woodland extra-locally and/or regionally is less diverse with tree pollen types such as Sorbus type, Taxus baccata, and Tilia cordata type disappearing. Picea abies and Corylus decline, but Betula pendula expands. 'Herbs of open ground' are abundant; most abundant herbs are the same as previously, with the exception of an increase in Artemisia. A mixed oak wood occurs at lower altitude. Burning of the catchment and regional vegetation is again suggested by the occurrence of abundant macroscopic and microscopic charcoal particles and the greater abundance of pollen of the early successional Betula pendula and Gramineae, and the occurrence of Pteridium aquilinum spores.

Between c. 480 and $80 \mathrm{BC}$ soil erosion declines further; minerogenic sediments are fine-grained, organic detritus increases, lake trophic level is meso-eutrophic, and organic nitrogen content is low. Water level probably declines and there may be greater seasonal fluctuation. A mixed marginal sedge and reed community surrounds the lake, with Carex hallerana type and Phragmites australis co-dominant. Vegetation of the catchment is now mostly beech wood with a much reduced pine component, but regionally pine is still dominant. 'Herbs of open ground' and the early successional types of Corylus and Betula 
pendula decline as the canopy of the catchment woodland becomes more closed. A mixed oak wood still occurs at lower altitude, but semi-evergreen oaks and species of the Ostrya pollen type have declined. An almost complete absence of charcoal particles and Pteridium aquilinum spores suggests burning of the catchment and regional vegetation has almost ceased.

At c. $80 \mathrm{BC}$ a volcanic ash fell; perhaps originating from an Italian volcanic eruption from Etna, Monte Albano, or Vulcano (Simkin et al. 1968).

Between c. $80 \mathrm{BC}$ and $330 \mathrm{AD}$ little soil erosion occurs; minerogenic sedimentation declines and the proportion of organic detritus increases until sediments are almost entirely organic. Trophic level of the lake water is meso-eutrophic, and organic nitrogen content is low. Water level increases and is probably at its highest levels with little seasonal fluctuation, as shown by the abundance of diatoms living in permanent water and an increase in pollen of aquatic macrophytes. The marginal vegetation is now dominated by plants of the Carex hallerana pollen type. Vegetation of the catchment is a closed-canopy beech wood; 'herbs of open ground' record the lowest relative pollen frequencies of the sequence. The regional highland vegetation is pine wood. At lower elevations a mixed oak wood is extant. Juglans regia and Secale cereale pollen occurs at very low abundance and may result from wild plants or from long-distance dispersal from cultivated areas. Burning of vegetation is not occurring, except perhaps at a long distance from the catchment.

Between c. 330 and $700 \mathrm{AD}$ more intense soil erosion occurs. In the wetland inorganic sedimentation occurs; alkaliphilous diatoms increase, the trophic level increases, and organicnitrogen content increases. Water level is probably high with little seasonal fluctuation. The lake-margin vegetation comprises a sedge-swamp community dominated by plants of the Carex hallerana pollen type and Cyperus michelianus with a small reed swamp growing on the lakeside. The beech wood in the catchment begins to decline at $c .330 \mathrm{AD}$. 'Herbs of open ground' increase; particularly the dryland grasses of Dactylis type and Festuca type, Pteridium aquilinum, Rumex acetosa group, Urtica dioica, and U. urens. Juglans regia and Secale cereale occur at the same very low abundance as previously. Pine wood replaces the beech wood, but then it declines at $c$. $600 \mathrm{AD}$, and an open canopy pine wood similar to that of today forms. This open pine wood is widespread. A mixed oak wood is extant at lower elevations. Burning of the catchment and regional vegetation occurs again from c. $500 \mathrm{AD}$, both locally and regionally, and almost reaches the levels of frequency/intensity of that between $c .1340$ and $480 \mathrm{BC}$.

Sediments younger than c. $700 \mathrm{AD}$ (above $25 \mathrm{~cm}$ ) are oxidized, and there is no accumulation of organic deposits. The palynological record is probably incomplete, thus no sample except the surface sample was pollen-analysed. Sediments of this horizon are 
disturbed by surface cracking when the lake bed dries out during summer and by cattle trampling so they are mixed, but they indicate that erosion of catchment soils has occurred. Diatom analysis of samples at 10 and $1 \mathrm{~cm}$ indicate that wetland conditions are similar to those at $35 \mathrm{~cm}$. The pollen analysed surface sample shows that the wetland vegetation is similar to that at $c .700 \mathrm{AD}$ : a much reduced wetland vegetation community mostly of plants indicative of high nutrient levels. The extra-local and regional vegetation differs from that at c. $700 \mathrm{AD}$ : Fagus, Quercus, and Abies are less abundant, and Betula pendula is absent, but Juniperus and plants of open dry rocky habitats are more abundant. Burning is much less than that at $c .700 \mathrm{AD}$. 


\section{Chapter 5. Anelia}

\subsection{Introduction}

This chapter includes a description of the site and sediments of the Anelia wetland coring site and then presents the results of pollen analysis of a sediment and peat sequence $250 \mathrm{~cm}$ long. The site was discovered on the 1:50 000 topographic map for the Metsovon area and is named after the name appearing on that map (Hellenic Army Geographic Service 1970).

\subsection{Site description}

Anelia bog is situated in the Pindos Mountains (latitude $39^{\circ} 57^{\prime} 30^{\prime \prime} \mathrm{N}$, longitude $21^{\circ} 05^{\prime}$ $30 " \mathrm{E}$ ) at an elevation of $1440 \mathrm{~m}$ asl (Figures 3.2, 5.1 \& 5.2). It is in Grevena Province about $3.5 \mathrm{~km}$ southwest of Perivolion, near the pass between Perivolion and Vovousa, which was used as a trading route during the Ottoman Period. It is located approximately $150 \mathrm{~m}$ north and $50 \mathrm{~m}$ below the Perivolion-Vovousa road and can be reached by turning off this road at the sign indicating the direction to Vovousa, then driving about $1.5 \mathrm{~km}$ eastwards along a track. It is about $500 \mathrm{~m}$ distant from the Katarramtis River, a tributary of the Aoos.

There is plenty of evidence that this area is often used by humans and animals. Cattle dung was noted on the surface of the bog, and the bog vegetation was grazed between our visits to it. On two occasions while we were visiting the site shepherds drove flocks of sheep and goats along the track at the northern end of the wetland (Figure 5.3). A dry area close to the track at the northern end appears to be a picnic area, perhaps used by shepherds. The remains of several small fires as well as plastic cups and food wrappers were seen in this area.

\subsubsection{Physiographic description}

The wetland at Anelia is currently a bog with a fringing fen (Figure 5.3). The surface of the bog has a slope of $2-5^{\circ}$ (inclinometer measurement) up to the south. On the slopes near the site are alternating ridges and ravines or hollows that are closely and relatively equally spaced, averaging $75-150 \mathrm{~m}$ between ridges. The bog is situated in a widening of a narrow valley of one of the ravines. There is a steep slope from the bog up to the Perivolion-Vovousa road (average slope of about $28^{\circ}$ (Savina 1990)). The bog has a shady N-NE aspect and is $90 \mathrm{~m}$ long (N/S) and $50 \mathrm{~m}$ wide (E/W), covering about $1 / 3$ ha.

A small stream emerges from a spring on the valley floor about 50 m upstream from the bog (Figure 5.3). At the southern margin of the mire the stream bifurcates and runs along either side of the bog. The western branch continues only a short distance while the eastern branch runs the full length of the bog then drains across the track at the northern end of the bog. The bog drains into the eastern stream, which is entrenched. The bog is mainly supplied 


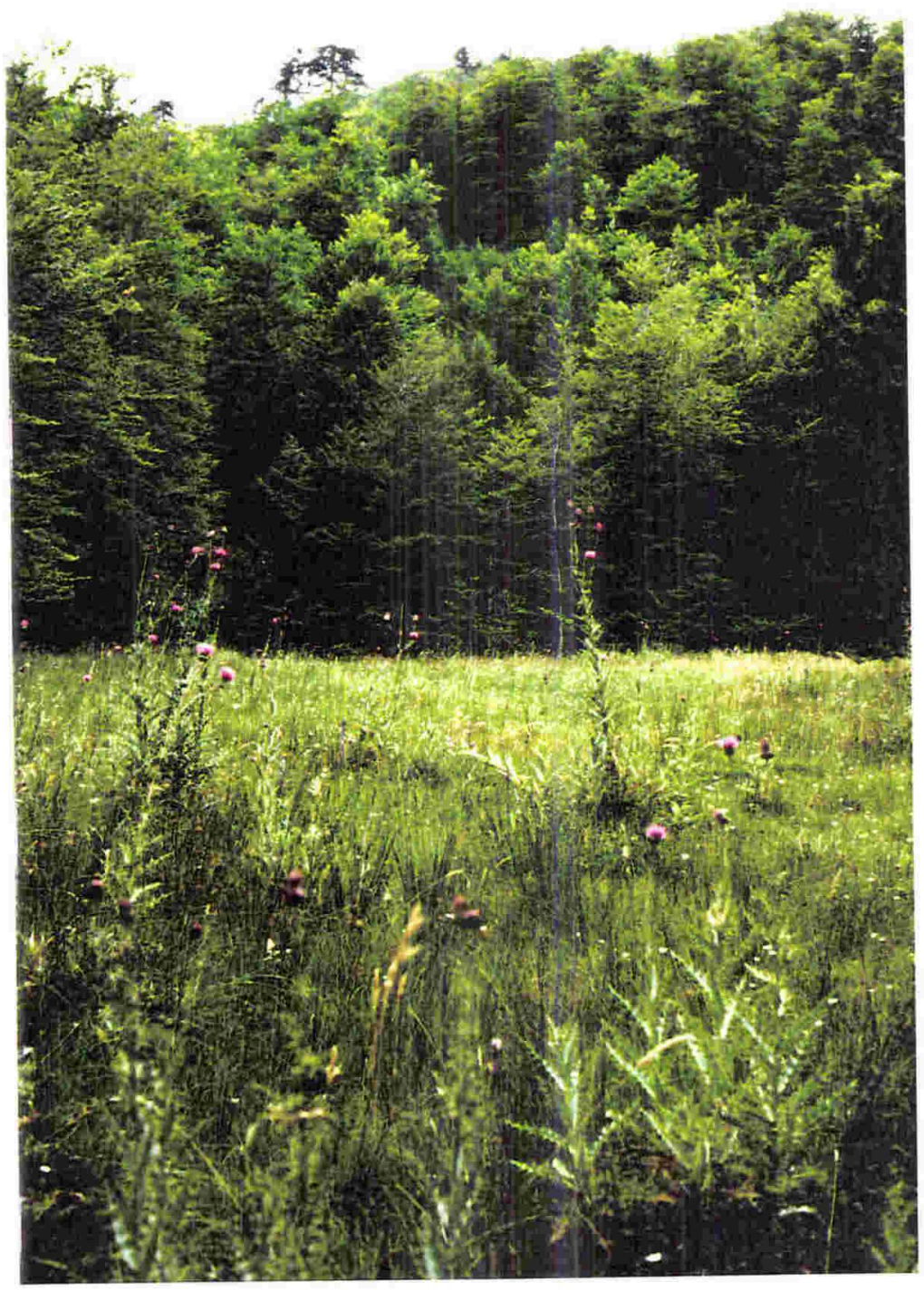

Figure 5.1 Anelia bog, looking SSW. A very lush bog vegetation can be seen with Cirsium dominating the foreground on relatively dry ground at the edge of the bog, Carex is dominant on the wetter central part of the bog. The pure Fagus sylvatica woods in the background grow close to the edge of the bog. 


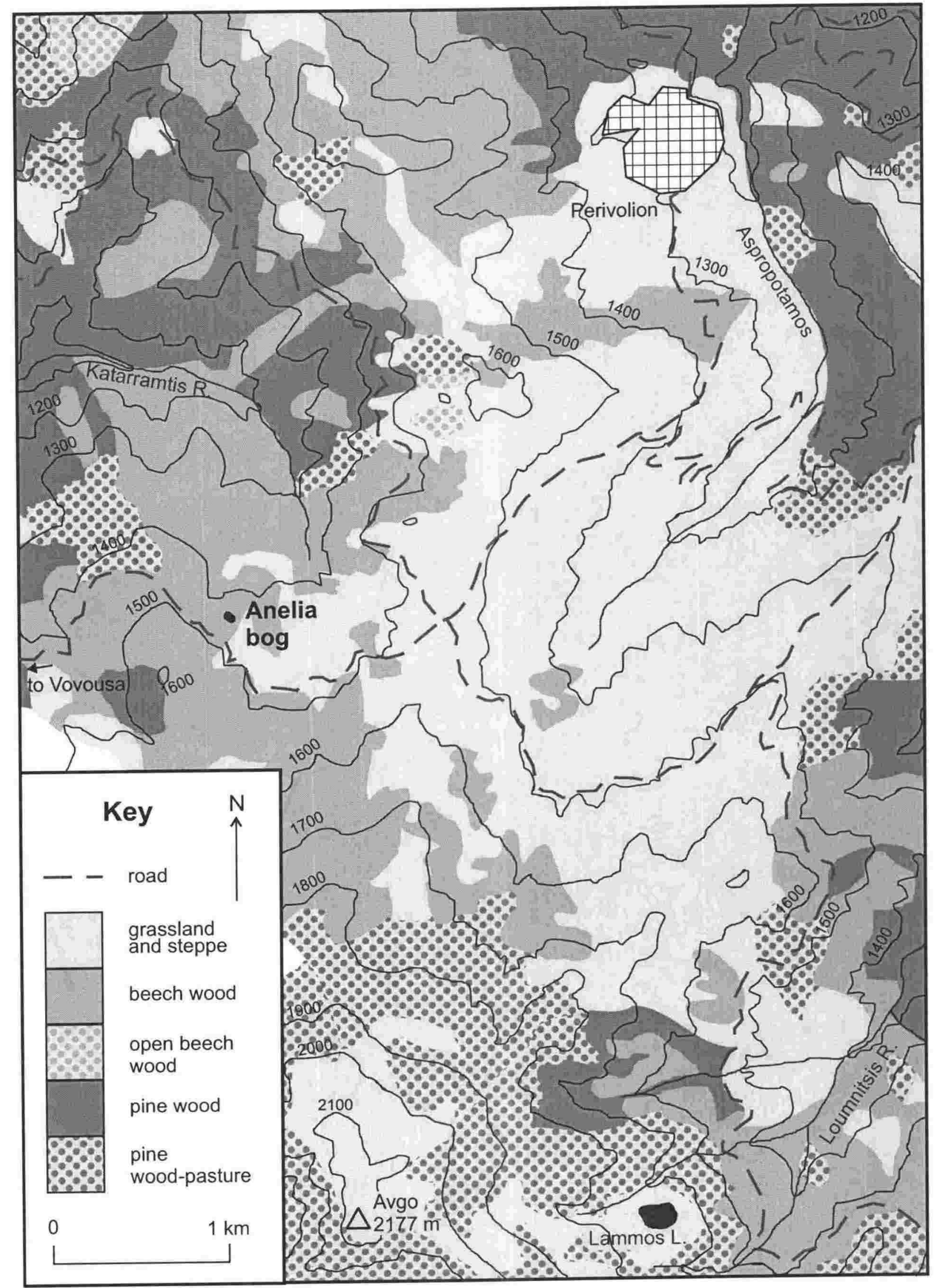

Figure 5.2 Location of Anelia bog in relation to local topography (contour interval $100 \mathrm{~m}$ ). Vegetation distribution is adapted from unpublished maps of the Greek Forest Service. 


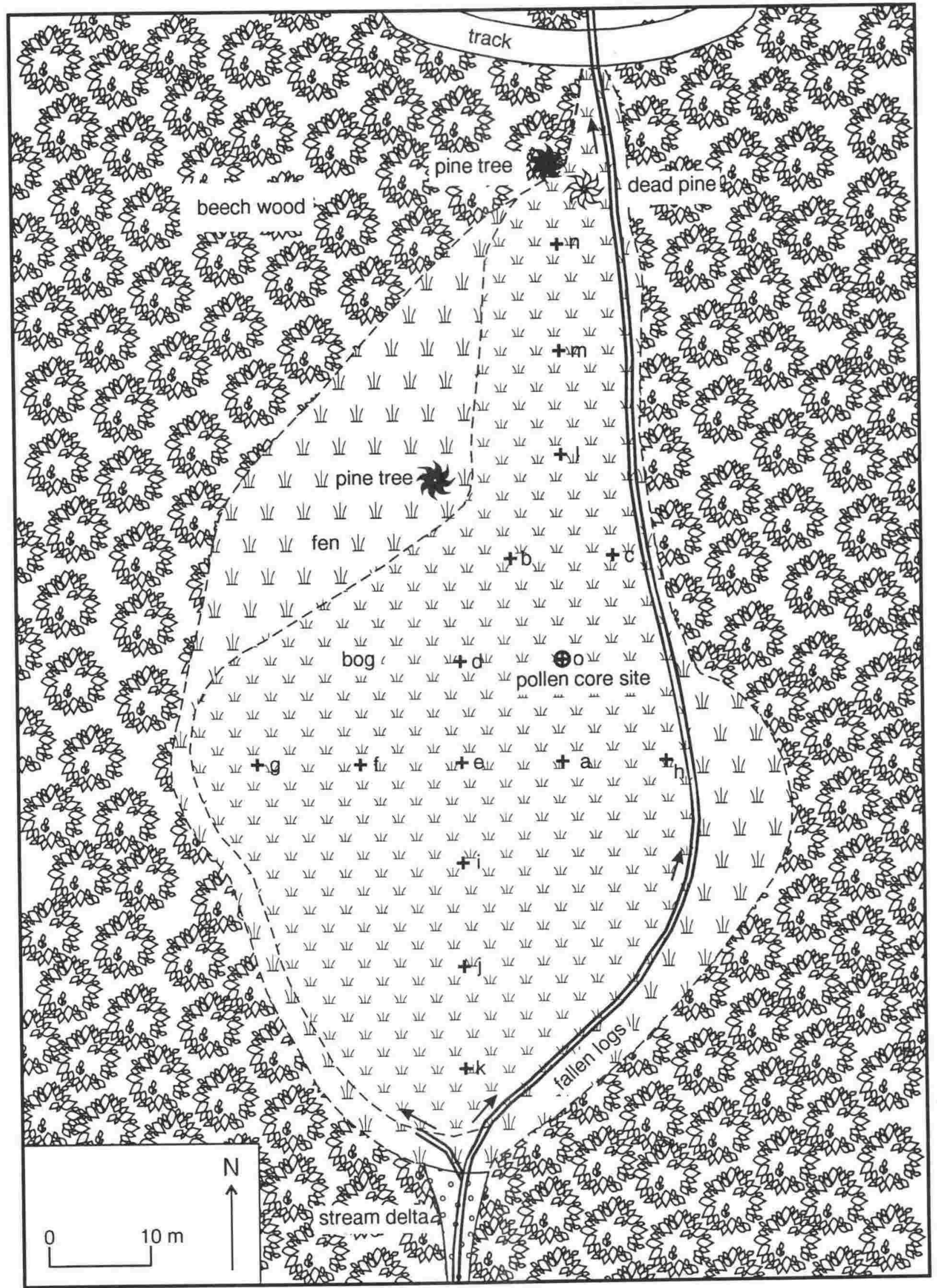

Figure 5.3 Anelia mire with location of exploratory borings and pollen core site. 
with water from the spring, but the central part is slightly raised and is thus somewhat ombrogenous, relying partly on precipitation in the form of rain and snow. It is probably perennially waterlogged. We visited it at the end of summer, the driest season, and the water table was at the surface of the bog. Precipitation is higher at this elevation than at lower elevations - it rained several times when we were visiting the site, when no rain had fallen at lower elevations.

\subsubsection{Regional and local vegetation}

The bog is situated in the mountain beech vegetation zone and is almost completely surrounded by pure beech (Fagus sylvatica) wood with an almost completely closed canopy (Figures 5.1 \& 5.2), described in Chapter 3 and Appendix VIII. Open beech wood, pine wood, and pine wood-pasture also occur in the vicinity, but a large proportion of the vegetation in the east, especially around the Aspropotamos catchment, is grassland and steppe, which is used by transhumant pastoralists for grazing sheep and goats (Figure 5.2).

The central bog is covered by a mixed vegetation of mosses and various sedges, grasses, herbs, and related vascular species (Figure 5.1). It is carpeted with the mosses Acrocladium cuspidatum and Cratoneuron sp., comprising together about $70 \%$ of the vegetation cover in the wetter areas of the bog and about $30 \%$ in the drier. In the drier areas of the bog Carex spp. are dominant ( $60 \%$ of the vegetation cover). This vegetation would form a mixed sedge and moss peat. The vegetation of the bog has been described in Appendix VIII.

Around the edge of the bog the influence of supplementary nutrients from surface and ground water and perhaps from grazing animals can be seen in the herb communities dominated by Caltha palustris (near spring drainage) and Cirsium (on fen).

Many ruderals grow on the dry land on the edge of the beech wood.

\subsubsection{Geology of site}

Bedrock is not exposed at or near the site, but is mapped as the Pindos ophiolite ultramafics, peridotites, and serpentinites (Institute for Geology and Subsurface Research 1959), the same as that at the Gomara site. About $300 \mathrm{~m}$ upslope of the site the bedrock type changes to Pindos flysch, which continues to the top of the saddle and ridge above the bog. The contact between these two bedrock units is a major thrust fault. An important feature of areas underlain by the Pindos ophiolite and flysch is the abundance of landslides observable on both the ground and on air photographs.

A geological survey of the area surrounding this site was undertaken by Dr M. Savina, geologist of the Grevena Project (Savina 1990). The following data are extracted from her report. The Anelia site is surrounded by landslide colluvium that appears to be part of a large 
slump, about 5000-9000 years old, through which modern streams have eroded. Colluvium is exposed in ridges where erosion has removed the soil and vegetation. Most of the cobbles and pebbles in the colluvium near the site are ultramafic and some are dolerite. The maximum intermediate diameter of blocks in the colluvium is about $1.5 \mathrm{~m}$.

Recent large-scale debris movement in the bog valley has not occurred, although there is evidence for some in the next valley. The most important current mass-movement erosional processes in the wood above the site are creep, tree throws, and small debris slides. Creep and tree throw are in most places responsible for relatively steady downslope movement. Some more rapid erosion probably also occurs in the form of small debris slides, but most material transported to the bog area probably moves down the valley by stream transport rather than directly by mass movement, although mass movement supplies the sediment to the stream.

Soil developed on landslide colluvium on the west side of the bog valley about $8 \mathrm{~m}$ above and $20 \mathrm{~m}$ distant from the bog was about $90 \mathrm{~cm}$ deep, well-drained with medium-sized stones covering about $1 \%$ of the surface. The dominant lithology in clasts was serpentinite. The $\mathrm{pH}$ values of the soil ranged between 5.2 and 5.7 and that of the parent material was 6.3. There was evidence of mantle creep.

\subsection{Sediment analysis}

The results of the exploratory borings of the mire, the Troels-Smith (1955) classification and laboratory analyses of the physical properties of the pollen analysed core sequence are reported below. In the diagrams and text, datum for depth (given in $\mathrm{cm}$ ) is the bog surface.

\subsubsection{Wetland profile}

The location of the exploratory borings is shown in Figure 5.3. Main features of the stratigraphy of the exploratory cores are shown in Figure 5.4; detailed logs of the boreholes are in Appendix XIV. Exploratory cores were bored with a Hiller corer at $10 \mathrm{~m}$ intervals in two intersecting transects across the bog in N/S and E/W directions.

The bog consists of a thick upper horizon of up to $110 \mathrm{~cm}$ of fibrous peat, which is thickest in the centre of the bog. At core sites "k" and "j" at the southern end of the bog, the lower part of the peat is mixed with gravel, inwash from the stream feeding the bog, but to the north the peat is homogeneous. The peat layer is underlain by stratified clay, silt, sand, and fine gravel with peaty horizons. It is possible to trace some of these horizons through adjacent borings, but no extensive marker units were found.

The lower stratified unit is interpreted as a fan-like deposit of the stream resulting from a reduction in gradient below the steeper slope upstream. As transportation energy of the stream varied and the stream course changed location, so varied sediments were deposited 


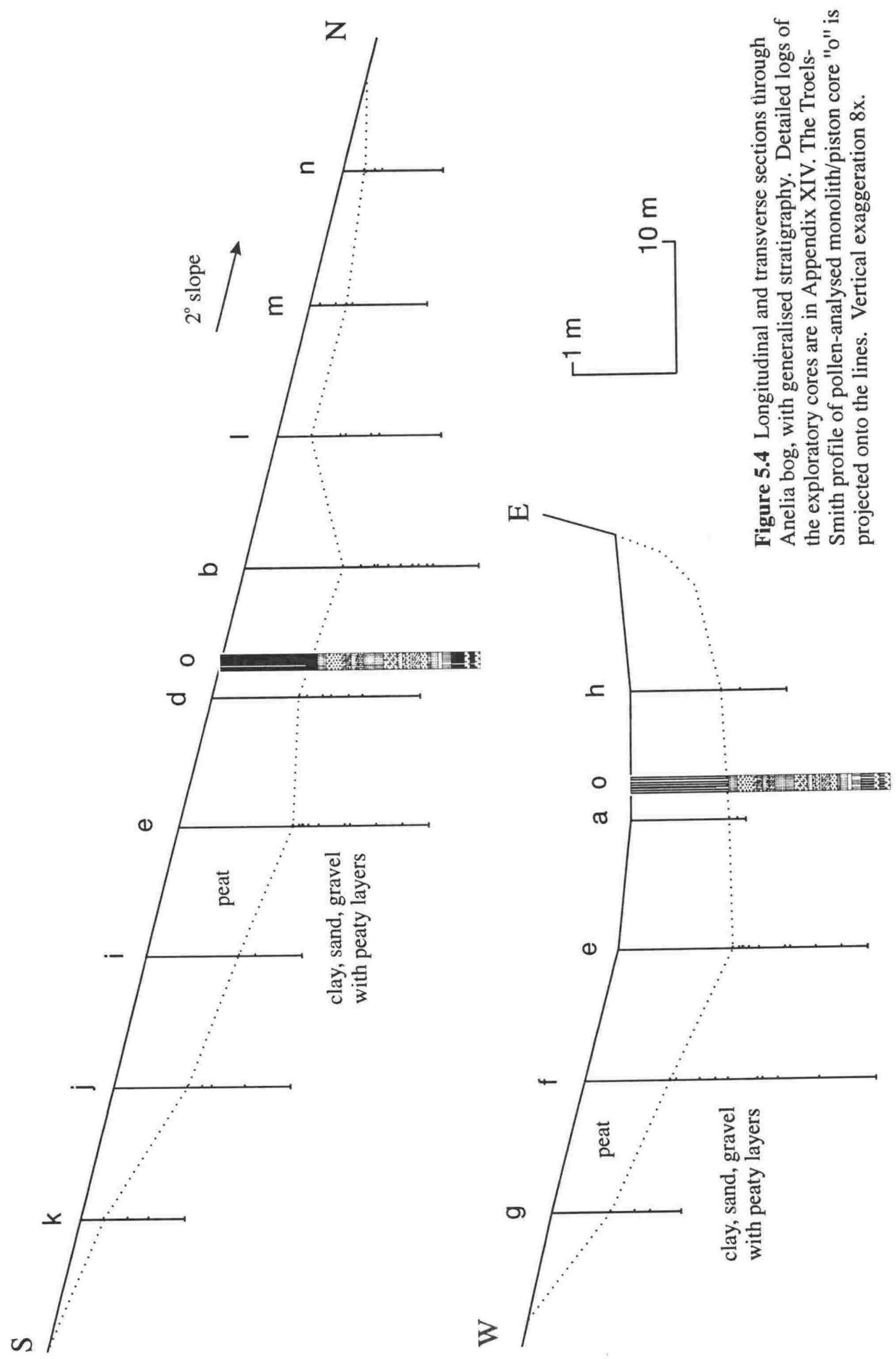


within the wetland. Eventually peat formation became general, with a permanently marginal stream.

After examination of these data it was decided that location ' $o$ ' was a suitable location for extracting a core for pollen analysis because it contained a near-maximum thickness of peat and minerogenic sediments. A central location also avoided gravel-rich sediments at the southern end of the bog, where the stream inlet is located.

\subsubsection{Stratigraphy}

An illustrated description of the deposits of the pollen-analysed core is given in Figure 5.5. The upper $110 \mathrm{~cm}$ were recovered by removing a monolith, and the lower sediments down to $240 \mathrm{~cm}$ were removed alternately with a Russian corer $(110-150$ and $162-200 \mathrm{~cm})$ and a piston corer (140-180 and 195-244 cm) in alternate holes $50 \mathrm{~cm}$ apart. The lowest section (240-250 cm) was recovered with a soil auger. The Troels-Smith (1955) classification scheme using symbols and formulas (Appendix III) is followed in the same manner as in Chapter 4. Colours are all fresh colours.

The deposits of this site are more complex than those of the Gomara wetland. They comprise a series of peat (Turfa herbacea) horizons separated by minerogenic horizons of varying grain size, indicating varied transport energy and depositional environment; gravel, sand, silt, and clay all occur in varying proportions. The upper $94 \mathrm{~cm}$ comprises fairly uniform peat. (Since the whole sequence is water saturated, siccitas is 2 throughout.) No samples effervesced in $10 \% \mathrm{HCl}$, indicating the absence of calcium carbonate.

250-245 cm Basal sediments are composed of Grana arenosa $(\mathrm{Ga})$ (mineral particles 0.06-0.6 mm), G. glareosa majora (Gg(maj)) (mineral particles 6.0-20.0 mm), and $G$. glareosa minora $(\mathrm{Gg}(\mathrm{min}))$ (mineral particles $2.0-6.0 \mathrm{~mm}$ ). The smallest mineral particles comprise half the sediment composition while the two larger-sized particle classes comprise the remaining half in equal proportions. The broad distribution of the grain size indicates a stream flood deposit, and the inclusion of large grains indicates high energy transport. The sediments are dark olive grey (2.5GY 4/1). Physical properties are nigror 2 , stratificatio 0 , and elasticitas 0 .

$245-240 \mathrm{~cm}$ A sharp boundary (limes 3) at $245 \mathrm{~cm}$ marks a change to a homogeneous brownish black (7.5YR 2/2) Turfa herbacea (Th) (root systems, and stems and leaves connected to the roots of herbaceous plants). The plant structure is partly decayed though distinct, humositas is equivalent to 2 , indicating that the bog surface was periodically dry. The darker colour of organic matter is reflected in nigror 3; elasticitas increases to 2 . Other physical properties remain unchanged (stratificatio 0 ). 
兽
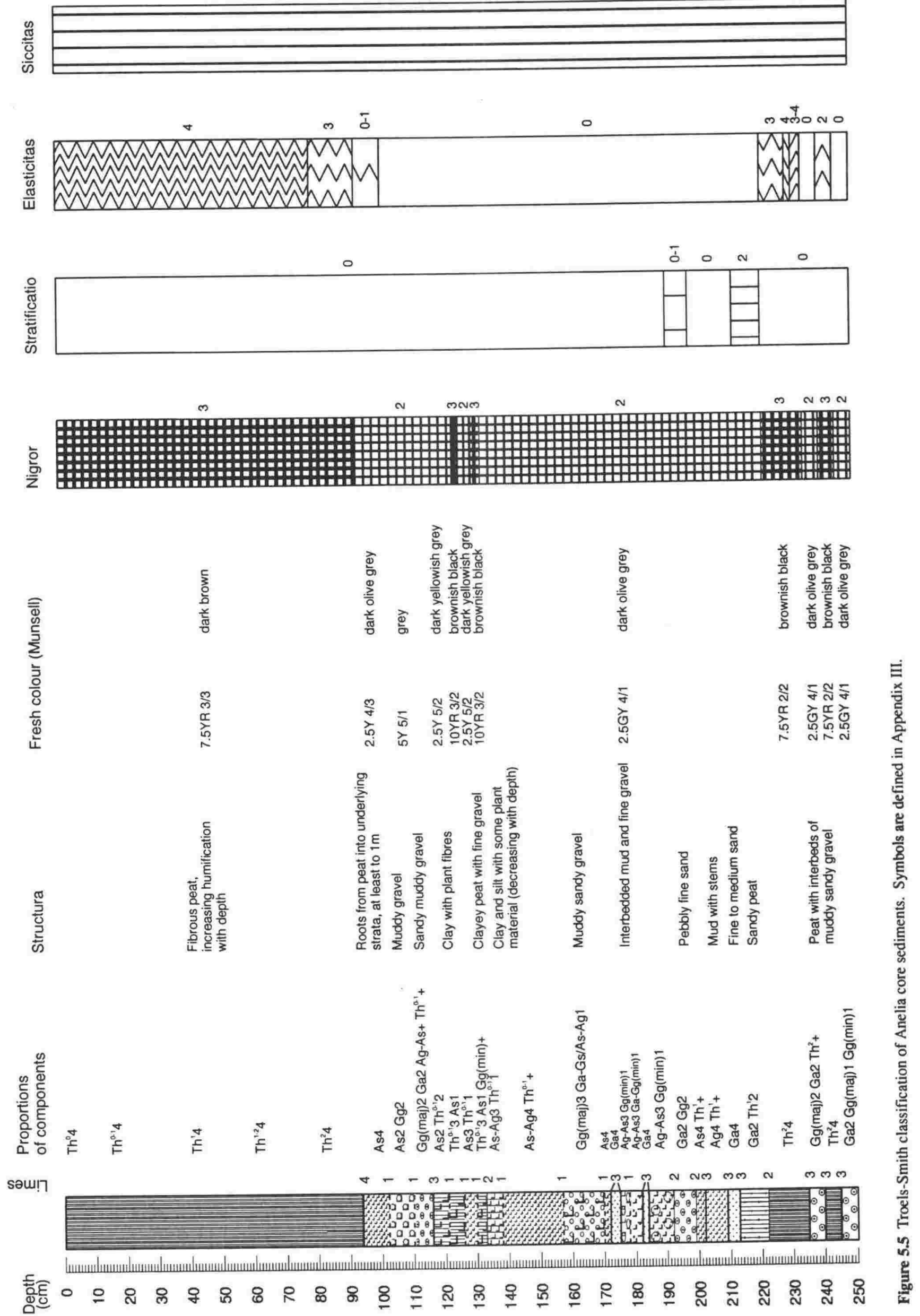
240-235 cm Another sharp boundary (limes 3) occurs at $240 \mathrm{~cm}$, where the sediment colour is again the same as that of the basal horizon, dark olive grey (2.5GY 4/1) (nigror 2). Sediment composition is very similar to that of the basal horizon, but the proportions of the mineral components differ slightly, being equal proportions of Grana glareosa majora and G. arenosa; Turfa herbacea of humositas 2 occurs as a trace. Stratificatio is 0 and elasticitas 0 .

235-222 $\mathrm{cm}$ The boundary at $235 \mathrm{~cm}$ is again sharp (limes 3 ) and signals a change to homogeneous brownish black (7.5YR 2/2) Turfa herbacea. This horizon is very similar to that at $245-240 \mathrm{~cm}$. Again the darker colour of the deposit (nigror 3 ) reflects the organic content. Stratificatio does not change, while elasticitas changes to 3-4.

222-213 $\mathrm{cm}$ The boundary at $222 \mathrm{~cm}$ is not as sharp as those below (limes 2). This horizon differs from that below by the inclusion of Grana arenosa. The two components, Turfa herbacea and Grana arenosa, occur in equal proportions. The plant structure of the Turfa herbacea is well preserved, with a homogeneous ground substance; humositas is 1 , indicating an increase in bog-surface wetness. The deposit colour becomes paler due to the addition of the mineral component, dark olive grey (2.5GY 4/1). Stratificatio is 2; it splits somewhat easily into very thin horizontal layers. It has no elasticity (elasticitas 0 ), and nigror is 2 .

213-209 $\mathrm{cm}$ The boundary at $213 \mathrm{~cm}$ is sharp, limes 3 . This narrow band comprises dark olive grey (2.5GY 4/1) Grana arenosa. Physical features include nigror 2, stratificatio 0 , and elasticitas 0 .

209-202 cm A change to finer-grained mineral sediment occurs at a sharp boundary (limes 3) at $209 \mathrm{~cm}$. Sediment of Argilla granosa (Ag) (particles of clay 0.002-0.06 mm) indicates lower-energy transportation of sediment and deposition. The trace of Turfa herbacea of humositas 1 has well preserved plant structure, indicating a continually wet environment. Colour of the deposit and physical properties are unchanged.

202-199 $\mathrm{cm}$ This narrow horizon is demarcated with a sharp lower boundary (limes 3). Sediment grain size decreases to that of Argilla steatodes (As) $(<0.002 \mathrm{~mm}$ ), otherwise components and physical properties remain unchanged.

199-192 cm A less sharp boundary (limes 2) at $199 \mathrm{~cm}$ signals an increase in particle grain size to that of Grana arenosa and G. glareosa, which occur in equal proportions. Colour remains unchanged, but there is a slight tendency towards splitting into very thin horizontal layers (stratificatio 0-1). Other physical properties remain unchanged.

192-184 cm Another conspicuous boundary (limes 2) occurs at $192 \mathrm{~cm}$, where the composition of the sediments changes to a mixture of Argilla granosa to A. steatodes, and 
Grana glareosa minora in the proportions of 3:1. Colour and elasticitas are unchanged. Stratificatio is 0 .

$184-170 \mathrm{~cm}$ Between 184 and $170 \mathrm{~cm}$ is a series of interbedded layers of Grana arenosa, and mixtures of Argilla granosa to A. steatodes, Grana arenosa, and G. glareosa. The colour of all these horizons is dark olive grey $(2.5 \mathrm{GY} 4 / 1)$. Physical properties are stratificatio 0 and elasticitas 0 . The definition of the boundaries between these layers varies: at $184 \mathrm{~cm}$ a sharp boundary (limes 3 ) signals a change to a very narrow band of Grana arenosa; at $182 \mathrm{~cm}$ another sharp boundary (limes 3) marks a change to a horizon of Argilla granosa to A. steatodes and other mineral particles ranging from Grana arenosa to G. glareosa minora in the ratio of $3: 1$; at $178 \mathrm{~cm}$ the boundary is diffuse (limes 1 ) and the composition of the sediments changes to Argilla granosa to A. steatodes (3) and Grana glareosa minora (1); at $175 \mathrm{~cm}$ the boundary is sharp (limes 3 ) and the sediment comprises Grana arenosa; and at $172 \mathrm{~cm}$ the boundary is sharp (limes 3 ) and sediment comprises Argilla steatodes.

$170-157 \mathrm{~cm}$ The boundary at $170 \mathrm{~cm}$ is diffuse (limes 1). Sediments of this thicker horizon are composed of Grana glareosa majora, and a mixture of Grana arenosa to G. saburralia (mineral particles from 0.6 to $2.0 \mathrm{~mm}$ ) and Argilla steatodes to A. granosa in the proportions of $3: 1$. Other properties remain unchanged.

$157-138 \mathrm{~cm}$ A diffuse boundary (limes 1 ) at $157 \mathrm{~cm}$ marks the base of another relatively thick horizon. Sediment comprises Argilla steatodes to A. granosa with a trace of Turfa herbacea of humositas $0-1$. Other properties remain unchanged.

$138-133 \mathrm{~cm}$ The boundary at $138 \mathrm{~cm}$ is diffuse (limes 1) as the deposit becomes increasingly organic. The deposit is characterised as Argilla steatodes to A. granosa and Turfa herbacea of humositas $0-1$, in the proportions of $3: 1$. Other properties remain unchanged.

$133-130 \mathrm{~cm}$ A conspicuous boundary at $133 \mathrm{~cm}$ marks the base of a narrow band of Turfa herbacea of humositas 0-1, and Argilla steatodes in the proportions of 3:1. Grana glareosa minora occurs as a trace. Colour becomes darker (brownish black, 10YR 3/2), and nigror increases to 3 . Other physical properties remain unchanged.

130-126 $\mathrm{cm}$ A diffuse boundary at $130 \mathrm{~cm}$ marks a narrow less organic horizon of Argilla steatodes and Turfa herbacea (humositas 0-1) in the proportions of 3:1. Colour becomes paler again (dark yellowish grey, $2.5 \mathrm{Y} 5 / 2$ ) and nigror reduces to 2 . Other properties remain unchanged.

126-121 cm Another diffuse boundary (limes 1) marks another highly organic horizon of Turfa herbacea (humositas 0-1) and Argilla steatodes in the proportions of 3:1. Colour becomes darker (brownish black, 10YR 3/2) and nigror increases to 3. Other properties remain unchanged. 
$121-116 \mathrm{~cm}$ A diffuse boundary (limes 1) at $121 \mathrm{~cm}$ signals a change in the proportions of the component elements, which are now represented in equal proportions. Colour becomes paler due to the reduction in organic matter (dark yellowish grey, $2.5 \mathrm{Y} 5 / 2$ ), and nigror reduces to 2 . Other properties remain unchanged.

116-110 $\mathrm{cm}$ A sharp boundary (limes 3) occurs at $116 \mathrm{~cm}$, and the deposit becomes much more minerogenic in composition, comprising Grana glareosa majora and G. arenosa in equal proportions with traces of Argilla granosa to A. steatodes and Turfa herbacea (humositas $0-1$ ). Colour of the deposit is now grey (5Y 5/1). Other properties are unchanged.

$110-102 \mathrm{~cm}$ A diffuse boundary (limes 1) at $110 \mathrm{~cm}$ marks a change to Argilla steatodes and Grana glareosa, which are represented in equal proportions. Other properties are the same as in the horizon below.

102-94 $\mathrm{cm}$ Another diffuse boundary (limes 1 ) at $102 \mathrm{~cm}$ marks a change to Argilla steatodes. The colour of the sediment changes to dark olive grey $(2.5 \mathrm{Y} 4 / 3)$, but nigror is unchanged. Elasticitas increases, 0-1. Stratificatio (0) remains unchanged. This horizon contains fine roots from the overlying Turfa herbacea.

94-0 cm A very sharp boundary (limes 4) occurs at the contact between the Argilla steatodes of the horizon below and the Turfa herbacea of this very thick horizon. Humositas decreases upwards from 2 to 0 , indicating increasing bog surface wetness (Blackford 1994). The peat is dark brown (7.5YR 3/3) and nigror is 3 . Elasticitas increases from 3 at the base to 4 at the top of the horizon. Stratificatio (0) remains unchanged.

\subsubsection{Physical properties}

Organic matter, inorganic matter, moisture content, and dry density are plotted against depth and stratigraphy in Figure 5.6. The first three properties are expressed as a percentage of the original wet weight of the sample. The results suggest a division of the sequence into 6 sections (A-F), described below.

Section F, 250-245 cm The sediment in this section is rich in inorganic matter (50.7\%) with a correspondingly high dry density of $1.03 \mathrm{~g} / \mathrm{cc}$. Organic matter is low $(2.0 \%)$ and moisture content is low (47.4\%).

Section $E, 245-213 \mathrm{~cm}$ Inorganic matter declines greatly (1.1\%) at the bottom of this section, but rises again at the top (53.9\%). Dry density has corresponding low $(0.07 \mathrm{~g} / \mathrm{cc})$ and high $(0.90 \mathrm{~g} / \mathrm{cc})$ values. Organic matter is high throughout this section, reaching a maximum of $12.8 \%$, which is almost as high as the highest value of the sequence, but is lower at the bottom and top ( 9.0 and $5.3 \%$ respectively). These high values of organic matter are indicative of peat formation. Moisture content is very high for most of the section, reaching a maximum of $89.9 \%$, but declines to $40.8 \%$ at the top. 


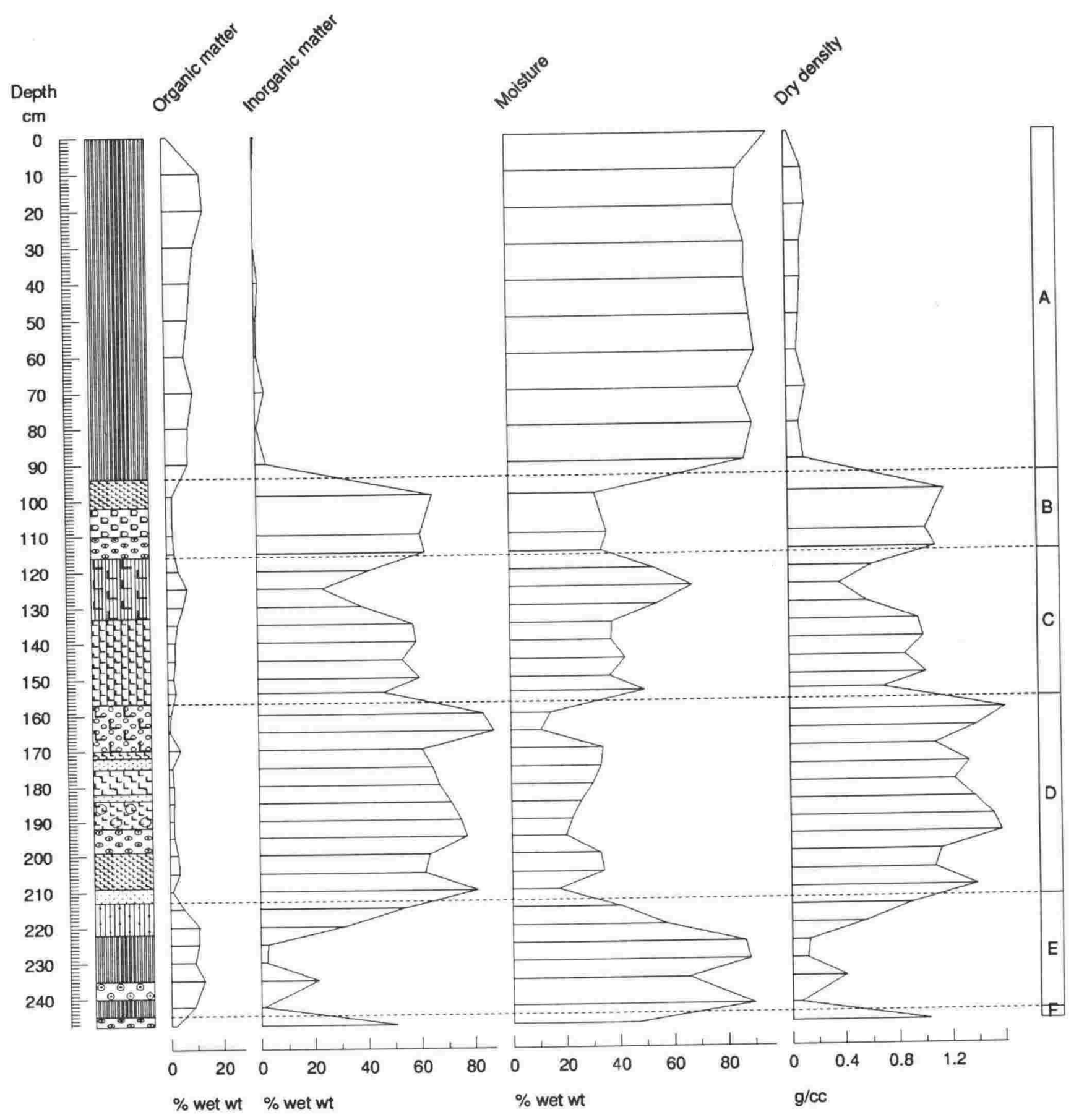

Figure 5.6 Anelia sediment physical properties: sediment type column (Troels-Smith classification 1955), organic matter, inorganic matter, moisture and bulk (dry) density. Sediment sections based on physical properties are indicated on the right. 
Section $D, 213-157 \mathrm{~cm}$ This section is characterised by the maxima of inorganic matter $(61.0-88.0 \%)$ and dry density $(1.08-1.61 \mathrm{~g} / \mathrm{cc})$ of the sequence. Organic matter is low $(0.8-3.8 \%)$ but reaches $4.5 \%$ at $170 \mathrm{~cm}$. Moisture content values are minima of the sequence (11.1-34.5\%). The characteristics of this section indicate deposition of allochthonous mineral material.

Section $C, 157-116 \mathrm{~cm}$ This section is characterised by intermediate values for all components. Organic matter ranges from $2.4-7.7 \%$, inorganic matter ranges from $24.2-60.4 \%$, moisture content ranges from 37.3-68.1\%, and dry density ranges from $0.38-1.02 \mathrm{~g} / \mathrm{cc}$. Deposition of allochthonous mineral material steadily decreases while peat formation increases.

Section B, 116-94 cm Inorganic matter (61.0-65.6\%) and dry density (1.10-1.20 g/cc) increase in these three samples, while organic matter (2.3-3.0\%) and moisture content decrease (32.1-36.5\%). The sediments of this section represent an influx of allochthonous mineral material.

Section $A, 94-0 \mathrm{~cm}$ This section is characterised by high organic-matter values and very high moisture content. Organic matter has consistently high values (with the exception of the surface sample, $1.8 \%$ ) of $7.3-11.4 \%$, reaching maxima of 15.2 and $14.1 \%$ at 20 and $10 \mathrm{~cm}$ respectively. Moisture content always exceeds $84.8 \%$, and the surface sample is almost entirely water $(97.6 \%)$. Inorganic matter declines to zero in three samples, while the maximum value reached is only $3.8 \%$. Dry-density values are very low throughout this section, never exceeding $0.15 \mathrm{~g} / \mathrm{cc}$. Initially deposition of allochthonous mineral matter declines sharply, then continues to decline until it ceases and deposition in the wetland is composed entirely of in situ peat formation.

\subsection{Chronostratigraphy}

\subsubsection{Radiocarbon dating}

An attempt was made to establish a chronology of the Anelia sequence based on radiocarbon ages of organic matter contained within the sediments. Intervals of the core containing considerable amounts of peat were selected to provide a higher percentage of carbon per sample, thus providing more precise radiocarbon ages, and also avoiding contamination of older soil carbon from alluvial inwash (Tolonen 1980). Therefore dated samples are not evenly spaced.

Radiocarbon dating results are tabulated in Table 5.1. Conventional radiocarbon ages are shown with an analytical error of one standard deviation. These ages have been calibrated by tree-rings (Appendix IV). 


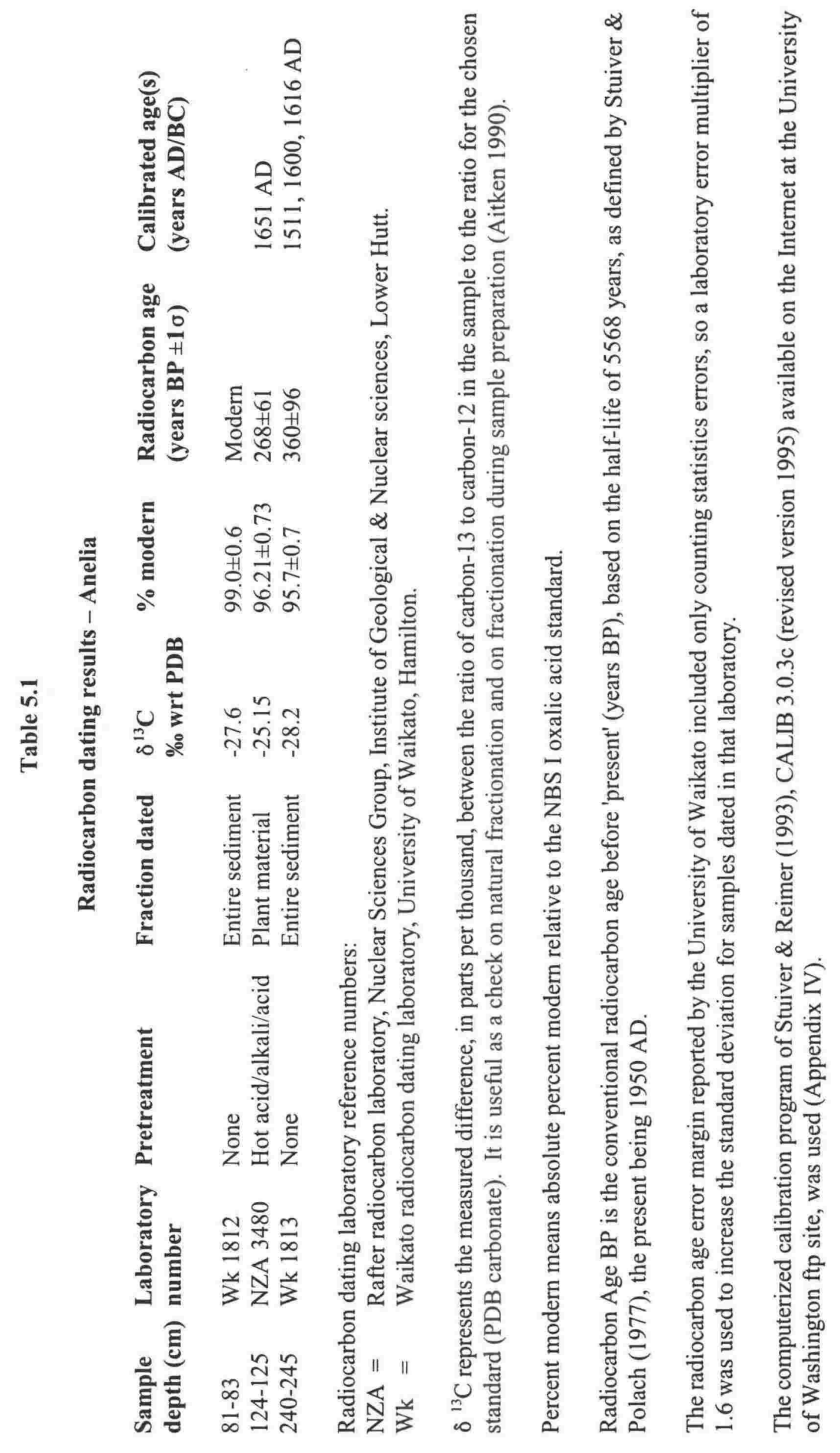


Initially, the basal and the uppermost samples were dated in the University of Waikato radiocarbon dating laboratory with the liquid scintillation counting method. Dated samples received no pretreatment. On receipt of the results of these samples the intermediate sample was submitted to the Rafter laboratory, Institute of Geological and Nuclear Sciences, for direct dating using accelerator mass spectrometry. Recognizable plant remains were removed for dating and given a pretreatment of alternating washes of hot acid, alkali, and acid. The radiocarbon ages of this sample are concordant with those of the first pair of samples dated and the ratio of carbon- 13 to carbon- $12\left(\delta^{13} \mathrm{C}\right)$ of all samples dated is consistent with average values for that of wood, charcoal, and peat (and the standard value used in radiocarbon age determinations) (Olsson 1986:286; Aitken 1990:63), thus the possibility of laboratory error or contamination, e.g. contamination within the measurement system or during sample preparation is minimal. Furthermore, these samples were collected in the same way as those of the Gomara site, which are considered to accurately reflect the age of the sediments of that sequence.

Despite these precautions, radiocarbon ages obtained for this wetland sequence are problematic. At $68 \%$ confidence level the basal and intermediate ages are not statistically different (Aitken 1990); nor are the intermediate and uppermost ages. Furthermore, the uppermost age could represent any calendar date in the last 250 years. Also at $68 \%$ level of confidence, the intermediate calibrated calendar date at $125-124 \mathrm{~cm}$ has four separate age ranges, the older two overlapping the calibrated calendar age range of the lowest dated sediments.

\subsubsection{Age/depth plot}

Mean sediment-accumulation rates were calculated in a manner similar to that of Chapter 4, that is, by linearly interpolating between contiguous calibrated dates (Figure 5.7).

Accumulation rates of clastic sediment can vary greatly depending upon type of material deposited and deposition regime. The clastic sediments of the lower part of the sequence indicate rapid episodic deposition, such as 'instantaneous' flood deposits separated by only partially decomposed peat. This suggests a relatively rapid accumulation rate, and therefore the high rate of $1.35 \mathrm{~cm} /$ year is considered possible.

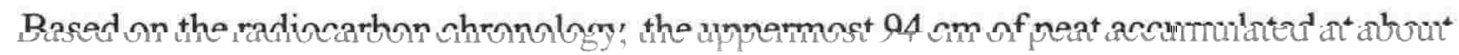
$0.37 \mathrm{~cm} /$ year. The peat decreases in decomposition from moderate to weak towards the surface, indicating relatively rapid accumulation due to the coarseness of incompletely decomposed peat and high moisture content. Also there is a tendency towards higher accumulation rates for younger peat layers due to lessening autocompaction (Aaby 1986). Berglund (1986b:119) has indicated that peat growth in Scandinavian bogs typically ranges 


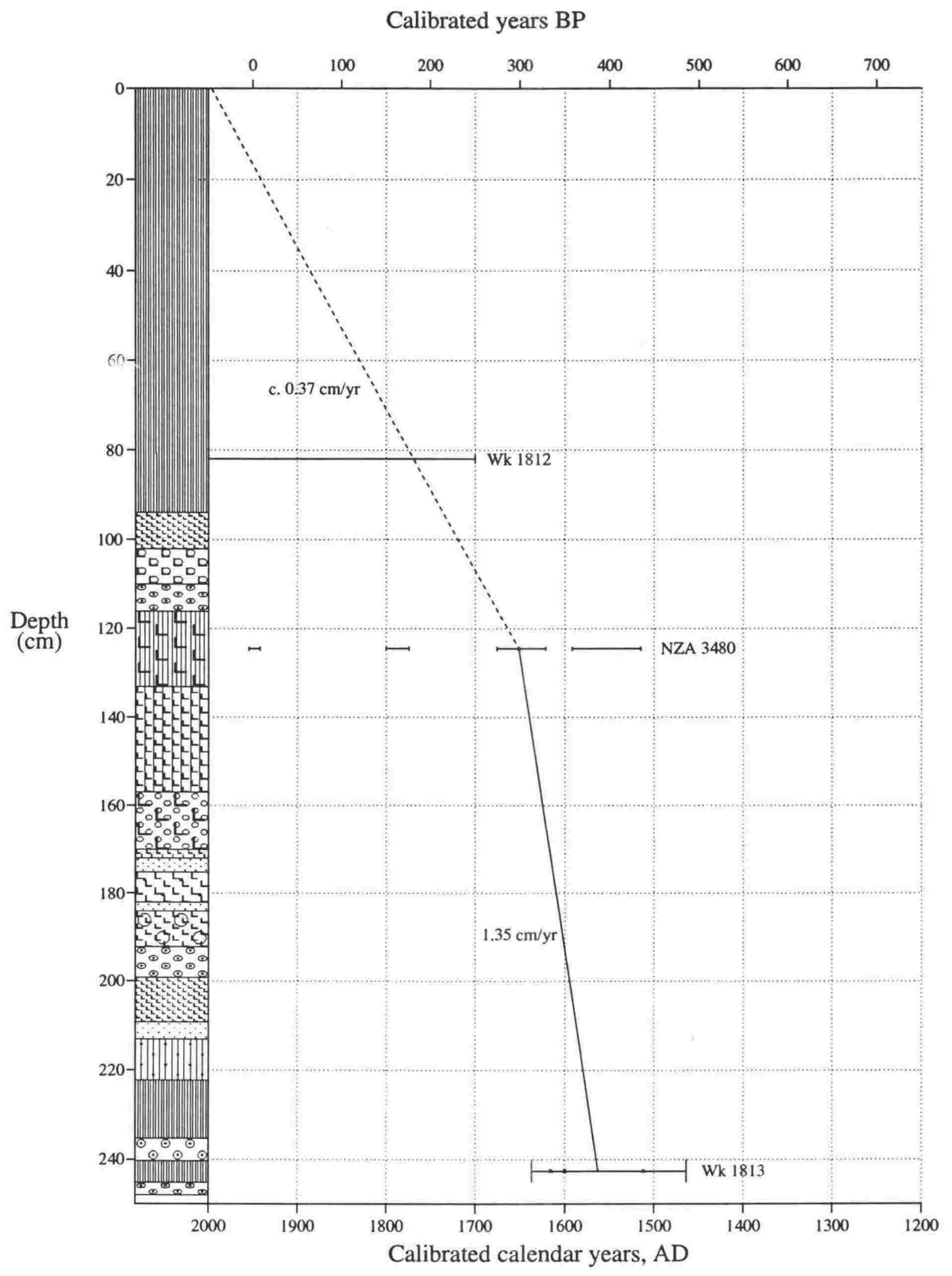

Figure 5.7 Age-depth plot. Calibrated radiocarbon ages (Table 5.1, Appendix IV) plotted against depth of sediment from Anelia. Length of cross-arms indicates uncertainty $( \pm 1 \sigma)$ of age measurement (horizontal) and thickness of dated sediment (vertical). Most probable dates are indicated by crosses: the calibrated calendar date at $124.5 \mathrm{~cm}$ depth has been joined to the median of the calibrated dates at $242.5 \mathrm{~cm}$, and the corresponding sediment accumulation rate is shown. A rate has also been calculated for the upper part of the section. 
between 0.02 and $0.10 \mathrm{~cm} /$ year, but Webb \& Webb (1988) indicated a wide range from 0.008 to $0.408 \mathrm{~cm} /$ year in deposition rate of organic sediments (detritus and peat) in mid-latitude North America; the Anelia site is in the upper part of this range.

A check on the duration of deposition of the Anelia sequence can be made with pollenconcentration figures. Influx rates have been calculated for the Gomara sequence (Chapter 4). Although the sediment types at Gomara differ from Anelia, the catchment size and terrestrial vegetation represented are in many ways similar, being a mosaic of beech, pine, and pasture. Applying the Gomara mean terrestrial pollen influx rate of 14748 grains $/ \mathrm{cm}^{2} /$ year to the Anelia sequence, the mean Anelia terrestrial pollen concentration of 19625 grains $/ \mathrm{cm}^{3}$ represents a deposition rate of $0.75 \mathrm{~cm} /$ year (or $1.33 \mathrm{yr} / \mathrm{cm}$ ). This leads to a duration of deposition of 330 years for the $250 \mathrm{~cm}$ sequence, in good agreement with the basal radiocarbon date.

A general chronology for the site based on radiocarbon dates is therefore accepted. Because of calibration uncertainties and the likelihood that the course of the interpolated age/depth function in the lower part of the sequence is complicated by irregular sedimentation, pollen influx rates have not been calculated. The timescale derived for this sequence should be regarded as tentative.

\subsection{Pollen analysis results}

\subsubsection{Introduction}

Relative frequency as well as concentration data are used to interpret the vegetation history of the Anelia area. Concentration data are not converted to influx rates because of the uncertainty of the reliability of the reported radiocarbon ages and erratic deposition of deposits. Only the exotic marker grain (tracer) method of calculating palynomorph concentrations was used for this sequence. Separate diagrams are drawn for local (wetland) taxa, extra-local and regional (terrestrial) taxa, and microscopic charcoal. Data used to calculate pollen and spore concentrations, all pollen and spore types counted, and charcoal abundances are tabulated in Appendix XIII.

\subsubsection{Pollen preservation/pollen abundance}

Palynomorph preservation is variable and closely related to lithology. The principal modes of deterioration of grains are breakage and degradation (thinning of exine) (Delcourt \& Delcourt 1980). There is a clear correlation between those spectra with the highest proportion of degraded and broken grains, and mineral sediments of larger particle size, that is, spectra at $160-210 \mathrm{~cm}$. Grains from peat are usually better preserved than those from the mineral sediments except where the surface of the peat has been intermittently dry, then 
oxidation of the exine occurs. Some palynomorphs from the humified peat have suffered from oxidation. Indeterminate grains are usually less than $3 \%$ and this is exceeded in only four spectra. These are from the lower (more humified) part of the upper horizon of peat -40 to $90 \mathrm{~cm}$. Unknown grains are rare, reaching $0.2 \%$ in only two spectra.

Concentration of total terrestrial pollen and spores fluctuates, but in general is highest in the lower part of the sequence (below $115 \mathrm{~cm}$ ), being usually more than $15 \times 10^{3}$ grains $/ \mathrm{cm}^{3}$ (Figure 5.12). There is no constant correlation between dry density of the sediments and terrestrial pollen and spore concentrations. Maximum concentrations are at $245-215 \mathrm{~cm}\left(23-52 \times 10^{3}\right.$ grains $\left./ \mathrm{cm}^{3}\right)$ and $155-115 \mathrm{~cm}\left(15-37 \times 10^{3}\right.$ grains $\left./ \mathrm{cm}^{3}\right)$ where sediments comprise mostly moderately humified peat and moderately-weakly humified peat mixed with fine grained sediment. Minimum concentrations occur at $210 \mathrm{~cm}$ in sand $\left(7 \times 10^{3}\right.$ grains $\left./ \mathrm{cm}^{3}\right), 190 \mathrm{~cm}$ in gravelly mud $\left(8 \times 10^{3}\right.$ grains $\left./ \mathrm{cm}^{3}\right), 165-160 \mathrm{~cm}$ in muddy sandy gravel $\left(4 \times 10^{3}\right.$ grains $\left./ \mathrm{cm}^{3}\right), 110 \mathrm{~cm}$ in muddy sandy gravel $\left(9 \times 10^{3}\right.$ grains $\left./ \mathrm{cm}^{3}\right), 50-20 \mathrm{~cm}$ in moderately-weakly humified peat $\left(7-10 \times 10^{3}\right.$ grains $\left./ \mathrm{cm}^{3}\right)$, and at the surface in unhumified peat $\left(5 \times 10^{3}\right.$ grains $\left./ \mathrm{cm}^{3}\right)$.

There is a an inverse correlation between dry density of sediment and wetland (local) pollen concentration; where values of dry density are lower, pollen concentration is higher. Concentration of wetland pollen and spores ranges from $1-20 \times 10^{3}$ grains $/ \mathrm{cm}^{3}$, being highest in spectra from moderately humified peat and moderately-weakly humified peat mixed with fine grained sediment $(225-215,155-115$ and $90-70 \mathrm{~cm}$, where concentrations usually range from 7-20 × $10^{3}$ grains $/ \mathrm{cm}^{3}$ ) and, in general, lowest in minerogenic deposits of sand, muddy sandy gravel, and clay (Figure 5.9). Lowest concentrations are recorded in the basal sample, at $210,165,160$, and $100 \mathrm{~cm}$, and the surface sample where concentrations range from $1-2 \times 10^{3}$ grains $/ \mathrm{cm}^{3}$.

\subsubsection{Wetland pollen analysis}

\subsubsection{Pollen diagram construction}

Pollen and spore relative frequencies are presented graphically in Figure 5.8 and concentration data in Figure 5.9. Style of the diagrams is as for Gomara site (Chapter 4). Pollen and spore types are arranged according to the degree of wetness of habitat of plant communities from which they derive. Habitat groups are the same as those in Chapter 4. Within these groups, types are arranged in order of appearance. The pollen sum on which the relative frequencies are based includes all wetland pollen and spore taxa. Relative frequencies of $0.2 \%$ or less are indicated with a dot.

A summary diagram is shown on the right of the relative frequency diagram showing the ratio of emergent types to aquatic types. A curve of palynological richness (rarefaction 


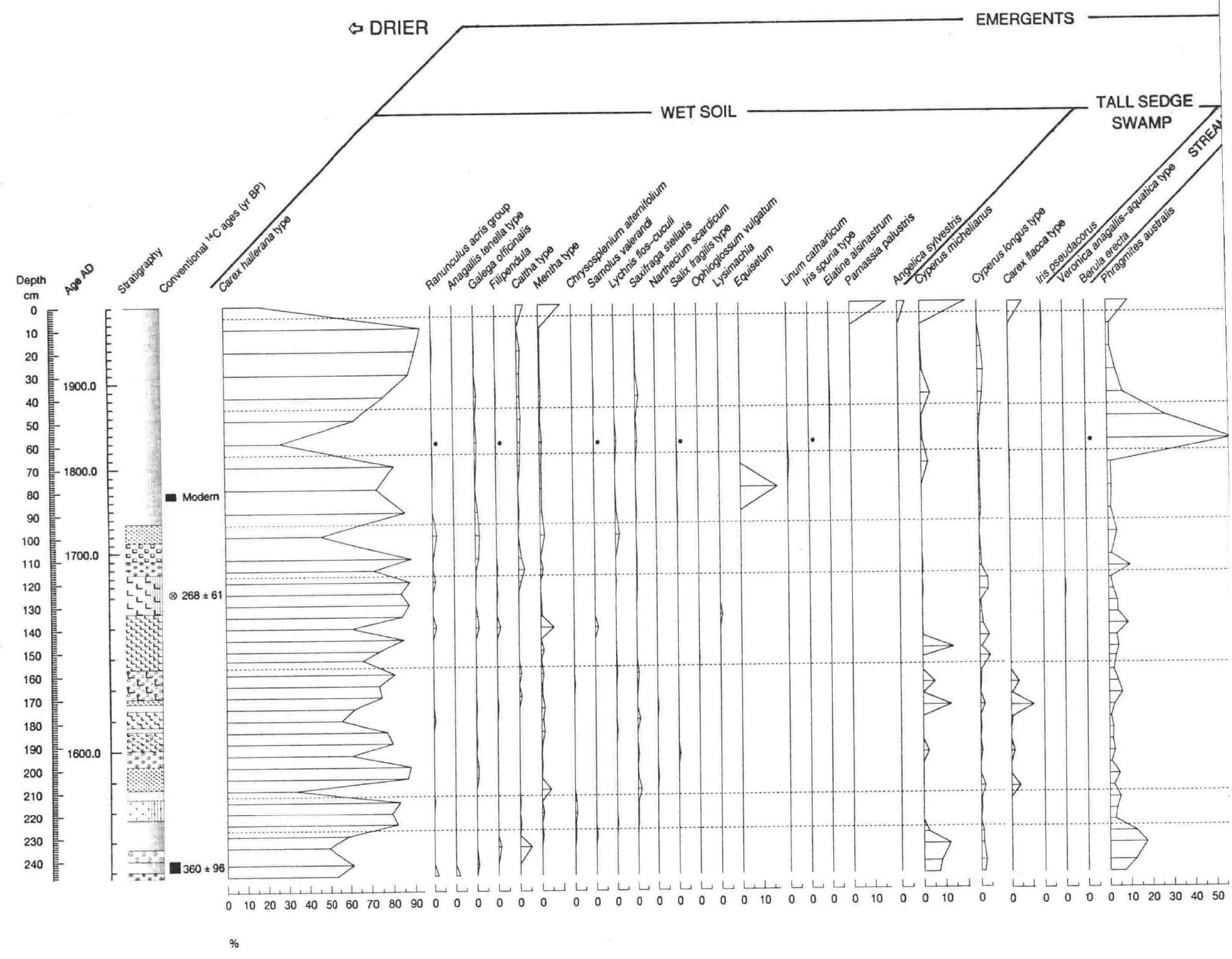

Figure 5.8 Anelia - relative frequency pollen diagram, wetland taxa (pollen sum is total wetland taxa) 


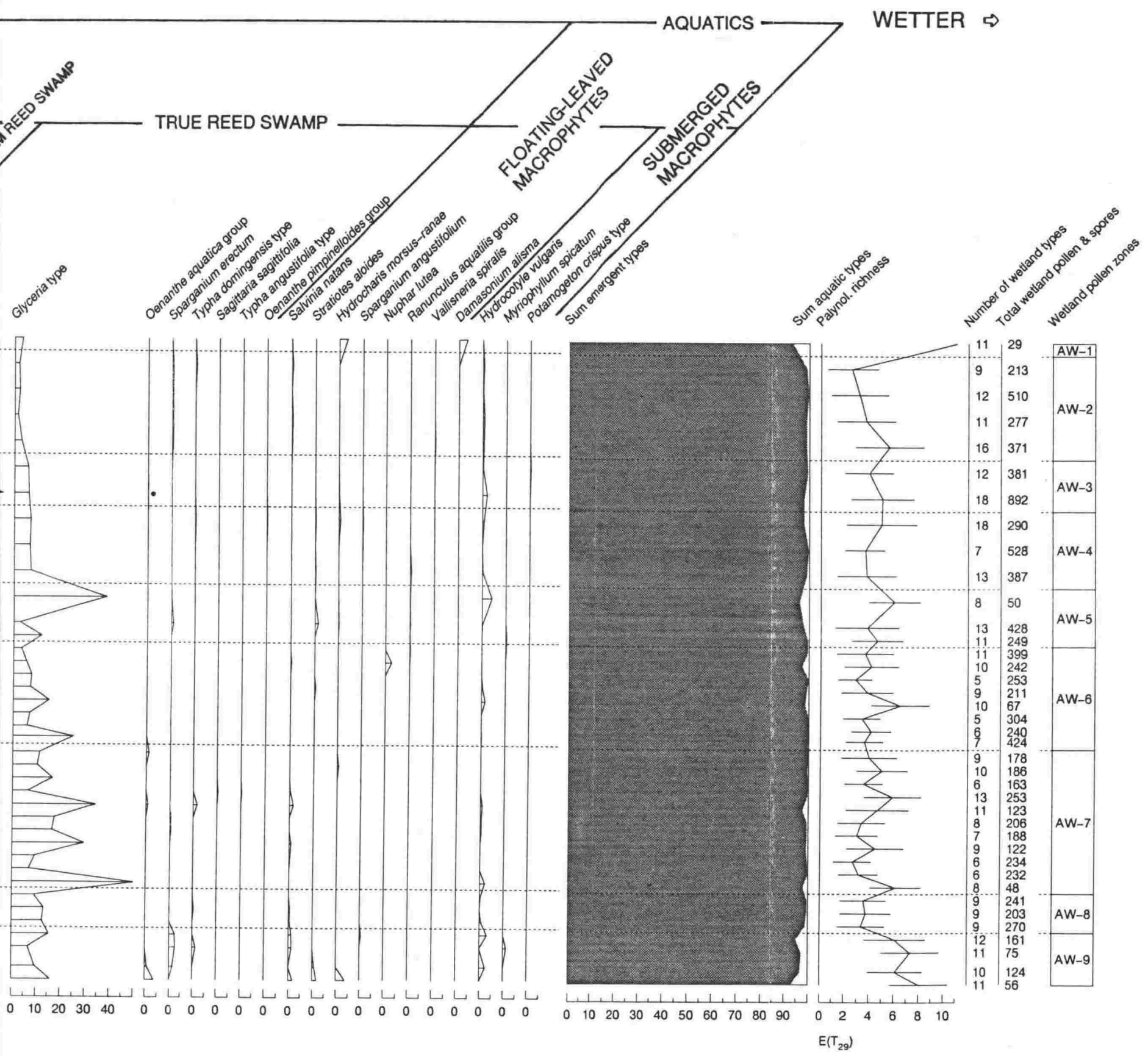

Figure 5.8 


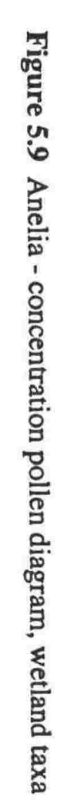

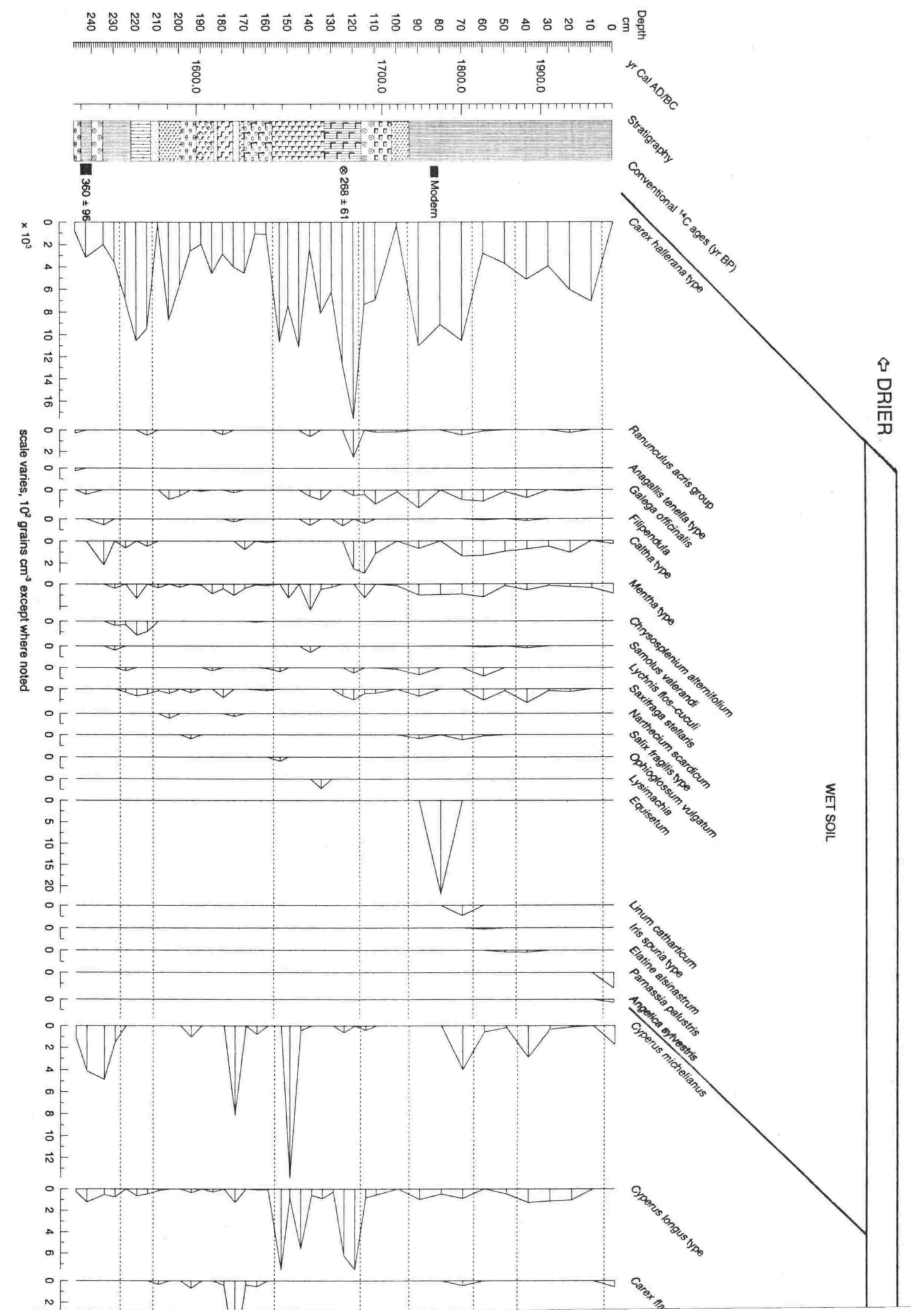




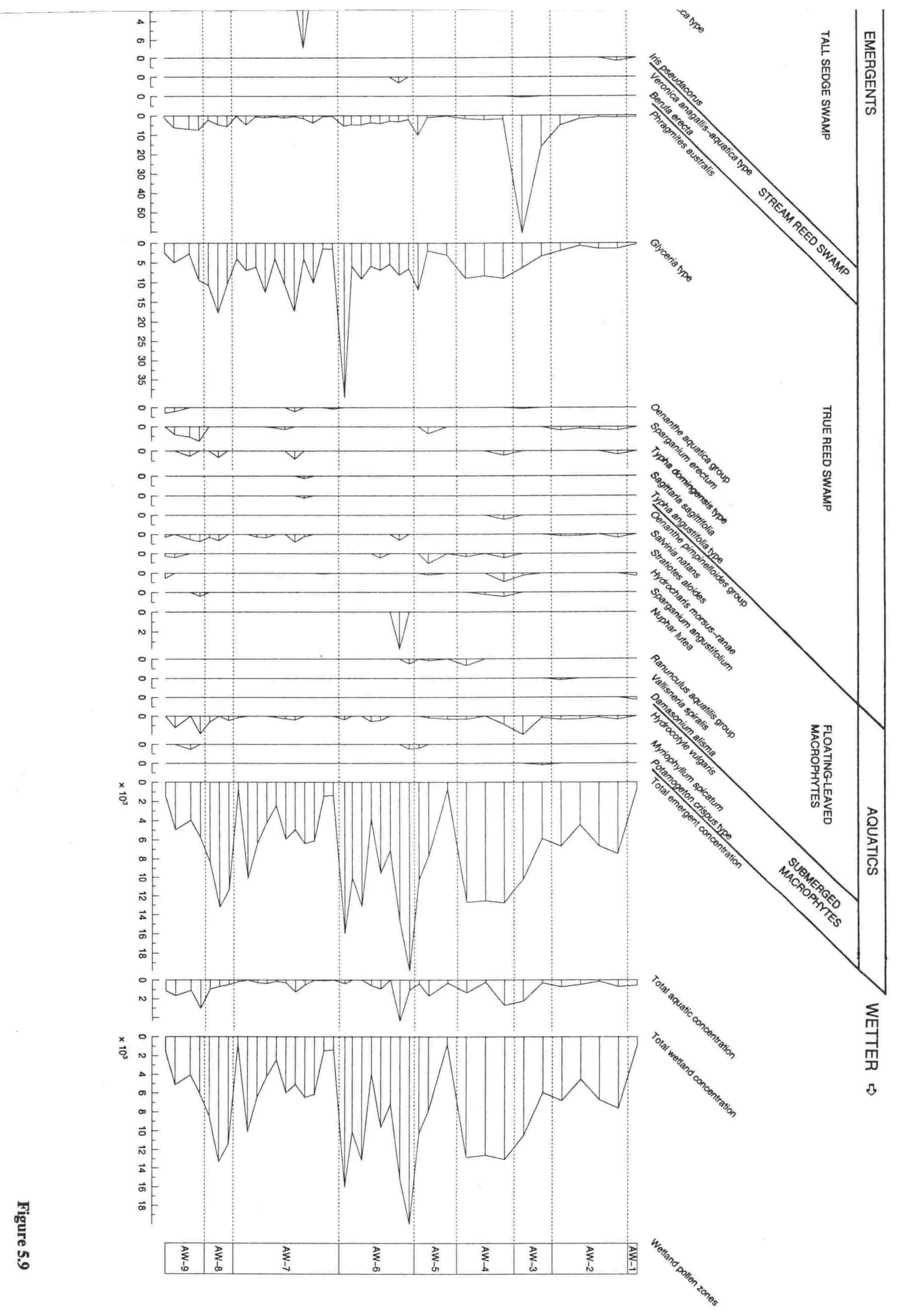


analysis) follows (Birks \& Line 1992; Bennett 1994); rarefaction analysis is based on the smallest total count of the wetland pollen and spores, 29 at the surface of the bog. The number of wetland types (not normalized for unequal pollen sums) in each spectra is shown next. Lastly, the total of all wetland pollen and spores counted is given.

Many weeds and ruderals were observed growing on the drier areas of the bog and on the marginal fen. These species are included in the Cirsium type, Dactylis type, Dianthus type, Helleborus, Plantago lanceolata type, Pteridium aquilinum, Scrophularia type, Scutellaria type, Trifolium repens type, Urtica dioica, U. pilulifera type, and U. urens type. These pollen and spore types have been included in the terrestrial pollen diagram as they are typically terrestrial in habitat. They are all included in the habitat group of 'herbs of open ground' with the exception of Helleborus, a genus typically of woodland herbs.

\subsubsection{Pollen record of wetland plant communities}

The wetland plant communities are represented by a few dominant pollen and spore types. Carex hallerana type is dominant throughout the sequence, with the exception of the spectrum at $60 \mathrm{~cm}$ where Phragmites australis is dominant. A maximum of only 18 pollen and spore types were identified in any spectrum; average is 10 . Estimates of palynological richness by rarefaction analysis range from 2-11 expected types.

Emergent communities The wetland pollen and spore sequence is dominated by types that derive from emergent plant communities. Pollen and spore types belonging to the 'wet soil' habitat group are most numerous; Carex hallerana type being the dominant type. Phragmites australis and Glyceria type of the 'true-reed swamp' group also play a prominent role.

See Chapter 4 for a discussion of the classification of wetland types into the ecological groups. Those types not included in the wetland pollen diagram of Chapter 4 , or that require further elucidation are discussed below.

Salix fragilis type includes many species; they belong to the order of Salicetalia purpureae, the willow communities of lowland flood plains (Ellenberg 1988). Only Lysimachia vulgaris of the five species included in the pollen type Lysimachia is mentioned by Ellenberg (1988), who places it in the order Molinietalia, but the other species in this type grow in similar habitats of damp ground (Polunin 1988). Parnassia palustris belongs to the vegetation class Scheuchzerio-Caricetea nigrae (fuscae), a small-sedge vegetation of intermediate mire and swamp swards. The type Equisetum includes a number of species that grow in a variety of habitats from wet meadows, stream banks, flood plains to reed swamps (Ellenberg 1988). 
Iris spuria type includes Iris spuria, and several other species that may belong to this pollen type (Iris reichenbachii, I. sintenisii, I. suaveolens). These species are not classified in Ellenberg (1988). Iris spuria grows in wet places, but the other species grow in dry habitats (Polunin 1988). Narthecium scardicum and Linum catharticum are also not classified by Ellenberg (1988), but they are known to grow in marshy places in the Pindos Mountains (Strid 1989; Strid \& Tan 1991).

Iris pseudacorus belongs to the tall-sedge-swamp community of the order Phragmitetalia. Oenanthe aquatica group also belongs to the order Phragmitetalia, but to the community of the true-reed swamp. The species included in the Oenanthe pimpinelloides group (Oenanthe pimpinelloides and possibly Oenanthe tenuifolia) are not classified by Ellenberg (1988). The habitat of Oenanthe pimpinelloides is not known, but $O$. tenuifolia grows in marshes.

Aquatic communities Pollen and spores of aquatic types occur sporadically throughout the sequence. Floating-leaved communities are better represented than submerged communities. Floating-leaved communities often grow in pools of water on bog surfaces.

Eight types that include species of floating-leaved communities are represented. Three of these, Hydrocharis morsus-ranae, Stratiotes aloides, and Salvinia natans belong to Ellenberg's (1988) Lemnetalia order of floating communities of waters more or less rich in nutrients. Nuphar lutea and most of the species of the Ranunculus aquatilis group belong to the order Potamogetonetalia of rooted water plant communities, while Sparganium angustifolium belongs to the shore hairgrass shallow-water swards and related communities, order Littorelletalia (Ellenberg 1988). Vallisneria spiralis and Damasonium alisma are not classified by Ellenberg (1988). The former grows submerged in still nutrient-rich water, but its flowers float to the surface when open and pollination occurs at the water surface (Willis 1951; Haslam et al. 1975; Polunin 1988). The latter grow in still shallow water usually as a floating plant, but sometimes as a submerged plant (Haslam et al. 1975; Polunin 1988).

Pollen types representing the submerged macrophyte group are: Hydrocotyle vulgaris, Myriophyllum spicatum, and Potamogeton crispus type. Representation of submerged macrophytes is low and only Myriophyllum spicatum is exclusively submerged (Ellenberg 1988).

\subsubsection{Wetland (local) pollen assemblage zones}

Wetland pollen diagrams are divided into 9 local pollen assemblage zones AW-1 to AW-9, based mostly on relative frequency data. These are defined below.

Zone $A W-9,250.0-227.5 \mathrm{~cm}$ This basal zone is characterised by high relative frequencies of Carex hallerana type (49.3-61.3\%), Phragmites australis (7.1-17.3\%), Cyperus 
michelianus (7.1-12.0\%, except the top spectrum at 2.5\%), and Glyceria type (6.7-16.1\%). The only other emergent type that occurs continuously is Cyperus longus type $(2.4 \%$ maximum). Sparganium erectum occurs in all spectra except the basal (2.7\% maxima). Caltha type occurs at $235 \mathrm{~cm}$ only, at $5.3 \%$. No other emergent type exceeds $1.8 \%$.

Aquatic types occur in all spectra and total relative frequency reaches $7.2 \%$ in the basal spectrum, which is the highest for the sequence. All aquatic types occur sporadically. Highest frequency of an aquatic type is that of Hydrocharis morsus-ranae, which reaches $3.6 \%$ in the basal spectrum. Hydrocotyle vulgaris occurs sporadically and reaches a maximum of $3.1 \%$ at $230 \mathrm{~cm}$. Several other aquatic types occur at more than 1.0\%: Myriophyllum spicatum $(1.3 \%$ at $235 \mathrm{~cm})$; Salvinia natans $(1.2,1.3$ and $1.8 \%)$; and Stratiotes aloides $(0.8$ and $1.8 \%)$.

Zone $A W-8,227.5-212.5 \mathrm{~cm}$ The boundary with the preceding zone is marked by an increase in the relative frequency of Carex hallerana type, the disappearance of Cyperus michelianus and Sparganium erectum, and a decline in Phragmites australis. Carex hallerana type is the dominant type, at around $80 \%$. Glyceria type is important $(9.1-13.3 \%)$. Phragmites australis declines to $2.6 \%$, then increases steadily to $5.0 \%$. No other emergent type exceeds $1.0 \%$.

Aquatic types occur in all spectra; maximum total frequency is only $1.1 \%$ at $225 \mathrm{~cm}$. No individual type exceeds $0.7 \%$.

Zone $A W-7,212.5-157.0 \mathrm{~cm}$ The boundary with the preceding zone is marked by a dramatic increase in the relative frequency of Glyceria type and a decline in Carex hallerana type. This zone is characterised by alternately fluctuating relative frequencies of Carex hallerana type and Glyceria type. Frequencies of Carex hallerana type are generally high (56.1-88.0\%, except the basal spectrum of 33.3\%). Glyceria type fluctuates between 6.3 and $50.0 \%$. Phragmites australis occurs continuously at 0.8-6.1\%. Carex flacca occurs sporadically, but reaches a maximum of $10.7 \%$ at $175 \mathrm{~cm}$. Cyperus michelianus also occurs sporadically, recording significant relative frequencies (maximum $12.6 \%$ at $175 \mathrm{~cm}$ ). Cyperus longus type and Saxifraga stellaris also occur sporadically and reach a maxima of $2.1 \%$ at $210 \mathrm{~cm}$. Mentha type occurs sporadically (maximum $4.2 \%$ at $210 \mathrm{~cm}$ ). No other emergent type exceeds $1.6 \%$.

Aquatic types occur in only 8 of the 11 spectra; total frequency does not exceed $2.4 \%$. Only Hydrocotyle vulgaris and Salvinia natans exceed $0.5 \%$, reaching $2.1 \%$ at $210 \mathrm{~cm}$ and $1.6 \%$ at $180 \mathrm{~cm}$, respectively.

Zone $A W-6,157.0-117.5 \mathrm{~cm}$ The boundary with the preceding zone is indistinct, but marked by an increase in the relative frequency of Cyperus longus type. The zone is characterised by an increase in Carex hallerana type (mostly more than $83.0 \%$ ) and Cyperus longus (mostly more than $1.5 \%$, with maximum of $4.7 \%$ ). Phragmites australis increases 
slightly (1.0-9.0\%, but usually more than $3.0 \%)$. Glyceria type fluctuates, but declines $(24.8 \%$ in the bottom spectrum and $3.3 \%$ in the top). Cyperus michelianus occurs sporadically at less than $0.5 \%$, except at $150 \mathrm{~cm}$ where $13.8 \%$ is recorded. Mentha type also occurs sporadically at low relative frequencies with the exception of $6.0 \%$ at $140 \mathrm{~cm}$. No other emergent type exceeds $1.5 \%$.

Aquatic types occur in 5 of the 8 spectra; total frequency does not exceed $2.9 \%$. Only two types exceed $0.5 \%$ : Hydrocotyle vulgaris reaches $1.5 \%$ at $140 \mathrm{~cm}$ and Nuphar lutea reaches $2.5 \%$ at $125 \mathrm{~cm}$.

Zone $A W-5,117.5-95.0 \mathrm{~cm}$ This is a narrower zone comprising only three spectra. The interval between the two upper spectra has been increased from 5 to $10 \mathrm{~cm}$. The boundary with the preceding zone is marked by a decline of Carex hallerana type and Cyperus longus type, and an increase in Phragmites australis and Glyceria type. Carex hallerana type continues to be the dominant type (88.6-46.0\%). The distinct character of this zone is more apparent in the concentration diagram due to a dramatic decline in the concentration of Carex hallerana type. In the uppermost spectrum where concentration and relative frequency of Carex hallerana type is most reduced Glyceria type increases greatly $(38.0 \%)$. The frequency of Phragmites australis is also low where Glyceria type increases, but in the concentration diagram both follow similar curves with lower concentration values in the upper two spectra.

Other characteristics of this zone are the almost complete disappearance of Cyperus longus type (maximum $0.8 \%$ ) and of Cyperus michelianus, which occurs in only one spectrum at only $0.4 \%$. Caltha type becomes more prominent, recording 2.8 and $1.4 \%$ in two spectra. No other emergent type exceeds $2.0 \%$.

Aquatic types occur in all spectra; total frequencies are $0.4,2.0$, and $4.0 \%$. Only two types exceed $0.4 \%$ : Stratiotes aloides reaches $1.4 \%$ at $110 \mathrm{~cm}$ and Hydrocotyle vulgaris reaches $4.0 \%$ at $100 \mathrm{~cm}$.

Zone $A W-4,95.0-65.0 \mathrm{~cm}$ The boundary with the preceding zone is marked by an increase of Carex hallerana type, a slight decline in Phragmites australis and a more pronounced decline in Glyceria type.

This zone is characterised by relative frequencies of Carex hallerana type that are generally higher and fluctuate less than those in the zone below (72.9-86.0\%), and lower relative frequencies of Phragmites australis (1.3-1.7\%) and Glyceria type (6.6-7.0\%). Equisetum occurs in the centre of the zone at $80 \mathrm{~cm}$ where it reaches $17.4 \%$. Cyperus michelianus occurs in the uppermost spectrum only, at $3.1 \%$. No other emergent type exceeds $1.3 \%$.

Aquatic types occur in all spectra; total frequencies are only $1.1,0.2$, and $2.0 \%$. No individual type exceeds $0.7 \%$. 
Zone $A W-3,65.0-45.0 \mathrm{~cm}$ Only two spectra comprise this narrow zone. The boundary with the preceding zone is marked by a sharp decline in Carex hallerana type and a very dramatic increase of Phragmites australis. The zone is characterised by low relative frequencies of Carex hallerana type (26.9 and 62.7\%), very low relative frequencies of Cyperus michelianus (0.6 and 0.3\%), and high frequencies of Phragmites australis (58.4 and $27.3 \%$ ). Glyceria type remains almost unchanged at 6.1 and $5.8 \%$. Caltha type has a continuous curve, but records only 1.2 and $1.6 \%$. No other emergent type exceeds $1.1 \%$.

Aquatic types occur in both spectra; total frequencies of the spectra are only 0.9 and $2.1 \%$. One individual type exceeds $0.3 \%$; Hydrocotyle vulgaris reaches $1.9 \%$ at $60 \mathrm{~cm}$.

Zone $A W-2,45.0-5.0 \mathrm{~cm}$ Four spectra comprise this zone. The boundary with the preceding zone is marked by an increase of Carex hallerana type, a slight increase of Cyperus longus, and a decrease of Phragmites australis and Glyceria type. This zone is characterised by high relative frequencies of Carex hallerana type (76.3-93.9\%), increased frequencies of Cyperus longus (1.6-2.5\%, except in the uppermost spectrum where it disappears), declining frequencies of Phragmites australis (1.4-7.3\%), and low frequencies of Glyceria type (1.4-3.0\%). Caltha type persists at 1.1-1.6\%, until it disappears in the uppermost spectrum. Cyperus michelianus declines from $4.3 \%$ in the basal spectrum to zero in the uppermost. No other emergent type exceeds $1.9 \%$.

Aquatic types occur in all spectra; total frequencies are $1.4,1.1,0.2$, and $1.0 \%$. No individual type exceeds $0.7 \%$.

Zone $A W-1,5.0-0 \mathrm{~cm}$ Only one spectrum comprises this narrow zone. The boundary with the preceding zone is marked by a sharp decline in the relative frequency of Carex hallerana type (17.2\%); the appearance of Parnassia palustris $(17.2 \%)$; an increase in the relative frequencies of Mentha type (10.3\%), Cyperus michelianus (20.7\%), Carex flacca type (6.9\%), and Phragmites australis (10.3\%); and an increase in the relative frequencies of the aquatics Hydrocharis morsus-ranae (3.4\%) and Damasonium alisma $(3.4 \%)$. The emergents Angelica sylvestris (3.4\%), Caltha type (3.4\%), and Glyceria type (3.4\%) are also recorded in this spectrum. No other emergent or aquatic types are recorded.

\subsubsection{Sedimentary and local vegetation history}

Stratigraphic data indicate that the wetland formed in a hollow of a ravine, a typical landscape feature of this area. Broadening of the ravine and reduction in stream gradient caused reduction in velocity of spring-stream water flow, and deposition of sediments and formation of peat. The landform may owe its origin to tectonic movements associated with the fault upslope (Section 5.2.3). 
The wetland consists of a thick upper horizon of up to $110 \mathrm{~cm}$ of fibrous peat, which is thickest in the centre of the wetland. At the southern end, the lower part of the peat is mixed with gravel, inwash from the stream feeding the wetland, but to the north the peat is homogeneous. The peat layer is underlain by stratified clay, silt, sand, and fine gravel with peaty horizons, the oldest sediments recovered being dated at c. $1560 \mathrm{AD}$. This lower stratified unit is interpreted as a combination of fan-like deposits of the stream, stream-bed deposits, and the formation of peat on waterlogged deposits; as transport energy of the stream varied and the stream channel(s) location changed, so varied sediments were deposited across the wetland, with mire vegetation establishing itself on the stream margins. After c. $1750 \mathrm{AD}$ peat formation became general, with a permanently marginal stream channel.

In the pollen analysed core, deposits above $94 \mathrm{~cm}$ comprise homogeneous peat, while those below $94 \mathrm{~cm}$ are very heterogeneous and comprise inorganic sediments that alternate or are mixed into peat horizons. These deposits represent periods of run-off and erosion of the valley catchment above the wetland site manifest by minerogenic sediments, and periods of greater landscape stability manifest by organic deposits when peat formation occurred on the wetland. Sediments of sand and gravel indicating high energy sediment transportation occur at 250-245, 240-235, 222-209, 199-157, 133-130, and 116-102 cm. Peat formation occurs at 245-213 (between 240-235 muddy, sandy gravel is washed in and from $222 \mathrm{~cm}$ sand is washed in), 209-199 (trace of peat with fine grained sediments), 157-110 (varying proportions of peat and minerogenic sediments), and $94-0 \mathrm{~cm}$. Accumulation of these deposits was erratic and many of the boundaries between deposits of differing composition are conspicuous indicating rapid changes in deposition processes, and perhaps erosion of wetland deposits.

There is very little apparent variation in the local wetland vegetation. It is dominated throughout the sequence by sedges of the Carex hallerana type of the 'wet soil' habitat group, which are probably indicative of a small-sedge-swamp community, although they may also represent dry habitats such as open woodland (Appendix X). The reeds of the types Phragmites australis and Glyceria type are also prominent. Communities of floating-leaved and submerged macrophytes are poorly represented and probably derive from a few plants growing in small pools on the wetland. The influence of minerals supplied by water from a soft-water spring, which maintained a high level of available nutrients, is evident throughout the sequence. Additional nutrient supply from anthropogenic activities (e.g. manure from grazing) is therefore difficult to detect. Where peat formation occurred surface peat was either continuously wet or only intermittently dry (probably only during the driest part of summer).

The sedimentary history of the wetland area is closely linked to the local wetland vegetation history, thus major sedimentary units defined above usually coincide with the 
established wetland pollen zones. These zones are used, for convenience, in the discussion below.

Zone $A W-9,250.0-227.5 \mathrm{~cm}$, c. 1560-1575 AD The dominant plant community is the wet-soil community that includes moist meadow, stream bank, and dwarf plant communities that are inundated in winter but dry out in summer. A bog community resembling that of today is indicated by the dominance of the Carex hallerana type and the occurrence of Mentha type and Ranunculus acris group, which grow on the wetter areas of the bog today as well as on the marginal fen. A true-reed swamp community has also developed. Both floating-leaved and submerged communities of open water are represented by a few pollen and spore types.

This zone comprises two minerogenic sediment horizons with an interbedded peat horizon, and the lower part of an overlying peat horizon. The lowest horizon $(250-245 \mathrm{~cm})$ comprises muddy sandy gravel indicating high energy sediment transportation (stream flood) and accelerated erosion in the catchment, and corresponds to sediment section F, which is characterised by low organic and moisture content and high inorganic content. Ponding of water is suggested by the occurrence of spores and pollen of the floating-leaved aquatic macrophytes Salvinia natans, Stratiotes aloides, and Hydrocharis morsus-ranae, and the submerged macrophyte Hydrocotyle vulgaris in the basal spectrum. These macrophytes usually grow in shallow ponds; Hydrocotyle vulgaris may also be emergent (Ellenberg 1988). Total wetland pollen and spore concentration is very low $\left(2 \times 10^{3}\right.$ grains $\left./ \mathrm{cm}^{3}\right)$ and palynomorphs may be partly derived from the overlying peat.

The next horizon $(245-240 \mathrm{~cm})$ is included in sediment section E. It comprises peat with very high organic and moisture content and very low inorganic matter indicating a period of landscape stability and waterlogging of the cored area. The level of humicity of the peat indicates that there was intermittent drying of surface peat, probably seasonally during summer (Blackford 1994). Total wetland pollen and spore concentration increases $\left(5 \times 10^{3}\right.$ grains $/ \mathrm{cm}^{3}$ ), but is low by comparison with other peat horizons in this sequence. This may be because the local wetland vegetation covers a small area and is in a formative stage, or due to rapid deposition. The presence of the annual Cyperus michelianus and the early hydrosere Sparganium erectum is supporting evidence for a colonizing vegetation (Ellenberg 1988), and the species represented by the pollen type Cyperus longus type may be the colonizer Eriophorum angustifolium (Ellenberg 1988). High palynological richness (6 expected types) is supporting evidence for a colonizing community.

The overlying sediment horizon $(240-235 \mathrm{~cm})$ is very similar to that of the basal sandy gravel horizon, but it contains a trace of peat also. No analyses of the physical properties of this horizon were undertaken, but the Troels-Smith classification of the sediment suggests that this was another period of high energy sediment transportation (stream flood) and accelerated 
erosion in the catchment. Eroded sediments inundated the peat forming vegetation but did not halt peat formation entirely. The overlying peat horizon $(235.0-227.5 \mathrm{~cm})$ is similar to that below and again indicates a period of landscape stability in the catchment and waterlogging of the cored area. Humicity level of the peat is the same as that for the lower peat horizon, indicating seasonal drying of surface peat. Total concentration of pollen and spores increases again and may indicate an expanding wetland vegetation $\left(6 \times 10^{3}\right.$ grains $\left./ \mathrm{cm}^{3}\right)$.

Throughout the zone Carex hallerana type, Cyperus michelianus, Phragmites australis, and Glyceria type are the most abundant types (no sample from the upper gravel and sand horizon was pollen analysed) and plants represented by these types and mosses, as suggested by the presence of Caltha type pollen (Ellenberg 1988), probably formed the peat. The dominant type is Carex hallerana type that includes the species Carex hallerana, which grows in dry places, Scirpus holoschoenus and S. sylvaticus, which grow in damp, usually shady places, and probably other Carex and Scirpus species, and Blysmus compressus, which grow in a variety of habitats from marshes and other damp places to woods and dry grassland (Tutin et al. 1964-80). Pollen of this type may thus derive from a variety of habitats such as the local wetland, or more distant woodlands or grasslands (Chapter 3). Since Carex forms $60 \%$ of the vegetation cover in the drier areas of the bog today, it is highly likely that plants of this genus grew on the wetland in the past.

The level of available nutrients is high as indicated by the presence of Glyceria type, Oenanthe aquatica group, Sparganium erectum, Salvinia natans, Stratiotes aloides, Hydrocharis morsus-ranae, and Myriophyllum spicatum (Ellenberg 1988). Nutrients may be contributed to the wetland via washed in sediment, spring water or from direct or indirect human activity. Caltha palustris, which often grows in soft-water spring fed mires (Ellenberg 1988), occurs in this zone. Today Caltha palustris, which is included in the Caltha type, is the dominant plant (about 90\%) growing in the area around the spring-fed stream inlet (Chapter 3). Rapid establishment of a wetland community would be assisted by the high level of available nutrients (Ellenberg 1988), and perhaps some of the plants represented, e.g. species belonging to the Ranunculus acris group, had been already growing on the stream bank before waterlogging and peat formation occurred.

Zone $A W-8,227.5-212.5 \mathrm{~cm}$, c. $1575-1585 A D$ This zone corresponds to the upper part of the peat horizon of the zone below and an overlying horizon of sandy peat, and to the upper part of sediment section E. The lower part of this zone, composed entirely of peat, has high organic and moisture content and very low inorganic matter content. Towards the top of the zone organic matter and moisture content decline and inorganic matter increases. These changes occur as increasing amounts of sand are washed onto the peat forming vegetation. 
The communities of wet-soil habitats continue to be the dominant wetland communities. The true-reed swamp is still represented in about the same proportions but a shift in dominance from Phragmites australis to Glyceria type has occurred, perhaps as a response to increased nutrients. The floating-leaved and submerged aquatic communities are much reduced.

Humicity of peat decreases as sand is washed onto the peat forming vegetation, indicating increasing wetness of the peat surface. Aquatic plants decline, perhaps due to a constant flow of water close to the core site and former pools on the peat surface being filled in with sediment.

Total concentration of wetland pollen and spores representing emergent plants increases greatly, reaching a maximum of $13 \times 10^{3}$ grains $/ \mathrm{cm}^{3}$ at $220 \mathrm{~cm}$, (but declines slightly at the top of the zone) indicating expanded and well developed sedge and reed communities. The disappearance of the annual Cyperus michelianus and the early hydrosere Sparganium erectum is supporting evidence for a well developed reed community. Low palynological richness (4 expected types) is also indicative of the monospecific stands that well developed reedswamp communities of Glyceria and Phragmites australis can form. The dominant type is Carex hallerana type with Phragmites australis and Glyceria type also prominent; Glyceria type is now the more abundant of the latter two. The appearance of Saxifraga stellaris suggests that mosses are still part of the peat forming vegetation (Ellenberg 1988) in combination with sedges and reeds.

The available nutrient level is still high, as indicated by the presence of Glyceria and Salvinia natans. The presence of Saxifraga stellaris suggests that at least some of the nutrients derive from spring water (Ellenberg 1988).

Zone $A W-7,212.5-157.0 \mathrm{~cm}, \mathrm{c.}$. 1585-1625 AD This zone corresponds to the sediment section $\mathrm{D}$, which is characterised by low organic and moisture content and high inorganic content. Seven different sediment horizons have been identified. With the exception of two horizons at 209-202 and 202-199 cm where a trace of peat occurs all deposits are composed of sediment of varying grain size representing transportation and deposition of sediment into the wetland at varying levels of energy.

Again the plant communities of wet-soil habitats are the dominant wetland communities. A tall-sedge community has developed and the true-reed-swamp community has expanded, dominated by Glyceria type. The communities of open water have not changed significantly. Hydrocotyle vulgaris is the only submerged aquatic represented and it is not exclusively submerged.

At 213-209 $\mathrm{cm}$ peat formation ceases, perhaps due to inundation of sediment, and concentration of total wetland pollen and spores is extremely low $\left(1 \times 10^{3}\right.$ grains $\left./ \mathrm{cm}^{3}\right)$. 
Sediment comprises sand sized particles probably washed in by stream flood. Organic and moisture content of the sediment is very low and inorganic matter is very high. No aquatic types occur with the exception of Hydrocotyle vulgaris, which may grow as an emergent.

The two mud horizons between 209 and $199 \mathrm{~cm}$ indicate lower energy transport of sediments, which may derive from run-off of bared soils. Organic and moisture content of the sediment increases while inorganic matter decreases. Peat formation begins again and total wetland pollen concentration increases $\left(6-10 \times 10^{3}\right.$ grains $\left./ \mathrm{cm}^{3}\right)$, indicating greater landscape stability in the catchment and waterlogging of the wetland. The peat surface is continually wet but no aquatic types occur, with the exception of Salvinia natans, which occurs at $200 \mathrm{~cm}$ at very low abundance.

Between 199 and $192 \mathrm{~cm}$ another episode of erosion in the catchment is indicated by deposition of coarse grained sediment of pebbly fine sand. Peat formation ceases, due to inundation by sediments, and total concentration of wetland pollen and spores is low $\left(4 \times 10^{3}\right.$ grains $/ \mathrm{cm}^{3}$ ). At $192 \mathrm{~cm}$ the sediment changes to mud with some gravel, indicating slightly lower energy transport of sediment.

Several thin sediment horizons of coarse and mixed grain sizes between 184 and $170 \mathrm{~cm}$, and a thicker horizon of muddy sandy gravel above $170 \mathrm{~cm}$ indicate a period of landscape instability with multiple episodes of sediment influx. Many boundaries between these horizons are conspicuous indicating rapid changes in deposition processes. No peat forms, due to rapid sediment influx.

Total concentration of wetland pollen and spores fluctuates, but is generally low $\left(1-10 \times 10^{3}\right.$ grains $\left./ \mathrm{cm}^{3}\right)$. Almost all types represent emergent plants; Carex hallerana type, Carex flacca type, Phragmites australis, and Glyceria type are dominant, although their relative frequencies and concentrations fluctuate. Glyceria type is associated with deposition of larger grain-sized minerogenic sediment (sand and larger). This type includes the species Glyceria maxima, which is known to tolerate influx of minerogenic sediment (Ellenberg 1988), although the association may also be interpreted as pollen of the terrestrial species included in the type being washed into the wetland. Peaks in relative frequency of Carex hallerana type coincide with sediments of smaller grain size and periods of lesser sediment influx reflecting quick recolonisation after inundation of relicts of the previous community. In this zone, Carex flacca type probably represents species such as Eriophorum, which can tolerate fluctuating water levels and grow on bare soil (Ellenberg 1988). Fluctuations in the annual Cyperus michelianus reflect the behaviour of a coloniser. Concentration values of Phragmites australis are very low during the periods of greatest instability. A fluctuating (3-6 expected types) palynological richness curve also suggests periods of greater instability on the wetland. 
The continued presence of Saxifraga stellaris and Caltha type suggest continued influence of a soft-water spring. The water level of the wetland area may be quite low as pollen of floating-leaved or submerged macrophytes is rare. Humicity of the peat at 209-199 cm indicates that the surface of the peat was continuously wet. The appearance of the mostly entomophilous Salix fragilis type at $195 \mathrm{~cm}$ may indicate that a part of the surface of the wetland was dry enough to allow willow to grow on it.

The level of available nutrients is still high as suggested by the presence of Glyceria type, Oenanthe aquatica group, Sparganium erectum, Typha domingensis type, Typha angustifolium type, Salvinia natans, and Hydrocharis morsus-ranae (Ellenberg 1988).

Zone AW-6, 157.0-117.5 cm, c. 1625-1670 AD This zone corresponds to sediment section $\mathrm{C}$, which is characterised by intermediate values for all components and represents a period of much greater landscape stability in the catchment. Deposition of allochthonous mineral material steadily decreases while peat formation increases. Several horizons comprise this zone, they are all mixtures of peat and minerogenic sediment; grain size of sediments is never larger than silt size, with the exception of a trace of fine gravel between $126-130 \mathrm{~cm}$.

The lowest horizon comprises clay and silt with a trace of peat, which increases upwards, suggesting landscape stability when only fine-grained mineral sediments were washed into the wetland. The proportion of peat increases further in the overlying horizon and continues increasing up to the top of the next horizon. In this horizon $(133-130 \mathrm{~cm})$ some fine gravel is washed in, suggesting a stream flood event of short duration. The overlying horizon comprises an increased proportion of minerogenic sediment and a decreased proportion of peat indicating an increased rate of sediment influx. The two uppermost horizons both comprise at least half peat and indicate landscape stability in the catchment and waterlogging of the wetland.

Wet-soil communities continue to be the dominant communities and the tall-sedgeswamp community has expanded. The true-reed-swamp community continues about the same, but Phragmites australis has expanded slightly. Aquatic communities occur only sporadically, but the obligate submerged plant Myriophyllum spicatum occurs in the uppermost spectrum.

Humicity of the peat indicates that the peat surface is continuously wet. Aquatic plants are very sparse, but occur in the horizons with the highest proportion of peat suggesting that they are growing in pools within a bog.

Total wetland pollen and spore concentration fluctuates greatly $\left(4-20 \times 10^{3}\right.$ grains $\left./ \mathrm{cm}^{3}\right)$, being highest at the base and top of the zone where it reaches the maximum for the sequence. Again almost all pollen and spores are contributed by types representing emergent plants. The dominant types are Carex hallerana type, Cyperus longus type, Phragmites australis, and Glyceria type, and parent plants and mosses probably form the peat. The most abundant type; 
Carex hallerana type, is very abundant, suggesting that a well developed small-sedge swamp has developed; the annual Cyperus michelianus occurs in significant abundance in only the second to bottom spectrum, indicating that colonization of bare ground declines. The concentration of Glyceria type increases greatly at the base of the zone, perhaps due to the influx of fine sediment with abundant nutrients. Cyperus longus type increases, indicating an expanded tall-sedge community and a more stable environment where perennials can establish. Palynological richness fluctuates but is generally low at about 4 expected types, which indicates a stable environment.

The presence of Lysimachia, Glyceria type, Salvinia natans, Stratiotes aloides, Nuphar lutea, and Ranunculus aquatilis group indicate a high available nutrient level (Ellenberg 1988).

Zone $A W-5,117.5-95.0 \mathrm{~cm}$, c. $1670-1730 A D$ This zone corresponds to sediment section $\mathrm{B}$, which is characterised by high inorganic matter and low organic content, corresponding to influx of allochthonous mineral material. Three sediment horizons occur; the two lower horizons contain coarse grained sediments and indicate high energy stream input and perhaps accelerated erosion in the catchment, while the uppermost horizon comprises small grain-size mineral particles and represents declining stream energy or sediment yield from the catchment. The basal horizon, a mixture of peat and sandy muddy gravel, is transitional from the zone below: peat is inundated by coarse sediment until it is eventually overwhelmed. Humicity of the peat in the basal horizon indicates that surface peat is continuously wet. An increase in the occurrence of aquatic types suggests that the water level has risen.

Plant communities of wet-soil habitats continue to be dominant. The true-reed-swamp community continues about the same and then expands with an increase of Glyceria type in the uppermost spectrum. The tall-sedge-swamp community almost disappears. Aquatic communities expand slightly and pollen of Myriophyllum spicatum occurs in the basal spectrum.

Total concentration of wetland pollen and spores declines to a very low value at the top of the zone $\left(10 \times 10^{3}\right.$ grains $/ \mathrm{cm}^{3}$ in the basal spectrum and $1 \times 10^{3}$ grains $/ \mathrm{cm}^{3}$ in the top), probably as wetland vegetation is destroyed by inundation of sediments, and comprises mostly types representing emergent plants. Palynological richness is low at 4 expected types, until the top of the zone where it increases to 6 . The increase in richness probably reflects the colonization of fresh nutrient-rich sediment. The dominant types of Carex hallerana type, Phragmites australis, and Glyceria type plus mosses probably form the peat in the basal horizon. 
The presence of Glyceria type, Sparganium erectum, Stratiotes aloides, Hydrocharis morsus-ranae, and Ranunculus aquatilis group indicate a high available nutrient level (Ellenberg 1988).

Zone $A W-4,95.0-65.0 \mathrm{~cm}$, c. 1730-1810 AD This zone corresponds to the basal part of a peat horizon, which is continuous to the surface of the current bog. It also corresponds to the basal part of sediment section $\mathrm{A}$, which extends to the top of the sequence, and which is characterised by high organic and moisture content and low inorganic content, and is indicative of landscape stability in the catchment and waterlogging of the wetland area. Low palynological richness, ranging from 4-5 expected types, also indicates a stable environment. The level of humicity of the peat indicates that there was intermittent drying of surface peat, probably seasonally during summer. The occurrence of pollen of floating-leaved macrophytes suggests that pools existed on the peat surface, but the occurrence of the mostly entomophilous Salix fragilis type suggests that some part of the wetland was dry enough for willow growth.

Plant communities of wet-soil continue to be dominant with Carex hallerana type the dominant type, but a peak in Equisetum occurs in the centre of the zone. The tall-sedge community is poorly represented, but increases slightly at the top of the zone. The true-reedswamp community is reduced as well as the aquatic communities.

Total wetland pollen and spore concentration increases and the highest overall values for a zone are scored $\left(13 \times 10^{3}\right.$ grains $\left./ \mathrm{cm}^{3}\right)$. The dominant types are Carex hallerana type and Glyceria type and species represented by these types and mosses are the main peat forming plants.

A sudden peak in Equisetum may be related to the condition of the bog, but the source of the spores may also be from a greater distance; for example from the arable weed Equisetum arvense (Ellenberg 1988). The reduction in Phragmites australis may be due to grazing or harvesting.

The presence of Glyceria type, Typha domingensis type, Stratiotes aloides, Hydrocharis morsus-ranae, and Ranunculus aquatilis group indicate a high available nutrient level (Ellenberg 1988).

Zone $A W-3,65.0-45.0 \mathrm{~cm}, c .1810-1865 A D$ This zone corresponds to the central part of sediment section A where less minerogenic sediment is washed into the wetland than in the zone below. Palynological richness is low again, 4-5 expected types, suggesting a stable environment. Humicity of the peat indicates a higher water level as surface peat is now continuously wet, but representation of aquatic macrophytes remains extremely low.

Total concentration of wetland pollen and spores is high $\left(6-10 \times 10^{3}\right.$ grains $\left./ \mathrm{cm}^{3}\right)$, but declines from the preceding zone, perhaps due to greater waterlogging. 
The true-reed-swamp community becomes dominant with a large expansion of Phragmites australis. The wet-soil communities are reduced as well as the tall-sedge community. The dominant types are: Carex hallerana type, Phragmites australis, and Glyceria type. Species of these types plus mosses are the main peat forming plants.

The dramatic rise in Phragmites australis is probably a succession following a reduction in grazing; the usual hydrosere of spring swamps is from small-sedge communities to Phragmites australis (Ellenberg 1988).

The presence of Berula erecta, Glyceria type, Oenanthe aquatica group, Hydrocharis morsus-ranae, and Potamogeton crispus type indicate available nutrient level is high (Ellenberg 1988).

Zone $A W-2,45.0-5.0 \mathrm{~cm}, \mathrm{c.}$ 1865-1975 AD This zone corresponds to the upper part of sediment section $\mathrm{A}$, where organic and moisture content are exceptionally high, although the latter is slightly lower than in the preceding zone and almost no minerogenic sediments are washed into the wetland. This is indicative of landscape stability in the catchment and waterlogging of the wetland. Low palynological richness of 5-2 expected values reflects the landscape stability. Humicity of the peat indicates that the surface peat is continuously wet.

Carex hallerana type of the wet-soil communities regains its dominance. The tallsedge-swamp community expands slightly, but the true-reed swamp is greatly reduced. Representation of the floating-leaved aquatic community remains sparse and the submerged community almost disappears, being represented by only Hydrocotyle vulgaris.

Total concentration of wetland pollen and spores is similar to the top of the preceding zone $\left(4-8 \times 10^{3}\right.$ grains $\left./ \mathrm{cm}^{3}\right)$. Carex hallerana type is dominant. Peat is probably now formed by species represented by the Carex hallerana type and mosses. Glyceria type and Phragmites australis have declined, perhaps due to grazing on the bog.

The presence of Glyceria type, Sparganium erectum, Typha domingensis type, and Salvinia natans indicate available nutrient level is high (Ellenberg 1988).

Zone $A W-1,5.0-0.0 \mathrm{~cm}$, c. 1975-1989 AD This zone comprises the surface spectrum only. Moisture content increases sharply while organic matter decreases sharply and inorganic matter content is extremely low. Humicity of the peat indicates that surface peat is continuously wet. Several aquatic types occur, albeit at very low concentration values. A dead pine tree at the north end of the bog may be a response to increased wetness in the bog, waterlogging killing the tree by cutting off the oxygen supply to its roots.

The wet-soil communities decline and the tall-sedge-swamp community expands, while the true-reed swamp increases slightly. The floating-leaved aquatic community expands and the submerged aquatic community disappears. A high palynological richness, 11 expected types, may be due to differential preservation of grains normally destroyed in burial. 
Total concentration of wetland pollen and spores is low $\left(1 \times 10^{3}\right.$ grains $\left./ \mathrm{cm}^{3}\right)$, perhaps due to high non-pollen biomass or water content and perhaps also to wash-down of pollen into slightly lower peat. The dominant types are Mentha type, Parnassia palustris, Carex hallerana type, Carex flacca type, Cyperus michelianus, and Phragmites australis. Species of these types were noted growing on the wetland, with the exception of Cyperus michelianus and Phragmites australis (Appendix VIII). The main peat forming species are sedges and mosses. The presence of Glyceria type and Hydrocharis morsus-ranae indicate available nutrient level is high (Ellenberg 1988).

\subsubsection{Terrestrial pollen analysis}

\subsubsection{Pollen diagram construction}

Pollen and spore relative frequencies are presented in Figures 5.10 and 5.11 (in pocket). Pollen and spore types are arranged according to habitat groups defined in Chapter 2. The pollen sum on which the relative frequencies are based includes all identified terrestrial pollen and spore taxa and excludes wetland taxa. Relative frequencies of $0.2 \%$ or less are indicated with a dot. Style of the pollen diagrams is similar to that of the wetland diagram (Figure 5.8).

Figure 5.10, the summary diagram, shows the coniferous and deciduous canopy trees group in resolved form, then a summary column showing the ratio of terrestrial trees and shrubs to terrestrial herbaceous plants. This is followed by summary curves of each habitat group, then a curve of palynological richness derived from rarefaction analysis and based on the smallest total count of terrestrial pollen and spores, 166 (Birks \& Line 1992; Bennett 1994). This is followed by the number of terrestrial types in each spectrum (not normalized for unequal pollen sums). Finally, the number of pollen grains of trees and shrubs, the total number of all terrestrial pollen and spores and the total of all pollen and spores counted in this sequence (wetland and terrestrial) are given.

The fully resolved diagram, Figure 5.11 (in pocket), displays all terrestrial pollen and spore taxa recognised in the sequence, in order of habitat groups. Within these groups they are arranged alphabetically with the exception of the dominant tree types Pinus (Diploxylon), Fagus, Quercus frainetto type, and Quercus trojana type, which are placed at the beginning of the 'coniferous and deciduous canopy trees' group.

Pollen concentration data are presented in Figure 5.12. Only concentration of pollen and spore types recorded in significant abundance, habitat groups, and total pollen and spores are included. The concentration diagram takes a similar form to the relative frequency diagram. 


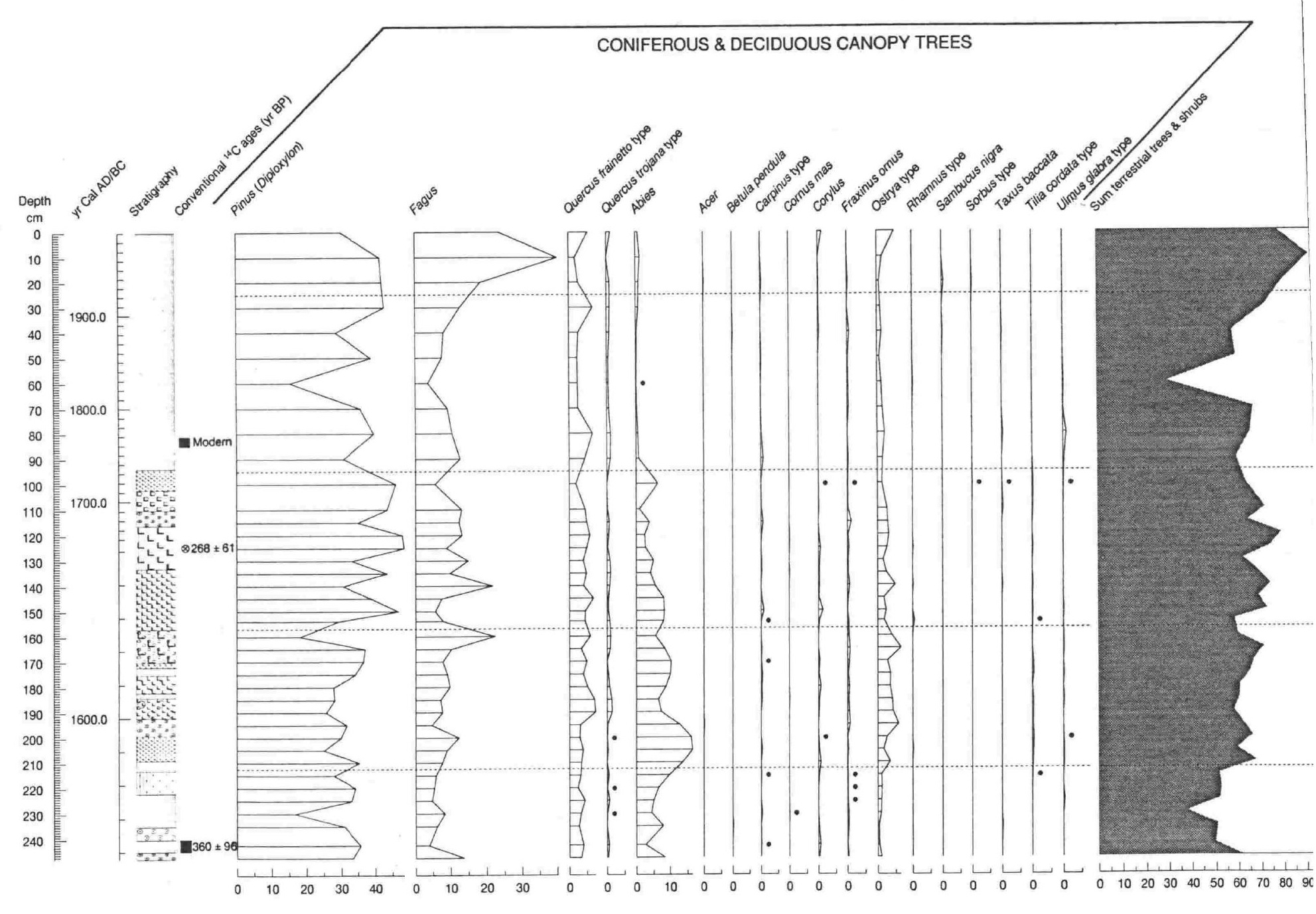

Figure 5.10 Anelia - summary relative frequency pollen diagram, terrestrial taxa; pollen sum is total terrestrial taxa; scale interval $5 \%$ for all taxa and groups (resolved canopy trees taxa have $1.5 \mathrm{x}$ scale of groups), percentages less than $0.2 \%$ shown by dots. 

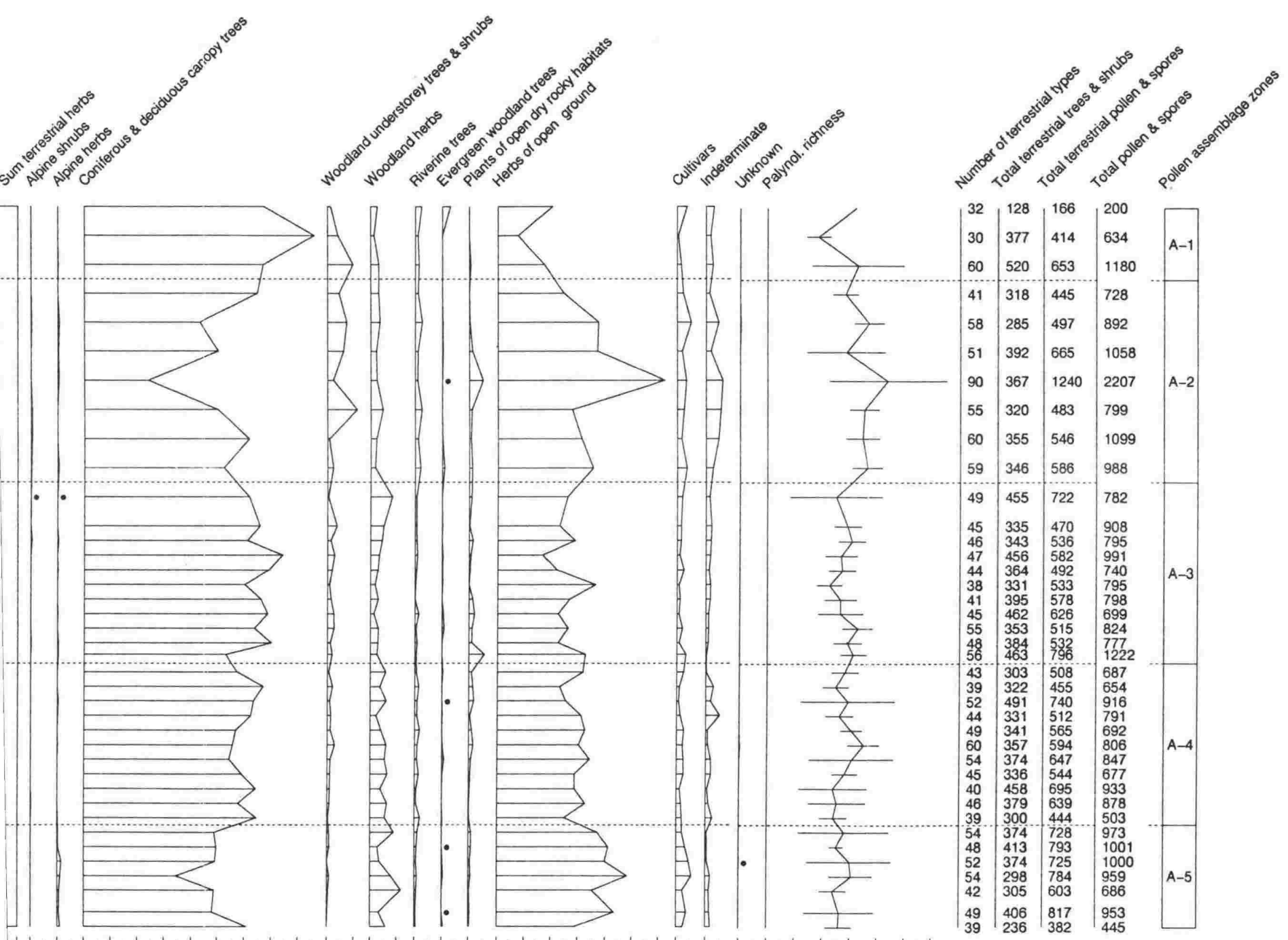

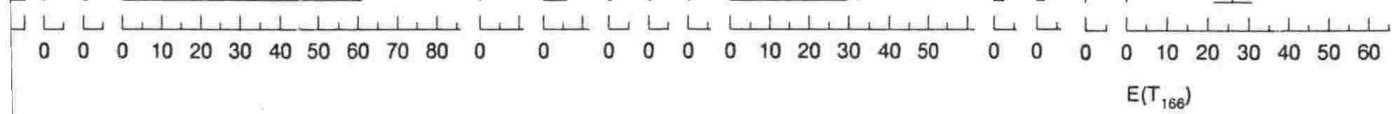

Figure 5.10 


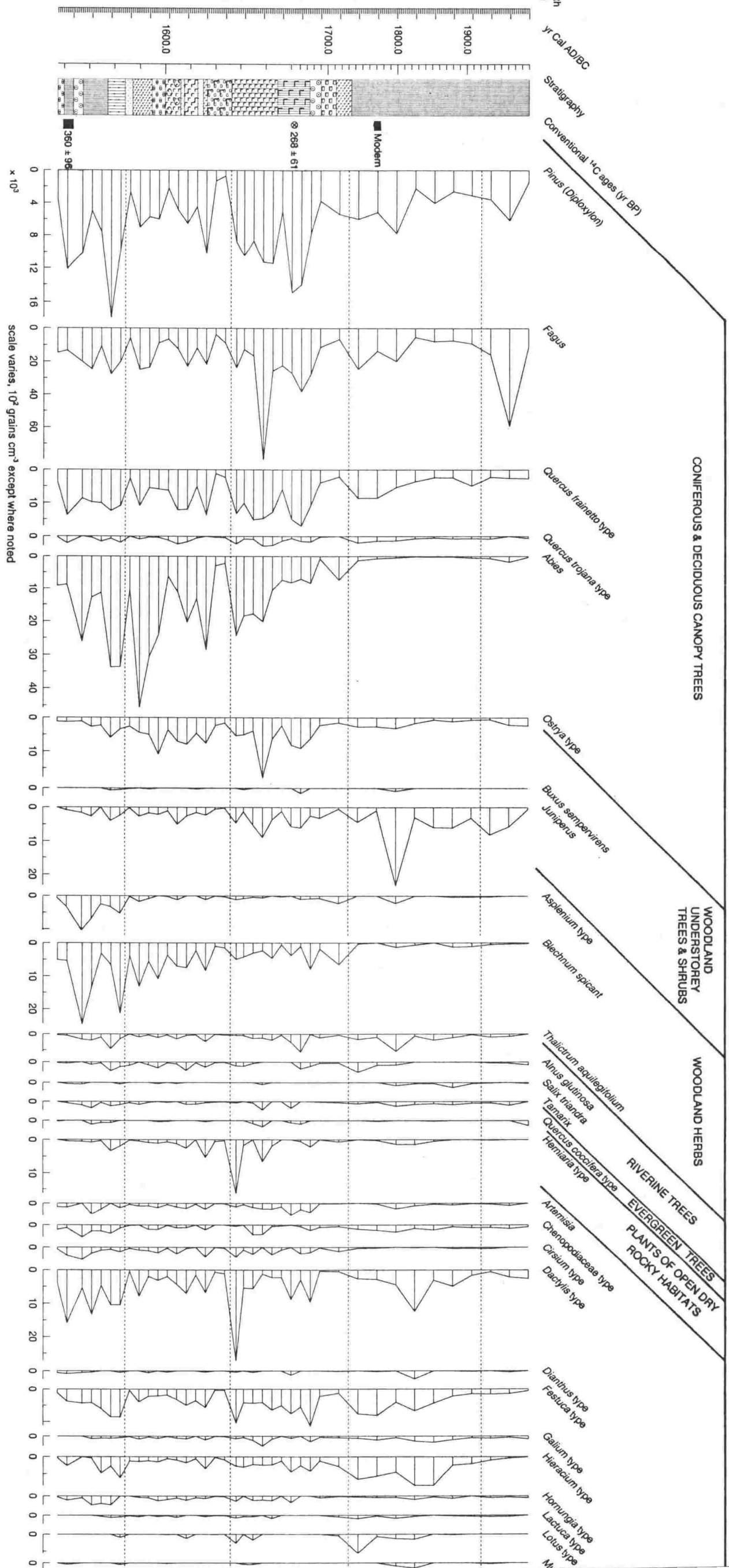




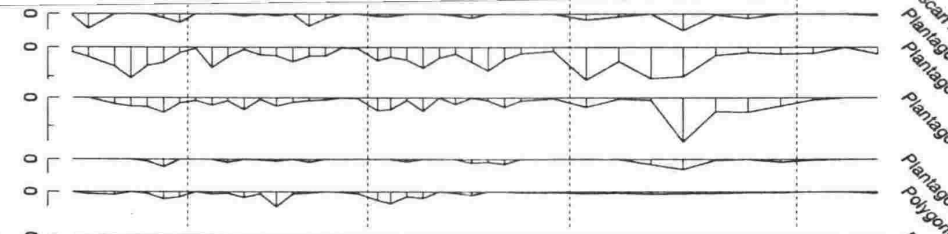

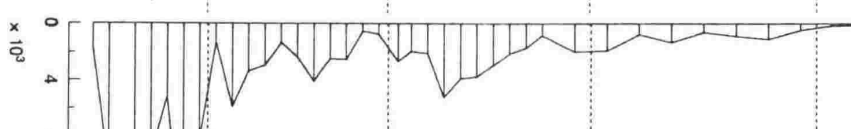

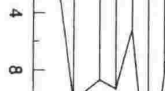

$\vec{N}+$

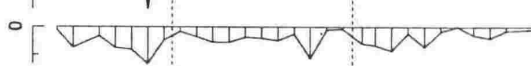

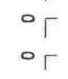

\begin{tabular}{l|l|l|l}
\hline & & & \\
\hline
\end{tabular}

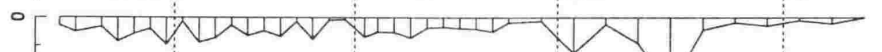

$$
\begin{array}{l|l|l|l} 
& & & V \\
0 & & & V \\
0 & &
\end{array}
$$

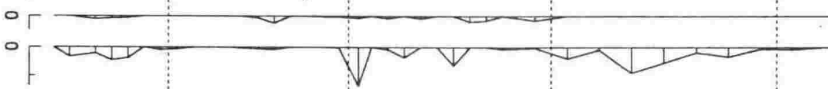$$
\text { ○ }
$$$$
0 \text { r }
$$$$
\text { 다 }
$$$$
\circ
$$$$
\text { or }
$$$$
\circ \text { एपम्य }
$$$$
\stackrel{x}{a}
$$
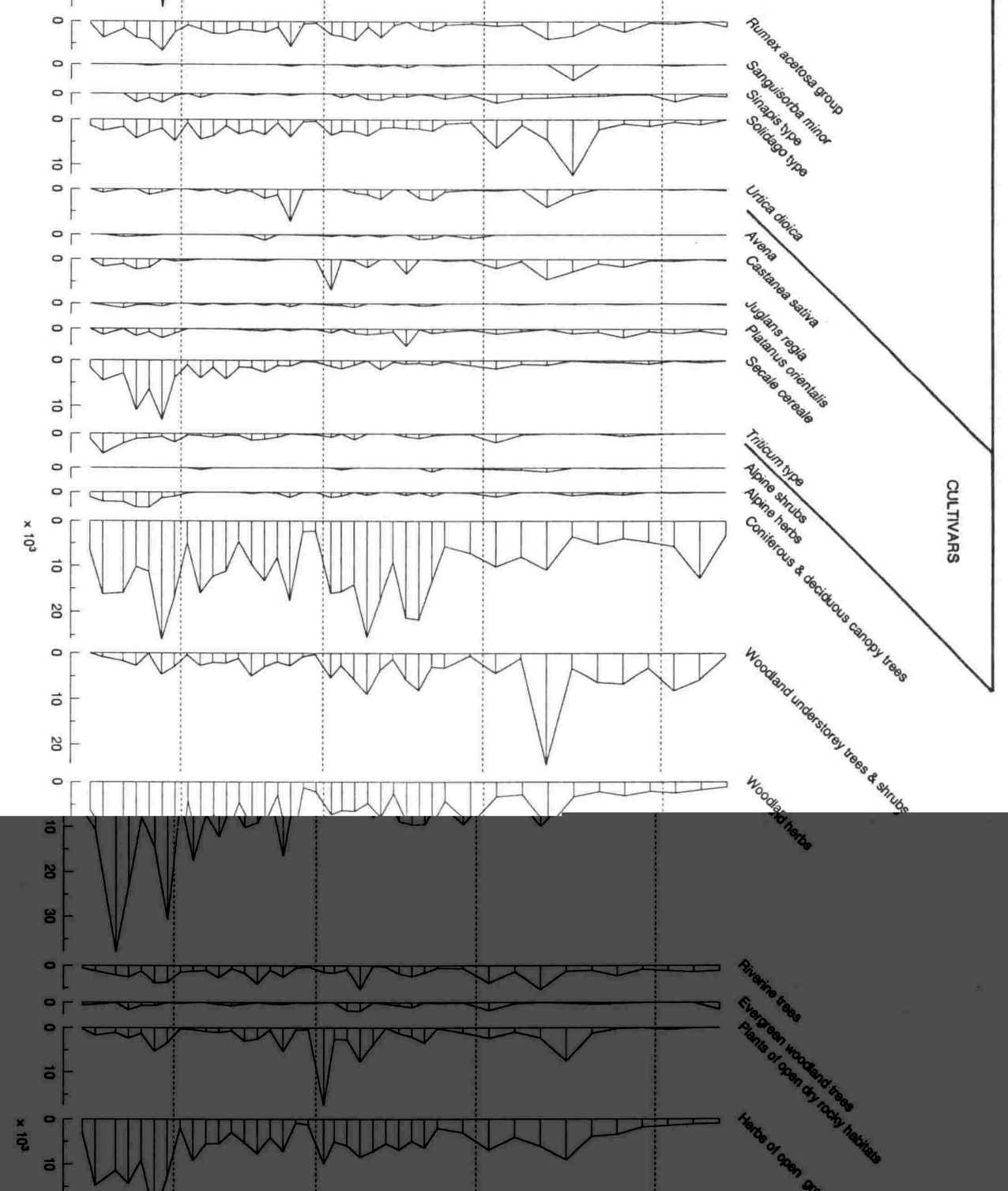
st

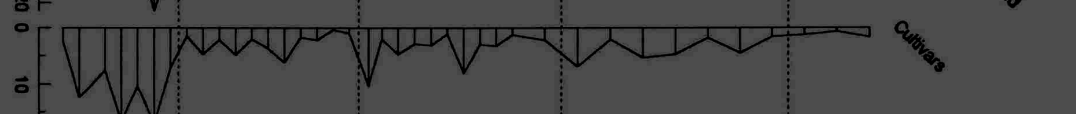

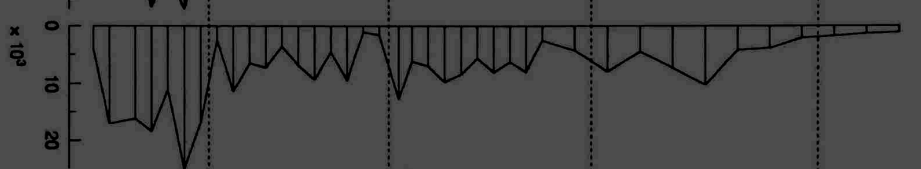

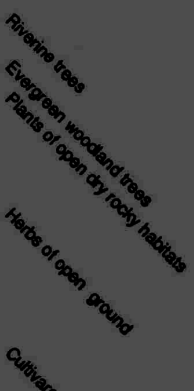

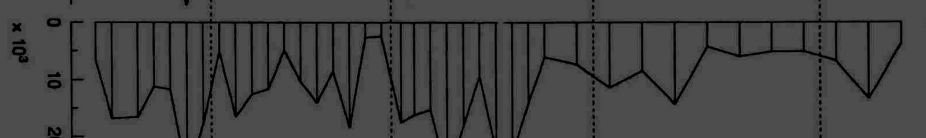
$\approx$. $V$ V

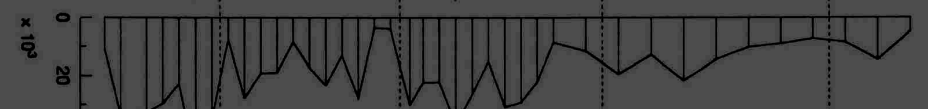
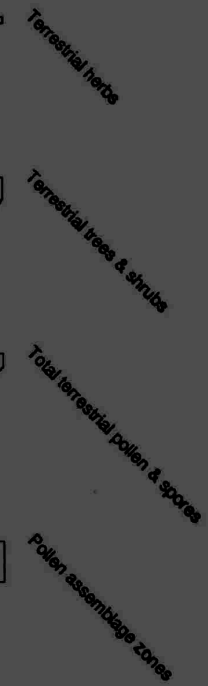


\subsubsection{Terrestrial pollen assemblage zones}

Terrestrial pollen diagrams are divided into 5 local pollen assemblage zones A-1 to A-5, based mostly on relative frequency data. These are defined below. Concentration data are discussed in the following section.

Zone A-5, 250.0-212.5 $\mathrm{cm}$ This basal zone is characterised by high relative frequencies of Pinus (Diploxylon) (16.7-35.7\%), and about average frequencies (of the sequence as a whole) of Fagus (3.9-13.6\%), Quercus frainetto type (2.4-4.4\%), and Abies (2.6-9.8\%). Ostrya type occurs at lowest frequencies (0.3-1.1\%) for the sequence. Other types belonging to the 'coniferous and deciduous canopy trees' habitat group do not exceed $0.7 \%$.

No 'alpine shrubs' are represented. Four types of 'alpine herbs' are represented; only Veratrum exceeds $0.3 \%$, and it reaches a maximum of only $1.5 \%$. 'Woodland understorey trees and shrubs' are represented by only the types Buxus sempervirens $(0.1 \%$ maximum) and Juniperus $(0-2.9 \%)$. 'Woodland herbs' are better represented, reaching the maximum relative frequency of the sequence of $11.6 \%$ at $235 \mathrm{~cm}$. Most abundant are the ferns Asplenium type (0.5-3.2\%) and Blechnum spicant (1.3-7.5\%). Other groups poorly represented are 'riverine trees' (1.1\% maximum), 'evergreen woodland trees' (no type exceeds $0.4 \%$ ), and 'plants of open dry rocky habitats' (no type exceeds $0.6 \%$ ).

'Herbs of open ground' are almost as abundant (28.8-48.1\%) as the group 'coniferous and deciduous canopy trees', and have a curve the inverse of that of the latter group. Many of the types of this group are represented, the most important are: Dactylis type (1.7-4.7\%), Festuca type (1.0-2.6\%), and Pteridium aquilinum (lowest in the basal spectrum at $15.9 \%$, then rises to $22.3-31.4 \%$ ). Artemisia, Chenopodiaceae type, Cirsium type, Hieracium type, Hornungia type, Plantago lanceolata type, Rumex acetosa group, and Solidago type are also quite important, but do not exceed $2.1 \%$.

'Cultivars' reach the maximum of the sequence of $5.6 \%$ at $230 \mathrm{~cm}$. Types that are most abundant are cereals: Secale cereale (0.8-3.7\%) and Triticum type (0.1-1.3\%). Avena occurs in the three central spectra, but does not exceed $0.2 \%$. Cereals are known to have been cultivated in the Pindos Mountains in historic times (Chapter 1). Secale cereale may have been grown mostly for fodder. Castanea sativa, Juglans regia, and Platanus orientalis occur sporadically and do not exceed $0.8 \%$. Vitis vinifera occurs in one spectrum, but at $0.1 \%$ only.

Zone $A-4,212.5-157.0 \mathrm{~cm}$ The boundary with the preceding zone is marked by an increase in the relative frequencies of Abies and Ostrya type, a decline in Pteridium aquilinum and an increase in the ratio of terrestrial trees and shrubs to terrestrial herbs. The zone is characterised by high relative frequencies of Pinus (Diploxylon) and Pteridium aquilinum; increased relative frequencies of Fagus, Abies, Quercus frainetto type, and Ostrya type; and increased ratio of terrestrial trees and shrubs to terrestrial herbs. 
'Alpine shrubs' and 'alpine herbs' are very poorly represented; no type exceeds $0.2 \%$.

'Coniferous and deciduous canopy trees' have increased (54.4-67.0\%). Pinus (Diploxylon) has high relative frequencies (18.1-37.0\%) and Fagus has increased $(7.1-22.2 \%$, with the exception of one spectrum of $4.6 \%$ ). Quercus frainetto type increases slightly from the centre of the zone (2.9-7.4\%). The curve of Abies has the reverse shape to that of Quercus frainetto type. Initially Abies increases to the maximum of the sequence of $16.4 \%$ in the second spectrum, then declines gradually to $5.5 \%$. Ostrya type is represented at $1.6-6.4 \%$, but mostly about $3.0-4.0 \%$. No other type of this group exceeds $1.4 \%$.

'Woodland understorey trees and shrubs' are again poorly represented, although Arceuthobium oxycedri is represented as well as Buxus sempervirens and Juniperus. Arceuthobium oxycedri and Buxus sempervirens occur sporadically and do not exceed $0.2 \%$. Juniperus occurs continuously and increases slightly to reach a maximum of $2.9 \%$ in the centre of the zone. 'Woodland herbs' decline slightly towards the top of the zone (2.1-6.4\%). Blechnum spicant is the most abundant type at $1.8-5.7 \%$. Asplenium type has declined to less than $1.0 \%$. No other type in this group exceeds $0.8 \%$. Other groups poorly represented are 'riverine trees' (2.0\% maximum), 'evergreen woodland trees' (no type exceeds $0.5 \%$ ), and 'plants of open dry rocky habitats' (2.4\% maximum).

'Herbs of open ground' decline, but remain the second most abundant habitat group (23.3-34.3\%). Again the curve is more or less the inverse of that of 'coniferous and deciduous canopy trees' and again a wide variety of herbs are represented. Dactylis type, Festuca type, and Pteridium aquilinum remain the most abundant types, but Pteridium aquilinum has declined (9.1-21.1\%). Frequencies of other types in this group do not change significantly.

'Cultivars' decline (0.8-2.7\%), due mostly to a decline in Secale cereale $(0.4-2.2 \%)$. Triticum type, and Avena occur sporadically ( 0.8 and $0.5 \%$ maximum, respectively). Castanea sativa, Juglans regia, and Platanus orientalis occur sporadically again and decline to a maximum of $0.4 \%$. Vitis vinifera has disappeared.

Zone $A-3,157.0-95.0 \mathrm{~cm}$ The boundary with the preceding zone is marked by an increase in Pinus (Diploxylon) and a decline in Ostrya type. This zone is characterised by an increase in Pinus (Diploxylon), Fagus, and the ratio of terrestrial trees and shrubs to terrestrial herbs; and a decrease in Abies, Ostrya type, Blechnum spicant, and Pteridium aquilinum.

'Alpine shrubs' and 'alpine herbs' are extremely poorly represented; $0.4 \%$ maximum.

In the habitat group 'coniferous and deciduous canopy trees' Pinus (Diploxylon) reaches the maximum of the sequence (48.4\%). Relative frequencies of Fagus fluctuate, but are generally higher than in all other zones except the uppermost (5.5-21.6\%). Quercus frainetto type sustains frequencies around $4.5 \%$ until the uppermost spectrum, where it declines to $1.8 \%$. The curve of Abies declines from around $8.0 \%$ to $0.9 \%$, but then increases in the 
uppermost spectrum to $6.2 \%$. Frequencies of Ostrya type are generally lower (usually less than $3.0 \%$ ). No other type in this group exceeds $1.3 \%$.

'Woodland understorey trees and shrubs' increase slightly (maximum 3.6\%). The number of types represented has increased (6) and the relative frequencies of Juniperus have generally increased slightly (maximum 3.6\%). 'Woodland herbs' decline in the lower half of the zone $(1.3 \%$ minimum), although they increase again in the upper half where they reach $8.0 \%$. Blechnum spicant continues as the most abundant type in this group $(0.3-5.5 \%)$. Thalictrum aquilegifolium increases in the upper half of the zone, reaching a maximum of $1.9 \%$. All other types of this group occur at less than $1.0 \%$ with the exception of Asplenium type in the uppermost spectrum (1.9\%). Other groups poorly represented are 'riverine trees' (decline slightly to a maximum of only $1.4 \%$ ), 'evergreen woodland trees' (no pollen count exceeds $0.8 \%$ ), and 'plants of open dry rocky habitats' (usually less than $2 \%$, although a peak in Herniaria type in the basal spectrum reaches 5.4\%).

'Herbs of open ground' decline slightly, but remain the second most abundant habitat group (16.8-36.4\%), and again the curve is more or less the inverse of that of 'coniferous and deciduous canopy trees'. There is little change in the representation of the individual types, with the exception that, in general, Pteridium aquilinum continues to decline $(7.2-16.8 \%$, except for the spectrum at $130 \mathrm{~cm}$ in which it reaches $25.0 \%$ ) and Hieracium type increases towards the top of the zone ( $2.4 \%$ maximum).

'Cultivars' decline slightly $(0.8-2.6 \%$, except in the basal spectrum $3.4 \%)$ due to a slight decline in Secale cereale (0-0.8\%). Triticum type and Avena continue to occur sporadically ( 0.6 and $0.7 \%$ maximum, respectively). Castanea sativa, Juglans regia, and Platanus orientalis continue to occur sporadically, but reach higher frequencies $(2.3,0.4$, and $1.2 \%$, respectively).

Zone $A-2,95.0-25.0 \mathrm{~cm}$ The boundary with the preceding zone is marked by a decline in Pinus (Diploxylon), the near disappearance of Abies, Ostrya type, and Blechnum spicant, and an increase in Plantago lanceolata type and Solidago type. This zone is characterised by a decline in the relative frequencies of Pinus (Diploxylon) and Fagus, and the near disappearance of Abies and Ostrya type. Other characteristics are the increase in Juniperus and several types of 'herbs of open ground' and the decreased ratio of terrestrial trees and shrubs to terrestrial herbs.

'Alpine shrubs' and 'alpine herbs' are again extremely poorly represented; $0.6 \%$ maximum.

In the habitat group 'coniferous and deciduous canopy trees' Pinus (Diploxylon) declines to $15.3 \%$, the minimum of the sequence, in the centre of the zone. Relative frequencies of Fagus describe a curve similar to that of Pinus, reaching the zonal minimum value of $3.6 \%$ 
in the centre of the zone. Relative frequencies of Quercus frainetto type remain about the same except that they drop to about $2.5 \%$ in the central spectra. Abies almost disappears, recording a maximum of only $0.7 \%$. Ostrya type also declines (maximum $2.0 \%$ ). No other type of the group exceeds $1.1 \%$.

'Woodland understorey trees and shrubs' increase to a maximum of $11.2 \%$, due to an increase in Juniperus, which reaches a maximum of 10.8\%. 'Woodland herbs' decline (1.7$4.3 \%$ ). Blechnum spicant is greatly reduced, being represented only sporadically and reaching a maximum of only $1.6 \%$. Thalictrum aquilegifolium increases slightly, now occurring continuously and reaching a maximum of $2.5 \%$. No other type of this group exceeds $1.0 \%$.

'Riverine trees' increase slightly, due to a slight increase in several tree types: Alnus glutinosa, Salix triandra, and Tamarix; but frequencies of these types do not exceed $1.6 \%$. 'Evergreen woodland trees' are very poorly represented; no type exceeds $0.7 \%$. Relative frequencies of 'plants of open dry rocky habitats' are also extremely low although a peak in the centre of the zone reaches $5.2 \%$ due to increased frequencies of several types including Campanula erinus type, Helianthemum, Herniaria type, and Jasione, but no type exceeds $1.2 \%$.

'Herbs of open ground' increase in the centre of the zone to the maximum of the sequence of $61.8 \%$ at $60 \mathrm{~cm}$, and in this spectrum are more abundant than 'coniferous and deciduous canopy trees'. Several types increase including Dactylis type, Festuca type, Hieracium type, Lotus type, Plantago coronopus, Plantago lanceolata type, Plantago major, Plantago media, Rumex acetosa group, Solidago type, and Urtica dioica. Pteridium aquilinum fluctuates, but in general declines.

'Cultivars' increase slightly (1.6-5.0\%), due mostly to an increase in Castanea sativa, which now occurs continuously and reaches a maximum of $2.1 \%$. The other trees, Juglans regia and Platanus orientalis, continue occurring sporadically. Secale cereale and Triticum type occur sporadically ( 0.9 and $1.0 \%$ maximum, respectively). Hordeum type occurs in the basal spectrum only at $0.2 \%$. Avena disappears.

Zone A-1, 25.0-0.0 cm This zone comprises only three spectra, including the spectrum from the surface sample. The boundary with the preceding zone is marked by an increase in Fagus, a decline in Pteridium aquilinum, and a decline in 'herbs of open ground'. This zone is characterised by an increase in the relative frequencies of Pinus (Diploxylon) and Fagus, high frequencies of Juniperus and a decline in several types of the habitat group of 'herbs of open ground', and an increase in the ratio of terrestrial trees and shrubs to terrestrial herbs.

'Alpine shrubs' are not represented and 'alpine herbs' are represented in one spectrum at $0.2 \%$. 
In the habitat group 'coniferous and deciduous canopy trees' Pinus (Diploxylon) records about $42.0 \%$ in the two lower spectra, then declines to $30.1 \%$. Fagus rises sharply from $18.4 \%$ at the base of the zone to $40.1 \%$ in the centre, then falls to $23.5 \%$. Quercus frainetto type remains about the same. Abies reaches a maximum of $1.2 \%$, while Ostrya type increases from $0.6 \%$ at the base of the zone to $4.8 \%$ at the top. No other type of the group exceeds $1.2 \%$.

'Woodland understorey trees and shrubs' maintain a high relative frequency $(9.5 \%)$ in the basal spectrum, then decline to $1.2 \%$ (values are due entirely to Juniperus). 'Woodland herbs' decline slightly and reach a maximum of only $2.5 \%$. All types in this group decline. 'Riverine trees' remain more or less unchanged (1.0-2.4\%), although Salix triandra declines slightly. Quercus coccifera type, the only type of the 'evergreen woodland trees' group represented, records $3.0 \%$ in the surface spectrum. Frequencies of 'plants of open dry rocky habitats' are extremely low and no type exceeds $0.5 \%$.

'Herbs of open ground' decline to the lowest values of the sequence (7.5-20.5\%). Pteridium aquilinum declines sharply to a maximum of $1.8 \%$ in the basal spectrum. All other taxa of the group either remain unchanged or decline. 'Cultivars' decline slightly, then increase again in the surface spectrum. Secale cereale is the only cereal occurring in this zone and it occurs in the middle spectrum only at $0.2 \%$. Castanea sativa and Juglans regia occur sporadically at low values. Platanus orientalis occurs continuously but reaches a maximum of only $2.4 \%$ in the surface spectrum.

\subsubsection{Terrestrial vegetation history}

This sequence records the vegetation history of the Anelia area over the past c. 450 years. Pollen of the habitat group 'coniferous and deciduous canopy trees' is the most abundant throughout the sequence with the exception of two spectra, at 230 and $60 \mathrm{~cm}$, when pollen of 'herbs of open ground', is more abundant. Pinus (Diploxylon) is the most abundant pollen type in the former habitat group, with Fagus, Abies (below $100 \mathrm{~cm}$ ), and Quercus frainetto type the next most abundant. 'Woodland herbs' and 'cultivars' are well-represented throughout the sequence and 'woodland understorey trees and shrubs' well-represented above $75 \mathrm{~cm}$.

There is close correspondence between changes in the composition of the sediment and changes in the terrestrial pollen profile, indicating that changes in the pollen assemblages are largely a response to changes in the catchment. This correspondence implies that much of the pollen and spores in the sediments derive from the catchment, so the pollen record reflects primarily the extra-local vegetation. However, the primary source of the pollen and spores preserved in this sequence would vary according to the amount of allochthonous material 
being washed into the wetland and the amount of canopy cover in the catchment. Where catastrophic erosion has occurred most palynomorphs would be washed-in from a wide area of the catchment. During peat formation a higher proportion of pollen and spores (apart from local pollen) would arrive from the air, possibly with a larger regional component.

Lack of corrosive deterioration suggests that pollen derived from erosion of older sediments is minimal and inwashed pollen is derived from surface materials containing pollen derived from plants living at or shortly before the time of redeposition.

The terrestrial vegetation history of the Anelia area is discussed below according to the local pollen assemblage zones.

Zone A-5, 250.0-212.5 cm, c. 1560-1585 AD Pinus (Diploxylon) occurs at high relative frequencies (apart from one spectrum at $230 \mathrm{~cm}$ ); values are similar to those of the surface sample from this site. Today pine does not grow in abundance close to the Anelia site, so perhaps the Pinus (Diploxylon) pollen in this zone is derived mostly from a regional pollen source. Concentration data indicate that initially concentration was very low (but about twice that of the surface sample). There are two peaks in the concentration curve separated by two spectra with values slightly more than the initial value. The peaks reach values three and four times greater than that of the initial value. Values of Fagus are about average for the sequence in both relative frequency and concentration, although relative frequencies are much lower than those of the surface sample concentration values are very similar and suggest an extralocal pollen source. Both the relative frequency and concentration values of Quercus frainetto type (deciduous oaks) are also about average for the sequence and relative frequencies are very similar to those of the surface sample, which probably indicates a regional pollen source. Relative frequency and concentration values of Abies are also about average for this sequence, but much greater than those of the surface sample. Estimation of pollen source for Abies is difficult because of its current absence from the vicinity of the site and its current sparseness in Grevena Province. However, from the limited surface sample investigation of this pollen type, and considering the size of the pollen, long distance transport is probably not likely to result in such high values. Ostrya type occurs at its lowest values; pollen of this type is anemophilous, has high relative dispersal, and is over-represented (Appendix XI). It may derive from trees that grow in a variety of habitats (but mostly in damp environments) at various elevations (Chapter 3, Appendix X). The pollen source may be extra-local or regional, or both.

Alpine shrub and herb types are extremely poorly represented in this zone, and indeed throughout this sequence, which is probably indicative of the absence of alpine vegetation in the vicinity and their low pollen production. 'Woodland understorey trees and shrubs' are poorly represented in this zone indicating a poorly developed woodland understorey, but the 
'woodland herbs' are quite well-represented. However, the most abundant members of the latter group, the ferns Asplenium and Blechnum spicant may actually be growing on the bog and not under the cover of a woodland canopy. The relative frequency and concentration values of 'riverine trees' are so low that an extra-local presence is very unlikely; the pollen may derive from trees growing near more distant streams. 'Evergreen woodland trees' and 'plants of open rocky habitats' are also extremely poorly represented indicating the absence of a Mediterranean-type vegetation. The relative frequency and concentration values of 'herbs of open ground' are very high, with highest concentration values for the sequence, but this may be due, in part, to higher total concentrations. Of particular interest is the occurrence of Centaurea cyanus type, Galium type, and Sinapis type, albeit at low abundance, which include species that are weeds of cereal fields.

'Cultivars' are abundant and have the highest concentration values for the sequence, perhaps in part due to higher total concentrations. The most abundant pollen type in this group is the cereal Secale cereale, but Triticum is also abundant, and the cereal Avena is also represented. The trees Castanea sativa, Juglans regia, and Platanus orientalis, which may have been planted or profited from human induced changes in habitats, are also present (Chapter 3). Vitis vinifera is also represented, but it may have been growing wild on or near the wetland (Appendix X). Distinction between different cereal pollen types is difficult; the identification of cereal type pollen is more certain. The abundance of the cereal type pollen suggests that cereals were grown close by. Dispersal of pollen of the two cereals Avena and Triticum is very poor (Chapter 3 ) so they must have been cultivated extra-locally. Dispersal of pollen of the cereal Secale cereale is better (being anemophilous), thus extra-local cultivation is not necessary. This cereal was introduced into Greece during the Roman Period (Renfrew, J. 1969, 1973a), suggesting that the base of this zone is not older than 146 BC, in conformity with suggested dating.

Great abundance of 'herbs of open ground' and moderately high palynological richness values (expected number 25-30) suggest the possibility of an open canopy extra-locally. The ratio of terrestrial trees and shrubs to terrestrial herbs is low; the sum of terrestrial trees and shrubs represents $38.0-61.8 \%$ of total terrestrial pollen and spores. However, it is lowest in the centre of the zone coinciding with peat formation and many of the herbs contributing to these high values may have been growing on the bog.

The extra-local vegetation surrounding the Anelia site at this time was probably a woodland mosaic of beech and fir with an open canopy. The open canopy may be due in part to coppicing - beech coppices at least 300 years old are locally common around the wetland (Chapter 1). Modern analogues of this type of woodland can be found at intermediate elevations in the Pindos Mountains today (Chapter 3). Pine woods probably grew at a higher 
elevation, while deciduous oak woods grew at lower elevations on the foothills. Cereals were cultivated extra-locally and the nut trees of Castanea sativa and Juglans regia may have been planted either sparsely in the vicinity of the site or more abundantly at a greater distance. A habitat suitable for Platanus orientalis may have been created by human disturbance of the natural extra-local vegetation, or this tree may have been growing wild either extra-locally or at a greater distance from the site (Chapter 3).

Zone A-4, 212.5-157.0 cm, c. 1585-1625 AD Relative frequencies of Pinus (Diploxylon) remain almost unchanged apart from a decline in the uppermost spectrum, but concentration values are much lower than in the zone below and are extremely low in the two uppermost spectra. These lower concentration values are reflected in the total concentration values and are probably related to the influx of minerogenic sediments (and therefore more rapid deposition). Relative frequencies of Fagus increase, but concentration values remain about the same, except for lower values in the basal spectrum in a sand horizon, and in two gravel horizons in the centre and at the top of the zone. The relative frequencies of Quercus frainetto type remain unchanged until the centre of the zone where they increase slightly. However, concentration values of Quercus frainetto type remain very low and decline in the same manner as those of Fagus. Relative frequency and concentration values of Abies both increase; concentration values are again low where minerogenic sediments occur. Relative frequencies and concentrations of Ostrya type increase; again the influence of the influx of the minerogenic sediments can be seen in the concentration curve.

'Alpine shrubs' and 'alpine herbs' are again very poorly represented. The relative frequencies of 'woodland understorey trees and shrubs' have increased slightly, due to an increase in Juniperus although the increase in this type is not enough to be significant and there is no change in concentration values. Relative frequency and concentration values of 'woodland herbs' decline, due mostly to declining values of Asplenium and Blechnum spicant. The values of 'riverine trees', 'evergreen woodland trees', and 'plants of open dry rocky habitats' remain unchanged. Relative frequency and concentration values of 'herbs of open ground' are reduced and the concentration curve displays the influence of the influx of the minerogenic sediments. The reduction in this group is due mostly to reductions in the values of Pteridium aquilinum, the concentration curve of which follows the same pattern as the habitat group. Of particular interest is the occurrence of Agrostemma githago, Centaurea cyanus type, Galium type, Nigella, and Sinapis type, albeit at low abundance, which include species that are weeds of cereal fields. 'Cultivars' decline, due mostly to a reduction in Secale cereale, but values are still high.

The great abundance of 'herbs of open ground' and moderately high palynological richness suggest an open canopy extra-local woodland. However, the ratio of terrestrial trees 
and shrubs to terrestrial herbs has increased; trees and shrubs represent $57.8-70.8 \%$ of total terrestrial pollen. Fagus and Abies, which are interpreted as comprising the main components of the extra-local woodland, both increase and the habitat group 'herbs of open ground' declines. This is thought to indicate either an incompletely closed canopy or an expanded extra-local woodland.

The regional pine woods of the mountains decline while the deciduous oak woods of the foothills are unchanged. The extra-local woodland mosaic of Fagus and Abies expands or becomes more dense. Cultivation of Secale cereale may have declined slightly, while the other cereals are still cultivated extra-locally and the nut trees, Castanea sativa and Juglans regia, and the shade-tree Platanus orientalis continue to grow (either planted or encouraged by human disturbance) either sparsely extra-locally or perhaps more abundantly at a greater distance from the site.

Zone A-3, 157.0-95.0 cm, c. 1625-1730 AD Relative frequencies of Pinus (Diploxylon) increase. Concentration values also increase, except in the two uppermost spectra where sediments of sandy muddy gravel occur. (These fluctuations in concentrations are also seen in total concentration.) Relative frequencies of Fagus also increase in general, but are lower in the three lowest spectra and in the uppermost. The curve of the concentration diagram also follows this pattern except that the two uppermost spectra, where the minerogenic sediments occur, are lower in value. Relative frequency values of Quercus frainetto type remain almost constant, but the concentration values are lower in the spectra where the minerogenic sediments occur. Both relative frequency and concentration values of Abies and Ostrya type are lower and decline steadily upwards.

'Alpine shrubs' and 'alpine herbs' are again very poorly represented. Relative frequencies of 'woodland understorey trees and shrubs' increase slightly due to an increase in Juniperus, and there is a slight increase in concentration values. Relative frequency and concentration values of 'woodland herbs' increase towards the top of the zone due mostly to an increase in the values of Asplenium and Blechnum spicant, which may have been growing on the bog. The values of 'riverine trees', 'evergreen woodland trees', and 'plants of open dry rocky habitats' remain unchanged, apart from an increase in the 'plants of open dry rocky habitats' in the lowest spectrum due to an increase in Herniaria type. Species of this pollen type may have been growing on eroded gravel.

Relative frequencies of 'herbs of open ground' are in general lower than in the zone below, due to lower values of Pteridium aquilinum, but concentration values remain unchanged except for reduced values in the two uppermost spectra where minerogenic sediments occur. The concentration curve of Pteridium aquilinum follows the curve of the habitat group to which it belongs. Of particular interest is the occurrence of Galium type and 
Sinapis type, albeit at low abundance, which include species that are weeds of cereal fields. The values of 'cultivars' remain unchanged, and there is no change in pollen type representation.

Palynological richness is between 25 and 30 expected types except for a decrease in the centre of the zone where it declines to 20. These values are similar to those of the basal zone, where supporting evidence indicates an open canopy, however in this zone (A-3) other evidence points towards a partially closed canopy. The ratio of terrestrial trees and shrubs to herbs increases. Trees and shrubs represent $58.2-78.4 \%$ of total terrestrial pollen. The habitat group of 'coniferous and deciduous canopy trees' increases, in general Fagus increases and the habitat group 'herbs of open ground' declines. These changes are interpreted as indicative of a further closing of canopy and/or an expansion of extra-local woodland, but not to a degree affecting the diversity of types.

The regional pine woods of the mountains expand and the deciduous oak woods of the foothills remain unchanged. The proportion of the Fagus component of the extra-local woods increases while that of Abies steadily declines. The level of intensity of extra-local cereal cultivation remains unchanged. The possible small-scale extra-local or more distant largerscale cultivation of Castanea sativa, Juglans regia, and Platanus orientalis continues at about the same level of intensity also.

Zone A-2, 95.0-25.0 cm, c. 1730-1920 AD Relative frequencies of Pinus (Diploxylon), Fagus, and Quercus frainetto type decline in the centre of the zone displaying very low values in the spectrum at $60 \mathrm{~cm}$. Concentration values of these types continue more or less the same as those of the preceding zone until $60 \mathrm{~cm}$ where they decline. They remain at lower values to the top of the zone. This pattern of concentration values is reflected in total concentration. Lower concentration values at the top of the zone are probably due to more rapid peat accumulation. Ostrya type declines to very low values, but follows the same pattern as the types above. Abies is greatly reduced at the base of the zone and almost disappears.

'Alpine shrubs' and 'alpine herbs' are again very poorly represented. 'Woodland understorey trees and shrubs' increase significantly from $70 \mathrm{~cm}$, due to an increase in Juniperus. The concentration curve also shows this increase, although not as prominently due to the more rapid peat accumulation. Relative frequency values of 'woodland herbs' are generally slightly higher than in the zone below, although concentration values are lower. Relative frequencies of 'riverine trees' increase slightly, but concentration values are very low. The values of 'evergreen woodland trees' remain extremely low as do those of 'plants of open dry rocky habitats', apart from an increase at $60 \mathrm{~cm}$ due to the appearance (at low value) or slight increase in several types. 
Relative frequencies of 'herbs of open ground' are higher than those of the zone below and a sharp peak occurs in the relative frequency curve at $60 \mathrm{~cm}$. Frequencies of individual types of this group are in general higher than in the zone below, i.e. in Dactylis type, Festuca type, Hieracium type, Lotus type, several Plantago pollen types, Rumex acetosa group, Solidago type, and Urtica dioica. Concentration values follow the total concentration curve; they increase in the lower half of the zone, then decline. Both relative frequency and concentration values of Pteridium aquilinum decline steadily. Of particular interest is the occurrence of Buglossoides arvensis type, Centaurea cyanus type, Galium type, Nigella, Ranunculus arvensis type, and Sinapis type, which include species that are weeds of cereal fields.

Relative frequencies of 'cultivars' increase, although concentration values of the group are almost unchanged. However, changes have occurred in the representation of individual types. Cereal types have declined slightly with Secale cereale now occurring sporadically. Triticum type continues to occur sporadically and Avena disappears; Hordeum type appears once. Castanea sativa increases and now occurs continuously. Juglans regia and Platanus orientalis continue to occur sporadically.

Palynological richness increases to values mostly about 35 expected types and above indicating an open canopy. The ratio of terrestrial trees and shrubs to herbs declines, with a sharp decline in the centre of the zone where trees and shrubs represent only $29.6 \%$ of total terrestrial pollen. When this spectrum is excluded terrestrial trees and shrubs represent from $57.3-71.5 \%$ of total terrestrial pollen. Other supporting evidence for an open canopy is the decline of 'coniferous and deciduous canopy trees', the near disappearance of Abies, and an increase of 'herbs of open ground'.

Regional pine woods of the mountains are reduced. The deciduous oak woods of the foothills are probably unchanged. The Fagus component of the extra-local woods is reduced and Abies declines sharply at $90 \mathrm{~cm}$. Representation values of Abies are similar to those of the surface sample suggesting that only scattered trees remain (Chapter 3 ). The decline in Abies may be due to lumbering, which is known to have been well established in the early 19th century (Leake 1835). Juniperus increases significantly above $70 \mathrm{~cm}$. Today Juniperus is ubiquitous in Grevena growing at elevations from the lowlands to the mountains. The source of this pollen may be extra-local and/or regional. The level of intensity of extra-local cereal cultivation probably declines slightly. Extra-local or regional cultivation of the nut tree Castanea sativa may have increased. Juglans regia and Platanus orientalis continue to grow either sparsely extra-locally or perhaps more abundantly at a greater distance from the site.

Zone A-1, 25.0-0.0 cm, c. 1920-1989 AD Relative frequency and concentration values of Pinus (Diploxylon) and Fagus increase, while those of Quercus frainetto type continue at 
about the same values. Abies does not recover, but Ostrya type increases a little in the surface sample.

The habitat group 'alpine shrubs' is not represented and 'alpine herbs' are represented in only one spectrum at an extremely low value. 'Woodland understorey trees and shrubs' decline towards the surface sample, due to declining values in Juniperus, while 'woodland herbs' remain constant at comparatively low values. 'Riverine trees' also remain constant at low values. 'Evergreen woodland trees' increase in the surface sample to reach the maximum of the sequence. This is due entirely to a count of Quercus coccifera type. Values of 'plants of open dry rocky habitats' are extremely low in the basal spectra then the group disappears.

'Herbs of open ground' record the lowest values of the sequence; all types either decline or disappear. Galium type, Nigella (in the basal spectrum only), and Sinapis type, which include species that are weeds of cereal fields, occur. 'Cultivars' decline slightly, due mostly to a reduction in Castanea sativa, the near disappearance of cereals, and an increase in Platanus orientalis. The only cereal present is Secale cereale, which occurs in the central spectrum only at low abundance. Pollen of Secale cereale is anemophilous so this pollen may be long-distance transported, or may derive from wild plants, thus no cereal cultivation may be occurring extra-locally at this time. Juglans regia continues to occur sporadically.

Palynological richness fluctuates between 30 and 20, which is indicative of a closed canopy. The very high ratio of terrestrial trees and shrubs to terrestrial herbs also supports the establishment of a closed canopy. Trees and shrubs contribute about $75-90 \%$ of total terrestrial pollen. A closed canopy is also indicated by the low values of 'herbs of open ground'.

The regional pine woods of the mountains decline in the surface sample. The deciduous oak woods of the foothills probably continue about the same. Fagus increases and an extralocal beech wood with scattered Abies and a closed canopy is indicated. Extra-local cultivation of cereals has probably ceased (no cereal cultivation occurs in the vicinity of this wetland today). Castanea sativa and Juglans regia are scarce. Platanus orientalis increases.

\subsection{Charcoal and fire history}

\subsubsection{Microscopic charcoal analysis}

Results of the microscopic charcoal particle analyses are presented as relative frequencies in Figure 5.13. The local zonation scheme of the terrestrial pollen and spores is used for convenience of correlation. The charcoal record shows high total charcoal frequencies in zones A-5 and A-3, extremely high total charcoal frequencies in zone A-4, and almost none in zones A-2 and A-1. An extremely high peak in total charcoal frequency (more than three times the value in any other spectrum) occurs at $160 \mathrm{~cm}$ in zone A-4. Visual 


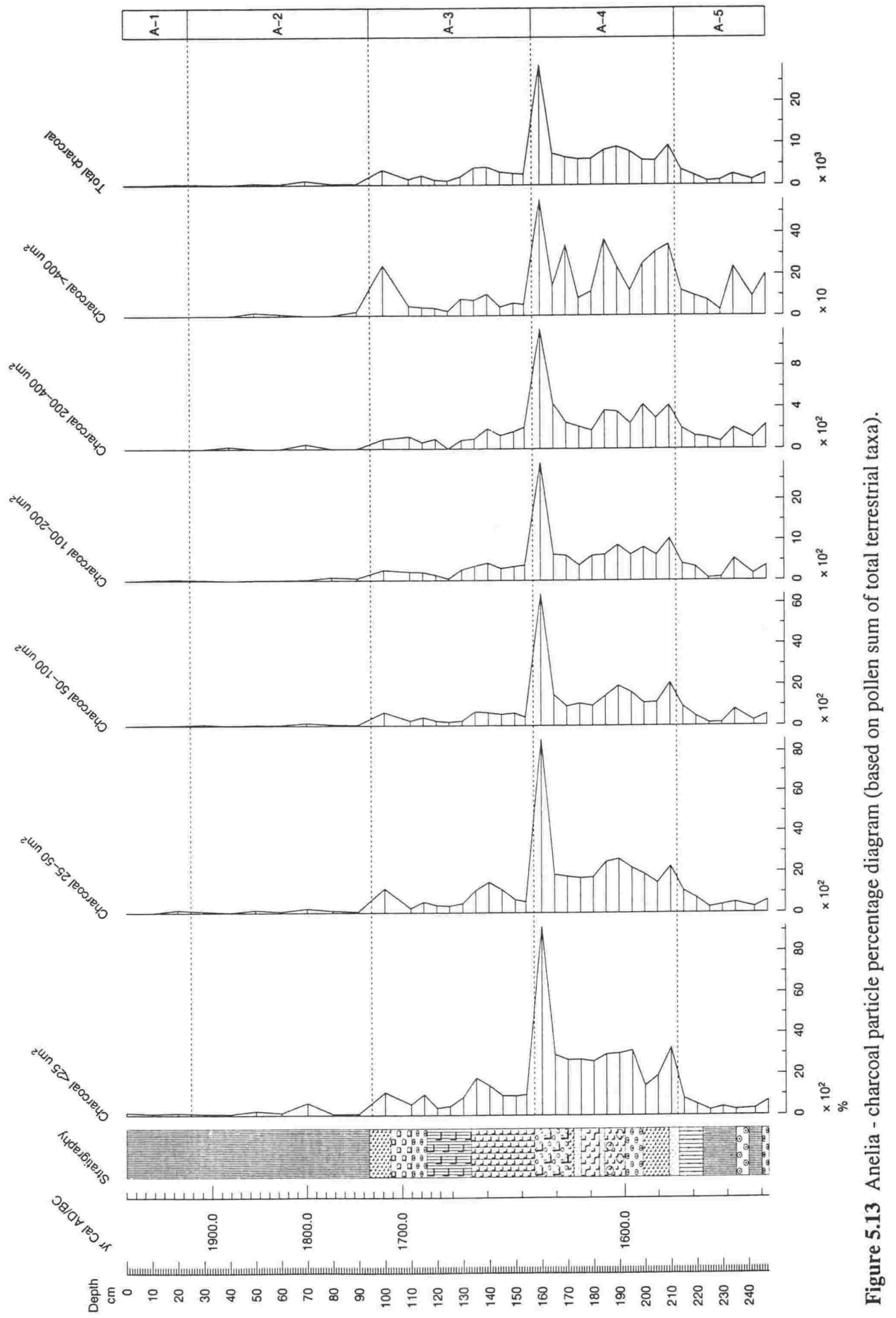


inspection of residues retained by the $260 \mu \mathrm{m}$ mesh sieve during sample preparation, showed that optically visible charcoal occurred in only those samples where the largest sized particles were observed through the microscope.

In zone A-5 all size classes are represented in every spectra. Charcoal particles are generally more abundant in the smaller size classes up to $200 \mu \mathrm{m}^{2}$, but are also abundant in the larger size classes including that $>400 \mu \mathrm{m}^{2}$, suggesting that fires occurred in the catchment (Chapter 3). Peaks in total charcoal abundance occur in the basal spectrum and at $235 \mathrm{~cm}$. These peaks occur in all size classes, with the exception of the peak at $235 \mathrm{~cm}$ in the smallest size class.

Zone A-4 records extremely high charcoal particle abundance and again all size classes are represented in every spectrum. Again charcoal particles are most abundant in the smaller size classes (that is less than $200 \mu \mathrm{m}^{2}$ ) and abundance has increased three fold from that in zone A-5, but the larger size classes are also abundant, being generally approximately twice the value of those in zone A-5. A peak occurs in the basal spectrum in all size classes and again at $160 \mathrm{~cm}$. A peak occurs between 190 and $185 \mathrm{~cm}$ in the $>400 \mu \mathrm{m}^{2}$ size class, which is not recorded in the smallest size class but is reflected in all other size classes. Another peak occurs at $170 \mathrm{~cm}$ in the $>400 \mu \mathrm{m}^{2}$ size class, which is not reflected in any other size class.

Zone A-3 displays moderate charcoal particle abundance, which is much lower than that of the zone below. Total charcoal abundance is similar to that in the basal zone. Again all size classes are represented, with the exception of the $200-400 \mu \mathrm{m}^{2}$ size class at $125 \mathrm{~cm}$. The smaller size classes are again more abundant than the larger, but the smallest size class has increased in proportion to the largest size classes. A peak occurs in the size class $25-50 \mu \mathrm{m}^{2}$ at $140 \mathrm{~cm}$, which is reflected poorly in all larger size classes. Another peak occurs at $130 \mathrm{~cm}$, but is recorded in the smallest size class only. A peak in the largest size class in the uppermost spectrum is reflected in all other size classes except that of $200-400 \mu \mathrm{m}^{2}$.

Total charcoal abundance is extremely low in zone A-2. Only the smallest size classes, up to $100 \mu \mathrm{m}^{2}$ are continuously represented. Charcoal particle abundance in the largest two size classes is very low. A peak at $70 \mathrm{~cm}$ is recorded in the smallest size class.

Total charcoal abundance in the uppermost zone is extremely low: no charcoal is recorded in the largest two size classes, and a continuous record exists for only the smallest size class.

\subsubsection{Fire history}

Today fire is not a major ecological factor, but charcoal analysis suggests greater importance in the past. There is continuous accumulation of microscopic charcoal particles of all size classes from the base of the sequence up to $100 \mathrm{~cm}$, with the exception of the 
$200-400 \mu \mathrm{m}^{2}$ size class at $125 \mathrm{~cm}$; above this depth microscopic charcoal particles of all size classes are sparse (Figure 5.13). Interpretation of charcoal data is complicated by the nonuniform sedimentation of this sequence because erosion after burning of the catchment vegetation could produce smoothed peaks or even anomalous peaks. In this sequence higher charcoal abundance coincides with influx of allochthonous minerogenic sediments into the wetland, but charcoal particles of the largest size class occur in the interbedded peat indicating that charcoal abundance is not entirely due to that washed in.

Microscopic charcoal evidence suggests that the fire history of the Anelia wetland sequence can be divided into four phases according to frequency/intensity and location. Most intense/frequent fires both extra-locally and regionally occur between c. 1585-1625 AD (terrestrial pollen assemblage zone A-4), although charcoal abundance is probably enhanced due to greater influx of minerogenic sediments. Between c. 1560-1585 AD (zone A-5) and c. 1625-1730 AD (zone A-3) fire regimes are very similar in intensity/frequency and location, being of high intensity/frequency, but not as high as that of the period between, and occurring both extra-locally and regionally. Between c. 1730-1989 AD (zones A-2 and A-1) fires do not occur extra-locally and are much reduced regionally.

Pollen evidence also suggests a high frequency of fires in the Anelia area in the lower part of the sequence. The lower pollen concentration of 'coniferous and deciduous canopy trees' between c. 1585-1625 AD (zone A-4) is thought to result from the depletion of the pollen source through the burning of the surrounding woodland vegetation (unfortunately no influx data are available to check this). Also during this period, lowered concentration values of Pinus (Diploxylon) are interpreted as due to reduction in the source of regional pine pollen through burning. Pteridium aquilinum spores are more abundant in the lower part of the sequence between c. 1560-1730 AD (below $95 \mathrm{~cm}$ ), being highest in the basal zone (c. 1560$1585 \mathrm{AD}$ ), and may indicate greater frequency of extra-local fires as Pteridium aquilinum spores have only moderate relative dispersal (Chapter 3 ). Fire sensitive Juniperus is most abundant in the upper part of the sequence, especially above $70 \mathrm{~cm}$.

\subsection{Synthesis of environmental changes}

A summary of the major environmental changes in the Anelia wetland and the surrounding area from c. 1989-1560 AD is given in Table 5.2.

There is closer correspondence between changes in the composition of the deposits and changes in the pollen profiles of wetland taxa than of the pollen profiles of terrestrial taxa. The lack of exact correspondence is due to deposition of pollen and spore types from both taxa washed in from the catchment and of taxa deposited from the atmosphere from regional and 


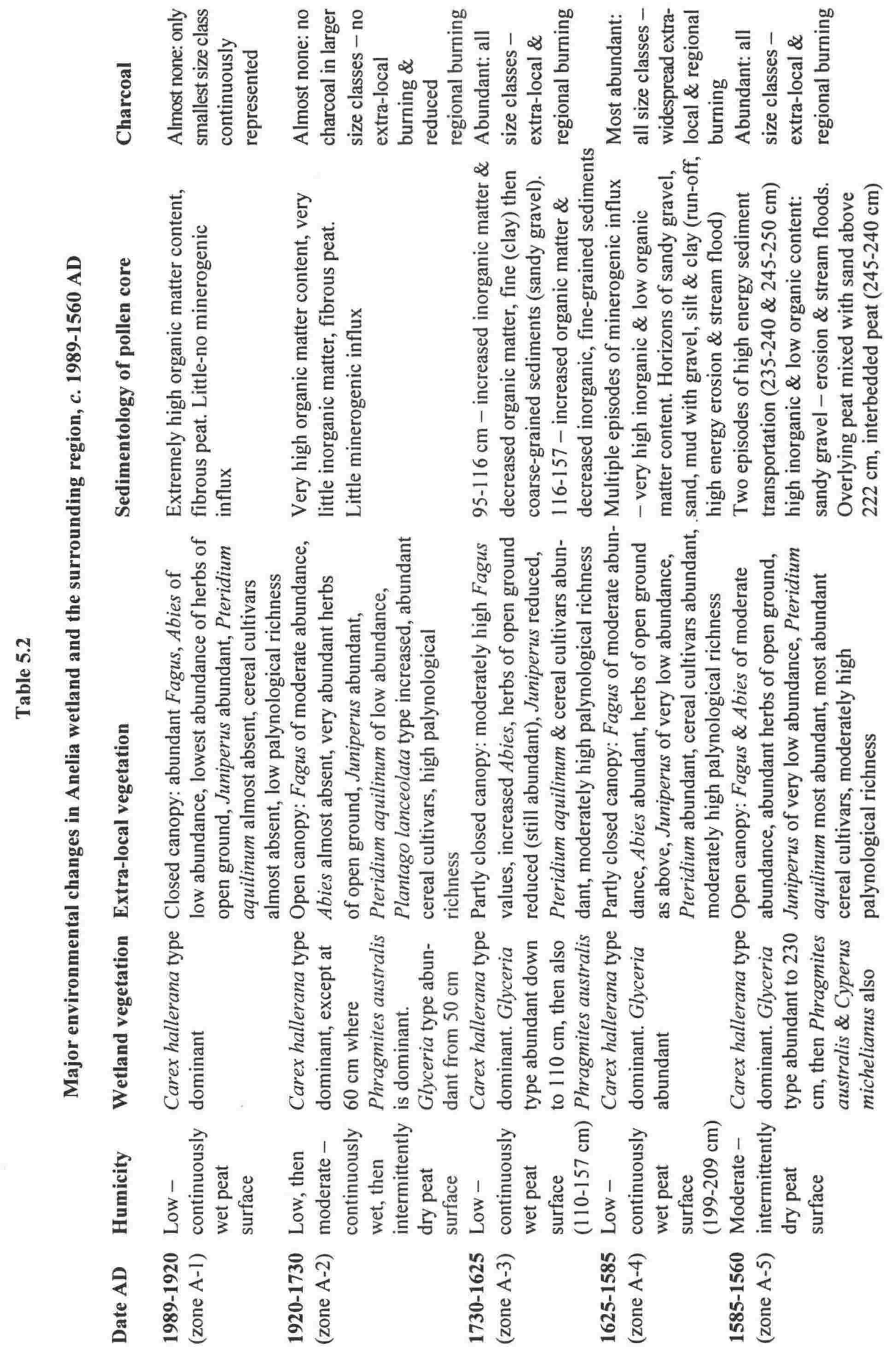


extra-local source areas. Thus the terrestrial pollen record reflects both the extra-local and regional vegetation.

The wetland formed in a hollow of a ravine a typical landscape feature of this area, which may owe its origin to tectonic movements associated with a fault about $300 \mathrm{~m}$ upslope. Broadening of the ravine and reduction in gradient caused impedance of spring-stream water flow with consequent deposition of sediments and formation of peat. As transport energy of the stream varied and the stream course changed location, so varied sediments were deposited across the wetland. Thus between $c .1560$ and $1730 \mathrm{AD}$ the wetland was in-filled with stratified clay, silt, sand, fine gravel, and peat, and mire vegetation established itself temporarily away from the stream course. At $c$. $1730 \mathrm{AD}$ peat formation became general with a permanently marginal stream channel. This situation has persisted until today.

Water level of the wetland varied as indicated by humicity of peat horizons indicating that some surface peat was continuously wet while other surface peat was intermittently dry, probably seasonally during the driest part of summer. Pollen evidence indicates that available nutrient level of the wetland is high throughout the sequence and nutrients are supplied from a soft-water spring. Nutrients were probably also contributed to the wetland via washed in sediments and from direct or indirect human activities.

There is very little apparent variation in the local wetland vegetation. It is dominated throughout the sequence (with the exception of the spectrum at $60 \mathrm{~cm}$ where Phragmites australis is dominant) by sedges of the Carex hallerana type of wet-soil plant communities, which are probably indicative of a small-sedge-swamp community similar to that growing on the bog today. Reeds of Phragmites australis and Glyceria type of true-reed-swamp communities are also prominent. Communities of floating-leaved and submerged macrophytes are poorly represented and usually represent a few plants growing in small pools on the surface of peat. Sedges, reeds, and mosses probably formed the peat deposits.

Terrestrial pollen evidence suggests that pine woods grew at higher elevations on the Pindos Mountains and deciduous oak woods grew on the foothills for the duration of the sequence. Between c. 1560 and $1730 \mathrm{AD}$ the extra-local vegetation is dominated by the canopy trees Fagus and Abies, but after c. $1730 \mathrm{AD}$ it is dominated by Fagus only. A variety of cereals were cultivated extra-locally for the duration of the sequence, except perhaps after c. $1920 \mathrm{AD}$, and may have been rotated (Chapter 1). The nut trees Castanea sativa and Juglans regia, which occur sporadically from the base of the sequence, may have been planted or growing wild either sparsely in the vicinity of the site or more abundantly at a greater distance. The shade tree Platanus orientalis, which also occurs sporadically from the base of the sequence, may also have been planted or growing wild either sparsely extra-locally or 
more abundantly at a greater distance from the site. These three trees may have profited from changes in habitats caused by humans.

There is continuous accumulation of microscopic charcoal particles of all size classes including the largest size class $\left(>400 \mu \mathrm{m}^{2}\right)$, with the exception of the size class of 200 $400 \mu \mathrm{m}^{2}$ at $125 \mathrm{~cm}$, from $c .1560 \mathrm{AD}$ (the base of the sequence) until c. $1730 \mathrm{AD}$. After this microscopic charcoal particles of all size classes are sparse.

A synthesis of environmental changes as indicated by the pollen analysed core follows. The genesis of the wetland, radiocarbon dated at c. $1560 \mathrm{AD}$, is coincident with high energy erosion in the catchment as evidenced by sediments containing a high proportion of inorganic matter, low organic matter, and comprising a mixture of sand size and larger particles. This deposit (250-245 cm) may represent a single catastrophic event such as a severe storm of very short duration on a landscape with an open-canopy vegetation. The occurrence of pollen and spores of floating-leaved and submerged aquatic macrophytes, at highest relative frequency of the sequence, in this sediment suggests that shallow ponding of water occurred. Between 245 and $213 \mathrm{~cm}$ peat formed at the coring site, which suggests a period of landscape stability in the catchment and waterlogging of the wetland. At $240-235 \mathrm{~cm}$ coarse grained sediments were again deposited at the core site, indicating another episode of high energy erosion in the catchment and stream flooding. This erosion was probably also of very short duration as peat formation continued and the pollen record does not change, and may have resulted because the tree canopy was open. Increasing amounts of sand are washed onto the peat surface from $222 \mathrm{~cm}$ until at $213 \mathrm{~cm}$ peat formation ceases, probably due to inundation by the sand, and the deposit is composed entirely of fine to medium sand. Humicity level of the peat indicates that there was intermittent drying of surface peat, until sand is washed onto the peat surface, then humicity decreases indicating continuously wet surface peat, perhaps due to stream water flowing across the peat.

Coincidentally, between c. 1560-1585 AD $(250.0-212.5 \mathrm{~cm})$ a bog community resembling that of today establishes. The dominant local plant communities are wet soil communities with sedges of the Carex hallerana type dominant. Below $230 \mathrm{~cm}$ reeds of Phragmites australis and Glyceria type of the true-reed-swamp community are equally abundant and the early hydrosere Sparganium erectum is prominent, while the annual Cyperus michelianus of the tall-sedge-swamp community is very abundant. Above this sedges of the Carex hallerana type become much more abundant, Cyperus michelianus and Sparganium erectum disappear and reeds of Glyceria type become more abundant than Phragmites australis. The extra-local vegetation at this time was probably a woodland mosaic of beech and fir with an open canopy. The open canopy may be due in part to coppicing - beech coppices at least 300 years old are locally common around the wetland (Chapter 1). Modern 
analogues of this type of woodland can be found today at intermediate elevations on the Pindos. Herbs of open ground are almost as abundant as coniferous and deciduous canopy trees and Pteridium aquilinum is more abundant than at any other time. Other herbs of open ground that are very abundant are the terrestrial grasses Dactylis type and Festuca type, Artemisia, Chenopodiaceae type, Cirsium type, Hieracium type, Hornungia type, Plantago lanceolata type, Rumex acetosa group, and Solidago type. Pollen types including species that are likely to have been cultivated reach maximum concentration of the sequence at $245-220 \mathrm{~cm}$; the most abundant are the cereal types Secale cereale and Triticum type. Secale cereale may have been grown mostly for fodder. Avena also occurs. Castanea sativa, Juglans regia, and Platanus orientalis occur sporadically and Vitis vinifera occurs in one spectrum. At this time pine woods probably grew at a higher elevations than that of the Anelia site on the Pindos Mountains, while deciduous oak woods grew at lower elevations on the foothills. Burning of the catchment and regional vegetation is suggested by the abundance of microscopic charcoal particles of all size classes and of spores of Pteridium aquilinum. Firesensitive Juniperus occurs at very low abundance. Peaks in microscopic charcoal particle abundance correspond to influx of sediments.

Between c. 1585 and $1625 \mathrm{AD}$ there is greater instability in the catchment landscape as evidenced by continuous influx of minerogenic sediments of varying grain size, and peat forms at the core site only at $209-199 \mathrm{~cm}$. Fine to medium sand is deposited up to $209 \mathrm{~cm}$, during moderate energy stream flow over the core site, then fine-grained sediments (clay and silt) are deposited, indicating low energy transportation and deposition, while peat forms. Humicity of the peat indicates that surface peat is continuously wet. These fine-grained sediments probably derive from run-off from bared soils in the catchment. At $199 \mathrm{~cm}$ coarse-grained inorganic sediment is deposited. This probably represents another catastrophic event of high energy erosion in the catchment and stream flood. Erosion, perhaps of longer duration, follows as indicated by the mixture of coarse- and fine-grained inorganic sediment from 192-157 cm, signalling a period of increased landscape instability in the catchment, when an open canopy allows accelerated erosion. Interbedded sand deposits at 184-182 and $175-172 \mathrm{~cm}$ represent periods when the stream flowed over the core site at moderate levels of energy flow.

During this period local plant communities of wet soil habitats are dominant, with sedges of the Carex hallerana type dominant. A tall-sedge community develops dominated by sedges of Carex flacca type, Cyperus michelianus, and Cyperus longus type (the pollen of each type occurs sporadically, due to unstable conditions on the wetland) and the true-reed swamp is dominated by reeds of Glyceria type; Phragmites australis has declined, probably due to a lack of tolerance to 'silting', which the former type tolerates. Aquatic macrophytes 
occur rarely at very low abundance and the appearance of Salix fragilis type pollen at $195 \mathrm{~cm}$ suggests that part of the surface of the wetland was dry enough to allow willow growth. The extra-local woodland mosaic of Fagus and Abies expands or becomes more dense with greater canopy cover. Herbs of open ground decline, but are still abundant. The most abundant types in this group are the same as previously. Pteridium aquilinum declines, but is still abundant. Cultivation of Secale cereale may have declined slightly, but it and the other cereals of the Triticum type and Avena are still cultivated extra-locally. The nut trees, Castanea sativa and Juglans regia, and the shade-tree Platanus orientalis continue to grow (either planted or encouraged by human disturbance) either sparsely extra-locally or perhaps more abundantly at a greater distance from the site. The regional mountain pine woods decline while the deciduous oak woods of the foothills are unchanged. Most abundant microscopic charcoal particles occur at this time; values increase two-three times those below, but abundances are enhanced by sediment influx. Lowered concentration values of Pinus (Diploxylon) are interpreted as due to the source of regional pine pollen being reduced through burning. High abundance of Pteridium aquilinum spores supports extra-local burning of vegetation and Juniperus occurs at very low abundance.

Between c. 1625 and $1730 \mathrm{AD}$ greater landscape stability in the catchment and waterlogging of the wetland is indicated as peat formation begins again while fine-grained sediments are deposited. Deposition of allochthonous mineral material steadily decreases up to $130 \mathrm{~cm}$ and grain size is never larger than silt size, with the exception of a trace of fine gravel between 130-126 cm, which may represent a stream flood event of short duration. The fine-grained sediments are indicative of low energy transport and are probably deposited as run-off. Influx of fine-grained sediment increases between $130-126 \mathrm{~cm}$, but declines sharply at $126 \mathrm{~cm}$. At $116 \mathrm{~cm}$ another period of high energy stream input and accelerated erosion of the catchment begins when sediment of mostly coarse-grained particles begins inundating the peat until at $110 \mathrm{~cm}$ peat forming vegetation is overwhelmed. Humicity of the peat indicates that the peat surface is continuously wet. Erosion of the catchment and deposition of sediment of a mixture of fine- and coarse-grained particles at the core site continues until $102 \mathrm{~cm}$ then fine-grained inorganic sediment representing declining stream energy or sediment yield from the catchment is deposited.

Plant communities of wet soil habitats continue to be the dominant wetland communities and sedges of the Carex hallerana type are by far the most abundant plants represented suggesting the establishment of a well developed small-sedge-swamp community. The tallsedge-swamp community is dominated by sedges of the Cyperus longus type up to $120 \mathrm{~cm}$ when it almost disappears and Cyperus michelianus declines indicating a more stable wetland vegetation. Reeds of Glyceria type and Phragmites australis are the most abundant plants of 
the true-reed-swamp community. Those of Glyceria type are dominant and Phragmites australis is less abundant above $110 \mathrm{~cm}$ where peat formation ceases and influx of sediments increases. Pollen of aquatic macrophytes is generally very sparse and probably represents aquatic plants growing in pools on peat surface, with the exception of an increase at $100 \mathrm{~cm}$, which may indicate ponding of water. The Fagus component of the extra-local mosaic woods increases while that of Abies steadily declines, perhaps due to lumbering, and the canopy may become slightly more closed. Herbs of open ground decline slightly, but are still abundant and the most abundant types of this group are the same as previously. Pteridium aquilinum declines. but is still abundant. Secale cereale and the other cereals of the Triticum type and Avena are still cultivated extra-locally. Castanea sativa, Juglans regia, and Platanus orientalis continue to grow either sparsely extra-locally or perhaps more abundantly at a greater distance from the site. The regional mountain pine woods expand or become more dense, while the deciduous oak woods of the foothills are unchanged. Abundance of microscopic charcoal particles of all size classes is reduced to an abundance similar to that between $c .1560$ and $1585 \mathrm{AD}$, but indicate that extra-local and regional burning is occurring. Pteridium aquilinum spores are abundant and Juniperus is of low abundance, supporting extra-local and regional burning of vegetation.

Landscape stability of the catchment and on the wetland is indicated between $c .1730$ and $1920 \mathrm{AD}$ when the lower part, $95.0-25.0 \mathrm{~cm}$, of the fibrous peat horizon of the current bog formed. It is characterised by very high organic and low inorganic content and very little minerogenic influx occurs. The level of humicity of the peat declines from 2 at the base of the horizon, where surface peat is intermittently dry, to 1 , where surface peat is continuously wet. The occurrence of pollen of floating-leaved macrophytes suggests that pools existed on the peat surface, but the occurrence of pollen of Salix fragilis type suggests that some part of the wetland was dry enough for willow growth. Plant communities of wet soil continue to be dominant on the wetland, and sedges of the Carex hallerana type are dominant, with the exception of the spectrum at $60 \mathrm{~cm}$ when the true-reed-swamp community becomes dominant with a large increase in Phragmites australis. The dramatic rise in Phragmites australis is probably a succession following a reduction in grazing on the wetland or other limiting factors. The tall-sedge community is poorly represented and the true-reed-swamp community is dominated by reeds of Glyceria type, with the exception of Phragmites australis at 60 and $55 \mathrm{~cm}$. This community is much reduced above $40 \mathrm{~cm}$. The extra-local woodland is now an open canopy beech wood with scattered Abies trees. The canopy is probably open due to lumbering of Abies trees and the coppicing of Fagus for fuel and fodder. Lumbering was well established in the early 19th century (Leake 1835). Herbs of open ground increase at $60 \mathrm{~cm}$ to the maximum of the sequence, and in this spectrum are more abundant than coniferous and 
deciduous canopy trees. Types of this group that increase are the grasses Dactylis type and Festuca type, and Hieracium type, Lotus type, several Plantago pollen types, Rumex acetosa group, Solidago type, and Urtica dioica, suggesting increased grazing. Pteridium aquilinum fluctuates, but in general declines. Extra-local cereal cultivation may decline as Secale cereale pollen now occurs sporadically and Avena disappears. Triticum type continues occurring sporadically and Hordeum type appears once. Castanea sativa now occurs continuously and the other trees, Juglans regia and Platanus orientalis, continue occurring sporadically. Regional mountain pine woods are reduced, perhaps through increased grazing pressure. The deciduous oak woods of the foothills are probably unchanged. Almost no microscopic charcoal was recorded during this period and only the smallest size classes, up to $100 \mu \mathrm{m}^{2}$ are continuously represented. No extra-local burning of vegetation occurs and regional burning is much reduced. Declining abundance of Pteridium aquilinum spores and increased abundance of Juniperus pollen at $70 \mathrm{~cm}$ supports reduced burning of vegetation.

Between c. 1920 and 1989 AD stability of the catchment landscape and on the wetland is again indicated. The upper part of the fibrous peat horizon, $25.0-0 \mathrm{~cm}$, is characterised by almost no inorganic matter content - very little or no minerogenic influx occurs - and extremely high organic matter content (with the exception of the surface sample where the proportion of organic matter content falls due to an increase in moisture content). An increase in wetness of the bog surface is also indicated by an increase in the floating-leaved aquatic macrophytes in the surface sample (although submerged aquatic macrophytes disappear) and a recently dead pine tree at the north end of the bog may be a response to increased wetness in the bog. This increased wetness may be due to greater humidity caused by a closed canopy beech wood growing close to the wetland, as it does today. Humicity of the peat indicates that surface peat is continuously wet. Wetland plant communities are the same as those below until the surface sample where wet soil communities decline slightly, perhaps as a response to greater wetness of the bog surface. Within this community changes occur in the abundances of pollen types: Carex hallerana type declines and pollen of Parnassia palustris is equally abundant, while Mentha type increases to its highest relative frequency of the sequence. This is probably due to plants of the entomophilous Parnassia palustris and Mentha aquatica, which are abundant on the bog today, growing near the sampling site. The tall-sedge-swamp community expands with Cyperus michelianus and Carex flacca type increasing, while the true-reed swamp increases due to an increase in Phragmites australis, which becomes more abundant than Glyceria type. In the surface sample, palynological richness increases to the highest number of expected types for the sequence but this may be due to differential preservation of grains normally destroyed in burial. The extra-local woodland is a closed canopy beech wood with scattered Abies. Coppicing of beech for fodder for draft animals has 
probably ceased. Herbs of open ground decline to the lowest abundances of the sequence; Pteridium aquilinum is much reduced. Extra-local cultivation of cereals has probably ceased - only the cereal Secale cereale occurs and only at very low abundance at $10 \mathrm{~cm}$. This is consistent with a known reduction of cereal cultivation in the Pindos Mountains during this century due to the introduction of mechanized agriculture (Chapter 1). Otherwise possible cultivars are represented by low abundance of Castanea sativa, Juglans regia, and Platanus orientalis. Pine woods persist at higher elevations on the Pindos Mountains and the deciduous oak woods of the foothills probably continue unchanged. An almost complete absence of microscopic charcoal particles and Pteridium aquilinum spores, and increased Juniperus pollen suggest burning of the catchment and regional vegetation has ceased.

About $15 \%$ of the bog surface was open water when field work was undertaken. The central bog is covered by a mixed vegetation of various sedges, grasses, herbs, and related vascular species as indicated by the surface pollen spectrum, and mosses. It is carpeted with the mosses Acrocladium cuspidatum and Cratoneuron sp., which are dominant in the wetter areas of the bog, and in the drier areas Carex spp. are dominant. The similarity of the pollen spectrum of the surface sample with those throughout the sequence and the high level of available nutrients as indicated by pollen evidence suggest that mosses were part of the peat forming vegetation in the past as they are today - the current vegetation forms a mixed sedge and moss peat. Around the edge of the bog the influence of supplementary nutrients from surface and ground water, and perhaps from grazing animals can be seen in the herb communities dominated by Caltha palustris (near spring drainage) and Cirsium (on fen) and many ruderals grow on the dry land on the edge of the beech wood. The bog is situated in the mountain beech vegetation zone and is almost completely surrounded by pure beech (Fagus sylvatica) wood with an almost completely closed canopy. Beech wood grows to the edge of the wetland. Open beech wood, pine wood, and pine wood-pasture occur at a greater distance from the bog, and most of the vegetation in the east, especially around the Aspropotamos catchment is grassland and steppe, which is used by transhumant pastoralists for grazing sheep and goats. No cereal cultivation occurs in the vicinity of the wetland today and no burning of extra-local and regional vegetation occurs today. 


\section{Chapter 6. Kellia}

\subsection{Introduction}

This chapter includes a description of the site and deposits of the Kellia wetland coring site and then presents the results of pollen analysis of a $396 \mathrm{~cm}$ long sediment and peat sequence. The wetland was first identified as potentially suitable for pollen analysis by Moody \& Rackham (1988). Preliminary palynological investigations suggested that the sediments of this wetland were suitable for a full palynological study; but fossil pollen and spore content was 'scarce and preservation fair' (Huber \& Rapp 1988).

\subsection{Site description}

Kellia fen is situated in the lowland plains about $2 \mathrm{~km} \mathrm{NW}$ of the modern city of Grevena (latitude $40^{\circ} 06^{\prime} \mathrm{N}$, longitude $21^{\circ} 25^{\prime} \mathrm{E}$ ) at an elevation of $580 \mathrm{~m}$ asl (Figures 3.2, 6.1, 6.2 \& 6.3). The well-travelled main road between Grevena and Megalo Seirini runs along the western boundary of the fen and a minor road runs along the northern margin. The name of this site is taken from a 1:50 000 topographic map, Grevena sheet (Hellenic Army Geographic Service 1970). This name appears at this location, although there is now no village here. The origin of the name is unknown.

The fen is adjacent to a designated archaeological site, number 216 on the Grevena Project register of sites, which covers an area of $>10,000 \mathrm{~m}^{2}$ on the north and northeast facing slopes of the fen catchment (Wilkie 1995). The site was used for settlement: prehistoric and historic periods represented by archaeological evidence are Early to Middle Bronze Age, Early Iron Age, Late Archaic to Classical, Classical to Hellenistic, Roman, and possibly Early Medieval. Artefacts recorded at the site include a shaft hole axe and scattered pottery sherds. The density of pottery scatter is variable, but mainly light.

It is evident that the fen is occasionally grazed: a shepherd drove his flock onto the fen while we were doing fieldwork. A stock water trough catching water from a spring close by is used frequently.

\subsubsection{Physiographic description}

Kellia fen is situated in an alluviated valley. It is rheotrophic, receiving water not only directly from the atmosphere, but also from run-off from the surrounding hillslopes and a spring. The fen is in a major side valley in the lower Leipsokouki catchment, which has been subject to much erosion in the recent past (Doyle 1990). Gentle hillslopes surround Kellia fen and the catchment covers about $2.5 \mathrm{~km}^{2}$ (Figure 6.2). 


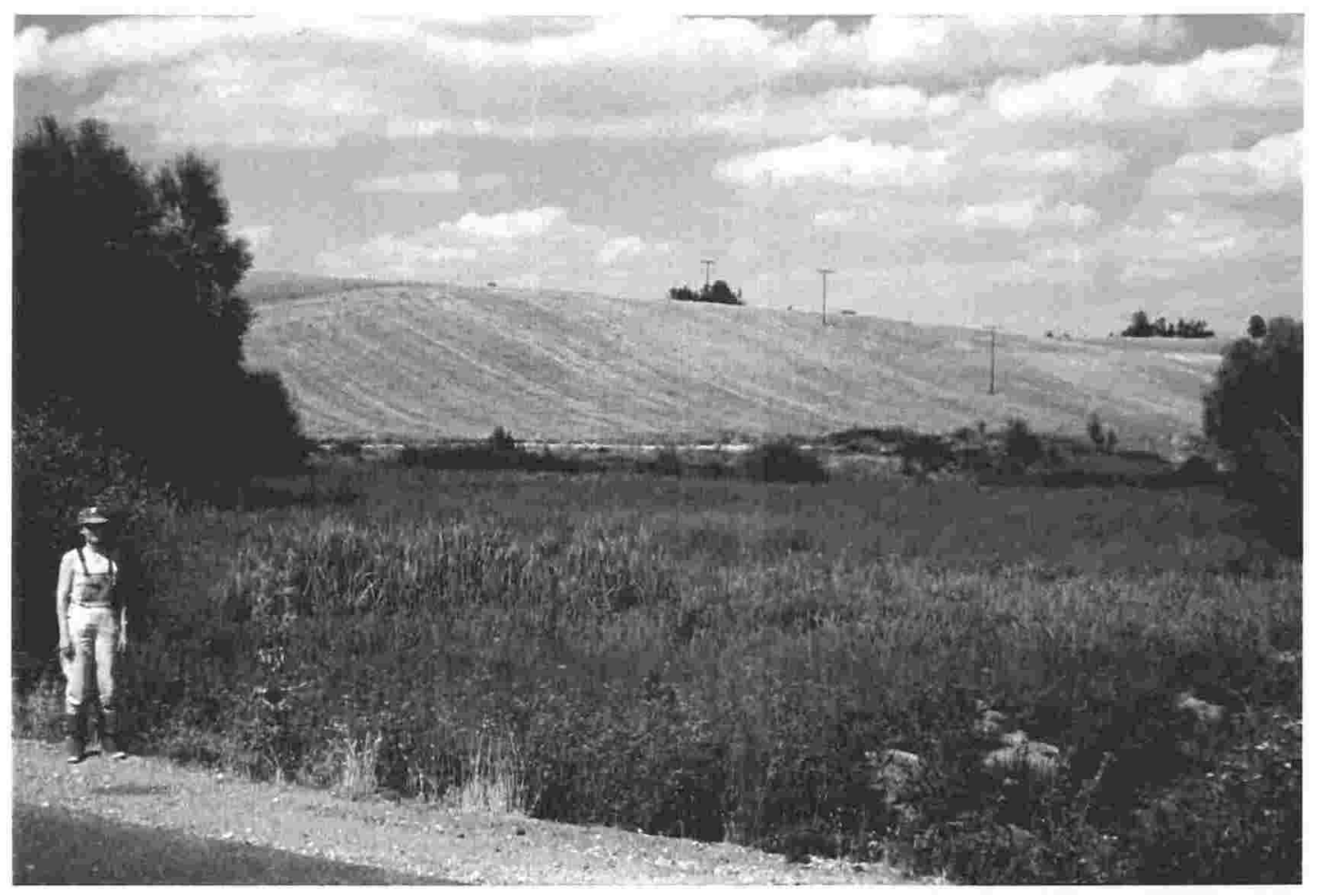

Figure 6.1 Kellia fen. Many members of the lush mixed fen vegetation can be seen in this view looking north across the fen. Cirsium is prominent in the foreground. Prominent genera on the fen are Typha, Cyperus, Juncus, Equisetum, Scirpus and Sparganium. The cultivated cereal field in the background has been harvested. 


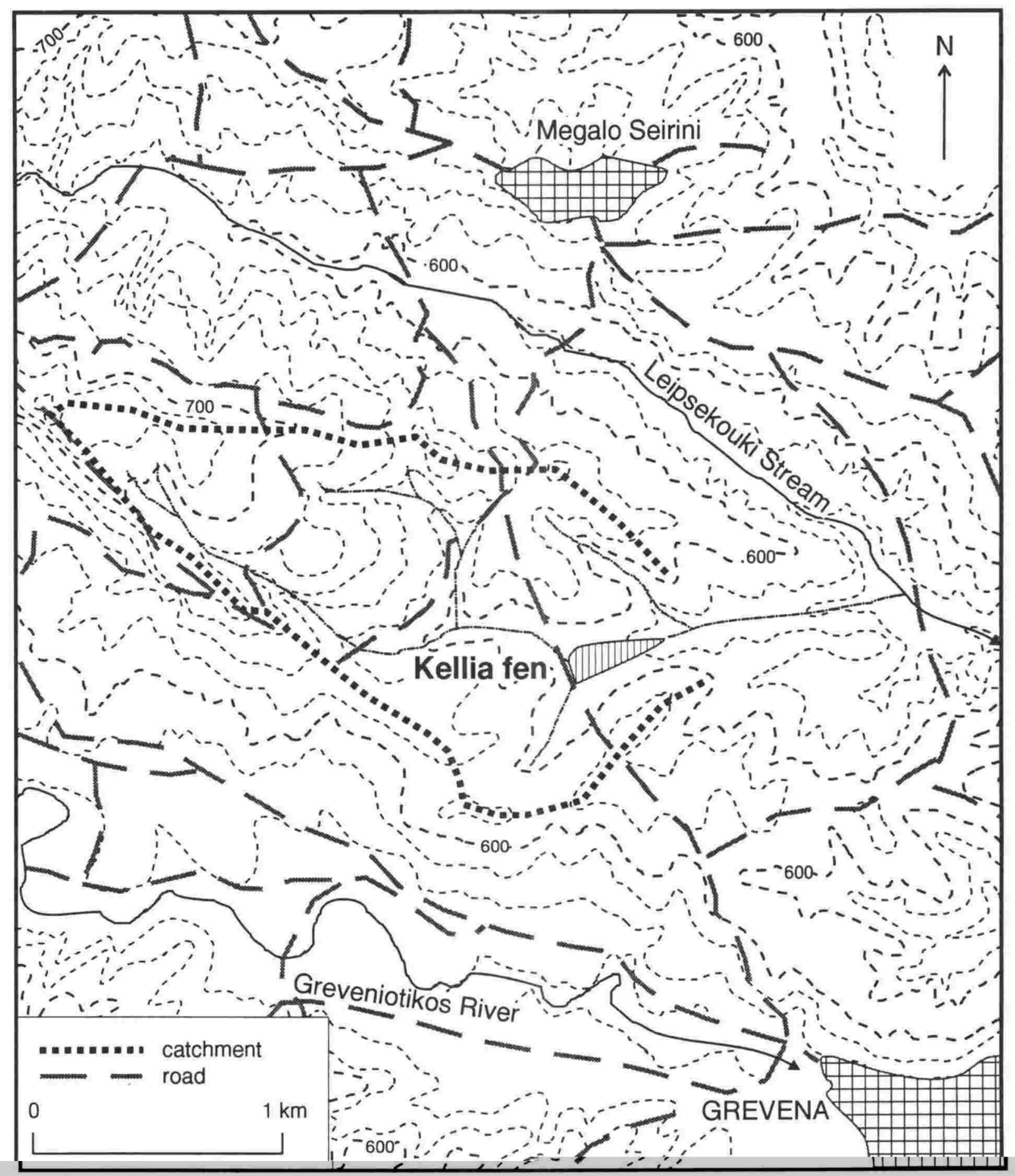

Figure 6.2 Location of Kellia fen in relation to local topography. Contour interval $20 \mathrm{~m}$. 


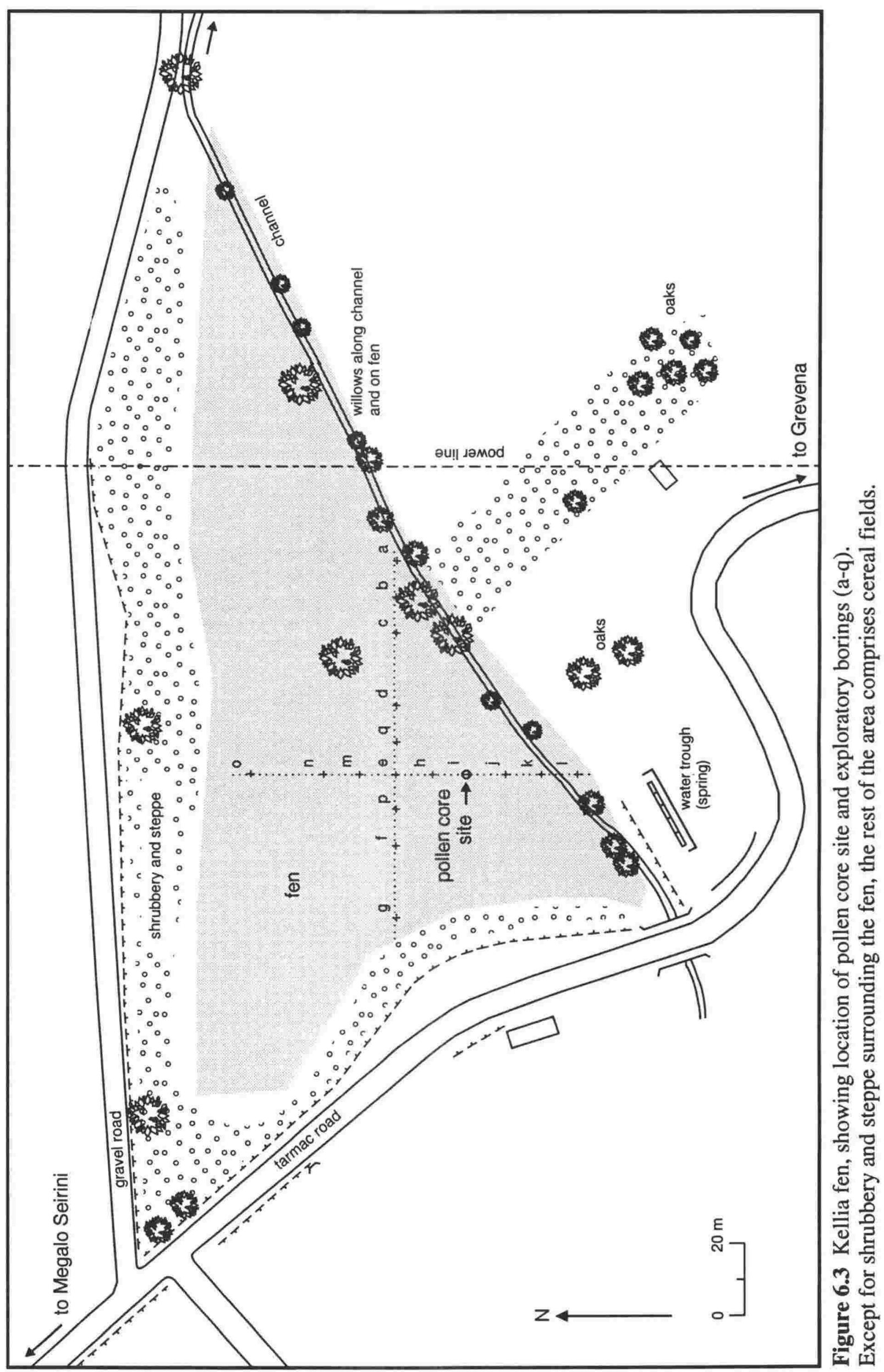


The fen has a gentle slope of about $0.5^{\circ}$ (inclinometer measurement) up to the north and west where water is supplied to the fen by two streams. A channel running along its southern boundary was dry from halfway along the edge of the fen when fieldwork was undertaken. A spring to the south of the channel has been tapped and a concrete tank constructed for watering animals.

\subsubsection{Regional and local vegetation}

Kellia fen is surrounded by cultivated fields of mostly cereals with a few scattered pollarded oaks. The typical regional vegetation of the lowlands has been described in Chapter 3.

The fen is well vegetated with a mixture of brushwood and tall luxuriant marsh plants (Figure 6.1). Numerous small plots surveyed on the fen and on the edge of the fen (Appendix VIII) showed that the bryophyte Eurhynchium praelongum and the rush Juncus inflexus were most abundant with small and poorly developed Typha in the wetter areas on the fen, but in wet areas close to the spring Typha was lush and dominant, with Sparganium very abundant. In drier areas on the fen Cirsium, Cyperus, and Equisetum were most abundant. Other important plants on the fen are: Scirpus, Mentha aquatica, Rumex conglomeratus, and Potentilla. Two herbaceous species were noted as indicative of disturbance on the fen: Pulicaria dysenterica and Lythrum salicaria. Brushwood on the fen includes Pteridium aquilinum, Rubus caesius, Prunus spinosa, and Salix.

Scattered Salix trees also grow along the channel to the south of the fen (Figure 6.3). Immediately surrounding the fen is shrubbery and steppe. To the south of the fen, on the north facing slope where many steppe plants grow, Centaurea and the bryophyte Camptothecium lutescens were almost equally dominant. To the north the shrubbery and steppe is taller and includes the brushwood plants of the fen.

\subsubsection{Geology of site}

Bedrock in this area is mapped as Pliocene-Pleistocene fluvial and lacustrine deposits in terraces: loose conglomerates, blue-green clays, sands, and loose sandstones of variable grain size and also conglomerates and red clays in their upper parts (Institute for Geology and Subsurface Research 1972). These terraces are up to $120 \mathrm{~m}$ thick.

Fine sands and silts that comprise the alluvial substrate have been washed into the fen from the surrounding slopes. A thick $(15 \mathrm{~cm})$ organic horizon covers most of the fen and is indicative of a low sedimentation rate. 


\subsection{Sediment analysis}

The results of the exploratory borings of the fen, the Troels-Smith (1955) classification and laboratory analyses of the physical properties of the pollen analysed core sequence are reported below. In the diagrams and text, datum for depth (given in $\mathrm{cm}$ ) is the fen surface.

\subsubsection{Wetland profile}

The location of the exploratory borings is shown in Figure 6.3, and main features of the stratigraphy of the exploratory cores in Figure 6.4. Detailed logs of the boreholes are in Appendix XIV. Exploratory cores were bored with a Hiller corer and a soil auger at mostly $10 \mathrm{~m}$, occasionally $20 \mathrm{~m}$, intervals in two intersecting transects across the fen in N/S and E/W directions. All exploratory cores were bored to $100 \mathrm{~cm}$ initially, then a few were selected for deeper boring.

Three major units can be distinguished. The uppermost c. $20 \mathrm{~cm}$ consists of silty peat in cores from the western and southern part of the fen, i.e. those closest to the spring. The organic content of this unit is generally higher than that of horizons rich in organic matter lower in the sequence. Towards the northern edge and the eastern (downstream) end of the fen, the unit becomes less peaty and is replaced by brown clay with a sandy layer at the base. This unit may be related to establishment of the modern sealed road and its causeway that may have reduced transportation of sediment into the fen from the catchment upstream.

Below the uppermost unit, up to $450 \mathrm{~cm}$ of greyish olive to olive black clay with variable proportions of calcareous grit, peat, and organic detritus was penetrated. Thin $(5-15 \mathrm{~cm})$ non-calcareous sand horizons are rare. Some of the peaty or sandy horizons can be traced through adjacent boreholes, but the disturbance and sometimes poor recovery of Hiller and soil auger samples limits the extent to which this can be done. Persistent organic-rich horizons occur at about 100 and $230 \mathrm{~cm}$. Below about $300 \mathrm{~cm}$, there is little organic matter in the stiff to soft greyish olive clay comprising the basal part of the unit. Occasional small mollusc shells and fruits or corms were noted in some cores, but no pattern to their occurrence was determined.

The lowest unit, somewhat heterogeneous, consists of muddy yellow sand and yellowbrown silt and clay with ferruginous mottling and nodules. In the most easterly borehole (a), gravel occurs at the base of sequence. The depth of the top of this unit varies, possibly reflecting a stream-bank topography established on partially weathered stream sediments of this unit that were later overtopped by the calcareous fen deposits of the middle unit. In this interpretation, the fen developed in an old stream course, which ran from west to east with a terrace on the northern side. An alternative is that the ferruginous mottling is due to subsurface water circulation and not weathering. The unit may then include all sediments 

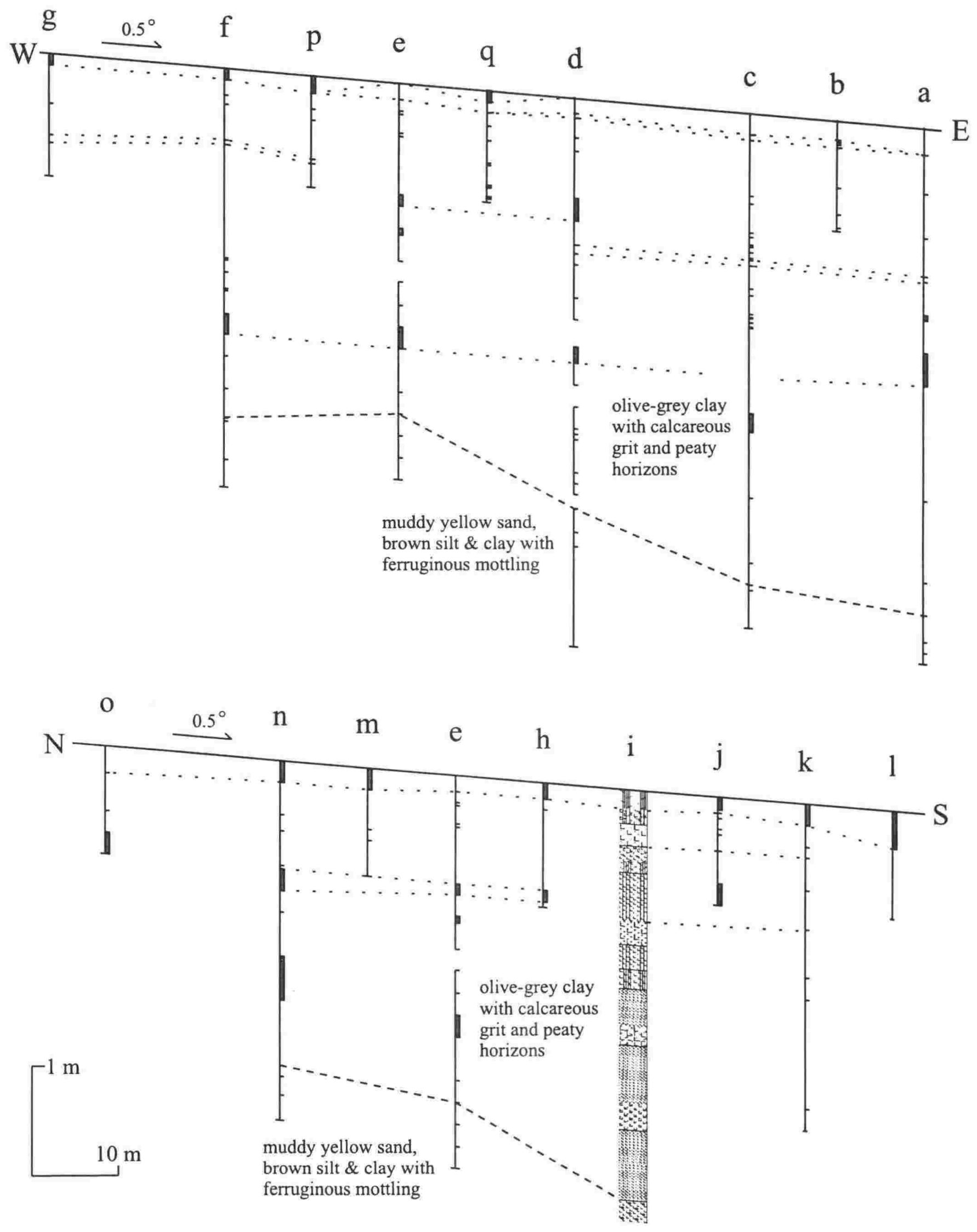

Figure 6.4 Longitudinal (E-W) and transverse (N-S) sections through Kellia fen. Columns a-q are based on Hiller sampler and soil auger cores (see Fig. 6.3), with the exception of site 'i', which is based on the monolith and piston core collected for pollen analysis. Peaty horizons in the exploratory cores are shaded, and matching sediment features joined by dashed lines. Vertical exaggeration $8 \mathrm{x}$. 
below $c .300 \mathrm{~cm}$, including the organic-poor clay mentioned above. These would represent a combination of fen and low-energy stream deposits.

After examination of these data it was decided that location ' $i$ ' was suitable for extracting a core for pollen analysis because it contained a relatively thick accumulation of sediments rich in peaty horizons and was thus likely to contain a pollen record.

\subsubsection{Stratigraphy}

An illustrated description of the sediments of the pollen-analysed core is given in Figure 6.5. The upper $215 \mathrm{~cm}$ were recovered by removing four overlapping monoliths from two contiguous columns $(0-90,70-115,110-150$, and $142-215 \mathrm{~cm})$. The piston corer was used to extract a core from 2-3 m. Below this the deposits were very stiff, so the soil auger was used to retrieve the remainder of the core. The Troels-Smith (1955) classification scheme using symbols and formulas (Appendix III) is followed in the same manner as in Chapter 4. Colours are all fresh colours. The whole sequence is water saturated, so siccitas is 2 throughout.

The major component of the sediments of the fen is stiff greyish-olive clay (Argilla steatodes and A. granosa) with varying amounts of silt, sand, and plant fragments that have been washed into the wetland from the surrounding catchment. Calcareous grit is common (all samples in this sequence effervesced in $10 \% \mathrm{HCl}$ ), but the source of it is uncertain; it may be allochthonous (derived from calcic soils of the catchment) or autochthonous (precipitated from the spring water) or both. Two horizons of a mixture of peat (Turfa herbacea) and minerogenic sediment (sand, clay and silt) occur above $183.0 \mathrm{~cm}(0-32.5$ and $67.5-183.0 \mathrm{~cm})$.

Macrofossils were found scattered throughout the deposits. No systematic study of these was undertaken, but those that were identified were of Equisetum; others were probably from swamp plants currently growing on the fen. Scattered gastropods occurred at $312.0-376.0 \mathrm{~cm}$. No ostracods were found in this sequence.

396.0-376.0 cm Basal sediments are composed of a mixture of Argilla steatodes (As) (particles of clay $<0.002 \mathrm{~mm}$ ) to A. granosa (Ag) (particles of clay 0.002-0.06 mm) and Grana saburralia (Gs) (mineral particles $0.6-2.0 \mathrm{~mm}$ ) in a ratio of $3: 1$, the larger particles being calcareous and ferruginous grit. The sediment is mottled, but the overall colour is $2.5 \mathrm{Y} 4 / 2$ (dark greyish yellow) which is a medium shade and nigror is 2 . The sediment is very stiff and homogeneous (stratificatio 0 ). The clay is plastic (elasticitas 0 ).

$376.0-312.0 \mathrm{~cm}$ A very diffuse boundary of limes 0 separates this horizon from that below. The sediments are now Argilla granosa to A. steatodes with only a trace of Grana saburralia and Detritus herbosus (Dh) (fragments of herbaceous plants $>2 \mathrm{~mm}$ ). The colour is 7.5 Y $4 / 2$ (greyish olive). Nigror remains at 2. Stratificatio increases to 1 as the deposit tends to split into thin horizontal layers due to horizontal layering of plant material. The clay 


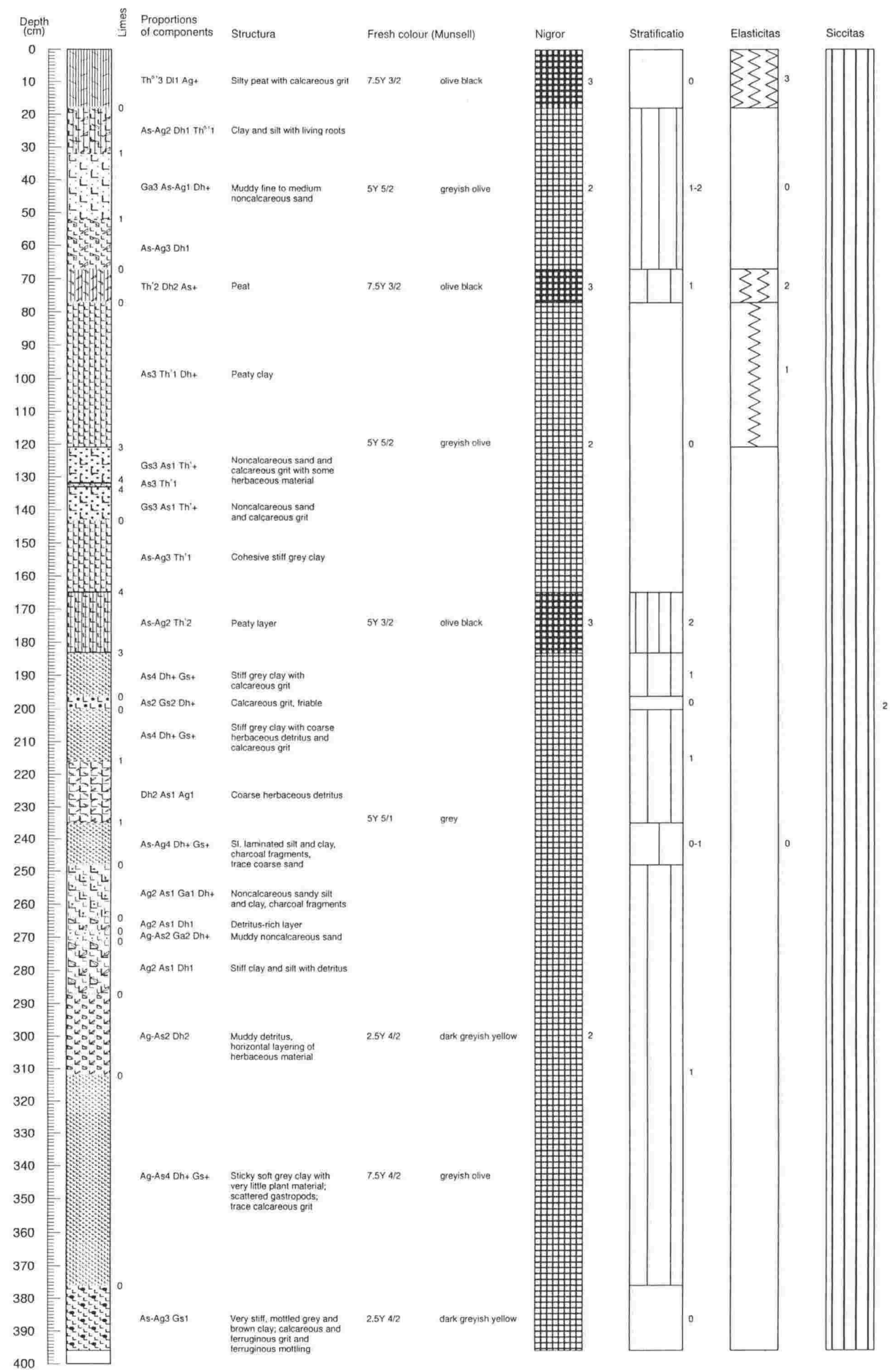

Figure 6.5 Troels-Smith classification of Kellia core sediments. Symbols are defined in Appendix III. 
is plastic (elasticitas 0), soft, and sticky. There are scattered gastropods throughout this horizon.

312.0-287.5 $\mathrm{cm}$ Another very diffuse boundary (limes 0 ) separates this horizon from that below. Plant fragments now form a higher proportion of the sediment; Argilla granosa to A. steatodes, and Detritus herbosus are in equal proportions. The colour returns to that of the basal horizon, $2.5 \mathrm{Y} 4 / 2$ (dark greyish yellow); nigror remains at 2. Stratificatio remains at 1 due to horizontal layering of herbaceous material. The deposit remains plastic (elasticitas $0)$.

287.5-271.0 cm Another very diffuse boundary (limes 0 ) separates this horizon from that below. The proportion of plant fragments has declined slightly (Detritus herbosus 1) and smaller grained mineral particles have become more abundant (Argilla granosa 2 and A. steatodes 1). The colour changes to $5 Y$ 5/1 (grey), but nigror remains at 2 . The sediment is stiff and plastic (elasticitas 0 ). Stratificatio (1) is unchanged.

271.0-268.0 $\mathrm{cm}$ This thin sandy horizon is separated from the horizon below by a very diffuse boundary (limes 0 ) and comprises a mixture of equal proportions of Argilla granosa to A. steatodes and Grana arenosa $(\mathrm{Ga})$ (mineral particles $0.06-0.6 \mathrm{~mm}$ ) plus a trace of Detritus herbosus. This larger grain size indicates higher energy transport. Physical properties remain unchanged (nigror 2, stratificatio 1 , and elasticitas 0 ).

268.0-264.0 $\mathrm{cm}$ This is another thin horizon separated from the horizon below by a very diffuse boundary (limes 0 ). It is composed of sediments the same as those in the horizon below the sandy horizon.

264.0-248.0 $\mathrm{cm}$ This is a thicker horizon separated from the horizon below by a very diffuse boundary (limes 0). It is a mixture of Argilla granosa, A. steatodes, and Grana arenosa in the proportions of 2:1:1 plus a trace of Detritus herbosus. Physical properties are unchanged. Charcoal particles were noted.

248.0-235.0 $\mathrm{cm}$ Another very diffuse boundary (limes 0 ) separates this horizon from that below. The sediments are composed of Argilla steatodes to A. granosa plus traces of Detritus herbosus and Grana saburralia. Physical properties are unchanged apart from stratificatio, which is slightly reduced, $0-1$. Charcoal particles were noted.

235.0-216.0 $\mathrm{cm}$ The boundary between this horizon and that below is diffuse, but slightly sharper than all the boundaries below being limes 1 . Detritus herbosus increases to 2, and Argilla steatodes and A. granosa share the remaining half of the mixture. Physical properties are unchanged except that stratificatio returns to a score of 1.

216.0-200.0 $\mathrm{cm}$ The boundary between this horizon and that below is again diffuse (limes 1). The sediments are now composed of Argilla steatodes with traces of Detritus 
herbosus and Grana saburralia. The clay is stiff and the grit is calcareous. Physical properties are unchanged.

200.0-196.0 $\mathrm{cm}$ This thin horizon is separated from the horizon below by a very diffuse boundary (limes 0 ). Sediments comprise a mixture of equal proportions of Argilla steatodes and Grana saburralia plus a trace of Detritus herbosus. The sediment is homogeneous (stratificatio 0 ) and friable. The grit is calcareous. Other physical properties are unchanged.

196.0-183.0 $\mathrm{cm}$ Another very diffuse boundary (limes 0 ) separates this horizon from that below. Sediment in this horizon is the same as that at $216.0-200.0 \mathrm{~cm}$.

183.0-165.0 cm A sharp boundary (limes 3 ) between this horizon and that below marks the beginning of peat formation. The deposit is a mixture of Argilla steatodes to A. granosa, and Turfa herbacea in equal parts. The colour of this horizon is 5Y 3/2 (olive black); nigror is 3. Stratificatio increases to 2 , but elasticitas remains at 0 . The plant structure of the peat is well-preserved; humositas is 1 indicating that the surface of the peat was probably continuously wet.

165.0-143.0 $\mathrm{cm}$ A very sharp boundary of limes 4, possibly indicating erosion of wetland sediments marks a change to increased Argilla steatodes to A. granosa, and decreased Turfa herbacea (3:1). Humositas remains at 1 . Colour of the sediment is paler (5Y 5/2, greyish olive), probably due to the increase in the minerogenic component; nigror has reduced to 2. The clay is cohesive and stiff. The deposit becomes homogeneous again (stratificatio $0)$, but elasticitas $(0)$ is unchanged.

$143.0-133.0 \mathrm{~cm}$ A very diffuse boundary (limes 0 ) at $143.0 \mathrm{~cm}$ separates this horizon from the peaty horizon below. The sediments of this horizon are a mixture of Grana saburralia (non-calcareous coarse to very coarse sand and calcareous grit) and Argilla steatodes in the proportions of 3:1. Turfa herbacea declines to a trace, but humositas remains at 1 . Physical properties are unchanged.

$133.0-132.0 \mathrm{~cm}$ This very narrow band is demarcated by very sharp boundaries (limes 4). The sediment comprises Argilla steatodes and Turfa herbacea in the proportions of $3: 1$. Humositas is 1 . No physical properties change.

132.0-121.0 $\mathrm{cm}$ This horizon is composed of the same sediment as that at $143.0-133.0 \mathrm{~cm}$.

121.0-77.5 $\mathrm{cm}$ This thick horizon is separated from the horizon below by a sharp boundary of limes 3. The deposit is a mixture of Argilla steatodes and Turfa herbacea in the proportions of 3:1 plus a trace of Detritus herbosus. Humositas is 1. Physical properties are unchanged with the exception of elasticitas, which increases to 1 .

77.5-67.5 $\mathrm{cm}$ A very diffuse boundary limes 0 separates this horizon from that below. Deposits are now a mixture of Turfa herbacea and Detritus herbosus in equal proportions plus 
a trace of Argilla steatodes. Humositas is 1 . Colour of the deposit is darker at 7.5Y $3 / 2$ (olive black). Stratificatio increases to 1 and elasticitas to 2 .

67.5-52.0 $\mathrm{cm}$ Another very diffuse boundary (limes 0 ) marks a change in the deposit to one that includes more inorganic material. Sediments comprise Argilla steatodes to A. granosa, and Detritus herbosus in the proportions of 3:1. Colour is paler (5Y 5/2, greyish olive) and nigror reduces to 2. Stratificatio increases to 1-2 and elasticitas decreases to 0 .

52.0-32.5 $\mathrm{cm}$ A diffuse boundary (limes 1) marks a change to sediments of larger grain size. They comprise a mixture of Grana arenosa and, Argilla steatodes to A. granosa in the proportions of 3:1 plus a trace of Detritus herbosus. Physical properties including colour are unchanged.

32.5-18.0 $\mathrm{cm}$ Another diffuse boundary of limes 1 marks the boundary between this horizon and that below. The deposit is a mixture of Argilla steatodes to A. granosa, Detritus herbosus, and Turfa herbacea in the proportions of 2:1:1. Humositas is 0-1. Physical properties are unchanged. Living roots of plants growing on the fen penetrate into this horizon.

18.0-0 $\mathrm{cm}$ The uppermost horizon is separated from the horizon below by a very diffuse boundary (limes 0 ). The deposit comprises a mixture of Turfa herbacea and Detritus lignosus (fragments of ligneous plants $>2 \mathrm{~mm}$ ) in the proportions of 3:1 plus a trace of Argilla granosa. Humositas is $0-1$. The colour is darker (7.5Y 3/2, olive black) and nigror increases to 3 . Stratificatio decreases to 0 and elasticitas increases to 3 .

\subsubsection{Physical properties}

Organic matter, inorganic matter, moisture content, and dry density are plotted against depth and stratigraphy in Figure 6.6. The first three properties are expressed as a percentage of the original wet weight of the sample. The results suggest a division of the sequence into 14 sections (A-N), described below.

Section $N, 396.0-376.0 \mathrm{~cm}$ This sediment is very rich in inorganic matter (73.8-78.4\%), with correspondingly high dry density values $(1.1-1.5 \mathrm{~g} / \mathrm{cc})$. Organic matter is very low $(0.8 \%)$, being minima of the sequence. Moisture content is also very low recording the minimum of the sequence in the basal sample (19.6-24.4\%). Carbonate content is very low $(1.0-1.6 \%)$.

Section M, 376.0-312.0 $\mathrm{cm}$ Physical properties are very similar to those in the section below, but inorganic matter and dry density decline upwards (65.0-76.0\% and 1.1-1.5 g/cc, respectively), while organic matter and moisture content increase $(0.8-1.6 \%$ and $22.0-32.2 \%$, respectively). Carbonate content continues to be very low (0.9-1.6\%). 


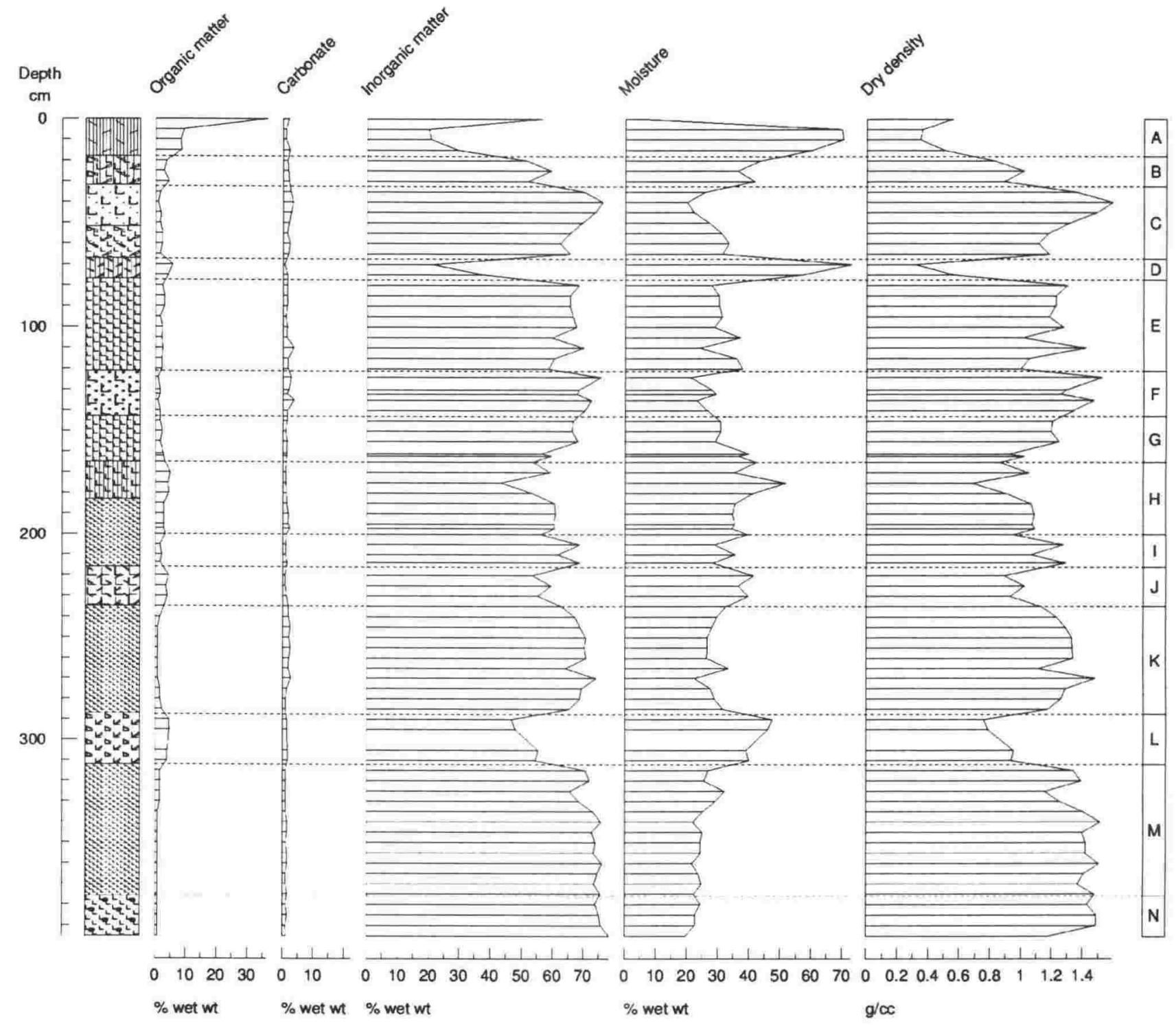

Figure 6.6 Kellia sediment physical properties: sediment type column (Troels-Smith classification 1955), organic matter, carbonate, inorganic matter, moisture and bulk (dry) density. Sediment sections based on physical properties are indicated on the right. 
Section $\mathbf{L}, 312.0-287.5 \mathrm{~cm}$ This section is characterised by significantly decreased inorganic matter (46.7-55.3\%) and dry density (0.7-0.9 g/cc), while organic matter and moisture content increase $(3.8-4.6 \%$ and $39.1-47.4 \%$, respectively). Carbonate content is moderate (1.4-1.6\%).

Section $\mathrm{K}, 287.5-235.0 \mathrm{~cm}$ This section is characterised by very high inorganic matter and dry density (64.0-70.8\% and 1.2-1.5 g/cc, respectively) and corresponding low organic matter and moisture content $(0.8-2.2 \%$ and $22.4-33.4 \%$, respectively). Carbonate content is generally slightly higher $(0.9-2.6 \%)$.

Section J, 235.0-216.0 cm This section includes the sample at $235.0 \mathrm{~cm}$. The section is characterised by increased organic content (2.9-4.4\%) and correspondingly higher moisture content (32.4-41.4\%). Inorganic matter and dry density decline (53.4-62.8\% and 0.9-1.1 g/cc). Carbonate content declines $(0.8-1.8 \%)$.

Section I, 216.0-200.0 $\mathrm{cm}$ This narrow section comprises three samples (excluding the sample at $200.0 \mathrm{~cm})$. Organic matter and moisture content decline $(1.5-2.0 \%$ and $26.6-35.6 \%$, respectively), and inorganic matter and dry density increase $(61.5-70.4 \%$ and $1.1-1.3 \mathrm{~g} / \mathrm{cc}$, respectively). Carbonate content remains low (0.9-1.5\%).

Section $\boldsymbol{H}, 200.0-165.0 \mathrm{~cm}$ This section is characterised by increased organic content $(2.7-4.8 \%)$. Moisture content increases (34.4-51.7\%). Inorganic matter and dry density decline (43.2-60.8\% and $0.7-1.1 \mathrm{~g} / \mathrm{cc}$, respectively). Carbonate content increases slightly at the base of this section, but then declines upwards (0.9-2.2\%).

Section $G, 165.0-143.0 \mathrm{~cm}$ Again this section demonstrates a reversal to lower organic matter and moisture content (1.5-2.7\% and 29.0-39.9\%) to higher inorganic and dry density values (56.3-68.0\% and 0.9-1.2 g/cc, respectively). Carbonate content remains low (0.9$1.4 \mathrm{~g} / \mathrm{cc})$.

Section F, 143.0-121.0 cm Inorganic matter (67.8-75.5\%) and dry density (1.2-1.5 g/cc) are very high and continue to increase, while organic matter and moisture content remain very low (0.82-1.6\% and 21.0-29.2\%, respectively). Carbonate content increases reaching the maximum of the sequence at $135.0 \mathrm{~cm}(1.4-3.7 \%)$.

Section $E, 121.0-77.5 \mathrm{~cm}$ Organic matter and moisture content increase slightly (1.5-3.0\% and 24.0-37.8\%, respectively). Inorganic matter and dry density decline (58.5$70.0 \%$ and $1.0-1.4 \mathrm{~g} / \mathrm{cc}$ ). Carbonate values remain high at first, but then decline to low values $(0.9-3.6 \%)$.

Section $D, 77.5-67.5 \mathrm{~cm}$ This narrow section comprises two samples of organic-rich matter (4.1 and 5.5\%). Maximum moisture content of the sequence of $72.9 \%$ is reached at $65.0 \mathrm{~cm}$ (the other sample records $57.0 \%$ ). Inorganic matter and dry density decline 
dramatically (21.0-37.6\% and 0.3-0.6 g/cc, respectively). Carbonate values remain low (0.5-1.5\%).

Section $C, 67.5-32.5 \mathrm{~cm}$ Inorganic matter dramatically increases to values similar to those of the section below the preceding section (62.2-76.0\%). Dry density also increases dramatically $(1.1-1.6 \mathrm{~g} / \mathrm{cc})$. Organic matter and moisture content decline to low levels $(0.8$ $2.2 \%$ and $19.8-33.3 \%$, respectively). Carbonate values increase steadily upwards (1.4-3.3\%) almost reaching the maxima of the sequence.

Section $B, 32.5-18.0 \mathrm{~cm}$ Organic content and moisture content increase again (2.7-4.3\% and $36.0-43.5 \%$, respectively), and inorganic matter and dry density decline (51.3-59.4\% and 0.82-1.0 g/cc, respectively). Carbonate values decline slightly (1.5-2.0\%).

Section $A, 18.0-0 \mathrm{~cm}$ This section is characterised by highest organic matter content of the sequence; increasing to $9.3 \%$, then to $36.3 \%$ at the surface. Moisture content rises to almost the maxima of the sequence at 10.0 and $5.0 \mathrm{~cm}(70.1$ and $69.7 \%$, respectively), but declines to only $5.4 \%$ in the surface sample. Inorganic matter declines sharply in samples at $15.0,10.0$, and $5.0 \mathrm{~cm}(29.4,20.6$, and $19.9 \%$, respectively), but increases in the surface sample to $56.4 \%$. Dry density is very low $(0.3-0.5 \mathrm{~g} / \mathrm{cc})$. Carbonate values continue to decline $(1.0-2.2 \%)$.

\subsection{Chronostratigraphy}

\subsubsection{Radiocarbon dating}

Samples for radiocarbon dating were selected to provide ages for various depositional events inferred from lithologic characteristics. Where possible samples were taken from intervals of the core containing considerable amounts of peat to provide a higher percentage of carbon per sample, thus providing more precise radiocarbon ages, and also avoiding contamination of older soil carbon from alluvial inwash (Tolonen 1980), so they are not evenly spaced.

Radiocarbon dating results are tabulated in Table 6.1. Conventional radiocarbon ages are shown with an analytical error of one standard deviation. These ages have been calibrated by tree-rings (Appendix IV).

The sequence contains few in situ peat horizons. The two uppermost samples dated radiometrically were taken from deposits that contained peat. As no peat horizons occurred below the lower of these samples less reliable sediment material had to be dated. Between 296 and $307 \mathrm{~cm}$ sediment from an organic detritus-rich horizon was dated. The two lowest samples are clay-rich sediment containing very little organic matter. The humin fraction of these sediments was used for dating. All samples received a pretreatment with hot acid, which removes calcium carbonate and fulvic acid, and three samples were also treated with an alkali to remove humic acids (Taylor 1987). 


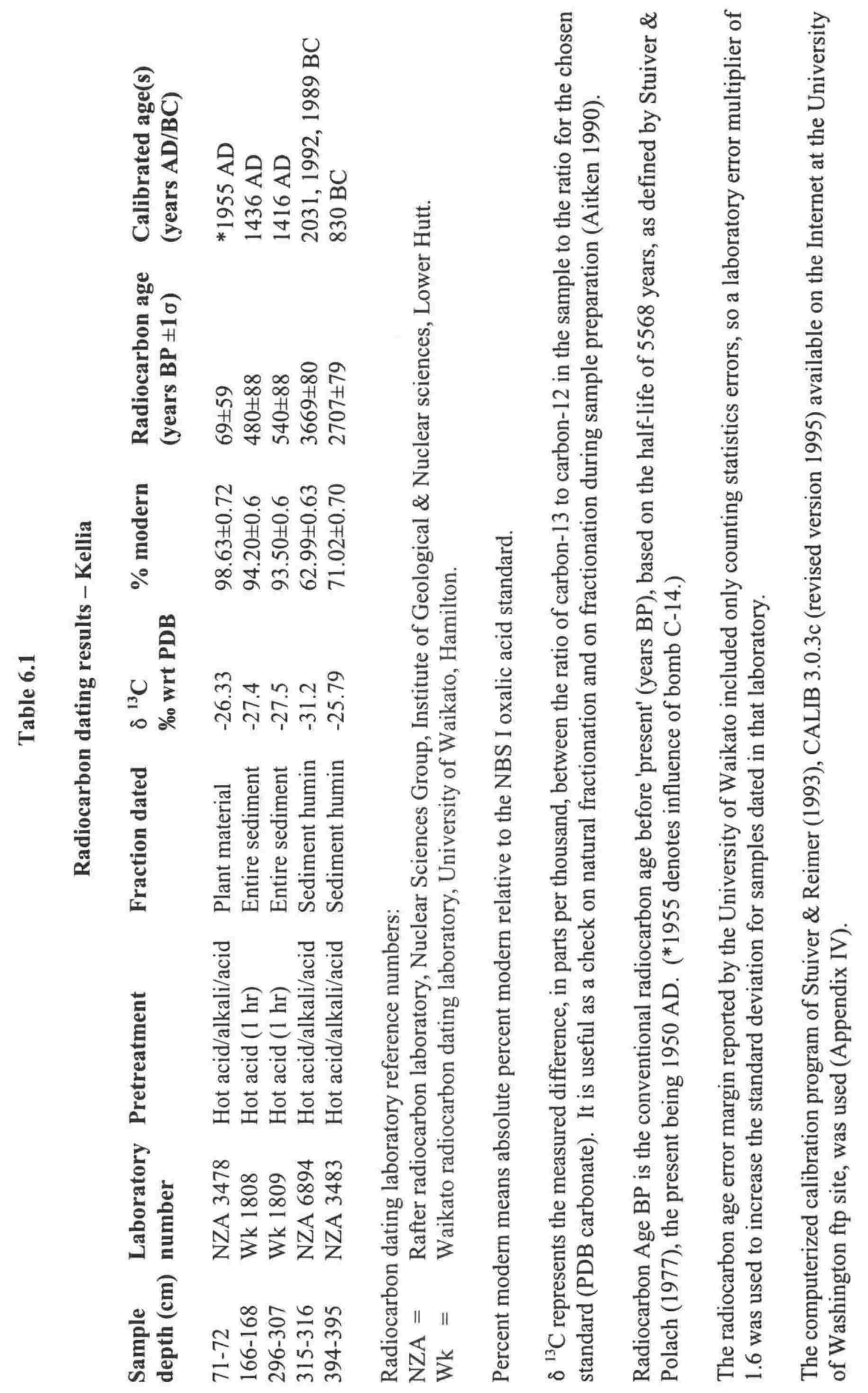


The uppermost sample $(71-72 \mathrm{~cm})$ is close to modern but has calibrated age ranges (Appendix IV), at 68\% confidence level, of 1698-1722 and 1817-1920 AD. After calibration the radiocarbon sample at $166-168 \mathrm{~cm}$ gives three age ranges at $68 \%$ confidence level (1323-1338, 1393-1514, and 1593-1620 AD), with a most probable calibrated calendar date of $1436 \mathrm{Cal} \mathrm{AD}$. The radiocarbon sample at $296-307 \mathrm{~cm}$ gave a younger most probable calibrated age than expected, $1416 \mathrm{Cal} \mathrm{AD}$, not statistically different from the one above. However $68 \%$ confidence level calibrated age range for this sample is $1324-1447$ AD.

The lower two radiocarbon ages are in reverse order and much older than those above. Although the reversal may indicate unreliability of these dates, it is considered that these lower sediments are older than those above and that an hiatus occurs at $312 \mathrm{~cm}$, also there is the possibility that sediments from $312-376 \mathrm{~cm}$ derive from older soils than those below. The sample at $315-316 \mathrm{~cm}$ shows a higher negative value for the ratio of carbon-13 to carbon-12 $\left(\delta{ }^{13} \mathrm{C}\right)$ than the sample at $394-395 \mathrm{~cm}$, even though it contains more organic matter. This may indicate a contribution of inorganic carbonates (Jansen 1984:36). Thus the date obtained for the lowest sample is considered the more reliable.

\subsubsection{Age/depth plot}

An age/depth plot (Figure 6.7) has been drawn in the same manner as those of the two preceding chapters to illustrate the relationship between the dates obtained from radiocarbon dating and the depths of the deposits. As a first approximation, a straight line has been fitted to the three dates above $312 \mathrm{~cm}$. If deposition of the sediments occurred at a constant rate, this would suggest that either Wk1808 is older than expected, or that Wk1809 is younger than expected. Contamination of these samples by older or younger material sufficient to cause such deviation is unlikely (Taylor 1987). However the line passes either within, or close to the $68 \%$ confidence level age ranges, and within the $95 \%$ ranges.

The variation in organic matter content suggests that sediment deposition rate may have been variable. A check on this was made by plotting cumulative Pinus pollen concentration against depth (Figure 6.8), after the manner of Middeldorp (1982). As a long-distance transported component of the pollen rain, Pinus would be expected to have relatively constant pollen deposition at the site. The plot shows variation in the depth rate of accumulation, but also considerable intervals of uniform accumulation. Low rates are associated with organicrich sediment, and high rates with sandy and organic-poor sediment, consistent with low sedimentation rate of sediment during periods of peat accumulation and vice versa. The radiocarbon date Wk1809 falls at about twice the accumulated Pinus pollen value as Wk1808, 


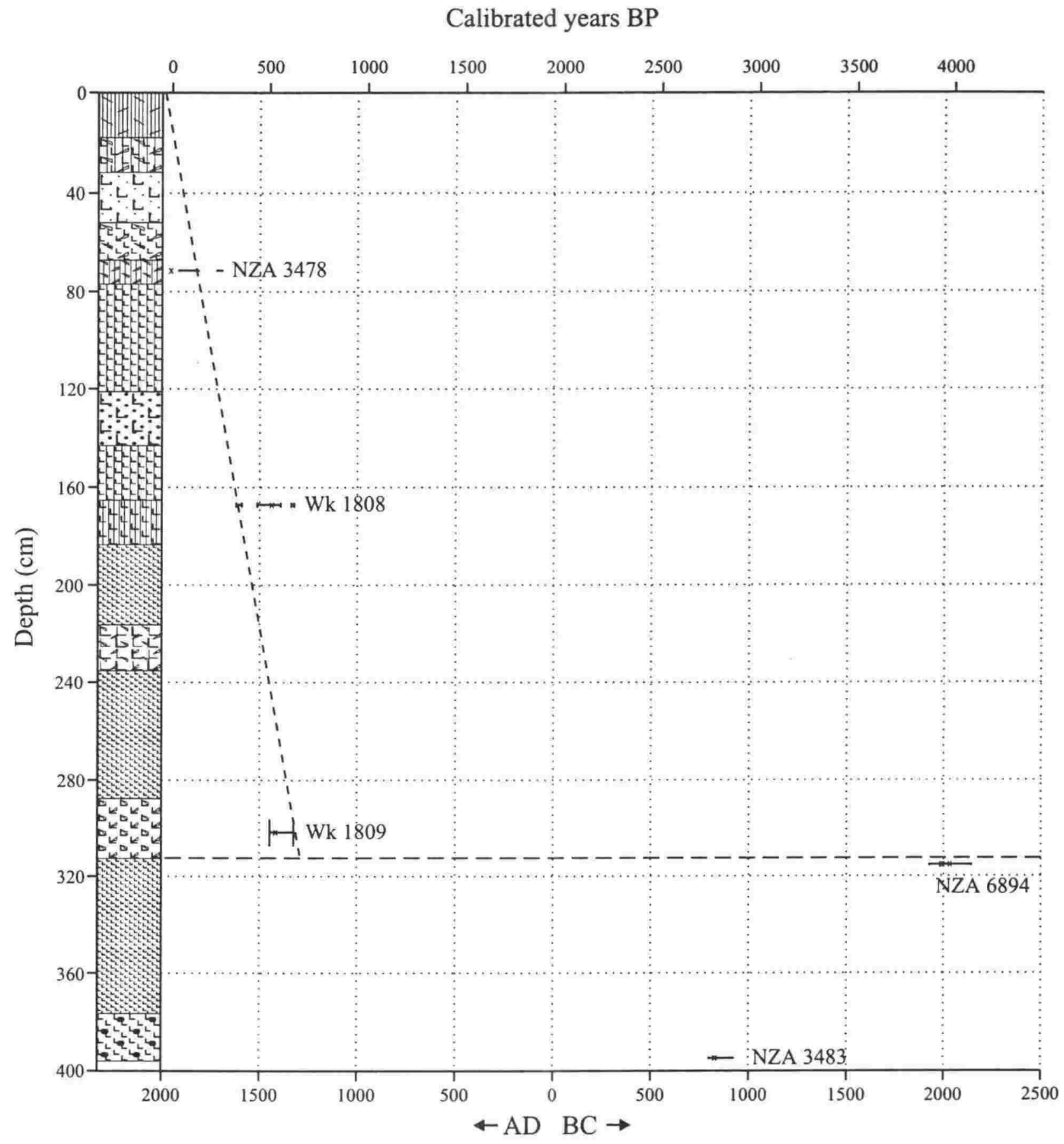

Calibrated calendar years

Figure 6.7 Age-depth plot. Calibrated radiocarbon ages (Table 6.1, Appendix IV) plotted against depth of sediment from Kellia. Length of cross-arms indicates uncertainty $( \pm 1 \sigma)$ of age measurement (horizontal) and thickness of dated sediment (vertical). Most probable dates are indicated by crosses. The horizontal line at $312 \mathrm{~cm}$ depth indicates a hiatus in sedimentation deduced from other data. A tentative line of correlation is shown above this level. 


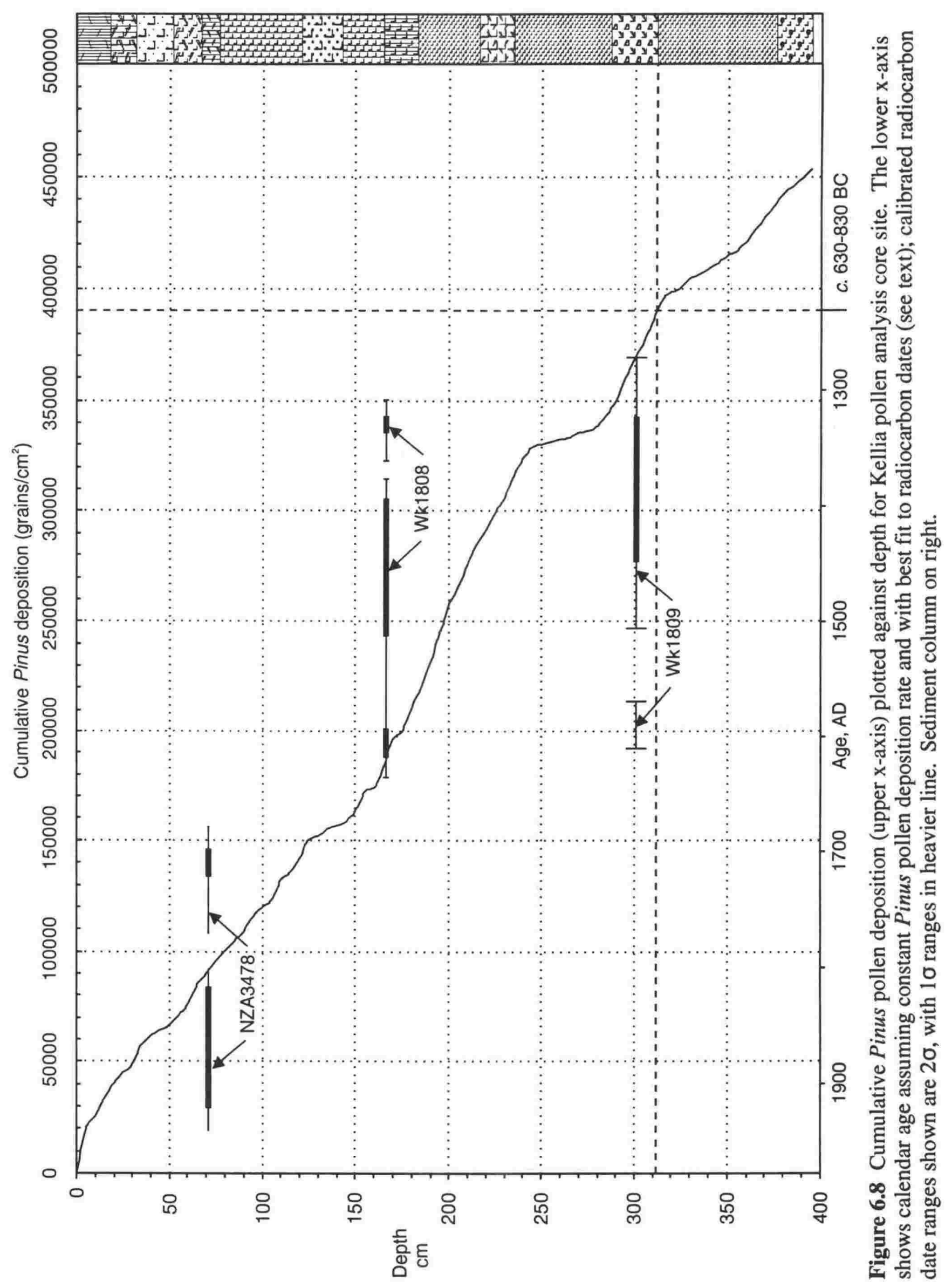


in agreement with the linear approximation of Figure 6.7. A linear calendrical scale has therefore been fitted to Figure 6.8. Although the depositional regime is not a simple closed system like the autochthonous peats treated by Middeldorp (1982), the pollen accumulation curve can be treated as an age-depth plot. Since it takes into account variations in sedimentation rate, it is used as a best estimate of ages of pollen zones.

The exact dating of the core interval below $312 \mathrm{~cm}$ is uncertain, as indicated above, although the lower radiocarbon date of $830 \mathrm{BC}$ is preferred. Because of the uncertainty, no ages are assigned to pollen zones from this interval. Figure 6.8 indicates that Pinus pollen accumulation rate in the interval below $312 \mathrm{~cm}$ is similar to that obtained in the interval 120$30 \mathrm{~cm}$. The section $396-312 \mathrm{~cm}$ therefore probably accumulated over a time interval of approximately 200 years.

\subsection{Pollen analysis results}

\subsubsection{Introduction}

Relative frequency and concentration data are used to interpret the vegetation history of the Kellia area. Concentration data are not converted to influx rates because of the uncertainty of the reliability of the reported radiocarbon ages and possible erratic deposition of deposits. Only the exotic marker grain (tracer) method of calculating palynomorph concentrations was used for this sequence. Separate diagrams are drawn for local (wetland) taxa, extra-local and regional (terrestrial) taxa, and microscopic charcoal. Data used to calculate pollen and spore concentrations, all pollen and spore types counted, and charcoal abundances are tabulated in Appendix XIII.

\subsubsection{Pollen preservation/pollen abundance}

Palynomorph preservation is generally good, but abundance is very low. The principal modes of deterioration of grains are breakage and crumpling, leaving the exine in good condition. In samples below $312 \mathrm{~cm}$ some exines have been degraded by oxidation (Delcourt \& Delcourt 1980). Only one spectrum records more than $4.0 \%$ indeterminate grains $(4.2 \%$ at $124 \mathrm{~cm}$ ). Unknown grains and unidentified monolete spores are rare, each reaching a maximum of $0.2 \%$ in two spectra.

Concentration values of total terrestrial pollen and spores are comparatively constant (Figure 6.13). It is highest between the base of the sequence and 355, 310-285, and 235$145 \mathrm{~cm}$ where it generally exceeds $7 \times 10^{3}$ grains $/ \mathrm{cm}^{3}$; and in the surface spectrum $\left(22 \times 10^{3}\right.$ grains $/ \mathrm{cm}^{3}$ ). With the exception of the surface sample, no spectrum exceeds $16 \times 10^{3}$ grains $/ \mathrm{cm}^{3}$. The lowest concentrations occur from 270-245, 140-135, 75-70, and at 45 and $25 \mathrm{~cm}$, where concentrations are less than $4 \times 10^{3}$ grains $/ \mathrm{cm}^{3}$. There is no obvious correlation 
between dry density of the sediments and terrestrial pollen and spore concentrations. There is some correlation between concentration and sediment type; in general, low concentrations occur in sand and grit horizons, and high concentrations in clay that is rich in plant detritus.

Concentration values of total wetland pollen and spores are highly variable $\left(0.4-45 \times 10^{3}\right.$ grains $\left./ \mathrm{cm}^{3}\right)$ (Figure 6.10). Highest concentrations occur 310-290 cm $\left(26-42 \times 10^{3}\right.$ grains $\left./ \mathrm{cm}^{3}\right)$, at $230 \mathrm{~cm}\left(39 \times 10^{3}\right.$ grains $\left./ \mathrm{cm}^{3}\right)$ (both plant detritus-rich clay sediment) and the surface $\left(45 \times 10^{3}\right.$ grains $\left./ \mathrm{cm}^{3}\right)$. With the exception of three spectra (all less than $10 \times 10^{3}$ grains $/ \mathrm{cm}^{3}$ ) all remaining concentrations are only about $5 \times 10^{3}$ grains $/ \mathrm{cm}^{3}$ or less. There is no correlation between dry density of the sediments and wetland pollen and spore concentration.

\subsubsection{Wetland pollen analysis}

\subsubsection{Pollen diagram construction}

Pollen and spore relative frequencies are presented graphically in Figure 6.9 and pollen concentration data in Figure 6.10. Style of the resolved and summary relative frequency diagram is as for the Anelia site (Chapter 5). In both diagrams, pollen and spore types are arranged in the same manner as in Chapter 5. Similarly, within habitat groups, types are arranged in order of appearance. The pollen sum includes all wetland pollen and spore taxa. Relative frequencies of $0.2 \%$ or less are indicated with a dot. Rarefaction analysis is based on the smallest total count of the wetland pollen and spores, 55 in the fourth spectrum from the top of the sequence.

Many weeds and ruderals were observed growing on the drier and disturbed areas of the fen. These species are included in the pollen types Calystegia, Cirsium type, Cuscuta types, Galium type, Hypericum perforatum type, Lactuca type, Nigella, Potentilla, Rubus type, Rumex conglomeratus group, Solidago type, Sonchus type, and Teucrium and are not included in the wetland diagram as they are typically terrestrial in habitat. With the exception of Rubus type, which is included in the 'woodland understorey trees and shrubs' habitat group, all these pollen types are included in the habitat group of 'herbs of open ground'.

\subsubsection{Pollen record of wetland plant communities}

The wetland communities are represented by several dominant pollen and spore taxa: Carex hallerana type, Equisetum, Schoenus nigricans, Carex flacca type, Cyperus longus type, Cyperus michelianus, Glyceria type, Phragmites australis, Sparganium erectum, Scirpus, and Sparganium angustifolium. The dominance of these types alternates. A maximum of 23 pollen and spore types were identified in any spectrum; average is 14 . Estimates of palynological richness by rarefaction analysis range between 4 and 16 expected types. 


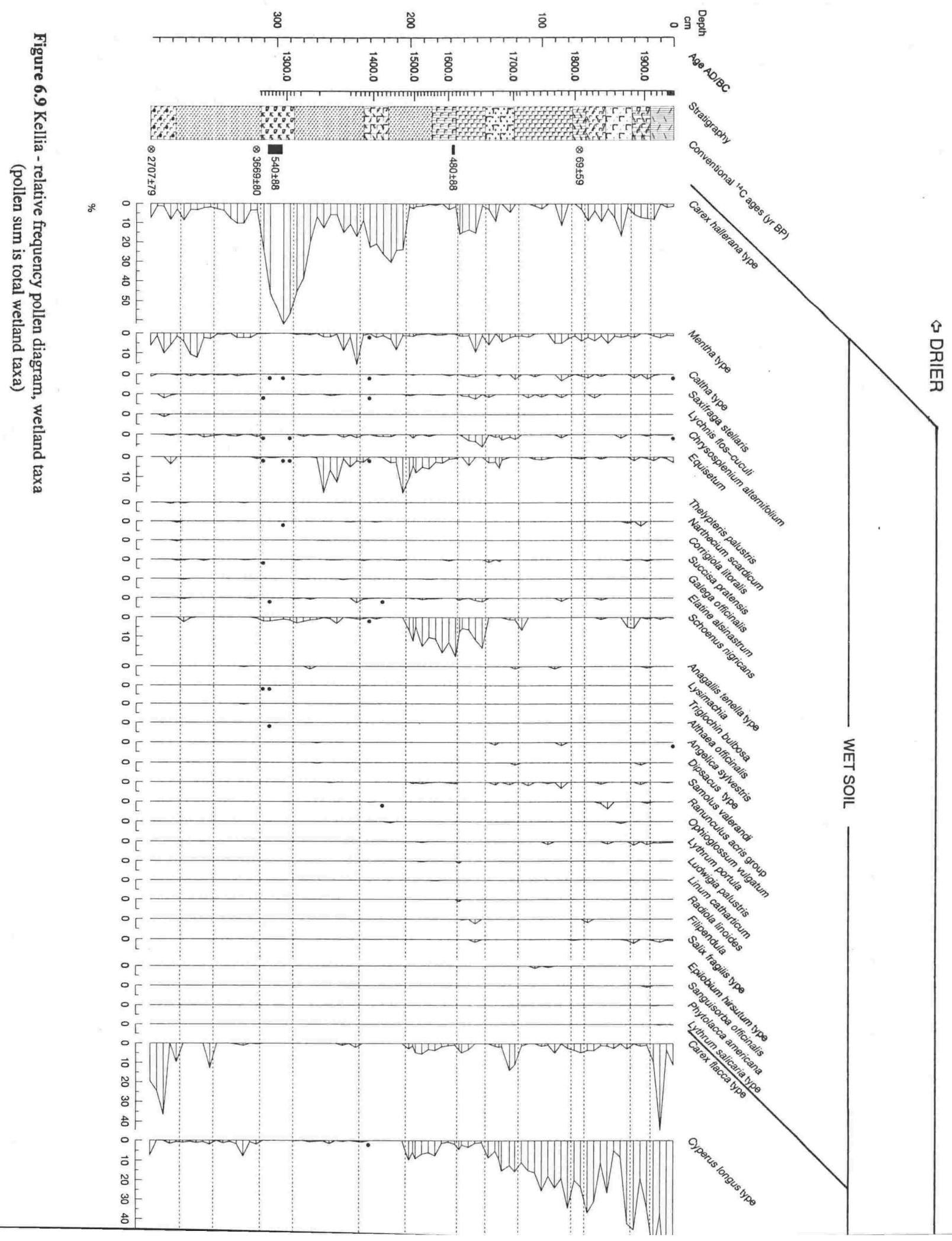




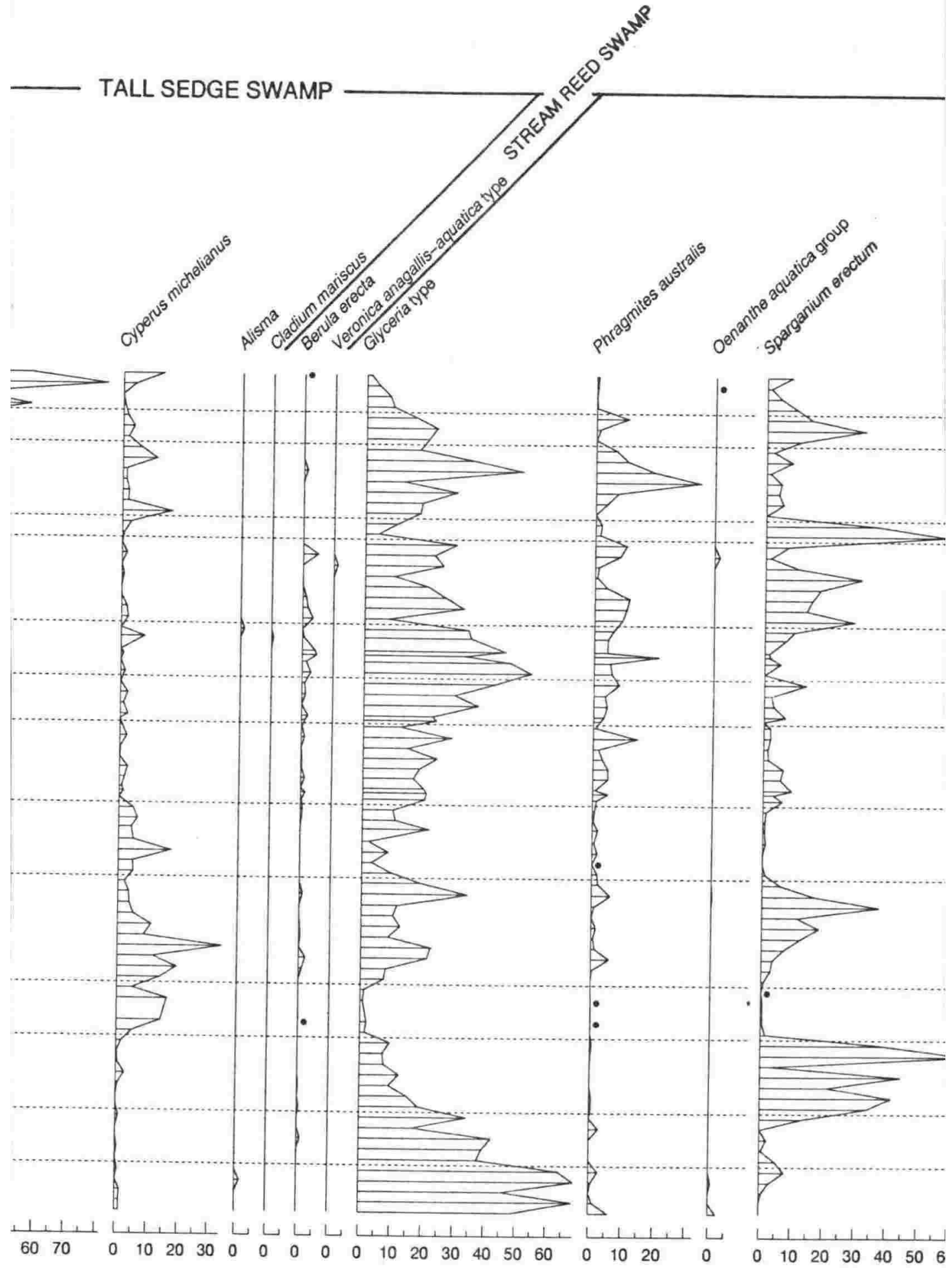




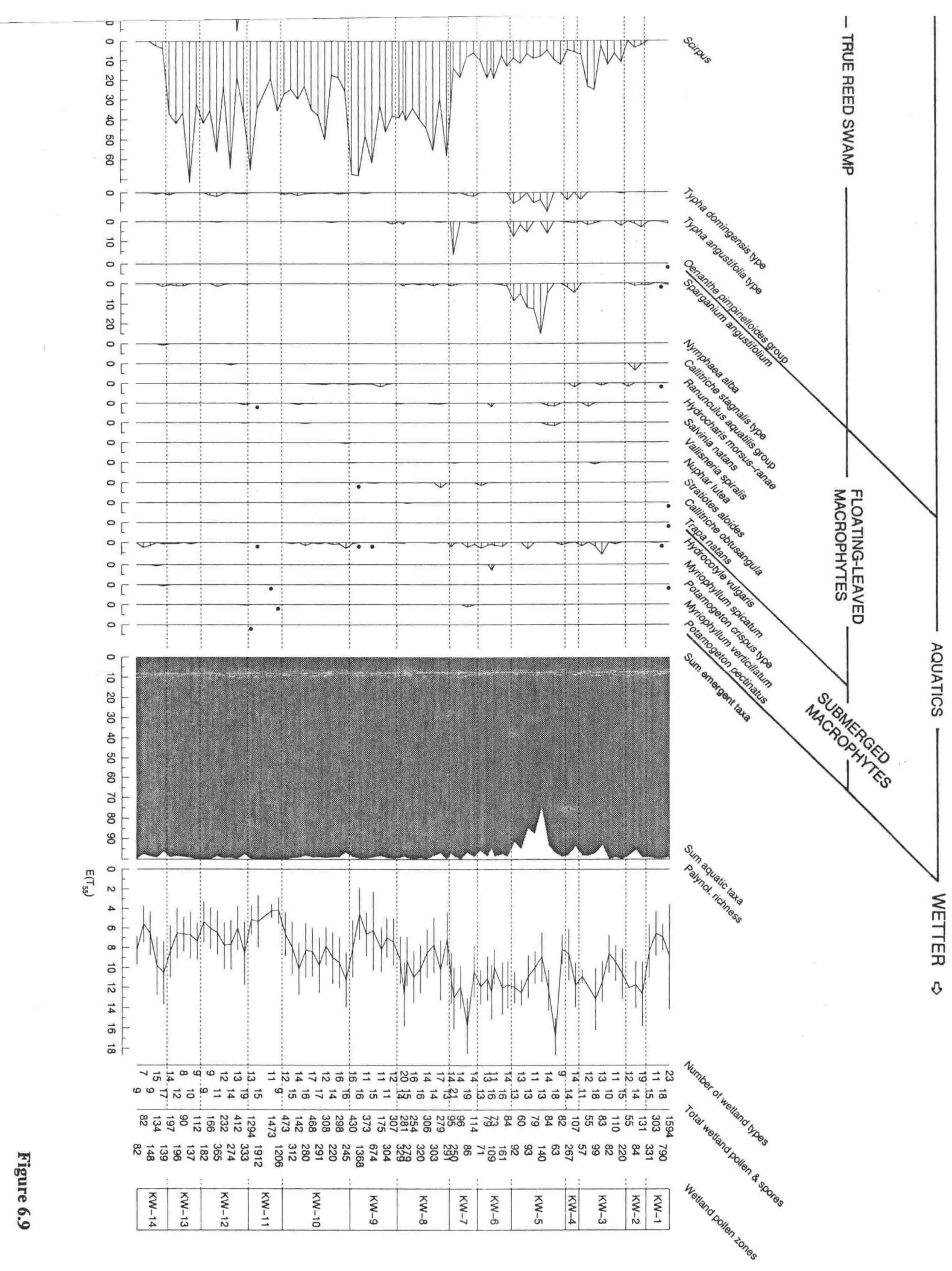




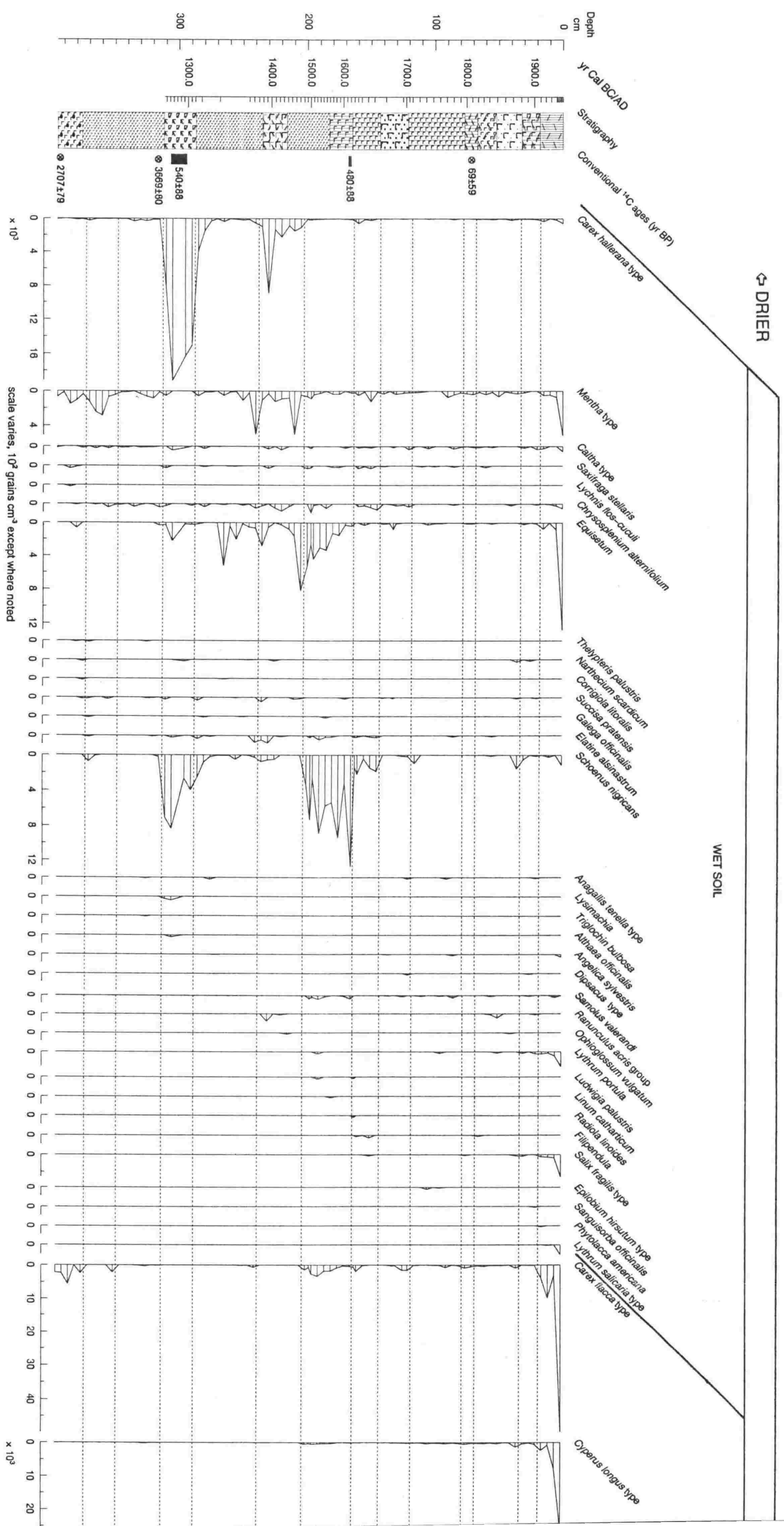




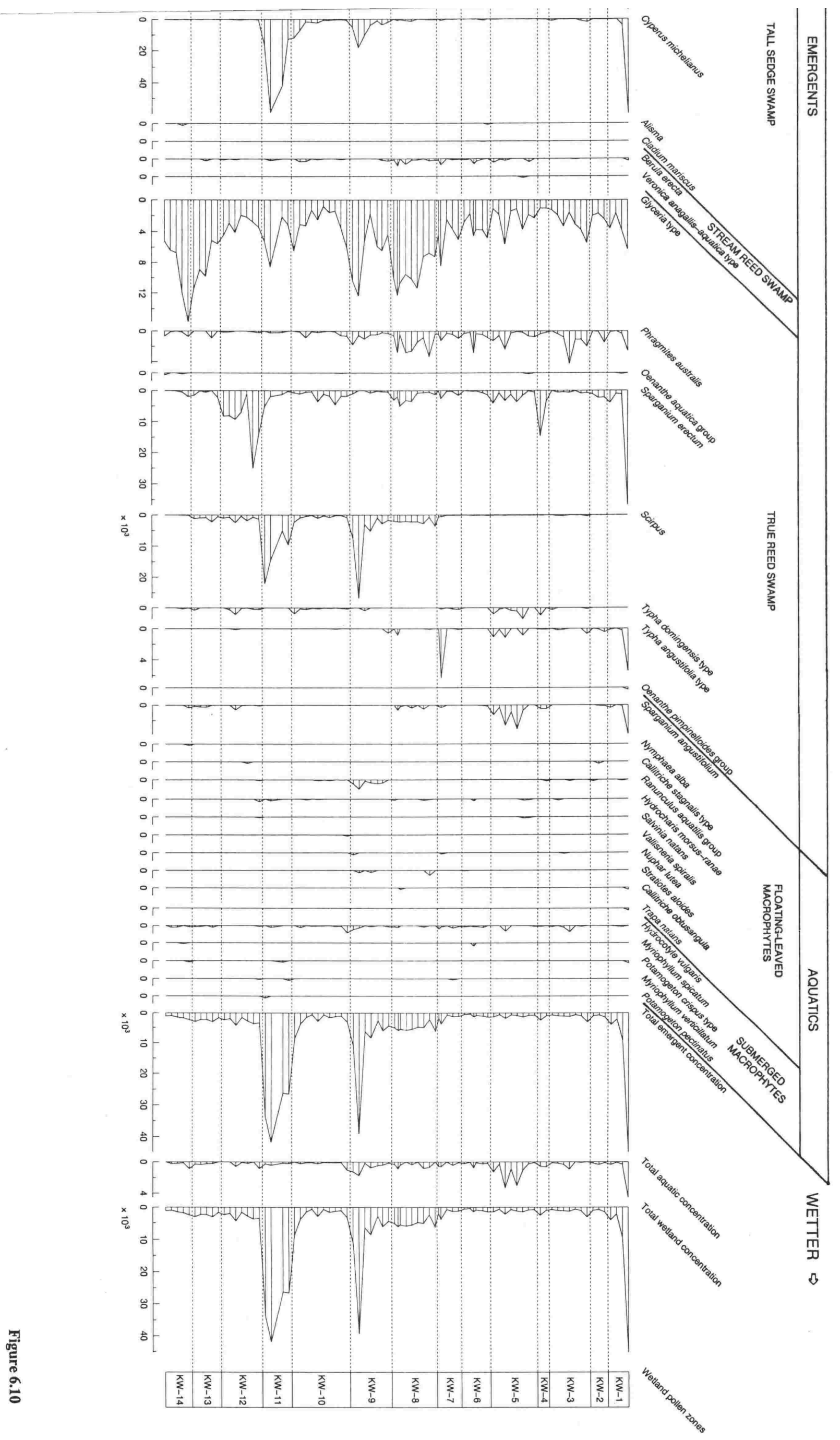


Emergent communities The wetland pollen and spore sequence is dominated by types that derive from emergent plant communities. Pollen and spore types belonging to the 'wet soil' habitat group are most numerous, although relative frequency values of the 'true reed swamp' habitat group are highest throughout the sequence except from $305-280 \mathrm{~cm}$, where the relative frequencies of the 'wet soil' habitat group are higher, and at $35,30,20 \mathrm{~cm}$, and the surface, where relative frequencies of the 'tall sedge swamp' habitat group are higher.

Chapters 4 and 5 include discussions on the classification of wetland pollen and spore types into ecological groups. Only types not discussed previously are discussed below.

Several types of the habitat group 'wet soil' identified in the Kellia sequence were not identified in the other two sequences. Succisa pratensis is placed in the order Molinietalia (moist meadow and stream bank communities) and Ludwigia palustris and Radiola linoides in the order Cyperetalia fusci (dwarf plant communities, which are inundated in winter but dry out) (Ellenberg 1988). Many other types of the 'wet soil' habitat group also belong to these orders. Schoenus nigricans is placed in the order Tofieldietalia of calcareous small-sedge beds and related communities. Corrigiola litoralis is placed in the class Chenopodietea of communities of waste ground and related arable and garden weed communities and described as a damp-site indicator that grows mainly on constantly damp, but not wet, soils (Ellenberg 1988). Although usually growing at low altitudes, it has been found at $c .1850 \mathrm{~m}$ in mainland Greece (Strid 1989). Thelypteris palustris belongs to the order Alnetalia of alder swamp woods (Ellenberg 1988).

Dipsacus type includes the two species Dipsacus fullonum and D. laciniatus, and possibly also Cephalaria ambrosioides, C. flava, and C. transylvanica. Dipsacus fullonum is placed in the order Calystegio-Alliarietalia of hedgerow veil communities and semi-shade communities (Ellenberg 1988) but it, as well as D. laciniatus sometimes also grows in marshes (Polunin 1988), while the species of the genus Cephalaria probably grow in semishade communities (Polunin 1988).

Epilobium hirsutum type includes several species (Epilobium hirsutum, E. montanum, E. palustre, E. parviflorum, E. tetragonum) and probably several others (Epilobium adenocaulon, E. alsinifolium, E. anagallidifolium, E. dodonaei, E. gemmascens, E. lanceolatum, E. obscurum, E. roseum). The species Epilobium hirsutum, E. montanum, and E. parviflorum that definitely belong to this type are classified by Ellenberg (1988) into a variety of plant communities. Epilobium hirsutum is placed in the order Brometalia erecti of suboceanic more or less arid swards, E. montanum is placed in the order Fagetalia of noble broadleaved woods and related communities, and E. parviflorum is placed in the order Calystegio-Alliarietalia of hedgerow veil communities and semi-shade communities. These species are indicated as growing in water conditions ranging from damp soils to water- 
saturated soils. E. palustre is a wet-site indicator, often in water-saturated badly aerated soils and E. tetragonum is a moist-site indicator (Ellenberg 1988). Several species of Epilobium were noted growing on Kellia fen; Epilobium hirsutum, E. cf. parviflorum, and E. cf. adenocaulon. In Grevena the genus Epilobium was observed growing mostly in wet places, especially along margins of water courses, on fens, and in disturbed places.

The following species are not classified by Ellenberg (1988): Phytolacca americana grows in marshes and ruderal places, Triglochin bulbosa grows in marshes (freshwater- or salt-marshes), Althaea officinalis grows in damp places and field verges, and Lythrum portula in wet places and in shallow pools (Polunin 1988; Strid 1989).

Only one additional pollen type of the 'tall sedge swamp' habitat group occurs, that of Alisma. Three species are included in this type: Alisma gramineum, A. lanceolatum, and A. plantago-aquatica. A plantago-aquatica is placed in the order Phragmitetalia. Alisma gramineum and A. lanceolatum are not classified by Ellenberg (1988). They grow in damp places and shallow water (Haslam et al. 1975; Polunin 1988).

Aquatic communities Aquatic pollen and spore types are poorly represented, although types of the 'floating-leaved macrophytes' habitat group are more numerous and better represented than the habitat group of 'submerged macrophytes'.

'Floating-leaved macrophytes' are represented in almost all spectra, although usually at very low relative frequencies. Three of these taxa, Hydrocharis morsus-ranae, Stratiotes aloides, and Salvinia natans belong to Ellenberg's (1988) Lemnetalia order of floating communities of waters more or less rich in nutrients. With the exception of Sparganium angustifolium of the order Littorelletalia and Vallisneria spiralis, which is not classified but grows submerged in still nutrient-rich water (Willis 1951; Haslam et al. 1975; Polunin 1988), the remainder of the taxa in this habitat group belong to the order Potamogetonetalia of rooted water plant communities; this includes three types (Callitriche stagnalis type, Callitriche obtusangula, and Trapa natans), which were not identified in the sequences from the Gomara and Anelia wetlands (Ellenberg 1988). Sparganium angustifolium (floating bur-reed) is abundant in zone KW-5, being more abundant than any other aquatic type in this sequence. It grows in still water in lakes of oligotrophic to dystrophic nutrient levels or pools at a water depth of 0.5-2.0 $\mathrm{m}$ and roots in fine organic soil (Haslam et al. 1975).

The only macrophytes included in the 'submerged macrophyte' habitat group that are exclusively submerged are those included in the pollen types of Myriophyllum spicatum, Myriophyllum verticillatum, and Potamogeton pectinatus. These types occur sporadically at low relative frequencies. Potamogeton crispus type, which includes species capable of living in either deep or shallow water, either submerged or floating, and subterrestrially in dried-up 
situations, also occurs infrequently at low relative frequencies. Hydrocotyle vulgaris, which may be submerged or emergent, occurs more often and at higher relative frequencies.

\subsubsection{Wetland (local) pollen assemblage zones}

Wetland pollen diagrams are divided into 14 local pollen assemblage zones KW-1 to $\mathrm{KW}-14$, based mostly on relative frequency data. These are defined below.

Zone $K \boldsymbol{W}-14,396.0-372.5 \mathrm{~cm}$ This basal zone is characterised by high relative frequencies of Glyceria type (45.3-68.7\%) and Carex flacca type (19.5-36.5\% in the three lowest spectra, but then at $0-9.4 \%$ ). Carex hallerana type fluctuates from $1.2-8.2 \%$ and Mentha type from 1.2-10.1\%. Several emergent types occur sporadically, but reach significant abundance when they occur: Phragmites australis reaches $6.1 \%$ in the basal spectrum, Scirpus and Sparganium erectum reach zonal maxima in the uppermost spectrum $(3.6 \%$ and $7.9 \%$, respectively), Cyperus longus type reaches $7.3 \%$ in the basal spectrum, and Equisetum occurs at $380 \mathrm{~cm}$ only at $3.7 \%$. All other emergents are represented at $2.0 \%$ or less.

Although poorly represented aquatic types occur in all spectra except the basal one, but total relative frequency does not exceed $2.8 \%$ (recorded in the top spectrum). Five types occur. Two types exceed 1\%; Sparganium angustifolium reaches $1.4 \%$ at $375 \mathrm{~cm}$ and Hydrocotyle vulgaris reaches $2.4 \%$ at $390 \mathrm{~cm}$ and $1.4 \%$ at $385 \mathrm{~cm}$.

Zone $\mathrm{KW}-13,372.5-347.5 \mathrm{~cm}$ The boundary with the preceding zone is marked by a decline in the relative frequency of Glyceria type and an increase in Scirpus. This zone is characterised by the co-dominance of Scirpus and Glyceria type. Both types generally record from about $32-42 \%$ with the exception of the spectrum second from the top where Scirpus increases to $71.5 \%$ and Glyceria type declines to $16.8 \%$. Carex hallerana type records $8.6 \%$ in the basal spectrum, but then drops to $3.1 \%$ and then declines further to $1.8 \%$ in the uppermost spectrum. Frequencies of Mentha type are highest in the centre of the zone reaching a maximum of $12.2 \%$, but the basal spectrum records $4.1 \%$ and the two uppermost record 2.2 and $2.7 \%$. Several emergent types occur sporadically: Carex flacca type is absent apart from the uppermost spectrum where it records $12.5 \%$, Phragmites australis is absent apart from the spectrum second from the top where $2.9 \%$ is recorded, Schoenus nigricans occurs in only the basal and following spectra (2.5 and 0.5\%), and Sparganium erectum reaches highest values in the basal and uppermost spectra (5.1 and 13.4\%, respectively), but other values are only $2.2 \%$ or less. No other emergent type exceeds $1.8 \%$.

Representation of aquatic types is very low; total frequency does not exceed $1.5 \%$. Sparganium angustifolium and Hydrocotyle vulgaris only are represented at $1.1 \%$ or less.

Zone $K W-12,347.5-312.5 \mathrm{~cm}$ The boundary with the preceding zone is marked by a further decline in Glyceria type and a dramatic increase in Sparganium erectum. The zone is 
characterised by the co-dominance of Sparganium erectum $(21.1-66.0 \%$, except at $325 \mathrm{~cm}$ where only $3.6 \%$ is recorded) and Scirpus (18.4-64.6\%). Relative frequencies of Glyceria type are still comparatively high (7.3-18.7\%). Carex hallerana type ranges from $2.7-10.3 \%$, being highest in the centre three spectra. Mentha type has declined (2.2\% and less). Cyperus longus type occurs sporadically, but reaches $7.7 \%$ at $325 \mathrm{~cm}$. Cyperus michelianus also occurs sporadically, but reaches $2.6 \%$ at $330 \mathrm{~cm}$. No other emergent type exceeds $2.0 \%$.

Aquatic types are again very rare, occurring in 4 of the 7 spectra of the zone; total frequency does not exceed $2.5 \%$. More types are represented (7) than in the zone below, but only Sparganium angustifolium exceeds $1.0 \%$, reaching $1.4 \%$ at $335 \mathrm{~cm}$.

Zone $K W-11,312.5-287.5 \mathrm{~cm}$ The boundary with the preceding zone is marked by an increase in the relative frequency of Carex hallerana type and Cyperus michelianus, and a decrease in Glyceria type and Sparganium erectum. The zone is characterised by high relative frequencies of Carex hallerana type (24.0-62.1\%) and Scirpus (19.3-65.3\%), with Cyperus michelianus (4.9-14.0\%) also abundant. Glyceria type declines to $2.0 \%$ and less. No other emergent type exceeds $2.0 \%$.

Aquatic types are again very rare but occur in all spectra; total frequency does not exceed $0.2 \%$. Five types are represented, but no individual type exceeds $0.1 \%$.

Zone $K \boldsymbol{W}-10,287.5-237.5 \mathrm{~cm}$ The boundary with the preceding zone is marked by a decline in the relative frequency of Carex hallerana type and an increase in Glyceria type. The zone is characterised by high relative frequencies of Cyperus michelianus, Glyceria type, Sparganium erectum, and Scirpus. Carex hallerana type is also abundant. Frequencies of these types fluctuate, being most constant in Scirpus (17.7-50.0\%). Glyceria type records 7.4-33.6\%, Cyperus michelianus $2.0-18.9 \%$ (with one exceptionally high value of $33.9 \%$ ), Sparganium erectum $0.8-37.3 \%$, and Carex hallerana type 5.8-45.2\%. Mentha type and Equisetum occur sporadically, but reach $15.5 \%$ and $17.9 \%$, respectively. Schoenus nigricans and Phragmites australis also occur sporadically and reach lower values of $3.2 \%$ and $5.7 \%$, respectively. No other emergent type exceeds $2.1 \%$.

Aquatic types occur in all spectra except the basal spectrum; total frequency exceeds $2.0 \%$ at only $240 \mathrm{~cm}$ where it reaches $3.3 \%$. Five types are represented, but only Hydrocotyle vulgaris exceeds $1.0 \%$, reaching $1.4 \%$ at $270 \mathrm{~cm}$ and $2.9 \%$ at $240 \mathrm{~cm}$.

Zone $K W-9,237.5-202.5 \mathrm{~cm}$ The boundary with the preceding zone is marked by an increase in the relative frequency of Scirpus and a decline in Glyceria type and Sparganium erectum. The zone is characterised by high relative frequencies of Carex hallerana type (20.9-30.3\% with the exception of the basal spectrum, which is 8.6\%) and Scirpus (33.168.0\%). Cyperus michelianus and Glyceria type are comparatively abundant recording 4.0$5.9 \%$ (with one exceptionally high value of $16.6 \%$ ) and $2.1-10.5 \%$ (with one exceptionally 
high value of $21.1 \%$ ), respectively. Mentha type occurs in all spectra usually at less than $3.0 \%$, but increases to $8.2 \%$ at $210 \mathrm{~cm}$. Equisetum occurs in all spectra except one at 0.1 $2.9 \%$, with the exception of the uppermost spectrum where it increases to $18.2 \%$. No other emergent type exceeds $1.7 \%$.

Aquatic types occur in all spectra, but total frequency does not exceed $1.7 \%$. Four types are represented; only Ranunculus aquatilis exceeds $0.5 \%$, reaching $1.7 \%$ at $214 \mathrm{~cm}$.

Zone $K \boldsymbol{W}-8,202.5-163.5 \mathrm{~cm}$ The boundary with the preceding zone is marked by an increase in the relative frequency of Schoenus nigricans, Carex flacca type, Cyperus longus type, and Sparganium erectum, and a decline in Carex hallerana type and Cyperus michelianus. The zone is characterised by high relative frequencies of Schoenus nigricans, Glyceria type, and Scirpus. Schoenus nigricans shows a general increase from the basal spectrum to the uppermost (6.7-20.6\%). Scirpus also shows a general increase from the basal spectrum to the uppermost (39.2-58.4\%) with the exception of a decline to $29.4 \%$ at $170 \mathrm{~cm}$, while Glyceria type is reasonably constant (11.7-28.0\%). Carex flacca type reaches its highest value of $5.5 \%$ at $190 \mathrm{~cm}$. Cyperus longus type increases in the lower part of the zone (5.7$9.7 \%)$, but then declines (0.7-1.7\%). Frequencies of Sparganium erectum increase in the lower part of the zone (3.2-9.0\%), but then decline (1.3-2.2\%). Phragmites australis fluctuates from $0.7-4.7 \%$ generally, but at $170 \mathrm{~cm}$ increases to $13.6 \%$. Equisetum shows a steadily declining trend from $10.3 \%$ in the basal spectrum to $0.7 \%$ in the uppermost. Carex hallerana type and Cyperus michelianus have declined and occur sporadically. They exceed $2.0 \%$ in only two spectra and neither exceeds $3.0 \%$. No other emergent type exceeds $1.8 \%$.

Aquatic types occur in 6 of the 9 spectra of this zone. Total frequency exceeds $2.0 \%$ only at $170 \mathrm{~cm}$, reaching $2.9 \%$. Five types are represented, but only Sparganium angustifolium and Stratiotes aloides exceed 1.0\%, reaching $1.1 \%$ at $197 \mathrm{~cm}$ and $2.5 \%$ at $170 \mathrm{~cm}$, respectively.

Zone $K W-7,163.5-142.5 \mathrm{~cm}$ The boundary with the preceding zone is marked by an increase in the relative frequency of Carex hallerana type and a decline in Scirpus. The zone is characterised by high relative frequencies of Carex hallerana type, Schoenus nigricans, Glyceria type, and Scirpus. Glyceria type records the highest frequencies (22.0-42.1\%). Carex hallerana type records $13.5-15.8 \%$ with the exception of the uppermost spectrum where it declines to $0.9 \%$. Schoenus nigricans records slightly lower frequencies (5.6-11.6\%), except in the uppermost spectrum, where it increases (15.8\%). Scirpus declines from a basal value of $35.8 \%$ to $6.1 \%$ in the uppermost spectrum.

Other emergent types that record $2.0 \%$ or more are: Phragmites australis (2.1-7.9\%); Mentha type (0.9-9.3\%); Cyperus longus type, which has declined (0.9-4.2\%); Carex flacca type, which occurs in the three lower spectra only (3.1-4.8\%); Saxifraga stellaris, which 
occurs in the three centre spectra only (0.8-2.3\%); Cyperus michelianus, which also occurs in the three centre spectra only (1.0-2.4\%); Chrysosplenium alternifolium, which occurs in the four upper spectra (0.8-6.1\%); Sparganium erectum, occurs in the four upper spectra also (2.3$13.2 \%$ ); Berula erecta, which occurs sporadically, but reaches $2.0 \%$ at $160 \mathrm{~cm}$; Filipendula, which occurs in two spectra (0.4 and $2.3 \%)$; Equisetum, which occurs in one spectrum only (4.2\%); and Typha angustifolia type, which occurs in two spectra ( 0.9 and $16.4 \%)$.

Aquatic taxa are represented in 4 of the 5 spectra; total frequency exceeds $2.0 \%$ in only 2 spectra, $3.2 \%$ at $162 \mathrm{~cm}$ and $3.5 \%$ at $150 \mathrm{~cm}$. Four types are represented, only 3 types exceed $1.0 \%$ : Hydrocotyle vulgaris $(2.1 \%$ at $162 \mathrm{~cm}$ and $2.3 \%$ at $150 \mathrm{~cm}$ ), Myriophyllum verticillatum $(1.2 \%$ at $150 \mathrm{~cm})$, and Sparganium angustifolium $(1.1 \%$ at $162 \mathrm{~cm})$.

Zone $K \boldsymbol{W}-6,142.5-117.5 \mathrm{~cm}$ The boundary with the preceding zone is marked by an increase in the relative frequency of Cyperus longus type and decrease in Carex hallerana type and Schoenus nigricans. The zone is characterised by high relative frequencies of Cyperus longus type, Glyceria type, and Scirpus. Glyceria type has the highest frequencies of the zone (32.1-53.5\%). Cyperus longus type rises steadily from $8.5 \%$ in the basal spectrum (with the exception of $5.1 \%$ in the second spectrum) to $15.5 \%$ in the uppermost spectrum. Scirpus rises from $9.9 \%$ in the basal spectrum to a maximum of $19.2 \%$ in the centre of the zone, then declines to $13.1 \%$ in the uppermost spectrum. Phragmites australis is comparatively abundant, recording 4.1-6.0\%, with a peak in the centre of the zone at $132 \mathrm{~cm}$ of $20.2 \%$.

Many emergent types occur at low frequencies: Berula erecta (5 spectra at $0.6-4.6 \%$ ); Carex flacca type (first 4 spectra at 0-1.8\%, then 13.7 and 10.7\%); Carex hallerana type (sporadic occurrence, but reaches $8.9 \%$ at $135 \mathrm{~cm}$ ); Chrysosplenium alternifolium (sporadic occurrence, reaches 2.7 and $2.4 \%$ in 2 spectra); Cyperus michelianus (sporadic occurrence, reaches $7.5 \%$ at $124 \mathrm{~cm})$; Equisetum (1.4-5.5\% in lower 4 spectra); Mentha type (sporadic occurrence, but exceeds $2.0 \%$ in 2 spectra -5.6 and $4.1 \%$ ); Sparganium erectum (sporadic occurrence, reaches $9.5 \%$ at $120 \mathrm{~cm}$ ); and Caltha type (sporadic occurrence, but reaches $2.4 \mathrm{~cm}$ at $120 \mathrm{~cm}$ ). No other emergent type exceeds $2.0 \%$.

Aquatic types are represented in all spectra. Total frequency exceeds $2.0 \%$ in 3 of the 6 spectra; $4.2 \%$ at $140 \mathrm{~cm}, 5.5 \%$ at $132 \mathrm{~cm}$, and $2.5 \%$ at $124 \mathrm{~cm}$. Only five aquatic types are represented, but frequencies have increased. Five types exceed $1.0 \%$ : Hydrocotyle vulgaris $(2.8 \%$ at $140 \mathrm{~cm}, 1.3 \%$ at $135 \mathrm{~cm}, 1.4 \%$ at $130 \mathrm{~cm}$, and $1.9 \%$ at $124 \mathrm{~cm})$, Myriophyllum spicatum $(2.8 \%$ at $132 \mathrm{~cm})$, Sparganium angustifolium $(1.2 \%$ at $120 \mathrm{~cm})$, Hydrocharis morsus-ranae $(1.8 \%$ at $132 \mathrm{~cm})$, and Stratiotes aloides $(1.4 \%$ at $140 \mathrm{~cm})$.

Zone $K \boldsymbol{W}-5,117.5-77.5 \mathrm{~cm}$ The boundary with the preceding zone is marked by an increase in the relative frequency of Sparganium erectum and Sparganium angustifolium. The zone is characterised by high relative frequencies of Cyperus longus type, Glyceria type, 
Sparganium erectum, and Sparganium angustifolium. The first three types represent emergent plants while the last represents a plant that is usually a floating-leaved aquatic. Although Glyceria type has declined, in general it has the highest relative frequencies of the zone usually from $20.3-31.7 \%$ (2 spectra record only 7.6 and 9.3\%). Sparganium erectum fluctuates also, but in general records $10.7-30.7 \%$ (only $1.6 \%$ and $7.3 \%$ in the two uppermost spectra). Cyperus longus type displays a general increase (10.9-34.1\%). Sparganium angustifolium ranges from $4.8-25.0 \%$ in the lower 6 spectra, then disappears.

Other emergents that are abundant are: Scirpus (4.8-12.2\%), Phragmites australis recording mostly $3.8-10.8 \%$ (but declining to 0.7 and $1.2 \%$ in two spectra), and Typha angustifolia type and Typha domingensis type both occurring sporadically but recording comparatively high frequencies where they occur (maximums of $7.6 \%$ and $9.5 \%$, respectively). Other emergents that are less abundant, but record $2.0 \%$ or more are: Berula erecta (occurs sporadically, but reaches 3.3 and $4.8 \%$ in 2 spectra); Caltha type (occurs sporadically, reaches $3.2 \%$ at $85 \mathrm{~cm}$ ); Carex flacca type (occurs sporadically, reaches 4.8 and $2.4 \%$ in 2 spectra); Carex hallerana type (occurs sporadically, reaches 11.1 and $2.5 \%$ in two spectrum); Cyperus michelianus (occurs sporadically, reaches $2.2 \%$ in one spectrum); Mentha type (occurs sporadically, reaches $4.8 \%$ in 2 spectra and $2.4 \%$ in one spectrum); Samolus valerandi (occurs sporadically, reaches $3.2 \%$ in one spectrum); and Schoenus nigricans (occurs in only the basal spectrum at $6.5 \%$ ).

Aquatic types are represented in all spectra; total frequency exceeds $2.0 \%$ in all spectra except the surface spectrum. Only four types are represented, but frequencies have increased again. All types represented exceed 1.0\%: Hydrocotyle vulgaris $(3.2 \%$ at $105 \mathrm{~cm}$ and $1.2 \%$ at $80 \mathrm{~cm})$, Salvinia natans $(1.2 \%$ at $90 \mathrm{~cm}$ and $1.6 \%$ at $85 \mathrm{~cm})$, Sparganium angustifolium (discussed above), and Hydrocharis morsus-ranae (1.2\% at $90 \mathrm{~cm}$ and $1.6 \%$ at $85 \mathrm{~cm}$ ).

Zone $K W-4,77.5-67.5 \mathrm{~cm}$ The boundary with the preceding zone is marked by an increase in the relative frequency of Sparganium erectum and a decline in Cyperus longus type and Glyceria type. The zone is characterised by high relative frequencies of Cyperus longus type (19.9 and 23.4\%) and Sparganium erectum (57.7 and 35.5\%). Other emergents exceeding 2.0\% are: Carex flacca type (3.4 and 4.7\%), Carex hallerana type (one spectrum only at $2.8 \%$ ), Cyperus michelianus (0.7 and 2.8\%), Glyceria type (4.1 and 10.3\%), Mentha type (1.1 and 3.7\%), Scirpus (4.5 and 5.6\%), and Typha domingensis type (basal spectrum only at $3.7 \%$ ).

Aquatic types are represented in both spectra; total frequency exceeds $2.0 \%$ in both. Only three types are represented. Two types exceed 1.0\%; Ranunculus aquatilis type (1.9\% at $70 \mathrm{~cm})$ and Sparganium angustifolium $(1.9 \%$ at $75 \mathrm{~cm}$ and $4.7 \%$ at $70 \mathrm{~cm})$. 
Zone $K W-3,67.5-32.5 \mathrm{~cm}$ The boundary with the preceding zone is marked by increases in the relative frequency of Carex hallerana type and Glyceria type and a decline in Sparganium erectum. The zone is characterised by high relative frequencies of Carex hallerana type, Cyperus longus type, Glyceria type, Phragmites australis, and Scirpus. Glyceria type has the highest overall relative frequencies (12.0-50.0\%). Scirpus records an initial low relative frequency $(7.0 \%)$, increases to $25.3 \%$, then declines $(2.4-12.2 \%$ in the upper four spectra). Cyperus longus type declines irregularly from 36.8 to $4.9 \%$ before a final increase to $42.3 \%$. Carex hallerana type fluctuates also (1.8-16.4\%). Phragmites australis is absent from the basal spectrum, records low values in the next two spectra and the two uppermost spectra (3.6-10.0\%), and peaks in the two central spectra (32.5-18.3\%).

Other emergent types that occur at $2.0 \%$ or greater are: Carex flacca $(0-3.6 \%)$, Cyperus michelianus (5.9-15.8\% at the top and bottom of the zone, but $1.2-2.0 \%$ in the centre), Mentha type (0.9-4.8\%), Ranunculus acris group (0 except for two central spectra, which record 1.0 and $3.6 \%$ ), Schoenus nigricans (0 except for two spectra recording 1.0 and 5.0\%), Sparganium erectum ( 0 in one spectrum, elsewhere 1.2-8.2\%), Typha angustifolia type (0-2.3\%), and Typha domingensis type ( 0 and $0.5 \%$, but one spectrum reaches $3.5 \%$ ).

Aquatic types are represented in 5 of the 7 spectra; total frequency exceeds $2 \%$ at only 55 and $50 \mathrm{~cm}$. Four aquatic types are represented, but only three of these exceed $1.0 \%$; Hydrocharis morsus-ranae $(1.8 \%$ at $60 \mathrm{~cm})$, Hydrocotyle vulgaris $(1.8 \%$ at $65 \mathrm{~cm}, 2.0 \%$ at $55 \mathrm{~cm}$, and $6.0 \%$ at $50 \mathrm{~cm})$ and Ranunculus aquatilis type $(1.2 \%$ at $50 \mathrm{~cm})$.

Zone $K W-2,32.5-17.5 \mathrm{~cm}$ The boundary with the preceding zone is marked by an increase in the relative frequency of Sparganium erectum and decreases in Carex hallerana type, Glyceria type, and Scirpus. The zone is characterised by high relative frequencies of Cyperus longus type (19.0-45.5\%), Glyceria type (16.0-22.6\%), and Sparganium erectum (10.9-31.0\%). Carex hallerana type is comparatively abundant, recording $5.5-7.6 \%$.

Other emergent types that exceed $2.0 \%$ are: Caltha type (the uppermost spectra reaches $2.3 \%$ ), Cyperus michelianus (reaches $3.6 \%$ in the central spectrum), Narthecium scardicum (occurs in the central spectrum only at $2.4 \%$ ), Schoenus nigricans (occurs sporadically, but reaches $5.5 \%$ in one spectrum), Scirpus (occurs in only two spectra at 3.6 and $2.3 \%$ ), and Typha angustifolia type (occurs sporadically, but reaches $3.1 \%$ in the uppermost spectrum).

Aquatic types are represented in all spectra; total frequency exceeds $2.0 \%$ only at $25 \mathrm{~cm}$. Only three types are represented, and all exceed 1.0\%: Callitriche stagnalis type $(3.6 \%$ at $25 \mathrm{~cm})$, Ranunculus aquatilis type $(1.8 \%$ at $30 \mathrm{~cm})$, and Sparganium angustifolium $(1.2 \%$ at $25 \mathrm{~cm}$ ).

Zone $K W-1,17.5-0 \mathrm{~cm}$ The boundary with the preceding zone is marked by an increase in the relative frequency of Carex flacca type and Cyperus longus type, and a decrease in 
Glyceria type, Phragmites australis, Sparganium erectum, and Scirpus. The zone is characterised by high relative frequencies of Cyperus longus type (37.3-81.4\%) and Carex flacca type (3.3-11.0\%, with a sharp increase in the second spectrum to $44.6 \%)$. Other emergent types represented are: Cyperus michelianus, which occurs sporadically but increases to $13.1 \%$ in the surface sample, Glyceria type, which is comparatively abundant but declines from 8.8-1.4\%, and Sparganium erectum, which fluctuates from 1.3-9.7\%. Less abundant emergent types are: Carex hallerana type (occurs sporadically but records a maximum of $7.9 \%$ in the basal spectrum), Equisetum (occurs sporadically but reaches $2.8 \%$ in the surface spectrum), and Mentha type (reaches a maximum of $2.0 \%$ ). No other emergent type exceeds $2.0 \%$.

Aquatic types are poorly represented, but occur in all spectra; total frequency does not exceed $1.1 \%$. The number of types represented has increased to six, but no type exceeds $1.0 \%$.

\subsubsection{Sedimentary and local vegetation history}

This sequence records the local (wetland) vegetation and sedimentary history of the Kellia wetland site. Radiocarbon dating suggests that it covers two periods of alluviation, an older period below $312 \mathrm{~cm}$ (occurring perhaps after $830 \mathrm{BC}$ and spanning 200 years, see section 6.4.2) and a younger one above, which may span about the last 760 years beginning c. $1230 \mathrm{AD}$.

Stratigraphic data indicate that Kellia fen may have formed in an old stream course that ran from west to east with a terrace on the northern side (Figures $6.2 \& 6.4$ ). The entire sequence represents a combination of fen and stream deposits and consists of three major units. The uppermost c. $20 \mathrm{~cm}$ consists of silty peat on the western and southern part of the fen, close to the spring. The organic content of this unit is generally higher than that of horizons rich in organic matter lower in the sequence. Towards the northern edge and the eastern (downstream) end of the fen, the unit becomes less peaty and is replaced by brown clay with a sandy layer at the base. This unit may be related to establishment of the modern sealed road and its causeway, which may have reduced the transport of sediment into the fen from the catchment upstream. Below this uppermost unit are up to $450 \mathrm{~cm}$ of greyish olive to olive black clay with variable proportions of calcareous grit, peat, and organic detritus. Thin $(5-15 \mathrm{~cm})$ noncalcareous sand horizons are rare. Persistent organic-rich horizons occur at c. 100 and $230 \mathrm{~cm}$. Below about $300 \mathrm{~cm}$, there is little organic matter in the stiff to soft greyish olive clay comprising the basal part of the unit. Occasional small mollusc shells and fruits or corms were noted in some cores, but no pattern to their occurrence was determined. The lowest unit, somewhat heterogeneous, consists of muddy yellow sand, yellow-brown silt, 
and clay with ferruginous mottling and nodules. In the most easterly borehole, gravel occurs at the base of sequence.

In the pollen analysed core, sediments are mostly greyish olive calcareous clay. Deposits of the current fen area are partly derived from soils of the catchment area, with a proportion being contributed by internal wetland productivity, i.e. autochthonous peat. Changes in the non-carbonate content of sediments reflect past changes in the catchment area related to differing rates of soil erosion. Peat developed during periods of relative soil stability in the catchment when water level was sufficient, at 183.0-67.5 and 32.5-0 cm. However, during these periods of relative stability some minerogenic sediments were washed into the fen area. Organic rich deposits containing organic detritus also indicate periods of comparative landscape stability when plants were able to become established in the catchment. These occur from 312.0-287.5 and 235.0-216.0 cm. Periods of high energy stream flow are indicated where minerogenic sediments of larger grain size (sand) occur; 271.0-268.0, $264.0-235.0,143.0-133.0,132-121$, and $52.0-32.5 \mathrm{~cm}$. The source of carbonate in the sediments is uncertain; it may be allochthonous (derived from calcic soils of the catchment) or autochthonous (precipitated from the spring water).

Several changes occur in the dominant local vegetation. At the base of the sequence local vegetation is dominated by the wetland reed-grass pollen type of Glyceria type. However, oxidation may have selectively removed some pollen types. In the overlying sediment horizon Glyceria type is co-dominant with Scirpus species, then Scirpus species and Sparganium erectum become co-dominant. When influx of minerogenic sediments declines at $312.0 \mathrm{~cm}$, species belonging to the Carex hallerana type, Cyperus michelianus and Scirpus are co-dominant. At $287.5 \mathrm{~cm}$ where influx of minerogenic sediments increases the concentration of all pollen and spore types declines dramatically and Scirpus species become dominant again, with Cyperus michelianus, Glyceria, and Sparganium erectum also abundant. At $237.0 \mathrm{~cm}$ where minerogenic influx declines again and total concentration of pollen and spores increases, Scirpus species increase dramatically, but species of Carex hallerana type, Cyperus michelianus, and wetland reed-grasses of the Glyceria type are again prominent. During the next phase of minerogenic influx Scirpus species and the wetland reed-grasses maintain their dominance and Schoenus nigricans also becomes prominent. These species continue to be dominant during the formation of peat at $183 \mathrm{~cm}$, but at $165 \mathrm{~cm}$ when additional minerogenic sediment is deposited Scirpus declines. Schoenus nigricans continues to be important up to $145 \mathrm{~cm}$ and Carex hallerana type increases between 162 and $150 \mathrm{~cm}$. Glyceria type increases from $162 \mathrm{~cm}$ and Cyperus longus type begins steadily increasing at $140 \mathrm{~cm}$. Wetland reed-grasses and species of the Cyperus longus type dominate the remainder of the sequence from $140 \mathrm{~cm}$, with the exception of Sparganium erectum becoming prominent 
during peat formation at $121.0-67.5 \mathrm{~cm}$ and reed-grasses almost disappearing at the surface. At the surface Cyperus longus type is dominant, and Carex flacca type, Cyperus michelianus, and Sparganium erectum increase.

The sedimentary history of the fen is intimately linked to the local wetland vegetation history, thus major sedimentary units defined above usually coincide with the established wetland pollen zones. These zones are used, for convenience, in the discussion below.

Zone $\mathrm{KW}-14,396.0-372.5 \mathrm{~cm}$, within range c. 830-630 BC The lowest sediment section plus a small part of the overlying section correspond to this zone (sections $\mathrm{N}$ and $\mathrm{M}$ ). The basal section at $396-376 \mathrm{~cm}$ comprises very stiff mottled grey and brown clay with calcareous and ferruginous grit. Ferruginous grit and mottling may indicate weathering prior to deposition of an overlying unit or subsurface water circulation. The sediments contain little organic matter and carbonate content is low.

At the base of the sequence the dominant plant community is the true-reed swamp, which is represented almost entirely by Glyceria type. A tall-sedge-swamp community has also developed, but declines towards the top of the zone. A small wet-soil community exists with Carex hallerana type and Mentha type being most abundant. Both floating-leaved and submerged communities of open water are represented by a few pollen and spore types.

The dominant type, Glyceria type, includes the reed-grasses Glyceria maxima and $G$. spicata as well as many species of the genus Bromus. The Glyceria species are perennials that grow in marshy ground or shallow water and currently grow on the seasonally dry lakebed at the Gomara core site. The Bromus species grow on dryland and are mostly annual weeds and ruderals, but also grow in grassland. However, the presence of members of the tall-sedgeswamp community suggests that the environment of deposition at this time was that of a flood plain with seasonal flooding; a river-bank reed strip (Ellenberg 1988). Of the two submerged aquatic macrophytes that are represented, Hydrocotyle vulgaris and Myriophyllum spicatum, the former may be submerged or emergent, while the latter is exclusively submerged and may have been growing in holes within the reed strip, i.e. in shallow and relatively humus-rich water. Concentration of wetland pollen and spores is low (total concentration of this horizon does not exceed $1.7 \times 10^{3}$ grains $/ \mathrm{cm}^{3}$ ) and selective oxidation in this horizon may complicate the picture by removing particular pollen and spore types. The low palynological richness (mostly 6-8 expected types) of this horizon may be a result of this. Nutrient level is probably very high as Glyceria maxima grows in eutrophic conditions and Carex species of the Carex flacca type grow in meso- to eutrophic conditions (Ellenberg 1988).

The overlying sediment horizon, above $376 \mathrm{~cm}$ comprises sticky soft grey clay with scattered gastropods and a trace of calcareous grit. Carbonate and organic content are unchanged from the horizon below, and there is little visible plant material. Total 
concentration of wetland pollen and spores increases to the maximum of the zone of $2.4 \times 10^{3}$ grains $/ \mathrm{cm}^{3}$ at $375 \mathrm{~cm}$ and palynological richness is slightly greater than 10 expected types. The concentration of Glyceria type increases at the top of the zone while that of Carex flacca type declines, perhaps due to more rapid influx of minerogenic sediments (Glyceria is able to tolerate silting, often growing on clay or alluvium where silting occurs (Haslam et al. 1975)). The appearance of the aquatic plants of Nymphaea alba (floating-leaved macrophyte) and Potamogeton crispus type (probably the submerged macrophyte Potamogeton natans) suggests the presence of eutrophic standing water with a community of MyriophylloNupharetum (Ellenberg 1988). This standing water may be on the side of a stream or on a fen.

Zone $\mathrm{KW}-13,372.5-347.5 \mathrm{~cm}$, within range c. 830-630 BC The sediments of this zone are included in sediment section $\mathrm{M}$ and are the same as those described above, but the vegetation changes. The true-reed-swamp community continues to be the dominant community, but Scirpus is now abundant and is co-dominant type with Glyceria type. The wet-soil community continues to be represented at about the same level, although Menthatype has become more important than Carex hallerana type. The tall-sedge community has almost disappeared and the stream-reed-swamp community has very low representation. Representation of the aquatic communities has declined slightly at the base of the zone and continues to decline towards the top. Both the floating-leaved and submerged macrophytes are represented; the former by Sparganium angustifolium and the latter by Hydrocotyle vulgaris.

The Scirpus pollen type includes two species of the genus Scirpus: Scirpus lacustris and S. maritimus. These species grow in lakes, ponds, and streams; in still to moderately fast flowing water. In still sheltered water they grow in water depths between one and two metres, but in flowing water they usually grow in water between 30 and $80 \mathrm{~cm}$ deep. They grow in eutrophic conditions in flowing water to oligotrophic in still water. They can be submerged or emergent. Scirpus maritimus can tolerate summer drought (Haslam et al. 1975), while Scirpus lacustris can grow in water and carry out photosynthesis while submerged (Ellenberg 1988). Scirpus maritimus usually grows in saline conditions so the species represented here is probably S. lacustris (Tutin et al. 1964-80). Scirpus lacustris (Common Clubrush) has two subspecies S. lacustris subsp. lacustris grows in fresh water while S. lacustris subsp. tabernaemontani grows in brackish water (Tutin et al. 1964-80). In calm lakes Scirpus is known to be a pioneer (Ellenberg 1988). Plants of the genus Scirpus are abundant on the fen today (Chapter 3). Scirpus may be colonising newly deposited sediment.

Available nutrient level is high as indicated by the abundance of Glyceria type and Scirpus and the occurrence of Sparganium erectum (Haslam et al. 1975; Ellenberg 1988). 
Zone $\mathrm{KW}-12,347.5-312.5 \mathrm{~cm}$, within range c. 830-630 BC The sediments of this zone are included in sediment section $\mathrm{M}$ and are the same as those of zone $\mathrm{KW}-13$, but organic content increases slightly, inorganic matter declines steadily, and the moisture content increases. The true-reed-swamp community continues to be the dominant community, but Sparganium erectum is now co-dominant with Scirpus. Glyceria type has declined further, but continues to be prominent. The wet-soil community continues to be represented at about the same level, but there is a shift from Mentha type to Carex hallerana type being the most abundant. The tall-sedge-swamp community continues to have low representation, and the stream-reed-swamp community has declined. Aquatic communities have declined further (but increase in the uppermost spectrum) and are represented only sporadically.

Sparganium erectum (the Branched Bur-reed) grows in lakes, ponds, ditches, and streams, in still to moderately fast flowing water rooted on margins up to $1 \mathrm{~m}$ deep, and also just above mean water level (Haslam et al. 1975). It is a widely distributed stream plant and flourishes in a weak current (Ellenberg 1988). It grows in mesotrophic to eutrophic conditions on mineral or organic soil, but often on clay. It is usually emergent but sometimes is submerged or floating (Haslam et al. 1975). Sparganium erectum does not form dense stands and seldom exceeds $1 \mathrm{~m}$ in height. Thus it plays only a subordinate role in established reed swamps, and is succeeded by large-sedge swamps (Ellenberg 1988). Here, it may be forming part of a succession of fen plants with the pioneer Scirpus. Sparganium erectum is abundant on the fen today (Chapter 3). It is one of the most productive plants in the temperate zone (Ellenberg 1988), so may contribute much of the organic material recorded in the sediments of this zone.

The number of aquatic types increases to 6 in the uppermost spectrum. This may be indicative of an expansion of fen aquatic vegetation. Palynological richness increases from the base of the zone to the top (6-8 expected types). Total wetland pollen and spore concentration increases slightly reaching a maximum of $4.3 \times 10^{3}$ grains $/ \mathrm{cm}^{3}$ at $335 \mathrm{~cm}$.

Available nutrients are high as indicated by the abundance of Glyceria type, Sparganium erectum, and Scirpus and the presence of Callitriche stagnalis type (which here may be growing in temporary pools (Haslam et al. 1975)), Ranunculus aquatilis group, Hydrocharis morsus-ranae, which grows in nutrient rich shallow ponds of lowland flood plains, and Salvinia natans, which grows in holes within a flooded sedge fen, i.e. in shallow and relatively humus-rich water (Haslam et al. 1975; Ellenberg 1988).

Zone $K W-11,312.5-287.5 \mathrm{~cm}$, c. 1230-1320 AD This zone corresponds to sediment section $\mathrm{L}$. The lower boundary of this zone coincides with a change in sediment composition to one of muddy detritus with horizontal layering of herbaceous material. Organic content increases significantly, inorganic matter declines, and carbonate content increases slightly. 
Community dominance has abruptly shifted from the Sparganium erectum-Scirpus dominated true-reed swamp of KW-12 to the wet-soil community where Carex hallerana type is the dominant type. All other types in this community are extremely poorly represented, with the exception of Schoenus nigricans. The second most important community is that of the true-reed swamp, with Scirpus the dominant type. The tall-sedge-swamp community has expanded and is next most abundant, with Cyperus michelianus the only type represented. The stream-reed-swamp community continues to be extremely poorly represented. Aquatic communities have declined even further and have almost disappeared.

Total wetland pollen and spore concentration increases to more than eight times that of the preceding zone $\left(26-42 \times 10^{3}\right.$ grains $\left./ \mathrm{cm}^{3}\right)$ and reaches a maximum similar to that of today. A sudden increase in concentration, at the boundary with the preceding zone, to more than five times that of the preceding zone, together with the change in wetland communities suggests that a catastrophic event took place. This evidence, as well as two radiocarbon dates (one at the top of the preceding zone and one at the base of this zone, which differ by perhaps $c .1800$ 3300 years), suggests that a hiatus occurs at the boundary between this and the zone below. This hiatus may be caused by erosion, or sediment by-passing due to a deeper stream channel.

Emergent plants that the dominant types probably represent: Scirpus lacustris (a pioneer), Cyperus michelianus (an annual), and Scirpus holoschoenus and S. sylvaticus (of the pollen type Carex hallerana type); and the aquatic plants represented suggest an environment of an establishing sedge and reed fen swamp with pools of water.

Available nutrients are high as suggested by the abundance of Scirpus and the presence of Hydrocharis morsus-ranae, Myriophyllum verticillatum, Potamogeton crispus type, and Potamogeton pectinatus (Haslam et al. 1975; Ellenberg 1988).

Zone $K W-10,287.5-237.5 \mathrm{~cm}, \mathrm{c.}$ 1320-1370 AD This zone corresponds to sediment section $\mathrm{K}$. The lower boundary of this zone coincides with a change in sediment composition to stiff clay and silt with detritus that continues up to $271 \mathrm{~cm}$. Three further complete sediment horizons and part of another are also included. These horizons comprise a series of minerogenic sediments (some sandy) with high inorganic content, high carbonate content, and low organic and moisture content, and represent a period of accelerated erosion and stream flooding. Between $264 \mathrm{~cm}$ and the top of the zone charcoal particles were observed in the sediments, suggesting that land clearance by fire led to the erosion. Sandy horizons occur at 271-268 and $264 \mathrm{~cm}$ to the top of the zone, perhaps due to unusually heavy rain falling on bared soils and/or stream flooding.

The dominant community is again the true-reed-swamp community. Although Scirpus is the dominant type, Glyceria type and Sparganium erectum are also important with Phragmites australis playing a minor but significant role. The wet-soil and tall-sedge-swamp 
communities are almost equally abundant. The dominant types in the wet-soil community are Carex hallerana type and Equisetum, with Mentha type less abundant. In the tall-sedgeswamp community the annual Cyperus michelianus is again the only type of any importance, suggesting an unstable environment. The stream-reed-swamp community has gained slightly, with Berula erecta increasing. The frequency of the aquatic types increases slightly towards the top of the zone, due mostly to an increase in Hydrocotyle vulgaris.

Available nutrient level is high as indicated by the abundance of Scirpus, Sparganium erectum, and Glyceria type, and the occurrence of Ranunculus aquatilis group, Hydrocharis morsus-ranae, Salvinia natans, and Vallisneria spiralis (Haslam et al. 1975; Ellenberg 1988).

Total concentration of wetland pollen and spores is very low $\left(0.6-3.9 \times 10^{3}\right.$ grain $/ \mathrm{cm}^{3}$ except in the lowest spectrum, which records $8.7 \times 10^{3}$ grain $/ \mathrm{cm}^{3}$ ), suggesting rapid accumulation of sediments and reduced wetland vegetation. Scirpus is the only type with many pollen grains represented and even this type reaches a maximum of only $2 \times 10^{3}$ grain $/ \mathrm{cm}^{3}$.

Palynological richness increases from the base of the zone to the top to comparatively moderate values for this sequence (6-11 expected types) due to an increase in types representing the wet-soil communities.

Zone $K W-9,237.5-202.5 \mathrm{~cm}, \mathrm{c.}$ 1370-1480 AD The sediments of this zone correspond to the sediment sections of $\mathrm{J}$ and I. A change in sediment composition occurs at $235 \mathrm{~cm}$ where sediments become highly organic and moisture content increases; carbonate content and inorganic matter decline. This horizon consists of a mixture of equal proportions of coarse herbaceous detritus and clay. At $216 \mathrm{~cm}$ sediment composition changes to one with a higher proportion of inorganic sediment, slightly more carbonate, and lower organic matter and moisture content: this is sediment section I-stiff grey clay with a trace of coarse herbaceous detritus and calcareous grit.

The dominant community continues to be the true-reed-swamp community with Scirpus the dominant type. Glyceria type is still important, but Sparganium erectum has almost disappeared and Phragmites australis has declined. The wet-soil community is the second most prominent community; Carex hallerana type is dominant with Mentha type and Equisetum playing minor but significant roles. The tall-sedge-swamp community has declined in importance and the stream-reed-swamp community has also declined. Aquatic communities have also declined again especially the submerged macrophytes community, which has almost disappeared.

Total concentration of wetland pollen and spores increases quickly in the lower part of the zone to a maximum almost as high as that of the preceding organic detritus-rich zone $(\mathrm{KW}-11)$, a level similar to that of today $\left(39 \times 10^{3}\right.$ grains $\left./ \mathrm{cm}^{3}\right)$. The dominant types Carex 
hallerana type, Cyperus michelianus, and Scirpus are also the same as those of the preceding detritus-rich zone. Rapid increase in the concentration of these types suggests that the plants represented by these types have recolonised the fresh sediment surface from a few relicts of the former vegetation on the site able to survive the deposition of sediment.

Total concentration of wetland pollen and spores declines rapidly with the influx of minerogenic sediment in the upper part of the zone $\left(3-8 \times 10^{3}\right.$ grains $\left./ \mathrm{cm}^{3}\right)$, indicating very rapid sediment accumulation.

Available nutrient level is high as indicated by the abundance of Scirpus, Glyceria type, and the presence of Ranunculus aquatilis group, Nuphar lutea, and Stratiotes aloides (Haslam et al. 1975; Ellenberg 1988).

Zone $K W-8,202.5-163.5 \mathrm{~cm}, c$. 1480-1640 AD This zone corresponds to sediment section $\mathrm{H}$, which is characterised by high organic and moisture content and low inorganic matter and carbonate content. Several changes in sediment composition occur. Up to $200 \mathrm{~cm}$ sediments are stiff grey clay, as in the zone below. Between 200 and $196 \mathrm{~cm}$ the sediment comprises a mixture of equal proportions of friable calcareous grit and clay. It then changes to a stiff grey clay. At $183 \mathrm{~cm}$ peat formation begins indicating greater landscape stability. Initially peat is mixed with clay in equal proportions, then at $165 \mathrm{~cm}$ the clay content increases indicating increased inwashed sediment.

The true-reed-swamp community continues to be dominant, with Scirpus the dominant type. Glyceria type is the next most abundant, with Phragmites australis and Sparganium erectum playing equally significant roles. The wet-soil community is next most important with Schoenus nigricans the dominant type and Equisetum also important. Carex hallerana type and Mentha type have almost disappeared. The tall-sedge-swamp community is the next most important community. Carex flacca type and Cyperus longus type are now the most important types in this community; Cyperus michelianus has declined. The stream-reedswamp community has expanded due to the increase of Berula erecta. Aquatic communities continue to be poorly represented with the exception of a peak of Stratiotes aloides in the second to uppermost spectrum. Otherwise, the floating-leaved macrophytes community is represented almost completely by Sparganium angustifolium.

Concentration of total wetland pollen and spores remains at about the same value $\left(6.2 \times 10^{3}\right.$ grains $/ \mathrm{cm}^{3}$ maximum) as in the upper part of the preceding zone, which is relatively high for this sequence. Palynological richness increases to comparatively moderate values for this sequence, fluctuating between 8 and 12, except for the uppermost spectrum, which is about 7 .

Available nutrients remain high, as indicated by the presence of the reed and sedge fen plants such as Glyceria, Scirpus, and Sparganium erectum, and the aquatic macrophytes of 
Callitriche obtusangula, Hydrocharis morsus-ranae, and Stratiotes aloides (Haslam et al. 1975; Ellenberg 1988).

Vegetation on the wetland indicates a more stable environment, with lower values of the pioneer Scirpus and the annual Cyperus michelianus and greater abundance of Carex flacca type and Cyperus longus type of the tall-sedge-swamp community, which occur continuously. The early hydrosere Sparganium erectum has also declined. Schoenus nigricans of the smallsedge fen community has also increased significantly, indicating the establishment of the typical zonation of a sedge fen.

Zone $K W-7,163.5-142.5 \mathrm{~cm}, c$. 1640-1680 AD This zone corresponds to sediment section $\mathrm{G}$, where organic and moisture content decline and inorganic content increases. Carbonate content is low. The sediment of this zone is a mixture of mostly clay with some peat, with the exception of the uppermost $0.5 \mathrm{~cm}$ where the sediment comprises mostly coarse to very coarse noncalcareous sand with some calcareous grit and includes some clay and a trace of peat.

The true-reed-swamp community continues to be dominant, but Glyceria type is now the dominant type. Scirpus is now only slightly more important than Phragmites australis and Sparganium erectum. Typha angustifolia type displays a sudden peak in the basal spectrum. The wet-soil community is the next most prominent community; Schoenus nigricans continues to be important, but Carex hallerana type has expanded and is equally important. Mentha type and Chrysosplenium alternifolium are also important and Equisetum occurs only sporadically. The tall-sedge-swamp community has declined; Carex flacca type and Cyperus longus type have both declined and Cyperus michelianus continues at the same low level of representation. The stream-reed-swamp community increases very slightly due to an increase in Berula erecta. Aquatic communities increase due to an increase in Hydrocotyle vulgaris.

Available nutrients remain high, as indicated by the presence of the reed and sedge fen plants such as Glyceria, Scirpus, Sparganium erectum, Typha domingensis type, and Typha angustifolia type, and the aquatic macrophytes of Nuphar lutea and Myriophyllum verticillatum (Haslam et al. 1975; Ellenberg 1988).

Concentration of total wetland pollen and spores declines upwards and becomes very low at the top of the zone $\left(0.7-3.8 \times 10^{3}\right.$ grains $\left./ \mathrm{cm}^{3}\right)$. This is probably due to increasing amounts of minerogenic soil being washed in and diluting the pollen concentration. The dominance of Glyceria may be due to the greater tolerance of that type to 'silting'.

Minerogenic inwash, however, is not sufficient to interrupt development of the reed and sedge fen vegetation, although it is reduced; a variety of reed and sedge plants are represented and the pioneer Scirpus declines. Salix fragilis type now appears (today Salix alba grows on the fen and along the channel to the south of the fen (Chapter 3)). 
Zone $K W-6,142.5-117.5 \mathrm{~cm}$, c. 1680-1720 AD This zone corresponds to sediment section $\mathrm{F}$, which is characterised by high inorganic and carbonate content and low organic and moisture content. The sediments comprise mostly coarse to very coarse noncalcareous sand with some calcareous grit and includes some clay and a trace of peat. A narrow band at $133-132 \mathrm{~cm}$ comprises clay with some peat. Above $121 \mathrm{~cm}$ is peaty clay (see zone $\mathrm{KW}-5$ ).

The true-reed-swamp community is again the dominant community and Glyceria type the dominant type, perhaps being favoured again by the influx of minerogenic sediment. Scirpus retains its position of next most important before Phragmites australis and Sparganium erectum. The next most important community now shifts to the tall-sedge-swamp community with Cyperus longus type the dominant type increasing steadily. Carex flacca type becomes prominent in the upper part of the zone. The wet-soil community is next most important, but its importance has declined. Several types occur sporadically, but reach significant abundance. These include Carex hallerana type, Mentha type, and Equisetum. The stream-reed-swamp community has again increased slightly; again due to an increase in Berula erecta. The aquatic communities increase slightly, due to an increase in Hydrocotyle vulgaris.

Available nutrients remain high, as indicated by the presence of the reed and sedge fen plants such as Glyceria, Scirpus, and Sparganium erectum, and the aquatic macrophytes of Hydrocharis morsus-ranae, Stratiotes aloides, and Myriophyllum spicatum (Haslam et al. 1975; Ellenberg 1988).

Concentration of total wetland pollen and spores is very low with total concentration reaching only $1.4 \times 10^{3}$ grains $/ \mathrm{cm}^{3}$. This is probably due to increasing amounts of minerogenic soil being washed in and diluting the pollen concentration. The dominance of Glyceria may be due to the greater tolerance of that type to 'silting'.

Zone $K W-5,117.5-77.5 \mathrm{~cm}$, c. 1720-1790 AD This zone corresponds to sediment section $\mathrm{E}$, which is characterised by comparatively high organic matter and moisture content and low inorganic content. Carbonate content declines upwards. The sediments are peaty clay, as at the top of the zone below. Total concentration of the wetland pollen and spores is again very low (only $2.2 \times 10^{3}$ grains $/ \mathrm{cm}^{3}$ maximum).

The true-reed-swamp community continues to be the dominant community. Glyceria type and Sparganium erectum are co-dominant. Phragmites australis and Scirpus are about equally important. Typha domingensis type and Typha angustifolia type have gained in importance. The tall-sedge-swamp community is second most prominent, with Cyperus longus type the dominant type. Carex flacca type and Cyperus michelianus have both declined. The wet-soil community continues to be represented sporadically by a few types: Carex hallerana type, Mentha type, and Schoenus nigricans. The stream-reed swamp is 
represented sporadically. As a whole the aquatic communities increase significantly due to a large increase in Sparganium angustifolium.

Available nutrients remain high, as indicated by the presence of the reed and sedge fen plants such as Cyperus longus type, Glyceria, Scirpus, Sparganium erectum, Typha domingensis type, and Typha angustifolia type, and the aquatic macrophytes of Hydrocharis morsus-ranae and Salvinia natans (Haslam et al. 1975; Ellenberg 1988).

The decline in Glyceria type is probably due to a reduction in minerogenic sediments being washed onto the fen, which allows other fen plants to be more competitive. Cyperus longus type increases steadily and is indicative of an expanding tall-sedge community. The increase in Sparganium erectum may be a result of the fen vegetation recovering from the reduction sustained during influx of sediment in the preceding zone. Sparganium angustifolium is usually a floating-leaved aquatic, but may also be submerged. It grows in still water in lakes or ponds of oligotrophic to dystrophic nutrient level on fine organic soil at 0.5-2 $\mathrm{m}$ depth. The abundance of Sparganium angustifolium suggests that pools of water at least $0.5 \mathrm{~m}$ deep are extant on the fen.

Zone $K W-4,77.5-67.5 \mathrm{~cm}$, c. 1790-1810 AD This zone corresponds to sediment section $\mathrm{D}$, which is characterised by high organic matter, very high moisture content, and very low inorganic matter and carbonate content. This high organic content may indicate that the core site was a low area of the fen and water had pooled in it. The sediment comprises equal quantities of peat and organic detritus.

The true-reed-swamp community is again the dominant community, now due to a large increase in Sparganium erectum. Glyceria type and Scirpus have both declined and are about equally important, while Phragmites australis, Typha domingensis type, and Typha angustifolia type have declined to insignificant values. The tall-sedge-swamp community is the second most important community, with Cyperus longus the dominant type. The wet-soil community has almost disappeared and the stream-reed-swamp community is not represented. Aquatic communities have declined again, but Sparganium angustifolium continues to be represented by significant values.

Available nutrients remain high, as indicated by the presence of the reed and sedge fen plants such as Cyperus longus type, Glyceria, Scirpus, Sparganium erectum, Typha domingensis type, and Typha angustifolia type, and the aquatic macrophyte of Ranunculus aquatilis type (Haslam et al. 1975; Ellenberg 1988).

Total concentration of pollen and spores increases slightly $\left(2.5 \times 10^{3}\right.$ grains $\left./ \mathrm{cm}^{3}\right)$ due mostly to high concentration of Sparganium erectum, which may have been growing close to the site of deposition. The concentration of Glyceria type has declined and that of Cyperus 
longus type increased. This zone appears to represent a well developed sedge and reed swamp vegetation with little sediment influx.

Zone $K W-3,67.5-32.5 \mathrm{~cm}, \mathrm{c.} 1810-1880 \mathrm{AD}$ This zone corresponds to sediment section $\mathrm{C}$, which is characterised by low organic matter and moisture content, high inorganic matter, and comparatively high carbonate content. Sediments up to $52 \mathrm{~cm}$ comprise mostly clay with some organic detritus, with muddy fine to medium sand above this level.

The true-reed-swamp community is again the dominant community. The dominant types are now Glyceria type, Phragmites australis, and Scirpus. Sparganium erectum is also important. The second most important community is that of the tall-sedge swamp. The dominant type is Cyperus longus type. Cyperus michelianus fluctuates. The wet-soil community increases in importance with an increase in Carex hallerana type. The streamreed-swamp community has only minor representation. The aquatic communities display a peak in the centre of the zone due to an increase in Hydrocotyle vulgaris, then decline towards the top of the zone.

Available nutrients remain high, as indicated by the presence of the reed and sedge fen plants such as Cyperus longus type, Glyceria, Scirpus, Sparganium erectum, Typha domingensis type, and Typha angustifolia type and the aquatic macrophytes of Ranunculus aquatilis type, Hydrocharis morsus-ranae, and Nuphar lutea (Haslam et al. 1975; Ellenberg 1988).

Total concentration of wetland pollen and spores is low $\left(3.1 \times 10^{3}\right.$ grains $/ \mathrm{cm}^{3}$ maximum). The concentration of Glyceria type increases as well as that of Phragmites australis and Scirpus. The increases in Glyceria type and Scirpus are probably responses to the increase in mineral influx, Glyceria expanding because of its competitive advantage due to a tolerance of minerogenic influx and Scirpus as a pioneer on freshly deposited sediment. The annual Cyperus michelianus also increases, which indicates unstable soil conditions. Cyperus longus type declines where Glyceria type increases, perhaps due to inundation with sediments.

Zone $K W-2,32.5-17.5 \mathrm{~cm}, \mathrm{c} .1880-1910 A D$ This zone corresponds to sediment section $\mathrm{B}$, which is characterised by high organic and moisture content, low inorganic matter, and moderately high carbonate content. Sediment consists of one horizon of clay and silt with some detritus and peat, and living roots.

Tall-sedge-swamp and true-reed-swamp communities are co-dominant. In the tallsedge-swamp community Cyperus longus type contributes almost all the pollen, while in the true-reed-swamp community several types contribute significant amounts of pollen - Glyceria type, Phragmites australis, and Sparganium erectum. The wet-soil community declines, with 
only Carex hallerana type contributing significant amounts of pollen. The stream-reedswamp community is not represented. Aquatic communities continue to be poorly represented, but Callitriche stagnalis type displays a small peak in the centre of the zone.

Available nutrients remain high, as indicated by the presence of the reed and sedge fen plants such as Cyperus longus type, Glyceria, Scirpus, Sparganium erectum, and Typha angustifolia type, and the aquatic macrophytes of Callitriche stagnalis type and Ranunculus aquatilis type (Haslam et al. 1975; Ellenberg 1988).

Total concentration of wetland pollen and spores is low $\left(1.5 \times 10^{3}\right.$ grains $\left./ \mathrm{cm}^{3}\right)$. Cyperus longus type is the most abundant with Glyceria type and Sparganium erectum also abundant. As before Sparganium erectum succeeds Scirpus as the fen vegetation recovers from an influx of minerogenic sediment, and Cyperus longus type recovers. Glyceria type declines as the other types increase.

Zone $K W-1,17.5-0 \mathrm{~cm}, \mathrm{c}$. 1910-1989 AD This zone corresponds to sediment section $\mathrm{A}$, which is characterised by the highest organic and moisture content of the sequence, lowest inorganic matter, and low to moderately high carbonate content. One sediment horizon coincides with this zone comprising slightly silty peat with woody detritus and calcareous grit.

The tall-sedge-swamp community becomes dominant, with Cyperus longus dominant and Carex flacca type also contributing a large amount of pollen. Cyperus michelianus contributes a significant amount in the surface sample. All other communities have declined greatly.

Available nutrients remain high, as indicated by the abundance of the reed and sedge fen plants such as Carex flacca type, Cyperus longus, and Typha angustifolia type (Ellenberg 1988). No aquatic macrophytes indicating high nutrient levels occur.

Total concentration of wetland pollen and spores increases from low at the base of the zone $\left(4.1 \times 10^{3}\right.$ grains $\left./ \mathrm{cm}^{3}\right)$ to the maximum recorded of this sequence at the top of the zone $\left(45 \times 10^{3}\right.$ grains $\left./ \mathrm{cm}^{3}\right)$. Several types record significant concentration values, especially in the surface sample. These are Carex hallerana type, Mentha type, Equisetum, Carex flacca type, Cyperus longus type, Cyperus michelianus, Glyceria type, Sparganium erectum, Typha angustifolia, Sparganium angustifolium, and Salix fragilis type. Species of Mentha type, Equisetum, Cyperus longus type, Sparganium erectum, and Typha angustifolia were identified growing abundantly on the fen and species that may belong to Carex hallerana type, Carex flacca type, Cyperus michelianus, Glyceria type, Sparganium angustifolium, and Salix fragilis type were also identified (Appendix VIII). 


\subsubsection{Terrestrial pollen analysis}

\subsubsection{Pollen diagram construction}

Pollen and spore relative frequencies are presented in Figures 6.11 and 6.12 (in pocket). Pollen and spore types are arranged according to habitat groups defined in Chapter 2. The pollen sum on which the relative frequencies are based includes all identified terrestrial pollen and spore taxa and excludes mire and aquatic taxa. Relative frequencies of $0.2 \%$ or less are indicated with a dot. Style of the summary and fully resolved relative frequency diagrams and the arrangement of pollen and spore types is the same as in Chapter 5. Rarefaction analysis is based on the smallest total count of terrestrial pollen and spores, 323 .

Pollen concentration data are presented in Figure 6.13, which takes a similar form to the relative frequency diagram. Only concentrations of pollen and spore types recorded in significant abundance, habitat groups, and total pollen and spores are included.

\subsubsection{Terrestrial pollen assemblage zones}

Terrestrial pollen diagrams are divided into 14 local pollen assemblage zones K-1 to $\mathrm{K}-14$, based mostly on relative frequency data. These are defined below. Concentration data are discussed in the following section.

Zone $K-14,396.0-372.5 \mathrm{~cm}$ This basal zone is characterised by high relative frequencies of the habitat groups of 'herbs of open ground' and 'cultivars'. The former group records $62.8-72.4 \%$ of terrestrial taxa, the latter 1.2-6.1\%. The most important type in the 'herbs of open ground' group is Pteridium aquilinum (8.4-21.9\%). Other types of importance in this habitat group recording more than $2.0 \%$ are: Centaurea solstitialis type (3.0-6.9\%), Cichorium type (1.8-4.6\%), Dactylis type (1.8-11.3\%), Festuca type (1.4-2.9\%), Galium type (0.5-2.7\%), Hieracium type (11.6-29.2\%), Lactuca type (1.7-3.2\%), Plantago lanceolata type (0.5-2.9\%), Solidago type (1.2-3.2\%), Sonchus type (0.0-9.1\%), and Taraxacum type (0.1$4.1 \%$ ). The most abundant types represented in the habitat group 'cultivars' are the cereals Secale cereale (maximum 3.5\%) and Triticum type (maximum 1.8\%). Other 'cultivars' represented are Avena (maximum 0.4\%), Castanea sativa (maximum 0.2\%), Hordeum type (maximum $0.2 \%$ ), Juglans regia (maximum $0.1 \%$ ), Platanus orientalis (maximum $0.4 \%$ ), and Vicia sativa type (maximum $0.3 \%$ ).

The dominant canopy tree type is Quercus trojana type (7.5-12.1\%). Other important types of the habitat group 'coniferous and deciduous canopy trees' are Pinus (Diploxylon) (7.0-9.5\%), Quercus frainetto type (4.1-7.5\%), and Ostrya type (2.7-1.0\%). No types of 'alpine shrubs' are represented. Only the types Ligusticum and Veratrum of 'alpine herbs' are represented recording only $0.2 \%$. The habitat group of 'woodland understorey trees and shrubs' is also poorly represented with only the types Ilex aquifolium and Juniperus 


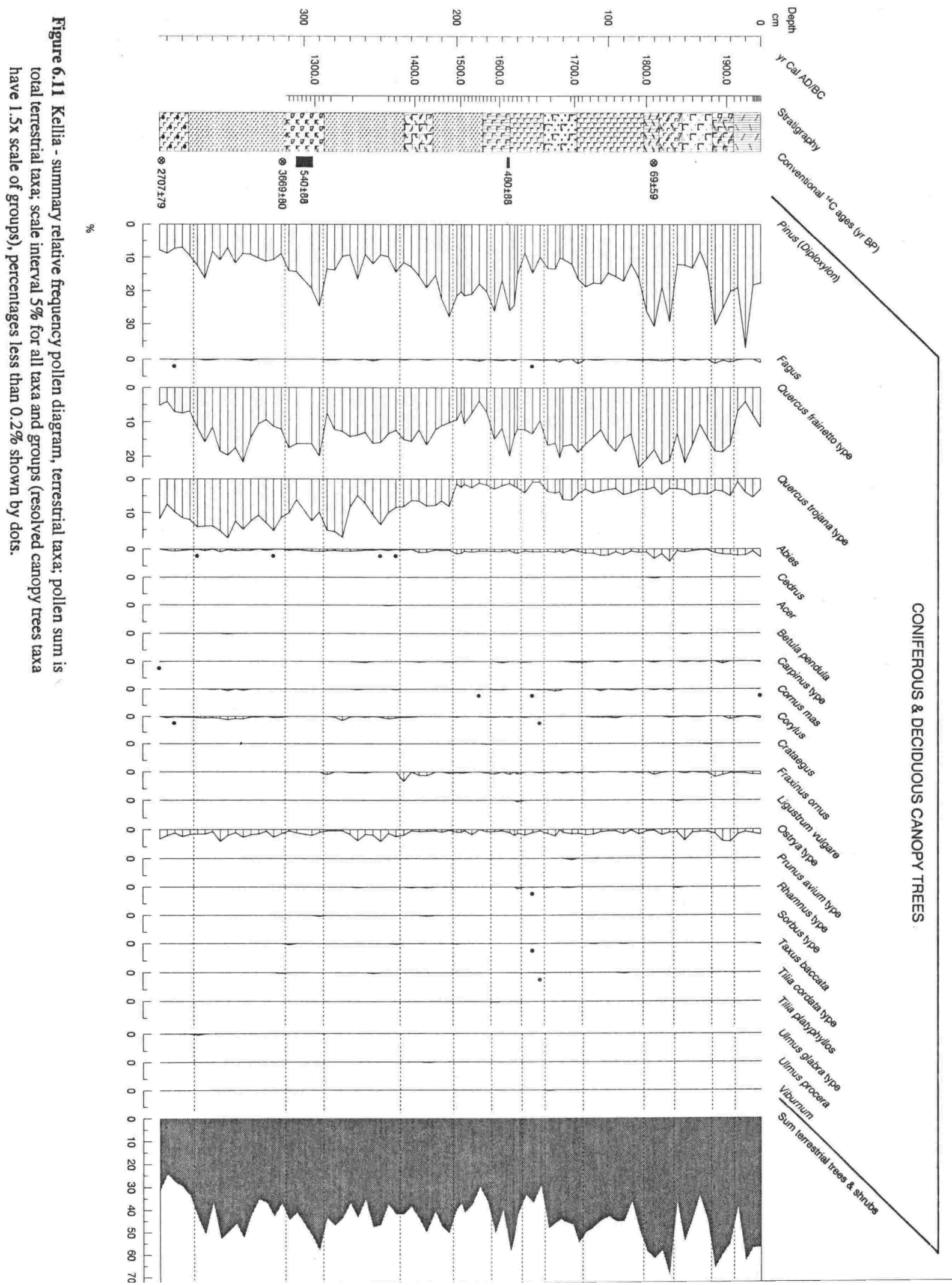




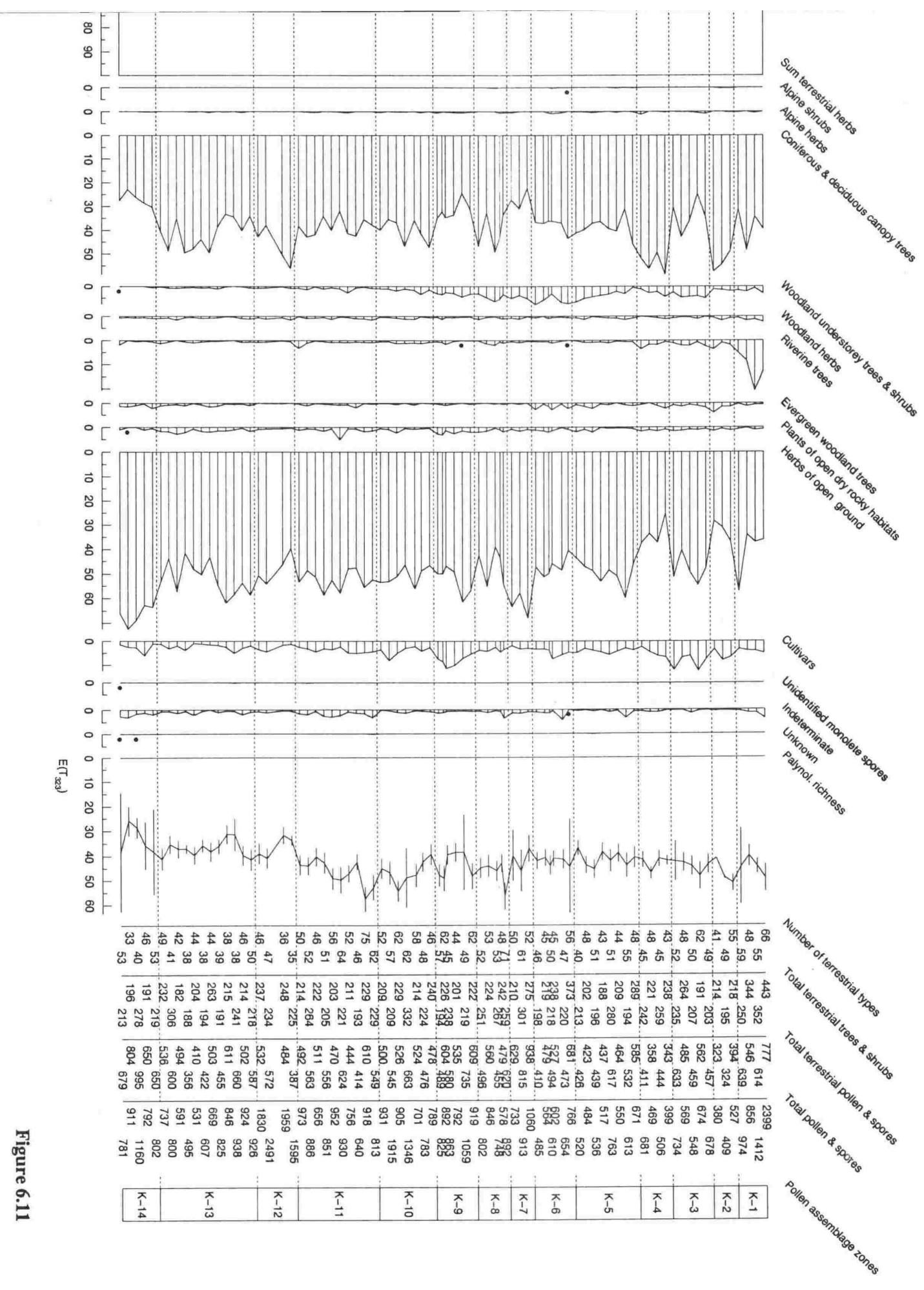



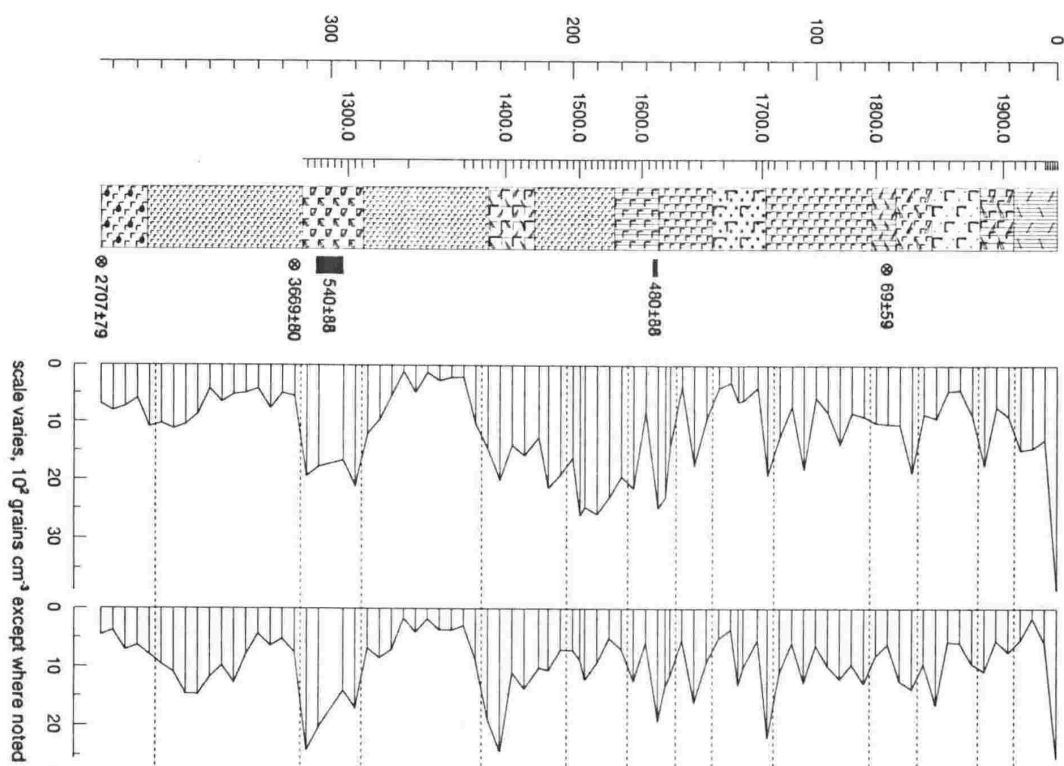

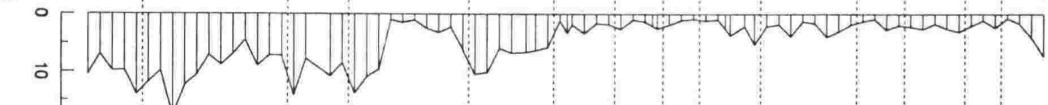

兽

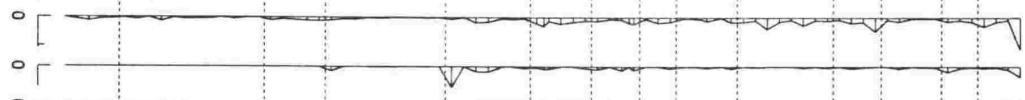

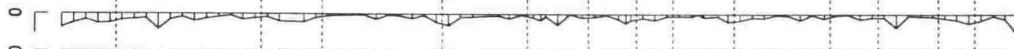
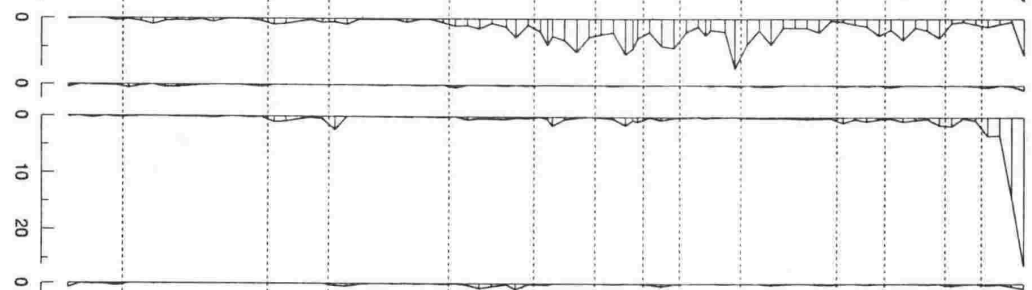

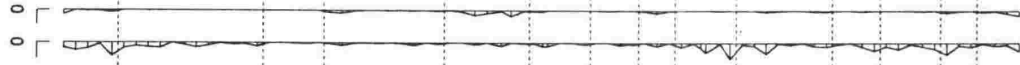

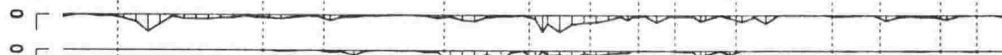

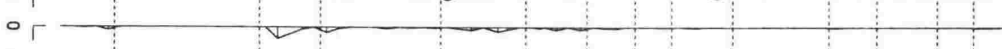

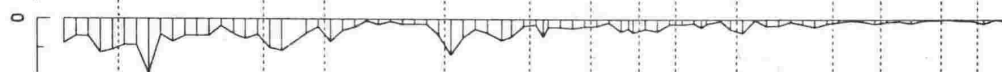

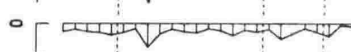

낭

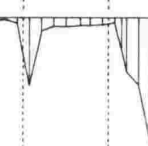

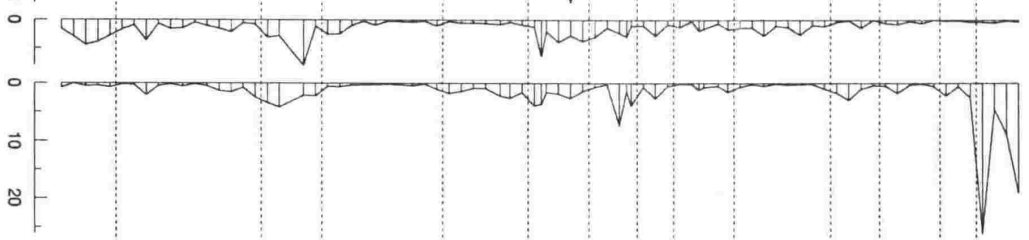
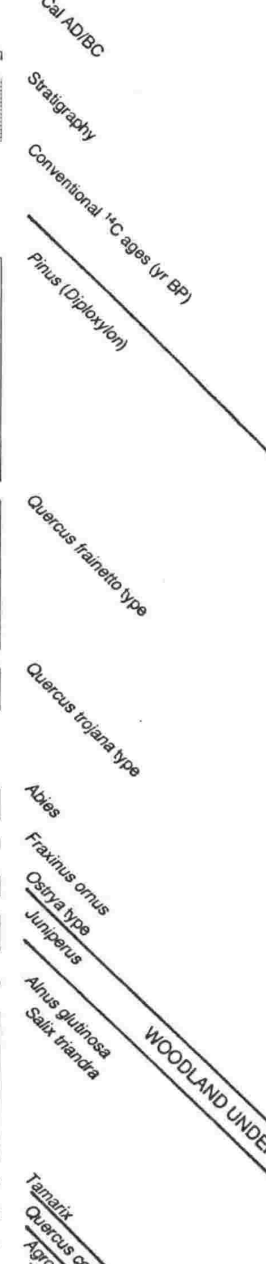

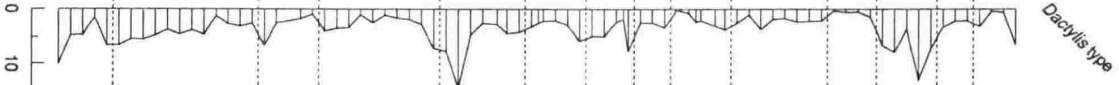

N

[F (1)

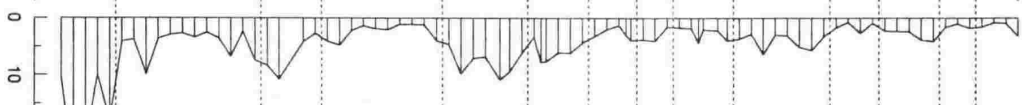
ㅁN $\sqrt{ }$

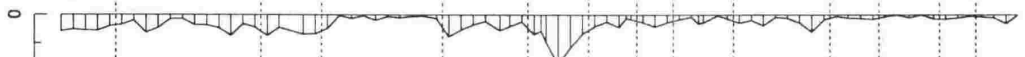

[

o
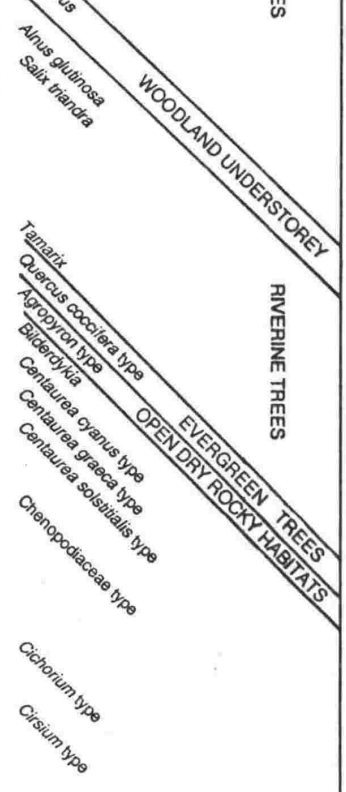

F

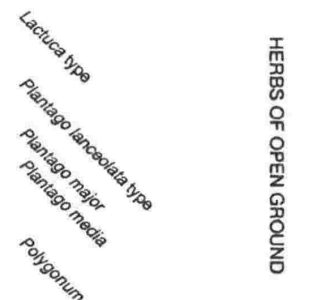


represented (with a maximum of $0.3 \%$ ). 'Woodland herbs' are also poorly represented, recording less than $1.0 \%$. 'Riverine trees' reach a maximum of $1.8 \%$, with Alnus glutinosa (maximum 0.6\%), Salix triandra (maximum 0.3\%), and Tamarix (maximum 0.9\%) most abundant. Pollen types of 'evergreen woodland trees' record $0.2 \%$ or less with the exception of Quercus coccifera type, which records from 1.0-2.0\%, except in one spectrum where only $0.3 \%$ is recorded. No type of 'plants of open dry rocky habitats' exceeds $0.5 \%$.

Zone $K-13,372.5-312.5 \mathrm{~cm}$ The boundary with the preceding zone is marked by increases in the relative frequencies of Pinus (Diploxylon), Quercus frainetto type, Quercus trojana type; a decline in Pteridium aquilinum and 'herbs of open ground'; and an increase in the ratio of terrestrial trees and shrubs to terrestrial herbs. This zone is characterised by high relative frequencies of the habitat groups of 'herbs of open ground' and 'cultivars' and of Pteridium aquilinum, and increased relative frequencies of the habitat group 'coniferous and deciduous canopy trees' and the pollen types Quercus frainetto type and Quercus trojana type.

'Herbs of open ground' have declined, but relative frequencies are still high (41.5$61.8 \%$ ). Pteridium aquilinum has declined but is still abundant at 5.8-12.6\%. Other types in this group exceeding $2.0 \%$ are the same as those in the preceding zone, and record similar frequencies. Exceptions are Chenopodiaceae type (1.3-3.1\%) and Polygonum aviculare type $(1.2-4.5 \%$ except the basal spectrum, which is $0.6 \%)$, which increase; Cirsium type, which increases in the upper part of the zone to $1.6-3.9 \%$; Plantago lanceolata type, which declines to less than $2.0 \%$, and Hieracium type, which has also declined (4.5-11.8\%).

'Cultivars' continue at similar relative frequencies (1.0-5.1\%), but changes occur within the group: Secale cereale declines (maximum 2.2\%); Triticum type disappears; Avena, Hordeum type, and Platanus orientalis increase slightly, recording maximum relative frequencies of $1.8 \%, 1.4 \%$, and $1.4 \%$, respectively; and Zea mays was identified in one spectrum at $0.3 \%$. (Zea mays probably represents Triticum type or Avena, see explanation below.) Castanea sativa and Juglans regia remain unchanged (they occur sporadically and do not exceed $0.3 \%$ ) and Vicia sativa type disappears.

In the habitat group 'coniferous and deciduous canopy trees' Quercus frainetto type and Quercus trojana type increase significantly. In the centre of the zone Quercus frainetto type reaches $21.6 \%$, which is close to the maximum of the sequence. Quercus trojana type displays its overall zonal maxima, reaching $17.2 \%$ at $350 \mathrm{~cm}$. Pinus (Diploxylon) increases slightly reaching its maximum of the zone $(16.2 \%)$ in the second spectrum from the base. The only other type in this group that exceeds $1.0 \%$ is Ostrya type (maximum $3.4 \%$ ).

Again 'alpine shrubs' are not represented. Six types of 'alpine herbs' occur, but these are all represented once only and at $0.2 \%$. 'Woodland understorey trees and shrubs' are represented by only Ilex aquifolium ( $0.4 \%$ in one spectrum) and Juniperus (maximum $0.8 \%$ ). 
'Woodland herbs' reach a maximum of only $1.6 \%$. 'Riverine trees' have declined slightly, reaching a maximum of $1.3 \%$; Alnus glutinosa and Salix triandra are unchanged, but Tamarix disappears. 'Evergreen woodland trees' are represented by only Ephedra fragilis (maximum $0.4 \%)$ and Quercus coccifera type (0.0-1.4\%). No type of 'plants of open dry rocky habitats' exceeds $0.8 \%$, with the exception of Agropyron type (0.4-2.2\%).

Zone $\mathrm{K}-12,312.5-287.5 \mathrm{~cm}$ The boundary with the preceding zone is marked by an increase in the relative frequencies of Pinus (Diploxylon) and Quercus frainetto type, and a decline in Quercus trojana type and Pteridium aquilinum. This zone is characterised by high relative frequencies of the habitat groups of 'herbs of open ground' and 'cultivars' and the types of Pinus (Diploxylon), Quercus frainetto type, and Quercus trojana type.

Frequencies of 'herbs of open ground' are comparatively low (39.5-54.0\%). Pteridium aquilinum has declined (2.3-4.5\%). Other types in this group exceeding $2.0 \%$ are the same as those in the preceding zone with the exception that Plantago lanceolata type has increased (uppermost spectrum reaches $2.6 \%$ ), and Galium type has declined to less than $2.0 \%$.

'Cultivars' continue at similar frequencies (1.3-4.5\%). Frequencies of individual types within this group are more or less constant except for Platanus orientalis, which declines $(0-0.2 \%)$. Secale cereale remains the most abundant cereal, while other cereals, including Zea mays, continue occurring sporadically at low values.

'Coniferous and deciduous canopy trees' increase from $42.7 \%$ in the basal spectrum to $56.1 \%$ in the uppermost. Pinus (Diploxylon) also increases from the basal spectrum $(14.0 \%)$ to the uppermost (24.6\%). Quercus frainetto type displays constantly high frequencies (16.3-19.9\%). Quercus trojana type has declined (6.1-12.4\%). No other type in this group exceeds $2.0 \%$.

'Alpine shrubs' and 'alpine herbs' are not represented. 'Woodland understorey trees and shrubs' are represented by Juniperus only (maximum $0.8 \%$ ). 'Woodland herbs' reach a maximum of $1.6 \%$. 'Riverine trees' have declined and only Salix triandra is represented (maximum 0.8\%). 'Evergreen woodland trees' are represented by Ephedra fragilis and Quercus coccifera type; both record $0.2 \%$ in one spectrum. 'Plants of open dry rocky habitats' have declined; no type exceeds $0.5 \%$.

Zone $K-11.287 .5-237.5 \mathrm{~cm}$ The boundary with the preceding zone is marked by an increase in the relative frequencies of Quercus trojana type, and a decrease in Pinus (Diploxylon) and Quercus frainetto type. This zone is characterised by high relative frequencies of the habitat groups of 'herbs of open ground' and 'cultivars' and the types Quercus frainetto type and Quercus trojana type.

Frequencies of 'herbs of open ground' are about average for this sequence (47.5-58.6\%) and those of Pteridium aquilinum are unchanged from the preceding zone (2.0-4.8\%). Other 
types in this group exceeding $2.0 \%$ are the same as those in the preceding zone with the exception of Galium type, which increases to a maximum of $2.4 \%$.

'Cultivars' increase (2.4-5.1\%). The cereals Avena and Secale cereale occur continuously and reach $1.1 \%$ and $2.7 \%$, respectively. Other cereals occur sporadically Hordeum type $0.7 \%$ maximum and Zea mays $0.9 \%$ maximum. Castanea sativa, Juglans regia, and Platanus orientalis occur more often than in the preceding zone, but at low frequencies $(0.9 \%, 0.5 \%$, and $0.7 \%$ maxima, respectively). Vitis vinifera appears at $260 \mathrm{~cm}$ for the first time then again at 255 and $240 \mathrm{~cm}$, at $0.2 \%$.

'Coniferous and deciduous canopy trees' fluctuate from 31.8-42.8\%. Pinus (Diploxylon) has declined (9.0-16.5\%). Quercus frainetto type declines in the lower part of the zone (7.5\%), but reaches a maximum of $16.2 \%$ near the top. Quercus trojana type increases in the lower part of the zone to $17.2 \%$, declines to $4.9 \%$ in the centre of the zone, then increases again to $13.5 \%$. Ostrya type increases from the centre of the zone, recording overall maxima of the sequence (3.4\% maximum). No other type in this group exceeds $2.0 \%$.

'Alpine shrubs' are not represented and no type of 'alpine herbs' exceeds $0.5 \%$. 'Woodland understorey trees and shrubs' are represented by more types (4), but only Juniperus, which reaches a maximum of $2.7 \%$, exceeds $0.2 \%$. 'Woodland herbs' reach a maximum of $1.6 \%$. 'Riverine trees' are represented by 7 types but none exceed $0.7 \%$ except Salix triandra, which reaches $2.6 \%$ in the lowest spectrum. 'Evergreen woodland trees' ( $1.9 \%$ maximum), are also represented by more types (Ephedra fragilis, Phillyrea, Pistacia, and Quercus coccifera type). Only Quercus coccifera type, which reaches a maximum of $1.9 \%$, exceeds $0.3 \%$. 'Plants of open dry rocky habitats' increase slightly (maximum $5.1 \%$ at $260 \mathrm{~cm}$ ), due mostly to an increase in Agropyron type (but this type never exceeds $1.0 \%$ ) and Crupina (one exceptional spectrum records $4.5 \%$ ).

Zone $K-10,237.5-202.5 \mathrm{~cm}$ The boundary with the preceding zone is marked by an increase in Quercus frainetto type and decline in Quercus trojana type. This zone is characterised by high relative frequencies of the habitat groups of 'herbs of open ground' and 'cultivars'; high and increasing relative frequencies of Pinus (Diploxylon); and high relative frequencies of Quercus frainetto type and Quercus trojana type.

Frequencies of 'herbs of open ground' are again about average for this sequence (46.3$56.1 \%$ ). Pteridium aquilinum has declined (1.5-3.4\%). Other types in this group exceeding $2.0 \%$ are the same as those in the preceding zone with the exception of Bilderdykia, which increases to 2.0 and $2.1 \%$ in two spectra and Cichorium type which declines to less than $2.0 \%$.

'Cultivars' increase again (2.3-8.1\%), due to increases in Avena (maximum 2.2\%) and Platanus orientalis (maximum 1.5\%). The cereals Avena and Secale cereale (maximum $3.5 \%$ ) occur continuously, while Hordeum type and Zea mays occur sporadically. Hordeum 
type increases at the base of the zone to $1.4 \%$, then declines. Zea mays has declined and reaches only $0.2 \%$ in three of the seven spectra. Castanea sativa continues to occur at low values (maximum $0.6 \%$ ). Juglans regia disappears in the upper part of the zone. Vitis vinifera occurs only at $225 \mathrm{~cm}$ at $0.6 \%$.

'Coniferous and deciduous canopy trees' increase slightly, but fluctuate (35.5-47.1\%). Pinus (Diploxylon) increases steadily from the relative frequencies of the preceding zone (basal spectrum $11.5 \%$ ) to $27.6 \%$ in the uppermost spectrum. Quercus frainetto type maintains frequencies similar to those of the preceding zone to the centre of this zone, then declines slightly to $10.2 \%$. Quercus trojana type declines (6.4-8.2\%). Ostrya type has declined to less than $2.0 \%$. No other type in this group exceeds $2.0 \%$ except for Fraxinus ornus, which reaches $2.8 \%$ in the basal spectrum.

'Alpine shrubs' are not represented and no type of 'alpine herbs' exceeds $0.2 \%$. 'Woodland understorey trees and shrubs' are represented by only three types: Juniperus records $0.7-3.4 \%$; Buxus sempervirens $0.4 \%$ in one spectrum; Ilex aquifolium $0.2 \%$ in one spectrum. 'Woodland herbs' reach a maximum of only $1.3 \%$. 'Riverine trees' are represented by fewer types (4) than in the preceding zone but none exceed $0.6 \%$ with the exception of Tamarix, which reaches $1.0 \%$ in two spectra. 'Evergreen woodland trees' decline ( $0.8 \%$ maximum). Ephedra distachya type, Ephedra fragilis, and Quercus coccifera type are represented, but only Quercus coccifera type exceeds $0.2 \%$ (it occurs sporadically and reaches $0.8 \%$ ). 'Plants of open dry rocky habitats' decline ( $2.3 \%$ maximum); only Agropyron type (maximum $1.1 \%$ ) exceeds $0.4 \%$.

Zone $K-9,202.5-177.5 \mathrm{~cm}$ The boundary with the preceding zone is marked by increases in the relative frequencies of the habitat groups of 'plants of open dry rocky habitats' and 'cultivars', a decrease in 'coniferous and deciduous canopy trees', and a sharp decrease in Quercus trojana type. This zone is characterised by very high relative frequencies of 'cultivars'; high relative frequencies of 'herbs of open ground', Pinus (Diploxylon), and Chenopodiaceae type; increasing frequencies of Juniperus; and low frequencies of 'coniferous and deciduous canopy trees', Quercus frainetto type, and Quercus trojana type.

'Herbs of open ground' again display about average relative frequencies, but in general these increase from the bottom of the zone to the top (ranging from 49.9-61.3\%). Pteridium aquilinum increases slightly from the preceding zone (2.9-4.8\%). Other types in this group exceeding $2.0 \%$ are the same as those in the preceding zone with the exception of Cichorium type, which increases to a maximum of $5.0 \%$. Notable changes in the relative frequencies of types in this group are a decline in Bilderdykia to $0.2 \%$ and less, a decline in Centaurea solstitialis type (only one spectrum exceeds $2.0 \%$ ), and an increase in Chenopodiaceae type (maximum $17.1 \%$ at $185 \mathrm{~cm}$ ). 
'Cultivars' increase significantly (5.4-11.4\%), due to increases in the cereals Avena, Hordeum type, Secale cereale, and Zea mays (2.6\%, 2.9\%, 3.8\%, and $1.5 \%$ maxima, respectively). All these types are continuously represented. The cereal Triticum type occurs in only one spectrum at $0.2 \%$. Castanea sativa continues to occur sporadically at low values ( $0.9 \%$ maximum) and Humulus lupulus appears in the uppermost spectrum at $0.2 \%$. Platanus orientalis declines again ( $0.8 \%$ maximum). Vicia sativa type occurs in one spectrum at $0.2 \%$.

'Coniferous and deciduous canopy trees' decline to almost the minima of the sequence (24.4-35.0\%). Pinus (Diploxylon) has comparatively high relative frequencies $(20.2-21.4 \%$, with one spectrum recording only $17.9 \%$ ). Quercus frainetto type declines (6.8-10.5\%, with the exception of one spectrum 3.9\%). Quercus trojana type declines even further (1.2-2.6\%). No other type in this group exceeds $2.0 \%$.

'Alpine shrubs' are not represented and no type of 'alpine herbs' exceeds $0.6 \%$. 'Woodland understorey trees and shrubs' are represented by only two types and only Juniperus exceeds $0.2 \%$; it increases (2.6-4.5\%). 'Woodland herbs' reach a maximum of only $1.3 \%$. 'Riverine trees' are represented by 5 types but none exceeds $0.3 \%$ except for Salix triandra, which reaches $1.4 \%$ at $195 \mathrm{~cm}$. 'Evergreen woodland trees' remains at the reduced value (maximum 0.8\%). Only Ephedra fragilis and Quercus coccifera type are represented and neither exceed $0.5 \%$ in any spectrum. 'Plants of open dry rocky habitats' increase (maximum $3.1 \%$ ) due to an increase in Agropyron type (2.2\% maximum).

Zone $K-8,177.5-157.5 \mathrm{~cm}$ The boundary with the preceding zone is marked by increases in the relative frequencies of 'coniferous and deciduous canopy trees' and Quercus frainetto type; and a decline in 'herbs of open ground', 'cultivars' and Chenopodiaceae type. This zone is characterised by high relative frequencies of 'herbs of open ground', 'cultivars', Pinus (Diploxylon), Quercus frainetto type, and Juniperus; increased relative frequencies of 'coniferous and deciduous canopy trees'; and low relative frequencies of Pteridium aquilinum.

'Herbs of open ground' decline (38.7-54.8\%). Pteridium aquilinum also declines (1.8-2.7\%, except in the uppermost spectrum where it increases to $5.8 \%)$. Other types in this group recording more than $2.0 \%$ are the same as those in the preceding zone with the exception of Daucus, reaches $3.3 \%$ at $162 \mathrm{~cm}$. Notable changes in the relative frequencies of types are the general increase in Centaurea solstitialis type, which exceeds $2.0 \%$ in two of the five spectra, and the decline in Chenopodiaceae type $(5.2 \%$ at the base to $1.3 \%$ in the spectrum second from the top).

'Cultivars' decline, but still record high frequencies (2.6-4.6\%). Reductions in this group are seen mostly in the cereals. Avena, Hordeum type, and Secale cereale although still occurring in all spectra, decline $(0.2-0.9 \%, 0.2-1.3 \%$, and $0.2-2.0 \%$, respectively). Zea mays does not occur in the basal spectrum, but occurs in all other spectra at $0.2 \%$. Triticum type 
does not occur. Castanea sativa is unchanged. Juglans regia occurs in the uppermost three spectra at a maximum of only $0.4 \%$. Morus nigra appears for the first time at 165 and $170 \mathrm{~cm}$ at 0.4 and $0.5 \%$. Platanus orientalis occurs in two spectra only; $160 \mathrm{~cm}(1.6 \%)$ and $170 \mathrm{~cm}$ $(0.2 \%)$.

'Coniferous and deciduous canopy trees' increase to comparatively high frequencies (32.6-49.0\%). In general, Pinus (Diploxylon) has comparatively high frequencies (24.1-25.9\%, but declines to $16.7 \%$ in the second spectrum and to $15.0 \%$ in the uppermost spectrum). Quercus frainetto type increases (11.9-19.8\%), while Quercus trojana type remains at low frequencies (1.3-3.0\%). No other type in this group exceeds $2.0 \%$.

One type of 'alpine shrubs' is represented but at only $0.2 \%$, and only two types of 'alpine herbs' are represented. These do not exceed $0.6 \%$. 'Woodland understorey trees and shrubs' are represented by Juniperus only, which reaches $6.4 \%$ in the centre of the zone. 'Woodland herbs' reach a maximum of $1.4 \%$. 'Riverine trees' are represented by 6 types but none exceeds $0.4 \%$ with the exception of Salix triandra, which reaches $1.5 \%$ at $165 \mathrm{~cm}$. 'Evergreen woodland trees' reach only $0.5 \%$. 'Plants of open dry rocky habitats' decline slightly (maximum 2.1\%) due to a decline in Agropyron type (maximum 1.6\%).

Zone $\mathrm{K}-7,157.5-142.5 \mathrm{~cm}$ The boundary with the preceding zone is marked by an increase in the relative frequencies of 'herbs of open ground'; and a decline in 'coniferous and deciduous canopy trees', Pinus (Diploxylon), and Quercus frainetto type. The zone is characterised by high relative frequencies of 'herbs of open ground', 'cultivars', Quercus frainetto type, Juniperus, and Polygonum aviculare type.

'Herbs of open ground' increase to the second highest overall values for a zone (57.7$67.9 \%$ ) (the basal zone is higher). Pteridium aquilinum increases slightly (2.0-4.5\%). Other types in this group exceeding $2.0 \%$ are the same as those in the preceding zone with the exception of Chenopodiaceae type, Daucus, and Festuca type, which have declined to less than 2.0\%; Galium type, which increases (basal spectrum records $2.5 \%$ ); and Polygonum aviculare type, which increases to its maximum of the sequence (41.2\%).

'Cultivars' decline further, but still record high relative frequencies $(1.9 \%$ in the basal spectrum, then 3.3 and $3.0 \%$ ). The cereals Avena, Hordeum type, and Secale cereale all continue to be represented continuously, but their maximum frequencies have declined; $0.6 \%$, $0.7 \%$, and $1.0 \%$, respectively. Zea mays does not occur in the basal spectrum, but increases to 0.6 and $0.3 \%$ in the upper two spectra. Triticum type occurs in the basal spectrum $(0.2 \%)$. Castanea sativa occurs in the basal spectrum only at $0.5 \%$. Juglans regia and Morus nigra disappear. Platanus orientalis increases from $0.2 \%$ in the basal spectrum to $1.3 \%$ in the uppermost. 
'Coniferous and deciduous canopy trees' decline to the lowest overall values for a zone (22.4-30.9\%). Pinus (Diploxylon) declines (8.5-14.6\%). Quercus frainetto type declines also (9.6-13.4\%), and Quercus trojana type remains at low frequencies declining from $4.1 \%$ in the basal spectrum to $0.9 \%$ in the two uppermost. No other type in this group exceeds $2.0 \%$.

'Alpine shrubs' are not represented and 'alpine herbs' are represented by one type at $0.5 \%$. 'Woodland understorey trees and shrubs' are again represented by Juniperus only, which reaches $5.2 \%$. 'Woodland herbs' reach a maximum of $1.2 \%$. 'Riverine trees' decline to reach a maximum of only $0.9 \%$. Three types are represented; Salix triandra is the most abundant at $0.5 \%$ maximum. 'Evergreen woodland trees' are represented in one spectrum only at $0.5 \%$. 'Plants of open dry rocky habitats' decline slightly (1.6\% maximum).

Zone $\mathrm{K}-6,142.5-117.5 \mathrm{~cm}$ The boundary with the preceding zone is marked by increases in the relative frequencies of 'coniferous and deciduous canopy trees', Quercus frainetto type, Quercus trojana type, and Quercus coccifera type; and a decline in 'herbs of open ground'. This zone is characterised by high relative frequencies of 'herbs of open ground', 'cultivars', and Quercus frainetto type; and increased relative frequencies of Quercus trojana type, Juniperus, Quercus coccifera type, and Pteridium aquilinum.

'Herbs of open ground' decline (40.3-51.1\%). Pteridium aquilinum increases (3.0-7.7\%). Other types in this group exceeding $2.0 \%$ are the same as those in the preceding zone, with the exception of those types that decline to less than $2.0 \%$ in all spectra (Cirsium type, Galium type, and Sonchus type); and those types where at least one spectrum reaches or exceeds $2.0 \%$ (Bilderdykia, Centaurea cyanus type, Rumex acetosa group, Chenopodiaceae type, and Festuca type). Polygonum aviculare type declines (2.5-6.3\%).

'Cultivars' increase in the upper half of the zone (3.4\% in the basal spectrum and $4.7 \%$ in the uppermost spectrum). Avena occurs sporadically and fluctuates (maximum $2.5 \%$ ). Frequencies of Hordeum type also fluctuate (0.2-2.5\%). Secale cereale increases in the upper part of the zone $(0.2-1.6 \%)$. Zea mays occurs in all spectra with the exception of the second from the bottom $(0.2-0.9 \%)$. Triticum type occurs in the second to bottom spectrum only, at $0.2 \%$. Castanea sativa occurs in the upper four spectra $(0.2-0.8 \%)$. Platanus orientalis occurs at $0.7-1.7 \%$.

'Coniferous and deciduous canopy trees' increase to $36.3-37.2 \%$, then increase again to $43.4 \%$ in the uppermost spectrum. Pinus (Diploxylon) continues to record low frequencies (10.1-13.4\%, and in the uppermost spectrum 16.4\%). Quercus frainetto type increases to comparatively high frequencies (16.1-20.4\%). Quercus trojana type also increases, but remains at comparatively low frequencies (3.7-6.3\%). No other type in this group exceeds $2.0 \%$. 
One type only of 'alpine shrubs' is represented, recording $0.2 \%$ maximum. Two types of 'alpine herbs' are represented, but do not exceed $0.9 \%$. 'Woodland understorey trees and shrubs' are again represented by Juniperus only, which increases to a maximum of $7.6 \%$. 'Woodland herbs' decline to a maximum of $1.0 \%$. 'Riverine trees' remain at very low frequencies and reach a maximum of only $1.1 \%$. Six types are represented, but all occur sporadically with Ulmus minor recording the maximum of only $0.8 \%$. Salix triandra occurs most often, but never exceeds $0.5 \%$. 'Evergreen woodland trees' increase due to an increase in Quercus coccifera type, which fluctuates from $0.4-2.8 \%$. 'Plants of open dry rocky habitats' remain at low values (maximum $2.0 \%$ ).

Zone $K-5,117.5-77.5 \mathrm{~cm}$ The boundary with the preceding zone is marked by increases in the relative frequencies of Pinus (Diploxylon) and Abies, and decreases in Quercus frainetto type and Quercus trojana type. This zone is characterised by high and increasing relative frequencies of 'herbs of open ground'; high relative frequencies of 'cultivars'; increased relative frequencies of Pteridium aquilinum; and declining relative frequencies of 'woodland understorey trees and shrubs', and Juniperus.

'Herbs of open ground' increase from frequencies similar to those of the preceding zone (43.6\% in the basal spectrum) to comparatively high values (59.7\% maximum). Pteridium aquilinum increases (4.1-9.0\%). Other types in this group exceeding $2.0 \%$ are the same as those in the preceding zone, with the exception of Bilderdykia and Centaurea cyanus (the former type disappears and the latter occurs in one spectrum only at $0.2 \%$ ). Of significance is an increase in Polygonum aviculare type (11.8\% maximum).

'Cultivars' remain at the elevated frequencies of the upper part of the preceding zone (3.0-5.7\%). Avena occurs in all except the basal spectrum (0.2-1.6\%). Hordeum type declines, recording the highest frequency of $1.6 \%$ in the basal spectrum. Secale cereale records $0.2-1.9 \%$. Triticum type and Zea mays occur sporadically, both recording a maximum of $0.8 \%$. Castanea sativa continues to occur sporadically at low frequencies $(0.7 \%$ maximum). Juglans regia reappears and occurs sporadically at low values ( $0.3 \%$ maximum) and Platanus orientalis now occurs sporadically (maximum 1.7\%). Vitis vinifera occurs in one spectrum only at $0.2 \%$.

'Coniferous and deciduous canopy trees' fluctuate from 30.9-45.8\%. Pinus (Diploxylon) increases to $11.8-18.7 \%$. Quercus frainetto type fluctuates from $12.3-18.6 \%$, then increases to $23.2 \%$. Quercus trojana type declines (2.9-4.7\%). Abies increases to reach a maximum of $1.9 \%$. All other types in this group score lower relative frequencies.

One pollen type only of 'alpine shrubs' is represented ( $0.2 \%)$. Several types of 'alpine herbs' are represented, but none exceeds $0.2 \%$. 'Woodland understorey trees and shrubs' are represented by Ilex aquifolium and Juniperus. Ilex aquifolium occurs sporadically and reaches 
a maximum of only $0.5 \%$ while Juniperus is continuously represented but declines from 6.6-0.5\%. 'Woodland herbs' increase slightly to a maximum of $1.5 \%$. 'Riverine trees' remain at very low values and again reach only $1.1 \%$. Five types are represented, but all are represented sporadically with the exception of Salix triandra, which records $0.2-0.5 \%$. Other types do not exceed $0.5 \%$. 'Evergreen woodland trees' decline in the upper part of the zone due to a decline in Quercus coccifera type, which fluctuates from $0.2-1.8 \%$ (with the exception of one spectrum where it does not occur). The group never exceeds $2.3 \%$ in any spectrum. 'Plants of open dry rocky habitats' remain poorly represented (maximum 2.0\%).

Zone $\mathrm{K}-4,77.5-57.5 \mathrm{~cm}$ The boundary with the preceding zone is marked by increases in the relative frequencies of 'coniferous and deciduous canopy trees', Pinus (Diploxylon), and Salix triandra; and a decline in the relative frequencies of 'herbs of open ground' and Pteridium aquilinum. This zone is characterised by high relative frequencies of 'herbs of open ground', 'cultivars', Pinus (Diploxylon), and Quercus frainetto type; increased relative frequencies of Abies and Salix triandra; and decreased Juniperus and Pteridium aquilinum.

'Herbs of open ground' decline to the minima of the sequence (25.1-37.4\%). Pteridium aquilinum declines to the minimum of the sequence of $1.1 \%$ at $70 \mathrm{~cm}$ (3.4\% maximum). Other types in this group exceeding $2.0 \%$ are the same as those in the preceding zone with the exception of those that decline to less than $2.0 \%$ - Centaurea solstitialis type, Festuca type, Lactuca type, Rumex acetosa group, and Solidago type; and those that increase to more than $2.0 \%$ - Cirsium type (maximum $8.6 \%$ at $70 \mathrm{~cm}$ ), Galium type $(2.3 \%$ at $65 \mathrm{~cm}$ ), and Plantago major $(3.8 \%$ at $60 \mathrm{~cm})$. Of note is the decline of Polygonum aviculare type, which now records $1.2-5.3 \%$ and Rumex acetosa group, which now occurs in only the two central spectra at 0.5 and $0.8 \%$.

'Cultivars' increase from $2.4 \%$ in the basal spectrum to $6.7 \%$ in the uppermost. Avena declines, but occurs in all spectra (0.2-0.6\%). Hordeum type occurs in the two central spectra only at 0.2 and $0.6 \%$. Secale cereale does not occur in the basal spectrum, but increases to a maximum of $2.3 \%$ in the uppermost. Triticum type occurs at $70 \mathrm{~cm}$ only, at $0.8 \%$. Zea mays is represented in every spectrum $(0.5-0.6 \%)$. Castanea sativa is also represented continuously (0.2-1.2\%). Juglans regia occurs sporadically ( $0.3 \%$ maximum). Platanus orientalis is again represented continuously and increases (3.4\% maximum).

'Coniferous and deciduous canopy trees' increase to the maximum of the sequence; $58.6 \%$ at $60 \mathrm{~cm}$. Pinus (Diploxylon) also increases to very high frequencies; 26.0-30.6\%, with a decline to $18.7 \%$ at $65 \mathrm{~cm}$. Frequencies of Quercus frainetto type are the overall highest of the sequence (18.1-22.3\%). Quercus trojana type continues unchanged at 2.5-4.7\%. Abies increases again to a maximum of $3.6 \%$ in the uppermost spectrum. No other type in this group exceeds $2.0 \%$. 
'Alpine shrubs' are not represented and only three types of 'alpine herbs' are represented, but only one exceeds $0.3 \%$; Soldanella records $1.0 \%$ at $75 \mathrm{~cm}$. 'Woodland understorey trees and shrubs' are represented by Juniperus only, which increases from $1.2 \%$ in the basal spectrum to $4.4 \%$ in the uppermost. 'Woodland herbs' decline slightly and occur sporadically (maximum 1.2\%). 'Riverine trees' increase to reach a maximum of $3.6 \%$ in the basal spectrum, but decline to $0.6 \%$ in the uppermost. The increase is due to an increase in Salix triandra, which records $2.7 \%$ in the basal spectrum and then declines to $0.6 \%$. All other types of this group occur sporadically and do not exceed $0.5 \%$. 'Evergreen woodland trees' occur sporadically, again at low values. Quercus coccifera type contributes almost all pollen, reaching a maximum of $2.0 \%$ in the uppermost spectrum. 'Plants of open dry rocky habitats' record $1.7 \%$ maximum.

Zone $\mathrm{K}-3,57.5-32.5 \mathrm{~cm}$ The boundary with the preceding zone is marked by increased relative frequencies of 'herbs of open ground', 'cultivars', Dactylis type, Avena, and Secale cereale; and a decline in 'coniferous and deciduous canopy trees', Pinus (Diploxylon), and Abies. This zone is characterised by high relative frequencies of 'herbs of open ground', 'cultivars', Quercus frainetto type, Dactylis type, Avena, and Secale cereale; increased relative frequencies of Juniperus and Quercus coccifera type; and low relative frequencies of Pinus (Diploxylon), Quercus trojana type, and Pteridium aquilinum.

'Herbs of open ground' increase (40.2-54.4\%). Pteridium aquilinum continues at low values (2.0-4.4\%). Other types in this group exceeding $2.0 \%$ are the same as those in the preceding zone with the exception of Cichorium type, which declines and in no spectrum reaches $2.0 \%$; Festuca type, which increases, exceeding $2.0 \%$ in several spectra and reaching a maximum of $2.4 \%$; Galium type, which reaches $2.0 \%$ at $45 \mathrm{~cm}$ and $2.2 \%$ at $35 \mathrm{~cm}$; and Plantago media, which occurs sporadically, but increases sharply to $14.5 \%$ at $55 \mathrm{~cm}$. A notable change is the sharp increase in Dactylis type (9.0-23.1\%).

'Cultivars' increase to the maximum relative frequencies of the sequence (6.1-12.3\%), mostly due to the cereal types of Avena and Secale cereale increasing to their maximum of the sequence, $5.0 \%$ and $5.4 \%$ respectively. Hordeum type occurs sporadically again and reaches only $0.5 \%$. Triticum type also occurs sporadically, but reaches $1.5 \%$ in the uppermost spectrum. Zea mays is represented continuously (0.2-1.1\%). Castanea sativa is also represented continuously $(0.2-0.4 \%$ with the exception of one spectrum, which increases to $2.3 \%)$. Juglans regia is represented in the two uppermost spectra only $(0.2$ and $0.4 \%)$. Lathyrus sativus and Morus alba are both represented in the basal spectrum only, at $0.2 \%$ and $0.6 \%$, respectively. Platanus orientalis declines to $0.4 \%$ in the uppermost spectrum.

'Coniferous and deciduous canopy trees' decline to almost the minimum of the sequence at $40 \mathrm{~cm}$. Overall, relative frequencies are very low (24.7-42.7\%). Pinus (Diploxylon) 
declines to low frequencies (8.0-13.6\%). Quercus frainetto type shows high overall frequencies, but fluctuates (10.7-21.9\%). Quercus trojana type continues unchanged at $3.0-4.8 \%$. Abies declines and reaches $0.9 \%$ only. Ostrya type records $0.7-0.9 \%$ except at $50 \mathrm{~cm}$ where it increases to $3.1 \%$. No other type in this group exceeds $0.4 \%$.

'Alpine shrubs' are not represented and only 3 types of 'alpine herbs' are represented, but none exceeds $0.7 \%$. 'Woodland understorey trees and shrubs' are represented by 3 types but only Juniperus exceeds $0.4 \%$. The overall frequencies of Juniperus have increased (2.5$5.0 \%$ ). 'Woodland herbs' occur continuously, but continue at low values (1.1\% maximum). 'Riverine trees' continue at slightly elevated values (0.7-2.4\%) with Salix triandra contributing most of the pollen. Other types in this group occur sporadically and at $0.5 \%$ or less. All pollen of 'evergreen woodland trees' $(0.7-1.8 \%)$ is contributed by Quercus coccifera type with the exception of $0.2 \%$ contributed by Ephedra fragilis in one spectrum. 'Plants of open dry rocky habitats' record $1.6 \%$ maximum.

Zone $K-2,32.5-17.5 \mathrm{~cm}$ The boundary with the preceding zone is marked by increases in the relative frequencies of 'coniferous and deciduous canopy trees', Pinus (Diploxylon), Abies, and Quercus coccifera type; and decreases in 'herbs of open ground', Juniperus, Dactylis type, and Avena. This zone is characterised by high relative frequencies of 'coniferous and deciduous canopy trees', 'herbs of open ground', 'cultivars', Pinus (Diploxylon), and Quercus frainetto type; comparatively high relative frequencies of Quercus coccifera type; and low relative frequencies of Juniperus.

'Herbs of open ground' decline to almost the lowest values of the sequence (28.2-36.7\%). Pteridium aquilinum declines to very low values (1.2-2.8\%). Other types in this group exceeding $2.0 \%$ are the same as in the preceding zone with the exception of Galium type, Plantago major, Plantago media, Polygonum aviculare type, and Taraxacum type, which decline to less than 2.0\%; and Solidago type, which increases to $2.5 \%$ mid-zone.

'Cultivars' decline but frequencies remain high (3.4-8.0\%), with cereal types contributing most pollen. However, Avena has declined and occurs in the two lower spectra only $(0.6$ and $1.2 \%$ ), while Hordeum type is represented in the two upper spectra only (maximum $0.8 \%$ ). Secale cereale is represented continuously (0.9-2.8\%). Triticum type occurs in the two upper spectra only (1.2 and 3.0\%). Zea mays increases slightly, occurring in all spectra (0.5-1.9\%). The tree types Castanea sativa, Juglans regia, and Platanus orientalis all occur in the two uppermost spectra, but at only $0.3-0.6 \%$.

'Coniferous and deciduous canopy trees' increase to $57.3 \%$ (almost the maximum of the sequence) in the basal spectrum, then decline to $48.8 \%$. Pinus (Diploxylon) also records high values in the basal spectrum (30.1\%), then declines to $20.1 \%$. Quercus frainetto type records high frequencies (16.7-18.8\%) and Quercus trojana type continues unchanged at 3.4-5.1\%. 
Abies increases, but to only $1.6 \%$. Ostrya type increases (1.2-3.5\%). No other type in this group exceeds $1.5 \%$.

Two types of 'alpine shrubs' and one of 'alpine herbs' are represented at $0.3 \%$. 'Woodland understorey trees and shrubs' decline and are represented by Juniperus only, which reaches a maximum of $2.0 \%$. 'Woodland herbs' occur continuously, but remain at low values (1.8\% maximum). 'Riverine trees' continue at slightly elevated values (0.9-3.7\%) and Salix triandra continues to contribute most of the pollen. Other types in this group occur sporadically ( $0.6 \%$ maximum). 'Evergreen woodland trees' increase due to an increase in Quercus coccifera type (1.5-3.4\%). Ephedra fragilis ( $0.6 \%$ in one spectrum) is the only other type represented in this group.

Zone $K-1,17.5-0 \mathrm{~cm}$ The boundary with the preceding zone is marked by increases in the relative frequencies of Salix triandra and Cirsium type; and a decline in the relative frequencies of 'coniferous and deciduous canopy trees' and Quercus frainetto type. This zone is characterised by high relative frequencies of 'herbs of open ground', 'cultivars', and Pinus (Diploxylon); increased relative frequencies of Salix triandra; and decreased relative frequencies of 'coniferous and deciduous canopy trees' and Quercus frainetto type.

'Herbs of open ground' increase to a moderate value (57.1\%), then decline to low values (33.9-36.0\%). Pteridium aquilinum remains at low abundance (0.8-2.5\%). Other types in this group exceeding $2.0 \%$ are the same as those in the preceding zone with the exception of Festuca type, which declines (no spectrum reaches 2.0\%); and those that increase to exceed $2.0 \%$ - Galium type (1.4-5.0\%), Lactuca type (0.0-2.1\%), and Polygonum aviculare type (2.2\% maximum). A notable change is the increase in Cirsium type, which increases to $33.3 \%$ in the basal spectrum then declines to $8.6 \%$ in the uppermost.

'Cultivars' decline but frequencies remain high (3.3-5.0\%). Cereal types still contribute most pollen of this group, although Avena has almost disappeared, being represented in the uppermost spectrum only at $0.3 \%$. Hordeum type is also very poorly represented; only the basal and uppermost spectra are represented (0.3 and 0.1\%). Secale cereale is represented continuously, but has declined (0.6-0.7\%). Triticum type is also continuously represented $(0.3-1.9 \%)$. Zea mays declines occurring sporadically and reaching only $0.8 \%$. Castanea sativa increases to reach $1.6 \%$ in the second spectrum, but disappears in the uppermost. Elaeagnus angustifolia appears for the first time in the uppermost spectrum at $0.1 \%$. Juglans regia occurs in one spectrum, at $0.2 \%$. Platanus orientalis is represented continuously and increases slightly (1.2\% maximum). Vicia sativa type occurs in the basal spectrum at $0.2 \%$.

'Coniferous and deciduous canopy trees' decline, fluctuating from 30.9-48.2\%. Pinus (Diploxylon) fluctuates from $19.0 \%$ in the basal spectrum to $37.0 \%$ at $10 \mathrm{~cm}$, then to $17.6 \%$ in the uppermost. Quercus frainetto type has declined (4.2-11.6\%). Quercus trojana type is 
unchanged (3.2-5.5\%) with the exception of the basal spectrum value of $0.9 \%$. Abies fluctuates from $0.6-2.4 \%$. No other type in this group exceeds $1.5 \%$.

'Alpine shrubs' are not represented. Three types of 'alpine herbs' are represented, but at $0.3 \%$ or less. All pollen of 'woodland understorey trees and shrubs' $(0.7-2.8 \%)$ is contributed by Juniperus with the exception of $0.1 \%$ of Frangula in the uppermost spectrum. 'Woodland herbs' occur continuously, remaining at low frequencies ( $2.4 \%$ maximum). 'Riverine trees' increase dramatically $(5.0-20.5 \%)$ due to an increase in Salix triandra, which reaches the maximum of $19.7 \%$ in the spectrum second from the surface. Other types in this group occur sporadically and at $0.7 \%$ or less. 'Evergreen woodland trees' decline due to a decline in Quercus coccifera type, which contributes almost all the pollen of this group (0.3-1.3\%).

\subsubsection{Terrestrial vegetation history}

This sequence records two periods of the vegetation history of the Kellia area, an older one of about 200 years duration beginning perhaps $c .830 \mathrm{BC}$ (see section 6.4.2) and a younger one, which may span about the last 760 years beginning c. $1230 \mathrm{AD}$.

The habitat groups of 'herbs of open ground' and 'coniferous and deciduous canopy trees' are the most abundant groups in this sequence, as demonstrated in both the relative frequency and concentration diagrams (Figures $6.11 \& 6.13$ ). The former group is usually the more abundant, and other habitat groups are much less abundant. Total abundance of 'herbs of open ground' is shared mostly by 22 types, the most abundant of which are Centaurea solstitialis type, Chenopodiaceae type, Cichorium type, Cirsium type, Dactylis type, Festuca type, Galium type, Hieracium type, Lactuca type, Plantago lanceolata type, Polygonum aviculare type, Pteridium aquilinum, Solidago type, Sonchus type, and Taraxacum type. Total abundance of the habitat group 'coniferous and deciduous canopy trees' is shared mostly between the three types of Pinus (Diploxylon), Quercus frainetto type (deciduous oaks), and Quercus trojana type (deciduous/semi-evergreen oaks); Abies and Ostrya type also contribute significant amounts of pollen. The habitat group of 'cultivars' is well represented throughout the sequence and that of 'woodland understorey trees and shrubs' above $210 \mathrm{~cm}$.

There is some correspondence between changes in the composition of the sediments and changes in the pollen profiles of both wetland and terrestrial pollen and spore types. Absence of total synchrony of these three factors is due to a significant contribution of pollen and spores derived from outside the catchment. This lowland site is today surrounded by cultivated fields and redeposition of cultivated soils is an important factor. The open fields are subject to sheet erosion and if the surface layers incorporate older pollen material it can be redeposited in the wetland basin. 
The terrestrial vegetation history of the Kellia area is discussed below according to the local pollen assemblage zones. In this discussion pollen and spore types of the habitat groups 'herbs of open ground' and 'cultivars' are mentioned first because of their importance in the vegetation history of this site.

Zone $\mathrm{K}-14,396.0-372.5 \mathrm{~cm}$, within range c. 830-630 BC 'Herbs of open ground' is the dominant habitat group: two types Pteridium aquilinum and Hieracium type record maxima concentrations of this zone. They also record maxima concentrations of their respective types of the sequence and these are at much higher values than those of the surface sample. Hieracium type is entomophilous, has medium relative dispersal, and can be over-represented (Chapter 3). It grows mostly in dry open habitats on disturbed ground, often as a weed. In New Zealand it is a well known pasture weed in open dry land. The source of pollen for this type is probably extra-local, from within the catchment. Pteridium aquilinum has medium relative dispersal and is well-represented, so the distance of the source cannot be determined. Pteridium aquilinum is a cosmopolitan pioneer. It is pyrophytic, not affected by cattle grazing, and often gregarious in woodland clearings.

Other types in this habitat group that are abundant are Centaurea solstitialis type, Cichorium type, Galium type, Lactuca type, Plantago lanceolata type, Solidago type, Sonchus type, Taraxacum type, and the grasses Dactylis type and Festuca type. Species of the Compositae family and those represented by Galium type are entomophilous and thus usually not dispersed long distances, while those of Plantago lanceolata type are anemophilous as well as entomophilous and the grasses are anemophilous (Knuth 1906-9), thus having the potential to disperse their pollen further. The species of these types are ecologically heterogeneous, but comprise mostly weeds or ruderals and grow on disturbed waste land and cultivated fields (Appendix X). Of particular interest is the occurrence of Agrostemma githago, Buglossoides arvensis type, Centaurea cyanus type, Galium type, and Sinapis type, which include species that are weeds of cereal cultivation. Some of the species represented also grow in damp places such as fens and marshes and may have been growing on the fen, e.g. species included in Galium type, Solidago type, and Taraxacum type.

'Cultivars' are abundant. The most abundant type in this group is the cereal Secale cereale, but Triticum is also abundant. The cereals Avena and Hordeum type are also represented. The nut trees Castanea sativa and Juglans regia, and Platanus orientalis (often grown for shade), which may have been planted (Bottema 1980), are also represented. Vicia sativa type is represented, but this pollen type includes several wild species as well as cultivated species of Vicia sativa, and probably also the cultivated species Vicia ervilia and the weed of cultivation Vicia pannonica. Vicia sativa (vetch) was cultivated in the Pindos foothills and on the Grevena plains before 1940 for fodder on a subsistence basis. It is no 
longer cultivated to the same extent. Seeds of domesticated Vicia ervilia (bitter vetch) were found in Macedonia at the archaeological sites of Nea Nikomedeia and Sitagroi in contexts dating to the Neolithic (Hansen 1988). The seeds are thought to have been used for human consumption (Renfrew, J. 1973a:116).

Identification of cereal pollen types is difficult and the types Avena and Hordeum type include some wild species as well as cultivars. Dispersal of pollen of Avena, Hordeum, and Triticum is very poor (Chapter 3 ) so they were either cultivated extra-locally or in the case of the former two may have grown wild in the vicinity. Dispersal of the pollen of the cereal Secale cereale is better, being anemophilous, thus extra-local cultivation is not necessarily indicated. This cereal was introduced into Greece during the Roman Period (Renfrew, J. $1969,1973 \mathrm{a}$ ), suggesting that the base of this zone is not older than $146 \mathrm{BC}$. This is more recent than the $830 \mathrm{BC}$ date calculated from radiocarbon dating. Van Zeist (1976) has indicated that prior to introduction domesticated rye was inadvertently dispersed by prehistoric agriculturalists as a field weed.

The second most abundant habitat group is that of 'coniferous and deciduous canopy trees'. The most abundant canopy tree type is Quercus trojana type (deciduous/semievergreen oak); values of both concentration data and relative frequencies are higher than those of the surface sample. Quercus frainetto type (deciduous oak) is also abundant, but occurs at lower concentrations and relative frequencies than in the surface spectrum. Comparison between former abundance of oak trees and the current abundance, based on pollen representation, is complicated by the woodland management practice of coppicing, which tends to decrease pollen yield. Coppicing is carried out extensively today, and although many ancient stools have been noted the exact extent of former coppicing is not known. Deciduous and semi-evergreen oaks are the dominant trees in the lowlands and foothills of Grevena today. The constant occurrence in the pollen diagram of types representing these trees suggests that the source of pollen has not changed significantly. Pinus (Diploxylon) occurs at lower concentrations and relative frequencies than in the surface spectrum. Today there are no sources of Pinus (Diploxylon) pollen on the lowland with the exception of a small (planted) stand near Grevena city. Thus the source of Pinus (Diploxylon) is considered to be from a regional source. Ostrya type occurs at about the same relative frequencies and concentrations as it does in the surface spectrum, and indeed throughout the sequence. Trees comprising this pollen type are scarce in Grevena today and grow in a variety of habitats (but mostly in damp environments) at various elevations (Chapter 3, Appendix X). The pollen source may be extra-local or regional, or both.

The habitat groups of alpine shrubs and herbs are extremely poorly represented in this zone and indeed throughout the sequence, reflecting the long distance of alpine vegetation 
from the site and its low pollen production. 'Woodland understorey trees and shrubs' and 'woodland herbs' are poorly represented, indicating a poorly developed woodland understorey vegetation. The relative frequencies and concentrations of 'riverine trees' are so low that a local or an extra-local presence is unlikely; the pollen probably derives from more distant streams. 'Evergreen woodland trees' are very poorly represented. Quercus coccifera type (evergreen oak) is the most abundant type in the group. The concentrations of this type are about the same as those of the surface spectrum. Today Quercus coccifera grows in a small stand of pseudo-maquis in the southeast corner of Grevena. Quercus coccifera is anemophilous, has high relative dispersal, and is over represented (Chapter 3 ). It is pyrophytic and takes advantage of destruction of primary forests (Behre 1990). The poor representation of this habitat group and that of 'plants of open rocky habitats' suggests that a Mediterraneantype vegetation does not occur extra-locally, and forms only a small part of the regional vegetation, as at present.

The sum of terrestrial trees and shrubs represents from $24.4-33.7 \%$ of total terrestrial pollen and spores; the minima of the sequence. The low ratio of terrestrial trees and shrubs to terrestrial herbs, high palynological richness values (25-40 expected types), and high relative frequencies and concentrations of the habitat group of 'herbs of open ground' are indicative of an open canopy.

The extra-local and regional lowland vegetation surrounding the Kellia wetland at this time was probably a highly disturbed vegetation with scattered open canopy deciduous and semi-evergreen oak woodland with a poorly developed understorey of herb-rich steppe, rather than shrubs and woodland herbs. The occurrence of Pteridium aquilinum suggests the presence of a pioneer vegetation, perhaps created by the burning of oak woodland. Cereals were cultivated extra-locally and the pulses Vicia sativa and Vicia ervilia may have been also. The nut trees Castanea sativa and Juglans regia may have been planted either extra-locally or further from the site. A habitat suitable for Platanus orientalis may have been created by human disturbance of the natural vegetation extra-locally or further from the wetland, or this tree may have been growing wild on flood plains. Riverine trees grew on more distant flood plains and pine woods grew at higher elevations further from the site in the Pindos Mountains.

Zone $\mathrm{K}-13,372.5-312.5 \mathrm{~cm}$, within range c. $830-630 \mathrm{BC}$ 'Herbs of open ground' remain the most abundant group, although concentration values are lower due to a decline in Hieracium type and Pteridium aquilinum. These types still record higher values than those of the surface sample, but have concentrations similar to those of Centaurea solstitialis type and Dactylis type whose concentrations are unchanged from the preceding zone. Cichorium type, Festuca type, Galium type, Lactuca type, Solidago type, Sonchus type, and Taraxacum type continue to be abundant. Chenopodiaceae type, Cirsium type, and Polygonum aviculare 
type now become abundant while Plantago lanceolata type declines. Of particular interest is the occurrence of Centaurea cyanus type, Galium type, Nigella, and Sinapis type, which include species that are weeds of cereal cultivation.

'Cultivars' are still abundant, but concentration values have declined slightly with the disappearance of Triticum type and Vicia sativa type, and a decline in Secale cereale. Secale cereale is still the most abundant cultivar type and the cereal types Avena and Hordeum type are abundant, increasing slightly. The pollen type Zea mays occurs at low abundance at $330 \mathrm{~cm}$, and small numbers of this pollen type were counted in samples at higher levels. Identification of these large Gramineae pollen grains followed the key set out in Appendix VI (and references therein). Zea mays was introduced into Europe from the New World after $1500 \mathrm{AD}$, and did not become widespread in cultivation until c. $1700 \mathrm{AD}$ (Huber 1962 in Bottema 1980; Bottema 1981). Radiocarbon dating indicates that these pollen grains cannot be Zea mays. It is most likely that they should be attributed to Triticum type or Avena, which although usually having smaller pollen grains, also have relatively large porus annuli, and are closest in morphology among the cereal group. The shade tree Platanus orientalis increases slightly and the nut trees Castanea sativa and Juglans regia remain unchanged. The occurrence of Castanea sativa and Juglans regia in this and the zone below suggests that the vegetation of these two zones grew at a time before 1628-1626 BC (Bottema 1980; Bottema 1981; Bottema 1994; Bottema \& Woldring 1990). This is not consistent with the radiocarbon age of c. $2000 \mathrm{BC}$ at the top of this zone, but the estimated date $(830 \mathrm{BC})$ from the radiocarbon date at the base of the sequence is more recent.

The habitat group of 'coniferous and deciduous canopy trees' has increased in relative frequency and concentration values, but is still the second most abundant habitat group. The increases are due to increases in Pinus (Diploxylon), Quercus frainetto type, and Quercus trojana type. Relative frequencies and concentration values of Pinus (Diploxylon) increase slightly to about the average of the sequence, which is much less than the values of the surface sample. Deciduous and semi-evergreen oaks are co-dominant and increase to relative frequency maxima of the sequence and concentrations that are also close to the maxima of the sequence. Relative frequencies and concentrations of Ostrya type are about the same as those of the preceding zone.

Alpine shrub and herb types are again extremely poorly represented. 'Woodland understorey trees and shrubs' and 'woodland herbs' are also extremely poorly represented. 'Riverine trees' decline slightly. 'Evergreen woodland trees' are again poorly represented; Quercus coccifera type declines slightly. 'Plants of open dry rocky habitats' are poorly represented with the exception of Agropyron type, which becomes abundant. This pollen type includes mostly wild grasses that grow in dry habitats with the exception of one species that 
may belong to this type, Panicum miliaceum (broomcorn millet). This small grained species was formerly widely cultivated as a cereal (Tutin et al. 1964-80).

The sum of terrestrial trees and shrubs represents from $35.2-52.9 \%$ of total terrestrial pollen and spores. The low ratio of terrestrial trees and shrubs to terrestrial herbs, high palynological richness values (30-40 expected types), and high relative frequencies and concentrations of the habitat group of 'herbs of open ground' (especially of the pioneer Pteridium aquilinum) are indicative of an open canopy.

The extra-local and regional lowland vegetation is still highly disturbed with an open canopy deciduous and semi-evergreen oak woodland with an understorey of herb-rich steppe, rather than of shrubs and woodland herbs. An increase in the concentrations of both the deciduous and deciduous/semi-evergreen oak pollen types suggest that either the oak wood has expanded or become more dense. A decline in concentration values of 'herbs of open ground' and Pteridium aquilinum, and an increase in the ratio of terrestrial trees and shrubs to terrestrial herbs support the latter. Cultivation of cereals is carried out extra-locally and the nut trees of Castanea sativa and Juglans regia, and the shade-tree Platanus orientalis continue to grow either sparsely extra-locally or more abundantly at a greater distance. Distant high elevation pine woods continue at about the same abundance.

Zone $\mathrm{K}-12,312.5-287.5 \mathrm{~cm}, \mathrm{c.}$ 1230-1320 AD 'Herbs of open ground' are co-dominant with the habitat group of 'coniferous and deciduous canopy trees'. The relative frequencies of 'herbs of open ground' are low for the sequence, but concentrations are very high. This is due to an increase in total concentration. Pteridium aquilinum declines further to very low concentration values at the top of the zone, where concentrations are almost as low as those of the surface sample. The pollen types Polygonum aviculare type, Solidago type, Sonchus type, and Taraxacum type with increased relative frequencies from the preceding zone; and Centaurea solstitialis type, Chenopodiaceae type, Cichorium type, Cirsium type, Hieracium type, Lactuca type, Plantago lanceolata type, Dactylis type, and Festuca type are abundant. Galium type has declined and the only other pollen type, Sinapis type, which includes species that are weeds of cereal cultivation occurs in the basal spectrum only.

'Cultivars' are again abundant, occurring at similar relative frequencies as in the preceding zone. Concentration values are increased in the lower part of the zone due to an increase in Secale cereale type and the presence of 'Zea mays' type. Secale cereale remains the most abundant cereal type. Other cereals of Avena and Hordeum type occur sporadically at about the same abundance as in the preceding zone. The nut trees Castanea sativa and Juglans regia continue at similar abundance while Platanus orientalis declines.

The habitat group of 'coniferous and deciduous canopy trees' has increased in relative frequency and concentration values, due to increases in Pinus (Diploxylon) and Quercus 
frainetto type. The frequency of Quercus trojana type has declined, but concentration values are high, probably due to the increase in total concentration. Deciduous oaks (Quercus frainetto type) are now more abundant than deciduous/semi-evergreen oaks (Quercus trojana type). Ostrya type has declined slightly.

Alpine shrubs and herbs are not represented. 'Woodland understorey trees and shrubs' and 'woodland herbs' are again extremely poorly represented. 'Riverine trees' have declined further and only Salix triandra is represented, at extremely low values. 'Evergreen woodland trees' and 'plants of open dry rocky habitats' have almost disappeared.

The sum of terrestrial trees and shrubs represents from $40.9-58.1 \%$ of total terrestrial pollen and spores. The low ratio of terrestrial trees and shrubs to terrestrial herbs, high palynological richness values (30-40 expected types), and high relative frequencies and concentrations of 'herbs of open ground' are indicative of an open canopy.

The extra-local and regional lowland vegetation is still highly disturbed and has an open canopy and an understorey of herb-rich steppe. The oak woodland now comprises more deciduous oaks than deciduous/semi-evergreen oaks. The higher proportion of deciduous oaks may be due to a reduction of coppicing (Quercus frainetto, which today is the preferred coppice-tree, takes at least 50 years to produce flowers after coppicing whereas other oaks produce flowers in the later part of each coppicing cycle (30-40 years)), selective preservation of deciduous oaks for the purpose of coppicing or to an increase in climatic wetness. Increased concentrations of the pollen types representing the oak wood may be due to a denser or expanded woodland and/or to slower sediment accumulation. A decline in the frequencies of 'herbs of open ground' and Pteridium aquilinum and an increase in the ratio of terrestrial trees and shrubs to terrestrial herbs support the first two, while sediments rich in organic detritus support the last. The near disappearance of Quercus coccifera type may be due to a reduction in direct or indirect human disturbance of the vegetation (e.g. burning), or to more humid climatic conditions. The reduction in Pteridium aquilinum suggests a reduction in burning of the woodland. Cereals are cultivated extra-locally. The trees Castanea sativa, Juglans regia, and Platanus orientalis continue to grow naturally or are planted, either sparsely extra-locally or more abundantly regionally. Mountain pine woods may have increased slightly.

Zone $\mathrm{K}-11,287.5-237.5 \mathrm{~cm}, \mathrm{c.}$ 1320-1370 AD 'Herbs of open ground' are dominant in the relative frequency diagram, while in the concentration diagram they are co-dominant with 'coniferous and deciduous canopy trees'. Relative frequencies of 'herbs of open ground' have increased but concentration values are low, due to low total concentration values. Relative frequencies and concentrations of Pteridium aquilinum have now declined to values as low as those of the surface sample. Types of this habitat group that are abundant are the same as 
in the preceding zone, with the addition of Galium type, which has increased. Of particular interest is the occurrence of Agrostemma githago, Buglossoides arvensis, Centaurea cyanus type, Galium type, Nigella, and Sinapis type, which include species that are weeds of cereal cultivation.

'Cultivars' are again abundant; relative frequencies have increased due to increases in Avena, Platanus orientalis, and Secale cereale types. Concentration values have declined due to a decline in total concentration. The cereal types Avena and Secale cereale occur continuously while those of Hordeum type and Zea mays occur sporadically. Vitis vinifera occurs for the first time at low abundance. Castanea sativa, Juglans regia, and Platanus orientalis continue to occur sporadically at low abundances, although they now occur more often.

The habitat group of 'coniferous and deciduous canopy trees' declines in relative frequency due to decreases in Pinus (Diploxylon) and Quercus frainetto type. The relative frequencies of Quercus trojana type fluctuate but in general they have increased and are high for this sequence. Concentration values re low due to low total concentration. Ostrya type occurs at low abundance, but at highest relative frequencies of the sequence.

Alpine shrubs and herbs are extremely poorly represented. 'Woodland understorey trees and shrubs' and 'woodland herbs' are poorly represented but the number of types represented has increased and Juniperus has increased slightly in relative frequency. 'Riverine trees' are also represented by more types, but at very low values. 'Evergreen woodland trees' and 'plants of open dry rocky habitats' are also represented by more types but at very low values.

The sum of terrestrial trees and shrubs represents from $35.5-47.6 \%$ of total terrestrial pollen and spores. The low ratio of terrestrial trees and shrubs to terrestrial herbs, very high palynological richness values (40-60 expected types), and high relative frequencies and concentrations of 'herbs of open ground' are indicative of an open canopy.

The extra-local and regional lowland vegetation surrounding the wetland continues to be highly disturbed open canopy oak woodland with a herb-rich steppe understorey. Deciduous and deciduous/semi-evergreen oaks occur in about the same proportion due to a decline in Quercus frainetto type and an increase in Quercus trojana type. These changes may be related to increased coppicing of the deciduous oaks or to drier climatic conditions. An increase in the relative frequencies of 'herbs of open ground' and in palynological richness, and a decline in the ratio of terrestrial trees and shrubs to terrestrial herbs may indicate increased disturbance of the woodland. Concentrations of Quercus frainetto type and Quercus trojana type are very low and is probably due to rapid sedimentation of minerogenic sediments. Quercus coccifera type occurs at extremely low abundance. Cultivation of cereals continues in the vicinity and perhaps viticulture has begun, but Vitis vinifera may be growing wild on 
the wetland. The nut trees and Platanus orientalis may be growing wild or are planted either extra-locally or regionally. High elevation pine woods may have declined.

Zone K-10, 237.5-202.5 cm, c. 1370-1480 AD 'Herbs of open ground' are dominant again and relative frequencies are high, being the same as those of the preceding zone; concentrations have increased due to an increase in total concentration. The relative frequencies of Pteridium aquilinum have decreased slightly, while concentrations have increased slightly. Types of this group that are abundant are the same as those in the preceding zone, with the addition of Bilderdykia, which has increased. The following types that occur include species that are weeds of cereal cultivation: Centaurea cyanus type, Galium type, Nigella, and Sinapis type.

'Cultivars' are again abundant; relative frequencies and concentrations have increased due to increases in Avena, Hordeum type, Platanus orientalis, and Secale cereale types. The cereal types Avena and Secale cereale occur continuously while Hordeum type and Zea mays occur sporadically. Vitis vinifera occurs in one spectrum only. Castanea sativa and Juglans regia occur sporadically at low values.

The habitat group of 'coniferous and deciduous canopy trees' is the second most abundant group. Relative frequencies are unchanged but concentration values increase due to an increase in total concentration. The concentrations of Pinus (Diploxylon) are comparatively high and relative frequencies increase. Relative frequencies of Quercus frainetto type are unchanged to the centre of the zone but then they decline, concentrations have increased due to an increase in total concentration. The relative frequencies of Quercus trojana type have declined while concentrations have increased. The deciduous oaks (Quercus frainetto type) are again more abundant than the deciduous/semi-evergreen (Quercus trojana type). Ostrya type has declined while Fraxinus ornus occurs at low but significant abundance.

Alpine shrubs and herbs are extremely poorly represented. 'Woodland understorey trees and shrubs' and 'woodland herbs' are poorly represented; the number of types represented by the former group declines to three. Juniperus increases steadily but still occurs at low values. 'Riverine trees' are poorly represented and the number of types represented declines. 'Evergreen woodland trees' and 'plants of open dry rocky habitats' decline to very low abundance.

The sum of terrestrial trees and shrubs represents from 38.4-50.3\% of total terrestrial pollen and spores. The low ratio of terrestrial trees and shrubs to terrestrial herbs, very high palynological richness values (40-55 expected types), and high relative frequencies and concentrations of the habitat group of 'herbs of open ground' are indicative of an open canopy. 
The extra-local and regional lowland vegetation surrounding the wetland continues to be highly disturbed open canopy oak woodland with an understorey of herb-rich steppe; although Juniperus increases slightly. Deciduous oaks are more abundant than deciduous/semi-evergreen oaks due to a decline in Quercus trojana type. This decline may be caused by wetter climatic conditions. Quercus coccifera type continues at extremely low abundance. Slightly lower abundance of Pteridium aquilinum may indicate a reduction in burning. An increase in total pollen concentration, unchanged relative frequencies of the habitat group 'herbs of open ground', and the absence of a change in the ratio of terrestrial trees and shrubs to terrestrial herbs may indicate greater stability in the landscape. Cultivation of cereals continues in the vicinity, perhaps at increased intensity. Cultivation of Vitis vinifera may occur and the nut trees of Castanea sativa and Juglans regia, and Platanus orientalis may be planted or grow naturally either extra-locally or regionally. Mountain pine woods increase.

Zone $\mathrm{K}-9,202.5-177.5 \mathrm{~cm}, \mathrm{c.}$ 1480-1580 AD 'Herbs of open ground' increase in the upper half of the zone and are dominant again. Relative frequencies are high in the lower half of the zone and become very high in the upper half. Concentrations are also high and increase. These increases are partly due to a dramatic increase in Chenopodiaceae type. Relative frequencies and concentrations of Pteridium aquilinum increase slightly. Types of this group that are abundant are the same as those in the preceding zone. Several types that occur include species that are weeds of cereal cultivation: Agrostemma githago, Centaurea cyanus type, Galium type, Nigella, and Sinapis type.

'Cultivars' increase to highest values of the sequence in relative frequency and concentration, due to increases in Avena, Hordeum type, Secale cereale, and Zea mays types, which are continuously represented, and the representation of more types - Triticum type, Humulus lupulus, and Vicia sativa type occur in one spectrum. Trees that may have been planted decline: Castanea sativa occurs sporadically, Juglans regia disappears, and Platanus orientalis declines.

The habitat group of 'coniferous and deciduous canopy trees' is the second most abundant group. Relative frequencies have declined to almost the lowest of the sequence, due to a dramatic decline in Quercus trojana type and a less pronounced decline in Quercus frainetto type. Concentration values of the group are unchanged because the decline in the two oak types is off-set by an increase in Pinus (Diploxylon). Deciduous oaks continue to be more abundant than deciduous/semi-evergreen oaks.

Alpine shrubs and herbs are extremely poorly represented. 'Woodland understorey trees and shrubs' and 'woodland herbs' are poorly represented: the only type that occurs in significant abundance is Juniperus, which continues to increase in relative frequency and concentration. 'Riverine trees' are poorly represented. 'Evergreen woodland trees' are also 
poorly represented, but 'plants of open dry rocky habitats' increase slightly due to an increase in Agropyron type.

The sum of terrestrial trees and shrubs represents $29.9-41.1 \%$ of total terrestrial pollen and spores. The very low ratio of terrestrial trees and shrubs to terrestrial herbs, high palynological richness values (about 37-47 expected types), and high relative frequencies and concentrations of 'herbs of open ground' are indicative of an open canopy.

The extra-local and regional lowland vegetation continues to be highly disturbed open canopy oak woodland of deciduous and semi-evergreen oaks, with deciduous oaks dominant. The understorey may now include Juniperus as well as a herb-rich steppe. A reduction in canopy cover is suggested by a dramatic decline in deciduous/semi-evergreen oaks, and an increase in 'herbs of open ground', particularly in the pioneer Pteridium aquilinum. The cause of the reduction in the deciduous/semi-evergreen oaks may be due to more humid climatic conditions, although deciduous oaks decline also. Quercus coccifera type continues at extremely low abundance. A dramatic increase in Chenopodiaceae type may result from increased clearance for cultivation fields (extra-locally) or increased grazing. Increases in the cereal pollen types supports the former, while the latter is supported by an increase in Juniperus. The increase in Agropyron type may be due to Panicum miliaceum (broomcorn millet) being cultivated, or to wild grasses growing in dry habitats. Wetter climatic conditions are supported by the development of peat on the wetland. Mountain pine woods have increased.

Zone $\mathrm{K}-8,177.5-157.5 \mathrm{~cm}, \mathrm{c.}$ 1580-1650 AD 'Herbs of open ground' decline and overall relative frequencies and concentration are about the same as those of 'coniferous and deciduous canopy trees'. Relative frequencies and concentrations of Pteridium aquilinum decline to very low values, almost as low as those of the surface sample. No significant changes occur in the other most abundant types of this group, with the exception of Chenopodiaceae type returning to an abundance similar to that of zone K-10. Four types that occur include species that are weeds of cereal cultivation: Centaurea cyanus type, Galium type, Nigella, and Sinapis type.

'Cultivars' decline in relative frequency and concentration, due to reductions in all constituent taxa, but are still abundant. The decline in concentration is partially due to a decline in total concentration. Avena, Hordeum type, and Secale cereale types occur continuously while the type Zea mays occurs in all except the basal spectrum. Triticum type disappears. Morus nigra appears in two spectra at low values. The trees Castanea sativa, Juglans regia (reappears), and Platanus orientalis all occur sporadically at low values.

Relative frequencies of 'coniferous and deciduous canopy trees' increase to comparatively high values due to an increase in Quercus frainetto type. Pinus (Diploxylon) 
continues at comparatively high values, while Quercus trojana type continues at low relative frequencies. Concentrations of Quercus frainetto type increase slightly, those of Pinus (Diploxylon) fluctuate and decline slightly, while those of Quercus trojana type continue at similar values to those of the zone below.

Alpine shrubs and herbs are extremely poorly represented. 'Woodland understorey trees and shrubs' and 'woodland herbs' are poorly represented with the exception of Juniperus, which continues to increase, perhaps encouraged by grazing. 'Riverine trees' are poorly represented 'Evergreen woodland trees' and 'plants of open dry rocky habitats' decline slightly, due to a decline in Agropyron type.

The sum of terrestrial trees and shrubs increases to $40.1-58.1 \%$ of total terrestrial pollen and spores. The low ratio of terrestrial trees and shrubs to terrestrial herbs, very high palynological richness values (about 42-55 expected types), and high relative frequencies and concentrations of 'herbs of open ground' are indicative of an open canopy.

The extra-local and regional lowland vegetation continues to be highly disturbed open canopy oak woodland dominated by deciduous oaks, semi-evergreen oaks play a minor role. The understorey may now include more Juniperus. Steadily increasing Juniperus may be indicative of grazing in the area, or a reduction in extra-local burning. A slight increase in canopy cover of the oak wood is suggested by an increase in the concentration of Quercus frainetto type and a slight decrease in 'herbs of open ground', particularly in Pteridium aquilinum. The decline of cereal pollen suggests a reduction of cultivation. Quercus trojana type does not recover and peat continues to form on the wetland. Quercus coccifera type continues at extremely low abundance. Pine woods may decline slightly.

Zone $\mathrm{K}-7,157.5-142.5 \mathrm{~cm}, \mathrm{c}$. 1650-1680 AD 'Herbs of open ground' again become dominant and relative frequencies are the highest of the sequence with the exception of the basal zone. Pteridium aquilinum increases slightly. No significant changes occur in the most abundant types of this group. A dramatic increase in Polygonum aviculare type in two spectra are probably due to species of this type growing on the wetland close to the coring site. Three types that occur include species that are weeds of cereal cultivation: Centaurea cyanus type, Galium type, and Nigella.

'Cultivars' are abundant, but their relative frequencies decline slightly, due to a slight decline in all cereal types. Concentrations are similar to those in the preceding zone. The types of Avena, Hordeum type, and Secale cereale are represented continuously, while those of Triticum type and Zea mays occur sporadically. Platanus orientalis occurs continuously at low values. Castanea sativa occurs sporadically and Juglans regia disappears.

Relative frequencies and concentrations of 'coniferous and deciduous canopy trees' decline and are the minimum overall values for a zone. Concentration values are 
comparatively low, but not minima of the sequence. This decline is due to a decline in relative frequencies and concentrations of Pinus (Diploxylon), Quercus frainetto type, and Quercus trojana type.

Alpine shrubs and herbs are extremely poorly represented. 'Woodland understorey trees and shrubs' and 'woodland herbs' are also poorly represented with the exception of Juniperus, which continues at the same abundance as in the preceding zone. 'Riverine trees' decline to very low abundance. 'Evergreen woodland trees' and 'plants of open dry rocky habitats' continue at very low abundance.

The sum of terrestrial trees and shrubs declines to very low values of $29.4-36.9 \%$ of total terrestrial pollen and spores. The low ratio of terrestrial trees and shrubs to terrestrial herbs, high palynological richness values (35-45 expected types), and very high relative frequencies and concentrations of the habitat group of 'herbs of open ground' are indicative of an open canopy.

Extra-local and regional lowland vegetation continues to be highly disturbed open canopy oak woodland dominated by deciduous oaks with semi-evergreen oaks now a very minor component. An understorey the same as in the preceding zone is indicated. A decrease in canopy cover of the oak wood is suggested by the increase in 'herbs of open ground', particularly of Pteridium aquilinum, and a decrease in the ratio of terrestrial trees and shrubs to terrestrial herbs. Evergreen oaks continue at extremely low abundance. A slight decrease in cereal pollen types suggests that cultivation of cereals has declined slightly. The abundance of Juniperus suggests that grazing of the woodland is occurring.

Zone $K-6,142.5-117.5 \mathrm{~cm}$, c. $1680-1720 \mathrm{AD}$ 'Herbs of open ground' decline to comparatively low relative frequencies and concentration values. Low concentration values are partly due to low total concentration values. Relative frequencies of Pteridium aquilinum increase while concentration values decrease but this decrease is due to a decrease in total concentration values. No significant changes occur in the most abundant types of this group. Four types that occur include species that are weeds of cereal cultivation: Agrostemma githago, Centaurea cyanus type, Galium type, and Sinapis type.

'Cultivars' are abundant and increase in the upper part of the zone in both values due to increases in Avena, Hordeum type, and Secale cereale. Hordeum type and Secale cereale occur continuously, while Avena, Triticum type, and Zea mays occur sporadically. Platanus orientalis occurs continuously at low values, while Castanea sativa occurs sporadically.

Relative frequencies of 'coniferous and deciduous canopy trees' increase, while concentrations of this group decline, then increase in the uppermost sample. The decline in concentration is due to the decline in total concentration. The increase in relative frequency is due to increases in Quercus frainetto type and Quercus trojana type. Quercus frainetto type 
increases to comparatively high relative frequencies for this sequence while Quercus trojana type increases to comparatively low relative frequencies and remains less abundant than the former type.

Alpine shrubs and herbs are extremely poorly represented. 'Woodland understorey trees and shrubs' and 'woodland herbs' are also poorly represented with the exception of Juniperus, which increases to highest relative frequencies of the sequence. 'Riverine trees' are poorly represented. 'Evergreen woodland trees' and 'plants of open dry rocky habitats' are poorly represented, but the pollen type Quercus coccifera type fluctuates and reaches its highest relative frequencies of the sequence in several spectra.

The sum of terrestrial trees and shrubs increases to $44.2-54.8 \%$ of total terrestrial pollen and spores, about average values for the sequence. The low ratio of terrestrial trees and shrubs to terrestrial herbs, high palynological richness values (about 40 expected types), and very high relative frequencies and concentrations of 'herbs of open ground' are indicative of an open canopy.

Extra-local and regional lowland vegetation continues to be highly disturbed open canopy oak woodland dominated by deciduous oaks. Semi-evergreen oaks have increased importance but are still a minor component and Juniperus is an important part of the understorey. An increase in canopy cover, at least extra-locally, is suggested by the decrease in 'herbs of open ground', an increase in the relative frequencies of deciduous and deciduous/semi-evergreen oaks, and an increase in the ratio of terrestrial trees and shrubs to terrestrial herbs, but the abundance of Juniperus suggests that the canopy is still open. Pteridium aquilinum increases; the source of the spores may be local, extra-local or regional. An increase in cereal cultivar types in the upper part of the zone suggests that cultivation of cereals has expanded. The increase in Quercus coccifera type may be due to degradation of the deciduous and semi-evergreen oak woodland, either extra-locally or regionally, into pseudo-maquis or to long distance transport (Chapter 3). Mountain pine woods remain at the reduced abundance of the preceding zone.

Zone $K-5,117.5-77.5 \mathrm{~cm}$, c. 1720-1790 AD 'Herbs of open ground' are dominant. Relative frequencies increase towards the top of the zone. Concentrations fluctuate, following the curve of total concentration, and are slightly higher in general due to an increase in total concentration. Relative frequencies and concentrations of Pteridium aquilinum increase. No significant changes occur in the other most abundant types of this group. An increase in Polygonum aviculare type in two spectra may be due to species of this type growing on the wetland close to the coring site. Three types that occur include species that are weeds of cereal cultivation: Centaurea cyanus type, Galium type, and Sinapis type. 
'Cultivars' are abundant. In general relative frequencies and concentrations are the same as those in the preceding zone. Hordeum type and Secale cereale occur continuously, while Avena, Triticum type, and Zea mays occur sporadically. Vitis vinifera occurs in one spectrum. Castanea sativa continues occurring sporadically at low abundance, Juglans regia reappears and occurs sporadically, and Platanus orientalis declines and occurs only sporadically.

Relative frequencies of 'coniferous and deciduous canopy trees' increase again due to increases in Pinus (Diploxylon) and Abies. Abies increases to relative frequencies similar to those of the surface sample. Concentrations increase; there is an increase in total concentration also. Relative frequencies and concentrations of Quercus frainetto type and Quercus trojana type decline slightly and Quercus frainetto type remains the dominant type.

Alpine shrubs and herbs are extremely poorly represented. 'Woodland understorey trees and shrubs' and woodland herbs' are also poorly represented with the exception of Juniperus, which now begins to decline. 'Riverine trees' are poorly represented. 'Evergreen woodland trees' and 'plants of open dry rocky habitats' are poorly represented with the exception of Quercus coccifera type, which has declined slightly.

The sum of terrestrial trees and shrubs increases to $36.5-50.1 \%$ of total terrestrial pollen and spores. The low ratio of terrestrial trees and shrubs to terrestrial herbs, high palynological richness values (35-45 expected types), and very high relative frequencies and concentrations of 'herbs of open ground' are indicative of an open canopy.

Extra-local and regional lowland vegetation continues to be highly disturbed open canopy oak woodland with trees represented by Quercus frainetto type dominant, and those of Quercus trojana type playing a minor role. The importance of Juniperus in the understorey is declining. A slight decrease in cancopy cover may oceur as suggested by an increase in 'herbs of open ground', a slight decline in the abundance of deciduous and semi-evergreen oaks, and a decline in the ratio of terrestrial trees and shrubs to terrestrial herbs. An increase in Pteridium aquilinum may be due to the reduction in canopy cover, although declining Juniperus suggests a closing canopy. Cultivation of cereals appears to continue at about the same level of intensity. The increase in the montane conifers Pinus (Diploxylon) and Abies may be due to a reduction in burning of the montane vegetation. A mixed Pinus/Abies wood similar to that growing in part of the Pindos today may have developed (Chapter 3).

Zone K-4, 77.5-57.5 cm, c. 1790-1840 AD 'Herbs of open ground' are the second most abundant habitat group; relative frequencies decrease to the lowest of the sequence. Concentrations decline, but total concentrations also decline. Relative frequencies and concentrations of Pteridium aquilinum decline. No significant changes occur in the other most abundant types of this group. Pollen types including species that are weeds of cereal cultivation have declined and include only Galium type and Sinapis type. 
'Cultivars' are abundant. Relative frequencies and concentrations increase through the zone due to an increase in Platanus orientalis. All cereal types have declined with the exception of Zea mays, which occurs continuously at similar abundance to that in the preceding zone. Platanus orientalis occurs continuously at increased levels of abundance. Castanea sativa occurs continuously at low abundance and Juglans regia occurs sporadically at low abundance.

Relative frequencies of 'coniferous and deciduous canopy trees' increase to the highest of the sequence, due to a large increase in Pinus (Diploxylon) and small increases in Quercus frainetto type and Abies. This is the first zone where 'coniferous and deciduous canopy trees' are the dominant habitat group. Concentration values of these pollen types remain about the same although total concentration declines. Relative frequencies of Quercus trojana type are unchanged and concentrations decline slightly.

Alpine shrubs and herbs are extremely poorly represented. 'Woodland understorey trees and shrubs' are poorly represented. Juniperus declines to relative frequencies slightly more and concentrations less than those of the surface sample. 'Riverine trees' increase due to an increase in Salix triandra, which is part of the current riverine flood plain vegetation of Grevena. It occurs at much lower abundance than that of the surface sample. 'Evergreen woodland trees' and 'plants of open dry rocky habitats' are poorly represented; Quercus coccifera type occurs sporadically.

The sum of terrestrial trees and shrubs increases to $58.4-69.4 \%$ of total terrestrial pollen and spores, which is the maximum of the sequence. The low ratio of terrestrial trees and shrubs to terrestrial herbs, high palynological richness values (40-45 expected types), and high relative frequencies and concentrations of 'herbs of open ground' are indicative of an open canopy.

Extra-local and regional lowland vegetation continues to be highly disturbed open canopy oak woodland with deciduous oaks dominant and semi-evergreen oaks playing a minor role. An increase in canopy cover is suggested by a decline in 'herbs of open ground', an increase in the abundance of deciduous oaks, and a decline in the ratio of terrestrial trees and shrubs to terrestrial herbs. A decrease in Pteridium aquilinum and Juniperus may be due to more canopy cover. Cultivation of cereals has declined. An increase in Platanus orientalis and Salix triandra suggests an expansion of the riverine flood plain vegetation of the province. Salix may also be growing on the fen, as it does today. The distant Pinus (Diploxylon)/Abies woodland, probably mostly on the Pindos Mountains, expands.

Zone $\mathrm{K}-3,57.5-32.5 \mathrm{~cm}, \mathrm{c}$. 1840-1880 AD 'Herbs of open ground' are dominant. Relative frequencies increase to high values. Concentrations increase, but total concentration increases also. Relative frequencies and concentrations of Pteridium aquilinum remain at low 
values. No significant changes occur in the other most abundant types of this group, with the exception of an increase in Dactylis type. Three types that occur include species that are weeds of cereal cultivation: Centaurea cyanus type, Galium type, and Sinapis type.

'Cultivars' are abundant, have increased, and almost reached the maxima of the sequence (zone K-9) due to increases in the types Avena and Secale cereale, which reach their maximum relative frequencies of the sequence. Zea mays occurs continuously. Hordeum type and Triticum type occur sporadically. Lathyrus sativus and Morus alba both occur in the basal spectrum only. Platanus orientalis occurs continuously but declines steadily. Castanea sativa also occurs continuously and Juglans regia occurs sporadically at about the same level of abundance as previously.

Relative frequencies of 'coniferous and deciduous canopy trees' decline to almost the minimum of the sequence due to a dramatic decline in Pinus (Diploxylon) and Abies, and a smaller decline in Quercus frainetto type. Quercus trojana type continues unchanged.

Alpine shrubs and herbs are extremely poorly represented. 'Woodland understorey trees and shrubs' are poorly represented; Juniperus increases slightly. 'Riverine trees' continue at the increased values of the preceding zone with Salix triandra the dominant type. 'Evergreen woodland trees' and 'plants of open dry rocky habitats' are poorly represented; Quercus coccifera type occurs continuously but at low values.

The sum of terrestrial trees and shrubs declines but fluctuates: $34.0-54.4 \%$ of total terrestrial pollen and spores. The very low ratio of terrestrial trees and shrubs to terrestrial herbs, high palynological richness values (40-45 expected types), and high relative frequencies and concentrations of 'herbs of open ground' are indicative of an open canopy.

Extra-local and regional lowland vegetation continues to be highly disturbed open canopy oak woodland with deciduous oaks dominant and semi-evergreen oaks playing a minor role. Juniperus probably forms a significant part of the understorey. A decrease in canopy cover is indicated by an increase in the habitat group of 'herbs of open ground', a decline in the abundance of deciduous oaks, and a decline in the ratio of terrestrial trees and shrubs to terrestrial herbs. An increase in Juniperus also supports a decrease in canopy cover. Cultivation of cereals increases greatly. The riverine flood plain vegetation may have declined slightly as indicated by a decline in Platanus orientalis although concentration values of the habitat group of 'riverine trees' are unchanged. The distant higher elevation Pinus (Diploxylon)/Abies woodland declines.

Zone $\mathrm{K}-2,32.5-17.5 \mathrm{~cm}, \mathrm{c.}$ 1880-1910 AD 'Herbs of open ground' are the second most abundant habitat group; relative frequencies and concentrations decrease. Pteridium aquilinum declines. No significant changes occur in the other most abundant types of this 
group. Four types that occur include species that are weeds of cereal cultivation: Centaurea cyanus type, Galium type, Nigella, and Sinapis type.

'Cultivars' are abundant, although they have declined. The decline is due to reductions in Avena and Platanus orientalis. Hordeum type occurs sporadically. Triticum type and Zea mays increase. Secale cereale still occurs continuously. Castanea sativa and Juglans regia occur sporadically.

Relative frequencies of 'coniferous and deciduous canopy trees' increase to almost the maximum of the sequence due to increases in Pinus (Diploxylon) and Abies. Concentrations also increase. The oaks remain unchanged.

Alpine shrubs and herbs are extremely poorly represented. 'Woodland understorey trees and shrubs' are poorly represented; Juniperus declines to relative frequencies similar to those of the surface sample, although concentrations are much lower (total concentrations are lower). 'Riverine trees' continue at the increased values of the preceding two zones, with Salix triandra the dominant type. 'Evergreen woodland trees' and 'plants of open dry rocky habitats' are poorly represented; Quercus coccifera type occurs continuously and increases slightly.

Although the sum of terrestrial trees and shrubs is very high for this sequence, 55.4$66.3 \%$ of total terrestrial pollen and spores, the ratio of terrestrial trees and shrubs to terrestrial herbs is low. This low ratio, high palynological richness values (40-50 expected numbers), and high relative frequencies and concentrations of the habitat group 'herbs of open ground' are indicative of an open canopy.

Extra-local and regional lowland vegetation surrounding the wetland continues to be highly disturbed open canopy oak woodland with deciduous oaks dominant and semievergreen oaks playing a minor role. The understorey comprises mostly herb-rich steppe. Extra-local canopy cover probably remains about the same, or increases as suggested by a decrease in the 'herbs of open ground' and a decrease in Juniperus. Cultivation of cereals may have declined but the cereal types of Avena and Hordeum type have become less important while those of Triticum type and Zea mays increase in importance. Secale cereale is still important. The riverine flood plain vegetation continues unchanged. The distant higher elevation Pinus (Diploxylon)/Abies woodland increases.

Zone $K-1,17.5-0.0 \mathrm{~cm}$, c. 1910-1989 AD 'Herbs of open ground' are co-dominant with 'coniferous and deciduous canopy trees'. Relative frequencies and concentrations of 'herbs of open ground' increase. Pteridium aquilinum continues at the same low abundance. No significant changes occur in the other most abundant types of this group, with the exception of a sharp increase in Cirsium type. Four types that occur include species that are weeds of cereal cultivation: Centaurea cyanus type, Galium type, Nigella, and Sinapis type. 
'Cultivars' are abundant, although they have declined again in relative frequency and concentration, with the exception of an increase in concentration of the surface sample due to an increase in total concentration. The decline is due to a reduction of all the cereal types. Avena almost disappears, while Hordeum type and Zea mays occur sporadically. Secale cereale and Triticum type occur continuously. Elaeagnus angustifolia and Vicia sativa type both occur in one spectrum only. Castanea sativa and Juglans regia occur sporadically. Platanus orientalis occurs continuously and increases slightly.

Relative frequencies of 'coniferous and deciduous canopy trees' decline due to a decline in Quercus frainetto type. The relative frequencies of Pinus (Diploxylon) fluctuate but are about the same as in the preceding zone. Those of Abies continue at similar values. Quercus trojana type continues at similar values also. Concentrations of all these pollen types continue at similar values as those in the preceding zone and then increase following the curve of the total concentration curve.

Alpine shrubs and herbs are extremely poorly represented. 'Woodland understorey trees and shrubs' are poorly represented; Juniperus continues at the low relative frequencies and concentrations of the preceding zone (it increases sharply in concentration in the surface sample but this is due to total concentration increasing greatly). The low abundance of Juniperus in this and the preceding zone may be related to increased burning of extra-local vegetation and to a closing canopy - dead remains (which may persist for many decades) observed in oak woods in the lowlands (Chapter 3) are evidence that the woods were once more open. In some parts of the lowland oak woods it was noted that Juniper was absent. This is probably due to the burning of steppe understorey or the leaf-litter of the woods. Juniper is much more sensitive to fire that oaks. Oaks resprout after burning while Juniper dies. 'Riverine trees' increase dramatically due to an increase in Salix triandra. 'Evergreen woodland trees' and 'plants of open dry rocky habitats' are poorly represented; Quercus coccifera type declines.

The sum of terrestrial trees and shrubs increases from the comparatively low value of $39.2 \%$ in the basal spectrum to comparatively high values from $57.0-63.0 \%$ of total terrestrial pollen and spores. The low ratio of terrestrial trees and shrubs to terrestrial herbs, high palynological richness values (40-50 expected types), and high relative frequencies and concentrations of 'herbs of open ground' are indicative of an open canopy.

Extra-local and regional lowland vegetation continues to be highly disturbed open canopy oak woodland. Deciduous and semi-evergreen oaks are almost equally abundant. The change in the proportions of these oaks may be due to more coppicing of the trees represented by Quercus frainetto type. The understorey is herb-rich steppe. Extra-local canopy cover probably declines, as indicated by the increased abundance of 'herbs of open ground', and a 
decline in the deciduous oaks. Cultivation of cereals may have declined further. The dramatic increase in Salix triandra may be due to it growing on the fen: today willows grow on the fen and along the channel on the southern boundary of the fen. The montane Pinus (Diploxylon)/Abies woodland remains unchanged.

\subsection{Charcoal analysis and fire history}

\subsubsection{Microscopic charcoal analysis}

Results of the microscopic charcoal particle analyses are presented as relative frequencies in Figure 6.14. The local zonation scheme of the terrestrial pollen and spores is used for convenience of correlation. The charcoal record shows high total charcoal relative frequencies in all zones with the exception of zone $\mathrm{K}-12$ where relative frequencies are very low. Extremely high total charcoal relative frequencies occur in zones $\mathrm{K}-14, \mathrm{~K}-13$, and $\mathrm{K}-11$. Most residues retained by the $260 \mu \mathrm{m}$ mesh sieve during sample preparation included charcoal particles except between $310-265,240-160$, and 105-55 cm.

In zones K-14 and K-13 charcoal particles of all size classes is very abundant in every spectrum. Peaks of total charcoal abundance which may reflect periods of more intense/frequent burning occur at $380,355-350$, and $330 \mathrm{~cm}$ and are reflected in the curves of all size classes except the uppermost peak in the largest size class. The largest size class displays a peak at $315 \mathrm{~cm}$ which is reflected in the $200-400 \mu \mathrm{m}^{2}$ size class. In comparison with other zones in the sequence, these two zones record the highest relative frequencies in the three smallest size classes (particles less than $100 \mu \mathrm{m}^{2}$ ) while those of the middle size range (particles $\left.100-400 \mu \mathrm{m}^{2}\right)$ are very abundant and the largest size class $\left(>400 \mu \mathrm{m}^{2}\right)$, although abundant, is not as abundant as in some higher zones (especially the two uppermost zones).

In zone K-12 total charcoal particle abundance is extremely low and all size class frequencies are extremely low; no charcoal was recorded in the $>400 \mu \mathrm{m}^{2}$ size class at $305 \mathrm{~cm}$.

Total charcoal particle abundance increases again in zone K-11 and in general records frequencies higher than in other zones with the exception of $\mathrm{K}-14$ and $\mathrm{K}-13$. Three peaks occur in the total charcoal frequencies. The highest peak, which is exceeded only by the three highest peaks of the two basal zones, occurs at $270 \mathrm{~cm}$. The two lower peaks occur at 260 and $250 \mathrm{~cm}$. A general decline from $270 \mathrm{~cm}$ displayed in the total charcoal curve is reflected in the size classes of 100-200 $\mu \mathrm{m}^{2}$ and smaller. The two largest size classes do not decline.

In zones $\mathrm{K}-10$ to $\mathrm{K}-8$ total charcoal particle abundance declines to about the average frequency for the remainder of the sequence. These three zones maintain a constant level of abundance of total particle frequency with the exception of a small peak at $170 \mathrm{~cm}$, which is reflected in the smallest size classes (up to $200 \mu \mathrm{m}^{2}$ ). The smaller size classes are still 


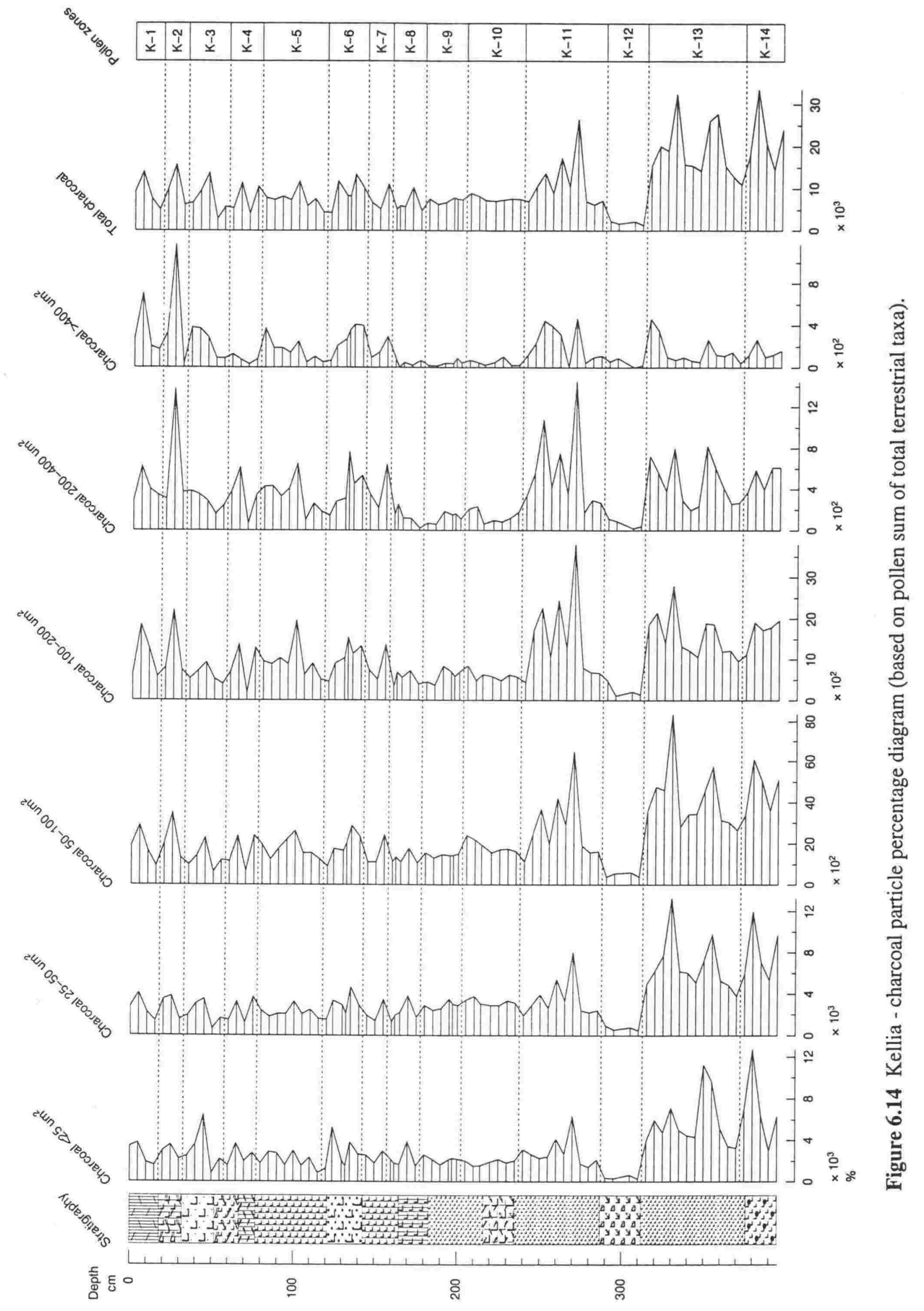


relatively abundant while the largest size class $\left(>400 \mu \mathrm{m}^{2}\right)$ has declined to very low frequencies.

In zone K-7 charcoal particle abundance increases in the two largest size classes while in the smaller size classes it remains unchanged. A peak at $155 \mathrm{~cm}$ is seen in all size classes. In zone K-6 total charcoal abundance increases and displays two peaks at 135 and $125 \mathrm{~cm}$. The lower peak is due to a peak recorded in the three smaller size classes and the largest size class while the second is due to a peak in the two smallest size classes. Particle frequency is very high in the four largest size classes in the lower part of the zone then declines, while the two smallest size classes are high throughout the zone.

In zone K-5 total charcoal particle abundance declines slightly, but displays a small peak at $100 \mathrm{~cm}$. This peak is reflected in all size classes. The largest sized particles $\left(>400 \mu \mathrm{m}^{2}\right)$ have low abundance at the base of the zone, but increase to relatively high frequencies in the uppermost spectrum. Charcoal particles of the middle size classes show the same general increasing trend as that of the largest size class but display a more pronounced peak at $100 \mathrm{~cm}$. The two smallest size classes have declined and maintain relatively constant frequencies.

In zone K-4 total charcoal particle abundance declines slightly again, but fluctuates in concordance with the frequencies of all the size classes except the largest and smallest. The largest size class is much diminished, while other size classes remain at about the same abundance. In zone K-3 total charcoal particle abundance increases from the centre of the zone. This increase is reflected in the two largest size classes. A peak at $45 \mathrm{~cm}$ is reflected in the smaller size classes. In zone K-2 total charcoal particle abundance peaks in the centre of the zone. This peak is reflected in all size classes; a very prominent peak occurs in the larger size classes, but only a small peak occurs in the smaller size classes. Similar curves are repeated in zone $\mathrm{K}-1$, but the peak of the larger size classes in the centre of the zone is not so prominent.

\subsubsection{Fire history}

There is continuous accumulation of microscopic charcoal particles throughout the sequence. The non-uniform sedimentation of this sequence may influence charcoal particle abundance but a general history of the frequency and intensity of fires on an extra-local and regional basis is possible based on microscopic charcoal particle frequencies. There are two phases when more intense/frequent fires occurred in the Kellia region and were of major ecological significance. These are between the base of the sequence, radiocarbon dated at c. $830 \mathrm{BC}$, and $315 \mathrm{~cm}$ (radiocarbon dated at c. $2000 \mathrm{BC}$, but probably younger c. $630 \mathrm{BC}$ ) and between $c .1340-1350 \mathrm{AD}$. 
In the former phase the charcoal record shows continuously high values of charcoal particles in all size classes and indicates widespread extra-local and regional burning. In the latter phase total charcoal is slightly lower than that of the lower phase but the smaller size classes are less abundant while the larger size classes are more abundant. Greater abundance of the largest size class suggests more intense/frequent extra-local burning than in the basal phase. Optically visible charcoal particles in the sediments between c. 1340-1370 AD (264$235 \mathrm{~cm}$ ) provide supporting evidence for the importance of fires during the latter phase.

Between these two phases when fires were of major ecological significance is a period between c. 1230-1340 AD (312-270 cm) when fires were more distant and/or sporadic and were of little ecological significance in the vicinity of Kellia. Between c. 1370-1650 AD (240-160 cm; zones K-10 to K-8) extra-local burning declines and is of little significance, while regional burning also declines it is probably still important ecologically. At c. 1650-1690 AD (155-125 cm; zones K-7 and most of K-6) extra-local burning increases and regional burning increases slightly from c. $1680 \mathrm{AD}(140 \mathrm{~cm})$. At c. 1710-1740 AD (120$105 \mathrm{~cm}$; top of zone K-6 and bottom of zone K-5) extra-local and regional burning decline. At c. 1750-1790 AD (100-80 cm; upper part of zone K-5) both extra-local and regional burning increase again. At c. $1800 \mathrm{AD}(75 \mathrm{~cm}$; base of zone $\mathrm{K}-4)$ extra-local burning is greatly reduced, while regional burning continues. Extra-local and regional burning increase at c. $1860 \mathrm{AD}(45 \mathrm{~cm})$. Fluctuating abundances in the largest and middle size classes above this depth suggest two periods of intense/frequent fires extra-locally and more distant, at c. $1890 \mathrm{AD}(25 \mathrm{~cm})$ and c. $1940 \mathrm{AD}(5 \mathrm{~cm})$. The former period has an assigned date that is close to the $1908 \mathrm{AD}$ date of known land clearance in the Lipsokouki Valley (Chapter 1). The most recent increase in microscopic charcoal particle abundance could be related to the return of Greek refugees in 1924 and the subsequent land clearance, and the introduction of stubble burning with the mechanization of agriculture (Chapter 1).

Pollen evidence also suggests high frequency/intensity of fires both extra-locally and regionally during two phases in the lower part of the sequence; the pyrophytes Pteridium aquilinum and Quercus coccifera type are more abundant below $315 \mathrm{~cm}$ and concentration values (unfortunately no influx data are available) of Pinus (Diploxylon) are lower below $315 \mathrm{~cm}$ and between $c$. 1340-1350 AD (275-245 cm). Pteridium aquilinum increases between c. $1350-1360 \mathrm{AD}(255-240 \mathrm{~cm})$ and the fire-sensitive Juniperus is less abundant in the lower part of the sequence before c. 1370 AD (below $235 \mathrm{~cm}$ ). After c. 1370 AD Juniperus increases. Pteridium aquilinum becomes abundant from c. 1640-1790 AD (160-80 cm). Quercus coccifera type is more abundant before c. $1680 \mathrm{AD}$ (above $140 \mathrm{~cm}$ ), but fluctuations do not correspond exactly to those of the charcoal curve; other factors such as climatic conditions and human disturbance of the vegetation such as grazing, cultivation, lumbering 
or various woodland practices may, particularly in this situation, also affect the abundance of this pyrophyte. Low concentration values of Pinus (Diploxylon), although subject to fluctuations in sedimentation rates, appear to correspond to peaks in the small size classes of microscopic charcoal particles at c. $1600 \mathrm{AD}, 1650 \mathrm{AD}, 1680 \mathrm{AD}, 1690 \mathrm{AD}, 1750 \mathrm{AD}$, and $1860 \mathrm{AD}(170,155,135,125,100$, and $45 \mathrm{~cm})$.

\subsection{Synthesis of environmental changes}

A summary of the major environmental changes in the Kellia wetland and its surrounding area between perhaps $c .830-630 \mathrm{BC}$ and from $c .1230-1989 \mathrm{AD}$ is given in Table 6.2 .

The sedimentary history of the fen is intimately linked to the local wetland vegetation history and there is close correspondence between the composition of deposits and changes in the pollen profiles of the wetland but there is less correspondence between sediment composition and terrestrial pollen and spore profiles. Absence of total synchrony of these three factors is due to a significant contribution of pollen and spores derived from outside the catchment.

Stratigraphic data indicate that Kellia fen probably formed at the side of a stream course that ran from west to east on the southern side of the fen. Radiocarbon dating of the pollenanalysed core suggests that two periods of alluviation are recorded in the fen sediments, an older period below $312 \mathrm{~cm}$ (beginning perhaps c. $830 \mathrm{BC}$ and spanning about 200 years, see section 6.4.2) and a younger one above, which may span about the last 760 years beginning c. $1230 \mathrm{AD}$, although pollen evidence (presence of Secale cereale type pollen) suggests a more recent age, of perhaps after $146 \mathrm{BC}$, for the lower older period. A hiatus occurs in the sequence between these two periods of alluviation, perhaps caused by erosion or sediment bypassing due to a deeper stream channel. The fen sediments comprise greyish olive to olive black clay with variable proportions of calcareous grit, peat, and organic detritus. Thin $(5-15 \mathrm{~cm})$ noncalcareous sand horizons are rare. Persistent organic-rich horizons occur at about 100 and $230 \mathrm{~cm}$. Below about $300 \mathrm{~cm}$, there is little organic matter in the stiff to soft greyish olive clay comprising the older period of alluviation. The uppermost c. $20 \mathrm{~cm}$ (c. 1900-1989 AD) of the fen consists of silty peat on the western and southern part of the fen, close to the spring. Towards the northern edge and the eastern (downstream) end of the fen, the unit becomes less peaty and is replaced by brown clay with a sandy layer at the base. This unit may be related to the establishment of the modern sealed road and its causeway, which may have reduced the transport of sediment into the fen from the catchment upstream. Occasional small mollusc shells and fruits or corms were noted in some sediment exploratory 


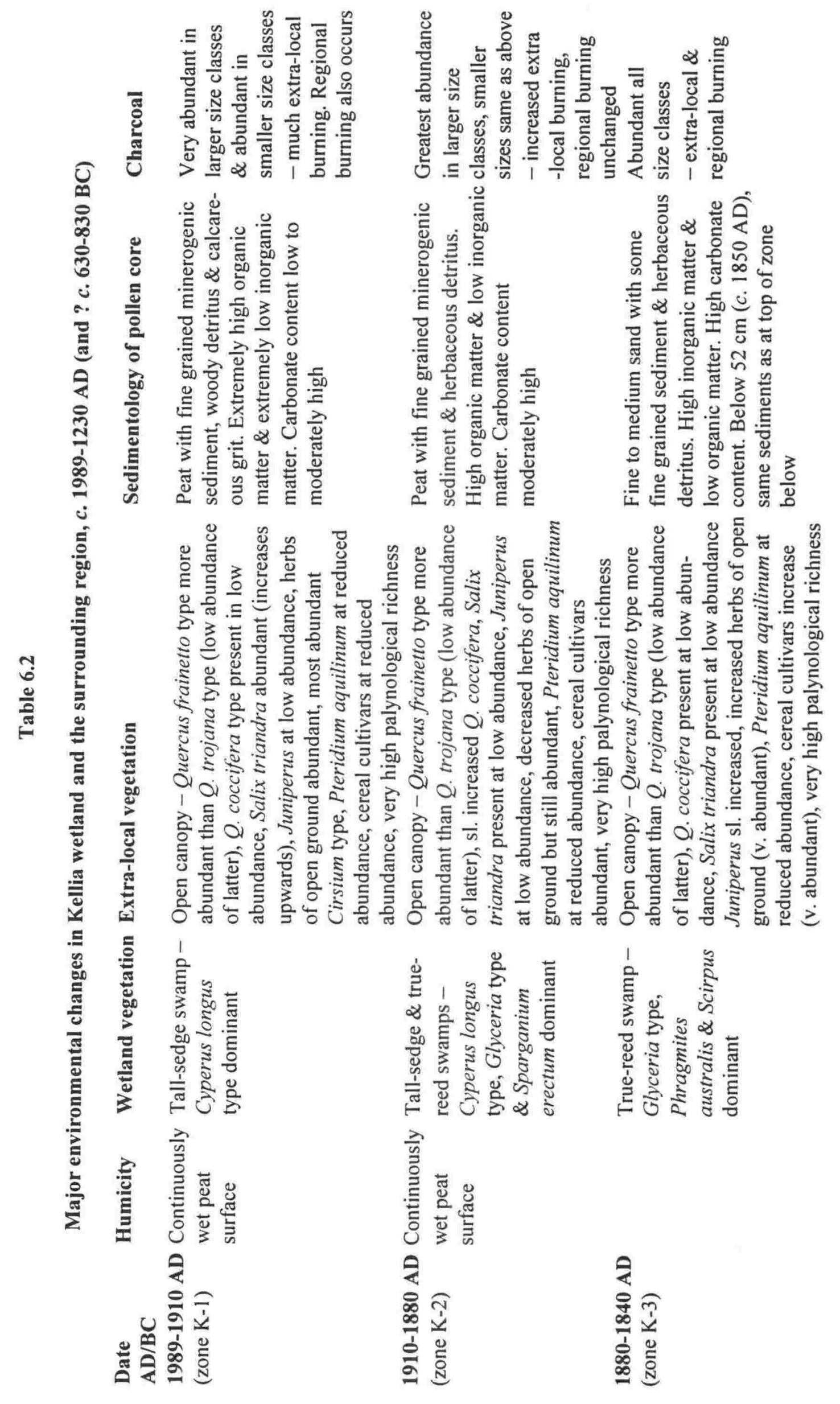




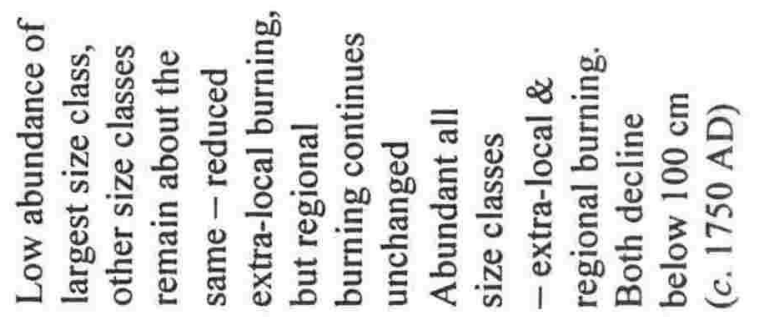

言

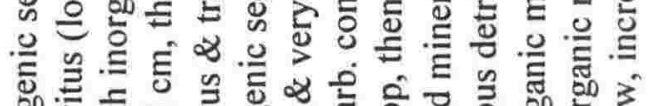

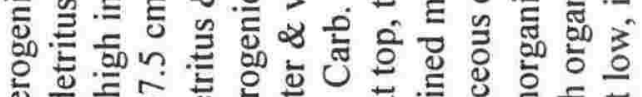

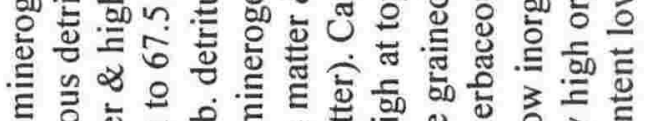

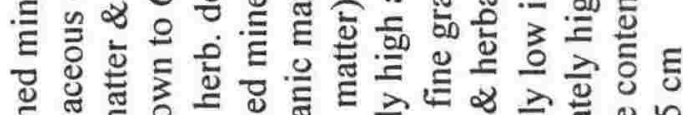

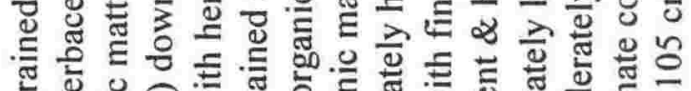

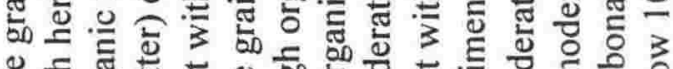

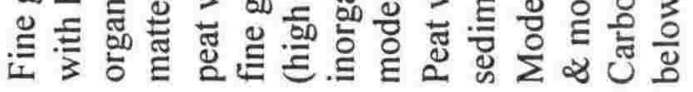

总善

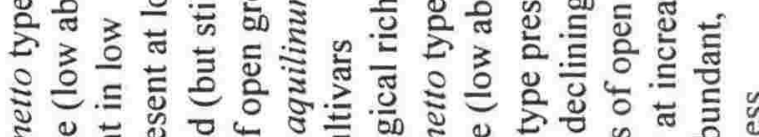

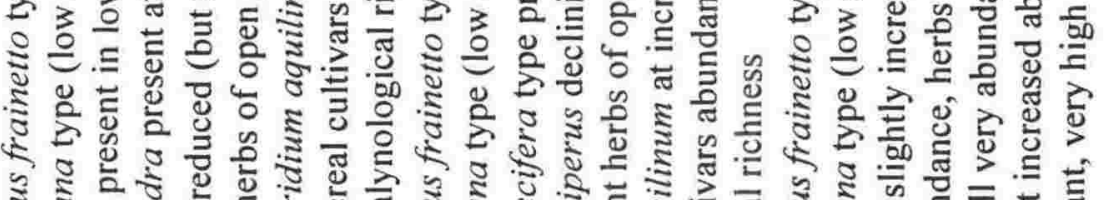

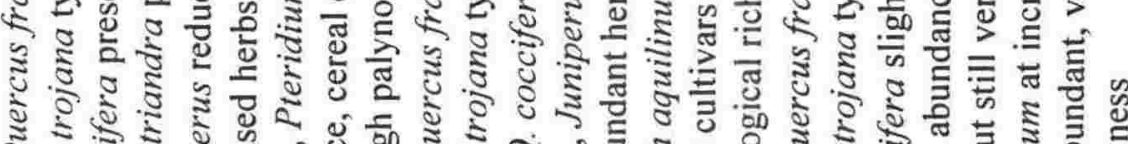

O10

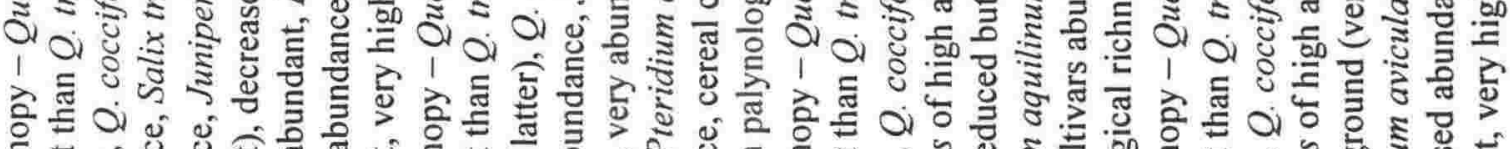

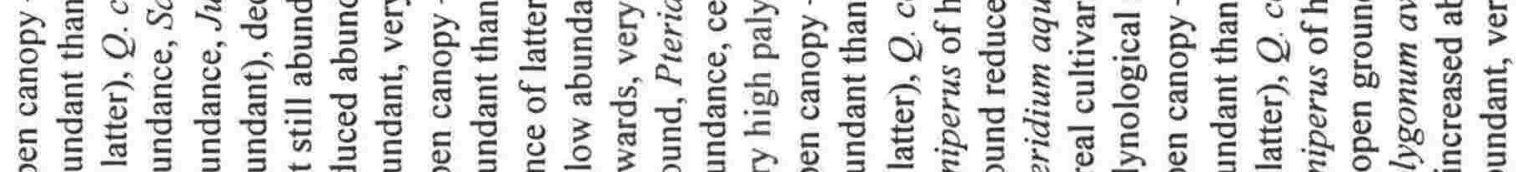

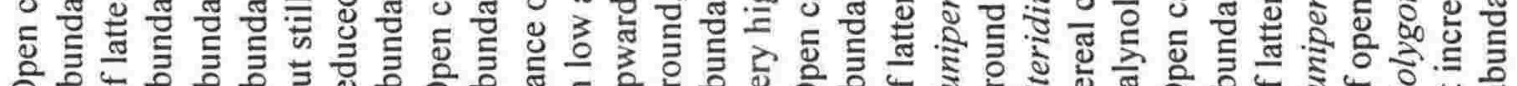

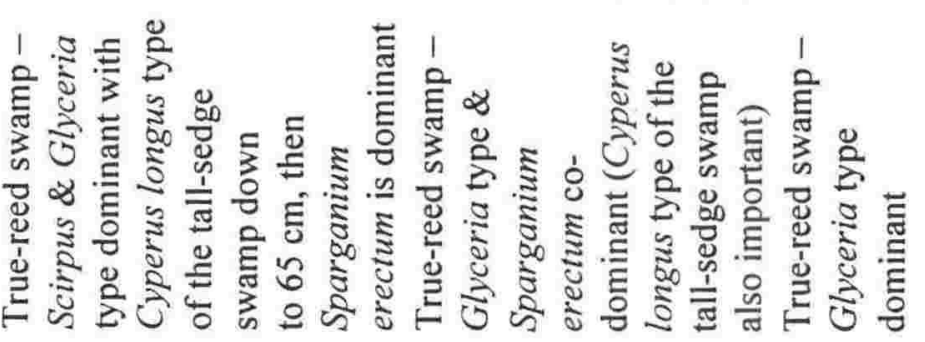
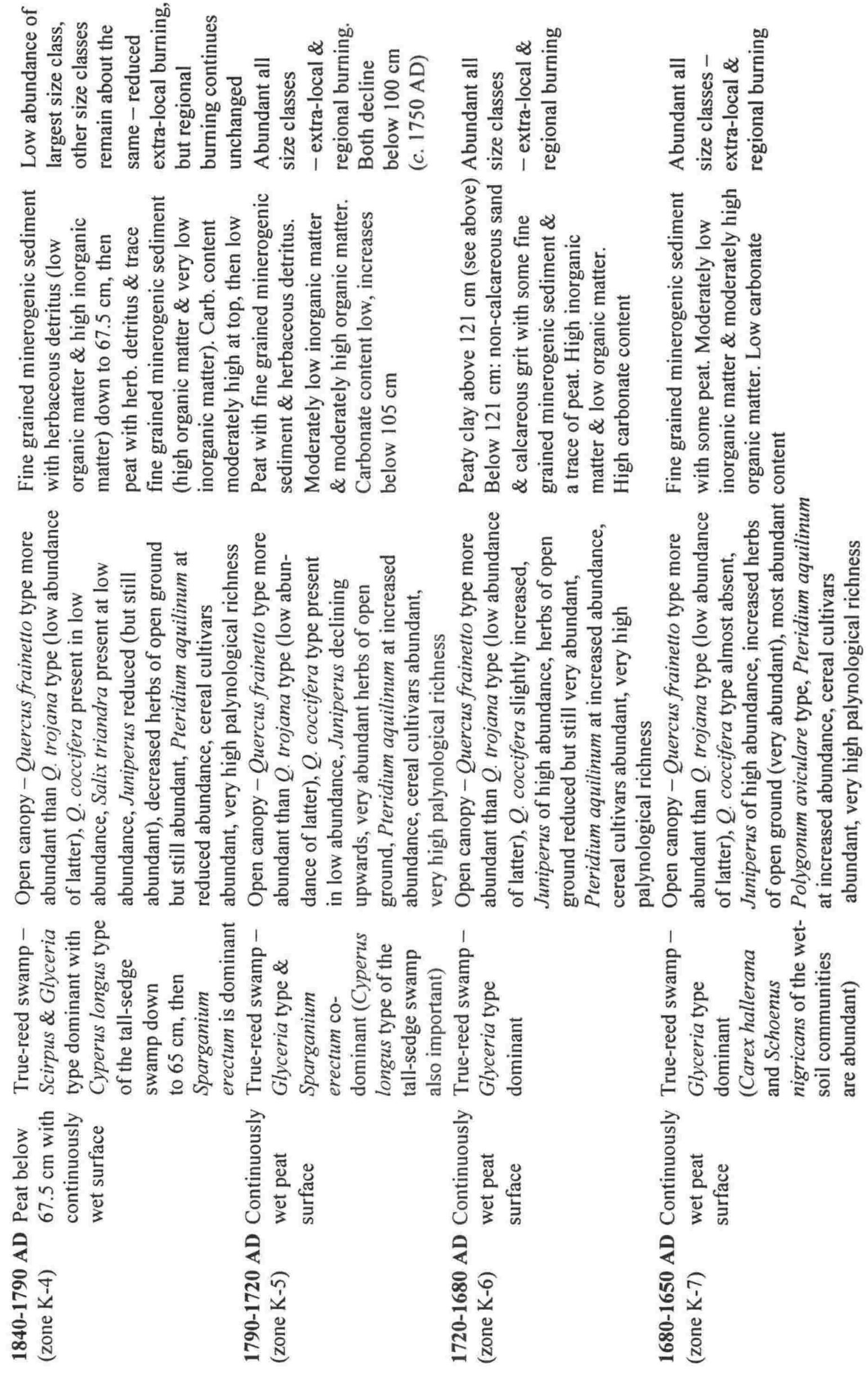

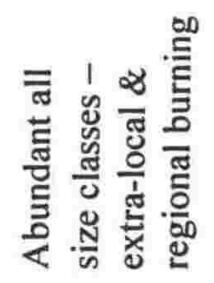

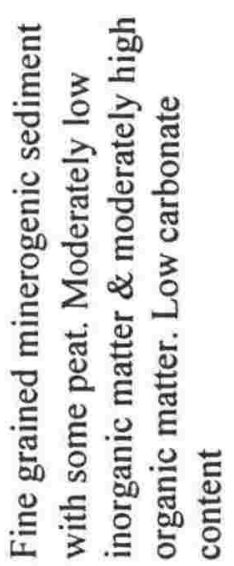

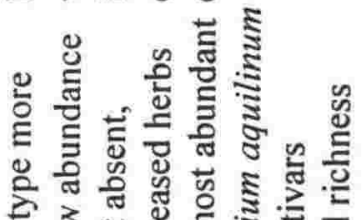

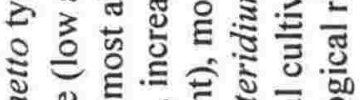

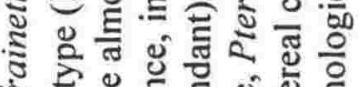

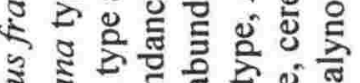
ปิ (0) 


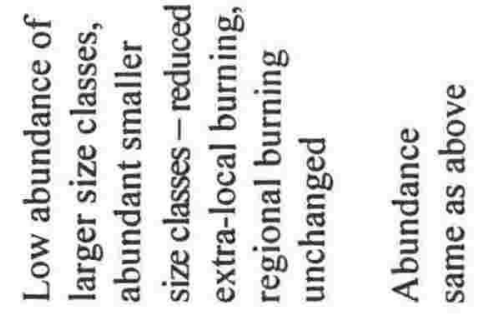

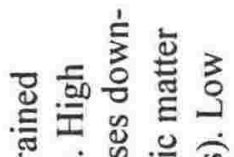

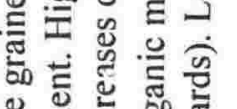

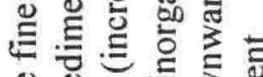

确谤言言言

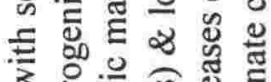

等

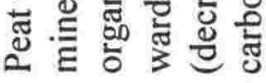

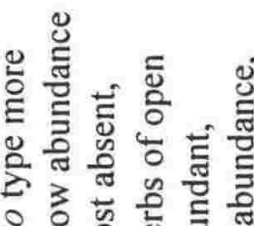

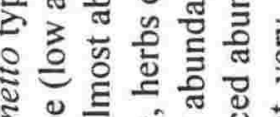

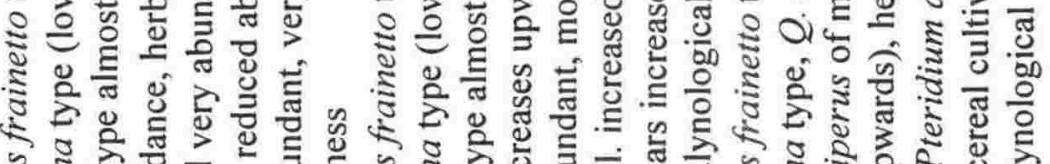

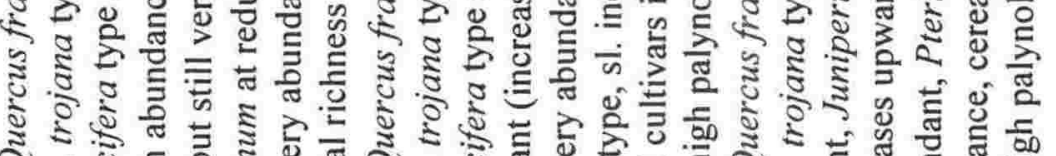

\%)

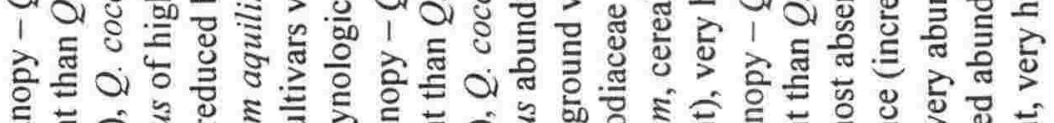

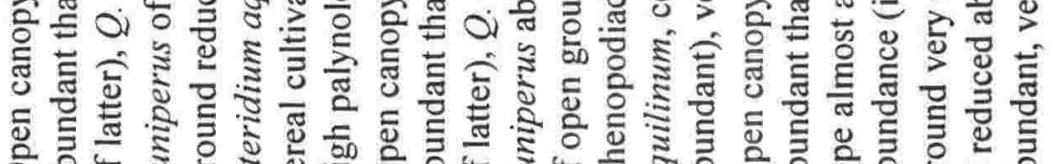

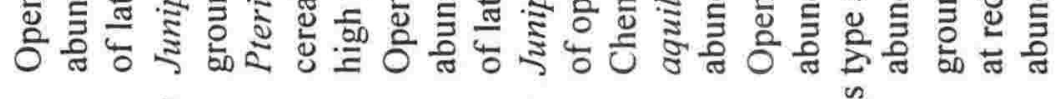
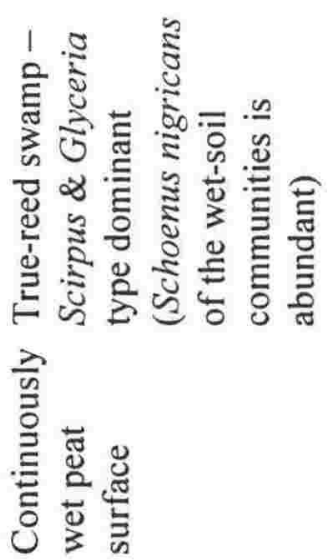

离到

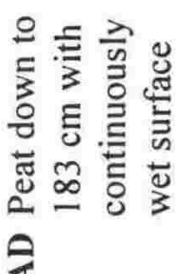

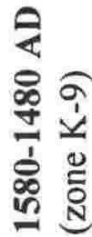

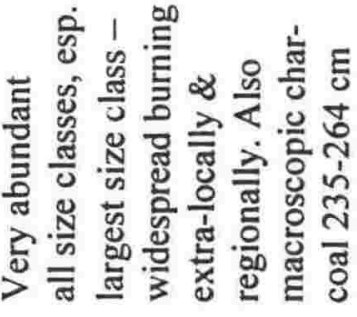
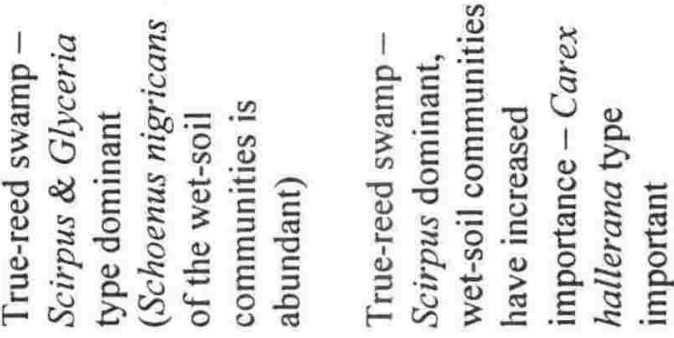

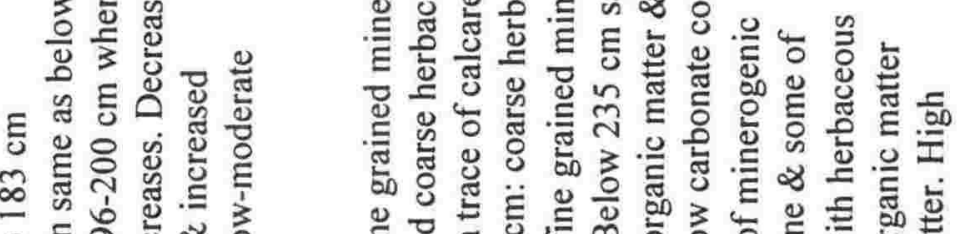

๘

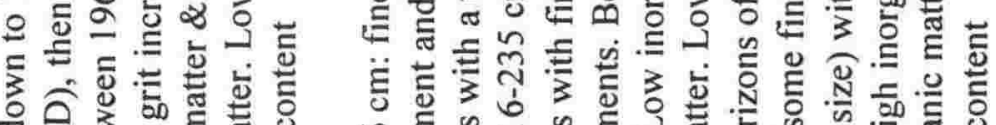

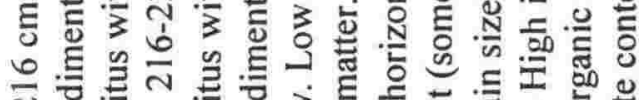

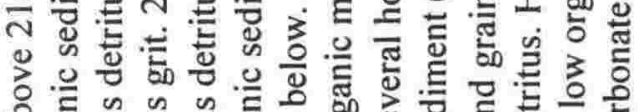

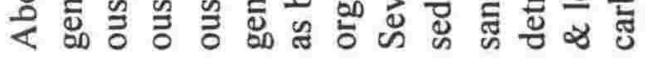

(2)

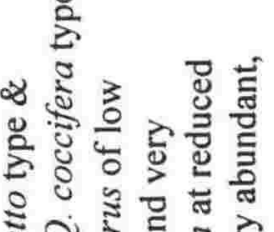

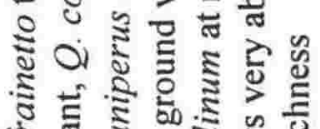

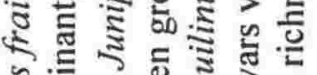

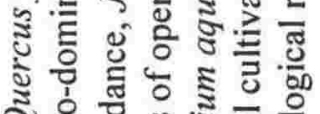

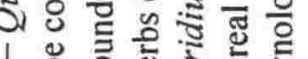
।

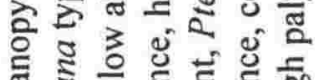

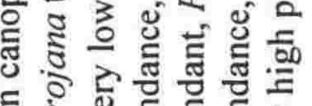

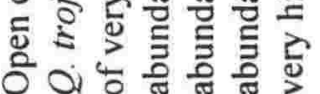
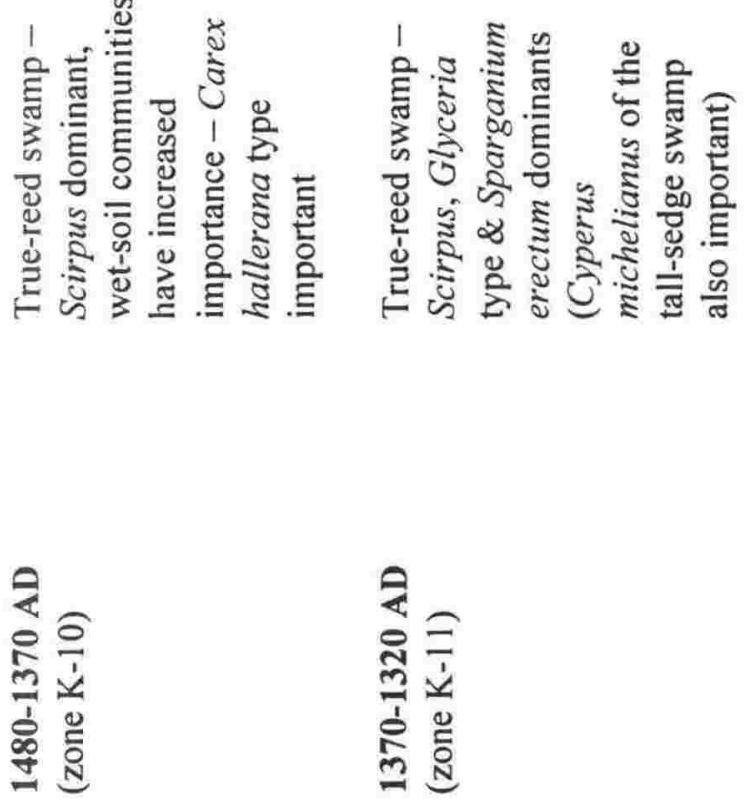


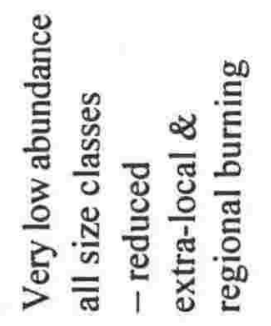

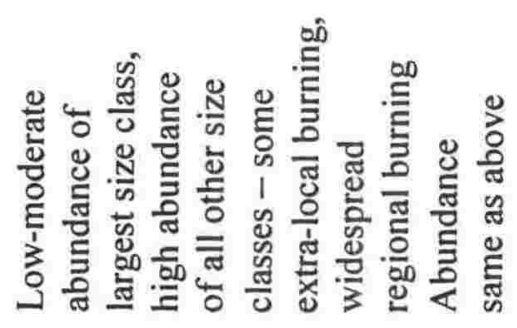

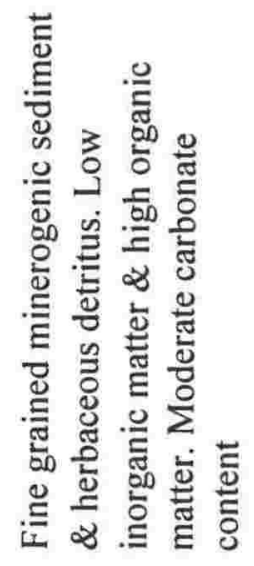
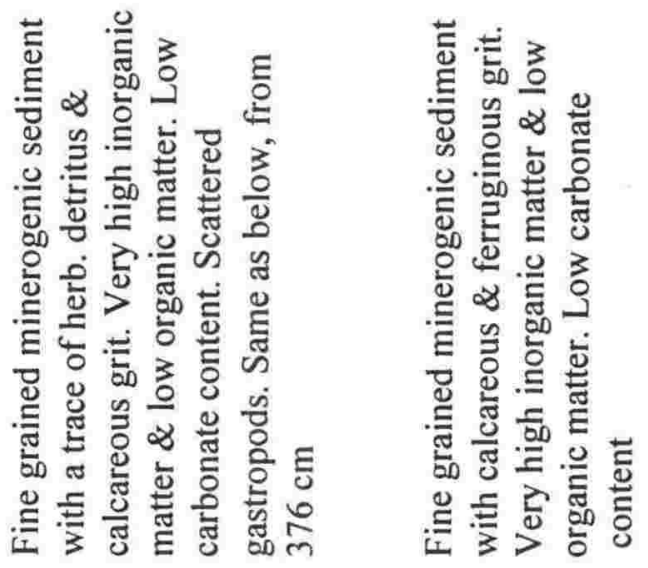

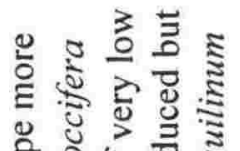

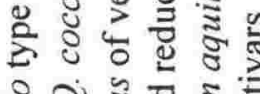

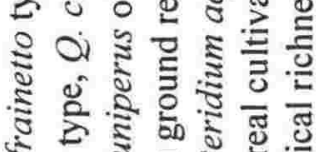

5.

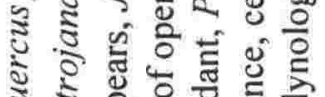

今。

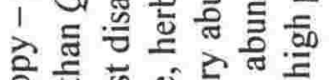

势

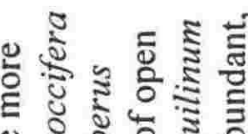

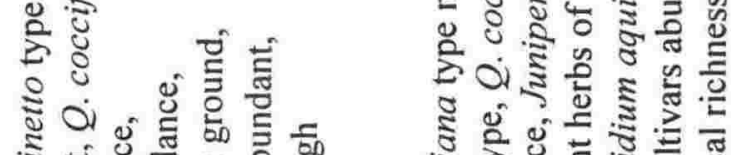

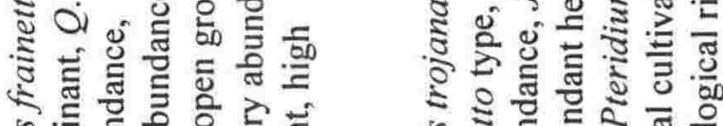

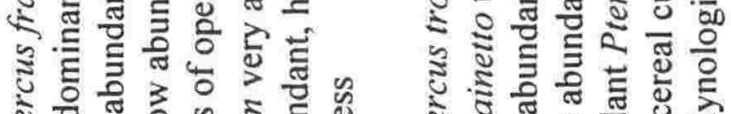

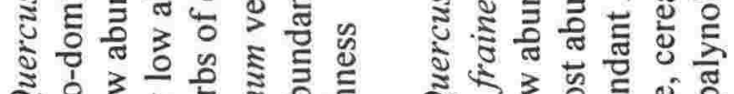

すั่

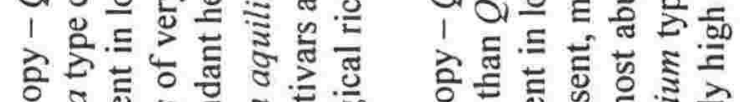

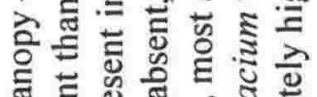

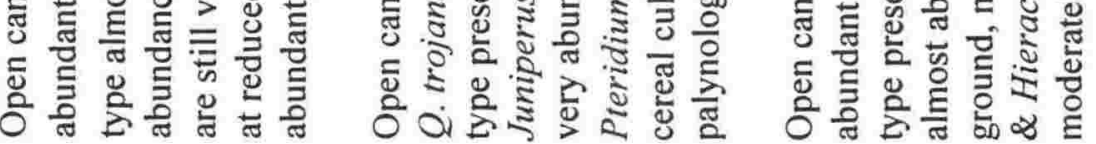

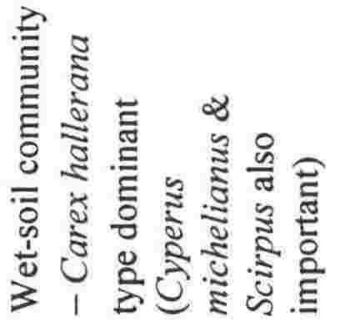

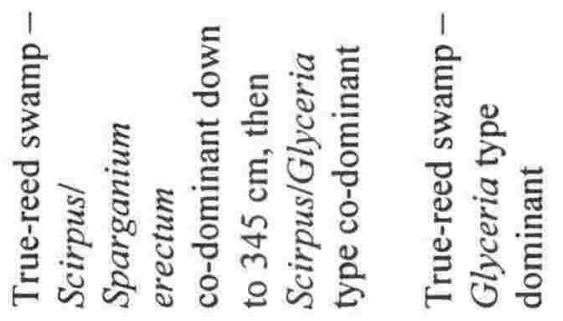

ن̈

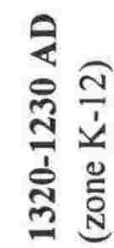

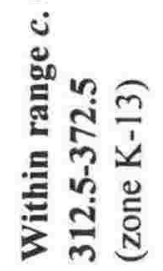

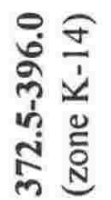


cores, but no pattern to their occurrence was determined although in the pollen analysed core they appeared to be more abundant between $376-312 \mathrm{~cm}$. No ostracods were found in the pollen analysed core. Where peaty horizons occur in the pollen analysed core at 183.0-67.5 and $32.5-0 \mathrm{~cm}$, humicity of peat indicates that surface peat was continuously wet.

The local wetland vegetation is dominated by pollen and spore types that derive from emergent plant communities of wet-soil, tall-sedge-swamp and true-reed-swamp communities. Pollen and spore types of wet-soil plant communities are most numerous, although total relative frequencies of the true-reed-swamp communities are highest throughout the sequence except at 305-280 cm, where frequencies of types of wet-soil communities are higher, and in several spectra above $35 \mathrm{~cm}(35,30,20$, and $0 \mathrm{~cm})$, where frequencies of pollen types of the tall-sedge-swamp communities are higher. The true-reed-swamp communities are dominated by reeds of the Glyceria type and Scirpus mostly, and to a lesser extent by Phragmites australis and Sparganium erectum. The wet-soil communities are dominated by sedges of the Carex hallerana type and Schoenus nigricans, and Equisetum occurs sporadically throughout the sequence and displays two peaks, at 265 and $205 \mathrm{~cm}$. Pollen types that dominate the tallsedge-swamp communities are Carex flacca type, Cyperus longus type, and Cyperus michelianus. Communities of floating-leaved and submerged macrophytes are poorly represented, the former being better represented than the latter and are more abundant above $185 \mathrm{~cm}$. They probably represent a few plants growing in small pools on the surface of peat.

The available nutrient level is high throughout the sequence as evidenced by the abundance of several pollen types representing emergent plants, which are indicative of high trophic status, such as Carex flacca type, Cyperus longus type, Glyceria type, Scirpus, Sparganium erectum, Typha angustifolia type, and Typha domingensis type; and the occurrence of several pollen and spore types of aquatic macrophytes indicative of high trophic status such as Callitriche stagnalis type, Hydrocharis morsus-ranae, Nuphar lutea, Nymphaea alba, Ranunculus aquatilis group, Salvinia natans, Stratiotes aloides, Vallisneria spiralis, Myriophyllum spicatum, Myriophyllum verticillatum, Potamogeton crispus type, and Potamogeton pectinatus. Nutrients may be contributed to the wetland via washed in sediments, spring water or from direct or indirect human activities, e.g. land clearance, cultivation or grazing.

Terrestrial pollen evidence suggests that pine woods grew at higher elevations on the Pindos Mountains for the duration of the sequence, perhaps mixed with Abies at intermediate elevations. The extra-local vegetation is open canopy deciduous/semi-evergreen oak woods 
dominated by the canopy trees of Quercus frainetto type (deciduous oaks) and $Q$. trojana type (deciduous/semi-evergreen oaks). Ostrya type occurs at low abundance almost continuously. Quercus coccifera type (evergreen oaks) occurs sporadically; greatest abundance is from 396$315 \mathrm{~cm}$ (perhaps c. 830-630 BC) and from 140-0 cm (c. 1680-1989 AD). The shrub Juniperus is more abundant above $235 \mathrm{~cm}$ (c. $1370 \mathrm{AD}$ ), while Pteridium aquilinum is most abundant below $315 \mathrm{~cm}$ (perhaps before c. $630 \mathrm{BC}$ ) and from 160-80 cm (c. 1650-1790 AD). Several herbs of open ground occur almost continuously throughout the sequence at significant abundance, the most abundant being: Centaurea solstitialis type, Chenopodiaceae type, Cichorium type, Cirsium type, Dactylis type, Festuca type, Galium type, Hieracium type, Lactuca type, Plantago lanceolata type, Polygonum aviculare type, Solidago type, Sonchus type, and Taraxacum type. A variety of cereals were cultivated extra-locally for the duration of the sequence and the nut trees Castanea sativa and Juglans regia, which occur sporadically from the base of the sequence, may have been planted, or growing wild, either sparsely in the vicinity of the site or more abundantly at a greater distance. The shade tree Platanus orientalis, which also occurs sporadically from the base of the sequence, may also have been planted or growing wild either sparsely extra-locally or more abundantly at a greater distance from the site. These three trees may have profited from changes in habitats caused by humans.

There is continuous accumulation of microscopic charcoal particles throughout the sequence. Two phases when more intense/frequent fires are indicated in the Kellia region are between the base of the sequence $(396 \mathrm{~cm}$ ) and $315 \mathrm{~cm}$ (perhaps c. 830-630 BC) and from 270-250 cm (c. 1340-1350 AD). In the former phase the charcoal record shows continuously high values of charcoal particles in all size classes and indicates some extra-local and widespread regional burning. In the latter phase total charcoal abundance is slightly lower but greater abundance of the largest size class indicates more intense/frequent extra-local burning than in the basal phase. Optically visible charcoal particles in the sediments at $264-235 \mathrm{~cm}$ provide supporting evidence for the importance of fires during the latter phase.

A synthesis of environmental changes as indicated by the pollen analysed core follows. The age of sediments and thus the period of vegetation history represented below $312.0 \mathrm{~cm}$ is uncertain but the basal deposits of the pollen analysed core have been radiocarbon dated at c. $830 \mathrm{Cal} \mathrm{BC}$ at $394-395 \mathrm{~cm}$. Between $396-376 \mathrm{~cm}$ deposits comprise very stiff mottled grey and brown clay with calcareous and ferruginous grit. Calcareous grit may be allochthonous (derived from calcic soils of the catchment) or autochthonous (precipitated from the spring water), or both. Sediments contain little organic matter and low carbonate content. They are 
small grain sized (clay) representing low energy transported sediments and are indicative of relative landscape stability in the catchment. They are distinct because of their ferruginous mottling and inclusion of ferruginous grit, which may be due to weathering or subsurface water circulation. Above $376 \mathrm{~cm}$ sediments are again fine grained and represent low energy transport comprising sticky soft grey clay with scattered gastropods and a trace of calcareous grit; carbonate and organic content are unchanged from the horizon below, and there is little visible plant material.

At this time wetland palynomorph concentration and palynological richness are very low, and the dominant local plant community of the true-reed swamp is represented almost entirely by Glyceria type, which is able to tolerate 'silting'. A small wet-soil community also exists with Carex hallerana type and Mentha type being most abundant. At the base of the sequence a tall-sedge-swamp community, dominated by Carex flacca type also occurs, but soon declines. Both floating-leaved and submerged macrophyte communities are represented by a few types, especially at the top of the zone. Of the two submerged aquatic macrophytes represented Hydrocotyle vulgaris and Myriophyllum spicatum the former may be submerged or emergent while the latter is exclusively submerged and may have been growing in holes within the reed strip, i.e. in shallow and relatively humus-rich water. The extra-local and regional lowland vegetation is open canopy oak woods dominated by canopy trees of Quercus trojana type (deciduous/semi-evergreen oaks), but canopy trees of Quercus frainetto type (deciduous oaks) are also abundant. The understorey comprises mostly herb-rich steppe; woodland understorey woody and herbaceous plants are poorly represented - Juniperus occurs at extremely low abundance. Quercus coccifera type occurs at an abundance, which although low, is comparatively high for this sequence. Herbs of open ground are the dominant habitat group and occur at the highest relative frequencies of the sequence; Pteridium aquilinum and Hieracium type are particularly prominent - both occur at their greatest abundance for the sequence. Cereal types of Secale cereale, Triticum type, Avena, and Hordeum type are represented and may have been cultivated extra-locally, although radiocarbon dating would suggest that the time period is too early for the cultivation of Secale cereale. The pulses Vicia sativa and $V$. ervilia may also have been cultivated. The nut trees Castanea sativa and Juglans regia may have been planted either extra-locally or further from the site. Platanus orientalis may have been growing in a disturbed or natural habitat, such as flood plains, extralocally or further from the wetland. Riverine trees grew on more distant flood plains and pine woods grew at higher elevations distant from the site in the Pindos Mountains. Microscopic 
charcoal particles are very abundant in the smaller size classes and of moderate abundance in the largest size class indicating widespread regional burning and also some extra-local burning. The very high abundance of spores of Pteridium aquilinum suggests burning of extra-local and regional vegetation. The occurrence of Quercus coccifera type may be due to burning of semi-evergreen oak woods. Lowered concentration values of Pinus (Diploxylon) may be due to the source of regional pine pollen being reduced through burning. Firesensitive Juniperus has very low abundance.

The age of sediments between 372.5 and $312.0 \mathrm{~cm}$ is uncertain, as sediments at $315-316 \mathrm{~cm}$ were radiocarbon dated to $c$. $2000 \mathrm{Cal} \mathrm{BC}$, but cumulative concentration of Pinus (Diploxylon) (Figure 6.8) implies that sediments took 200 years to accumulate between the base of the sequence, radiocarbon dated at $830 \mathrm{BC}$, and $312 \mathrm{~cm}$, which would suggest that sediments at this level are $c .630 \mathrm{BC}$. Sediments are the same as those below. Concentration of wetland palynomorphs continues to be very low. Up to $345 \mathrm{~cm}$ the true-reed-swamp community continues to be the dominant wetland plant community but the pioneer Scirpus is now co-dominant with Glyceria type, perhaps due to slower influx of sediments into the fen. The wet-soil community continues to be represented at about the same level, although Mentha type has become more important than Carex hallerana type. The tall-sedge community has almost disappeared and the stream-reed-swamp community has very low representation. Aquatic communities decline but both floating-leaved and submerged macrophytes are represented; the former by Sparganium angustifolium and the latter by Hydrocotyle vulgaris. Above $345 \mathrm{~cm}$ the true-reed-swamp community continues to be the dominant plant community, but the early hydrosere Sparganium erectum is now co-dominant with Scirpus. Glyceria type declines further, but continues to be prominent. The wet-soil community continues to be represented at similar abundance but Carex hallerana type is now more abundant than Mentha type. The tall-sedge-swamp community continues to have low representation and the stream-reed-swamp community declines. Aquatic communities decline further, although the number of aquatic types represented increases in the uppermost spectrum. Pollen of the extra-local and regional lowland canopy trees of Quercus trojana type and Quercus frainetto type is now equally abundant. A similar understorey to that below is indicated: Juniperus occurs at very low abundance. Quercus coccifera type continues at a similar abundance. Herbs of open ground remain the dominant habitat group, although abundance has declined. Pteridium aquilinum declines, but remains very abundant. 'Cultivars' are still abundant and cereals are cultivated extra-locally. Platanus orientalis increases 
slightly and Castanea sativa and Juglans regia remain unchanged. Mountain pine woods continue at similar abundance. Abundance of microscopic charcoal particles is very similar to that below indicating widespread regional burning and some extra-local burning. High abundance of spores of Pteridium aquilinum supports burning of extra-local and regional vegetation, while the abundance of Quercus coccifera type, which is similar to that below, suggests burning of semi-evergreen oak wood. Again lowered concentration values of Pinus (Diploxylon) may be due to the source of regional pine pollen being reduced through burning and fire-sensitive Juniperus remains at low abundance.

Between c. 1230-1320 AD (312.0-287.5 cm) greater landscape stability in the catchment is suggested by a large increase in the concentration of wetland palynomorphs, slow accumulation rate of the deposits, and by the inclusion of abundant fragments of herbaceous plants (thought to derive from plants growing in the catchment) in fine-grained mineral sediments. Sediments comprise muddy detritus with horizontal layering of herbaceous material; organic content increases significantly, inorganic matter declines, and carbonate content increases slightly. Local plant communities are dominated by a wet-soil community and sedges of Carex hallerana type are dominant. All other types in this community are extremely poorly represented, with the exception of Schoenus nigricans. The second most important community, that of the true-reed swamp, is dominated by species of the Scirpus type. The tall-sedge-swamp community increases and is next most abundant; only the annual Cyperus michelianus is represented suggesting some instability in the catchment. The streamreed-swamp community continues to be extremely poorly represented. Aquatic communities have almost disappeared. Pollen of the extra-local and regional lowland canopy trees of Quercus frainetto type is now more abundant than that of Quercus trojana type. A similar understorey to that below is indicated; Juniperus occurs at very low abundance. Quercus coccifera type almost disappears. Herbs of open ground remain the dominant habitat group, although relative frequencies decline. Pteridium aquilinum declines, but remains abundant. 'Cultivars' are still abundant. Extra-local cultivation of the cereals continues. The trees Castanea sativa, Juglans regia, and Platanus orientalis continue to grow or are planted, either sparsely extra-locally or more abundantly regionally. Mountain pine woods increase. Microscopic charcoal particle abundance is extremely low in all size classes and no particles in the $>400 \mu \mathrm{m}^{2}$ size class were recorded at $305 \mathrm{~cm}$. Declining abundance of Pteridium aquilinum spores supports reduced burning of extra-local and regional vegetation, while the near disappearance of Quercus coccifera type suggests a reduction in burning of semi- 
evergreen oak woods (but the coinciding decline in Quercus coccifera type and Quercus trojana type could also be interpreted as indicative of an increase in humidity). Increased concentrations of Pinus (Diploxylon) may be due to a reduction of burning of mountain pine woods.

Between c. $1320-1370 \mathrm{AD}(287.5-237.5 \mathrm{~cm})$ is a period of greater landscape instability in the catchment: pollen concentration of wetland taxa is again very low. Sediments are characterised by high inorganic matter and carbonate content, and low organic matter content. At c. $1320 \mathrm{AD}(287.5 \mathrm{~cm})$ accumulation rate of deposits begins to increase and influx of fine grained sediments increases, while that of herbaceous detritus declines. Between c. $1340-1350 \mathrm{AD}(271-248 \mathrm{~cm})$ the accumulation rate of deposits increases sharply and influx of larger grain sized particles (fine-medium noncalcareous sand) suggests greater energy of transport of sediments. Between c. $1350-1370 \mathrm{AD}(248-235 \mathrm{~cm})$ the accumulation rate of deposits declines and influx of fine grained (silt and clay) increases, although some influx of noncalcareous sand still occurs. The dominant local plant community is again the true-reed swamp. Although Scirpus is the dominant type represented, Glyceria type and Sparganium erectum are also important. The wet-soil and tall-sedge-swamp communities are almost equally abundant. The dominant types in the wet-soil community are Carex hallerana type and Equisetum, with Mentha type less abundant. In the tall-sedge-swamp community Cyperus michelianus is again the only type of any importance. The stream-reed-swamp community has gained slightly, with Berula erecta increasing. The frequency of the aquatic types increases slightly towards the top of the zone, due mostly to an increase in Hydrocotyle vulgaris. Pollen of the extra-local and regional lowland canopy trees of Quercus frainetto type and Quercus trojana type is almost equal in abundance. A similar understorey to that below is indicated; Juniperus still occurs at very low abundance. Quercus coccifera type remains at the very low abundance. Herbs of open ground remain the dominant habitat group and relative frequencies increase to very high values. Pteridium aquilinum continues at the reduced abundance of below. 'Cultivars' are still abundant. Extra-local cereal cultivation continues. Vitis vinifera occurs for the first time at low abundance. Castanea sativa, Juglans regia, and Platanus orientalis continue to occur sporadically at low abundances, although they now occur more often. Mountain pine woods decline. Abundance of microscopic charcoal particles is very similar to that below $312 \mathrm{~cm}$, although particles of the largest size class are more abundant (especially between 270-250 cm, c. 1340-1350 AD) suggesting more intense/frequent extralocal burning; widespread regional burning also occurs. Macroscopic charcoal particles were 
noted from $264-235 \mathrm{~cm}$. Although Pteridium aquilinum spores occur at reduced abundance, they are still abundant and indicative of burning of extra-local and regional vegetation. Lowered concentration values of Pinus (Diploxylon) may, in part, be due to the source of regional pine pollen being reduced through burning. Fire-sensitive Juniperus occurs at low abundance.

Between c. 1370-1480 AD $(237.5-202.5 \mathrm{~cm})$ is a period of relative stability in the catchment: sediments are characterised by low inorganic and high organic matter, and low carbonate content. Between c. 1370-1430 AD (235-216 cm) influx of herbaceous detritus increases and concentration of wetland palynomorphs increases sharply to very high values at $230 \mathrm{~cm}$, then declines but remains comparatively high indicating an expanded and less disturbed wetland vegetation. Accumulation rate of deposits declines slightly. Between c. 1430-1480 AD (216-200 cm) influx of herbaceous detritus declines and influx of small mineral particles (clay) increases. Calcareous grit also occurs in small amount but the origin of this is unknown and may be allochthonous or autochthonous, or both. The dominant local plant community continues to be the true-reed swamp with Scirpus the dominant type. Glyceria type is still important, although reduced, and Sparganium erectum has almost disappeared. The wet-soil community is the second most prominent community; Carex hallerana type is dominant with Mentha type and Equisetum playing minor but significant roles. The tall-sedge swamp, represented by Cyperus michelianus, and the stream-reed swamp decline. Aquatic communities have also declined again especially the submerged macrophytes community, which has almost disappeared. Pollen of the extra-local and regional lowland canopy trees of Quercus frainetto type is more abundant than that of Quercus trojana type. A similar understorey to that below is indicated, but Juniperus increases slightly. Quercus coccifera type continues at the very low abundance. Herbs of open ground remain the dominant habitat group. Pteridium aquilinum continues at reduced abundance. 'Cultivars' are again abundant. Extra-local cereal cultivation continues. Vitis vinifera occurs in one spectrum only. Platanus orientalis increases and Castanea sativa and Juglans regia occur sporadically at low values. Pollen of mountain pine woods steadily increases. Abundance of microscopic charcoal particles declines in all size classes. The largest size class declines to very low frequencies, but the smaller size classes are still relatively abundant indicating that regional burning is still widespread while extra-local burning is reduced. Pteridium aquilinum declines slightly and Juniperus increases, perhaps as a response to a reduction in burning extra-local and regional vegetation. Quercus coccifera type occurs at very low abundance and 
concentration of Pinus (Diploxylon) increases, suggesting a reduction in burning of regional vegetation.

Between c. 1480-1580 AD (202.5-177.5 cm) increasing landscape stability in the catchment is indicated by the occurrence of peat from c. $1550 \mathrm{AD}(183 \mathrm{~cm})$. Accumulation rate of deposits is the slowest of the sequence and sediments are characterised by high organic and low inorganic matter, and low carbonate content. Sediments are the same as those above until peat formation begins with the exception of a narrow band (200-196 cm) where sediments comprise a mixture of equal proportions of friable calcareous grit and clay. Influx of fine grained (clay) sediments continues as peat forms. Locally the true-reed-swamp community continues to be dominant, with Scirpus the dominant type; Glyceria type increases and is next most abundant. The wet-soil community is next most important with Schoenus nigricans the dominant type and Equisetum also important. Carex hallerana type and Mentha type have almost disappeared. The tall-sedge-swamp community is the next most important community. Carex flacca type and Cyperus longus type are now the most important types in this community; Cyperus michelianus has declined. The stream-reed-swamp community has increased due to an increase of Berula erecta. Aquatic communities continue to be poorly represented with the exception of a peak of Stratiotes aloides in the second to uppermost spectrum. Otherwise, the floating-leaved macrophytes community is represented almost completely by Sparganium angustifolium. Pollen of the extra-local and regional lowland canopy trees of Quercus frainetto type continues to be more abundant than that ouercus trojana type. Both types have declined; the latter type more than the former. The understorey shrub Juniperus continues increasing and is now a significant component in the understorey. Quercus coccifera type continues at the very low abundance. Herbs of open ground remain the dominant habitat group; relative frequencies increase slightly due to a slight increase in Pteridium aquilinum and a dramatic increase in Chenopodiaceae type. Cereal pollen types increase to the highest values of the sequence indicating extensive extra-local cereal cultivation. Humulus lupulus and Vicia sativa type occur in one spectrum. Trees that may have been planted decline: Castanea sativa occurs sporadically, Juglans regia disappears, and Platanus orientalis declines. An increase in Agropyron type may be due to Panicum miliaceum (broomcorn millet) being cultivated, or to wild grasses growing in dry habitats. Pinus (Diploxylon) is abundant. Abundance of microscopic charcoal particles suggests that extra-local and regional burning continues at the same level of intensity/frequency as below. Pollen evidence supports continued reduced burning of vegetation; Pteridium aquilinum and 
Juniperus increase slightly, Quercus coccifera type remains at very low abundance, and concentrations of Pinus (Diploxylon) increase slightly.

Between c. 1580-1650 AD (177.5-157.5 cm) represents a period of relative stability in the catchment landscape with continuous peat formation, although influx of fine grained sediment (clay) increases at $165 \mathrm{~cm}$. Sediments are characterised by high organic and low inorganic matter content, and low carbonate content, but above $165 \mathrm{~cm}$ organic matter declines and inorganic matter increases. An apparent change in the accumulation rate of deposits at c. $170 \mathrm{~cm}$ corresponds to a peak in the small size classes of microscopic charcoal particle frequencies suggesting that the changes in the cumulative Pinus deposition (Figure 6.8) are due to changes in the deposition of Pinus (Diploxylon) pollen rather than changes in accumulation rate of deposits (this is supported by concentration data of Pinus pollen). Accumulation of the fen deposits are probably reasonably steady and comparatively slow. The true-reed-swamp community continues to be dominant locally, with Scirpus the dominant type and Glyceria type next most abundant up to $162 \mathrm{~cm}$, where Scirpus declines and Glyceria type becomes dominant probably as a response to the increased influx of fine grained sediments. The wet-soil community is next most important with Schoenus nigricans the dominant type and Equisetum also important, but declining. Carex hallerana type and Mentha type have almost disappeared, but Carex hallerana type increases from $162 \mathrm{~cm}$. The tall-sedge-swamp community declines; Carex flacca type and Cyperus longus type have both declined and Cyperus michelianus continues at the same low values. The stream-reed-swamp community increases very slightly due to an increase in Berula erecta. Aquatic communities continue to be poorly represented with the exception of a peak of Stratiotes aloides at $170 \mathrm{~cm}$. Otherwise, the floating-leaved macrophytes community is represented almost completely by Sparganium angustifolium and the submerged communities by Hydrocotyle vulgaris. Pollen of the extralocal and regional lowland canopy trees of Quercus frainetto type continues to be more abundant than that of Quercus trojana type; the former type increases while the latter is unchanged. Juniperus increases, now occurring at high abundance and probably forms a significant part of the understorey along with herb-rich steppe. Quercus coccifera type continues at the very low abundance. Herbs of open ground remain the dominant habitat group. Pteridium aquilinum declines to values almost as low as those of the surface sample. 'Cultivars' decline, due to reductions in all constituent taxa, but extra-local cereal cultivation continues. Morus nigra appears in two spectra at low values. The trees Castanea sativa, Juglans regia (reappears), and Platanus orientalis all occur sporadically at low values. Pollen 
of mountain pine woods is abundant. Abundance of microscopic charcoal particles suggests that extra-local and regional burning continues at similar intensity/frequency as previously. In general pollen evidence again supports burning of vegetation at similar frequency/intensity; Pteridium aquilinum declines slightly, while Juniperus continues increasing and Quercus coccifera type remains at very low abundance. Concentration of Pinus (Diploxylon) declines slightly; very low concentration value is displayed at $170 \mathrm{~cm}$ where a peak occurs in smaller sized microscopic charcoal particles suggesting that more intense/frequent burning of regional vegetation occurred at that time.

Between c. $1650-1680 \mathrm{AD}(157.5-143.0 \mathrm{~cm})$ is a period of comparative landscape stability in the catchment but increasing instability is suggested by the greater influx of sediment beginning at $165 \mathrm{~cm}$ (see above) and concentration values of wetland palynomorphs is again very low from $155 \mathrm{~cm}$. Sediments are the same as those previously and are characterised by moderately low inorganic and moderately high organic matter content, and low carbonate content. An apparent sudden increase in the accumulation rate of deposits at c. $155 \mathrm{~cm}$ corresponds to a peak in the frequencies of small size classes of microscopic charcoal particles suggesting that the changes in the cumulative Pinus deposition (Figure 6.8) are due to changes in the deposition of Pinus (Diploxylon) pollen rather than changes in accumulation rate of deposits (this is supported by concentration data of Pinus pollen). However, the accumulation rate of the fen deposits probably does increase to a moderate rate. Locally the true-reed-swamp community continues to be dominant; Glyceria type continues to be the dominant type. Scirpus is now only slightly more important than Phragmites australis and Sparganium erectum. The wet-soil community is the next most prominent community; Schoenus nigricans continues to be important, and Carex hallerana type continues to be equally important. Mentha type and Chrysosplenium alternifolium are also important and Equisetum occurs sporadically. The tall-sedge-swamp community continues at decreased abundance and the stream-reed-swamp community continues at slightly increased values due to an increase in Berula erecta. Aquatic communities increase due to an increase in Hydrocotyle vulgaris. Extra-local and regional lowland vegetation is very similar to previously. Pollen of the extra-local and regional lowland canopy trees of Quercus frainetto type continues to be more abundant than that of Quercus trojana type. Juniperus continues at high abundance and probably forms a significant part of the understorey along with herbrich steppe. Quercus coccifera type continues at extremely low abundance. Herbs of open ground remain the dominant habitat group and increase to almost the highest relative 
frequencies of the sequence, those of the basal zone. Pteridium aquilinum increases slightly. Polygonum aviculare type displays a peak in this zone. 'Cultivars' are still abundant and extralocal cereal cultivation continues. Platanus orientalis and Castanea sativa occur sporadically. Juglans regia disappears. Mountain pine woods decline. Abundance of microscopic charcoal particles of the largest two size classes increase while that of smaller size classes is unchanged, indicating increased extra-local burning and continued regional burning. A slight increase in Pteridium aquilinum may be indicative of increased burning of the extra-local vegetation, while high abundance of Juniperus suggests a lack of burning of extra-local and regional vegetation at least in some areas. Concentration of Pinus (Diploxylon) declines and a very low concentration value is displayed at $155 \mathrm{~cm}$, where a peak occurs in smaller-sized microscopic charcoal particles suggesting that more intense/frequent burning of regional vegetation occurred at that time.

Between $c .1680-1720 \mathrm{AD}(143.0-117.5 \mathrm{~cm})$ is a period of instability in the catchment landscape as indicated by influx of large grain sized minerogenic particles until c. $1710 \mathrm{AD}$ $(121.0 \mathrm{~cm})$, although peat formation continues. Two episodes of influx of coarse grained sediment occur separated by a narrow band $(133-132 \mathrm{~cm})$ of fine grained (clay) sediments. Non-calcareous coarse and very coarse sand forms the greatest proportion of the coarse grained minerogenic deposits while calcareous grit is less abundant. Unfortunately the source of the calcareous grit is unknown - it may be allochthonous or autochthonous, or both. Accumulation rate of the deposits increases sharply and they were probably deposited over a short period of time. Concentration values of wetland palynomorphs are the lowest of the sequence. Locally the true-reed-swamp community is again dominant and Glyceria type the dominant type. Scirpus retains its position as next most important before Phragmites australis and Sparganium erectum. The tall-sedge swamp becomes the next most abundant community with Cyperus longus type the dominant type increasing steadily. Carex flacca type becomes prominent at $c .1690 \mathrm{AD}(130$ and $125 \mathrm{~cm})$. The wet-soil community is next most important but it has declined with Carex hallerana type, Mentha type, and Equisetum occurring sporadically. The stream-reed-swamp community has again increased slightly; again due to an increase in Berula erecta. Aquatic communities increase slightly due to an increase in Hydrocotyle vulgaris. Extra-local and regional lowland vegetation is very similar to that previously. Pollen of the extra-local and regional lowland canopy trees of Quercus frainetto type continue to be more abundant than that of Quercus trojana type, but Quercus trojana type now plays a slightly more important role. Juniperus occurs at highest relative frequencies of 
the sequence and must be an important part of the understorey; herb-rich steppe also occurs. Quercus coccifera type occurs sporadically, but when it occurs abundance is greater than that below $315 \mathrm{~cm}$ and reaches highest relative frequencies of the sequence in several spectra. Herbs of open ground remain the dominant habitat group, although relative frequencies decline. Pteridium aquilinum increases to comparatively high relative frequencies. 'Cultivars' are abundant and increase in the upper part of the zone due to increases in several cereal pollen types. Extra-local cereal cultivation continues. Platanus orientalis occurs continuously at low values and Castanea sativa occur sporadically. Mountain pine woods remain at reduced abundance. Microscopic charcoal particles increase in all size classes, but mostly in the largest two size classes, indicating increased extra-local burning and slightly increased regional burning. Increases in Pteridium aquilinum and Quercus coccifera type may be indicative of increased burning of the extra-local and regional vegetation, while high abundance of Juniperus suggests an absence of burning at least in some areas. Low concentrations of Pinus (Diploxylon) pollen at c. 1680-1690 AD (135 and $125 \mathrm{~cm}$ ) where peaks occur in the frequencies of the smaller sized microscopic charcoal particles suggest that increased regional burning of vegetation occurred at that time.

Between c. 1720-1790 AD (117.5-77.5 cm) comparative stability in the catchment landscape is again indicated as peat continues to form while fine grained sediment and herbaceous detritus are washed into the fen. Accumulation rate of deposits declines and becomes moderate. Sediments are peaty clay and are characterised by comparatively high organic matter and low inorganic matter content. Carbonate content is high below $105 \mathrm{~cm}$ but declines upwards. Concentration values of wetland palynomorphs is very low. Locally the true-reed-swamp community continues to be the dominant plant community. Glyceria type and Sparganium erectum are co-dominant. Phragmites australis and Scirpus are about equally important. Typha domingensis type and Typha angustifolia type have gained in importance. The tall-sedge-swamp community is second most prominent, with Cyperus longus type dominant. Carex flacca type and Cyperus michelianus have both declined. The wet-soil community continues to be represented sporadically by a few types: Carex hallerana type, Mentha type, and Schoenus nigricans. The stream-reed swamp is represented sporadically. Aquatic communities increase significantly due to a large increase in Sparganium angustifolium. Extra-local and regional lowland vegetation is very similar to previously. Pollen of the extra-local and regional lowland canopy trees of Quercus frainetto type continues to be more abundant than that of Quercus trojana type. Although declining 
Juniperus still forms a major part of the understorey. Quercus coccifera type occurs at similar abundance to that below $315 \mathrm{~cm}$. Herbs of open ground remain the dominant habitat group and increase in relative frequency. Pteridium aquilinum increases to relative frequencies almost as great as those between $372-312 \mathrm{~cm}$. 'Cultivars' are abundant and extra-local cereal cultivation continues. Vitis vinifera occurs in one spectrum. Castanea sativa continues to occur sporadically at low abundance, Juglans regia reappears and occurs sporadically, and Platanus orientalis declines and occurs only sporadically. The montane conifers Pinus (Diploxylon) and Abies increase; Abies to relative frequencies similar to those of the surface sample. Abundance of microscopic charcoal particles of all size classes remains high and suggests that extra-local and regional burning is occurring. High abundance of Pteridium aquilinum spores supports extra-local and regional burning of vegetation and declining Juniperus may be due to a declining pollen source due to burning of vegetation. The abundance of Quercus coccifera type suggests some degradation of semi-evergreen oak woods, perhaps also through burning.

Between c. 1790-1840 AD (77.5-57.5 cm) represents an initial period of stability in the catchment landscape and then one of reduced stability. Until c. $1810 \mathrm{AD}(67.5 \mathrm{~cm})$ deposits comprise equal quantities of peat and herbaceous detritus and are characterised by high organic matter, and very low inorganic matter and carbonate content. After this, peat formation ceases, perhaps due to inundation of fine grained mineral sediments, and deposits comprise mostly clay with some herbaceous detritus suggesting that the catchment was vegetated. These sediments are characterised by low organic matter, high inorganic matter, and comparatively high carbonate content. Accumulation rate of deposits declines and becomes slow. Concentration values of wetland palynomorphs are again low. Locally the true-reed-swamp community is again dominant. Before c. $1810 \mathrm{AD}$ (below $67.5 \mathrm{~cm}$ ) Sparganium erectum is dominant. After this, Glyceria type and Scirpus are dominant. Phragmites australis, Typha domingensis type, and Typha angustifolia type have declined to insignificant values. The tall-sedge-swamp community is the second most important community, with Cyperus longus the dominant type but a peak occurs in Cyperus michelianus at c. $1820 \mathrm{AD}(65 \mathrm{~cm})$. The wet-soil community almost disappears but Carex hallerana type increases at c. 1820-1840 AD (65 and $60 \mathrm{~cm})$. The stream-reed-swamp community is not represented. Aquatic communities have declined again but Sparganium angustifolium continues to be represented by significant values. Extra-local and regional lowland vegetation is very similar to that below. Relative frequencies of pollen of the extra-local and regional 
lowland canopy trees of Quercus frainetto type increase and are again higher than those of Quercus trojana type. Although reduced Juniperus still forms a major part of the understorey. Quercus coccifera type occurs sporadically at low values similar to those below. Relative frequencies of herbs of open ground decline to the lowest of the sequence and are now lower than those of the habitat group coniferous and deciduous canopy trees. Pteridium aquilinum declines to low relative frequencies similar to those of the surface sample. Although all cereals have declined, extra-local cereal cultivation is still indicated. Castanea sativa and Platanus orientalis occur continuously, the former at low abundance and the latter at increased abundance. Juglans regia occurs sporadically at low abundance. Salix triandra displays a significant increase. Coniferous and deciduous trees become the dominant habitat group for the first time. This is due mostly to increases in Pinus (Diploxylon) and Abies, which reach their highest relative frequencies of the sequence. Low abundance of microscopic charcoal particles of the largest size class suggests that extra-local burning has declined while unchanged abundances of all other size classes suggests that regional burning continues at similar frequency/intensity. Decreased Pteridium aquilinum and increasing Juniperus support a decline in extra-local and regional burning of vegetation. However, the abundance of Quercus coccifera type suggests some degradation of semi-evergreen oak woods, perhaps through burning.

Between c. 1840-1880 AD (57.5-32.5 cm) greater instability in the catchment landscape is indicated by the influx of larger grain sized particles. Until c. $1850 \mathrm{AD}(52 \mathrm{~cm})$ sediments are the same as those previously, then fine to medium noncalcareous sand, indicating greater energy of transport, is deposited with a small amount of fine grained (mud) sediment and a trace of herbaceous detritus. This was probably deposited over a short time span (accumulation rate of deposits is fast). Sediments are characterised by low organic matter, high inorganic matter, and comparatively high carbonate content. Concentration of wetland palynomorphs is again low. Locally the true-reed-swamp community is again dominant. The dominant types are now Glyceria type, Phragmites australis, and Scirpus; Sparganium erectum is also important. The second most important community is that of the tall-sedge swamp, where the dominant type is Cyperus longus type; Cyperus michelianus fluctuates. The wet-soil community increases in importance with increasing Carex hallerana type. The stream-reed-swamp community has only minor representation. Aquatic communities display a peak at $50 \mathrm{~cm}$ due to an increase in Hydrocotyle vulgaris, then decline. Extra-local and regional lowland vegetation is very similar to that previously. Pollen of the extra-local and 
regional lowland canopy trees of Quercus frainetto type continues to be more abundant than that of Quercus trojana type. Juniperus increases and Quercus coccifera type occurs continuously at low values. Herbs of open ground regain dominance; relative frequencies increase to high values. Pteridium aquilinum remains at the reduced values. 'Cultivars' increase and almost reach the maxima of the sequence due to increases in cereal pollen types; extra-local cereal cultivation is indicated. Lathyrus sativus and Morus alba both occur at $55 \mathrm{~cm}$ only. Platanus orientalis occurs continuously but declines steadily. Castanea sativa also occurs continuously and Juglans regia occurs sporadically at similar abundance as previously. Salix triandra continues at the same abundance as previously. The montane Pinus (Diploxylon)/Abies woodland declines sharply. Increasing abundance of microscopic charcoal particles of the larger size classes and unchanged abundance of the smaller size classes suggests that extra-local burning is increasing, while the intensity/frequency of regional burning remains the same. Reduced Pteridium aquilinum and increased Juniperus suggests a decline in extra-local and regional burning of vegetation. However, the abundance of Quercus coccifera type suggests some degradation of semi-evergreen oak woods, perhaps through burning. Concentration values of Pinus (Diploxylon) decline at c. $1860 \mathrm{AD}(45 \mathrm{~cm})$ where the smaller size classes of microscopic charcoal particles display a peak suggesting that increased burning of regional vegetation occurred at that time.

Between c. $1880-1910 \mathrm{AD}(32.5-18 \mathrm{~cm})$ is a period of comparative landscape stability in the catchment. Peat formation begins again at $32.5 \mathrm{~cm}$ indicating greater landscape stability in the catchment and water logging of the wetland. Fine grained sediments (clay and silt) are deposits coincidentally with a small amount of herbaceous detritus. Sediments are characterised by high organic and low inorganic matter and moderately high carbonate content. Accumulation rate of the deposits declines and is slow. Concentration of wetland palynomorphs is again low. Locally tall-sedge-swamp and true-reed-swamp communities are co-dominant. Cyperus longus type contributes almost all pollen of the tall-sedge-swamp community, while several types of the true-reed-swamp community contribute significant amounts of pollen - Glyceria type, Phragmites australis, and Sparganium erectum. The wetsoil community declines and only Carex hallerana type contributes significant amounts of pollen. The stream-reed-swamp community is not represented. Aquatic communities continue to be poorly represented, but Callitriche stagnalis type displays a small peak at $25 \mathrm{~cm}$. Extra-local and regional lowland vegetation is very similar to that previously. Pollen of the extra-local and regional lowland canopy trees of Quercus frainetto type continues to be 
more abundant than that of Quercus trojana type. Juniperus has declined to relative frequencies similar to that of the surface sample. Quercus coccifera type occurs continuously and increases slightly to relative frequencies greater than that of the surface sample. Relative frequencies of herbs of open ground decline to almost the lowest values of the sequence and are the second most abundant habitat group after coniferous and deciduous canopy trees. Pteridium aquilinum declines. 'Cultivars' are abundant, although they have declined due to reduced Avena and Platanus orientalis. Extra-local cereal cultivation is still indicated. Castanea sativa and Juglans regia occur sporadically. Salix triandra continues at the same abundance as previously. Coniferous and deciduous trees are the dominant habitat group and increase to almost the maximum relative frequencies of the sequence due to increases in Pinus (Diploxylon) and Abies. Abundance of microscopic charcoal particles of the largest size class increases to the highest values of the sequence at c. $1890 \mathrm{AD}(25 \mathrm{~cm})$, suggesting most intense/frequent extra-local burning took place at that time. Abundance of other size classes, except the smallest also increases to high values. Decreased Juniperus pollen suggests the possibility of an increase in extra-local and regional burning of vegetation, while reduced abundance of Pteridium aquilinum spores suggests a decline in the burning of vegetation. However, increased abundance of Quercus coccifera type suggests degradation of semievergreen oak woods, which may be caused by burning.

Between c. 1910-1989 AD (18-0 cm) is a period of apparent stability in the catchment. Peat formation continues while only a very small amount of fine-grained (silt) sediment and woody detritus is washed in. The apparent stability in the catchment is thought to be due to the establishment of the modern sealed road and its causeway, which may have reduced the transport of sediment into the fen from the catchment upstream. Woody detritus probably derives from shrubby plants growing on drier parts of the fen. A very small amount of calcareous grit was noted in these deposits. The sediments are characterised by the highest organic and lowest inorganic matter of the sequence and low to moderately high carbonate content. Accumulation rate of these deposits is very slow and concentration values of wetland palynomorphs increase until very high. Locally the tall-sedge-swamp community becomes dominant with Cyperus longus dominant. Species of Carex flacca type also contribute a large amount of pollen and Cyperus michelianus contributes a significant amount in the surface sample. All other wetland communities have declined greatly. Pollen of the extra-local and regional lowland canopy trees of Quercus frainetto type continues to be more abundant than that of Quercus trojana type, although Quercus frainetto type has declined. Juniperus 
continues at the same low values as previously. Quercus coccifera type declines. Herbs of open ground increase and are co-dominant with coniferous and deciduous canopy trees. Pteridium aquilinum occurs at low abundance while Cirsium increases sharply. 'Cultivars' are abundant although they have declined again. The decline is due to a reduction of all the cereals but extra-local cereal cultivation is indicated. Elaeagnus angustifolia and Vicia sativa type both occur once only. Castanea sativa and Juglans regia occur sporadically. Platanus orientalis occurs continuously and increases slightly. A dramatic increase in Salix triandra occurs. The montane Pinus (Diploxylon)/Abies woodland remains unchanged. Abundance of microscopic charcoal particles of the largest size class increases to the second highest values of the sequence at $5 \mathrm{~cm}$, suggesting intense/frequent extra-local burning. This is perhaps due to the introduction of mechanized agriculture and the practise of stubble burning. Abundance of other size classes are lower than below, but they are still abundant. Low abundance of Pteridium aquilinum does not support widespread burning of vegetation while low abundance of Juniperus pollen suggests the possibility of extra-local and regional burning of vegetation. However, low abundance of Quercus coccifera type suggests degradation of semi-evergreen oak woods, which may be caused by burning. 


\section{Chapter 7. Discussion and conclusions}

Preceding chapters set out the basis of the chronology and vegetation history of the three sites studied. In addition to summarizing these results, this chapter attempts to interpret the data in terms of the history of climate and land use over the past 3000 years. Since the lower part (below $312 \mathrm{~cm}$ ) of the Kellia sequence is not dated satisfactorily it is not included in the discussions.

\subsection{Vegetation and interpretation of fossil pollen sequences}

The extant vegetation of Grevena is dominated by woodland, which covers over half the entire land area; grassland and cultivation cover the remainder. The landscape is intensively used and little, if any, unmodified vegetation remains. Today land use includes agriculture; pastoralism; removal of trees by cutting; and harvesting of trees by coppicing, pollarding, and shredding. In spite of this intensive land use, the composition and structure of the woodlands varies according to elevation and moisture, and forms distinct vegetation zones dominated by only a few tree genera. The highest elevations are occupied by alpine meadows and Pinus woods; intermediate elevations by a mosaic of Pinus woods, Fagus woods and mixed coniferous/deciduous woods; the lower slopes of the Pindos foothills by deciduous Quercus woods; and the plains by a mosaic of deciduous Quercus woods and steppe, and agricultural land. The west-to-east moisture gradient results in the dry eastern Vourinos Mountains supporting few trees. The trees that dominate the landscape, Pinus, Fagus, and Quercus, are also dominant in the surface pollen spectra, and the major vegetation zones that these trees dominate are characterized by corresponding high pollen relative frequencies: $51-84 \%$ Pinus in pine wood, 23.5-29.4\% Fagus in beech woods, and 26.5-40.7\% Quercus frainetto and Quercus trojana types combined in oak woods.

As has been found in many other surface pollen studies, the proportion of pollen recorded in the pollen spectra is different from that of corresponding plant taxa in vegetation. An obvious feature of the results of this study is the over-representation of pine pollen, which suppresses the relative frequencies of all other pollen types. This extreme over-representation of Pinus necessitates that a high pollen sum be counted in samples collected from pine vegetation zones, so that the frequencies of other pollen taxa may be reasonably accurately estimated, and it should be taken into account when the fossil sequences are interpreted.

The relative dispersal and representation of pollen of other less abundant trees varies (as demonstrated by the two surface samples collected from beneath the canopy of the relic mixed woods growing on limestone), but they all have lower relative dispersal and representation than Pinus, Fagus and Quercus. In general the taller anemophilous trees contribute most to 
the pollen rain. Many trees of the mixed woods are entomophilous and contribute almost no pollen to the pollen rain, e.g. Acer, Cornus, and Tilia and genera of Rosaceae. Fraxinus ornus is also primarily entomophilous. If pollen of these trees is found in the pollen record it is likely to have a source close to the sampling site. Poor representation of pollen of these trees makes detection of greater diversity of trees in woodlands difficult. Corylus avellana and Carpinus betulus are anemophilous, but although dispersal is good often little pollen is produced. Corylus is known to produce varying amounts of pollen depending upon its structural position in woods, i.e. it produces little pollen as an understorey shrub, but produces much as a canopy tree (Iversen 1941; Moore 1976). A few pollen grains of Corylus may thus arise from an extra-local or regional source.

One of the biggest problems in interpreting pollen data is defining the size of the area that contributes to the pollen rain. It is often impossible to tell whether a small percentage comes from a few trees in the vicinity or from stands at a distance. In a basin such as Grevena with distinct belts of vegetation zones at different elevations, it is possible to measure pollen dispersal distances from source vegetation. Long-distance transport of some pollen types identified in the surface pollen samples is apparent. The occurrence of pollen of trees that grow in the mountains, such as Pinus and Fagus, in surface samples of the lowlands are evidence of long-distance transport; as are pollen of trees that grow in the lowlands and foothills, e.g. Quercus, in surface samples collected in the mountains. A Pinus relative frequency of as much as $13 \%$, in samples from the lowland oak woods of Grevena, is not indicative of pine growing on or near the site at the time of deposition of the pollen. Threshold values for Fagus and Quercus (Q. frainetto and Q. trojana types combined) of $1 \%$ and $6.5 \%$ respectively are proposed for Grevena. Another pollen type that clearly displays long-distance dispersal is Quercus coccifera, whose appearance in samples collected in the Pindos Mountains must be a result of long-distance transport from the western side of the mountains, taking into account the prevailing westerly winds.

\subsection{Chronology}

An attempt was made to develop a chronology by radiometrically dating material within the sediments of the pollen-analysed cores and by identification of stratigraphic markers; a number of problems were encountered.

The radiocarbon dating and derived chronology for the Gomara sequence is thought to be reasonable, on the basis of sediment-accumulation rates and palynology, and they span the sidereal years from $1340 \mathrm{BC}$ at the base of the sequence to $700 \mathrm{AD}$ at the top of the undisturbed sediments. In the Gomara sequence a sediment horizon identified as tephra by the presence of glass shards could not be correlated by chemical analysis with known 
eruptions because of alkaline etching of the glass shards. Thus the tephra could not be used as a chronological marker, although a radiocarbon date of $105 \mathrm{Cal} \mathrm{BC}$ just below the tephra suggests that it derives from an Italian volcanic eruption at Etna, Monte Albano, or Vulcano (Simkin et al. 1968).

In the Anelia sequence radiocarbon dating provided ages that, at $60 \%$ confidence, were not statistically different for contiguous samples; one sample included four separate calibrated age ranges, and the uppermost sample could represent any calendar date in the last 250 years. Two different laboratories using two different techniques of radiocarbon dating were used. The radiocarbon ages obtained by the two laboratories were compatible and each laboratory carried out pretreatments appropriate to the material being dated, to minimize erroneous results (Chapter 2). Thus the possibility of laboratory error or contamination, e.g. contamination within the measurement system or during sample preparation, is minimal. Mean sediment-accumulation rates for contiguous samples were calculated and checked by applying the mean terrestrial pollen influx rate of the Gomara sequence to the mean terrestrial pollen concentration of the Anelia sequence. A mean deposition rate resulted that implied a basal age for the Anelia sequence in good agreement with the basal radiocarbon date of c. $1560 \mathrm{Cal} \mathrm{AD}$. This chronology is in conformity with pollen data, and a general chronology for the site based on radiocarbon dates is therefore accepted, although the timescale derived for this sequence should be regarded as tentative.

Radiocarbon ages of the Kellia sequence suggest that sediments represent two periods of deposition with a hiatus between. The upper period spans c. 1230-1989 AD. The two lowest radiocarbon ages, based on humin extracted from slight organic deposits, are much older than those of the richly organic deposits above, and they occur in reverse order, giving calendar dates of $830 \mathrm{Cal} \mathrm{BC} \mathrm{(lower} \mathrm{sample)} \mathrm{and} \mathrm{2031,} 1992$ or $1989 \mathrm{Cal} \mathrm{BC} \mathrm{(upper} \mathrm{sample).}$ The reversal may be due to older deposits being washed in on top of sediments from younger deposits. However, the occurrence of Castanea sativa and Juglans regia in the basal pollen assemblage zone suggests that the base of the sequence is younger than 1628-1626 BC (Athanasiadis 1975; Bottema 1980; Bottema 1981; Bottema \& Woldring 1990; Bottema 1994), while the occurrence of Secale cereale, introduced during the Roman Period (Renfrew, J. 1969, 1973a), suggests that the sediments may have been deposited after 146 BC. Identification of individual cereal pollen types is difficult. Although pollen of rye is reasonably distinctive, the pollen identified as rye (Secale cereale) may actually represent another cereal, perhaps one that has been cultivated in Grevena for a longer period, such as wheat or barley, the seeds of which have been found associated with Hellenistic sites in Grevena (Chapter 1), or oats. Evidence of the cultivation of wheat, barley, and oats dates back to the Neolithic in west Macedonia and Thessaly (Renfrew, J. 1969, 1973a \& b; Hansen 1988). 
Another possibility is that it was introduced as a field weed (Stutz 1972; van Zeist 1976). The age of the lower period of deposition is thus uncertain. Variations in the composition of deposits of the Kellia sequence suggest variation in sediment-deposition rate, thus cumulative concentration of Pinus pollen (as a constant component of long-distance transport unlikely to be affected by climatic variation in the time span being considered) was plotted against depth (Middeldorp 1982) and a calendrical scale fitted in compatibility with that suggested by the linearly interpolated radiocarbon age/depth plot. Since this plot takes into account variations in sedimentation rate it was used as a best estimate for the chronology of the younger period of the sequence. This plot also implies that the older period of deposition lasted over about 200 years.

Thus the highland site of Gomara spans the period $1340 \mathrm{BC}$ to $700 \mathrm{AD}$. Then there is a gap in the time sequence until $1230 \mathrm{AD}$ when the lowland site of Kellia is dated reliably. From $1560 \mathrm{AD}$ to the present, the sequences from the Kellia and Anelia sites overlap, offering a comparison between highland and lowland sites. It must be remembered that, although calendrical chronologies are presented, dates are based on interpolation between radiocarbon ages. Because of statistical uncertainties in sampling and calibration associated with radiocarbon dates and variations in sedimentation rates, all given calendar dates must be taken as approximate. The magnitude of the uncertainty varies according to the sequence and depth, and it is difficult to attach precise figures. However, the combination of multiple radiocarbon dates with sedimentation and pollen deposition data reduce the uncertainty below levels associated with single radiocarbon dates.

\subsection{Climatic reconstruction}

As vegetation is related in a broad way to climate, a vegetation history can be used to reconstruct an approximate climatic history. Because of the severe impact that humans have had on the vegetation of Grevena, the small number of samples of the modern-pollen deposition study and the lack of modern climatic data, transfer functions (Birks 1981) could not be determined for the reconstruction of former climates. Thus a method similar to that of Iversen (1944), using a limited number of indicator pollen and spore taxa of known ecological and climatic preferences, is employed. Unfortunately, autecological data are difficult to obtain, and much comes from outside the region of study, where climatic factors that may affect plant distribution such as solar radiation are different. Thus the climatic reconstruction is very approximate. For this study, the use of indicator species is more appropriate than plant communities because communities have been altered by human disturbance (Birks 1981), but ecological preferences of major vegetation types are used. 
There are limitations to this approach. Plant distributions may be caused in part by nonclimatic factors such as competition or edaphic conditions. Also, vegetation may not be in equilibrium with climate at the relevant scales of time and space (Davis 1978; Birks 1981; Prentice 1983; Davis \& Botkin 1985; Gajewski 1993). Moreover, temperature and precipitation do not vary independently of each other, and the interdependence of these two factors is not so well known that we can precisely separate their individual effects in the vegetation (Prentice et al. 1992). Finally, there is a limit to the resolution that can be recorded in pollen diagrams, due to sedimentation rates and post-depositional mixing.

Since no meteorological data are available for this part of the Pindos Mountains, approximate modern-day climatic parameters of the highland site at Gomara have been calculated by use of the 1978-1987 meteorological data collected and analysed by Doyle (1990) from the lowland station at Grevena (550 m asl) and of the altitudinal temperature lapse rate of $5.7^{\circ} \mathrm{C}$ per $1000 \mathrm{~m}$ (Savina 1993). An approximate estimate of the mean temperature of the warmest month (July) at Gomara is $15.9^{\circ} \mathrm{C}$. This does not take into account the probable cooling effects of prolonged snow lie and greater cloudiness in the mountains. The mean temperature of the coolest month (January), calculated on the same basis, is about $-3.6^{\circ} \mathrm{C}$. However, in winter there is commonly a temperature inversion in valleys, and mountain slopes and peaks at the same height are $2-3^{\circ} \mathrm{C}$ warmer (Furlan 1977:196), so the January mean at Gomara may be up to $3^{\circ} \mathrm{C}$ higher. These data make it possible to reconstruct some approximate climatic parameters in the highlands from $1340 \mathrm{BC}$ to $700 \mathrm{AD}$ and deduce minimal deviations from modern values. The climatic reconstruction for the lowlands is based mostly on a Quercus index. This depends on the relative abundance of different groups of Quercus species with different temperature requirements.

1340-890 BC (pollen zone G-6) The climate in the Pindos at this time appears to have been warm and dry. Ilex aquifolium, Viscum album, and Hedera helix are ideal indicator species because their pollen is identifiable to species level, they are entomophilous so problems of long-distance transport do not arise, and the plants are rapidly dispersed by birds eating their berries, hence their distribution is likely to be in equilibrium with the climate (Knuth 1906, 1908, 1909; Iversen 1944). Also they are evergreen, so their survival depends on winter as well as summer temperatures (Birks 1981). Hedera helix, Ilex aquifolium, and Viscum album all occur during this period; the former two species occur near the base of the sequence (c.1300-1200 BC), while the last occurs at $c$. $1000 \mathrm{BC}$. These species occur at very low abundance, but this is to be expected because they are entomophilous and because of the over-representation of Pinus pollen. Ilex aquifolium (holly) is intolerant of cold winters, as its cambium is killed by frost. Iversen (1944), working in Denmark, correlated the northern and eastern limit of Ilex aquifolium with the $-0.5^{\circ} \mathrm{C}$ isotherm of the coldest month of the year. 
Hedera helix is tolerant of lower winter temperatures. It cannot flower or produce fruit below $-1.5^{\circ} \mathrm{C}$, but it requires warmer summers than Ilex (Iversen 1944). Viscum album (mistletoe) is tolerant of cold winter temperatures, the limiting temperature being $c .-8^{\circ} \mathrm{C}$, but it requires warmer summers. The limiting summer temperature is $c .15 .5^{\circ} \mathrm{C}$ (Faegri \& Iversen 1989). The presence of Ilex aquifolium suggests that the mean temperature of the coldest month was warmer than $-0.5^{\circ} \mathrm{C}$, perhaps $3.2^{\circ} \mathrm{C}$ warmer than today, while Hedera helix would require that the coldest month was about $2^{\circ} \mathrm{C}$ warmer than at present. These estimates are very approximate and do not take the possibility of inversions into account; if the modern January mean at Gomara were estimated $2-3^{\circ} \mathrm{C}$ higher, taking into account the probable topographic inversion at Grevena station, these taxa would be close to their climatic limit. Thus their occurrence in this period may reflect slightly warmer winter temperatures than at present. The current estimated temperature for the warmest month of the year $\left(15.9^{\circ} \mathrm{C}\right)$ would allow marginal growth of Viscum album, which requires that the mean temperature of the warmest month be greater than $15.5^{\circ} \mathrm{C}$.

Woodland pollen data suggest that other thermophilous species absent today were growing at this highland site. The occurrence of such primarily entomophilous pollen types as Tilia cordata, Sorbus, Cornus sanguinea, Crataegus, and Fraxinus ornus suggests that they were growing in the catchment. Current distribution data from Grevena are not useful in establishing the ecological limits of these taxa as they occur largely as relics in places inaccessible to grazing animals. Prentice et al. (1992) indicate that temperate deciduous woods occur where the relative moisture availability index $(\mathrm{E} / \mathrm{PE}, \mathrm{E}=$ actual evapotranspiration, $\mathrm{PE}=$ potential evapotranspiration) is greater than 0.65 and winter temperatures are between $-2^{\circ} \mathrm{C}$ and $5^{\circ} \mathrm{C}$. Assuming that $\mathrm{E} / \mathrm{PE}>0.65$, the occurrence of these trees in the catchment gives support to the postulated slightly warmer winter temperature, although the trees would require a temperature only $1.7^{\circ} \mathrm{C}$ higher than that estimated for the present day. Further, the occurrence of these trees indicates that the mean summer temperature (July) was above $13^{\circ} \mathrm{C}$ (Iversen 1954); this is lower than the present estimated temperature and does not imply a difference.

The presence of Picea abies, whose current distribution range is more northern, does not appear to be in accord with a warmer climate (Tutin et al. 1964:31; Strid 1989:41). Today Picea abies (Norway spruce) grows only in NE Greece near the Bulgarian border in the KaraDere woodland in the Rhodope Mountains (c. $300 \mathrm{~km}$ northeast of Gomara), where it forms extensive woodland at c. 1400-1900 m, partly in pure stands and partly together with Pinus sylvestris and Fagus sylvatica. This is its current southern limit (Sfikas 1978; Strid 1989). Ecological and palynological studies suggest that the distribution of some species of Picea is more strongly influenced by competition with other species than by climatic factors (Bormann 
et al. 1970; Davis 1978). Data from Norway indicate that for seeds to mature a mean July temperature of over $18-19^{\circ} \mathrm{C}$ is injurious and a mean January temperature below $-2^{\circ} \mathrm{C}$ to $3^{\circ} \mathrm{C}$ is required (Tallantire 1972). This is lower than the temperature required by Ilex aquifolium. Since Ilex aquifolium is entomophilous it seems that it was growing close to the site, so explanations for the presence of Picea abies need to be sought. Long-distance transport is an obvious explanation and indeed occurrences of very long-distance transport of Picea pollen has been reported (Tyldesley 1973), but a different prevailing wind direction would be required to transport it from northern localities. It could be a relic of an earlier period when winter temperatures were colder or snow cover provided the cold temperatures required. If Picea abies were growing close to the site it seems that it was close to its environmental tolerance. At the end of this time period it disappeared. Not only warm winter temperatures but early summer drought or strong winds due to an open canopy (as seedlings require protection from wind - Tallantire 1972) may have been the prevailing conditions unfavourable to Picea. Disease also cannot be discounted (Tallantire 1977). Human influence does not seem a likely cause of its disappearance, as it is not palatable and does not burn easily.

Palynologically it is difficult to estimate precipitation by analogy with today's conditions. At this time the extra-local vegetation was probably an open canopy pine wood mixed with a few deciduous trees, but not as open as today. Land-use practices in the highlands included burning, which is absent today. The predominance of Pinus, which is more drought tolerant than Fagus, and the very low relative frequencies of Fagus (which are lower than those of the surface sample) suggest that summer precipitation was lower than that of today. Pollen of the xeric habitat group, plants of open dry rocky habitats, is most diverse in this part of the sequence. This may also be indicative of slightly lower summer precipitation.

From c. $1250 \mathrm{BC}$ diatom evidence indicates that the water level of the Gomara lake fluctuated seasonally and the average water level declined, indicating a progressively drying and/or warming climate, although lower water levels may also result from increased evaporation due to an increasingly open woodland structure, or from use by grazing domesticated animals. On the other hand, an open canopy would reduce the amount of moisture taken up in evapotranspiration and the relative amount of run-off would increase. In climatic terms, seasonal fluctuation of the water level implies dry summers. Representation of aquatic macrophyte taxa declines at this time: the only aquatic plant represented, Hydrocotyle vulgaris, can live as an emergent. The addition of coarser particles of mineral sediments may be due to increased storminess but could also be caused by greater exposure of mineral soils through anthropogenic reduction in vegetation cover. Reduced organic matter 
may result not only from less litter being produced by a reduced canopy but also from an increased oxidation rate of litter in drier soils.

890-480 BC (pollen zone $G$-5) During this period the climate appears to have been wetter and colder than the preceding period. In the Gomara diagram Fagus expands at the expense of Pinus. It exhibits an increase in both relative frequency and in absolute abundance. Fagus is not a pioneer tree and is not light-demanding. Today Fagus overlaps with the pine zone, but this is only in damp areas. The slopes of the Gomara site have a sunny SSW aspect and are open to the prevailing westerly winds. Today this creates a local climate marginal for beech growth due to droughty conditions in summer; a few small Fagus sylvatica trees grow in a small valley close to the lake, where soil moisture is greater.

The temperatures determined for the preceding period combined with an open canopy of mostly pine suggests that, if wind conditions were the same as today, summer drought conditions would have been similar to or worse than those of today. Soils of this area have low water-holding capacity. The growth of more extensive beech wood at this site would require wetter summers without droughty conditions (Ellenberg 1988). Therefore an increase in summer precipitation must have occurred and perhaps also an increase in summer cloudiness.

Anthropogenic disturbance is less intense than in the preceding period (less erosion and less burning are indicated). This reduction in anthropogenic disturbance in combination with increased summer precipitation resulted in the rapid expansion of Fagus, whose competitive advantage over pine, given suitable climatic conditions, is well known (Turrill 1929) and can be seen today in Grevena.

Increases in Fraxinus ornus and Ostrya type also support an increase in precipitation. The abundance and diversity of pollen of the xeric taxa of the habitat group 'plants of open dry rocky habitats' declined. Sediment organic-matter content rose steadily, perhaps as a closing canopy (and one that included Fagus) produced more litter, and oxidation rate of litter declined in wetter soils. A denser canopy would have reduced evaporation by shielding the lake from strong winds. Diatom evidence indicates that water level in the lake increased. No seasonal fluctuations are indicated, suggesting that summer precipitation was greater than in the preceding period.

Betula pendula pollen increased during this period and was probably growing extralocally (based on its abundance). Betula pendula (silver birch) is not known in Grevena today and currently grows only in north Greece in the west Rhodopi mountain range close to the northeast border of Greece. Turrill (1929:143) recorded it in the montane beech and fir forests further north on the Balkan Peninsula. It is the only species of Betula growing in Greece today. Pollen was identified to species level based on its current distribution (Tutin et al. 
1964-80; Sfikas 1978) and size of pollen grains (Birks 1968; Prentice 1981; Roure 1985). It was not identified in the surface samples (Chapter 3). The species occurs throughout most of Europe, from $69^{\circ} \mathrm{N}$ in Norway to Sicily; it is local in the south and confined to mountains (Tutin et al. 1964:58; Sfikas 1978; Polunin 1988; Strid 1989). Since Betula pendula now has a more northern distribution it is likely that the current climatic regime of Gomara is too warm for it, suggesting that the increase in pollen in this zone is due a climatic change to conditions cooler than in the preceding period, and cooler than today.

The alpine shrub Vaccinium myrtillus (Ericaceae) increased slightly, supporting a change to cooler conditions. Disappearance of Ilex aquifolium, Hedera helix, and the entomophilous temperate trees (with the exception of Fraxinus ornus, which is partly anemophilous) in this zone suggests that winter temperatures declined. The absence of these species cannot be attributed to anthropogenic disturbance, as this is a period of reduced disturbance. Ulmus glabra type also declined slightly, perhaps also owing to cooler winter temperatures.

Viscum album occurs at very low abundance at c. $700 \mathrm{BC}$, suggesting climatic limits higher than $c .-8^{\circ} \mathrm{C}$ for minimum winter temperature and a minimum summer temperature of at least $15.5^{\circ} \mathrm{C}$.

480-80 BC (pollen zone G-4) The climate appears to have been warmer and perhaps wetter than the preceding period. During this period the regional pine woods and extra-local beech wood expanded and/or became denser. This may have been partly due to increased precipitation and also to a further reduction in anthropogenic disturbance. An increase in Abies and Fraxinus ornus may be related to increased precipitation. Organic-matter content of the lake deposits continued to rise steadily as litter production rose and oxidation rates declined in moist soils beneath an extensive canopy cover. A decline in the steppe taxa of Artemisia and Chenopodiaceae supports moister soil conditions in the catchment, and these heliophytes may also have declined due to greater shading by a denser canopy cover. Diatom and wetland pollen evidence points to a reduction in lake water level and seasonal fluctuation, which may indicate reduced precipitation.

A sharp decline in Betula pendula occurs at the lower boundary of this zone. As discussed above, Betula pendula currently has a more northerly geographic range. Thus its near disappearance in this zone suggests that summer temperatures increased. However, its extra-local disappearance may also be partly due to shading by the expanding Fagus woodland. An increase in Typha angustifolia type may also be a response to warmer temperatures (Iversen 1954).

At c. 80 BC (pollen zone $G$-3) a tephra was deposited. There are no significant differences in palynomorph content of the sediments directly above and below the tephra, 
indicating that the ash caused insufficient climate change to affect vegetation growth either extra-locally or regionally.

$80 B C-330 A D$ (pollen zone $G-2$ ) The climate was probably wetter than today and warmer than the preceding period. A closed canopy Fagus woodland surrounded Gomara lake, replacing the former pine wood of the catchment. Abies and Fagus gradually increased and a parallel gradual increase in precipitation may have occurred, although Fagus may have been still responding to a former increase in precipitation. The vegetation may have reached a particular threshold where greater vegetation cover and greater wetness were self-sustaining, i.e., that there was more convection and therefore more rainfall (Lamb 1982:320).

Water level increased and was probably at its highest level, with little seasonal fluctuation, as shown by the abundance of diatoms living in permanent water and an increase in pollen of aquatic macrophytes. The lake deposits are highly organic, suggesting the soils in the catchment were very moist and that little oxidation of litter was occurring. The combination of organic lake deposits and increased lake water level also argues for increased precipitation.

The reappearance of Ilex aquifolium and entomophilous temperate tree pollen types such as Acer, Crataegus, Cornus sanguinea, Cornus mas, Sorbus, and Tilia cordata suggests that winter temperatures were warmer than today and were higher than in the preceding period. At c. $200 \mathrm{AD}$ the presence of Ilex aquifolium suggests that the mean temperature of the coldest month was warmer than $-0.5^{\circ} \mathrm{C}$, perhaps $3.2^{\circ} \mathrm{C}$ warmer than today. Viscum album occurs at $85 \mathrm{~cm}$, suggesting at least $15.5^{\circ} \mathrm{C}$ minimum mean summer temperature (Iversen 1944).

330-700 AD (pollen zone G-1) The climate was probably similar to that of today. In the catchment pine wood expanded at the expense of Fagus, and the canopy was open. The decline in Fagus suggests reduced summer precipitation: Fagus cannot tolerate summer drought like Pinus. This is a period when anthropogenic disturbance is evident, so the decline in Fagus may be partly due to the direct exploitation of Fagus for fodder and grazing and/or clearance to create pastureland. Pine is a more favoured tree in pasture, as it allows light to penetrate to the ground and pasture plants to grow. Also pine is not as palatable as Fagus so has a better chance of regeneration in pastureland. A reduction in soil moisture, unfavourable to Fagus, may also have been caused by the reduced canopy cover, which would allow greater evaporation. A reduction in soil moisture is supported by the increased abundance in pollen of the habitat group 'plants of open dry rocky habitats'.

The pollen of a few entomophilous temperate tree pollen types such as Cornus mas, Sorbus, and Tilia cordata occurs, suggesting a mild climate, but Hedera helix and Ilex aquifolium, which were present in the preceding period, are absent. Their absence could be due to a decline in winter temperature or to anthropogenic disturbance. An increase in 
Quercus coccifera type during this period suggests a regional increase in temperature (as this pollen must be transported from low elevations), or alternatively greater anthropogenic disturbance such as grazing at lower elevations. An increase in temperature would have increased evaporation rates.

Lake-sediment organic-matter content remained high. This may have been due to an increased rate of erosion caused by greater exposure of soils due to reduced canopy cover. Lake water level was probably high, with little seasonal fluctuation. The level probably increased due to increased run-off.

1230 AD to present Data are available from the lowland site of Kellia (580 m asl), which is close to Grevena meteorological station (550 m asl) (Chapter 1). The Anelia site (1440 $\mathrm{m}$ asl) spans the period from c. $1560 \mathrm{AD}$ to present.

Anelia lies within the beech wood zone and is not at present close to the climatic limit of major tree species. Also, the record of woodland vegetation at the site displays the effects of clearance and other anthropogenic disturbance in the vicinity of the site, and it is therefore difficult to discern climatically linked vegetation changes.

The oak woodlands of the lowlands provide a better opportunity for investigation of climatic change. Prentice et al. (1992) note that temperate deciduous forests occur in winter temperatures (mean coldest month) from $-2^{\circ} \mathrm{C}$ to $5^{\circ} \mathrm{C}$ where $\mathrm{E} / \mathrm{PE}>0.65$, whereas evergreen trees require winter temperatures of more than $5^{\circ} \mathrm{C}$ where $\mathrm{E} / \mathrm{PE}>0.65$. At Grevena meteorological station the mean temperature of the coldest month (January) is $3.2^{\circ} \mathrm{C}$, and the mean temperature of the warmest month (July) is $22.7^{\circ} \mathrm{C}$. In the lowlands of Grevena today three different types of oaks occur, that is, deciduous, semi-evergreen, and evergreen. The distribution of these oak types varies according to temperature, with deciduous oaks occurring in the coldest sites and evergreen oaks occurring in the warmest sites, which are restricted to the southeast corner of the province. Ellenberg (1988) points out that evergreen oaks are more susceptible to frost than deciduous oaks. Semi-evergreen oaks occupy intermediate sites.

Pollen types were established on the basis of the pollen-morphological descriptions of van Benthem et al. (1984) and examination of reference pollen. The main distinguishing features are the surface texture, which varies from scabrate/verrucate (deciduous), to scabrate (semi-evergreen), and indistinctly scabrate/rugulate (evergreen), as well as size of the grain. It is easier to distinguish deciduous and semi-evergreen species from evergreen species than to distinguish deciduous species from semi-evergreen species. Quercus frainetto type includes deciduous species only, Quercus trojana type includes a semi-evergreen species, two semievergreen or deciduous species and one deciduous species, and $Q$. coccifera type includes only evergreen species (Appendix VI). 


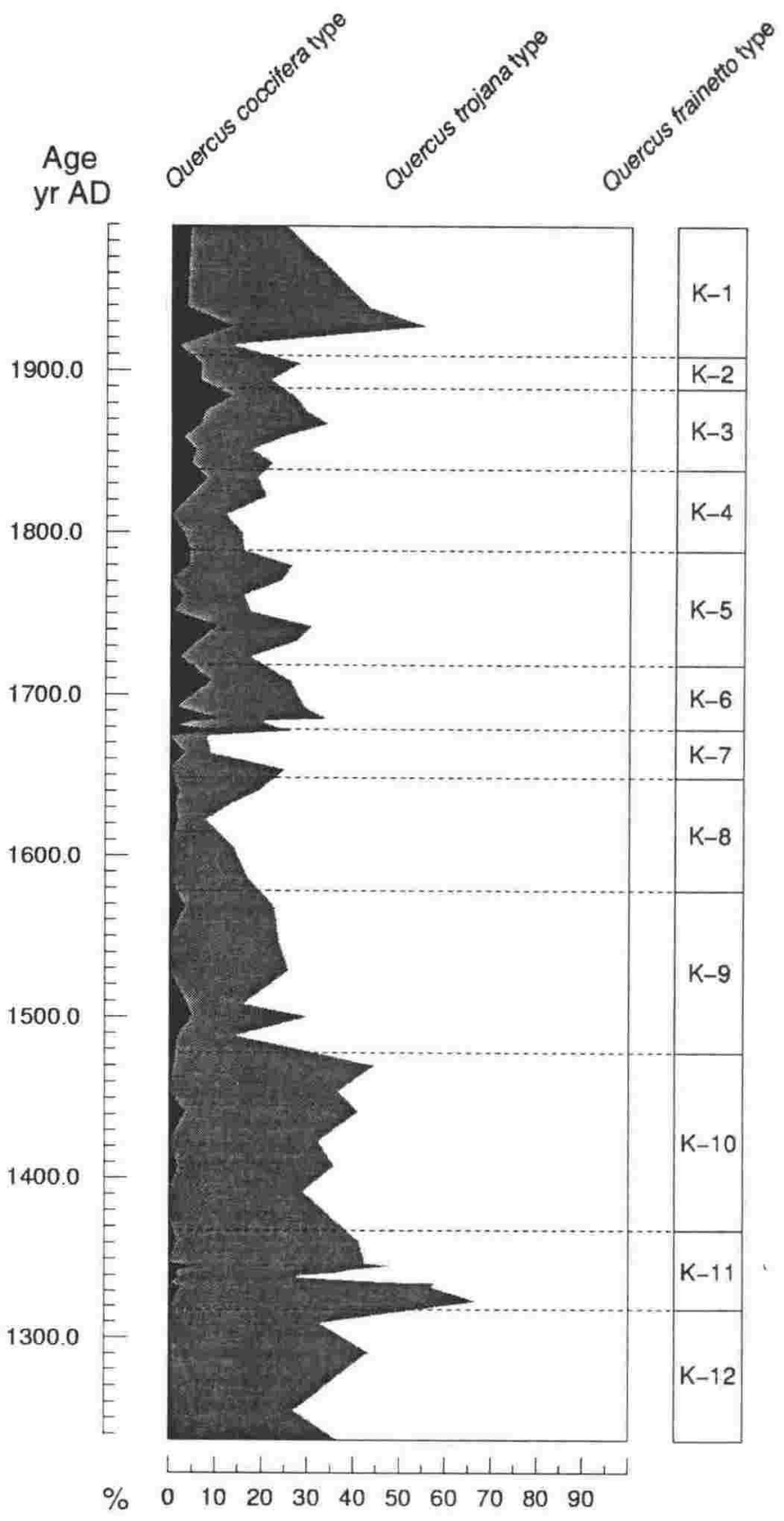

Mean annual temperature, England

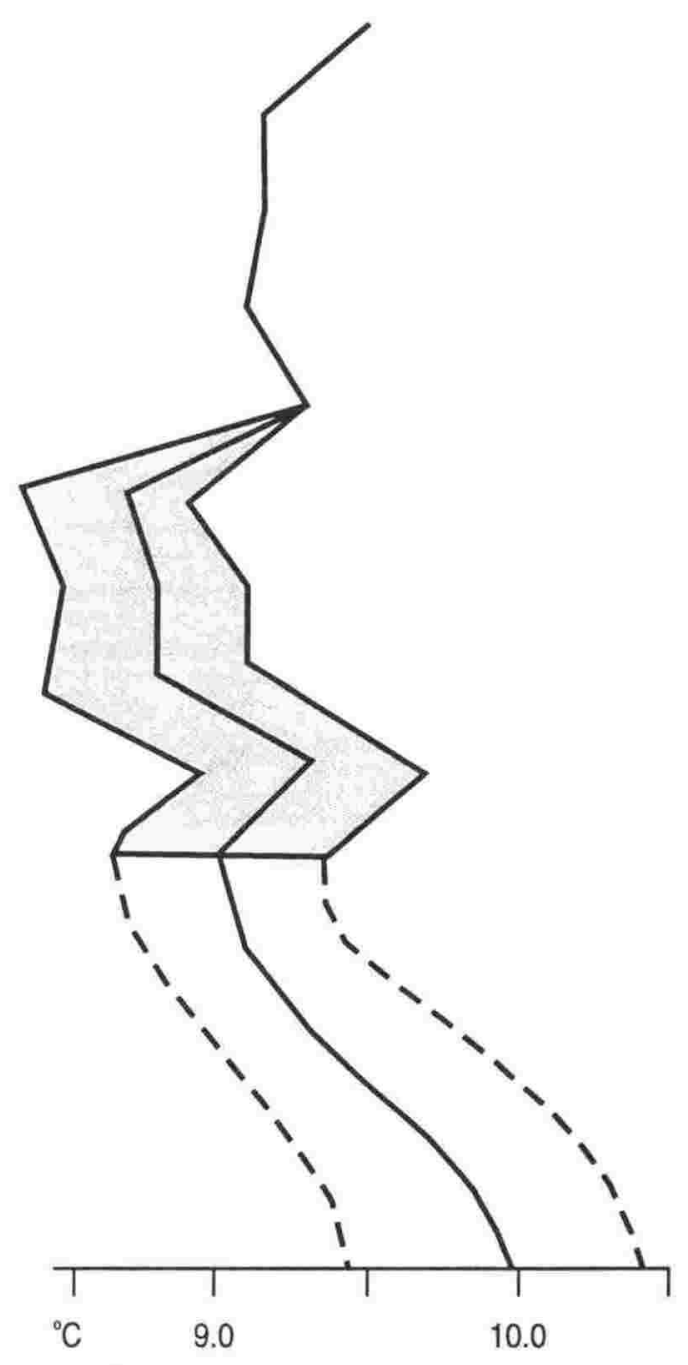

Figure 7.1 Relative abundance of the three Quercus pollen types (coccifera - evergreen, trojana - semi-evergreen/deciduous, and frainetto - deciduous) since $1230 \mathrm{AD}$ at Kellia site, compared with estimated course of annual temperatures in central England over the same period (this latter curve adapted from Lamb 1982, fig. 30a - the shaded area indicates a range of uncertainty). In the Quercus pollen curves the inferred sense of temperature increase is towards the right (increasing proportion of evergreen and semi-evergreen/deciduous oak pollen, decreasing proportion of deciduous oak pollen). 
Curves have been drawn for the Kellia site (Figure 7.1) showing the relative abundances (i.e. one to another, with total oak pollen count taken as $100 \%$ ) of the three oak pollen types Quercus frainetto type (deciduous oaks), Quercus trojana type (semi-evergreen/deciduous oaks), and Quercus coccifera type (evergreen oaks). Set against these curves is the estimated course of temperatures prevailing in central England based on fifty-year averages for the whole year as derived from historical records (Lamb 1982). These curves include the period known as the Little Ice Age, which is a period of lower global temperatures, although there are fluctuations and extreme conditions were more frequent, following a period of warmth in the Middle Ages (Grove 1988). There is no agreement on when precisely the Little Ice Age started; it ended at the end of the nineteenth century (Whyte 1995). After a decline in temperature in the thirteenth and fourteenth centuries there was an interval of more favourable conditions before a return of more severe weather between the mid-sixteenth and midnineteenth centuries (Grove 1988). Absolute temperatures from central England reflect a more oceanic climate and are not expected to correspond exactly to those of Grevena. Nevertheless, the two sets of data show the same general trends. The period of greatest abundance of Quercus frainetto type pollen, lowest abundance of Quercus trojana type pollen, and low abundance of Quercus coccifera type between c. 1580-1680 AD corresponds well with the low temperatures recorded in England between 1550-1690 AD. Two other periods when colder temperatures are indicated for Grevena by the greater abundance of Quercus frainetto type, lower abundance of Quercus trojana type, and low abundance of Quercus coccifera type are between $c$. 1480-1520 AD and c. 1790-1850 AD. These also correspond well with periods of low temperatures in England. Furthermore, the greatest abundance of the deciduous oaks (Quercus frainetto type) and the lowest abundance of semi-evergreen/deciduous oaks (Quercus trojana type) occurs at c. $1680 \mathrm{AD}$ and shows good correspondence with the lowest temperatures indicated at $1690 \mathrm{AD}$ and the culmination of the Little Ice Age, which occurred c. 1660-1680 AD (Lamb 1982; Grove 1988).

The curve of the evergreen oaks (Quercus coccifera type) does not closely follow the curves of the other two types, although it is to some degree positively correlated with that of the semi-evergreen oaks. This is thought to be due partly to the different pollen-source areas. It was found in Chapter 3 that Quercus coccifera type is over-represented, and much pollen is contributed from a long distance. Bottema (1974) also noted this phenomenon while researching pollen dispersal in northern Greece. The other two pollen types contribute pollen mostly from extra-local and regional source areas. Another possible cause for the difference in the curves may be due to anthropogenic disturbance. It has been found that evergreen oaks are favoured by human disturbance such as forest clearance and grazing (Trabaud 1970; Reille \& Pons 1992; Chapter 3). 
The correlation between the Quercus curves of lowland Grevena and the temperature curves of Lamb (1982) suggests that the relative abundances of the different groups of oak trees are changing according to broadscale climatic changes. It also appears that these oak species are responding quickly to climatic change, and there is little or no time lag between changes in temperature and the production of oak pollen. While longer-term climatic change (over several decades or longer) could be expected to be reflected in migration of oak communities, a more likely explanation for the short-term rapid response is that flowering of the more sensitive species is temporarily suppressed by cold spells.

\subsection{Land use}

By the seventh millennium $\mathrm{BC}$ small farming communities were established in the lowlands of mainland Greece, including Thessaly and Macedonia (Renfrew, J. 1973a \& b; Halstead 1981). In the fossil pollen record the first signs of anthropogenic impact on the vegetation in the Balkan region have been recognized as a reduction or change in woodland composition, accompanied by the expansion of open-ground herbaceous types typical of disturbed agricultural ground in southeast Europe, and the presence of cereals (in combination with supporting evidence, required by the difficulty in distinguishing wild from domesticated grasses) (Willis \& Bennett 1994). Forest clearance and plant cultivation is not recorded in Greek pollen diagrams until c. 3500 years ago, when major reduction in tree pollen occurred, along with expansion of agricultural weeds and increase or appearance of such cultivated types as Juglans (walnut), Castanea (chestnut), Olea (olive), and Vitis (grape). Today the landscape of Grevena is intensively used. Current land-use practices are chiefly grazing (including transhumance pastoralism); agriculture; and tree felling and other woodland management practices such as coppicing, pollarding, and shredding.

\subsubsection{Woodland clearance}

Agricultural economies require land on which to grow crops, while pastoralists require grassland to feed the animals - woodland is an obstacle. Woodland can be destroyed by cutting or by burning, and perhaps both methods have been employed in Grevena. In this study several lines of evidence are used to identify woodland clearance: the abundance of charcoal particles; appearance of burned-ground successional species (pyrophytes); reduction of woodland species; and appearance/increase in light-demanding herbs and cultigens. Increased erosion reflected in increased sediment and nutrient influx to depositional sites has also been used to indicate woodland clearance: these are discussed in the following section.

Fire has frequently been used as a tool by people to clear land for pastoralism and cultivation, to promote palatability of grazing, and to drive game from forests (Turrill 1929; 
Mellars 1975, 1976; Rackham 1982, 1983; Stevenson \& Moore 1988). The intentional or accidental spread of fire when grazing areas were burnt off, or deliberate clearance of trees by means of fire, both contributed to the destruction of woodland. On the other hand (and this seems less likely), Chambers (1993) pointed out that the association of human artifacts with stratified charcoal does not necessarily imply that fire had a human cause, for human groups may have moved into areas locally devastated by natural fire. To assist with establishing the causes of deforestation, a fire history of Grevena was established by obtaining the fire history of each pollen site from counts of microscopic charcoal particles.

Many researchers have used charcoal particles incorporated in sediments as evidence of anthropogenic woodland clearance by burning (Iversen 1941; Tolonen 1978), although the only Greek pollen diagram that displays microscopic charcoal abundance is that from Korone moor, coastal Epirus (Yasuda 1985). Clark (1988) examined charcoal transport in relation to particle size and considered that fine particles (such as those found on microscope slides for pollen analysis) are transported over long distances and do not necessarily reflect fires in the immediate catchment. A comparison of the abundance of microscopic charcoal particles found in surface samples with the known current fire regime of Grevena suggested that particles of $400 \mu \mathrm{m}^{2}$ and greater represent charcoal from a very local source, while those of less than $25 \mu \mathrm{m}^{2}$ represent charcoal derived from a distant source. These data are used in determining the location of fires.

The extant wild vegetation of Grevena is not very flammable. In the pine woods of Grevena, soil conditions and the small amount of ground litter minimize the incidence of natural fires begun by lightning strikes. Evidence of frequent lightning strike on pine trees without subsequent conflagration is abundant, for about half the ancient pines having been struck by lightning, some of them many times (Chapter 3). According to Wilson (1979), in the Mediterranean region only $2-3 \%$ of fires are lightning-induced. In times past denser pine woods would have produced more litter, thus perhaps making them more vulnerable to burning. Also if the climate were warmer and drier this may have increased vulnerability. Both Fagus sylvatica and Abies cephalonica do not burn easily, and in the oak woods there is little evidence of fires. These do not burn easily; their litter can be ignited by adjacent stubble or grass fires and young oaks are killed to the ground, but these later sprout from surviving root collars (Moody \& Rackham, in prep.). Juniperus is killed by fire and its absence may indicate the presence of fires; it is widespread in Grevena today, occurring in many different vegetation communities, but pollen representation is not very high. Steppe burns occasionally and this could limit trees, by setting back juniper which would protect young trees from browsing. 
Since mechanized cultivation was introduced cereal stubble has been burnt (Figure 1.5). Although controlled by regulations these fires often spread into oak wood or steppe. The fires are lit when much of the vegetation is dormant, during summer drought, but plants that have an autumn growing season would be expected to be set back. Fire frequency has thus increased on the plain, but this does not seem to have changed the vegetation much, apart from reduction in the fire-sensitive juniper.

In pollen diagrams, clearance of woodland by fire may be indicated by an increase in pyrophytic plants such as Betula pendula and Pteridium aquilinum. Betula pendula possesses the ability to sprout from root collars; in an experiment Iversen (1973) found that it sprouted in quantity after burning, but only very sparsely outside the burned area. Thus in Denmark Iversen (1941) and in France Reille \& de Beaulieu (1988) found it to be the tree that profited most from the clearance fire. After non-fire woodland clearances in fertile regions Betula pendula does not usually appear: its seed germinates only when the soil conditions are favourable, especially on ashy soil. Betula pendula is a pioneer requiring light for growth and is typically short-lived (about 100 years). It therefore is a poor competitor and succumbs in competition with other trees (Iversen 1960, 1973; Rackham 1980). In Grevena, Betula has occurred only at the Gomara site, where its presence may have been limited by climate, as discussed in the preceding section. Pteridium aquilinum is also encouraged by fire, regrowing quickly from deep-lying rhizomes after being burnt (Iversen 1973). It is under-represented in the pollen record, so a continuous record may indicate anthropogenic burning, although as a light-demanding species other forms of clearance would also favour it.

Initial reduction of the canopy allows more light to penetrate to the woodland floor, allowing herbaceous plants that require light to grow (herbs of open ground). Thus the pollen ratio of terrestrial trees and shrubs to that of herbs has been used as a guide to changes in the density of woodland cover. However, a reduction in canopy cover may result either from climatic factors or land-use practices such as clearance by fire, wood cutting, pastoralism and fodder production. In the modern pollen deposition part of this study higher ratios were found in the mountain and foothill woods than in open vegetation, but the ratio varied according to tree genera forming the canopy; the ratio being higher in the mountain and foothill pine and beech woods than in the lowland oak woods. The lowest ratio was recorded, as expected, in the most open vegetation in the cultivated area of the lowlands. In the fossil sequences, extent and density of woodland is also interpreted from changes in abundance (concentration, influx) of the major tree species.

Palynological richness is another measure used to indicate openness of the canopy. Results from the present study show some correlation between openness of sites and palynological richness interpreted from estimated numbers of pollen and spore taxa 
standardized to a common count size using rarefaction analysis (Birks \& Line 1992). The values of expected number of taxa were highest for most open sites, while woods with closed canopies have the lowest values. Woods with incomplete canopies have intermediate palynological richness. Intensive land use, such as the current grazing at Polinerion and the agriculture at Asprokampos, lowered palynological richness. Other researchers have found intermediate levels of disturbance, either natural or anthropogenic, important in maximizing richness at the landscape scale (Birks \& Line 1992). Changes in palynological richness are interpreted as reflecting predominantly the changing floristic richness of the vegetation types in the pollen source area. These changes may be the result of many and varied causes. A reduction, or small openings, in a canopy would allow a wider variety of pollen taxa from regional sources to fall on the deposition site and thus increase palynological richness. Regeneration of a more diverse woodland following cessation of grazing may also increase the palynological richness.

The scale of the woodland mosaic (i.e. size of openings in the canopy) might be interpreted from indicator species. For example Hedera helix cannot flower under the closed canopy of a Fagus wood, and the presence of its pollen suggests that the canopy was open (Troels-Smith 1960). The canopy trees Fagus, Ulmus, and Tilia are among the group growing in the weakest light; Corylus, Fraxinus, Alnus, and Quercus require more light; Betula pendula and Pinus require the most light and do not tolerate any shade (Iversen 1941). Juniperus and Pteridium are other light-demanding indicator species.

Light-demanding herbs that occur in Greek pollen diagrams spanning the Würm glacial period (van der Hammen et al. 1965; Bottema 1967; Wijmstra 1969; Turner \& Greig 1975; Allan 1986, 1990; Willis 1992a, 1992b) include Gramineae, Polygonum aviculare, Plantago, Xanthium, Rumex, Chenopodiaceae and Compositae. All of these relatively well-represented (and for the most part anemophilous) taxa continue to be important indicators of open ground in the Holocene. Distinction among species of some of the genera mentioned should be made, e.g. Plantago lanceolata demands full sunlight, in contrast to Plantago major, and will not grow in grazed woodland (Iversen 1973). Spread of these indigenous plants (apophytes) is influenced by human activity, but of course also occurs naturally.

Pollen of most cultigens is direct evidence of local cultivation, as is that of other plants introduced by man, including weeds of cultivation, ornamentals, etc. (anthropochores), although associated clearance may not necessarily be of a large scale. The significance of various species is discussed further below.

Deforestation increases run-off volume and variability, and this has an expression in lake water level and wetland saturation. Similarly, grazing and agriculture may be expected to influence wetland vegetation. To some degree, then, the relative proportions of wetland plant 
communities (e.g. submerged and floating versus emergent) can be interpreted in relation to the history of the terrestrial vegetation.

\subsubsection{Erosion}

Factors which are important for erosion to occur are local topography, vegetation cover, climate (both general and extreme weather conditions), soil conditions and tectonics (earthquake shaking and local displacements). Of these, vegetation cover and soil conditions are subject to human influence.

The connection of valley-floor alluviation with erosion and its causes, and the chronology of episodes of alluviation have been the subject of much debate. In 1969 VitaFinzi proposed that in the Mediterranean region two phases of alluviation could be recognised, both with a climatic origin: an Older Fill deposited in late-glacial time, and a Younger Fill laid down in a single phase of alluviation from around $400 \mathrm{AD}$ until about $1800 \mathrm{AD}$. Since then a large number of studies in Greece (Bintliff 1977; Davidson 1980; Pope \& van Andel 1984; van Andel et al. 1986; Demitrack 1986; Finke 1988; van Andel \& Zangger 1990; Doyle 1990; Savina 1993) have shown that there has been more than one phase of aggradation and incision during the timespan of the Younger Fill and that phases of aggradation occurred between the 'Older' and 'Younger' phases of Vita-Finzi. Explanation of alluviation phases has fluctuated between schools of climatic and cultural determinism (Bintliff 1992). In Grevena, one or more periods of alluviation and more or less synchronous colluviation occurred in most catchments during the last 2300 years (Doyle 1990; Savina 1993).

The chronology of slope erosion and valley alluviation in response to natural factors and human activities (forest clearance, forest exploitation, agriculture, and pastoralism) was investigated through pollen analysis (of both wetland and terrestrial vegetation), sedimentaccumulation rates, wetland-sediment organic/inorganic content, size of sediment particles, and diatom indications of lake nutrient level.

It is well known that removal of woodland vegetation accelerates erosion by reducing rain interception and evapotranspiration, so enhancing run-off. This increases the loss of particulate and dissolved materials from catchments (Leopold 1956; Bormann et al. 1969a, 1969b, 1974; Davis 1976; Stumm \& Baccini 1978). Mass movements or slope wash as a result of the aftermath of woodland fire may be severe (Bloemendal et al. 1979). Increased sediment load and supply of nutrients have profound effects both on the hydrology and on the biology of downstream aquatic systems (Mackereth 1966).

Deforestation markedly increases erodibility, but according to Bormann et al. (1974) its full expression occurs only after a considerable lag period. This suggests that organic decomposition, particularly in the upper few centimetres of the soil, requires several years to 
surpass the boundary limits of stability inherent from residual organic matter. Primary factors involved in this decrease are soil moisture and soil temperature conditions more favourable to rapid decomposition, and a major increase in the process of nitrification.

Bormann et al. (1974) found that deforestation has a pronounced effect on the amount and size of particulate matter exported from a catchment, the proportion of organic to inorganic material, and the origin of organic material from within the catchment. Brief intense run-off is the major factor in removing particulate matter from both forested and deforested catchments - storm periods greatly increase removal of particulate matter. In the Grevena sites studied, increased rates of inorganic-sediment accumulation and greater sediment particle size are used as a guide to accelerated erosion. In the wetland sites, varying inorganicsediment influx is partly expressed in the inorganic-to-organic sediment ratio. Relatively pure peat and organic detritus sedimentation, in conjunction with palynological data, have been interpreted to represent periods of low erosion, landscape stability, and extensive vegetation cover.

Increased sedimentation rates led to enrichment of wetlands with nutrients, followed by changes in chemical and biological composition of the aquatic habitats. With deforestation concentrations of most dissolved nutrients in stream water increase several fold ( Bormann et al. 1974). However, this increase in nutrients declines as the source of organic matter reduces. Diatoms were used to interpret the trophic history of the lake basin at Gomara. Hydroseral succession is also encouraged by greater input of nutrients to wetlands, and this was considered in interpretation of wetland vegetation. At Anelia and Kellia the situation for some wetland species is complicated by a contribution of nutrients from spring water.

\subsubsection{Pastoralism}

The modern pastoral economy in Grevena is based on the herding of sheep and goats. Koster (1987) identified eight different zones of pastoral exploitation based on degree of dependence on transhumance or 'village pastoralism', ethnicity, vegetation types, and stocking rates, but two main groups can be distinguished: the well-known "Vlachohoria" in the Pindos, which are strictly transhumant, practice virtually no agriculture, use pastures seasonally, and have moderate to high stocking rates; and the lowland agricultural areas, which are grazed by year-round 'village pastoralists' using smaller flocks (Chapter 1).

Pasture is often temporarily improved by burning of grassland or open woodland. Trees are browsed by many kinds of livestock, and the cropping of leaves for cattle feed. Overstocking - beyond the carrying-capacity of pasture at its seasonal minimum productivity - is bad with all species. Sheep have the additional trait of grazing down to root level, often destroying beneficial grasses and permanently opening up the ground mat, with only partial 
recolonization by shrubby vegetation. Goats are notorious for indiscriminate grazing, resulting in destruction of trees and their seedlings. Large aggregations of livestock in fenced plots or through nightly collecting of herds in enclosures also serves to destroy the plant cover, not least through trampling. In many ways, therefore, pastoralism can promote accelerated soil erosion on a scale that vies with the impact of cultivation (Butzer 1974).

Pastoralism is dependent on gaining access to adequate grazing. Forest grazing without effective woodland clearance is difficult to detect in pollen diagrams. Once woodland clearance takes place and is followed by grazing, openings occur in the woodland canopy, allowing illumination by sunlight, and indicator plants for rough grazed pasture appear. Palynological evidence for pastoralism in Grevena consists of partial or complete woodland clearance in situations not consistent with agriculture (Section 7.4.1), decrease in abundance of palatable plants (with resultant loss in diversity), rise in abundance of plants favoured by grazing, and systematic variations in abundance of plants harvested for fodder (Sections 7.4.4 - crops, 7.4.5 - woodland leaf harvesting). Burning to increase pasture extent and palatability has been mentioned in the preceding section and has been interpreted from charcoal-particle abundance. Increased nutrient run-off resulting from stocking has been detected from its effects on the trophic status of wetlands, although nutrient flux is also increased temporarily by forest clearance (Section 7.4.2). Kabailiené (1988) found that the diatom flora of sediments of small lakes fed by surface water from small catchments under intense agriculture is dominated by species characteristic of eutrophication of human origin. High concentrations of phosphate are produced by the decay of human and animal excreta (Shackley 1975).

In Grevena there is greater floristic diversity in woods that are inaccessible to grazing animals (Chapter 3); the more palatable trees remain, e.g. Fraxinus, Tilia, and Ulmus. In woods that are grazed palatable species such as these are rare (Rackham 1982). Iversen (1941) also noted that Tilia and Ulmus are more palatable than oak. In the pollen record, a decline in the variety of deciduous trees, and especially of the more palatable species, could be due to grazing.

The dominant trees of Grevena woodlands are all resistant to grazing. Both Fagus sylvatica and Abies cephalonica can survive long periods of browsing when young. Pines are not very palatable and are not used as fodder. Grazing also favours Juniperus, but other understorey shrubs are destroyed. Intensive grazing in the dry southeast corner of Grevena has led to the development of pseudo-maquis. Unfortunately, the extent and location of a degraded vegetation of pseudo-maquis is difficult to determine from the pollen record. The only pollen type indicative of this vegetation with reasonable representation is Quercus coccifera, which is over-represented and is transported long distances. 
Palynological evidence for pastoral activity is supported by the identification of pollen from plant taxa indicative of pastureland: non-cultivated grasses (Gramineae) and the ribwort plantain (Plantago lanceolata) are usually included in lists of pastoral indicators (Buckland \& Edwards 1984). Rumex acetosalacetosella, Artemisia, Trifolium repens, Chenopodiaceae, are other taxa recognised by various authors (Iversen 1941, 1960, 1973; Tinsley \& Smith 1974; van Zeist \& Woldring 1978; Bottema 1982; Stevenson 1985; Behre 1990; Reille \& de Beaulieu 1988; Bottema \& Woldring 1990).

All of the taxa mentioned are also weeds of agricultural lands (e.g. the cereal field at Asprokampos - Chapter 3). Some researchers have devised ratios of arable to pastoral taxa in order to evaluate more objectively changing trends in prehistoric farming practices (reviewed in Behre 1981). Steckhan (1961) and Lange (1975) use the ratio of cereals to Plantago lanceolata pollen, Kramm (1978) employs cereal:non-cultivated Gramineae, while Turner's arable/pastoral index expresses the number of Plantago grains as 'a percentage of the total number of Plantago, Compositae, cereal, Cruciferae, Artemisia, and Chenopodiaceae pollen grains' (Turner 1964:81). Problems in interpretation may arise. Pastoral indicator pollen types may represent the growth of field-edge weeds or even grassland lying fallow between bouts of cultivation. Donaldson \& Turner (1977) use Rumex acetosalacetosella type as a pastoral indicator, whilst Riezebos \& Slotboom (1978) use undifferentiated Rumex as an arable indicator. Behre (1981) considers Rumex cf. acetosa to be particularly indicative of wet meadows and pasture, while Rumex cf. acetosella may not only be an important weed of winter cereal crops but of various types of grassland also.

It might fairly be assumed that a pastoral episode would be indicated by pollen spectra displaying high grass and plantain values with an absence of cereals and reduced values for arable weed 'indicators' and woodland taxa. Plantago lanceolata demands full sunlight, in contrast to Plantago major, and will not grow in grazed woodland (Iversen 1973). Trifolium repens (white clover), on the other hand, will grow in grazed woodland if the shade is not too close, but it flowers more richly and is more frequent on open pasture. If pollen of Trifolium repens occurs it must have been growing locally (Iversen 1973). Ruderals, plants that favour ground manured by stock, include Urtica species. The occurrence of these taxa in the Gomara sequence coincides with other evidence for grazing.

\subsubsection{Cultivation}

On mainland Greece the earliest evidence for agriculture, in the form of carbonised seeds of cultivated plants, comes from several archaeological sites: Franchthi cave (the Argolid), Gediki, Achilleion, Sesklo, Argissa, Soufli (Thessaly), Nea Nikomedeia (western Macedonia), and Elateia (Boetia) dated to c. 6000 BC (Renfrew, J. 1973a \& b; Hansen 1988). 
In Grevena, archaeological surface surveys undertaken by the Grevena Project have recorded wheat/barley seeds in association with Hellenistic sites. In the pollen record, apart from evidence for clearance of natural vegetation, the most important indicator plants for agriculture are the cultivated plants themselves. Weeds associated with cultivation are also of significance.

A list of plants that may have been cultivated in Grevena since the Late Bronze Age was constructed based on ethnographic studies in Grevena (Aschenbrenner 1987, 1988, in prep.) and from botanical macrofossil studies in mainland Greece (Renfrew, J. 1969, 1973a \& b; van Zeist \& Bottema 1971; Hansen 1978, 1985, 1988; Hansen \& Renfrew, J. 1978; Hubbard 1979; Jones 1981). These include the cereals Triticum monococcum (einkorn), T. dicoccon (emmer), T. aestivum (bread wheat), Hordeum distichon (two-row barley), H. vulgare (six-row barley), Avena sativa (oats) and Panicum miliaceum (millet), which have all been found in Neolithic contexts. Secale cereale (rye) and Zea mays (maize) are more recent introductions, the former in the Roman Period and the latter probably c. 1700 AD (Huber 1962 in Bottema 1980; Bottema 1981). The pulses include Pisum sativum (peas), Lens culinaris (lentils), Vicia ervilia (bitter vetch), Vicia faba (broad bean), and Cicer arietinum (chickpea), which have all been found in Neolithic contexts. Other crops may have included Vicia sativa (vetch), Lathyrus sativus (pea hay/grass pea), Humulus lupulus (hop), and Linum usitatissimum (flax). Other species such as Urtica dioica (nettle), Polygonum aviculare, and species of Chenopodiaceae, which are commonly found with seeds of domesticated plants, may also have been cultivated, collected from the wild for human consumption, or grown as weeds of cultivation. These are not included in the list of cultivars. Macrofossil evidence indicates that Vitis vinifera (grape) was domesticated by the Late Bronze Age in Macedonia (Hansen 1988). Macrofossils collected from archaeological sites often include stones of fruit and nut trees such as Quercus (acorns), Corylus (hazel nut), Prunus sp., and Cornus mas, but it is not known if these trees were planted. These are also not included in the list. Several other trees that are thought to have been cultivated include the nut trees Castanea sativa and Juglans regia; a tree that today is often planted for shade, Platanus orientalis; and Elaeagnus angustifolia (Bottema 1980). Morus alba and M. nigra may have been cultivated in recent times.

The macrofossil data suggest that the most important cultigens were the cereals Triticum monococcum (einkorn), T. dicoccon (emmer), Hordeum distichon (two-row barley), and $H$. vulgare (six-row barley), and the pulses Pisum sativum (pea), Lens culinaris (lentil), and Vicia ervilia (bitter vetch). In the Bronze Age, in northern Greece, Triticum monococcum (einkorn) was the most common cultivated wheat, and $H$. vulgare (six-row barley) appears to have replaced Hordeum distichon (two-row barley) by the beginning of the Bronze Age. 
In the sequences from Grevena no pollen of the pulses Vicia faba (broad bean), Pisum sativum (pea), Lens culinaris (lentil), and Cicer arietinum (chick peas) was identified. These species all belong to the family Leguminosae and are entomophilous (Knuth 1908). Thus their absence from the pollen record may be a result of their poor pollen production and dispersal: it is impossible to say if they were cultivated or not.

The following pollen types, which include prehistoric and historic cultivars, were identified: Lathyrus sativus, Medicago sativa, Vicia sativa type, Elaeagnus angustifolia, Humulus lupulus, Morus alba, Morus nigra, Linum usitatissimum type, Castanea sativa, Juglans regia, Platanus orientalis, Vitis vinifera, Avena, Hordeum type, Secale cereale, Triticum type, Zea mays, and Agropyron type. It was possible to identify some cultigens to species level, but many of these species are severely under-represented in the pollen record, e.g. the legume Lathyrus sativus, which before 1940 was cultivated in the Pindos foothills and the Grevena plains for fodder on a subsistence basis. Seeds of $L$. sativus (grass pea) are found quite widely in archaeological sites as old as early Neolithic and may have been cultivated for both human consumption and fodder. Another legume, Medicago sativa subsp. sativa (alfalfa), is still grown as a forage crop in Grevena. In addition to being severely underrepresented it cannot be distinguished from other subspecies of Medicago sativa that are wild, or from the wild Trifolium montanum (Appendix VI.2).

Vicia sativa type includes several wild species as well as cultivated species of Vicia sativa, and probably also the cultivated species Vicia ervilia and the weed of cultivation Vicia pannonica. Vicia sativa (vetch) was cultivated for fodder on a subsistence basis in the Pindos foothills and on the Grevena plains before 1940, but is no longer cultivated to the same extent. Seeds of domesticated $V$. ervilia (bitter vetch) were found in Macedonia at the archaeological sites of Nea Nikomedeia and Sitagroi in contexts dating to the Neolithic (Hansen 1988). The seeds are thought to have been used for human consumption (Renfrew 1973a:116). This pollen type is also entomophilous and therefore under-represented. As it occurs very sparsely in the pollen record, no conclusions about cultivation of leguminous fodder and forage can be made.

Morus alba and Morus nigra are cultivated in the Balkans (Polunin 1988) but were not seen growing in Grevena. They each occur only once in the pollen diagrams, and at very low abundance. No details of their pollen representation are known, but as they are anemophilous their occurrence is quite possibly due to long-distance transport, and they perhaps were never cultivated in Grevena. Humulus lupulus is widely cultivated in Europe, but it grows naturally in hedges, bushy places and on river banks. It was not seen growing in Grevena and is very sparse in the pollen record. Elaeagnus angustifolia is today planted for ornamentation and is probably a recent introduction. It was identified in the surface sample only at one site. 
The Linum usitatissimum pollen type includes two species: Linum bienne and $L$. usitatissimum. Linum bienne is a wild biennial to perennial of grassy, stony places, native to south and west Europe, while L. usitatissimum (flax) is cultivated and sometimes naturalized and of uncertain origin. Flax was formerly cultivated throughout most of Europe for fibre and oil from the seed (linseed). It is now much less commonly grown but is still recorded as a casual throughout Europe. A large number of flax seeds were found in Early Bronze Age deposits at Lerna, in the Argolid, and flax cultivation at Servia in Thessaly during the early Neolithic was suggested in a preliminary report (Hubbard 1979). It is uncertain if flax was cultivated in Grevena, so this pollen type was not placed in the cultivars group in the pollen diagrams. It occurs in one spectrum only, at very low abundance.

Only a very few pollen grains of Vitis vinifera were recorded, even from the lowland site where settlements have existed in the past. Vine pollen has been found to be poorly dispersed and is probably under-represented (Chapter 3), although it has been found at comparatively high relative frequencies where derived from a local source (Stevenson \& Moore 1988). Today grapes are grown in household gardens, but ethnographic studies suggest that grapes were grown on a much larger scale in the past (Chapter 1).

The three trees Castanea sativa, Juglans regia, and Platanus orientalis are of interest. It has been demonstrated that an increase in the pollen of these taxa is associated with anthropogenic disturbance of woodlands in Greece (Bottema 1974, 1980, 1982; Bottema \& Woldring 1990). There is no evidence for Juglans regia or Castanea sativa appearing postglacially in Greece before about 3500 BP (Bottema 1980). (Juglans regia occurred in the Balkans during the Eem Interglacial but disappeared during the Würm Glacial - Bottema 1980.) The average time of arrival or increase of Juglans, Castanea, and Platanus in western Macedonia is about 3100-3300 BP for those areas adjacent to the coast or not very far inland (Bottema 1974). Castanea and Juglans appear in Litochoro (altitude $25 \mathrm{~m}$ ) at about $3000 \mathrm{BP}$, in Pertouli (1275 m) about $1000 \mathrm{BP}$, although rare grains appear before $3200 \mathrm{BP}$ (Athanasiadis 1975). At Xinias ( $500 \mathrm{~m}$ ) pollen of this group is found in only the top sample (Bottema 1979). Pollen of these trees also appears late at Tenaghi Philippon (40 m) (Wijmstra 1969). At Trikhonis Juglans appears at either about $3425 \mathrm{BP}$ or $79 \mathrm{AD}$, according to interpretations of different authors (Bottema 1980). Juglans regia and Platanus orientalis are anemophilous, but pollen production is not very high. These trees are thought to have either profited from human-induced changes in habitats or were planted in prehistoric times. Thus low relative percentages in the pollen record may be due to long-distance dispersal or to a few trees growing in the vicinity of the sampling site, and they may or may not have been cultivated but perhaps established spontaneously. Castanea sativa is entomophilous primarily, but from the results of this study it appears capable of long-distance dispersal. Long-distance dispersal of 
Castanea pollen has been reported by others (Ruffaldi 1994). Ethnographic studies suggest that fruit and nut trees would be planted around vineyards close to settlements (Chapter 1). At the Gomara site low abundances of Castanea sativa and Platanus orientalis occur from the base of the sequence $c$. $1340 \mathrm{BC}$, but Juglans regia does not appear until c. $450 \mathrm{BC}$. At Kellia and Anelia Juglans regia occurs at low abundance throughout, while Castanea sativa and especially Platanus orientalis increase at c. $1800 \mathrm{AD}$, probably reflecting regional increase. However, abundance is not sufficient to imply local planting of these trees near any of the sites.

The poor dispersal properties of cereals have been discussed (Chapter 3). Another problem associated with pollen of cereal crop plants is the distinction of their pollen from one genus to another and from wild species. The identification of cereal pollen to the genus level is difficult, but the distinction from wild grass is usually easier. Cereal-type pollen differs from wild grass in being much larger and having a relatively large porus annulus (Appendix VI). The pollen types Zea mays, Triticum (except T. monococcum), Secale cereale, and Avena are reasonably distinct from wild grasses, but the Avena pollen type includes some wild species of Avena. Hordeum type, which includes Triticum monococcum, is less distinct from wild grasses, and the wild genus Elymus cannot be distinguished from the cultivated cereals included in this type. The wild grass pollen type Agropyron is very similar to the Hordeum type. Panicum miliaceum (millet) unfortunately cannot be distinguished from wild grasses (Andersen 1979). Poor dispersal, under-representation and difficulties in distinguishing cereal grains makes interpretation of cereal cropping strategies difficult. Hordeum may never be represented in the pollen record, and any grains attributed to this type are probably either Triticum monococcum (einkorn) or a wild genus. Avena type pollen may represent cereal crop species or weeds of disturbed and waste ground, including cultivated ground. If identified correctly, the occurrence of pollen grains of Triticum type, Secale cereale, and Zea mays probably indicates cultivation of species included in those pollen types. From the pollen production and dispersal characteristics, cultivation of Avena, Triticum, and Zea mays would likely be close to the pollen-occurrence site, while that of Secale cereale could be more distant. At the Gomara site in the lower part of the sequence there is sporadic low abundance of cereal-type pollen, which may be due to large-grained wild grasses, minor nearby cultivation, or long-distance transport from cultivated areas. The most notable feature of the cereal group distribution in this sequence is the relatively high abundance of Secale cereale in late Roman times, which may reflect widespread cultivation of rye in the region. At Anelia the only cereal pollen present in significant abundance is that of Secale cereale and Triticum type; these are present from the base of the sequence $c .1560 \mathrm{AD}$ until $c .1920 \mathrm{AD}$. The record is interpreted as indicating nearby cultivation of wheat and possibly other cereals. At Kellia 
there is a continuous record of most cereal pollen types. Avena, Hordeum type, and Secale cereale occur in greater abundance throughout the sequence than at the other two sites, while Triticum type is mainly present after c. $1870 \mathrm{AD}$. Zea mays occurs sporadically, and its earlier occurrences in the sequence are difficult to reconcile with its known date of introduction into Europe, after $1500 \mathrm{AD}$. The pollen record indicates that cereal cultivation was an important land use in the vicinity of this site since $c .1230 \mathrm{AD}$.

In conclusion, while ethnographic studies suggest that prior to mechanization of agriculture, each household grew a diversity of crops for human consumption (wheat; barley, probably mainly for livestock; maize; rye; beans; chickpeas; vegetables; fruits; nuts; and vines for table grapes, wine, and tsipouro) and for fodder (barley, oats, and several legume varieties, esp. vetch, pea hay, and alfalfa), few of these crops are represented in the pollen record. Cereals are a group better represented than others, but due to differences in pollen representation and dispersal, and the difficulty of reliably distinguishing between the pollen of different cereals the relative importance of cereal crops can be assessed only tentatively. Nevertheless, different patterns of cultivation at different times are suggested.

Among weeds associated with agriculture are a number of anemophilous species with a large pollen production, and these are prominent in the pollen flora, but most of these do not occur exclusively in cultivated fields. The surface sample from the cereal field at Asprokampos contained the pollen types of Polygonum aviculare type, Plantago lanceolata, Rumex acetosa group, and Chenopodiaceae. All except the first are anemophilous, and all have medium to high relative dispersal and are well- to over-represented. Pollen of several Compositae species were also identified in this sample. These pollen types are entomophilous, dispersing their pollen locally. They are included in Bottema's (1982) and Bottema \& Woldring's (1990) lists of anthropogenic indicator species. As these weeds are native to Greece, their occurrence is not by itself sufficient indication of human activity.

However, agriculture can perhaps be identified by 'specialist' weeds closely associated with cultivation. Extant weeds of cereals in Grevena include Agrostemma githago, Asperula arvensis, Brassica nigra, Buglossoides arvensis subsp. sibthorpiana, Centaurea cyanus, Consolida regalis subsp. paniculata, Dasypyrum villosum, Erysimum cheiranthoides, Galium tricornutum, Nigella arvensis, Orlaya kochii, Ranunculus arvensis, Sinapis arvensis, and Vicia pannonica subsp. striata (Moody \& Rackham, in prep.). Of these, Agrostemma githago, a classic weed of cultivation (Tutin et al. 1964-80), is the only species belonging to its pollen type. Asperula arvensis and Galium tricornutum belong to the pollen type Galium, which includes a large number of herbs of various habitats (Appendix VI). Brassica nigra and Erysimum cheiranthoides belong to the Sinapis type, another type which includes many species of varied habitat (Appendix VI). Buglossoides arvensis subsp. sibthorpiana, belongs 
to the $B$. arvensis type, which includes two species of dry open habitats as well as cultivated ground. Centaurea cyanus belongs to the Centaurea cyanus type, which includes two other species also weeds of cultivation and dry stony places (Tutin et al. 1964-80). Pollen of Consolida regalis subsp. paniculata and Orlaya kochii was not identified in the fossil pollen sequences. The grass Dasypyrum villosum could not be distinguished from other wild grasses. Nigella arvensis belongs to the pollen type Nigella, which also includes two cultivated species, sometimes naturalized (Tutin et al. 1964-80). Ranunculus arvensis belongs to Ranunculus arvensis type, which may include species of varied habitats (Appendix VI). Vicia pannonica subsp. striata is included in Vicia sativa type, which includes several wild species, so its presence is not a reliable indication of cultivation (Appendix X).

In summary indicator pollen types which are best indicators of cultivation are those which include only species of similar preference, i.e., Agrostemma githago, Centaurea cyanus type, Nigella, and Buglossoides arvensis type. These unfortunately are all entomophilous so occur in low abundance in the pollen diagrams, but where they do occur they may be presumed to grow nearby. For example, Centaurea cyanus type occurs in the set of surface samples only at the cereal field at Asprokampos and at Kellia fen, which is in close proximity to cereal fields.

At Anelia site, Agrostemma githago and Centaurea cyanus type both occur in the lower part of the sequence where cereal pollen is most abundant. Buglossoides arvensis type and Nigella also occur sporadically in low abundance. At Kellia site, all four pollen types occur throughout the sequence from c. $1230 \mathrm{AD}$ to the present but most abundantly between $c .1320$ and $1720 \mathrm{AD}$. At Gomara site, only Buglossoides arvensis type and Nigella occur, both rarely and at very low abundance. As noted above these two pollen types include some species not so exclusively associated with agriculture.

\subsubsection{Woodland management}

Grevena has very extensive woodlands for the size of its population (Chapter 3). All woodlands have a history of intensive management, which includes tree-felling, coppicing, pollarding, shredding, and grazing (Section 7.4.3) and little, if any, unmodified woodland remains. Pines are felled but not usually coppiced, pollarded, or shredded, while beech and oak and less dominant woodland trees may be felled, coppiced, pollarded, or less often shredded. Trees in woods are usually coppiced; free-standing oaks in pastures or fields are pollarded.

Coppicing, pollarding, and shredding are all ways of increasing the number of stems per tree. In coppice-woods, the majority of trees are cut down every so many years, then allowed to sprout again from the stump, while scattered timber trees are allowed to grow longer to 
produce beams and planks. Pollards are cut like coppice stools but at $4 \mathrm{~m}$ above ground, while shredding is the removal of side branches so that successive crops of stems are produced.

It is likely that systematic woodland management, the management of wild trees as a renewable resource to produce a permanent succession of crops of timber and underwood, began in the Neolithic in Greece (Rackham 1982:180). In Grevena coppicing has been widespread for many centuries, e.g. Tilia (lime) stools in the Aetia gorge measure $2 \frac{1}{2} \mathrm{~m}$ diameter, and at Anelia annual rings indicate that a beech stool was about 300 years old when last cut about 20 years ago. The practice still continues but on a diminished scale (Moody \& Rackham, in prep.). Coppice is used for many purposes, but usually for fuel.

Pollarding and shredding are both commonly used to provide leaf fodder. Pollarding has been practised at least since the Middle Ages: in Aetia the stump of an oak was found which was first pollarded in $1556 \mathrm{AD}$ (from annual tree-ring count). Pollarding and shredding has occurred at very irregular intervals, from 4 to 75 years, with no apparent pattern. Leaffodder production is a major industry, and almost all oaks have been cut or pollarded within the last 50 years (Moody \& Rackham, in prep.).

Pollen production would be severely reduced after cutting of a coppice-wood, although the scattered timber trees left standing might produce more pollen per individual tree than when previously surrounded by other trees (Bonny 1976). Two oak woods that were sampled in the lowlands had been coppiced. Quercus relative frequencies in these woods were high ( 33.4 and $40.7 \%$ ), but it is impossible to say if coppicing has depressed pollen deposition, as no data are available against which to compare these frequencies. Oaks produce flowers in the later part of each coppicing cycle (30-40 years), except Quercus frainetto, which takes at least 50 years. The unusually long interval (40-75 years) between cutting of coppice-woods in Grevena may therefore have reduced the effect in pollen sequences. Coppicing could not only reduce the pollen production of the trees coppiced but would also reduce the canopy of the woods, allowing in more light, so that light-demanding species could grow. Both the regional pollen component of the pollen rain, and the woodland understorey component would assume greater prominence in the pollen spectra. At Anelia, more intense use of the contemporary beech woodland in the past is suggested by extant beech trees which, formerly coppiced, have been left uncoppiced for many years. The resulting increased flowering is probably reflected in the rise in beech pollen at the top of the sequence.

Timber production was well established 180 years ago (Leake 1835). In the recent past the main export of high-altitude villages such as Smixi and Samarina was milled pine planks. Beech and oak are also felled for timber - many local houses and out-buildings contain beams of oak. Abies is also a well known timber tree elsewhere (Tutin et al. 1964-80). A sudden and sustained decline in Abies in the Anelia pollen sequence at c. $1730 \mathrm{AD}(90 \mathrm{~cm})$ is similar to 
that seen in other pollen diagrams from northern Greece ((Bottema 1974; Gerasimidis \& Athanasiadis 1995). This decline occurs at different times in different localities, suggesting human-induced destruction rather than climate or other natural factor as the cause. At Anelia it is a local event as abundance of Abies in the Kellia sequence increases slightly at this time, indicating that the regional pollen source area remains almost uniform. The decline is interpreted as selective logging of Abies at Anelia. Selective exploitation of Abies is also known from other parts of the Mediterranean (Reille 1992).

\subsection{Synthesis of environmental history}

Details of vegetation change at the three sites studied have been given in preceding chapters, and various aspects of the interpretation of these sequences in terms of climatic and cultural history have been discussed above. This section draws together these aspects in interpreting the ecological history of each site and evidence for cultural activity, summarized in Table 7.1.

\subsubsection{Gomara c. $1340 \mathrm{BC}-700 \mathrm{AD}$}

As noted in Chapter 4, there is close correspondence between changes in the composition of the sediment and changes in the pollen profiles of both wetland and terrestrial pollen and spore types. This close correspondence is explained by these three elements responding to environmental changes in the catchment. Thus the boundaries of the wetland and terrestrial pollen zones are synchronous. Further, this synchronism implies that many of the pollen and spores in the sediments are derived from the catchment: the terrestrial pollen record reflects primarily the extra-local rather than regional vegetation.

The combination of evidence from deposited sediments and the wetland pollen sequence indicates a hydroseral succession from a permanently wet environment (small lake) to a seasonally wet fen as the deposition basin filled in from c. $1340 \mathrm{BC}$ to $700 \mathrm{AD}$. Diatom results are in general agreement with those of pollen analysis. The permanent water was shallow, probably fluctuating between 1 and $2 \mathrm{~m}$ depth. Water condition throughout the sequence was fresh, slightly alkaline, and rich in oxygen. A marginal swamp vegetation of sedges and reeds, with a Carex swamp community on the landward side of the reed swamp, surrounded the lake. Only occasional submerged and floating-leaved aquatic macrophytes from a deeper water zone inside the Phragmites australis belt were identified. The wetland has never been nutrient-poor. Trophic level is highest at the base of the sequence (eutrophic), and organic nitrogen compounds are most abundant at the base and top. In the centre of the sequence the trophic level drops to meso-eutrophic, and organic nitrogen levels are low. 


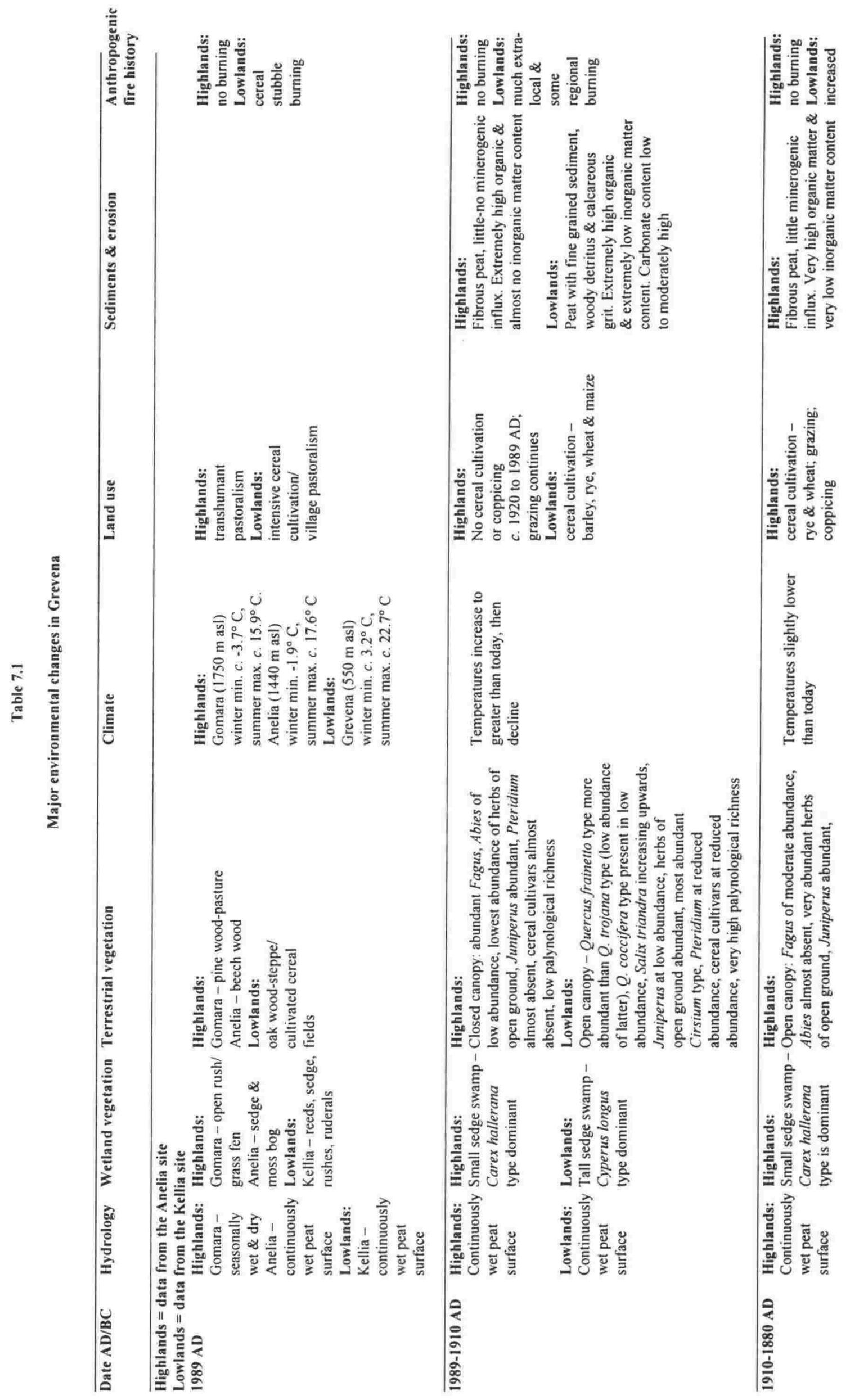




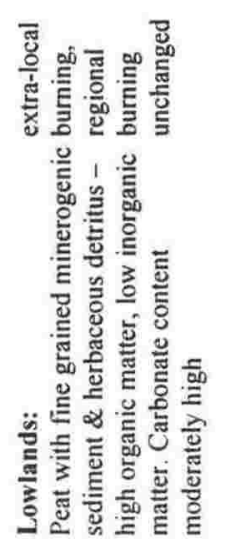

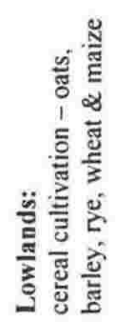
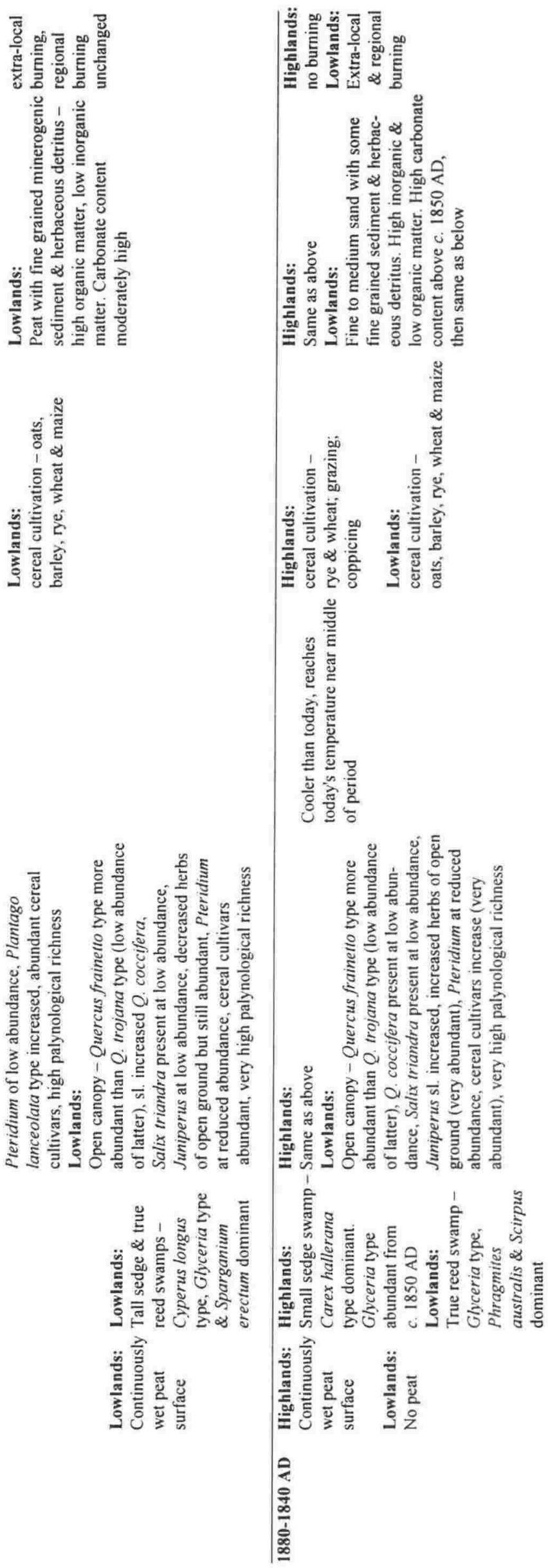

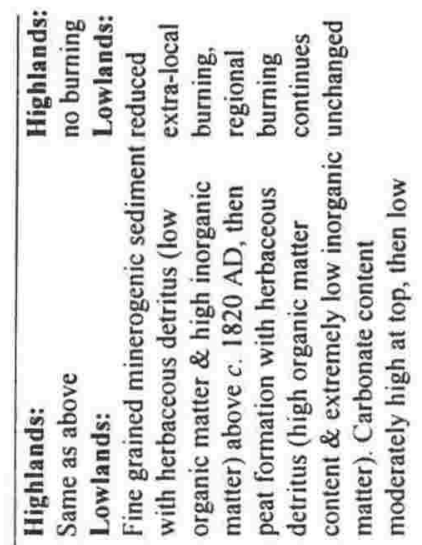

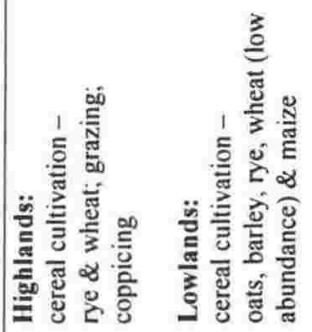

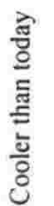

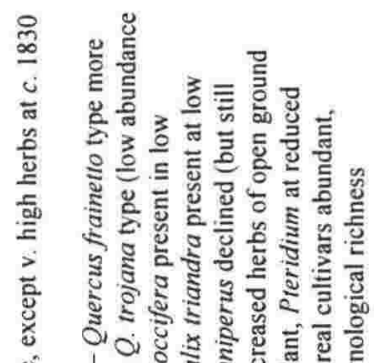

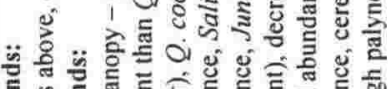

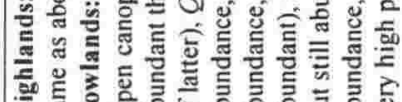

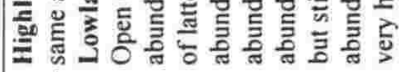

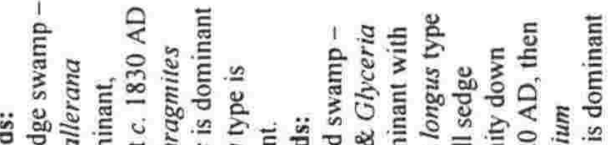

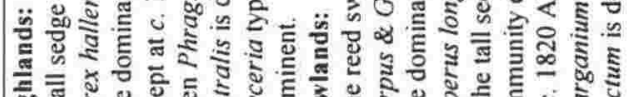

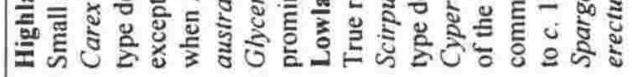

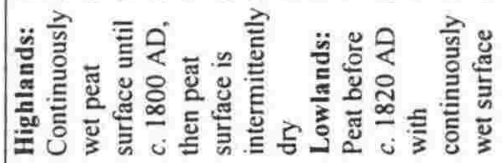




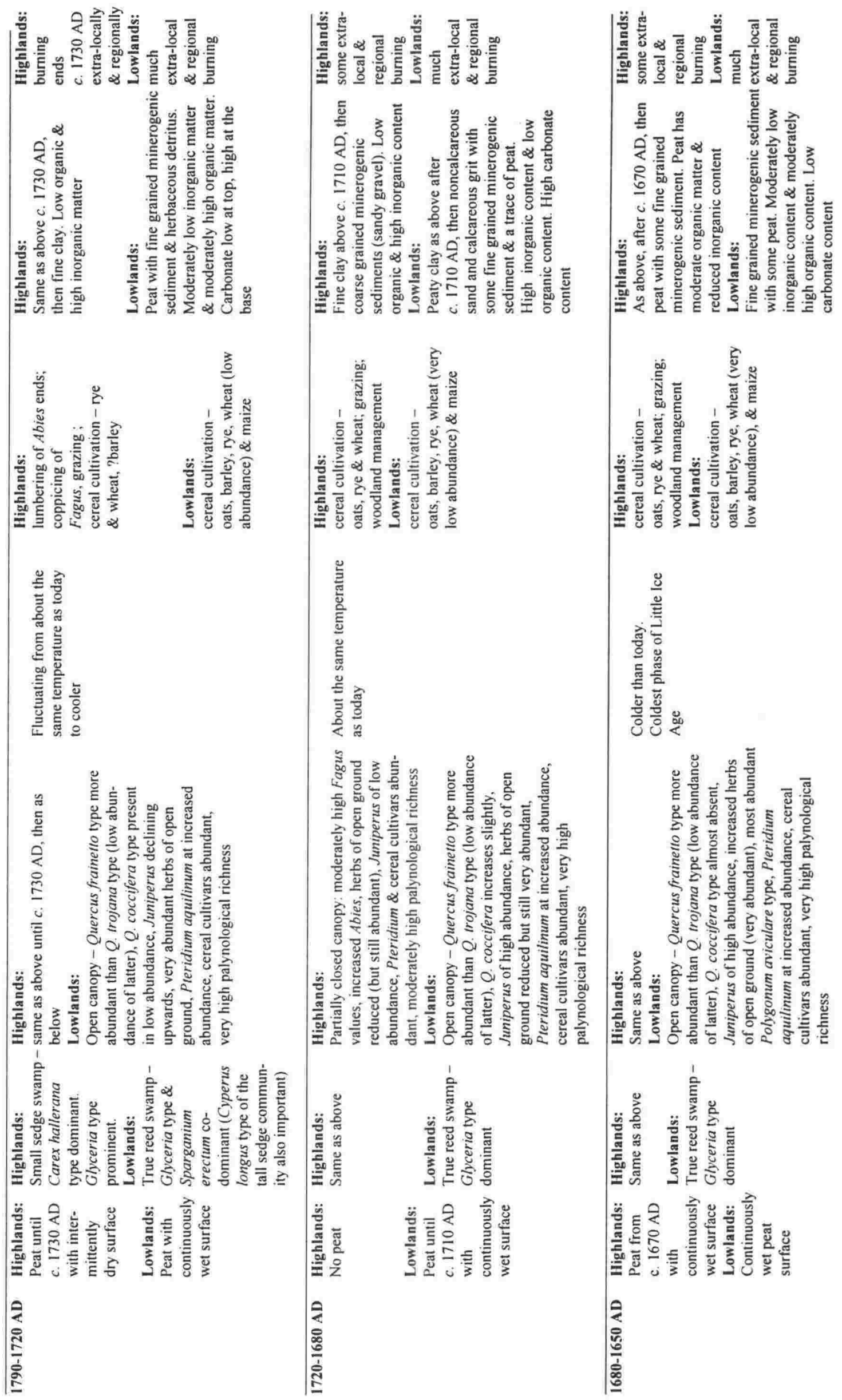




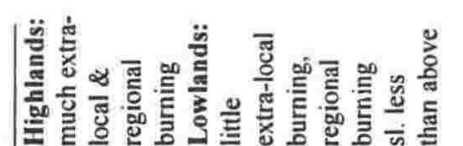

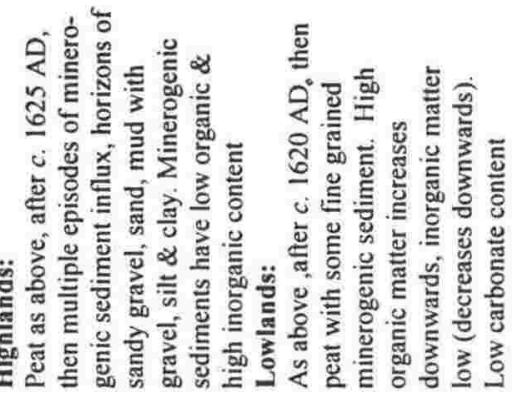

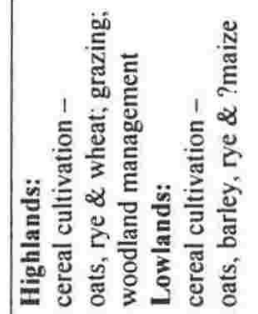

亚

高

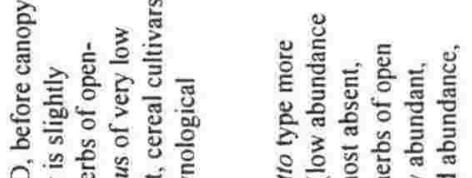

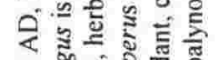

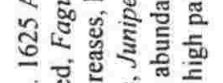

ن

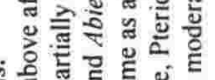

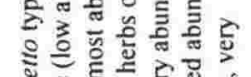

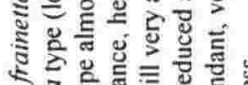

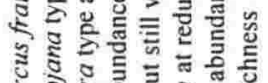

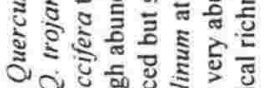
10,0 .

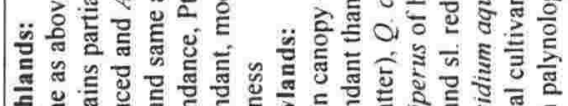
MIMIMInIm

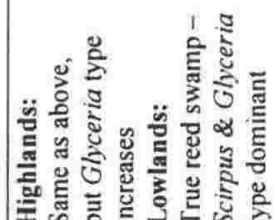

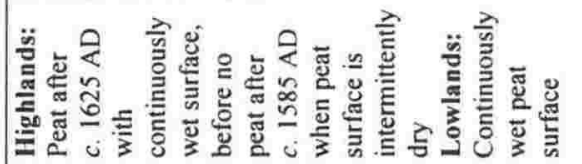

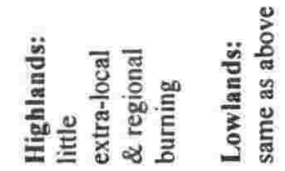

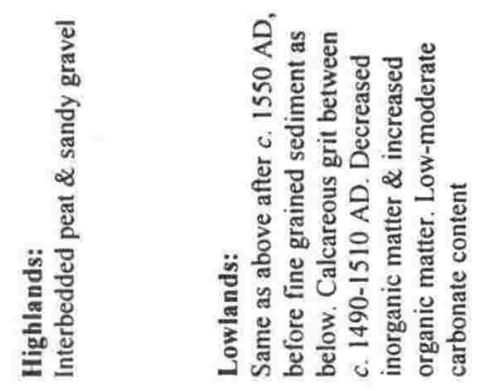

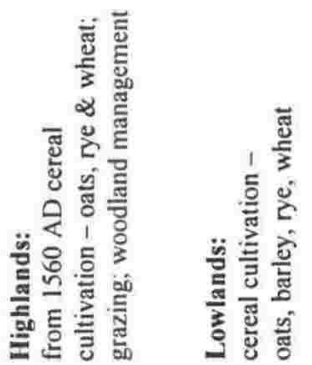

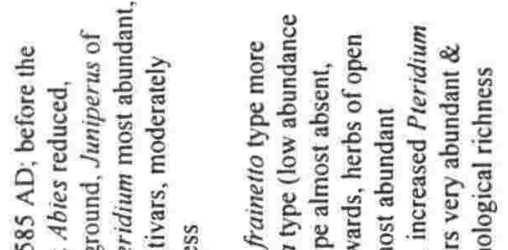

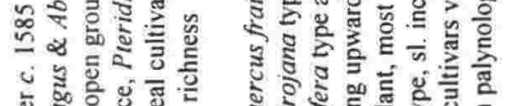

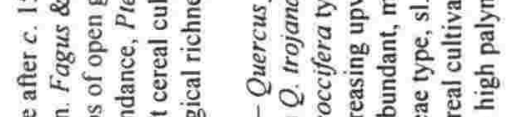

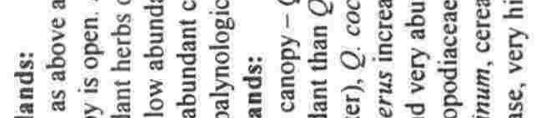

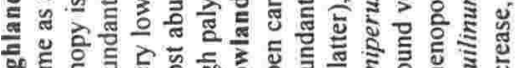

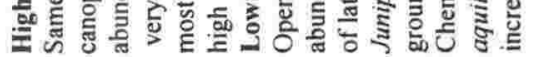

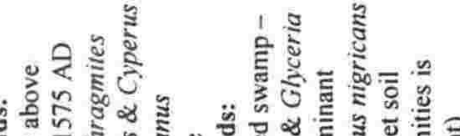

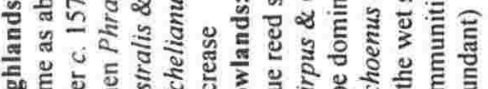

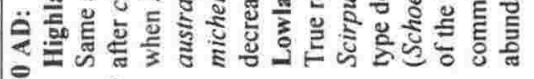

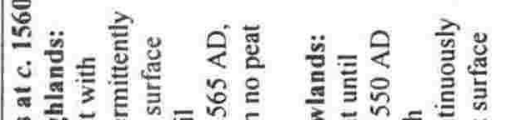

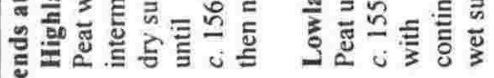

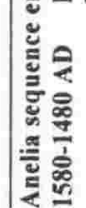

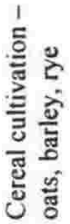

覀

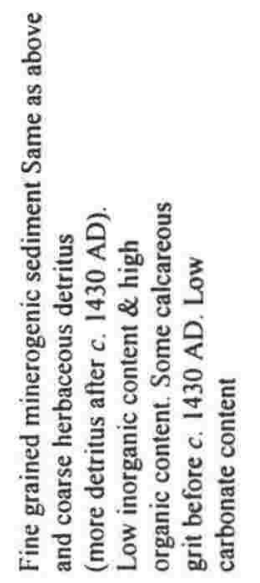

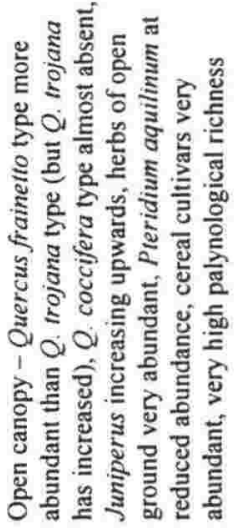

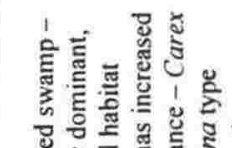

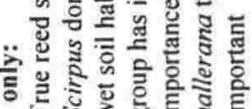

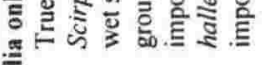




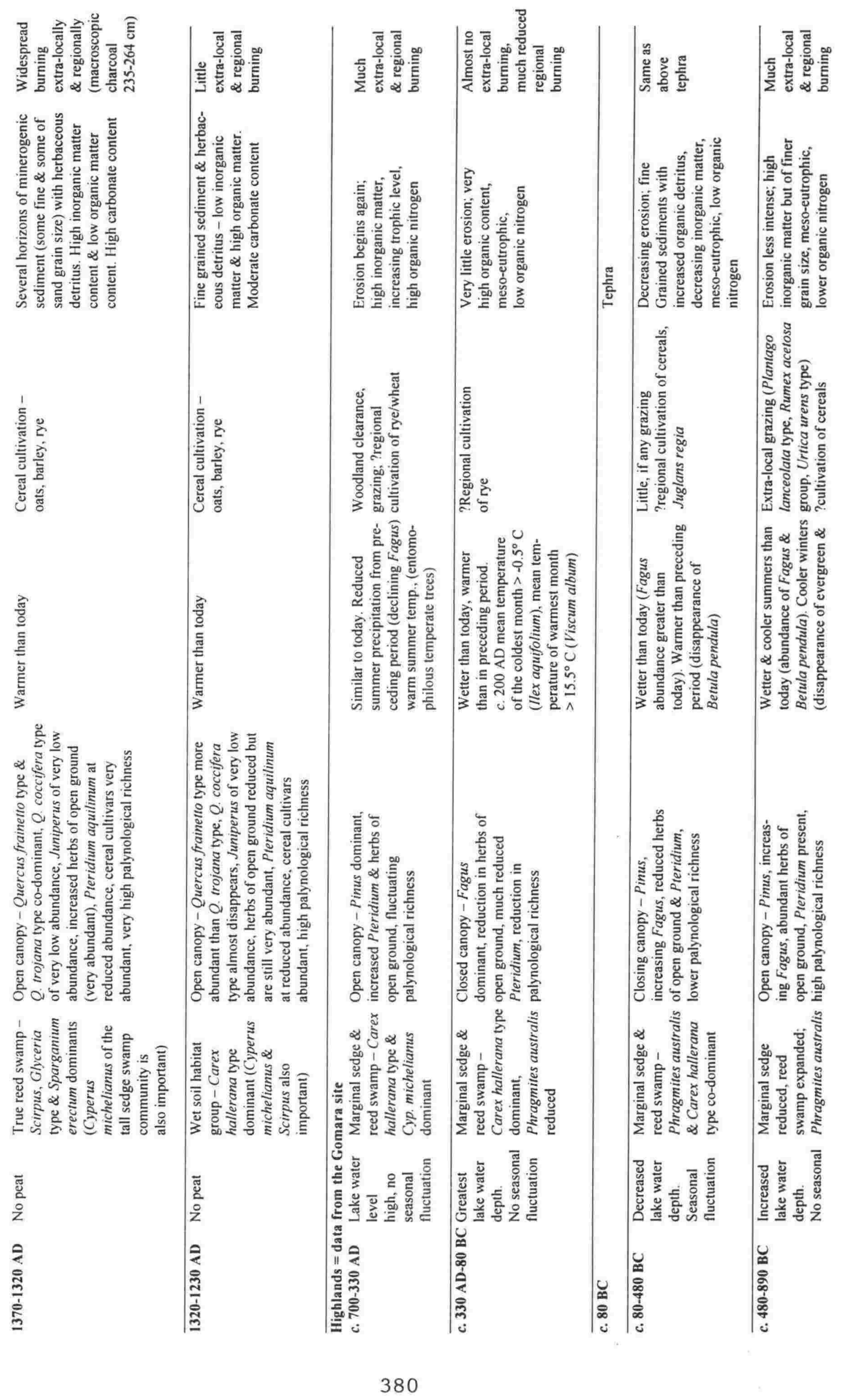




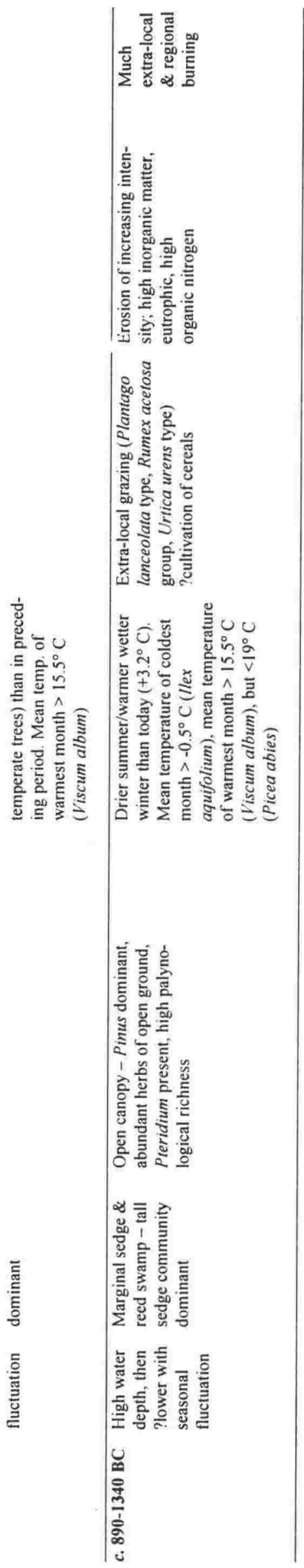


Broadly, the sediments represent two episodes of higher soil erosion rate, represented by sediments with a high proportion of inorganic matter, separated by a period of little soil erosion from $c .80 \mathrm{BC}$ to $330 \mathrm{AD}$ when sediments were highly organic. Only one abrupt stratigraphical change occurs; that at $107.2 \mathrm{~cm}, c .80 \mathrm{BC}$, where a catastrophic event is represented, that of a volcanic eruption. All other changes in lithology are indistinct and represent slower environmental changes.

Abundant microscopic charcoal particles were deposited during the period 1340-890 $\mathrm{BC}$, indicating frequent/intense regional burning of vegetation. After $890 \mathrm{BC}$ regional burning continued at a reduced but high level of frequency/intensity until c. $500 \mathrm{BC}$ at the end of the Archaic period. The largest microscopic charcoal particles, which probably derive from the catchment, were most abundant during the Late Bronze Age (1340-1100 BC), then declined sharply during the Iron Age until c. $850 \mathrm{BC}$, when local burning increased again and continued through the Archaic period. During the Classical and Hellenistic periods and most of the Roman Period little burning of regional highland and local vegetation around the Gomara site is indicated, with the exception of a short period at c. 200-250 AD, until the end of the Roman Period, c. $475 \mathrm{AD}$, when both regional highland and local burning around the Gomara site increased. During the Early Medieval, from c. 580-700 AD regional and local burning increased. Relative frequency data suggest that the intensity/frequency of burning at this time is similar to that of the Late Bronze Age, the most recent half of the Iron Age, and the Archaic, while influx data suggest lower intensity/frequency.

The highland regional vegetation was predominantly pine wood throughout the sequence, but the vegetation in the catchment changed greatly. Between c. 1340 and $890 \mathrm{BC}$ the catchment vegetation was an open canopy pine wood with a few deciduous trees, not as open as today. After $890 \mathrm{BC}$ there was increasing Fagus in the catchment, and the lake was surrounded by beech wood soon after this time. The beech wood was dominant in the catchment from c. $480 \mathrm{BC}$ until $330 \mathrm{AD}$, forming a very dense cover from $80 \mathrm{BC}$. At about $330 \mathrm{AD}$ a rapid decline in Fagus occurred, and pine wood-pasture similar to that of today is indicated.

Between c. 1340 and $890 \mathrm{BC}$ various herbs that may be indicative of grazing are abundant, especially terrestrial grasses, Chenopodiaceae type, Plantago lanceolata type, Rumex species, Solidago type, and Urtica species. Urtica in particular is nitrophilous and highly suggestive of animal-fertilised areas. The coincidence of evidence of burning of local vegetation, soil erosion, high lake trophic status and nitrogen content, and weeds possibly indicative of grazing all point to a landscape subject to active pastoralism. In addition the presence of cereal pollen in low abundance may indicate minor local cereal cultivation. 
Between c. 890 and $480 \mathrm{BC}$ less intense soil erosion is suggested by finer grain size of inorganic matter, a reduced average sedimentation accumulation rate, a decline in trophic level to meso-eutrophic and lower organic nitrogen content of the lake. However, herbs associated with pastoralism continued to occur in abundance, with an increase in Artemisia. Burning of the catchment and regional vegetation is again suggested by the occurrence of abundant macroscopic and microscopic charcoal particles and the greater abundance of pollen of the early-successional Betula pendula, and the occurrence of Pteridium aquilinum spores. It is inferred that pastoral activity continued, with repeated clearance of beech and pine woodland.

From c. $480 \mathrm{BC}$ to $80 \mathrm{BC}$ soil erosion declined further; minerogenic sediments are fine-grained, organic detritus increases, lake trophic level is meso-eutrophic and organic nitrogen content is low. Vegetation of the catchment was now mostly beech wood, with a much reduced pine component. The early successional types of Corylus and Betula pendula decline as the canopy of the catchment woodland becomes more closed. An almost complete absence of charcoal particles and Pteridium aquilinum spores suggests that burning of the catchment and the regional vegetation had almost ceased. Herbs associated with pastoralism declined, especially Urtica, but continued to be present. Pastoralism had effectively ceased during this period, although abundance of light-demanding herbs suggests that some open ground surrounded the lake, perhaps occasionally visited by stock being herded via the nearby mountain pass.

Between c. $80 \mathrm{BC}$ and $330 \mathrm{AD}$ little soil erosion occurs; minerogenic sedimentation declines and the proportion of organic detritus increases until sediments are almost entirely organic. Trophic level of the lake water is meso-eutrophic, and organic nitrogen content is low. Vegetation of the catchment is a closed-canopy beech wood. Burning of vegetation is not occurring, except perhaps at a long distance from the catchment. Light-demanding herbs record the lowest relative pollen frequencies of the sequence, and human cultural activity is inferred to be very minor. Juglans regia and Secale cereale pollen occurs at very low abundance, but at increased levels compared with previously; both may result from wild plants or from long-distance dispersal from cultivated areas.

Between c. 330 and $700 \mathrm{AD}$ more intense soil erosion occurred. In the wetland, inorganic sedimentation occurred; alkaliphilous diatoms increased, the trophic level increased, and organic-nitrogen content increased. The beech wood in the catchment began to decline at c. $330 \mathrm{AD}$. Pine wood replaced the beech wood, but then it declined at c. $600 \mathrm{AD}$, and an open canopy pine wood similar to that of today formed. Burning of the catchment and regional vegetation occurred again from c. $475 \mathrm{AD}$, both locally and regionally, and almost reached the levels of frequency/intensity of that between $c .1340$ and 
480 BC. Light-demanding herbs increase; particularly Urtica dioica and U. urens. These changes are interpreted to result from woodland clearance, initially by cutting, later by burning associated with pastoral activity.

Earliest finds of loomweights in Grevena date to the Hellenistic Period, and sheep/goat bone was found in association with a Hellenistic site in the lowlands, Grevena Project 318 (Wilkie 1995). The earliest archaeological evidence of occupation above $1000 \mathrm{~m}$ in the Pindos Mountains in Early Medieval times is from sites distributed in a pattern similar to that of modern transhumant villages, which suggests the existence of a pastoral economy. From historical information, transhumant pastoralism is known to have been practised in the Pindos Mountains and foothills in the Ottoman Period. The palynological interpretation presented above indicates two periods of pastoralism at Gomara: one of Late Bronze Age to Archaic, spanning c. $1340 \mathrm{BC}$ (or earlier) to $480 \mathrm{BC}$, the other Late Roman to Early Medieval or later, $c$. 330-700+ AD. The latter is coincident with the earliest Grevena Project highland archaeological sites, but the earlier period is so far unknown from archaeological data. However, from palynological study Willis (1992) has described woodland clearance and ensuing pastoralism dating from c. 4250-1900 BP at Rezina Marsh (1800 m asl) in the Pindos Mountains to the southwest.

During the period of pastoral abandonment of the site, c. $480 \mathrm{BC}$ to $330 \mathrm{AD}$, Fagus was dominant in the catchment. Bottema (1994:58) noted that the initial appearance or expansion of Fagus "all over Europe is always connected with human activity ... it profits from the appearance of abandoned, impoverished land, and clearings resulting from lumbering or from meadows that are no longer grazed". However, in the same paper Bottema ( $c f$. Bottema \& Woldring 1990) concludes that a change to wetter conditions may have lasted from c. 3200 to $1500 \mathrm{BP}$.

The climatic pattern interpreted from this site (Section 7.3) is a warm dry period from 1340 to $890 \mathrm{BC}$ (possibly with drier summers and warmer wetter winters than today), followed by a cooler period with wetter summers, then from $480-80 \mathrm{BC}$ a period that was wetter and warmer than today. From $80 \mathrm{BC}$ to $330 \mathrm{AD}$ the climate is interpreted as wetter than today and warmer than the preceding period. Between 330-700 AD climatic conditions were similar to today; summer precipitation was reduced from the preceding period and summer temperatures were warmer. This pattern of climatic change accords with the north and central European climatic sequence during the Sub-boreal/Sub-atlantic transition and early Sub-atlantic period described by Lamb (1982:132-135). Detailed pollen-analytical records from Greece and from sites of climatic sensitivity for the period in question are few, most sequences studied being from the lowlands and insufficiently dated (Chapter 1). However, Gerasimidis \& Athanasiadis (1995) have also described vegetation change in 
northern Greek mountains in terms of Blytt-Sernander climatic zones, noting an expansion of Fagus in the early Sub-atlantic. More well-dated pollen sequences are required to confirm a general pattern. At Gomara, while the expansion of beech woodland appears to have been aided by human abandonment, the primary role of climate is favoured.

\subsubsection{Anelia c. 1560 - 1989 AD}

At this site there is closer correspondence between changes in the composition of the deposits and changes in the pollen profiles of wetland taxa than of the pollen profiles of terrestrial taxa. The lack of exact correspondence is due to deposition of pollen and spore types both of taxa washed in from the catchment and of taxa deposited from the atmosphere from regional and extra-local source areas. Thus the terrestrial pollen record reflects both the extra-local and the regional vegetation.

There is very little apparent variation in the local wetland vegetation. It is dominated throughout the sequence by sedges of the Carex hallerana type of the 'wet soil' habitat group, which are probably indicative of a small sedge swamp community, although they may also represent dry habitats such as open woodland. The reeds of the types Phragmites australis and Glyceria type are also prominent. Communities of floating-leaved and submerged macrophytes are poorly represented and probably derive from a few plants growing in small pools on the wetland. The influence of minerals supplied by water from a soft-water spring, which maintained a high level of available nutrients, is evident throughout the sequence. Additional nutrient supply from anthropogenic activities (e.g. manure from grazing) is therefore difficult to detect. Where peat formation occurred surface peat was either continuously wet or only intermittently dry (probably only during the driest part of summer).

The sedimentary history of the wetland area is closely linked to the local wetland vegetation history, so major sedimentary units usually coincide with wetland pollen zones. The wetland formed in a hollow of a ravine. Broadening of the ravine and reduction in gradient caused impedance of spring-stream water flow, with consequent deposition of sediments. Between c. 1560 and $1730 \mathrm{AD}$ the wetland was in-filled with stratified clay, silt, sand, fine gravel, and peat, and mire vegetation established itself temporarily away from the stream course. At c. $1730 \mathrm{AD}$ peat formation became general, with a permanently marginal stream channel. This situation has persisted until today.

Microscopic charcoal evidence suggests that the most intense/frequent fires, both extralocally and regionally, occurred between $c$. 1585-1625 AD. Between $c$. 1560-1585 AD and c. 1625-1730 AD fire regimes were very similar in intensity/frequency and location, being of high intensity/frequency, but not as high as that of the period between, and occurring both extra-locally and regionally. From $c .1730 \mathrm{AD}$ to the present fires did not occur extra-locally 
and were much reduced regionally. The charcoal data are supported by pollen analysis. Fireencouraged Pteridium aquilinum is more abundant in the lower part of the sequence between c. 1560-1730 AD, being highest in the basal zone, c. 1560-1585 AD. In contrast, fire sensitive Juniperus was most abundant in the upper part of the sequence, especially after $1800 \mathrm{AD}$.

Between $c .1560$ and $1730 \mathrm{AD}$ the extra-local vegetation was a woodland dominated by Fagus and Abies, but after $c$. $1730 \mathrm{AD}$ it was dominated by Fagus only. For most of the period, the woodland canopy was open, but after $1920 \mathrm{AD}$ the beech woodland assumed a denser character.

For the period 1560-1730 AD, rapid and coarse-grained sedimentation and evidence for extra-local fires suggest woodland disturbance in the vicinity and possibly also regionally, although woodland surrounded the site itself. Pollen of light-demanding herbs is abundant, especially Artemisia, Chenopodiaceae type, Cirsium type, Dactylis type, Festuca type, Hieracium type, Plantago lanceolata type, Rumex acetosa group, and Solidago type. Together with abundant pollen of the ruderal Urtica this suggests grazing in the neighbourhood of the site.

From $1730 \mathrm{AD}$ absence of anthropogenic fires coincides with steady peat formation, suggesting reduction in soil erosion in the catchment. Pastoralism continued unabated, however, judging from the continued high abundance of herbs associated with grazing. The steady increase in Fagus after c. 1920 AD is thought to be due to cessation of woodland coppicing; evidence for former practise of this activity has been noted (Chapter 1).

A steady decline in Abies from c. 1600 AD to 1730 AD, followed by abrupt near disappearance is interpreted as result of selective lumbering. The coincidence of the latter date with cessation of burning and erosion may indicate that lumbering activities contributed to these effects, or that the lumbering formed part of a cultural land-use system, which changed after that date.

Cereal pollen, particularly Secale cereale and Triticum type, occur throughout the sequence before c. $1920 \mathrm{AD}$; Avena occurs until $1730 \mathrm{AD}$. The highly characteristic weeds of cultivation Agrostemma githago and Centaurea cyanus type also occur in a number of samples prior to c. $1920 \mathrm{AD}$. Together, these suggest nearby cultivation of wheat and oats, and possibly rye (although the last could be more distant). The trees Castanea sativa and Platanus orientalis were more abundant after c. $1730 \mathrm{AD}$, but not in such frequency to suggest extra-local cultivation. The increase is seen at Kellia (and other sites in northern Greece-Bottema 1974), and is probably due to increased planting regionally.

The association of grazing, lumbering, coppicing, and cereal cultivation reflects a highly exploited landscape and a cultural style similar to that reported for the Vlach villages 
by Leake (1835). The reduction in woodland management and cereal cultivation since c. $1920 \mathrm{AD}$ also matches well the changes in land use that have accompanied the introduction of mechanised transport and farming methods and disruption caused by war.

\subsubsection{Kellia c. 1230 - 1989 AD}

The sedimentary history of Kellia fen is intimately linked to the local wetland vegetation history, and there is close correspondence between the composition of deposits and changes in the pollen profiles of the wetland, but there is less correspondence between sediment composition and terrestrial pollen and spore profiles. Absence of total synchrony of these three factors is due to a significant contribution of pollen and spores derived from outside the catchment.

Stratigraphic data indicate that Kellia fen probably formed at the side of a stream course, which ran from west to east on the southern side of the fen. Radiocarbon dating of the pollen-analysed core suggests that two periods of alluviation are recorded in the fen sediments, an older period below $312 \mathrm{~cm}$ (beginning perhaps $c .830 \mathrm{BC}$ and spanning about 200 years, see Section 6.4.2) and a younger one above which may span about the last 760 years, beginning c. $1230 \mathrm{AD}$. Since the earlier period is not securely dated, it is not further discussed here.

The fen sediments comprise greyish olive to olive-black clay with variable proportions of calcareous grit, peat, and organic detritus. Thin $(5-15 \mathrm{~cm})$ noncalcareous sand horizons are rare. Persistent organic horizons occur at about 100 and $230 \mathrm{~cm}$. The uppermost c. $20 \mathrm{~cm}$ (c. 1900-1989 AD) of the fen consists of silty peat and brown clay with a sandy layer at the base. This unit may be related to the establishment of the modern sealed road and its causeway, which may have reduced the transport of sediment into the fen from the catchment upstream.

The local wetland vegetation is dominated by pollen and spore types derived from emergent plant communities of wet-soil, tall-sedge-swamp and true-reed-swamp communities, the last contributing the most pollen. The true-reed-swamp communities are dominated by reeds of Glyceria type and Scirpus and to a lesser extent by Phragmites australis and Sparganium erectum. The wet-soil communities are dominated by sedges of the Carex hallerana type and Schoenus nigricans, and Equisetum occurs sporadically. Pollen types that dominate the tall-sedge-swamp communities are Carex flacca type, Cyperus longus type, and Cyperus michelianus. Communities of floating-leaved and submerged macrophytes are poorly represented; they probably comprise a few plants growing in small pools on the surface of peat. The available nutrient level is high throughout the sequence, 
as evidenced by the abundance of several pollen types representing wetland plants indicative of high trophic status.

From microscopic charcoal particles, during the period c. 1230-1320 AD fires were apparently uncommon either extra-locally or regionally. After c. $1320 \mathrm{AD}$ until c. $1370 \mathrm{AD}$ there was considerable extra-local and probably also regional burning. From $c .1370$ AD regional burning continued at a fairly uniform frequency/intensity, but extra-local burning was at a low level until c.1650 AD. From this date, extra-local burning fluctuates, with peaks during the periods c. $1650-1690 \mathrm{AD}$, c. $1750-1790 \mathrm{AD}$, c. $1860-1880 \mathrm{AD}$, and at c. $1890 \mathrm{AD}$ and c. $1940 \mathrm{AD}$. Pteridium aquilinum increased between c. 1350-1360 AD, and the fire-sensitive Juniperus was less abundant in the lower part of the sequence before c. 1370 AD. After c. 1370 AD Juniperus increased. Pteridium aquilinum again became abundant from c. 1640 to $1790 \mathrm{AD}$.

The extra-local and regional lowland vegetation is open canopy deciduous/semievergreen oak woods dominated by the canopy trees of Quercus frainetto type (deciduous oaks) and $Q$. trojana type (deciduous/semi-evergreen oaks). Ostrya type occurs at low abundance almost continuously. The ratio of tree and shrub pollen to that of terrestrial herbs fluctuates through the period c. $1230 \mathrm{AD}$ to present, but generally high herb levels indicate a low density of trees and extensive steppe and open land. This is supported by high levels of palynological richness.

Weeds typical of human disturbance occur continuously throughout the sequence at significant abundance, especially: Centaurea solstitialis type, Chenopodiaceae type, Cichorium type, Cirsium type, Galium type, Hieracium type, Lactuca type, Plantago lanceolata type, Polygonum aviculare type, Solidago type, Sonchus type, and Taraxacum type.

A variety of cereals was cultivated extra-locally for the duration of the sequence, pollen types identified being Avena, Hordeum type, Secale cereale, Triticum type, and Zea mays. A notable peak in frequency in most types (except Triticum) occurred between c. 1480$1580 \mathrm{AD}$. Triticum type occurs in very low abundance before $c .1730 \mathrm{AD}$ but is relatively common after c. $1870 \mathrm{AD}$, while Avena almost disappears after c. $1900 \mathrm{AD}$. As noted in Section 7.4.4, weeds of cultivation are prevalent, particularly Centaurea cyanus type. Low frequencies of Castanea sativa and Juglans regia occur sporadically from c. $1230 \mathrm{AD}$ in insufficient abundance to suggest any extensive planting in the vicinity of the site. The shade tree Platanus orientalis increases, although rather irregularly, after c. 1650 AD. The increased abundance of pollen of this species regionally has already been noted in discussion of the Anelia results. 
The abundance of cereal pollen supports intensive extra-local cereal cultivation since c. $1230 \mathrm{AD}$. In the presence of cultivation, it is difficult to distinguish the signature of pastoralism, as most weeds of disturbance occur in association with both activities.

A climate sequence, based on the ratio of pollen of Quercus frainetto type (deciduous oaks) to Quercus trojana type (semi-evergreen/deciduous oaks) and Quercus coccifera type (evergreen oaks) discussed in Section 7.3, has been used to interpret temperature changes in the lowlands (Table 7.1). The record of the deciduous/semi-evergreen Quercus pollen curve agrees in its general features with tree-ring and documentary records from Europe (Lamb 1982). For example, the coldest periods indicated by the Kellia oak curve match the cold periods indicated by tree-ring density from Lauenen, Switzerland (Stuiver 1980). The greatest abundance of the deciduous oaks (Quercus frainetto type) and the lowest abundance of semi-evergreen/deciduous oaks (Quercus trojana type) occurs at $c .1680 \mathrm{AD}$ and shows good correspondence with the lowest temperatures indicated at $1690 \mathrm{AD}$ and the culmination of the Little Ice Age, which occurred between c. 1660 and 1680 AD (Lamb 1982; Grove 1988). 


\section{References}

Aaby, B. 1986. Palaeoecological studies of mires. In, B.E. Berglund (ed), Handbook of Holocene palaeoecology and palaeohydrology: 145-164. Reprinted 1991. John Wiley \& Sons Ltd, New York.

Aaby, B. and G. Digerfeldt 1986. Sampling techniques for lakes and bogs. In, B.E. Berglund (ed), Handbook of Holocene palaeoecology and palaeohydrology: 181-194. Reprinted 1991. John Wiley \& Sons Ltd, New York.

Adamakopoulos, P. 1989. Contribution to the study of the flora of the NE Pindos. Unpublished report to the Forest Service of Grevena.

Aitken, M.J. 1990. Science-based dating in archaeology. Longman Inc., New York.

Allen, H. 1986. Late Quaternary of the Kopais Basin, Greece: sedimentary and environmental history. Unpublished $\mathrm{PhD}$ thesis. University of Cambridge, Cambridge.

Allen, H. 1990. A Postglacial record from the Kopais Basin, Greece. In, S. Bottema, G. Entjes-Nieborg and W. van Zeist (eds), Man's role in the shaping of the Eastern Mediterranean landscape: 173-182. A.A. Balkema, Rotterdam.

Andersen, S.T. 1960. Silicone oil as a mounting medium for pollen grains. Danmarks Geologiske Undersфgelse. Rakke IV, 4 (1): 6-24.

Andersen, S.T. 1965. Mounting media and mounting techniques. In, B. Kummel and D. Raup (eds) 1965. Handbook of paleontological techniques: 587-598. W.H. Freeman and Company, San Francisco and London.

Andersen, S.T. 1967. Tree-pollen rain in a mixed deciduous forest in south Jutland (Denmark). Review of palaeobotany and palynology 3: 267-275.

Andersen, S.T. 1970. The relative pollen productivity and pollen representation of north European trees, and correction factors for tree pollen spectra. Determined by surface pollen analyses from forests. Danmarks Geologiske Unders $\emptyset$ gelse. Rakke II, (96).

Andersen, S.T. 1973. The differential pollen productivity of trees and its significance for the interpretation of a pollen diagram from a forested region. In, H.J. B. Birks and R.G. West (eds), Quaternary plant ecology: 109-115. Blackwell scientific publications, London.

Andersen, S.T. 1974. Wind conditions and pollen deposition in a mixed deciduous forest. Grana 14: 64-77.

Andersen, S.T. 1978. On the size of Corylus avellana L. pollen mounted in silicone oil. Grana 17: 5-13.

Andersen, S.T. 1979. Identification of wild grass and cereal pollen. Danmarks Geologiske Undersøgelse, årbog 1978: 69-92.

Andersen, S.T. 1986. Palaeoecological studies of terrestrial soils. In, B.E. Berglund (ed), Handbook of Holocene palaeoecology and palaeohydrology: 165-177. Reprinted 1991. John Wiley \& Sons Ltd, New York.

Art, H.W. (ed) 1993. The dictionary of ecological and environmental science. Henry Holt \& Company, New York.

Aschenbrenner, S.E. 1987. Ethnoarchaeology of agricultural economy. Unpublished Grevena Project report.

Aschenbrenner, S.E. 1988. Field report - Grevena Project. Unpublished Grevena Project report.

Aschenbrenner, S.E. in prep. Ethnoarchaeology of agricultural economy in the nomos of Grevena.

Athanasiadis, N. 1975. Zur postglazialen vegetationsentwicklung von Litochoro Katerinis und Pertouli Trikalon (Griechenland). Flora 164: 99-132. (In German with English summary.) 
Athanasiadis, N. and A. Gerasimidis 1986. Zur postglazialen vegetationsentwicklung von Boras - Gebirge (Almopia - Griechenland). Scientific annals of the Department of Forestry and Natural Environment, Aristotelian University of Thessaloniki 29 (4): 211-249. (In Greek with German summary.)

Athanasiadis, N. and A. Gerasimidis 1987. Zur postglazialen vegetationsentwicklung des Paikon-Gebirges (Nord Griechenland). Scientific annals of the Department of Forestry and Natural Environment, Aristotelian University of Thessaloniki 30 (11): 403-445. (In Greek with German summary.)

Athanasiadis, N., A. Gerasimidis, E. Eleftheriadou and K. Theodoropouulos 1993. Zur postglazialen vegetationsentwicklung des Rhodopi-Gebirges (Elatia DramasGriechenland.). Dissertationes botanicae 196: 427-437. (In German with English summary.)

Atherden, M. 1989. Notes on the Strymon 1 core. Unpublished notes.

Atherden, M., J. Hall and J.C. Wright 1993. A pollen diagram from the northeast Peloponnese, Greece: implications for vegetation history and archaeology. Holocene 3,4: 351-356.

Baillie, M.G.L. and M.A.R. Munro 1988. Irish tree rings, Santorini and volcanic dust veils. Nature 332: 344-346.

Ball, D.F. 1964. Loss-on-ignition as an estimate of organic matter and organic carbon in noncalcareous soils. Journal of soil science 15 (1): 84-92.

Barkley, F.A. 1934. The statistical theory of pollen analysis. Ecology 15: 283-289.

Bayliss-Smith T.P. 1982. The ecology of agricultural systems. Cambridge University Press, London.

Behre, K.-E. 1981. The interpretation of anthropogenic indicators in pollen diagrams. Pollen et spores 23: $225-245$.

Behre, K.-E. 1990. Some reflections on anthropogenic indicators and the record of prehistoric occupation phases in pollen diagrams from the Near East. In, S. Bottema, G. EntjesNieborg and W. van Zeist (eds), Man's role in the shaping of the Eastern Mediterranean landscape: 219-230. A.A. Balkema, Rotterdam.

Belmonte, J., R. Pérez-Obiol and J.M. Roure 1986. Claves para la determinación de los pólenes de las principales especies melíferas de la Península Ibérica. Orsis 2: 27-54.

Bengtsson, L. and M. Enell 1986. Chemical analysis. In, B.E. Berglund (ed), Handbook of Holocene palaeoecology and palaeohydrology: 423-451. Reprinted 1991. John Wiley \& Sons Ltd, New York.

Bennett, K.D. 1994. Psimpoll 2.23. Department of Plant Sciences, Cambridge University, Cambridge.

Bennett, K.D. 1996. Determination of the number of zones in a biostratigraphical sequence. New phytologist 132: 155-170.

Bennett, K.D. and R.W. Humphry 1995. Analysis of late-glacial and Holocene rates of vegetational change at two sites in the British Isles. Review of palaeobotany and palynology 85: 263-287.

Benninghoff, W.S. 1960. Pollen spectra from bryophytic polsters, Inverness Mud Lake bog, Cheboygan County, Michigan. Papers of the Michigan Academy of Science, Arts, and Letters 45: 41-60.

Benninghoff, W.S. 1962. Calculation of pollen and spores density in sediments by addition of exotic pollen in known quantities. Pollen et spores 4 (2): 332-333.

Berglund, B.E. 1986a. Handbook of Holocene palaeoecology and palaeohydrology. Reprinted 1991. John Wiley \& Sons, New York. 
Berglund, B.E. 1986b. Palaeoecological reference areas and reference sites. In, B.E. Berglund (ed), Handbook of Holocene palaeoecology and palaeohydrology: 111-126. Reprinted 1991. John Wiley \& Sons, New York.

Berglund, B.E. and M. Ralska-Jasiewiczowa 1986. Pollen analysis and pollen diagrams. In, B.E. Berglund (ed), Handbook of Holocene palaeoecology and palaeohydrology: 455-484. Reprinted 1991. John Wiley \& Sons, New York.

Berry, L.G. and B. Mason 1959. Mineralogy: concepts, descriptions, determinations: 188-189. W.H. Freeman \& Co., San Francisco.

Beug, H.-J. 1961a. Leitfaden der Pollenbestimmung für Mitteleuropa und angrenzende Gebiete. Gustav Fischer Verlag, Stuttgart.

Beug, H.-J. 1961b. Beitrage zur Postglazialen Floren- und Vegetationsgeschichte in Suddalmatien; der See "Malo Jezero" auf Mljet. Flora 150 (4): 631-659.

Biel, E.R. 1944. Climatology of the Mediterranean area. The University of Chicago Press, Chicago.

Bintliff, J. 1977. Natural environment and human settlement in prehistoric Greece. British archaeological reports, supplementary series 28,2 vols.

Bintliff, J. 1992. Erosion in the Mediterranean lands: a reconsideration of pattern, process and methodology. In, M. Bell and J. Boardman (eds), Past and present soil erosion. Archaeological and geographical perspectives. Oxbow monograph 22: 125-132. Oxbow Books, Oxford.

Birks, H.J.B. 1968. The identification of Betula nana pollen. New phytologist 67: 309-314.

Birks, H.J.B. 1973. Past and present vegetation of the Isle of Skye. A palaeoecological study. Cambridge University Press, Cambridge.

Birks, H.J.B. 1977. Modern pollen rain and vegetation of the St. Elias Mountains, Yukon Territory. Canadian journal of botany 55: 2367-2382.

Birks, H.J.B. 1981. Pollen analysis and climate reconstruction. In, T.M.L. Wigley, M.J. Ingram and G. Farmer, Climate and history:111-138. Cambridge University Press, Cambridge.

Birks, H.J.B 1986. Late-Quaternary biotic changes in terrestrial and lacustrine environments, with particular reference to north-west Europe. In, B.E. Berglund, Handbook of Holocene palaeoecology and palaeohydrology: 3-8. Reprinted 1991. John Wiley \& Sons Ltd, New York.

Birks, H.J.B. and H.H. Birks 1980. Quaternary Palaeoecology. Edward Arnold, London.

Birks, H.J.B. and A.D. Gordon 1985. Numerical methods in Quaternary pollen analysis. Academic Press, London.

Birks, H.J.B. and J.M. Line 1992. The use of rarefaction analysis for estimating palynological richness from Quaternary pollen-analytical data. The Holocene 2 (1): 1-10.

Birks, H.J.B. and R.G. West (eds) 1973. Quaternary plant ecology. Blackwell scientific publications, Oxford.

Blackford, J. 1994. Peat bogs as sources of proxy climatic data: past approaches and future research. In, F.M. Chambers (ed), Climate change and human impact on the landscape: 47-65. Chapman \& Hall, London.

Bloemendal, J., F. Oldfield and R. Thompson 1979 . Magnetic measurements used to assess sediment influx at Llyn Goddionduon. Nature 280:50-53

Boelter, D.H. 1969. Soil and water management and conservation. Soil Science Society of America proceedings 33: 606-609.

Bonny, A.P. 1972. A method for determining absolute pollen frequencies in lake sediments. New phytologist 71 : 393-405.

Bonny, A.P. 1976. Recruitment of pollen to the seston and sediment of some lake district lakes. Journal of ecology 64: 859-887. 
Bormann, F.H., G.E. Likens and J.S. Eaton 1969a. Biotic regulation of particulate and solution losses from a forest ecosystem. Bio-science 19: 600-610.

Bormann, F.H., G.E. Likens, D.W. Fisher and R.S. Pierce 1969b. Nutrient loss accelerated by clear-cutting of a forest ecosystem. Science 159: 882-884.

Bormann, F.H., G.E. Likens, T.G. Siccama, R.S. Pierce and J.S. Eaton 1974. The export of nutrients and recovery of stable conditions following deforestation at Hubbard Brook. Ecological monographs 44 (3): 255-277.

Bormann, F.H., T.G. Siccama, G.E. Likens and R.H. Whitaker 1970. The Hubbard Brook ecosystem study: composition and dynamics of the tree stratum. Ecological monographs 40: 373-388.

Bottema, S. 1967. A Late Quaternary pollen diagram from Ioannina, north-western Greece. Appendix in, E.S. Higgs, C. Vita-Finzi, D.R. Harris and A.E. Fagg, The climate, environment and industries of Stone Age Greece: Part III. Proceedings of the Prehistoric Society 33: 26-29.

Bottema, S. 1974. Late Quaternary vegetation history of northwestern Greece. Doctoral dissertation, Rijsuniversiteit te Gronigen, Gronigen, Netherlands.

Bottema, S. 1975. Reconstruction of the Late Quaternary vegetation of Northwestern Greece. Problems of Balkan flora and vegetation: 58-63.

Bottema, S. 1978. The Late Glacial in the Eastern Mediterranean and the Near East. In, W.C. Brice (ed), The environmental history of the Near and Middle East since the last Ice Age: 15-28. Academic Press, London.

Bottema, S. 1979. Pollen analytical investigations in Thessaly (Greece). Palaeohistoria 21: 20-40.

Bottema, S. 1980. On the history of the walnut (Juglans regia L.) in southeastern Europe. Acta botanica Neerlandica 29 (5/6): 343-349.

Bottema, S. 1981. Palynological dating of some young Holocene sediments from three Greek lakes. Appendix. In, K.M. Creer, P.M. Readman and S. Papamarinopoulos, Geomagnetic secular variations in Greece through the last 6000 years obtained from lake sediment studies. Geophysical journal of the Royal Astronomical Society 66: 217-219.

Bottema, S. 1982. Palynological investigations in Greece with special reference to pollen as an indicator of human activity. Palaeohistoria 24: 257-289.

Bottema, S, 1988. A reconstruction of the Halos environmenton the basis of palynological information. Appendix I. In, H.R. Reindeers, New Halos, a Hellenistic town in Thessalia, Greece: 216-226. Doctoral dissertation, Rijsuniversiteit te Groningen, Groningen, Netherlands.

Bottema, S. 1990. Holocene environment of the Southern Argolid: a pollen core from Kiladha Bay. In, T.J. Wilkinson and S.T. Duhon (with contributions by J.A. Gifford and S. Bottema), Franchthi Paralia: 117-138. Indiana University Press, Bloomington.

Bottema, S. 1994. The prehistoric environment of Greece: a review of the palynological record. In, P.N. Kardulias (ed), Beyond the site: regional studies in the Aegean: 45-68. University Press of America, Lanham (MD).

Bottema, S. and H. Woldring 1990. Anthropogenic indicators in the pollen record of the Eastern Mediterranean. In, S. Bottema, G. Entjes-Nieborg and W. van Zeist (eds), Man's role in the shaping of the Eastern Mediterranean landscape: 231-264. A.A. Balkema, Rotterdam.

Boyd, W.E. 1986. The role of mosses in modern pollen analysis: the influence of moss morphology on pollen entrapment. Pollen et spores 28 (2): 243-256.

Bradshaw, R.H.W. 1981. Modern pollen-representation factors for woods in south-east England. Journal of ecology 69: 45-70. 
Bradshaw, R.H.W. and T. Webb III 1985. Relationships between contemporary pollen and vegetation data from Wisconsin and Michigan, USA. Ecology 66 (3): 721-737.

Brookes, D. and K.W. Thomas 1968. The distribution of pollen grains in microscope slides. Part I. The non-randomness of the distribution. Pollen et spores 9 (1967): 621-630.

Brown, C.A. 1960. Palynological techniques. Privately published, Baton Rouge.

Brubaker, L.B. 1986. Responses of tree populations to climatic change. Vegetatio 67: 119-130.

Butzer, K.W. 1974. Accelerated soil erosion: a problem of man-land relationships. In, I.R. Manners and M.W. Mikesell (eds), Perspectives on the environment: 57-78. Association of American geographers, Washington, D.C.

Carroll, G. 1943. The use of bryophytic polsters and mats in the study of recent pollen deposition. American journal of botany 30: 361-366.

Cerceau, M.-Th. 1959. Clé de détermination d'Ombellifères de France et d'Afrique du Nord d'après leurs grains de pollen. Pollen et spores 1: 145-190.

Chanda, S. 1962. On the pollen morphology of some Scandinavian Caryophyllaceae. Grana palynologica 3: 67-89.

Chappell, J. 1978. Landscape changes involving biologic interaction. In, D. Walker and J. C. Guppy (eds), Biology and Quaternary environments: 1-34. Australian Academy of Science, Canberra.

Chester, P.I. (Compiler) 1989a. Species lists for pollen surface sample collection sites. Unpublished Grevena Project report.

Chester, P.I. (Compiler) 1989b. Species lists for reconnaissance vegetation surveys (where no surface pollen sample was collected). Unpublished Grevena Project report.

Chester, P.I. (Compiler) 1989c. Herbarium specimens collected in Grevena Province. Unpublished Grevena Project report.

Chester, P.I. 1991. Recent vegetation history of Mana Island: palynological investigations. Science \& research series 28, New Zealand Department of Conservation.

Clark, H.E. 1951. An atmospheric pollen survey of four centres in the North Island, New Zealand, 1949-50. New Zealand journal of science and technology, section B 33 (2): 73-91.

Clark, J.S. 1988. Particle motion and the theory of charcoal analysis: source area, transport, deposition, and sampling. Quaternary research 30: 67-80.

Clark, M.H. 1988. The region of Grevena modern settlement and population history II. The regional trade system. Unpublished Grevena Project report.

Clark, R.L. 1984. Effects on charcoal of pollen preparation procedures. Pollen et spores 26 (3-4): 559-576.

Creer, K.M., P.M. Readman and S. Papamarinopoulos 1981. Geomagnetic secular variations in Greece through the last 6000 years obtained from lake sediment studies. Geophysical journal of the Royal Astronomical Society 66: 193-210.

Cruise, G.M. 1990. Holocene peat initiation in the Ligurian Apennines, northern Italy. Review of palaeobotany and palynology 63: 173-182.

Cushing, E.J. 1967. Evidence for differential pollen preservation in Late Quaternary sediments in Minnesota. Review of palaeobotany and palynology 4: 87-101.

Cushing, E.J. and H.E. Wright 1967. Introduction. In, E.J. Cushing and H.E. Wright (eds), Quaternary paleoecology: 1-8. Yale University Press, New Haven.

Davidson, D.A. 1980. Erosion in Greece during the first and second millenia BC. In, R.A. Cullingford, D.A. Davidson and J. Lewin (eds), Time scales in geomorphology: 143-158. John Wiley \& Sons Ltd, London. 
Davis, M.B. 1963. On the theory of pollen analysis. American journal of science 261: 897-912.

Davis, M.B. 1965. A method for determination of absolute pollen frequncy. In, B. Kummel and D. Raup (eds), Handbook of paleontological techniques: 674-686. W.H. Freeman and Company, San Francisco and London.

Davis, M.B. 1966. Determination of absolute pollen frequency. Ecology 47 (2): 310-311.

Davis, M.B. 1967. Pollen accumulation rates at Rogers Lake, Connecticut, during Late and Postglacial time. Review of palaeobotany and palynology 2: 219-230

Davis, M.B. 1969. Palynology and environmental history during the Quaternary Period. American scientist 57: 317-332.

Davis, M.B. 1976. Erosion rates and land-use history in southern Michigan. Environmental conservation 3 (2): 139-148.

Davis, M.B. 1978. Climatic interpretation of pollen in Quaternary sediments. In, D. Walker and J.C. Guppy (eds), Biology and Quaternary environments: 35-51. Australian Academy of Science, Canberra.

Davis, M.B. and D.B. Botkin 1985. Sensitivity of cool temperature forests and their fossil pollen record to rapid temperature changes. Quaternary research 23: 327-340.

Davis, M.B. and E.S. Deevey, Jr 1964. Pollen accumulation rates: estimates from late-glacial sediments of Rogers Lake. Science 145: 1293-1295.

Davis, M.B. and M.S. Ford 1982. Sediment focusing in Mirror Lake, New Hampshire. Limnology and oceanography 27 (1): 137-150.

Dean, W.D. Jr. 1974. Determination of carbonate and organic matter in calcareous sediments and sedimentary rocks by loss on ignition: comparison with other methods. Journal of sedimentary petrology 44 (1): 242-248.

Dearing, J.A. 1986. Core correlation and total sediment influx. In, B.E. Berglund (ed), Handbook of Holocene palaeoecology and palaeohydrology: 247-270. Reprinted 1991. John Wiley \& Sons Ltd, New York.

Dearing, J.A., J.K. Elner and C.M. Happey-Wood 1981. Recent sediment flux and erosional processes in a Welsh upland lake-catchment based on magnetic susceptibility measurements. Quaternary research 16: 356-372.

de Beaulieu, J.L. 1982. Palynological subdivision of the Holocene in France. Striae 16: 106-109.

Deevey, E.S., Jr. 1965. Sampling lake sediments by use of the Livingstone sampler. In, B. Kummel and D. Raup (eds) 1965. Handbook of paleontological techniques: 521-529. W.H. Freeman and Company, San Francisco and London.

Delcourt, P.A. and H.R. Delcourt 1980. Pollen preservation and Quaternary environmental history in the southeastern United States. Palynology 4: 215-231.

Delorme, L.D. 1969. Ostracodes as Quaternary paleoeclogical indicators. Canadian journal of earth science 6: 1471-1476.

Delorme, L.D., S.C. Zoltai and L.L. Kalas 1977. Freshwater shelled invertebrate indicators of paleoclimate in northwestern Canada during late glacial times. Canadian journal of earth science 14: 2029-2046.

Demitrack, A. 1986. The late Quaternary geologic history of the Larissa Plain Thessaly, Greece: tectonic, climatic, and human impact on the landscape. Unpublished $\mathrm{PhD}$ thesis, Stanford University.

Dennell, R.W. 1972. The interpretation of plant remains: Bulgaria. In E.S Higgs (ed), Papers in economic prehistory: 149-159. Cambridge University Press, Cambridge.

Digerfeldt, G. 1972. The post-glacial development of Lake Trummen: regional vegetation history, water level changes and palaeolimnology. Folia limnologica Scandinavica 16: 1-104. 
Dimbleby, G.W. 1985. The palynology of archaeological sites. Academic Press, London.

Donaldson, A.M. and J. Turner 1977. A pollen diagram from Holowell Moss, near Durham City, U.K. Journal of biogeography 4: 25-33.

Doyle, R. 1990. The soils, geomorphology and erosion history of the Leipsokouki catchment, Nomos of Grevena, Greece. Unpublished Masters Degree thesis. Victoria University of Wellington.

Ellenberg, H. 1988 (4th edition). Vegetation ecology of Central Europe. Cambridge University Press, Cambridge.

Erdtman, G. 1943. An introduction to pollen analysis. Chronica Botanica Company, Waltham, Mass.

Erdtman, G. 1952. An introduction to palynology I. Pollen morphology and plant taxonomy. Angiosperms. Almqvist \& Wiksell, Stockholm.

Erdtman, G. 1957. Pollen and spore morphology/plant taxonomy. Gymnospermae, pteridophyta, bryophyta (Illustrations). (An intropduction to palynology. II.). Almqvist \& Wiksell, Stockholm.

Erdtman, G. 1960. The acetolysis method. A revised description. Svensk botanisk tidskrift 54 (4): $561-564$.

Erdtman, G. 1965. Pollen and spore morphology/plant taxonomy. Gymnospermae, Bryophyta (Text). (An introduction to palynology. III.). Almqvist \& Wiksell, Stockholm.

Erdtman, G. 1969. Handbook of Palynology. Morphology. Taxonomy. Ecology. Munksgaard, Copenhagen.

Erdtman, G. and P. Sorsa 1971. Pollen and spore morphology/plant taxonomy IV. Pteridophyta. (Text and additional illustrations.). Almqvist and Wiksell, Stockholm.

Erdtman, G., B. Berglund and J. Praglowski 1961. An introduction to a Scandinavian pollen flora. Almqvist \& Wiksell, Stockholm.

Erdtman, G., J. Praglowski and S. Nilsson 1963. An introduction to a Scandinavian pollen flora. Vol. II. Almqvist \& Wiksell, Stockholm.

Espenshade, E.B. Jr. (ed) 1990 (18th edition). Goode's world atlas. Rand McNally \& Company, Chicago.

Eurola, S., S. Hicks and E. Kaakinen 1984. Key to Finnish mire types. In, P.D. Moore (ed), European mires: 11-117. Academic Press, London.

Faegri, K. and J. Iversen 1950. Textbook of modern pollen analysis. Munksgaard, Copenhagen.

Faegri, K. and J. Iversen 1964 Textbook of pollen analysis. (2nd edition) Munksgaard, Copenhagen.

Faegri, K. and J. Iversen 1965. Field Techniques. In, B. Kummel and D. Raup (eds), Handbook of paleontological techniques: 482-494. W.H. Freeman and Company, San Francisco and London.

Faegri, K. and J. Iversen 1975. Textbook of pollen analysis. (3rd rev. edition) Hafner Press, New York.

Faegri, K. and J. Iversen 1989. Textbook of pollen analysis. 4th edition by K. Faegri, P.E. Kaland, and K. Krzywinski. Reprinted 1992. John Wiley \& Sons Ltd, Chichester.

Fall, P. 1992. Pollen accumulation in a montane region of Colorado, USA: a comparison of moss polsters, atmospheric traps, and natural basins. Review of palaeobotany and palynology 72: 169-197.

Farnham, R.S. and H.R. Finney 1965. Classification and properties of organic soils. Advances in agronomy 17: 115-162. 
Ferrarini, E., F. Ciampolini, R.E.G. Pichi Sermolli and D. Marchetti 1986. Iconographia palynologica pteridophytorum Italiae. Webbia 40: 1-202.

Finke, E.A.W. 1988. Landscape evolution of the Argive Plain, greece: paleoecology, Holocene depositional history, and coastline changes. Unpublished $\mathrm{PhD}$ thesis, Stanford University.

Flenley, J.R. 1973. The use of modern pollen rain samples in the study of the vegetation history of tropical regions. In, H.J.B Birks and R.G West (eds), Quaternary plant ecology: 131-142. Blackwell scientific publications, Oxford.

Folk, R.L. 1965. Petrology of sedimentary rocks. Hemphill's, Austin, Texas.

Forbes, H.A. and H.A. Koster 1976. Fire, axe, and plow: human influence on local plant communities in Southern Argolid. Annals of the New York Academy of Science 268: 109-126.

Fosberg, F.R. 1960. Plant collecting as an anthropolocical field method. El palacio 67 (4): 125-139.

Furlan, D. 1977. The climate of southeast Europe. In, C.C. Wallen (ed), World survey of climatology 6: climates of Central and Southern Europe: 185-235. Elsevier, Amsterdam.

Gajewski, K. 1993. The role of paleoecology in the study of global climatic change. Review of palaeobotany and palynology 79: 141-151.

Gamper, M. and J. Suter 1982. Postglaziale Klimageschichte der Schweizer Alpen. Geographica Helvetica 37: 105-114.

Garcia Novo, F. 1977. The effects of fire on the vegetation of the Doñana National Park. Proceedings of the symposium on the consequences of fire and fuel management in Mediterranean ecosystems. General technical report WO-3: 318-325, USDA Forest Service.

Gennett, J. 1982. Three Holocene pollen records from southern Greece. Palynology 4: 282.

Gerasimidis, A. 1985. Standortkundliche verhaltnisse und postglaziale vegetationsentwicklung der Walder Lailias, Bez. Serres und Katafygi im Pieria-Gebirge (Nordgreichenland). Scientific annals of the Department of Forestry and Natural Environment, Aristotelian University of Thessaloniki 26 (7): 1-133. (In Greek with German summary.)

Gerasimidis, A. and N. Athanasiadis 1995. Woodland history of northern Greece from the mid Holocene to recent time based on evidence from peat pollen profiles. Vegetation history and archaeobotany 4: 109-116.

Glaser, P.H. 1981. Transport and deposition of leaves and seeds on tundra: a late-glacial analog. Arctic and alpine research 13: 173-182.

Godwin, H. 1934. Pollen analysis. An outline of the problems and potentialities of the method. New phytologist 33: 278-305.

Godwin, H. 1960. The history of weeds in Britian. In, J.L. Harper (ed), The biology of weeds: 1-10. Blackwell, Oxford.

Goh, K.M. and B.P.J. Molloy 1972. Reliability of radiocarbon dates from buried charcoals. Proceedings of the 8th International Conference on Radiocarbon Dating, Royal Society of New Zealand: 2.

Gray, J. 1965. Palynological techniques. In, B. Kummel and D. Raup (eds), Handbook of paleontological techniques: 471-481. W.H. Freeman and Company, San Francisco and London.

Gray, J. 1965. Extraction techniques. In, B. Kummel and D. Raup (eds), Handbook of paleontological techniques: 530-587. W.H. Freeman and Company, San Francisco and London. 
Green, D.G. and G.S. Dolman 1988. Fine resolution pollen analysis. Journal of biogeography 15: 685-701.

Greig, J.R.A. and J. Turner 1974. Some pollen diagrams from Greece and their archaeological significance. Journal of archaeological science 1: 177-194.

Greig-Smith, P. 1983. Quantitative plant ecology. Blackwell scientific publications, London.

Griffin, J.J. and E.D. Goldberg 1979. Morphologies and origin of elemental carbon in the environment. Science 206: 563-565.

Grosse-Brauckmann, G. 1986. Analysis of vegetative plant macrofossils. In, B.E. Berglund (ed), Handbook of Holocene palaeoecology and palaeohydrology: 590-618. Reprinted 1991. John Wiley \& Sons Ltd, New York.

Grove, J.M. 1988. The Little Ice Age. Methuen, London.

Hall, V. 1989. A comparison of grass foliage, moss polsters and soil surfaces as pollen traps in modern pollen studies. Circaea 6 (1): 63-69.

Halstead, P. 1981. Counting sheep in Neolithic and Bronze Age Greece. In, I. Hodder, G. Isaac and N. Hammond (eds), Pattern of the past: studies in honour of David Clarke: 307-339. Cambridge University Press, Cambridge.

Hamilton, A.C. 1972. The interpretation of pollen diagrams from highland Uganda. Palaeoecology of Africa 7: 45-149.

Hansen, J.M. 1978. The earliest seed remains from Greece: Palaeolithic through Neolithic at Franchthi Cave. Berichte-Deutsche botanishe Gesellschaft 91: 39-46.

Hansen, J.M. 1985. Palaeoethnobotany in Greece: past, present and future. In, N.C. Wilkie and W.D.E. Coulson (eds), Contributions to Aegean Archaeology (Studies in Honour of William A. McDonald). Center for Ancient Studies University of Minnesota Publications in Ancient Studies (1): 171-181.

Hansen, J.M. 1988. Agriculture in the prehistoric Aegean: data versus speculation. American journal of archaeology 92: 39-52.

Hansen, J.M. and J. Renfrew 1978. Palaeolithic-Neolithic seed remains at Franchthi Cave, Greece. Nature 271: 349-352.

Haslam, S.M., C.A. Sinker and P.A. Wolseley 1975. British water plants. Field studies 4: 243-351.

Havinga, A.J. 1964. Investigation into the differential corrosion susceptibility of pollen and spores. Pollen et spores 6 (2): 621-635.

Havinga, A.J. 1967. Palynology and pollen preservation. Review of palaeobotany and palynology 2: 81-98.

Havinga, A.J. 1984. A 20-year experimental investigation into the differential corrosion susceptibility of pollen and spores in various soil types. Pollen et spores 26 (3-4): 541-558.

Hedberg, H.D. 1976. International stratigraphic guide: a guide to stratigraphic classification, terminology, and procedure. John Wiley \& Sons, New York.

Hellenic Army Geographic Service 1970. Map of Greece 1:50 000, Grevena.

Hellenic Army Geographic Service 1970. Map of Greece 1:50 000, Metsovon.

Hellenic Army Geographic Service 1970. Map of Greece 1:50 000, Pendalofos.

Heywood, V.H. 1978. Flowering plants of the World. Oxford University Press, Oxford.

Hionides, H.T. 1987. Collins gem Greek dictionary. William Collins Sons \& Co. Ltd, London.

Hogg, A. 1989. Radiocarbon dating. The University of Waikato, Hamilton.

Horn, S.P., R.D. Horn and R. Byrne 1992. An automated charcoal scanner for paleoecological studies. Palynology 16: 7-12. 
Hubbard, R.N.L.B. 1979. Ancient agriculture and ecology at Servia. In, C. Ridley and K.A. Wardle, Rescue excavations at Servia 1971-1973: a preliminary report. Annual of the British School at Athens 74: 226-228.

Huber, J.K. and G. Rapp Jr. 1988. Results of a preliminary pollen study from Grevena Province, northwest Greece. Unpublished Grevena Project report.

Huntley, B. and T. Webb III (eds) 1988. Vegetation history. Kluwer Academic Publishers, London.

Huxley, A. and W. Taylor 1989. Flowers of Greece and the Aegean. The Hogarth Press, London.

Ikuse, M. 1956. Pollen grains of Japan. Hirokawa Publishing Company, Tokyo.

Ingram. H.A.P. 1982. Size and shape in raised mire ecosystems: a geophysical model. Nature 297: 300-303.

Institute of Geology and Mineral Exploration (I.G.M.E.) 1980. Geological map of Greece 1:50000, Panayia Sheet.

Institute of Geology and Mineral Exploration (I.G.M.E.) 1987a. Geological map of Greece 1:50000, Dheskati Sheet.

Institute of Geology and Mineral Exploration (I.G.M.E.) 1987b. Geological map of Greece 1:50000, Konitsa Sheet.

Institute for Geological and Mining Research (I.G.M.R.) 1979. Geological Map of Greece 1:50000, Ayiofillon Sheet.

Institute for Geology and Subsurface Research (I.G.S.R.) 1959. Geological map of Greece 1:50000, Metsovon Sheet.

Institute for Geology and Subsurface Research (I.G.S.R.) 1960. Geological map of Greece 1:50000, Pentalofon Sheet.

Institute for Geology and Subsurface Research (I.G.S.R.) 1972. Geological Map of Greece 1:50000, Grevena Sheet.

Iversen, J. 1941. Land occupation in Denmark's Stone Age. Danmarks Geologiske Unders申gelse. Rakke II, (66).

Iversen, J. 1944. Viscum, Hedera and Ilex as climate indicators. A contribution to the study of the post-glacial temperature climate. Geologiska föreningens $i$ Stockholm. Förhanndlingar 66: 463-483.

Iversen, J. 1954. The late-glacial flora of Denmark and its relation to climate and soil.

Iversen, J. 1960. Problems of the early postglacial forest development in Denmark. Danmarks Geologiske Undersøgelse. Rakke IV, 4 (3): 1-32.

Iversen, J. 1964. Plant indicators of climate, soil and other factors during the Quaternary. Report of the sixth international congress on Quaternary, Warsaw 1961, Vol. 2, palaeobotanical section: 421-428.

Iversen, J. 1973. The development of Denmark's nature since the Last Glacial. Danmarks Geologiske Undersøgelse. Rakke VII-C: 1-126.

Jacobson, G.L., Jr. and R.H. Bradshaw 1981. The selection of sites for paleovegetational studies. Quaternary research 16: 80-96.

Jahns, S. 1990. Preliminary note on human influence and the history of vegetation in southern Dalmatia and southern Greece. In, S. Bottema, G. Entjes-Nieborg and W. van Zeist, Man's role in the shaping of the Eastern Mediterranean landscape: 333-340. A.A. Balkema, Rotterdam.

Jahns, S. 1993. On the Holocene vegetation history of the Argive Plain (Peloponnese, southern Greece). Vegetation history and archaeobotany 2: 187-203. 
Jansen, H.S. 1984. Radiocarbon for contributors. Institute of Nuclear Sciences, Lower Hutt, N.Z.

Janssen, C.R. 1972. The palaeoecology of plant communities in the Dommel Valley, north Brabant, Netherlands. Journal of ecology 60: 411-437.

Janssen, C.R. 1973. Local and regional pollen deposition. In, H.J.J. Birks and R.G. West (eds) Quaternary plant ecology: 31-42. Blackwell scientific publications, Oxford.

Jones, G. 1981. Crop processing at Assiros Toumba - a taphonomic study. Zeitschrift fur Archaologie 15: 105-111.

Kabailiené, M. 1988. On the reconstruction of past cultural landscape by pollen and diatom analytical data of lacustrine deposits in Lithuania. In, H.H. Birks, H.J.B. Birks, P.E. Kaland and D. Moe (eds), The cultural landscape - past, present and future: 474. Cambridge University Press, Cambridge.

Karasavvidis, G. (unpub.) 1:50 000 vegetation map of Grevena. Unpublished, Greek Forest Service, Grevena.

Keys, D. 1982. Effect of peat type and decomposition on the calorific value of some New Brunswick peats. In, P.M. Jarrett (ed), Testing of peats and organic soils. American Society for Testing and Materials, special technical publication 820: 111-121.

Kivinen, E. 1980. Proposal for a general classification of virgin peat. Proceedings of the 6 th International peat congress. International peat society.

Klemetti, V. and D. Keys 1982. Relationships between dry density, moisture content, and decomposition of some New Brunswick peats. In, P.M. Jarrett (ed), Testing of peats and organic soils. American Society for Testing and Materials, special technical publication 820: 72-82.

Knuth, P. 1906-9. Handbook of flower pollination. 3 Vols. Clarendon Press, Oxford. Translated by J.R. Ainsworth Davis.

Koster, H. 1987. Ethnography of herding in the Grevena area. Unpublished Grevena Project report.

Kraft, J.C., G.R. Rapp, Jr and S.E. Aschenbrenner 1980. Late Holocene palaeogeomorphic reconstructions in the area of the Bay of Navarino: Sandy Pylos. Journal of archaeological science 7: 187-210.

Kramm, E. 1978. Pollenanalytische Hochmooruntersuchungen zur floren und siedlungsgeschichte zwischen Ems und Hase. Abhandlungen. Landesmuseum fuer Naturkunde zu Münster in Westfalen 40: 445.

Kummel, B. and D. Raup (eds) 1965. Handbook of paleontological techniques. W.H. Freeman and Company, San Francisco and London.

Lamb, H.H. 1982. Climate, history and the modern world. Methuen, London.

Lange, E. 1975. The development of agriculture during the first millenium A.D. Geologiska föreningens $i$ Stockholm. Förhanndlingar 97: 115-124.

Leake, W.M. 1835. Travels in northern Greece. Reprinted 1967. Adolf M. Hakkert, Amsterdam.

Leopold, L.B. 1956. Land use and sediment yield. In, W.L. Thomas, Jr. (ed), Man's role in changing the face of the earth: 639-647. University of Chicago Press, Chicago.

Lewis, D.M. and E.C. Ogden 1965. Trapping methods for modern pollen rain studies. In, B. Kummel and D. Raup (eds), Handbook of paleontological techniques: 613-626. W.H. Freeman and Company, San Francisco and London.

Lichti-Federovich and J.C. Ritchie 1965. Contemporary pollen spectra in Central Canada. II. The forest-grassland transition in Manitoba. Pollen et spores 7 (1): 63-87. 
Licitis, R. 1953. Air-borne pollen and spores sampled at five New Zealand stations, 1951-52. New Zealand journal of science and technology, B34: 289-316.

Likens, G. E., F.H. Bormann, N.M. Johnson, D.W. Fisher and R.S. Pierce 1969. Effects of forest cutting and herbicide treatment on nutrient budgets in the Hubbard Brook watershed ecosystem. Ecological monographs 40: 23-47.

Lowe, D.C. and W.J. Judd 1987. Graphite target preparation for radiocarbon dating by accelerator mass spectrometry. Nuclear instruments and methods in physics research, B28: 113-116.

MacDonald, G.M., C.P.S. Larsen, J.M. Szeicz and K.A. Moser 1991. The reconstruction of boreal forest fire history from lake sediments: a comparison of charcoal, pollen, sedimentological, and geochemical indices. Quaternary science reviews 10: 53-71.

Mackereth, F.J.H. 1966. Some chemical observations of post-glacial lake sediments. Philosophical transactions. Royal Society of London. Series B. Biological Sciences 250: 165-213.

Magri, D. 1995. Some questions on the late-Holocene vegetation of Europe. The Holocene 5 (3): 354-360.

Maher, L.J. 1972. Absolute pollen diagram of Redrock Lake, Boulder County, Colorado. Quaternary research 2: 531-553.

Maher, L.J. 1981. Statistics for microfossil concentration measurements. Review of paleobotany and palynology 32: 153-191.

Markgraf, V. 1980. Pollen dispersal in a mountain area. Grana 19: 127-146.

Marshall, S. 1981. Handbook of toxic and hazardous chemicals. Noyes Publications, New Jersey.

Martin, P.S. 1963. The Last 10,000 years. The University of Arizona Press, Tucson.

Martyn, D. 1992. Climates of the World. Elsevier, London.

Matthews, J. 1969. The assessment of a method for the determination of absolute pollen frequencies. New phytologist 68: 161-166.

Matthews, J. 1994. Radiocarbon dating of arctic-alpine palaeosols and the reconstruction of Holocene palaeoenvironmental change. In, F.M. Chambers (ed), Climate change and human impact on the landscape: 81-96. Chapman \& Hall, London.

Mehringer, P.J., Jr., E. Blinman and K.L. Petersen 1977. Pollen influx and volcanic ash. Science 198: 257-261.

Mellars, P.A. 1975. Ungulate populations, economic patterns and the Mesolithic landscape. In, J.G. Evans, S. Limbrey and H. Cleere (eds), The effect of man on the landscape: the highland zone. Council for British Archaeology research report 11: 49-56.

Mellars, P.A. 1976. Fire ecology, animal populations and man: a study of some ecological relationships in prehistory. Proceedings of the Prehistoric Society 42: 15-45.

Middeldorp, A.A. 1982. Pollen concentration as a basis for indirect dating and quantifying net organic and fungal production in a peat bog ecosystem. Review of palaeobotany and palynology 37: 225-282.

Mirams, R.V. 1953. A comparison of the gravity and impact methods of collecting spores and pollen grains from the atmosphere at Wellington, New Zealand. New Zealand journal of science and technology, B34 (5): 378-383.

Moody, J. and O. Rackham 1988. Grevena report. Unpublished Grevena Project report.

Moody, J. and O. Rackham in prep. Observations on the historical ecology of Grevena.

Moore, P.D. 1973. The influence of prehistoric cultures upon the initiation and spread of blanket bog in upland Wales. Nature 241: 350-353.

Moore, P.D. 1976. How far does pollen travel? Nature 260: 388-389. 
Moore, P.D. and S.B. Chapman (eds) 1986. Methods in plant ecology. Blackwell scientific publications, London.

Moore, P.D. and J.A. Webb 1978. An illustrated guide to pollen analysis. Hodder and Stoughton, London.

Moore, P.D., J.A. Webb and M.E. Collinson 1991 (2nd edition). Pollen analysis. Blackwell scientific publications, Oxford.

Muir, G.D. (ed) 1977 (2nd edition). Hazards in the chemical laboratory. The Chemical Society, London.

Muller, J.M. 1982. Selected climatic data for a global set of standard stations for vegetation science. Dr W. Junk, The Hague.

Nelson, D.W. and L.E. Sommers 1982 (2nd edition). Total carbon, organic carbon, and organic matter. In, A.L. Page, R.H. Miller and D.R. Keeney (eds), Methods of soil analysis. Part 2 - Chemical and microbiological properties: 539-579. American Society of Agronomy Inc. and Soil Science of America Inc., Madison, Wisconsin, USA.

Nilsson, S., J. Praglowski and L. Nilsson 1977. Atlas of airborne pollen grains and spores in northern Europe. Natur och Kultur, Stockholm.

O'Connell, M. 1986. Reconstruction of local development in the post-Atlantic based on palaeoecological investigations at Carrownaglogh prehistoric field system, County Mayo, Ireland. Review of palaeobotany and palynology 49: 117-176.

Oikonomou, D., N., Koroxenithe, Z. Sarri, H. Parousi, A. Spiropulou and T. Sarri 1988. Soil studies of cultivatable land in the region of Nomos Grevena. Ministry of Agriculture Soils Institute, Thessaloniki. (In Greek. Translated by M. Savina.)

Oldfield, F. 1970. Some aspects of scale and complexity in pollen-analytically based palaeoecology. Pollen et spores 12 (2): 163-171.

Oldfield, F. 1977. Lakes and their drainage basins as units of sediment based ecological study. Progress in physical geography 1: 460-504.

Olsson, I. U. 1986. Radiometric dating. In, B.E. Berglund (ed), Handbook of Holocene palaeoecology and palaeohydrology: 273-312. Reprinted 1991. John Wiley \& Sons, Chichester.

Opdyke, N.D., D. Ninkovich, W. Lowrie and J.D. Hays 1972. The palaeomagnetism of two Aegean deep-sea cores. Earth and planetary science letters 14: 145-159.

Orvedal, A.C. 1990. World soils. In, E.B. Espenshade, Jr. (ed) (18th edition), Goode's world atlas: 18 \& 19. Rand McNally \& Company, Chicago.

Oyama, M. and H. Takehara 1967. Revised standard soil colour charts. Ministry of Agriculture and Forestry, Japan.

Parker, S.P. (ed) 1995. World geographical encyclopedia. Vol.4. Europe: 14-17. McGrawHill Inc., New York.

Patterson, W.A.III, K.J. Edwards and D.J. Maguire 1987. Microscopic charcoal as a fossil indicator of fire. Quaternary science reviews 6: 3-23.

Pearson, G.W. and M. Stuiver 1993. High-precision bidecadal calibration of the radiocarbon time scale, 500-2500 BC. Radiocarbon 35: 25-33.

Pennington, W. and A.P. Bonny 1970. An absolute pollen diagram from the British lateglacial. Nature 226: 871-873.

Pennington, W. and J.P. Lishman 1971. Iodine in lake sediments in northern England and Scotland. Biological reviews 46: 279-313. 
Pennington, W., E.Y. Haworth, A.P. Bonny and J.P. Lishman 1972. Lake sediments in northern Scotland. Philosophical transactions. Royal society of London. Series B. Biological sciences 264: 191-294.

Perry, I. and P.D. Moore 1987. Dutch elm disease as an analogue of Neolithic elm decline. Nature 326: 72-73.

Polunin, O. 1988. Flowers of Greece and the Balkans : a field guide. Oxford University Press, Oxford.

Polunin, O. and A. Huxley 1987 (3rd edition). Flowers of the Mediterranean. The Hogarth Press, London.

Pope, K.O. and Th. van Andel 1984. Late Quaternary alluviation and soil formation in the southern Argolid: its history, causes and archaeological implications. Journal of archaeological science 11: 281-306.

Potter, L.D. and J. Rowley 1960. Pollen rain and vegetation, San Augustin Plains, New Mexico. Botanical gazette 122 (1): 1-25.

Pouqueville, F.C.H.L. 1826. Voyage de la Grece. [No publisher given], Paris.

Praglowski, J. 1962. Notes on the pollen morphology of Swedish trees and shrubs. Grana palynologica 3 (2): 45-65.

Prentice, I.C. 1981. Quantitative birch (Betula L.) pollen separation by analysis of size frequency data. New phytologist 89: 145-157.

Prentice, I.C. 1983. Postglacial climatic change: vegetation dynamics and the pollen record. Progress in physical geography 7: 273-286.

Prentice, I.C. 1985. Pollen representation, source area, and basin size: toward a unified theory of pollen analysis. Quaternary research 23: 76-86.

Prentice, I.C. 1986a. Forest-composition calibration of pollen data. In, B.E. Berglund (ed), Handbook of Holocene palaeoecology and palaeohydrology: 799-816. Reprinted 1991. John Wiley \& Sons Ltd, New York.

Prentice, I.C. 1986b. Vegetation responses to past climatic variation. Vegetatio 67: 131-141. Prentice, I.C. 1988. Records of vegetation in time and space: the principles of pollen analysis. In, B. Huntley and T. Webb III (eds), Vegetation history: 17-42. Kluwer Academic Publishers, London.

Prentice, I.C., J. Guiot and S.P. Harrison 1992. Mediterranean vegetation, lake levels and palaeoclimate at the Last Glacial maximum. Nature 360: 658-660.

Proctor, J. and S. R. J. Woodell 1971. The ecology of serpentine soils. Advanced ecological research 9: 255-366.

Punt,W. 1976-1991. Northwest European Pollen Flora. Vols 1-6. Elsevier, Amsterdam.

Rackham, O. 1980. Ancient woodland. Edward Arnold, London.

Rackham, O. 1982. Land use and the native vegetation of Greece. In, M. Bell and S. Limbrey (eds), Archaeological aspects of woodland ecology. British archaeological reports. Initial series 146: 177-198.

Rackham, O. 1983. Observations on the historical ecology of Boetia. Annual of the British School at Athens 78: 291-351.

Rackham, O. 1991. Flora Greveniotica. Unpublished Grevena Project report.

Raine, J.I., J.S. Crampton, E.M. Crouch, C.H. Hollis, W. St George, G.H. Scott 1996. Manual of digital image capture and processing techniques in Paleontology Section, IGNS. Institute of Geological \& Nuclear Sciences science report 96/20.

Raynor, G.S., E. C. Ogden and J.V. Hayes 1972. Dispersion and deposition of corn pollen from experimental sources. Agronomy journal 64: 420-427.

Readman, P.W., J.W.M. Coey, C. Moser and F. Weber 1976. Analysis of some lake sediments from Greece. Journal de physique 37 (no. 12, supplement C6): 845-848. 
Reille, M. 1992. New pollen-analytical researches in Corsica: the problem of Quercus ilex L. and Erica arborea L., the origin of Pinus halepensis Miller forests. New phytologist 122: 359-378.

Reille, M. and J.L. de Beaulieu 1988. History of the Würm and Holocene vegetation in western Velay (Massif Central, France): a comparison of pollen analysis from three corings at Lac du Bouchet. Review of palaeobotany and palynology 54: 233-248.

Reille, M. and A. Pons 1992. The ecological significance of sclerophyllous oak forests in the western part of the Mediterranean basin: a note on pollen analytical data. Vegetatio 99 100: 13-17.

Reinders, H.R. and S. Bottema 1983. Investigations at Halos and Zileria. Preliminary report 1982. Bulletin Antieke Beschaving 58: 91-100.

Reitsma, Tj. 1969. Size modification of recent pollen grains under different treatments. Review of palaeobotany and palynology 9 (3/4): 175-202.

Reitsma, Tj. 1970. Suggestions towards unification of descriptive terminology of angiosperm pollen grains. Review of palaeobotany and palynology 10: 39-60.

Renfrew, C. 1971. Sitagroi, radiocarbon and prehistory of southeast Europe. Antiquity 45: 275-282.

Renfrew, J. 1969. Palaeoethnobotany and the Neolithic Period in Greece and Bulgaria. Unpublished $\mathrm{PhD}$ thesis. Cambridge University, Cambridge.

Renfrew, J. 1973a. Palaeoethnobotany. The prehistoric food plants of the Near East and Europe. Methuen \& Co. Ltd, London.

Renfrew, J. 1973b. Agriculture. In, D. Theocharis (ed), Neolithic Greece: 147-164. National Bank of Greece, Athens.

Riezebos, P.A. and R.T. Slotboom 1978. Pollen analysis of the Husterbaach peat (Luxembourg): its significance for the study of subrecent geomorphological events. Boreas 7: 75-82.

Ritchie, J.C. and S. Lichti-Federovich 1963. Contemporary pollen spectra in central Canada. Pollen et spores 5: 95-114.

Rodden, R.J. 1972. An early Neolithic village in Greece. In, C.C. Lamberg-Karlovsky (ed), Old World archaeology: foundations of civilization: 95-104. W.H. Freeman and Company, San Francisco.

Roure, J.M. 1985. Palinología Ibérica. Fam. 1 a 20. Cupressaceae a Betulaceae. Orsis 1: 3-69.

Ruffaldi, P. 1994. Relationship between recent pollen spectra and current vegetation around the cerin peat bog (Ain, France). Review of palaeobotany and palynology 82: 97-112.

Rull, V. 1987. A note on pollen counting in palaeoecology. Pollen et spores 29: 471-481.

Rymer, L. 1978. The use of uniformitarianism and analogy in palaeoecology, particularly in pollen analysis. In, D. Walker and J.C. Guppy (eds), Biology and Quaternary environments: 245-257. Australian Academy of Science, Canberra.

Ryvarden, L. 1971. Studies in seed dispersal I: trapping of diaspores in the alpine zone at Finse, Norway. Norwegian journal of botany 18: 215-226.

Ryvarden, L. 1975. Studies in seed dispersal. II: winter-dispersed species at Finse, Norway. Norwegian journal of botany 22: 21-24.

Sakellariou, M.B. (ed) 1988. Macedonia: 4000 years of Greek history and civilization. Ekdotike Athenon S.A., Athens.

Savina, M. 1989. Archaeological geology of Grevena Nomos. Unpublished Grevena Project report.

Savina, M. 1990. Geology and geomorphology of the Anelia site. Unpublished Grevena Project report. 
Savina, M. 1993. Some aspects of the geomorphology and Quaternary geology of Grevena Nomos, western Macedonia, Greece. Salzburger geographische arbeiten 22: 57-75.

Savina, M. 1995. Archaeological sites in Grevena. Unpublished notes on Grevena Project.

Scott, H.G. and C.J. Stojanovich 1963. Digestion of juniper pollen by Collembola. Florida entomologist 46: 189-191.

Sfikas, G. 1978. Trees and shrubs of Greece. P. Efstathiadis \& Sons S.A., Athens.

Shackley, M.L. 1975. Archaeological sediments. Butterworth \& Co. Ltd, London.

Shackley, M. 1981. Environmental archaeology. Allen \& Unwin, London.

Sheehan, M.C. 1979. The Postglacial vegetational history of the Argolid Peninsula, Greece. Unpublished $\mathrm{PhD}$ thesis. Indiana University.

Sheehan, M.C. and D.R. Whitehead 1973. Pollen analysis of Franchthi Cave sediments: preliminary results. In, T.W. Jacobsen, Excavations in the Franchthi Cave, 1969-1971, part I. Hesperia 42: 68-72.

Simkin, T., L. Siebert, L. McClelland, D. Bridge, C. Newhall and J.H. Latter 1968. Volcanoes of the world. Hutchinson Ross Publishing Company, Stroudsburg, Pennsylvania.

Spence, D.H.N. 1957. Studies on the vegetation of Shetland. I. The serpentine debris vegetation in Unst. Journal of ecology 45: 917-945.

Spence, D.H.N. 1982. The zonation of plants in freshwater lakes. Advances in ecological research 12: 37-125.

Starkel, L. 1987. Anthropogenic sedimentological changes in Central Europe. Striae 26: 21-29.

Steckhan, H.U. 1961. Pollenanalytisch-vegetationsgeschichtliche Undersuchungen sur früten Siedlungsgeschichte im Vogelsberg, Knüll und Solling. Flora 150: 514-551.

Stein, J.K. 1985. Interpreting sediments in cultural settings. In, J.K. Stein and W.R. Farrand (eds), Archaeological sediments in context: 5-19. University of Maine, Orono.

Stevenson, A.J. (ed) 1977. Webster's new geographical dictionary. G. \& C. Merriam Company, Springfield, Massachusetts.

Stevenson, A.C. 1985. Studies in the vegetational history of S.W. Spain. II. Palynological investigations at Laguna de las Madres, S.W. Spain. Journal of biogeography 12: 293-314.

Stevenson, A.C. and P.D. Moore 1988. Studies in the vegetation history of S.W. Spain. IV. Palynological investigations of a valley mire at El Acebron, Huelva. Journal of biogeography 15: 339-361.

Stockmarr, J. 1973. Determination of spore concentration with an electronic particle counter. Danmarks geologiske undersфgelse, årbog 1972: 87-89.

Strid, A. (ed) 1989. Mountain flora of Greece. Volume 1. First published 1986, reprinted with corrections 1989. Cambridge University Press, Cambridge.

Strid, A. and K. Tan (eds) 1991. Mountain Flora of Greece. Volume 2. Edinburgh University Press, Edinburgh.

Stuiver, M. 1971. Evidence for the variation of atmospheric ${ }^{14} \mathrm{C}$ content in the Late Quaternary. In, K.K. Turekian (ed), The late Cenozoic glacial ages: 57-70. Yale University Press, New Haven.

Stuiver, M. 1978. Radiocarbon timescale tested against magnetic and other dating methods. Nature 273: 271-274.

Stuiver, M. 1980. Solar variability and climatic change during the current millennium. Nature 286 (5776): 868-871.

Stuiver, M. and G.W. Pearson 1986. High-precision calibration of the radiocarbon time scale, AD 1950-500 BC. Radiocarbon 28: 805-838.

Stuiver, M. and G.W. Pearson 1993. High-precision bidecadal calibration of the radiocarbon time scale, AD 1950-500 BC and 2500-6000 BC. Radiocarbon 35: 1-23. 
Stuiver, M. and H.A. Polach 1977. Discussion reporting of ${ }^{14} \mathrm{C}$ data. Radiocarbon 19: 355-363.

Stuiver, M. and P.J. Reimer 1993. Extended ${ }^{14} \mathrm{C}$ data base and revised CALIB radiocarbon age calibration program. Radiocarbon 35: 215-230.

Stuiver, M. and P.J. Reimer 1995. CALIB user's guide rev. 3.0.3c. Quaternary isotope laboratory, University of Washington.

Stumm, W. and P. Baccini 1978. Man-made chemical perturbation of lakes. In, A. Lerman (ed), Lakes: chemistry, geology and physics: 91-126. Springer-Verlag, New York.

Stutz, H.C. 1972. On the origin of cultivated rye. American journal of botany 59 (1): 59-70.

Tallantire, P.A. 1972. The regional spread of spruce (Picea abies (L.) Karst.) within Fennoscandia: a reassessment. Norwegian journal of botany 19: 1-16.

Tauber, H. 1965. Differential pollen dispersion and interpretation of pollen diagrams. Danmarks geologiske unders $\phi$ gelse. Rakke II, (89).

Tauber, H. 1967. Differential pollen dispersion and filtration. In, E.J. Cushing and H.E. Wright Jr. (eds), Quaternary paleoecology: 131-141. Yale University Press, New Haven.

Taylor, R.E. 1987. Radiocarbon dating - an archaeological perspective. Academic Press, New York.

Theocharis, D.R. 1973. Neolithic Greece. National Bank of Greece, Athens.

Thurston, J.M. 1982. Wild oats as successful weeds. In, W. Holzner and M. Numata (eds), Biology and ecology of weeds: 191-199. Dr W. Junk, The Hague.

Tinsley, H.M. and R.T. Smith 1974. Surface pollen studies across a woodland heath transition and their application to the interpretation of pollen diagrams. New phyologist 73: 547565.

Tolonen, M. 1980. Post glacial pollen stratigraphy of Lake Lamminjarvi, South Finland. Annales botanici Fennici 17: 15-25.

Trabaud, L. 1970. Quelques valeurs et observations sur la phytodynanamique des surfaces incindiées dans le bas Languedoc (Premiers résultats). Naturalia monspeliensia. Serie botanique 21: 231-242.

Traverse, A. 1965. Preparation of modern pollen and spores for palynological reference collections. In, B. Kummel and D. Raup (eds), Handbook of paleontological techniques: 598-613. W.H. Freeman and Company, San Francisco and London.

Trewartha, G.T. 1990. Climatic regions. In, E.B. Espenshade Jr. (ed) (18th edition), Goode's world atlas: 8 -9. Rand McNally \& Company, Chicago.

Troels-Smith, J. 1955. Karakterisering af løse jordater. Characterisation of unconsolidated sediments. Danmarks geologiske unders $\phi$ gelse. Rakke IV, 3 (10).

Troels-Smith, J. 1960. Ivy, mistletoe and elm: climate indicators - fodder plants. Danmarks Geologiske Undersøgelse. Rakke IV, 4 (4).

Turner, J. 1964. The anthropogenic factor and vegetational history. I. Tregaron and Whixall Mosses. New phytologist 63: 73-90.

Turner, J. and J.R.A. Grieg 1975. Some Holocene pollen diagrams from Greece. Review of paleobotany and palynology 20: 171-204.

Turrill, W.B. 1929. The plant-life of the Balkan Peninsula. A phytogeographical study. Clarendon Press, Oxford.

Tutin, T.G., V.H. Heywood, N.A. Burgess, D.H. Valentine, S.M. Walters and D.A. Webb (eds) 1964-1980. Flora Europaea. 5 Vols. University Press, Cambridge.

Tyldesley, J.B. 1973. Long-range transmission of tree pollen to Shetland. New phytologist 72: $175-181$. 
Ubera, J.L., C. Galán and F.H. Guerrero 1988. Palynological study of the genus Plantago in the Iberian Peninsula. Grana 27: 1-15.

Valdes, D., M.J. Diez and I. Fernandez 1987. Atlas pollinico de Andalucia Occidental. Instituto de Desarrollo. Regional 43 Universidad de Seville, Spain.

van Andel, Th. and E. Zangger 1990. Landscape stability and destabilisation in the prehistory of Greece. In, S. Bottema, G. Entjes-Nieborg and W. van Zeist (eds), Man's role in shaping the Eastern Mediterranean landscape: 139-157. Balkema, Rotterdam.

van Benthem, F., G.C.S. Clarke and W. Punt 1984. Fagaceae. In, W. Punt and G.C.S. Clarke, The Northwest European pollen flora IV: 87-110. Elsevier, Oxford.

van Dam, H., A. Mertens and J. Sinkeldam 1994. A coded checklist and ecological indicator values of freshwater diatoms from the Netherlands. Netherlands journal of aquatic ecology 28 (1): 117-133.

van der Hammen, T., T.A. Wijmstra and W.H. van der Molen 1965. Palynological study of a very thick peat section in Greece, and the Würm-glacial vegetation in the Mediterranean region. Geologie en Mijnbouw 44e: 37-39.

van Zeist, W. 1976. Two early rye finds from the Netherlands. Acta botanica Neerlandica 25 (1): 71-79.

van Zeist, W. and S. Bottema 1971. Plant husbandry in early neolithic Nea Nikomedeia, Greece. Acta botanica Neerlandica 20 (5): 524-538.

van Zeist, W. and H. Woldring 1978. A postglacial pollen diagram from Lake Van in east Anatolia. Review of palaeobotany and palynology 26: 249-276.

Vidal, G. 1988. A palynological preparation method. Palynology 12: 215-220.

Vita-Finzi, C. 1969. The Mediterranean valleys - geological changes in historical times. Cambridge University Press, Cambridge.

Vuorela, I. 1972. Human influence on the vegetation of Katinhäntä bog, southern Finland. Acta botanica Fennica 98: 1-21.

Vuorela, I. 1973. Relative pollen rain around cultivated fields. Acta botanica Fennica 102: $1-27$.

Wace, A.J.B. and M.S. Thompson 1972. The Nomads of the Balkans. An account of life and customs among the Vlachs of northern Pindus. Methuen \& Co. Ltd, London.

Walker, D. 1964. A modified Vallentyne mud sampler. Ecology 45: 642-644.

Walker, D. 1970. Direction and rate in some British post-glacial hydroseres. In, D. Walker and R.G. West (eds), Studies in the vegetational history of the British Isles: 117-139. Cambridge University Press, Cambridge.

Wallace, G., R.J. Sparks, D.C. Lowe and K.P. Pohl 1987. The New Zealand accelerator mass spectrometry facility. Nuclear instruments and methods om physics research B29: 124-128.

Walter, H., E. Harnickell and D. Mueller-Dombois 1975. Climate-diagram maps. SpringerVerlag, Berlin.

Wasylikowa, K. 1986. Analysis of fossil fruits and seeds. In, B.E. Berglund (ed), Handbook of Holocene palaeoecology and palaeohydrology: 571-590. Reprinted 1991. John Wiley \& Sons Ltd, New York.

Watson E.V. 1968 (2nd edition). British mosses and liverworts. Cambridge University Press, Cambridge.

Watts, W.A. 1978. Plant macrofossils and Quaternary palaeoecology. In, D. Walker and J.C. Guppy (eds), Biology and Quaternary environments: 53-67. Australian Academy of Science, Canberra. 
Webb, R.S. and T. Webb III 1988. Rates of sediment accumulation in pollen cores from small lakes and mires of eastern North America. Quaternary research 30: 284-297.

Webb, T. III, R.A. Laseski and J.C. Bernabo 1978. Sensing vegetational patterns with pollen data: choosing the data. Ecology 59 (6): 1151-1163.

Webb, T. III, S. Howe, R.H.W. Bradshaw and K.M. Heide 1981. Estimating plant abundances from pollen percentages: the use of regression analysis. Review of palaeobotany and palynology 34: 269-300.

Whitehead, D.R. 1961. A note on silicone oil as a mounting medium for fossil and modern pollen. Ecology 42: 591.

Whitehead, D.R. 1965. Pollen morphology in the Juglandaceae, 2. Survey of the family. Journal Arnold Arboretum (Harvard University) 46: 369-410.

Whitehead, D.R. and M.C. Sheehan 1981. The Late-Postglacial vegetational history of the Argolid Peninsula, Greece. National Geographic Society research report 13: 693-708.

Whittaker, R.H. 1962. Classification of natural communities. The botanical review 28 (1): 1-239.

Whyte, I.D. 1995. Climate change and human society. Arnold, London.

Wijmstra, T.A. 1969. Palynology of the first 30 metres of a $120 \mathrm{~m}$ deep section in northern Greece. Acta botanica Neerlandica 18: 511-527.

Wilkie, N. 1987. The Grevena project. Unpublished Grevena Project report.

Wilkie, N. 1988. Results of the 1988 archaeological survey. Unpublished Grevena Project report.

Wilkie, N. 1995. Grevena Project - List of archaeological sites from surface survey. Unpublished Grevena Project report.

Willis, J.C. 1951 (6th edition). A dictionary of the flowering plants and ferns. Cambridge University Press, Cambridge.

Willis, K.J. 1989. Late Quaternary vegtational history of Epirus, northwestern Greece. Unpublished $\mathrm{PhD}$ thesis. Cambridge University, Cambridge.

Willis, K.J. 1992a. The late Quaternary vegetational history of northwest Greece. I. Lake Gramousti. New phytologist 121: 101-117.

Willis, K.J. 1992b. The late Quaternary vegetational history of northwest Greece. II. Rezina marsh. New phytologist 121: 119-138.

Willis, K.J. 1992c. The late Quaternary vegetational history of northwest Greece. III. A comparative study of two contrasting sites. New phytologist. 121: 139-155.

Willis, K.J. 1994. The vegetational history of the Balkans. Quaternary science reviews 13: 769-788.

Willis, K.J. and K.D. Bennett 1994. The Neolithic transition - fact or fiction? Palaeoecological evidence from the Balkans. The Holocene 4: 326-333.

Wilson, C.C. 1979. Management problems and solutions at the interface between man and Mediterranean wildlands. In, Fire and fuel management in Mediterranean-climate ecosystems: research priorities and programmes. MAB technical note 11, UNESCO, Paris.

Wodehouse, R.P. 1935. Pollen grains. McGraw Hill Book Co., New York.

Wright, H.E. 1972. Vegetation History. In, W.A. McDonald and G.R. Rapp (eds), The Minneapolis Messenia expedition: reconstructing a Bronze Age regional environment: 188-199. Minnesota University, Minneapolis.

Wright, H.E. 1985. Palaeoecology, climatic change, and Aegean prehistory. In, Wilkie, N.C. and W.D.E. Coulson (eds), Contributions to Aegean Archaeology (Studies in Honour of William A. McDonald). Center for Ancient Studies University of Minnesota Publications in Ancient Studies (1): 183-195. 
Wright, H.E., E.J. Cushing and D.A. Livingstone 1965. Coring devices for lake sediments. In, B. Kummel and D. Raup (eds), Handbook of paleontological techniques: 494-520. W.H. Freeman and Company, San Francisco and London.

Wright, H.E., J.H. McAndrews and W. van Zeist 1967. Modern pollen rain in western Iran, and its relation to plant geography and Quaternary vegetational history. Journal of ecology 55: 415-443.

Yasuda, Y. 1985. The study of the vegetational history in Greece II. Research related to UNESCO's Man in the Biosphere Programme, 1984-1985, Tokyo. 


\title{
LATE HOLOCENE VEGETATION HISTORY OF GREVENA PROVINCE, NORTHWESTERN GREECE
}

\author{
Volume II - Appendices
}

by

Pamela I. Chester

\author{
A thesis \\ submitted to the Victoria University of Wellington \\ in fulfilment of the \\ requirements for the degree of \\ Doctor of Philosophy \\ in Geology
}

Victoria University of Wellington, New Zealand. 


\section{Appendices}

I Herbarium specimens collected in Grevena Province $\ldots \ldots \ldots \ldots \ldots \ldots \ldots$ 1-10

II Laboratory preparation techniques for pollen and charcoal analysis $\ldots \ldots \ldots$ 1-12

II.1 Laboratory preparation of moss polsters and litter, soil

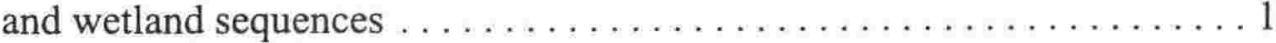

II.1.1 Laboratory preparation of moss polsters and litter samples . . . . . 2

II.1.2 Laboratory preparation of soil samples $\ldots \ldots \ldots \ldots \ldots \ldots \ldots$

II.1.3 Laboratory preparation of wetland samples $\ldots \ldots \ldots \ldots \ldots \ldots$

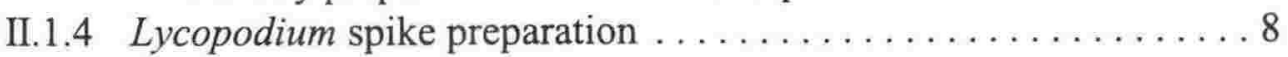

II.1.5 Laboratory procedure for diatom extraction $\ldots \ldots \ldots \ldots \ldots$

II.2 Laboratory preparation of pollen reference material $\ldots \ldots \ldots \ldots \ldots 10$

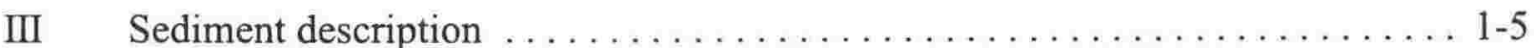

III.1 Troels-Smith characterization of unconsolidated sediments $\ldots \ldots \ldots \ldots \ldots$

III.2 Comparison of grain size class scales of inorganic sediments $\ldots \ldots \ldots \ldots 5$

IV Radiocarbon results and calibration $\ldots \ldots \ldots \ldots \ldots \ldots \ldots \ldots \ldots \ldots \ldots \ldots \ldots \ldots .17$

V Modern pollen and spore reference collection $\ldots \ldots \ldots \ldots \ldots \ldots \ldots \ldots$ 1-53

VI Pollen key ........

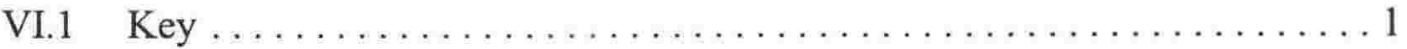

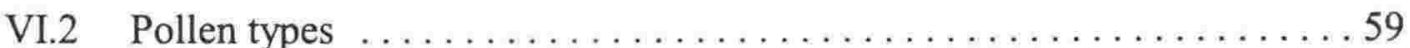

VII Species lists for reconnaissance vegetation surveys $\ldots \ldots \ldots \ldots \ldots \ldots \ldots 1-4$

VIII Modern vegetation composition at pollen surface sample collection sites ... 1-33

IX Surface samples - pollen and charcoal counts $\ldots \ldots \ldots \ldots \ldots \ldots \ldots \ldots \ldots \ldots$

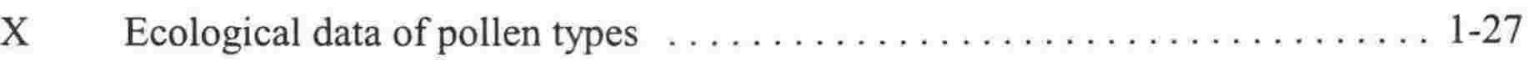

XI Relative dispersal and representation $\ldots \ldots \ldots \ldots \ldots \ldots \ldots \ldots \ldots \ldots \ldots \ldots$

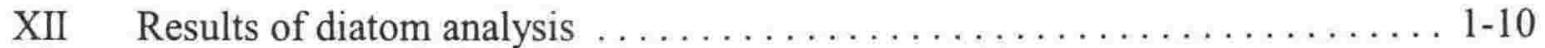

XIII Sediment sequences - pollen and charcoal counts $\ldots \ldots \ldots \ldots \ldots \ldots \ldots$ 1-60

XIV Stratigraphy of exploratory boreholes in Anelia \& Kellia wetlands . . . . . . . 1-7 


\section{Appendix I}

\section{Herbarium specimens collected in Grevena Province}

Taxonomic names follow :

Tutin, T. G. et al. (eds) 1964-80. Flora Europaea. Volumes 1-5. Cambridge University Press, Cambridge.

Common names follow:

Polunin, O. 1988. Flowers of Greece and the Balkans. Oxford University Press, Oxford.

Identifications were made by Dr Oliver Rackham, Cambridge University.

Herbarium specimens were collected from the following geographic areas and vegetation:

1) Perivolion/Vovousa (mountains) - understorey (beech wood)

2) Anelia (mountains) - bog (beech wood)

- dry land on edge of bog (beech wood)

- road verge (beech wood)

3) Kellia (lowland plains) - - margin of fen (agricultural fields)

- field margin steppe (agricultural fields)

- fen (agricultural fields)

4) Mavranaioi (lowland plains) - - road verge (oak wood-pasture)

5) Polynerion (foothills) - steppe on ridge top (oak wood-pasture)

- steppe on east facing hillslope (oak wood-pasture)

6) Aetia (foothill limestone gorge) - limestone gorge (mixed wood)

7) Mirsina (lowland plains) - Phragmites fen (oak wood)

- margin of Phragmites fen (oak wood)

- steppe near Phragmites fen in oak wood

- ground cover (oak wood)

- understorey (oak wood)

8) Gomara (mountains)

- dried lakebed (pine, Pinus leucodermis, wood-pasture)

- alpine pasture below lake (pine, Pinus leucodermis, wood-pasture)

9) Zakas to Agios Nikolaos (mountains) - road verge (pine wood-pasture)

10) Perivolion/Avdella (mountains) - understorey (pine/oak wood)

11) Lowland plains $\quad$ - cultivated fields

12) Agios Nikolaos (mountains) - understorey (Pinus nigra/oak wood)

- understorey (Pinus nigra wood)

13) Above Zakas (foothill limestone - mixed wood on limestone (mixed wood) outcrop)

- road verge (pine wood-pasture)

14) Asprokampos (lowland plains) - weeds (cultivated barley field)

- road verge (agricultural fields)

- understorey (oak wood)

- oaks (oak wood)

15) Avgo (mountains)

- pine (mountain pine, Pinus leucodermis, wood-pastures)

Note: A "P" in the left margin indicates that a pollen reference slide from this specimen has been placed in the Geology Department, Victoria University of Wellington, European pollen reference collection. 
Perivolion/Vovousa - understorey in beech wood: .

$\begin{array}{lll}\text { P } 1 & \text { Myosotis sp. } & \\ \text { P } 2 & \text { Digitalis grandiflora Miller } & \text { Large yellow foxglove } \\ \text { P } 3 & \text { Prunella laciniata (L.) L. } & \text { Cut-leaved self-heal } \\ \text { P } 4 & \text { Dianthus sp. } & \text { Pink } \\ \text { P } 5 & \text { Centaurea } \text { sp. } & \text { Knapweed } \\ \text { P } 6 & \text { Campanula sp. } & \text { Bellflower } \\ \text { P } 7 & \text { Leontodon } \text { or related genus } & \\ \text { P } 8 & \text { Lotus sp. } & \text { Birdsfoot-trefoil } \\ \text { P } 9 & \text { Lathyrus sp. } & \text { Pea, Vetch } \\ \text { P 10 } & \text { Viola } \text { sp. } & \text { Violet, Pansy } \\ \text { P 11 } & \text { Euphrasia sp. } & \end{array}$

Boraginaceae

Scrophulariaceae

Labiatae

Caryophyllaceae

Compositae

Campanulaceae

Compositae

Leguminosae

Leguminosae

Violaceae

Scrophulariaceae

Grass of Parnassus

Willow-herb

Red clover

Water mint

Rush

Rush

Parnassiaceae

Onagraceae

Leguminosae

Labiatae

Juncaceae

Gramineae

Juncaceae

Kellia - margin of fen in agricultural fields:

P 19 Verbena officinalis L.

Vervain

Verbenaceae

P 20 Ononis spinosa L.

Spiny restharrow

Leguminosae

Kellia - disturbed area on fen in agricultural fields:

P 21 Pulicaria dysenterica (L.) Bernh.

Fleabane

Compositae

Kellia - margin of fen in agricultural fields:

P 22 Potentilla reptans $\mathrm{L}$.

Creeping cinquefoil

Bur-reed

P 23 Sparganium erectum L.

Kellia - margin of fen in agricultural fields:

P 24 Mentha aquatica L.

P 25 Torilis japonica (Houtt.) DC

P 26 Daucus carota L.

P 27 Cichorium intybus L.

Chicory

Water mint

Labiatae

Umbelliferae

Umbelliferae

Compositae

Kellia - on fen in agricultural fields:

$\begin{array}{ll}\text { P } 28 \text { Epilobium hirsutum L. } & \text { Great hairy willow-herb, } \\ & \text { Codlins \& cream }\end{array}$

P 29 Epilobium cf. adenocaulon Hausskn.

Onagraceae

Onagraceae

Kellia - disturbed area on fen in agricultural fields:

P 30 Lythrum salicaria L. Purple loosestrife Lythraceae

Kellia - margin of fen in agricultural fields:

P 31 Centaurea ?napulifera Rochel

P 32 Colchicum ?autumnale L.

Compositae

P 33 Consolida sp.

Meadow saffron, Autumn crocus

Liliaceae

Ranunculaceae 
Kellia - on fen in agricultural fields:

P 34 Teucrium scordium L.

P 35 Galium rivale (Sibth. \& Sm.) Griseb.

Water germander

Bedstraw

Dewberry

Gipsy-wort

Love-in-a-mist

Greater bindweed

Fluellen

Dodder

Bedstraw

Water-parsnip

Dock, Sorrel

Woolly thistle

Fluellen

Lady's bedstraw

Wild basil

Birdsfoot-trefoil

Bastard toadflax

Knotgrass

Globe thistle

Scabious

Knapweed

Pliant lettuce

Wild basil

Wild service tree

Hawksbeard

Cocklebur

Ribwort, Plantain

Chamomile

Spurge

Ribwort, Plantain

P 68 Plantago lanceolata $\mathrm{L}$.

69 Convolvulus arvensis L.

P 70 Chondrilla juncea L.

\section{Labiatae \\ Rubiaceae \\ Rosaceae \\ Labiatae \\ Ranunculaceae \\ Convolvulaceae \\ Scrophulariaceae \\ Convolvulaceae \\ Rubiaceae \\ Umbelliferae \\ Primulaceae \\ Polygonaceae}

Compositae

Scrophulariaceae

Compositae

Rubiaceae

Labiatae

Leguminosae

Leguminosae

Santalaceae

Polygonaceae

Compositae

Dipsacaceae

Compositae

Compositae

Cruciferae

Leguminaceae

Labiatae

Rosaceae

Compositae

Compositae

Plantaginaceae

Compositae

Euphorbiaceae

Plantaginaceae

Convolvulaceae

Compositae

Labiatae

Caryophyllaceae

Labiatae

Geraniaceae

Compositae

Compositae

Compositae 
P 78 Xanthium spinosum L.

P 79 Eryngium amethystinum L.

80 Euphorbia myrsinites L.

P 81 Cyclamen sp.

82 No specimen
Spiny cocklebur

Blue eryngo

Broad-leaved glaucous spurge
Compositae

Umbelliferae

Euphorbiaceae

Primulaceae

Polynerion - steppe on east facing hillslope in oakwood-pasture:

P 83 Filago pyramidata L.

84 Hieracium/Pilosella agg.

85 Leontodon sp. (or could be Crepis sp.) Hawkbit

86 Convolvulus arvensis L.

87 Cynodon dactylon (L.) Pers.

P 88 Sanguisorba minor Scop.

89 Galium rivale (Sibth. \& Sm.) Griseb.

P 90 Cynosurus elegans Desf.

91. Piptatherum miliaceum (L.) Cosson

92 Festuca cf. glauca Vill.

93 Lolium perenne L.

94 Agropyron sp.

95 Bromus hordeaceus

96 Taeniatherum caput-medusae (L.) Nevski

97 Lactuca viminea (L.) J. \& C. Presl.

P 98 Lotus cf. corniculatus L.

99 Aegilops neglecta Req. ex Bertol.

P100 Polygonum aviculare L.

Bermuda grass

Bedstraw

P101 Cirsium sp.

102 Rumex tenuifolius (Wallr.) A. Love

103 Prunella grandiflora (L.) Scholler

P104 Trifolium campestre Schreber

105 Trisetum sp. ?flavescens (L.) Beauv.

106 Cynosurus cristatus L.

107 Phleum sp. ?pratense L.

108 Carex sp.

109 Piptatherum miliaceum (L.) Cosson

110 Helianthemum nummularium (L.) Miller

111 Potentilla sp.

112 Agrostis cf. tenuis Sibth.

113 Lolium perenne L.

114 Carex sp.

115 Juncus inflexus L.

Aetia - limestone gorge in mixed wood:

116 Euonymus verrucosus Scop.

117 Ostrya sp.

Ryegrass

Pliant lettuce

Knotgrass

Thistle

Dock, Sorrel

Large self-heal

Hop trefoil

Sedge

Cinquefoil

Sedge

Rush
Compositae

Compositae

Compositae

Convolvulaceae

Gramineae

Rosaceae

Rubiaceae

Gramineae

Gramineae

Gramineae

Birdsfoot-trefoil

Warted spindle-tree

Celastraceae

Corylaceae

Gramineae

Cyperaceae

Labiatae

Onagraceae

Equisetaceae 
Mirsina - margin of Phragmites fen in oakwood:

P123 Mentha aquatica L.

P124 Teucrium scordium L.

P125 Pulicaria dysenterica (L.) Bernh.

P126 Cirsium creticum (Lam.) D'Urv.

P127 Cyperus cf. longus L.

P128 Juncus inflexus L.
Water mint

Water germander

Fleabane

Sedge

Rush
Labiatae

Labiatae

Compositae

Compositae

Cyperaceae

Juncaceae

Mirsina - steppe near Phragmites fen in oakwood:

129 Corylus avellana L. Hazel

P130 Agrimonia eupatoria L.

Agrimony

P131 Dorycnium graecum (L.) Ser.

P132 Daucus carota L.

P133 Crepis sp.

Hawksbeard

134 Brachypodium sylvaticum (Hudson) Beauv.

P135 Teucrium polium L.

Felty germander

136 Buglossoides purpurocaerulea

$$
\text { (L.) I. M. Johnston }
$$

137 Teucrium chamaedrys L.

138 Cuscuta sp.

139 Carex sp

140 Taraxacum sp. (glaucus)

141 Teucrium polium $\mathrm{L}$.

P142 Petrorhagia fasciculata (Margot \& Reuter) P.W. Ball \& Heywood

P143 Alyssum murale Waldst. \& Kit.

144 Thymus sp.

145 Galium verum $\mathrm{L}$.

146 Dactylis glomerata (agg.)

147 Phleum sp.

P148 Scabiosa sp.

P149 Scilla autumnalis L.

P150 Anchusa azurea Miller

151 Melica ciliata L.

P152 Cichorium intybus L.

153 Salvia ?viridis L.

Wall germander

Dodder

Sedge

Felty germander

Thyme

Lady's bedstraw

Scabious

Large blue alkanet

Chicory

Red-topped sage

Mirsina - ground cover in oak wood:

154 Cyclamen sp.

155 ?Thalictrum sp.

156 Elymus ?caninus (L.) L.

157 Tussilago farfara L.

P158 Bupleurum asperuloides Heldr. ex Boiss

Anelia - road verge in beech wood:

P159 Verbascum macrurum Ten.

Anelia - dry land on edge of bog in beech wood:

P160 ?Stachys sp.

P161 Lychnis coronaria (L.) Desr. in Lam.

P162 Geranium sp.

P163 Digitalis ferruginea L.
Woundwort

Rose campion

Cranesbill

Rusty foxglove
Corylaceae

Rosaceae

Leguminosae

Umbelliferae

Compositae

Gramineae

Labiatae

Boraginaceae

Labiatae

Convolvulaceae

Cyperaceae

Compositae

Labiatae

Caryophyllaceae

Cruciferae

Labiatae

Rubiaceae

Gramineae

Gramineae

Dipsacaceae

Liliaceae

Boraginaceae

Gramineae

Compositae

Labiatae

Primulaceae

Ranunculaceae

Gramineae

Compositae

Umbelliferae

Scrophulariaceae

Labiatae

Caryophyllaceae

Geraniaceae

Scrophulariaceae 
Mirsina - steppe near Phragmites fen in oak wood:

P164 Opopanax hispidus (Friv.) Griseb.

Umbelliferae

Gomara - dried lakebed in pine, Pinus leucodermis, wood-pasture:

165 Convolvulus arvensis L.

166 Euphorbia myrsinites L.

Broad-leaved glaucous spurge

Convolvulaceae

P167 Trifolium repens $\mathrm{L}$.

168 Juncus sp.

Rush

Euphorbiaceae

Leguminosae

169 Glyceria sp.

Juncaceae

Gramineae

Gomara - below lake in pine, Pinus leucodermis, wood-pasture:

P170 Echinops cf. ?microcephalus Sibth. \& Sm. Globe thistle

Compositae

Zakas to Agios Nikolaos - road verge in pine wood-pasture:

171. Cornus mas L.

Comelian cherry

Cornaceae

Perivolion/Avdella - pine/oak wood understorey:

P172 Hedera helix L. Ivy

P173 Lotus aegaeus (Griseb.) Boiss

P174 Dorycnium pentaphyllum Scop.

P175 Cuscutasp.

176 Veronica $\mathrm{cf}$. chamaedrys L.

P177 Teucrium sp.

Dodder

Germander speedwell

Germander

P178 Alyssum murale Waldst. \& Kit.

179 Dactylis sp.

180 ?Festuca sp.

181 Centaurea sp.

182 ?Hieracium/Pilosella agg.

183 Pyrus communis L.

184 Crataegus sp.

Knapweed

Pear

Hawthorn

Lowland plains - cultivated fields:
185 Triticum durum Desf.
186 Triticum durum Desf.
Durum wheat
Durum wheat
187 Hordeum distichon $\mathrm{L}$.
Two-rowed barley

Agios Nikolaos - understorey Pinus nigra wood:

$\begin{array}{lll}188 & \text { Stachys cf. officinalis (L.) Trevisan } & \text { Woundwort } \\ 189 & \text { Prunella } \text { ?vulgaris } \text { L. } & \text { Common self-heal } \\ 190 & \text { Potentilla sp. } & \text { Cinquefoil } \\ 191 & \text { Rubus caesius } \text { L. } & \text { Dewberry } \\ 192 & \text { Viola } \text { sp. } & \text { Violet, Pansy } \\ 193 \text { cf. } \text { Plantago } & \\ 194 \text { ?Daucus } \text { sp. } & \\ 195 \text { Echinops } \text { sp. } & \text { Globe thistle } \\ 196 & \text { Thymus sp. } & \text { Thyme } \\ 197 & \text { Festuca ovina } \text { L. } & \\ 198 \text { Festuca ovina } \text { L. } & \\ 199 & \text { Lathyrus sp. } & \text { Pea, Vetch }\end{array}$

\section{Araliaceae \\ Leguminosae \\ Leguminosae \\ Convolvulaceae \\ Scrophulariaceae \\ Labiatae \\ Cruciferae \\ Gramineae \\ Gramineae \\ Compositae \\ Compositae \\ Rosaceae \\ Rosaceae}

Gramineae

Gramineae

Gramineae

Labiatae

Labiatae

Rosaceae

Rosaceae

Violaceae

Umbelliferae

Compositae

Labiatae

Gramineae

Gramineae

Leguminosae 
Above Zakas - mixed wood on limestone:

200 Rubus corylifolius Sm.

201 Veronica cf. chamaedrys L.

202 Euphorbia cf. amygdaloides L.

203 ?Lathyrus laxiflorus (Desf.) O. Kuntze

204 Potentilla sp. ?sterilis (L.) Garcke

205 Viola sp.

206 Quercus cf. petraea (Mattuschka) Liebl.

207 Pteridium aquilinum (L.) Kuhn in Decken

208 Acer opalus Miller

209 Galium sp.

210 Fagus sylvatica L.

211 Abies cephalonica Loudon

212 Primula elatior (L.) Hill

213A Indeterminate

213B Luzula sylvatica (Hudson) Gaudin

214 Brachypodium sylvaticum (Hudson) Beauv.

214A. Festuca sp., rubra group

215 Festuca sp., rubra group
Germander speedwell

Violet, Pansy

Maple
Bedstraw
Beech
Greek fir
Oxlip, Paigle

Wood rush

Above Zakas - road verge in pine wood-pasture:

216 Populus tremula L.

P217 Campanula trachelium subsp. athoa

(Boiss. \& Heldr.) Hayek
Rosaceae

Scrophulariaceae

Euphorbiaceae

Leguminosae

Rosaceae

Violaceae

Fagaceae

Hypolepidaceae

Aceraceae

Rubiaceae

Fagaceae

Pinaceae

Primulaceae

Juncaceae

Gramineae

Gramineae

Gramineae

Salicaceae

Campanulaceae

Ranunculaceae

Polygonaceae

Gramineae

Gramineae

Gramineae

Gramineae

Gramineae

Gramineae

Plantaginaceae

Boraginaceae

Malvaceae

Primulaceae

Solanaceae

Primulaceae

Scrophulariaceae

Umbelliferae

Chenopodiaceae

Plantaginaceae

Compositae

Cruciferae

Geraniaceae

Convolvulaceae

Salicaceae

Chenopodiaceae

Oraches

Pliant lettuce

Compositae

Compositae 
245 Lactuca virosa $\mathrm{L}$.

246 Cuscuta sp.
Poisonous lettuce

Dodder
Compositae

Convolvulaceae

Asprokampos - road verge in agricultural fields:

$\begin{array}{rll}\text { P247 } & \text { Centaurea sp. } & \text { Knapweed } \\ \text { P248 } & & \\ \text { P249 } & \text { Mentha suaveolens Ehrh. } & \text { Mint } \\ \text { P250 } & \text { Verbena officinalis L. } & \text { Vervain } \\ 251 & \text { Lactuca viminea } \text { (L.) J. \& C. Presl. } & \text { Pliant lettuce } \\ \text { P252 } & \text { Salvia } \text { sp. } & \text { Clary, Sage } \\ \text { P253 } & \text { ?Sinapis alba } \text { L. } & \text { White mustard } \\ \text { P254 } & \text { ?Scorzonera } \text { sp. } & \text { ?Vipergrass } \\ 255 & \text { Lactuca viminea } \text { (L.) J. \& C. Presl. } & \text { Pliant lettuce }\end{array}$
Compositae
Dipsacaceae
Labiatae
Verbenaceae
Compositae
Labiatae
Cruciferae
Compositae
Compositae

Asprokampos - oak wood understorey :

256. Alyssum murale Waldst. \& Kit.

257 Potentilla sterilis (L.) Garcke

258 Scilla autumnalis L.

259 Viola sp.

260 Carlina corymbosa L.

261 Dactylis sp.

262 Agrimonia sp.

263 Teucrium chamaedrys L.

265 Veronica chamaedrys L.

266 Crataegus monogyna Jacq.

267 Rosa sp.

268 Buglossoides purpurocaerulea

269 Veronica sp.

(L.) I.M. Johnston

Violet, Pansy

Flat-topped carline thistle

Agrimony

Wall germander

Germander speedwell

Hawthorn

Cruciferae

Rosaceae

Liliaceae

Violaceae

Compositae

Gramineae

Rosaceae

Labiatae

Leguminosae

Scrophulariaceae

Rosaceae

Rosaceae

Gromwell

Speedwell

Thyme

Cinquefoil

Felty germander

Rock-cress

Chamomile

Germander speedwell

Wild basil

Boraginaceae

Scrophulariaceae

Leguminosae

Labiatae

Rosaceae

Gramineae

Labiatae

Cruciferae

Labiatae

Compositae

Scrophulariaceae

Labiatae

Gramineae

Gramineae

Gramineae

Gramineae

Gramineae

Gramineae

Gramineae

Leguminosae

Compositae

Rosaceae

Gramineae

Gramineae

Gramineae

Lady's bedstraw 
Avgo - mountain pine, Pinus leucodermis, wood-pasture:

P306 Pinus leucodermis Antoine

Pinaceae

\section{Asprokampos - oak wood}

\section{Quercus leaves}

\begin{tabular}{|c|c|c|c|}
\hline a & Quercus cerris L. & Turkey oak & Fagaceae \\
\hline b & Q. ?brachyphylla Kotschy & Oak & Fagaceae \\
\hline c & Q. cerris L. hybrid & Turkey oak & Fagaceae \\
\hline d & Q. cf. frainetto, ?cerris L. $\mathrm{x}$ frainetto $\mathrm{Ten}$. & Oak & Fagaceae \\
\hline $\mathrm{e}$ & Q.brachyphylla Kotschy & Oak & Fagaceae \\
\hline $\mathrm{f}$ & $Q . \mathrm{sp}$. & Oak & Fagaceae \\
\hline $\mathrm{g}$ & $Q$. sp. & Oak & Fagaceae \\
\hline $\mathrm{h}$ & $Q$. sp. & Oak & Fagaceae \\
\hline $\mathrm{i}$ & $Q$. sp. & Oak & Fagaceae \\
\hline $\mathrm{j}$ & $Q$. sp. & Oak & Fagaceae \\
\hline $\mathrm{k}$ & Q. cerris $\mathrm{L}$. & Turkey oak & Fagaceae \\
\hline 1 & $Q$. sp. & Oak & Fagaceae \\
\hline $\mathrm{m}$ & Q. cf. cerris $\mathrm{L}$. & cf. Turkey oak & Fagaceae \\
\hline $\mathrm{n}$ & Q. cf. cerris L. & cf. Turkey oak & Fagaceae \\
\hline o & Q. brachyphylla Kotschy & Oak & Fagaceae \\
\hline $\mathrm{p}$ & Q. cerris $\mathrm{L}$. & Turkey oak & Fagaceae \\
\hline $\mathrm{q}$ & Q. cerris $\mathrm{L}$. [x virgiliana? (Ten.) Ten.] & Oak & Fagaceae \\
\hline $\mathrm{r}$ & Q. brachyphylla Kotschy [x cerris L.?] & Oak & Fagaceae \\
\hline $\mathrm{s}$ & Q. pubescens Willd., hybrid & White or Downy oak & Fagaceae \\
\hline $\mathrm{t}$ & $Q$. sp. & Oak & Fagaceae \\
\hline $\mathrm{u}$ & $Q$. cerris $\mathrm{L}$. & Turkey oak & Fagaceae \\
\hline $\mathrm{v}$ & Q. cerris L., ?hybrid & Turkey oak & Fagaceae \\
\hline w & $Q$. sp. & Oak & Fagaceae \\
\hline $\mathrm{x}$ & Q. sp. & Oak & Fagaceae \\
\hline $\mathrm{y}$ & Q. sp. & Oak & Fagaceae \\
\hline $\mathrm{z}$ & Q. cerris $\mathrm{L}$. & Turkey oak & Fagaceae \\
\hline aa & Q. cerris $\mathrm{L}$. & Turkey oak & Fagaceae \\
\hline $\mathrm{bb}$ & Q. sp. & Oak & Fagaceae \\
\hline $\mathrm{cc}$ & $Q . \mathrm{sp}$. & Oak & Fagaceae \\
\hline $\mathrm{dd}$ & Q. sp. & Oak & Fagaceae \\
\hline ee & Q. cerris L., hybrid & Turkey oak & Fagaceae \\
\hline $\mathrm{ff}$ & Q. cerris L. & Turkey oak & Fagaceae \\
\hline gg & Q. cerris $\mathrm{L}$. & Turkey oak & Fagaceae \\
\hline hh & Q. cerris L., hybrid & Turkey oak & Fagaceae \\
\hline ii & Q. sp. [cf. cerris L.] & Oak & Fagaceae \\
\hline $\mathrm{jj}$ & Q. sp. [cf. cerris L.] & Oak & Fagaceae \\
\hline $\mathrm{kk}$ & Q. cerris $\mathrm{L}$. & Turkey oak & Fagaceae \\
\hline 11 & $Q$. sp. & Oak & Fagaceae \\
\hline $\mathrm{mm}$ & $Q$. sp. & Oak & Fagaceae \\
\hline $\mathrm{nn}$ & Q. cerris $\mathrm{L}$. & Turkey oak & Fagaceae \\
\hline oo & $Q$.sp. & Oak & Fagaceae \\
\hline $\mathrm{pp}$ & $Q$. sp. & Oak & Fagaceae \\
\hline $\mathrm{qq}$ & Q. cerris $\mathrm{L}$. & Turkey oak & Fagaceae \\
\hline$\pi$ & $Q$. sp. & Oak & Fagaceae \\
\hline ss & $Q$. sp. & Oak & Fagaceae \\
\hline
\end{tabular}


Family

\begin{tabular}{|c|c|c|c|}
\hline $\mathrm{tt}$ & Q. cerris L., hybrid [mainly cerris L.] & Turkey oak & Fagaceae \\
\hline uu & Q. cerris L. & Turkey oak & Fagaceae \\
\hline vv & Q. cerris L., hybrid & Turkey oak & Fagaceae \\
\hline ww & Q. cerris $\mathrm{L}$. & Turkey oak & Fagaceae \\
\hline$x x$ & Q. cerris L. & Turkey oak & Fagaceae \\
\hline yy & Q. sp. & Oak & Fagaceae \\
\hline $\mathrm{zz}$ & Q. sp. & Oak & Fagaceae \\
\hline aаa & Q. brachyphylla Kotschy & Oak & Fagaceae \\
\hline bbb & Q. cerris L. & Turkey oak & Fagaceae \\
\hline $\operatorname{ccc}$ & Q. trojana Webb in Loudon & Oak & Fagaceae \\
\hline ddd & Q. sp. & Oak & Fagaceae \\
\hline eee & Q. sp. & Oak & Fagaceae \\
\hline $\mathrm{fff}$ & Q. sp. & Oak & Fagaceae \\
\hline ggg & $Q$. sp. & Oak & Fagaceae \\
\hline
\end{tabular}




\section{Appendix II}

\section{Laboratory preparation techniques for pollen and charcoal analysis}

The object of these preparation techniques was to concentrate the palynomorphs and microscopic charcoal and remove extraneous matter. The process involves the step-by-step removal of carbonates, humic acids, silica, and cellulose by appropriate methods, separated by washing with distilled water and concentration using a centrifuge, followed by mounting and storage. Sieving to remove both coarse and fine unwanted material was also used.

Texts consulted for routine techniques of preparation of pollen samples were: Brown 1960; Kummel \& Raup 1965; Erdtman 1969; Reitsma 1969; Faegri \& Iversen 1975 \& 1992; Moore \& Webb 1978; Shackley 1981; Dimbleby 1985; Vidal 1988; Moore et al 1991 and Raine \& Tremain 1992.

In the laboratory the highest standards of safety were followed (Muir 1977; Marshall 1981). A laboratory coat, rubber gloves and protective shoes were worn during all laboratory procedures. In addition, a face shield was worn while hydrofluoric acid (HF) and sulphuric acid $\left(\mathrm{H}_{2} \mathrm{SO}_{4}\right)$ were being used. Both of these chemicals were handled under a fume hood. A paste of glycerin and magnesium oxide for the treatment of hydrofluoric acid burns/suspected burns was kept next to the fume cupboard.

Slides used throughout were 1-1.5 mm thick and $26 \times 76 \mathrm{~mm}$. Coverslips were number $1,22 \times 22 \mathrm{~mm}$.

\section{II.1 Laboratory preparation of moss polsters and litter, soil and wetland sequences}

These laboratory preparations were carried out in the Palynology Laboratory, Geology Department, Victoria University of Wellington. Care was taken to ensure that samples were not contaminated with pollen circulating in the air. Ideally, the laboratory would be pollen-sterile with non-opening windows and any air ventilation should be through window spaces covered with filters. Before processing began, as a check on the sterility of the laboratory, microscope slides with glycerine jelly on their surface were left exposed in the laboratory for a month and then sealed. These slides were checked for any contaminant pollen circulating in the laboratory air. This was repeated several times. Initially the test slides captured significant numbers of pollen grains, so all the shelves and glassware were washed. Plastic tape was put around the windows, latex closed cell foam was placed around the door to stop draughts and an air filter was fitted to the only air inlet, a window into an internal hallway. This was particularly important since air extractors used under the fume hoods draw air into the room. Atmospheric pollen surveys carried out in Wellington show that Pinus, Quercus, Cupressus, Populus, Fraxinus, Salix, Rumex, Chenopodiaceae, Plantago and Gramineae are all present in the air in significant quantities at various times of the year (Clark 1951; Licitis 1953; Mirams 1953). These pollen types represent plant taxa extant in Grevena. After carrying out the above precautions contaminant pollen was minimal.

Polypropylene heat resistant Falcon 2070, $50 \mathrm{ml}$ screw cap, conical based centrifuge tubes were used throughout the laboratory processing. A 5338 adapter was fitted to a Heraeus centrifuge to accommodate the tubes.

Samples were washed between each chemical treatment by adding distilled water to the centrifuge tube, centrifuging and decanting, twice. Samples were centrifuged at $3000 \mathrm{rpm}$ for 4 minutes, unless otherwise stated, before being decanted to get rid of unwanted liquid. The distilled water was tested from time to time for contaminant pollen; none were found

Extreme care was taken to avoid contamination. Each sample was given a laboratory number and equipment was used for that particular sample only, except the sieve, which was cleaned between each sample use. Glass rods were not allowed to come into contact with each other and care was also taken to ensure that cross contamination did not occur when centrifuge tubes were boiling together in water baths. All glassware and containers were washed in very hot water and laboratory grade detergent. 
Only minor details differ between the extraction procedures used to extract palynomorphs from the moss polsters and litter, soil and wetland sequences. The subsurface samples from soil profiles and wetland sequences were prepared for absolute as well as relative counting procedures, so a vortex mechanical stirrer was used, where possible, in preference to stirring rods.

\section{II.1.1 Laboratory preparation of moss polsters and litter samples}

Seven steps were used to extract pollen, spores and microscopic charcoal from moss polsters and litter samples; hot potassium hydroxide $(\mathrm{KOH})$ treatment, wet sieving, cold dilute hydrochloric acid $(\mathrm{HCl})$ treatment, cold hydrofluoric acid (HF) treatment, acetolysis, cold potassium hydroxide and distilled water wash, and filtration. This was followed by dehydration in alcohol and silicone oil mounting.

The procedure used:

\section{1) Potassium hydroxide treatment}

This treatment was carried out to remove humic acids and effect deflocculation. The main purpose of the treatment was to break up the moss and allow effective sieving.

An amount of moss about the size of a matchbox was placed in $100 \mathrm{ml}$ pyrex beaker, $80 \mathrm{ml} 10 \%$ potassium hydroxide solution was added to the beaker before it was placed on a hot plate and boiled for 10 minutes while being stirred vigorously with a glass stirring rod to break up the moss.

The sample was not washed at this stage, but sieved first.

\section{2) Wet sieving}

This procedure was undertaken to remove the coarse inorganic and organic particles.

The samples were sieved and washed through a $260 \mu \mathrm{m}$ aperture diameter sieve into $50 \mathrm{ml}$ centrifuge tubes by squirting distilled water onto the residue on the sieve.

The same sieve was used for each sample so care was taken to clean the sieve thoroughly between each sample. This was done by squirting the sieve with a jet of water, then burning any residual organic matter by placing the sieve over a bunsen burner until the sieve was red hot. Distilled water was then squirted on the sieve to cool it.

The samples were then centrifuged and decanted, and washed in distilled water until the supernatant was clear.

The residue retained on the sieve was put into a glass vial with a mixture of 1:1 distilled water and glycerol with a small amount of phenol, for later inspection.

\section{3) Cold hydrochloric acid treatment}

This treatment was undertaken to dissolve carbonates. This must be done before hydrofluoric acid treatment to ensure that insoluble calcium fluoride $\left(\mathrm{CaF}_{2}\right)$ precipitates do not form.

$20 \mathrm{ml} 10 \%$ hydrochloric acid was added to each sample in the centrifuge tubes, stirred and left to stand overnight.

Next morning the samples were centrifuged and decanted. 


\section{4) Hydrofluoric acid digestion}

This removed siliceous material and reduced the expansion of grains during acetolysis (Faegri \& Iversen 1992:78).

$15 \mathrm{ml}$ of $40 \%$ hydrofluoric acid was added to the centrifuge tubes. This was stirred with teflon stirring rods (glass rods cannot be used as hydrofluoric acid corrodes glass) and left to stand overnight. The samples were centrifuged and decanted in the morning.

$15 \mathrm{ml} \mathrm{10 \%} \mathrm{hydrochloric} \mathrm{acid} \mathrm{was} \mathrm{added} \mathrm{to} \mathrm{the} \mathrm{centrifuge} \mathrm{tubes} \mathrm{which} \mathrm{were} \mathrm{placed} \mathrm{in} \mathrm{a} \mathrm{water} \mathrm{bath} \mathrm{and}$ gently heated to remove the silicofluorides and effect deflocculation. The samples were left in the water bath until the supernatant had turned a greenish-yellow colour. They were left in the water bath a few more minutes until the supernatant had become clear, then centrifuged and decanted. If the supernatant was not completely clear the hydrochloric acid treatment was repeated.

\section{5) Acetolysis}

This treatment destroyed cellulose and some other organic compounds.

The standard procedure for acetolysis was followed. Firstly, the samples were dehydrated (because sulphuric acid reacts with water) by adding $15 \mathrm{ml}$ glacial acetic acid to the centrifuge tube, centrifuging and decanting. The acetolysis mixture of 1:9, concentrated sulphuric acid: acetic anhydride was then mixed by pouring the sulphuric acid very slowly into the acetic anhydride, as the procedure is exothermic and generates heat very rapidly to great temperatures. $15 \mathrm{ml}$ of this mixture (it must be used fresh) was added to the centrifuge tubes which were then placed in a boiling water bath for 4 minutes while they were stirred. The tubes were removed from the bath and $5 \mathrm{ml}$ glacial acetic acid was added to stop acetolysis. The samples were then centrifuged and decanted.

$15 \mathrm{ml}$ glacial acetic acid was then added to the centrifuge tubes and left to stand for 5 minutes to ensure that acetolysis had stopped.

The samples were then centrifuged and decanted, and washed in distilled water.

\section{6) Potassium hydroxide wash}

This neutralized any residual acid and washed the sample.

$20 \mathrm{ml} 10 \%$ potassium hydroxide solution was added to the centrifuge tube which was then centrifuged and decanted. The samples then had $20 \mathrm{ml}$ distilled water added, and were centrifuged and decanted thrice.

\section{7) Filtration}

To remove very fine organic and mineral particles, and insoluble fluorides, and concentrate the pollen.

The samples were filtered through a $6.5 \mu$ m nylon monofilament mesh filter cloth with distilled water using a Sartorius membrane filtering unit SM165-10, fitted to a laboratory stand, and supplied with normal/inverted vacuum suction (Raine \& Tremain 1992; Vidal 1988).

\section{8) Dehydration}

The samples required treatment with absolute alcohol so that evaporation would be complete when mounting in silicone oil. 
Samples were washed in 95\% ethanol, then tertiary butyl alcohol $=2$-methyl propan-2-ol (TBA), in the following manner:

$30 \mathrm{ml}$ of $95 \%$ ethanol was added to the centrifuge tubes, stirred on a mechanical stirrer, then centrifuged and decanted. This was repeated.

$20 \mathrm{ml}$ tertiary butyl alcohol was added to the centrifuge tubes, stirred on a mechanical stirrer, then centrifuged and decanted. This was repeated.

\section{9) Mounting}

Silicone oil, refractive index 1.40, was used as the mounting media. Silicone oil "200 Fluid" at 1000 centistokes and " 200 Fluid" at 12500 centistokes was supplied by Ajax Chemicals, Auckland. These two viscosities were blended by weight to achieve the required $2000 \mathrm{cs}$ viscosity.

A microscope slide was heated on a histology hotplate to about $110^{\circ} \mathrm{C}$. About 4 drops of silicone oil was deposited on the centre of the slide, using a $0.5 \mathrm{ml}$ disposable insulin syringe. The required amount of oil varied according to the thickness of the thickest particles on the slide. The oil has to completely cover the area under a $22 \mathrm{~mm}$ square coverslip without oozing out the sides of it.

Using a micropipetter (Finnpipette, 200-1000 $\mu \mathrm{l}$ ) with disposable polypropylene tips (Finntip 61, for volume range 200-1000 $\mu$ l), a volume of agitated suspension was transferred to the drop of silicone oil on the slide (the volume did not need to be known as these slides were prepared for relative pollen frequency calculations only). All the suspension in the micropipette tip was dispensed very slowly onto the slide and the tip discarded. The tertiary butyl alcohol was allowed to completely evaporate. The slide was removed from the hotplate and allowed to cool a little before the cover slip was placed over the oil. The slide was returned to the hotplate for the oil to completely fill the space under the coverslip.

The slide was cooled and sealed carefully with clear nail vamish. Slides were kept horizontal.

\section{II.1.2 Laboratory preparation of soil samples}

The laboratory preparation of these samples was undertaken in the Palynology Section laboratory of the Institute of Geological and Nuclear Sciences, Lower Hutt.

These samples were prepared for the calculation of the absolute concentration of palynomorphs as well as relative frequency, therefore they were dried then volumetrically measured and weighed prior to processing. To minimize sample loss, a mechanical stirrer was used where possible in preference to using stirring rods and the samples remained in the same centrifuge tubes for the entire extraction procedure except when wet sieving was carried out.

The extraction procedure was very similar to that carried out for the moss polster and litter samples. The differences were that two steps were added at the beginning of the procedure, one to disaggregate the samples and the other to remove carbonates, and stronger hydrochloric acid was used to effect deflocculation after the hydrofluoric acid treatment.

\section{1) Disaggregation}

$20 \mathrm{ml} 5 \%$ Calgon (sodium hexametaphosphate) solution was added to the centrifuge tubes. The tubes were placed on a rotating shaker table overnight, centrifuged and decanted the following morning, then washed. 


\section{2) Hydrochloric acid treatment}

To remove carbonates.

$20 \mathrm{ml} 10 \%$ hydrochloric acid was added to the centrifuge tube and the amount of effervescence recorded, as an indication of the amount of calcareous material present. When the effervescing ceased, samples were centrifuged and decanted, then washed twice in distilled water.

\section{3) Potassium hydroxide treatment}

To remove humic acids and effect deflocculation.

$20 \mathrm{ml} 10 \%$ potassium hydroxide solution was added to the centrifuge tube. A glass rod was used to disperse the sample. The centrifuge tubes were placed in a hot water bath and stirred for 10 minutes.

\section{4) Wet sieving}

To remove the coarse inorganic and organic particles.

The samples were sieved and washed through a $260 \mu \mathrm{m}$ aperture diameter sieve into $50 \mathrm{ml}$ centrifuge tubes in the same manner as for the moss polster and litter samples.

\section{5) Hydrofluoric acid digestion}

To remove siliceous material and reduce the expansion of grains during acetolysis.

Hydrofluoric acid treatment for the soil samples was different from that for the moss polster and litter samples in that the sample was treated with hot hydrofluoric acid.

The samples were first washed in cold $10 \%$ hydrochloric acid (to ensure the removal of carbonates and prevent the formation of calcium fluoride precipitate), then $10 \mathrm{ml} 40 \%$ hydrofluoric acid was added to the centrifuge tubes. The tubes were then placed in a hot water bath for one hour. The water bath was turned off, but the samples were left in it overnight. In the morning fresh hydrofluoric acid was added and the samples stirred, then the water bath was turned on again for one hour. If the silicates had not dissolved, further treatment was given, by adding fresh hydrofluoric acid and heating. When all the silicates had dissolved, the samples were centrifuged and decanted and given a hot hydrochloric acid treatment similar to that given to the moss polster and litter samples, except $25 \%$ hydrochloric acid was used initially to effect deflocculation, then the samples had cold $10 \%$ hydrochloric acid added before centrifugation and washing.

\section{6) Acetolysis}

Acetolysis destroys cellulose and some other organic compounds.

This was carried out in the same manner as for the moss polster and litter samples.

\section{7) Filtration}

To remove very fine organic and mineral particles, and insoluble fluorides, and concentrate the pollen.

The samples were filtered in the same manner as for the moss polster and litter samples.

As a check the filtrate which passed through the filter cloth was retained and examined for palynomorphs; none were found. 


\section{8) Dehydration}

The samples were washed in $95 \%$ ethanol twice, then tertiary butyl alcohol twice in the same manner as for the moss polster and litter sampies.

\section{9) Mounting}

Silicone oil, blended as described above, was used as the mounting media.

A standard microscope slide was heated on a histology hotplate to about $110^{\circ} \mathrm{C}$. About 4 drops of silicone oil was deposited on the centre of the slide, using a $0.5 \mathrm{ml}$ disposable insulin syringe. The required amount of oil varied according to the thickness of the thickest particles on the slide. The oil had to completely cover the area under a $22 \mathrm{~mm}$ square coverslip without oozing out the sides of it.

Using a micropipetter (Finnpipette, 200-1000 $\mathrm{\mu l}$ ) with disposable polypropylene tips (Finntip 61, for volume range 200-1000 $\mu \mathrm{l}$ ), a known volume ("aliquot") of agitated suspension was transferred to the drop of silicone oil on the slide. All the suspension in the micropipette tip was dispensed very slowly onto the slide and the tip discarded. The tertiary butyl alcohol was allowed to completely evaporate. The slide was removed from the hotplate and allowed to cool a little before the cover slip was placed over the oil. The slide was returned to the hotplate for the oil to completely fill the space under the coverslip.

The slide was cooled and sealed carefully with clear nail varnish. Slides were kept horizontal.

\section{II.1.3 Laboratory preparation of wetland samples}

These laboratory preparations were carried out in the Palynology laboratory, Geology Department, Victoria University of Wellington.

These samples were prepared for the calculation of the absolute concentration of palynomorphs as well as relative frequency, therefore they were volumetrically measured and weighed prior to processing. To minimize sample loss, a mechanical stirrer was used where possible in preference to using stirring rods and the samples remained in the same centrifuge tubes for the entire extraction procedure except when wet sieving was carried out.

All samples were processed uniformly.

The extraction procedure was very similar to that carried out for the soil samples. The differences were that a spike of Lycopodium spores was added to the sample before extraction began, so that pollen, spore and charcoal concentrations could be calculated using the exotic marker grain method; no initial disaggregation treatment was given, the initial hydrochloric acid treatment included heating the samples, and no filtering was done.

\section{1) Spike added}

A known volume of a prepared spike of Lycopodium spores was added to the $1 \mathrm{cc}$ wet sample in the $50 \mathrm{ml}$ centrifuge tube, so that pollen concentration could be calculated.

\section{2) Hydrochloric acid treatment}

$20 \mathrm{ml} \mathrm{10 \%} \mathrm{hydrochloric} \mathrm{acid} \mathrm{was} \mathrm{added} \mathrm{to} \mathrm{the} \mathrm{centrifuge} \mathrm{tubes} \mathrm{and} \mathrm{the} \mathrm{amount} \mathrm{of} \mathrm{effervescence}$ recorded, as an indication of the amount of calcareous material present. When the effervescing had ceased, the tubes were placed in a hot water bath either for 5 minutes or until the effervescing stopped. Centrifuged and decanted. $15 \mathrm{ml}$ of $10 \%$ hydrochloric acid was then added to the tubes. The sample was centrifuged and decanted and washed twice in distilled water. 


\section{3) Potassium hydroxide treatment}

This was carried out using the same procedures as for the soil samples except the colour of the supernatant, after heating, was compared to the Munsell Chart and recorded, as a measure of humification.

\section{4) Wet sieving}

Wet sieving was carried out in the same manner, as for the soil samples.

\section{5) Hydrofluoric acid digestion}

Hydrofluoric acid digestion was effected in the same manner as for the soil samples.

However, flocculation of some of the samples persisted after the hot hydrochloric acid treatment, especially in samples from the Gomara sequence. It was found that the mechanical stirrer did not effect dispersal. When this occurred the centrifuge tube was suspended in a Unisonics $40 \mathrm{KHz}$ ultrasonic transducer tank for a few seconds only, until dispersal was achieved (Gray 1965:534-5). Although prolonged ultrasonic treatment may damage delicate pollen (McIntyre \& Norris 1964; Dodson 1983), it appeared that in the Grevena samples only pine grains may have been subject to breakage, bladders being broken off bodies, but it is not certain that this occurred during ultrasonic irradiation. The following treatment with $95 \%$ ethanol assisted with dispersal of the organic particles.

\section{6) Acetolysis}

Acetolysis was carried out in the same manner as for the soil samples.

\section{7) Dehydration}

The samples were washed in $95 \%$ ethanol twice, then tertiary butyl alcohol twice, in the same manner as the soil samples.

\section{8) Mounting}

Samples from the Anelia and Kellia sites where the exotic marker grain technique for pollen and spore concentration calculates only was used were mounted using the technique described above for the soil samples.

Samples from the Gomara site where the aliquot technique for calculating pollen and spore concentrations was used, as well as the exotic marker grain technique, were mounted with precisely measured known volumes (aliquots) from a suspension of known volume, as outlined below.

Special technique of measuring aliquots from a suspension for pollen and spore concentration calculations:

a) The residue was carefully washed into a volumetric flask (either 10 or $25 \mathrm{ml}$ depending on the volume of residue), using a small glass funnel and wash bottle with warm tertiary butyl alcohol. The volume of the suspension was made up to the graduation mark with tertiary butyl alcohol, using a micropipette for fine control. The flask was stoppered and the suspension mixed by shaking the flask. Once the suspension was completely mixed it was quickly tipped into a labelled, straight sided, soda glass Samco specimen tube with polythene stopper. This is the first dilution, and when the residue was sufficiently diluted it was used directly for slide making. When further dilution was necessary, this was achieved by the method outlined in (b) below. 
b) The residue in the specimen tube vial was agitated to completely suspend it, then $1 \mathrm{ml}$ was promptly withdrawn using a graduated, spring-loaded measuring syringe (Manostat Varipet). The $1 \mathrm{ml}$ was discharged into a $10 \mathrm{ml}$ volumetric flask and the volume made up to the graduation mark. This second dilution was then $1 / 10$ of the concentration of the initial dilution.

c) A standard $26 \times 76 \mathrm{~mm}$ microscope slide was heated on a histology hotplate to about $110^{\circ} \mathrm{C}$. About 4 drops of $2000 \mathrm{cs}$ viscosity silicone oil (Dow Coming "200 Fluid") was deposited on the centre of the slide, using a $0.5 \mathrm{ml}$ disposable insulin syringe. The amount of oil varied according to the thickness of the thickest particles on the slide. The oil had to completely cover the area under a $22 \mathrm{~mm}$ square coverslip without oozing out the sides. This was most important because the aliquot method of calculating pollen concentration requires that the total number of palynomorphs placed on the slide be counted.

d) Using a micropipetter (Finnpipette, 200-1000 $\mu \mathrm{l}$ ) with disposable polypropylene tips (Finntip 61, for volume range 200-1000 $\mu \mathrm{l}$ ), a known volume ("aliquot") of agitated suspension was transferred to the drop of silicone oil on the slide. All the suspension in the micropipette tip was dispensed very slowly onto the slide and the tip discarded. The tertiary butyl alcohol was allowed to completely evaporate. The slide was removed from the hotplate and allowed to cool a little before the cover slip was placed over the oil. The slide was returned to the hotplate for the oil to completely fill the space under the coverslip. The slide was cooled and sealed carefully with clear nail varnish. Slides were kept horizontal.

\section{II.1.4 Lycopodium spike preparation}

Preparation, calibration, and addition of the spike to samples are all quantitative procedures so special care was taken at each step to ensure accuracy. Statistical calculations are treated by Maher (1981).

\section{Preparation of spike}

$0.5 \mathrm{~g}$ of Lycopodium spores, donated by Greer Laboratories, Inc., North Carolina, U.S.A. were placed in a $50 \mathrm{ml}$ conical bottom centrifuge tube.

\section{1) Wetting}

The Lycopodium spores were washed $2 \mathrm{x}$ in distilled water with a very small amount of liquid detergent added. Centrifuged and decanted after each.

\section{2) Acetolysis}

The spores were dehydrated in glacial acetic acid. Centrifuged and decanted.

The acetolysis mixture of $9 \mathrm{ml}$ acetic anhydride followed by $1 \mathrm{ml}$ concentrated sulfuric acid was mixed. This was poured onto the spores in the tube, stirred, and placed in an actively boiling water bath for 2 minutes only; centrifuged and decanted.

Rinsed $1 \mathrm{x}$ with glacial acetic acid. Centrifuged and decanted.

Rinsed $4 \mathrm{x}$ with distilled water. Centrifuged and decanted after each.

\section{3) Staining}

About 10 drops of $0.5 \%$ safranin were added with a little water and left for one or two minutes. The spores were then washed with $95 \%$ ethanol, then distilled water. 


\section{4) Suspension}

The stained Lycopodium spores were washed into a $250 \mathrm{ml}$ glass conical flask containing a magnetic flea. The flask was filled with a 1:1 mixture of glycerine and water with 3 or 4 grains of phenol to act as a preservative.

\section{5) Calibration}

To determine the exact concentration of Lycopodium spores in the spike, aliquots of known volume were mounted on microscope slides. The Lycopodium spores were then counted.

The following procedure was followed:

The suspension was thoroughly stirred on a magnetic stirrer, then one $\mathrm{ml}$ was withdrawn with a Manostat Varipet dispenser (a calibrated syringe, 1-10 ml). This was diluted to $10 \mathrm{mls}$ in a volumetric flask. Two hundred microlitres was put on a microscope slide using a micropipetter (Finnpipette, 200$1000 \mu \mathrm{l}$ ) with disposable polypropylene tips (Finntip 61, for volume range 200-1000 $\mathrm{ll}$ ) and all the Lycopodium on the slide were counted. Five microscope slides were made from this dilution (Dilution 1). Another dilution was made as above and 5 more slides were made, then the Lycopodium counted (Dilution 2).

\section{6) Storage}

The standardized mixture was stored in the $250 \mathrm{ml}$ glass conical flask it was mixed in. The magnetic flea was left in the flask. The flask was covered tightly with parafilm, to minimize evaporation, and stored in a refrigerator. The calibration was checked from time to time.

\section{7) Usage}

An aliquot of the spike was added to each sample at the beginning of pollen extraction procedure. The following procedure was followed:

(a) Mixing: The flask was placed on a magnetic stirring plate for $3 / 4$ hour before dispensing. Just before using, the flask was hand-swirled so that no grains were trapped on the sides or in vortex patterns.

(b) Dispensing: The Manostat Varipet or the Finnpipette as described above were used for dispensing.

\section{II.1.5 Laboratory procedure for diatom extraction}

Ten samples were prepared for diatom analysis by removing organic matter and sand in the following way:

\section{1) Weighing of sample}

Samples were weighed so that approximate concentration calculations could be made. $0.5 \mathrm{~g}$ of wet sample was weighed and placed in $50 \mathrm{ml}$ centrifuge tubes.

\section{2) Oxidation to remove organic matter}

The samples were boiled in $30 \%$ hydrogen peroxide $\left(\mathrm{H}_{2} \mathrm{O}_{2}\right)$ for 4 hours. The samples were then washed in distilled water twice, being centrifuged at $3000 \mathrm{rpm}$ for 8 minutes each time.

\section{3) Removal of sand by decantation}

The $50 \mathrm{ml}$ centrifuge tubes were filled to the $45 \mathrm{ml}$ graduation mark with distilled water. The tubes 
were shaken for about $1 / 4$ minute (until no sediment was adhering to the base of the tube), then let stand for $3 / 4$ minute, before being decanted into another $50 \mathrm{ml}$ centrifuge tube. (The diatoms were then in the decanted suspension). The sand was discarded from original tube and the process repeated, returning the suspension to the original tube. Tubes were centrifuged for 8 minutes at $3000 \mathrm{rpm}$ and decanted.

\section{4) Dilution}

The samples were diluted with $5 \mathrm{ml}$ of ethanol.

\section{5) Mounting}

Diatom suspensions were mounted in Hyrax diluted with toluene (methyl benzene).

\section{II.2 Laboratory preparation of pollen reference material}

The laboratory preparation of the reference collection was undertaken in the Palynology Section laboratory of the Institute of Geological and Nuclear Sciences, Lower Hutt.

The main objectives of this procedure are the removal of protoplasm and expansion of the palynomorph to make the morphology of the exine clearly visible. This also leaves the grains in a physical condition more similar to fossilized grains.

Sixteen samples were processed simultaneously, in $15 \mathrm{ml}$ conical bottom polypropylene centrifuge tubes with self-attached press-on tops. Centrifuging was done at 2500 revolutions per minute for three minutes.

Both herbarium and fresh plant material were treated in the same manner except that, in the case of fresh material, an initial step was added, the samples being soaked in $95 \%$ ethanol for about 10 minutes to remove any oils or fats from the plant material.

\section{1) Wetting}

Each sample was soaked overnight in $3 \mathrm{ml}$ of wetting agent (a few drops of liquid detergent added to a $400 \mathrm{ml}$ squirt bottle of distilled water), to assist the removal of cell contents by hydrating and softening the plant material.

The sample was then boiled in a water bath (lid open) for at least 10 minutes to further soften and wet the plant material, then centrifuged and decanted.

\section{2) Acetolysis}

Most importantly this step removes the protoplasm of the grains. It also destroys cellulose and some other organic compounds, thus removing organic debris.

Three to four $\mathrm{ml}$ of glacial acetic acid $\left(\mathrm{CH}_{3} \mathrm{COOH}\right)$ was added to the centrifuge tubes to dehydrate the plant material and prevent a reaction between the water and the acetolysis mixture. This was centrifuged then decanted. The acetolysis mixture was then mixed under a fume cupboard.

The acetolysis mixture consists of 9 parts $(90 \mathrm{ml})$ acetic anhydride $\left(\left(\mathrm{CH}_{3} \mathrm{CO}\right)_{2} \mathrm{O}\right)$ and 1 part $(10 \mathrm{ml})$ concentrated sulphuric acid $\left(\mathrm{H}_{2} \mathrm{SO}_{4}\right)$. The sulphuric acid is added to the acetic anhydride very slowly to prevent overheating. The mixture was poured into a $250 \mathrm{ml}$ glass beaker to mix the two liquids. The quantities given above made a double quantity of the mixture. Since this mixture needs to be used fresh it was important that two batches of samples were processed close together. 
Three $\mathrm{ml}$ of the acetolysis mixture was drawn-up into a graduated glass pipette, then added to the centrifuge tube which was placed in a boiling waterbath for 4 minutes. The sample was stirred frequently with a glass rod. The tubes were then removed and glacial acetic acid was added to the tubes up to $5 \mathrm{ml}$ to stop acetolysis. The samples were immediately centrifuged and decanted.

Three $\mathrm{ml}$ glacial acetic acid was added to the centrifuge tube to dissolve the soluble products of acetolysis (e.g. cellulose acetate). This was stirred with a vortex mechanical mixer, then centrifuged and decanted.

\section{3) Rinsing}

The samples were rinsed at least twice, usually thrice, in distilled water by adding $3 \mathrm{ml}$ distilled water, stirring, centrifuging and decanting.

\section{4) Bleaching}

In a very few cases, that is, with thick walled pollen such as that of Geraniaceae, the standard acetolysis procedure followed rendered the pollen too dark for identification. In these cases the pollen was bleached. This also had the benefit of removing some extraneous plant material. The following procedure was used:

1. $10 \mathrm{ml}$ stock bleach $(31 \mathrm{~g} \mathrm{NaOCl}$ (sodium hypochlorite)/1000 $\mathrm{ml}$ distilled water) was added to $90 \mathrm{ml}$ distilled water.

2. $1 \mathrm{ml}$ of this diluted bleaching solution was added to the centrifuge tube of the pollen to be bleached. This was stirred on a mechanical stirrer and allowed to stand for 5 minutes.

3. The centrifuge tube was then filled up to $10 \mathrm{mls}$ with distilled water to slow the bleaching action, then immediately centrifuged.

4. The residue was washed twice in distilled water.

\section{5) Mounting and sealing}

No stain was added to the pollen.

Glycerine jelly, refractive index less than 1.47 (about 1.43), was used as a mounting media. The following glycerine jelly mixture was used: $35 \mathrm{~g}$ sheet gelatin, $95 \mathrm{ml}$ distilled water, $165 \mathrm{~g}$ glycerol and $5 \mathrm{~g}$ phenol.

A drop of residue was placed on a microscope slide with a disposable pipette which was then discarded. The slide was warmed on a hot-plate $\left(80-100^{\circ} \mathrm{C}\right)$ and the water was allowed to almost completely evaporate. A drop of warm melted glycerine jelly was added to the residue and gently mixed with a toothpick until uniformly spread. The mixture was allowed to spread to almost the size of the coverslip before the coverslip was placed over the residue.

When the slide had cooled, clear nail-varnish was painted around the coverslip to seal the edges.

\section{6) Storage of residue}

Excess residue was left in the centrifuge tubes. A small amount of 1:1 distilled water and glycerol with a small amount of phenol (a fungicide) was added, then the centrifuge tubes were sealed. 


\section{References}

Brown, C.A. 1960. Palynological techniques. Baton Rouge, Privately Published.

Clark, H.E. 1951. An atmospheric pollen survey of four centres in the North Island, New Zealand, 1949-50. New Zealand journal of science and technology, Section B 33(2): 73-91.

Dimbleby, G.W. 1985. The palynology of archaeological sites. Academic Press, London.

Dodson, J.R. 1983. Pollen recovery from organic lake clays: a comparison of two techniques. Pollen et spores 25(1): 131-138.

Erdtman, G. 1969. Handbook of Palynology. Morphology. Taxonomy. Ecology. Munksgaard, Copenhagen.

Faegri, K. and J. Iversen 1975 (3rd ed). Textbook of pollen analysis. Hafner Press, New York.

Faegri, K. and J. Iversen 1992. Textbook of pollen analysis. 4th edition by K. Faegri, P.E. Kaland, and K. Krzywinski. John Wiley \& Sons Ltd, Chichester.

Gray, J. 1965. Extraction techniques. In, B. Kummel \& D. Raup (eds), Handbook of paleontological techniques: 530-587. W.H. Freeman and Company, San Francisco and London.

Kummel, B. \& D. Raup (eds) 1965. Handbook of paleontological techniques. W.H. Freeman and Company, San Francisco and London.

Licitis, R. 1953. Air-borne pollen and spores sampled at five New Zealand stations, 1951-52. New Zealand journal of science and technology 34B: 289-316.

Maher, L. J. 1981. Statistics for microfossil concentration measurements employing samples spiked with marker grains. Review of paleobotany and palynology 32: 153-191.

Marshall, S. 1981. Handbook of toxic and hazardous chemicals. Noyes Publications, New Jersey.

McIntyre, D.J. and G. Norris 1964. Effect of ultrasound on recent spores and pollen. New Zealand journal of science 7(2): 242-57.

Mirams, R.V. 1953. A comparison of the gravity and impact methods of collecting spores and pollen grains from the atmosphere at Wellington, New Zealand. New Zealand journal of science and technology 34B: 378-383.

Moore, P.D. and J.A. Webb 1978. An illustrated guide to pollen analysis. Hodder and Stoughton, London.

Moore, P.D., J.A. Webb and M.E. Collinson 1991 (2nd ed). Pollen analysis. Blackwell Scientific Publications, London.

Muir, G.D. (ed) 1977 (2nd ed). Hazards in the chemical laboratory. The Chemical Society, London.

Raine, J.I. and R. Tremain 1992. Apparatus for rapid sieving of palynological residues. N.Z. Geological Survey report PAL 151.

Reitsma, Tj. 1969. Size modification of recent pollen grains under different treatments. Review of palaeobotany and palynology 9(3/4): 175-202.

Shackley, M. 1981. Environmental archaeology. Allen \& Unwin, London.

Vidal, G. 1988. A palynological preparation method. Palynology 12: 215-220. 



\section{Appendix III}

\section{Sediment description}

\section{III.1 Troels-Smith characterization of unconsolidated sediments}

Troels-Smith, J. 1955. Karakterisering af løse jordater. Characterisation of unconsolidated sediments. Danm. Geol. Unders. Ser. IV, 3(10), 73pp.

Basic terms of this classification system are explained under the following headings:

1) Component elements

2) Accessory elements

3) Physical properties and limes

4) Humicity

Characterization is based on a 5 - class scale, 0 indicating a lack of the feature and 4 indicating maximum expression of that feature. The values are:

$\begin{array}{ll}\text { Class value } & 0 \text { - absence of } \\ & 1 \text { - minor presence of } \\ & 2 \text { - medium presence of } \\ & 3 \text { - major presence of } \\ & 4 \text { - maximum, or sole, presence of } \\ \text { Traces } & +- \text { slight presence of }\end{array}$

1) Component elements

Substantia humosa (Sh) - humous substance, consists of completely disintegrated, or nearly disintegrated, or decomposed, organic substances or precipitated humic acids. Completely humified Turfa (peat) may be characterized as Substantia humosa.

Turfa $(T)$ - macroscopic structure consisting of mosses, or roots of woody or herbaceous plants. Stumps, trunks, stems, belong to Turfa if connected with the root system.

T. bryophytica $\left(T b^{0-4}\right)$ - moss peat. $+/$ - humous substance (superscript indicates degree of humicity).

T. lignosa $\left(T l^{0-4}\right)$ - root systems of woody plants, and stumps, trunks, branches, etc connected to the roots. $+/$ - humous substance.

T. herbacea $\left(T h^{0-4}\right)$ - root systems and stems and leaves connected to the roots of herbaceous plants. +/- humous substance.

Detritus $(D)$ - consists of fragments, varying in size , of the superterranean parts of plants not connected to root systems, thus forming a contrast to Turfa.

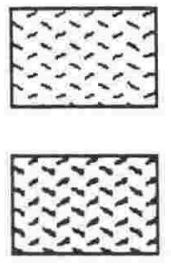

D. lignosus $(D l)$ - fragments of ligneous plants $>2 \mathrm{~mm}$.

D. herbosus $(D h)$ - fragments of herbaceous plants $>2 \mathrm{~mm}$.

D. granosus $(D g)$ - fragments of ligneous and herbaceous plants, and, sometimes, of animal fossils (except molluscs) $>$ c. $0.1<2 \mathrm{~mm}$. 
Limus (L) - mudlike (gyttja), homogeneous deposit, consisting of particles or colloids $<0.1 \mathrm{~mm}$.

$L$. detritus $\left(L d^{0}\right)$ - plants and animals (except diatoms, needles of spongi, siliceous skeletons, etc, of organic origin), or fragments of these. Particles < c. $0.1 \mathrm{~mm}$. +/humous substance.

L. humosus $\left(L h=L d^{4}\right)$ - 'dy' i.e. completely humified, colloidal material.

L. siliceus organogenes (Lso) - diatoms, needles of spongi, siliceous skeletons, etc, of organic origin, or parts of these. Particles < c. $0.1 \mathrm{~mm}$.

L. calcareus $(L c)$ - marl, not hardened like calcareous tufa; lime and the like. Particles $<$ c. $0.1 \mathrm{~mm}$.

$\because \because \because \because$

L. ferrugineus $(L f)$ - rust, non-hardened. Particles <c. $0.1 \mathrm{~mm}$.

Argilla $(A)$ - mineral particles $<0.06 \mathrm{~mm}$.

A. steatodes (As) - particles of clay $<0.002 \mathrm{~mm}$.

A. granosa $(\mathrm{Ag})$ - particles of clay 0.002 to $0.06 \mathrm{~mm}$.

Grana $(G)$ - solid particles $>0.06 \mathrm{~mm}$.

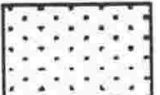

G. arenosa $(G a)$ - mineral particles 0.06 to $0.6 \mathrm{~mm}$.

G. saburralia $(G s)$ - mineral particles 0.6 to $2.0 \mathrm{~mm}$.

G. glareosa minora $(G g(\min ))$ - mineral particles 2.0 to $6.0 \mathrm{~mm}$.

00

G. glareosa majora $(G g(m a j))$ - mineral particles 6.0 to $20.0 \mathrm{~mm}$.

2) Accessory elements

Trunci et rami - trunks, branches, and twigs

(0) $\begin{array}{lll}\text { IRoman numerals I to IV ind } & =<5 \mathrm{~mm} \\ \text { II } & = & 5-20 \mathrm{~mm} \\ \text { III } & = & 20-50 \mathrm{~mm} \\ \text { IV } & = & <50 \mathrm{~mm}]\end{array}$


3) Physical properties and limes

Nigror - degree of darkness.

nigror 0 the lightest shades occurring in deposits, e.g.clear quartz sand.

nigror $1 \quad$ light shades, e.g. calcareous clay.

nigror 2 medium shades, e.g. fresh swamp peat.

nigror 3 dark shades, e.g. partly decayed swamp peat.

nigror $4 \quad$ darkest shades occurring in deposits, e.g. completely decomposed peat.

Stratificatio - degree of stratification. May manifest itself in the ease with which the deposit splits horizontally, in the horizontal position of macro-detritus, or in the division of the deposit into minor layers.

stratificatio 0

indicates complete homogeneity of the deposit in question, or/and that the deposit breaks with equal ease in all directions.

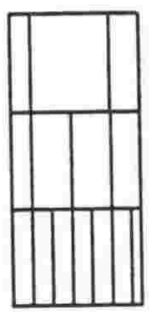

stratificatio 1 to $3 \quad$ indicates intermediate stages between stratificatio 0 and 4 .

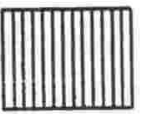

stratificatio 4

indicates that the deposit in question consists of very thin minor layers, or/and that it splits very easily in very thin horizontal layers.

Elasticitas

degree of elasticity. The ability of the sediment to regain its original form after being exposed to pressure, squeezing, or bending; the greater its power of resistance, the greater its degree of elasticity.

elasticitas 0

indicates total absence of elasticity: e.g. plastic clay, sand, diatom deposits, fen peat, completely disintegrated swamp peat.

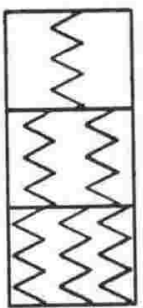

elasticitas 1 to 3 indicate intermediate stages between elasticitas 0 and 4 .

elasticitas 4

indicates the highest degree of elasticity occurring in deposits, e.g. fresh sphagnum peat, and swamp peat. 
- degree of dryness. The basis of classification here is dryness (dry substance), not humidity (water).

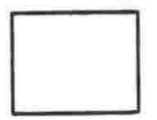

siccitas 0

siccitas 1

siccitas 2

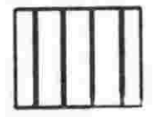

IIIII

siccitas 3

siccitas 4 indicates clear water.

indicates a deposit thoroughly saturated with water, its consistency being very soft, or like thick gruel.

indicates a deposit saturated with water, i.e. the condition in which deposits normally occur below the ground water level. It should be noted that the percentage of water content may vary considerably in different deposits having siccitas 2 .

indicates a deposit not saturated with water.

indicates a deposit air-dry.

Structura - the 5 - class scale is not used for this, but notes should include properties such as plasticity, cohesion, adhesion.

Limes

- the boundary between two sediment units which are different as regards physical conditions. The smaller the zone of mixing between two deposits, the more well-defined the boundary. Sharpness of boundary is indicated in the following way: lim. 0 boundary area $>1 \mathrm{~cm}$

lim. 1 - diffusus boundary area $<1 \mathrm{~cm} \&>2 \mathrm{~mm}$

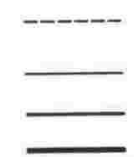

lim. 2 - conspicuus boundary area $<2 \mathrm{~mm} \&>1 \mathrm{~mm}$

lim. 3 - manifestus boundary area $<1 \mathrm{~mm} \mathrm{\&}>0.5 \mathrm{~mm}$

lim. 4 - acutus boundary area $<0.5 \mathrm{~mm}$

4) Humicity

Humositas - the degree of disintegration or decomposition observable in the organic substance of all kinds of Turfa and Limus detritus - indicates the quantity of humic acids (soluble in potassium hydroxide). Increasing degrees of humositas are shown symbolically by increasing the thickness of the strokes. In formula it is shown in superscript. In this category, 4 indicates total disintegration, whereas 0 indicates that disintegration processes have not yet begun.

humositas $0 \quad$ indicates plant structure fresh and well-preserved, no homogeneous ground substance present. A lump of peat, when squeezed in the hand, yields clear, colourless water.

humositas 1 indicates plant structure well-preserved, homogeneous ground substance present in slight quantity. Squeezing yields more or less dark-coloured, perhaps turbid, water. One-fourth, at most, of the mass is squeezed out between the fingers, in the form of a homogeneous ground substance.

humositas 2 indicates plant structure partly decayed, though distinct. Squeezing yields up to half the mass in the form of a homogeneous substance. 
humositas 3 indicates plant structure in advanced stage of decay, and indistinct. Squeezing yields up to three-fourths of the mass in the form of a homogeneous ground substance.

humositas 4 indicates plant structure hardly discemible, or completely absent. By squeezing, the whole of the peat mass, or nearly the whole of it, passes out between the fingers.

III.2 Comparison of grain size class scales of inorganic sediments

\section{Troels-Smith}

Argilla

A. steatodes As Clay particles $<0.002 \mathrm{~mm}$

A. granosa Ag

Clay particles

0.002 to $0.06 \mathrm{~mm}$

Grana

G. arenosa $\quad G a \quad$ Mineral particles

0.06 to $0.6 \mathrm{~mm}$

G. saburralia Gs Mineral particles

0.6 to $2.0 \mathrm{~mm}$

G.glareosa minora Mineral particles

$G g(\min ) \quad 2.0$ to $6.0 \mathrm{~mm}$

G. glareosa majora

Gg(maj)
Mineral particles

6.0 to $20.0 \mathrm{~mm}$
Udden/Wentworth

Mud

Clay $<0.0039 \mathrm{~mm}$

Silt; coarse, medium, fine, v.fine 0.0039 to $0.0625 \mathrm{~mm}$

\section{Sand}

Sand; v.fine, fine, medium

0.0625 to $0.5 \mathrm{~mm}$

Sand; coarse, v.coarse

0.5 to $2.00 \mathrm{~mm}$

\section{Gravel}

Granule

2.0 to $4.0 \mathrm{~mm}$

Pebble

4.0 to $64.0 \mathrm{~mm}$ 


\section{Appendix IV}

\section{Radiocarbon dating results and calibration}

Guide:

Radiocarbon dating laboratory reference numbers:

NZA = Rafter radiocarbon laboratory, Nuclear Sciences Group, Institute of Geological \& Nuclear sciences, Lower Hutt.

$\mathrm{Wk}=$ Waikato radiocarbon dating laboratory, University of Waikato, Hamilton.

Calibrated ages were calculated using the CALIB program of Stuiver \& Reimer (1995).

Radiocarbon Age BP is the conventional radiocarbon age before 'present' (years BP), based on the half-

life of 5568 years, as defined by Stuiver and Polach (1977), the present being AD1950.

$\nmid$ This standard deviation (error) includes a laboratory error multiplier.

$1 \sigma=$ square root of (sample std. dev. ${ }^{2}+$ curve std. dev. ${ }^{2}$ )

$2 \sigma=2 \times$ square root of (sample std. dev. $^{2}+$ curve std. dev. ${ }^{2}$ )

*1955 denotes influence of bomb C-14

NOTE: Calibrated ("Cal") ages and ranges are rounded to the nearest year which is too precise since all the standard deviations in the radiocarbon age are greater than 50 years. Rounding to the nearest 10 years would be more appropriate.

Following the textual reporting of the results and calibration are graphical representations.

\section{Gomara $52-53 \mathrm{~cm}$}

NZA 3481

Radiocarbon Age BP $\quad 1747 \pm 64$

Calibrated ages: $\mathrm{Cal} \mathrm{AD} 261,286,325$

Reference: Stuiver and Pearson 1993

$\mathrm{Cal} \mathrm{AD} / \mathrm{BC}$ age ranges (Cal ages as above) from probability distribution:

$\%$ area enclosed $\quad \mathrm{Cal} \mathrm{AD}$ age ranges

$68.3(1 \sigma)$

$95.4(2 \sigma)$
Cal AD 239 - 387

Cal AD 135 - 426 relative area under probability distribution

1.00

1.00

\section{Gomara 81-87 cm}

Wk 1810

Radiocarbon Age BP $1940 \pm 96+$

Calibrated age: Cal AD 76

Reference: Stuiver and Pearson 1993

(40 year moving average)

Cal $\mathrm{AD} / \mathrm{BC}$ age ranges ( $\mathrm{Cal}$ ages as above) from probability distribution:

$\%$ area enclosed

$68.3(1 \sigma)$

$95.4(2 \sigma)$
$\mathrm{Cal} \mathrm{AD} / \mathrm{BC}$ age ranges

Cal BC 25-Cal AD 160

Cal AD 161 - 203

Cal BC 166 - Cal AD 269

Cal AD 288 - 325 relative area under probability distribution

0.85

0.15

0.98

0.02 
Gomara 111-112 cm

NZA 3482

Radiocarbon Age BP $2103 \pm 73$

Calibrated age: Cal BC 105

Reference: Stuiver and Pearson 1993

$\mathrm{Cal} \mathrm{AD} / \mathrm{BC}$ age ranges ( $\mathrm{Cal}$ ages as above) from probability distribution:

$\%$ area enclosed

$\mathrm{Cal} \mathrm{AD} / \mathrm{BC}$ age ranges

relative area under

$68.3(1 \sigma)$

Cal BC 195 - 30

probability distribution

$27-7$

0.93

0.07

$95.4(2 \sigma)$
Cal BC 359 - 283
255 - Cal AD 60

0.11

0.89

\section{Gomara 156-157 cm}

NZA 3477

Radiocarbon Age BP $2749 \pm 66$

Calibrated ages: Cal BC 897, 871, 867

Reference: Pearson and Stuiver 1993

$\mathrm{Cal} \mathrm{AD} / \mathrm{BC}$ age ranges (Cal ages as above) from probability distribution:

$\%$ area enclosed

Cal $\mathrm{BC}$ age ranges

$68.3(1 \sigma)$

Cal BC 973 - 967

$933-818$

$95.4(2 \sigma)$

Cal BC1028 - 800

relative area under

probability distribution

0.03

0.97

1.00

\section{Gomara $186-190 \mathrm{~cm}$}

Wk 1811

Radiocarbon Age BP $3070 \pm 144$

Calibrated ages: Cal BC 1367, 1354, 1314

Reference: Pearson and Stuiver 1993

(40 year moving average)

$\mathrm{Cal} \mathrm{AD} / \mathrm{BC}$ age ranges (Cal ages as above) from probability distribution:

$\%$ area enclosed

$\mathrm{Cal} \mathrm{BC}$ age ranges

$68.3(1 \sigma)$

$95.4(2 \sigma)$
Cal BC1501 - 1487

$1466-1118$

Cal BCl617 - 925 relative area under probability distribution

$$
\begin{aligned}
& 0.03 \\
& 0.97 \\
& 1.00
\end{aligned}
$$


Anelia 124-125 cm

NZA 3480

Radiocarbon Age BP $\quad 268 \pm 61$

Calibrated age: Cal AD 1651

Reference: Stuiver and Pearson 1993

$\mathrm{Cal} \mathrm{AD} / \mathrm{BC}$ age ranges ( $\mathrm{Cal}$ ages as above) from probability distribution:

$\%$ area enclosed

Cal $\mathrm{AD}$ age ranges

$68.3(1 \sigma)$

$95.4(2 \sigma)$
Cal ADI515 - 1592

$1621-1676$

$1774-1800$

$1942 * 1955$

Cal AD 469 - 1693

$1727-1815$

1922 **1955 relative area under probability distribution

0.41

0.39

0.13

0.07

0.73

0.19

0.08

\section{Anelia 240-245 cm}

Wk 1813

Radiocarbon Age BP $360 \pm 96+$

Calibrated ages: Cal AD 1511, 1600, 1616

Reference: Stuiver and Pearson, 1993

$\mathrm{Cal} \mathrm{AD} / \mathrm{BC}$ age ranges (Cal ages as above) from probability distribution:

$\%$ area enclosed

$\mathrm{Cal} \mathrm{AD}$ age ranges

$68.3(1 \sigma)$

$95.4(2 \sigma)$
Cal AD 464 - 1638

Cal ADI 404 - 1684

$1743-1807$

$1932 * 1955$ relative area under probability distribution 1.00

0.93

0.05

0.02

Kellia 71-72 cm

NZA 3478

Radiocarbon Age BP $\quad 69 \pm 59$

Calibrated age: Cal AD *1955

Reference: Stuiver and Pearson 1993

$\mathrm{Cal} \mathrm{AD} / \mathrm{BC}$ age ranges ( $\mathrm{Cal}$ ages as above) from probability distribution:

$\%$ area enclosed

$\mathrm{Cal} \mathrm{AD}$ age ranges

$68.3(1 \sigma)$

$95.4(2 \sigma)$
Cal AD1698 - 1722

$1817-1920$

Cal AD $679-1768$

$1802-1939$ relative area under probability distribution

0.18

0.82

0.31

0.69 


\section{Kellia 166-168 cm}

Wk 1808

Radiocarbon Age BP $\quad 480 \pm 88 \dagger$

Calibrated age: Cal AD 1436

Reference: Stuiver and Pearson 1993

$\mathrm{Cal} \mathrm{AD} / \mathrm{BC}$ age ranges ( $\mathrm{Cal}$ ages as above) from probability distribution:

$\%$ area enclosed

$68.3(1 \sigma)$

$95.4(2 \sigma)$
Cal $\mathrm{AD}$ age ranges

Cal AD1323 - 1338

$1393-1514$

$1593-1620$

Cal ADI $307-1362$

$1377-1635$ relative area under probability distribution

0.06

0.83

0.11

0.12

0.88

Kellia 296-307 cm

Wk 1809

Radiocarbon Age BP $\quad 540 \pm 88+$

Calibrated age: Cal AD 1416

Reference: Stuiver and Pearson 1993

(80 year moving average)

$\mathrm{Cal} \mathrm{AD} / \mathrm{BC}$ age ranges (Cal ages as above) from probability distribution:

$\%$ area enclosed

$\mathrm{Cal} \mathrm{AD}$ age ranges

$68.3(1 \sigma)$

Cal ADI 324 - 1447

$95.4(2 \sigma)$

Cal ADI 272 - 1506

$1571-1611$

relative area under probability distribution
1.00
0.97
0.03

\section{Kellia 315-316 cm}

NZA 6894

Radiocarbon Age BP $3669 \pm 80$

Calibrated age(s) Cal BC 2031, 1992, 1989

Reference: Pearson and Stuiver 1993

$\mathrm{Cal} \mathrm{AD} / \mathrm{BC}$ age ranges ( $\mathrm{Cal}$ ages as above) from probability distribution:

$\%$ area enclosed

$68.3(1 \sigma)$

$95.4(2 \sigma)$
Cal $\mathrm{BC}$ age ranges

Cal BC2140 - 1922

Cal BC2280 - 2210

$2210-1872$

$1840-1811$

$1809-1780$ relative area under probability distribution

1.00

0.05

0.91

0.02

0.02 
Kellia 394-395 cm

NZA 3483

Radiocarbon Age BP $2707 \pm 79$

Calibrated age: Cal BC 830

Reference: Pearson and Stuiver 1993

$\mathrm{Cal} \mathrm{AD} / \mathrm{BC}$ age ranges ( $\mathrm{Cal}$ ages as above) from probability distribution:

$\%$ area enclosed

Cal $\mathrm{BC}$ age ranges

relative area under

$68.3(1 \sigma)$

Cal BC $921-800$

probability distribution

$95.4(2 \sigma)$

Cal BC1112 - 1102

1.00

0.00

$1063-761$

0.99

$626-596$

0.01

$572-569$

0.00

\section{References}

Pearson, G.W. and M. Stuiver 1993. High-precision bidecadal calibration of the radiocarbon time scale, 500-2500 BC. Radiocarbon 35: 25-33.

Stuiver, M. and G.W. Pearson 1993. High-precision bidecadal calibration of the radiocarbon time scale, AD 1950-500 BC and 2500-6000 BC. Radiocarbon 35: 1-23.

Stuiver, M. and H.A. Polach 1977. Discussion reporting of ${ }^{14} \mathrm{C}$ data. Radiocarbon 19(3): 355-363.

Stuiver, M. and P.J. Reimer 1993. Extended ${ }^{14} \mathrm{C}$ data base and revised CALIB radiocarbon age calibration program. Radiocarbon 35: 215-230.

Stuiver, M. and P.J. Reimer 1995. CALIB user's guide rev. 3.0.3c. Quaternary isotope laboratory, University of Washington. 


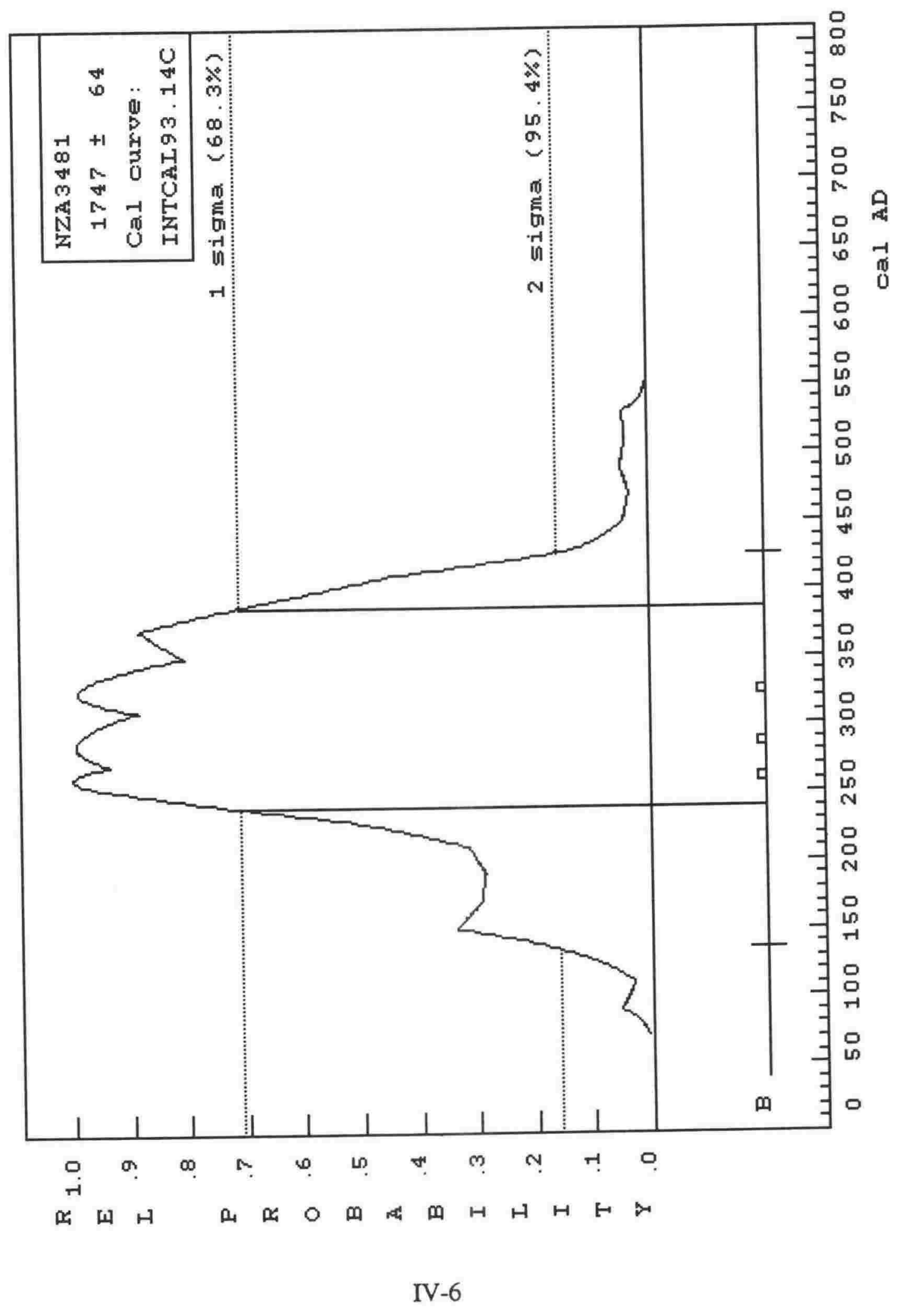




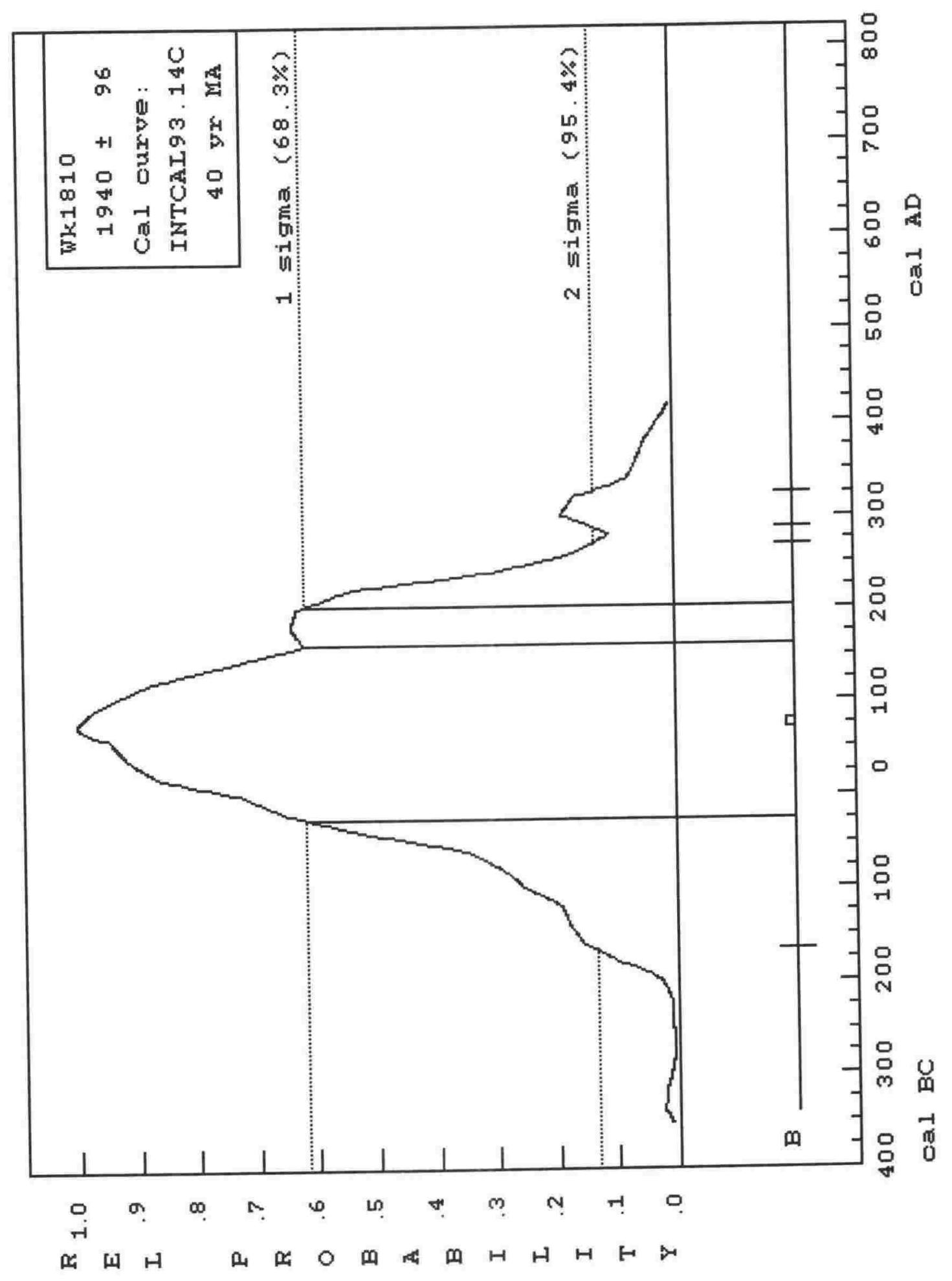




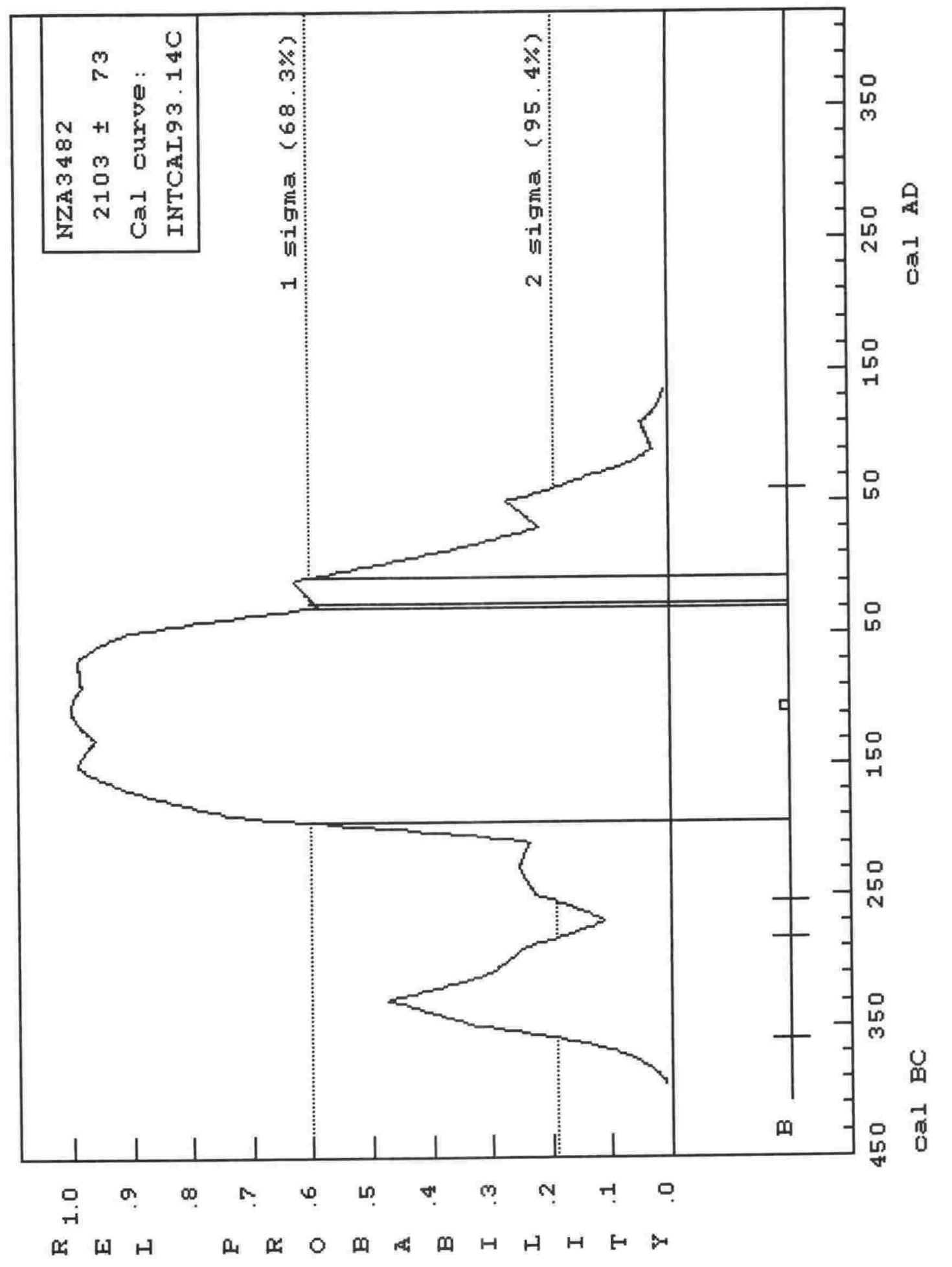




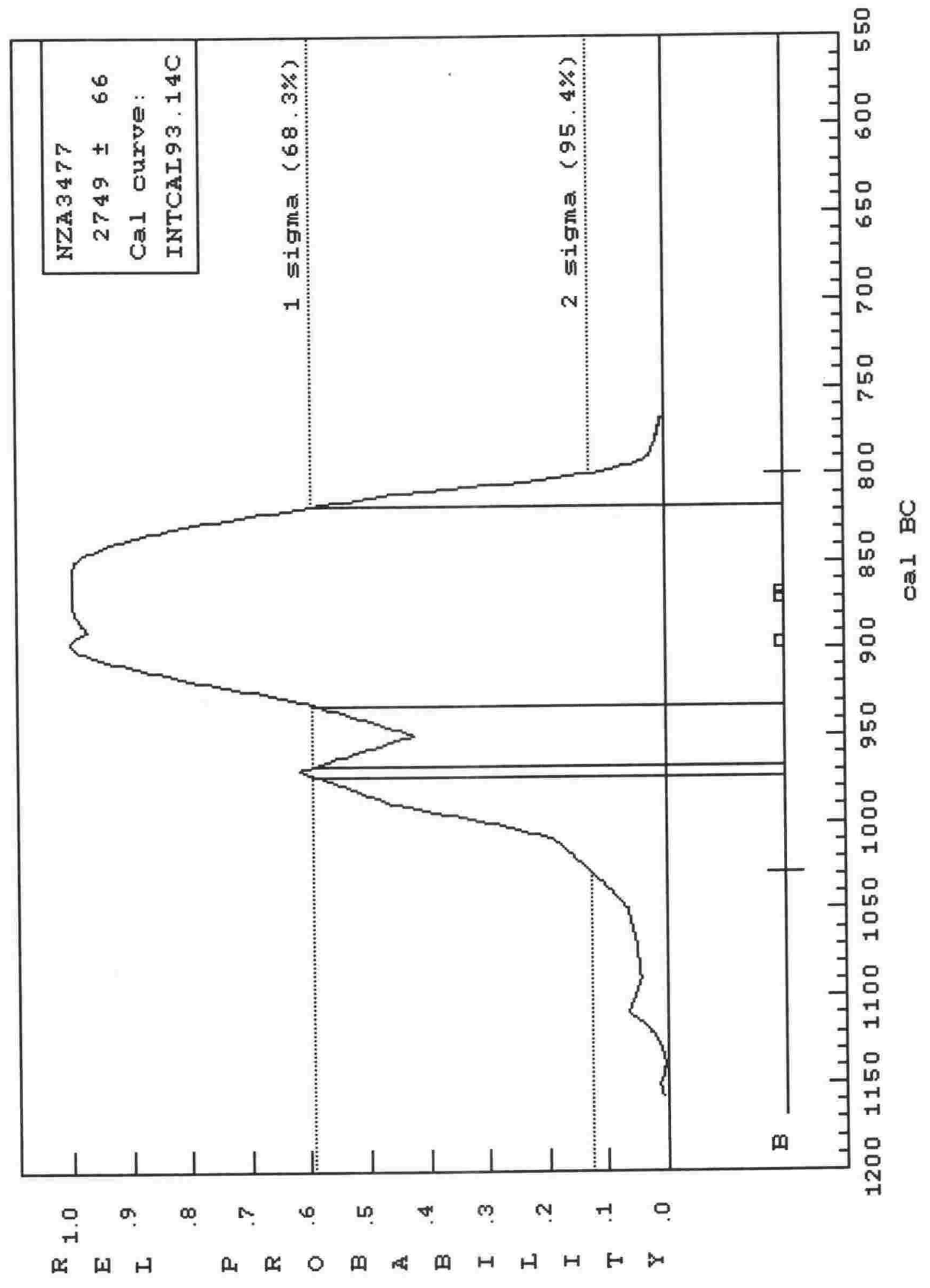




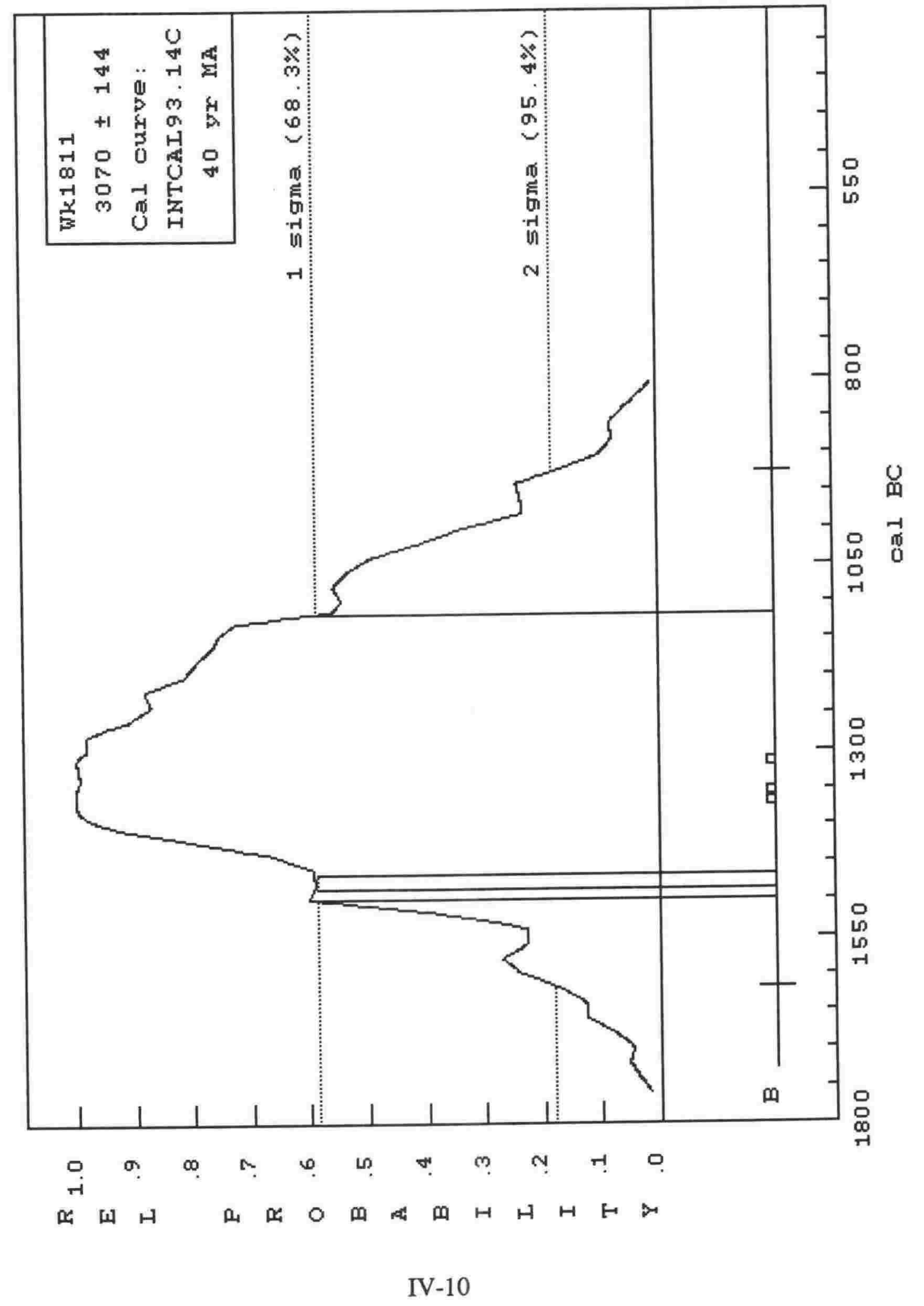




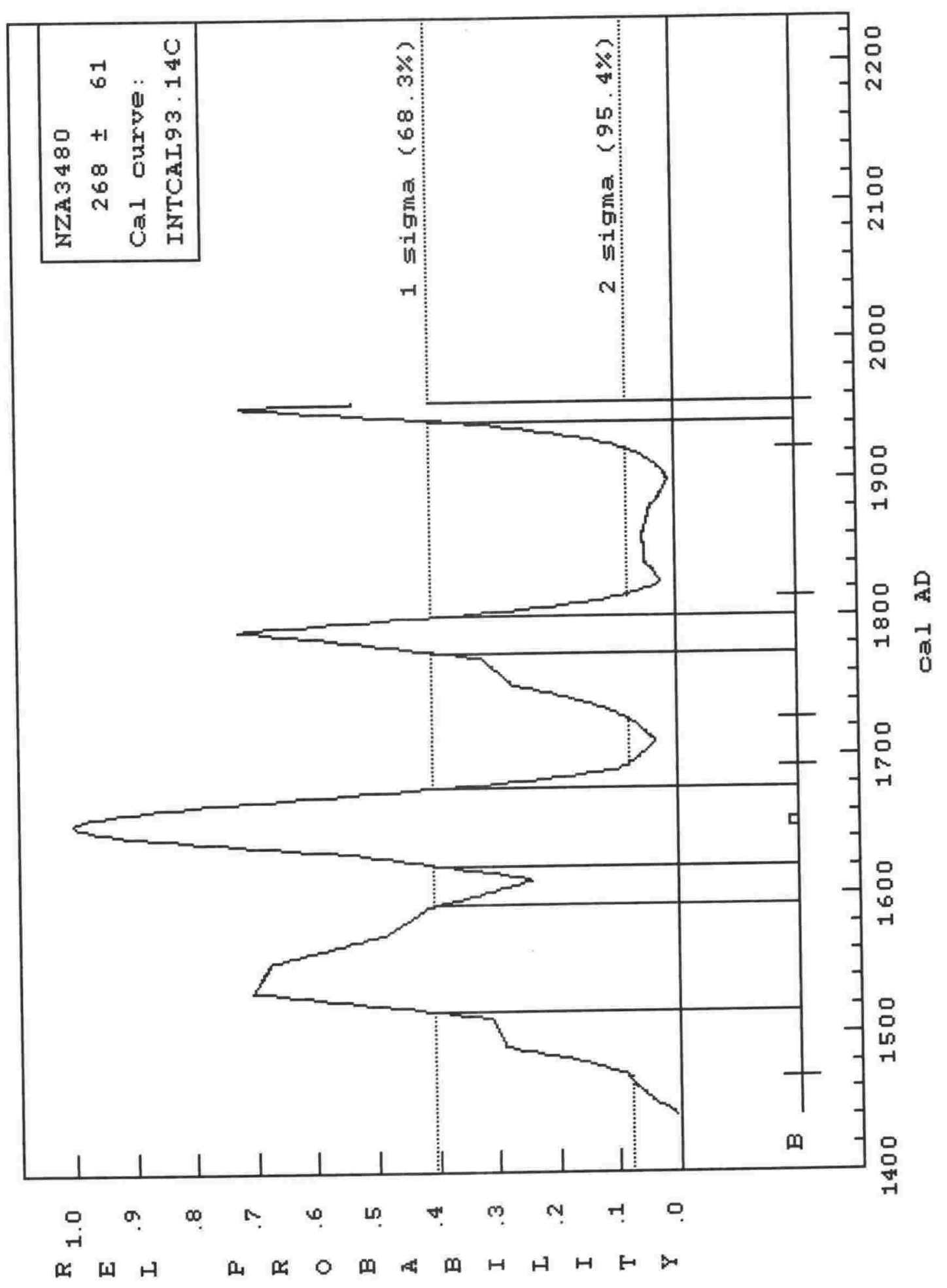




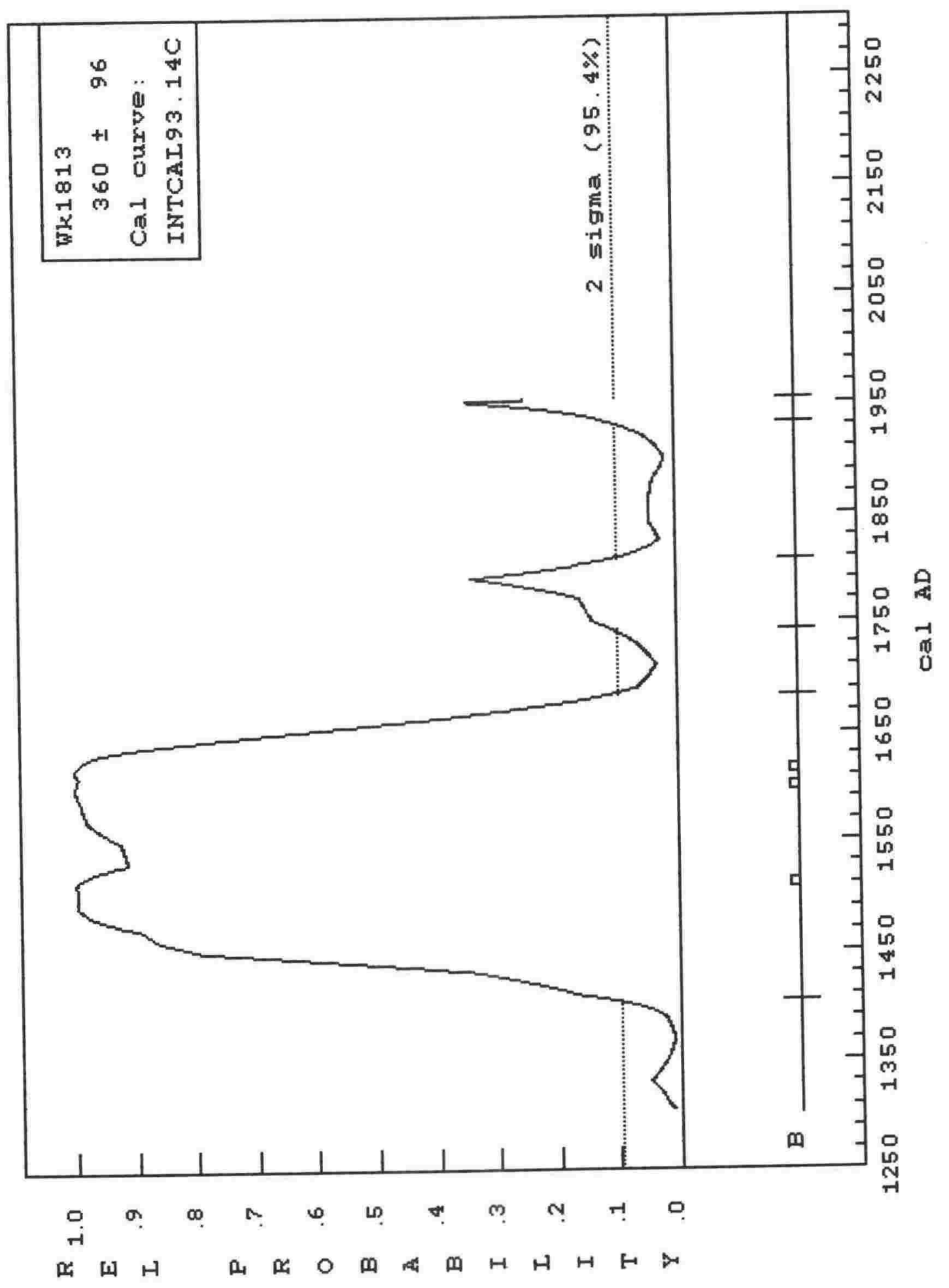




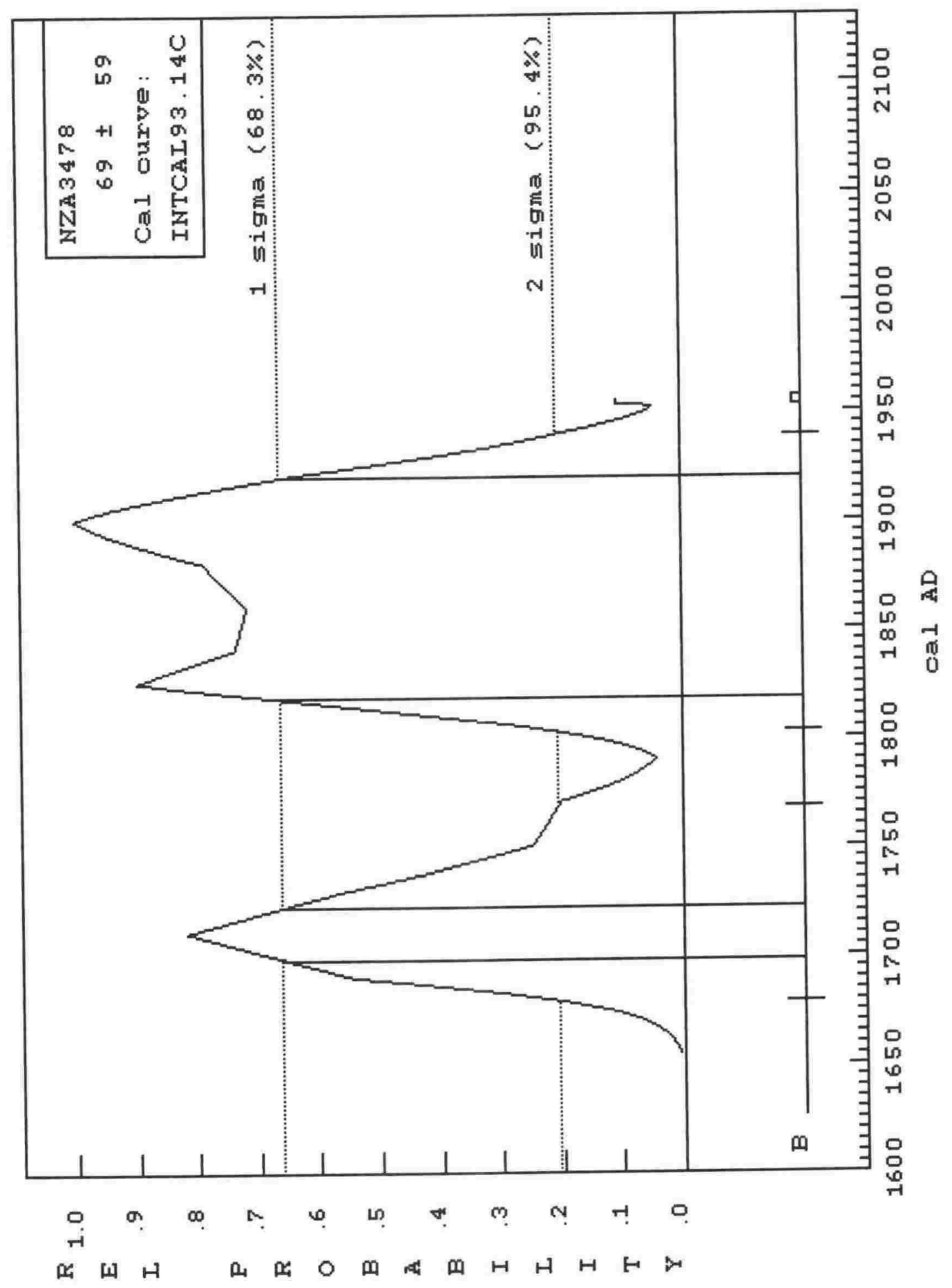




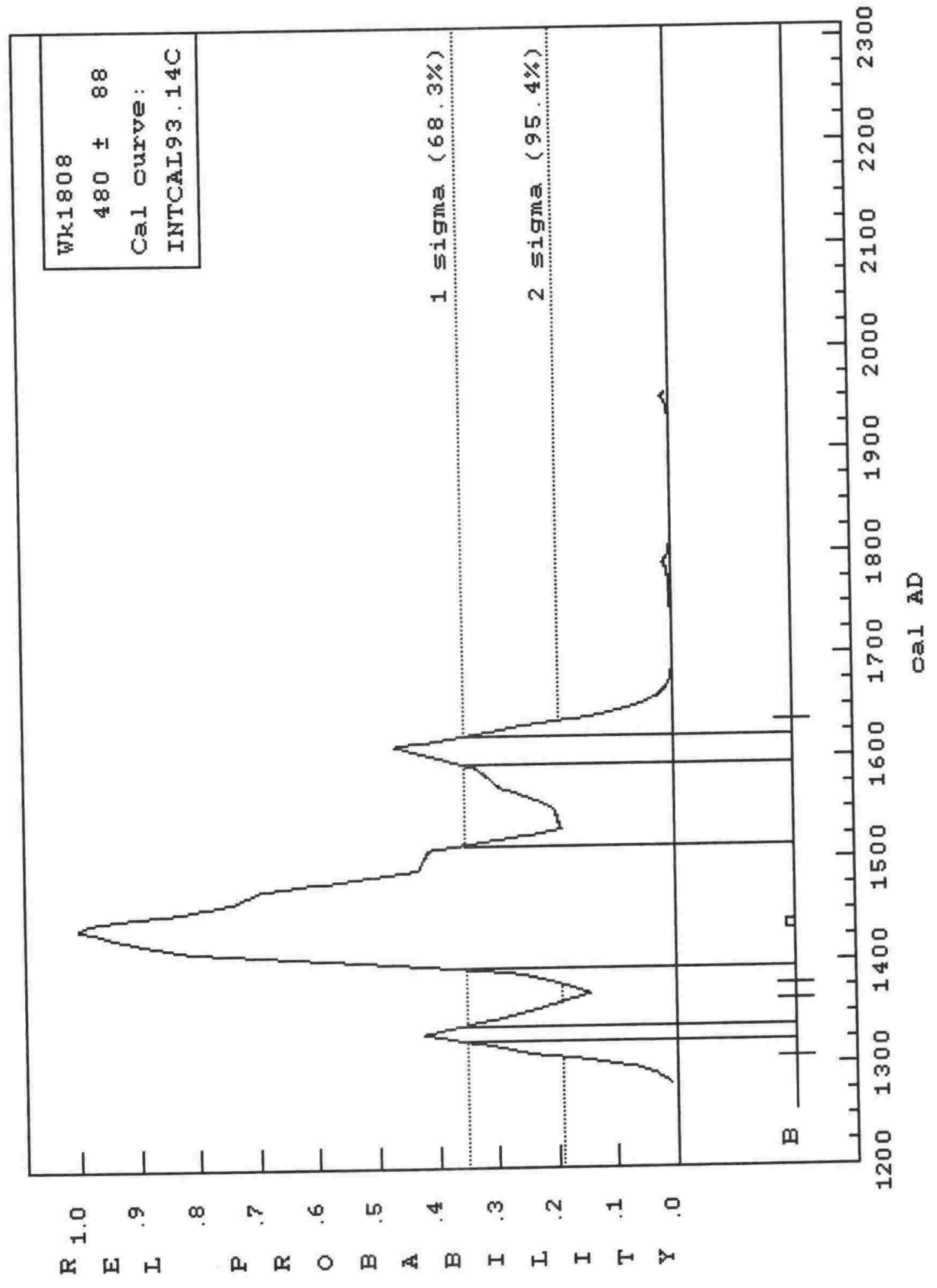




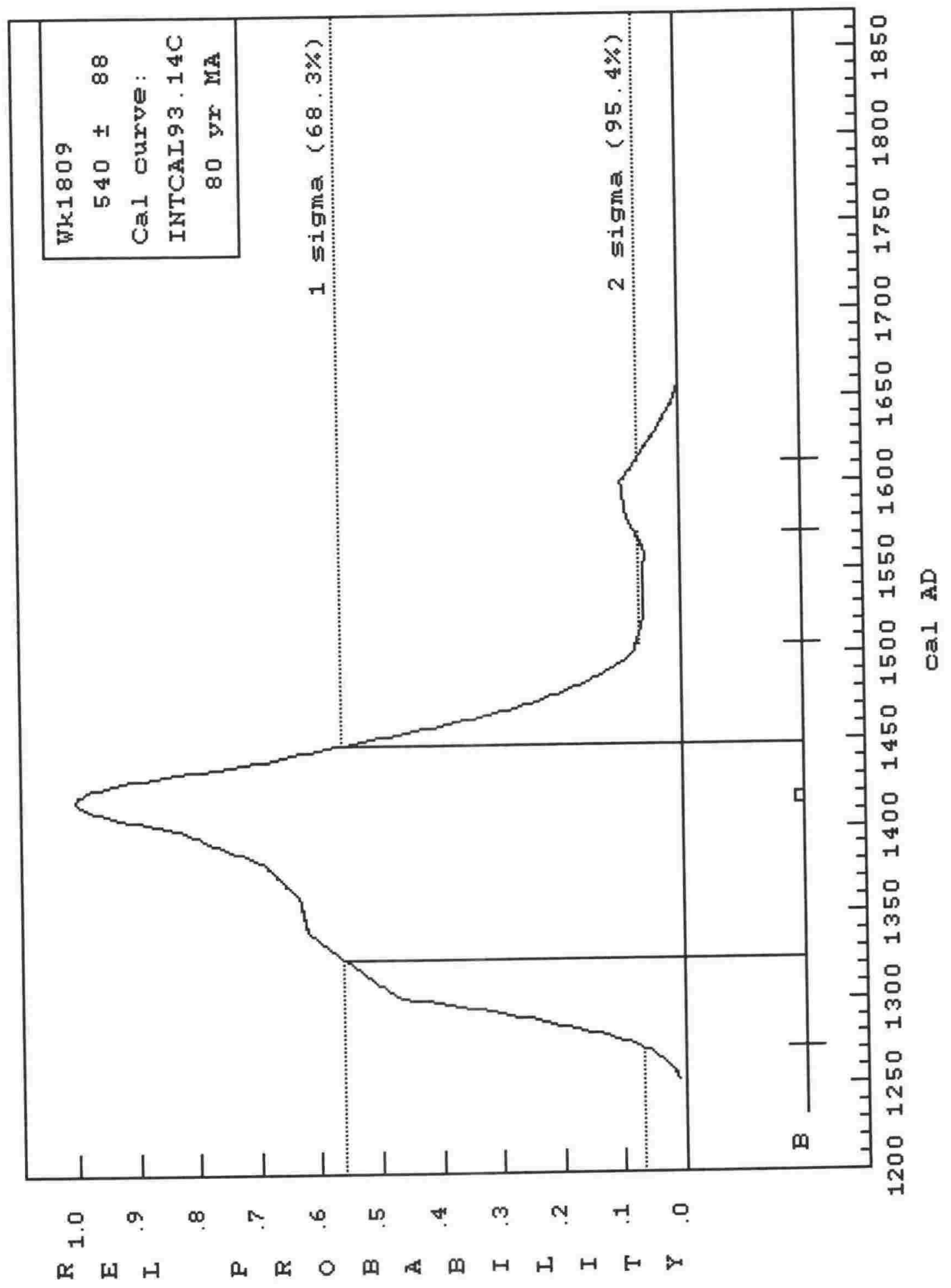




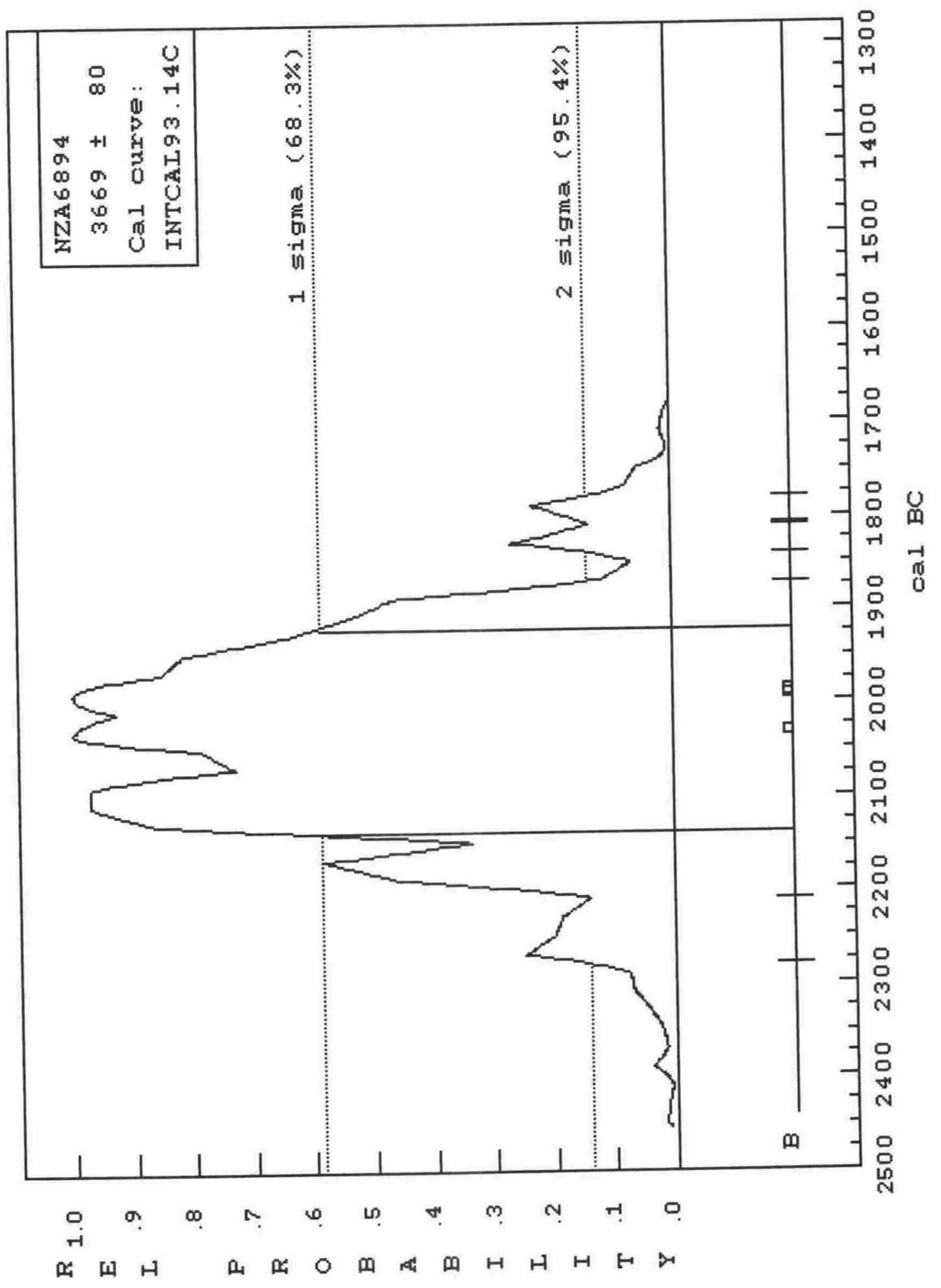




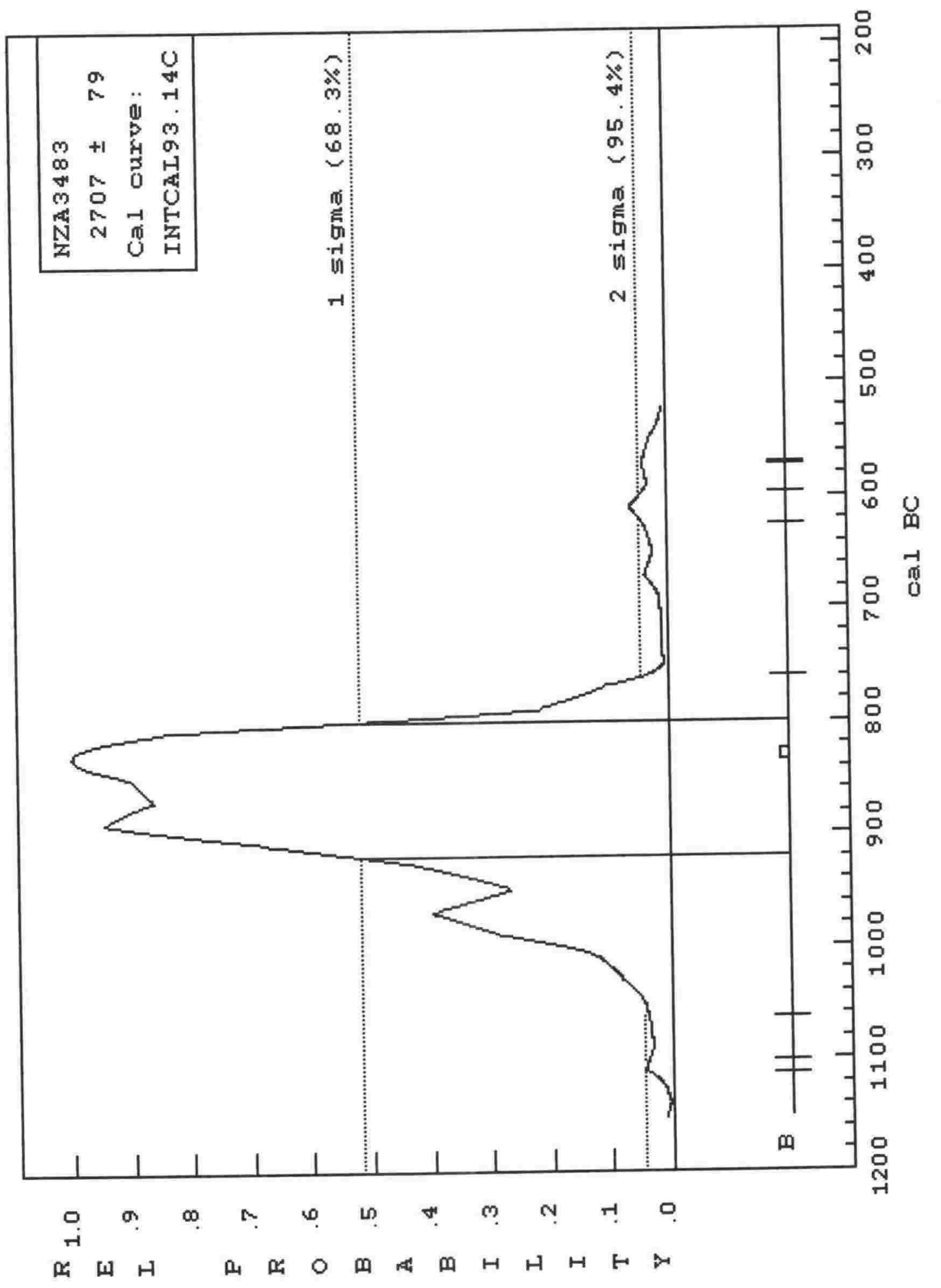





\section{Appendix V}

\section{Modern pollen and spore reference collection}

This is a register of all the pollen and spore reference slides used to assist with making a pollen key and identifying fossil pollen and spores in surface samples and wetland sequences collected in Grevena Province. The collection has been ordered alphabetically according to family.

Slide numbers prefixed 'VUWG' are held in the Geology Department of Victoria University of Wellington, those prefixed 'NUNG', 'DUDB' or 'CRYG' were made in England from reference pollen residues held in the following respective universities: Newcastle Upon Tyne (Department of Geography), Durham (Department of Botany) and the College of Ripon \& York (Department of Geography). Permission to use these residues was given by the following individuals who had prepared the residues: Drs A. Stevenson, B. Huntley and M. Atherden.

Herbaria are identified thus:

WELT = Natural History Unit, National Museum of New Zealand, Wellington, New Zealand.

WELTU = Biological Sciences, Victoria University of Wellington, Wellington, New Zealand.

Taxonomic names are taken from the herbarium sheets, where applicable, and because some of the specimens were collected last century they do not always follow Flora Europaea (1964-80). The taxonomic names of pollen and spores collected from living plants follow texts as outlined: taxonomic names of vascular European species follow Tutin et al. (1964-80), except where taxa have been described more recently in Strid (1989) and Strid \& Tan (1991). Taxonomic names of non-vascular species follow Watson (1968).

\begin{tabular}{|c|c|c|c|}
\hline $\begin{array}{l}\text { Pollen/spore } \\
\text { reference } \\
\text { number }\end{array}$ & Family & Genus \& species & $\begin{array}{l}\text { Collector of herbarium specimen } \\
\text { (if known)/herbarium,slide } \\
\text { herbarium collection number } \\
\text { (if known) \& place of collection } \\
\text { of herbarium specimen }\end{array}$ \\
\hline
\end{tabular}

\section{PTERIDOPHYTA}

SPHENOPSIDA

$\begin{array}{lrll}\text { NUNG } & 4 & \text { Equisetaceae } & \text { Equisetum arvense } \\ \text { VU } & 775 & \text { Equisetaceae } & \text { Equisetum palustre }\end{array}$

FILICOPSIDA

$\begin{array}{lrll}\text { NUNG } & 174 & \text { Aspleniaceae } & \text { Asplenium septentrionale } \\ \text { NUNG } & 90 & \text { Aspleniaceae } & \text { ?Asplenium } \mathrm{sp} .\end{array}$

SPERMATOPHYTA

GYMNOSPERMAE

$\begin{array}{lrll}\text { NUNG } & 34 & \text { Cupressaceae } & \text { ?Cupressus macrocarpa } \\ \text { DUDB } & 309 & \text { Cupressaceae } & \text { Cupressus } \mathrm{sp} .\end{array}$

Stevenson, Newcastle Upon Tyne University, England.

Chester 122. Mirsina, Grevena Pr., NW Greece.
Stevenson, Newcastle Upon Tyne University, England.

Stevenson, Newcastle Upon Tyne University, England. 


\begin{tabular}{|c|c|c|c|}
\hline $\begin{array}{l}\text { Pollen/s } \\
\text { referenc } \\
\text { slide \# }\end{array}$ & $\begin{array}{l}\text { pore } \\
\text { ce }\end{array}$ & Family & Genus \& species \\
\hline DUDB & 310 & Cupressaceae & Cupressus sp. \\
\hline VUWG & 1290 & Cupressaceae & Juniperus virginiana \\
\hline NUNG & 93 & Cupressaceae & Juniperus sp. \\
\hline NUNG & 94 & Ephedraceae & Ephedra fragilis \\
\hline VUWG & 1287 & Pinaceae & Abies cephalonica \\
\hline VUWG & 810 & Pinaceae & Abies ?nordmanniano \\
\hline NUNG & 231 & Pinaceae & Abies pinsapo \\
\hline NUNG & 226 & Pinaceae & Cedrus \\
\hline NUNG & 58 & Pinaceae & Picea abies \\
\hline NUNG & 218 & Pinaceae & Picea abies \\
\hline NUNG & 100 & Pinaceae & Picea marina \\
\hline CRYG & 294 & Pinaceae & Pinus halepensis \\
\hline VUWG & 774 & Pinaceae & Pinus leucodermis \\
\hline VUWG & 786 & Pinaceae & Pinus mugo \\
\hline NUNG & 179 & Pinaceae & Pinus mugo \\
\hline NUNG & 10 & Pinaceae & Pinus nigra \\
\hline DUDB & 295 & Pinaceae & Pinus nigra \\
\hline VUWG & 824 & Pinaceae & Pinus sp. \\
\hline NUNG & 219 & Taxaceae & Taxus baccata \\
\hline
\end{tabular}

\section{ANGIOSPERMAE Dicotyledones}

\begin{tabular}{|c|c|c|c|}
\hline VUWG & 1420 & Acanthaceae & Acanthus mollis \\
\hline VUWG & 317 & Acanthaceae & Acanthus mollis \\
\hline VUWG & 318 & Acanthaceae & Acanthus mollis \\
\hline VUWG & 319 & Acanthaceae & Acanthus mollis \\
\hline VUWG & 320 & Acanthaceae & Acanthus mollis \\
\hline VUWG & 1139 & Acanthaceae & Aclathoda vesica \\
\hline VUWG & 1140 & Acanthaceae & Barleria cristata \\
\hline VUWG & 957 & Aceraceae & Acer campestre \\
\hline VUWG & 405 & Aceraceae & Acer parviflorum \\
\hline
\end{tabular}

Collector (if known)/herbarium, herbarium \# (if known), place of collection
Huntley, Durham University, England.

WELTU box 204; Rotorua, N.Z.

Stevenson, Newcastle Upon Tyne University, England.

Stevenson, Newcastle Upon Tyne University, England.

WELTU 15257; Rotorua, N.Z.

Chester 331. Ewen Bridge, Lower Hutt, N.Z.

Stevenson, Newcastle Upon Tyne University, England.

Stevenson, Newcastle Upon Tyne University, England.

Stevenson, Newcastle Upon Tyne University, England.

Stevenson, Newcastle Upon Tyne University, England.

Stevenson, Newcastle Upon Tyne University, England.

Atherden, College of Rippon \& York, England.

Chester 306. Lakkos, Grevena Pr., NW Greece.

Chester 307. Wellington Botanic

Gardens, N.Z.

Stevenson, Newcastle Upon Tyne University, England.

Stevenson, Newcastle Upon Tyne University, England.

Huntley, Durham University, England.

Chester 345. Victoria University, Wellington, N.Z.

Stevenson, Newcastle Upon Tyne University, England.
WELTU; Wellington, N.Z. WELT 69900a; Wairau Ck, Milford, N.Z.

WELT 60469c; Kelburn, Wellington, N.Z.

WELT no \#; Seatoun Rd, Wellington, N.Z.

WELT 60469b; Kelburn, Wellington, N.Z. WELTU; Old Delhi, India. WELTU; India.

WELTU box 97; Holland. WELT no \#. 


\begin{tabular}{|c|c|c|c|c|}
\hline $\begin{array}{l}\text { Pollen/s } \\
\text { referenc } \\
\text { slide \# }\end{array}$ & & Family & Genus \& species & $\begin{array}{l}\text { Collector (if known)/herbarium } \\
\text { herbarium \# (if known), } \\
\text { place of collection }\end{array}$ \\
\hline VUWG & 404 & Aceraceae & Acer pseudoplatanus & $\begin{array}{l}\text { WELT } 61195 ; \text { S Karori Rd, } \\
\text { Wellington, N.Z. }\end{array}$ \\
\hline VUWG & 809 & Aceraceae & Acer pseudoplatanus & $\begin{array}{l}\text { Chester } 330 \text {. Ewen Bridge, Lower } \\
\text { Hutt, N.Z. }\end{array}$ \\
\hline DUDB & 296 & Aceraceae & Acer pseudoplatanus & $\begin{array}{l}\text { Huntley, Durham University, } \\
\text { England. }\end{array}$ \\
\hline VUWG & 956 & Aceraceae & Acer tegmentosum & WELTU box 97; Russia. \\
\hline VUWG & 610 & Amaranthaceae & Amaranthus sp. & $\begin{array}{l}\text { WELT 62832; Kaiti Hill, } \\
\text { Gisborne, N.Z. }\end{array}$ \\
\hline VUWG & 611 & Amaranthaceae & Amaranthus sp. & WELT 62642; Auckland City, N.Z. \\
\hline VUWG & 612 & Amaranthaceae & Amaranthus sp. & $\begin{array}{l}\text { WELT 70543; Pukerua Bay, } \\
\text { Wellington, N.Z. }\end{array}$ \\
\hline VUWG & 613 & Amaranthaceae & Amaranthus sp. & $\begin{array}{l}\text { WELT } 69990 ; \text { Pukerua Bay, } \\
\text { Wellington, N.Z. }\end{array}$ \\
\hline VUWG & 614 & Amaranthaceae & Amaranthus sp. & $\begin{array}{l}\text { WELT 70063; Rongotai, } \\
\text { Wellington, N.Z. }\end{array}$ \\
\hline NUNG & 173 & Anacardiaceae & Pistacia lentiscus & $\begin{array}{l}\text { Stevenson, Newcastle Upon Tyne } \\
\text { University, England. }\end{array}$ \\
\hline NUNG & 192 & Anacardiaceae & Pistacia lentiscus & $\begin{array}{l}\text { Stevenson, Newcastle Upon Tyne } \\
\text { University, England. }\end{array}$ \\
\hline CRYG & 281 & Anacardiaceae & Pistacia lentiscus & $\begin{array}{l}\text { Atherden, College of Rippon \& } \\
\text { York, England. }\end{array}$ \\
\hline NUNG & 53 & Anacardiaceae & Pistacia terebinthus & $\begin{array}{l}\text { Stevenson, Newcastle Upon Tyne } \\
\text { University, England. }\end{array}$ \\
\hline NUNG & 18 & Anacardiaceae & Rhus typhina & $\begin{array}{l}\text { Stevenson, Newcastle Upon Tyne } \\
\text { University, England. }\end{array}$ \\
\hline NUNG & 162 & Anacardiaceae & Schinus molle & $\begin{array}{l}\text { Stevenson, Newcastle Upon Tyne } \\
\text { University, England. }\end{array}$ \\
\hline VUWG & 396 & Apocynaceae & Nerium oleander & $\begin{array}{l}\text { WELT 59811; Muriwai, Auckland, } \\
\text { N.Z. }\end{array}$ \\
\hline VUWG & 392 & Apocynaceae & Vinca major & $\begin{array}{l}\text { WELT } 60724 \text {; S Karori Rd, } \\
\text { Wellington, N.Z. }\end{array}$ \\
\hline VUWG & 393 & Apocynaceae & Vinca major & $\begin{array}{l}\text { WELT } 60705 ; \text { Raroa Rd, } \\
\text { Wellington, N.Z. }\end{array}$ \\
\hline VUWG & 394 & Apocynaceae & Vinca major & $\begin{array}{l}\text { WELT } 63331 \text {; Lake Pupuke, } \\
\text { Milford, N.Z. }\end{array}$ \\
\hline VUWG & 395 & Apocynaceae & Vinca minor & $\begin{array}{l}\text { WELT } 60864 \text {; Karori, Wellington, } \\
\text { N.Z. }\end{array}$ \\
\hline VUWG & 744 & Araliaceae & Hedera helix & $\begin{array}{l}\text { Chester 172. Agios Nicholas, } \\
\text { Grevena Pr., NW Greece. }\end{array}$ \\
\hline VUWG & 932 & Araliaceae & Hedera helix & WELTU box 95; Holland. \\
\hline DUDB & 306 & Betulaceae & Alnus cordata & $\begin{array}{l}\text { Huntley, Durham University, } \\
\text { England. }\end{array}$ \\
\hline VUWG & 1292 & Betulaceae & Alnus glutinosa & WELTU 10304; Wanganui, N.Z. \\
\hline VUWG & 423 & Betulaceae & Alnus glutinosa & $\begin{array}{l}\text { WELT 70134; near Tuakau, } \\
\text { Franklin Co., N.Z. }\end{array}$ \\
\hline VUWG & 778 & Betulaceae & Alnus glutinosa & WELTU 10304; Wanganui, N.Z. \\
\hline VUWG & 859 & Betulaceae & Alnus glutinosa & $\begin{array}{l}\text { WELTU box } 86 \text {; Whakarara, } \\
\text { Hawke Bay, N.Z. }\end{array}$ \\
\hline VUWG & 814 & Betulaceae & Alnus ?glutinosa & Chester 335. Wellington City, N.Z. \\
\hline DUDB & 304 & Betulaceae & Alnus incana & $\begin{array}{l}\text { Huntley, Durham University, } \\
\text { England. }\end{array}$ \\
\hline
\end{tabular}




\begin{tabular}{|c|c|c|c|c|}
\hline \multicolumn{2}{|c|}{$\begin{array}{l}\text { Pollen/spore } \\
\text { reference } \\
\text { slide \# }\end{array}$} & \multirow{2}{*}{$\begin{array}{l}\text { Family } \\
\text { Betulaceae }\end{array}$} & \multirow{2}{*}{$\begin{array}{l}\text { Genus \& species } \\
\text { Alnus incana }\end{array}$} & \multirow{2}{*}{$\begin{array}{l}\text { Collector (if known)/herbarium, } \\
\text { herbarium \# (if known), } \\
\text { place of collection } \\
\text { Huntley, Durham University, } \\
\text { England. }\end{array}$} \\
\hline DUDB & 305 & & & \\
\hline VUWG & 858 & Betulaceae & Alnus ?viridis & WELTU box 86 ; Switzerland. \\
\hline VUWG & 777 & Betulaceae & Betulus alba & $\begin{array}{l}\text { WELTU 10305; Botanic Gardens, } \\
\text { Wellington, N.Z. }\end{array}$ \\
\hline VUWG & 860 & Betulaceae & Betula manshurica & WELTU box 86 ; Russia. \\
\hline VUWG & 1291 & Betulaceae & Betula pendula & $\begin{array}{l}\text { WELTU; Wellington Botanic } \\
\text { Gardens, N.Z. }\end{array}$ \\
\hline VUWG & 812 & Betulaceae & Betula pendula & $\begin{array}{l}\text { Chester 333. Riddiford Gardens, } \\
\text { Lower Hutt, N.Z. }\end{array}$ \\
\hline CRYG & 291 & Betulaceae & Betula sp. & $\begin{array}{l}\text { Atherden, College of Rippon \& } \\
\text { York, England. }\end{array}$ \\
\hline VUWG & 733 & Boraginaceae & Anchusa azurea & $\begin{array}{l}\text { Chester 150. Mirsina, Grevena Pr., } \\
\text { NW Greece. }\end{array}$ \\
\hline VUWG & 946 & Boraginaceae & Anchusa officinalis & WELTU box 97; Holland. \\
\hline VUWG & 198 & Boraginaceae & Borago officinalis & $\begin{array}{l}\text { WELT } 60707 \text {; Norway St, } \\
\text { Wellington, N.Z. }\end{array}$ \\
\hline VUWG & 199 & Boraginaceae & Borago officinalis & $\begin{array}{l}\text { WELT } 61247 \text {; Island Bay, } \\
\text { Wellington, N.Z. }\end{array}$ \\
\hline VUWG & 818 & Boraginaceae & Borago officinalis & $\begin{array}{l}\text { Chester } 339 . \text { Garden, Johnsonville, } \\
\text { Wellington, N.Z. }\end{array}$ \\
\hline VUWG & 200 & Boraginaceae & Cerinthe major & $\begin{array}{l}\text { WELT } 60831 \text {; Dr Thomson's } \\
\text { Garden, Hawera, N.Z. }\end{array}$ \\
\hline VUWG & 201 & Boraginaceae & Cerinthe major & $\begin{array}{l}\text { WELT } 62660 \text {; E Coast Rd, } \\
\text { Milford, Waitemata Co., N.Z. }\end{array}$ \\
\hline VUWG & 202 & Boraginaceae & Echium plantagineum & $\begin{array}{l}\text { WELT 69892a; Wairau Ck } \\
\text { Estuary, Milford, N.Z. }\end{array}$ \\
\hline VUWG & 203 & Boraginaceae & Echium plantagineum & $\begin{array}{l}\text { WELT 69892b; Wairau Ck } \\
\text { Estuary, Milford, N.Z. }\end{array}$ \\
\hline VUWG & 204 & Boraginaceae & Echium plantagineum & WELT 60815 \\
\hline VUWG & 205 & Boraginaceae & Echium vulgare & WELT 9217 \\
\hline VUWG & 206 & Boraginaceae & Echium vulgare & $\begin{array}{l}\text { WELT 59819; Desert Rd, Central } \\
\text { Nth Is., N.Z. }\end{array}$ \\
\hline VUWG & 207 & Boraginaceae & Echium vulgare & $\begin{array}{l}\text { WELT 63485; Te Awanga, } \\
\text { Hawkes Bay, N.Z. }\end{array}$ \\
\hline VUWG & 947 & Boraginaceae & Heliotropium ellipticum & WELTU box 97; India. \\
\hline VUWG & 755 & Boraginaceae & Heliotropium hirsutissimum & $\begin{array}{l}\text { Chester 227. Asprokampos, } \\
\text { Grevena Pr., NW Greece. }\end{array}$ \\
\hline VUWG & 208 & Boraginaceae & Lithospermum arvense & WELT (no\#) \\
\hline VUWG & 209 & Boraginaceae & Lithospermum arvense & WELT 60829; Otago, N.Z. \\
\hline VUWG & 210 & Boraginaceae & Lithospermum arvense & WELT 60828; Auckland, N.Z. \\
\hline VUWG & 805 & Boraginaceae & Lithospermum ?diffusa & $\begin{array}{l}\text { Chester } 326 . \text { Wellington Botanic } \\
\text { Gardens, N.Z. }\end{array}$ \\
\hline VUWG & 948 & Boraginaceae & Lithospermum sp. & WELTU box 97; Switzerland. \\
\hline VUWG & 1399 & Boraginaceae & Myosotis arvensis & $\begin{array}{l}\text { WELTU } 15241 \text {; Karori, } \\
\text { Wellington, N.Z. }\end{array}$ \\
\hline VUWG & 214 & Boraginaceae & Myosotis arvensis & $\begin{array}{l}\text { WELT } 63225 \text {; nr Mangatuna, } \\
\text { Waiapu County, N.Z. }\end{array}$ \\
\hline VUWG & 215 & Boraginaceae & Myosotis arvensis & WELT 9210; Waikanae, N.Z. \\
\hline NUNG & 50 & Boraginaceae & Myosotis arvensis & $\begin{array}{l}\text { Stevenson, Newcastle Upon Tyne } \\
\text { University, England. }\end{array}$ \\
\hline VUWG & 216 & Boraginaceae & Myosotis discolor & $\begin{array}{l}\text { WELT } 62881 \text {; Mitimiti, Nth } \\
\text { Hokianga, N.Z. }\end{array}$ \\
\hline
\end{tabular}




\begin{tabular}{|c|c|c|c|c|}
\hline $\begin{array}{l}\text { Pollen/sp } \\
\text { reference } \\
\text { slide \# }\end{array}$ & & Family & Genus \& species & $\begin{array}{l}\text { Collector (if known)/herbarium } \\
\text { herbarium \# (if known), } \\
\text { place of collection }\end{array}$ \\
\hline VUWG & 217 & Boraginaceae & Myosotis discolor & $\begin{array}{l}\text { WELT } 60820 \text {; Crib Ck, Kaikoura, } \\
\text { N.Z. }\end{array}$ \\
\hline VUWG & 218 & Boraginaceae & Myosotis discolor & $\begin{array}{l}\text { WELT } 60819 ; \text { Hills above Crib } \\
\text { Ck, Kaikoura, N.Z. }\end{array}$ \\
\hline VUWG & 219 & Boraginaceae & Myosotis laxa caespitosa & $\begin{array}{l}\text { WELT 2494; Western Bay, Taupo, } \\
\text { N.Z. }\end{array}$ \\
\hline VUWG & 220 & Boraginaceae & Myosotis laxa caespitosa & WELT 42845 \\
\hline VUWG & 222 & Boraginaceae & Myosotis scorpioides & $\begin{array}{l}\text { WELT 63496; Lake Wairarapa, } \\
\text { N.Z. }\end{array}$ \\
\hline VUWG & 223 & Boraginaceae & Myosotis scorpioides & $\begin{array}{l}\text { WELT } 62587 \text {; Kenepuru Valley, } \\
\text { N.Z. }\end{array}$ \\
\hline VUWG & 225 & Boraginaceae & Myosotis scorpioides & WELT 45433c; Wellington, N.Z. \\
\hline VUWG & 226 & Boraginaceae & Myosotis scorpioides & WELT 45431; Wellington, N.Z. \\
\hline VUWG & 221 & Boraginaceae & Myosotis scorpioides & $\begin{array}{l}\text { WELT 70396; Clinton to Mataura } \\
\text { Rd, N.Z. }\end{array}$ \\
\hline VUWG & 224 & Boraginaceae & $\begin{array}{l}\text { Myosotis scorpioides } \\
\text { subsp. strigulosa }\end{array}$ & WELT 60825; Wellington, N.Z. \\
\hline VUWG & 227 & Boraginaceae & Myosotis sylvatica & $\begin{array}{l}\text { WELT } 70361 \text {; Kitchener Rd, } \\
\text { Milford, N.Z. }\end{array}$ \\
\hline VUWG & 228 & Boraginaceae & Myosotis sylvatica & $\begin{array}{l}\text { WELT 60366; Sth Karori Rd, } \\
\text { Wellington, N.Z. }\end{array}$ \\
\hline VUWG & 229 & Boraginaceae & Myosotis sylvatica & $\begin{array}{l}\text { WELT } 60365 \text {; Karori, Wellington, } \\
\text { N.Z. }\end{array}$ \\
\hline VUWG & 639 & Boraginaceae & Myosotis sp. & $\begin{array}{l}\text { Chester 1. Anelia, Grevena Pr., } \\
\text { NW Greece. }\end{array}$ \\
\hline VUWG & 211 & Boraginaceae & Symphytum officinale & WELT 70181; Rotorua, N.Z. \\
\hline VUWG & 212 & Boraginaceae & Symphytum officinale & $\begin{array}{l}\text { WELT } 61316 \text {; Johnsonville, } \\
\text { Wellington, N.Z. }\end{array}$ \\
\hline VUWG & 213 & Boraginaceae & Symphytum officinale & $\begin{array}{l}\text { WELT } 60368 \text {; Karori, Wellington, } \\
\text { N.Z. }\end{array}$ \\
\hline VUWG & 950 & Boraginaceae & Symphytum officinale & WELTU box 97 ; Holland. \\
\hline VUWG & 951 & Boraginaceae & Symphytum officinale & WELTU box 97 ; Holland. \\
\hline VUWG & 952 & Boraginaceae & Symphytum officinale & WELTU box 97; Holland. \\
\hline VUWG & 382 & Butomaceae & Butomus umbellatus & $\begin{array}{l}\text { WELT no \#; Medway Tanbridge, } \\
\text { Kent, UK. }\end{array}$ \\
\hline VUWG & 416 & Buxaceae & Buxus sempervirens & WELT no \#; Surrey, England. \\
\hline VUWG & 57 & Calyceraceae & Acicarpha tribuloides & WELT 63172 \\
\hline VUWG & 1167 & Campanulaceae & Campanula latifolia & $\begin{array}{l}\text { WELTU; Voguez nr Strasburg, } \\
\text { France. }\end{array}$ \\
\hline VUWG & 1168 & Campanulaceae & Campanula persicifolia & WELTU. \\
\hline VUWG & 1434 & Campanulaceae & Campanula persicifolia & WELTU; Wellington, N.Z. \\
\hline VUWG & 1169 & Campanulaceae & Campanula rapunculoides & WELTU; Holland. \\
\hline VUWG & 191 & Campanulaceae & Campanula rapunculoides & $\begin{array}{l}\text { WELT } 63154 a ; \text { Miramar, } \\
\text { Wellington, N.Z. }\end{array}$ \\
\hline VUWG & 192 & Campanulaceae & Campanula rapunculoides & $\begin{array}{l}\text { WELT } 60733 \mathrm{a} \text {; Norway St, } \\
\text { Wellington, N.Z. }\end{array}$ \\
\hline VUWG & 193 & Campanulaceae & Campanula rapunculoides & $\begin{array}{l}\text { WELT } 60733 \mathrm{~b} \text {; Norway St, } \\
\text { Wellington, N.Z. }\end{array}$ \\
\hline VUWG & 1170 & Campanulaceae & Campanula rapunculus & WELTU; Holland. \\
\hline VUWG & 1171 & Campanulaceae & Campanula rotundifolia & WELTU. \\
\hline NUNG & 207 & Campamulaceae & Campanula rotundifolia & $\begin{array}{l}\text { Stevenson, Newcastle Upon Tyne } \\
\text { University, England. }\end{array}$ \\
\hline
\end{tabular}




\begin{tabular}{|c|c|c|c|c|}
\hline \multicolumn{2}{|c|}{$\begin{array}{l}\text { Pollen/spore } \\
\text { reference } \\
\text { slide \# }\end{array}$} & \multirow{2}{*}{$\begin{array}{l}\text { Family } \\
\text { Campanulaceae }\end{array}$} & \multirow{2}{*}{$\begin{array}{l}\text { Genus \& species } \\
\text { Campanula trachelium }\end{array}$} & \multirow{2}{*}{$\begin{array}{l}\text { Collector (if known)/herbarium, } \\
\text { herbarium \# (if known), } \\
\text { place of collection }\end{array}$} \\
\hline NUNG & 188 & & & \\
\hline VUWG & 750 & Campanulaceae & $\begin{array}{l}\text { Campanula trachelium } \\
\text { subsp. athoa }\end{array}$ & $\begin{array}{l}\text { Chester 217. Above Ziakas, } \\
\text { Grevena Pr., NW Greece. }\end{array}$ \\
\hline VUWG & 1166 & Campanulaceae & Campanula sp. & WELTU; Germany. \\
\hline VUWG & 644 & Campanulaceae & Campanula sp. & $\begin{array}{l}\text { Chester 6. Anelia, Grevena Pr., } \\
\text { NW Greece. }\end{array}$ \\
\hline VUWG & 194 & Campanulaceae & Solenopsis laurentia & $\begin{array}{l}\text { WELT 70717; Houhora Cemetery, } \\
\text { Northland, N.Z. }\end{array}$ \\
\hline VUWG & 195 & Campanulaceae & Trachelium caeruleum & $\begin{array}{l}\text { WELT } 62677 \text {; Symonds St } \\
\text { Cemetery, Auckland, N.Z. }\end{array}$ \\
\hline VUWG & 196 & Campanulaceae & Trachelium caeruleum & $\begin{array}{l}\text { WELT 59812; Muriwai, Auckland, } \\
\text { N.Z. }\end{array}$ \\
\hline VUWG & 197 & Campanulaceae & Wahlenbergia marginata & $\begin{array}{l}\text { WELT 70621; Taylors Mistake, } \\
\text { Canterbury, N.Z. }\end{array}$ \\
\hline VUWG & 869 & Cannabaceae & Cannabis sativa & WELTU box 86; Holland. \\
\hline VUWG & 38 & Caprifoliacea & Leycesteria formosa & WELT 62579; Picton, N.Z. \\
\hline VUWG & 1436 & Caprifoliaceae & Lonicera japonica & $\begin{array}{l}\text { WELTU; Victoria University, } \\
\text { Wellington, N.Z. }\end{array}$ \\
\hline VUWG & 33 & Caprifoliaceae & Lonicera japonica & $\begin{array}{l}\text { WELT } 60467 \text {; Sth Karori, } \\
\text { Wellington, N.Z. }\end{array}$ \\
\hline VUWG & 34 & Caprifoliaceae & Lonicera japonica & WELT 8401; Opunake, N.Z. \\
\hline VUWG & 35 & Caprifoliaceae & Lonicera japonica & $\begin{array}{l}\text { WELT } 60637 \text {; Wadestown, } \\
\text { Wellington, N.Z. }\end{array}$ \\
\hline VUWG & 36 & Caprifoliaceae & Lonicera japonica & $\begin{array}{l}\text { WELT } 60468 \text {; Karori, Wellington, } \\
\text { N.Z. }\end{array}$ \\
\hline VUWG & 37 & Caprifoliaceae & Lonicera nitida & $\begin{array}{l}\text { WELT } 60721 \text {; Sth Karori Rd, } \\
\text { Wellington, N.Z. }\end{array}$ \\
\hline VUWG & 1155 & Caprifoliaceae & Lonicera periclymenum & WELTU; Holland. \\
\hline VUWG & 1156 & Caprifoliaceae & Sambucus ebulus & WELTU; Switzerland. \\
\hline VUWG & 1157 & Caprifoliaceae & Sambucus nigra & WELTU; Holland. \\
\hline VUWG & 1437 & Caprifoliaceae & Sambucus nigra & WELTU; Wellington, N.Z. \\
\hline VUWG & 1438 & Caprifoliaceae & Sambucus nigra & WELTU 10397; Dunedin, N.Z. \\
\hline VUWG & 39 & Caprifoliaceae & Sambucus nigra & WELT 63177 \\
\hline VUWG & 40 & Caprifoliaceae & Sambucus nigra & $\begin{array}{l}\text { WELT 63017; Pukerua Bay, } \\
\text { Wellington, N.Z. }\end{array}$ \\
\hline VUWG & 41 & Caprifoliaceae & Sambucus nigra & WELT 63016; Wellington, N.Z. \\
\hline NUNG & 227 & Caprifoliaceae & Sambucus nigra & $\begin{array}{l}\text { Stevenson, Newcastle Upon Tyne } \\
\text { University, England. }\end{array}$ \\
\hline NUNG & 157 & Caprifoliaceae & Sambucus nigra & $\begin{array}{l}\text { Stevenson, Newcastle Upon Tyne } \\
\text { University, England. }\end{array}$ \\
\hline VUWG & 1158 & Caprifoliaceae & Sambucus racemosa & WELTU; Holland. \\
\hline VUWG & 42 & Caprifoliaceae & Viburnum tinus & $\begin{array}{l}\text { WELT } 60900 \text {; Sth Karori Rd, } \\
\text { Wellington, N.Z. }\end{array}$ \\
\hline VUWG & 43 & Caprifoliaceae & Viburnum tinus & $\begin{array}{l}\text { WELT 60774; Tinakori Rd, } \\
\text { Wellington, N.Z. }\end{array}$ \\
\hline VUWG & 892 & Caryophyllaceae & Agrostemma githago & WELTU box 87 ; Holland. \\
\hline VUWG & 1314 & Caryophyllaceae & Arenaria serpyllifolia & $\begin{array}{l}\text { WELTU 7654; Granity Pass, } \\
\text { Owen Ra.,N.Z. }\end{array}$ \\
\hline VUWG & 622 & Caryophyllaceae & Arenaria serpyllifolia & WELT 62133. \\
\hline VUWG & 623 & Caryophyllaceae & Arenaria serpyllifolia & WELT 61881. \\
\hline VUWG & 624 & Caryophyllaceae & Arenaria serpyllifolia & WELT 13644. \\
\hline VUWG & 1315 & Caryophyllaceae & Cerastium arvense & WELTU 10274; Taieri Plain, N.Z. \\
\hline
\end{tabular}




\begin{tabular}{|c|c|c|c|}
\hline $\begin{array}{l}\text { Pollen/spore } \\
\text { reference } \\
\text { slide \# }\end{array}$ & Family & Genus \& species & $\begin{array}{l}\text { Collector (if known)/herbarium, } \\
\text { herbarium \# (if known), } \\
\text { place of collection }\end{array}$ \\
\hline
\end{tabular}

\begin{tabular}{|c|c|c|c|c|}
\hline VUWG & 625 & Caryophyllaceae & Cerastium arvense & $\begin{array}{l}\text { WELT 13627; Broken River } \\
\text { Basin, Canterbury, N.Z. }\end{array}$ \\
\hline VUWG & 626 & Caryophyllaceae & Cerastium fontanum triviale & $\begin{array}{l}\text { WELT 61886; Campbell Island, } \\
\text { N.Z. }\end{array}$ \\
\hline VUWG & 628 & Caryophyllaceae & Cerastium glomeratum & $\begin{array}{l}\text { WELT } 70098 \mathrm{a} \text {; Milford, } \\
\text { Waitemata Co., N.Z. }\end{array}$ \\
\hline VUWG & 629 & Caryophyllaceae & Cerastium glomeratum & $\begin{array}{l}\text { WELT } 60934 \text {; Hokio Beach, } \\
\text { Levin, N.Z. }\end{array}$ \\
\hline VUWG & 630 & Caryophyllaceae & Cerastium glomeratum & $\begin{array}{l}\text { WELT } 60935 \text {; Hokio Beach, } \\
\text { Levin, N.Z. }\end{array}$ \\
\hline NUNG & 65 & Caryophyllaceae & ?Cerastium glomeratum & $\begin{array}{l}\text { Stevenson, Newcastle Upon Tyne } \\
\text { University, England. }\end{array}$ \\
\hline VUWG & 893 & Caryophyllaceae & Cerastium semidecandrum & WELTU box 87 ; Holland. \\
\hline VUWG & 627 & Caryophyllaceae & Cerastium vulgare & WELT 13626; Waikanae, N.Z. \\
\hline VUWG & 1316 & Caryophyllaceae & Dianthus armeria & $\begin{array}{l}\text { WELTU 15219; Mt Richmond, } \\
\text { N.Z. }\end{array}$ \\
\hline VUWG & 1317 & Caryophyllaceae & Dianthus barbatus & $\begin{array}{l}\text { WELTU 10272; Cape Wanbrow, } \\
\text { Oamaru, N.Z. }\end{array}$ \\
\hline VUWG & 894 & Caryophyllaceae & Dianthus carthusianorum & $\begin{array}{l}\text { WELTU box } 87 \text {; Black Forest, } \\
\text { Germany. }\end{array}$ \\
\hline VUWG & 631 & Caryophyllaceae & Dianthus deltoides & $\begin{array}{l}\text { WELT 70417; Rakaia River, } \\
\text { Canterbury, N.Z. }\end{array}$ \\
\hline VUWG & 642 & Caryophyllaceae & Dianthus sp. & $\begin{array}{l}\text { Chester 4. Anelia, Grevena Pr., } \\
\text { NW Greece. }\end{array}$ \\
\hline VUWG & 700 & Caryophyllaceae & Dianthus sp. & $\begin{array}{l}\text { Chester } 72 \text {. Polineri, Grevena Pr., } \\
\text { NW Greece. }\end{array}$ \\
\hline VUWG & 832 & Caryophyllaceae & Dianthus sp. & $\begin{array}{l}\text { Chester } 353 \text {. Victoria University, } \\
\text { Wellington, N.Z. }\end{array}$ \\
\hline VUWG & 802 & Caryophyllaceae & Dianthus sp. [cultivar] & $\begin{array}{l}\text { Chester } 323 \text {. Wellington Botanic } \\
\text { Gardens, N.Z. }\end{array}$ \\
\hline VUWG & 803 & Caryophyllaceae & Dianthus sp. [cultivar] & $\begin{array}{l}\text { Chester 324. Wellington Botanic } \\
\text { Gardens, N.Z. }\end{array}$ \\
\hline VUWG & 804 & Caryophyllaceae & Dianthus sp. [cultivar] & $\begin{array}{l}\text { Chester } 325 \text {. Wellington Botanic } \\
\text { Gardens, N.Z. }\end{array}$ \\
\hline VUWG & 632 & Caryophyllaceae & Holosteum umbellatum & WELT 78162; Central Otago, N.Z. \\
\hline VUWG & 738 & Caryophyllaceae & Lychnis coronaria & $\begin{array}{l}\text { Chester } 161 . \text { Anelia, Grevena Pr., } \\
\text { NW Greece. }\end{array}$ \\
\hline VUWG & 895 & Caryophyllaceae & Lychnis diurna & WELTU box 87 ; Holland. \\
\hline VUWG & 896 & Caryophyllaceae & Lychnis flore alba & WELTU box 87 ; Holland. \\
\hline VUWG & 898 & Caryophyllaceae & Lychnis vespertina & WELTU box 87 ; Holland. \\
\hline VUWG & 897 & Caryophyllaceae & Lychnis viscida & WELTU box 87; Holland. \\
\hline VUWG & 899 & Caryophyllaceae & Moehringia muscosa & WELTU box 87; Switzerland. \\
\hline VUWG & 729 & Caryophyllaceae & Petrorhagia fasciculata & $\begin{array}{l}\text { Chester 142. Mirsina, Grevena Pr., } \\
\text { NW Greece. }\end{array}$ \\
\hline VUWG & 633 & Caryophyllaceae & Sagina procumbens & $\begin{array}{l}\text { WELT 70913; Rakaia River, } \\
\text { Canterbury, N.Z. }\end{array}$ \\
\hline VUWG & 634 & Caryophyllaceae & Sagina procumbens & $\begin{array}{l}\text { WELT 70813; Belmont, Hutt } \\
\text { Valley, N.Z. }\end{array}$ \\
\hline VUWG & 635 & Caryophyllaceae & Sagina procumbens & $\begin{array}{l}\text { WELT } 60540 \text {; S Karori, } \\
\text { Wellington, N.Z. }\end{array}$ \\
\hline VUWG & 900 & Caryophyllaceae & Saponaria officinalis & WELTU box 87 ; Holland. \\
\hline VUWG & 901 & Caryophyllaceae & Saponaria officinalis & WELTU box 87. \\
\hline VUWG & 902 & Caryophyllaceae & Saponaria officinalis & WELTU box 87. \\
\hline
\end{tabular}




\begin{tabular}{|c|c|c|c|}
\hline $\begin{array}{l}\text { Pollen/spore } \\
\text { reference } \\
\text { slide \# }\end{array}$ & Family & Genus \& species & $\begin{array}{l}\text { Collector (if known)/herbarium, } \\
\text { herbarium \# (if known), } \\
\text { place of collection }\end{array}$ \\
\hline
\end{tabular}

\begin{tabular}{|c|c|c|c|c|}
\hline VUWG & 903 & Caryophyllaceae & Scleranthus annuus & WELTU box 87; Holland. \\
\hline VUWG & 638 & Caryophyllaceae & Silene alba & WELT 13617. \\
\hline NUNG & 139 & Caryophyllaceae & Silene alpestris & $\begin{array}{l}\text { Stevenson, Newcastle Upon Tyne } \\
\text { University, England. }\end{array}$ \\
\hline VUWG & 637 & Caryophyllaceae & Silene armeria & $\begin{array}{l}\text { WELT } 61803 ; \text { S Karori, } \\
\text { Wellington, N.Z. }\end{array}$ \\
\hline VUWG & 636 & Caryophyllaceae & Silene dioica & $\begin{array}{l}\text { WELT } 60872 ; \text { S Karori, } \\
\text { Wellington, N.Z. }\end{array}$ \\
\hline VUWG & 1318 & Caryophyllaceae & Silene gallica & $\begin{array}{l}\text { WELTU 10267; Andersons Bay, } \\
\text { Dunedin, N.Z. }\end{array}$ \\
\hline VUWG & 1319 & Caryophyllaceae & Silene gallica & WELTU. \\
\hline VUWG & 1320 & Caryophyllaceae & Silene gallica & WELTU 15217; Wellington, N.Z. \\
\hline NUNG & 201 & Caryophyllaceae & Silene gallica & $\begin{array}{l}\text { Stevenson, Newcastle Upon Tyne } \\
\text { University, England. }\end{array}$ \\
\hline NUNG & 217 & Caryophyllaceae & Silene micropetala & $\begin{array}{l}\text { Stevenson, Newcastle Upon Tyne } \\
\text { University, England. }\end{array}$ \\
\hline VUWG & 890 & Caryophyllaceae & Silene nutans & WELTU box 87; Holland. \\
\hline NUNG & 196 & Caryophyllaceae & Silene otites & $\begin{array}{l}\text { Stevenson, Newcastle Upon Tyne } \\
\text { University, England. }\end{array}$ \\
\hline VUWG & 889 & Caryophyllaceae & Silene vulgaris & WELTU box 87; Holland. \\
\hline VUWG & 1321 & Caryophyllaceae & Silene vulgaris maritima & WELTU. \\
\hline VUWG & 794 & Caryophyllaceae & $\begin{array}{l}\text { Silene vulgaris subsp. } \\
\text { maritima }\end{array}$ & $\begin{array}{l}\text { Chester } 315 \text {. Wellington Botanic } \\
\text { Gardens, N.Z. }\end{array}$ \\
\hline VUWG & 1322 & Caryophyllaceae & Silene sp. & WELTU; Karori, Wellington, N.Z. \\
\hline VUWG & 905 & Caryophyllaceae & Stellaria alsine & WELTU box 87 ; Holland. \\
\hline VUWG & 1323 & Caryophyllaceae & Stellaria graminea & WELTU 10277; Taieri Plain, N.Z. \\
\hline VUWG & 904 & Caryophyllaceae & Stellaria graminea & WELTU box 87 ; Holland. \\
\hline CRYG & 284 & Caryophyllaceae & Stellaria holostea & $\begin{array}{l}\text { Atherden, College of Rippon \& } \\
\text { York, England. }\end{array}$ \\
\hline VUWG & 906 & Caryophyllaceae & Stellaria media & WELTU box 87 ; India. \\
\hline NUNG & 48 & Caryopyyllaceae & Stellaria sp. & $\begin{array}{l}\text { Stevenson, Newcastle Upon Tyne } \\
\text { University, England. }\end{array}$ \\
\hline VUWG & 1324 & Chenopodiaceae & Atriplex patula & $\begin{array}{l}\text { WELTU 11569; Cape Turakirae, } \\
\text { Wellington, N.Z. }\end{array}$ \\
\hline VUWG & 1325 & Chenopodiaceae & Atriplex patula & WELTU 10289; Waipiro Bay, N.Z. \\
\hline VUWG & 603 & Chenopodiaceae & Atriplex patula & WELT 70720; Taieri, Otago, N.Z. \\
\hline NUNG & 174 & Chenopodiaceae & Atriplex sp. & $\begin{array}{l}\text { Stevenson, Newcastle Upon Tyne } \\
\text { University, England. }\end{array}$ \\
\hline NUNG & 43 & Chenopoiaceae & Beta vulgaris & $\begin{array}{l}\text { Stevenson, Newcastle Upon Tyne } \\
\text { University, England. }\end{array}$ \\
\hline VUWG & 1326 & Chenopodiaceae & Chenopodium album & WELTU 15311; Waikanae, N.Z. \\
\hline VUWG & 1327 & Chenopodiaceae & Chenopodium album & WELTU 10286; Dunedin, N.Z. \\
\hline VUWG & 1328 & Chenopodiaceae & Chenopodium album & WELTU 10285; Napier, N.Z. \\
\hline VUWG & 604 & Chenopodiaceae & Chenopodium album & $\begin{array}{l}\text { WELT 70875; Wakanui, } \\
\text { Ashburton, N.Z. }\end{array}$ \\
\hline VUWG & 605 & Chenopodiaceae & Chenopodium album & WELT 70719; Nelson, N.Z. \\
\hline VUWG & 606 & Chenopodiaceae & Chenopodium album & $\begin{array}{l}\text { WELT no \#; Pukerua Bay, } \\
\text { Wellington, N.Z. }\end{array}$ \\
\hline VUWG & 886 & Chenopodiaceae & Chenopodium album & WELTU box 87 ; India. \\
\hline VUWG & 759 & Chenopodiaceae & Chenopodium cf. album & $\begin{array}{l}\text { Chester 234. Asprokampos, } \\
\text { Grevena Pr., NW Greece. }\end{array}$ \\
\hline $\begin{array}{l}\text { VUWG } \\
\text { VUWG }\end{array}$ & $\begin{array}{l}887 \\
607\end{array}$ & Chenopodiaceae & Chenopodium bonus-henricus & $\begin{array}{l}\text { WELTU box } 87 \text {; Switzerland. } \\
\text { WELT } 70562 \text {; Mana Island, N.Z. }\end{array}$ \\
\hline $\mathrm{J}$ & 607 & Chenopodiaceae & Chenopodium murale & WELT 70562; Mana Island. \\
\hline
\end{tabular}




\begin{tabular}{|c|c|c|c|c|}
\hline \multicolumn{2}{|c|}{$\begin{array}{l}\text { Pollen/spore } \\
\text { reference } \\
\text { slide \# }\end{array}$} & \multirow{2}{*}{$\begin{array}{l}\text { Family } \\
\text { Chenopodiaceae }\end{array}$} & \multirow{2}{*}{$\begin{array}{l}\text { Genus \& species } \\
\text { Chenopodium murale }\end{array}$} & \multirow{2}{*}{$\begin{array}{l}\text { Collector (if known)/herbarium } \\
\text { herbarium \# (if known), } \\
\text { place of collection }\end{array}$} \\
\hline vUWG & 608 & & & \\
\hline VUWG & 609 & Chenopodiaceae & Chenopodium murale & $\begin{array}{l}\text { WELT 52295; Breaker Bay, } \\
\text { Wellington, N.Z. }\end{array}$ \\
\hline NUNG & 59 & Cistaceae & Cistus albidus & $\begin{array}{l}\text { Stevenson, Newcastle Upon Tyne } \\
\text { University, England. }\end{array}$ \\
\hline NUNG & 109 & Cistaceae & Cistus crispus & $\begin{array}{l}\text { Stevenson, Newcastle Upon Tyne } \\
\text { University, England. }\end{array}$ \\
\hline NUNG & 129 & Cistaceae & Cistus incanus & $\begin{array}{l}\text { Stevenson, Newcastle Upon Tyne } \\
\text { University, England. }\end{array}$ \\
\hline NUNG & 124 & Cistaceae & Cistus monspeliensis & $\begin{array}{l}\text { Stevenson, Newcastle Upon Tyne } \\
\text { University, England. }\end{array}$ \\
\hline NUNG & 190 & Cistaceae & Cistus populifolius & $\begin{array}{l}\text { Stevenson, Newcastle Upon Tyne } \\
\text { University, England. }\end{array}$ \\
\hline NUNG & 41 & Cistaceae & Cistus psilosepalus & $\begin{array}{l}\text { Stevenson, Newcastle Upon Tyne } \\
\text { University, England. }\end{array}$ \\
\hline VUWG & 1313 & Cistaceae & Cistus purpureus & $\begin{array}{l}\text { WELTU; Victoria University, } \\
\text { Wellington, N.Z. }\end{array}$ \\
\hline NUNG & 142 & Cistaceae & Cistus salvifolius & $\begin{array}{l}\text { Stevenson, Newcastle Upon Tyne } \\
\text { University, England. }\end{array}$ \\
\hline NUNG & 271 & Cistaceae & Cistus salvifolius & $\begin{array}{l}\text { Stevenson, Newcastle Upon Tyne } \\
\text { University, England. }\end{array}$ \\
\hline NUNG & 277 & Cistaceae & Cistus salvifolius & $\begin{array}{l}\text { Stevenson, Newcastle Upon Tyne } \\
\text { University, England. }\end{array}$ \\
\hline NUNG & 87 & Cistaceae & Cistus varius & $\begin{array}{l}\text { Stevenson, Newcastle Upon Tyne } \\
\text { University, England. }\end{array}$ \\
\hline NUNG & 258 & Cistaceae & Cistus sp. & $\begin{array}{l}\text { Stevenson, Newcastle Upon Tyne } \\
\text { University, England. }\end{array}$ \\
\hline NUNG & 140 & Cistaceae & Fumana procumbens & $\begin{array}{l}\text { Stevenson, Newcastle Upon Tyne } \\
\text { University, England. }\end{array}$ \\
\hline VUWG & 853 & Cistaceae & Helianthemum nummularium & WELTU box 93 ; Germany. \\
\hline VUWG & 772 & Cistaceae & Helianthemum ?nummularium & $\begin{array}{l}\text { Chester 304. Mirsina, Grevena Pr., } \\
\text { NW Greece. }\end{array}$ \\
\hline VUWG & 795 & Cistaceae & Helianthemum sp. & $\begin{array}{l}\text { Chester 316. Wellington Botanic } \\
\text { Gardens, N.Z. }\end{array}$ \\
\hline VUWG & 703 & Compositae & ?Anthemis sp. & $\begin{array}{l}\text { Chester 75. Polineri, Grevena Pr., } \\
\text { NW Greece. }\end{array}$ \\
\hline VUWG & 767 & Compositae & ?Anthemis sp. & $\begin{array}{l}\text { Chester 277. Asprokampos, } \\
\text { Grevena Pr., NW Greece. }\end{array}$ \\
\hline VUWG & 727 & Compositae & ?Crepis sp. & $\begin{array}{l}\text { Chester 133. Mirsina, Grevena Pr., } \\
\text { NW Greece. }\end{array}$ \\
\hline VUWG & 645 & Compositae & ?Leontodon sp. & $\begin{array}{l}\text { Chester 7. Anelia, Grevena Pr., } \\
\text { NW Greece. }\end{array}$ \\
\hline VUWG & 766 & Compositae & ?Scorzonera sp. & $\begin{array}{l}\text { Chester 254. Asprokampos, } \\
\text { Grevena Pr., NW Greece. }\end{array}$ \\
\hline VUWG & 1172 & Compositae & Achillea millefolium & WELTU; Holland. \\
\hline VUWG & 1173 & Compositae & Achillea millefolium & WELTU; Holland. \\
\hline VUWG & 1442 & Compositae & Achillea millefolium & $\begin{array}{l}\text { WELTU; Tinakori Hill, } \\
\text { Wellington, N.Z. }\end{array}$ \\
\hline VUWG & 1443 & Compositae & Achillea millefolium & $\begin{array}{l}\text { WELTU; Tinakori Hill, } \\
\text { Wellington, N.Z. }\end{array}$ \\
\hline VUWG & 1444 & Compositae & Achillea millefolium & $\begin{array}{l}\text { WELTU 15254; Victoria } \\
\text { University, Wellington, N.Z. }\end{array}$ \\
\hline
\end{tabular}




\begin{tabular}{|c|c|c|c|c|}
\hline \multicolumn{2}{|c|}{$\begin{array}{l}\text { Pollen/spore } \\
\text { reference } \\
\text { slide \# }\end{array}$} & \multirow{2}{*}{$\begin{array}{l}\text { Family } \\
\text { Compositae }\end{array}$} & \multirow{2}{*}{$\begin{array}{l}\text { Genus \& species } \\
\text { Achillea millefolium }\end{array}$} & \multirow{2}{*}{$\begin{array}{l}\text { Collector (if known)/herbarium, } \\
\text { herbarium \# (if known), } \\
\text { place of collection }\end{array}$} \\
\hline VUWG & 58 & & & \\
\hline VUWG & 59 & Compositae & Achillea millefolium & $\begin{array}{l}\text { WELT } 61785 \text {; Castlepoint, } \\
\text { Wairarapa, N.Z. }\end{array}$ \\
\hline VUWG & 60 & Compositae & Achillea millefolium & $\begin{array}{l}\text { WELT } 61451 \text {; Seatoun, } \\
\text { Wellington, N.Z. }\end{array}$ \\
\hline VUWG & 1174 & Compositae & Achillea nobilis & $\begin{array}{l}\text { WELTU; Kaiserstuhl nr Freiburg, } \\
\text { Germany. }\end{array}$ \\
\hline VUWG & 1175 & Compositae & Achillea ptarmica & WELTU; Holland. \\
\hline NUNG & 161 & Compositae & Achillea ptarmica & $\begin{array}{l}\text { Stevenson, Newcastle Upon Tyne } \\
\text { University, England. }\end{array}$ \\
\hline VUWG & 61 & Compositae & Anthemis cotula & $\begin{array}{l}\text { WELT } 62624 \text {; Keith George Pk, } \\
\text { Hutt Valley, N.Z. }\end{array}$ \\
\hline VUWG & 62 & Compositae & Anthemis cotula & $\begin{array}{l}\text { WELT } 62627 \text {; Pukemokemoke, } \\
\text { Hamilton, N.Z. }\end{array}$ \\
\hline VUWG & 63 & Compositae & Anthemis cotula & $\begin{array}{l}\text { WELT } 60429 \text {; Makara, } \\
\text { Wellington, N.Z. }\end{array}$ \\
\hline VUWG & 696 & Compositae & Anthemis sp. & $\begin{array}{l}\text { Chester 66. Mavrani, Grevena Pr., } \\
\text { NW Greece. }\end{array}$ \\
\hline NUNG & 66 & Compositae & Anthemis or Chamomilla & $\begin{array}{l}\text { Stevenson, Newcastle Upon Tyne } \\
\text { University, England. }\end{array}$ \\
\hline VUWG & 65 & Compositae & Arctium minus & $\begin{array}{l}\text { WELT } 61401 \text {; Cliffs towards } \\
\text { Waimea, Nelson, N.Z. }\end{array}$ \\
\hline VUWG & 66 & Compositae & Arctium minus & $\begin{array}{l}\text { WELT 70714a; Waipapa Bay, } \\
\text { Marlborough, N.Z. }\end{array}$ \\
\hline VUWG & 1446 & Compositae & Artemisia absinthium & WELTU 10399; Taieri Plain, N.Z. \\
\hline VUWG & 68 & Compositae & Artemisia absinthium & WELT 61405; Auckland, N.Z. \\
\hline VUWG & 69 & Compositae & Artemisia absinthium & WELT 61404; Auckland, N.Z. \\
\hline VUWG & 70 & Compositae & Artemisia absinthium & $\begin{array}{l}\text { WELT (Ex CHR 420888); Waitaki } \\
\text { Valley, Otago, N.Z. }\end{array}$ \\
\hline VUWG & 67 & Compositae & Artemisia arborescens & $\begin{array}{l}\text { WELT } 60442 \text {; Karori, Wellington, } \\
\text { N.Z. }\end{array}$ \\
\hline NUNG & 151 & Compositae & Artemisia genipi & $\begin{array}{l}\text { Stevenson, Newcastle Upon Tyne } \\
\text { University, England. }\end{array}$ \\
\hline NUNG & 146 & Compositae & Artemisia umbelliformis & $\begin{array}{l}\text { Stevenson, Newcastle Upon Tyne } \\
\text { University, England. }\end{array}$ \\
\hline VUWG & 1177 & Compositae & Artemisia vulgaris & WELTU; Holland. \\
\hline NUNG & 32 & Compositae & ?Aster tripolium & $\begin{array}{l}\text { Stevenson, Newcastle Upon Tyne } \\
\text { University, England. }\end{array}$ \\
\hline VUWG 1 & 1447 & Compositae & Bellis perennis & $\begin{array}{l}\text { WELTU 15183; Victoria } \\
\text { University, Wellington, N.Z. }\end{array}$ \\
\hline VUWG & 71 & Compositae & Bellis perennis & $\begin{array}{l}\text { WELT } 70838 \text {; Belmont, Hutt } \\
\text { Valley, N.Z. }\end{array}$ \\
\hline VUWG & 796 & Compositae & Bellis perennis & $\begin{array}{l}\text { Chester 317. Wellington Botanic } \\
\text { Gardens, N.Z. }\end{array}$ \\
\hline VUWG & 819 & Compositae & Bellis perennis & $\begin{array}{l}\text { Chester } 340 . \text { Garden, Johnsonville, } \\
\text { Wellington, N.Z. }\end{array}$ \\
\hline NUNG & 3 & Compositae & Carduus acanthoides & $\begin{array}{l}\text { Stevenson, Newcastle Upon Tyne } \\
\text { University, England. }\end{array}$ \\
\hline NUNG & 13 & Compositae & Carduus acanthoides & $\begin{array}{l}\text { Stevenson, Newcastle Upon Tyne } \\
\text { University, England. }\end{array}$ \\
\hline VUWG & 1178 & Compositae & Carduus crispus & WELTU; Holland. \\
\hline VUWG 1 & 1449 & Compositae & Carduus crispus & WELTU 10403; Oamaru, N.Z. \\
\hline
\end{tabular}




\begin{tabular}{|c|c|c|c|c|}
\hline \multicolumn{2}{|c|}{$\begin{array}{l}\text { Pollen/spore } \\
\text { reference } \\
\text { slide \# }\end{array}$} & \multirow{2}{*}{$\begin{array}{l}\text { Family } \\
\text { Compositae }\end{array}$} & \multirow{2}{*}{$\begin{array}{l}\text { Genus \& species } \\
\text { Carduus lanceolatus }\end{array}$} & \multirow{2}{*}{$\begin{array}{l}\text { Collector (if known)/herbarium } \\
\text { herbarium \# (if known), } \\
\text { place of collection }\end{array}$} \\
\hline VUWG & 1448 & & & \\
\hline VUWG & 1179 & Compositae & Carduus marianus & WELTU; Switzerland. \\
\hline VUWG & 72 & Compositae & Carduus nutans & $\begin{array}{l}\text { WELT 16169; Hakataramea } \\
\text { Valley, N.Z. }\end{array}$ \\
\hline VUWG & 73 & Compositae & Carduus pycnocephalus & WELT 61444; Otago, N.Z. \\
\hline VUWG & 74 & Compositae & Carduus pycnocephalus & $\begin{array}{l}\text { WELT 70626; Sydenham, } \\
\text { Christchurch, N.Z. }\end{array}$ \\
\hline VUWG & 75 & Compositae & Carduus pycnocephalus & WELT 61335 \\
\hline VUWG & 1180 & Compositae & Carlina acaulis & WELTU; Switzerland. \\
\hline VUWG & 76 & Compositae & Centaurea calcitrapa & WELT 61339a; Akaroa, N.Z. \\
\hline VUWG & 1182 & Compositae & Centaurea cyanus & WELTU; Holland. \\
\hline VUWG & 77 & Compositae & Centaurea cyanus & $\begin{array}{l}\text { WELT } 61188 \text {; Sth Karori Rd, } \\
\text { Wellington, N.Z. }\end{array}$ \\
\hline VUWG & 1181 & Compositae & Centaurea jacea & WELTU; Holland. \\
\hline NUNG & 154 & Compositae & Centaurea jacea & $\begin{array}{l}\text { Stevenson, Newcastle Upon Tyne } \\
\text { University, England. }\end{array}$ \\
\hline VUWG & 78 & Compositae & Centaurea melitensis & $\begin{array}{l}\text { WELT 61344; Island Bay, } \\
\text { Wellington, N.Z. }\end{array}$ \\
\hline VUWG & 79 & Compositae & Centaurea melitensis & $\begin{array}{l}\text { WELT 78173; Huangarua River, } \\
\text { Martinborough, N.Z. }\end{array}$ \\
\hline VUWG & 669 & Compositae & Centaurea ?napulifera & $\begin{array}{l}\text { Chester } 31 . \text { Kellia, Grevena Pr., } \\
\text { NW Greece. }\end{array}$ \\
\hline NUNG & 198 & Compositae & Centaurea nigra & $\begin{array}{l}\text { Stevenson, Newcastle Upon Tyne } \\
\text { University, England. }\end{array}$ \\
\hline NUNG & 166 & Compositae & Centaurea scabiosa & $\begin{array}{l}\text { Stevenson, Newcastle Upon Tyne } \\
\text { University, England. }\end{array}$ \\
\hline NUNG & 8 & Compositae & Centaurea sphaerocephala & $\begin{array}{l}\text { Stevenson, Newcastle Upon Tyne } \\
\text { University, England. }\end{array}$ \\
\hline VUWG & 1451 & Compositae & Centaurea solstitialis & $\begin{array}{l}\text { WELTU 10405; Clutha Valley, } \\
\text { N.Z. }\end{array}$ \\
\hline VUWG & 705 & Compositae & Centaurea solstitialis & $\begin{array}{l}\text { Chester 77. Polineri, Grevena Pr., } \\
\text { NW Greece. }\end{array}$ \\
\hline NUNG & 80 & Compositae & Centaurea triumfetti & $\begin{array}{l}\text { Stevenson, Newcastle Upon Tyne } \\
\text { University, England. }\end{array}$ \\
\hline VUWG & 643 & Compositae & Centaurea sp. & $\begin{array}{l}\text { Chester 5. Anelia, Grevena Pr., } \\
\text { NW Greece. }\end{array}$ \\
\hline VUWG & 689 & Compositae & Centaurea sp. & $\begin{array}{l}\text { Chester 57. Mavrani, Grevena Pr., } \\
\text { NW Greece. }\end{array}$ \\
\hline VUWG & 760 & Compositae & Centaurea sp. & $\begin{array}{l}\text { Chester 247. Asprokampos, } \\
\text { Grevena Pr., NW Greece. }\end{array}$ \\
\hline VUWG & 769 & Compositae & Centaurea sp. & $\begin{array}{l}\text { Chester 299. Mirsina, Grevena Pr., } \\
\text { NW Greece. }\end{array}$ \\
\hline VUWG & 80 & Compositae & Centaurea sp. & $\begin{array}{l}\text { WELT 70914; Tauherenikau } \\
\text { Valley, Tararua Ra., N.Z. }\end{array}$ \\
\hline VUWG & 789 & Compositae & Centaurea sp. [cultivar] & $\begin{array}{l}\text { Chester } 310 \text {. Wellington Botanic } \\
\text { Gardens, N.Z. }\end{array}$ \\
\hline VUWG & 1445 & Compositae & Chamaemelum nobile & $\begin{array}{l}\text { WELTU 10400; Port Chalmers, } \\
\text { N.Z. }\end{array}$ \\
\hline VUWG & 698 & Compositae & Chondrilla juncea & $\begin{array}{l}\text { Chester 70. Mavrani, Grevena Pr., } \\
\text { NW Greece. }\end{array}$ \\
\hline VUWG & 81 & Compositae & Chondrilla juncea & $\begin{array}{l}\text { WELT 63329; Gt Nth Rd, New } \\
\text { Lynn, Auckland, N.Z. }\end{array}$ \\
\hline VUWG & 82 & Compositae & Chrysanthemum coronarium & WELT 70342; Auckland, N.Z. \\
\hline
\end{tabular}




\begin{tabular}{|c|c|c|c|c|}
\hline $\begin{array}{l}\text { Pollen/sp } \\
\text { referenc } \\
\text { slide \# }\end{array}$ & & Family & Genus \& species & $\begin{array}{l}\text { Collector (if known)/herbarium, } \\
\text { herbarium \# (if known), } \\
\text { place of collection }\end{array}$ \\
\hline VUWG & 83 & Compositae & Chrysanthemum coronarium & $\begin{array}{l}\text { WELT 16142; Seatoun, } \\
\text { Wellington, N.Z. }\end{array}$ \\
\hline VUWG & 84 & Compositae & Chrysanthemum coronarium & $\begin{array}{l}\text { WELT } 61431 \text {; Near Railway Stn, } \\
\text { Wellington, N.Z. }\end{array}$ \\
\hline VUWG & 116 & Compositae & $\begin{array}{l}\text { Chrysanthemum } \\
\text { leucanthemum }\end{array}$ & $\begin{array}{l}\text { WELT } 60428 \text {; Sth Karori Rd, } \\
\text { Wellington, N.Z. }\end{array}$ \\
\hline VUWG & 85 & Compositae & Chrysanthemum segetum & $\begin{array}{l}\text { WELT 60426; Sth Karori Rd, } \\
\text { Wellington, N.Z. }\end{array}$ \\
\hline VUWG & 86 & Compositae & Chrysanthemum segetum & $\begin{array}{l}\text { WELT } 60427 \text {; Happy Valley, } \\
\text { Wellington, N.Z. }\end{array}$ \\
\hline VUWG & 87 & Compositae & Chrysanthemum segetum & $\begin{array}{l}\text { WELT } 60743 \text {; Karori Cemetery, } \\
\text { Wellington, N.Z. }\end{array}$ \\
\hline VUWG & 1450 & Compositae & Cichorium intybus & $\begin{array}{l}\text { WELTU; Mt Victoria, Wellington, } \\
\text { N.Z. }\end{array}$ \\
\hline VUWG & 665 & Compositae & Cichorium intybus & $\begin{array}{l}\text { Chester 27. Kellia, Grevena Pr., } \\
\text { NW Greece. }\end{array}$ \\
\hline VUWG & 734 & Compositae & Cichorium intybus & $\begin{array}{l}\text { Chester 152. Mirsina, Grevena Pr., } \\
\text { NW Greece. }\end{array}$ \\
\hline VUWG & 1184 & Compositae & Cirsium arvense & WELTU; Holland. \\
\hline VUWG & 1185 & Compositae & Cirsium arvense & WELTU; Holland. \\
\hline VUWG & 1452 & Compositae & Cirsium arvense & WELTU; Wellington, N.Z. \\
\hline VUWG & 1453 & Compositae & Cirsium arvense & $\begin{array}{l}\text { WELTU 10407; Queen Charlotte } \\
\text { Sound, N.Z. }\end{array}$ \\
\hline VUWG & 88 & Compositae & Cirsium arvense & WELT 16118; Opunake, N.Z. \\
\hline VUWG & 721 & Compositae & Cirsium creticum & $\begin{array}{l}\text { Chester 126. Mirsina, Grevena Pr., } \\
\text { NW Greece. }\end{array}$ \\
\hline VUWG & 1186 & Compositae & Cirsium eriophorum & WELTU; Holland. \\
\hline NUNG & 108 & Compositae & Cirsium oleraceum & $\begin{array}{l}\text { Stevenson, Newcastle Upon Tyne } \\
\text { University, England. }\end{array}$ \\
\hline NUNG & 118 & Compositae & Cirsium palustra & $\begin{array}{l}\text { Stevenson, Newcastle Upon Tyne } \\
\text { University, England. }\end{array}$ \\
\hline NUNG & 60 & Compositae & Cirsium spinosissimum & $\begin{array}{l}\text { Stevenson, Newcastle Upon Tyne } \\
\text { University, England. }\end{array}$ \\
\hline VUWG & 1454 & Compositae & Cirsium vulgare & $\begin{array}{l}\text { WELTU 11309; Cape Turakirae, } \\
\text { Wellington, N.Z. }\end{array}$ \\
\hline VUWG & 90 & Compositae & Cirsium vulgare & WELT 61356 \\
\hline VUWG & 682 & Compositae & Cirsium sp. & $\begin{array}{l}\text { Chester } 48 . \text { Kellia, Grevena Pr., } \\
\text { NW Greece. }\end{array}$ \\
\hline VUWG & 715 & Compositae & Cirsium sp. & $\begin{array}{l}\text { Chester 101. Polineri, Grevena Pr., } \\
\text { NW Greece. }\end{array}$ \\
\hline NUNG & 42 & Compositae & Cirsium sp. & $\begin{array}{l}\text { Stevenson, Newcastle Upon Tyne } \\
\text { University, England. }\end{array}$ \\
\hline VUWG & 1190 & Compositae & Conyza canadensis & WELTU; Holland. \\
\hline VUWG & 1457 & Compositae & Conyza canadensis & WELTU; Waikanae R., N.Z. \\
\hline VUWG & 1187 & Compositae & Crepis aurea & WELTU; Switzerland. \\
\hline VUWG & 1455 & Compositae & Crepis capillaris & $\begin{array}{l}\text { WELTU 11262; Cape Turakirae, } \\
\text { Wellington, N.Z. }\end{array}$ \\
\hline VUWG & 1456 & Compositae & Crepis capillaris & WELTU 10409; Kaingaroa, N.Z. \\
\hline VUWG & 91 & Compositae & Crepis capillaris & $\begin{array}{l}\text { WELT 70808b; Kelburn, } \\
\text { Wellington, N.Z. }\end{array}$ \\
\hline VUWG & 92 & Compositae & Crepis capillaris & $\begin{array}{l}\text { WELT 70752; Totaranui Bay, } \\
\text { Nelson, N.Z. }\end{array}$ \\
\hline VUWG & 93 & Compositae & Crepis capillaris & WELT 16120; Ihaia, N.Z. \\
\hline
\end{tabular}




\begin{tabular}{|c|c|c|c|c|}
\hline \multicolumn{2}{|c|}{$\begin{array}{l}\text { Pollen/spore } \\
\text { reference } \\
\text { slide \# }\end{array}$} & \multirow{2}{*}{$\begin{array}{l}\text { Family } \\
\text { Compositae }\end{array}$} & \multirow{2}{*}{$\begin{array}{l}\text { Genus \& species } \\
\text { Crepis sp. }\end{array}$} & \multirow{2}{*}{$\begin{array}{l}\text { Collector (if known)/herbarium, } \\
\text { herbarium \# (if known), } \\
\text { place of collection }\end{array}$} \\
\hline VUWG & 693 & & & \\
\hline VUWG & 1188 & Compositae & Doronium grandiflorum & WELTU; Switzerland. \\
\hline VUWG & 687 & Compositae & Echinops microcephalus & $\begin{array}{l}\text { Chester 55. Mavrani, Grevena Pr., } \\
\text { NW Greece. }\end{array}$ \\
\hline VUWG & 768 & Compositae & Echinops microcephalus & $\begin{array}{l}\text { Chester 297. Mirsina, Grevena Pr., } \\
\text { NW Greece. }\end{array}$ \\
\hline VUWG & 743 & Compositae & Echinops cf. microcephalus & $\begin{array}{l}\text { Chester } 170 . \text { Gomara, Grevena Pr., } \\
\text { NW Greece. }\end{array}$ \\
\hline VUWG & 1189 & Compositae & Erigeron alpinus & WELTU; Switzerland. \\
\hline VUWG & 1458 & Compositae & Erigeron karvinskianus & WELTU; Wellington, N.Z. \\
\hline VUWG & 1459 & Compositae & Erigeron karvinskianus & WELTU; Wellington, N.Z. \\
\hline VUWG & 799 & Compositae & Erigeron sp. & $\begin{array}{l}\text { Chester } 320 \text {. Wellington Botanic } \\
\text { Gardens, N.Z. }\end{array}$ \\
\hline VUWG & 709 & Compositae & Filago pyramidata & $\begin{array}{l}\text { Chester 83. Polineri, Grevena Pr., } \\
\text { NW Greece. }\end{array}$ \\
\hline VUWG & 1191 & Compositae & Filago sp. & WELTU; Switzerland. \\
\hline VUWG & 94 & Compositae & Hieracium aurantiacum & WELT 63391; Lake Rotoiti, N.Z. \\
\hline VUWG & 95 & Compositae & Hieracium aurantiacum & WELT 63391b; Lake Rotoiti, N.Z. \\
\hline VUWG & 96 & Compositae & Hieracium caespitosum & WELT 16185; Springfield, N.Z. \\
\hline VUWG & 1194 & Compositae & Hieracium pilosella & WELTU; Holland. \\
\hline VUWG & 1461 & Compositae & Hieracium pilosella & WELTU 7657; Owen Range, N.Z. \\
\hline VUWG & 100 & Compositae & Hieracium praealtum & $\begin{array}{l}\text { WELT 70705; Pivot Ck, Westland, } \\
\text { N.Z. }\end{array}$ \\
\hline VUWG & 101 & Compositae & Hieracium praealtum & $\begin{array}{l}\text { WELT 16182; Broken River } \\
\text { Basin, N.Z. }\end{array}$ \\
\hline VUWG & 99 & Compositae & Hieracium praealtum & $\begin{array}{l}\text { WELT 16172; Hakataramea } \\
\text { Valley, N.Z. }\end{array}$ \\
\hline VUWG & 1195 & Compositae & Hieracium umbellatum & WELTU; Holland. \\
\hline VUWG & 704 & Compositae & Hieracium /Pilosella agg. & $\begin{array}{l}\text { Chester 76. Polineri, Grevena Pr., } \\
\text { NW Greece. }\end{array}$ \\
\hline VUWG & 102 & Compositae & Hypochoeris glabra & $\begin{array}{l}\text { WELT 61089a; Hepburn St, } \\
\text { Auckland, N.Z. }\end{array}$ \\
\hline VUWG & 103 & Compositae & Hypochoeris glabra & WELT 70545; Pukerua Bay, N.Z. \\
\hline VUWG & 104 & Compositae & Hypochoeris radicata & $\begin{array}{l}\text { WELT 70684; Charles Bay, } \\
\text { Stephenson Is., N.Z. }\end{array}$ \\
\hline VUWG & 105 & Compositae & Hypochoeris radicata & $\begin{array}{l}\text { WELT 38850; Chatham Islands, } \\
\text { N.Z. }\end{array}$ \\
\hline VUWG & 106 & Compositae & Hypochoeris radicata & $\begin{array}{l}\text { WELT 51429; Red Rocks, } \\
\text { Wellington, N.Z. }\end{array}$ \\
\hline VUWG & 1196 & Compositae & Inula conyza & WELTU; Holland. \\
\hline VUWG & 107 & Compositae & Lactuca virosa & $\begin{array}{l}\text { WELT 63195; Raroa Reserve, } \\
\text { Pukerua Bay, N.Z. }\end{array}$ \\
\hline VUWG & 108 & Compositae & Lactuca virosa & $\begin{array}{l}\text { WELT } 63502 \text {; old Hutt River bed, } \\
\text { Heretaunga, N.Z. }\end{array}$ \\
\hline VUWG & 109 & Compositae & Lactuca virosa & $\begin{array}{l}\text { WELT 63503; old Hutt Rd, Hutt } \\
\text { Valley, N.Z. }\end{array}$ \\
\hline VUWG & 110 & Compositae & Lapsana communis & $\begin{array}{l}\text { WELT 61256; Sth Karori Rd, } \\
\text { Wellington, N.Z. }\end{array}$ \\
\hline VUWG & 111 & Compositae & Lapsana communis & WELT (no \#) \\
\hline VUWG & 112 & Compositae & Lapsana communis & $\begin{array}{l}\text { WELT 61102; Near Wellington, } \\
\text { N.Z. }\end{array}$ \\
\hline VUWG & 1198 & Compositae & Lapsana communis & WELTU; Holland. \\
\hline
\end{tabular}




\begin{tabular}{|c|c|c|c|}
\hline $\begin{array}{l}\text { Pollen/spore } \\
\text { reference } \\
\text { slide \# }\end{array}$ & Family & Genus \& species & $\begin{array}{l}\text { Collector (if known)/herbarium, } \\
\text { herbarium \# (if known), } \\
\text { place of collection }\end{array}$ \\
\hline
\end{tabular}

\begin{tabular}{|c|c|c|c|}
\hline VUWG & 1199 & Compositae & Lapsana communis \\
\hline VUWG & 1200 & Compositae & Lapsana communis \\
\hline VUWG & 1201 & Compositae & Leontodon hispidus danubialis \\
\hline VUWG & 113 & Compositae & Leontodon taraxacoides \\
\hline VUWG & 114 & Compositae & Leontodon taraxacoides \\
\hline VUWG & 115 & Compositae & Leontodon taraxacoides \\
\hline VUWG & 1202 & Compositae & Leontodon taraxacoides \\
\hline VUWG & 1462 & Compositae & Leontodon taraxacoides \\
\hline VUWG & 1192 & Compositae & Logfia arvensis \\
\hline VUWG & 117 & Compositae & Logfia gallica \\
\hline VUWG & 118 & Compositae & Matricaria chamomilla \\
\hline VUWG & 119 & Compositae & Matricaria chamomilla \\
\hline VUWG & 1197 & Compositae & Mycelis muralis \\
\hline VUWG & 120 & Compositae & Mycelis muralis \\
\hline VUWG & 121 & Compositae & Mycelis muralis \\
\hline VUWG & 1464 & Compositae & Mycelis muralis \\
\hline VUWG & 123 & Compositae & Olearia gunniana \\
\hline VUWG & 124 & Compositae & Olearia gunniana \\
\hline VUWG & 125 & Compositae & Olearia gunniana \\
\hline VUWG & 1465 & Compositae & Onopordum acanthium \\
\hline VUWG & 126 & Compositae & Picris echioides \\
\hline VUWG & 127 & Compositae & Picris echioides \\
\hline VUWG & 128 & Compositae & Picris echioides \\
\hline VUWG & 129 & Compositae & Picris hieracioides \\
\hline VUWG & 130 & Compositae & Picris hieracioides \\
\hline VUWG & 1204 & Compositae & Prenanthes purpurea \\
\hline VUWG & 1205 & Compositae & Pulicaria dysenterica \\
\hline VUWG & 659 & Compositae & Pulicaria dysenterica \\
\hline VUWG & 720 & Compositae & Pulicaria dysenterica \\
\hline VUWG & 1206 & Compositae & Senecio aquaticus \\
\hline VUWG & 143 & Compositae & Senecio cineraria \\
\hline VUWG & 144 & Compositae & Senecio jacobaea \\
\hline VUWG & 145 & Compositae & Senecio jacobaea \\
\hline VUWG & 1467 & Compositae & Senecio jacobaea \\
\hline NUNG & 138 & Compositae & Senecio paludosus \\
\hline
\end{tabular}

WELTU.

WELTU.

WELTU; Holland.

WELT 62837; Pukerua Bay, N.Z.

WELT 60449; Island Bay,

Wellington, N.Z.

WELT 61105; Kilburnie,

Wellington, N.Z.

WELTU; Holland.

WELTU; Victoria University,

Wellington, N.Z.

WELTU; Holland.

WELT 70713; Redwood Pass,

Marlborough, N.Z.

WELT 70843; Wakanui,

Ashburton, N.Z.

WELT 70536; Pukerua Bay,

Wellington, N.Z.

WELTU; Holland.

WELT 70171b; Tararua Ra.,

Palmerston Nth, N.Z.

WELT 61123; Ure River Valley,

Marlborough, N.Z.

WELTU 7659; Granity Pass,

Owen Range, N.Z.

WELT 39072; Invercargill, N.Z.

WELT 61125

WELT 61126; Near Fairfield,

Otago, N.Z.

WELTU 11337; Cape Turakirae,

Wellington, N.Z.

WELT 16165; Kapiti Is, N.Z.

WELT 60447; Makara,

Wellington, N.Z.

WELT 60435; Between Otaki \&

Waikanae, N.Z.

WELT 70117; Rakitu (Arid) Is., Nth Is., N.Z.

WELT 63293; Mahenotiti Is., Bay of Is., N.Z.

WELTU; Switzerland.

WELTU; Holland.

Chester 21. Kellia, Grevena Pr.,

NW Greece.

Chester 125. Mirsina, Grevena Pr.,

NW Greece.

WELTU; Holland.

WELT 61025; Westshore, N.Z.

WELT 24172A; Hawkes Bay, N.Z.

WELT 24172B; Hawke Bay, N.Z.

WELTU; Wellington, N.Z.

Stevenson, Newcastle Upon Tyne University, England. 


\begin{tabular}{|c|c|c|c|}
\hline $\begin{array}{l}\text { Pollen/spore } \\
\text { reference } \\
\text { slide \# }\end{array}$ & Family & Genus \& species & $\begin{array}{l}\text { Collector (if known)/herbarium, } \\
\text { herbarium \# (if known), } \\
\text { place of collection }\end{array}$ \\
\hline
\end{tabular}

\begin{tabular}{|c|c|c|c|c|}
\hline NUNG & 39 & Compositae & Senecio squalidos & $\begin{array}{l}\text { Stevenson, Newcastle Upon Tyne } \\
\text { University, England. }\end{array}$ \\
\hline VUWG & 1466 & Compositae & Senecio sylvaticus & $\begin{array}{l}\text { WELTU } 15296 \text {; Victoria } \\
\text { University, Wellington, N.Z. }\end{array}$ \\
\hline VUWG & 1207 & Compositae & Senecio vulgaris & WELTU; Holland. \\
\hline VUWG & 131 & Compositae & Siegesbeckia orientalis & WELT $61142 ;$ Near Fairbum, N.Z. \\
\hline VUWG & 132 & Compositae & Siegesbeckia orientalis & $\begin{array}{l}\text { WELT } 61151 \text {; Coral Bay, Sunday } \\
\text { Is. }\end{array}$ \\
\hline VUWG & 133 & Compositae & Siegesbeckia orientalis & WELT 14376; Shruts Bay. \\
\hline VUWG & 134 & Compositae & Silybum marianum & $\begin{array}{l}\text { WELT } 51428 ; \text { Red Rocks, } \\
\text { Wellington, N.Z. }\end{array}$ \\
\hline VUWG & 135 & Compositae & Silybum marianum & $\begin{array}{l}\text { WELT } 61153 \text {; Near Wellington, } \\
\text { N.Z. }\end{array}$ \\
\hline VUWG & 136 & Compositae & Silybum marianum & $\begin{array}{l}\text { WELT 60908a; Waikanae Rd, } \\
\text { Paraparaumu, N.Z. }\end{array}$ \\
\hline VUWG & 1209 & Compositae & Solidago canadensis & WELTU; Holland. \\
\hline VUWG & 1210 & Compositae & Solidago gigantea & WELTU; Black Forest, Germany. \\
\hline VUWG & 1211 & Compositae & Solidago virgaurea & WELTU; Holland. \\
\hline VUWG & 1212 & Compositae & Solidago virgaurea & WELTU; Holland. \\
\hline VUWG & 1213 & Compositae & Solidago virgaurea & WELTU; Holland. \\
\hline VUWG & 137 & Compositae & Sonchus asper & $\begin{array}{l}\text { WELT } 70675 \text {; Orongorongo } \\
\text { Valley, Wellington, N.Z. }\end{array}$ \\
\hline VUWG & 138 & Compositae & Sonchus asper & $\begin{array}{l}\text { WELT } 61322 \mathrm{a} \text {; Sth Karori Rd, } \\
\text { Wellington, N.Z. }\end{array}$ \\
\hline VUWG & 139 & Compositae & Sonchus asper & WELT 9577; Waikaremoana, N.Z. \\
\hline VUWG & 1468 & Compositae & Sonchus asper & WELTU 15548; Auckland Island. \\
\hline VUWG & 1469 & Compositae & Sonchus asper & $\begin{array}{l}\text { WELTU } 15181 \text {; Waikanae River } \\
\text { estuary, N.Z. }\end{array}$ \\
\hline NUNG & 88 & Compositae & Sonchus asper & $\begin{array}{l}\text { Stevenson, Newcastle Upon Tyne } \\
\text { University, England. }\end{array}$ \\
\hline VUWG & 140 & Compositae & Sonchus oleraceus & $\begin{array}{l}\text { WELT 70840; Kelburn, } \\
\text { Wellington, N.Z. }\end{array}$ \\
\hline VUWG & 141 & Compositae & Sonchus oleraceus & $\begin{array}{l}\text { WELT } 63400 \text {; Little Barrier Is., } \\
\text { N.Z. }\end{array}$ \\
\hline VUWG & 142 & Compositae & Sonchus oleraceus & $\begin{array}{l}\text { WELT } 60430 \text {; Sth Karori, } \\
\text { Wellington, N.Z. }\end{array}$ \\
\hline NUNG & 28 & Compositae & Sonchus oleraceus & $\begin{array}{l}\text { Stevenson, Newcastle Upon Tyne } \\
\text { University, England. }\end{array}$ \\
\hline VUWG & 1214 & Compositae & Tanacetum sp. & WELTU; Holland. \\
\hline VUWG & 1215 & Compositae & Tanacetum sp. & WELTU; Switzerland. \\
\hline VUWG & 1471 & Compositae & Tanacetum vulgare & WELTU 10429; Taieri Plain, N.Z. \\
\hline VUWG & 1208 & Compositae & Taraxacum officinale & WELTU. \\
\hline VUWG & 1470 & Compositae & Taraxacum officinale & $\begin{array}{l}\text { WELTU; Victoria University, } \\
\text { Wellington, N.Z. }\end{array}$ \\
\hline VUWG & 162 & Compositae & Tripleurospermum inodorum & $\begin{array}{l}\text { WELT 70625; Ross Rd, } \\
\text { Canterbury Plains, N.Z. }\end{array}$ \\
\hline VUWG & 163 & Compositae & Tripleurospermum inodorum & $\begin{array}{l}\text { WELT 70842; Wakanui, } \\
\text { Ashburton, N.Z. }\end{array}$ \\
\hline VUWG & 164 & Compositae & Tripleurospermum inodorum & WELT 61116 \\
\hline VUWG & 706 & Compositae & Xanthium spinosum & $\begin{array}{l}\text { Chester } 78 . \text { Polineri, Grevena Pr., } \\
\text { NW Greece. }\end{array}$ \\
\hline VUWG & 1217 & Compositae & Xanthium strumarium & WELTU; India. \\
\hline
\end{tabular}




\begin{tabular}{|c|c|c|c|}
\hline $\begin{array}{l}\text { Pollen/spore } \\
\text { reference } \\
\text { slide \# }\end{array}$ & Family & Genus \& species & $\begin{array}{l}\text { Collector (if known)/herbarium, } \\
\text { herbarium \# (if known), } \\
\text { place of collection }\end{array}$ \\
\hline
\end{tabular}

\begin{tabular}{|c|c|c|c|c|}
\hline VUWG & 694 & Compositae & Xanthium strumarium & $\begin{array}{l}\text { Chester 64. Mavrani, Grevena Pr., } \\
\text { NW Greece. }\end{array}$ \\
\hline NUNG & 44 & Compositae & Xeranthemum annuum & $\begin{array}{l}\text { Stevenson, Newcastle Upon Tyne } \\
\text { University, England. }\end{array}$ \\
\hline VUWG & 240 & Convolvulaceae & Calystegia sepium & $\begin{array}{l}\text { WELT } 60398 \text {; Days Bay, } \\
\text { Wellington, N.Z. }\end{array}$ \\
\hline VUWG & 783 & Convolvulaceae & Calystegia sepium & $\begin{array}{l}\text { WELTU 7328; Lake Wairarapa, } \\
\text { N.Z. }\end{array}$ \\
\hline VUWG & 677 & Convolvulaceae & Calystegia, prob. sepium & $\begin{array}{l}\text { Chester } 39 . \text { Kellia, Grevena Pr., } \\
\text { NW Greece. }\end{array}$ \\
\hline VUWG & 237 & Convolvulaceae & Calystegia silvatica & $\begin{array}{l}\text { WELT 70828; Belmont, Hutt } \\
\text { Valley, N.Z. }\end{array}$ \\
\hline VUWG & 238 & Convulvulaceae & Calystegia silvatica & WELT 4637; Waikanae, N.Z. \\
\hline VUWG & 239 & Convolvulaceae & Calystegia silvatica & WELT 4649; Wellington, N.Z. \\
\hline VUWG & 1400 & Convolvulaceae & Convolvulus arvensis & WELTU 10370. \\
\hline VUWG & 241 & Convolvulaceae & Convolvulus arvensis & $\begin{array}{l}\text { WELT 63505; Eastbourne, } \\
\text { Wellington, N.Z. }\end{array}$ \\
\hline VUWG & 242 & Convolvulaceae & Convolvulus arvensis & WELT 61724; Wellington, N.Z. \\
\hline VUWG & 243 & Convolvulaceae & Convolvulus arvensis & $\begin{array}{l}\text { WELT } 63500 ; \text { Brooklyn, } \\
\text { Wellington, N.Z. }\end{array}$ \\
\hline VUWG & 770 & Convolvulaceae & Convolvulus arvensis & $\begin{array}{l}\text { Chester } 300 \text {. Mirsina, Grevena Pr., } \\
\text { NW Greece. }\end{array}$ \\
\hline VUWG & 943 & Convolvulaceae & Convolvulus arvensis & WELTU box 97; Holland. \\
\hline NUNG & 236 & Convolvulaceae & Convolvulus arvensis & $\begin{array}{l}\text { Stevenson, Newcastle Upon Tyne } \\
\text { University, England. }\end{array}$ \\
\hline VUWG & 944 & Convolvulaceae & Convolvulus pluricaulis & WELTU box 97; India. \\
\hline VUWG & 244 & Convolvulaceae & Cuscuta epithymum & WELT 62914; Picton, N.Z. \\
\hline VUWG & 245 & Convolvulaceae & Cuscuta epithymum & WELT 62923; Picton, N.Z. \\
\hline VUWG & 945 & Convolvulaceae & Cuscuta epithymum & WELTU box 97; Holland. \\
\hline VUWG & 235 & Convolvulaceae & Cuscuta suaveolens & $\begin{array}{l}\text { WELT 61727; Near Christchurch, } \\
\text { N.Z. }\end{array}$ \\
\hline VUWG & 236 & Convolvulaceae & Cuscuta suaveolens & WELT 61725 \\
\hline VUWG & 784 & Convolvulaceae & Cuscuta suaveolens & WELTU 5952; Tereinakau, N.Z. \\
\hline VUWG & 679 & Convolvulaceae & Cuscuta sp. & $\begin{array}{l}\text { Chester 41. Kellia, Grevena Pr., } \\
\text { NW Greece. }\end{array}$ \\
\hline VUWG & 747 & Convolvulaceae & Cuscuta sp. & $\begin{array}{l}\text { Chester 175. Agios Nicholas, } \\
\text { Grevena Pr., NW Greece. }\end{array}$ \\
\hline VUWG & 785 & Convolvulaceae & Dichondra brevifolia & $\begin{array}{l}\text { WELTU 5945; Nydia Bay, } \\
\text { Marlborough Sounds, N.Z. }\end{array}$ \\
\hline VUWG & $247 b$ & Convolvulaceae & Ipomoea congestus & $\begin{array}{l}\text { WELT } 70111 \text {; Waiwera, Rodney } \\
\text { County, N.Z. }\end{array}$ \\
\hline VUWG & 1401 & Convolvulaceae & Ipomoea purpurea & $\begin{array}{l}\text { WELTU 15242; Victoria } \\
\text { University, Wellington, N.Z. }\end{array}$ \\
\hline VUWG & 1382 & Cornaceae & Cornus capitata & WELTU; New Plymouth, N.Z. \\
\hline VUWG & 933 & Cornaceae & Cornus capitata & WELTU 9383. \\
\hline VUWG & 406 & Cornaceae & Cornus sanguinea & WELT no \#; Uppsala, Sweden. \\
\hline NUNG & 22 & Cornaceae & Cornus suecica & $\begin{array}{l}\text { Stevenson, Newcastle Upon Tyne } \\
\text { University, England. }\end{array}$ \\
\hline DUDB & 313 & Corylaceae & Carpinus betulus & $\begin{array}{l}\text { Huntley, Durham University, } \\
\text { England. }\end{array}$ \\
\hline VUWG & 425 & Corylaceae & Corylus avellana & $\begin{array}{l}\text { WELT no \#; ex Otago Museum, } \\
\text { N.Z. }\end{array}$ \\
\hline VUWG & 861 & Corylaceae & Corylus avellana & WELTU box 86; Belmont. \\
\hline
\end{tabular}




\begin{tabular}{|c|c|c|c|c|}
\hline $\begin{array}{l}\text { Pollen/s/ } \\
\text { referenc } \\
\text { slide \# }\end{array}$ & $\begin{array}{l}\text { pore } \\
\text { e }\end{array}$ & Family & Genus \& species & $\begin{array}{l}\text { Collector (if known)/herbarium } \\
\text { herbarium \# (if known), } \\
\text { place of collection }\end{array}$ \\
\hline CRYG & 286 & Corylaceae & Corylus avellana & $\begin{array}{l}\text { Atherden, College of Rippon \& } \\
\text { York, England. }\end{array}$ \\
\hline DUDB & 298 & Corylaceae & Ostrya carpinifolia & $\begin{array}{l}\text { Huntlet, Durham University, } \\
\text { England. }\end{array}$ \\
\hline NUNG & 96 & Crassulaceae & Pistorinia hispania & $\begin{array}{l}\text { Stevenson, Newcastle Upon Tyne } \\
\text { University, England. }\end{array}$ \\
\hline VUWG & 1367 & Crassulaceae & Sedum acre & $\begin{array}{l}\text { WELTU 10339; Cardrona R., } \\
\text { Otago, N.Z. }\end{array}$ \\
\hline VUWG & 962 & Crassulaceae & Sedum acre & WELTU box 97; Holland. \\
\hline VUWG & 963 & Crassulaceae & Sedum ?acre & WELTU box 97; Holland. \\
\hline VUWG & 964 & Crassulaceae & Sedum album & WELTU box 97; Belgium. \\
\hline VUWG & 965 & Crassulaceae & Sedum reflexum & WELTU box 97; Switzerland. \\
\hline VUWG & 960 & Crassulaceae & Sedum sp. & WELTU box $97 ;$ Holland. \\
\hline VUWG & 961 & Crassulaceae & Sedum sp. & WELTU box 97; Holland. \\
\hline VUWG & 1301 & Cruciferae & Alyssum alyssoides & $\begin{array}{l}\text { WELTU 10307; Waiareka Valley, } \\
\text { N.Z. }\end{array}$ \\
\hline VUWG & 997 & Cruciferae & Alyssum montanum & WELTU box 97; Holland. \\
\hline VUWG & 749 & Cruciferae & Alyssum murale & $\begin{array}{l}\text { Chester 178. Agios Nicholas, } \\
\text { Grevena Pr., NW Greece. }\end{array}$ \\
\hline VUWG & 730 & Cruciferae & ?Alyssum murale & $\begin{array}{l}\text { Chester 143. Mirsina, Grevena Pr., } \\
\text { NW Greece. }\end{array}$ \\
\hline VUWG & 690 & Cruciferae & ?Alyssum cf. murale & $\begin{array}{l}\text { Chester 59. Mavrani, Grevena Pr., } \\
\text { NW Greece. }\end{array}$ \\
\hline VUWG & 996 & Cruciferae & Arabis blepharophylla & $\begin{array}{l}\text { WELTU box 97; Sonoma Co., } \\
\text { California, USA. }\end{array}$ \\
\hline VUWG & 1302 & Cruciferae & Barbarea vulgaris & $\begin{array}{l}\text { WELTU 10308; Maori Hill, } \\
\text { Dunedin, N.Z. }\end{array}$ \\
\hline VUWG & 999 & Cruciferae & Brassica napus & WELTU box 97; Holland. \\
\hline VUWG & 998 & Cruciferae & Brassica nigra & WELTU box 97; Holland. \\
\hline VUWG & 1304 & Cruciferae & Brassica oleracea & $\begin{array}{l}\text { WELTU } 11018 \text {; North Harbour, } \\
\text { Auckland, N.Z. }\end{array}$ \\
\hline VUWG & 1000 & Cruciferae & Brassica rapa & WELTU box 97; Holland. \\
\hline VUWG & 1303 & Cruciferae & Brassica rapa & $\begin{array}{l}\text { WELTU 10309; Maori Hill, } \\
\text { Dunedin, N.Z. }\end{array}$ \\
\hline VUWG & 1305 & Cruciferae & Brassica rapa & $\begin{array}{l}\text { WELTU } 15209 \text {; Victoria } \\
\text { University, Wellington, N.Z. }\end{array}$ \\
\hline VUWG & 1001 & Cruciferae & Cardamine hirsuta & WELTU box 97; Holland. \\
\hline VUWG & 1306 & Cruciferae & Cardamine hirsuta & $\begin{array}{l}\text { WELTU 10315; Khandallah, } \\
\text { Wellington, N.Z. }\end{array}$ \\
\hline VUWG & 1307 & Cruciferae & Cardamine hirsuta & $\begin{array}{l}\text { WELTU 10312; Wellington } \\
\text { Botanic Gardens, N.Z. }\end{array}$ \\
\hline VUWG & 1308 & Cruciferae & Cardamine hirsuta & $\begin{array}{l}\text { WELTU; Victoria University, } \\
\text { Wellington, N.Z. }\end{array}$ \\
\hline VUWG & 1002 & Cruciferae & Cardamine pratensis & WELTU box 97; Holland. \\
\hline VUWG & 806 & Cruciferae & Erysimum sp. & $\begin{array}{l}\text { Chester } 327 . \text { Wellington Botanic } \\
\text { Gardens, N.Z. }\end{array}$ \\
\hline VUWG & 1004 & Cruciferae & Lunaria annua & WELTU box 97; Holland. \\
\hline VUWG & 1005 & Cruciferae & Lunaria annua & WELTU box 97; Germany. \\
\hline VUWG & 1309 & Cruciferae & Lunaria annua & $\begin{array}{l}\text { WELTU 15203; Karori, } \\
\text { Wellington, N.Z. }\end{array}$ \\
\hline NUNG & 14 & Cruciferae & Lunaria sp. & $\begin{array}{l}\text { Stevenson, Newcastle Upon Tyne } \\
\text { University, England. }\end{array}$ \\
\hline
\end{tabular}




\begin{tabular}{|c|c|c|c|}
\hline $\begin{array}{l}\text { Pollen/spore } \\
\text { reference } \\
\text { slide \# }\end{array}$ & Family & Genus \& species & $\begin{array}{l}\text { Collector (if known)/herbarium, } \\
\text { herbarium \# (if known), } \\
\text { place of collection }\end{array}$ \\
\hline
\end{tabular}

\begin{tabular}{|c|c|c|c|c|}
\hline VUWG & 1310 & Cruciferae & Nasturtium officinale & $\begin{array}{l}\text { WELTU 11355; Cape Turakirae, } \\
\text { Wellington, N.Z. }\end{array}$ \\
\hline VUWG & 1311 & Cruciferae & Nasturtium officinale & WELTU 15202. \\
\hline VUWG & 765 & Cruciferae & ?Sinapis alba & $\begin{array}{l}\text { Chester 253. Asprokampos, } \\
\text { Grevena Pr., NW Greece. }\end{array}$ \\
\hline VUWG & 1006 & Cruciferae & Thlaspi arvense & WELTU box 97; Germany. \\
\hline VUWG & 1007 & Cruciferae & Thlaspi arvense & WELTU box 97; Holland. \\
\hline NUNG & 119 & Dioscoreaceae & Borderea pyrenaica & $\begin{array}{l}\text { Stevenson, Newcastle Upon Tyne } \\
\text { University, England. }\end{array}$ \\
\hline VUWG & 761 & Dipsacaceae & & $\begin{array}{l}\text { Chester 248. Asprokampos, } \\
\text { Grevena Pr., NW Greece. }\end{array}$ \\
\hline VUWG & 52 & Dipsacaceae & Dipsacus fullonum & $\begin{array}{l}\text { WELT 59833; Albany, Auckland, } \\
\text { N.Z. }\end{array}$ \\
\hline VUWG & 1163 & Dipsacaceae & Dipsacus pilosus & WELTU; Holland. \\
\hline VUWG & 53 & Dipsacaceae & Dipsacus sylvestris & $\begin{array}{l}\text { WELT 62809; Grays Hill, } \\
\text { Gisborne, N.Z. }\end{array}$ \\
\hline VUWG & 1164 & Dipsacaceae & Knautia arvensis & WELTU; Switzerland. \\
\hline VUWG & 55 & Dipsacaceae & Scabiosa atropurpurea & $\begin{array}{l}\text { WELT } 63169 \text {; Near Wellington, } \\
\text { N.Z. }\end{array}$ \\
\hline VUWG & 56 & Dipsacaceae & Scabiosa atropurpurea & $\begin{array}{l}\text { WELT 84425; Near Port Ahuriri, } \\
\text { N.Z. }\end{array}$ \\
\hline VUWG & 688 & Dipsacaceae & Scabiosa ?atropurpurea & $\begin{array}{l}\text { Chester 56. Mavrani, Grevena Pr., } \\
\text { NW Greece. }\end{array}$ \\
\hline VUWG & 1441 & Dipsacaceae & Scabiosa arvensis & $\begin{array}{l}\text { WELTU 10398; Roslyn, Dunedin, } \\
\text { N.Z. }\end{array}$ \\
\hline VUWG & 1165 & Dipsacaceae & Scabiosa succisa & WELTU; Holland. \\
\hline VUWG & 731 & Dipsacaceae & Scabiosa sp. & $\begin{array}{l}\text { Chester 148. Mirsina, Grevena Pr., } \\
\text { NW Greece. }\end{array}$ \\
\hline VUWG & 621 & Droseraceae & Drosera anglica & $\begin{array}{l}\text { WELT no \#; Tollstad, } \\
\text { Ostergotland, Sweden. }\end{array}$ \\
\hline NUNG & 45 & Ericaceae & Erica arborea & $\begin{array}{l}\text { Stevenson, Newcastle Upon Tyne } \\
\text { University, England. }\end{array}$ \\
\hline NUNG & 105 & Ericaceae & Erica australis & $\begin{array}{l}\text { Stevenson, Newcastle Upon Tyne } \\
\text { University, England. }\end{array}$ \\
\hline NUNG & 163 & Ericaceae & Erica ciliaris & $\begin{array}{l}\text { Stevenson, Newcastle Upon Tyne } \\
\text { University, England. }\end{array}$ \\
\hline VUWG & 1071 & Ericaceae & Erica herbacea & WELTU box 91; Switzerland. \\
\hline VUWG & 1394 & Ericaceae & Erica lusitanica & WELTU; Wellington, N.Z. \\
\hline VUWG & 1395 & Ericaceae & Erica lusitanica & $\begin{array}{l}\text { WELTU 11299; Cape Turakirae, } \\
\text { Wellington, N.Z. }\end{array}$ \\
\hline NUNG & 73 & Ericaceae & Erica lusitanica & $\begin{array}{l}\text { Stevenson, Newcastle Upon Tyne } \\
\text { University, England. }\end{array}$ \\
\hline NUNG & 178 & Ericaceae & Erica lusitanica & $\begin{array}{l}\text { Stevenson, Newcastle Upon Tyne } \\
\text { University, England. }\end{array}$ \\
\hline NUNG & 75 & Ericaceae & Erica multiflora & $\begin{array}{l}\text { Stevenson, Newcastle Upon Tyne } \\
\text { University, England. }\end{array}$ \\
\hline NUNG & 256 & Ericaceae & Erica scoparia & $\begin{array}{l}\text { Stevenson, Newcastle Upon Tyne } \\
\text { University, England. }\end{array}$ \\
\hline VUWG & 1073 & Ericaceae & Erica tetralix & WELTU box 91; Holland. \\
\hline NUNG & 205 & Ericaceae & Erica tetralix & $\begin{array}{l}\text { Stevenson, Newcastle Upon Tyne } \\
\text { University, England. }\end{array}$ \\
\hline NUNG & 191 & Ericaceae & Erica umbellata & $\begin{array}{l}\text { Stevenson, Newcastle Upon Tyne } \\
\text { University, England. }\end{array}$ \\
\hline
\end{tabular}




\begin{tabular}{|c|c|c|c|c|}
\hline $\begin{array}{l}\text { Pollen/s! } \\
\text { referenc } \\
\text { slide \# }\end{array}$ & $\begin{array}{l}\text { pore } \\
\text { ce }\end{array}$ & Family & Genus \& species & $\begin{array}{l}\text { Collector (if known)/herbarium } \\
\text { herbarium \# (if known), } \\
\text { place of collection }\end{array}$ \\
\hline NUNG & 63 & Ericaceae & Erica sp. & $\begin{array}{l}\text { Stevenson, Newcastle Upon Tyne } \\
\text { University, England. }\end{array}$ \\
\hline VUWG & 1066 & Euphorbiaceae & Euphorbia amygdaloides & $\begin{array}{l}\text { WELTU box } 91 ; \text { Lac de la } \\
\text { Gilleppe, Belgium. }\end{array}$ \\
\hline VUWG & 511 & Euphorbiaceae & Euphorbia helioscopia & $\begin{array}{l}\text { WELT 60898; Karori, Wellington, } \\
\text { N.Z. }\end{array}$ \\
\hline NUNG & 247 & Euphorbiaceae & Euphorbia helioscopia & $\begin{array}{l}\text { Stevenson, Newcastle Upon Tyne } \\
\text { University, England. }\end{array}$ \\
\hline VUWG & 1069 & Euphorbiaceae & Euphorbia hirta & WELTU box 91; India. \\
\hline VUWG & 512 & Euphorbiaceae & Euphorbia lathyris & $\begin{array}{l}\text { WELT 70764; Ohariu Valley, } \\
\text { Wellington, N.Z. }\end{array}$ \\
\hline VUWG & 1070 & Euphorbiaceae & Euphorbia palustris & WELTU box 91; Holland. \\
\hline NUNG & 121 & Euphorbiaceae & Euphorbia paralias & $\begin{array}{l}\text { Stevenson, Newcastle Upon Tyne } \\
\text { University, England. }\end{array}$ \\
\hline VUWG & 1383 & Euphorbiaceae & Euphorbia peplus & $\begin{array}{l}\text { WELTU; Stokes Valley, Lower } \\
\text { Hutt, N.Z. }\end{array}$ \\
\hline VUWG & 1384 & Euphorbiaceae & Euphorbia peplus & $\begin{array}{l}\text { WELTU 11302; Cape Turakirae, } \\
\text { Wellington, N.Z. }\end{array}$ \\
\hline VUWG & 513 & Euphorbiaceae & Euphorbia peplus & $\begin{array}{l}\text { WELT 63321; Wairau Ck, } \\
\text { Milford, N.Z. }\end{array}$ \\
\hline VUWG & 514 & Euphorbiaceae & Euphorbia peplus & $\begin{array}{l}\text { WELT 60731; Webb St, } \\
\text { Wellington, N.Z. }\end{array}$ \\
\hline VUWG & 515 & Euphorbiaceae & Euphorbia peplus & $\begin{array}{l}\text { WELT 63357; Pukerua Bay, } \\
\text { Wellington, N.Z. }\end{array}$ \\
\hline VUWG & 516 & Euphorbiaceae & Euphorbia platyphyllos & WELT 70695; Auckland City, N.Z. \\
\hline VUWG & 517 & Euphorbiaceae & Euphorbia sp. & $\begin{array}{l}\text { WELT 70119; Oakura Bay, } \\
\text { Whangarei, N.Z. }\end{array}$ \\
\hline NUNG & 2 & Fagaceae & Castanea sativa & $\begin{array}{l}\text { Stevenson, Newcastle Upon Tyne } \\
\text { University, England. }\end{array}$ \\
\hline NUNG & 21 & Fagaceae & Castanea sativa & $\begin{array}{l}\text { Stevenson, Newcastle Upon Tyne } \\
\text { University, England. }\end{array}$ \\
\hline NUNG & 55 & Fagaceae & Quercus cerris & $\begin{array}{l}\text { Stevenson, Newcastle Upon Tyne } \\
\text { University, England. }\end{array}$ \\
\hline NUNG & 110 & Fagaceae & Quercus cerris & $\begin{array}{l}\text { Stevenson, Newcastle Upon Tyne } \\
\text { University, England. }\end{array}$ \\
\hline NUNG & 54 & Fagaceae & Quercus coccifera & $\begin{array}{l}\text { Stevenson, Newcastle Upon Tyne } \\
\text { University, England. }\end{array}$ \\
\hline NUNG & 177 & Fagaceae & Quercus coccifera & $\begin{array}{l}\text { Stevenson, Newcastle Upon Tyne } \\
\text { University, England. }\end{array}$ \\
\hline CRYG & 287 & Fagaceae & Quercus coccifera & $\begin{array}{l}\text { Atherden, College of Rippon \& } \\
\text { York, England. }\end{array}$ \\
\hline NUNG & 64 & Fagaceae & Quercus faginea & $\begin{array}{l}\text { Stevenson, Newcastle Upon Tyne } \\
\text { University, England. }\end{array}$ \\
\hline NUNG & 68 & Fagaceae & Quercus faginea & $\begin{array}{l}\text { Stevenson, Newcastle Upon Tyne } \\
\text { University, England. }\end{array}$ \\
\hline NUNG & 135 & Fagaceae & Quercus fruticosa & $\begin{array}{l}\text { Stevenson, Newcastle Upon Tyne } \\
\text { University, England. }\end{array}$ \\
\hline CRYG & 288 & Fagaceae & Quercus macrolepis & $\begin{array}{l}\text { Atherden, College of Rippon \& } \\
\text { York, England. }\end{array}$ \\
\hline DUDB & 297 & Fagaceae & Quercus petraea & $\begin{array}{l}\text { Huntley, Durham University, } \\
\text { England. }\end{array}$ \\
\hline NUNG & 224 & Fagaceae & Quercus pyrenaica & $\begin{array}{l}\text { Stevenson, Newcastle Upon Tyne } \\
\text { University, England. }\end{array}$ \\
\hline
\end{tabular}




\begin{tabular}{|c|c|c|c|c|}
\hline $\begin{array}{l}\text { Pollen/s } \\
\text { referenc } \\
\text { slide \# }\end{array}$ & & Family & Genus \& species & $\begin{array}{l}\text { Collector (if known)/herbarium, } \\
\text { herbarium \# (if known), } \\
\text { place of collection }\end{array}$ \\
\hline VUWG & 811 & Fagaceae & Quercus robur & $\begin{array}{l}\text { Chester 332. Wellington Botanic } \\
\text { Gardens, N.Z. }\end{array}$ \\
\hline NUNG & 175 & Fagaceae & Quercus suber & $\begin{array}{l}\text { Stevenson, Newcastle Upon Tyne } \\
\text { University, England. }\end{array}$ \\
\hline NUNG & 158 & Fagaceae & Quercus suber & $\begin{array}{l}\text { Stevenson, Newcastle Upon Tyne } \\
\text { University, England. }\end{array}$ \\
\hline NUNG & 79 & Fagaceae & Quercus suber & $\begin{array}{l}\text { Stevenson, Newcastle Upon Tyne } \\
\text { University, England. }\end{array}$ \\
\hline VUWG & 165 & Gentianaceae & Blackstonia perfoliata & $\begin{array}{l}\text { WELT 70741; Paihia, Bay of Is., } \\
\text { N.Z. }\end{array}$ \\
\hline VUWG & 166 & Gentianaceae & Centaurium erythraea & $\begin{array}{l}\text { WELT 70335; Hen \& Chickens Is., } \\
\text { N.Z. }\end{array}$ \\
\hline VUWG & 167 & Gentianaceae & Centaurium erythraea & $\begin{array}{l}\text { WELT 70468; Poor Knight Is., } \\
\text { N.Z. }\end{array}$ \\
\hline VUWG & 170 & Gentianaceae & Centaurium erythraea & $\begin{array}{l}\text { WELT } 63290 \text {; Forest Hill, } \\
\text { Waitemata Co., N.Z. }\end{array}$ \\
\hline VUWG & 171 & Gentianaceae & Centaurium erythraea & $\begin{array}{l}\text { WELT } 60378 \text {; Karori, Wellington, } \\
\text { N.Z. }\end{array}$ \\
\hline NUNG & 234 & Gentianaceae & Centaurium erythraea & $\begin{array}{l}\text { Stevenson, Newcastle Upon Tyne } \\
\text { University, England. }\end{array}$ \\
\hline VUWG & 169 & Gentianaceae & Centaurium minus & $\begin{array}{l}\text { WELT } 6234 \text {; Seatoun, Wellington, } \\
\text { N.Z. }\end{array}$ \\
\hline VUWG & 168 & Gentianaceae & Centaurium tenuiflorum & $\begin{array}{l}\text { WELT 63493; Lake Ellesmere } \\
\text { Flat, N.Z. }\end{array}$ \\
\hline VUWG & 934 & Gentianaceae & Centaurium venustum & $\begin{array}{l}\text { WELTU 9378; Amador Co., } \\
\text { California, USA. }\end{array}$ \\
\hline VUWG & 935 & Gentianaceae & Gentiana andrewsii & $\begin{array}{l}\text { WELTU 9387; Shelburne Falls, } \\
\text { Mass., USA. }\end{array}$ \\
\hline VUWG & 936 & Gentianaceae & Gentiana asclepiadea & WELTU box 97; England. \\
\hline VUWG & 938 & Gentianaceae & Gentiana lutea & WELTU box 97. \\
\hline VUWG & 941 & Gentianaceae & Gentiana pneumonanthe & WELTU box 97; Switzerland. \\
\hline VUWG & 940 & Gentianaceae & Gentiana utriculosa & WELTU box 97; Switzerland. \\
\hline VUWG & 939 & Gentianaceae & Gentiana verna gr. & WELTU box 97; Switzerland. \\
\hline VUWG & 937 & Gentianaceae & Gentianella campestris & WELTU box 97; Switzerland. \\
\hline VUWG & 1048 & Geraniaceae & Erodium cicutarium & $\begin{array}{l}\text { WELTU box 91; Amsterdam, } \\
\text { Holland. }\end{array}$ \\
\hline VUWG & 1329 & Geraniaceae & Erodium cicutarium & $\begin{array}{l}\text { WELTU 11300; Cape Turakirae, } \\
\text { Wellington, N.Z. }\end{array}$ \\
\hline VUWG & 1330 & Geraniaceae & Erodium cicutarium & WELTU 10341; Moeraki, N.Z. \\
\hline VUWG & 567 & Geraniaceae & Erodium cicutarium & $\begin{array}{l}\text { WELT 63481; Riverside Rd, } \\
\text { Havelock North, N.Z. }\end{array}$ \\
\hline VUWG & 568 & Geraniaceae & Erodium cicutarium & $\begin{array}{l}\text { WELT 62598; Keith George Park, } \\
\text { Hutt Valley, N.Z. }\end{array}$ \\
\hline VUWG & 569 & Geraniaceae & Erodium cicutarium & $\begin{array}{l}\text { WELT } 60380 ; \text { S Karori Rd, } \\
\text { Wellington, N.Z. }\end{array}$ \\
\hline NUNG & 228 & Geraniaceae & Erodium cicutarium & $\begin{array}{l}\text { Stevenson, Newcastle Upon Tyne } \\
\text { University, England. }\end{array}$ \\
\hline VUWG & 702 & Geraniaceae & Erodium cf. cicutarium & $\begin{array}{l}\text { Chester 74. Polineri, Grevena Pr., } \\
\text { NW Greece. }\end{array}$ \\
\hline VUWG & 570 & Geraniaceae & Erodium moschatum & $\begin{array}{l}\text { WELT } 60895 \text {; S Karori Rd, } \\
\text { Wellington, N.Z. }\end{array}$ \\
\hline VUWG & 559 & Geraniaceae & Geranium dissectum & WELT 62851; Auckland City, N.Z. \\
\hline
\end{tabular}




Pollen/spore Family $\quad$ Genus \& species
reference
slide \#

\begin{tabular}{|c|c|c|c|c|}
\hline VUWG & 560 & Geraniaceae & Geranium dissectum & $\begin{array}{l}\text { WELT } 61318 \text {; S Karori Rd, } \\
\text { Wellington, N.Z. }\end{array}$ \\
\hline NUNG & 19 & Geraniaceae & Geranium dissectum & $\begin{array}{l}\text { Stevenson, Newcastle Upon Tyne } \\
\text { University, England. }\end{array}$ \\
\hline VUWG & 1331 & Geraniaceae & Geranium molle & WELTU. \\
\hline VUWG & 1332 & Geraniaceae & Geranium molle & WELTU 11275. \\
\hline VUWG & 561 & Geraniaceae & Geranium molle & WELT 62886; Auckland City, N.Z. \\
\hline VUWG & 562 & Geraniaceae & Geranium molle & $\begin{array}{l}\text { WELT 6059; Karaka Bay, } \\
\text { Wellington, N.Z. }\end{array}$ \\
\hline VUWG & 563 & Geraniaceae & Geranium molle & $\begin{array}{l}\text { WELT } 60379 ; \text { S Karori, } \\
\text { Wellington, N.Z. }\end{array}$ \\
\hline VUWG & 826 & Geraniaceae & Geranium molle & $\begin{array}{l}\text { Chester } 347 . \text { Garden, Johnsonville, } \\
\text { Wellington, N.Z. }\end{array}$ \\
\hline VUWG & 1054 & Geraniaceae & Geranium ?molle & WELTU box 91 ; Holland. \\
\hline VUWG & 1049 & Geraniaceae & Geranium pusillum & WELTU box 91 ; Holland. \\
\hline VUWG & 1051 & Geraniaceae & Geranium pyrenaicum & $\begin{array}{l}\text { WELTU box } 91 \text {; Kaiserstuhl nr. } \\
\text { Freiburg, Germany. }\end{array}$ \\
\hline VUWG & 564 & Geraniaceae & Geranium robertianum & $\begin{array}{l}\text { WELT 63389; Willis St, } \\
\text { Wellington, N.Z. }\end{array}$ \\
\hline VUWG & 565 & Geraniaceae & Geranium robertianum & $\begin{array}{l}\text { WELT no \#; Karori, Wellington, } \\
\text { N.Z. }\end{array}$ \\
\hline VUWG & 566 & Geraniaceae & Geranium robertianum & $\begin{array}{l}\text { WELT 60376; Karori, Wellington, } \\
\text { N.Z. }\end{array}$ \\
\hline VUWG & 828 & Geraniaceae & Geranium robertianum & $\begin{array}{l}\text { Chester } 349 . \text { Garden, Johnsonville, } \\
\text { Wellington, N.Z. }\end{array}$ \\
\hline NUNG & 222 & Geraniaceae & Geranium robertianum & $\begin{array}{l}\text { Stevenson, Newcastle Upon Tyne } \\
\text { University, England. }\end{array}$ \\
\hline NUNG & 220 & Geraniaceae & Geranium robertianum & $\begin{array}{l}\text { Stevenson, Newcastle Upon Tyne } \\
\text { University, England. }\end{array}$ \\
\hline VUWG & 1053 & Geraniaceae & Geranium ?sylvaticum & WELTU box 91; Holland. \\
\hline VUWG & 739 & Geraniaceae & Geranium sp. & $\begin{array}{l}\text { Chester 162. Anelia, Grevena Pr., } \\
\text { NW Greece. }\end{array}$ \\
\hline VUWG & 321 & Globulariaceae & Globularia alypum & WELT no \#. \\
\hline VUWG & 548 & Guttiferae & Hypericum androsaemum & WELT no \#, Upper Hutt, N.Z. \\
\hline VUWG & 843 & Guttiferae & Hypericum androsaemum & WELTU box 93; Holland. \\
\hline NUNG & 208 & Guttiferae & Hypericum calycinum & $\begin{array}{l}\text { Stevenson, Newcastle Upon Tyne } \\
\text { University, England. }\end{array}$ \\
\hline VUWG & 844 & Guttiferae & Hypericum elodes & WELTU box 93; Holland. \\
\hline NUNG & 76 & Guttiferae & Hypericum elodes & $\begin{array}{l}\text { Stevenson, Newcastle Upon Tyne } \\
\text { University, England. }\end{array}$ \\
\hline VUWG & 845 & Guttiferae & Hypericum hirsutum & WELTU box 93 ; Holland. \\
\hline NUNG & 132 & Guttiferae & Hypericum hirsutum & $\begin{array}{l}\text { Stevenson, Newcastle Upon Tyne } \\
\text { University, England. }\end{array}$ \\
\hline VUWG & 846 & Guttiferae & Hypericum montanum & WELTU box 93; Holland. \\
\hline VUWG & 847 & Guttiferae & Hypericum nummularium & WELTU box 93; Holland. \\
\hline VUWG & 550 & Guttiferae & Hypericum perforatum & WELT 69885; Wellington, N.Z. \\
\hline VUWG & 551 & Guttiferae & Hypericum perforatum & $\begin{array}{l}\text { WELT } 60627 \text {; nr Atimuri, Taupo, } \\
\text { N.Z. }\end{array}$ \\
\hline VUWG & 848 & Guttiferae & Hypericum perforatum & WELTU box 93; Holland. \\
\hline NUNG & 24 & Guttiferae & Hypericum sp. & $\begin{array}{l}\text { Stevenson, Newcastle Upon Tyne } \\
\text { University, England. }\end{array}$ \\
\hline VUWG & 1369 & Hydrangeaceae & Deutzia scabra & WELTU; Wellington, N.Z. \\
\hline
\end{tabular}

\author{
Collector (if known)/herbarium, \\ herbarium \# (if known), \\ place of collection
}




\begin{tabular}{|c|c|c|c|c|}
\hline $\begin{array}{l}\text { Pollen/s } \\
\text { referenc } \\
\text { slide \# }\end{array}$ & & Family & Genus \& species & $\begin{array}{l}\text { Collector (if known)/herbarium, } \\
\text { herbarium \# (if known), } \\
\text { place of collection }\end{array}$ \\
\hline VUWG & 1368 & Hydrangeaceae & Philadelphus mexicanus & $\begin{array}{l}\text { WELTU; Stokes Valley, Lower } \\
\text { Hutt, N.Z. }\end{array}$ \\
\hline VUWG & 1295 & Juglandaceae & Juglans regia & $\begin{array}{l}\text { WELTU 10303; Wellington } \\
\text { Botanic Gardens, N.Z. }\end{array}$ \\
\hline VUWG & 325 & Labiatae & Acinos arvensis & $\begin{array}{l}\text { WELT } 61687 \text {; Porter Rd, Castle } \\
\text { Hill, N.Z. }\end{array}$ \\
\hline VUWG & 326 & Labiatae & Acinos arvensis & $\begin{array}{l}\text { WELT 14205; Broken River } \\
\text { Basin, Canterbury, N.Z. }\end{array}$ \\
\hline VUWG & 701 & Labiatae & Acinos suaveolens & $\begin{array}{l}\text { Chester 73. Polineri, Grevena Pr., } \\
\text { NW Greece. }\end{array}$ \\
\hline VUWG & 375 & Labiatae & Ajuga australis & $\begin{array}{l}\text { WELT no \#; King River, Victoria, } \\
\text { Australia. }\end{array}$ \\
\hline VUWG & 1080 & Labiatae & Ajuga genevensis & $\begin{array}{l}\text { WELTU box } 91 \text {; Black Forest, } \\
\text { Germany. }\end{array}$ \\
\hline VUWG & 376 & Labiatae & Ajuga genevensis & WELT no \#. \\
\hline VUWG & 1081 & Labiatae & Ajuga reptans & WELTU box 91 ; Holland. \\
\hline VUWG & 1419 & Labiatae & Ajuga reptans & WELTU; Karori, Wellington, N.Z. \\
\hline VUWG & 327 & Labiatae & Ajuga reptans & $\begin{array}{l}\text { WELT 70349; Waikanae Rd, } \\
\text { Wellington, N.Z. }\end{array}$ \\
\hline VUWG & 822 & Labiatae & Ajuga reptans & $\begin{array}{l}\text { Chester } 343 \text {. Garden, Johnsonville, } \\
\text { Wellington, N.Z. }\end{array}$ \\
\hline NUNG & 99 & Labiatae & Ajuga reptans & $\begin{array}{l}\text { Stevenson, Newcastle Upon Tyne } \\
\text { University, England. }\end{array}$ \\
\hline VUWG & 790 & Labiatae & Ajuga sp. & $\begin{array}{l}\text { Chester } 311 \text {. Wellington Botanic } \\
\text { Gardens, N.Z. }\end{array}$ \\
\hline VUWG & 377 & Labiatae & Calamintha grandiflora & WELT no \#; Transylvania. \\
\hline VUWG & 1082 & Labiatae & Clinopodium vulgare & WELTU box 91; Switzerland. \\
\hline VUWG & 328 & Labiatae & Clinopodium vulgare & $\begin{array}{l}\text { WELT 63178; Banks Peninsula, } \\
\text { Canterbury, N.Z. }\end{array}$ \\
\hline VUWG & 692 & Labiatae & Clinopodium vulgare & $\begin{array}{l}\text { Chester 61. Mavrani, Grevena Pr., } \\
\text { NW Greece. }\end{array}$ \\
\hline VUWG & 378 & Labiatae & Galeopsis tetrahit & WELT no \#; Transylvania. \\
\hline VUWG & 379 & Labiatae & Galeopsis versicolor & $\begin{array}{l}\text { WELT no \#; Bothwell Spott, } \\
\text { Haddingtonshire, UK. }\end{array}$ \\
\hline VUWG & 1083 & Labiatae & Lamium album & WELTU box 91; Holland. \\
\hline VUWG & 1084 & Labiatae & Lamium album & WELTU box 91; Holland. \\
\hline VUWG & 1085 & Labiatae & Lamium maculatum & WELTU box 91 ; Holland. \\
\hline VUWG & 330 & Labiatae & Lamium purpureum & $\begin{array}{l}\text { WELT 70399; Mt Roskill, } \\
\text { Auckland, N.Z. }\end{array}$ \\
\hline VUWG & 331 & Labiatae & Lamium purpureum & $\begin{array}{l}\text { WELT 69977; Pukerua Bay, } \\
\text { Wellington, N.Z. }\end{array}$ \\
\hline VUWG & 791 & Labiatae & ?Lamium sp. & $\begin{array}{l}\text { Chester } 312 \text {. Wellington Botanic } \\
\text { Gardens, N.Z. }\end{array}$ \\
\hline VUWG & 833 & Labiatae & Lavandula angustifolia & $\begin{array}{l}\text { Chester } 354 \text {. Victoria University, } \\
\text { Wellington, N.Z. }\end{array}$ \\
\hline VUWG & 821 & Labiatae & Lavandula sp. & $\begin{array}{l}\text { Chester } 342 \text {. Garden, Johnsonville, } \\
\text { Wellington, N.Z. }\end{array}$ \\
\hline VUWG & 1086 & Labiatae & Lycopus europaeus & WELTU box 91 ; Holland. \\
\hline VUWG & 675 & Labiatae & Lycopus europaeus & $\begin{array}{l}\text { Chester 37. Kellia, Grevena Pr., } \\
\text { NW Greece. }\end{array}$ \\
\hline VUWG & 333 & Labiatae & Marrubium vulgare & $\begin{array}{l}\text { WELT } 61675 \text {; Onehunga, } \\
\text { Auckland, N.Z. }\end{array}$ \\
\hline VUWG & 334 & Labiatae & Marrubium vulgare & WELT 61678. \\
\hline
\end{tabular}




\begin{tabular}{|c|c|c|c|c|}
\hline \multicolumn{2}{|c|}{$\begin{array}{l}\text { Pollen/spore } \\
\text { reference } \\
\text { slide \# }\end{array}$} & Family & Genus \& species & $\begin{array}{l}\text { Collector (if known)/herbarium } \\
\text { herbarium \# (if known), } \\
\text { place of cullection }\end{array}$ \\
\hline VUWG & 335 & Labiatae & Marrubium vulgare & $\begin{array}{l}\text { WELT 61680; Pukerua Bay, } \\
\text { Wellington, N.Z. }\end{array}$ \\
\hline VUWG & 336 & Labiatae & Marrubium vulgare & $\begin{array}{l}\text { WELT 62666; Devonport, } \\
\text { Auckland, N.Z. }\end{array}$ \\
\hline VUWG & 1087 & Labiatae & Melissa officinalis & WELTU box 91; Mediterranean. \\
\hline VUWG & 337 & Labiatae & Melissa officinalis & $\begin{array}{l}\text { WELT } 69884 ; \text { W Hutt Rd, } \\
\text { Wellington, N.Z. }\end{array}$ \\
\hline VUWG & 1088 & Labiatae & Melittis melissophyllum & $\begin{array}{l}\text { WELTU box } 91 \text {; Black Forest, } \\
\text { Germany. }\end{array}$ \\
\hline VUWG & 1089 & Labiatae & Mentha aquatica & WELTU box 91; Holland. \\
\hline VUWG & 1090 & Labiatae & Mentha aquatica & WELTU box 91 \\
\hline VUWG & 1422 & Labiatae & Mentha aquatica & WELTU; Wellington, N.Z. \\
\hline VUWG & 662 & Labiatae & Mentha aquatica & $\begin{array}{l}\text { Chester 24. Kellia, Grevena Pr., } \\
\text { NW Greece. }\end{array}$ \\
\hline VUWG & 718 & Labiatae & Mentha aquatica & $\begin{array}{l}\text { Chester 123. Mirsina, Grevena Pr., } \\
\text { NW Greece. }\end{array}$ \\
\hline NUNG & 189 & Labiatae & Mentha longifolia & $\begin{array}{l}\text { Stevenson, Newcastle Upon Tyne } \\
\text { University, England. }\end{array}$ \\
\hline VUWG & 363 & Labiatae & Mentha x piperita & $\begin{array}{l}\text { WELT 70227a; Birchfield, Buller, } \\
\text { N.Z. }\end{array}$ \\
\hline VUWG & 364 & Labiatae & Mentha x piperita & $\begin{array}{l}\text { WELT 70227b; Birchfield, Buller, } \\
\text { N.Z. }\end{array}$ \\
\hline VUWG & 365 & Labiatae & Mentha x piperita & $\begin{array}{l}\text { WELT 69932a; Inverness Rd, } \\
\text { Waitemata, N.Z. }\end{array}$ \\
\hline VUWG & 1092 & Labiatae & Mentha pulegium & WELTU box 91; Holland. \\
\hline VUWG & 1424 & Labiatae & Mentha pulegium & $\begin{array}{l}\text { WELTU 11369; Cape Turakirae, } \\
\text { Wellington, N.Z. }\end{array}$ \\
\hline VUWG & 367 & Labiatae & Mentha pulegium & $\begin{array}{l}\text { WELT 59823; Piha, Auckland, } \\
\text { N.Z. }\end{array}$ \\
\hline VUWG & 368 & Labiatae & Mentha pulegium & $\begin{array}{l}\text { WELT 59831; Karekare, } \\
\text { Auckland, N.Z. }\end{array}$ \\
\hline VUWG & 369 & Labiatae & Mentha pulegium & $\begin{array}{l}\text { WELT 61781; Castlepoint, } \\
\text { Wairarapa, N.Z. }\end{array}$ \\
\hline VUWG & 1093 & Labiatae & Mentha rotundifolia & WELTU box 91 ; Holland. \\
\hline VUWG & 370 & Labiatae & Mentha spicata & $\begin{array}{l}\text { WELT 70135; Kaiwhata Rd, Te } \\
\text { Wharua, N.Z. }\end{array}$ \\
\hline VUWG & 371 & Labiatae & Mentha spicata & $\begin{array}{l}\text { WELT 69908a; East Coast Rd, } \\
\text { Milford, N.Z. }\end{array}$ \\
\hline VUWG & 372 & Labiatae & Mentha spicata & $\begin{array}{l}\text { WELT } 69908 \text { b; East Coast Rd, } \\
\text { Milford, N.Z. }\end{array}$ \\
\hline VUWG & 373 & Labiatae & Mentha suaveolens & WELT 70386; L. Wairarapa, N.Z. \\
\hline VUWG & 762 & Labiatae & Mentha suaveolens & $\begin{array}{l}\text { Chester 249. Asprokampos, } \\
\text { Grevena Pr., NW Greece. }\end{array}$ \\
\hline VUWG & 653 & Labiatae & Mentha sp. & $\begin{array}{l}\text { Chester } 15 . \text { Anelia, Grevena Pr., } \\
\text { NW Greece. }\end{array}$ \\
\hline VUWG & 338 & Labiatae & Nepeta cataria & $\begin{array}{l}\text { WELT 62693; Stronvar, } \\
\text { Wairarapa, N.Z. }\end{array}$ \\
\hline VUWG & 339 & Labiatae & Nepeta cataria & $\begin{array}{l}\text { WELT 60792; Kenepuru Valley, } \\
\text { N.Z. }\end{array}$ \\
\hline VUWG & 340 & Labiatae & Nepeta cataria & $\begin{array}{l}\text { WELT 61673; Tarakohe, Golden } \\
\text { Bay, N.Z. }\end{array}$ \\
\hline VUWG & 341 & Labiatae & Origanum vulgare & $\begin{array}{l}\text { WELT 62553; Standen St, Karori, } \\
\text { Wellington, N.Z. }\end{array}$ \\
\hline
\end{tabular}




\begin{tabular}{|c|c|c|c|c|}
\hline $\begin{array}{l}\text { Pollen/sr } \\
\text { referenc } \\
\text { slide \# }\end{array}$ & & Family & Genus \& species & $\begin{array}{l}\text { Collector (if known)/herbarium } \\
\text { herbarium \# (if known), } \\
\text { place of collection }\end{array}$ \\
\hline VUWG & 342 & Labiatae & Origanum vulgare & $\begin{array}{l}\text { WELT } 62647 \text {; Silverstream Rd, } \\
\text { Wellington, N.Z. }\end{array}$ \\
\hline VUWG & 343 & Labiatae & Origanum vulgare & WELT 60851. \\
\hline NUNG & 98 & Labiatae & Phlomis purpurea & $\begin{array}{l}\text { Stevenson, Newcastle Upon Tyne } \\
\text { University, England. }\end{array}$ \\
\hline VUWG & 1094 & Labiatae & Prunella grandiflora & $\begin{array}{l}\text { WELTU box } 91 \text {; Kaiserstuhl nr. } \\
\text { Freiburg, Germany. }\end{array}$ \\
\hline VUWG & 641 & Labiatae & Prunella laciniata & $\begin{array}{l}\text { Chester 3. Anelia, Grevena Pr., } \\
\text { NW Greece. }\end{array}$ \\
\hline VUWG & 1426 & Labiatae & Prunella vulgaris & $\begin{array}{l}\text { WELTU 10374; Kennedy Bay, } \\
\text { Coromandel Peninsula, N.Z. }\end{array}$ \\
\hline VUWG & 1427 & Labiatae & Prunella vulgaris & $\begin{array}{l}\text { WELTU 11437; Cape Turakirae, } \\
\text { Wellington, N.Z. }\end{array}$ \\
\hline VUWG & 344 & Labiatae & Prunella vulgaris & $\begin{array}{l}\text { WELT 70166a; Kahuteraura Rd, } \\
\text { Tararua Ra., N.Z. }\end{array}$ \\
\hline VUWG & 345 & Labiatae & Prunella vulgaris & $\begin{array}{l}\text { WELT 63231; Tokomaru Bay, } \\
\text { Mangatuna, N.Z. }\end{array}$ \\
\hline VUWG & 346 & Labiatae & Prunella vulgaris & $\begin{array}{l}\text { WELT 62614; Keith George Park, } \\
\text { Hutt Valley, N.Z. }\end{array}$ \\
\hline NUNG & 16 & Labiatae & Rosmarinus officinalis & $\begin{array}{l}\text { Stevenson, Newcastle Upon Tyne } \\
\text { University, England. }\end{array}$ \\
\hline VUWG & 1095 & Labiatae & Salvia glutinosa & WELTU box 91; Switzerland. \\
\hline NUNG & 102 & Labiatae & Salvia glutinosa & $\begin{array}{l}\text { Stevenson, Newcastle Upon Tyne } \\
\text { University, England. }\end{array}$ \\
\hline VUWG & 1098 & Labiatae & Salvia pratensis & $\begin{array}{l}\text { WELTU box 91; Donau R., } \\
\text { Germany. }\end{array}$ \\
\hline VUWG & 1097 & Labiatae & Salvia roemeriana & $\begin{array}{l}\text { WELTU } 9413 \text {; Mt Bonnel, Travis } \\
\text { Co., USA. }\end{array}$ \\
\hline VUWG & 352 & Labiatae & Salvia sclarea & $\begin{array}{l}\text { WELT 14203; Seatoun, } \\
\text { Wellington, N.Z. }\end{array}$ \\
\hline VUWG & 349 & Labiatae & Salvia verbenacea & WELT 61691; Auckland, N.Z. \\
\hline VUWG & 350 & Labiatae & Salvia verbenacea & WELT 61693; Kaikoura, N.Z. \\
\hline VUWG & 351 & Labiatae & Salvia verbenacea & WELT 60846; Kaikoura, N.Z. \\
\hline VUWG & 1096 & Labiatae & Salia verticillata & WELTU box 91; Switzerland. \\
\hline VUWG & 764 & Labiatae & Salvia sp. & $\begin{array}{l}\text { Chester 252. Asprokampos, } \\
\text { Grevena Pr., NW Greece. }\end{array}$ \\
\hline VUWG & 788 & Labiatae & Salvia sp. [cultivar] & $\begin{array}{l}\text { Chester } 309 \text {. Wellington Botanic } \\
\text { Gardens, N.Z. }\end{array}$ \\
\hline VUWG & 353 & Labiatae & Stachys arvensis & $\begin{array}{l}\text { WELT } 62616 \text {; Keith George Park, } \\
\text { Hutt Valley, N.Z. }\end{array}$ \\
\hline VUWG & 354 & Labiatae & Stachys arvensis & $\begin{array}{l}\text { WELT } 60700 ; \text { S Karori Rd, } \\
\text { Wellington, N.Z. }\end{array}$ \\
\hline VUWG & 1099 & Labiatae & Stachys palustris & $\begin{array}{l}\text { WELTU box 91; De Steeg, } \\
\text { Holland. }\end{array}$ \\
\hline VUWG & 355 & Labiatae & Stachys palustris & WELT 61704; Whanganui, N.Z. \\
\hline VUWG & 356 & Labiatae & Stachys palustris & WELT 61704; Whanganui, N.Z. \\
\hline VUWG & 1100 & Labiatae & Stachys recta & WELTU box 91 ; Holland. \\
\hline NUNG & 145 & Labiatae & Stachys recta & $\begin{array}{l}\text { Stevenson, Newcastle Upon Tyne } \\
\text { University, England. }\end{array}$ \\
\hline VUWG & 1101 & Labiatae & Stachys sylvatica & $\begin{array}{l}\text { WELTU box } 91 \text {; Zandvoorl, } \\
\text { Holland. }\end{array}$ \\
\hline VUWG & 1428 & Labiatae & Stachys sylvatica & $\begin{array}{l}\text { WELTU 15190; Karori, } \\
\text { Wellington, N.Z. }\end{array}$ \\
\hline
\end{tabular}




\begin{tabular}{|c|c|c|c|c|}
\hline \multicolumn{2}{|c|}{$\begin{array}{l}\text { Pollen/spore } \\
\text { reference } \\
\text { slide \# }\end{array}$} & \multirow{2}{*}{$\begin{array}{l}\text { Family } \\
\text { Labiatae }\end{array}$} & \multirow{2}{*}{$\begin{array}{l}\text { Genus \& species } \\
\text { Stachys sylvatica }\end{array}$} & \multirow{2}{*}{$\begin{array}{l}\text { Collector (if known)/herbarium, } \\
\text { herbarium \# (if known), } \\
\text { place of collection }\end{array}$} \\
\hline VUWG & 1429 & & & \\
\hline VUWG & 357 & Labiatae & Stachys sylvatica & $\begin{array}{l}\text { WELT 70734; Banks Peninsula, } \\
\text { Canterbury, N.Z. }\end{array}$ \\
\hline VUWG & 358 & Labiatae & Stachys sylvatica & $\begin{array}{l}\text { WELT 60373; S Karori Rd, } \\
\text { Wellington, N.Z. }\end{array}$ \\
\hline VUWG & 359 & Labiatae & Stachys sylvatica & $\begin{array}{l}\text { WELT 51421; Red Rock, } \\
\text { Wellington, N.Z. }\end{array}$ \\
\hline NUNG & 78 & Labiatae & Stachys sylvatica & $\begin{array}{l}\text { Stevenson, Newcastle Upon Tyne } \\
\text { University, England. }\end{array}$ \\
\hline VUWG & 737 & Labiatae & ?Stachys sp. & $\begin{array}{l}\text { Chester } 160 \text {. Anelia, Grevena Pr., } \\
\text { NW Greece. }\end{array}$ \\
\hline VUWG & 102 & Labiatae & Teucrium botrys & WELTU box 91; Holland. \\
\hline VUWG & 1103 & Labiatae & Teucrium ?montanum & WELTU box 91; Switzerland. \\
\hline VUWG & 728 & Labiatae & Teucrium polium & $\begin{array}{l}\text { Chester 135. Mirsina, Grevena Pr., } \\
\text { NW Greece. }\end{array}$ \\
\hline VUWG & 1430 & Labiatae & Teucrium scordium & $\begin{array}{l}\text { WELTU 10373; Canterbury } \\
\text { Plains, N.Z. }\end{array}$ \\
\hline VUWG & 672 & Labiatae & Teucrium scordium & $\begin{array}{l}\text { Chester 34. Kellia, Grevena Pr., } \\
\text { NW Greece. }\end{array}$ \\
\hline VUWG & 719 & Labiatae & Teucrium scordium & $\begin{array}{l}\text { Chester 124. Mirsina, Grevena Pr., } \\
\text { NW Greece. }\end{array}$ \\
\hline VUWG & 1104 & Labiatae & Teucrium scorodonia & WELTU box 91; Holland. \\
\hline NUNG & 183 & Labiatae & Teucrium scorodonia & $\begin{array}{l}\text { Stevenson, Newcastle Upon Tyne } \\
\text { University, England. }\end{array}$ \\
\hline VUWG & 748 & Labiatae & Teucrium sp. & $\begin{array}{l}\text { Chester 177. Agios Nicholas, } \\
\text { Grevena Pr., NW Greece. }\end{array}$ \\
\hline VUWG & 1105 & Labiatae & Thymus serpyllum & WELTU box 91; Germany. \\
\hline VUWG & 360 & Labiatae & Thymus vulgaris & $\begin{array}{l}\text { WELT 70409; Roxburgh, Central } \\
\text { Otago, N.Z. }\end{array}$ \\
\hline VUWG & 699 & Labiatae & Thymus sp. & $\begin{array}{l}\text { Chester 71. Polineri, Grevena Pr., } \\
\text { NW Greece. }\end{array}$ \\
\hline NUNG & 38 & Labiatae & & $\begin{array}{l}\text { Stevenson, Newcastle Upon Tyne } \\
\text { University, England. }\end{array}$ \\
\hline VUWG & 1013 & Leguminosae & Astragalus massiliensis & WELTU box 91 \\
\hline NUNG & 7 & Leguminosae & Ceratonia siliqua & $\begin{array}{l}\text { Stevenson, Newcastle Upon Tyne } \\
\text { University, England. }\end{array}$ \\
\hline NUNG & 1 & Leguminosae & Cercis siliquastrum & $\begin{array}{l}\text { Stevenson, Newcastle Upon Tyne } \\
\text { University, England. }\end{array}$ \\
\hline NUNG & 84 & Leguminosae & Colutea arborescens & $\begin{array}{l}\text { Stevenson, Newcastle Upon Tyne } \\
\text { University, England. }\end{array}$ \\
\hline NUNG & 107 & Leguminosae & Coronilla repanda & $\begin{array}{l}\text { Stevenson, Newcastle Upon Tyne } \\
\text { University, England. }\end{array}$ \\
\hline VUWG & 429 & Leguminosae & Coronilla varia & $\begin{array}{l}\text { WELT 69894; Grafton, Auckland, } \\
\text { N.Z. }\end{array}$ \\
\hline NUNU & 101 & Leguminosae & Cytisus grandiflorus & $\begin{array}{l}\text { Stevenson, Newcastle Upon Tyne } \\
\text { University, England. }\end{array}$ \\
\hline VUWG & 428 & Leguminosae & Cytisus scoparius & $\begin{array}{l}\text { WELT 60776; S Karori Rd, } \\
\text { Wellington, N.Z. }\end{array}$ \\
\hline NUNG & 47 & Leguminosae & Cytisus scoparius & $\begin{array}{l}\text { Stevenson, Newcastle Upon Tyne } \\
\text { University, England. }\end{array}$ \\
\hline NUNG & 114 & Leguminosae & Cytisus sessilifolius & $\begin{array}{l}\text { Stevenson, Newcastle Upon Tyne } \\
\text { University, England. }\end{array}$ \\
\hline
\end{tabular}




\begin{tabular}{|c|c|c|c|c|}
\hline \multicolumn{2}{|c|}{$\begin{array}{l}\text { Pollen/spore } \\
\text { reference } \\
\text { slide \# }\end{array}$} & \multirow{2}{*}{$\begin{array}{l}\text { Family } \\
\text { Leguminosae }\end{array}$} & \multirow{2}{*}{$\begin{array}{l}\text { Genus \& species } \\
\text { Dorycnium graecum }\end{array}$} & \multirow{2}{*}{$\begin{array}{l}\text { Collector (if known)/herbarium } \\
\text { herbarium \# (if known), } \\
\text { place of collection }\end{array}$} \\
\hline VUWG & 683 & & & \\
\hline VUWG & 725 & Leguminosae & Dorycnium graecum & $\begin{array}{l}\text { Chester 131. Mirsina, Grevena Pr., } \\
\text { NW Greece. }\end{array}$ \\
\hline VUWG & 691 & Leguminosae & Dorycnium hirsutum & $\begin{array}{l}\text { Chester } 60 . \text { Mavrani, Grevena Pr., } \\
\text { NW Greece. }\end{array}$ \\
\hline VUWG & 746 & Leguminosae & Dorycnium pentaphyllum & $\begin{array}{l}\text { Chester 174. Agios Nicholas, } \\
\text { Grevena Pr., NW Greece. }\end{array}$ \\
\hline NUNG & 165 & Leguminosae & Echinospartun boisseri & $\begin{array}{l}\text { Stevenson, Newcastle Upon Tyne } \\
\text { University, England. }\end{array}$ \\
\hline VUWG & 1014 & Leguminosae & Genista anglica & WELTU box $91 ;$ Holland. \\
\hline VUWG & 1015 & Leguminosae & Genista lineus & WELTU box 91; Germany. \\
\hline VUWG & 1016 & Leguminosae & Genista pulegium & WELTU box 91 ; Germany. \\
\hline VUWG & 1017 & Leguminosae & Genista tinctoria & WELTU box 91; Germany. \\
\hline VUWG & 1018 & Leguminosae & Genista tinctoria & WELTU box 91 . \\
\hline VUWG & 1019 & Leguminosae & Laburnum anagyroides & WELTU box 91; Holland. \\
\hline VUWG & 1020 & Leguminosae & Laburnum anagyroides & WELTU box 91; Holland. \\
\hline VUWG & 1021 & Leguminosae & Lathyrus aphaca & WELTU box 91; India. \\
\hline VUWG & 434 & Leguminosae & Lathyrus latifolius & $\begin{array}{l}\text { WELT } 60575 \text {; Karori, Wellington, } \\
\text { N.Z. }\end{array}$ \\
\hline VUWG & 435 & Leguminosae & Lathyrus latifolius & $\begin{array}{l}\text { WELT 62600; Keith George Park, } \\
\text { Hutt Valley, N.Z. }\end{array}$ \\
\hline VUWG & 436 & Leguminosae & Lathyrus latifolius & $\begin{array}{l}\text { WELT 61329; McKays Crossing, } \\
\text { Paekakariki, N.Z. }\end{array}$ \\
\hline VUWG & 1339 & Leguminosae & Lathyrus latifolius & $\begin{array}{l}\text { WELTU 15312; Avalon, Lower } \\
\text { Hutt, N.Z. }\end{array}$ \\
\hline VUWG & 1340 & Leguminosae & Lathyrus latifolius & WELTU; Wellington, N.Z. \\
\hline VUWG & 1022 & Leguminosae & Lathyrus palustris & WELTU box 91; Holland. \\
\hline VUWG & 437 & Leguminosae & Lathyrus pratensis & $\begin{array}{l}\text { WELT 70405; Waikari, N } \\
\text { Canterbury, N.Z. }\end{array}$ \\
\hline VUWG & 1023 & Leguminosae & Lathyrus pratensis & WELTU box 91; Holland. \\
\hline NUNG & 49 & Leguminosae & Lathyrus pratensis & $\begin{array}{l}\text { Stevenson, Newcastle Upon Tyne } \\
\text { University, England. }\end{array}$ \\
\hline VUWG & 1024 & Leguminosae & Lathyrus sulphureus & $\begin{array}{l}\text { WELTU } 9379 ; \text { nr. Cool, Eldorado } \\
\text { Co., USA. }\end{array}$ \\
\hline VUWG & 1341 & Leguminosae & Lathyrus sylvestris & $\begin{array}{l}\text { WELTU; Victoria University, } \\
\text { Wellington, N.Z. }\end{array}$ \\
\hline VUWG & 438 & Leguminosae & Lathyrus tingitanus & $\begin{array}{l}\text { WELT 63489a; Raroa Rd, Karori, } \\
\text { Wellington, N.Z. }\end{array}$ \\
\hline VUWG & 439 & Leguminosae & Lathyrus tingitanus & $\begin{array}{l}\text { WELT 60581; S Karori Rd, } \\
\text { Wellington, N.Z. }\end{array}$ \\
\hline VUWG & 440 & Leguminosae & Lathyrus tingitanus & $\begin{array}{l}\text { WELT 61533; Pukerua Bay, } \\
\text { Wellington, N.Z. }\end{array}$ \\
\hline VUWG & 1025 & Leguminosae & Lathyrus tuberosus & WELTU box 91; Holland. \\
\hline VUWG & 647 & Leguminosae & Lathyrus sp. & $\begin{array}{l}\text { Chester 9. Anelia, Grevena Pr., } \\
\text { NW Greece. }\end{array}$ \\
\hline VUWG & 745 & Leguminosae & Lotus aegaeus & $\begin{array}{l}\text { Chester 173. Agios Nicholas, } \\
\text { Grevena Pr., NW Greece. }\end{array}$ \\
\hline VUWG & 430 & Leguminosae & Lotus angustissimus & $\begin{array}{l}\text { WELT 70774; Pukerua Bay, } \\
\text { Wellington, N.Z. }\end{array}$ \\
\hline VUWG & 431 & Leguminosae & Lotus angustissimus & $\begin{array}{l}\text { WELT 70646; Great Island, Three } \\
\text { Kings Is, N.Z. }\end{array}$ \\
\hline
\end{tabular}




\begin{tabular}{|c|c|c|c|c|}
\hline \multicolumn{2}{|c|}{$\begin{array}{l}\text { Pollen/spore } \\
\text { reference } \\
\text { slide \# }\end{array}$} & Family & Genus \& species & $\begin{array}{l}\text { Collector (if known)/herbarium, } \\
\text { herbarium \# (if known), } \\
\text { place of collection }\end{array}$ \\
\hline VUWG & 432 & Leguminosae & Lotus angustissimus & $\begin{array}{l}\text { WELT 70087a; Rodney Cnty, N of } \\
\text { Waiwera, N.Z. }\end{array}$ \\
\hline VUWG & 433 & Leguminosae & Lotus corniculatus & $\begin{array}{l}\text { WELT 70414; Milton, S Otago, } \\
\text { N.Z. }\end{array}$ \\
\hline VUWG & 1008 & Leguminosae & Lotus corniculatus & WELTU box 91; Holland. \\
\hline NUNG & 11 & Leguminosae & Lotus corniculatus & $\begin{array}{l}\text { Stevenson, Newcastle Upon Tyne } \\
\text { University, England. }\end{array}$ \\
\hline NUNG & 266 & Leguminosae & Lotus corniculatus & $\begin{array}{l}\text { Stevenson, Newcastle Upon Tyne } \\
\text { University, England. }\end{array}$ \\
\hline VUWG & 684 & Leguminosae & Lotus cf. corniculatus & $\begin{array}{l}\text { Chester 52. Kellia, Grevena Pr., } \\
\text { NW Greece. }\end{array}$ \\
\hline VUWG & 713 & Leguminosae & Lotus cf. corniculatus & $\begin{array}{l}\text { Chester 98. Polineri, Grevena Pr., } \\
\text { NW Greece. }\end{array}$ \\
\hline NUNG & 149 & Leguminosae & Lotus creticus & $\begin{array}{l}\text { Stevenson, Newcastle Upon Tyne } \\
\text { University, England. }\end{array}$ \\
\hline VUWG & 1342 & Leguminosae & Lotus parviflorus & WELTU; Kaingaroa, N.Z. \\
\hline VUWG & 1343 & Leguminosae & Lotus pedunculatus & WELTU; Wellington, N.Z. \\
\hline VUWG & 1344 & Leguminosae & Lotus pedunculatus & WELTU; Wellington, N.Z. \\
\hline VUWG & 1009 & Leguminosae & Lotus uliginosus & WELTU box 91; Holland. \\
\hline VUWG & 646 & Leguminosae & Lotus sp. & $\begin{array}{l}\text { Chester 8. Anelia, Grevena Pr., } \\
\text { NW Greece. }\end{array}$ \\
\hline VUWG & 441 & Leguminosae & Lupinus angustifolius & $\begin{array}{l}\text { WELT } 61288 \text {; Milford, Waitemata } \\
\text { Co., N.Z. }\end{array}$ \\
\hline VUWG & 442 & Leguminosae & Lupinus angustifolius & $\begin{array}{l}\text { WELT } 61292 \text {; Milford, Waitemata } \\
\text { Co., N.Z. }\end{array}$ \\
\hline VUWG & 443 & Leguminosae & Lupinus angustifolius & $\begin{array}{l}\text { WELT } 60917 \text {; Hokio Beach, } \\
\text { Levin, N.Z. }\end{array}$ \\
\hline NUNG & 91 & Leguminosae & Lupinus hispanicus & $\begin{array}{l}\text { Stevenson, Newcastle Upon Tyne } \\
\text { University, England. }\end{array}$ \\
\hline NUNG & 131 & Leguminosae & Lupinus polyphyllus & $\begin{array}{l}\text { Stevenson, Newcastle Upon Tyne } \\
\text { University, England. }\end{array}$ \\
\hline VUWG & 446 & Leguminosae & Medicago arabica & WELT 63267; Hikurangi, N.Z. \\
\hline VUWG & 447 & Leguminosae & Medicago arabica & WELT 61471; Cuvier Island, N.Z. \\
\hline VUWG & 448 & Leguminosae & Medicago arabica & $\begin{array}{l}\text { WELT } 60920 ; \text { S Karori Rd, } \\
\text { Wellington, N.Z. }\end{array}$ \\
\hline VUWG & 444 & Leguminosae & Medicago arborea & $\begin{array}{l}\text { WELT 61246; Island Bay, } \\
\text { Wellington, N.Z. }\end{array}$ \\
\hline VUWG & 445 & Leguminosae & Medicago arborea & $\begin{array}{l}\text { WELT 60570; Island Bay, } \\
\text { Wellington, N.Z. }\end{array}$ \\
\hline VUWG & 1010 & Leguminosae & Medicago falcata & WELTU box 91 ; Holland. \\
\hline VUWG & 449 & Leguminosae & Medicago lupulina & $\begin{array}{l}\text { WELT 70859; Wakanui, } \\
\text { Ashburton, N.Z. }\end{array}$ \\
\hline VUWG & 450 & Leguminosae & Medicago lupulina & $\begin{array}{l}\text { WELT 70076a; Weranui Rd, } \\
\text { Waiwera, N.Z. }\end{array}$ \\
\hline VUWG & 451 & Leguminosae & Medicago lupulina & $\begin{array}{l}\text { WELT } 63478 \text {; Riverside Rd, } \\
\text { Havelock North, N.Z. }\end{array}$ \\
\hline VUWG & 1345 & Leguminosae & Medicago lupulina & $\begin{array}{l}\text { WELTU 11372; Cape Turakirae, } \\
\text { Wellington, N.Z. }\end{array}$ \\
\hline VUWG & 1346 & Leguminosae & Medicago lupulina & WELTU 14934; Raumati, N.Z. \\
\hline VUWG & 1347 & Leguminosae & Medicago lupulina & WELTU 10333; N.Z. \\
\hline VUWG & 452 & Leguminosae & Medicago minima & $\begin{array}{l}\text { WELT 70725; Waipara River, } \\
\text { Canterbury, N.Z. }\end{array}$ \\
\hline
\end{tabular}




\begin{tabular}{|c|c|c|c|c|}
\hline $\begin{array}{l}\text { Pollen/sp } \\
\text { referenc } \\
\text { slide \# }\end{array}$ & & Family & Genus \& species & $\begin{array}{l}\text { Collector (if known)/herbarium, } \\
\text { herbarium \# (if known), } \\
\text { place of collection }\end{array}$ \\
\hline VUWG & 453 & Leguminosae & Medicago polymorpha & $\begin{array}{l}\text { WELT } 61255 \text {; S Karori Rd, } \\
\text { Wellington, N.Z. }\end{array}$ \\
\hline VUWG & 454 & Leguminosae & Medicago polymorpha & WELT 61487 \\
\hline VUWG & 455 & Leguminosae & Medicago polymorpha & $\begin{array}{l}\text { WELT 61485; Pukerua Bay, } \\
\text { Wellington, N.Z. }\end{array}$ \\
\hline VUWG & 456 & Leguminosae & Medicago sativa & $\begin{array}{l}\text { WELT 70888; Vivian St, } \\
\text { Wellington, N.Z. }\end{array}$ \\
\hline VUWG & 457 & Leguminosae & Medicago sativa & $\begin{array}{l}\text { WELT 70442; Island Bay, } \\
\text { Wellington, N.Z. }\end{array}$ \\
\hline VUWG & 458 & Leguminosae & Medicago sativa & $\begin{array}{l}\text { WELT 70103; Browns Bay, } \\
\text { Waitemata Co., N.Z. }\end{array}$ \\
\hline VUWG & 1011 & Leguminosae & Medicago sativa & WELTU box 91; Holland. \\
\hline VUWG & 1012 & Leguminosae & Medicago sativa & WELTU box 91 . \\
\hline VUWG & 1348 & Leguminosae & Medicago sativa & WELTU; New Plymouth, N.Z. \\
\hline NUNG & 143 & Leguminosae & Medicago sativa & $\begin{array}{l}\text { Stevenson, Newcastle Upon Tyne } \\
\text { University, England. }\end{array}$ \\
\hline VUWG & 459 & Leguminosae & Melilotus alba & $\begin{array}{l}\text { WELT 70861; Pokaka, central N } \\
\text { Island, N.Z. }\end{array}$ \\
\hline VUWG & 460 & Leguminosae & Melilotus alba & WELT 61504; Napier, N.Z. \\
\hline VUWG & 1026 & Leguminosae & Melilotus alba & WELTU box 91; Holland. \\
\hline VUWG & 1027 & Leguminosae & Melilotus altissima & WELTU box 91; Holland. \\
\hline VUWG & 1028 & Leguminosae & Melilotus altissima & WELTU box 91 . \\
\hline VUWG & 461 & Leguminosae & Melilotus indica & $\begin{array}{l}\text { WELT 70096; Waiwera, Rodney } \\
\text { Co., N.Z. }\end{array}$ \\
\hline VUWG & 462 & Leguminosae & Melilotus indica & $\begin{array}{l}\text { WELT } 69893 \text {; Milford, Waitemata } \\
\text { Co., N.Z. }\end{array}$ \\
\hline VUWG & 463 & Leguminosae & Melilotus indica & $\begin{array}{l}\text { WELT } 61512 \text {; Seatoun, } \\
\text { Wellington, N.Z. }\end{array}$ \\
\hline VUWG & 1349 & Leguminosae & Melilotus indica & WELTU; Wellington, N.Z. \\
\hline VUWG & 1350 & Leguminosae & Melilotus indica & WELTU; Wellington, N.Z. \\
\hline VUWG & 1351 & Leguminosae & Melilotus indica & WELTU; Wellington, N.Z. \\
\hline VUWG & 464 & Leguminosae & Melilotus officinalis & $\begin{array}{l}\text { WELT 70422; Islington, } \\
\text { Christchurch, N.Z. }\end{array}$ \\
\hline VUWG & 465 & Leguminosae & Melilotus officinalis & $\begin{array}{l}\text { WELT 62807; Tatapouri Bay, } \\
\text { Gisborne, N.Z. }\end{array}$ \\
\hline VUWG & 1029 & Leguminosae & Melilotus officinalis & WELTU box 91; Holland. \\
\hline VUWG & 1030 & Leguminosae & Melilotus officinalis & WELTU box 91; Holland. \\
\hline VUWG & 1031 & Leguminosae & Onobrychis viciifolia & WELTU box 91 ; Holland. \\
\hline VUWG & 1032 & Leguminosae & Onobrychis viciifolia & WELTU box 91; Holland. \\
\hline VUWG & 1033 & Leguminosae & Onobrychis viciifolia & WELTU box 91; Switzerland. \\
\hline NUNG & 123 & Leguminosae & Ononis baetica & $\begin{array}{l}\text { Stevenson, Newcastle Upon Tyne } \\
\text { University, England. }\end{array}$ \\
\hline VUWG & 466 & Leguminosae & Ononis spinosa & $\begin{array}{l}\text { WELT 70420; Templeton, } \\
\text { Canterbury, N.Z. }\end{array}$ \\
\hline VUWG & 658 & Leguminosae & Ononis spinosa & $\begin{array}{l}\text { Chester } 20 . \text { Kellia, Grevena Pr., } \\
\text { NW Greece. }\end{array}$ \\
\hline VUWG & 1034 & Leguminosae & Ononis spinosa & WELTU box 91; Holland. \\
\hline VUWG & 1035 & Leguminosae & Ononis spinosa & WELTU box 91 ; Holland. \\
\hline NUNG & 124 & Leguminosae & Ononis subspicata & $\begin{array}{l}\text { Stevenson, Newcastle Upon Tyne } \\
\text { University, England. }\end{array}$ \\
\hline VUWG & 468 & Leguminosae & Ornithopis pinnatus & $\begin{array}{l}\text { WELT 70699; Stevenson Island, } \\
\text { Whangaroa Co., N.Z. }\end{array}$ \\
\hline VUWG & 467 & Leguminosae & Ornithopus pinnatus & WELT 70696; Auckland City, N.Z. \\
\hline
\end{tabular}




Pollen/spore Family $\quad$ Genus \& species
reference
slide \#

\begin{tabular}{|c|c|c|c|c|}
\hline VUWG & 470 & Leguminosae & Teline monspessulanus & $\begin{array}{l}\text { WELT 60885; Bowen St, } \\
\text { Wellington, N.Z. }\end{array}$ \\
\hline VUWG & 471 & Leguminosae & Teline monspessulanus & $\begin{array}{l}\text { WELT } 63153 \text {; Karori, Wellington, } \\
\text { N.Z. }\end{array}$ \\
\hline VUWG & 469 & Leguminosae & Teline $\mathrm{x}$ spachianus & $\begin{array}{l}\text { WELT 63188; Pukerua Bay, } \\
\text { Wellington, N.Z. }\end{array}$ \\
\hline VUWG & 1036 & Leguminosae & Trifolium alpinum & WELTU box 91; Switzerland. \\
\hline NUNG & 52 & Leguminosae & Trifolium alpinum & $\begin{array}{l}\text { Stevenson, Newcastle Upon Tyne } \\
\text { University, England. }\end{array}$ \\
\hline VUWG & 472 & Leguminosae & Trifolium arvense & WELT 63311; Nelson City, N.Z. \\
\hline VUWG & 473 & Leguminosae & Trifolium arvense & $\begin{array}{l}\text { WELT 61603; Karori, Wellington, } \\
\text { N.Z. }\end{array}$ \\
\hline VUWG & 474 & Leguminosae & Trifolium arvense & WELT 60580; Paraparaumu, N.Z. \\
\hline VUWG & 1037 & Leguminosae & Trifolium arvense & WELTU box 91; Velp, Holland. \\
\hline NUNG & 125 & Leguminosae & Trifolium badium & $\begin{array}{l}\text { Stevenson, Newcastle Upon Tyne } \\
\text { University, England. }\end{array}$ \\
\hline VUWG & 476 & Leguminosae & Trifolium campestre & WELT 70866; Ashburton, N.Z. \\
\hline VUWG & 716 & Leguminosae & Trifolium campestre & $\begin{array}{l}\text { Chester 104. Polineri, Grevena Pr., } \\
\text { NW Greece. }\end{array}$ \\
\hline NUNG & 250 & Leguminosae & Trifolium campestre & $\begin{array}{l}\text { Stevenson, Newcastle Upon Tyne } \\
\text { University, England. }\end{array}$ \\
\hline VUWG & 477 & Leguminosae & Trifolium dubium & $\begin{array}{l}\text { WELT 61776; Orongorongo } \\
\text { Valley, Wellington, N.Z. }\end{array}$ \\
\hline VUWG & 478 & Leguminosae & Trifolium dubium & WELT 61609; Cuvier Island, N.Z. \\
\hline VUWG & 479 & Leguminosae & Trifolium dubium & WELT 61607; Pukerua Bay, N.Z. \\
\hline NUNG & 97 & Leguminosae & Trifolium dubium & $\begin{array}{l}\text { Stevenson, Newcastle Upon Tyne } \\
\text { University, England. }\end{array}$ \\
\hline VUWG & 480 & Leguminosae & Trifolium glomeratum & $\begin{array}{l}\text { WELT 61212; Somes Island, } \\
\text { Wellington, N.Z. }\end{array}$ \\
\hline VUWG & 481 & Leguminosae & Trifolium glomeratum & $\begin{array}{l}\text { WELT 63187; Eastbourne, } \\
\text { Wellington, N.Z. }\end{array}$ \\
\hline VUWG & 482 & Leguminosae & Trifolium glomeratum & $\begin{array}{l}\text { WELT } 62651 \text {; Waipoua State } \\
\text { Forest, N.Z. }\end{array}$ \\
\hline VUWG & 1352 & Leguminosae & Trifolium glomeratum & $\begin{array}{l}\text { WELTU 11431; Cape Turakirae, } \\
\text { Wellington, N.Z. }\end{array}$ \\
\hline VUWG & 1353 & Leguminosae & Trifolium glomeratum & WELTU; Wellington, N.Z. \\
\hline vUWG & 483 & Leguminosae & Trifolium hybridium & $\begin{array}{l}\text { WELT 70863; Palmerston North, } \\
\text { N.Z. }\end{array}$ \\
\hline VUWG & 484 & Leguminosae & Trifolium incarnatum & WELT 61637a; Wellington, N.Z. \\
\hline VUWG & 1038 & Leguminosae & Trifolium montanum & WELTU box 91; Switzerland. \\
\hline NUNG & 122 & Leguminosae & Trifolium montanum & $\begin{array}{l}\text { Stevenson, Newcastle Upon Tyne } \\
\text { University, England. }\end{array}$ \\
\hline VUWG & 485 & Leguminosae & Trifolium pratense & $\begin{array}{l}\text { WELT 63322; Forest Hill, } \\
\text { Waitemata Co., N.Z. }\end{array}$ \\
\hline VUWG & 486 & Leguminosae & Trifolium pratense & $\begin{array}{l}\text { WELT 61620; Pukerua Bay, } \\
\text { Wellington, N.Z. }\end{array}$ \\
\hline VUWG & 652 & Leguminosae & Trifolium pratense & $\begin{array}{l}\text { Chester 14. Anelia, Grevena Pr., } \\
\text { NW Greece. }\end{array}$ \\
\hline VUWG & 1039 & Leguminosae & Trifolium pratense & WELTU box 91; Holland. \\
\hline VUWG & 1354 & Leguminosae & Trifolium pratense & WELTU; Wellington, N.Z. \\
\hline VUWG & 1355 & Leguminosae & Trifolium pratense & WELTU; Wellington, N.Z. \\
\hline NUNG & 213 & Leguminosae & Trifolium pratense & $\begin{array}{l}\text { Stevenson, Newcastle Upon Tyne } \\
\text { University, England. }\end{array}$ \\
\hline
\end{tabular}

Collector (if known)/herbarium, herbarium \# (if known), place of collection 


\begin{tabular}{|c|c|c|c|c|}
\hline $\begin{array}{l}\text { Pollen/s } \\
\text { referenc } \\
\text { slide \# }\end{array}$ & pore & Family & Genus \& species & $\begin{array}{l}\text { Collector (if known)/herbarium } \\
\text { herbarium \# (if known), } \\
\text { place of collection }\end{array}$ \\
\hline VUWG & 487 & Leguminosae & Trifolium repens & $\begin{array}{l}\text { WELT 70358a; Milford, } \\
\text { Waitemata Co., N.Z. }\end{array}$ \\
\hline VUWG & 488 & Leguminosae & Trifolium repens & $\begin{array}{l}\text { WELT } 60564 ; \text { S Karori Rd, } \\
\text { Wellington, N.Z. }\end{array}$ \\
\hline VUWG & 489 & Leguminosae & Trifolium repens & $\begin{array}{l}\text { WELT } 61261 \text {; S Karori Rd, } \\
\text { Wellington, N.Z. }\end{array}$ \\
\hline VUWG & 742 & Leguminosae & Trifolium repens & $\begin{array}{l}\text { Chester 167. Gomara, Grevena Pr., } \\
\text { NW Greece. }\end{array}$ \\
\hline VUWG & 834 & Leguninosae & Trifolium repens & $\begin{array}{l}\text { Chester } 355 \text {. Victoria University, } \\
\text { Wellington, N.Z. }\end{array}$ \\
\hline VUWG & 1041 & Leguminosae & Trifolium repens & WELTU box 91; Velp, Holland. \\
\hline VUWG & 1356 & Leguminosae & Trifolium repens & $\begin{array}{l}\text { WELTU; Victoria University, } \\
\text { Wellington, N.Z. }\end{array}$ \\
\hline VUWG & 1357 & Leguminosae & Trifolium repens & $\begin{array}{l}\text { WELTU; Cape Turakirae, } \\
\text { Wellington, N.Z. }\end{array}$ \\
\hline VUWG & 1040 & Leguminosae & Trifolium rubens & $\begin{array}{l}\text { WELTU box 91; Kaiserstuhl nr. } \\
\text { Freiburg, Germany. }\end{array}$ \\
\hline VUWG & 490 & Leguminosae & Trifolium striatum & $\begin{array}{l}\text { WELT 61654; Cashmere Hills, } \\
\text { Christchurch, N.Z. }\end{array}$ \\
\hline VUWG & 491 & Leguminosae & Trifolium striatum & $\begin{array}{l}\text { WELT 61646; Pukerua Bay, } \\
\text { Wellington, N.Z. }\end{array}$ \\
\hline VUWG & 492 & Leguminosae & Trifolium subterraneum & $\begin{array}{l}\text { WELT 70363a; Milford, } \\
\text { Waitemata Co., N.Z. }\end{array}$ \\
\hline VUWG & 493 & Leguminosae & Trifolium subterraneum & $\begin{array}{l}\text { WELT } 60871 \mathrm{~b} \text {; S Karori Rd, } \\
\text { Wellington, N.Z. }\end{array}$ \\
\hline VUWG & 494 & Leguminosae & Trifolium subterraneum & $\begin{array}{l}\text { WELT } 61211 \text {; Somes Island, } \\
\text { Wellington, N.Z. }\end{array}$ \\
\hline NUNG & 257 & Leguminosae & Trifolium subterraneum & $\begin{array}{l}\text { Stevenson, Newcastle Upon Tyne } \\
\text { University, England. }\end{array}$ \\
\hline VUWG & 495 & Leguminosae & Trifolium tomentosum & WELT 70413; Christchurch, N.Z. \\
\hline VUWG & 496 & Leguminosae & Trifolium tomentosum & $\begin{array}{l}\text { WELT } 61650 \text {; Worser Bay, } \\
\text { Wellington, N.Z. }\end{array}$ \\
\hline VUWG & 1358 & Leguminosae & Ulex europaeus & $\begin{array}{l}\text { WELTU 11325; Cape Turakirae, } \\
\text { Wellington, N.Z. }\end{array}$ \\
\hline NUNG & 6 & Leguminosae & Ulex europaeus & $\begin{array}{l}\text { Stevenson, Newcastle Upon Tyne } \\
\text { University, England. }\end{array}$ \\
\hline VUWG & 500 & Leguminosae & Vicia angustifolia & $\begin{array}{l}\text { WELT } 63185 ; \text { Eastbourne, } \\
\text { Wellington, N.Z. }\end{array}$ \\
\hline VUWG & 501 & Leguminosae & Vicia angustifolia & WELT 62904; Kenepuru, N.Z. \\
\hline VUWG & 502 & Leguminosae & Vicia angustifolia & $\begin{array}{l}\text { WELT } 61224 \text {; S Karori Rd, } \\
\text { Wellington, N.Z. }\end{array}$ \\
\hline VUWG & 497 & Leguminosae & Vicia hirsuta & $\begin{array}{l}\text { WELT 70384; Pukerua Bay, } \\
\text { Wellington, N.Z. }\end{array}$ \\
\hline VUWG & 498 & Leguminosae & Vicia hirsuta & $\begin{array}{l}\text { WELT } 61577 \text {; Seatoun, } \\
\text { Wellington, N.Z. }\end{array}$ \\
\hline VUWG & 499 & Leguminosae & Vicia hirsuta & $\begin{array}{l}\text { WELT } 60875 \text {; S Karori Rd, } \\
\text { Wellington, N.Z. }\end{array}$ \\
\hline VUWG & 1044 & Leguminosae & Vicia hirsuta & WELTU box 91; Holland. \\
\hline VUWG & 1359 & Leguminosae & Vicia hirsuta & WELTU 10366; Dunedin, N.Z. \\
\hline VUWG & 1360 & Leguminosae & Vicia hirsuta & WELTU 14935; Raumati, N.Z. \\
\hline VUWG & 1361 & Leguminosae & Vicia hirsuta & $\begin{array}{l}\text { WELTU; Victoria University, } \\
\text { Wellington, N.Z. }\end{array}$ \\
\hline VUWG & 1045 & Leguminosae & Vicia ?melanops & WELTU box 91; Holland. \\
\hline
\end{tabular}




\begin{tabular}{|c|c|c|c|c|}
\hline $\begin{array}{l}\text { Pollen/s| } \\
\text { referenc } \\
\text { slide \# }\end{array}$ & & Family & Genus \& species & $\begin{array}{l}\text { Collector (if known)/herbarium } \\
\text { herbarium \# (if known), } \\
\text { place of collection }\end{array}$ \\
\hline VUWG & 503 & Leguminosae & Vicia sativa & $\begin{array}{l}\text { WELT 63450; Stephens Island, } \\
\text { N.Z. }\end{array}$ \\
\hline VUWG & 504 & Leguminosae & Vicia sativa & WELT 63193; Westport, N.Z. \\
\hline VUWG & 505 & Leguminosae & Vicia sativa & $\begin{array}{l}\text { WELT } 61273 \text {; S Karori Rd, } \\
\text { Wellington, N.Z. }\end{array}$ \\
\hline VUWG & 1046 & Leguminosae & Vicia sativa & WELTU box 91; Holland. \\
\hline VUWG & 1042 & Leguminosae & Vicia sativa subsp. nigra & WELTU box 91; Holland. \\
\hline VUWG & 1047 & Leguminosae & Vicia sepium & WELTU box 91; Holland. \\
\hline NUNG & 115 & Leguminosae & Vicia sepium & $\begin{array}{l}\text { Stevenson, Newcastle Upon Tyne } \\
\text { University, England. }\end{array}$ \\
\hline VUWG & 506 & Leguminosae & Vicia tetrasperma & $\begin{array}{l}\text { WELT } 63277 \text {; Milford, Waitemata } \\
\text { Co., N.Z. }\end{array}$ \\
\hline VUWG & 507 & Leguminosae & Vicia tetrasperma & WELT 63273; Hamilton, N.Z. \\
\hline VUWG & 510 & Leguminosae & Vicia villosa & $\begin{array}{l}\text { WELT } 63271 \text {; nr Spye, N } \\
\text { Canterbury, N.Z. }\end{array}$ \\
\hline VUWG & 508 & Leguminosae & Vicia villosa varia & $\begin{array}{l}\text { WELT 63272; North Canterbury, } \\
\text { N.Z. }\end{array}$ \\
\hline VUWG & 509 & Leguminosae & Vicia villosa varia & $\begin{array}{l}\text { WELT } 63266 \text {; HW1 between Spye } \\
\text { \& Motunui, N.Z. }\end{array}$ \\
\hline VUWG & 1137 & Lentibulariaceae & Utricularia stellaris & WELTU; Najabgarh, India. \\
\hline VUWG & 1136 & Lentibulariaceae & Utricularia vulgaris & $\begin{array}{l}\text { WELTU } 9872 \text {; Zeya R., Amur } \\
\text { Territory, USSR. }\end{array}$ \\
\hline VUWG & 552 & Linaceae & Linum bienne & $\begin{array}{l}\text { WELT 63473; Havelock North, } \\
\text { N.Z. }\end{array}$ \\
\hline VUWG & 553 & Linaceae & Linum bienne & WELT 61778; Wairarapa, N.Z. \\
\hline VUWG & 554 & Linaceae & Linum bienne & $\begin{array}{l}\text { WELT } 61223 \text {; S Karori Rd, } \\
\text { Wellington, N.Z. }\end{array}$ \\
\hline NUNG & 167 & Linaceae & Linum campanulatum & $\begin{array}{l}\text { Stevenson, Newcastle Upon Tyne } \\
\text { University, England. }\end{array}$ \\
\hline VUWG & 1057 & Linaceae & Linum catharticum & $\begin{array}{l}\text { WELTU 7653; Granity Pass, E } \\
\text { Owen Ra., N.Z. }\end{array}$ \\
\hline VUWG & 1058 & Linaceae & Linum catharticum & WELTU box 91; Holland. \\
\hline VUWG & 555 & Linaceae & Linum catharticum & $\begin{array}{l}\text { WELT } 6002 \text {; Takaka Hill, Nelson, } \\
\text { N.Z. }\end{array}$ \\
\hline VUWG & 556 & Linaceae & Linum catharticum & WELT 63365; Mamaku, N.Z. \\
\hline VUWG & 1334 & Linaceae & Linum marginale & WELTU. \\
\hline NUNG & 210 & Linaceae & Linum maritimum & $\begin{array}{l}\text { Stevenson, Newcastle Upon Tyne } \\
\text { University, England. }\end{array}$ \\
\hline NUNG & 200 & Linaceae & Linum narbonense & $\begin{array}{l}\text { Stevenson, Newcastle Upon Tyne } \\
\text { University, England. }\end{array}$ \\
\hline NUNG & 137 & Linaceae & Linum strictum & $\begin{array}{l}\text { Stevenson, Newcastle Upon Tyne } \\
\text { University, England. }\end{array}$ \\
\hline NUNG & 212 & Linaceae & Linum suffruticosum & $\begin{array}{l}\text { Stevenson, Newcastle Upon Tyne } \\
\text { University, England. }\end{array}$ \\
\hline NUNG & 209 & Linaceae & Linum tenuifolium & $\begin{array}{l}\text { Stevenson, Newcastle Upon Tyne } \\
\text { University, England. }\end{array}$ \\
\hline VUWG & 557 & Linaceae & Linum trigynum & $\begin{array}{l}\text { WELT 70115; Waitemata Co., } \\
\text { N.Z. }\end{array}$ \\
\hline NUNG & 185 & Linaceae & Linum viscosum & $\begin{array}{l}\text { Stevenson, Newcastle Upon Tyne } \\
\text { University, England. }\end{array}$ \\
\hline NUNG & 202 & Linaceae & Linum sp. & $\begin{array}{l}\text { Stevenson, Newcastle Upon Tyne } \\
\text { University, England. }\end{array}$ \\
\hline
\end{tabular}




\begin{tabular}{|c|c|c|c|}
\hline $\begin{array}{l}\text { Pollen/spore } \\
\text { reference } \\
\text { slide \# }\end{array}$ & Family & Genus \& species & $\begin{array}{l}\text { Collector (if known)/herbarium, } \\
\text { herbarium \# (if known), } \\
\text { place of collection }\end{array}$ \\
\hline
\end{tabular}

\begin{tabular}{|c|c|c|c|c|}
\hline VUWG & 872 & Loranthaceae & Viscum album & $\begin{array}{l}\text { WELTU box } 86 \text {; Seine valley, } \\
\text { France. }\end{array}$ \\
\hline VUWG & 1378 & Lythraceae & Lythrum hyssopifolia & WELTU 10354. \\
\hline VUWG & 1379 & Lythraceae & Lythrum hyssopifolia & WELTU; Wellington, N.Z. \\
\hline VUWG & 1380 & Lythraceae & Lythrum hyssopifolia & WELTU 10352; Napier, N.Z. \\
\hline VUWG & 579 & Lythraceae & Lythrum hyssopifolium & $\begin{array}{l}\text { WELT 69979; Pukerua Bay, } \\
\text { Wellington, N.Z. }\end{array}$ \\
\hline VUWG & 580 & Lythraceae & Lythrum hyssopifolium & $\begin{array}{l}\text { WELT } 63480 \text {; Riverside Rd, } \\
\text { Havelock North, N.Z. }\end{array}$ \\
\hline VUWG & 581 & Lythraceae & Lythrum hyssopifolium & $\begin{array}{l}\text { WELT } 62609 \text {; Keith George Park, } \\
\text { Hutt Valley, N.Z. }\end{array}$ \\
\hline VUWG & 582 & Lythraceae & Lythrum salicaria & $\begin{array}{l}\text { WELT 70019a; nr Hokio Beach, } \\
\text { Levin, N.Z. }\end{array}$ \\
\hline VUWG & 583 & Lythraceae & Lythrum salicaria & $\begin{array}{l}\text { WELT } 70019 b \text {; nr Hokio Beach, } \\
\text { Levin, N.Z. }\end{array}$ \\
\hline VUWG & 668 & Lythraceae & Lythrum salicaria & $\begin{array}{l}\text { Chester } 30 . \text { Kellia, Grevena Pr., } \\
\text { NW Greece. }\end{array}$ \\
\hline VUWG & 879 & Lythraceae & Lythrum salicaria & WELTU box 94 ; Holland. \\
\hline NUNG & 25 & Lythraceae & Lythrum salicaria & $\begin{array}{l}\text { Stevenson, Newcastle Upon Tyne } \\
\text { University, England. }\end{array}$ \\
\hline VUWG & 880 & Lythraceae & Lythrum virgatum & WELTU box 94; Switzerland. \\
\hline VUWG & 838 & Malvaceae & Abutilon indicum & WELTU box 93; Okhla, India. \\
\hline VUWG & 756 & Malvaceae & Hibiscus trionum & $\begin{array}{l}\text { Chester 228. Asprokampos, } \\
\text { Grevena Pr., NW Greece. }\end{array}$ \\
\hline VUWG & 534 & Malvaceae & Lavatera arborea & $\begin{array}{l}\text { WELT 63504; Buckle St, } \\
\text { Wellington, N.Z. }\end{array}$ \\
\hline VUWG & 535 & Malvaceae & Lavatera arborea & $\begin{array}{l}\text { WELT no \#; Buckle St, } \\
\text { Wellington, N.Z. }\end{array}$ \\
\hline VUWG & 536 & Malvaceae & Lavatera arborea & $\begin{array}{l}\text { WELT } 61270 ; \text { Happy Valley Rd, } \\
\text { Wellington, N.Z. }\end{array}$ \\
\hline VUWG & 537 & Malvaceae & Lavatera cretica & $\begin{array}{l}\text { WELT 70895; Pukerua Bay, } \\
\text { Wellington, N.Z. }\end{array}$ \\
\hline VUWG & 538 & Malvaceae & Lavatera cretica & $\begin{array}{l}\text { WELT 70666; Milford, Waitemata } \\
\text { Co., N.Z. }\end{array}$ \\
\hline VUWG & 539 & Malvaceae & Lavatera cretica & $\begin{array}{l}\text { WELT 62656; Waimauku, West } \\
\text { Auckland, N.Z. }\end{array}$ \\
\hline NUNG & 150 & Malvaceae & Malva alcea & $\begin{array}{l}\text { Stevenson, Newcastle Upon Tyne } \\
\text { University, England. }\end{array}$ \\
\hline VUWG & 540 & Malvaceae & Malva nicaeensis & $\begin{array}{l}\text { WELT 70473; Pukerua Bay, } \\
\text { Wellington, N.Z. }\end{array}$ \\
\hline VUWG & 541 & Malvaceae & Malva nicaeensis & $\begin{array}{l}\text { WELT 63494; Wellington City, } \\
\text { N.Z. }\end{array}$ \\
\hline VUWG & 542 & Malvaceae & Malva nicaeensis & $\begin{array}{l}\text { WELT 63468; Te Awanga, } \\
\text { Hawkes Bay, N.Z. }\end{array}$ \\
\hline VUWG & 841 & Malvaceae & Malva pusilla & WELTU box 93; Holland. \\
\hline VUWG & 543 & Malvaceae & Malva sylvestris & $\begin{array}{l}\text { WELT 63499; Rongotai, } \\
\text { Wellington, N.Z. }\end{array}$ \\
\hline VUWG & 544 & Malvaceae & Malva sylvestris & $\begin{array}{l}\text { WELT 60488; nr Paekakariki, } \\
\text { Wellington, N.Z. }\end{array}$ \\
\hline VUWG & 545 & Malvaceae & Malva sylvestris & $\begin{array}{l}\text { WELT 63232; Main Road, } \\
\text { Gisborne, N.Z. }\end{array}$ \\
\hline VUWG & 839 & Malvaceae & Malva sylvestris & WELTU box 93. \\
\hline
\end{tabular}




\begin{tabular}{|c|c|c|c|c|}
\hline $\begin{array}{l}\text { Pollen/sp } \\
\text { reference } \\
\text { slide \# }\end{array}$ & & Family & Genus \& species & $\begin{array}{l}\text { Collector (if known)/herbarium, } \\
\text { herbarium \# (if known), } \\
\text { place of cullection }\end{array}$ \\
\hline NUNG & 211 & Malvaceae & Malvella shererdiana & $\begin{array}{l}\text { Stevenson, Newcastle Upon Tyne } \\
\text { University, England. }\end{array}$ \\
\hline VUWG & 842 & Malvaceae & Sidalcea oregana & $\begin{array}{l}\text { WELTU box } 93 \text {; Enzler 152, nr } \\
\text { Placerville, California. }\end{array}$ \\
\hline VUWG & 415 & Meliaceae & Melia azederach & $\begin{array}{l}\text { WELT no \#; Auckland Domain, } \\
\text { N.Z. }\end{array}$ \\
\hline VUWG & 574 & Menyanthaceae & Nymphoides ?peltata or ?alba & $\begin{array}{l}\text { WELT 792; Herb. University of } \\
\text { California, USA. }\end{array}$ \\
\hline VUWG & 427 & Moraceae & Morus alba & WELT no \#; Transylvania. \\
\hline NUNG & 253 & Musaceae & Musa sp. & $\begin{array}{l}\text { Stevenson, Newcastle Upon Tyne } \\
\text { University, England. }\end{array}$ \\
\hline NUNG & 159 & Myoporaceae & Myoporum tenuifolium & $\begin{array}{l}\text { Stevenson, Newcastle Upon Tyne } \\
\text { University, England. }\end{array}$ \\
\hline VUWG & 882 & Myrtaceae & Eucalyptus stuartiana & WELTU box 94; NSW, Australia. \\
\hline VUWG & 1376 & Myrtaceae & Eucalyptus sp. & $\begin{array}{l}\text { WELTU 10356; Wellington } \\
\text { Botanic Gardens, N.Z. }\end{array}$ \\
\hline VUWG & 1377 & Myrtaceae & Eucalyptus sp. & $\begin{array}{l}\text { WELTU 10355; Wellington } \\
\text { Botanic Gardens, N.Z. }\end{array}$ \\
\hline VUWG & 546 & Myrtaceae & Myrtus communis & WELT 62448; Wellington, N.Z. \\
\hline VUWG & 547 & Myrtaceae & Myrtus communis & WELT 62449; Wellington, N.Z. \\
\hline VUWG & 390 & Oleaceae & Jasminium fruticans & WELT no \#. \\
\hline VUWG & 391 & Oleaceae & Jasminium fruticans & WELT no\#. \\
\hline NUNG & 95 & Oleaceae & Jasminum nudiflorum & $\begin{array}{l}\text { Stevenson, Newcastle Upon Tyne } \\
\text { University, England. }\end{array}$ \\
\hline VUWG & 831 & Oleaceae & Jasminum ?polyanthum & $\begin{array}{l}\text { Chester } 352 \text {. Victoria University, } \\
\text { Wellington, N.Z. }\end{array}$ \\
\hline DUDB & 308 & Oleaceae & Ligustrum cf. lucidum & $\begin{array}{l}\text { Huntley, Durham University, } \\
\text { England. }\end{array}$ \\
\hline VUWG & 388 & Oleaceae & Ligustrum ovalifolium & $\begin{array}{l}\text { WELT 60455; S Karori Rd, } \\
\text { Wellington, N.Z. }\end{array}$ \\
\hline VUWG & 387 & Oleaceae & Ligustrum sinense & $\begin{array}{l}\text { WELT } 63497 \text {; Raroa Rd, Kelburn, } \\
\text { Wellington, N.Z. }\end{array}$ \\
\hline VUWG & 1397 & Oleaceae & Ligustrum vulgare & $\begin{array}{l}\text { WELTU; Victoria University, } \\
\text { Wellington, N.Z. }\end{array}$ \\
\hline VUWG & 389 & Oleaceae & Ligustrum vulgare & $\begin{array}{l}\text { WELT 45560; Porirua, } \\
\text { Wellington, N.Z. }\end{array}$ \\
\hline VUWG & 955 & Oleaceae & Ligustrum vulgare & WELTU box 97 ; Holland. \\
\hline NUNG & 193 & Oleaceae & Olea europaea & $\begin{array}{l}\text { Stevenson, Newcastle Upon Tyne } \\
\text { University, England. }\end{array}$ \\
\hline NUNG & 230 & Oleaceae & Olea europaea & $\begin{array}{l}\text { Stevenson, Newcastle Upon Tyne } \\
\text { University, England. }\end{array}$ \\
\hline CRYG & 292 & Oleaceae & Olea europaea & $\begin{array}{l}\text { Atherden, College of Rippon \& } \\
\text { York, England. }\end{array}$ \\
\hline NUNG & 82 & Oleaceae & Olea euripaea & $\begin{array}{l}\text { Stevenson, Newcastle Upon Tyne } \\
\text { University, England. }\end{array}$ \\
\hline NUNG & 187 & Oleaceae & Phillyrea angustifolia & $\begin{array}{l}\text { Stevenson, Newcastle Upon Tyne } \\
\text { University, England. }\end{array}$ \\
\hline NUNG & 240 & Oleaceae & Phillyrea angustifolia & $\begin{array}{l}\text { Stevenson, Newcastle Upon Tyne } \\
\text { University, England. }\end{array}$ \\
\hline NUNG & 37 & Oleaceae & Phillyrea angustifolia & $\begin{array}{l}\text { Stevenson, Newcastle Upon Tyne } \\
\text { University, England. }\end{array}$ \\
\hline
\end{tabular}




\begin{tabular}{|c|c|c|c|c|}
\hline $\begin{array}{l}\text { Pollen/s } \\
\text { referenc } \\
\text { slide \# }\end{array}$ & & Family & Genus \& species & $\begin{array}{l}\text { Collector (if known)/herbarium, } \\
\text { herbarium \# (if known), } \\
\text { place of collection }\end{array}$ \\
\hline NUNG & 243 & Oleaceae & Phillyrea angustifolia & $\begin{array}{l}\text { Stevenson, Newcastle Upon Tyne } \\
\text { University, England. }\end{array}$ \\
\hline NUNG & 35 & Oleaceae & Phillyrea latifolia & $\begin{array}{l}\text { Stevenson, Newcastle Upon Tyne } \\
\text { University, England. }\end{array}$ \\
\hline NUNG & 57 & Oleaceae & Phillyrea latifolia & $\begin{array}{l}\text { Stevenson, Newcastle Upon Tyne } \\
\text { University, England. }\end{array}$ \\
\hline CRYG & 293 & Oleaceae & Phillyrea latifolia & $\begin{array}{l}\text { Atherden, College of Rippon \& } \\
\text { York, England. }\end{array}$ \\
\hline NUNG & 81 & Oleaceae & Phillyrea latifolia & $\begin{array}{l}\text { Stevenson, Newcastle Upon Tyne } \\
\text { University, England. }\end{array}$ \\
\hline DUDB & 312 & Oleaceae & Phillyrea cf. media & $\begin{array}{l}\text { Huntley, Durham University, } \\
\text { England. }\end{array}$ \\
\hline VUWG & 589 & Onagraceae & Circaea lutetiana & $\begin{array}{l}\text { WELT no \#; nr Nysted, Lolland, } \\
\text { Denmark. }\end{array}$ \\
\hline VUWG & 1381 & Onagraceae & Epilobium adenocaulon & WELTU; Waikanae, N.Z. \\
\hline VUWG & 651 & Onagraceae & Epilobium ?adenocaulon & $\begin{array}{l}\text { Chester 13. Anelia, Grevena Pr., } \\
\text { NW Greece. }\end{array}$ \\
\hline VUWG & 667 & Onagraceae & Epilobium cf. adenocaulon & $\begin{array}{l}\text { Chester 29. Kellia, Grevena Pr., } \\
\text { NW Greece. }\end{array}$ \\
\hline VUWG & 584 & Onagraceae & Epilobium angustifolium & WELT no \#; Japan. \\
\hline VUWG & 883 & Onagraceae & Epilobium angustifolium & $\begin{array}{l}\text { WELTU 9385; Yellowstone Park, } \\
\text { USA. }\end{array}$ \\
\hline VUWG & 585 & Onagraceae & Epilobium collinum & $\begin{array}{l}\text { WELT no \#; nr Strommen, } \\
\text { Ostergotland, Sweden }\end{array}$ \\
\hline VUWG & 666 & Onagraceae & Epilobium hirsutum & $\begin{array}{l}\text { Chester 28. Kellia, Grevena Pr., } \\
\text { NW Greece. }\end{array}$ \\
\hline VUWG & 881 & Onagraceae & Epilobium hirsutum & WELTU box 94 ; Holland. \\
\hline VUWG & 586 & Onagraceae & Epilobium hornemanni & $\begin{array}{l}\text { WELT no \#; Jukkasjarvi, L. } \\
\text { Tornetrask, Sweden. }\end{array}$ \\
\hline VUWG & 593 & Onagraceae & Epilobium montanum & $\begin{array}{l}\text { WELT 40967; Campbell Island, } \\
\text { N.Z. }\end{array}$ \\
\hline VUWG & 594 & Onagraceae & Epilobium montanum & WELT 39499; Motueka, N.Z. \\
\hline VUWG & 595 & Onagraceae & Epilobium montanum & WELT 42095b; Mt Egmont, N.Z. \\
\hline VUWG & 587 & Onagraceae & Epilobium palustre & $\begin{array}{l}\text { WELT no \#; Boda, nr Grankulla, } \\
\text { Oland, Sweden. }\end{array}$ \\
\hline VUWG & 884 & Onagraceae & Epilobium palustre & WELTU box 94 ; Holland. \\
\hline VUWG & 885 & Onagraceae & Epilobium parviflorum & WELTU box 94 ; Holland. \\
\hline VUWG & 588 & Onagraceae & Epilobium rubescens & $\begin{array}{l}\text { WELT no \#; Boda, nr Grankulla, } \\
\text { Oland, Sweden. }\end{array}$ \\
\hline VUWG & 590 & Onagraceae & Fuchsia magellanica & WELT no \#; Hawaii. \\
\hline VUWG & 591 & Onagraceae & Fuchsia magellanica & WELT no \#; Hawaii. \\
\hline VUWG & 592 & Onagraceae & Oenothera biennis & WELT no \#; Lancashire, UK. \\
\hline VUWG & 314 & Orobanchaceae & Orobanche minor & $\begin{array}{l}\text { WELT 63173; Pukerua Bay, } \\
\text { Wellington, N.Z. }\end{array}$ \\
\hline VUWG & 315 & Orobanchaceae & Orobanche minor & $\begin{array}{l}\text { WELT } 61744 \text {; Otaki Forks, } \\
\text { Tararua Rge, N.Z. }\end{array}$ \\
\hline VUWG & 316 & Orobanchaceae & Orobanche minor & $\begin{array}{l}\text { WELT } 61228 \text {; Karori, Wellington, } \\
\text { N.Z. }\end{array}$ \\
\hline VUWG & 1338 & Oxalidaceae & Oxalis acetosella & WELTU; Wellington, N.Z. \\
\hline VUWG & 1337 & Oxalidaceae & Oxalis articulata & $\begin{array}{l}\text { WELTU; Victoria University, } \\
\text { Wellington, N.Z. }\end{array}$ \\
\hline VUWG & 1336 & Oxalidaceae & Oxalis pes-caprae & $\begin{array}{l}\text { WELTU; Mount St, Wellington, } \\
\text { N.Z. }\end{array}$ \\
\hline
\end{tabular}




Pollen/spore Family $\quad$ Genus \& species
reference
slide \#

slide \#

\author{
Collector (if known)/herbarium, \\ herbarium \# (if known), \\ place of collection
}

\begin{tabular}{|c|c|c|c|}
\hline VUWG & 1335 & Oxalidaceae & Oxalis rubra \\
\hline VUWG & 1056 & Oxalidaceae & Oxalis stricta \\
\hline VUWG & 571 & Oxalidaceae & Oxalis sp. \\
\hline VUWG & 572 & Oxalidaceae & Oxalis sp. \\
\hline VUWG & 573 & Oxalidaceae & Oxalis sp. \\
\hline NUNG & 194 & Paeoniaceae & Paeonia broteroi \\
\hline NUNG & 23 & Papaveraceae & Fumaria sp. \\
\hline VUWG & 995 & Papaveraceae & Papaver rhoeas \\
\hline VUWG & 994 & Papaveraceae & Papaver somniferum \\
\hline VUWG & 650 & Parnassiaceae & Parnassia palustris \\
\hline NUNG & 33 & Plantaginaceae & Plantago afra \\
\hline NUNG & 127 & Plantaginaceae & Plantago afra \\
\hline NUNG & 203 & Plantaginaceae & Plantago afra \\
\hline NUNG & 89 & Plantaginaceae & Plantago albicans \\
\hline VUWG & 1141 & Plantaginaceae & Plantago amplexicaulis \\
\hline NUNG & 155 & Plantaginaceae & Plantago arenaria \\
\hline NUNG & 111 & Plantaginaceae & Plantago arenaria \\
\hline VUWG & 179 & Plantaginaceae & Plantago australis \\
\hline VUWG & 180 & Plantaginaceae & Plantago australis \\
\hline VUWG & 181 & Plantaginaceae & Plantago australis \\
\hline VUWG & 176 & Plantaginaceae & Plantago coronopus \\
\hline VUWG & 178 & Plantaginaceae & Plantago coronopus \\
\hline NUNG & 233 & Plantaginaceae & Plantago coronopus \\
\hline NUNG & 279 & Plantaginaceae & Plantago coronopus \\
\hline NUNG & 164 & Plantaginaceae & Plantago lagopus \\
\hline VUWG & 1431 & Plantaginaceae & Plantago lanceolata \\
\hline VUWG & 183 & Plantaginaceae & Plantago lanceolata \\
\hline VUWG & 184 & Plantaginaceae & Plantago lanceolata \\
\hline VUWG & 695 & Plantaginaceae & Plantago lanceolata \\
\hline
\end{tabular}

WELTU 10342; Mount St, Wellington, N.Z. WELTU box 91; Holland. WELT 70429c; Hokio Beach, nr Levin, N.Z. WELT 70429b; Hokio Beach, nr Levin, N.Z. WELT 60884b; Waikanae, Wellington, N.Z.

Stevenson, Newcastle Upon Tyne University, England.

Stevenson, Newcastle Upon Tyne University, England.

WELTU box 97; Holland.

WELTU box 97; Holland.

Chester 12. Anelia, Grevena Pr., NW Greece.

Stevenson, Newcastle Upon Tyne University, England.

Stevenson, Newcastle Upon Tyne University, England.

Stevenson, Newcastle Upon Tyne University, England.

Stevenson, Newcastle Upon Tyne University, England.

WELTU; Roshamara Garden, India.

Stevenson, Newcastle Upon Tyne University, England.

Stevenson, Newcastle Upon Tyne University, England.

WELT (no \#); Te Rewarewa Pt, Plimmerton, N.Z.

WELT 63046; Black Rocks, N.Z. WELT 63043a; Ngaruawahia, N.Z. WELT (CHR 420600); Birdling's Flat, Canterbury, N.Z.

WELT 63039; Awatere, N.Z.

Stevenson, Newcastle Upon Tyne University, England.

Stevenson, Newcastle Upon Tyne University, England.

Stevenson, Newcastle Upon Tyne University, England.

WELTU; Victoria University,

Wellington, N.Z.

WELT 70107b; Wairau Ck

Estuary, Milford, N.Z.

WELT 60560; Sth Karori Rd,

Wellington, N.Z.

Chester 65. Mavrani, Grevena Pr., NW Greece. 


\begin{tabular}{|c|c|c|c|c|}
\hline \multicolumn{2}{|c|}{$\begin{array}{l}\text { Pollen/spore } \\
\text { reference } \\
\text { slide \# }\end{array}$} & \multirow{2}{*}{$\begin{array}{l}\text { Family } \\
\text { Plantaginaceae }\end{array}$} & \multirow{2}{*}{$\begin{array}{l}\text { Genus \& species } \\
\text { Plantago lanceolata }\end{array}$} & \multirow{2}{*}{$\begin{array}{l}\text { Collector (if known)/herbarium, } \\
\text { herbarium \# (if known), } \\
\text { place of collection }\end{array}$} \\
\hline VUWG & 697 & & & \\
\hline VUWG & 754 & Plantaginaceae & Plantago lanceolata & $\begin{array}{l}\text { Chester 226. Asprokampos, } \\
\text { Grevena Pr., NW Greece. }\end{array}$ \\
\hline NUNG & 263 & Plantaginaceae & Plantago lanceolata & $\begin{array}{l}\text { Stevenson, Newcastle Upon Tyne } \\
\text { University, England. }\end{array}$ \\
\hline VUWG & 1433 & Plantaginaceae & Plantago major & $\begin{array}{l}\text { WELTU 11264; Cape Turakirae, } \\
\text { Wellington, N.Z. }\end{array}$ \\
\hline VUWG & 185 & Plantaginaceae & Plantago major & $\begin{array}{l}\text { WELT 70837; Belmont, Hutt } \\
\text { Valley, N.Z. }\end{array}$ \\
\hline VUWG & 186 & Plantaginaceae & Plantago major & $\begin{array}{l}\text { WELT 69968; Pukerua Bay } \\
\text { Primary School, N.Z. }\end{array}$ \\
\hline VUWG & 187 & Plantaginaceae & Plantago major & $\begin{array}{l}\text { WELT 69910a; Wairau Ck } \\
\text { Estuary, Milford, N.Z. }\end{array}$ \\
\hline NUNG & 237 & Plantaginaceae & Plantago media & $\begin{array}{l}\text { Stevenson, Newcastle Upon Tyne } \\
\text { University, England. }\end{array}$ \\
\hline NUNG & 92 & Plantaginaceae & Plantago nivalis & $\begin{array}{l}\text { Stevenson, Newcastle Upon Tyne } \\
\text { University, England. }\end{array}$ \\
\hline NUNG & 9 & Plantaginaceae & Plantago serraria & $\begin{array}{l}\text { Stevenson, Newcastle Upon Tyne } \\
\text { University, England. }\end{array}$ \\
\hline VUWG & 188 & Plantaginaceae & Plantago varia & WELT 63022; Wellington, N.Z. \\
\hline VUWG & 189 & Plantaginaceae & Plantago varia & WELT 63025; Happy Valley, N.Z. \\
\hline VUWG & 190 & Plantaginaceae & Plantago varia & WELT 63026; Happy Valley, N.Z. \\
\hline CRYG & 285 & Plantaginaceae & Plantago sp. & $\begin{array}{l}\text { Atherden, College of Rippon \& } \\
\text { York, England. }\end{array}$ \\
\hline VUWG & 813 & Platanaceae & Platanus ?x hispanica & $\begin{array}{l}\text { Chester 334. Riddiford Gardens, } \\
\text { Lower Hutt, N.Z. }\end{array}$ \\
\hline NUNG & 270 & Platanaceae & Platanus orientalis & $\begin{array}{l}\text { Stevenson, Newcastle Upon Tyne } \\
\text { University, England. }\end{array}$ \\
\hline VUWG & 175 & Plumbaginaceae & Limonium sp. & $\begin{array}{l}\text { WELT 70568; Crofton Downs, } \\
\text { Wellington, N.Z. }\end{array}$ \\
\hline VUWG & 1062 & Polygalaceae & Polygala amara & WELTU box 91; Switzerland. \\
\hline VUWG & 1063 & Polygalaceae & Polygala comosa & WELTU box 91; Switzerland. \\
\hline VUWG & 1064 & Polygalaceae & Polygala serpyllifolia & WELTU box $91 ;$ Holland. \\
\hline VUWG & 1385 & Polygonaceae & Polygonum aviculare & $\begin{array}{l}\text { WELTU; Stokes Valley, Lower } \\
\text { Hutt, N.Z. }\end{array}$ \\
\hline VUWG & 1386 & Polygonaceae & Polygonum aviculare & $\begin{array}{l}\text { WELTU 11450; Cape Turakirae, } \\
\text { Wellington, N.Z. }\end{array}$ \\
\hline VUWG & 1387 & Polygonaceae & Polygonum aviculare & WELTU 10300. \\
\hline VUWG & 1388 & Polygonaceae & Polygonum aviculare & WELTU; Dunedin, N.Z. \\
\hline VUWG & 597 & Polygonaceae & Polygonum aviculare & $\begin{array}{l}\text { WELT } 62593 \text {; Keith George Park, } \\
\text { Hutt Valley, N.Z. }\end{array}$ \\
\hline VUWG & 598 & Polygonaceae & Polygonum aviculare & $\begin{array}{l}\text { WELT } 60504 \text {; S Karori, } \\
\text { Wellington, N.Z. }\end{array}$ \\
\hline VUWG & 599 & Polygonaceae & Polygonum aviculare & $\begin{array}{l}\text { WELT 62204; Pukerua Bay, } \\
\text { Wellington, N.Z. }\end{array}$ \\
\hline VUWG & 714 & Polygonaceae & Polygonum aviculare & $\begin{array}{l}\text { Chester } 100 . \text { Polineri, Grevena Pr., } \\
\text { NW Greece. }\end{array}$ \\
\hline VUWG & 752 & Polygonaceae & Polygonum aviculare & $\begin{array}{l}\text { Chester 219. Asprokampos, } \\
\text { Grevena Pr., NW Greece. }\end{array}$ \\
\hline VUWG & 874 & Polygonaceae & Polygonum aviculare & WELTU box 86; Holland. \\
\hline VUWG & 686 & Polygonaceae & Polygonum cf. aviculare & $\begin{array}{l}\text { Chester 54. Kellia, Grevena Pr., } \\
\text { NW Greece. }\end{array}$ \\
\hline
\end{tabular}




\begin{tabular}{|c|c|c|c|c|}
\hline $\begin{array}{l}\text { Pollen/s } \\
\text { referenc } \\
\text { slide \# }\end{array}$ & & Family & Genus \& species & $\begin{array}{l}\text { Collector (if known)/herbarium } \\
\text { herbarium \# (if known), } \\
\text { place of collection }\end{array}$ \\
\hline VUWG & 600 & Polygonaceae & Polygonum persicaria & $\begin{array}{l}\text { WELT } 62698 \mathrm{a} \text {; S Karori Rd, } \\
\text { Wellington, N.Z. }\end{array}$ \\
\hline VUWG & 601 & Polygonaceae & Polygonum persicaria & $\begin{array}{l}\text { WELT 62594; Keith George Park, } \\
\text { Hutt Valley, N.Z. }\end{array}$ \\
\hline VUWG & 602 & Polygonaceae & Polygonum persicaria & $\begin{array}{l}\text { WELT 62804; Pilmer Rd, } \\
\text { Gisborne, N.Z. }\end{array}$ \\
\hline NUNG & 172 & Polygonaceae & Polygonum viviparum & $\begin{array}{l}\text { Stevenson, Newcastle Upon Tyne } \\
\text { University, England. }\end{array}$ \\
\hline VUWG & 1391 & Polygonaceae & Rumex acetosella & WELTU 10296; Kaingaroa, N.Z. \\
\hline VUWG & 1392 & Polygonaceae & Rumex acetosella & WELTU 10294; Kaingaroa, N.Z. \\
\hline VUWG & 1393 & Polygonaceae & Rumex acetosella & WELTU 15314; Wellington, N.Z. \\
\hline VUWG & 875 & Polygonaceae & Rumex acetosella & WELTU 10291; Wellington, N.Z. \\
\hline NUNG & 248 & Polygonaceae & Rumex bucephalophorus & $\begin{array}{l}\text { Stevenson, Newcastle Upon Tyne } \\
\text { University, England. }\end{array}$ \\
\hline NUNG & 268 & Polygonaceae & Rumex conglomeratus & $\begin{array}{l}\text { Stevenson, Newcastle Upon Tyne } \\
\text { University, England. }\end{array}$ \\
\hline VUWG & 1406 & Polygonaceae & Rumex crispus & WELTU; Wellington, N.Z. \\
\hline DUDB & 299 & Polygonaceae & Rumex crispus & $\begin{array}{l}\text { Huntley, Durham University, } \\
\text { England. }\end{array}$ \\
\hline DUDB & 300 & Polygonaceae & Rumex crispus & $\begin{array}{l}\text { Huntley, Durham University, } \\
\text { England. }\end{array}$ \\
\hline DUDB & 303 & Polygonaceae & Rumex crispus & $\begin{array}{l}\text { Huntley, Durham University, } \\
\text { England. }\end{array}$ \\
\hline VUWG & 876 & Polygonaceae & Rumex dentatus & $\begin{array}{l}\text { WELTU box } 86 \text {; Jumna canal, } \\
\text { India. }\end{array}$ \\
\hline VUWG & 1405 & Polygonaceae & Rumex obtusifolius & WELTU; Wellington, N.Z. \\
\hline VUWG & 877 & Polygonaceae & Rumex scutatus & WELTU box $86 ;$ Switzerland. \\
\hline NUNG & 12 & Polygonaceae & Rumex tenuifolius & $\begin{array}{l}\text { Stevenson, Newcastle Upon Tyne } \\
\text { University, England. }\end{array}$ \\
\hline VUWG & 1404 & Polygonaceae & Rumex sp. & $\begin{array}{l}\text { WELTU; Endeavour Inlet, Queen } \\
\text { Charlotte Sound, N.Z. }\end{array}$ \\
\hline VUWG & 1074 & Primulaceae & Anagallis arvensis & WELTU box 91; Holland. \\
\hline VUWG & 1396 & Primulaceae & Anagallis arvensis & WELTU 10362; Dunedin, N.Z. \\
\hline VUWG & 172 & Primulaceae & Anagallis arvensis & $\begin{array}{l}\text { WELT 70791; Wakanui, } \\
\text { Ashburton, N.Z. }\end{array}$ \\
\hline VUWG & 173 & Primulaceae & Anagallis arvensis & $\begin{array}{l}\text { WELT (Ref 435532); Pukerua } \\
\text { Bay, N.Z. }\end{array}$ \\
\hline VUWG & 174 & Primulaceae & Anagallis arvensis & $\begin{array}{l}\text { WELT } 62618 \text {; Keith George Pk, } \\
\text { Hutt Valley, N.Z. }\end{array}$ \\
\hline NUNG & 69 & Primulaceae & Cyclamen hederifolium & $\begin{array}{l}\text { Stevenson, Newcastle Upon Tyne } \\
\text { University, England. }\end{array}$ \\
\hline VUWG & 708 & Primulaceae & Cyclamen sp. & $\begin{array}{l}\text { Chester 81. Polineri, Grevena Pr., } \\
\text { NW Greece. }\end{array}$ \\
\hline VUWG & 1078 & Primulaceae & Lysimachia nummularia & WELTU box 91; Holland. \\
\hline VUWG & 1076 & Primulaceae & Lysimachia thyrsiflora & $\begin{array}{l}\text { WELTU box } 91 \text {; Black Forest, } \\
\text { Germany. }\end{array}$ \\
\hline VUWG & 1077 & Primulaceae & Lysimachia vulgaris & WELTU box 91; Holland. \\
\hline VUWG & 1079 & Primulaceae & Soldanella alpina & WELTU box 91; Switzerland. \\
\hline NUNG & 204 & Primulaceae & Soldanella alpina & $\begin{array}{l}\text { Stevenson, Newcastle Upon Tyne } \\
\text { University, England. }\end{array}$ \\
\hline NUNG & 251 & Rafflesiaceae & Cytinus ruber & $\begin{array}{l}\text { Stevenson, Newcastle Upon Tyne } \\
\text { University, England. }\end{array}$ \\
\hline
\end{tabular}




\begin{tabular}{|c|c|c|c|}
\hline $\begin{array}{l}\text { Pollen/spore } \\
\text { reference } \\
\text { slide\# }\end{array}$ & Family & Genus \& species & $\begin{array}{l}\text { Collector (if known)/herbarium, } \\
\text { herbarium \# (if known), } \\
\text { place of cullection }\end{array}$ \\
\hline
\end{tabular}

\begin{tabular}{|c|c|c|c|c|}
\hline VUWG & 815 & Ranunculaceae & Aquilegia vulgaris [cultivar] & $\begin{array}{l}\text { Chester 336. Garden, Johnsonville, } \\
\text { Wellington, N.Z. }\end{array}$ \\
\hline CRYG & 290 & Ranunculaceae & Aquilegia sp. & $\begin{array}{l}\text { Atherden, College of Rippon \& } \\
\text { York, England. }\end{array}$ \\
\hline VUWG & 1296 & Ranunculaceae & Caltha palustris & $\begin{array}{l}\text { WELTU; Massey University, } \\
\text { Palmerston North, N.Z. }\end{array}$ \\
\hline VUWG & 1297 & Ranunculaceae & Clematis vitalba & WELTU 15193. \\
\hline VUWG & 908 & Ranunculaceae & Clematis vitalba & WELTU box 88 ; Holland. \\
\hline VUWG & 671 & Ranunculaceae & Consolida sp. & $\begin{array}{l}\text { Chester 33. Kellia, Grevena Pr., } \\
\text { NW Greece. }\end{array}$ \\
\hline VUWG & 751 & Ranunculaceae & Consolida sp. & $\begin{array}{l}\text { Chester 218. Asprokampos, } \\
\text { Grevena Pr., NW Greece. }\end{array}$ \\
\hline NUNG & 5 & Ranunculaceae & Consolida sp. & $\begin{array}{l}\text { Stevenson, Newcastle Upon Tyne } \\
\text { University, England. }\end{array}$ \\
\hline VUWG & 909 & Ranunculaceae & Helleborus hyemalis & WELTU box 88 ; Germany. \\
\hline VUWG & 910 & Ranunculaceae & Nigella sativa & WELTU box 88 ; Germany. \\
\hline VUWG & 676 & Ranunculaceae & Nigella sp. & $\begin{array}{l}\text { Chester } 38 . \text { Kellia, Grevena Pr., } \\
\text { NW Greece. }\end{array}$ \\
\hline VUWG & 911 & Ranunculaceae & Ranunculus acris & WELTU box 88; Holland. \\
\hline NUNG & 239 & Ranunculaceae & Ranunculus arvensis & $\begin{array}{l}\text { Stevenson, Newcastle Upon Tyne } \\
\text { University, England. }\end{array}$ \\
\hline VUWG & 914 & Ranunculaceae & Ranunculus bulbosus & WELTU box 88 ; Holland. \\
\hline NUNG & 31 & Ranunculaceae & Ranunculus bulbosus & $\begin{array}{l}\text { Stevenson, Newcastle Upon Tyne } \\
\text { University, England. }\end{array}$ \\
\hline VUWG & 807 & Ranunculaceae & Ranunculus ficaria & $\begin{array}{l}\text { Chester } 328 \text {. Wellington Botanic } \\
\text { Gardens, N.Z. }\end{array}$ \\
\hline VUWG & 915 & Ranunculaceae & Ranunculus ?hirsutus & WELTU box 88 ; Holland. \\
\hline VUWG & 916 & Ranunculaceae & Ranunculus lingua & WELTU box 88 ; Holland. \\
\hline CRYG & 280 & Ranunculaceae & Ranunculus lingua & $\begin{array}{l}\text { Atherden, College of Rippon \& } \\
\text { York, England. }\end{array}$ \\
\hline NUNG & 40 & Ranunculaceae & Ranunculus ophioglossifolius & $\begin{array}{l}\text { Stevenson, Newcastle Upon Tyne } \\
\text { University, England. }\end{array}$ \\
\hline VUWG & 1299 & Ranunculaceae & Ranunculus repens & WELTU 15195; Wellington, N.Z. \\
\hline VUWG & 1300 & Ranunculaceae & Ranunculus repens & WELTU 10262; Dunedin, N.Z. \\
\hline VUWG & 820 & Ranunculaceae & Ranunculus repens & $\begin{array}{l}\text { Chester } 341 \text {. Garden, Johnsonville, } \\
\text { Wellington, N.Z. }\end{array}$ \\
\hline VUWG & 917 & Ranunculaceae & Ranunculus repens & WELTU box 88 ; Holland. \\
\hline DUDB & 301 & Ranunculaceae & Ranunculus repens & $\begin{array}{l}\text { Huntley, Durham University, } \\
\text { England. }\end{array}$ \\
\hline VUWG & 918 & Ranunculaceae & Ranunculus sceleratus & WELTU box 88; Holland. \\
\hline NUNG & 153 & Ranunculaceae & Ranunculus sceleratus & $\begin{array}{l}\text { Stevenson, Newcastle Upon Tyne } \\
\text { University, England. }\end{array}$ \\
\hline NUNG & 216 & Ranunculaceae & Ranunculus trilobus & $\begin{array}{l}\text { Stevenson, Newcastle Upon Tyne } \\
\text { University, England. }\end{array}$ \\
\hline VUWG & 919 & Ranunculaceae & Ranunculus ?trilobus & WELTU box 88; Switzerland. \\
\hline VUWG & 920 & Ranunculaceae & Ranunculus ?trilobus & WELTU box 88 ; Switzerland. \\
\hline VUWG & 808 & Ranunculaceae & $\begin{array}{l}\text { Ranunculus sp. } \\
\text { 'Bloomingdale' [cult.] }\end{array}$ & $\begin{array}{l}\text { Chester 329. Wellington } \\
\text { Botanic Gardens, N.Z. }\end{array}$ \\
\hline VUWG & 922 & Ranunculaceae & Thalictrum aquilegifolium & ELTU box 88; Switzerland. \\
\hline VUWG & 921 & Ranunculaceae & Thalictrum flavum & WELTU box 88 . \\
\hline VUWG & 959 & Rhamnaceae & Rhamnus alaternus & WELTU 12636. \\
\hline VUWG & 412 & Rhamnaceae & Rhamnus alpina & WELT no \#; Switzerland. \\
\hline
\end{tabular}




\begin{tabular}{|c|c|c|c|}
\hline $\begin{array}{l}\text { Pollen/spore } \\
\text { reference } \\
\text { slide \# }\end{array}$ & Family & Genus \& species & $\begin{array}{l}\text { Collector (if known)/herbarium, } \\
\text { herbarium \# (if known), } \\
\text { place of collection }\end{array}$ \\
\hline
\end{tabular}

\begin{tabular}{|c|c|c|c|c|}
\hline VUWG & 413 & Rhamnaceae & Rhamnus catharticus & $\begin{array}{l}\text { WELT no \#; Cirencester, } \\
\text { Gloucester, UK. }\end{array}$ \\
\hline VUWG & 958 & Rhamnaceae & Trymalium daltonii & $\begin{array}{l}\text { WELTU box 97; Victoria, } \\
\text { Australia. }\end{array}$ \\
\hline VUWG & 724 & Rosaceae & Agrimonia eupatoria & $\begin{array}{l}\text { Chester 130. Mirsina, Grevena Pr., } \\
\text { NW Greece. }\end{array}$ \\
\hline VUWG & 971 & Rosaceae & Agrimonia eupatoria & WELTU box 97; Holland. \\
\hline VUWG & 972 & Rosaceae & Agrimonia eupatoria & WELTU box 97; Holland. \\
\hline VUWG & 973 & Rosaceae & Alchemilla alpina & WELTU box 97; Switzerland. \\
\hline VUWG & 974 & Rosaceae & Alchemilla alpina & WELTU box 97; Switzerland. \\
\hline VUWG & 976 & Rosaceae & Alchemilla xanthochlora & WELTU box 97; Switzerland. \\
\hline VUWG & 1362 & Rosaceae & Cotoneaster harroviana & WELTU; Wellington, N.Z. \\
\hline VUWG & 518 & Rosaceae & Crataegus monogyna & WELT 62493 \\
\hline VUWG & 977 & Rosaceae & Crataegus monogyna & WELTU box 97; Holland. \\
\hline NUNG & 85 & Rosaceae & Crataegus monogyna & $\begin{array}{l}\text { Stevenson, Newcastle Upon Tyne } \\
\text { University, England. }\end{array}$ \\
\hline DUDB & 307 & Rosaceae & Crataegus monogyna & $\begin{array}{l}\text { Huntley, Durham University, } \\
\text { England. }\end{array}$ \\
\hline VUWG & 978 & Rosaceae & Fragaria vesca & WELTU box 97; Finland. \\
\hline NUNG & 117 & Rosaceae & Malus sylvestris & $\begin{array}{l}\text { Stevenson, Newcastle Upon Tyne } \\
\text { University, England. }\end{array}$ \\
\hline NUNG & 67 & Rosaceae & Malus sp. & $\begin{array}{l}\text { Stevenson, Newcastle Upon Tyne } \\
\text { University, England. }\end{array}$ \\
\hline CRYG & 289 & Rosaceae & Malus sp. & $\begin{array}{l}\text { Atherden, College of Rippon \& } \\
\text { York, England. }\end{array}$ \\
\hline VUWG & 979 & Rosaceae & Potentilla anserina & WELTU box 97; Holland. \\
\hline NUNG & 17 & Rosaceae & Potentilla anserina & $\begin{array}{l}\text { Stevenson, Newcastle Upon Tyne } \\
\text { University, England. }\end{array}$ \\
\hline VUWG & 519 & Rosaceae & Potentilla argentea & WELT 70406; S Canterbury, N.Z. \\
\hline VUWG & 520 & Rosaceae & Potentilla argentea & $\begin{array}{l}\text { WELT 70392a; Clyde, Central } \\
\text { Otago, N.Z. }\end{array}$ \\
\hline NUNG & 20 & Rosaceae & Potentilla fruticosa & $\begin{array}{l}\text { Stevenson, Newcastle Upon Tyne } \\
\text { University, England. }\end{array}$ \\
\hline VUWG & 980 & Rosaceae & Potentilla montana & $\begin{array}{l}\text { WELTU box } 97 \text {; Colmar, } \\
\text { Alsace-Lorraine, France. }\end{array}$ \\
\hline VUWG & 660 & Rosaceae & Potentilla reptans & $\begin{array}{l}\text { Chester 22. Kellia, Grevena Pr., } \\
\text { NW Greece. }\end{array}$ \\
\hline VUWG & 981 & Rosaceae & Potentilla reptans & WELTU box 97; Holland. \\
\hline VUWG & 982 & Rosaceae & Potentilla supina & $\begin{array}{l}\text { WELTU box 97; Jumna River, } \\
\text { India. }\end{array}$ \\
\hline VUWG & 711 & Rosaceae & Poterium minus & $\begin{array}{l}\text { Chester 88. Polineri, Grevena Pr., } \\
\text { NW Greece. }\end{array}$ \\
\hline VUWG & 521 & Rosaceae & Prunus avium & WELT 70509; Nelson, N.Z. \\
\hline NUNG & 30 & Rosaceae & Prunus cerasifera & $\begin{array}{l}\text { Stevenson, Newcastle Upon Tyne } \\
\text { University, England. }\end{array}$ \\
\hline VUWG & 522 & Rosaceae & Prunus domestica & $\begin{array}{l}\text { WELT } 60737 \text {; Raroa Rd, } \\
\text { Wellington, N.Z. }\end{array}$ \\
\hline VUWG & 523 & Rosaceae & Prunus domestica & $\begin{array}{l}\text { WELT } 60777 \text {; S Karori Rd, } \\
\text { Wellington, N.Z. }\end{array}$ \\
\hline NUNG & 26 & Rosaceae & Prunus spinosa & $\begin{array}{l}\text { Stevenson, Newcastle Upon Tyne } \\
\text { University, England. }\end{array}$ \\
\hline DUDB & 302 & Rosaceae & Prunus sp. & $\begin{array}{l}\text { Huntley, Durham University, } \\
\text { England. }\end{array}$ \\
\hline
\end{tabular}




\begin{tabular}{|c|c|c|c|}
\hline $\begin{array}{l}\text { Pollen/spore } \\
\text { reference } \\
\text { slide \# }\end{array}$ & Family & Genus \& species & $\begin{array}{l}\text { Collector (if known)/herbarium, } \\
\text { herbarium \# (if known), } \\
\text { place of collection }\end{array}$ \\
\hline
\end{tabular}

\begin{tabular}{|c|c|c|c|c|}
\hline VUWG & 983 & Rosaceae & Rosa arizonica & $\begin{array}{l}\text { WELTU 9390; Baker Butte, } \\
\text { Arizona, USA. }\end{array}$ \\
\hline VUWG & 985 & Rosaceae & Rosa arvensis & WELTU box 97; Holland. \\
\hline VUWG & 984 & Rosaceae & Rosa canina & WELTU box 97; Holland. \\
\hline VUWG & 524 & Rosaceae & Rosa eglanteria & WELT 70818; Lake Ohau, N.Z. \\
\hline VUWG & 1363 & Rosaceae & Rosa rubiginosa & WELTU; Wellington, N.Z. \\
\hline VUWG & 1364 & Rosaceae & Rosa rubiginosa & $\begin{array}{l}\text { WELTU } 11560 \text {; Cape Turakirae, } \\
\text { Wellington, N.Z. }\end{array}$ \\
\hline VUWG & 986 & Rosaceae & Rosa rubiginosa & WELTU box 97 ; Holland. \\
\hline NUNG & 130 & Rosaceae & Rosa rugosa & $\begin{array}{l}\text { Stevenson, Newcastle Upon Tyne } \\
\text { University, England. }\end{array}$ \\
\hline VUWG & 987 & Rosaceae & Rubus adaeus & WELTU box 97; Holland. \\
\hline VUWG & 674 & Rosaceae & Rubus caesius & $\begin{array}{l}\text { Chester 36. Kellia, Grevena Pr., } \\
\text { NW Greece. }\end{array}$ \\
\hline NUNG & 83 & Rosaceae & Rubus caesius & $\begin{array}{l}\text { Stevenson, Newcastle Upon Tyne } \\
\text { University, England. }\end{array}$ \\
\hline VUWG & 1365 & Rosaceae & Rubus discolor & WELTU 10325; Dunedin, N.Z. \\
\hline VUWG & 1366 & Rosaceae & Rubus fruticosus & $\begin{array}{l}\text { WELTU; Karori Park, Wellington, } \\
\text { N.Z. }\end{array}$ \\
\hline VUWG & 525 & Rosaceae & Rubus fruticosus & $\begin{array}{l}\text { WELT 69967; Pukerua Bay, } \\
\text { Wellington, N.Z. }\end{array}$ \\
\hline VUWG & 526 & Rosaceae & Rubus fruticosus & $\begin{array}{l}\text { WELT 63491; Raroa Rd, Karori, } \\
\text { Wellington, N.Z. }\end{array}$ \\
\hline VUWG & 527 & Rosaceae & Rubus fruticosus & $\begin{array}{l}\text { WELT 62613; Keith George Park, } \\
\text { Hutt Valley, N.Z. }\end{array}$ \\
\hline VUWG & 989 & Rosaceae & Rubus fruticosus & WELTU box 97; Holland. \\
\hline NUNG & 223 & Rosaceae & Rubus fruticosus & $\begin{array}{l}\text { Stevenson, Newcastle Upon Tyne } \\
\text { University, England. }\end{array}$ \\
\hline NUNG & 225 & Rosaceae & Rubus fruticosus & $\begin{array}{l}\text { Stevenson, Newcastle Upon Tyne } \\
\text { University, England. }\end{array}$ \\
\hline VUWG & 528 & Rosaceae & Rubus laciniatus & $\begin{array}{l}\text { WELT } 62611 \text {; Keith George Park, } \\
\text { Hutt Valley, N.Z. }\end{array}$ \\
\hline VUWG & 529 & Rosaceae & Rubus laciniatus & WELT 62526; Tua marina. \\
\hline VUWG & 988 & Rosaceae & Rubus nessensis & WELTU box 97; Holland. \\
\hline VUWG & 530 & Rosaceae & Rubus ulmifolius & $\begin{array}{l}\text { WELT } 6049 \mathrm{a} \text {; Karori Cemetery, } \\
\text { Wellington, N.Z. }\end{array}$ \\
\hline VUWG & 531 & Rosaceae & Rubus ulmifolius & $\begin{array}{l}\text { WELT 78155; South Auckland, } \\
\text { N.Z. }\end{array}$ \\
\hline VUWG & 771 & Rosaceae & Rubus ulmifolius $\mathrm{x}$ caesius & $\begin{array}{l}\text { Chester 301. Mirsina, Grevena Pr., } \\
\text { NW Greece. }\end{array}$ \\
\hline VUWG & 992 & Rosaceae & Sanguisorba minor & WELTU box 97; Holland. \\
\hline VUWG & 990 & Rosaceae & Sanguisorba officinalis & WELTU box 97; Germany. \\
\hline VUWG & 991 & Rosaceae & Sanguisorba officinalis & WELTU box 97; Switzerland. \\
\hline NUNG & 199 & Rosaceae & Sorbus aucuparia & $\begin{array}{l}\text { Stevenson, Newcastle Upon Tyne } \\
\text { University, England. }\end{array}$ \\
\hline NUNG & 215 & Rosaceae & Sorbus aucuparia & $\begin{array}{l}\text { Stevenson, Newcastle Upon Tyne } \\
\text { University, England. }\end{array}$ \\
\hline VUWG & 1153 & Rubiaceae & Asperula cynanchica & WELTU; Switzerland. \\
\hline VUWG & 1154 & Rubiaceae & Asperula glaucum & WELTU; Switzerland. \\
\hline VUWG & 1144 & Rubiaceae & Galium aparine & WELTU; Holland. \\
\hline VUWG & 1145 & Rubiaceae & Galium laevipes & WELTU; Holland. \\
\hline VUWG & 1148 & Rubiaceae & Galium palustre & WELTU; Holland. \\
\hline
\end{tabular}




\begin{tabular}{|c|c|c|c|}
\hline $\begin{array}{l}\text { Pollen/spore } \\
\text { reference } \\
\text { slide \# }\end{array}$ & Family & Genus \& species & $\begin{array}{l}\text { Collector (if known)/herbarium, } \\
\text { herbarium \# (if known), } \\
\text { place of collection }\end{array}$ \\
\hline
\end{tabular}

\begin{tabular}{|c|c|c|c|c|}
\hline VUWG & 399 & Rubiaceae & Galium palustre & $\begin{array}{l}\text { WELT 70427a; Hokio Beach, } \\
\text { Levin, N.Z. }\end{array}$ \\
\hline VUWG & 400 & Rubiaceae & Galium palustre & $\begin{array}{l}\text { WELT } 70427 \mathrm{~b} \text {; Hokio Beach, } \\
\text { Levin, N.Z. }\end{array}$ \\
\hline VUWG & 673 & Rubiaceae & Galium rivale & $\begin{array}{l}\text { Chester } 35 . \text { Kellia, Grevena Pr., } \\
\text { NW Greece. }\end{array}$ \\
\hline VUWG & 680 & Rubiaceae & Galium rivale & $\begin{array}{l}\text { Chester } 42 . \text { Kellia, Grevena Pr., } \\
\text { NW Greece. }\end{array}$ \\
\hline VUWG & 780 & Rubiaceae & Galium trilobum & $\begin{array}{l}\text { WELTU 4853; Wanganui Inlet, } \\
\text { NW Nelson, N.Z. }\end{array}$ \\
\hline VUWG & 1146 & Rubiaceae & Galium uliginosum & WELTU; Holland. \\
\hline VUWG & 1147 & Rubiaceae & Galium uliginosum & WELTU; Holland. \\
\hline VUWG & 1150 & Rubiaceae & Galium verum & WELTU; Holland. \\
\hline VUWG & 1151 & Rubiaceae & Galium verum & WELTU; Switzerland. \\
\hline VUWG & 1152 & Rubiaceae & Galium verum & WELTU; Holland. \\
\hline VUWG & 401 & Rubiaceae & Sherardia arvensis & $\begin{array}{l}\text { WELT 51346; Red Rocks, } \\
\text { Wellington, N.Z. }\end{array}$ \\
\hline VUWG & 402 & Rubiaceae & Sherardia arvensis & $\begin{array}{l}\text { WELT 63309; Marsh Ave, } \\
\text { Milford, N.Z. }\end{array}$ \\
\hline VUWG & 403 & Rubiaceae & Sherardia arvensis & $\begin{array}{l}\text { WELT 63190; Pencarrow, } \\
\text { Eastbourne, N.Z. }\end{array}$ \\
\hline NUNG & 269 & Rutaceae & Citrus sp. & $\begin{array}{l}\text { Stevenson, Newcastle Upon Tyne } \\
\text { University, England. }\end{array}$ \\
\hline VUWG & 1059 & Rutaceae & Eriostemon correifolius & $\begin{array}{l}\text { WELTU box } 91 \text {; upper Yarra, } \\
\text { Victoria, Australia. }\end{array}$ \\
\hline VUWG & 1060 & Rutaceae & Phellodendron amurense & WELTU box 91; Russia. \\
\hline VUWG & 1061 & Rutaceae & Zieria laevigata & WELTU box 91; NSW, Australia. \\
\hline NUNG & 61 & Salicaceae & Populus x euramericana & $\begin{array}{l}\text { Stevenson, Newcastle Upon Tyne } \\
\text { University, England. }\end{array}$ \\
\hline NUNG & 62 & Salicaceae & Populus nigra & $\begin{array}{l}\text { Stevenson, Newcastle Upon Tyne } \\
\text { University, England. }\end{array}$ \\
\hline CRYG & 283 & Salicaceae & Populus sp. & $\begin{array}{l}\text { Atherden, College of Rippon \& } \\
\text { York, England. }\end{array}$ \\
\hline VUWG & 417 & Salicaceae & Salix cinerea & $\begin{array}{l}\text { WELT } 60865 \text {; Banks Peninsula, } \\
\text { Canterbury, N.Z. }\end{array}$ \\
\hline VUWG & 418 & Salicaceae & Salix cinerea & $\begin{array}{l}\text { WELT } 60734 ; \text { S Karori, } \\
\text { Wellington, N.Z. }\end{array}$ \\
\hline VUWG & 419 & Salicaceae & Salix fragilis & $\begin{array}{l}\text { WELT } 63335 \text {; Milford, Waitemata } \\
\text { Co., N.Z. }\end{array}$ \\
\hline VUWG & 420 & Salicaceae & Salix fragilis & WELT 63436. \\
\hline NUNG & 156 & Salicaceae & Salix herbacea & $\begin{array}{l}\text { Stevenson, Newcastle Upon Tyne } \\
\text { University, England. }\end{array}$ \\
\hline VUWG & 422 & Salicaceae & Salix purpurea & $\begin{array}{l}\text { WELT } 60716 ; \text { S Karori Stm, } \\
\text { Wellington, N.Z. }\end{array}$ \\
\hline VUWG & 863 & Salicaceae & Salix sitchensis & $\begin{array}{l}\text { WELTU 9404; Marin Co., } \\
\text { California, USA. }\end{array}$ \\
\hline VUWG & 685 & Santalaceae & Thesium sp. & $\begin{array}{l}\text { Chester 53. Kellia, Grevena Pr., } \\
\text { NW Greece. }\end{array}$ \\
\hline VUWG & 615 & Saxifragaceae & Chrysosplenium sp. & $\begin{array}{l}\text { WELT no \#; Westfalen, West } \\
\text { Germany. }\end{array}$ \\
\hline VUWG & 616 & Saxifragaceae & Deutzia sp. & WELT 8002; Levin, N.Z. \\
\hline VUWG & 617 & Saxifragaceae & Saxifraga aizoides & $\begin{array}{l}\text { WELT no \#; Vallee de la Broye, } \\
\text { Switzerland. }\end{array}$ \\
\hline
\end{tabular}




\begin{tabular}{|c|c|c|c|}
\hline $\begin{array}{l}\text { Pollen/spore } \\
\text { reference } \\
\text { slide \# }\end{array}$ & Family & Genus \& species & $\begin{array}{l}\text { Collector (if known)/herbarium, } \\
\text { herbarium \# (if known), } \\
\text { place of collection }\end{array}$ \\
\hline
\end{tabular}

\begin{tabular}{|c|c|c|c|c|}
\hline VUWG & 966 & Saxifragaceae & Saxifraga aizoides & WELTU box 97; Switzerland. \\
\hline VUWG & 967 & Saxifragaceae & Saxifraga ?aizoides & WELTU box 97; Switzerland. \\
\hline VUWG & 618 & Saxifragaceae & Saxifraga aizoon & $\begin{array}{l}\text { WELT no \#; Alpes de Bex, } \\
\text { Switzerland. }\end{array}$ \\
\hline NUNG & 152 & Saxifragaceae & Saxifraga moschata & $\begin{array}{l}\text { Stevenson; Newcastle Upon Tyne } \\
\text { University, England. }\end{array}$ \\
\hline VUWG & 969 & Saxifragaceae & Saxifraga paniculata & WELTU box 97; Switzerland. \\
\hline NUNG & 128 & Saxifragaceae & Saxifraga paniculata & $\begin{array}{l}\text { Stevenson; Newcastle Upon Tyne } \\
\text { University, England. }\end{array}$ \\
\hline VUWG & 968 & Saxifragaceae & Saxifraga ?paniculata & WELTU box 97; Switzerland. \\
\hline VUWG & 970 & Saxifragaceae & Saxifraga rotundifolia & WELTU box 97; Switzerland. \\
\hline VUWG & 620 & Saxifragaceae & Saxifraga stellaris & $\begin{array}{l}\text { WELT no \#; Jukkasjarvi, L. } \\
\text { Tornetrask, Sweden. }\end{array}$ \\
\hline NUNG & 77 & Saxifragaceae & Saxifraga sp. & $\begin{array}{l}\text { Stevenson, Newcastle Upon Tyne } \\
\text { University, England. }\end{array}$ \\
\hline VUWG & 288 & Scrophulariaceae & Antirrhinum majus & $\begin{array}{l}\text { WELT no \#; Gloucestershire, } \\
\text { England. }\end{array}$ \\
\hline VUWG & 259 & Scrophulariaceae & Antirrhinum orontium & WELT 62653; Auckland, N.Z. \\
\hline VUWG & 260 & Scrophulariaceae & Antirrhinum orontium & $\begin{array}{l}\text { WELT 63304; Selwyn Pl., Nelson } \\
\text { City, N.Z. }\end{array}$ \\
\hline VUWG & 261 & Scrophulariaceae & Cymbalaria muralis & $\begin{array}{l}\text { WELT 70167; Kahuterawa Rd, } \\
\text { Palmerston North, N.Z. }\end{array}$ \\
\hline VUWG & 262 & Scrophulariaceae & Cymbalaria muralis & $\begin{array}{l}\text { WELT } 60730 \text {; Norway St, } \\
\text { Wellington, N.Z. }\end{array}$ \\
\hline VUWG & 263 & Scrophulariaceae & Cymbalaria muralis & $\begin{array}{l}\text { WELT } 60513 \text {; Happy Valley, } \\
\text { Wellington, N.Z. }\end{array}$ \\
\hline VUWG & 280 & Scrophulariaceae & Digitalis ferruginea & WELT no \#. \\
\hline VUWG & 740 & Scrophulariaceae & Digitalis ferruginea & $\begin{array}{l}\text { Chester 163. Anelia, Grevena Pr., } \\
\text { NW Greece. }\end{array}$ \\
\hline VUWG & 1107 & Scrophulariaceae & Digitalis grandiflora & $\begin{array}{l}\text { WELTU box } 91 \text {; Black Forest, } \\
\text { Germany. }\end{array}$ \\
\hline VUWG & 281 & Scrophulariaceae & Digitalis grandiflora & WELT no \#; Transylvania. \\
\hline VUWG & 640 & Scrophulariaceae & Digitalis grandiflora & $\begin{array}{l}\text { Chester 2. Anelia, Grevena Pr., } \\
\text { NW Greece. }\end{array}$ \\
\hline VUWG & 1109 & Scrophulariaceae & Digitalis lutea & $\begin{array}{l}\text { WELTU box } 91 \text {; Black Forest, } \\
\text { Germany. }\end{array}$ \\
\hline VUWG & 282 & Scrophulariaceae & Digitalis lutea & WELT no \#. \\
\hline VUWG & 283 & Scrophulariaceae & Digitalis lutea & WELT no \#. \\
\hline VUWG & 284 & Scrophulariaceae & Digitalis lutea & WELT no \#. \\
\hline VUWG & 1108 & Scrophulariaceae & Digitalis purpurea & $\begin{array}{l}\text { WELTU box } 91 \text {; Black Forest, } \\
\text { Germany. }\end{array}$ \\
\hline VUWG & 1411 & Scrophulariaceae & Digitalis purpurea & $\begin{array}{l}\text { WELTU; Red Rocks, Wellington, } \\
\text { N.Z. }\end{array}$ \\
\hline VUWG & 1412 & Scrophulariaceae & Digitalis purpurea & $\begin{array}{l}\text { WELTU 15244; Fairlie Tce, } \\
\text { Wellington, N.Z. }\end{array}$ \\
\hline VUWG & 264 & Scrophulariaceae & Digitalis purpurea & $\begin{array}{l}\text { WELT } 63119 \text {; Mount Eden, } \\
\text { Auckland, N.Z. }\end{array}$ \\
\hline VUWG & 265 & Scrophulariaceae & Digitalis purpurea & $\begin{array}{l}\text { WELT 60516; Happy Valley, } \\
\text { Wellington, N.Z. }\end{array}$ \\
\hline VUWG & 266 & Scrophulariaceae & Digitalis purpurea & $\begin{array}{l}\text { WELT } 70093 \text { b; Waiwera, Rodney, } \\
\text { N.Z. }\end{array}$ \\
\hline VUWG & 285 & Scrophulariaceae & Digitalis purpurea & WELT no \#. \\
\hline VUWG & 286 & Scrophulariaceae & Digitalis purpurea & WELT no \#; Durham, England. \\
\hline
\end{tabular}




\begin{tabular}{|c|c|c|c|}
\hline $\begin{array}{l}\text { Pollen/spore } \\
\text { reference } \\
\text { slide \# }\end{array}$ & Family & Genus \& species & $\begin{array}{l}\text { Collector (if known)/herbarium, } \\
\text { herbarium \# (if known), } \\
\text { place of collection }\end{array}$ \\
\hline
\end{tabular}

\begin{tabular}{|c|c|c|c|c|}
\hline VUWG & 287 & Scrophulariaceae & Digitalis purpurea & WELT no \#; Norway. \\
\hline NUNG & 134 & Scrophulariaceae & Digitalis sp. & $\begin{array}{l}\text { Stevenson, Newcastle Upon Tyne } \\
\text { University, England. }\end{array}$ \\
\hline VUWG & 294 & Scrophulariaceae & Erimus alpinus & WELT no \#; Switzerland. \\
\hline VUWG & 1111 & Scrophulariaceae & Euphrasia micrantha & WELTU box 91; Holland. \\
\hline VUWG & 1110 & Scrophulariaceae & Euphrasia rostkoviana & WELTU box 91; Holland. \\
\hline VUWG & 289 & Scrophulariaceae & Euphrasia salisburgensis & WELT no \#; Switzerland. \\
\hline VUWG & 1113 & Scrophulariaceae & Euphrasia sp. & WELTU box 91 ; Switzerland. \\
\hline VUWG & 649 & Scrophulariaceae & Euphrasia sp. & $\begin{array}{l}\text { Chester 11. Anelia, Grevena Pr., } \\
\text { NW Greece. }\end{array}$ \\
\hline NUNG & 74 & Scrophulariaceae & Euphrasia sp. & $\begin{array}{l}\text { Stevenson, Newcastle Upon Tyne } \\
\text { University, England. }\end{array}$ \\
\hline VUWG & 293 & Scrophulariaceae & Gratiola officinalis & WELT no \#; Prussia. \\
\hline VUWG & 678 & Scrophulariaceae & Kickxia elatine & $\begin{array}{l}\text { Chester } 40 . \text { Kellia, Grevena Pr., } \\
\text { NW Greece. }\end{array}$ \\
\hline VUWG & 681 & Scrophulariaceae & Kickxia spuria & $\begin{array}{l}\text { Chester } 47 . \text { Kellia, Grevena Pr., } \\
\text { NW Greece. }\end{array}$ \\
\hline VUWG & 1114 & Scrophulariaceae & Linaria alpina & WELTU box 91; Switzerland. \\
\hline VUWG & 290 & Scrophulariaceae & Linaria alpina & WELT no \#; Bavarian Alps. \\
\hline VUWG & 269 & Scrophulariaceae & Linaria arvensis & $\begin{array}{l}\text { WELT } 70401 \text {; Middleton, } \\
\text { Christchurch, N.Z. }\end{array}$ \\
\hline VUWG & 291 & Scrophulariaceae & Linaria arvensis & WELT no \#; Transylvania. \\
\hline VUWG & 292 & Scrophulariaceae & Linaria arvensis & WELT no \#. \\
\hline VUWG & 268 & Scrophulariaceae & Linaria genistifolia dalmatica & $\begin{array}{l}\text { WELT 70727; Govt Gardens, } \\
\text { Queenstown, N.Z. }\end{array}$ \\
\hline VUWG & 1414 & Scrophulariaceae & Linaria purpurea & WELTU; Wellington, N.Z. \\
\hline VUWG & 1415 & Scrophulariaceae & Linaria purpurea & WELTU; Wellington, N.Z. \\
\hline VUWG & 270 & Scrophulariaceae & Linaria vulgaris & $\begin{array}{l}\text { WELT 70729; Arrowtown, } \\
\text { Queenstown, N.Z. }\end{array}$ \\
\hline VUWG & 1413 & Scrophulariaceae & Linaria sp. & $\begin{array}{l}\text { WELTU 15315; Stokes Valley, } \\
\text { Lower Hutt, N.Z. }\end{array}$ \\
\hline NUNG & 206 & Scrophulariaceae & Mimulus luteus & $\begin{array}{l}\text { Stevenson, Newcastle Upon Tyne } \\
\text { University, England. }\end{array}$ \\
\hline VUWG & 296 & Scrophulariaceae & Odontites lutea & WELT no \#; Florence, Italy. \\
\hline VUWG & 1112 & Scrophulariaceae & Odontites verna & WELTU box 91; Holland. \\
\hline VUWG & 295 & Scrophulariaceae & Odontites verna & WELT no \#. \\
\hline VUWG & 272 & Scrophulariaceae & Parentucellia viscosa & $\begin{array}{l}\text { WELT 61777; Castlepoint, } \\
\text { Wairarapa, N.Z. }\end{array}$ \\
\hline VUWG & 273 & Scrophulariaceae & Parentucellia viscosa & $\begin{array}{l}\text { WELT 63134; Pukerua Bay, } \\
\text { Wellington, N.Z. }\end{array}$ \\
\hline VUWG & 274 & Scrophulariaceae & Parentucellia viscosa & $\begin{array}{l}\text { WELT 60509; Crofton Downs, } \\
\text { Wellington, N.Z. }\end{array}$ \\
\hline VUWG & 1115 & Scrophulariaceae & Pedicularis ?alpina & WELTU box 91; Switzerland. \\
\hline VUWG & 1117 & Scrophulariaceae & Pedicularis sylvatica & WELTU box 91; Holland. \\
\hline VUWG & 297 & Scrophulariaceae & Pedicularis verticillata & WELT no \#; Hungary. \\
\hline VUWG & 298 & Scrophulariaceae & Pedicularis verticillata & WELT no \#; Switzerland. \\
\hline VUWG & 1118 & Scrophulariaceae & Rhinanthus sp. & WELTU box 91; Holland. \\
\hline NUNG & 168 & Scrophulariaceae & Scrophularia auriculata & $\begin{array}{l}\text { Stevenson, Newcastle Upon Tyne } \\
\text { University, England. }\end{array}$ \\
\hline VUWG & 1119 & Scrophulariaceae & Scrophularia canina & $\begin{array}{l}\text { WELTU box } 91 \text {; nr Neu Breenach, } \\
\text { Rhineland, Germany. }\end{array}$ \\
\hline VUWG & 299 & Scrophulariaceae & Scrophularia canina & WELT no \#. \\
\hline VUWG & 300 & Scrophulariaceae & Scrophularia nodosa & WELT no \#; Transylvania. \\
\hline
\end{tabular}




\begin{tabular}{|c|c|c|c|}
\hline $\begin{array}{l}\text { Pollen/spore } \\
\text { reference } \\
\text { slide \# }\end{array}$ & Family & Genus \& species & $\begin{array}{l}\text { Collector (if known)/herbarium, } \\
\text { herbarium \# (if known), } \\
\text { place of collection }\end{array}$ \\
\hline
\end{tabular}

\begin{tabular}{|c|c|c|c|c|}
\hline VUWG & 301 & Scrophulariaceae & Scrophularia nodosa & WELT no \#. \\
\hline VUWG & 302 & Scrophulariaceae & Scrophularia nodosa & $\begin{array}{l}\text { WELT no \#; Dudstone Blantyre, } \\
\text { UK. }\end{array}$ \\
\hline VUWG & 303 & Scrophulariaceae & Scrophularia peregrina & WELT no \#. \\
\hline VUWG & 1120 & Scrophulariaceae & Verbascum blattaria & WELTU box 91; Alsace, France. \\
\hline VUWG & 1416 & Scrophulariaceae & Verbascum blattaria & WELTU 10388; West Taieri, N.Z. \\
\hline VUWG & 275 & Scrophulariaceae & Verbascum blattaria & WELT 63126. \\
\hline VUWG & 305 & Scrophulariaceae & Verbascum blattaria & WELT no \#; Vienna, Austria. \\
\hline VUWG & 306 & Scrophulariaceae & Verbascum chaixii & WELT no \#; Vienna, Austria. \\
\hline VUWG & 1121 & Scrophulariaceae & Verbascum lychnitis & WELTU box 91; Holland. \\
\hline VUWG & 736 & Scrophulariaceae & Verbascum macrurum & $\begin{array}{l}\text { Chester 159. Anelia, Grevena Pr., } \\
\text { NW Greece. }\end{array}$ \\
\hline VUWG & 1122 & Scrophulariaceae & Verbascum nigrum & WELTU box 91; Holland. \\
\hline VUWG & 1123 & Scrophulariaceae & Verbascum nigrum & WELTU box 91; Holland. \\
\hline VUWG & 1124 & Scrophulariaceae & Verbascum thapsus & $\begin{array}{l}\text { WELTU box 91; Kowai Valley, } \\
\text { N.Z. }\end{array}$ \\
\hline VUWG & 277 & Scrophulariaceae & Verbascum thapsus & $\begin{array}{l}\text { WELT 62622; Keith George Park, } \\
\text { Hutt Valley, N.Z. }\end{array}$ \\
\hline VUWG & 278 & Scrophulariaceae & Verbascum thapsus & $\begin{array}{l}\text { WELT 63128; Pukerua Bay, } \\
\text { Wellington, N.Z. }\end{array}$ \\
\hline VUWG & 279 & Scrophulariaceae & Verbascum thapsus & $\begin{array}{l}\text { WELT } 60512 \text {; Makara Beach, } \\
\text { Wellington, N.Z. }\end{array}$ \\
\hline VUWG & 249 & Scrophulariaceae & Veronica agrestis & WELT 63090; Hawkes Bay, N.Z. \\
\hline NUNG & 180 & Scrophulariaceae & Veronica alpina & $\begin{array}{l}\text { Stevenson, Newcastle Upon Tyne } \\
\text { University, England. }\end{array}$ \\
\hline VUWG & 1125 & Scrophulariaceae & Veronica alpina & WELTU box 91; Switzerland. \\
\hline VUWG & 246 & Scrophulariaceae & Veronica anagallis-aquatica & $\begin{array}{l}\text { WELT 70762; Waipatiki Bush } \\
\text { Reserve, Napier, N.Z. }\end{array}$ \\
\hline VUWG & $247 a$ & Scrophulariaceae & Veronica anagallis-aquatica & $\begin{array}{l}\text { WELT 69901; Rainbow Springs, } \\
\text { Rotorua, N.Z. }\end{array}$ \\
\hline VUWG & 248 & Scrophulariaceae & Veronica anagallis-aquatica & $\begin{array}{l}\text { WELT 8406; Te Araroa Swamp, } \\
\text { N.Z. }\end{array}$ \\
\hline VUWG & 1126 & Scrophulariaceae & Veronica anagallis-aquatica & WELTU box 91; Okhla, India. \\
\hline VUWG & 309 & Scrophulariaceae & Veronica austriaca & WELT no \#; Hungary. \\
\hline VUWG & 310 & Scrophulariaceae & Veronica austriaca & WELT no \#; Transylvania. \\
\hline VUWG & 311 & Scrophulariaceae & Veronica austriaca & WELT no \#; Transylvania. \\
\hline VUWG & 1131 & Scrophulariaceae & $\begin{array}{l}\text { Veronica austriaca } \\
\text { subsp. teucrium }\end{array}$ & WELTU; Germany. \\
\hline VUWG & 307 & Scrophulariaceae & Veronica beccabunga & WELT no \#; Hungary. \\
\hline VUWG & 1128 & Scrophulariaceae & Veronica chamaedrys & WELTU; Holland. \\
\hline NUNG & 182 & Scrophulariaceae & Veronica chamaedrys & $\begin{array}{l}\text { Stevenson, Newcastle Upon Tyne } \\
\text { University, England. }\end{array}$ \\
\hline VUWG & 1129 & Scrophulariaceae & Veronica ?officinalis & WELTU; Holland. \\
\hline VUWG & 253 & Scrophulariaceae & Veronica persica & $\begin{array}{l}\text { WELT } 60517 \text {; Karori, Wellington, } \\
\text { N.Z. }\end{array}$ \\
\hline VUWG & 254 & Scrophulariaceae & Veronica persica & $\begin{array}{l}\text { WELT } 60701 \text {; Sth Karori Rd, } \\
\text { Wellington, N.Z. }\end{array}$ \\
\hline VUWG & 255 & Scrophulariaceae & Veronica persica & $\begin{array}{l}\text { WELT 70139a; Milford, } \\
\text { Waitemata Co., N.Z. }\end{array}$ \\
\hline VUWG & 313 & Scrophulariaceae & Veronica persica & WELT no \#. \\
\hline VUWG & 1417 & Scrophulariaceae & Veronica serpyllifolia & WELTU 13082. \\
\hline VUWG & 1418 & Scrophulariaceae & Veronica serpyllifolia & WELTU 10389; Taieri Plain, N.Z. \\
\hline
\end{tabular}




\begin{tabular}{|c|c|c|c|}
\hline $\begin{array}{l}\text { Pollen/spore } \\
\text { reference } \\
\text { slide \# }\end{array}$ & Family & Genus \& species & $\begin{array}{l}\text { Collector (if known)/herbarium, } \\
\text { herbarium \# (if known), } \\
\text { place of collection }\end{array}$ \\
\hline
\end{tabular}

\begin{tabular}{|c|c|c|c|}
\hline VUWG & 257 & Scrophulariaceae & Veronica serpyllifolia \\
\hline VUWG & 258 & Scrophulariaceae & Veronica serpyllifolia \\
\hline VUWG & 312 & Scrophulariaceae & Veronica triphyllos \\
\hline NUNG & 71 & Solanaceae & Cestrum parqui \\
\hline NUNG & 106 & Solanaceae & Datura stramonium \\
\hline VUWG & 234 & Solanaceae & Hyoscyamus niger \\
\hline VUWG & 782 & Solanaceae & Solanum aviculare \\
\hline VUWG & 1132 & Solanaceae & Solanum dulcamara \\
\hline VUWG & 1134 & Solanaceae & Solanum nigrum \\
\hline VUWG & 1135 & Solanaceae & Solanum nigrum \\
\hline VUWG & 1407 & Solanaceae & Solanum nigrum \\
\hline VUWG & 1408 & Solanaceae & Solanum nigrum \\
\hline VUWG & 1409 & Solanaceae & Solanum nigrum \\
\hline VUWG & 1410 & Solanaceae & Solanum nigrum \\
\hline VUWG & 230 & Solanaceae & Solanum nigrum \\
\hline VUWG & 231 & Solanaceae & Solanum nigrum \\
\hline VUWG & 232 & Solanaceae & Solanum nigrum \\
\hline VUWG & 233 & Solanaceae & Solanum nigrum \\
\hline VUWG & 757 & Solanaceae & Solanum nigrum \\
\hline NUNG & 36 & Tamaricaceae & Tamarix africana \\
\hline NUNG & 56 & Tamaricaceae & Tamarix africana \\
\hline VUWG & 856 & Tamaricaceae & Tamarix articulata \\
\hline VUWG & 857 & Tamaricaceae & Tamarix gallica \\
\hline VUWG & 878 & Thymelaeaceae & Daphne cneorum \\
\hline NUNG & 70 & Thymelaeaceae & Daphne gnidioides \\
\hline VUWG & 596 & Thymelaeaceae & Daphne striata \\
\hline NUNG & 229 & Thymelaeaceae & Thymelaea hirsuta \\
\hline NUNG & 184 & Tiliaceae & Tilia cordata \\
\hline VUWG & 532 & Tiliaceae & Tilia europaea \\
\hline VUWG & 837 & Tiliaceae & Tilia manshurica \\
\hline VUWG & 533 & Tiliaceae & Tilia vulgaris \\
\hline VUWG & 1517 & Tiliaceae & Tilia $\mathrm{x}$ vulgaris \\
\hline
\end{tabular}

WELT 63062; Raroa Reserve, Pukerua Bay, N.Z.

WELT 60874; Sth Karori Rd, Wellington, N.Z.

WELT no \#; Hungary.

Stevenson, Newcastle Upon Tyne University, England.

Stevenson, Newcastle Upon Tyne University, England.

WELT 60996; Wellington, N.Z. WELT 5926; Te Akatea, Nelson, N.Z.

WELTU; Holland.

WELTU; Holland.

WELTU; Holland.

WELTU 15178; Victoria

University, Wellington, N.Z.

WELTU 13582; Titahi Bay,

Wellington, N.Z.

WELTU 8765; Middle Trio Island, Cook Strait, N.Z.

WELTU 10377; Makara,

Wellington, N.Z.

WELT 70800; Belmont, Hutt

Valley, N.Z.

WELT 23512

WELT 70168a; Kahuteraura Rd,

Tararua Ra., N.Z.

WELT 70168b; Kahuteraura Rd, Tararua Ra., N.Z.

Chester 230. Asprokampos,

Grevena Pr., NW Greece.

Stevenson, Newcastle Upon Tyne University, England.

Stevenson, Newcastle Upon Tyne

University, England.

WELTU box 93; India.

WELTU box 93; India.

WELTU box 94; Black Forest,

Germany.

Stevenson, Newcastle Upon Tyne

University, England.

WELT no \#; Alpe Buffalora,

Switzerland.

Stevenson, Newcastle Upon Tyne

University, England.

Stevenson, Newcastle Upon Tyne

University, England.

WELT no \#; Denmark.

WELTU box 93; Russia.

WELT no \#.

Chester 357; St Orans College;

Lower Hutt, N.Z. 


\begin{tabular}{|c|c|c|c|c|}
\hline $\begin{array}{l}\text { Pollen/s } \\
\text { referenc } \\
\text { slide \# }\end{array}$ & $\begin{array}{l}\text { pore } \\
\text { ce }\end{array}$ & Family & Genus \& species & $\begin{array}{l}\text { Collector (if known)/herbarium, } \\
\text { herbarium \# (if known), } \\
\text { place of collection }\end{array}$ \\
\hline VUWG & 836 & Tiliaceae & Tilia $\mathrm{x}$ vulgaris & WELTU box 93; Holland. \\
\hline DUDB & 311 & Ulmaceae & Ulmus cf. canescens & $\begin{array}{l}\text { Huntley, Durham University, } \\
\text { England. }\end{array}$ \\
\hline VUWG & 1294 & Ulmaceae & Ulmus glabra & $\begin{array}{l}\text { WELTU 10301; Wellington } \\
\text { Botanic Gardens, N.Z. }\end{array}$ \\
\hline VUWG & 1293 & Ulmaceae & Ulmus sp. & WELTU; Wellington, N.Z. \\
\hline VUWG & 779 & Ulmaceae & Ulmus sp. & $\begin{array}{l}\text { WELTU 10302; Upland Rd, } \\
\text { Wellington, N.Z. }\end{array}$ \\
\hline CRYG & 282 & Ulmaceae & Ulmus sp. & $\begin{array}{l}\text { Atherden, College of Rippon \& } \\
\text { York, England. }\end{array}$ \\
\hline VUWG & 19 & Umbelliferae & Ammi majus & WELT 22887 \\
\hline VUWG & 20 & Umbelliferae & Ammi majus & $\begin{array}{l}\text { WELT } 62661 \mathrm{a} ; \text { Mt Eden, } \\
\text { Auckland, N.Z. }\end{array}$ \\
\hline VUWG & 21 & Umbelliferae & Angelica sylvestris & $\begin{array}{l}\text { WELT 70885b; Newlands, } \\
\text { Wellington, N.Z. }\end{array}$ \\
\hline VUWG & 22 & Umbelliferae & Anthriscus caucalis & $\begin{array}{l}\text { WELT 70225; Adams Estate, } \\
\text { Greendale, Canterbury, N.Z. }\end{array}$ \\
\hline VUWG & 23 & Umbelliferae & Apium graveolens & WELT 62930; Ship Cove, N.Z. \\
\hline VUWG & 24 & Umbelliferae & Apium graveolens & WELT 60852; Chatham Is., N.Z. \\
\hline VUWG & 25 & Umbelliferae & Apium nodiflorum & $\begin{array}{l}\text { WELT 70426a; Ngarara Rd, } \\
\text { Waikanae, N.Z. }\end{array}$ \\
\hline VUWG & 26 & Umbelliferae & Apium nodiflorum & WELT $70426 \mathrm{~b}$ \\
\hline VUWG & 27 & Umbelliferae & Apium nodiflorum & $\begin{array}{l}\text { WELT } 63244 \text {; Kerikeri Stm, } \\
\text { Whangarei, N.Z. }\end{array}$ \\
\hline NUNG & 232 & Umbelliferae & Apium nodiflorum & $\begin{array}{l}\text { Stevenson, Newcastle Upon Tyne } \\
\text { University, England. }\end{array}$ \\
\hline VUWG & 924 & Umbelliferae & Berula erecta & WELTU box 95; Holland. \\
\hline VUWG & 735 & Umbelliferae & Bupleurum asperuloides & $\begin{array}{l}\text { Chester 158. Mirsina, Grevena Pr., } \\
\text { NW Greece. }\end{array}$ \\
\hline VUWG & 28 & Umbelliferae & Bupleurum lancifolium & WELT 61817; Auckland, N.Z. \\
\hline VUWG & 29 & Umbelliferae & Bupleurum lancifolium & WELT 61817; Auckland, N.Z. \\
\hline VUWG & 925 & Umbelliferae & Bupleurum rotundifolium & WELTU box 95; Germany. \\
\hline VUWG & 926 & Umbelliferae & Bupleurum ?stellatum & WELTU box 95; Switzerland. \\
\hline VUWG & 30 & Umbelliferae & Conium maculatum & $\begin{array}{l}\text { WELT 69903a; Wairau Ck } \\
\text { Estuary, Milford, N.Z. }\end{array}$ \\
\hline VUWG & 31 & Umbelliferae & Conium maculatum & $\begin{array}{l}\text { WELT 69903b; Wairau Ck } \\
\text { Estuary, Milford, N.Z. }\end{array}$ \\
\hline VUWG & 32 & Umbelliferae & Coriandrum sativum & WELT 60857; near Dunedin, N.Z. \\
\hline VUWG & 1370 & Umbelliferae & Daucus carota & WELTU; Taupiri, N.Z. \\
\hline VUWG & 1371 & Umbelliferae & Daucus carota & $\begin{array}{l}\text { WELTU; Stokes Valley, Lower } \\
\text { Hutt, N.Z. }\end{array}$ \\
\hline VUWG & 1372 & Umbelliferae & Daucus carota & WELTU; Taupiri, N.Z. \\
\hline VUWG & 4 & Umbelliferae & Daucus carota & $\begin{array}{l}\text { WELT 70066; The Terrace, } \\
\text { Wellington, N.Z. }\end{array}$ \\
\hline VUWG & 664 & Umbelliferae & Daucus carota & $\begin{array}{l}\text { Chester 26. Kellia, Grevena Pr., } \\
\text { NW Greece. }\end{array}$ \\
\hline VUWG & 726 & Umbelliferae & Daucus carota & $\begin{array}{l}\text { Chester 132. Mirsina, Grevena Pr., } \\
\text { NW Greece. }\end{array}$ \\
\hline VUWG & 927 & Umbelliferae & Daucus carota & WELTU box 95; Holland. \\
\hline NUNG & 112 & Umbelliferae & Daucus carota & $\begin{array}{l}\text { Stevenson, Newcastle Upon Tyne } \\
\text { University, England. }\end{array}$ \\
\hline NUNG & 214 & Umbelliferae & Echinophora spinosa & $\begin{array}{l}\text { Stevenson, Newcastle Upon Tyne } \\
\text { University, England. }\end{array}$ \\
\hline
\end{tabular}




\begin{tabular}{|c|c|c|c|c|}
\hline \multicolumn{2}{|c|}{$\begin{array}{l}\text { Pollen/spore } \\
\text { reference } \\
\text { slide \# }\end{array}$} & Family & Genus \& species & $\begin{array}{l}\text { Collector (if known)/herbarium } \\
\text { herbarium \# (if known), } \\
\text { place of collection }\end{array}$ \\
\hline VUWG & 928 & Umbelliferae & Eryngium alpinum & WELTU box 95; Switzerland. \\
\hline VUWG & 707 & Umbelliferae & Eryngium amethystinum & $\begin{array}{l}\text { Chester } 79 . \text { Polineri, Grevena Pr., } \\
\text { NW Greece. }\end{array}$ \\
\hline VUWG & 929 & Umbelliferae & Eryngium maritimum & WELTU box 95 ; Holland. \\
\hline NUNG & 15 & Umbelliferae & Eryngium maritimum & $\begin{array}{l}\text { Stevenson, Newcastle Upon Tyne } \\
\text { University, England. }\end{array}$ \\
\hline VUWG & 5 & Umbelliferae & Foeniculum vulgare & WELT 70849; Wellington, N.Z. \\
\hline VUWG & 6 & Umbelliferae & Foeniculum vulgare & $\begin{array}{l}\text { WELT } 70372 \mathrm{a} \text {; Wairau Ck, } \\
\text { Waitemata Co, N.Z. }\end{array}$ \\
\hline VUWG & 7 & Umbelliferae & Foeniculum vulgare & $\begin{array}{l}\text { WELT 63226; nr Mangatuna, } \\
\text { Waiapu County, N.Z. }\end{array}$ \\
\hline NUNG & 235 & Umbelliferae & Foeniculum vulgare & $\begin{array}{l}\text { Stevenson, Newcastle Upon Tyne } \\
\text { University, England. }\end{array}$ \\
\hline NUNG & 86 & Umbelliferae & Ligusticum mutellina & $\begin{array}{l}\text { Stevenson, Newcastle Upon Tyne } \\
\text { University, England. }\end{array}$ \\
\hline VUWG & 9 & Umbelliferae & Oenanthe aquatica & $\begin{array}{l}\text { WELT 63247; Tua Marina, } \\
\text { Marlborough, N.Z. }\end{array}$ \\
\hline VUWG & 741 & Umbelliferae & Opopanax hispidus & $\begin{array}{l}\text { Chester 164. Mirsina, Grevena Pr., } \\
\text { NW Greece. }\end{array}$ \\
\hline VUWG & 10 & Umbelliferae & Pastinaca sativa & $\begin{array}{l}\text { WELT 61779a; Waterside Rd, } \\
\text { Greytown, N.Z. }\end{array}$ \\
\hline NUNG & 46 & Umbelliferae & Pastinaca sativa & $\begin{array}{l}\text { Stevenson, Newcastle Upon Tyne } \\
\text { University, England. }\end{array}$ \\
\hline VUWG & 11 & Umbelliferae & Petroselinum crispum & $\begin{array}{l}\text { WELT } 60417 \text {; Island Bay, } \\
\text { Wellington, N.Z. }\end{array}$ \\
\hline VUWG & 12 & Umbelliferae & Petroselinum crispum & $\begin{array}{l}\text { WELT } 60416 \text {; Sth Karori Rd, } \\
\text { Wellington, N.Z. }\end{array}$ \\
\hline NUNG & 197 & Umbelliferae & Pseudorlaya pumila & $\begin{array}{l}\text { Stevenson, Newcastle Upon Tyne } \\
\text { University, England. }\end{array}$ \\
\hline VUWG & 930 & Umbelliferae & Sanicula europaea & WELTU box 95 ; Holland. \\
\hline VUWG & 13 & Umbelliferae & Scandix geniculata & $\begin{array}{l}\text { WELT 69938; Wainuiomata, } \\
\text { Wellington, N.Z. }\end{array}$ \\
\hline VUWG & 14 & Umbelliferae & Sison amomum & $\begin{array}{l}\text { WELT } 63263 \text {; Kerikeri Stm, } \\
\text { Whangarei, N.Z. }\end{array}$ \\
\hline VUWG & 15 & Umbelliferae & Sison amomum & $\begin{array}{l}\text { WELT 59806; Waiwera Hill, } \\
\text { Auckland, N.Z. }\end{array}$ \\
\hline VUWG & 16 & Umbelliferae & Torilis arvensis & $\begin{array}{l}\text { WELT 63212; Riverside Rd, } \\
\text { Gisborne, N.Z. }\end{array}$ \\
\hline VUWG & 17 & Umbelliferae & Torilis arvensis & $\begin{array}{l}\text { WELT } 63242 ; \text { HW1, Sth of } \\
\text { Silverdale, N.Z. }\end{array}$ \\
\hline VUWG & 1373 & Umbelliferae & Torilis japonica & $\begin{array}{l}\text { WELTU; Tapuwaeroa R., East } \\
\text { Cape, N.Z. }\end{array}$ \\
\hline VUWG & 1374 & Umbelliferae & Torilis japonica & $\begin{array}{l}\text { WELTU; Tapuwaeroa R., East } \\
\text { Cape, N.Z. }\end{array}$ \\
\hline VUWG & 1375 & Umbelliferae & Torilis japonica & WELTU; Napier, N.Z. \\
\hline VUWG & 18 & Umbelliferae & Torilis japonica & $\begin{array}{l}\text { WELT 63239; Coast Nth of } \\
\text { Hundalee Hills, N.Z. }\end{array}$ \\
\hline VUWG & 663 & Umbelliferae & Torilis japonica & $\begin{array}{l}\text { Chester } 25 . \text { Kellia, Grevena Pr., } \\
\text { NW Greece. }\end{array}$ \\
\hline VUWG & 931 & Umbelliferae & Torilis japonica & WELTU box 95; Holland. \\
\hline VUWG & 865 & Urticaceae & Parietaria debilis & $\begin{array}{l}\text { WELTU box } 86 \text {; Krabe Peninsula, } \\
\text { Khasansky, Primorye, USSR }\end{array}$ \\
\hline
\end{tabular}




\begin{tabular}{|c|c|c|c|c|}
\hline \multicolumn{2}{|c|}{$\begin{array}{l}\text { Pollen/spore } \\
\text { reference } \\
\text { slide\# }\end{array}$} & \multirow{2}{*}{$\begin{array}{l}\text { Family } \\
\text { Urticaceae }\end{array}$} & \multirow{2}{*}{$\begin{array}{l}\text { Genus \& species } \\
\text { Parietaria diffusa }\end{array}$} & \multirow{2}{*}{$\begin{array}{l}\text { Collector (if known)/herbarium, } \\
\text { herbarium \# (if known), } \\
\text { place of collection }\end{array}$} \\
\hline VUWG & 408 & & & \\
\hline VUWG & 409 & Urticaceae & Parietaria diffusa & $\begin{array}{l}\text { WELT 70065a; Brooklyn, } \\
\text { Wellington, N.Z. }\end{array}$ \\
\hline VUWG & 410 & Urticaceae & Parietaria diffusa & $\begin{array}{l}\text { WELT } 60630 ; \text { Wadestown, } \\
\text { Wellington, N.Z. }\end{array}$ \\
\hline VUWG & 867 & Urticaceae & Urtica dioica & WELTU box 86 ; Holland. \\
\hline NUNG & 254 & Urticaceae & Urtica dioica & $\begin{array}{l}\text { Stevenson, Newcastle Upon Tyne } \\
\text { University, England. }\end{array}$ \\
\hline NUNG & 242 & Urticaceae & Urtica dubia & $\begin{array}{l}\text { Stevenson, Newcastle Upon Tyne } \\
\text { University, England. }\end{array}$ \\
\hline NUNG & 160 & Urticaceae & Urtica pilulifera & $\begin{array}{l}\text { Stevenson, Newcastle Upon Tyne } \\
\text { University, England. }\end{array}$ \\
\hline NUNG & 103 & Urticaceae & Urtica urens & $\begin{array}{l}\text { Stevenson, Newcastle Upon Tyne } \\
\text { University, England. }\end{array}$ \\
\hline VUWG & 411 & Urticaceae & Urtica urens (?) & $\begin{array}{l}\text { WELT 69937; Pukerua Bay, } \\
\text { Wellington, N.Z. }\end{array}$ \\
\hline VUWG & 1439 & Valerianaceae & Centranthus ruber & $\begin{array}{l}\text { WELTU; Victoria University, } \\
\text { Wellington, N.Z. }\end{array}$ \\
\hline VUWG & 1440 & Valerianaceae & Centranthus ruber & WELTU; Wellington, N.Z. \\
\hline VUWG & 50 & Valerianaceae & Centranthus ruber & $\begin{array}{l}\text { WELT 60528; Happy Valley, } \\
\text { Wellington, N.Z. }\end{array}$ \\
\hline VUWG & 51 & Valerianaceae & Centranthus ruber & $\begin{array}{l}\text { WELT 60526; Karori, Wellington, } \\
\text { N.Z. }\end{array}$ \\
\hline VUWG & 792 & Valerianaceae & Centranthus ruber & $\begin{array}{l}\text { Chester 313; Wellington Botanic } \\
\text { Gardens, N.Z. }\end{array}$ \\
\hline VUWG & 829 & Valerianaceae & Centranthus ruber & $\begin{array}{l}\text { Chester 356; Victoria University, } \\
\text { Wellington, N.Z. }\end{array}$ \\
\hline VUWG & 1159 & Valerianaceae & Centranthus sp. & WELTU. \\
\hline VUWG & 1160 & Valerianaceae & Valeriana calcitrapa & WELTU; Holland. \\
\hline VUWG & 1161 & Valerianaceae & Valeriana dioica & WELTU; Holland. \\
\hline VUWG & 1162 & Valerianaceae & Valeriana officinalis & WELTU; Holland. \\
\hline VUWG & 44 & Valerianaceae & Valerianella carinata & $\begin{array}{l}\text { WELT 61294; Mairangi Bay, } \\
\text { Waitemata Co., N.Z. }\end{array}$ \\
\hline VUWG & 45 & Valerianaceae & Valerianella carinata & $\begin{array}{l}\text { WELT 78171; Sth of Port } \\
\text { Waikato, N.Z. }\end{array}$ \\
\hline VUWG & 46 & Valerianaceae & Valerianella locusta & $\begin{array}{l}\text { WELT 63163; Three Kings Is., } \\
\text { N.Z. }\end{array}$ \\
\hline VUWG & 47 & Valerianaceae & Valerianella locusta & WELT 63161; Whakatane, N.Z. \\
\hline VUWG & 1421 & Verbenaceae & Verbena bonariensis & $\begin{array}{l}\text { WELTU 10369; Kennedy Bay, } \\
\text { Coromandel Peninsula, N.Z. }\end{array}$ \\
\hline VUWG & 322 & Verbenaceae & Verbena officinalis & $\begin{array}{l}\text { WELT } 60634 a \text {; Taihape Rd, } \\
\text { Mangaweka, N.Z. }\end{array}$ \\
\hline VUWG & 323 & Verbenaceae & Verbena officinalis & $\begin{array}{l}\text { WELT 63210; Riverside Rd, } \\
\text { Gisborne, N.Z. }\end{array}$ \\
\hline VUWG & 324 & Verbenaceae & Verbena officinalis & $\begin{array}{l}\text { WELT } 63475 \text {; Riverside Rd, } \\
\text { Havelock North, N.Z. }\end{array}$ \\
\hline VUWG & 657 & Verbenaceae & Verbena officinalis & $\begin{array}{l}\text { Chester 19. Kellia, Grevena Pr., } \\
\text { NW Greece. }\end{array}$ \\
\hline VUWG & 763 & Verbenaceae & Verbena officinalis & $\begin{array}{l}\text { Chester 250. Asprokampos, } \\
\text { Grevena Pr., NW Greece. }\end{array}$ \\
\hline VUWG & 953 & Verbenaceae & Verbena officinalis & WELTU box 97; Holland. \\
\hline VUWG & 954 & Verbenaceae & Verbena officinalis & WELTU box 97; Holland. \\
\hline
\end{tabular}




\begin{tabular}{|c|c|c|c|}
\hline $\begin{array}{l}\text { Pollen/spore } \\
\text { reference } \\
\text { slide \# }\end{array}$ & Family & Genus \& species & $\begin{array}{l}\text { Collector (if known)/herbarium, } \\
\text { herbarium \# (if known), } \\
\text { place of collection }\end{array}$ \\
\hline
\end{tabular}

\begin{tabular}{|c|c|c|c|}
\hline NUNG & 186 & Verbenaceae & Vitex agnus-castus \\
\hline VUWG & 849 & Violaceae & Viola lutea var. calaminariae \\
\hline VUWG & 850 & Violaceae & Viola palustris \\
\hline NUNG & 29 & Violaceae & Viola reichenbachiana \\
\hline VUWG & 1312 & Violaceae & Viola tricolor \\
\hline VUWG & 851 & Violaceae & Viola tricolor \\
\hline VUWG & 852 & Violaceae & Viola tricolor \\
\hline VUWG & 648 & Violaceae & Viola sp. \\
\hline VUWG & 797 & Violaceae & Viola sp. \\
\hline VUWG & 830 & Violaceae & Viola sp. [cultivar] \\
\hline VUWG & 414 & Vitaceae & Vitis vinifera \\
\hline VUWG & 835 & Vitaceae & Vitis vinifera \\
\hline NUNG & 126 & Vitaceae & Vitis vinifera \\
\hline VUWG & 558 & Zygophyllaceae & Tribulus terrestris \\
\hline
\end{tabular}

Stevenson, Newcastle Upon Tyne

University, England.

WELTU box 93; Limburg,

Holland.

WELTU box 93; Holland.

Stevenson, Newcastle Upon Tyne

University, England.

WELTU 10351.

WELTU box 93; Holland.

WELTU box 93; Holland.

Chester 10. Anelia, Grevena Pr., NW Greece.

Chester 318. Wellington Botanic

Gardens, N.Z.

Chester 351; Victoria University, Wellington, N.Z.

WELT no \#; Etrusia.

WELTU box 93; Germany.

Stevenson, Newcastle Upon Tyne

University, England.

WELT no \#; Adelaide Plains, South Australia.

\section{Monocotyledones}

\begin{tabular}{|c|c|c|c|}
\hline VUWG & 380 & Alismaceae & Alisma natans \\
\hline VUWG & 381 & Alismaceae & Alisma plantago-aquatica \\
\hline NUNG & 51 & Alismaceae & Alisma plantago-aquatica \\
\hline VUWG & 383 & Alismaceae & Sagittaria sagittifolia \\
\hline VUWG & 1276 & Amaryllidaceae & Beschorneria yuccoides \\
\hline VUWG & 1277 & Amaryllidaceae & Galanthus nivalis \\
\hline VUWG & 1516 & Araceae & Zantedeschia aethiopica \\
\hline NUNG & 221 & Cyperaceae & Carex arenaria \\
\hline VUWG & 1224 & Cyperaceae & Carex elata elata \\
\hline VUWG & 1221 & Cyperaceae & Carex flacca flacca \\
\hline VUWG & 1484 & Cyperaceae & Carex ovalis \\
\hline VUWG & 1485 & Cyperaceae & Carex ovalis \\
\hline VUWG & 1486 & Cyperaceae & Carex ovalis \\
\hline VUWG & 1222 & Cyperaceae & Carex paniculata \\
\hline VUWG & 1223 & Cyperaceae & Carex pseudocyperus \\
\hline VUWG & 1483 & Cyperaceae & Cyperus congestus \\
\hline VUWG & 1478 & Cyperaceae & Cyperus eragrostis \\
\hline VUWG & 1482 & Cyperaceae & Cyperus eragrostis \\
\hline
\end{tabular}

WELT no \#; Transylvania. WELT 60620; Hunterville, Rangitikei, N.Z.

Stevenson, Newcastle Upon Tyne University, England.

WELT no\#.

WELTU; Cerro Tres Picos, Chiapas, Mexico.

WELTU; Castle Douglas, Scotland.

Chester 356; Johnsonville, Wellington, N.Z.

Stevenson, Newcastle Upon Tyne University, England.

WELTU; Holland.

WELTU; Holland.

WELTU 14929.

WELTU 10256; Ketetahi Springs

track, N.Z.

WELTU 8930; Taurewa, N.Z.

WELTU; Holland.

WELTU; Holland.

WELTU 9023; Paturau, NW

Nelson, N.Z.

WELTU; Waikanae, N.Z.

WELTU 11490; Cape Turakirae, Wellington, N.Z. 


\begin{tabular}{|c|c|c|c|c|}
\hline $\begin{array}{l}\text { Pollen/s } \\
\text { referens } \\
\text { slide \# }\end{array}$ & $\begin{array}{l}\text { spore } \\
\text { ce }\end{array}$ & Family & Genus \& species & $\begin{array}{l}\text { Collector (if known)/herbarium, } \\
\text { herbarium \# (if known), } \\
\text { place of collection }\end{array}$ \\
\hline VUWG & 722 & Cyperaceae & Cyperus cf. longus & $\begin{array}{l}\text { Chester } 127 . \text { Mirsina, Grevena Pr., } \\
\text { NW Greece. }\end{array}$ \\
\hline VUWG & 1225 & Cyperaceae & Cyperus rotundus & WELTU; Gazibad, India. \\
\hline VUWG & 1479 & Cyperaceae & Cyperus tenellus & $\begin{array}{l}\text { WELTU 9507; Lake Taharoa, nr } \\
\text { Dargaville, N.Z. }\end{array}$ \\
\hline VUWG & 1481 & Cyperaceae & Cyperus tenellus & WELTU 406 \\
\hline VUWG & 1226 & Cyperaceae & Eriophorum angustifolium & WELTU; Holland. \\
\hline VUWG & 1227 & Cyperaceae & Scirpus holoschoenus & WELTU; Holland. \\
\hline VUWG & 1228 & Cyperaceae & Scirpus lacustris & WELTU; Holland. \\
\hline VUWG & 1229 & Cyperaceae & Scirpus maritimus & WELTU; Gazibad, India. \\
\hline VUWG & 1230 & Cyperaceae & Scirpus maritimus & WELTU; Holland. \\
\hline VUWG & 1477 & Cyperaceae & Scirpus setaceus & $\begin{array}{l}\text { WELTU 10257; Horokiwi R., } \\
\text { Pauatahanui, N.Z. }\end{array}$ \\
\hline VUWG & 1491 & Gramineae & ?Agropogon littoralis & $\begin{array}{l}\text { WELTU 15307; Vernon Lagoons, } \\
\text { Marlborough, N.Z. }\end{array}$ \\
\hline VUWG & 1232 & Gramineae & Agrostis alpina & WELTU; Switzerland. \\
\hline VUWG & 1231 & Gramineae & Agrostis ?alpina & WELTU; Switzerland. \\
\hline VUWG & 1487 & Gramineae & Agrostis stolonifera & $\begin{array}{l}\text { WELTU 15306; Waikanae R., } \\
\text { N.Z. }\end{array}$ \\
\hline VUWG & 1488 & Gramineae & Agrostis stolonifera & WELTU; Wellington, N.Z. \\
\hline VUWG & 1489 & Gramineae & Agrostis stolonifera & $\begin{array}{l}\text { WELTU 10220; Longwood Range, } \\
\text { N.Z. }\end{array}$ \\
\hline VUWG & 1235 & Gramineae & Aira caryophyllea & WELTU; Holland. \\
\hline VUWG & 1234 & Gramineae & Anthoxanthum odoratum & WELTU; Holland. \\
\hline VUWG & 1492 & Gramineae & Anthoxanthum odoratum & $\begin{array}{l}\text { WELTU; Victoria University, } \\
\text { Wellington, N.Z. }\end{array}$ \\
\hline VUWG & 1493 & Gramineae & Anthoxanthum odoratum & $\begin{array}{l}\text { WELTU 10982; Erebus Cove, } \\
\text { Auckland Island. }\end{array}$ \\
\hline NUNG & 133 & Gramineae & Anthoxanthum odoratum & $\begin{array}{l}\text { Stevenson, Newcastle Upon Tyne } \\
\text { University, England. }\end{array}$ \\
\hline VUWG & 1494 & Gramineae & Avena gonzaloi & WELTU 10226 \\
\hline VUWG & 753 & Gramineae & Avena cf. sterilis & $\begin{array}{l}\text { Chester 224. Asprokampos, } \\
\text { Grevena Pr., NW Greece. }\end{array}$ \\
\hline VUWG & 1237 & Gramineae & Brachypodium pinnatum & WELTU; Holland. \\
\hline VUWG & 1238 & Gramineae & Brachypodium sylvaticum & WELTU; Holland. \\
\hline VUWG & 1239 & Gramineae & Bromus arvensis & WELTU; Holland. \\
\hline VUWG & 1495 & Gramineae & Bromus hordaceus hordaceus & WELTU; Wellington, N.Z. \\
\hline VUWG & 1496 & Gramineae & Bromus hordaceus hordaceus & $\begin{array}{l}\text { WELTU 7722; Granity Pass, } \\
\text { Owen Range, N.Z. }\end{array}$ \\
\hline VUWG & 1240 & Gramineae & Bromus muralis & WELTU; Holland. \\
\hline VUWG & 1241 & Gramineae & Bromus sterilis & WELTU; Holland. \\
\hline VUWG & 1497 & Gramineae & Bromus sterilis & $\begin{array}{l}\text { WELTU 8790; Trio Island, Cook } \\
\text { Strait, N.Z. }\end{array}$ \\
\hline VUWG & 773 & Gramineae & Calamagrostis arundinacea & $\begin{array}{l}\text { Chester } 305 . \text { Mirsina, Grevena Pr., } \\
\text { NW Greece. }\end{array}$ \\
\hline VUWG & 1242 & Gramineae & Calamagrostis canescens & WELTU; Holland. \\
\hline VUWG & 1498 & Gramineae & Cynodon dactylon & WELTU; Wellington, N.Z. \\
\hline VUWG & 1499 & Gramineae & Cynodon dactylon & $\begin{array}{l}\text { WELTU 10229; Upland Road, } \\
\text { Wellington, N.Z. }\end{array}$ \\
\hline VUWG & 1243 & Gramineae & Cynosurus cristatus & WELTU; Holland. \\
\hline VUWG & 1500 & Gramineae & Cynosurus cristatus & WELTU; Taupo, N.Z. \\
\hline VUWG & 1501 & Gramineae & Cynosurus cristatus & WELTU. \\
\hline
\end{tabular}




\begin{tabular}{|c|c|c|c|c|}
\hline \multicolumn{2}{|c|}{$\begin{array}{l}\text { Pollen/spore } \\
\text { reference } \\
\text { slide \# }\end{array}$} & \multirow{2}{*}{$\begin{array}{l}\text { Family } \\
\text { Gramineae }\end{array}$} & \multirow{2}{*}{$\begin{array}{l}\text { Genus \& species } \\
\text { Cynosurus cristatus }\end{array}$} & \multirow{2}{*}{$\begin{array}{l}\text { Collector (if known)/herbarium, } \\
\text { herbarium \# (if known), } \\
\text { place of collection } \\
\text { Chester } 17 . \text { Anelia, Grevena Pr., } \\
\text { NW Greece. }\end{array}$} \\
\hline VUWG & 655 & & & \\
\hline NUNG & 181 & Gramineae & Cynosurus cristatus & $\begin{array}{l}\text { Stevenson, Newcastle Upon Tyne } \\
\text { University, England. }\end{array}$ \\
\hline VUWG & 1502 & Gramineae & Cynosurus echinatus & WELTU; Wellington, N.Z. \\
\hline VUWG & 712 & Gramineae & Cynosurus elegans & $\begin{array}{l}\text { Chester } 90 . \text { Polineri, Grevena Pr., } \\
\text { NW Greece. }\end{array}$ \\
\hline VUWG & 1503 & Gramineae & Dactylis glomerata & WELTU 10231 \\
\hline VUWG & 1504 & Gramineae & Dactylis glomerata & $\begin{array}{l}\text { WELTU 7708; Granity Pass, } \\
\text { Owen Range, N.Z. }\end{array}$ \\
\hline VUWG & 1252 & Gramineae & Elymus repens & WELTU; Holland. \\
\hline VUWG & 1505 & Gramineae & Festuca ovina & WELTU 10237. \\
\hline NUNG & 170 & Gramineae & Festuca ovina & $\begin{array}{l}\text { Stevenson, Newcastle Upon Tyne } \\
\text { University, England. }\end{array}$ \\
\hline VUWG & 1506 & Gramineae & Festuca rubra & $\begin{array}{l}\text { WELTU 10234; Hooker Valley, } \\
\text { Mt Cook, N.Z. }\end{array}$ \\
\hline VUWG & 1245 & Gramineae & Glyceria fluitans & WELTU; Holland. \\
\hline VUWG & 1507 & Gramineae & Glyceria fluitans & $\begin{array}{l}\text { WELTU 15320; Belmont Park, } \\
\text { Lower Hutt, N.Z. }\end{array}$ \\
\hline VUWG & 1246 & Gramineae & Holcus lanatus & WELTU; Holland. \\
\hline NUNG & 120 & Gramineae & Holcus lanatus & $\begin{array}{l}\text { Stevenson, Newcastle Upon Tyne } \\
\text { University, England. }\end{array}$ \\
\hline NUNG & 113 & Gramineae & Hordeum distichon & $\begin{array}{l}\text { Stevenson, Newcastle Upon Tyne } \\
\text { University, England. }\end{array}$ \\
\hline VUWG & 1508 & Gramineae & Hordeum marinum & WELTU; Wellington, N.Z. \\
\hline VUWG & 1244 & Gramineae & Leymus arenarius & WELTU; Holland. \\
\hline VUWG & 1247 & Gramineae & Lolium perenne & WELTU; New Zealand. \\
\hline VUWG & 1509 & Gramineae & Lolium perenne & $\begin{array}{l}\text { WELTU 11514; Cape Turakirae, } \\
\text { Wellington, N.Z. }\end{array}$ \\
\hline VUWG & 1248 & Gramineae & Melica ciliata & WELTU; Eiffel, Germany. \\
\hline VUWG & 1249 & Gramineae & Melica ciliata & WELTU; Holland. \\
\hline VUWG & 1250 & Gramineae & Milium effusum & WELTU; Holland. \\
\hline VUWG & 1510 & Gramineae & Phleum pratense & WELTU; Waikanae River, N.Z. \\
\hline NUNG & 176 & Gramineae & Phleum pratense & $\begin{array}{l}\text { Stevenson, Newcastle Upon Tyne } \\
\text { University, England. }\end{array}$ \\
\hline VUWG & 717 & Gramineae & Phragmites australis & $\begin{array}{l}\text { Chester 118. Mirsina, Grevena Pr., } \\
\text { NW Greece. }\end{array}$ \\
\hline VUWG & 1511 & Gramineae & Setaria viridis & WELTU 10252; Tauranga, N.Z. \\
\hline VUWG & 776 & Gramineae & Triticum aestivum & $\begin{array}{l}\text { Chester 225. Asprokampos, } \\
\text { Grevena Pr., NW Greece. }\end{array}$ \\
\hline NUNG & 144 & Gramineae & Zea mays & $\begin{array}{l}\text { Stevenson, Newcastle Upon Tyne } \\
\text { University, England. }\end{array}$ \\
\hline VUWG & 800 & Iridaceae & Ixia sp. & $\begin{array}{l}\text { Chester } 321 \text {. Wellington Botanic } \\
\text { Gardens, N.Z. }\end{array}$ \\
\hline VUWG & 1472 & Juncaceae & Juncus articulatus & $\begin{array}{l}\text { WELTU 8822; Seatoun, } \\
\text { Wellington, N.Z. }\end{array}$ \\
\hline VUWG & 1473 & Juncaceae & Juncus articulatus & WELTU 10204; Taurewa, N.Z. \\
\hline VUWG & 1475 & Juncaceae & Juncus articulatus & WELTU 15187; Waikanae, N.Z. \\
\hline VUWG & 656 & Juncaceae & Juncus articulatus & $\begin{array}{l}\text { Chester 18. Anelia, Grevena Pr., } \\
\text { NW Greece. }\end{array}$ \\
\hline VUWG & 1253 & Juncaceae & Juncus bufonius & WELTU; New Zealand. \\
\hline VUWG & 1254 & Juncaceae & Juncus bufonius & WELTU; Jumna R., India. \\
\hline VUWG & 1256 & Juncaceae & Juncus bulbosus & WELTU. \\
\hline
\end{tabular}




\begin{tabular}{|c|c|c|c|c|}
\hline \multicolumn{2}{|c|}{$\begin{array}{l}\text { Pollen/spore } \\
\text { reference } \\
\text { slide \# }\end{array}$} & \multirow{2}{*}{$\begin{array}{l}\text { Family } \\
\text { Juncaceae }\end{array}$} & \multirow{2}{*}{$\begin{array}{l}\text { Genus \& species } \\
\text { Juncus inflexus }\end{array}$} & \multirow{2}{*}{$\begin{array}{l}\text { Collector (if known)/herbarium } \\
\text { herbarium \# (if known), } \\
\text { place of collection }\end{array}$} \\
\hline VUWG & 654 & & & \\
\hline VUWG & 723 & Juncaceae & Juncus inflexus & $\begin{array}{l}\text { Chester } 128 . \text { Mirsina, Grevena Pr., } \\
\text { NW Greece. }\end{array}$ \\
\hline VUWG & 1255 & Juncaceae & Juncus squarrosus & WELTU; Holland. \\
\hline VUWG & 1257 & Juncaceae & Luzula luzuloides & WELTU; Black Forest, Germany. \\
\hline VUWG & 1258 & Juncaceae & Luzula luzuloides & WELTU; Black Forest, Germany. \\
\hline VUWG & 1260 & Juncaceae & Luzula pilosa & WELTU; Holland. \\
\hline VUWG & 1259 & Juncaceae & Luzula sylvatica & WELTU; Holland. \\
\hline VUWG & 1220 & Juncaginaceae & Triglochin palustris & WELTU; England. \\
\hline VUWG & 576 & Liliaceae & Allium neapolitanum & $\begin{array}{l}\text { WELT 63151; Wadestown, } \\
\text { Wellington, N.Z. }\end{array}$ \\
\hline VUWG & 575 & Liliaceae & Allium roseum bulbiferum & $\begin{array}{l}\text { WELT 70649; Mt Wellington, } \\
\text { Auckland, N.Z. }\end{array}$ \\
\hline VUWG & 1263 & Liliaceae & Allium schoenoprasum & WELTU; Switzerland. \\
\hline VUWG & 825 & Liliaceae & Allium schoenoprasum & $\begin{array}{l}\text { Chester } 346 . \text { Garden, Johnsonville, } \\
\text { Wellington, N.Z. }\end{array}$ \\
\hline VUWG & 1476 & Liliaceae & Allium triquetrum & WELTU; Wellington, N.Z. \\
\hline NUNG & 171 & Liliaceae & Allium triquetrum & $\begin{array}{l}\text { Stevenson, Newcastle Upon Tyne } \\
\text { University, England. }\end{array}$ \\
\hline NUNG & 169 & Liliaceae & ?Allium ursinum & $\begin{array}{l}\text { Stevenson, Newcastle Upon Tyne } \\
\text { University, England. }\end{array}$ \\
\hline VUWG & 1262 & Liliaceae & Allium vineale & WELTU; Switzerland. \\
\hline VUWG & 1261 & Liliaceae & Allium sp. & WELTU; Switzerland. \\
\hline NUNG & 104 & Liliaceae & Allium sp. & $\begin{array}{l}\text { Stevenson, Newcastle Upon Tyne } \\
\text { University, England. }\end{array}$ \\
\hline VUWG & 1264 & Liliaceae & Anthericum ramosum & WELTU; Switzerland. \\
\hline VUWG & 1265 & Liliaceae & Anthericum ramosum & WELTU; Switzerland. \\
\hline VUWG & 1266 & Liliaceae & Anthericum ramosum & $\begin{array}{l}\text { WELTU; Kaiserstuhl nr Freiburg, } \\
\text { Germany. }\end{array}$ \\
\hline VUWG & 577 & Liliaceae & Asparagus asparagoides & $\begin{array}{l}\text { WELT 70434; River Road, } \\
\text { Havelock North, N.Z. }\end{array}$ \\
\hline VUWG & 1267 & Liliaceae & Asphodelus fistulosus & WELTU; Delhi, India. \\
\hline NUNG & 136 & Liliaceae & Asphodelus ramosus & $\begin{array}{l}\text { Stevenson, Newcastle Upon Tyne } \\
\text { University, England. }\end{array}$ \\
\hline NUNG & 141 & Liliaceae & Colchium autumnale & $\begin{array}{l}\text { Stevenson, Newcastle Upon Tyne } \\
\text { University, England. }\end{array}$ \\
\hline VUWG & 670 & Liliaceae & Colchicum ?autumnale & $\begin{array}{l}\text { Chester 32. Kellia, Grevena Pr., } \\
\text { NW Greece. }\end{array}$ \\
\hline VUWG & 1268 & Liliaceae & Convallaria majalis & WELTU; Holland. \\
\hline VUWG & 827 & Liliaceae & Convallaria majalis & $\begin{array}{l}\text { Chester } 348 . \text { Garden, Johnsonville, } \\
\text { Wellington, N.Z. }\end{array}$ \\
\hline NUNG & 264 & Liliaceae & Fritillaria lusitanica & $\begin{array}{l}\text { Stevenson, Newcastle Upon Tyne } \\
\text { University, England. }\end{array}$ \\
\hline VUWG & 1269 & Liliaceae & Fritillaria sp. & WELTU; Holland. \\
\hline NUNG & 72 & Liliaceae & Hyacinthus orientalis & $\begin{array}{l}\text { Stevenson, Newcastle Upon Tyne } \\
\text { University, England. }\end{array}$ \\
\hline VUWG & 578 & Liliaceae & Ipheion uniflorum & WELT 60940; Paraparaumu, N.Z. \\
\hline VUWG & 1270 & Liliaceae & Lilium martagon & $\begin{array}{l}\text { WELTU; Kaiserstuhl nr Freiburg, } \\
\text { Germany. }\end{array}$ \\
\hline VUWG & 798 & Liliaceae & Muscari armeniacum & $\begin{array}{l}\text { Chester 319. Wellington Botanic } \\
\text { Gardens, N.Z. }\end{array}$ \\
\hline
\end{tabular}




\begin{tabular}{|c|c|c|c|c|}
\hline \multicolumn{2}{|c|}{$\begin{array}{l}\text { Pollen/spore } \\
\text { reference } \\
\text { slide \# }\end{array}$} & \multirow{2}{*}{$\begin{array}{l}\text { Family } \\
\text { Liliaceae }\end{array}$} & \multirow{2}{*}{$\begin{array}{l}\text { Genus \& species } \\
\text { Muscari armeniacum }\end{array}$} & \multirow{2}{*}{$\begin{array}{l}\text { Collector (if known)/herbarium, } \\
\text { herbarium \# (if known), } \\
\text { place of collection }\end{array}$} \\
\hline VUWG & 817 & & & \\
\hline VUWG & 1271 & Liliaceae & Ornithogalum umbellatum & WELTU; Holland. \\
\hline VUWG & 1272 & Liliaceae & Paris quadrifolia & WELTU; Germany. \\
\hline VUWG & 1273 & Liliaceae & Polygonatum odoratum & WELTU; Holland. \\
\hline VUWG & 1274 & Liliaceae & Polygonatum verticillatum & WELTU; Holland. \\
\hline VUWG & 732 & Liliaceae & Scilla autumnalis & $\begin{array}{l}\text { Chester 149. Mirsina, Grevena Pr., } \\
\text { NW Greece. }\end{array}$ \\
\hline NUNG & 116 & Liliaceae & Smilax aspera & $\begin{array}{l}\text { Stevenson, Newcastle Upon Tyne } \\
\text { University, England. }\end{array}$ \\
\hline VUWG & 1275 & Liliaceae & Veratrum album & WELTU; Holland. \\
\hline VUWG & 1278 & Orchidaceae & Anacamptis pyramidalis & WELTU; nr Freiburg, Germany. \\
\hline VUWG & 1279 & Orchidaceae & Cephalanthera damasonium & WELTU; Black Forest, Germany. \\
\hline VUWG & 1282 & Orchidaceae & Epipactis helleborine & WELTU; Holland. \\
\hline VUWG & 1283 & Orchidaceae & Epipactis ?palustris & WELTU; Holland. \\
\hline VUWG & 1284 & Orchidaceae & Neottia nidus-avis & WELTU; Black Forest, Germany. \\
\hline VUWG & 384 & $\begin{array}{l}\text { Potamogeton- } \\
\text { aceae }\end{array}$ & Potamogeton alpinus & WELT no \#. \\
\hline VUWG & 385 & $\begin{array}{l}\text { Potamogeton- } \\
\text { aceae }\end{array}$ & Potamogeton obtusifolius & WELT no \#; Funbo, Switzerland. \\
\hline VUWG & 386 & $\begin{array}{l}\text { Potamogeton- } \\
\text { aceae }\end{array}$ & Potamogeton perfoliatus & $\begin{array}{l}\text { WELT no \#; Westfalen, West } \\
\text { Germany. }\end{array}$ \\
\hline VUWG & 661 & Sparganiaceae & Sparganium erectum & $\begin{array}{l}\text { Chester 23. Kellia, Grevena Pr., } \\
\text { NW Greece. }\end{array}$ \\
\hline VUWG & 1218 & Sparganiaceae & Sparganium minimum & WELTU; Finland. \\
\hline VUWG & 1515 & Sparganiaceae & Sparganium subglobosum & $\begin{array}{l}\text { WELTU 06439; nr. Normandy, } \\
\text { Taranaki, N.Z. }\end{array}$ \\
\hline NUNG & 252 & Sparganiaceae & Sparganium sp. & $\begin{array}{l}\text { Stevenson, Newcastle Upon Tyne } \\
\text { University, England. }\end{array}$ \\
\hline NUNG & 241 & Typhaceae & Typha latifolia & $\begin{array}{l}\text { Stevenson, Newcastle Upon Tyne } \\
\text { University, England. }\end{array}$ \\
\hline VUWG & 1514 & Typhaceae & Typha orientalis & WELTU 8846; Foxton, N.Z. \\
\hline
\end{tabular}

\section{References}

Strid, A. (ed) 1989. Mountain Flora of Greece. Volume 1. Cambridge University Press, Cambridge.

Strid, A. and K. Tan (eds) 1991. Mountain Flora of Greece. Volume 2. Edinburgh University Press, Edinburgh.

Tutin, T.G., V.H. Heywood, N.A. Burges, D.H. Valentine, S.M. Walters and D.A. Webb (eds) 1964-80. Flora Europaea. Cambridge University Press, Cambridge. 5 volumes.

Watson, E.V. 1968 (2nd ed). British mosses and liverworts. Cambridge University Press, Cambridge.. 



\section{Appendix VI}

\section{Pollen key}

\section{VI.1 Key}

In this key pollen and spores of extant plants (those occurring naturally and in cultivation) in Grevena Province, northwestern Greece are described at the lowest possible morphological rank, using a light microscope. Descriptions of the grains were collected from many sources, the main literary sources being the major morphological works of Erdtman (1943, 1952, 1957, 1965 \& 1969); Erdtman, Berglund \& Praglowski (1961); Erdtman, Praglowski \& Nilsson (1963); Erdtman \& Sorsa (1971); Faegri \& Iversen (1975 \& 1992); Nilsson, Praglowski \& Nilsson (1977); Belmonte, Perez-Obiol \& Roure (1986); Ferrarini, Ciampolini, Sermolli \& Marchetti (1986); Moore, Webb \& Collinson (1991); various articles in The Northwest European Pollen Flora, volumes 1-6, edited by Punt (1976-1991; in the key these are referred to by "NW" followed by the volume number); Roure (1985); Valdes, Diez \& Fernandez (1987); and smaller works in the journals of Grana palynologica and Grana; and Review of paleobotany and palynology. In addition, to assist with the description of pollen and spores reference was made to a comprehensive pollen and spore reference collection of local species from plants collected in Grevena, and from herbarium specimens. Although this key was made to cover extant species in northwestern Greece, it is likely it would cover most common pollen and spores encountered in Quaternary deposits in the Central Balkans. A full list of species included in each pollen type is given in Appendix VI.2 at the end of this key. Unfortunately, the morphology of many species still remains unexamined, but the probable morphology of these species can be guessed at based on plant taxonomy, allowing them to be placed in the most likely pollen type. These species are listed at the end of each species list and can be identified by the '?' which precedes them. Nomenclature follows the Flora Europaea.

The purpose of this key is to supply a practical means of identifying pollen and spores without specialist pollen morphological knowledge. The key is intended for use with a light microscope, using mostly a X40 objective and occasionally an oil immersion X1000 objective. Special attention has been paid to closely identifying pollen and spores of particular interest to the archaeologist, e.g. cultivated plants, weeds of cultivation, and plants indicating disturbance of the natural vegetation.

The key has been modelled on the key in Faegri \& Iversen (1992) and a glossary to terms used can be found in that text. In principle the key is binary, but more than two entries at the same level have been used where taxa are very similar. The number, shape and arrangement of apertures are the major entries in the key. Exine structure and sculpturing are considered next. Because these features vary and are sometimes difficult to discern, some pollen taxa occur in more than one place in the key. Measurements given should be considered after the above features have been considered, and used cautiously. During the course of researching the morphology of the pollen and spores included in this key many widely differing measurements were found for the same pollen and spore taxa. Also different chemical treatments will affect the size of pollen and spores variously (Reitsma 1969). Thus, no particular attention has been paid to the mounting mediums used and the measurements should be considered approximate. Measurements do however give a rough guide to whether the grains are large or small. Occasionally size is used as a distinguishing feature, as for example in Quercus, where it has been used prudently. Particular distinguishing features have been italicized.

The right-hand column gives the ultimate breakdown to taxa. A genus name alone refers to species of that genus only; similarly a species name signifies only that species. The addition of the term 'type', abbreviated "t.", implies a pollen morphological type common to several families, genera or species, as the case may be. 


\section{Master key}

A2 Grains with air-sacs

Key A

A1 Grains without air-sacs.

B2 Composite grains (tetrads, polyads) Key B

B1 Grains single.

C2 Grains bilateral Key C

C1 Grains isodiametric or one axis longer/shorter, rarely heteropolar.

D2 Grains subtetrahedral Key D

D1 Grains rotational Key E

\section{Key A. Vesiculate}

A1 Distinct, subglobular or pouch-shaped air-sacs with internal, 3-dimensional reticulum, usually two.

B2 Proximal part of the exine ('crest') more than $5 \mu$ m thick

C2 Body of the grain (excl. air-sacs) $80-100 \mu$ m. Air-sacs more than semiglobular . Abies

C1 Body of the grain c. $50 \mu \mathrm{m}$. Air-sacs semiglobular PINACEAE

1 Proximal part of exine less than $5 \mu \mathrm{m}$ thick.

D2 Proximal entry angle of air-sac very blunt; no obvious constriction between air-sac and body of the grain

E2 Gradual transition between structure of the air-sac and that of the wall of the body of the grain. Colpus membrane smooth ................. Picea abies PINACEAE

E1 Abrupt transition between structure of air-sac and that of the wall of the body of the grain. Colpus membrane dotted Pinus (Haploxylon) PINACEAE

D1 Proximal entry angle of air-sac sharp; distinct constriction between air-sac and body of the grain. Body of the grain c. $50 \mu \mathrm{m}$. Colpus membrane smooth ...Pinus (Diploxylon) PINACEAE

\section{Key B. Composite grains}

The 'normal' position of a tetrahedral tetrad is the one in which the polar axis of one grain is parallel with the optical axis of the microscope. 
Key B1. Tetrads

A2 Tetrads linear, flat (quadrangular) or irregular. Grains reticulate or rugulate.

B4 Grains with one pore, c. $3 \mu \mathrm{m}$ wide, away from join between grains. Tetrad linear or flat. Typha spp.

C2 Rugulate

Typha angustifolia t.

TYPHACEAE

C1 Reticulate, lumina c. $2 \mu \mathrm{m}$

Typha domingensis t.

TYPHACEAE

B3 Tetrad flat. Grains with two pores................. Cytinus hypocistis

RAFFLESIACEAE

B2 Tetrad irregular.Grain with single pore $6 \mu \mathrm{m}$ wide (Epipactis palustris) or two apertures. Reticulate, mesh 3-5 $\mu \mathrm{m}$ wide

Epipactis t.

ORCHIDACEAE

B1 Tetrad irregular. Grain with 3-6 pores situated on dividing walls between grains ...

Periploca graeca ASCLEPIADACEAE

A1 Tetrads tetrahedral, sometimes irregularly so.

D2 Grains psilate, inaperturate. Exine extremely thin, normally not preserved/recognized in sediments Juncaceae

D1 Grains scabrate or verrucate.

JUNCACEAE

E2 Triporate, pores with vestibulum. Grains loosely connected. Cf. Key E15: Triporate Epilobium hirsutum t.

ONAGRACEAE

E1 Tricolpate/tricolporate. Tetrads compact, subglobular. Grains united by their proximal faces, but usually not in a strictly normal position (Y-figure of inner walls not rectilinear). Colpi regular. Pyrolaceae, Ericaceae.

F2 Colpi very short. Costae indistinct

Moneses uniflora

PYROLACEAE

F1 Colpi long, usually with margo. Interior walls with few or no perforations. Psilate to irregularly scabrate-verrucate.

G2 Costae absent/indistinct.

Pyrola

G1 Costae distinct.

PYROLACEAE

H2 Thin exine

Vaccinium myrtillus ERICACEAE

H1 Thick exine

Erica herbacea ERICACEAE

Key B2. Polyads

Polyads irregular; tetrads usually indistinguishable, densely crowded in massulae.

A2 Monoporate grains. 


\section{Key C. Bilateral grains}

Some monocolpate and biporate grains are faintly bilateral or pear-shaped (heteropolar). They are found in Key E.

A2 With more than one aperture.

B2 Bi-aperturate: two pores; scabrate Cytinus hypocistis RAFFLESIACEAE

B1 Tetra-aperturate, reticulate Impatiens noli-tangere BALSAMINACEAE

A1 With one or no aperture.

C2 With columellae. Grain reticulate. Heterobrochate, semitectate, columellae forming muri. Liliaceae, Amaryllidaceae, etc.: see Key E4.

C1 No columellae. Mostly Pteridophyte spores.

D2 Perine loose, crested or folded, not echinate but in some spores spinulose.

E2 Perine saccate

F3 Perine folded into numerous vesiculae, surface smooth or minutely vermiculate (SEM)

Dryopteris filix-mas t.

ASPIDIACEAE

F2 Perine folded into numerous vesiculae, foveolate-reticulate . . . . . . . . .

Gymnocarpium dryopteris ASPIDIACEAE

F1 Perine with widely spaced folds, minutely echinate Dryopteris expansa ASPIDIACEAE

E1 Perine lophate-cristate.

G2 Crests few, $1-4$, long, sometimes as long as the spore Thelypteris phegopteris THELYPTERIDACEAE

G1 Usually more than 4 crests, which are shorter.

H2 Crests with few or no spinules Athyrium distentifolium

H1 Crests densely spinulose.

ATHYRIACEAE

I2 Perine entire or sparsely perforate. Includes Ceterach, Phyllitis. Keys to species are to be found in Ferrarini et al. (1986) ... Asplenium t.

I1 Perine densely perforate (lacy) . Polystichum ASPIDIACEAE

D1 No loose outer perine or, if present, not folded or crested.

J4 Verrucate, sculpturing elements coarse, more than $3 \mu \mathrm{m}$ high $\ldots$... Polypodium POLYPODIACEAE 
J3 Scabrate. (Najas marina might be included here, but its exine is not preserved in acetolysis, or, presumably, in fossilisation)

K2 No perine visible Athyrium filix-femina

K1 Perine thin, slightly wrinked ATHYRIACEAE Blechnum spicant BLECHNACEAE

J2 Psilate. (Like Najas, Ceratophyllum is not preserved in acetolysis)

L2 Wall double with loose outer cover Isoetes ISOETACEAE

L1 Wall one-layered or outer cover not loose, smooth, structureless. Unidentifiable fern spores that have lost their perispore.

J1 Echinate, with echinae and (blunt) papillae.

M2 Mainly papillae Cystopteris fragilis

ATHYRIACEAE

M1 Sculpturing elements variable Thelypteris palustris THELYPTERIDACEAE

Key D. Subtetrahedral grains (spores)

A2 With an outer, loose, folded perine

Equisetum

A1 No loose perine. Cf. Thesium, Key E18.

EQUISETACEAE

B3 Grain subglobular.

C3 Spore psilate; aperture obscure, telaters Equisetum

C2 Spore wall undulose to scabrate (perine flakes off) EQUISETACEAE

$\mathrm{C} 1$ Spore baculate-clavate (tending to rugulate distally) SINOPTERIDACEAE

Spore triangular, semi-lobate. Corners rounded

D2 Verrucate . Anogramma leptophylla GYMNOGRAMMACEAE

D1 Scabrate-fimbriate, usually smooth by loss of ornamentation.

E3 Average diameter $40-50 \mu \mathrm{m}$, sides in polar view convex, laesurae margins conspicuously ridged $\ldots \ldots \ldots \ldots \ldots \ldots \ldots \ldots$ Adiantum capillus-veneris

ADIANTACEAE

E2 Average diameter $28-36 \mu \mathrm{m}$, sides in polar view concave, laesurae margins not ridged $\ldots \ldots \ldots \ldots \ldots \ldots \ldots \ldots \ldots \ldots \ldots \ldots \ldots \ldots \ldots \ldots \ldots$ Pteridium aquilinum HYPOLEPIDACEAE

E1 Average diameter $<25 \mu \mathrm{m}$. Spores similar to Adiantum and Pteridium, but significantly smaller

B1 Equatorial limb triangular-subcircular. Polar axis shorter than equatorial diameter. 
F4 Rugulate/verrucate to reticulate. Equatorial ridge present.

G2 Distally rugulate

Pteris cretica

G1 Distally reticulate with verrucae in lumina PTERIDACEAE

Pteris vittata PTERIDACEAE

SELAGINELLACEAE

F3 Foveolate/reticulate with rounded muri. No equatorial ridge. Ophioglossum.

H2 Tiny punctae

Ophioglossum lusitanicum

OPHIOGLOSSACEAE

H1 Coarsely reticulate

Ophioglossum vulgatum

OPHIOGLOSSACEAE

F2 Verrucate. Verrucae rounded, sometimes spinulate. Spore 43-58 $\mu \mathrm{m}$. Sculpturing on proximal face as distinct as on the distal

Botrychium

F1 Minutely echinate or granulate

Selaginella

OPHIOGLOSSACEAE

Key E. Rotational ellipsoidic (or ovate) grains: main key

A3 No distinct aperture.

B2 With meridional grooves and ridges

El Polyplicate

B1 No meridional grooves or ridges

E2 Inaperturate

A2 With one aperture.

C3 Aperture Y-shaped

E3 Trichotomocolpate

C2 Aperture elongate E4 Monocolpate

C1 Aperture circular E5 Monoporate

A1 More than one aperture.

D2 No lacunae or, if present, not in a fixed geometric pattern.

E2 Apertures not fused.

F3 With colpi, no pores or transverse endexinous colpi (colpate)

G3 Two colpi E6 Dicolpate

G2 Three colpi E7 Tricolpate

G1 More than three colpi.

H2 All colpi meridional E8 Stephanocolpate

H1 Some or all colpi not meridional E9 Pericolpate 
F2 Both colpi and distinct pores or transverse colpi present (colporate). Usually one pore per colpus, occasionally missing; in some taxa more than one pore per colpus (not in our material).

13 Two colpi

E10 Dicolporate

I2 Three colpi E11 Tricolporate

I1 More than three colpi.

J2 All colpi meridional E12 Stephanocolporate

J1 Some or all colpi not meridional E13 Pericolporate

F1 Free pores present, no colpi (porate)

K3 Two pores

E14 Diporate

K2 Three pores E15 Triporate

K1 More than three pores.

L2 Pores confined to a circular (sub-)equatorial belt E16 Stephanoporate

L1 Pores \pm uniformly distributed on the surface of the grain E17 Periporate

El Apertures fused to rings or spirals

E18 Syncolpate

D1 With lacunae in a fixed geometric pattern

M2 Lacunae elongate, meridional (pseudocolpi)

E19 Heterocolpate

M1 Lacunae not elongate

E20 Fenestrate

\section{Key E1. Polyplicate}

A2 Grooves branched

Ephedra distachya t.

EPHEDRACEAE

A1 Grooves simple. Meridional ridges high, distinct in polar view

Ephedra fragilis

EPHEDRACEAE

\section{Key E2. Inaperturate}

Many cryptogram spores, cysts, etc., are also inaperturate. They are, on the whole, distinguished from pollen grains by the (absence of) structure of the wall. They are not included in the key.

A5 Striate. Araceae

A4 Echinate or verrucate. 
B3 Echinate. Cf. also Lemnaceae, Nymphaea: Key E5 (Monoporate); and Nuphar, Stratiotes: Key E4 (Monocolpate) Hydrocharis morsus-ranae HYDROCHARITACEAE

B2 Verrucate. Grain $>40 \mu \mathrm{m}$. Obscure sulcus Vallisneria spiralis

B1 Verrucate. Grain $<40 \mu \mathrm{m}$. cf. Plantago, Key E17 (Periporate).

A3 Scabrate or frustillate (Najas is scabrate, bilateral, but its exine is not usually preserved).

C2 Grain heteropolar, with thin areas (lacunae). Cf. special key. Cyperaceae

C1 Isopolar, \pm spherical. No lacunae or perforations.

D5 Scabrate, tiny echinae. Grain $64-99 \mu \mathrm{m}$. Crocus

D4 Scabrate, tiny echinae. Grain 40-46 $\mu \mathrm{m}$. Exine 1-1.5 $\mu \mathrm{m}$. IRIDACEAE

D3 Scabrate. Grain 40-52 $\mu \mathrm{m}$. Exine c. $3 \mu \mathrm{m}$. Laurus nobilis

D2 Scabrate, micro-verrucate. Grain 18-25 $\mu \mathrm{m}$ LAURACEAE

D1 Scabrate, with minute dark dots. Grain $22-30 \mu \mathrm{m}$ Aristolochia ARISTOLOCHIACEAE Orobanche minor $\mathrm{t}$. OROBANCHACEAE silate or gemmate.

A2 Psilate or gemmate.

E2 Psilate.

F2 Grain more than $50 \mu \mathrm{m}$.

G2 Grain c. $60 \mu \mathrm{m}$. Obscurely trilete, wall simple or cavate with outer thin layer wrinked

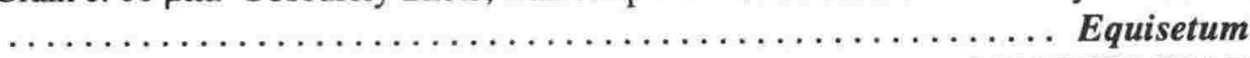

EQUISETACEAE

G1 Grain c. $90 \mu \mathrm{m}$ Pseudotsuga menziesii PINACEAE

F1 Grain less than $50 \mu \mathrm{m}$. Ceratophyllum (grain bilateral, c. 25-30 $\mu \mathrm{m}$ ) keys out here, but exine is not usually preserved. Similarly Juncaceae (spheroidal, very thin-walled).

H2 Grain $25-47 \mu \mathrm{m}$. Psilate with tiny echinae (often lost). Araceae.

I2 Grain bilateral Arum

I1 Grain spheroidal ARACEAE Dracunculus t. ARACEAE

El Gemmate

J2 Fossil grains usually two-split. Gemmae scattered, deciduous, rounded, with constricted base, mostly lost in fossil material. Cf. Pedicularis, Key E16 ..........Juniperus CUPRESSACEAE

J1 Gemmae rather crowded, size variable, not deciduous (SEM: microbaculate) ......

Taxus baccata TAXACEAE

A1 Clavate-baculate or reticulate.

K2 Grain bilateral, elliptical. Reticulate Callitriche obtusangula 
K1 Grain rotational.

L3 Rugulose-baculate. Grain 21-32 $\mu \mathrm{m}$

Limodorum abortivum

ORCHIDACEAE

L2 Isolated clavaelbaculae in reticulate pattern Callitriche stagnalis $t$.

L1 Reticulum distinct.

M2 Columellae distinct.

N2 Diameter of lumina subequal to breadth of muri Cephalanthera ORCHIDACEAE

N1 Diameter of lumina several times the breadth of muri. Potamogetonaceae.

$\mathrm{O} 2$ Columellae widely spaced, mostly confined to corners of brochi $\ldots . .$.

Potamogeton pectinatus POTAMOGETONACEAE

O1 Columellae denser, also between corners of brochi . ............

Potamogeton crispus t. POTAMOGETONACEAE

M1 Columellae indistinct.

P3 Diameter of lumina subequal to breadth of muri. Muri (indistinctly) duplicolumellate Daphne

THYMELAEACEAE

P2 Muri much narrower than width of lumina, simplicolumellate ...........

Triglochin bulbosa JUNCAGINACEAE

P1 Lumina c. $4 \mu \mathrm{m}$. No apparent columellae Zannichellia palustris ZANNICHELLIACEAE

Key E3. Trichotomocolpate

Triradiate scars are very common in cryptogam spores (cf. Key D). In angiosperm pollen they are found in some taxa, but in the area none is known, apart from occasional faint traces in some grains of Trapa natans.

\section{Key E4. Monocolpate}

Compare also Key E5, Monoporate, the pore of which may become colpus-like by collapse of the grain.

A6 Verrucate. Verrucae dimorphic, smaller on the proximal side Gagea pratensis LILIACEAE

A5 Clavate-baculate. Rounded clavae/baculae up to $2 \mu \mathrm{m}$ long Iris pumila A4 Echinate-baculate.

B2 Grains usually prolate. Echinate; echinae more than $5 \mu \mathrm{m}$ long Nuphar lutea NYMPHAEACEAE

B1 Grains subspherical. Baculate-echinate, large projections less than $5 \mu \mathrm{m}$ long. Aperture sometimes indistinct 
A3 Rugulate to striate. Allium.

$\mathrm{C} 2$ Colpus extends to the proximal side of the grain.

D2 Grain more than $50 \mu \mathrm{m}$

Allium roseum $\mathrm{t}$.

LILIACEAE

D1 Grain less than $50 \mu \mathrm{m}$ Allium sphaerocephalon t.

LILIACEAE

C1 Colpus restricted to the distal side of the grain. Grain less than $50 \mu \mathrm{m} \ldots \ldots \ldots \ldots \ldots \ldots$

Allium paniculatum $\mathrm{t}$.

LILIACEAE

A2 Reticulate (brochi equal to or more than $1 \mu \mathrm{m}$ ).

E2 Brochi more than $3 \mu \mathrm{m}$ diameter.

F2 Heterobrochate. Margo very broad

Anthericum liliago

F1 Homobrochate or brochi not falling into discrete size groups.

LILIACEAE

G2 Columellae \pm cylindrical. Extremely coarsely reticulate, brochi $4-18 \mu \mathrm{m} \ldots \ldots$.

Iris germanica

G1 Muri consisting of distinct transverse elements. No free columellae in

IRIDACEAE lumina. Margo present

LILIACEAE

E1 Brochi 1-3 $\mu$ m diameter.

H3 Colpus extends to the proximal side of the grain. Muscari.

I2 Grain more than $40 \mu \mathrm{m}$ Muscari comosum

LILIACEAE

I1 Grain less than $40 \mu \mathrm{m}$ Muscari neglectum $\mathrm{t}$. LILIACEAE

H2 Colpus completely covers the distal pole. Colpus membrane covered with irregularly shaped islands of columellate exine. Grain $4455 \mu \mathrm{m}$

Gynandriris sisyrinchium

$\mathrm{H} 1$ Colpus restricted to the distal side of the grain.

IRIDACEAE

J2 Heterobrochate.

K2 Grains long, narrow.

L2 Few large brochi, mostly on the proximal side of the grain. ... Scilla t. LILIACEAE

L1 Lumina variable, rounded, muri about the same size as lumina ......

Narcissus papyraceus t.

AMARYLLIDACEAE

K1 Grain almost circular in polar view. Perforate-reticulate with muri and lumina almost the same size over the distal pole, remainder of surface reticulate with lumina twice width of muri

Asphodelus fistulosus t.

LILIACEAE

J1 Homobrochate. 
M3 Grain less than $30 \mu \mathrm{m}$. Brochi c. $1 \mu \mathrm{m}$; reticulum decreases towards colpus Narthecium scardicum

LILIACEAE

M2 Grain 30-50 $\mu \mathrm{m}$. Margo present. Muri narrow. Lumina up to $3 \mu \mathrm{m}$. Incl. Butomus, Veratrum.

N2 Lumina in mesocolpium decrease sharply to a broad colpus margin that is clearly tectate-perforate. Grain often circular in outline

Butomus umbellatus

BUTOMACEAE

N1 Lumina decrease more gradually to colpus margin, which is not tectateperforate, i.e. some lumina are still detectable at the very edge. Grain more often elliptic

Veratrum

LILIACEAE

M1 Grain more than $50 \mu \mathrm{m}$.

$\mathrm{O} 2$ Pollen often ruptured with ragged colpus edges. No margo. Free columellae in some brochi.

P3 Brochi 2-3 $\mu \mathrm{m}$

Hermodactylus tuberosus

P2 Brochi 1-2.5 $\mu \mathrm{m}$ IRIDACEAE

P1 Brochi 1-1.5 $\mu \mathrm{m}$ Iris pseudacorus IRIDACEAE

O1 Margo present. Columellae distinct Iris spuria t. IRIDACEAE

A1 Microreticulate (brochi less than $1 \mu \mathrm{m}$ ), psilate or scabrate.

. Fritillaria

Q2 Colpus extends to the proximal side of the grain. Allium - see above.

Q1 Colpus restricted to the distal side of the grain.

R2 Grain less than $30 \mu \mathrm{m}$.

S2 Columellae imperceptible.

T2 Brochi c. $1 \mu \mathrm{m}$ Narthecium scardicum LILIACEAE

T1 Psilate (perforate) Galanthus t.

S1 Columellae distinct. Brochi less than $1 \mu \mathrm{m}$ AMARYLLIDACEAE

R1 Grain more than $30 \mu \mathrm{m}$.

Asparagus

U2 Grain shaped like an orange segment.

V4 Microreticulate, brochi c. $1 \mu \mathrm{m}$. Grain 50-60 $\mu \mathrm{m}$ Sternbergia AMARYLLIDACEAE

V3 Perforate to reticulate, brochi $<1 \mu \mathrm{m}$. Grain $25-50 \mu \mathrm{m} \ldots$ Narcissus tazetta AMARYLLIDACEAE

V2 Perforate. Polar outline \pm circular Asphodelus albus LILIACEAE

V1 Psilate to faintly perforate Polygonatum t. LILIACEAE 
U1 Grain not segment-shaped.

W2 Reticulate, muri broader than width of lumina.

X2 Homobrochate

Tulipa sylvestris

X1 Heterobrochate

LILIACEAE

Ornithogalum t.

LILIACEAE

W1 Psilate-scabrate, perforate.

Y2 Grain about $60 \mu \mathrm{m}$. Psilate-perforate and micro-echinate. 2 operculi over colpus (giving appearance of being 3-colpate)

Gladiolus t.

IRIDACEAE

Y2 Grain 47-70 $\mu \mathrm{m}$. Granulate and perforate Urginea maritima

LILIACEAE

Y1 Grain less than $50 \mu \mathrm{m}$.

Z2 Colpus edges ragged

Gagea lutea t.

Z1 Colpus edges not ragged

LILIACEAE

Convallaria majalis

LILIACEAE

\section{Key E5. Monoporate}

A3 Pore large, diameter more than half that of the grain, with operculum.

B2 Grain gemmate

Nymphaea alba

NYMPHAEACEAE

B1 Grain granulate with echinae c. $1 \mu \mathrm{m}$ projecting above densely crowded granules. Smilax

A2 Pore diameter about half grain diameter. Grain reticulate or rugulate-verrucate.

C2 Grain reticulate

Cephalanthera

ORCHIDACEAE

C1 Grain with verrucae and short rugulae

Limodorum abortivum

ORCHIDACEAE

A1 Pore smaller, diameter usually much less than half that of the grain.

D2 With distinct anulus. Grain tectate, psilate, scabrate, verrucate. Cf. special key Gramineae

D1 No anulus. Cf. Juniperus, Key E2.

E4 Grain clavate-baculate

Callitriche stagnalis $\mathrm{t}$.

CALLITRICHACEAE

E3 Grain psilate, densely perforate with lacunae, one of which forms a rudimentary pore at broad end of usually pear-shaped grain. Cf. special key $\ldots \ldots \ldots \ldots$ Cyperaceae

E2 Grain reticulate/rugulate, heterobrochate. Pore not always distinct.

F4 Microreticulate (lumina $<1 \mu \mathrm{m})$

F3 Reticulate, lumina 1-2 $\mu \mathrm{m}$ 
F2 Reticulate, lumina c. $2 \mu \mathrm{m}$

F1 Rugulate. Often in tetrads Typha angustifolia $\mathrm{t}$.

TYPHACEAE

E1 Grain echinate, echinae c. $1.5 \mu \mathrm{m}$ with broad conical base. Pore may be indistinct. Cf. algal cysts and other microfossils of unknown origin

\section{Key E6. Dicolpate}

A2 Columellae distinct. Per-reticulate. Margo present

Tamus communis

A1 Columellae indistinct. No margo.

B2 Grain sometimes syncolpate. Grain densely microechinate

Hypecoum

(B1 Grain usually two-split (syncolpate), edges ragged. Structure and sculpturing very faint or nil. Cf. Key E18

Pedicularis SCROPHULARIACEAE

A2 Tectate

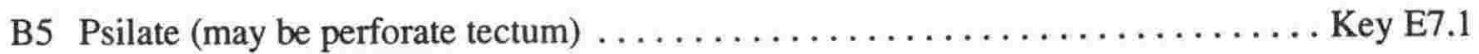

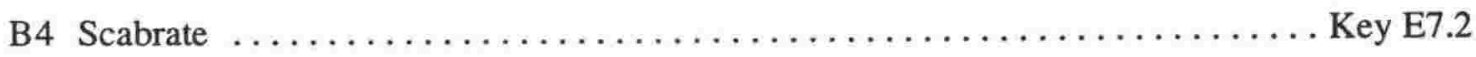

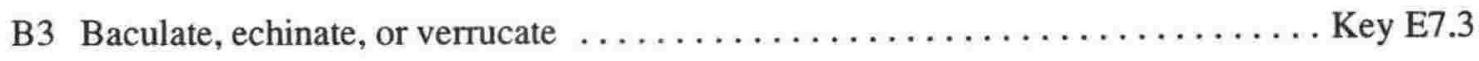

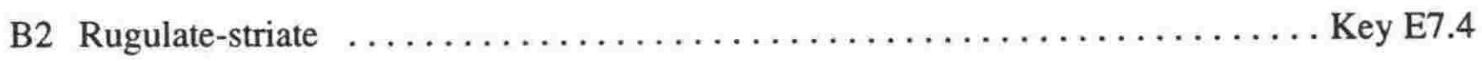

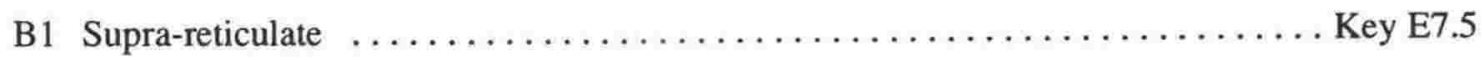

A1 Semitectate or intectate

C2 Per-reticulate Key E7.6

C1 Intectate (clavate, gemmate or baculate) Key E7.7

The crested grains of Trapa are not included in this key; see Key A.

Key E7.1 Tricolpate, psilate (may be perforate tectum)

A3 Colpi very short with nexinous ridges crossing underneath, never gaping Monotropa hypopitys

A2 Colpus without equatorial constriction

B4 Exine equal to or thicker than $3 \mu \mathrm{m}$. Columellae shorter and exine thinner next to colpus.

C2 Exine 4-6 $\mu \mathrm{m}$ thick. Columellae branched. Grain 45-67 $\mu \mathrm{m}$ 
C1 Exine c. $3 \mu \mathrm{m}$ thick. Columellae simple.

D2 Grain $>30 \mu \mathrm{m}$

D1 Grain $\leq 30 \mu \mathrm{m}$. Cuscuta.

E2 In centre of mesocolpium sexine about as thick as nexine; columellae short and rather indistinct Cuscuta epithymum t. CONVOLVULACEAE

E1 In centre of mesocolpium sexine thicker than nexine; columellae longer, easily visible .. Cuscuta europaea t. CONVOLVULACEAE

B3 Exine thinner in middle of intercolpium. Colpus narrow, straight, edges sharp. Grain c. $20 \mu \mathrm{m}$ Melampyrum SCROPHULARIACEAE

B2 Exine 2-3 $\mu \mathrm{m}$, even thickness. Edge of colpus clear, not ragged. Grain 21-30 $\mu \mathrm{m}$ Phytolacca americana PHYTOLACCACEAE

B1 Exine equal to or thinner than $2 \mu \mathrm{m}$. Edge of colpus ragged.

F2 Exine thinner next to colpus, micro-echinate. Columellae distinct and/or irregularly distributed.

G2 Grain globular-prolate. Exine thicker in polar area, without perforations. Columellae present in colpus, grouped .............Veronica triphyllos t.

G1 Grain globular-oblate. No polar thickening of exine. Tectum with perforations. Columellae in colpus, if present, scattered.

H2 Distinctly micro-echinate. Number of perforations and columellae approximately equal. Columellae in a dense, regular pattern ..... Spergula

CARYOPHYLLACEAE

H1 Not echinate. Perforations fewer than columellae. Columellae irregularly distributed. Spergularia t.

CARYOPHYLLACEAE

F1 Exine same thickness throughout, c. $1 \mu \mathrm{m}$. Surface smooth

I2 Grains oblate to spheroidal. Polar area small, index below 0.3

J2 Grain c. $30 \mu \mathrm{m}$. Structure imperceptible Myricaria germanica TAMARICACEAE

J1 Grain 40-50 $\mu \mathrm{m}$. Structure distinct. Sometimes 4-colpate .... Euphrasia

I1 Grains subglobular/prolate, 30-40 $\mu \mathrm{m}$. Surface uniform.

K3 Supratectal granules grouped into circles Odontites t. SCROPHULARIACEAE

K2 Supratectal granules grouped only as rugulae ......... Bellardia trixago SCROPHULARIACEAE

K1 Minutely superficially strio-reticulate. Incl. Orobanche purpurea $\ldots \ldots \ldots$

Rhinanthus t. SCROPHULARIACEAE

A1 Colpus with equatorial constriction. Cf. Key E11.1

L2 Colpus with prominent, undulating edges. Special key Rosaceae 
L1 Colpus edges flat, straight.

M2 Microreticulate. Columellae imperceptible Saxifraga stellaris

M1 Exine not reticulate.

N2 Columellae imperceptible. Exine thin Viola hirta t.

N1 Columellae distinct.

VIOLACEAE

O2 Columellae coarse Saxifraga bulbifera

O1 Columellae irregularly dispersed, minute. Perforations scattered, irregular Myricaria germanica TAMARICACEAE

Key E7.2. Tricolpate, scabrate

A2 Colpus with equatorial constriction

B2 Costae colpi distinct. Equatorial limb triangular. Cornus

C2 Grain more than $40 \mu \mathrm{m}$, scabrate. Tectum perforate C. sanguinea CORNACEAE

C1 Grain less than $35 \mu \mathrm{m}$, with scattered microverrucae. Tectum imperforate ... C. mas

B1 No distinct costae colpi. Columellae crowded, minute CORNACEAE

A1 Colpus without equatorial constriction

D3 Nexine very thin. Sexine thicker than nexine, distinctly columellate, tectate. Usually syncolpate

D2 Nexine distinct. Exine thick, at any rate in the polar area.

E3 Exine thick in mesocolpia $(3.5-5 \mu \mathrm{m})$; structure of sexine complex, with 2 layers of columellae

E2 Exine more than $3 \mu \mathrm{m}$ thick, crescentic. Nexine heavy. Columellae distinct, their mutual distance 1-2 times diameter. Distinct, regularly and widely spaced microechinae. Colpus membrane with columella-like granules.

F2 Grain $>40 \mu \mathrm{m}$. Some echinae larger than $1 \mu \mathrm{m}$. Exine thicker at poles $(6 \mu \mathrm{m})$.

F1 Grain $<40 \mu \mathrm{m}$. Only microechinae. Valerianella.

G2 Sexine as thick as nexine in mesocolpium; columellae more scattered and somewhat larger at poles than elsewhere Valerianella rimosa $\mathrm{t}$. VALERIANACEAE

G1 Sexine thicker than nexine in mesocolpia; columellae not differentiated at poles 
E1 Exine thinner except in polar area.

H2 Exine thickest adjacent (but not actually at) poles; endocracks absent. Granulate colpus operculum. Colpus edges straight $\ldots \ldots \ldots \ldots \ldots \ldots \ldots$ Teucrium

LABIATAE

H1 Exine thickest at poles; endocracks present. Colpus with granulate membrane, but not operculate.

I3 Colpus narrow, infolded, margins very prominent with adhering granules, traversed by prominent endocracks; colpus membrane not usually visible. Columellae distinct . . . . . . . . . . . . . . . . . Adonis

RANUNCULACEAE

I2 Colpus broad; margins ragged, not prominent, traversed by endocracks (but not prominently); colpus membrane covered with numerous distinct granules. Columellae distinct $\ldots \ldots \ldots \ldots \ldots \ldots \ldots \ldots \ldots \ldots \ldots \ldots \ldots$ Consolida t.

RANUNCULACEAE

I1 Colpus broad, slightly sunken; margin ragged and indistinct, traversed by faint endocracks; colpus membrane granular. Columellae indistinct ... Caltha t.

RANUNCULACEAE

D1 Not so.

J3 Without spinules.

K2 Exine perforate. Grain $27-36 \mu \mathrm{m} \ldots \ldots \ldots \ldots \ldots$ Veronica triphyllos $\mathrm{t}$. SCROPHULARIACEAE

K1 Not perforate. Grain $17-23 \mu \mathrm{m} \ldots \ldots \ldots \ldots \ldots \ldots$ Orobanche ramosa t.

OROBANCHACEAE

J2 Low ridges at base of spinules form a reticuloid pattern. Exine with multiple perforations. Spinules (scabrae) regularly distributed. Detailed key to species within this type ( $P$. dubium, $P$. hybridum, $P$. rhoeas, $P$. somniferum) in NW2 . Papaver rhoeas $\mathbf{t}$.

PAPAVERACEAE

J1 No ridges between bases of spinules.

L2 Structure and sculpturing very fine. Grain oblate ....... Myricaria germanica

TAMARICACEAE

L1 Structure and sculpturing distinct. Spinules/verrucae irregularly distributed, size variable.

M3 Tectum \pm smooth with very scattered verrucae

Glinus t.

MOLLUGINACEAE

M2 Tectum without perforations. Columellae distinct, uniform, rather crowded. Colpus narrow. Quercus.

N3 Indistinctly scabrate/rugulate. Polar diameter 22-28 $\mu \mathrm{m}$.Q. coccifera t. FAGACEAE

N2 Scabrate. Polar diameter $30-37 \mu \mathrm{m} \ldots \ldots \ldots \ldots \ldots$ Q. trojana $\mathrm{t}$. FAGACEAE

N1 Distinctly scabrate/verrucate $\ldots \ldots \ldots \ldots \ldots \ldots \ldots$. frainetto t. FAGACEAE

M1 Tectum with perforations, (sub-)verrucate-echinate. Colpus open, membrane granulate. Grains often pericolpate, cf. Key E9. Columellae in open pattern, forming a bright area ('halo') around each spinule. Perforations follow the same pattern. See key to species and groups in NW6. Incl. Ranunculus p.p., Pulsatilla, Anemone, Ceratocephalus \& Clematis .... Ranunculus acris t. 
Key E7.3. Tricolpate; baculate, echinate or verrucate

A3 Baculate, baculae c. $2.5 \mu \mathrm{m}$ long, in groups of 2 and 3. Exine $2-4 \mu \mathrm{m}$, tectum complete, infratectum columellate. Grain $42-60 \mu \mathrm{m} \ldots \ldots \ldots \ldots \ldots \ldots \ldots$. Plumbago europaea

A2 Echinate. Grain large, $40 \mu \mathrm{m}$ or more.

PLUMBAGINACEAE

B2 Echinae prominent, widely separated.

C2 Echinae 1-1.5 $\mu \mathrm{m}$ long, suppported on shield-shaped verrucae. Valeriana.

D2 Echinae considerably shorter than wall thickness, height greater than width .....

Valeriana officinalis t.

VALERIANACEAE

D1 Echinae about as wide as tall

Valeriana tuberosa

VALERIANACEAE

(C1 Echinae coarse, not on shield-shaped verrucae. Polar area large. Equatorial limb subtriangular. Lonicera. Tricolporate: see Key E11.2 )

B1 Echinae smaller, of different size classes. Exine 5-10 $\mu \mathrm{m}$. Columellae branched. Colpus short, broad, cf. Key E15 ( Triporate). Dipsacaceae p.p.

E3 Halo surrounding a very short colpus

Dipsacus t.

DIPSACACEAE

E2 Structural and sculpturing elements regularly distributed. Exine thicker at poles .....

Scabiosa columbaria t.

DIPSACACEAE

E1 Not so

Succisa pratensis

DIPSACACEAE

A1 Grains smaller.

F3 Echinae conical, low. Tectum thick. Colpi short, indistinct. Compositae, see Key E11.2: H2

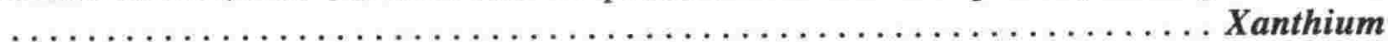

COMPOSITAE

F2 Grains with prominent mixed echinae and microechinae, irregularly and widely spaced ...

Actaea spicata

RANUNCULACEAE

F1 Grains microechinate, echinae evenly distributed $\ldots \ldots \ldots \ldots \ldots \ldots \ldots \ldots$ Nigella

RANUNCULACEAE

Key E7.4. Tricolpate, rugulate or striate

A2 Exine very thick, 5-7 $\mu \mathrm{m}$. Striae coarse, multilevelled. Colpi wide Erodium

GERANIACEAE

A1 Exine thickness and striation normal and fine.

B2 Exine with or without perforations. Edges of colpi undulating, often prominent. Rosaceae, see special key

B1 Exine perforate. Edges of colpi straight or ragged.

C3 Very finely rugulate-striate Veronica anagallis-aquatica t. SCROPHULARIACEAE 
C2 Rugulate-scabrate. Vallae short.

D2 Vallae in reticuloid pattern, may be perfectly reticulate. Colpus margins ragged .

Oxalis

OXALIDACEAE

D1 Vallae very faint. Colpus margins straight. Quercus coccifera t.

FAGACEAE

C1 Distinctly striate.

E3 Sculpturing fine, striae predominantly meridional. Grain $30 \mu \mathrm{m}$ or more ... Acer

E2 Direction of striae variable

F2 Grain smaller than $25 \mu \mathrm{m}$. Sculpturing fine ........ Trollius europaeus

RANUNCULACEAE

F1 Grain more than $25 \mu \mathrm{m}$. Sculpturing coarse. Refer NW2.

Saxifraga oppositifolia t.

SAXIFRAGACEAE

E1 Striation diffuse. Vallae in very low relief. S. granulata type of NW2.

G2 Vallae with granulae

Saxifraga tridactylites $\mathrm{t}$.

SAXIFRAGACEAE

G1 No granulae on vallae

Saxifraga sempervivum $\mathrm{t}$.

SAXIFRAGACEAE

\section{Key E7.5. Tricolpate, supra-reticulate}

A2 Colpus without equatorial constriction or poroid area. No costae.

B2 Meridional limb compressed oval, grain subcylindric, usually prolate-perprolate. Colpus narrow, not boat-shaped.

C3 Muri broader than lumina; lumina of even size, c. $1 \mu \mathrm{m} \ldots \ldots \ldots \ldots$. . . . . . . ACANTHACEAE

C2 Muri narrow; all brochi large, c. $1.5 \mu \mathrm{m} \ldots \ldots \ldots \ldots \ldots \ldots \ldots$ Onobrychis

LEGUMINOSAE

$\mathrm{C} 1$ Muri narrow, brochi small, c. $0.5 \mu \mathrm{m}$, or a median field of small brochi in each intercolpium

Hedysarum coronarium

LEGUMINOSAE

B1 Meridional limb circular, oval or rhomboidal, not compressed. Colpi boat-shaped, often ruptured. Labiatae p.p.

D2 Lumina \pm uniform in size all over the grain (may be so small as to be microreticulate).

E3 \pm Microreticulate.

F2 Grain $>25 \mu \mathrm{m}$

Stachys sylvatica t.

LABIATAE

F1 Grain $<25 \mu \mathrm{m}$. Brochi somewhat variable in size

Marrubium

E2 Reticulate.

LABIATAE

G2 Thick muri $(0.5-1.0 \mu \mathrm{m})$, baculae in lumina Stachys arvensis LABIATAE 
G1 Muri thin $(0.2-0.4 \mu \mathrm{m})$, punctae perforating the lumina $\ldots \ldots$ Stachys recta

E1 Doubly reticulate Phlomis

LABIATAE

D1 Lumina largest in the centre of each mesocolpium (where muri may appear winding), decrease in size gradually towards colpi $\ldots \ldots \ldots \ldots \ldots \ldots \ldots \ldots$. Scutellaria $\mathrm{t}$.

LABIATAE

A1 Equatorial part of colpus constricted or ruptured. Costae interrupted at equator.

H2 Poroid area large, usually ruptured. Branches of costae colpi short.

Genista t.

H1 Poroid area small. Branches of costae colpi long. Hypericum, see Key E11.5.

Key E7.6. Tricolpate, per-reticulate

(A3 Tri-sulcate, one sulcus oval and occupying the distal pole of the grain, the other two semicircular and located at the margins of the proximal face

Tulipa praecox t.)

LILIACEAE

A2 Colpi without equatorial constriction

B2 Grain large, more than $50 \mu \mathrm{m}$, thick-walled.

C3 Finely and uniformly reticulate, lumina c. $1 \mu \mathrm{m}$.

D2 Colpus membrane granulate

Leontice leontopetalum BERBERIDACEAE

D1 Not so. Muri broader than lumina

ACANTHACEAE

C2 Coarsely reticulate, brochi $4-14 \mu \mathrm{m}$. Columellae not branched, the heads standing above the muri spinulose

Armeria maritima t.

C1 Columellae branched, fused to a reticulum some distance below their heads, forming coarse clavae on top. See key to groups in NW6

Geranium

GERANIACEAE

B1 Grain smaller.

E2 Brochi small, less than $1 \mu \mathrm{m}$ (microreticulate).

F2 Heterobrochate, brochi larger in mesocolpia.

G2 Colpi long, margins diffuse, membrane rugate \pm granules. \pm Circular in polar view, prolate. Infratectum clearly columellate $\ldots \ldots \ldots \ldots \ldots \ldots$ Reseda

RESEDACEAE

G1 Colpi short. Angulaperturate, triangular in polar view; suboblate $\ldots \ldots \ldots \ldots$

F1 Homobrochate.

H2 Grain larger than $25 \mu \mathrm{m}$.

I2 Exine c. $4 \mu \mathrm{m}$ thick 
I1 Exine $2 \mu \mathrm{m}$ or less. Colpus margin indistinct. Colpus membrane granulate. Columellae indistinct ................. Chelidonium majus

H1 Grain smaller than $25 \mu \mathrm{m}$.

J2 Exine thicker than $1 \mu \mathrm{m}$, thinner at colpus margin and in apocolpia. Polar exine not differentiated. Columellae distinct in surface view.

K3 Exine 2-4 $\mu \mathrm{m}$ thick. Colpus margin distinct. Colpus membrane not granulate.

Arabis

CRUCIFERAE

K2 Exine $2 \mu \mathrm{m}$ or less. Colpus margin distinct. Colpus membrane not granulate

Hornungia t. CRUCIFERAE

K1 Exine 1-3 $\mu \mathrm{m}$. Colpus membrane granulate. Muri broad . Tamarix

TAMARICACEAE

J1 Exine $1 \mu \mathrm{m}$ or less, tectate-perforate at poles. Columellae indistinct.

L2 Exine c. $1 \mu \mathrm{m}$ thick. Polar area tectate-perforate, not thickened or reticulate Saxifraga stellaris SAXIFRAGACEAE

L1 Exine less than $1 \mu \mathrm{m}$ thick. Polar area exine thicker, tectate with few perforations. Obscurely tricolporate, with 2-6 endocolpi ......

E1 At least some brochi more than $1 \mu \mathrm{m}$.

M2 Colpus long

N2 Heterobrochate, brochi reduced in size towards colpi.

O2 Margo distinct, semitectate. Brochi c. $2 \mu \mathrm{m}$. Grain \pm spheroidal .....

O1 Margo thin, tectate.

P2 Endoaperture apparent. Grain prolate.

Q2 Brochi 1.5-2 $\mu \mathrm{m}$. Grain 16-20 $\mu \mathrm{m}$ Salix triandra SALICACEAE

Q1 Brochi 2-3 $\mu \mathrm{m}$. Grain 16-27 $\mu \mathrm{m} \ldots \ldots \ldots \ldots$. Salix fragilis $\mathrm{t}$.

SALICACEAE

P1 Endoaperture very indistinct. Grain \pm spheroidal ........ Oxalis

N1 Brochi not reduced in size towards colpi. No distinct margo. Columellae very distant, only their heads joined into a reticulum.

R2 Exine rather thick. Columellae clear in top view, length decreases next to colpi. Reticulum with narrow muri. Colpus without granules or with a delicate structure.

R1 Muri broad. Colpus with scattered granules

M1 Colpus short. Polar area rather large, index c. 0.4. 
S2 Colpus broad, with scattered granules. Grain c. $22 \mu \mathrm{m}$. Platanus orientalis

PLATANACEAE

S1 Colpus narrow, covered with granules, broader towards equator, geniculate. Fraxinus.

T2 Equatorial outline angular, sides sometimes nearly straight. Nexine about as thick as sexine. Reticulum fine, brochi $\pm 1 \mu \mathrm{m}$. Grain 18.5-23.5 $\mu \mathrm{m}$

Fraxinus angustifolia

OLEACEAE

T1 Equatorial outline circular. Nexine distinctly thinner than sexine. Reticulum slightly coarser, brochi $>1 \mu \mathrm{m}$. Grain $20-29.5 \mu \mathrm{m} \ldots \ldots \ldots$

Fraxinus excelsior t. OLEACEAE

A1 Colpi with equatorial constriction. See Key E11.5 (Tricolporate).

Key E7.7. Tricolpate, intectate (clavate, gemmate and baculate)

A2 Size of clavae variable.

B2 Intercolpium and polar area with scattered, large, free clavae in addition to many small ones, more or less fused. Colpus not distinct

C2 Grain c. $60 \mu \mathrm{m}$. Equatorial outline circular .............. Viscum album

$\mathrm{C} 1$ Grain c. $20 \mu \mathrm{m}$. Equatorial outline \pm triangular .......... Arceuthobium oxycedri

LORANTHACEAE

B1 All clavae free. Small clavae scattered beneath the large ones. Near the colpus there are only small clavae, forming a margo $\ldots \ldots \ldots \ldots \ldots \ldots \ldots \ldots \ldots$ Ilex aquifolium

AQUIFOLIACEAE

Al Clavae dimorphic or uniform. Linaceae.

D2 Clavae dense, almost contiguous, fine, 1-1.5 $\mu \mathrm{m}$, dimorphic, the smaller ones dominant. . .

Linum usitatissimum t.

LINACEAE

D1 Clavaelgemmae distinctly separate.

E2 Grain smaller than $25 \mu \mathrm{m}$. Colpus margins regular, not appearing ruptured. Gemma of \pm uniform diameter $\ldots \ldots \ldots \ldots \ldots \ldots \ldots \ldots \ldots \ldots \ldots \ldots \ldots \ldots \ldots \ldots \ldots$ Radiola linoides

E1 Grain large, $>35 \mu \mathrm{m}$. Colpus margins very irregular, ruptured. Clavae uniform or dimorphic. If dimorphic, both size classes subequally represented.

F3 Grain c. $80 \mu \mathrm{m}$. Clavae uniform. [Rogers 1984 suggests pericolpate] .........

Linum hologynum t.

LINACEAE

F2 Grain smaller, c. 50-65 $\mu$ m. Endocolpus lolongate, broad, distinct $\ldots \ldots \ldots \ldots$

Linum austriacum t.

LINACEAE

F1 Grain 35-70 $\mu \mathrm{m}$. Endoaperture not, or hardly visible, except at ends. Includes $L$. catharticum, L. tenuifolium, L. trigynum, L. strictum, L. hirsutum - See detailed key to groups and species in NW3

LINACEAE 
A2 Psilate-scabrate.

B4 Grain large, $60-80 \mu \mathrm{m}$. 9-11 broad colpi. Finely pilate-scabrate Sesamum indicum

B3 Grain medium size.

C2 $25-28 \mu \mathrm{m} .11-12$ colpi Sherardia arvensis RUBIACEAE

C1 20-35 $\mu \mathrm{m}$. 4 or 6 colpi. Detailed key to species within this type (P. dubium, P. hybridum, $P$. rhoeas, $P$. somniferum) in NW2 .

B2 Grain small.

D2 Grain 15-29 $\mu \mathrm{m}$. 6-10 narrow colpi. Ornament of circular perforations and scabrae .

Galium t.

RUBIACEAE

D1 Grain 15-21 $\mu$ m. c. 6 colpi. Ornament of small circular perforations regularly distributed, together with paired rugae giving a unique band and point pattern ........Valantia

RUBIACEAE

B1 Grain very small, 10-13 $\mu$ m. 3-4 colpi

Corrigiola litoralis p.p. CARYOPHYLLACEAE

A1 Reticulate.

E2 Polar area large, index higher than 0.6

F3 Supra-reticulate; no columellae discernible in muri. Colpi 4. Grain oblate in equatorial view, subrectangular in polar view.

Impatiens noli-tangere BALSAMINACEAE

F2 Recticulate-perforate. Colpi 4. Grain obtuse-rectangular or quadrangular in equatorial and polar views

LABIATAE

F1 Per-reticulate.

G3 5-10 colpi Primula

G2 46 colpi. Usually tricolpate, see E7.6

PRIMULACEAE

G1 4 colpi.

$\mathrm{H} 2$ Columellae indistinct

Fraxinus angustifolia

H1 Muri simpli -or duplicolumellate

OLEACEAE

Huri simpli -or duplicolumellate

Fraxinus excelsior $\mathrm{t}$.

OLEACEAE

E1 Polar area medium-small, index lower than 0.5 .

I2 Supra-reticulate.

J2 6 or more colpi. Free columellae in lumina

Prunella t.

J1 4 colpi. No free columellae (lumina punctate) Stachys angustifolia

I1 Per-reticulate. Columellae restricted to muri. 
K3 Muri scabrate. Microreticulate

K2 Muri beaded.

L2 Reticulum double

Salvia officinalis $\mathrm{t}$.

LABIATAE

L1 Reticulum single, \pm rugulate Salvia verticillata

LABIATAE

K1 Muri not beaded

Menthat.

LABIATAE

Key E9. Pericolpate

A3 Reticulate.

B2 Lumina deep, with free, coarse clavae. 30 short colpi in dodecahedral arrangement .....

Polygonum amphibium POLYGONACEAE

B1 No free elements in lumina. 4-6 colpi. Cf. Tricolpate, Keys E7.5 and E7.6.

A2 Echinate. Echinae small, irregularly distributed. Tectum perforate. Central area of intercolpium with coarse columellae in reticuloid pattern. 12 colpi

C2 Exine thickest in mesocolpium, thinning towards colpi Montia fontana PORTULACACEAE

C1 Exine \pm even thickness Portulaca oleracea PORTULACACEAE

A1 Psilate, microechinate, scabrate, verrucate.

D3 Irregularly distributed spinules, verrucae or scabrae of varying size. Tectum with perforations (phase, SEM): some Papaveraceae, Ranunculaceae more usually inaperturate or tricolpate (Keys E2, E7) may key out here.

E2 Psilate. 6- or 12-colpate, usually syncolpate Corydalis ochroleuca

E1 Verrucate. 6-colpate, not syncolpate PAPAVERACEAE

D2 Psilate-scabrate with uniform minute spinules and perforations Corydalis solida t.

D1 Psilate, spinules absent PAPAVERACEAE

DI Psilate, spinules absent Spergula

CARYOPHYLLACEAE Spergularia t. CARYOPHYLLACEAE

Key E10. Dicolporate

Prolate, equatorially constricted (bone-shaped). Colpi $2 / 3$ length of grain; ora rectangular, lalongate. Exine tectate, psilate. Includes Tordylium maximum 
The transition colpate-colpate with equatorial constriction-colporate is not easy to define in categorical terms. Consultation of Key E7 is recommended if Key E11 does not lead to a result.

\section{A3 Tectate.}

B4 Psilate or scabrate Key E11.1

B3 Echinate Key E11.2

B2 Striate or rugulate Key E11.3

B1 Foveolate or supra-reticulate Key E11.4

A2 Semitectate. Per-reticulate Key E11.5

A1 Intectate. Clavate Key E11.6

\section{Key E11.1 Tricolporate. Psilate-scabrate}

A2 With costae aequatoriales and transverse colpi

B4 Equatorial limb intersemiangular. Exine more than 4 m thick, crescentic. Columellae coarse, branched. Centaurea sect. Cyanus

C2 Meridional limb compressed oval. Costae aequatoriales sharply projecting in meridional optical section

C. cyanus t.

C1 Meridional limb oval. Costae aequatoriales less prominent COMPOSITAE

\section{Cl Meridional limb oval. Costae aequatoriales less prominent. .} C. napulifera t.

B3 Equatorial limb subcircular. Exine thinner, not crescentic. Columellae finer. Costae aequatoriales less prominent. Grain $22-39 \mu \mathrm{m}$, colpi long ...... Polygonum aviculare t.

B2 Equatorial limb intermediate between B4 and B3. Exine slightly crescentic .. Bilderdykia

B1 Equatorial limb triangular. Exine 1.25-1.5 $\mu \mathrm{m}$, not crescentic, short columellae. Costae aequatoriales stronger near ectoapertures, colpi short and narrow with parallel sides, often forking slightly at ends. Grain $45-56 \mu \mathrm{m}$

A1 No costae aequatoriales

D3 Heteropolar. Exine thin, psilate or punctate, columellae not discernible.

E2 Pear-shaped.

F2 Grain 10-16 $\mu \mathrm{m}$. Exine with scattered punctae $<1 \mu \mathrm{m}$ bordering the apertures ..

Alkanna t.

BORAGINACEAE

F1 Grain 17-24 $\mu \mathrm{m}$. Exine comprehensively but faintly micro-suprareticulate Echium

E1 Grain \pm spheroidal, one apocolpium very small, the other larger. Exine psilate. 
G2 Grain $>15 \mu \mathrm{m}$. Colpi constricted at equator; endoapertures indistinct $\ldots \ldots \ldots$.

Gratiola officinalis

SCROPHULARIACEAE

G1 Grain 10-15 $\mu \mathrm{m}$. Costae colpi present; endoapertures large and distinct, 8-shaped

D2 Meridional limb compressed oval.

$\mathrm{H} 2$ Grain distinctly prolate.

I2 Grain larger than $20 \mu \mathrm{m}$. Exine $>1 \mu \mathrm{m}$. For detailed key see NW4; additional taxa described by Cerceau-Larrival (1963 - Tordylium officinale; 1967 - Cachrys); van Zeist \& Bottema (1977 - Malabaila)

Umbelliferae

UMBELLIFERAE

I1 Grain $<20 \mu \mathrm{m}$. Exine $<1 \mu \mathrm{m}$ Androsace villosa

H1 Not so distinctly prolate.

J2 Exine more than $3 \mu \mathrm{m}$ thick. Grain more than $50 \mu \mathrm{m}$, dimorphic, one type with denser structure. Tectum with scattered perforations. Columellae branched, coarser at poles.

K2 Columellae evenly distributed, gaps between columellae less than their diameters Polygonum viviparum POLYGONACEAE

K1 Columellae irregularly distributed, more widely spaced Polygonum bistorta $\mathrm{t}$. POLYGONACEAE

J1 Exine thin.

L3 Grain more than $30 \mu \mathrm{m}$. Pore edges distinctly protruding. Scabrate, polar area

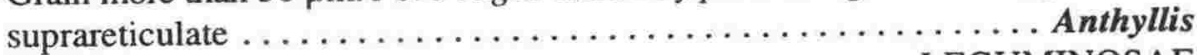

L2 Grain $20-30 \mu \mathrm{m}$.

LEGUMINOSAE

M2 Pores edges distinctly protruding $\ldots \ldots \ldots \ldots \ldots \ldots \ldots$ Dorycnium

LEGUMINOSAE

M1 Pores not protruding Ornithopus compressus LEGUMINOSAE

L1 Grain less than $20 \mu \mathrm{m}$, psilate Lotus t. LEGUMINOSAE

D1 Meridional limb oval or circular.

M2 Intercolpium flattened or concave in polar view.

N2 Exine thicker and columellae coarser at poles. Colpus narrow, slit-shaped. Grain subglobular

N1 Thickness of exine uniform. Colpus not slit-shaped.

$\mathrm{O} 2$ With transverse colpus

P2 Scabrate. Grain c. 35-45 $\mu \mathrm{m}$, angulaperturate. Tectum perforate 
Q2 Longitudinally oriented rugulae.

ANACARDIACEAE

Q1 Micro-foveolate/fossulate. Equatorial sexinal bridge over colpus ...
Styrax officinalis

STYRACACEAE

P1 Psilate. Tectum not perforate.

R2 Grain more than $20 \mu \mathrm{m}$.

S2 Irregular outline. H-shaped endoaperture

Verbena

VERBENACEAE

S1 Smooth outline. Endoaperture not $\mathrm{H}$-shaped, fastigiate ........

Solanum nigrum t. SOLANACEAE

R1 Grain smaller than $20 \mu \mathrm{m}$. Endoaperture fastigiate ............

Solanum dulcamara

SOLANACEAE

O1 No transverse colpus.

T2 Equatorial limb semilobate. Psilate

Frangula

RHAMNACEAE

T1 Equatorial limb semiangular. Scabrate.

U3 Grain 58-64 $\mu \mathrm{m}$, subprolate, circular equatorial outline. Colpi long. Exine c. $3 \mu \mathrm{m}$, tectum perforate ... Lagenaria siceraria

CUCURBITACEAE

U2 Grain more than $40 \mu \mathrm{m}$, angulaperturate ... Cornus sanguinea CORNACEAE

U1 Grain c. $25 \mu \mathrm{m}$, angulaperturate . Cornus mas CORNACEAE

M1 Intercolpium convex.

V2 Pore edges distinctly protruding.

W2 Endopore equatorially elongated.

X2 Grain $23 \mu \mathrm{m}$ or more.

Y2 Psilate to sparsely punctate Osyris alba

Y1 Densely punctate/microreticulate (see E11.5) ... Ricinus communis EUPHORBIACEAE

$\mathrm{X} 1$ Grain less than $25 \mu \mathrm{m}$.

Z3 Microechinate, vermiculate Filipendula ROSACEAE

Z2 Psilate, tectum not perforate Castanea sativa FAGACEAE

Z1 Scabrate or indistinctly suprareticulate.

A'2 Operculate Sanguisorba minor ROSACEAE

A'l No operculum. Incl. Medicago sativa p.p. ............... 
W1 Endopore circular. Colpus slit-shaped.

B'2 With vestibulum. Colpus very short, about twice the diameter of the pore

B'1 No vestibulum.

$\mathrm{C}^{\prime} 2$ Microechinate, columellae in vermiculoid pattern ..... Filipendula

ROSACEAE

C'1 Psilate. Colpi very short. Grain spheroidal to oblate .............

Elaeagnus angustifolia

ELAEAGNACEAE

V1 Edges of pore not protruding, endopore meridionally elongated, isodiametric or indistinct.

D'2 Grain perforate. Colpus slit-shaped, endopore small, completely covered.

E'2 With costae endopori. See key to species and groups in NW5. Incl. Oxyria digyna

POLYGONACEAE

E'1 No costae endopori.

F'2 Grain $33-45 \mu \mathrm{m} \ldots \ldots \ldots \ldots \ldots \ldots \ldots$ Spartium junceum

LEGUMINOSAE

F'1 Grain 16-19 $\mu \mathrm{m}$. Colpus interrupted at equator . Samolus valerandi PRIMULACEAE

D'1 Not so.

G'2 Distinctly prolate. Grain $<30 \mu \mathrm{m}$.

H'2 Scrabrate.

I'2 Scabrate/suprareticulate. Equatorial limb circular with deeply intruding colpi. Colpi long, wide, with densely granulate membrane. Exine thins towards colpi. ........ Globularia

GLOBULARIACEAE

I'1 Scabrate/granulate. Intercolpia flattened or slightly convex. Colpi long and narrow, with psilate membrane ...... Diospyros lotus

EBENACEAE

H'1 Psilate. Crassulaceae.

$\mathrm{J}^{\prime} 2$ Grain $>27 \mu \mathrm{m}$. Colpus membrane slightly granulate Jovibarba heuffelii CRASSULACEAE

$\mathrm{J}^{\prime} 1 \quad$ Grain $<27 \mu \mathrm{m}$ . Umbilicus CRASSULACEAE

G'1 Subprolate to spheroidal.

K'2 Colpi long, broad. Psilate to scabrate. H-endoaperture with equatorial bridge of sexine over colpus. Colpus membrane with coarse granules; margins not intruding, irregular ... Robinia pseudacacia

K'1 Colpi short, slit-like. 
L'2 Grain 44-50 $\mu \mathrm{m}$. Scabrate/rugulate. Endoaperture circular, with thickened sexine (marginate) . . . . . . . . . . Fagus

FAGACEAE

L'1 Grain 20-28 $\mu \mathrm{m}$. Scabrate/granulate. Endoaperture circular, with thickened nexine (costate) $\ldots \ldots \ldots \ldots \ldots$ Emex spinosa

POLYGONACEAE

\section{KeyE11.2. Tricolporate, Echinate}

This Key mainly comprises Compositae Asteroideae. Their exine is in typical cases multi-layered and consists of, from the inside outwards: (1) foot layer = nexine; sexine composed of (2) inner columella layer, usually with scattered, coarse, often branched columellae; (3) paratectum formed by partial fusion of heads of the inner columellae; (4) outer columellae layer; columellae thinner and more numerous than in the inner layer; (5) (outer) tectum, which is very thin and densely perforated.

A2 Columellae straight, not branched.

B2 Diameter of echinae and columellae equal. Echinae few, scattered. Lonicera.

C2 Sexine about as thick as nexine, columellae in surface view rather indistinct. Grain usually smaller than $60 \mu \mathrm{m} \ldots \ldots \ldots \ldots \ldots \ldots \ldots \ldots \ldots \ldots \ldots$. xylosteum $\mathrm{t}$.

CAPRIFOLIACEAE

C1 Sexine much thicker than nexine, columellae in surface view distinct. Grain usually larger than $60 \mu \mathrm{m}$.

D2 Echinae up to $2.5 \mu \mathrm{m}$. Ends of ectocolpi usually obtuse $\ldots \ldots \ldots \ldots$ L. alpigena

D1 Echinae up to $1.5 \mu \mathrm{m}$. Ends of ectocolpi usually acute ..... L. periclymenum t.

CAPRIFOLIACEAE

B1 Echinae much coarser than columellae, conical, broad at base. Short colpi, prominent endopores

Abutilon t.

MALVACEAE

A1 Columellae branched, echinae conical from base. Asteroideae.

E2 Columellae indistinct in optical section

E1 Columellae prominent in optical section.

F2 Echinae at least partly joined by low ridges.

G2 Echinae joined by smooth low ridges which form a coarse reticulum ...........

G1 Echinae large, partly confluent at bases. Inner columella layer variable, thicker

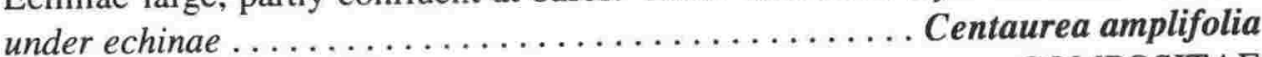

F1 Echinae discrete, not joined by ridges.

H2 Inner columella layer equal to or thicker than the outer.

I3 Echinae vestigial. 
J2 Grains large, prolate with longitudinal thickenings of intercolpia (intersemiangulate)

J1 Grains small, spherical to oblate

COMPOSITAE

Echinae blunt, length equal to or smaller than basal diameter. Prolate. Artemisia COMPOSITAE

........ COMPOSITAE

I1 Echinae long, sharp. Intercolpium in optical section distinctly crescentic. ... Achillea t. COMPOSITAE

H1 Inner columellae layer thinner than outer.

K2 Echinae vestigial Centaurea graeca t.

K1 Echinae distinct. COMPOSITAE

L2 Grains c. $30 \mu \mathrm{m}$ or smaller. Spherical. Colpi short. Echinae short (1-2 $\mu \mathrm{m})$. Cf. also Key E7.3 ................ Xanthium

L1 Grains larger. Colpi long.

M3 Prolate. Spines short

Centaurea solstitialis t. COMPOSITAE

M2 Spheroidal to subprolate. Numerous warts between spines. Size 40-62 $\mu \mathrm{m} \ldots \ldots \ldots \ldots \ldots \ldots$ Crupina COMPOSITAE

M1 Oblate. Spines long. Incl. Carduus Cirsium t. COMPOSITAE

Key E11.3. Tricolporate. Striate-rugulate

A2 Operculate. See Special key Rosaceae

A1 No operculum.

ROSACEAE

B2 Endoaperture a transversal colpus. Costae transversales present. Anacardiaceae.

C2 Colpus long, transverse colpus well defined.

D2 Nexine and sexine of equal thickness Schinus molle ANACARDIACEAE

D1 Nexine markedly thinner than sexine Rhus coriaria ANACARDIACEAE

C1 Colpus shorter, transverse colpus not so well defined, sometimes poroid. Nexine thinner than sexine

B1 Endoaperture not distinctly transversal. No costae transversales.

E3 Finely verrucate. Colpus very short, about twice the diameter of the pore

E2 Rugulate. 
F2 Colpus short. Distinctly rugulate

F1 Colpus long. Finely rugulate-perforate

E1 Striate-rugulate. Colpus much longer than the diameter of the pore.

G2 Pore indistinct.

H2 Edge of colpus undulating. See Special key Rosaceae

H1 Edge of colpus straight.

I2 Striae very distinct. Columellae fine.

J2 Costae colpi distinct. Strio-reticulate

Ailanthus altissima

J1 Costae colpi absent or very thin SIMAROUBACEAE

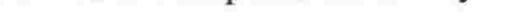
Saxifraga oppositifolia t. SAXIFRAGACEAE

I1 Striae indistinct Saxifraga tridactylites $\mathrm{t}$. SAXIFRAGACEAE

G1 Pore distinct

K2 Grain $25 \mu \mathrm{m}$ or larger.

L2 Polar area pointed. Pore covered. Striae fine Helianthemum

L1 Polar area rounded. Pore not or only partly covered. CISTACEAE

M2 All or almost all striae meridional.

N2 Grain c. $40 \mu \mathrm{m}$. Endoaperture colpoid.

$\mathrm{O} 2$ Fastigium large and distinct. Striate in mesocolpium, reticulate or microreticulate at poles ........Atropa $\mathrm{t}$. SOLANACEAE

O1 No fastigium. Faint striae throughout, or treticulate in mesocolpium and tectate at poles .......... Nicotiana

SOLANACEAE

N1 Grain less than $40 \mu \mathrm{m}$. Endoaperture poroid.

P2 Poles pointed. Endoaperture margins smooth, or with a few coarse granules. $\mathrm{P} / \mathrm{E}>1 \ldots \ldots \ldots$ Gentiana lutea $\mathrm{t}$. GENTIANACEAE

P1 Poles rounded/flattened. Endoaperture margins coarsely granular. P/E usually $<1 \ldots \ldots \ldots \ldots$ Centaurium

GENTIANACEAE

M1 Some striae not meridional.

Q2 Intercolpium median with predominantly meridional striae.

R2 Striae run together at pores and poles to form a reticulum .

Tuberaria guttata CISTACEAE 
R1 Striae mostly meridional in mesocolpia, sometimes abruptly changing direction, sometimes transverse next to colpi ...

Centaurium

GENTIANACEAE

Q1 Intercolpium median rugulate.

S3 Grain 47-56 $\mu \mathrm{m}$. Intercolpium edges meridionally striated. Vestibulae present ............... Haplophyllum

RUTACEAE

$\mathrm{S} 2$ Grain $<30 \mu \mathrm{m}$. Intercolpium edges meridionally striated. No vestibulae. ........ Blackstonia perfoliata GENTIANACEAE

$\mathrm{S} 1 \mathrm{Grain}<30 \mu \mathrm{m}$. Intercolpium edges with groups of non-meridional striae.

T2 Grain 19-26 $\mu \mathrm{m}$. Faint costae colpi; H-endoaperture ..

Cicendia filiformis

GENTIANACEAE

T1 Grain 22-32 $\mu \mathrm{m}$. Distinct costae colpi; endoaperture lalongate, not $\mathrm{H}$-shaped

Ruta chalepensis

RUTACEAE

K1 Grain smaller than $25 \mu \mathrm{m}$.

U2 Colpus with coarse echinae

Aesculus hippocastanum HIPPOCASTANACEAE

U1 Colpus membrane without sculptural elements.

V2 Sculpturing of intercolpium indistinct.

W2 Grain prolate, rectangular in equatorial view. Exine c. $1.5 \mu \mathrm{m}$.

X2 Rugulate pattern over all of grain. Each elliptic or 8-shaped endoaperture commonly with "horns" into mesocolpia ...

Coronilla scorpioides t. LEGUMINOSAE

$\mathrm{X} 1$ Rugulate pattern may disintegrate into small reticulations. Each elliptic or 8-shaped endoaperture without "horns" ..

Hippocrepis LEGUMINOSAE

W1 Grain prolate, oval in equatorial view.

Y2 Exine 3-5 $\mu \mathrm{m}$, thins towards colpi. Intercolpium edges with groups of non-meridional striae ..... Cicendia filiformis

GENTIANACEAE

Y1 Exine thinner, even thickness. Striae very indistinct (phase contrast) Crassula

CRASSULACEAE

V1 Sculpturing of intercolpium uniform, distinct.

Z2 Columellae distinctly visible Sempervivum

Z1 Columellae indistinct-imperceptible.

A'2 Endoapertures H-shaped 
Key E11.4. Tricolporate. Suprareticulate-foveolate

A2 Heteropolar. Pores near the broad end

Al Isopolar.

B3 Grain peroblate. Foveolate. Colpi very short. Costae colpi heavy. Exine more than $3 \mu \mathrm{m}$ thick. Tilia.

C2 $4-6$ brochi $/ 10 \mu \mathrm{m}$

Tilia platyphyllos

C1 $8-11$ brochi $/ 10 \mu \mathrm{m}$ TILIACEAE

C1 8-11 brochi/10 pm. Tilia cordata $\mathrm{t}$.

B2 Grain oblate, triangular. Exine $4 \mu \mathrm{m}$. Colpi short, endopores large.

D2 Finely rugulate/foveolate. Lumina with granules TILIACEAE

D1 Regular reticulum, brochi 4-7 $\mu \mathrm{m}$. Incl. Vigna LEGUMINOSAE

B1 Grain not peroblate. Colpus not very short.

LEGUMINOSAE

E2 Grain prolate. Exine more than $2 \mu \mathrm{m}$ thick. Columellae very coarse.

F2 Grain oval. Columellae uniform.

G2 Costae colpi thick; margo of thin sexine along colpi. Grain subprolate, pores often protruding. Exine c. $3 \mu \mathrm{m}$, with densely packed columellae; foveolate to reticulate

EUPHORBIACEAE

G1 Costae colpi absent, colpi long; endoaperture slightly lalongate. Grain subprolate to spheroidal, $60 \mu \mathrm{m}$; circular in polar view. Exine c. $3 \mu \mathrm{m}$, with wellspaced columellae; microrugulate to reticulate ....... Lagenaria siceraria

F1 Grain compressed oval. Columellae coarser and more scattered at poles.

H2 Channels between columellae at apocolpium \pm regular in width, narrow, no wider than columella diameter; branched columellae extending no more than $1 / 2$ way down grain from poles $\ldots \ldots \ldots \ldots \ldots \ldots \ldots$ Polygonum viviparum

POLYGONACEAE

H1 Channels between columellae at apocolpium irregular in width, often broader than columella diameter; branched columellae often extending from poles almost to equator

Polygonum bistorta t. POLYGONACEAE

E1 Grains variable. Exine less than $2 \mu \mathrm{m}$ thick. Columellae fine or indistinct.

I2 Equatorial limb subangular. Grain oblate. Polar area very small. Costae transversales and pores distinct $\ldots \ldots \ldots \ldots \ldots \ldots \ldots \ldots \ldots$ Rhamnus t.

RHAMNACEAE

I1 Equatorial limb circular or semiangular. Grain subspherical or prolate. Polar area medium to large. Mainly Leguminosae. 
J3 Reticulum indistinct.

K2 Grain prolate. Pore distinct. Costae colpi present.

L2 Endoaperture lalongate, 8-shaped, covered by a thin, structureless exine. Costae colpi well developed ........... Vicia cracca t.

LEGUMINOSAE

L1 Endoaperture \pm circular, not covered Punica granatum

PUNICACEAE

K1 Grain subspherical. Pore covered, more or less distinct.

M3 Grain more than $35 \mu \mathrm{m}$. Pore indistinct. Exine thin. Colpus with costae and equatorial bridge ..............Medicago sativa

M2 Grain more than $30 \mu \mathrm{m}$. Exine thin ........... Astragalus

LEGUMINOSAE

LEGUMINOSAE

M1 Grain $<35 \mu \mathrm{m}$. Exine thick

$\mathrm{N} 2$ Grain 30-35 $\mu \mathrm{m}$, compressed oval in equatorial view. Endoaperture lolongate ......... Trifolium montanum $\mathrm{t}$.

LEGUMINOSAE

N1 Grain $<30 \mu \mathrm{m}$, rhomboidal in equatorial view. Colpus with equatorial bridge. Endoaperture a colpus Solanum melongena

J2 Reticulum distinct, some lumina blocked.

O2 Polar area large

Coronilla emerus

LEGUMINOSAE

O1 Polar area medium Astragalus

LEGUMINOSAE

J1 Reticulum distinct. No lumina blocked.

P2 More than 13-15 equatorial brochi per intercolpium.

Q2 Lumina with free columellae Astragalus

Q1 No free columellae in lumina.

R2 Grain more than $27 \mu \mathrm{m}$

Ononis t.

R1 Grain smaller than $27 \mu \mathrm{m}$

R1 Grain smaller than $27 \mu m$. Galega officinalis

P1 Less than 13-15 equatorial brochi per intercolpium.

S2 Meridional limb compressed oval. Colpus with heavy costae. Pore distinct, covered by a thin, almost structureless sexine.

T2 Grain subspheroidal. Lumina with perforations. Breadth of muri less than diameter of lumina $\ldots \ldots \ldots \ldots$ Lathyrus sativus

LEGUMINOSAE

T1 Grain prolate.

U2 Grain more than $50 \mu \mathrm{m}$. 
V2 With distinct perforations in lumina .. Pisum sativum

V1 No perforations in lumina ............ Vicia faba

LEGUMINOSAE

U1 Grain smaller than $50 \mu \mathrm{m}$.

W2 Lumina with distinct perforations Lathyrus pratensis t. LEGUMINOSAE

W1 No perforations in lumina

$\mathrm{X} 2$ Ectocolpi usually $3 / 4$ length of polar axis .......

Vicia sativa t.

LEGUMINOSAE

$\mathrm{X} 1$ Ectocolpi rather short (c. $25 \mu \mathrm{m}) \ldots \ldots \ldots$ Lens

LEGUMINOSAE

S1 Meridional limb circular or more or less rhomboidal. Costae colpi variable. Pore covered by sexine with distinct structure. Trifolium.

Y2 Grain more than $40 \mu \mathrm{m}$

T. repens t.

Y1 Grain smaller than $40 \mu \mathrm{m} \ldots \ldots \ldots \ldots \ldots \ldots$ T. dubium $\mathrm{t}$.

LEGUMINOSAE

LEGUMINOSAE

Key E11.5. Tricolporate. Per-reticulate - frustillate

A3 Reticulum indistinct or frustillate-perforate. B3 Incomplete reticulum, composed of irregular muri ............ Cistus monspeliensis t.
CISTACEAE

B2 Columellae united only by their topmost parts. Colpus with equatorial bridge. Mercurialis.

C2 Exine more than $2 \mu \mathrm{m}$ thick. Columellae grouped/irregularly distributed. Colpus membrane not with a row of granules ................ Mercurialis perennis

EUPHORBIACEAE

$\mathrm{C} 1$ Exine less than $1.5 \mu \mathrm{m}$ thick. Columellae evenly distributed. Colpus operculum formed by a (single) row of granules ...................... Mercurialis annua t.

EUPHORBIACEAE

B1 Irregularly reticulate-frustillate-perforate, lumina larger in the polar area. Colpus narrow, slightly wider at both ends. Pore small, covered. Distinctive hexagonal outline in polar view

Vitis vinifera

VITACEAE

A2 Reticulate-striate. Columellae coarse, isolated at bottom, forming reticulum at middle level and striations at top; dupli- or simplicolumellate muri ............... Gentiana verna

A1 Reticulate.

D2 Brochi more than $1 \mu \mathrm{m}$.

E2 Sexine thick, usually more than $2 \mu \mathrm{m}$. Columellae coarse, distinct. 
F2 Muri distinctly duplicolumellate. Reticulum irregular, brochi smaller towards colpus. Muri beaded, breadth equal to or less than diameter of lumina. With transverse colpus

F1 Muri usually simplicolumellate.

G3 Lumina with distinct free columellae, those in muri crowded. Transverse colpus short.

H2 Reticulum very coarse except in the polar area. Equatorial limb subtriangular

Sambucus ebulus

CAPRIFOLIACEAE

H1 Reticulum in polar area not different, but finer in narrow margo to ectocolpus. Equatorial limb tricrescentic. Sometimes 2- or 4-colporate

Viburnum

CAPRIFOLIACEAE

G2 No free columellae in lumina. Endoaperture a transverse colpus ..........

Ligustrum vulgare

OLEACEAE

G1 No free columellae in lumina. Those in muri clearly separated. Endoaperture poroid.

I2 Grain more than $40 \mu \mathrm{m}$.

J2 Endoaperture circular, indistinct .

Jasminum

J1 Endoaperture circular, distinct.

OLEACEAE

K2 Muri spinose

Cistus salvifolius

CISTACEAE

K1 Muri not spinose. Exine thicker at apocolpium; sexine $>$ nexine

Gentiana verna

GENTIANACEAE

I1 Grain less than $40 \mu \mathrm{m}$, spheroidal to oblate.

L2 H-endoaperture. Exine of even thickness; sexine \pm as thick as nexine

Gentianella ciliata

GENTIANACEAE

L1 Lolongate endoaperture, $6 \mu \mathrm{m}$. Exine thins towards colpi .........

CELASTRACEAE

E1 Exine thin, usually less than $2 \mu \mathrm{m}$.

M2 Columellae distinct, coarse.

$\mathrm{N} 2$ Lumina $1.5-2 \mu \mathrm{m}$ Salix triandra

N1 Lumina 2-3 $\mu \mathrm{m}$ Salix fragilis t.

M1 Columellae fine or indistinct.

O2 Equatorial limb subangular. 
P2 Grain $>30 \mu \mathrm{m}$, prolate, polar area pointed. Lumina c. $1.5 \mu \mathrm{m}$, meridionally elongated in apocolpia ........... Dictamnus albus

RUTACEAE

P1 Grain 18-22 $\mu \mathrm{m}$, prolate. Lumina irregular in size, c. $1 \mu \mathrm{m}$. Endoaperture indistinct

O1 Equatorial limb circular.

Q2 Greatest diameter of grain $>20 \mu \mathrm{m}$. Transverse colpus/pore distinct.

R2 Costae equatoriales; "horns" on endoaperture Anagallis arvensis t. PRIMULACEAE

R1 Not so.

S2 Distinctly duplicolumellate

Lysimachia

S1 Simplicolumellate or columellae indistinct.

T2 Muri broad. With transverse colpus.

U2 Columellae indiscernible. Margo heavy .. Lysimachia PRIMULACEAE

U1 Columellae distinct. Margo thin .. Anagallis tenella t. PRIMULACEAE

T1 Muri narrow. Endoaperture poroid or indistinct.

V3 Grain large, $P=45-73 \mu \mathrm{m}$, prolate, equatorial outline subangular. Brochi c. 1.5-2 $\mu \mathrm{m}$, decreasing in size towards colpi. Endoaperture a pore ...... Bryonia

CUCURBITACEAE

V2 $\mathrm{P}<40 \mu \mathrm{m}$, prolate, equatorial outline variable, commonly interlobate. Brochi c. $1 \mu \mathrm{m}$. Colpus equat. constricted, endoaperture indistinct ...Scrophularia t.

SCROPHULARIACEAE

V1 $\mathrm{P}=19-25 \mu \mathrm{m}$, equatorial and polar outlines circular. Lumina $1.5-3.5 \mu \mathrm{m}$, smaller towards poles and colpi. Endoaperture a pore ........ Parnassia palustris PARNASSIACEAE

Q1 Grain smaller, $\mathrm{P}<20 \mu \mathrm{m}$.

W2 Endopore indistinct. Lumina not decreasing towards colpi Phillyrea OLEACEAE

W1 Endoaperture lalongate colpus, usually tapering, sometimes with small horns; margins sharp; costae \pm distinct. Lumina decreasing in size towards colpi and apocolpia ............ Anagallis tenella t. PRIMULACEAE

D1 Micro-reticulate; brochi less than $1 \mu \mathrm{m}$.

X2 Costae aequatoriales; "horns" on endoaperture. Grain 20-25 $\mu \mathrm{m}$ Anagallis arvensis t. PRIMULACEAE

$\mathrm{X} 1$ No costae aequatoriales.

Y2 Colpus slit-shaped

Z2 Endoaperture a transverse colpus. 
$\mathrm{A}^{\prime} 4$ Grain large: $\mathrm{P}=40 \mu \mathrm{m}, \mathrm{E}=25 \mu \mathrm{m}$. Endoaperture distinct, with abruptly pointed ends Andrachne telephioides EUPHORBIACEAE

$A^{\prime} 3 \mathrm{P}=14-17 \mu \mathrm{m}, \mathrm{E}=13-16 \mu \mathrm{m}$. Endoaperture distinct, with tapering ends Asterolinon linum-stellatum PRIMULACEAE $\mathrm{A}^{\prime} 2 \mathrm{P}=23-28 \mu \mathrm{m}, \mathrm{E}=22-28 \mu \mathrm{m}$. Endoaperture distinct, with blunt ends . .

Ricinus communis EUPHORBIACEAE

$\mathrm{A}^{\prime} 1 \mathrm{P}=17-22 \mu \mathrm{m}, \mathrm{E}=15-20 \mu \mathrm{m}$. Endoaperture indistinct . Ruta montana t. RUTACEAE

Z1 Endoaperture poroid.

$\mathrm{B}^{\prime} 2 \mathrm{P}=18-22 \mu \mathrm{m}, \mathrm{E}=18-22 \mu \mathrm{m}$. Endopore indistinct, without costae $\ldots$.

Fraxinus ornus

OLEACEAE

B'1 Various sizes. Endopore covered, with distinct costae. Refer E11.1; See key to species and groups in NW5. Incl. Oxyria digyna ..... Rumex t. POLYGONACEAE

Y1 Colpus not slit-shaped.

C'2 Exine $2 \mu \mathrm{m}$ or thicker. Transverse endocolpus prominent, oblong. Ectocolpus sunken. $\mathrm{P}=13-28 \mu \mathrm{m}$

C'1 Exine thinner than $2 \mu \mathrm{m}$.

D'3 Endoaperture \pm circular.

E'3 Muri supra-microechinate

Fumana

CISTACEAE

E'2 Muri not microechinate. Prolate. Colpus equatorially constricted. Very similar - refer to reference slides.

$F^{\prime} 4 \quad P=15-23 \mu \mathrm{m}, E=10-21 \mu \mathrm{m}$

Elatine alsinastrum ELATINACEAE

$F^{\prime} 3 P=26 \mu \mathrm{m}, E=18 \mu \mathrm{m}$ Cercis siliquastrum

$F^{\prime} 2 \quad P<40 \mu \mathrm{m}$ LEGUMINOSAE

$F^{\prime} 1 \quad P=15-23 \mu \mathrm{m}, E=11-15 \mu \mathrm{m}$ Scrophularia t. SCROPHULARIACEAE $\mathrm{F} 1 \mathrm{P}=15-23 \mu \mathrm{m}, \mathrm{E}=11-15 \mu \mathrm{m}$ Hypericum perforatum $\mathrm{t}$. GUTTIFERAE

E'1 Muri not microechinate. Colpus fusiform, not constricted, with smooth membrane. $\mathrm{P}=20-25 \mu \mathrm{m}$, subprolate, equatorial outline circular $\ldots$

Kickxia

SCROPHULARIACEAE

D'2 Endoaperture lalongate

G'2 Endoaperture 11-16 $\mu \mathrm{m}$ broad, with short costae near colpus. $\mathrm{P}=28$ $41 \mu \mathrm{m} \ldots \ldots \ldots \ldots \ldots \ldots \ldots \ldots \ldots \ldots \ldots \ldots \ldots$ Hyoscyamus niger

SOLANACEAE

G'1 Endoaperture 7-10 $\mu \mathrm{m}$ broad, without costae. $P=21-24 \mu \mathrm{m} \ldots \ldots$. Hypericum hircinum $\mathrm{t}$. GUTTIFERAE

D'1 Endoaperture area with 2-6 small , \pm distinct, lalongate narrow endocolpi. Saxifragaceae p.p. 
$\mathrm{H}^{\prime} 2 \mathrm{P}>16.5 \mu \mathrm{m}, \mathrm{E}>14 \mu \mathrm{m}$
$\mathrm{H}^{\prime} 1 \mathrm{P}<16.5 \mu \mathrm{m}, \mathrm{E}<14 \mu \mathrm{m}$ Saxifraga stellaris

SAXIFRAGACEAE enium alternifolium SAXIFRAGACEAE

Key E11.6. Tricolporate. Intectate-clavate.

Ilex aquifolium is the only taxon properly keying out here, although Linum spp. may be considered to have a lolongate endoaperture paralleling the smaller ectoaperture - see Key E7.7 (Tricolpate, clavate).

\section{Key E12. Stephanocolporate}

All grains are psilate-scabrate except where otherwise indicated. The transverse colpus in Boraginaceae is blunt-ended, pore-like. The number of colpi varies individually; numbers indicated represent averages.

A2 More than 4 colpi. Cf. also Key E19.

B2 Grain larger than $20 \mu \mathrm{m}$.

C2 Endoapertures colpoid.

D2 (Endo-)colpus aequatorialis present. No costae aequatoriales

E2 More than 10 colpi. Grain barrel-like (depressed oval) with an equatorial ridge.

F2 Polar area with several isodiametric lacunae. Polygala.

G2 7 lacunae in apocolpium $(2-5 \mu \mathrm{m})$

Polygala monspeliaca t. POLYGALACEAE

G1 15 lacunae in apocolpium $(4-7 \mu \mathrm{m})$ Polygala vulgaris t. POLYGALACEAE

F1 Polar area coarsely vermiculate, no round lacunae ....... Utricularia LENTIBULARIACEAE

E1 9-10 colpi. Grain spherical. No equatorial ridge ...... Borago officinalis

D1 Endocolpi distinctly separate.

H2 7-11 meridional colpi. Grain compressed-oval, $\mathrm{P}=23-32 \mu \mathrm{m}$. Symphytum

H1 Mostly 7 meridional colpi. Grain bone-shaped, $\mathrm{P}=14-25 \mu \mathrm{m} \ldots \ldots \ldots \ldots$.

BORAGINACEAE

BORAGINACEAE

C1 Endoapertures poroid or obscure.

I2 6-7 colpi.

J2 Microreticulate. Grain spherical ................. Pinguicula LENTIBULARIACEAE

J1 Psilate to striate. Grain prolate. Tricolporate with broad colpal operculi, thus appearing 6-colporate Sanguisorba officinalis ROSACEAE

I1 5 colpi (more commonly 4 ). 
K2 Grain more than $40 \mu \mathrm{m}$, subprolate, angulaperturate. Tectate, superficially scabrate except in polar area which has an intectate field of coarse columellae

Viola arvensis $\mathrm{t}$. VIOLACEAE

K1 Grain 30-40 $\mu \mathrm{m}$, spheroidal, circular in polar view. Reticulate ..... Citrus RUTACEAE

B1 Grain smaller than $20 \mu \mathrm{m}$.

L2 Grain bone-shaped. Meridional colpi very short, hardly discernible. Protruding poroid area with free granules $\sim$ operculum.

M2 Colpi 4-6. $\mathrm{P}=14-17 \mu \mathrm{m}$

Buglossoides arvensis t.

M1 Colpi 7. $P=14-25 \mu \mathrm{m}$

BORAGINACEAE

L1 Grain compressed oval, $\mathrm{P}=18-21 \mu \mathrm{m}$. Meridional colpi distinct, $\begin{array}{r}\text { BORAGINACEAE } \\ 7-8 \ldots \ldots \ldots \ldots\end{array}$

BORAGINACEAE

A1 Four colpi.

N2 Grain larger than $40 \mu \mathrm{m}$.

O2 Transverse colpus distinct.

P2 Grain prolate, oval in equatorial view. Psilate

Anchusa arvensis t. BORAGINACEAE

P1 Grain oblate. Echinate. See key to species in Key E11.2 ......... Lonicera

CAPRIFOLIACEAE

O1 No transverse colpus. Viola.

Q2 Grain depressed oval (barrel-shaped) to polyedric. Polar area with an intectate field of coarse columellae

Viola arvensis $\mathrm{t}$.

VIOLACEAE

Q1 Grain oval. Exine structure uniform

Viola riviniana $\mathrm{t}$.

VIOLACEAE

N1 Grain smaller than $40 \mu \mathrm{m}$.

R3 Grain elliptic or circular in equatorial view.

S2 H-endoaperture. Reticulum striate near colpus

Blackstonia perfoliata

S1 Endoaperture indistinct in surface view. Finely reticulate.

T2 Colpi slightly sunken in polar view. $\mathrm{P}=15-25 \mu \mathrm{m}$. (Usually tricolporate) ...

OLEACEAE

T1 Colpi not sunken. $\mathrm{P}=23-29 \mu \mathrm{m}$

\section{Ceratonia siliqua}

LEGUMINOSAE

R2 Grain compressed oval. Equatorial exine reticulate. Transverse colpus present.

U2 Exine thinner in polar area. Equatorial reticulum coarse

Nonea

U1 Exine thickness uniform. Grain more than $20 \mu \mathrm{m}$ 
V2 Meridional colpi with margo of coarse columellae

V1 Meridional colpi not with coarse columellae.

W2 Colpi short. Equator constricted. Psilate to punctate

Anchusa officinalis t. BORAGINACEAE

W1 Colpi long. Equator not constricted. Reticulate Citrus RUTACEAE

R1 Grain constricted oval. No transverse colpi. Pores (but not constriction) nearer one pole.

$\mathrm{X} 2$ Poles normally of equal width. Grain $>13 \mu \mathrm{m}$.. Buglossoides purpurocaerulea BORAGINACEAE

$\mathrm{X} 1$ One pole usually considerably wider than the other. Grain $<13 \mu \mathrm{m}$

Lithospermum BORAGINACEAE

\section{Key E13. Pericolporate}

Tri- or stephanocolporate grains sometimes appear with more apertures than normal, and are then usually imperfectly isometric. The most important cases are those set out below.

A2 Echinate.

B2 Apertures arranged in spirals

Gossypium

MALVACEAE

B1 Apertures not arranged in spirals. Cf. Key E11.2 Compositae Asteroideae COMPOSITAE

A1 Psilate or reticulate.

C2 Psilate. Costae aequatoriales present. See Key E11.1 ........ Polygonum aviculare t. POLYGONACEAE

C1 Psilate with perforations and/or reticulate. Colpus slit-shaped. Pore covered. No costae aequatoriales. Cf. Key E11.1. See key to species and groups in NW5. Incl. Oxyria digyna

\section{Key E14. Diporate}

A2 Grains elongate with pores near ends

B2 Scabrate (usually in planar tetrads)

Cytinus ruber

RAFFLESIACEAE

B1 Reticulate

C2 Reticulate, muri much narrower than lumina. Pore $10 \mu \mathrm{m}$, edges ragged. $\mathrm{P}=30-32 \mu \mathrm{m}$, $\mathrm{E}=$ c. $60-85 \mu \mathrm{m}$

LILIACEAE

C1 Perforate to microreticulate, muri \pm as wide as lumina. Pore $5 \mu \mathrm{m}$, not with ragged edges. $\mathrm{P}=17-24 \mu \mathrm{m}, \mathrm{E}=\mathrm{c} .30-45 \mu \mathrm{m}$. 
Pollen grains of Ficus fit here, but given its highly specialized pollination mechanism it is unlikely that pollen would ever be found in sediments except in exceptional circumstances. The pollen grain is psilate and very small $(P=10 \mu \mathrm{m}, \mathrm{E}=16 \mu \mathrm{m})$.

D2 Pori distinctly protruding in optical section, annuli broad and distinct ... Humulus lupulus

D1 Pori not or only slightly protruding, annuli narrow or absent

E2 Pori slightly or not protruding, circular in outline, small (diameter 1-2.5 $\mu \mathrm{m}$ ). Grains small $(E=13-20 \mu \mathrm{m})$

Urtica dioica

E1 Pori not protruding, usually irregularly elliptic, rather large (diameter 2.5-5 $\mu \mathrm{m}$ ). Grains larger $(E=18-37 \mu \mathrm{m})$

Morus alba

MORACEAE

Key E15. Triporate

A2 With large, cylindrical vestibulum, more than $10 \mu \mathrm{m}$ deep. Onagraceae.

B2 Grain $50 \mu \mathrm{m}$ or larger

Epilobium angustifolium t. ONAGRACEAE

B1 Grain smaller than $50 \mu \mathrm{m}$

Circaea lutetiana

ONAGRACEAE

A1 Vestibulum smaller, conical, or absent.

C3 Reticulate. Grains large, $>50 \mu \mathrm{m}$.

D2 Pores annulate, not ragged

Cucumis melo CUCURBITACEAE

D1 Pore margins ragged (usually diporate) Colchicum LILIACEAE

C2 Distinctly echinate.

E2 Grain more than $50 \mu \mathrm{m}$. Exine thick, more than $4 \mu \mathrm{m}$. Echinae dimorphic, of two size classes. Dipsacaceae.

F2 Pore with thickened anulus surrounded by halo of thinner exine. Echinae short, broad-based, scattered.

G2 Exine \pm even thickness.

H2 Microechinae between echinae not distinct. Halo broad. Grain spheroidal

Dipsacus t. DIPSACACEAE

H1 Microechinae distinct. Halo narrow. Grain oblate ... Scabiosa sicula t. DIPSACACEAE

G1 Exine much thicker at poles than at equator, often partially detached, forming sacci 
F1 Anulus indistinct, no halo. Echinae slender, crowded; microechinae distinct ...

E1 Grain smaller. Echinae uniform, scattered. Campanulaceae.

H2 Echinae $\geq 1 \mu \mathrm{m}$. Grain $>25 \mu \mathrm{m}$.

I2 Echinae c. $1 \mu \mathrm{m}$. Pore annuli 3.5-4 $\mu \mathrm{m}$....... Campanula rotundifolia

I1 Echinae coarser, longer. Pore annuli thicker. Usually 4-porate ... Phyteuma

H1 Echinae $<1 \mu \mathrm{m}$.

J2 Tectum rugulate-perforate. Grain $>25 \mu \mathrm{m} \ldots \ldots$ Campanula erinus $\mathrm{t}$.

J1 Tectum psilate-perforate. Grain $<25 \mu \mathrm{m} \ldots \ldots \ldots \ldots \ldots \ldots \ldots$ Jasione

C1 Psilate-scabrate.

K2 Equatorial limb almost circular, without protruding pore edges.

L2 Scabrate; micro-sculpturing faintly rugulate with minute echinae.

M2 Columellae not visible, even at high magnification; exine 1-2 $\mu \mathrm{m}$. Morus

$\mathrm{N} 2 \mathrm{E}=19-25 \mu \mathrm{m}$. Outline in polar view circular. (Usually diporate, elliptical)

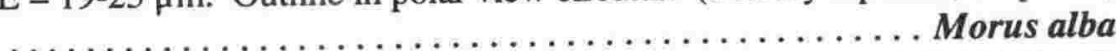

MORACEAE

N1 $E=26-37 \mu \mathrm{m}$. Outline in polar view often \pm angular. Pores not always equidistant, $3-4 \ldots \ldots \ldots \ldots \ldots \ldots \ldots \ldots \ldots \ldots \ldots \ldots \ldots \ldots$ Morus nigra

MORACEAE

M1 Columellae very distinct, scattered; exine c. $2 \mu \mathrm{m}$. Grain more than $20 \mu \mathrm{m}$..

ULMACEAE

L1 Psilate, columellae delicate.

$\mathrm{O} 2$ Exine very thin, but thickened around pores, with minute projections; pore margins a little protruding.

P2 Spinules scattered. 2-5 pores, usually $3-4 \ldots \ldots \ldots \ldots$ Urtica dioica

URTICACEAE

P1 Spinules closer together, more regularly distributed. (Urtica urens has 3-4 pores, Pilea microphylla 2-3) $\ldots \ldots \ldots \ldots \ldots \ldots \ldots$ Urtica urens $\mathrm{t}$.

URTICACEAE

O1 Exine slightly thicker, but not thickened around pores; pore margins not protruding. Projections slightly coarser. 3-4 pores ..........Parietaria

URTICACEAE

K1 Equatorial limb semiangular, or with protruding pore edges.

Q3 Psilate. Grain square in equatorial view

Q2 Scabrate, \pm verrucose or granular 
R2 Grain very large, prolate, polar axis c. $205 \mu \mathrm{m}$. Pores, trumpet-shaped with thickened edges, protrude from circular equatorial outline. Subtectal sexine "spongy", not columellate

R1 Grains $20-40 \mu \mathrm{m}$, spheroidal to oblate.

S2 Pores not protruding, annuli narrow. Outline in polar view often \pm angular (pores not always equidistant)

S1 Pores protruding, annuli broad. Incl. Cannabis sativa and Humulus lupulus, which may be distinguished on subtle characters of pore morphology, see NW4 Humulus t.

Q1 Micro-sculpturing and structure more or less rugulate, with scattered minute spinules on top of low vallae (phase!). Betulaceae.

T2 Equatorial limb circular with protruding pores. Nexine and tectum diverge in the pores, forming a vestibulum.

U2 Vestibulum distinct. Tectum sharply thickened at the pore.

Betula pendula

BETULACEAE

U1 Vestibulum not distinct. Tectum not appreciably thickened at the pore. Incl. some grains of Carpinus, which is usually 4-porate .... Ostrya t.

CORYLACEAE

T1 Equatorial limb semiangular, pore not protruding. Nexine and tectum do not diverge in the pore. Depth of pore not much greater than thickness of the regular exine

CORYLACEAE

\section{Key E16. Stephanoporate}

A4 Echinate. Echinae distinct, regularly distributed. Campanulaceae. See Key E15: E1. Species of Phyteuma, Asyneuma, and Legousia are usually 4-porate, but occasional grains of some Campanula spp. are also stephanoporate.

B1 Echinae $<1 \mu \mathrm{m}$. Incl. Legousia hybrida $\ldots \ldots \ldots \ldots \ldots \ldots \ldots \ldots$ Asyneuma t.

B2 Echinae $>1 \mu \mathrm{m}$

CAMPANULACEAE

CAMPANULACEAE

A3 Finely micro-suprareticulate and microechinate; exine $2.5 \mu \mathrm{m}$ with distinct columellae. 4-8 pores without annuli. $\mathrm{P}=23-26 \mu \mathrm{m} \ldots \ldots \ldots \ldots \ldots \ldots \ldots \ldots \ldots \ldots \ldots \ldots$ Theligonum cynocrambe

A2 Coarsely and softly rugulate or suprareticulate. Peroblate. 4-7 pores. Ulmus.

C3 Rugulate sculpture on both poles. Angular outline. 5-7 pores, mostly $6 \ldots \ldots$ U. procera

ULMACEAE

C2 Rugulate sculpture reduced on proximal pole. Angular outline. 4-6 pores, mostly 4-5 ...

U. glabra t.

ULMACEAE

C1 Rugulate sculpture reduced on proximal pole. Rounded outline, well-developed annuli lacking. $4-6$ pores, mostly $5 \ldots \ldots \ldots \ldots \ldots \ldots \ldots \ldots \ldots \ldots \ldots \ldots \ldots$ minor type

ULMACEAE

A1 Psilate, scabrate or very delicately rugulate. 
D2 Grain globular. Equatorial limb circular. Pores not distinctly protruding

E2 Rugulate, spinulose. 3-6 pores

E1 Psilate. Columellae delicate.

F3 Pores with large cylindrical vestibulum (>10 $\mu \mathrm{m}$ deep). Grain more than $50 \mu \mathrm{m}$

F2 Pores annulate, protruding; no vestibulum, Mostly 3-porate, cf. Key E15 .....

F1 Pores annulate, but not protruding or vestibulate.

CANNABACEAE

G2 Exine very thin, structure very delicate. See Key E15: N2 for distinction of species which are occasionally stephanoporate ............. Urtica

G1 Exine thicker, structure coarser.

H2 Pores 3 or 4

Parietaria

URTICACEAE

H1 Pores 6-12 Urtica pilulifera $\mathrm{t}$.

URTICACEAE

D1 Grain oblate. Equatorial limb more or less angular and/or pore edges protruding.

I2 Neighbouring pores (annuli) connected with thickened bands (arci). Pore with thick anulus. 46 pores

I1 No arci.

J1 4-6 "pores" meridionally elongated with endexinous annuli. Myriophyllum.

K2 Anulus distinct, protruding. Pores circular or elliptic M. spicatum

K1 Anulus externally flat, not protruding. Pores colpoid

HALORAGACEAE

J1 Exine thickness uniform. Pores circular, without endexinous annuli.

HALORAGACEAE

L2 Equatorial limb angular (square)

Vicia lathyroides

LEGUMINOSAE

L1 Equatorial limb more or less circular.

M2 Grain more than $40 \mu \mathrm{m}$. Microsculpturing faintly rugulate with minute spinules on vallae. Incl. some grains of Ostrya ........ Carpinus t.

CORYLACEAE

M1 Grain less than $40 \mu \mathrm{m}$. Microechinate. Pores with narrow annuli, not always equidistant 
Verrucate or echinate

Key E17.1

Rugulate, striate or reticulate

Key E17.2

Psilate or scabrate

Key E17.3

\section{Key E17.1. Periporate. Verrucate or echinate}

A2 Verrucate. Special key

Plantaginaceae

A1 Echinate.

B2 Grains large, $>60 \mu \mathrm{m}$

C2 c. 75 pores. Grain c. $80-130 \mu \mathrm{m}$. Sexine between echinae composed of crowded baculae. Echinae long $(8-11 \mu \mathrm{m})$, with rounded tips, situated at the corners of polygonal ridges formed by longer baculae, in the centre of each of which are situated the rather indistinct pores. Smaller baculae surround the pores. ................. Ipomoea

$\mathrm{C} 1$ Echinae not linked by ridges

D2 Pores 4-12, large (diameter 15-26 $\mu \mathrm{m}$ ), not spirally arranged, with operculi. Grain 90-190 $\mu \mathrm{m}$. Echinae regularly spaced over whole grain, including over the operculi, which bear a single central echina. Echinae not based on cushions. Numerous microprojections (baculae or microechinae) between echinae. For detailed key to species see Ayala-Nieto et al. $1988 \ldots \ldots \ldots \ldots \ldots \ldots \ldots \ldots \ldots$ Cucurbita

CUCURBITACEAE

D1 Pores 9 to $>100$, in some species arranged in a spiral, without echinate operculi. Grain $>60 \mu \mathrm{m}$. Echinae usually sharp, in some species elevated on cushions of thicker exine. See special key

Malvaceae

B1 Grains $<60 \mu \mathrm{m}$

E2 Pores with annuli. Special key

Caryophyllaceae p.p.

E1 Pores without annuli.

F2 $24-28$ indistinct pores usually covered by non-operculate membranes with echinae. Grain 44-65 $\mu \mathrm{m}$. Echinae conical, c. $3 \mu \mathrm{m}$ long, irregularly spaced, with broad irregular bases which sometimes coalesce in groups of $2-3$

\section{Ranunculus arvensis $\mathrm{t}$.}

RANUNCULACEAE

F1 Pores 10-15 (diameter 4-6 $\mu \mathrm{m}$ ). Grain 26-36 $\mu \mathrm{m}$. Echinae sharp, about as tall as broad, irregularly distributed but without broad bases and not coalescent ....... 
Key E17.2. Periporate. Rugulate, striate or reticulate

A2 Vermiculate-rugulate. Columellae fine. Fewer than 50 pores

Buxus sempervirens

BUXACEAE

A1 Reticulate.

B2 Pores with annuli. Special key

Caryophyllaceae p.p.

B1 Annuli absent or indistinct.

C3 Reticulum regular, brochi $48 \mu \mathrm{m}$, muri simplicolumellate. Pore in each lumen: 60-75 pores

Tribulus terrestris

ZYGOPHYLLACEAE

C2 Reticulum regular, brochi $>4 \mu \mathrm{m}$, muri duplicolumellate. Not every lumina with a pore: 12-27 pores. Lumina with pores are smaller than those without

Polygonum persicaria t.

POLYGONACEAE

C1 Reticulum imperfect, brochi smaller and not polygonal. Fewer than 50 pores

D2 Pore indistinct, included in a lumen. Lumen with a pore slightly larger than those without. Columellae fused at top into wedge-shaped elements in surface view, which are arranged to form a reticulum ("croton" pattern)

Daphne

THYMELAEACEAE

D1 Pore larger than brochi. Microreticulate. Columellae not fused into wedge-shaped elements ............................. Buxus sempervirens

BUXACEAE

KeyE17.3. Periporate. Psilate-scabrate.

A2 Grain irregular, usually pear-shaped. Pores indistinct. Special key.

Cyperaceae

A1 Grain regularly ellipsoidic.

B2 Microechinate, dotted.

C2 Columellae fine and evenly distributed, or not visible.

D2 Pores aggregated towards one pole.

Juglans regia

D1 Pores \pm evenly distributed.

E2 Columellae clear, exine surface undulating. Pores 5-6

Celtis

E1 Columellae invisible, exine surface smooth. Pores 6-12, usually 7-9 ......

Urtica pilulifera t.

URTICACEAE

C1 Columellae coarse and/or irregularly distributed. Pores evenly distributed.

F2 Anulus distinct. Special key

Caryophyllaceae p.p.

F1 Anulus absent or indistinct.

G2 Surface undulating. Special key

Plantaginaceae 
G1 Surface even.

H2 Grain more than $25 \mu \mathrm{m}$. Indistinctly intrareticulate.

I2 Grain sub-polyhedric. Exine between pores distinctly crescentic. Pores round.

J2 Pores with annuli .................. Alisma

ALISMATACEAE

J1 No annuli ...

Damasonium alisma

ALISMATACEAE

I1 Grain (sub-)globular. Exine not distinctly crescentic. Pores irregular, indistinct

Papaver argemone $\mathrm{t}$.

PAPAVERACEAE

H1 Grain 10-25 $\mu \mathrm{m}$. No intrareticulum. Thalictrum

K3 Grain $>17 \mu \mathrm{m}$. Columellae distinct. Porus margins clearly defined, regular

T. lucidum

RANUNCULACEAE

K2 Grain $>17 \mu \mathrm{m}$. Columellae distinct. Porus margins poorly defined, irregular $\ldots \ldots \ldots \ldots \ldots \ldots \ldots \ldots \ldots \ldots \ldots \ldots \ldots \ldots \ldots \ldots \ldots$. minus

K1 Grain $<17 \mu \mathrm{m}$. Columellae usually very indistinct $T$. aquilegifolium RANUNCULACEAE
tinct $T$. aquilegifolium RANUNCULACEAE

B1 Not microechinate, dotted.

L2 Areas without tectum, surrounding and connecting pores ............ Ribes

GROSSULARIACEAE

L1 Tectum not reduced.

M2 Grain large $(70-80 \mu \mathrm{m})$. Exine thick

Calystegia

CONVOLVULACEAE

M1 Grain smaller.

N2 Anulus present.

O2 Anulus distinct, but often narrow and without structure.

P2 Tectum without perforations. Number of pores usually higher than 50 . Incl. Amaranthaceae and Sagina (Caryophyllaceae)

Chenopodiaceae t.

P1 Tectum perforate (indistinct in Sagina). Pores fewer than 50.

Q2 6-12 large pores. Tectum very thick. Anulus prominent. Columellae very fine and short. Fumaria.

R2 Usually 12 pores F. parviflora

PAPAVERACEAE

R1 Usually 6 pores F. densiflora $\mathrm{t}$.

PAPAVERACEAE

Q1 Not so. Special key Caryophyllaceae p.p.

O1 Anulus diffuse. Columellae widely dispersed in interporia, dense on annuli. Grain usually polyhedric. Intrareticulate. Alismataceae. 
S2 Grain less than $25 \mu \mathrm{m}$

S1 Grain more than $25 \mu \mathrm{m}$ Alisma

ALISMATACEAE

N1 No anulus.

T3 Grain 23-42 $\mu \mathrm{m}$. 4-11 pores. Exine punctate/microreticulate ..Pistacia

T2 Grain $10-25 \mu \mathrm{m} .4-12$ pores.

U2 Grain rounded. Distinct minute echinae. See Key E17.3: H1 .....

Thalictrum

RANUNCULACEAE

U1 Grain sub-polyhedric. Indistinctly dotted. Incl. Polycnemum (Chenopodiaceae)

Paronychia t.

CARYOPHYLLACEAE

T1 Grain c. $10 \mu \mathrm{m}$. No echinae. 4 pores Herniaria t.

CARYOPHYLLACEAE

\section{Key E18. Syncolpate}

Colpi sometimes fuse at one pole also in grains not considered here to be syncolpate.

\section{A3 Heteropolar.}

B2 Tricolporate with large pores, the three colpi joining at one pole only.

$\mathrm{C} 2$ Coarsely reticulate

Thesium divaricatum $\mathrm{t}$. SANTALACEAE

$\mathrm{Cl}$ Psilate to finely verrucate

Thesium alpinum

SANTALACEAE

B1 Single encircling colpus, closer to one pole. Microechinate LILIACEAE

A1 Isopolar.

D3 Colpi meridional.

E2 Two colpi fused to a ring. Grain usually split, edges of colpus ragged. Cf. Juniperus, Key E2.

F2 Grain 17-21 $\mu \mathrm{m}$. Scabrate (densely microechinate) Hypecoum

F1 Grain 26-32 $\mu \mathrm{m}$. Psilate perforate-perfossulate.

E1 More than two colpi.

G2 Colpi bifurcate, fused with neighbouring colpi, delimiting a large polar field. Grain larger than $30 \mu \mathrm{m}$. Coarsely striated .............. Nymphoides peltata

G1 Colpi 3, not bifurcate.

MENYANTHACEAE

H4 Grain striate. Tricolporate. Prolate, c. $45 \mu \mathrm{m}$ 
H3 Grain scabrate. No endoapertures. Grain subprolate, 18-21 $\mu \mathrm{m}$. Soldanella PRIMULACEAE

H2 Grain microverrucate. Grain oblate ............ Loranthus europaeus

LORANTHACEAE

H1 Grain psilate. Oblate, triangular in polar view. Short transverse colpus present

D2 Colpi spirally oriented (various arrangements). Grain spheroidal.

I3 Grain microechinate, $60-100 \mu \mathrm{m}$

Crocus

LILIACEAE

I2 Grain with scattered clavae on top of irregularly granulate exine, $110-130 \mu \mathrm{m} \ldots \ldots$.

Iris planifolia

IRIDACEAE

I1 Grain irregularly perforate, $30-50 \mu \mathrm{m}$. Exine usually splits

Berberis vulgaris t.

BERBERIDACEAE

D1 Peri-syncolpate. Exine divided into angular plates, usually falling apart.

J3 Exine microechinate, 1.5-3 $\mu \mathrm{m}$ thick, columellae coarse. Colpi 12 or more. Grain spheroidal, 20-35 $\mu \mathrm{m}$. See also Key E9 ................... Montia fontana

PORTULACACEAE

J2 Exine psilate, pitted, $1.5 \mu \mathrm{m}$ thick. Colpi 6 to 16 . Grain spheroidal, $30-40 \mu \mathrm{m}$. .....

Mahonia aquifolium

BERBERIDACEAE

J1 Exine psilate, pitted, $2 \mu \mathrm{m}$ thick. Colpi 6 or 12 . Grain spheroidal or a convex tetrahedron, $40-50 \mu \mathrm{m}$

Corydalis ochroleuca

PAPAVERACEAE

\section{Key E19. Heterocolpate}

Grains with three colpi with pores which alternate with three similar without pores.

A2 Colpi approximately equally long, or aporate colpi longer than porate colpi. Grains flattened at poles, often dumbbell-shaped (constricted at equator), or compressed oval in equatorial view. Polar area index large, above 0.5. Boraginaceae. Also see detailed key in NW2.

B2 Endoapertures join together at equator in a narrow colpus aequatoriales. "Aporate" colpi longer than those in which the endoapertures are more strongly developed Cynoglossum t.

B1 Endoapertures discrete, not joined at equator.

BORAGINACEAE

$\mathrm{C} 2$ Grain $>30 \mu \mathrm{m}$, not constricted at equator

Heliotropium

C1 Grain $<20 \mu \mathrm{m}$, constricted at equator.

BORAGINACEAE

D2 Isopolar, endoapertures at equator

Myosotis t.

BORAGINACEAE

D1 Heteropolar, 2 endoapertures closer to one pole and 1 closer to the other Lappula

BORAGINACEAE

A1 Aporate colpi distinctly shorter than the porate ones. Polar area smaller. Grain circular to oval in equatorial view, not flattened at poles, or constricted at equator.

E2 Exine scabrate-psilate 
E1 Non-porate colpi separated from porate colpi by bands of thick, tectate striate exine. Lythrum

F2 Grain asymmetrical, with apertures often arranged irregularly. Non-porate colpi wide areas of intectate, granular exine

Lythrum portula

LYTHRACEAE

F1 Grain symmetrical, with apertures clearly zonally arranged. Non-porate colpi do not occupy wide areas, are narrower and shorter than porate colpi ... Lythrum salicaria t.

LYTHRACEAE

\section{Key E20. Fenestrate}

Grains echinate, lophate (ridged). COMPOSITAE Cichorioideae.

A1 Ectoapertures pores, surrounded by continuous lophae; paraporal lacunae 2 per mesocolpium; total of 15 lacunae

Scolymus

A2 Ectoapertures colpi, broadly rounded to angular and often divided into lacunae by constrictions or short paired ridges.

B2 Colpi each divided into 2 abporal lacunae by a central constriction or pair of ridges.

C2 One equatorial lacuna per mesocolpium. Paraporal lacunae absent, interporal lacunae present, total of 15 lacunae

Tragopogon

C1 Two equatorial lacunae per mesocolpium. Abporal lacunae extending to an expanded spiny polar area, or to a polar lacuna; total of 18-20 lacunae.

D2 Polar lacunae absent, extensive echinate polar areas present; total of 18 lacunae .

Scorzonera laciniata t.

D1 Polar lacunae 1 per pole, large, hexagonal; total of 20 lacunae

Scorzonera hispanica t.

B1 Colpi each divided into 1 poral and 2 abporal lacunae by 2 pairs of short continuous ridges. Paraporal lacunae 2 per mesocolpium.

E2 Polar lacunae absent, polar area ranging from a triradiate ridge to an extensive echinate region; total of 15 lacunae (20 in occasional 4-aperturate grains).

F2 Polar area not more than a triradiate ridge usually with one row of echinae, abporal and paraporal lacunae extending almost to the poles .......... Lactuca t.

F1 Polar area more extensive.

G2 Grains small, usually $\mathrm{E}<25 \mu \mathrm{m} \ldots \ldots \ldots \ldots \ldots \ldots \ldots$ Hieracium $\mathrm{t}$.

G1 Grains larger, E $>25 \mu \mathrm{m}$.

H2 Polar area small with 1-(2)-4 isolated central echinae ..... Cichorium t.

H1 Polar area extensive, 4-(5.5)-15 isolated central echinae .. Taraxacum t.

E1 Polar lacunae 3 in each of the extensive polar areas; total of 21 lacunae ( 28 in occasional 4-aperturate grains)

Sonchus t. 
A3 Tricolporate (Key E11.2)

Abutilon t.

A2 Pericolporate (apertures spirally arranged; Key E13)

Gossypium

A1 Periporate (apertures spirally or evenly arranged).

B2 Supratectal processes all conical echinae

C2 Bases of echinae subglobose; porus margins often indistinct

Althaea officinalis

C1 Bases of echinae not subglobose; porus margins distinct

D2 <100 pori per grain. Refer to reference material!

E3 $46-93$ pori $(3-9 \mu \mathrm{m})$. Echinae c. $18 \mu \mathrm{m}$

Hibiscus

E2 $60-80$ pori (c. $3 \mu \mathrm{m})$. Echinae $414 \mu \mathrm{m}$

Malva moschata

E1 $78-81$ pori $($ c. $10 \mu \mathrm{m})$. Echinae $22 \mu \mathrm{m}$

Abelmoschus esculentus

D1 $>100$ pori per grain; distance between pori c. $11 \mu \mathrm{m}$

F2 Echinae tapering, forming more than half of the thickness of the exine and frequently curved towards the apex; grains larger than $110 \mu \mathrm{m} \ldots \ldots \ldots \ldots$

Althaea hirsuta

F1 Echinae generally straight sided, forming approximately half of the thickness of the exine; grains $60-125 \mu \mathrm{m}$

Malva sylvestris $\mathbf{t}$.

B1 Supratectal processes other than conical echinae present including clavae, verrucae or bluntly rounded echinae

G2 Number of pori $>200$; echinae long, conical, acute, interspersed with smaller rounded clavae; porus diameter $1-2 \mu \mathrm{m}$; sexine-1 and sexine- $2<1 \mu \mathrm{m} \ldots \ldots \ldots \ldots$ Alcea $\mathrm{t}$.

G1 Number of pori $<150$; echinae either a mixture of conical and blunt, the latter may be almost parallel sided or with rounded verrucae only; porus diameter $2-3 \mu \mathrm{m}$; sexine-1 and sexine- $2>1 \mu \mathrm{m}$

Malva pusilla

\section{CARYOPHYLLACEAE (periporate)}

(Cf. Chanda 1962; Faegri \& Iversen 1992; McNeill \& Crompton 1978; Melzheimer 1975; Moore et al 1991)

The section Sperguleae has colpate grains, cf. Key E7.1.

A2 Grain c. $10 \mu \mathrm{m} .1-8$ pores. Incl. Corrigiola litoralis p.p.

Herniaria t.

A1 Grain larger than $10 \mu \mathrm{m}$. 
B2 Grains spheroidal

C2 Tectum with scattered distinct perforations similar in number to the columellae and spinules, or per-reticulate.

D2 10-30 pores. Incl. Arenaria, Cerastium, Dianthus, Lychnis p.p., Silene p.p., Stellaria

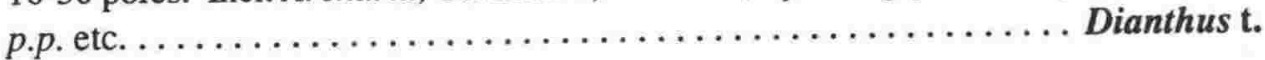

D1 Pores 30 or more.

F3 Grain $>50 \mu \mathrm{m}($ c. $63 \mu \mathrm{m})$. $>30$ pores (up to 45$), \pm 8 \mu \mathrm{m} \ldots \ldots \ldots \ldots \ldots$

Agrostemma githago

F2 Grain c. $50 \mu \mathrm{m}$. Pores 30-35, c. $4.5 \mu \mathrm{m}$. Echinate. ........ Silene alba $\mathrm{t}$.

F1 Grain $<45 \mu \mathrm{m}($ c. $40 \mu \mathrm{m})$. Pores $30-40$, c. $6 \mu \mathrm{m} \ldots \ldots$ Lychnis flos-cuculi

C1 Tectum with indistinct perforations, distinctly scabrate. Pores small, more than 24. Incl. Sagina and Amaranthaceae . . . . . . . .

B1 Grains rounded-polyhedral. Pores (+annuli) in depressions, surrounded by a zone without big columellae; the rest of the grain forming a hexagonal pattern of ridges.

G2 Ridges scabrate or with indistinct sculpturing.

H3 Grain 8-25 $\mu \mathrm{m}$. Pores 6-12 (2.5-4 $\mu \mathrm{m})$.

Paronychia t.

H2 Grain 20-35 $\mu$ m. Pores 16-19 (c. $4 \mu \mathrm{m}$ )

Scleranthus annuus t.

H1 Grain c. $36 \mu \mathrm{m}$. Pores 12 (c. $5 \mu \mathrm{m})$.

Scleranthus perennis

G1 Ridges echinate. Grain c. $42 \mu \mathrm{m}$. Pores $12(6 \mu \mathrm{m})$.

Stellaria holostea

\section{CYPERACEAE}

(Faegri \& Iversen 1992; Valdes et al 1987)

Grains with perforate/granulate tectum with lacunae, i.e. areas where the tectum is broken up into small frustillae, usually forming an areolar sculpturing. The form and arrangement of lacunae is of great diagnostic value. One or two of the lateral lacunae may be smaller than the others; the indices given below do not apply to these.

A3 Lateral lacunae oblong, length twice as long as the breadth or more. Grain ovoid or pear-shaped.

B2 Length of lateral lacunae more than half that of the grain.

C2 Grain 36-43 $\mu \mathrm{m}$

Schoenus nigricans

C1 Grain 27-37 $\mu \mathrm{m}$

Cyperus longus t.

B1 Length of lateral lacunae less than half of that of the grain. Grain more than $40 \mu \mathrm{m} \ldots \ldots$
Scirpus 
A2 Lateral lacunae more or less circular or slightly elongated. Grain ovoid or pear-shaped.

D2 2-3 small lacunae, length less than $20 \%$ that of the grain. Grain more than $40 \mu \mathrm{m}$, the narrow end usually projecting like a beak or a finger $\ldots \ldots \ldots \ldots \ldots \ldots$ Cladium mariscus

D1 4-6 lateral lacunae, their length normally more than $20 \%$ of that of the grain. Grain smaller than $40 \mu \mathrm{m}$, exceptionally (Carex hirta) 40-50 $\mu \mathrm{m}$. The narrow end rounded: Carex type.

E2 Lacunae poroid. Incl. Scirpus p.p. .................. Carex hallerana t.

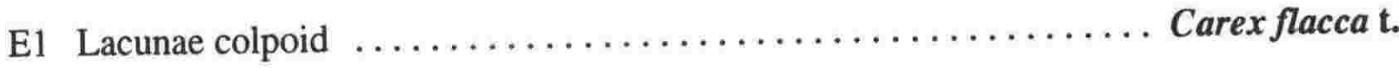

A1 Lacunae irregular, rather large. Grain almost isodiametric, less than $20 \mu \mathrm{m}$. Structure rather coarse, columellae distinct $\ldots \ldots \ldots \ldots \ldots \ldots \ldots \ldots \ldots \ldots \ldots \ldots \ldots \ldots \ldots \ldots$ Cyperus michelianus

\section{GRAMINEAE}

(Andersen 1979; Andersen \& Bertelsen 1972; Beug 1961; Faegri \& Iversen 1992)

Owing to the great importance of grasses, both in natural plant communities and in cultivated areas, and also the importance of certain taxa as cultivation indicators, it would be highly desirable to be able to identify grasses to species. However, the paucity of distinguishing characters limits separation. The Gramineae as a family are easily recognized, but also very monotonous in pollen morphology. The main identifying oharanters used in this key' are: diameter of grain. of pore and of anulus (the latter two being the most reliable). The measurements are taken from grains embedded in glycerol jelly. Where data from Anderson's key (grains embedded in silicon oil) has been used a conversion factor of 1.2 has been applied. However, for the same species, conversion factors may vary between 1.1 and 1.3 for grain size and between 1.1 and 1.5 for pore size.

To make the distinction between the two cereal types, Triticum and Avena, the less easily seen columellae pattern is used. Two different patterns can be detected. In single-grain structure columellae are individually free, and more or less crowded and aggregated. In the double-grain structure some or all columellae are united into small frustillae. These patterns are also used to make the less important distinction between the two wild grass types of grasslands, Dactylis and Festuca. The patterns can be more easily seen using phase contrast.

A2 Grain $>40 \mu \mathrm{m}$. Incl. mostly cereals and very few wild grasses. Cerealia type.

B3 Grain $>60 \mu \mathrm{m}$ Zea mays

B2 Grain between 40 and $60 \mu \mathrm{m}$.

C2 P/E ratio higher than 1.25 . Exine distinctly scabrate. Pore lateral ..... Secale cereale

C1 P/E ratio less than 1.25. Pore (sub-)apical.

D2 Anulus diameter larger than $12 \mu \mathrm{m}$. Exine verrucate.

E2 Exine with single-grain structure

Triticum t.

E1 Exine with double-grain structure

Avena

D1 Anulus diameter between 10 and $12 \mu \mathrm{m}$. Exine scabrate. Incl. some wild grasses. 
F1 Outer limit of anulus not sharp

Agropyron t.

A1 Grain smaller than $40 \mu \mathrm{m}$. Anulus diameter less than $10 \mu \mathrm{m}$. Incl. most wild-grasses. Gramineae

G2 Grain smaller than $26 \mu \mathrm{m}$. Columellae indistinct

Phragmites australis

G1 Grain between 26 and $40 \mu \mathrm{m}$.

H2 Double-grain structure. Columellae distinct.

Dactylis t.

H1 Single-grain structure.

I2 Exine thick

Festuca t.

I1 Exine thin. Some aggregation opposite the pore Glyceria t.

\section{PLANTAGINACEAE}

(Cf. Clarke \& Jones 1980 (NW2); Faegri \& Iversen 1992; Saad 1986; Ubera, Galan \& Guerrero 1988.)

A3 Pore with distinct anulus.

B2 Pore operculate (operculum may be lost, but then no granules on pore membrane). Markedly protruding anulus. $8-12$ pores, 1-3 $\mu \mathrm{m}$. Distinctly scabrate, verrucate, undulate. Grain $18-33 \mu \mathrm{m} \ldots \ldots \ldots \ldots \ldots \ldots \ldots$ lanceolata $\mathrm{t}$.

B1 Pore rarely operculate, but with isolated granules.

C2 Markedly protruding anulus. Verrucate, scabrate, undulate. 5-8 pores, 1-3 $\mu \mathrm{m}$.

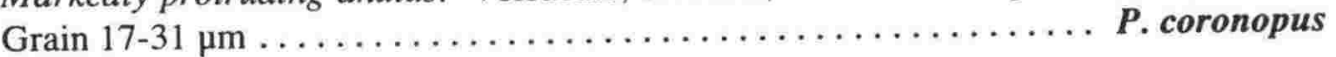

C1 Exine scabrate, undulate. 5-9 pores, $2-4 \mu \mathrm{m}$. Grain $20-29 \mu \mathrm{m} \ldots \ldots \ldots$. . alpina t.

A2 Pore with incipient anulus.

D2 Pore operculate.

E2 Exine psilate, slightly undulate. Grain $22-33 \mu \mathrm{m}$. 6-12 pores, 2-4 $\mu \mathrm{m} \ldots$. P. albicans

E1 Exine microechinate, not undulate. Grain 19-24 $\mu \mathrm{m}$. 8-9 pores, 2-3 $\mu \mathrm{m}$.P. argentea

D1 Pore not operculate, membrane granulate. Exine undulate-verrucate. Grain 22-28 $\mu \mathrm{m}$. 4-6 pores, 3-4 $\mu \mathrm{m}$.

P. amplexicaulis

A1 Pore without distinct anulus, with granules, no operculum.

F2 Grain $>25 \mu \mathrm{m}$.

G2 $8-9$ pores, 3-4 $\mu \mathrm{m}$. Verrucate, scabrate. Grain $27-36 \mu \mathrm{m}$ P. atrata 
G1 46 pores.

H2 Verrucae distinct. Pores not sharply defined, 2-4 $\mu \mathrm{m}$. Grain 21-28 $\mu \mathrm{m} P$. arenaria

H1 Verrucae indistinct. Pores 2-4 $\mu \mathrm{m}$. Grain $22-32 \mu \mathrm{m}$

P. bellardii

F1 Grain $<25 \mu \mathrm{m}$. Pores not sharply delimited. Microechinate, or verrucate/undulate.

I3 Distinctly micro-echinate. Pores 4-7, 1-2 $\mu \mathrm{m}$. Grain $14-25 \mu \mathrm{m} . \ldots \ldots$. . P. afra

I2 Verrucae very coarse, scabrae distinct. Pores 7-11, 2-5 $\mu \mathrm{m}$. Grain 18-27 $\mu \mathrm{m} \ldots$.

P. media

I1 Verrucae small, scabrae very weak. Pores 5-9, 1-3 $\mu \mathrm{m}$. Grain 16-22 $\mu \mathrm{m}$ P. major

\section{ROSACEAE}

(Cf. Faegri \& Iversen 1992; Moore et al 1991; Teppner 1966)

A2 With tectate operculum.

B2 Psilate-scabrate-echinate, oblate-globular. Sanguisorba.

C2 Operculum narrow, colpus very short $\ldots \ldots \ldots \ldots \ldots \ldots \ldots \ldots \ldots \ldots \ldots \ldots$ S. minor

$\mathrm{C} 1$ Operculum as broad as the intercolpium, colpus long

S. officinalis

B1 Striate.

D2 Vallae coarse. Perforations not visible. Grain size c. $20 \mu \mathrm{m}$

E2 Vallae broad, diffuse.

F2 Polar area flattened

Fragaria vesca t.

F1 Polar area not flattened

Potentilla

E1 Vallae narrow, distinct.

G2 Colpus ends rounded

Fragaria viridis $\mathrm{t}$.

G1 Colpus ends acute

Potentilla

D1 Vallae fine. Tectum perforate.

H2 Vallae transversal, extremely fine. Operculum extremely narrow ... Agrimonia t.

H1 Vallae submeridional to subtransversal. Operculum short

Rosa

A1 No tectate operculum, but colpus membrane occasionally with loose columellae.

I3 Microechinate-echinate. No perforations

Filipendula 
I2 Rugulate-microstriate/psilate.

J3 Rugulate-microstriate. No costae colpi nor granules on colpus membrane. Size 47-52 $\mu \mathrm{m}$ Mespilus germanica

J2 Rugulate to psilate, costae colpi and granules usually present $\ldots \ldots$ Cotoneaster t.

J1 Psilate. Polar projection usually subtriangular .............. Alchemilla t.

I1 Striate.

K2 Vallae coarse. Colpus with distinct bridge

Geum

K1 Vallae fine.

L2 Vallae paired.

M3 Striation weak. Size $29-42 \mu \mathrm{m}$

Crataegus

M2 Striation definite. Size $30-36 \mu \mathrm{m}$

Pyrus

M1 Size $42-58 \mu \mathrm{m}$

Cydonia oblonga

L1 Vallae simple.

N2 Vallae indistinct. Perforations large

Rubus t.

N1 Vallae distinct.

O2 Vallae high, frequently short Rosa

O1 Vallae low and narrow.

P2 Vallae straight, moderately branched.

Q2 Perforations distinct. Vallae without dense structure . Sorbus t.

Q1 Perforations may be unobservable. Vallae with dense structure

Prunus avium t.

P1 Vallae curved, branched, anastomosing.

R2 Vallae short, strongly curved

Prunus spinosa t.

R1 Vallae moderately curved. With costae colpi, size c. $27 \mu \mathrm{m} . .$. .

Malus 


\section{References}

Andersen, S. T. 1979. Identification of wild grass and cereal pollen. Danmarks Geologiske Unders фgelse Årbog, 1978: 69-92.

Andersen, S. T. and F. Bertelsen 1972. Scanning electronmicroscope studies of pollen of cereals and other grasses. Grana 12: 79-86.

Ayala-Nieto, M.L., R. Lira Saade, and J.L. Alvarado 1988. Morfologia polinica de las Cucurbitaceae de la Peninsula de Yucatan, Mexico. Pollen et spores 30 (1): 5-28.

Belmonte, J., R. Pérez-Obiol and J.M. Roure 1986. Claves para la determinación de los polenes de las principales especies melíferas de la Península Ibérica. Orsis 2: 27-54.

Beug, H-J. 1961. Leitfaden der pollenbestimmung für Mitteleuropa und angrenzende gebiete. Lief. 1. Gustav Fischer Verlag, Stuttgart. 63 p.

Blackmore, S. 1982. Palynology of subtribe Scorzonerinae (Compositae: Lactuceae) and its taxonomic significance. Grana 21: 149-160.

Cerceau-Larrival, M-Th. 1963. Le pollen d'ombellifères Méditerranéennes. II. Tordylinae Drude. Review of palaeobotany and palynology 5: 297-323.

Cerceau-Larrival, M-Th. 1967. Corrélations de caractères chez les grains de pollen d'ombellifères. Review of palaeobotany and palynology 4: 311-324.

Chanda, S. 1962. On the pollen morphology of some Scandinavian Caryophyllaceae. Grana palynologica 3: 67-89.

Clarke, G.C.S. and M.R. Jones 1980. Plantaginaceae. Chapter 15 In W. Punt and G.C.S. Clarke, The Northwest European pollen flora. Vol. 2: 129-154. Elsevier, Amsterdam.

Erdtman, G. 1943. An introduction to pollen analysis. Chronica Botanica Company, Waltham, U.S.A. $239 \mathrm{p}$.

Erdtman, G. 1957. Pollen and spore morphology/plant taxonomy. Gymnospermae, Pteridophyta, Bryophyta. Illustrations. An introduction to palynology, II. Almqvist \& Wiksell, Stockholm. 151 p.

Erdtman, G. 1965. Pollen and spore morphology/plant taxonomy. Gymnospermae, Bryophyta. Text. An introduction to palynology, III. Almqvist \& Wiksell, Stockholm. 191 pages, +24 plates.

Erdtman, G. 1969. Handbook of palynology. Morphology, taxonomy, ecology. An introduction to the study of pollen grains and spores. Munksgaard, Copenhagen. 486 pages.

Erdtman, G. 1971. Pollen morphology and plant taxonomy. Angiosperms. An introduction to palynology, I. [Corrected reprint of 1952 ed.] Hafner Publishing Company, New York. 553 p.

Erdtman, G., B. Berglund and J. Praglowski 1961. An introduction to a Scandinavian pollen flora. Almqvist \& Wiksell, Stockholm. 92 pages, +74 plates.

Erdtman, G. J. Praglowski and S. Nilsson 1963. An introduction to a Scandinavian pollen flora. Vol. II. Almqvist \& Wiksell, Stockholm. 89 p.

Erdtman, G. and P. Sorsa 1971. Pollen and spore morphology/plant taxonomy. Pteridophyta. Text and additional illustrations. An introduction to palynology, IV. Almqvist \& Wiksell, Stockholm. 211 p.

Faegri, K. and J. Iversen 1975. Textbook of pollen analysis. 3rd ed. Hafner Press, New York. 295 p.

Faegri, K. and J. Iversen 1989. Textbook of pollen analysis. John Wiley \& Sons, Chichester. 328 p. (4th ed. by K. Faegri, P.E. Kaland \& K. Krzywinski) 
Ferrarini, E., F. Ciampolini, R.E.G. Pichi Sermolli and D. Marchetti 1986. Iconographia palynologica pteridophytorum Italiae. Webbia 40(1): 1-202.

McNeill, J and C.W. Crompton 1978. Pollen dimorphism in Silene alba (Caryophyllaceae). Canadian Journal of Botany 56: 1280-1286.

Melzheimer, V. 1975. Pollensystematische untersuchungen in der gattung Silene L. (Caryophyllaceen). Bot. Jahrb. Syst. 95(2): 215-225.

Moore, P.D., J.A. Webb and M.E. Collinson 1991. Pollen analysis. 2nd ed. Blackwell Scientific Publications, Oxford. $216 \mathrm{p}$.

Nilsson, S., J. Praglowski and L. Nilsson 1977. Atlas of airborne pollen grains and spores in northern Europe. Bokförlaget Natur och Kultur, Stockholm. 159 p.

Punt, W. and S. Blackmore (eds) 1991. The Northwest European pollen flora. Vol. 6. Elsevier Scientific Publishing Company, Amsterdam. 275 p.

Punt, W., S. Blackmore and G.C.S. Clarke (eds) 1988. The Northwest European pollen flora. Vol. 5. Elsevier Scientific Publishing Company, Amsterdam. 154 p.

Punt, W. and G.C.S. Clarke (eds) 1980. The Northwest European pollen flora. Vol. 2. Elsevier Scientific Publishing Company, Amsterdam. 265 p.

Punt, W. and G.C.S. Clarke (eds) 1981. The Northwest European pollen flora. Vol. 3. Elsevier Scientific Publishing Company, Amsterdam. 138 p.

Punt, W. and G.C.S. Clarke (eds) 1984. The Northwest European pollen flora. Vol. 4. Elsevier Scientific Publishing Company, Amsterdam. 369 p.

Punt, W., C.R. Janssen, Tj. Reitsma and G.C.S. Clarke (eds) 1976. The Northwest European pollen flora. Vol. I. Elsevier Scientific Publishing Company, Amsterdam. 145 p.

Reitsma, Tj. 1969. Size modification of recent pollen grains under different treatments. Review of Palaeobotany and Palynology 9: 175-202.

Rogers, C.M. 1984. A further note on the relationship of the European Linum hologynum and the Australian species of Linum (Linaceae). Plant systematics and evolution 147: 327-328.

Roure, J.M. 1985. Palinología Ibérica. Fam. 1 a 20. Cupressaceae a Betulaceae. Orsis 1: 43-69.

Saad, S.I. 1986. Palynological studies in the genus Plantago L. (Plantaginaceae). Pollen et spores 28(1): 43-60.

Teppner, H. 1966. Zur kenntnis der Gattung Waldsteinia, 1. Schlüssel zum bestimmen von Rosaceenpollen einschlie $\beta$ lich ähnlicher pollen formen aus anderen familien. Phyton 11(3-4): 224-238.

Tomb, A.S. 1975. Pollen morphology in Tribe Lactuceae (Compositae). Grana 15:79-89.

Ubera, J.L., C. Galán and F.H. Guerrero 1988. Palynological study of the genus Plantago in the Iberian Peninsula. Grana 27: 1-15.

Valdes, B., M.J. Diez and I. Fernandez (eds) 1987. Atlas polinico de Andalucia Occidental. Instituto de Desarrollo Regional de la Universidad de Sevilla, Seville. 450 p.

van Zeist, W. and S. Bottema 1977. Palynological investigations in western Iran. Palaeohistoria 19: 1985. 


\section{VI.2 Pollen types}

The authors field observations and the following references were used to determine species that are either extant or likely to occur in the Grevena region:

Adamakopoulos, P. 1989. Contribution to the study of the flora of the NE Pindos. Report to the Forest Service of Grevena.

Aschenbrenner, S. 1987. Ethnoarchaeology of agricultural economy. Unpublished Grevena Project report.

Chester, P. I. (Compiler) 1989a. Species lists for pollen surface sample collection sites. Unpublished Grevena Project report.

Chester, P. I. (Compiler) 1989b. Species lists for reconnaissance vegetation surveys (where no surface pollen sample was collected). Unpublished Grevena Project report.

Chester, P. I. (Compiler) 1989c. Herbarium specimens collected in Grevena Province. Unpublished Grevena Project report.

Leake, W.M. 1835. Travels in northern Greece. Reprinted 1967, Adolf M. Hakkert, Amsterdam.

Moody, J. and O. Rackham 1988. Grevena report. Unpublished Grevena Project report.

Moody, J. and O. Rackham in prep. Observations on the historical ecology of Grevena.

Polunin, O. 1988. Flowers of Greece and the Balkans. Oxford University Press, Oxford.

Pouqueville, F.C.H.L. 1826. Voyage de la Grece. Paris.

Rackham, O. 1991. Flora Greveniotica. Unpublished Grevena Project report.

Sfikas, G. 1978. Trees and shrubs of Greece. P. Efstathiadis \& Sons S.A., Athens.

Strid, A. (ed) 1986. Mountain Flora of Greece. Volume 1. Cambridge University Press, Cambridge.

Strid, A. and K. Tan (eds) 1991. Mountain Flora of Greece. Volume 2. Edinburgh University Press, Edinburgh.

Tutin, T. G. et al (ed) 1964-80. Flora Europaea. Volumes 1-5. Cambridge University Press, Cambridge.

Wace, A.J.B. and M.S. Thompson 1972. The nomads of the Balkans. Methuen \& Co. Ltd, London.

Taxonomic names of vascular European species follow:

Tutin, T. G. et al (eds) 1964-80. Flora Europaea. Volumes 1-5. Cambridge University Press, Cambridge.

In some exceptional cases, for example where taxa have been described more recently than the publication of the above volumes, taxonomic names follow those used in the following volumes:

Strid, A. (ed) 1986. Mountain Flora of Greece. Volume 1. Cambridge University Press, Cambridge. Strid, A. and K. Tan (eds) 1991. Mountain Flora of Greece. Volume 2. Edinburgh University Press, Edinburgh.

Taxonomic names of non-vascular species follow:

Watson, E.V. 1968 (2nd ed.), British mosses and liverworts. Cambridge University Press, Cambridge. 


\title{
PTERIDOPHYTA \\ LYCOPSIDA
}

\section{SELAGINELLACEAE}

Selaginella

Selaginella denticulata, S. helvetica

\section{ISOETACEAE}

Isoetes

Isoetes lacustris

?Isoetes heldreichii

\section{SPHENOPSIDA}

\section{EQUISETACEAE}

Equisetum

Equisetum arvense, E. fluviatile, E. palustre, E. ramosissimum, E. sylvaticum, E. telmateia

\section{FILICOPSIDA}

OPHIOGLOSSACEAE

Ophioglossum lusitanicum

Ophioglossum lusitanicum

Ophioglossum vulgatum

Ophioglossum vulgatum

Botrychium

Botrychium lunaria, B. simplex

\section{OSMUNDACEAE}

Osmunda regalis

Osmunda regalis

\section{SINOPTERIDACEAE}

Cheilanthes

Cheilanthes persica

?Cheilanthes catanensis, $C$. fragrans, $C$. marantae

\author{
ADIANTACEAE \\ Adiantum capillus-veneris \\ Adiantum capillus-veneris \\ PTERIDACEAE \\ Pteris cretica \\ Pteris cretica \\ Pteris vittata \\ Pteris vittata \\ GYMNOGRAMMACEAE \\ Anogramma leptophylla \\ Anogramma leptophylla \\ HYPOLEPIDACEAE \\ Pteridium aquilinum \\ Pteridium aquilinum \\ THELYPTERIDACEAE \\ Thelypteris palustris \\ Thelypteris palustris
}


Thelypteris phegopteris

Thelypteris phegopteris

ASPLENIACEAE

Asplenium type

Asplenium adiantum-nigrum, A. cuneifolium, A. fissum, A. fontanum, A. lepidum, A. obovatum, A. onopteris, A. petrarchae, A. ruta-muraria, A. septentrionale, A. trichomanes, A. viride, Ceterach officinarum, Phyllitis sagittata, $P$. scolopendrium

?Asplenium bornmuelleri, A. javorkeanum, A. macedonicum

\author{
ATHYRIACEAE \\ Athyrium filix-femina \\ Athyrium filix-femina \\ Athyrium distentifolium \\ Athyrium distentifolium \\ Cystopteris fragilis \\ Cystopteris fragilis
}

\author{
ASPIDIACEAE \\ Polystichum \\ Polystichum aculeatum, $P$. lonchitis, $P$. setiferum \\ ?Polystichum $x$ illyricum \\ Dryopteris filix-mas type \\ Dryopteris filix-mas, D. villarii, Gymnocarpium robertianum \\ ?Cystopteris dickieana, Dryopteris $x$ tavelii \\ Dryopteris expansa \\ Dryopteris expansa \\ Gymnocarpium dryopteris \\ Gymnocarpium dryopteris

\section{BLECHNACEAE} \\ Blechnum spicant \\ Blechnum spicant \\ POLYPODIACEAE \\ Polypodium \\ Polypodium australe, $P$. vulgare

\section{SALVINIACEAE} \\ Salvinia natans \\ Salvinia natans
}




\title{
SPERMATOPHYTA
}

\section{GYMNOSPERMAE}

\section{PINACEAE}

Abies

Abies alba, A. borisii-regis, A. cephalonica

Pseudotsuga menziesii

Picea abies

$$
\text { Pseudotsuga menziesii }
$$

Picea abies

Cedrus

Cedrus atlantica, C. deodara

Pinus subgenus Diploxylon

Pinus heldreichii, $P$. leucodermis, $P$. nigra, $P$. sylvestris

Pinus subgenus Haploxylon

Pinus peuce

\section{CUPRESSACEAE \\ Juniperus}

Juniperus communis, J. excelsa, J. foetidissima, J. oxycedrus, J. sabina

TAXACEAE

Taxus baccata

Taxus baccata

EPHEDRACEAE

Ephedra fragilis

Ephedra fragilis

Ephedra distachya type

Ephedra distachya, E. major

\section{SALICACEAE}

\section{ANGIOSPERMAE}

Dicotyledones

\section{Salix triandra}

Salix triandra

Salix fragilis type

Salix alba, S. aurita, S. caprea, S. fragilis, S. pedicellata, S. pentandra, S. purpurea, S.

viminalis

?S. aegyptiaca, S. amplexicaulis, S. cinerea, S. elaeagnos, S. retusa

Populus

Populus alba, $P$. canescens, $P$. nigra, $P$. tremula

\author{
JUGLANDACEAE \\ Juglans regia \\ Juglans regia \\ BETULACEAE \\ Alnus glutinosa \\ Alnus glutinosa \\ Betula pendula
}

Betula pendula 


\section{CORYLACEAE}

Carpinus type

Carpinus betulus (mostly), C. orientalis (pp), Ostrya carpinifolia pp.

Ostrya type

Corylus

Carpinus betulus pp., C. orientalis (mostly), Ostrya carpinifolia (mostly)

Corylus avellana, C. colurna, C. maxima

\section{FAGACEAE}

Fagus

Fagus moesiaca, $F$. sylvatica

Castanea sativa

Castanea sativa

Quercus coccifera type

Quercus coccifera, $Q$. ilex

Quercus trojana type

Quercus cerris, $Q$. macrolepis, $Q$. trojana

?Quercus brachyphylla

Quercus frainetto type

$Q u e r c u s$ frainetto, $Q$. petraea, $Q$. pubescens, $Q$. robur, $Q$. virgiliana

$? Q u e r c u s$ dalechampii, $Q$. pedunculiflora, $Q$. polycarpa

ULMACEAE

Ulmus minor type

Ulmus minor

?Ulmus canescens

Ulmus procera

Ulmus procera

Ulmus glabra type

Ulmus glabra, U. laevis

Celtis

Celtis australis

?Celtis caucasia

\section{MORACEAE}

Morus nigra

Morus nigra

Morus alba

Morus alba

Ficus carica

Ficus carica

CANNABACEAE

Cannabis sativa

Cannabis sativa

Humulus lupulus

Humulus lupulus

\section{URTICACEAE}

Urtica dioica

Urtica dioica

Urtica urens type

Urtica urens, Pilea microphylla 
Urtica pilulifera type

Parietaria

Urtica dubia, U. pilulifera

Parietaria diffusa, $P$. lusitanica, $P$. officinalis

?Parietaria cretica

\title{
SANTALACEAE
}

Comandra elegans

Osyris alba

Comandra elegans

Osyris alba

Thesium divaricatum type

Thesium divaricatum, T. humile

?Thesium arvense, T. bavarum, T. bergeri, T. brachyphyllum, T. linophyllon, T. parnassi

Thesium alpinum

Thesium alpinum

\section{LORANTHACEAE}

Viscum album

Viscum album

Arceuthobium oxycedri

Arceuthobium oxycedri

Loranthus europaeus

Loranthus europaeus

\section{ARISTOLOCHIACEAE}

Aristolochia

Aristolochia clematitis, A. longa

?Aristolochia macedonica, A. pallida, A. rotunda

\author{
RAFFLESIACEAE \\ Cytinus hypocistis \\ Cytinus hypocistis \\ Cytinus ruber \\ Cytinus ruber
}

POLYGONACEAE

Polygonum bistorta type

Polygonum bistorta

?Polygonum alpinum

Polygonum viviparum

Polygonum viviparum

Polygonum amphibium

Polygonum amphibium

Polygonum aviculare type

Polygonum arenarium, $P$. arenastrum, $P$. aviculare, $P$. equisetiforme, $P$. patulum

?Polygonum longipes

Polygonum persicaria type

Polygonum hydropiper, P. lapathifolium, P. mite, P. persicaria

?Polygonum salicifolium

\section{Bilderdykia}

Bilderdykia convolvulus, B. dumetorum 


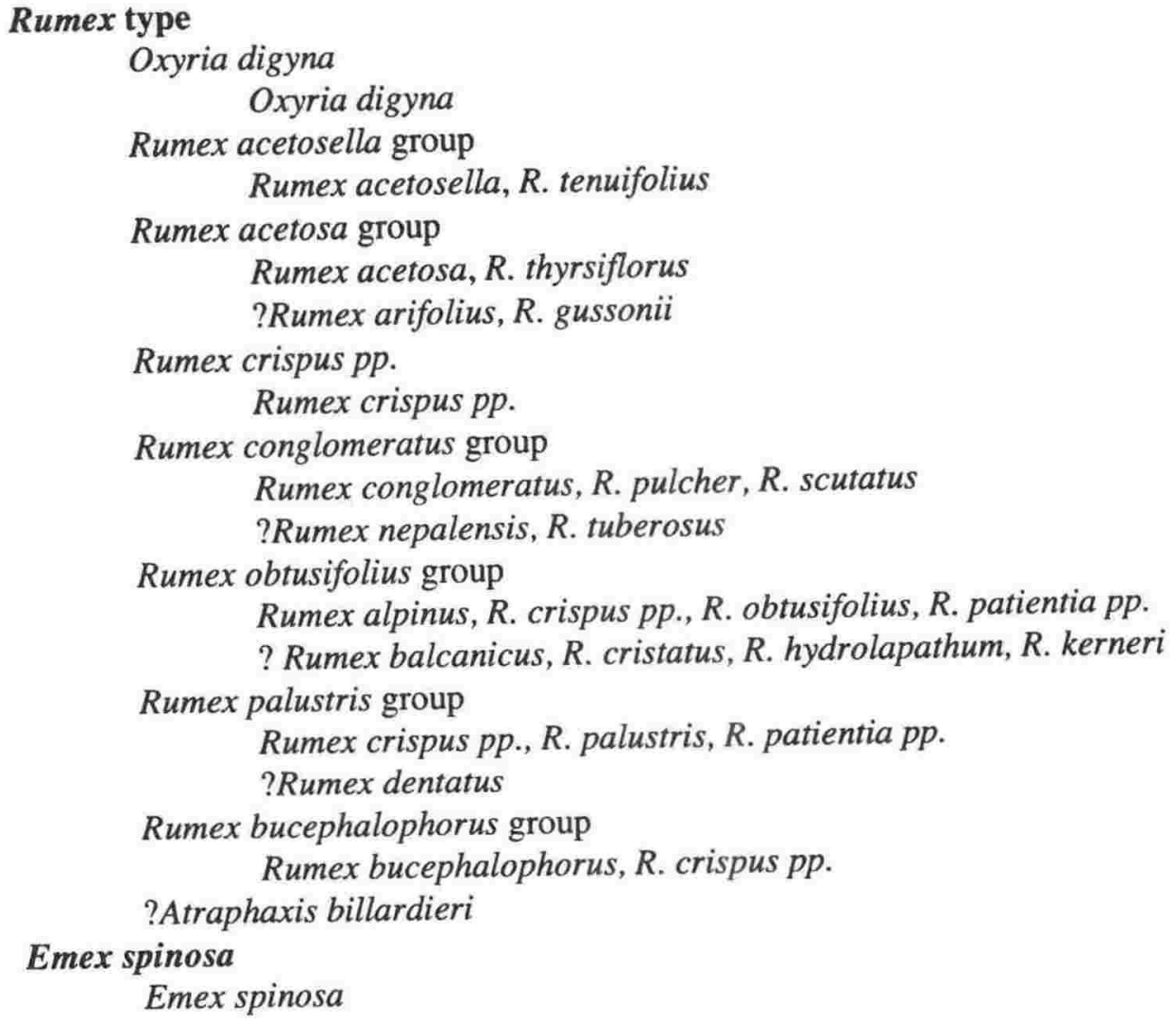

\section{CHENOPODIACEAE}

Chenopodiaceae type

Chenopodiaceae: Atriplex halimus, A. hastata, A. hortensis, A. littoralis, A. oblongifolia, A. patula, A. rosea, A. tatarica, Beta vulgaris, Camphorosma monspeliaca, Chenopodium album, C. ambrosioides, C. bonus-henricus, C. ficifolium, C. foliosum, C. glaucum, $C$. hybridum, C. murale, C. opulifolium, C. polyspermum, C. rubrum, C. urbicum, C. vulvaria, Corispermum nitidum, Noaea mucronata, Petrosimonia brachiata, P. triandra, Salsola kali, S. soda

Amaranthaceae: Amaranthus albus, A. deflexus, A. hybridus, A. lividus, A. retroflexus Caryophyllaceae: Sagina apetala, S. procumbens, S. saginoides, S. subulata

\section{PHYTOLACCACEAE}

Phytolacca americana

Phytolacca americana

\section{MOLLUGINACEAE \\ Glinus type}

Glinus lotoides, Mollugo cerviana

\section{PORTULACACEAE \\ Montia fontana \\ Montia fontana \\ Portulaca oleracea}

Portulaca oleracea

\section{CARYOPHYLLACEAE \\ Spergula}

Spergula arvensis, S. pentandra 


\section{Spergularia type}

Polycarpon tetraphyllum, Spergularia diandra, S. marina, S. media, S. rubra

Herniaria type Corrigiola litoralis pp., Herniaria cinerea, H. glabra, H. hirsuta, H. incana, $H$.

\section{Corrigiola litoralis} nigrimontium, $H$. parnassica

\section{Paronychia type}

Corrigiola litoralis pp.

Paronychia argentea, P. capitata, P. echinulata

Chenopodiaceae: Polycnemum arvense, $P$. heuffelii, P. majus

?Paronychia albanica, P. cephalotes, P. kapela, P. macedonica, P. polygonifolia, P. taurica

Dianthus type

Arenaria leptoclados, A. serpyllifolia, Cerastium alpinum, C. arvense, C. brachypetalum, C. cerastoides, C. diffusum, C. fontanum, C. glomeratum, C. gracile, C. pumilum, C. semidecandrum, Cucubalus baccifer, Dianthus deltoides, Holosteum umbellatum, Lychnis coronaria, $L$. viscaria, Minuartia recurva, M. verna, Moehringia pentandra, M. trinervia, Moenchia erecta, Myosoton aquaticum, Petrorhagia prolifera, $P$. saxifraga, Silene apetala, S. armeria, S. bupleuroides, S. cephallenia, S. colorata, S. congesta, S. dichotoma, S. fabarioides, S. frivaldszkyana, S. gallica, S. genistifolia, S. gigantea, S. italica, S. linifolia, S. multicaulis, S. nutans, S. oligantha, S. otites, S. paradoxa, S. parnassica, S. pindicola, S. radicosa, S. saxifraga, S. skorpilii, S. thessalonica, S. viridiflora, S. vulgaris, S. waldsteinii, Stellaria alsine, S. graminea, S. media, S. neglecta, S. nemorum, Vaccaria pyramidata ?Arenaria biflora, A. conferta, A. cretica, A. filicaulis, A. serpentini, Bolanthus graecus, $B$. thessalus, Bufonia paniculata, B. tenuifolia, Cerastium banaticum, C. decalvans, C. dinaricum, C. dubium, C. moesiacum, C. rectum, C. smolikanum, C. vourinense, Dianthus biflorus, $D$. brachyzonus, $D$. capitatus, $D$. cruentus, $D$. degenii, $D$. diffusus, $D$.

drenowskianus, D. formanekii, D. giganteus, D. gracilis, D. haematocalyx, D. leptopetalus, $D$. minutiflorus, D. myrtinervius, $D$. pallens, $D$. petraeus, $D$. pinifolius, $D$. pontederae, $D$. stenopetalus, $D$. sylvestris, $D$. tristis, $D$. viscidus, Minuartia baldaccii, M. eurytanica, $M$. garckeana, M. globulosa, M. glomerata, M. graminifolia, $M$. hirsuta, M. juniperina, $M$. setacea, $M$. stellata, Moenchia graeca, M. mantica, Petrorhagia cretica, $P$. fasciculata, $P$. glumacea, P. illyrica, Saponaria bellidifolia, S. calabrica, S. glutinosa, S. sicula, Silene asterias, S. balcanica, S. caesia, S. chromodonta, S. ciliata, S. compacta, S. cretica, S. damboldtiana, S. densiflora, S. haussknechtii, S. lerchenfeldiana, S. lydia, S. pusilla, S. roemeri, S. schwarzenbergeri, S. supina, S. trinervia, S. ventricosa

Silene alba type ??Drypis spinosa

Silene alba, S. conica

Lychnis flos-cuculi Lychnis flos-cuculi

Agrostemma githago Agrostemma githago

Scleranthus perennis Scleranthus perennis

Scleranthus annuus type Scleranthus annuus

?Scleranthus uncinatus

\section{Stellaria holostea}

Stellaria holostea

\section{NYMPHAEACEAE}

Nymphaea alba

Nymphaea alba 
Nuphar lutea

\section{CERATOPHYLLACEAE \\ Ceratophyllum}

Ceratophyllum demersum, C. submersum

\section{RANUNCULACEAE}

\section{Helleborus}

\section{Helleborus odorus}

?Helleborus cyclophyllus

\section{Nigella}

Nigella arvensis, $N$. damascena, $N$. sativa

\section{Trollius europaeus}

Trollius europaeus

\section{Actaea spicata}

Actaea spicata

\section{Consolida type}

Consolida orientalis, $C$. regalis, Delphinium fissum, D. peregrinum

?Consolida brevicornis, Delphinium balcanicum, D. hellenicum

\section{Adonis}

Adonis aestivalis, A. annua, A. flammea, A. microcarpa

\section{Caltha type}

Aquilegia vulgaris, Caltha palustris, Myosurus minimus

?Aquilegia amaliae, A. ottonis

\section{Ranunculus acris type}

Ranunculus acris group

Ranunculus acris, $R$. bulbosus, $R$. lanuginosus, $R$. muricatus, $R$. nemorosus, $R$. polyanthemos, $R$. repens, $R$. sardous

$?$ Ranunculus constantinopolitanus, $R$. serbicus, $R$. velutinus

Ranunculus lingua

Ranunculus lingua

Ranunculus aquatilis group

Clematis flammula, Ranunculus aquatilis, $R$. peltatus, $R$. pseudomontanus, $R$.

rionii, $R$. sphaerospermus, $R$. trichophyllus

?Ranunculus lateriflorus

Pulsatilla halleri

Pulsatilla halleri

Anemone nemorosa group

Anemone apennina, A. nemorosa

?Anemone blanda

Clematis vitalba group

Clematis vitalba, $C$. viticella

Ranunculus auricomus group

Ceratocephalus falcatus, Ranunculus auricomus, $R$. cassubicus, $R$. fallax, $R$. monophyllus

Ranunculus ficaria

Ranunculus ficaria

Ranunculus ophioglossifolius

Ranunculus ophioglossifolius

Ranunculus sceleratus group

Ranunculus platanifolius, $R$. sceleratus

?Ranunculus brevifolius, $R$. brutius, $R$. demissus, $R$. marginatus, $R$. neapolitanus, $R$. sartorianus 
Ranunculus arvensis type

Ranunculus arvensis

?Ranunculus chius, $R$. gracilis, $R$. miliarakesii, $R$. millefoliatus, $R$. millii, $R$. psilostachys,

$R$. rumelicus, $R$. spruneranus, $R$. subhomophyllus

Thalictrum aquilegifolium

Thalictrum aquilegifolium

Thalictrum minus

Thalictrum minus

Thalictrum lucidum

Thalictrum lucidum

\section{BERBERIDACEAE \\ Berberis vulgaris type \\ Berberis vulgaris \\ ?Berberis cretica \\ Mahonia aquifolium \\ Mahonia aquifolium \\ Leontice leontopetalum \\ Leontice leontopetalum}

LAURACEAE

Laurus nobilis

Laurus nobilis

PAPAVERACEAE

Chelidonium majus

Chelidonium majus

Papaver dubium

Papaver dubium

Papaver hybridum

Papaver hybridum

Papaver rhoeas type

Papaver rhoeas

?Papaver apulum, $P$. lecoqii

Papaver somniferum

Papaver somniferum

Glaucium

Glaucium corniculatum, G. leiocarpum

Papaver argemone type

Papaver argemone, Roemeria hybrida

Fumaria densiflora type

Fumaria densiflora, $F$. kralikii, $F$. officinalis, $F$. rostellata

?Fumaria petteri, F. schleicheri

Fumaria parviflora

Fumaria parviflora

Corydalis solida type

Corydalis bulbosa, C. solida

Corydalis ochroleuca

Corydalis ochroleuca

Hypecoum

Hypecoum imberbe, $H$. pendulum 


\title{
CRUCIFERAE
}

\section{Hornungia type}

Alyssum alyssoides, A. bertolonii, A. caliacrae, A. chalcidicum, A. chlorocarpum, A. corymbosoides, A. corymbosum, A. cuneifolium, A. desertorum, A. diffusum, A. doerfleri, A. foliosum, A. heldreichii, A. minus, A. minutum, A. montanum, A. murale, A. obtusifolium, A. petraeum, A. pichleri, A. pulvinare, A. repens, A. saxatile, A. sibiricum, A. smolikanum, A. strigosum, A. tortuosum, A. umbellatum, Arabidopsis thaliana, Berteroa incana, Capsella bursa-pastoris, Cardaria draba, Descurainia sophia, Hornungia petraea, Lepidium campestre, Thlaspi alpestre

?Aethionema saxatile, Berteroa mutabilis, B. obliqua, B. orbiculata, Capsella grandiflora, C. rubella, Lepidium graminifolium, $L$. hirtum, L. perfoliatum, $L$. sativum, Thlaspi bulbosum, T. epirotum, T. goesingense, T. microphyllum, T. ochroleucum, T. perfoliatum,

Arabis $T$. praecox, $T$. rivale

Arabis glabra, A. turrita

\section{Sinapis type}

Alliaria petiolata, Arabis alpina, Barbarea bracteosa, B. sicula, B. vulgaris, Brassica napus, B. nigra, B. rapa, Bunias erucago, Calepina irregularis, Camelina microcarpa, Cardamine bulbifera, C. hirsuta, C. pratensis, Diplotaxis muralis, D. viminea, Draba aizoides, D. athoa, D. lasiocarpa, D. muralis, D. scardica, Erophila minima, E. verna, Erysimum cephalonicum, E. cheiranthoides, E. comatum, E. cuspidatum, E. diffusum, E. linariifolium, E. microstylum, E. odoratum, E. pulchellum, E. pusillum, E. repandum, E. sylvestre, Iberis saxatilis, I. sempervirens, I. umbellata, Isatis lusitanica, I. tinctoria, $I$. vermia, Lepidium latifolium, L. ruderale, Lunaria annua, L. rediviva, Matthiola fruticulosa, Raphanus raphanistrum, Rorippa amphibia, $R$. prolifera, $R$. pyrenaica, $R$. sylvestris, Sinapis alba, S. arvensis, Sisymbrium altissimum, S. irio, S. loeselii, S. officinale, S. orientale, S. polyceratium, Teesdalia coronopifolia

?Alyssoides utriculata, Arabis allionii, A. bryoides, A. caucasica, A. collina, A. laxa, A. muralis, A. pseudoturritis, A. recta, A. sagittata, A. subflava, Aubrieta deltoidea, A. glabrescens, A. gracilis, A. intermedia, Bornmuellera baldaccii, B. tymphaea, Camelina alyssum, C. rumelica, C. sativa, Cardamine barbaraecoides, C. carnosa, C. glauca, C. graeca, C. hirsuta, C. pectinata, C. plumieri, C. raphanifolia, Clypeola jonthlaspi, Conringia austriaca, C. orientalis, Coronopus squamatus, Eruca vesicaria, Hesperis dinarica, $H$. laciniata, $H$. matronalis, $H$. rechingeri, $H$. sylvestris, $H$. theophrasti, Hirschfeldia incana, Hutchinsia alpina, Hymenolobus procumbens, Iberis odorata, I. pinnata, I. pruitii, I. umbellata, Lobularia maritima, Malcolmia africana, M. angulifolia, M. bicolor, $M$. graeca, $M$. maritima, $M$. orsiniana, Myagrum perfoliatum, Nasturtium officinale, Neslia paniculata, Peltaria emarginata, Ptilotrichum cyclocarpum, Rapistrum rugosum

\section{RESEDACEAE}

Reseda

Reseda lutea, $R$. luteola, $R$. phyteuma

\author{
CRASSULACEAE \\ Umbilicus \\ Umbilicus erectus, $U$. horizontalis, $U$. rupestris \\ Jovibarba heuffelii \\ Crassula \\ Jovibarba heuffelii \\ Crassula tillaea, C. vaillantii
}


Sedum acre, S. album, S. alpestre, S. annuum, S. athoum, S. atratum, S. cepaea, S. dasyphyllum, S. flexuosum, S. hispanicum, S. laconicum, S. litoreum, S. magellense, S. ochroleucum, S. pallidum, S. reflexum, S. sartorianum, S. sediforme, S. telephium, S.

\section{Sempervivum} tenuifolium

Sempervivum ballsii, S. ciliosum, S. kindingeri, S. marmoreum, S. reginae-amaliae, S. zeleborii

\section{SAXIFRAGACEAE}

Chrysosplenium alternifolium

Chrysosplenium alternifolium

Saxifraga stellaris

Saxifraga stellaris

Saxifraga tridactylites type

Saxifraga chrysosplenifolia, S. exarata, S. rotundifolia, S. tridactylites

Saxifraga oppositifolia type

Saxifraga adscendens, S. glabella, S. marginata, S. oppositifolia, S. paniculata

\section{Saxifraga bulbifera}

Saxifraga bulbifera

Saxifraga sempervivum type

Saxifraga porophylla, S. sempervivum, S. stribrnyi

?Saxifraga graeca, S. grisebachii, S. luteoviridis, S. scardica, S. spruneri, S. taygetea

\section{PARNASSIACEAE \\ Parnassia palustris \\ Parnassia palustris}

\section{GROSSULARIACEAE}

Ribes

Ribes alpinum, $R$. multiflorum

PLATANACEAE

Platanus orientalis

Platanus orientalis

\section{ROSACEAE}

Filipendula

Filipendula ulmaria, $F$. vulgaris

Rubus type

Rubus caesius, $R$. candicans, $R$. canescens, $R$. corylifolius, $R$. hirtus, $R$. idaeus, $R$. serpens,

$R$. thyrsiflorus, $R$. ulmifolius

?Rubus thessalus

?? Spiraea crenata

Sorbus type

Sorbus aria, S. aucuparia, S. domestica, S. graeca, S. torminalis, S. umbellata

?Amelanchier ovalis

Rosa

Rosa agrestis, $R$. andegavensis, $R$. arvensis, $R$. caesia, $R$. canina, $R$. corymbifera, $R$. elliptica, $R$. foetida, $R$. gallica, $R$. majalis, $R$. micrantha, $R$. montana, $R$. moschata, $R$. nitidula, $R$. obtusifolia, $R$. pendulina, $R$. pimpinellifolia, $R$. rubiginosa, $R$. squarrosa, $R$. villosa, $R$. vosagiaca ?Rosa caryophyllacea, $R$. glutinosa, $R$. heckeliana, $R$. orientalis, $R$. sempervirens, $R$. turcica 


\section{Cotoneaster type}

Cotoneaster integerrimus, $C$. nebrodensis

?Pyracantha coccinea

Mespilus germanica

Mespilus germanica

Prunus avium type

Prunus avium, P. mahaleb

Prunus spinosa type

Prunus cerasus, $P$. domestica, $P$. persica, $P$. spinosa

?Prunus armeniaca, $P$. cerasifera, $P$. cocomilia, $P$. dulcis, $P$. prostrata

Malus

Malus domestica, M. pumila, M. sylvestris

?Malus florentina

Pyrus

Pyrus amygdaliformis, $P$. communis, $P$. cordata, $P$. pyraster

?Pyrus eleagrifolia, $P$. salvifolia

\section{Crataegus}

Crataegus heldreichii, C. laciniata, C. monogyna, C. pentagyna

?Crataegus schraderana

Cydonia oblonga

Cydonia oblonga

Agrimonia type

Agrimonia eupatoria, Aremonia agrimonoides

Sanguisorba officinalis

Sanguisorba officinalis

Sanguisorba minor

Sanguisorba minor ssp. minor, S. minor ssp. muricata

Geum

Geum coccineum, G. molle, G. montanum, G. reptans, G. urbanum

\section{Potentilla}

Potentilla argentea, $P$. aurea, $P$. erecta, $P$. inclinata, $P$. micrantha, $P$. recta, $P$. reptans, $P$. sterilis, $P$. supina

?Potentilla astracanica, $P$. australis, $P$. detommasii, $P$. geoides, $P$. pedata, $P$. speciosa

Fragaria viridis type

Fragaria viridis

?Fragaria $\mathrm{x}$ ananassa

Fragaria vesca type

Fragaria vesca

?Sibbaldia parviflora

\section{Alchemilla type}

Alchemilla xanthochlora, Aphanes arvensis, A. microcarpa

?Alchemilla acutiloba, A. cinerea, A. fallax, A. gorcensis, A. heterotricha, A. incisa, A. plicatula, A. straminea, A. subglabra group

\section{LEGUMINOSAE}

Genista type

Cytisus decumbens, C. procumbens, C. villosus, Genista acanthoclada, G. albida, G. carinalis, G. hassertiana, G. januensis, G. radiata, G. tinctoria ?Chamaecytisus austriacus, C. ciliatus, C. heuffelii, C. hirsutus, C. polytrichus, $C$. spinescens, C. supinus, Lembotropis nigricans, Podocytisus caramanicus 
Onobrychis aequidentata, $O$. alba, $O$. arenaria, $O$. caput-galli, $O$. ebenoides,

$O$. hypargyrea, $O$. montana, $O$. oxyodonta, $O$. pindicola

\section{Hedysarum coronarium}

\section{Ononis type} Hedysarum coronarium

Colutea arborescens, Melilotus alba, M. altissima, M. indica, M. neapolitana, $M$. officinalis, $M$. segetalis, Ononis pusilla, $O$. reclinata, $O$. spinosa

Trifolium dubium type Medicago lupulina, Trifolium angustifolium, T. arvense, T. aureum, T. badium, T. campestre, T. dubium, T. lappaceum, T. micranthum, T. nigrescens, T. pallidum, T. resupinatum

?Medicago aculeata, M. ciliaris, $M$. coronata, M. disciformis, M. orbicularis, M. praecox, M. prostrata, M. rigidula, M. scutellata, $M$. turbinata, Trifolium alpestre, T. aurantiacum, $T$. bocconei, $T$. cherieri, $T$. dalmaticum, $T$. diffusum, T. dolopium, T. echinatum, $T$. glomeratum, $T$. heldreichianum, T. hirtum, T. latinum, T. leucanthum, T. noricum, $T$. ochroleucon, $T$. pannonicum, $T$. parnassi, $T$. patens, $T$. patulum, $T$. phleoides, $T$. physodes, T. pignantii, T. pilczii, T. purpureum, T. sebastianii, T. smyrnaeum, T. strictum, $T$.

Trifolium repens type Medicago arabica, M. minima, M. polymorpha, Scorpiurus muricatus, Trifolium fragiferum, $T$. globosum, $T$. hybridum, $T$. incarnatum, $T$. medium, $T$. pratense, $T$. repens, $T$. scabrum, $T$, squarrosum, $T$. striatum, $T$. subterraneum

\section{Lotus type}

Lotus aegaeus, $L$. angustissimus, $L$. corniculatus, L. ornithopodioides, $L$. palustris, $L$. parviflorus, L. stenodon, L. tenuis, L. uliginosus, Tetragonolobus biflorus, T. purpureus, Trigonella monspeliaca ?Glycyrrhiza echinata, G. glabra, Trigonella caerulea, T. foenum-graecum, T. procumbens, $T$. spicata, T. striata ??Hymenocarpus circinnatus, Securigera securidaca

Lathyrus pratensis type

Lathyrus alpestris, L. aphaca, L. cicera, L. digitatus, L. grandiflorus, L. hallersteinii, $L$. hirsutus, L. latifolius, L. laxiflorus, L. niger (mostly), L. nissolia, L. pallescens, $L$. pratensis, L. setifolius, $L$. sphaericus, $L$. sylvestris, L. tuberosus, $L$. venetus, $L$. vernus, Lupinus albus, Trigonella gladiata

?Lathyrus ochrus

Lens

Lens culinaris

?Lens ervoides, L. nigricans

Vicia sativa type

Vicia cracca (sometimes), V. hirsuta (mostly), V. hybrida, V. lutea, V. peregrina, V. sativa, V. sepium (mostly), V. tetrasperma (mostly)

?Vicia articulata, V. barbazitae, V. canescens, V. cassubica, V. dumetorum, V. ervilia, V. grandiflora, $V$. incana, V. melanops, $V$. narbonensis, V. onobrychioides, V. pannonica, $V$. pubescens, V. sibthorpii, V. villosa

Vicia lathyroides

Vicia faba

Vicia lathyroides

Vicia faba

Vicia cracca type

Lathyrus niger (sometimes), Vicia cracca (mostly), V. hirsuta (sometimes), V. sepium (sometimes), V. tenuifolia, V. tetrasperma (sometimes) 
Astragalus depressus, A. exscapus, A. glycyphyllos, A. hamosus, A. monspessulanus, A. onobrychis, $A$. purpureus, $A$. sesameus, $A$. vesicarius

?Astragalus angustifolius, A. baldaccii, A. creticus, A. glycyphylloides, A. mayeri, A. parnassi, A. pubiflorus, A. sinaicus, A. sirinicus, A. subuliformis

Galega officinalis

Galega officinalis

Coronilla scorpioides type

Coronilla scorpioides, C. valentina, C. varia

?Coronilla coronata, C. cretica, C. elegans, C. rostrata

Medicago sativa

Medicago sativa pp.

Ornithopus compressus

Dorycnium

Ornithopus compressus

Dorycnium graecum, D. hirsutum, D. pentaphyllum, D. rectum

Trifolium montanum type

Anthyllis

Medicago sativa pp., Trifolium montanum

Anthyllis aurea, A. montana, A. vulneraria

Coronilla emerus

Coronilla emerus

Spartium junceum

Spartium junceum

Psoralea type

Psoralea bituminosa, Vigna unguiculata

Phaseolus

Phaseolus vulgaris

?Phaseolus coccineus

??Cicer arietinum, Glycine $\max$

Pisum sativum

Pisum sativum

Lathyrus sativus

Lathyrus sativus

Ceratonia siliqua

Ceratonia siliqua

Cercis siliquastrum

Cercis siliquastrum

Hippocrepis

Hippocrepis ciliata, H. comosa, H. unisiliquosa

Robinia pseudacacia

Robinia pseudacacia

Arachis hypogaea

Arachis hypogaea

OXALIDACEAE

Oxalis

Oxalis acetosella, $O$. corniculata, $O$. pes-caprae

\section{GERANIACEAE}

Geranium

Geranium robertianum group

Geranium purpureum, G. pusillum, G. pyrenaicum, G. robertianum, $G$. rotundifolium 
Geranium bohemicum group

Geranium bohemicum, G. macrorrhizum

?Geranium aristatum, G. cinereum, G. reflexum

Geranium pratense group

Geranium pratense, G. sanguineum, G. sylvaticum

?Geranium asphodeloides, G. macrostylum, G. tuberosum

Geranium molle group

Geranium columbinum, G. dissectum, G. divaricatum, G. lucidum, G. molle, G.

versicolor

?Geranium brutium

Erodium

Erodium botrys, E. ciconium, E. cicutarium, E. hoefftianum, E. malacoides, E. moschatum

\author{
ZYGOPHYLLACEAE \\ Peganum harmala \\ Peganum harmala \\ Tribulus terrestris \\ Tribulus terrestris
}

LINACEAE

Linum usitatissimum type

Linum bienne, L. usitatissimum

Linum austriacum type

Linum austriacum, L. perenne, L. punctatum

Radiola linoides

Radiola linoides

Linum catharticum type

Linum catharticum

Linum catharticum

Linum tenuifolium

Linum tenuifolium

Linum trigynum

Linum trigynum

Linum strictum group

Linum hirsutum, $L$ strictum

?Linum pubescens, L. spathulatum

Linum hologynum type

Linum hologynum

?Linum aroanium, L. capitatum, L. elegans, L. nodiflorum L. tauricum, L. thracicum

\title{
EUPHORBIACEAE
}

Andrachne telephioides

Andrachne telephioides

Mercurialis perennis

Mercurialis perennis

Mercurialis annua type

Mercurialis annua

?Mercurialis ovata

\section{Euphorbia}

Euphorbia amygdaloides, E. brittingeri, E. chamaesyce, E. esula, E exigua, E. falcata, E. helioscopia, E. lathyris, E. nicaeensis, E. palustris, E. peplus, E. platyphyllos, E. pubescens, E. segetalis, E. seguierana, E. serrulata, E. spinosa, E. villosa ?Euphorbia agraria, E. aleppica, E. barrelieri, E. cyparissias, E. deflexa, E. epithymoides, E. flavicoma, E. glabriflora, E. heldreichii, E. herniariifolia, E. lathyris, E. lingulata, E. lucida, E. myrsinites, E. oblongata, E. phymatosperma, E. salicifolia, E. taurinensis 
Ricinus communis

Ricinus communis

RUTACEAE

Dictamnus albus

Dictamnus albus

Ruta chalepensis

Ruta chalepensis

Ruta montana type

Ruta montana

?Ruta graveolens

Haplophyllum

?Haplophyllum boissieranum, $H$. coronatum, $H$. patavinum

Citrus

Citrus aurantium, C. limon, C. sinensis

SIMAROUBACEAE

Ailanthus altissima

Ailanthus altissima

POLYGALACEAE

Polygala monspeliaca type

Polygala monspeliaca

?Polygala alpestris, P. major, P. nicaeensis, P. supina

Polygala vulgaris type

Polygala vulgaris

?Polygala comosa

ANACARDIACEAE

Rhus coriaria

Rhus coriaria

Schinus molle

Schinus molle

Pistacia

Pistacia lentiscus, $P$. terebinthus

Cotinus coggygria

Cotinus coggygria

\section{ACERACEAE}

Acer

Acer campestre, A. monspessulanum, A. opalus, A. platanoides, A. pseudoplatanus

?Acer heldreichii, A. hyrcanum, A. obtusatum, A. sempervirens, A. tataricum

HIPPOCASTANACEAE

Aesculus hippocastanum

Aesculus hippocastanum

BALSAMINACEAE

Impatiens noli-tangere

Impatiens noli-tangere 
AQUIFOLIACEAE

Ilex aquifolium

Ilex aquifolium

CELASTRACEAE

Euonymus europaeus type

Euonymus europaeus

?Euonymus latifolius, E. verrucosus

BUXACEAE

Buxus sempervirens

Buxus sempervirens

RHAMNACEAE

Frangula

Frangula alnus

?Frangula rupestris

Rhamnus type

Paliurus spina-christi, Rhamnus alpinus, R. saxatilis, Ziziphus jujuba, Z. lotus

?Paliurus microcarpus, Rhamnus prunifolius

VITACEAE

Vitis vinifera

Vitis vinifera subsp. sylvestris, $V$. vinifera subsp. vinifera

TILIACEAE

Tilia platyphyllos

Tilia platyphyllos

?Tilia rubra

Tilia cordata type

Tilia cordata, T. tomentosa

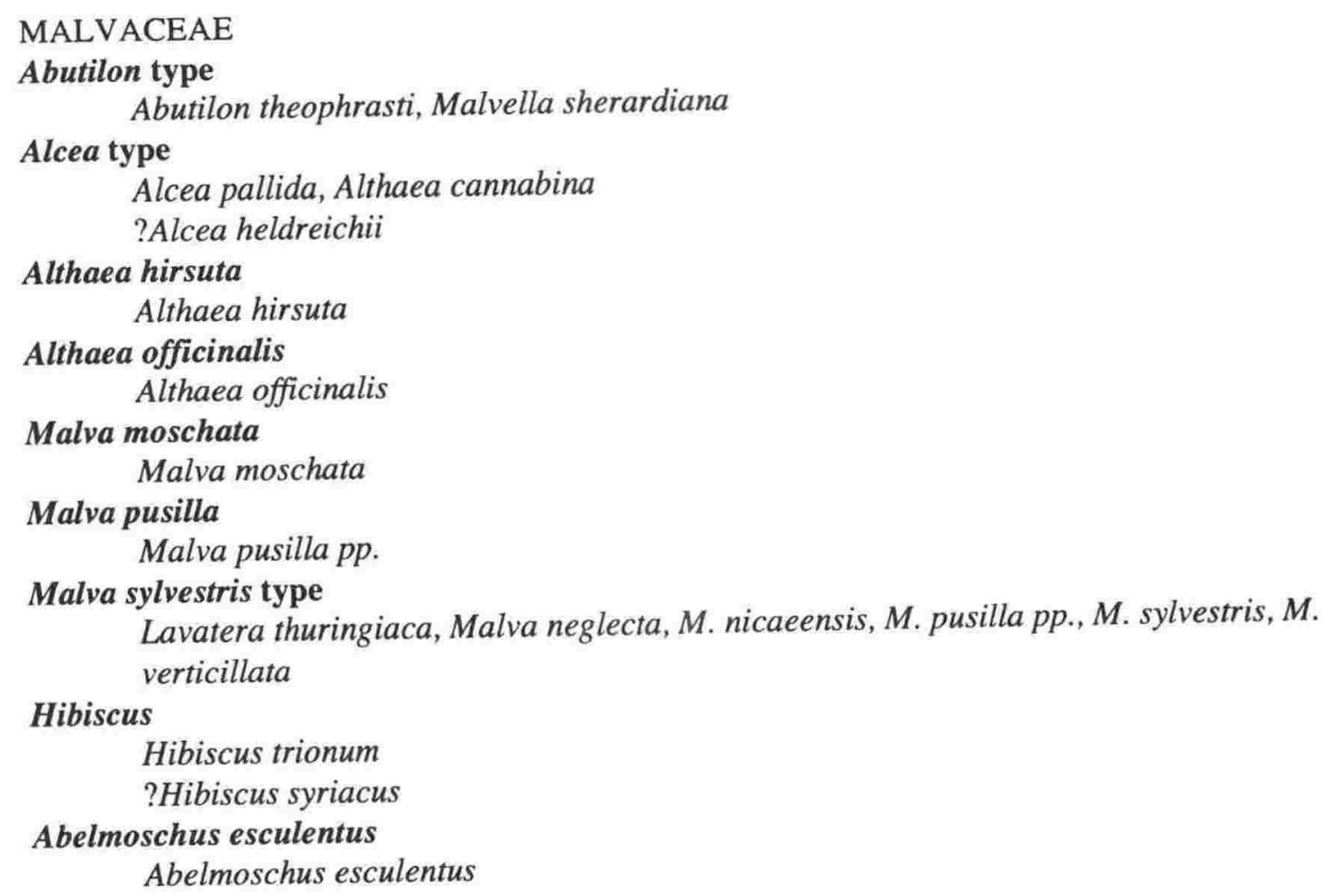


Gossypium

Gossypium herbaceum, G. hirsutum

THYMELAEACEAE

Daphne

Daphne blagayana, D. gnidium, D. laureola, D. mezereum, D. oleoides

\section{ELAEAGNACEAE}

Elaeagnus angustifolia

Elaeagnus angustifolia

\section{GUTTIFERAE}

Hypericum hircinum type

Hypericum hircinum

?Hypericum empetrifolium

Hypericum perforatum type

Hypericum hirsutum, $H$. perforatum, $H$. tetrapterum

?Hypericum annulatum, $H$. barbatum, $H$. linarioides, $H$. olympicum, $H$. rumeliacum, $H$. spruneri, $H$. thasium

\section{VIOLACEAE}

Viola hirta type

Viola alba, V. elatior, V. hirta, V. odorata, V. riviniana pp.

Viola riviniana type

Viola reichenbachiana, $V$. riviniana pp.

Viola arvensis type

Viola arvensis, V. kitaibeliana, V. tricolor

?Viola aetolica, V. allchariensis, V. brachyphylla, V. chelmea, V. dukadjinica, V. epirota, $V$. eximia, V. gracilis, V. heterophylla, V. magellensis, V. orphanidis, V. pyrenaica, $V$.

rupestris, $V$. sieheana

\section{CISTACEAE}

Helianthemum

Helianthemum apenninum, $H$. canum, $H$. ledifolium, H. nummularium, $H$. oelandicum, $H$. salicifolium

Tuberaria guttata

Tuberaria guttata

Cistus monspeliensis type

Cistus monspeliensis

?Cistus incanus, C. parviflorus

Cistus salvifolius

Cistus salvifolius

Fumana

Fumana aciphylla, F. arabica, F. bonapartei

\section{TAMARICACEAE}

Myricaria germanica

Tamarix

Myricaria germanica

Tamarix dalmatica, T. hampeana, T. parviflora, T. smyrnensis, T. tetrandra

\section{ELATINACEAE}

Elatine alsinastrum

Elatine alsinastrum 
CUCURBITACEAE

Cucumis melo

Cucumis melo

?Citrullus lanatus

Bryonia

Bryonia alba, B. cretica

Lagenaria siceraria

Cucurbita

Lagenaria siceraria

Cucurbita ficifolia, C. maxima, C. mixta, C. moschata, C. pepo

\section{LYTHRACEAE}

Lythrum salicaria type

Lythrum hyssopifolia, L. junceum, L. salicaria, L. tribracteatum

? Lythrum borysthenicum, $L$. virgatum

Lythrum portula

Lythrum portula

TRAPACEAE

Trapa natans

Trapa natans

MYRTACEAE

Myrtus communis

Myrtus communis

\section{PUNICACEAE}

Punica granatum

Punica granatum

ONAGRACEAE

Circaea lutetiana

Circaea lutetiana

Ludwigia palustris

Ludwigia palustris

Epilobium angustifolium type

Epilobium angustifolium, Oenothera biennis

Epilobium hirsutum type

Epilobium hirsutum, E. montanum, E. palustre, E. parviflorum, E. tetragonum ?Epilobium adenocaulon, E. alsinifolium, E. anagallidifolium, E. dodonaei, E. gemmascens, E. lanceolatum, E. obscurum, E. roseum

\section{HALORAGACEAE}

Myriophyllum verticillatum

Myriophyllum verticillatum

Myriophyllum spicatum

Myriophyllum spicatum

\section{THELIGONACEAE}

Theligonum cynocrambe

Theligonum cynocrambe 


\section{CORNACEAE}

Cornus sanguinea

Cornus sanguinea

Cornus mas

Cornus mas

\section{ARALIACEAE}

Hedera helix

Hedera helix

UMBELLIFERAE

Hydrocotyle vulgaris

Hydrocotyle vulgaris

Anthriscus caucalis

Anthriscus caucalis

Torilis nodosa

Torilis nodosa

Physospermum cornubiense

Physospermum cornubiense

Smyrnium

Smyrnium olusatrum, S. perfoliatum

Ligusticum

?Ligusticum lucidum, Ligusticum rhizomaticum

Bupleurum

Bupleurum asperuloides, B. falcatum, B. flavicans, B. fruticosum, B. gerardi, B.

glumaceum, B. karglii, B. lancifolium, B. praealtum, B. rotundifolium, B. tenuissimum

Sison amomum

Sison amomum

Carum

?Carum heldreichii, C. multiflorum, C. rigidulum

??Stefanoffia daucoides

Berula erecta

Berula erecta

Trinia

Trinia dalechampii, T. glauca

Sanicula europaea

Sanicula europaea

Conium maculatum

Conium maculatum

Cachrys

?Cachrys cristata, C. ferulacea

Cnidium silaifolium

Cnidium silaifolium

Seseli type

Opopanax chironium, Seseli libanotis, S. pallasii, S. parnassicum, S. peucedanoides, S.

rigidum, $S$. tortuosum

?Opopanax hispidus

\section{Oenanthe type}

Oenanthe aquatica group

Oenanthe aquatica, $O$. lachenalii

Oenanthe pimpinelloides group

Oenanthe pimpinelloides

?Oenanthe tenuifolia 
Oenanthe silaifolia group

Oenanthe silaifolia

?Athamanta macedonica, Oenanthe banatica

Oenanthe fistulosa

Oenanthe fistulosa

Cicuta virosa

Cicuta virosa

Chaerophyllum hirsutum type

Chaerophyllum hirsutum

?Chaerophyllum aromaticum

Chaerophyllum temulentum type

Chaerophyllum temulentum

??Myrrhoides nodosa

Ammi

Ammi majus, A. visnaga

Pimpinella saxifraga

Pimpinella saxifraga

Torilis arvensis

Torilis arvensis

Anethum graveolens

Anethum graveolens

Falcaria vulgaris

Falcaria vulgaris

Peucedanum austriacum type

Peucedanum austriacum

?Peucedanum aegopodioides, $P$. longifolium, $P$. oligophyllum, $P$. stridii, $P$. vittijugum, $P$.

vourinense

Angelica sylvestris

Angelica sylvestris

Petroselinum crispum

Petroselinum crispum

Peucedanum schotti

Peucedanum schotti

Bunium

Bunium ferulaceum

?Bunium tenerum

??Huetia cynapioides

Foeniculum vulgare ssp. vulgare

Foeniculum vulgare ssp. vulgare

??Ferulago campestris, $F$. nodosa, $F$. sylvatica

Anthriscus sylvestris type

Anthriscus sylvestris

?Anthriscus nemorosa

Orlaya

Orlaya grandiflora

?Orlaya daucorlaya, O. kochii, O. topaliana

Pastinaca sativa

Pastinaca sativa

Eryngium

Eryngium campestre

?Eryngium amethystinum, E. creticum, E. palmatum

Scandix type

Caucalis platycarpos, Scandix australis, S. pecten-veneris, Turgenia latifolia 
Heracleum sphondylium

Heracleum sphondylium

Pimpinella anisum type

Pimpinella anisum

?Pimpinella peregrina, $P$. tragium

Anthriscus cerefolium

Anthriscus cerefolium

Laserpitium siler

Laserpitium siler

Torilis japonica type

Torilis japonica

?Torilis leptophylla, T. ucranica

Daucus

Daucus broteri, D. carota

?Daucus guttatus

Coriandrum type

Bifora testiculata, Coriandrum sativum

Aegopodium podagraria

Aegopodium podagraria

Chaerophyllum aureum

Chaerophyllum aureum

Malabaila

Malabaila aurea, $M$. involucrata

Tordylium officinale

Tordylium officinale

Echinophora type

Echinophora tenuifolia, Tordylium maximum

Conopodium capillifolium (= Conopodium majus type in NW4)

?Conopodium capillifolium

PYROLACEAE

Monotropa hypopitys

Monotropa hypopitys

Pyrola

Pyrola chlorantha, P. minor

Moneses uniflora

Moneses uniflora

ERICACEAE

Erica herbacea

Erica herbacea

Vaccinium myrtillus

Vaccinium myrtillus

PRIMULACEAE

Soldanella

?Soldanella hungarica, S. pindicola

Cyclamen

Cyclamen hederifolium

?Cyclamen graecum, C. repandum

Androsace villosa

Androsace villosa

Androsace maxima

Androsace maxima 


\section{Primula}

Primula elatior, $P$. veris, $P$. vulgaris

?Primula halleri

Lysimachia

Lysimachia atropurpurea, L. dubia, L. nummularia, L. punctata, L. vulgaris

Anagallis arvensis type

Anagallis arvensis, A. foemina

Anagallis tenella type

Anagallis minima, A. tenella

Asterolinon linum-stellatum

Asterolinon linum-stellatum

Samolus valerandi

Samolus valerandi

PLUMBAGINACEAE

Armeria maritima type

Acantholimon androsaceum, Armeria canescens, A. maritima, A. rumelica, A. sancta, A. undulata, Goniolimon tataricum

Plumbago europaea

Plumbago europaea

\section{EBENACEAE}

Diospyros lotus

Diospyros lotus

\section{STYRACACEAE}

Styrax officinalis

Styrax officinalis

\section{OLEACEAE}

Fraxinus excelsior type

Fraxinus angustifolia ssp. oxycarpa, F. excelsior

Fraxinus angustifolia

Fraxinus angustifolia

Fraxinus ornus

Fraxinus ornus

Ligustrum vulgare

Phillyrea

Ligustrum vulgare

Jasminum

Phillyrea latifolia, P. media

Jasminum fruticans

?Jasminum humile

\section{GENTIANACEAE}

Gentiana verna

Gentiana verna

Gentianella ciliata

Gentianella ciliata

Cicendia filiformis

Cicendia filiformis

\section{Centaurium}

Centaurium erythraea, C. pulchellum, C. tenuiflorum 


\title{
Blackstonia perfoliata
}

Blackstonia perfoliata ssp. perfoliata, B. perfoliata ssp. serotina

\section{Gentiana lutea type}

Gentiana asclepiadea, G. cruciata, G. lutea

\section{MENYANTHACEAE \\ Nymphoides peltata}

Nymphoides peltata

\section{APOCYNACEAE \\ Vinca herbacea \\ Vinca herbacea}

\author{
ASCLEPIADACEAE \\ Periploca graeca \\ Periploca graeca \\ Vincetoxicum \\ Vincetoxicum hirundinaria \\ ?Vincetoxicum fuscatum, V. speciosum
}

\section{CONVOLVULACEAE \\ Convolvulus}

Convolvulus arvensis, C. betonicifolius, C. boissieri, C. cantabrica, C. holosericeus

Cuscuta epithymum type

Cuscuta epithymum, C. planiflora

?Cuscuta approximata, C. australis, C. suaveolens

Cuscuta europaea type

Cuscuta campestris, C. europaea, C. palaestina

Cuscuta monogyna

\section{Calystegia}

Cuscuta monogyna

Ipomoea

Calystegia sepium, C. silvatica

Ipomoea batatas, I. purpurea

\section{BORAGINACEAE}

Cynoglossum type

Cynoglossum creticum, C. officinale, Rindera graeca

?Cynoglossum hungaricum, C. nebrodense, Solenanthus albanicus

\section{Heliotropium}

Heliotropium europaeum, $H$. suaveolens, $H$. supinum

?Heliotropium hirsutissimum

Myosotis type

Asperugo procumbens, Myosotis arvensis, $M$. laxa ssp. caespitosa, $M$. nemorosa, $M$. ramosissima, $M$. sicula, $M$. stricta, $M$. sylvatica, Omphalodes verna

?Myosotis incrassata, $M$. refracta, $M$. sparsiflora, $M$. suaveolens, Rochelia disperma

Lappula

Lappula squarrosa

Echium

?Lappula marginata

Echium italicum, E. plantagineum, E. vulgare

\section{Alkanna type}

Alkanna tinctoria

?Alkanna corcyrensis, A. noneiformis, A. pindicola, Halacsya sendtneri 
Borago officinalis

Borago officinalis

Cerinthe type

elegantissima, $O$. graeca, $O$. helvetica, $O$. heterophylla, $O$. ??Moltkia petraea, Onosma pygmaeum, $O$, rhodopea, $O$. taurica, $O$. visianii mattiro

Symphytum

Symphytum bulbosum, S. ottomanum

Buglossoides arvensis type

Buglossoides arvensis

?Buglossoides tenuiflora

Neatostema apulum

Neatostema apulum

Anchusa arvensis type

Anchusa arvensis, A. azurea

?Anchusa cretica

Nonea

?Nonea pallens, $N$. pulla, $N$. ventricosa

Pulmonaria mollis

Pulmonaria mollis

Anchusa officinalis type

Anchusa officinalis, A. stylosa

?Anchusa macedonica, A. serpentinicola, A. thessala

Lithospermum

Lithospermum officinale

?Lithospermum goulandriorum

Buglossoides purpurocaerulea

Buglossoides purpurocaerulea

\section{VERBENACEAE}

Verbena

Verbena officinalis, V. supina

Vitex agnus-castus

Vitex agnus-castus

CALLITRICHACEAE

Callitriche obtusangula

Callitriche obtusangula

Callitriche stagnalis type

Callitriche stagnalis

?Callitriche truncata

\section{LABIATAE}

Teucrium

Teucrium chamaedrys, $T$. halacsyanum, T. montanum, $T$. polium, $T$. scordium

Stachys sylvatica type Lamium album, L. amplexicaule, L. Ilum. Stachys alopecuros, S. alpina, S. annua, S. marrubiastrum, Melittis melissophy, S. menthifolia, S. officinalis, S. palustris, S. sylvatica cretica, S. germanica, S. goulimyi, S. mifidum, L. garganicum, Stachys atherocalyx, S. ?Lamiastrum galeobdolon, Lamium, S. iva, S. leucoglossa, S. obliqua, S. parolini, S. balcanica, S. cassia, S. decumbens, S. inulosa, S. thirkei, S. tymphaea

Stachys arvensis

Stachys arvensis 
PEDALIACEAE

Sesamum indicum

Sesamum indicum

\title{
GESNERIACEAE
}

?Ramonda

?Ramonda nathaliae, $R$. serbica

\section{OROBANCHACEAE}

\section{Orobanche minor type}

Orobanche amethystea, $O$, crenata, $O$. gracilis, $O$. minor, $O$. reticulata

?Orobanche alba, $O$. cernua, $O$. elatior, $O$. grisebachii, $O$. hederae, $O$. loricata, $O$. lutea, $O$. oxyloba, $O$. pubescens, $O$. rechingeri, Scrophulariaceae: Rhynchocorys elephas

\section{Orobanche ramosa type}

Orobanche ramosa, Scrophulariaceae: Tozzia alpina

\author{
LENTIBULARIACEAE \\ Pinguicula \\ Pinguicula alpina, $P$. vulgaris \\ ?Pinguicula balcanica, $P$. hirtiflora \\ Utricularia \\ Utricularia intermedia, U. minor, U. vulgaris
}

\author{
RUBIACEAE \\ Putoria calabrica \\ Putoria calabrica \\ Crucianella \\ Crucianella angustifolia \\ ?Crucianella latifolia \\ Sherardia arvensis \\ Sherardia arvensis
}

\section{Galium type}

Asperula aristata, A. arvensis, A. cynanchica, A. laevigata, A. taurina, Cruciata laevipes, Galium album, G. aparine, G. corrudifolium, G. debile, G. divaricatum, G. elongatum, G. lucidum, G. odoratum, G. palustre, Galium x pomeranicum, G. rotundifolium, G. setaceum, G. spurium, G. tricornutum, $G$. verrucosum, $G$. verticillatum, $G$. verum, Rubia peregrina, R. tinctorum

?Asperula chlorantha, A. doerfleri, A. involucrata, A. lutea, A. purpurea, A. rumelica, A. tenella, Cruciata glabra, C. pedemontana, Galium absurdum, G. advenum, G.

anisophyllon, G. asparagifolium, G. breviramosum, G. circae, G. degenii, G. hellenicum, G. humifusum, G. incanum, G. intricatum, G. laconicum, G. monasterium, G. octonarium, G. ophiolithicum, G. oreophilum, G. pisoderium, G. procurrens, G. pseudaristatum, G. rhodopeum, G. rigidifolium, G. rivale, G. sacrorum, G. scabrifolium, G. speciosum, $G$.

\section{Valantia} tenuissimum

?Valantia aprica, V. hispida

\author{
PLANTAGINACEAE \\ Plantago major \\ Plantago major subsp. intermedia, $P$. major subsp. major \\ Plantago afra \\ Plantago afra \\ Plantago alpina type \\ Plantago alpina, $P$. serraria, $P$. subulata
}


Plantago lanceolata type

Plantago altissima, $P$. lagopus, $P$. lanceolata

?Plantago cretica, $P$. gentianoides, $P$. holosteum, $P$. reniformis, $P$. squarrosa

Plantago coronopus

Plantago coronopus

Plantago bellardii

Plantago bellardii

Plantago media

Plantago media

Plantago arenaria

Plantago arenaria

Plantago argentea

Plantago argentea

Plantago albicans

Plantago albicans

Plantago amplexicaulis

Plantago amplexicaulis

Plantago atrata

Plantago atrata

CAPRIFOLIACEAE

Sambucus nigra

Sambucus nigra

Sambucus ebulus

Sambucus ebulus

Lonicera alpigena

Lonicera alpigena

Lonicera periclymenum type

Lonicera periclymenum

?Lonicera etrusca

Lonicera xylosteum type

Lonicera nigra, L. xylosteum

Viburnum

Viburnum lantana, V. opulus, V. tinus

VALERIANACEAE

Valerianella rimosa type

Valerianella coronata, V. eriocarpa, V. rimosa

?Valerianella microcarpa

Valeriana tuberosa

Valeriana tuberosa

Valeriana officinalis type

Valeriana officinalis

?Valeriana crinii, $V$. dioscoridis

Centranthus

Centranthus calcitrapae

?Centranthus longiflorus

Valerianella locusta type

Valerianella carinata, V. locusta

?Valerianella costata, V. echinata, V. pumila, V. turgida

DIPSACACEAE

Succisa pratensis

Succisa pratensis 


\section{Scabiosa columbaria type}

Scabiosa atropurpurea, S. columbaria

?Scabiosa taygetea, S. tenuis, S. triniifolia, S. webbiana

\section{Dipsacus type}

Dipsacus fullonum, D. laciniatus

?Cephalaria ambrosioides, C. flava, C. transylvanica

\section{Knautia}

Knautia integrifolia

?Knautia ambigua, $K$. drymeia, $K$. longifolia, $K$. macedonica, $K$. magnifica, $K$. orientalis

\section{Scabiosa sicula type}

Scabiosa graminifolia, S. sicula

?Scabiosa argentea, S. crenata, S. epirota

Pterocephalus type

Pterocephalus papposus

?Pterocephalus perennis, Tremastelma palaestinum

Morina persica

Morina persica

\section{CAMPANULACEAE}

Jasione

?Jasione heldreichii, J. laevis

Campanula erinus type

Campanula erinus, C. glomerata, C. persicifolia, C. rapunculus, C. trachelium

?Campanula albanica, C. cervicaria, C. foliosa, C. formanekiana, C. hawkinsiana, C.

lingulata, C. macrostachya, C. patula, C. phrygia, C. ramosissima, C. scutellata, C.

sparsa, C. spatulata, C. trichocalycina, C. tymphaea, C. versicolor, Edraianthus

graminifolius, E. tenuifolius, L. speculum-veneris, Trachelium jacquinii

Campanula rotundifolia

Campanula rotundifolia

Asyneuma type

Asyneuma canescens, Campanula pp., Legousia hybrida

?Asyneuma limonifolium

\section{Phyteuma}

Phyteuma orbiculare

?Phyteuma confusum

\section{COMPOSITAE}

Subfamily Asteroidae ('Tubiliflorae type')

Solidago type

Tribe Heliantheae: Bidens cernua, B. tripartita, Helianthus annuus

Tribe Astereae: Aster alpinus, A. amellus, A. linosyris, A. novi-belgii, A. tripolium, Bellis annua, B. perennis, B. sylvestris, Conyza canadensis, Erigeron acer, E. alpinus, E. epiroticus, E. glabratus, Solidago virgaurea

Tribe Inuleae: Antennaria dioica, Dittrichia graveolens, $D$. viscosa, Filago pyramidata, $F$. vulgaris, Gnaphalium luteo-album, Inula britannica, I. conyza, I. germanica, I. helenium, I. salicina, Pallenis spinosa, Pulicaria dysenterica, P. vulgaris

Tribe Calendula: Calendula arvensis

Tribe Senecioneae: Petasites hybridus, Senecio aquaticus, S. doronicum, S. erucifolius, $S$. fluviatilis, S. gallicus, S. integrifolius, S. jacobaea, S. macedonicus, S. othonnae, S. papposus, S. scopolii, S. squalidus, S. thapsoides, S. vernalis, S. viscosus, S. vulgaris, Tussilago farfara

Tribe Eupatorieae: Eupatorium cannabinum

Tribe Lactuceae (Subfamily Cichorioideae): Scorzonera austriaca 
?Tribe Senecioneae: Adenostyles alliariae, Doronicum austriacum, D. clusii, D. columnae, D. orientale

Tribe Inuleae: Evax pygmaea, Filaginella uliginosa, Helichrysum italicum, H. plicatum, $H$. stoechas, Inula ensifolia, I. hirta, I. oculus-christi, I. verbascifolia, Logfia arvensis, $L$. gallica, L. minima, Omalotheca hoppeana, O. roeseri, O. supina, O. sylvatica, Phagnalon

Echinops graecum

Artemisia

Tribe Cardueae: Echinops bannaticus, E. mirocephalus, E. ritro, E. sphaerocephalus

Tribe Anthemideae: Artemisia abrotanum, A. absinthium, A. alba, A. campestris, A.

\section{Carlina type} eriantha, A. vulgaris

Tribe Cardueae: Arctium lappa, A. minus, A. tomentosum, Carlina acanthifolia, C. acaulis, C. corymbosa, C. frigida, C. vulgaris, Carthamus dentatus, C. lanatus, Onopordum acanthium, $O$. bracteatum, $O$. tauricum ?Jurinea glycacantha, J. mollis, Staehelina uniflosculosa

??Tribe Cardueae: Cnicus benedictus

\section{Picnomon acarna}

Tribe Cardueae: Picnomon acarna

Achillea type

Tribe Anthemideae: Achillea clypeolata, A. coarctata, A. millefolium, A. nobilis, A. ptarmica, A. setacea, Anthemis altissima, A. arvensis, A. carpatica, A. cotula, A. cretica, A. tinctoria, A triumfetti, Chamomilla recutita, Coleostephus myconis, Matricaria caucasica, M. perforata, M. tempskyana, M. trichophylla, Tanacetum corymbosum, $T$. vulgare Tribe Cardueae: Serratula tinctoria ?Tribe Anthemideae: Achillea abrotanoides, A. absinthoides, A. ageratifolia, A. chrysocoma, A. clavennae, A. clusiana, A. collina, A. crithmifolia, A. depressa, A. distans, A. fraasii, A. grandifolia, A. holosericea, A. lingulata, A. pannonica, A. pindicola, Anthemis auriculata, A. coelopoda, A. meteorica, A. parnassica, A. ruthenica, A. segetalis, A. tenuiloba, A. tomentosa, Leucanthemum praecox, $L$. vulgare, Tanacetum macrophyllum Tribe Cardueae: Xeranthemum annuum, $X$. cylindraceum, $X$. inapertum

Centaurea cyanus type

Tribe Cardueae: Centaurea cyanus, C. depressa, C. pinardii

\section{Centaurea napulifera type}

Tribe Cardueae: Centaurea napulifera, C. pindicola, C. triumfetti

Cirsium type

Tribe Cardueae: Carduus acanthoides, C. acicularis, C. candicans, C. cronius, C.

hamulosus, C. kerneri, C. pycnocephalus, C. thessalus, C. tmoleus, Cirsium

appendiculatum, C. arvense, C. candelabrum, C. creticum, C. eriophorum, C. erisithales,

C. heldreichii, C. ligulare, C. mairei, C. tymphaeum, C. vulgare

Xanthium

?Tribe Cardueae: Carduus macrocephalus, C. taygeteus, C. thoermeri

Tribe Heliantheae: Xanthium spinosum, X. strumarium

Centaurea solstitialis type

Tribe Cardueae: Centaurea affinis, C. alba, C. attica, C. calcitrapa, C. charrelii, C. cuneifolia, C. diffusa, C. grisebachii, C. iberica, C. jacea, C. kalambakensis, C. lactiflora, C. melitensis, C. nicopolitana, C. niederi, C. orphanidea, C. pallidior, C. pannosa, C. pelia, C. solstitialis, C. tymphaea, C. uniflora, C. zuccariniana, Cynara scolymus ?Tribe Cardueae: Amphoricarpos neumayeri, Centaurea deustiformis, C. epirota, C. immanuelis-loewii, C. macedonica, C. pannonica, C. pawlowski, C. stenolepis, C. stereophylla, C. triamularia, C. vlachorum, C. weldeniana, Ptilostemon afer, $P$. chamaepeuce, P. gnaphaloides, P. strictus

\section{Centaurea graeca type}

Tribe Cardueae: Centaurea graeca, C. psilacantha, C. rupestris, C. salonitana, C. spruneri 
Centaurea amplifolia

Tribe Cardueae: Centaurea amplifolia

Crupina

Tribe Cardueae: Crupina crupinastrum, C. vulgaris

Subfamily Cichorioideae ('Liguliflorae type', 'Taraxacum type')

Tragopogon

Tragopogon dubius, $T$. porrifolius, T. pratensis

?Tragopogon balcanicus, $T$. crocifolius, $T$. hybridus, T. tommasinii

Scorzonera laciniata type

Scorzonera doria, S. laciniata; S. purpurea

Scorzonera hispanica type

Scorzonera hispanica

?Scorzonera cana, S. mollis, S. parviflora

Sonchus type

Reichardia picroides, Sonchus arvensis, S. asper, S. oleraceus, S. tenerrimus

??Cephalorrhynchus tuberosus

Lactuca type

Lactuca quercina, L. saligna, L. sativa, L. serriola, L. viminea, $L$. virosa, Mycelis muralis,

Rhagadiolus stellatus

?Lactuca aurea, L. graeca

Scolymus

Scolymus hispanicus, S. maculatus

Cichorium type

Cichorium intybus, Crepis pulchra

Hieracium type

Hieracium/Pilosella agg., Hieracium amplexicaule, $H$. dasycraspedum, $H$. epirense, $H$.

ferdinandi-coburgi, $H$. lactucella, $H$. naegelianiforme, $H$. necopinum, $H$. pilosella, $H$.

trikalense, Tolpis barbata

?Hieraceum subgenus Pilosella: Hieracium x auriculoides, H. caespitosum, H. cymosum, $H$. $\mathrm{x}$ densiflorum, $H . \times$ fuscatrum, $H$. x halacsyi, $H$. hoppeanum, $H . \times$ macrotrichum, $H$. pavichii, $H$. piloselloides, $H$. praealtum, $H$. pseudopilosella, $H . \times$ ruprechtii, $H$. x spurium, H. x zizianum

Hieraceum subgenus Hieraceum: H. amplexicaule group (H. petraeum), H. bifidum group (H. caesiiflorum, H. stenolepis), H. bracteolatum group (H. bracteolatum), H. dolopicum group (H. dolopicum), H. eriobasis group, H. gaudryi group (H. gaudryi), H. graecum group (H. graecum), H. gymnocephalum group (H. gymnocephalum), $H$. heldreichii group, $H$. hypochoeroides group, $H$. latifolium group $(H$. brachyphyllum $), H$. lazistanum group $(H$. leithneri), H. marmoreum group (H. megalothecum), H. murorum group (H. grandidens, $H$. integratum, $H$. semisilvaticum), $H$. naegelianum group ( $H$. naegelianum), $H$. pannosum group ( $H$. friwaldii, H. pannosum), $H$. parnassi group ( $H$. parnassi, $H$.

pseudobracteolatum), $H$. pilosissimum group, $H$. pseudorieni group (H. scardicum), $H$. racemosum group (H. barbatum, $H$. crinitum, $H$. italicum, $H$. racemosum), $H$. sabaudum group (H. obliquum, $H$. sabaudum), $H$. sartorianum group, $H$. scapigerum group, $H$. schmidtii group ( $H$. pallidum), $H$. sericophyllum group, $H$. silesiacum group, $H$. umbrosum group ( $H$. umbrosum), $H$. waldsteinii group ( $H$. delpinoi, $H$. waldsteinii)

Taraxacum type

Crepis capillaris, C. foetida, C. vesicaria, Hypochoeris glabra, H. maculata, H. radicata, Lapsana communis, Leontodon autumnalis, L. crispus, L. hispidus, L. taraxacoides, Picris echioides, $P$. hieracioides, Taraxacum apenninum group, Taraxacum bithynicum group ( $T$. bithynicum, T. megalorrhizon, $T$. minimum), Taraxacum erythrospermum group ( $T$. pindicola), Taraxacum fulvum group, Taraxacum gasparrinii group (T. dorchocarpum, $T$. epirense, T. xanthiense), Taraxacum officinale group (T. copidophyllum, T. officinale), Taraxacum serotinum group (Taraxacum serotinum)

?Crepis aurea, C. baldaccii, C. dioscoridis, C. fraasii, C. geracioides, C. guioliana, 
C. merxmuelleri, C. neglecta, C. rubra, C. sancta, C. setosa, C. turcica, C. viscidula, Leontodon cichoraceus, Picris pauciflora, $P$. sprengerana, Taraxacum aganippeum, $T$. bulgaricum, $T$. gionense, Taraxacum glaciale, $T$. graecofontanum, Taraxacum hoppeanum group (T. amborum, T. hoppeanum, T. poliochlorum), T. nudum, Taraxacum palustre group (Taraxacum scaturiginosum), $T$. subolivaceum

?? Calycocorsus stipitatus, Chondrilla juncea, C. ramosissima, Hedypnois cretica,

Prenanthes purpurea

\author{
ALISMATACEAE \\ Sagittaria sagittifolia \\ Sagittaria sagittifolia \\ Baldellia ranunculoides \\ Baldellia ranunculoides \\ Alisma
}

Alisma gramineum, A. lanceolatum, A. plantago-aquatic

Damasonium alisma

Damasonium alisma

\title{
BUTOMACEAE \\ Butomus umbellatus
}

Butomus umbellatus

\section{HYDROCHARITACEAE}

Hydrocharis morsus-ranae

Hydrocharis morsus-ranae

Stratiotes aloides

Stratiotes aloides

Vallisneria spiralis

Vallisneria spiralis

JUNCAGINACEAE

Triglochin bulbosa

Triglochin bulbosa

\section{POTAMOGETONACEAE}

Potamogeton crispus type

Groenlandia densa, Potamogeton acutifolius, P. alpinus, $P$. berchtoldii, $P$. coloratus, $P$. compressus, $P$. crispus, $P$. lucens, $P$. natans, $P$. nodosus, $P$. obtusifolius, $P$. perfoliatus, $P$. polygonifolius, $P$. trichoides

Potamogeton pectinatus Potamogeton pectinatus

\section{ZANNICHELLIACEAE}

Zannichellia palustris

Zannichellia palustris

\section{NAJADACEAE}

Najas marina

Najas marina 


\section{LILIACEAE}

Colchicum

Colchicum autumnale, C. graecum, C. neapolitanum, C. triphyllum

?Colchicum bivonae, C. callicymbium, C. cupanii, C. hungaricum, C. lingulatum, C. turcicum

Merendera sobolifera

Merendera sobolifera

Narthecium scardicum

Narthecium scardicum

Veratrum

Veratrum album

?Veratrum nigrum

Asphodelus fistulosus type

Asphodelus aestivus, $A$. fistulosus

Asphodelus albus

Asphodelus albus

Ornithogalum type

Asphodeline liburnica, A. taurica, Ornithogalum arabicum, O. collinum, O. narbonense,

$O$. nutans, $O$. orthophyllum, $O$. pyrenaicum, $O$. umbellatum, Scilla hyacinthoides

? Ornithogalum armeniacum, $O$. comosum, $O$. divergens, $O$. exscapum, $O$. montanum, $O$.

oligophyllum, $O$. refractum, $O$. sphaerocarpum

Fritillaria

Fritillaria epirotica, F. gussichiae, F. messanensis, F. mutabilis, F. orientalis, F. pontica,

F. thessala

Gagea lutea type

Gagea arvensis, G. bohemica, G. lutea, Paris quadrifolia

?Gagea amblyopetala, G. dubia, G. fibrosa, G. fistulosa, G. minima, G. pusilla, G.

reticulata, G. saxatilis

Gagea pratensis

Gagea pratensis

Tulipa sylvestris

Tulipa sylvestris

Tupila praecox type

Tulipa gesnerana, T. praecox

?Tulipa boeotica, $T$. clusiana

Lilium

Lilium albanicum, L. candidum, L. carniolicum, L. chalcedonicum, L. martagon

Anthericum liliago

Scilla type

Anthericum liliago

Polygonatum verticillatum, Scilla autumnalis, S. bifolia, S. hyacinthoides

?Scilla albanica

Muscari comosum

Muscari comosum

Muscari neglectum type

Muscari neglectum

?Muscari armeniacum, M. botryoides, M. cummutatum, M. macrocarpum, M. parviflorum, M. tenuiflorum

??Bellevalia ciliata, B. dubia, B. romana, B. trifoliata, Hyacinthus orientalis, Hyacinthella leucophaea, Strangweia spicata

Allium roseum type

Allium neapolitanum, A. roseum 
Allium sphaerocephalon type

Allium ampeloprasum, A. atroviolaceum, A. guttatum, A. sativum, A. scorodoprasum, A. sphaerocephalon, A. vineale

Allium paniculatum type

Allium carinatum, A. cepa, A. chamaemoly, A. moschatum, A. nigrum, A. pallens, A. paniculatum, A. schoenoprasum, A. subhirsutum, A. ursinum

?Allium amethystinum, A amphipulchellum, A. bornmuelleri, A. breviradium, A.

chamaespathum, A. favosum, A. flavum, A. frigidum, A. heldreichii, A. hirtovaginum, A.

meteoricum, A. phthioticum, A. sipyleum, A. stamineum, A. suaveolens

Polygonatum type

Polygonatum multiflorum, $P$. odoratum, Ruscus aculeatus, $R$. hypoglossum

?Polygonatum latifolium

Convallaria majalis

Convallaria majalis

Asparagus

Asparagus acutifolius, A. officinalis

Urginea maritima

?Asparagus stipularis, A. tenuifolius, A. verticillatus

Urginea maritima

Smilax

Smilax aspera

?Smilax excelsa

AMARYLLIDACEAE

Narcissus papyraceus type

Narcissus papyraceus, $N$. poeticus, $N$. serotinus

Narcissus tazetta

Narcissus tazetta

Sternbergia

Sternbergia lutea

?Sternbergia colchiciflora

Galanthus type

Galanthus nivalis, Leucojum aestivum

?Leucojum valentinum

DIOSCOREACEAE

Tamus communis

Tamus communis

IRIDACEAE

Hermodactylus tuberosus

Hermodactylus tuberosus

Iris pseudacorus

Iris pseudacorus

Iris spuria type

Iris spuria

?Iris reichenbachii, I. sintenisii, I. suaveolens

Iris pumila

Iris pumila

Iris germanica

Iris germanica

Gladiolus type

Gladiolus communis, G. illyricus, G. italicus, Romulea bulbocodium, R. linaresii

?Gladiolus imbricatus 
Gynandriris sisyrinchium

Gynandriris sisyrinchium

Crocus

Crocus biflorus, C. chrysanthus, C. flavus

?Crocus boryi, C. cvijicii, C. hadriaticus, C. olivieri, C. pulchellus, C. robertianus, C. sieberi, $C$. veluchensis

Iris planifolia

Iris planifolia

\section{JUNCACEAE}

Juncaceae

Juncus alpinus, J. anceps, J. articulatus, J. bufonius, J. compressus, J. conglomeratus, J. effusus, J. fontanesii, J. hybridus, J. inflexus, J. minutulus, J. sphaerocarpus, J. striatus, J. subnodulosus, J. tenuis, J. trifidus, Luzula campestris, L. forsteri, L. luzulina, L. multiflora, L. pilosa, L. spicata, L. sylvatica

?Juncus atratus, J. heldreichianus, J. thomasii, Luzula italica, L. pindica, L. sudetica

\section{GRAMINEAE}

Festuca type

Bellardiochloa violacea, Desmazeria rigida, Festuca alpina, F. altissima, F. amethystina, $F$. arundinacea, $F$. callieri, $F$. circummediterranea, $F$. cyllenica, $F$. dalmatica, $F$. drymeja, $F$. glauca, $F$. graeca, $F$. grandiaristata, $F$. hercegovinica, $F$. heterophylla, $F$.

hirtovaginata, $F$. horvatiana, $F$. jeanpertii, $F$. korabensis, $F$. koritnicensis, $F$. loliacea, $F$. nigrescens, $F$. ovina, $F$. paniculata, $F$. penzesii, $F$. peristerea, $F$. pindica, $F$. polita, $F$. pratensis, $F$. rechingeri, $F$. rubra, $F$. spectabilis, $F$. thracica, $F$. valesiaca, $F$. valida, $F$. varia, $F$. violacea, $F$. vizzavonae, Lolium multiflorum, $L$. perenne, $L$. remotum, $L$. rigidum, L. temulentum, Micropyrum tenellum, Poa alpina, P. angustifolia, P. annua, P. bulbosa, P. cenisia, $P$. chaixii, $P$. glauca, $P$. macedonica, $P$. nemoralis, $P$. ophiolithica, $P$. palustris, $P$. perconcinna, $P$. pratensis, $P$. pumila, $P$. timoleontis, $P$. trivialis, $P$. versicolor, Puccinellia distans, Sclerochloa dura, Vulpia bromoides, V. ciliata, V. muralis, V. myuros ?Eleusine indica, Eragrostis cilianensis, E. minor, E. pilosa

??Aristida adscensionis, Cleistogenes serotina, Crypsis aculeata, C. alopecuroides, $C$. schoenoides, Danthonia alpina, $D$. decumbens, Molinia caerulea, Tragus racemosus

\section{Glyceria type}

Bromus alopecuros, B. arvensis, B. benekenii, B. cappadocicus, B. commutatus, $B$. diandrus, $B$. erectus, $B$. hordeaceus, $B$. intermedius, $B$. japonicus, $B$. lanceolatus, $B$. madritensis, $B$. pindicus, $B$. ramosus, B. rigidus, B. riparius, $B$. rubens, $B$. scoparius, $B$. secalinus, B. squarrosus, B. sterilis, B. tectorum, Glyceria maxima, G. spicata

\section{Dactylis type}

Apera spica-vent, Beckmannia eruciformis, Brachypodium distachyon, $B$. phoenicoides, $B$. pinnatum, B. retusum, B. sylvaticum, Briza humilis, B. maxima, B. media, B. minor, Catabrosa aquatica, Cynodon dactylon, Cynosurus cristatus, C. echinatus, C. elegans, Dactylis glomerata, Echinaria capitata, Festucopsis sancta, Lamarckia aurea, Melica altissima, M. ciliata, $M$. minuta, $M$. nutans, $M$ transsilvanica, Mibora minima, Nardus stricta, Psilurus incurvus, Sesleria bielzii, S. coerulans, S. korabensis, S. robusta, S. sadlerana, S. tenerrima, $S$. vaginalis ??Achnatherum calamagrostis, Agrostis canina, A. capillaris, A. castellana, A. gigantea, A. stolonifera, Aira caryophyllea, A. cupaniana, A. elegantissima, A. tenorii, Alopecurus aequalis, A. arundinaceus, A. creticus, A. myosuroides, A. rendlei, A. setarioides, Ampelodesmos mauritanica, Andropogon distachyos, Anthoxanthum gracile, A. odoratum, A. ovatum, Arrhenatherum elatius, Arundo donax, Avellinia michelii, Avenula cincinnata, A. compressa, A. pubescens, Brachiaria eruciformis, Calamagrostis arundinacea, C. varia, Chrysopogon gryllus, Corynephorus divaricatus, Danthoniastrum compactum, Dasypyrum villosum, Deschampsia cespitosa, D. flexuosa, D. media, Dichanthium ischaemum, Digitaria ischaemum, D. sanguinalis, Echinochloa crus-galli, Gastridium ventricosum, 
Hainardia cylindrica, Helictotrichon convolutum, Hemarthria altissima, Holcus lanatus, H. mollis, H. setiglumis, Hyparrhenia hirta, Imperata cylindrica, Koeleria lobata, K.

macrantha, K. pyramidata, K. splendens, Lagurus ovatus, Lophochloa cristata, L. hispida, Milium effusum, M. vernale, Molineriella minuta, Parapholis pycnantha, Parvotrisetum myrianthum, Paspalum paspalodes, Phacelurus digitatus, Phalaris arundinacea, $P$. brachystachys, $P$. canariensis, $P$. coerulescens, $P$. minor, $P$. paradoxa, $P$ hleum alpinum, $P$. arenarium, $P$. echinatum, $P$. graecum, $P$. montanum, $P$. paniculatum, $P$. phleoides, $P$. pratense, $P$. subulatum, Pholiurus pannonicus, Piptatherum coerulescens, $P$. holciformis, P. miliaceum, Polypogon maritimus, $P$. monspeliensis, $P$. viridis, Saccharum ravennae, $S$. strictum, Setaria italic, S. pumila, S. verticillata, S. viridis, Sorghum halepense, Stipa bromoides, S. capensis, S. capillata, S. epilosa, S. fontanesii, S. joannis, S. lessingiana, S. pennata, S. pulcherrima, S. rechingeri, S. thessala, S. tirsa, Trisetum flavescens, Trisetum ?flavescens, T. tenuiforme, Ventenata dubia

Agropyron type

Aegilops cylindrica, A. dichasians, A. geniculata, A. lorentii, A. neglecta, A. speltoides, A. triuncialis, A. uniaristata, Agropyron cristatum

?Panicum miliaceum, $P$. repens, Secale montanum

\section{Triticum type}

Triticum aestivum, T. compactum, T. dicoccon, T. durum, T. turgidum

Secale cereale

Secale cereale

Hordeum type

Elymus caninus, E. elongatus, E. hispidus, E. repens, Hordeum murinum, H. vulgare,

Triticum monococcum

?Hordelymus europaeus, Hordeum bulbosum, H. distichon, H. hystrix, H. marinum,

Taeniatherum caput-medusae, Triticum baeoticum

Avena

Avena byzantina, $A$. fatua, A. sativa

?Avena barbata, A. clauda, A. sterilis, A. strigosa

Phragmites australis

Zea mays

Phragmites australis

Zea mays

ARACEAE

Arum

Arum italicum, A. maculatum

?Arum orientale, A. petteri

Dracunculus type

Dracunculus vulgaris

?Biarum tenuifolium

Arisarum vulgare

Arisarum vulgare

LEMNACEAE

Lemnaceae

Lemna gibba, L. minor, L. trisulca, Spirodela polyrhiza

SPARGANIACEAE

Sparganium erectum

Sparganium erectum

Sparganium angustifolium

Sparganium angustifolium 
TYPHACEAE

Typha angustifolia type

Typha angustifolia, T. latifolia

Typha domingensis type

Typha domingensis

?Typha laxmannii

\section{CYPERACEAE}

Scirpus

Scirpus lacustris, S. maritimus

Cladium mariscus

Cladium mariscus

Cyperus michelianus

Cyperus michelianus

Cyperus longus type

Cyperus glomeratus, C. laevigatus, C. longus

?Cyperus difformis, C. esculentus, C. flavescens, C. flavidus, C. glaber

Schoenus nigricans

Schoenus nigricans

Carex hallerana type

Carex hallerana, Scirpus holoschoenus, S. sylvaticus

?Blysmus compressus, Carex depressa, C. ferruginea, C. liparocarpos, C. macrolepis, C. melanostachya, C. sempervirens, Scirpus cernuus, S. cespitosus, S. litoralis, S. mucronatus, S. setaceus, $S$. supinus

\section{Carex flacca type}

Carex acuta, C. acutiformis, C. appropinquata, C. atrata, C. caryophyllea, C.

chordorrhiza, C. depauperata, C. digitata, C. distachya, C. distans, C. disticha, C. divisa, C. divulsa, C. echinata, C. elata, C. elongata, C. ericetorum, C. flacca, C. hirta, C. hostiana, C. humilis, C. lepidocarpa, C. muricata, C. nigra, C. otrubae, C. ovalis, C. pallescens, C. panicea, C. paniculata, C. pendula, C. punctata, C. remota, C. riparia, C. rostrata, C. serotina, C. spicata, C. sylvatica, C. tomentosa, C. umbrosa, C. vulpina, Cyperus fuscus, C. rotundus, Eleocharis multicaulis, E. palustris, Eriophorum angustifolium, E. gracile, E. latifolium, E. vaginatum

?Carex davalliana, C. hispida, C. kitaibeliana, Eleocharis acicularis, E. quinqueflora, E. uniglumis, Fimbristylis bisumbellata

\section{ORCHIDACEAE}

Epipactis type

Subfamily Neottioideae: Epipactis atrorubens, E. helleborine, E. leptochila, E. microphylla, E. palustris, E. purpurata, Listera ovata, Neottia nidus-avis ?Cypripedium calceolus, Epipogium aphyllum

Limodorum abortivum

Subfamily Neottioideae: Limodorum abortivum

\section{Cephalanthera}

Subfamily Neottioideae: Cephalanthera damasonium, C. rubra

\section{Orchis type}

Subfamily Orchidoideae: Aceras anthropophorum, Anacamptis pyramidalis, Barlia robertiana, Coeloglossum viride, Dactylorhiza fuchsii, D. incarnata, D. sambucina, $D$. sulphurea, Gymnadenia conopsea, Himantoglossum hircinum, Neotinea maculata, Ophrys apifera, $O$. bombyliflora, $O$. fusca, $O$. reinholdii, $O$. scolopax, $O$. sphegodes, $O$. tenthredinifera, Orchis coriophora, $O$. italica, $O$. laxiflora, $O$. mascula, $O$. pallens, $O$. papilionacea, $O$. provincialis, $O$. purpurea, $O$. quadripunctata, $O$. tridentata, $O$. ustulata, Platanthera bifolia, P. chlorantha, Serapias cordigera, S. lingua, S. vomeracea Subfamily Neottioideae: Spiranthes aestivalis, S. spiralis

?Subfamily Orchidoideae: Dactylorhiza baumanniana, D. cordigera, D. iberica, 
D. kalopissii, D. saccifera, Ophrys carmeli, O. ferrum-equinum, O. fuciflora, O. spruneri, Orchis simia, O. spitzelii, Pseudorchis frivaldii

?Corallorhiza trifida 



\section{Appendix VII}

\section{Species lists for reconnaissance vegetation surveys}

Several reconnaissance vegetation surveys were undertaken at sites where no samples were collected for pollen analysis, but a note was made of the dominant species present. These vegetation surveys were undertaken to enhance the author's knowledge of the ecology of the indigenous vegetation of Grevena as well as to gain some knowledge of regeneration patterns, and for the collection of pollen reference material, particularly of herbs. Below are listed the species of interest at these sites, which are presented in order of elevation. The vegetation zone in which the site occurs follows the location of the site in brackets.

Taxonomic names of European species follow:

Tutin, T. G. et al. (eds) 1964-80. Flora Europaea. Volumes 1-5. Cambridge University Press, Cambridge.

The numbers in [ ] following the species names are herbarium specimen numbers.

An "**" preceding a taxonomic name denotes an exotic species.

\section{i) Zakas to Agios Nikolaos (mountain pine wood)}

Dominant tree species were noted at three locations on the road between Zakas and Agios Nikolaos in the mountain pine woods. At the highest elevation $(1100 \mathrm{~m})$, all three pine species, Pinus nigra (commonest), $P$. heldreichii, and $P$. leucodermis, that occur in Grevena grow together. They grow in a mosaic of small patches of same species and same age trees. The oldest pine tree here is about 150 years old.

Close to Agios Nikolaos, at a lower elevation of about $1025 \mathrm{~m}$ asl, a herbarium specimen of Cornus mas (collection \# 171) was collected on a slope of $15-20^{\circ}$ with a southerly aspect. Malus sylvestris (crab apple) and Cichorium intybus (chicory) were abundant here. There were occasional oaks by the roadside.

Near the church of Agios Nikolaos, in a riverine vegetation zone, are a few Salix spp. (willows) trees, some were large; and sparse oak trees.

Genus \& species
Common name

Black pine

Balkan pine

Bosnian pine

Cornelian cherry

Crabapple

Chicory

Willows
Family

Pinaceae

Pinaceae

Pinaceae

Cornaceae

Rosaceae

Compositae

Fagaceae

Salicaceae 


\section{ii) Above Zakas (mountain pine wood-pasture)}

This mountain pine wood-pasture occurs at 1000-1200 m, below the mountain pine woods of (i) above. Pinus nigra dominates this wood. Trees are relatively widely spaced. The wood is grazed, but ground cover is quite thick, comprising Pteridium aquilinum and dryish grassland (steppe). Many of the large pine trees in this area have been struck by lightning, but this has not killed them, nor did they burn.

\begin{tabular}{ccc}
\hline Genus \& species & Common name & Family \\
\hline $\begin{array}{c}\text { Pteridophyta: } \\
\text { Pteridium acquilinum (L.) Kuhn in Decken }\end{array}$ & Bracken & Hypolepidaceae \\
$\begin{array}{c}\text { Gymnospermae: } \\
\text { Pinus nigra Arnold }\end{array}$ & Black pine & Pinaceae \\
$\begin{array}{c}\text { Angiospermae: } \\
\text { Monocotyledones: } \\
\text { Gramineae spp. }\end{array}$ & Grasses & Gramineae \\
\hline
\end{tabular}

\section{iii) Perivolion/Vovousa (mountain beech wood)}

Plants are listed from a disturbed area in beech wood at $1480 \mathrm{~m}$ asl. The site is near the pass between Perivolion and Vovousa about $1.5 \mathrm{~km}$ west of the wetland, Anelia, where pollen analysis was undertaken. The site has stony brown earth and a northeast aspect with a slope of $5^{\circ}$. Particular note was made of herbaceous plants growing on a track along a ridge top under the beech wood. These plants include species that would grow in a coppiced beech wood.

$\begin{array}{lll}\text { Genus } \& \text { species } & \text { Common name } & \text { Family }\end{array}$

\begin{tabular}{lll}
\hline $\begin{array}{l}\text { Angiospermae: } \\
\text { Dicotyledones: }\end{array}$ & & \\
Campanula sp. [6] & Bellflower & Campanulaceae \\
Centaurea sp. [5] & Knapweed & Compositae \\
Dianthus sp. [4] & Pink & Caryophyllaceae \\
Digitalis grandiflora Miller [2] & Large yellow foxglove & Scrophulariaceae \\
Euphrasia sp. [11] & & Scrophulariaceae \\
Lathyrus sp. [9] & Pea, Vetch & Leguminosae \\
Lotus sp. [8] & Birdsfoot-trefoil & Leguminosae \\
Leontodon sp. or related sp. [7] & & Compositae \\
Myosotis sp. [1] & & Boraginaceae \\
Prunella laciniata (L.) L. [3] & Cut-leaved self-heal & Labiatae \\
Viola sp. [10] & Violet, Pansy & Violaceae \\
\hline
\end{tabular}


iv) Polinerion (foothill deciduous oak wood-pasture)

The area surveyed is about $1.5 \mathrm{~km}$ west of Polinerion at about $1200 \mathrm{~m}$ asl. The species listed grow in grassland on a ridge top in oak wood-pasture close to the quadrats that were quantitatively surveyed (see Appendix VIII).

Genus \& species

Common name

Family

ermae:

Dicotyledones:

Acinos suaveolens (Sibth. \& Sm.)

G. Don fil. in Loudon [73]

Anthemis sp. or Achillea sp. [75]

Centaurea solstitialis L. [77]

Cyclamen sp. [81]

Dianthus sp. [72]

Erodium cf. cicutarium (L.) L'Her [74]

Eryngium amethystinum L. [79]

Euphorbia myrsinites L. [80]

Hieracium/Pilosella agg. [76]

Thymus sp. [71]

Xanthium spinosum L. [78]

$\begin{array}{ll}\text { Chamomile } & \begin{array}{l}\text { Labiatae } \\ \text { Compositae }\end{array} \\ \text { St. Barnaby's thistle } & \begin{array}{l}\text { Compositae } \\ \text { Primulaceae }\end{array} \\ \text { Pink } & \text { Caryophyllaceae } \\ \text { Common storksbill } & \text { Geraniaceae } \\ \text { Blue eryngo } & \text { Umbelliferae } \\ \text { Broad-leaved glaucous spurge } & \text { Euphorbiaceae } \\ & \text { Compositae } \\ \text { Thyme } & \text { Labiatae } \\ \text { Spiny Cocklebur } & \text { Compositae }\end{array}$

v) Mavranaioi (lowland plains steppe and deciduous oak wood-pasture)

This site is close to Mavranaioi at about $700 \mathrm{~m}$ asl. A road passes close to a dense patch of gnarled oaks, typical of the patches that grow in the mosaic of steppe and deciduous oak woods of the lowland plains. The site has a SW aspect and the substrate was red earth on colluvium.

Oak species growing in this stand included Quercus cerris, $Q$. frainetto and $Q$. pubescens. Typically there were many oak hybrids and clinal variations. The oaks had been coppiced. In this vegetation zone, if coppicing stopped, the oaks would probably grow into tall trees and steppe plants would grow as undergrowth. Juniperus oxycedrus grows in this stand indicating that it was once more open. The roadside vegetation was a mixture of steppe and ruderal plants.
Genus \& species
Common name
Family

\section{Gymnospermae: \\ Juniperus oxycedrus L. \\ Angiospermae: \\ Dicotyledones: \\ Alyssum cf. murale Waldst. \& Kit. or ?Neslia paniculata (L.) Desv. [59]}

Anthemis sp. [66]

Centaurea sp. [57]

Chondrilla juncea L. [70]

Cichorium intybus $\mathrm{L}$.

Clinopodium vulgare L. [61]

Convolvulus arvensis L. [ 69]

Crepis sp. [63]

Dorycnium hirsutum (L.) Ser. in DC [60]

Echinops microcephalus Sibth. \& Sm. [55]

Euphorbia ?segetalis L. [67]

Lactuca viminea (L.) J. \& C. Presl [58]

Lotus sp.
Prickly juniper

Cupressaceae

Cruciferae

Compositae

Compositae

Compositae

Compositae

Chicory

Wild basil

Hawksbeard

Globe thistle

Spurge

Pliant lettuce

Labiatae

Convolvulaceae

Compositae

Leguminosae

Compositae

Euphorbiaceae

Compositae

Leguminosae 
Plantago lanceolata L. [65 \& 68]

Polygonum sp.

Quercus cerris L.

$Q$. frainetto Ten.

$Q$. pubescens Willd.

Scabiosa sp. ?atropurpurea L. [56]

Scrophularia canina L.

Sorbus torminalis (L.) Crantz [62]

*Xanthium strumarium L. [64]
Ribwort, Plantain

Dock

Turkey oak

Hungarian sak

White oak

Scabious

Figwort

Wild service tree

Cocklebur
Plantaginaceae

Polygonaceae

Fagaceae

Fagaceae

Fagaceae

Dipsacaceae

Scrophulariaceae

Rosaceae

Compositae

\title{
vi) Asprokampos (lowland plains steppe and deciduous oak wood)
}

Weeds were collected from the road verge near Asprokampos in the steppe and oak wood-pasture vegetation zone of the lowland plains at an elevation of $560 \mathrm{~m}$ asl. Much of the surrounding area is cultivated fields.

The most common weeds are Cichorium intybus, Mentha spp., Galium verum, Lotus spp., Ononis spinosa, Taraxacum sp. and Verbena officinalis.

$\begin{array}{lll}\text { Genus \& species } \quad \text { Common name } & \text { Family }\end{array}$

\author{
Angiospermae: \\ Dicotyledones: \\ Centaurea sp. [247] \\ Cichorium intybus L. \\ Dipsacaceae [248] \\ Galium verum $\mathrm{L}$. \\ Lactuca viminea (L.) J. \& C. Presl.

$$
[251,255]
$$ \\ Lotus sp. \\ Mentha suaveolens Ehrh. [249] \\ Ononis spinosa L. \\ Salvia sp. [252] \\ ?Scorzonera sp. [254] \\ ?Sinapis alba L. [253] \\ Taraxacum sp. \\ Verbena officinalis L. [250]
}

Knapweed

Compositae

Chicory

Compositae

Slender crosswort

Dipsacaceae

Pliant lettuce

Rubiaceae

Mint

Spiny restharrow

Clary, Sage

?Vipergrass

White mustard

Dandelion

Vervain

Compositae

Leguminosae

Labiatae

Leguminosae

Labiatae

Compositae

Cruciferae

Compositae

Verbenaceae 



\section{Appendix VIII}

\section{Modern vegetation composition at pollen surface sample collection sites}

Vegetation was surveyed at each site where a surface pollen sample was collected. These surveys were undertaken to gain some knowledge of the ecology of species growing in Grevena as well as to study the relationship between vegetational composition and pollen precipitation. This appendix tabulates the (mostly) vascular species growing at and near these sites. Quantitative surveys were undertaken at most sites to gain a rough estimate of relative abundance of species. Visual estimates were made of the percent cover of herbaceous species and woody plants (Andersen 1970, 1973; Birks 1973, 1977; Moore \& Chapman 1986). Where more than one vegetational stratum existed, each stratum was considered separately for estimating the relative values. Cover estimates have not been converted into classes but left in their raw percentage estimates as these are more easily compared to pollen relative percentages $(+=$ under $1 \%)$.

Since the main objective was to study the relationship between vegetational composition and pollen precipitation, the selection of survey areas was based on the major vegetation types described by Moody \& Rackham (in prep.). The aim was to survey a representation sample of these vegetation types. Thus, a transect from the western Pindos Mountains across the foothills to the lowlands was surveyed. Unfortunately, time precluded a survey of the slopes of the Vourinos Mountains.

Plot characteristics are given above each species list. Altitudes were recorded with an altimeter and checked against geological maps (see References). Aspect and slope were measured in the field with a hand-held compass. Height of canopy was estimated by eye. Life-form classes follow Raunkiaer (1937) (see Appendix X). Identifications were checked by Dr O. Rackham, Cambridge University. Taxonomic names of vascular species follow Tutin et al. (1964-80) and of non-vascular species Watson (1968).

Vegetation surveys are presented according to landforms from west to east across Grevena:

\section{PINDOS MOUNTAINS}

Pine wood-pasture, Pinus leucodermis

Beech woods

Pine woods, Pinus nigra

Pine/oak woods

PINDOS FOOTHILLS

Oak wood-pasture

Mixed woods

\section{LOWLAND PLAINS}

Cultivated fields

Oak woods

Steppe/oak woods 


\section{PINDOS MOUNTAINS}

\section{Pine wood-pasture, Pinus leucodermis}

\section{Avgo}

Grid reference: $39^{\circ} 55^{\prime} 05^{\prime \prime} \mathrm{N} 21^{\circ} 07^{\prime} \mathrm{E}$

Altitude: $1760 \mathrm{~m}$

Local vegetation: floating sedge mat

Slope: flat

Aspect: northeast

Substrate: peat of Lammos Lake margin

Reconnaisance survey

The site is named after the nearby peak of Avgo. The lake is almost entirely covered with a floating mat of $95 \%$ Cyperaceae. Most of the floating mat comprises two species of Eriophorum (50\%). Milium grows as high tussocks on the mat. Eleocharis grows in the lake rooted to the bottom while Utricularia floats in open water near the edge of the lake. At the peaty margin grow Eriophorum, Carex spp., Parnassia palustris, Juncus articulatus, Blysmus sp. and Pinguicula vulgaris. On the slightly drier margin there are Festuca sp., Nardus stricta and Agrostis sp..

The lake is surrounded by pasture with scattered large Pinus leucodermis trees estimated (from annual tree ring counts) to be about 700 years old. On more distant slopes Pinus leucodermis provides about $10 \%$ tree cover. These trees are estimated to be of two ages; 150 and 700 years old. Fagus sylvatica trees grow in small patches in gullies about $55 \mathrm{~m}$ from the lake edge with Buxus sempervirens understorey. This area is heavily grazed.

\begin{tabular}{|c|c|c|}
\hline $\begin{array}{l}\% \quad \text { Genus \& Species } \\
\text { cover }\end{array}$ & Family & Pollen type \\
\hline
\end{tabular}

\section{On lake}

FLOATING MAT

Angiospermae:

Dicotyledones:

Parnassia palustris L.

Pinguicula vulgaris L.

Utricularia sp.

Monocotyledones:

Agrostis sp.

Blysmus sp.

Carex spp. (3)

Eleocharis sp. ?multicaulis (Sm.) Desv.

Eleocharis sp.

Eriophorum spp. (2)

Festuca sp.

Juncus articulatus L.

Milium sp.

Nardus stricta L.

\section{Dryland around lake}

\section{CANOPY TREES}

Gymnospermae:

Pinus leucodermis Antoine

\section{GROUND COVER}

Angiospermae:

Dicotyledones:

Acinos sp.

Armeria maritima (Miller) Willd.
Parnassiaceae

Lentibulariaceae

Lentibulariaceae

Gramineae

Cyperaceae

Cyperaceae

Cyperaceae

Cyperaceae

Cyperaceae

Gramineae

Juncaceae

Gramineae

Gramineae
Parnassia palustris

Pinguicula

Utricularia

??Dactylis $\mathrm{t}$.

?Carex hallerana t.

Carex hallerana or C. flacca $\mathrm{t}$.

Prob. Carex flacca t.

Prob. Carex flacca t.

Carex flacca t.

Festuca t.

Juncaceae

??Dactylis $\mathrm{t}$.

Dactylis t.

Pinaceae

Pinus subgenus Diploxylon

Labiatae

Plumbaginaceae
Prob. Mentha t.

Armeria maritima t. 


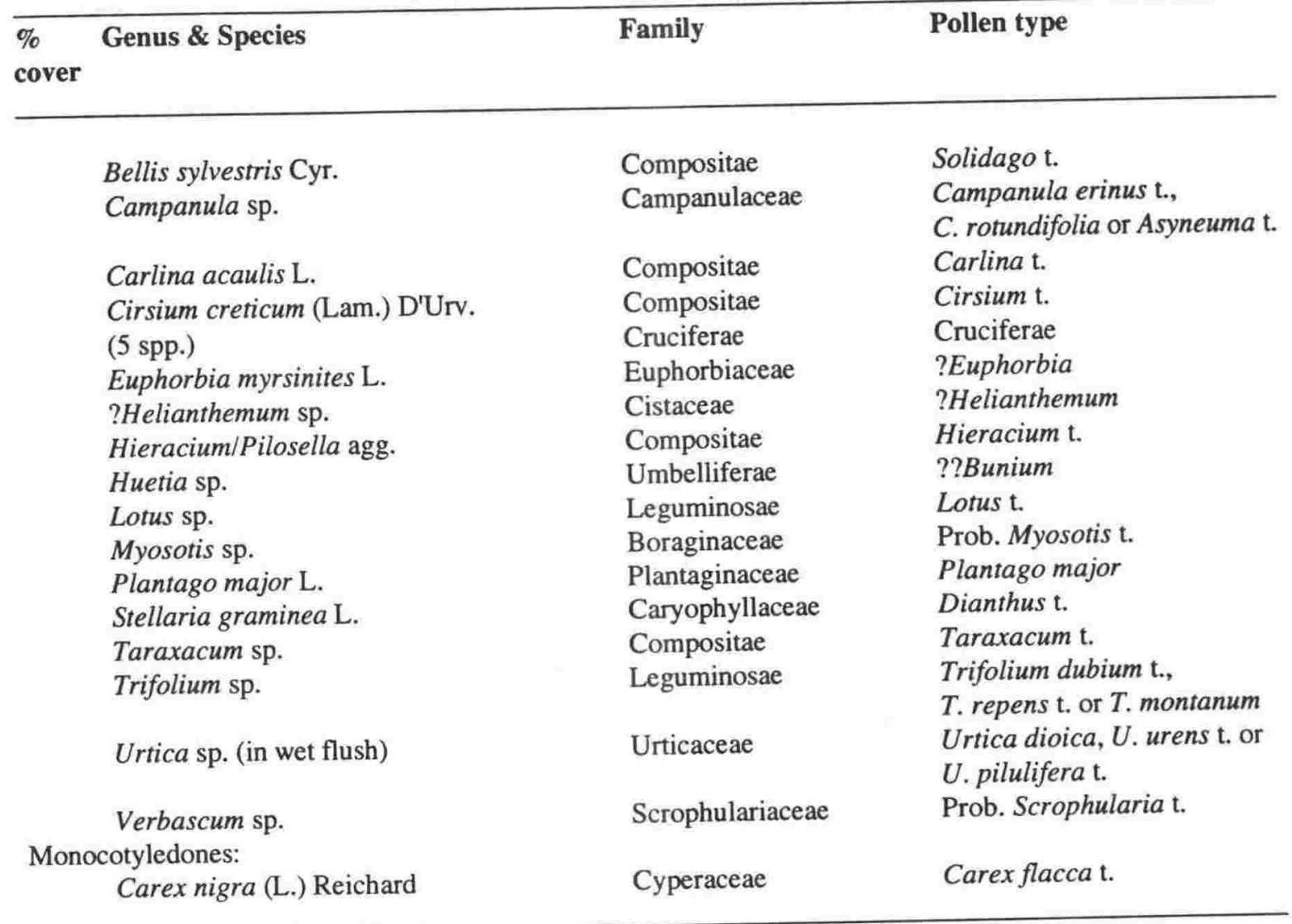




\section{Gomara fen}

Grid reference: $40^{\circ} 03^{\prime} \mathrm{N} 21^{\circ} 04^{\prime} \mathrm{E}$

Altitude: $1750 \mathrm{~m}$

Local vegetation: open rush/grass fen

Slope: small basin on gentle southerly slope

Aspect: south

Substrate: fen peat

$2 \times 2 \mathrm{~m}$ plots

The regional vegetation surrounding this site is very similar to that at Avgo.

The surface of the wetland was dry and cracked. It was covered by very sparse herbaceous plants dominated by Polygonum sp. Three $2 \times 2 \mathrm{~m}$ plots (a,b and c) within the rim of the ephemeral lake were surveyed to find out what pioneer plants grew on the lakebed during seasonal drying. These plots were selected on the basis of dryness. There appeared to be three distinct zones of increasing dryness, the outermost zone being the driest.

\begin{tabular}{llll}
\hline$\%$ & Genus \& Species & Family & Pollen type \\
cover & &
\end{tabular}

Plot a - outermost zone of dry lakebed ( $2 \times 2 \mathrm{~m}$ plot)

\section{GROUND COVER}

Angiospermae:

Dicotyledones:

$\begin{array}{llll}2 \% & \text { Convolvulus arvensis } \mathrm{L} . & \text { Convolvulaceae } & \text { Convolvulus } \\ + & \text { Euphorbia myrsinites } \mathrm{L} . & \text { Euphorbiaceae } & \text { ?Euphorbia } \\ 40 \% & \text { Polygonum } \text { sp. } & \text { Polygonaceae } & \text { Prob. Polygonum aviculare } \mathrm{t} . \\ + & \text { Trifolium repens } \mathrm{L} . & \text { Leguminosae } & \text { Trifolium repens } \mathrm{t} .\end{array}$

Plot b - intermediate zone of dry lakebed ( $2 \times 2 \mathrm{~m}$ plot)

GROUND COVER

Angiospermae:

Dicotyledones:

5\% Polygonum aviculare L. Polygonaceae Polygonum aviculare t.

Plot c - central zone of dry lakebed ( $2 \times 2 \mathrm{~m}$ plot)

GROUND COVER

Angiospermae:

Monocotyledones:

20\% Glyceria sp.

$10 \%$ Juncus sp.

$\begin{array}{ll}\text { Gramineae } & \text { Glyceria t. } \\ & \text { Juncaceae }\end{array}$

Close by: (alpine pasture below lake)

GROUND COVER

Angiospermae:

Dicotyledones:

Echinops cf. ?microcephalus

Sibth. \& Sm.

Compositae

Echinops 


\section{Beech woods}

\section{Anelia woods}

Grid reference: $39^{\circ} 57^{\prime} 30^{\prime \prime} \mathrm{N} 21^{\circ} 05^{\prime} 30^{\prime \prime} \mathrm{E}$

Altitude: $1445 \mathrm{~m}$

Local vegetation: beech wood + bracken

Canopy height: $15 \mathrm{~m}$

Slope: $12^{\circ}$

Aspect: east

Substrate: colluvium of mostly ultramafic cobbles and pebbles and some diabase, covered by a soil estimated to be about 5000-9000 years old (Savina 1990)

$30 \times 30$ m quadrat

The canopy cover is $100 \%$. This wood has been coppiced, but not for about 20 years. Pteridium aquilinum grew in a small clearing about $10 \mathrm{~m}$ to the west of the quadrat.

This quadrat is $30 \mathrm{~m}$ west of the bog (described below).

\begin{tabular}{|c|c|c|}
\hline$\% \quad$ Genus \& Species & Family & Pollen type \\
\hline
\end{tabular}

\section{CANOPY TREES}

Angiospermae:

Dicotyledones:

$100 \%$ Fagus sylvatica L.

Fagaceae

Fagus

\section{GROUND COVER}

Angiospermae:

Dicotyledones:

Fragaria sp.

Rosaceae

Fragaria viridis or $F$. vesca t.

Close by:

UNDERSTOREY TREES AND SHRUBS

Filicopsida:

Pteridium aquilinum (L.) Kuhn in Decken Hypolepidacea

Pteridium aquilinum 


\section{Anelia bog}

Grid reference: $39^{\circ} 57^{\prime} 30^{\prime \prime} \mathrm{N} 21^{\circ} 05^{\prime} 30^{\prime \prime} \mathrm{E}$

Altitude: $1440 \mathrm{~m}$

Local vegetation: sedge bog

Slope: $2-5^{\circ}$

Aspect: northeast

Substrate: sedge peat

$2 \times 2 \mathrm{~m}$ plots \& reconnaisance

Beech wood grows to the edge of the wetland. Three Pinus nigra trees (one recently dead), about 40 years old, grow on the edge of the bog. About $15 \%$ of the bog surface is open water.

Four $2 \times 2 \mathrm{~m}$ plots were quantitatively surveyed, two on the bog and two on the edge of the bog. These plots differed in wetness. The bog is carpeted with the bryophyte Acrocladium cuspidatum. The drier plot on the bog was dominated by Carex sp. The plots on the edge of the bog were drier than those on the bog, the wetter of the two was dominated by Caltha palustris while the drier one was dominated by Cirsium. A reconnaisance survey was also undertaken of the dry land between the edge of the bog and the beech wood.

\begin{tabular}{|c|c|c|}
\hline $\begin{array}{l}\% \quad \text { Genus \& Species } \\
\text { cover }\end{array}$ & Family & Pollen type \\
\hline
\end{tabular}

On bog ( $2 \times 2$ m plot)

GROUND COVER

Bryophyta:

$50 \%$ Acrocladium cuspidatum (Hedw.) Lindb. Hypnaceae

20\% Cratoneuron sp. Hypnaceae

Angiospermae:

Dicotyledones:

$+\quad$ Epilobium sp. ?hirsutum L.

$+\quad$ Hypericum sp. ?tetrapterum Fries

$+\quad$ Mentha aquatica L.

$+\quad$ Parnassia palustris L.

$3 \%$ Ranunculus repens $\mathrm{L}$.

Monocotyledones:

$+\quad$ Agrostis sp. ?canina L.

$3 \%$ Holcus lanatus L.

$+\quad$ Juncus articulatus L.

$3 \%$ Juncus inflexus L.

Close by:

CANOPY TREES

Fagus sylvatica L.

Drier area on bog ( $2 \times 2 \mathrm{~m}$ plot)

\section{GROUND COVER}

Bryophyta:

25\% Acrocladium cuspidatum (Hedw.) Lindb.

5\% Cratoneuron sp.

Angiospermae:

Dicotyledones:

$\begin{array}{ll}5 \% & \text { Alchemilla } \text { sp. } \\ + & \text { Cerastium } \text { sp. ?fontanum } \\ & \quad \text { subsp. triviale (Link) Jalas } \\ + & \text { Cirsium } \text { sp. } \\ + & \text { Epilobium ?adenocaulon Hausskn. } \\ + & \text { Euphrasia } \text { sp. }\end{array}$

Onagraceae

Guttiferae

Labiatae

Parnassiaceae

Ranunculaceae

Gramineae

Gramineae

Juncaceae

Juncaceae

Fagaceae

Fagus
Rosaceae

Caryophyllaceae

Compositae

Onagraceae

Scrophulariaceae
?Epilobium hirsutum t.

Prob. Hypericum perforatum t. Mentha t.

Parnassia palustris

Ranunculus acris gr.

??Dactylis t.

??Dactylis $\mathrm{t}$.

Juncaceae

Juncaceae

Hypnaceae

Hypnaceae

\section{Alchemilla t.}

Dianthus t.

Cirsium t.

?Epilobium hirsutum $\mathrm{t}$.

Euphrasia 


\begin{tabular}{|c|c|c|}
\hline $\begin{array}{l}\% \quad \text { Genus \& Species } \\
\text { cover }\end{array}$ & Family & Pollen type \\
\hline
\end{tabular}

\section{$+\quad$ Linum catharticum L. \\ 1\% Parnassia palustris L. \\ 2\% Plantago lanceolata $\mathrm{L}$. \\ 5\% Trifolium pratense $\mathrm{L}$. \\ $+\quad$ Viola sp.}

Monocotyledones:

60\% Carex sp.

$+\quad$ Cynosurus cristatus L.

$4 \% \quad$ Holcus lanatus L.

$1 \%$ Juncus effusus L.

$+$

Dry ground west side of bog ( $2 \times 2 \mathrm{~m}$ plot)

\section{SHRUB LAYER}

Filicopsida:

15\% Pteridium aquilinum (L.) Kuhn in Decken Hypolepidaceae

\section{GROUND COVER}

Sphenopsida:

$+\quad$ Equisetum palustre L.

Angiospermae:

Dicotyledones:

$+\quad$ Ajuga reptans $\mathrm{L}$.

50\% Cirsium sp.

$10 \%$ Mentha aquatica L.

$15 \%$ Ranunculus repens $\mathrm{L}$.

$+\quad$ Stellaria graminea L.

Monocotyledones:

$10 \%$ Brachypodium sylvaticum (Hudson) Beauv. Gramineae

$1 \%$ Carex hirta L.

$+\quad$ Holcus lanatus L.

\section{Cyperaceae \\ Gramineae \\ Labiatae \\ Compositae \\ Labiatae \\ Ranunculaceae \\ Caryophyllaceae}

Close by:

Angiospermae:

Monocotyledones:

Arum maculatum L.

Araceae

Arum

South end of bog by water inlet ( $2 \times 2 \mathrm{~m}$ plot)

\section{GROUND COVER}

Angiospermae:

Dicotyledones:

90\% Caltha palustris L.

$5 \% \quad$ Ranunculus repens $\mathrm{L}$.

$5 \%$ Veronica beccabunga $\mathrm{L}$.

Monocotyledones:

$1 \%$ Carex remota $\mathrm{L}$.

$1 \%$ Dactylorhiza fuchsii (Druce) Soo

Ranunculaceae

Ranunculaceae

Scrophulariaceae

Cyperaceae

Orchidaceae
Linum catharticum

Parnassia palustris

Plantago lanceolata t.

Trifolium repens $\mathrm{t}$.

Viola hirta t., $V$. riviniana $t$. or

$V$. arvensis t.

Carex hallerana t. or

C. flacca t.

Dactylis $\mathrm{t}$.

??Dactylis t.

Juncaceae

Orchidaceae

Pteridium aquilinum

Equisetum

Scutellaria t.

Cirsium t.

Mentha t.

Ranunculus acris gr.

Dianthus t.

Dactylis $\mathrm{t}$.

Carex flacca t.

??Dactylis $\mathrm{t}$.

Close by:

Angiospermae:

Dicotyledones:
Oxalis acetosella $\mathrm{L}$.

Oxalidaceae

Oxalis

Caltha t.

Ranunculus acris gr.

Veronica anagallis-aquatica $\mathrm{t}$.

Carex flacca $\mathrm{t}$.

Orchis t. 
Dryland on edge of beech wood around bog (reconnaisance survey)

\section{SHRUB LAYER}

Filicopsida:

Pteridium aquilinum (L.) Kuhn in Decken Hypolepidaceae

Pteridium aquilinum

\section{GROUND COVER}

Angiospermae:

Dicotyledones:

Digitalis ferruginea $\mathrm{L}$.

Geranium sp.

Helleborus sp.

Lychnis coronaria (L.) Desr. in Lam.

Salvia sp.

?Stachys sp.

Urtica sp.
Scrophulariaceae

Geraniaceae

Ranunculaceae

Caryophyllaceae

Labiatae

Labiatae

Urticaceae
?Scrophularia t.

Geranium

Prob. Helleborus

Dianthus t.

Salvia officinalis t. or

S. verticillata

Stachys arvensis, S. recta,

S. angustifolia or S. sylvatica t.

Urtica dioica, $U$. urens $\mathrm{t}$. or

$U$. pilulifera $\mathrm{t}$. 


\section{Agios Nikolaos}

Grid reference: $39^{\circ} 59^{\prime} \mathrm{N} 21^{\circ} 10^{\prime} \mathrm{E}$

Altitude: $1100 \mathrm{~m}$

Local vegetation: Pine wood + herbaceous understorey

Canopy height: $30 \mathrm{~m}$

Slope: $20^{\circ}$

Aspect: north

Substrate: rocky with skeletal stony soil on calcareous shist

$30 \times 30$ m quadrat

Open canopy. About $10 \%$ of the ground cover was rocks and $5 \%$ was covered with litter. There were abundant pine seedlings.

\begin{tabular}{|c|c|c|}
\hline $\begin{array}{l}\% \quad \text { Genus \& Species } \\
\text { cover }\end{array}$ & Family & Pollen type \\
\hline
\end{tabular}

\section{CANOPY TREE}

Gymnospermae:

$30 \% \quad$ P. nigra Arnold

Pinaceae

Pinus subgenus Diploxylon

\section{UNDERSTOREY TREES AND SHRUBS}

Filicopsida:

30\% Pteridium aquilinum (L.) Kuhn in Decken Hypolepidaceae Angiospermae:

Dicotyledones:

$+\quad$ Buxus sp.

\section{GROUND COVER}

Gymnospermae:

30\% Pinus nigra Arnold (seedlings)

Angiospermae:

Dicotyledones:

2\% Cyclamen sp.

$+\quad$ ?Daucus sp.

$+\quad$ Echinops sp.

10\% Helleborus sp.

$+\quad$ Lathyrus sp.

$+\quad c f$. Plantago

2\% Potentilla sp.

$+\quad$ Prunella sp. ?vulgaris L.

$2 \%$ Rubus caesius L.

2\% Stachys cf. officinalis (L.) Trevisan

5\% Thymus sp.

$1 \%$ Viola sp.

Monocotyledones:

$30 \%$ Festuca ovina L.
Buxaceae

Pinaceae

Primulaceae

Umbelliferae

Compositae

Ranunculaceae

Leguminosae

Plantaginaceae

Rosaceae

Labiatae

Rosaceae

Labiatae

Labiatae

Violaceae

Gramineae

Orchidaceae
Pteridium aquilinum

Prob. Buxus sempervirens

Pinus subgenus Diploxylon

Prob. Cyclamen

?Daucus

Echinops

Prob. Helleborus

Lathyrus pratensis t.,

L. sativus or Vicia cracca t.

Plantaginaceae

Prob. Potentilla

Prunellat.

Rubus t.

Prob. Stachys sylvatica t.

Prob. Mentha t.

Viola hirta t., V. riviniana t. or $V$. arvensis $\mathrm{t}$.

Festuca t.

Orchidaceae 


\section{Perivolion}

Grid reference: $39^{\circ} 59^{\prime} \mathrm{N} 21^{\circ} 09^{\prime} \mathrm{E}$

Altitude: $1100 \mathrm{~m}$

Local vegetation: Pine/oak wood + herbaceous understorey

Canopy height: $18 \mathrm{~m}$

Slope: $28^{\circ}$

Aspect: southwest

Substrate: skeletal stony soil on serpentine

$30 \times 30$ m quadrat

Total canopy cover is about $60 \%$. Ground cover is relatively dense with pine litter covering about $40 \%$ of the ground surface and rocks about $10 \%$. Grass is the dominant ground cover species.

\begin{tabular}{|c|c|c|}
\hline $\begin{array}{l}\% \quad \text { Genus \& Species } \\
\text { cover }\end{array}$ & Family & Pollen type \\
\hline
\end{tabular}

\section{CANOPY TREES}

Gymnospermae:

40\% Pinus nigra Amold

Pinaceae

Pinus subgenus Diploxylon

Angiospermae:

Dicotyledones:

20\% Quercus frainetto Ten.

Fagaceae

Quercus frainetto t.

\section{UNDERSTOREY TREES AND SHRUBS}

Filicopsida:

10\% Pteridium aquilinum (L.) Kuhn in Decken

Gymnospermae:

$2 \%$ Juniperus communis L.

Hypolepidaceae

Cupressaceae

Rosaceae

Rosaceae

Pinaceae

Compositae

Convolvulaceae

Leguminosae

Euphorbiaceae

Ranunculaceae

Fagaceae

Rosaceae

Labiatae

Scrophulariaceae

Violaceae

Gramineae

Gramineae
Pteridium aquilinum

Juniperus

Crataegus

Pyrus

Pinus subgenus Diploxylon

Cirsium t.

Cuscuta epithymum t.,

C. europaea t. or C. monogyna

Dorycnium

Prob. Euphorbia

Prob. Helleborus

Quercus

Prob. Rubus t.

Teucrium

Prob. Veronica triphyllos $\mathrm{t}$.

Viola hirta t., V. riviniana t. or $V$. arvensis $\mathrm{t}$.

Dactylis t.

?Festuca t. 


\begin{tabular}{|c|c|c|}
\hline $\begin{array}{l}\% \quad \text { Genus \& Species } \\
\text { cover }\end{array}$ & Family & Pollen type \\
\hline \multicolumn{3}{|l|}{ Close by: } \\
\hline $\begin{array}{l}\text { Angiospermae: } \\
\text { Dicotyledones: }\end{array}$ & & \\
\hline $\begin{array}{l}\text { Alyssum murale Waldst. \& Kit } \\
\text { Centaurea sp. }\end{array}$ & Cruciferae & $\begin{array}{l}\text { Hornungia t. } \\
\text { Centaurea cyanus t., }\end{array}$ \\
\hline Centaurea sp. & Compositae & $\begin{array}{l}\text { C. napulifera t., C. solstitialis t., } \\
\text { C. graeca t. or C. amplifolia }\end{array}$ \\
\hline Cichorium intybus $\mathrm{L}$. & Compositae & Cichorium t. \\
\hline Clematis sp. & Ranunculaceae & $\begin{array}{l}\text { Clematis vitalba gr. or } \\
\text { Ranunculus aquatilis gr. }\end{array}$ \\
\hline Cyclamen sp. & Primulaceae & Prob. Cyclamen \\
\hline Hedera helix $\mathrm{L}$. & Araliaceae & Hedera helix \\
\hline ?Hieracium/Pilosella agg. & Compositae & Hieracium t. \\
\hline Lotus aegaeus (Griseb.) Boiss. & Leguminosae & Lotus t. \\
\hline
\end{tabular}




\section{PINDOS FOOTHILLS}

\section{Oak wood-pasture}

\section{Polinerion}

Grid reference: $40^{\circ} 3.5^{\prime} \mathrm{N} 21^{\circ} 12^{\prime} \mathrm{E}$

Altitude: $1100 \mathrm{~m}$

Local vegetation: grassland + scattered oak \& juniper

Slope : $8-19^{\circ}$

Aspect for $100 \times 100$ m plot: southeast

Substrate: shallow stony soil on Pindos flysch

$2 \times 2 \mathrm{~m}$ plots within $100 \times 100 \mathrm{~m}$ plot

Oak wood-pasture covers about $20 \%$ of Grevena. A large quadrat of $100 \times 100 \mathrm{~m}$ was surveyed to get a general impression of the woody species and larger more common herbaceous species because woody plants are so sparse in this zone; grasses cover an estimated $90 \%$ of this quadrat. Smaller plots $(2 \times 2 \mathrm{~m})$ were surveyed within this larger one to estimate relative abundance of less obvious plants.

This area is heavily grazed and scattered Juniperus are grazed into $1 \mathrm{~m}$ diameter cone-shaped bushes. The tops of the juniper develop once the diameter of the bush reaches about $1.5 \mathrm{~m}$, when the bush is too broad for the grazing animals to reach the top. Maximum height of the junipers is $2 \mathrm{~m}$. Juniper also occurs in patches on steeper, eroded slopes with old trunks up to $10 \mathrm{~cm}$ diameter.

Three $2 \times 2 \mathrm{~m}$ plots within the larger quadrat were surveyed in different grassland types: post-cultivation (plot a); non-cultivation (plot b); and a moist non-cultivated area (plot c).

Oak wood-pasture (100 x $100 \mathrm{~m}$ quadrat)

\begin{tabular}{llll}
\hline$\%$ & Genus \& Species & Family & Pollen type \\
cover & & &
\end{tabular}

\section{TREES}

Angiospermae:

Dicotyledones:

$+\quad$ Malus sp.

5\% Quercus spp. (Q. brachyphylla,

$\begin{array}{ll}\text { Rosaceae } & \text { Malus } \\ \text { Fagaceae } & \text { Quercus }\end{array}$

$Q$. brachyphyllalfrainetto, $Q$. cerris,

$Q$. frainetto, $Q$. virgiliana)

SMALL TREES AND SHRUBS

Gymnospermae:

$3 \%$ Juniperus oxycedrus L.

Angiospermae:

Dicotyledones:

$+\quad$ Crataegus sp.

$+\quad$ Rosa sp.

Cupressaceae

Juniperus

GROUND COVER

Angiospermae:

Dicotyledones:

1\% Centaurea solstitialis $\mathrm{L}$. Compositae

Rosaceae

Crataegus

$+\quad$ Cirsium sp.

1\% Euphorbia myrsinites L.

Compositae

Euphorbiaceae

Prob. Rosa

$1 \%$ Helleborus cyclophyllus Boiss.

Ranunculaceae

$+\quad$ Lotus cf. corniculatus L.

Leguminosae

$+\quad$ Ononis spinosa L.

Leguminosae

Centaurea solstitialis t.

Cirsium t.

?Euphorbia

?Helleborus

Monocotyledones:

$90 \%$

Gramineae

Lotus t.

Ononis t.

Gramineae 
Grassland subplot a - ex-cultivation ( 2 × 2 m plot)

Aspect: southwest

Slope: $14^{\circ}$

This area was cultivated $40-50$ years ago, and has developed a suite of weeds associated with cultivation. About $2 \%$ of the ground surface was bare and about $1 \%$ of the ground was covered with rocks. The dominance of Taeniatherum caput-medusae is remarkable.

\begin{tabular}{|c|c|c|}
\hline $\begin{array}{ll}\% & \text { Genus \& Species } \\
\text { cover } & \end{array}$ & Family & Pollen type \\
\hline
\end{tabular}

\section{GROUND COVER}

\section{Angiospermae:}

Dicotyledones:

\begin{tabular}{|c|c|c|}
\hline+ & Cichorium intybus $\mathrm{L}$. & Compositae \\
\hline+ & Cirsium sp. & Compositae \\
\hline+ & Convolvulus arvensis $\mathrm{L}$. & Convolvulaceae \\
\hline+ & Dianthus sp. & Caryophyllaceae \\
\hline+ & Euphorbia myrsinites L. & Euphorbiaceae \\
\hline+ & Filago pyramidata L. & Compositae \\
\hline+ & Galium rivale (Sibth. \& Sm.) Griseb. & Rubiaceae \\
\hline $1 \%$ & Helleborus cyclophyllus Boiss. & Ranunculaceae \\
\hline $1 \%$ & Hieracium/Pilosella agg. & Compositae \\
\hline+ & Lactuca viminea (L.) J. \& C. Presl. & Compositae \\
\hline+ & Leontodon sp. or Crepis sp. & Compositae \\
\hline+ & Lotus cf. corniculatus L. & Leguminosae \\
\hline+ & Ononis spinosa $\mathrm{L}$. & Leguminosae \\
\hline+ & Plantago lanceolata $\mathrm{L}$. & Plantaginaceae \\
\hline+ & Polygonum aviculare $\mathrm{L}$. & Polygonaceae \\
\hline+ & Prunella grandiflora (L.) Scholler & Labiatae \\
\hline+ & Rumex tenuifolius (Wallr.) A. Love & Polygonaceae \\
\hline+ & Sanguisorba minor Scop. & Rosaceae \\
\hline+ & Taraxacum sp. & Compositae \\
\hline+ & Trifolium campestre Schreber & Leguminosae \\
\hline+ & Trifolium sp. & Leguminosae \\
\hline+ & Trifolium sp. & Leguminosae \\
\hline
\end{tabular}

Monocotyledones:

$\begin{array}{lll}+ & \text { Aegilops neglecta } \text { Reg. ex Bertol. } & \text { Gramineae } \\ 2 \% & \text { Agropyron sp. } & \text { Gramineae } \\ + & \text { Agrostis sp. } & \text { Gramineae } \\ 5 \% & \text { Bromus hordeaceus } & \text { Gramineae } \\ 1 \% & \text { Cynodon dactylon } \text { (L.) Pers. } & \text { Gramineae } \\ 5 \% & \text { Cynosurus elegans } \text { Desf. } & \text { Gramineae } \\ 10 \% & \text { Festuca cf. glauca Vill. } & \text { Gramineae } \\ + & \text { Lolium perenne } \text { L. } & \text { Gramineae } \\ + & \text { Piptatherum miliaceum } \text { (L.) Cosson } & \text { Gramineae } \\ 80 \% & \text { Taeniatherum caput-medusae }(\mathrm{L} .) \text { Nevski } & \text { Gramineae }\end{array}$

Cichorium t.

Cirsium t.

Convolvulus

Prob. Dianthus t.

?Euphorbia

Solidago t.

?Galium t.

?Helleborus

Hieracium t.

Lactuca t.

Cichorium t. or Taraxacum t.

Lotus t.

Ononis t.

Plantago lanceolata $\mathrm{t}$.

Polygonum aviculare $\mathrm{t}$.

Prunella t.

Rumex acetosella gr.

Sanguisorba minor

Taraxacum t.

Trifolium dubium $\mathrm{t}$.

Trifolium dubium t.,

$T$. repens t. or $T$. montanum

Trifolium dubium t.,

$T$. repens $\mathrm{t}$. or $T$. montanum $\mathrm{t}$.

Agropyron t.

Agropyron t.

??Dactylis t.

Glyceria t.

Dactylis t.

Dactylis t.

Festuca t.

Festuca t.

??Dactylis $\mathrm{t}$.

?Hordeum $\mathrm{t}$.

Close by plot a:

Angiospermae:

Dicotyledones:

Centaurea solstitialis L. 
Grassland subplot b - non-cultivated ( $2 \times 2 \mathrm{~m}$ plot)

Aspect: south east

Slope: $15^{\circ}$

About $3 \%$ of the ground surface was bare and about $1 \%$ of the ground surface was covered with rocks.

\begin{tabular}{|c|c|c|}
\hline $\begin{array}{l}\text { \% Genus \& Species } \\
\text { cover }\end{array}$ & Family & Pollen type \\
\hline
\end{tabular}

\section{GROUND COVER}

Angiospermae:

Dicotyledones:

$30 \%$ Achillea millefolium L.

$+\quad$ Acinos sp.

$+\quad$ Euphorbia myrsinites L.

$+\quad$ Helianthemum nummularium (L.) Miller

2\% Hieracium/Pilosella agg.

$+\quad$ Lotus sp.

$1 \%$ Lotus corniculatus L.

$+\quad$ Odontites verna (Bellardi) Dumort.

$+\quad$ Ononis spinosa L.

4\% Plantago lanceolata $\mathrm{L}$.

2\% Potentilla sp.

2\% Prunella sp.

$+\quad$ Sanguisorba sp.

3\% Taraxacum sp.

1\% Trifolium campestre Schreber

Monocotyledones:

$+\quad$ Anthoxanthum sp.

$+\quad$ Bromus sp.

$10 \%$ Carex sp.

2\% Cynosurus cristatus L.

$1 \%$ Cynosurus elegans Desf.

10\% Festuca sp. glauca Vill.

$+\quad$ Lolium perenne $\mathrm{L}$.

$+\quad$ Phleum sp. ?pratense L.

20\% Piptatherum miliaceum (L.) Cosson

$+\quad$ Taeniatherum caput-medusae (L.) Nevski

2\% Trisetum sp. ?flavescens (L.) Beauv.

Close by plot b:

Gymnospermae:

Juniperus sp.

Angiospermae:

Dicotyledones:

Crataegus sp.

Dianthus sp.

Helleborus sp.
Compositae

Labiatae

Euphorbiaceae

Cistaceae

Compositae

Leguminosae

Leguminosae

Scrophulariaceae

Leguminosae

Plantaginaceae

Rosaceae

Labiatae

Rosaceae

Compositae

Leguminosae

Gramineae

Gramineae

Cyperaceae

Gramineae

Gramineae

Gramineae

Gramineae

Gramineae

Gramineae

Gramineae

Gramineae

Cupressaceae

Rosaceae

Caryophyllaceae

Ranunculaceae
Achillea t.

Prob. Mentha t.

?Euphorbia

Helianthemum

Hieracium t.

Lotus t.

Lotus t.

Veronica anagallis-aquatica $\mathrm{t}$.

Ononis t.

Plantago lanceolata t.

Prob. Potentilla

Prunella t.

Sanguisorba officinalis or

$S$. minor

Taraxacum $\mathrm{t}$.

Trifolium dubium $\mathrm{t}$.

??Dactylis t.

Glyceria t.

Carex hallerana t. or

C. flacca t.

Dactylis t.

Dactylis t.

Festuca t.

Festuca t.

??Dactylis $\mathrm{t}$.

??Dactylis $\mathrm{t}$.

?Hordeum $\mathrm{t}$.

??Dactylis t.

Juniperus

Crataegus

Prob. Dianthus t.

Prob. Helleborus 
Grassland subplot c - moist ground ( $2 \times 2 \mathrm{~m}$ plot)

Aspect: east

Slope $19^{\circ}$

This type of grassland is less common in this area than those of the other two plots. Carex sp. is dominant. About $5 \%$ of the ground surface was bare.

\begin{tabular}{lll}
\hline$\%$ Genus \& Species & Family & Pollen type \\
cover & & \\
\hline
\end{tabular}

\section{GROUND COVER}

\section{Angiospermae:}

Dicotyledones:

$+\quad$ Acinos sp.

$+\quad$ Centaurium tenuiflorum

(Hoffmanns. \& Link.) Fritsch

4\% Hieracium/Pilosella agg.

$1 \% \quad$ Lotus sp.

2\% Plantago lanceolata L.

10\% Potentilla sp.

10\% Prunella sp.

$+\quad$ Taraxacum sp.

2\% Trifolium repens $\mathrm{L}$.

Monocotyledones:

$50 \%$ Carex sp.

$+\quad$ Agrostis cf. capillaris Sibth.

$+\quad$ Anthoxanthum sp.

$+\quad$ Cynodon sp.

4\% Cynosurus cristatus L.

2\% Festuca ovina $\mathrm{L}$.

$+\quad$ Lolium perenne L.

$+\quad$ Taeniatherum caput-medusae (L.) Nevski

4\% Trisetum $\mathrm{sp}$.

$15 \%$ Juncus inflexus L.
Labiatae

Gentianaceae

Compositae

Leguminosae

Plantaginaceae

Rosaceae

Labiatae

Compositae

Leguminosae

Cyperaceae

Gramineae

Gramineae

Gramineae

Gramineae

Gramineae

Gramineae

Gramineae

Gramineae

Juncaceae
Prob. Mentha t.

Centaurium

Hieracium t.

Lotus t.

Plantago lanceolata t.

Prob. Potentilla Prunella t.

Taraxacum t.

Trifolium repens $\mathrm{t}$.

Carex hallerana t. or

C. flacca t.

??Dactylis t.

??Dactylis t.

Dactylis t.

Dactylis t.

Festuca t.

Festuca t.

?Hordeum t.

??Dactylis t.

Juncaceae

Close by plot c:

Gymnospermae:

$$
\text { Juniperus oxycedrus L. }
$$

Cupressaceae

Juniperus

Angiospermae:

Dicotyledones:

Cirsium creticum (Lam.) D'Urv. Compositae

Cirsium t.

Monocotyledones:

Brachypodium pinnatum (L.) Beauv.

Gramineae

Dactylis t.

Scirpus holoschoenus $\mathrm{L}$.

Cyperaceae 


\section{Mixed woods}

Zakas

Grid reference: $40^{\circ} 02^{\prime} \mathrm{N} 21^{\circ} 16^{\prime} \mathrm{E}$

Altitude: $1160 \mathrm{~m}$

Local vegetation: mixed deciduous and coniferous wood

Canopy height: $15 \mathrm{~m}$

Slope: $30^{\circ}$

Aspect: north

Substrate: shallow stony soil on massive limestone

$30 \times 30$ m quadrat

This is one of the two areas in Grevena where mixed deciduous woods occur. Both woods grow on limestone. This type of wood differs from other woods in Grevena in the great diversity of trees that grow in it and it is also one of the few woods in Grevena dominated by Abies. Many trees were noted growing close to the quadrat that were not growing within it. The canopy cover is about $90 \%$. Ground cover is $80 \%$ litter.

\begin{tabular}{|c|c|c|}
\hline $\begin{array}{l}\% \quad \text { Genus \& Species } \\
\text { cover }\end{array}$ & Family & Pollen type \\
\hline
\end{tabular}

\section{CANOPY TREES}

Gymnospermae:

60\% Abies cephalonica Loudon

Pinaceae

Abies

Angiospermae:

Dicotyledones:

29\% Acer opalus Miller

1\% Fagus sylvatica L.

Aceraceae

Acer

$+\quad$ Quercus cf. frainetto (Mattuschka) Liebl.

Fagaceae

Fagaceae

Hypolepidaceae

Pinaceae

Rosaceae

Scrophulariaceae Euphorbiaceae

Rubiaceae Ranunculaceae Leguminosae

Rosaceae

Primulaceae

Scrophulariaceae

Violaceae
Fagus

Prob. Quercus frainetto t.

Pteridium aquilinum

Abies

Rubus t.

?Scrophularia t. Euphorbia

Prob. Galium t.

Prob. Helleborus

?Lathyrus pratensis $\mathrm{t}$.

Prob. Potentilla

Primula

Prob. Veronica triphyllos $\mathrm{t}$.

Viola hirta t., V. riviniana $\mathrm{t}$. or $V$, arvensis $\mathrm{t}$. 


\begin{tabular}{|c|c|c|c|}
\hline $\begin{array}{l}\% \\
\text { cover }\end{array}$ & Genus \& Species & Family & Pollen type \\
\hline \multicolumn{4}{|c|}{ Monocotyledones: } \\
\hline $1 \%$ & Brachypodium sylvaticum (Hudson) Beauv. & Gramineae & Dactylis $\mathrm{t}$. \\
\hline+ & Festuca sp. rubra group & Gramineae & Festuca t. \\
\hline+ & Luzula sylvatica (Hudson) Gaudin & Juncaceae & Juncaceae \\
\hline+ & & Orchidaceae & Orchidaceae \\
\hline \multicolumn{4}{|c|}{ Close by: } \\
\hline \multicolumn{4}{|c|}{ CANOPY TREES } \\
\hline \multicolumn{4}{|c|}{ Angiospermae: } \\
\hline \multicolumn{4}{|c|}{ Dicotyledones: } \\
\hline & Acer pseudoplatanus L. & Aceraceae & Acer \\
\hline & Carpinus betulus L. & Corylaceae & Carpinus t. or Ostrya t. \\
\hline & Ostrya carpinifolia Scop. & Corylaceae & Ostrya t. or Carpinus t. \\
\hline & Populus tremula L. & Salicaceae & Populus \\
\hline & Taxus baccata $\mathrm{L}$. & Taxaceae & Taxus baccata \\
\hline & Tilia rubra DC & Tiliaceae & ?Tilia platyphyllos $\mathrm{t}$. \\
\hline \multicolumn{4}{|c|}{ UNDERSTOREY TREES AND SHRUBS } \\
\hline \multicolumn{4}{|c|}{ Angiospermae: } \\
\hline Dicot & $\begin{array}{l}\text { yledones: } \\
\text { Buxus sempervirens L. }\end{array}$ & Buxaceae & Buxus sempervirens \\
\hline
\end{tabular}




\section{Aetia}

Grid reference: $40^{\circ} 4.5^{\prime} \mathrm{N} 21^{\circ} 12^{\prime} \mathrm{E}$

Altitude: $1000 \mathrm{~m}$

Local vegetation: mixed deciduous wood

Canopy height: $12 \mathrm{~m}$

Slope: $30^{\circ}$

Aspect: west

Substrate: shallow stony soil on massive limestone

Reconnaisance survey

\begin{tabular}{|c|c|c|}
\hline $\begin{array}{ll}\% & \text { Genus \& Species } \\
\text { cover } & \end{array}$ & Family & Pollen type \\
\hline
\end{tabular}

\section{CANOPY}

Angiospermae:

Dicotyledones:

Acer monspessulanum $\mathrm{L}$.

A. obtusatum Waldst. \& Kit. ex Willd

A. pseudoplatanus L.

Carpinus orientalis Miller

Cornus mas L.

Corylus colurna L.

Euonymus latifolius (L.)Miller

E. verrucosus Scop.

Fraxinus ornus $\mathrm{L}$.

Ostrya carpinifolia Scop.

Ostrya sp.

Prunus cerasifera Ehrh.

$P$. mahaleb L.

Sorbus graeca (Spach) Kotschy

S. torminalis (L.) Crantz

Taxus baccata $\mathrm{L}$.

Tilia rubra DC

T. tomentosa Moench

Ulmus glabra Hudson
Aceraceae

Aceraceae

Aceraceae

Corylaceae

Cornaceae

Corylaceae

Celastraceae

Celastraceae

Oleaceae

Corylaceae

Corylaceae

Rosaceae

Rosaceae

Rosaceae

Rosaceae

Taxaceae

Tiliaceae

Tiliaceae

Ulmaceae
Acer

Prob. Acer

Acer

Ostrya t. or Carpinus t.

Cornus mas

Corylus

?Euonymus europaeus $\mathrm{t}$.

?Euonymus europaeus t.

Fraxinus ornus

Ostrya t. or Carpinus t.

Ostrya t. or Carpinus t.

?Prunus spinosa t.

Prunus avium $\mathrm{t}$.

Sorbus t.

Sorbus t.

Taxus baccata

?Tilia platyphyllos $\mathrm{t}$.

Tilia cordata t.

Ulmus glabra t.

\section{UNDERSTOREY TREES AND SHRUBS}

Gymnospermae:

Juniperus excelsa Bieb.

Cupressaceae

Juniperus

$J$. foetidissima Willd.

Cupressaceae

Juniperus

Angiospermae:

Dicotyledones:

Buxus sempervirens L.

Buxus sempervirens 


\section{LOWLAND PLAINS}

\section{Cultivated fields}

\section{Kellia fen}

Grid reference: $40^{\circ} 06^{\prime} \mathrm{N} 21^{\circ} 25^{\prime} \mathrm{E}$

Altitude: $580 \mathrm{~m}$

Local vegetation: mixed fen

Slope: flat

Aspect: open

Substrate: silty peat

General survey of fen, 2 × 2 m plots \& reconnaisance survey

Most of the gently rolling hills surrounding this fen are cultivated with cereal crops. A survey of the fen as a whole was undertaken and relative frequencies noted. Common species on the fen were Cyperus longus, Typha, Juncus inflexus, Scirpus, Equisetum telmateia, Sparganium erectum, Rumex conglomeratus, Mentha aquatica, Potentilla and Pulicaria.

To get an idea of the variation of vegetation on the fen, three $2 \times 2 \mathrm{~m}$ subplots were quantitatively surveyed and percent cover estimated. Plot a was located $3 \mathrm{~m}$ west of the core site for the fossil pollen sequence on a drier part of the fen where Cirsium, Cyperus and Equisetum were almost equally abundant. Plot b was located about $20 \mathrm{~m}$ southeast of the core site in a wetter area where Juncus inflexus and Eurhynchium praelongum were equally abundant and Typha was small and poorly developed. Plot $\mathrm{c}$ was located near a spring on the very wet eastern side of the fen. It was the wettest area surveyed and Typha was dominant with Sparganium very abundant.

To the south of the fen on a north-facing slope that had not been used for cultivation recently a $2 \times 2 \mathrm{~m}$ plot was surveyed and percentage cover estimated. Many steppe species grow here; Centaurea and Camptothecium lutescens were almost equally abundant. About $1 \%$ of the ground surface was bare.

Reconnaisance surveys were undertaken to augment the quantitative surveys. Three areas were surveyed in this manner; a disturbed area on the fen, a disturbed area near the fen and the steppe to the south of the fen. Two species were noted as indicative of disturbance on the fen, Pulicaria dysenterica and Lythrum salicaria. In a disturbed area near the fen, a mixture of those species growing on the fen and ruderals were noted. In a dry area to the south of the fen a variety of steppe species were noted.

\begin{tabular}{llll}
\hline$\%$ & Genus \& Species & Family & Pollen type \\
cover & &
\end{tabular}

\section{On fen}

( $a=$ abundant; la=locally abundant; $f=$ frequent; $\mathrm{o}=$ occasional; $+=$ present)

Sphenopsida:

a Equisetum
Angiospermae:
Dicotyledones:

$+\quad$ Ajuga reptans $\mathrm{L}$.

f Berula erecta (Hudson) Coville

f Calystegia sp. ?sepium (L.) R. Br.

f Cirsium creticum (Lam.) D'Urv.

a Cuscuta sp.

$+\quad$ Epilobium hirsutum L.

$+\quad$ Epilobium cf. adenocaulon Hausskn.

o Epilobium cf. parviflorum Schreber

f Galium rivale (Sibth. \& Sm.) Griseb.

- Galium spurium L.

$+\quad$ Hypericum cf. tetrapterum Fries
Equisetaceae

Equisetum

Labiatae

Umbelliferae

Convolvulaceae

Compositae

Convolvulaceae

Onagraceae

Onagraceae

Onagraceae

Rubiaceae

Rubiaceae

Guttiferae
Scutellaria t.

Berula erecta

Calystegia

Cirsium t.

Cuscuta epithymum t.,

C. europaea t. or C. monogyna

Epilobium hirsutum t.

?Epilobium hirsutum t.

Prob. Epilobium hirsutum t.

?Galium t.

Galium t.

Prob. Hypericum perforatum $\mathrm{t}$. 


\begin{tabular}{|c|c|c|}
\hline $\begin{array}{l}\% \quad \text { Genus \& Species } \\
\text { cover }\end{array}$ & Family & Pollen type \\
\hline
\end{tabular}

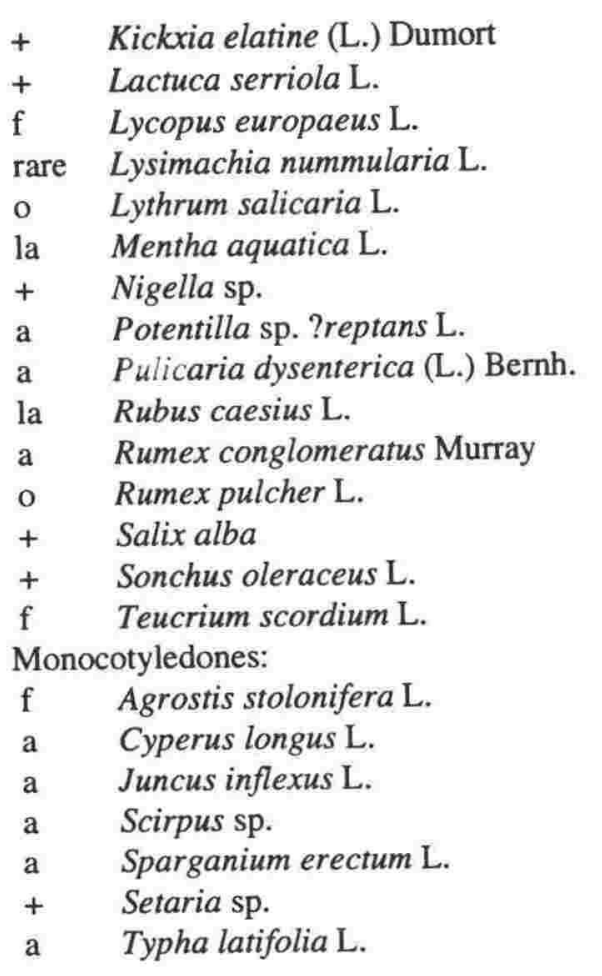

Scrophulariaceae
Compositae
Labiatae
Primulaceae
Lythraceae
Labiatae
Ranunculaceae
Rosaceae
Compositae
Rosaceae
Polygonaceae
Polygonaceae
Salicaceae
Compositae
Labiatae

Gramineae
Cyperaceae
Juncaceae
Cyperaceae
Sparganiaceae
Gramineae
Typhaceae

Close by:

Angiospermae:

Dicotyledones:

$$
\text { Prunus spinosa L. }
$$

Rosaceae

Equisetaceae

Compositae

Onagraceae

Rubiaceae

Labiatae

Lythraceae

Labiatae

Polygonaceae

Labiatae

Cyperaceae

Juncaceae
Kickxia

Lactuca t.

Mentha t.

Lysimachia

Lythrum salicaria t.

Mentha t.

Nigella

Prob. Potentilla

Solidago t.

Rubus t.

Rumex conglomeratus gr.

Rumex conglomeratus gr.

Salix fragilis t.

Sonchus t.

Teucrium

??Dactylis t.

Cyperus longus $\mathrm{t}$.

Juncaceae

Carex hallerana t. or Scirpus

Sparganium erectum

??Dactylis t.

Typha angustifolia $\mathrm{t}$.

Prunus spinosa t.

\section{Equisetum}

Cirsium t.

Prob. Epilobium hirsutum t. Galium t.

Prob. Mentha t.

Lythrum salicaria t. or

L. portula

Mentha t.

Rumex conglomeratus gr.

Teucrium

Cyperus michelianus or

C. longus t.

Juncaceae 


\begin{tabular}{|c|c|c|}
\hline $\begin{array}{l}\% \quad \text { Genus \& Species } \\
\text { cover }\end{array}$ & Family & Pollen type \\
\hline
\end{tabular}

\section{Subplot b - on fen ( $2 \times 2 \mathrm{~m}$ plot)}

Bryophyta:

80\% Eurhynchium praelongum (Hedw.) Hobk. Hypnaceae

Sphenopsida:

3\% Equisetum sp.

Equisetaceae

Equisetum

Angiospermae:

Dicotyledones:

$\begin{array}{ll}+ & \text { Berula erecta (Hudson) Coville } \\ 1 \% & \text { Cirsium } \text { sp. } \\ 8 \% & \text { Hypericum } \text { cf. tetrapterum Fries } \\ + & \text { Lycopus } \text { sp. } \\ + & \text { Lysimachia nummularia } \text { L. } \\ 5 \% & \text { Lythrum salicaria } \text { L. } \\ 1 \% & \text { Potentilla } \text { sp. }\end{array}$

Umbelliferae

Compositae

Guttiferae

Labiatae

Primulaceae

Lythraceae

Rosaceae

Monocotyledones:

$+\quad$ ?Agrostis sp.

$80 \%$ Juncus inflexus L.

$+\quad$ Typha sp.

\section{Subplot c - on fen ( $2 \times 2$ m plot)}

Sphenopsida:

2\% Equisetum telmateia Ehrh.

Angiospermae:

Dicotyledones:

$$
\begin{array}{ll}
1 \% & \text { Cirsium } \text { sp. } \\
+ & \text { Galium palustre } \mathrm{L} . \\
+ & \text { Galium spurium } \mathrm{L} . \\
2 \% & \text { Lycopus } \mathrm{sp} . \\
3 \% & \text { Lythrum } \mathrm{sp} .
\end{array}
$$

Monocotyledones:

$\begin{array}{lll}+ & \text { Juncus inflexus } \mathrm{L} . & \text { Juncaceae } \\ 30 \% & \text { Sparganium } \mathrm{sp} . & \text { Sparganiaceae } \\ & & \\ 60 \% & \text { Typha } \text { sp. } & \text { Typhaceae }\end{array}$

Disturbed area on fen (reconnaisance survey)

Angiospermae:

Dicotyledones:

Lythrum salicaria $\mathrm{L}$.

Pulicaria dysenterica (L.) Bernh.
Gramineae

Juncaceae

Typhaceae

Equisetaceae

Compositae

Rubiaceae

Rubiaceae

Labiatae

Lythraceae

Juncaceae

Typhaceae

Lythraceae

Compositae

\section{Berula erecta}

Cirsium t.

Prob. Hypericum perforatum $\mathrm{t}$.

Prob. Mentha t.

Lysimachia

Lythrum salicaria t.

Prob. Potentilla

??Dactylis t.

Juncaceae

Typha angustifolia t. or

$T$. domingensis $\mathrm{t}$.

\section{Equisetum}

Cirsium t.

Galium t.

Galium t.

Prob. Mentha t.

Lythrum salicaria t. or

L. portula

Juncaceae

Sparganium erectum or

S. angustifolium

Typha angustifolia $\mathrm{t}$. or

$T$. domingensis $\mathrm{t}$.

Lythrum salicaria t.

Solidago t. 


\begin{tabular}{|c|c|c|}
\hline $\begin{array}{l}\% \quad \text { Genus \& Species } \\
\text { cover }\end{array}$ & Family & Pollen type \\
\hline
\end{tabular}

Disturbed area near fen (reconnaisance survey)

Angiospermae:

Dicotyledones:

Centaurea sp. ?napulifera Rochel

Cichorium intybus L.

Consolida sp.

Daucus carota L.

Mentha aquatica $\mathrm{L}$.

Ononis spinosa L.

Potentilla reptans $\mathrm{L}$.

Torilis japonica (Houtt.) DC

Verbena officinalis L.

Monocotyledones:

Colchicum sp. ?autumnale L.

\section{Steppe south of Kellia fen ( 2 × 2 m plot)}

Bryophyta:

$60 \%$ Camptothecium lutescens (Hedw.) B.

Angiospermae:

Dicotyledones:

$40 \%$ Centaurea sp.

$+\quad$ Cuscuta sp.

$1 \%$ Daucus carota L.

$2 \%$ Dorycnium sp.

$+\quad$ Eryngium cf. campestre L.

$2 \% \quad$ Galium verum $\mathrm{L}$.

15\% Prunus spinosa $\mathrm{L}$.

$+\quad$ Sanguisorba minor Scop.

$+\quad$ Thesium sp.

Monocotyledones:

2\% Carex sp.

$+\quad$ Colchicum autumnale L.

$+\quad$ Cynosurus cristatus L.

5\% Festuca ovina L.
Compositae

Compositae

Ranunculaceae

Umbelliferae

Labiatae

Leguminosae

Rosaceae

Umbelliferae

Verbenaceae

Liliaceae

Hypnaceae

Compositae

Convolvulaceae

Umbelliferae

Leguminosae

Umbelliferae

Rubiaceae

Rosaceae

Rosaceae

Santalaceae

Cyperaceae

Liliaceae

Gramineae

Gramineae
?Centaurea napulifera $\mathrm{t}$.

Cichorium t.

Prob. Consolida t.

Daucus

Mentha t.

Ononis t.

Potentilla

Torilis japonica t.

Verbena

Colchicum

Steppe south of Kellia fen (reconnaisance survey)

Angiospermae:

Dicotyledones:

Cirsium eriophorum (L.) Scop.

Compositae

C. mairei Halacsy

Compositae

Cirsium sp. ?vulgare

Compositae

Cirsium cf. vulgare (Savi) Ten.

Clinopodium vulgare $\mathrm{L}$.

Compositae

Labiatae

Dorycnium graecum (L.) Ser.

Dorycnium sp.

Leguminosae

Leguminosae

Galium verum $\mathrm{L}$.

Rubiaceae

Cirsium t.

Cirsium t.

Cirsium t.

Cirsium t.

Mentha t.

Dorycnium

Dorycnium

Galium t.

Rubiaceae

Galium t. 


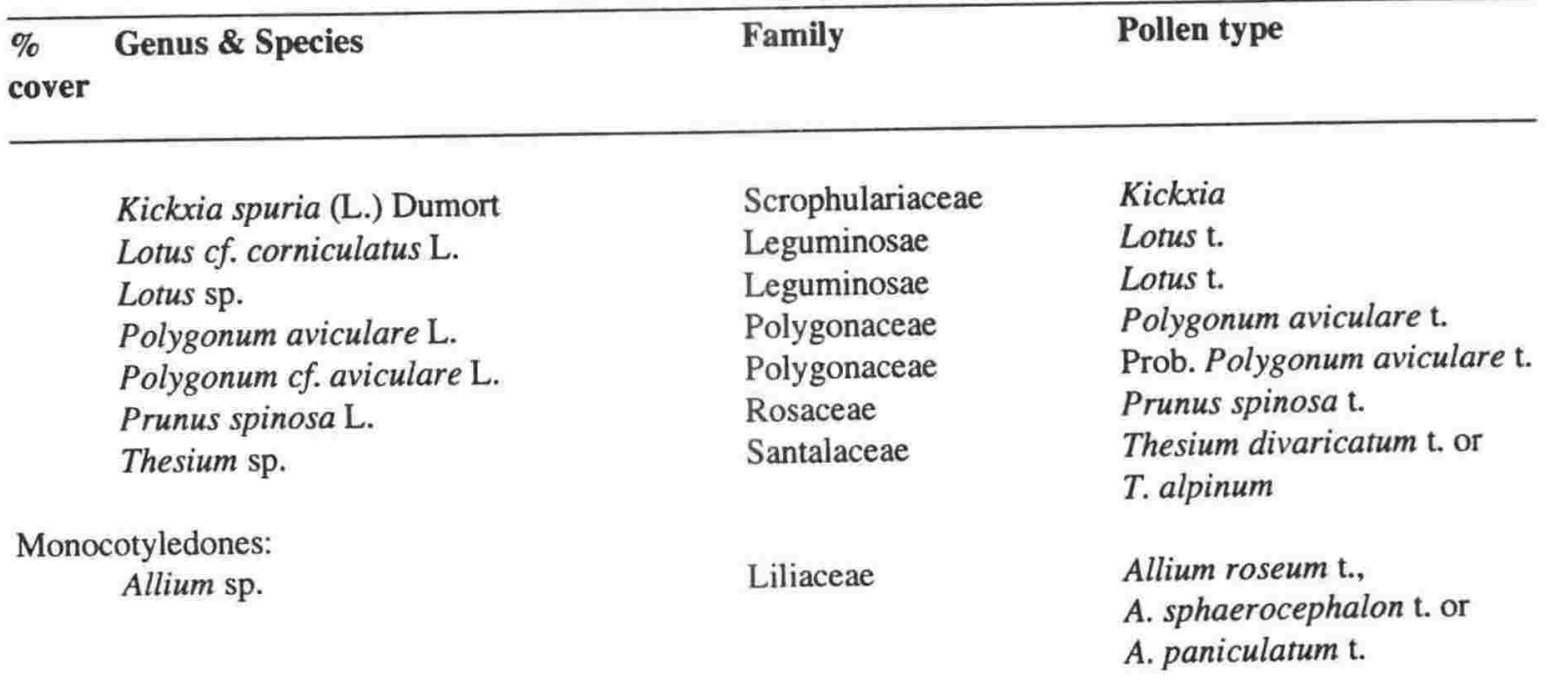




\section{Asprokampos field}

Grid reference: $40^{\circ} 08^{\prime} 30^{\prime \prime} \mathrm{N} 21^{\circ} 29^{\prime} 30^{\prime \prime} \mathrm{E}$

Altitude: $560 \mathrm{~m}$

Local vegetation: cereal agricultural field

Slope: flat

Aspect: open

Substrate: brown agricultural soil

Reconnaisance survey

This cereal field is surrounded by other cultivated fields. A reconnaisance survey was undertaken when weeds had begun to grow a few weeks after harvest. The dominant weed was Polygonum aviculare. Other abundant weeds were Consolida sp. and grasses.

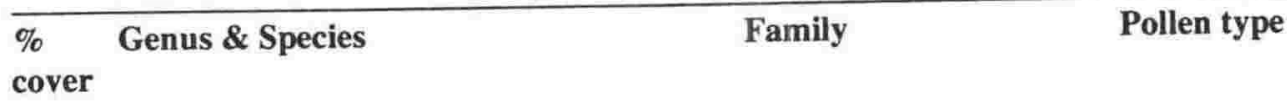

\section{TREE}

Angiospermae:

Dicotyledones:

Salix sp.

Salicaceae

Salix triandra or S. fragilis t.

\section{GROUND COVER}

Angiospermae:

Dicotyledones:

Anagallis sp.

?Atriplex sp.

Chenopodium cf. album $\mathrm{L}$.

Cirsium arvense (L.) Scop.

Consolida sp.

Convolvulus arvensis L.

Cuscuta sp.

Eryngium sp.

Geranium sp. ?molle L.

Heliotropium hirsutissimum Grauer

Hibiscus trionum L.

Kickxia sp.

Lactuca viminea (L.) J. \& C. Presl.

Lactuca virosa $\mathrm{L}$.

Plantago lanceolata L.

Polygonum aviculare $\mathrm{L}$.

Sinapis alba $\mathrm{L}$.

Solanum nigrum $\mathrm{L}$.

Monocotyledones:

Avena cf. sterilis L.

Setaria sp.

Triticum aestivum $\mathrm{L}$.
Primulaceae

Chenopodiaceae

Chenopodiaceae

Compositae

Ranunculaceae

Convolvulaceae

Convolvulaceae

Umbelliferae

Geraniaceae

Boraginaceae

Malvaceae

Scrophulariaceae

Compositae

Compositae

Plantaginaceae

Polygonaceae

Cruciferae

Solanaceae

Gramineae

Gramineae

Gramineae

Gramineae
Anagallis arvensis t. or

A. tenella t.

Chenopodiaceae t.

Chenopodiaceae $t$.

Cirsium t.

Prob. Consolida t.

Convolvulus

Cuscuta epithymum t.,

C. europaea t. or C. monogyna

Prob. Eryngium

Prob. Geranium molle gr.

?Heliotropium

Hibiscus

Kickxia

Lactuca t.

Lactuca t.

Plantago lanceolata t.

Polygonum aviculare t.

Sinapis t.

Solanum nigrum $\mathrm{t}$.

Avena t.

Gramineae

??Dactylis $\mathrm{t}$.

Triticum $\mathrm{t}$. 


\section{Oak woods}

\section{Asprokampos woods}

Grid reference: $40^{\circ} 08^{\prime} 30^{\prime \prime} \mathrm{N} 21^{\circ} 30^{\prime} \mathrm{E}$

Altitude: $550 \mathrm{~m}$

Local vegetation: oak wood

Canopy height: $8 \mathrm{~m}$

Slope: $20^{\circ}$

Aspect: south

Substrate: skeletal brown soil

$30 \times 30$ m quadrat

This oak wood is heavily grazed. The oaks have been coppiced (although not in the last few years) and some have several stems. Canopy cover is about $50 \%$. Oaks are much hybridized. Scattered Juniperus and Buxus form an understorey shrubbery. Grasses cover $80 \%$ of the ground and about $15 \%$ of the ground is bare.

\begin{tabular}{|c|c|c|}
\hline $\begin{array}{l}\% \text { Genus \& Species } \\
\text { cover }\end{array}$ & Family & Pollen type \\
\hline
\end{tabular}

\section{CANOPY TREES}

Angiospermae:

Dicotyledones:

50\% Quercus spp. (Q. brachyphylla,

Fagaceae

Quercus frainetto \& $Q$. trojana

Q. brachyphylla $\times$ ?cerris,

$Q$. cerris, $Q$. cerris hybrid,

$Q$. cerris x ?virgiliana, $Q$. cf frainetto,

$Q$. pubescens hybrid, $Q$. trojana

\section{UNDERSTOREY TREES AND SHRUBS}

Gymnospermae:

$1 \%$ Juniperus sp.

Cupressaceae

Juniperus

Angiospermae:

Dicotyledones:

$+\quad$ Buxus sempervirens L.

$+\quad$ Crataegus monogyna Jacq.

Buxaceae

Rosaceae

Buxus sempervirens

Crataegus

\section{GROUND COVER}

Angiospermae:

Dicotyledones:

\begin{tabular}{|c|c|}
\hline+ & Agrimonia sp. \\
\hline+ & Alyssum murale Waldst. \& Kit. \\
\hline+ & Anthemis sp. or Leucanthemum sp. \\
\hline+ & Arabis sp. \\
\hline+ & $\begin{array}{c}\text { Buglossoides purpurocaerulea } \\
\text { (L.) I.M. Johnston }\end{array}$ \\
\hline+ & Carlina corymbosa $\mathrm{L}$. \\
\hline+ & Clinopodium vulgare $\mathrm{L}$. \\
\hline+ & Fragaria vesca $\mathrm{L}$. \\
\hline+ & Galium rivale (Sibth. \& Sm.) Griseb. \\
\hline+ & Galium verum $\mathrm{L}$. \\
\hline+ & Hieracium/Pilosella agg. \\
\hline+ & Lotus or Dorycnium sp. \\
\hline+ & Potentilla sp. \\
\hline & Potentilla sterilis (L.) Garcke \\
\hline & Prunella sp. \\
\hline
\end{tabular}

Rosaceae
Cruciferae
Compositae
Cruciferae
Boraginaceae
Compositae
Labiatae
Rosaceae
Rubiaceae
Rubiaceae
Compositae
Leguminosae
Leguminosae
Rosaceae
Rosaceae
Labiatae

Rosaceae

Compositae

Cruciferae

Boraginaceae

Compositae

Labiatae

Rubiaceae

Rubiaceae

Compositae

Leguminosae

Rosaceae

Labiatae
Agrimonia t.

Hornungia t.

Prob. Achillea t.

Arabis or Sinapis t.

Buglossoides

purpurocaerulea

Carlina t.

Mentha t.

Fragaria vesca t.

?Galium t.

Galium t.

Hieracium $\mathrm{t}$.

Leguminosae

Lotus t. or Dorycnium

Prob. Potentilla

Potentilla

Prunella t. 


\begin{tabular}{|c|c|c|c|}
\hline $\begin{array}{l}\% \\
\text { cover }\end{array}$ & Genus \& Species & Family & Pollen type \\
\hline+ & Rosa sp. & Rosaceae & Prob. Rosa \\
\hline+ & Sanguisorba minor Scop. & Rosaceae & Sanguisorba minor \\
\hline+ & Stachys alopecuros (L.) Bentham & Labiatae & Stachys sylvatica t. \\
\hline+ & Teucrium chamaedrys $\mathrm{L}$. & Labiatae & Teucrium \\
\hline+ & Teucrium polium $\mathrm{L}$. & Labiatae & Teucrium \\
\hline+ & Thymus sp. & Labiatae & Prob. Mentha t. \\
\hline+ & Veronica chamaedrys $\mathrm{L}$. & Scrophulariaceae & Veronica triphyllos $\mathrm{t}$. \\
\hline+ & Veronica cf. chamaedrys L. & Scrophulariaceae & Prob. Veronica triphyllos t. \\
\hline+ & Veronica sp. & Scrophulariaceae & $\begin{array}{l}\text { Veronica anagallis-aquatica } \mathrm{t} \text {. } \\
\text { or Veronica triphyllos } \mathrm{t} \text {. }\end{array}$ \\
\hline+ & Viola sp. & Violaceae & $\begin{array}{l}\text { Viola hirta t., V. riviniana t. or } \\
\text { V. arvensis t. }\end{array}$ \\
\hline \multicolumn{4}{|c|}{ Monocotyledones: } \\
\hline+ & Carex sp. & Cyperaceae & $\begin{array}{l}\text { Carex hallerana t. or } \\
\text { C. flacca t. }\end{array}$ \\
\hline \multirow[t]{7}{*}{$80 \%$} & (total of taxa below) & Gramineae & \\
\hline & Dactylis sp. & Gramineae & Dactylis t. \\
\hline & Chrysopogon gryllus (L.) Trin. & Gramineae & Dactylis $\mathrm{t}$. \\
\hline & Festuca sp. & Gramineae & Festuca t. \\
\hline & Festuca ovina $\mathrm{L}$. & Gramineae & Festuca t. \\
\hline & Elymus sp. & Gramineae & Hordeum $\mathrm{t}$. \\
\hline & Koeleria sp. & Gramineae & ??Dactylis t. \\
\hline+ & Muscari sp. & Liliaceae & $\begin{array}{l}\text { Muscari comosum or } \\
\text { Muscari neglectum t. }\end{array}$ \\
\hline+ & & Orchidaceae & $\begin{array}{l}\text { Muscarl neglectum } \mathrm{t} \\
\text { Orchidaceae }\end{array}$ \\
\hline+ & Scilla autumnalis L. & Liliaceae & Scilla t. \\
\hline
\end{tabular}




\section{Mirsina woods}

Grid reference: $40^{\circ} 08^{\prime} \mathrm{N} 21^{\circ} 29^{\prime} 30^{\prime \prime} \mathrm{E}$

Altitude: $500 \mathrm{~m}$

Local vegetation: oak wood

Canopy height: about $7.5 \mathrm{~m}$

Slope: $12^{\circ}$

Aspect: south

Substrate: skeletal soil on Pleistocene gravels

$10 \times 20 \mathrm{~m}$ plot

This plot is irregular because it runs along the tread of a terrace about $40 \mathrm{~m}$ above the fen given the same name. The oak wood is of even age, about 30 years. The wood had been coppiced and the area is browsed. As a result of this several woody species are small shrub size. Canopy cover is $75 \%$. Oak seedlings and low coppice shoots covered about $15 \%$ of the ground surface and slightly more than $10 \%$ was bare.

\begin{tabular}{|c|c|c|}
\hline $\begin{array}{l}\% \quad \text { Genus \& Species } \\
\text { cover }\end{array}$ & Family & Pollen type \\
\hline
\end{tabular}

\section{CANOPY TREES}

Angiospermae:

Dicotyledones:

$+\quad$ Pyrus sp.

75\% Quercus spp. as below:

Quercus brachyphylla Kotschy

Rosaceae

Prob. Pyrus

Q. brachyphylla Kotschy x frainetto Ten. Fagaceae

Q. brachyphylla Kotschy x virgilliana (Ten.) Ten.

Fagaceae

Q. cerris $\mathrm{L}$.

Fagaceae

$Q$. frainetto Ten.

Fagaceae

Q. trojana Webb

Fagaceae

Q. virgiliana (Ten.) Ten

Fagaceae

$Q$. virgiliana (Ten.) Ten. x frainetto Ten.

Fagaceae

Quercus trojana t. or

$Q$. frainetto t.

Quercus trojana t. or

$Q$. frainetto $\mathrm{t}$.

Quercus trojana t. or $Q$. frainetto t.

Quercus trojana t.

Quercus frainetto $\mathrm{t}$.

Quercus trojana t. or

Q. frainetto $\mathrm{t}$.

Quercus frainetto t.

Quercus frainetto $\mathrm{t}$.

\section{SMALL SHRUBS AND GROUND COVER}

Angiospermae:

Dicotyledones:

$+\quad$ Agrimonia eupatoria $\mathrm{L}$.
$+\quad$ Buglossoides purpurocaerulea

$15 \%$ Carpinus sp.

(L.) I.M. Johnston

$+\quad$ Clinopodium sp.

$+\quad$ Cornus sanguinea L.

2\% Crataegus sp.

$+\quad$ Cyclamen sp.

3\% Dorycnium sp.

2\% Dorycnium sp.

$10 \%$ Fragaria vesca $\mathrm{L}$.

$2 \%$ Fraxinus ornus $\mathrm{L}$.

$1 \%$ Galium verum $\mathrm{L}$.

$+\quad$ Lathyrus sp.

$+\quad$ Prunus spinosa L.
Rosaceae

Boraginaceae

Corylaceae

Labiatae

Cornaceae

Rosaceae

Primulaceae

Leguminosae

Leguminosae

Rosaceae

Oleaceae

Rubiaceae

Leguminosae

Rosaceae
Agrimonia t.

Buglossoides

purpurocaerulea

Carpinus t. or Ostrya t.

Mentha t.

Cornus sanguinea

Prob. Crataegus

Prob. Cyclamen

Dorycnium

Dorycnium

Fragaria vesca t.

Fraxinus ornus

Galium t.

Lathyrus pratensis t.,

L. sativus or Vicia cracca t.

Prunus spinosa t. 


\begin{tabular}{|c|c|c|c|}
\hline $\begin{array}{l}\% \\
\text { cover }\end{array}$ & Genus \& Species & Family & Pollen type \\
\hline $5 \%$ & Rosa arvensis Hudson & Rosaceae & Rosa \\
\hline $3 \%$ & Stachys alopecuros (L.) Bentham & Labiatae & Stachys sylvatica t. \\
\hline $5 \%$ & ?Thalictrum sp. & Ranunculaceae & $\begin{array}{l}\text { Thalictrum aquilegifolium, } \\
T . \text { minus or T. lucidum }\end{array}$ \\
\hline+ & Thymus sp. & Labiatae & Prob. Mentha t. \\
\hline+ & Veronica chamaedrys $\mathrm{L}$. & Scrophulariaceae & Veronica triphyllos $\mathrm{t}$. \\
\hline \multicolumn{4}{|c|}{ Monocotyledones: } \\
\hline $5 \%$ & Carex sp. & Cyperaceae & $\begin{array}{l}\text { Carex hallerana t. or } \\
\text { C. flacca t. }\end{array}$ \\
\hline $15 \%$ & Chrysopogon gryllus (L.) Trin. & Gramineae & ??Dactylis t. \\
\hline $5 \%$ & Festuca glauca Vill. & Gramineae & Festuca t. \\
\hline+ & Fritillaria sp. & Liliaceae & Fritillaria \\
\hline+ & Scilla autumnalis $\mathrm{L}$. & Liliaceae & Scilla t. \\
\hline \multicolumn{4}{|c|}{ Close by: } \\
\hline \multicolumn{4}{|c|}{ Angiospermae: } \\
\hline \multicolumn{4}{|c|}{ Dicotyledones: } \\
\hline & $\begin{array}{l}\text { Bupleurum asperuloides Heldr. } \\
\text { ex Boiss }\end{array}$ & Umbelliferae & Bupleurum \\
\hline & Tussilago farfara $\mathrm{L}$. & Compositae & Solidago t. \\
\hline Monc & $\begin{array}{l}\text { cotyledones: } \\
\text { Elymus ?caninus (L.) L. }\end{array}$ & Gramineae & Hordeum t. \\
\hline
\end{tabular}




\section{Mirsina fen}

Grid reference: $40^{\circ} 08^{\prime} \mathrm{N} 21^{\circ} 30^{\prime} \mathrm{E}$

Altitude: $460 \mathrm{~m}$

Local vegetation: depends on plot

Slope: flat

Aspect: open

Substrate: silty peat

$2 \times 2 \mathrm{~m}$ plots \& reconnaisance

This is a small fen on a terrace about $20 \mathrm{~m}$ above and $40 \mathrm{~m}$ west of the Aliakmon River. It is covered with $4 \mathrm{~m}$ high Phragmites australis. The area was burnt recently (about 15 years before the survey was done). Current browsing is minimal, but there is evidence for heavier browsing in the past (Rackham pers. comm. 1989).

Four $2 \times 2$ m plots were quantitatively surveyed on and in the vicinity of the fen. The plots are located in areas of increasing dryness: plot a on the fen, plot b on the margin of the fen, plot $c$ on dryland near the fen and plot $d$ on dry ground (steppe) more distant from the fen. In plot b Phragmites is replaced mostly by Scirpus holoschoenus which covers $50 \%$ of the ground. Other prominent species are Cirsium creticum, Juncus inflexus and Pulicaria dysenterica. Plot $\mathrm{c}$ is influenced by the fen and damper than ground further from the fen. Equisetum telmateia is dominant in this plot. Carex is also abundant along with Crepis, Dorycnium and Ononis spinosa. The tallest plants here were $60 \mathrm{~cm}$ and the average height of the vegetation was $15 \mathrm{~cm}$.

Plot d is located in open dry grassland (steppe) further from the fen. This dryness is typical of the general area. The plot has a northerly aspect with a $10-15^{\circ}$ slope. The dominant species in this plot was Thymus. Other abundant species were Carex, Festuca, Teucrium chamaedrys and Teucrium polium. Equisetum palustre was suckering into this area. About $5 \%$ of the ground was bare in this plot.

A reconnaisance survey was undertaken of the species growing between plots $\mathrm{c}$ and $\mathrm{d}$. Several shrubby species, for example, Paliurus spina-christi ( $2.5 \mathrm{~m}$ tall), Crataegus sp. (up to $4 \mathrm{~m}$ tall), Fraxinus ornus $(0.5 \mathrm{~m}$ tall), Euonymus europaeus $(0.20 \mathrm{~m}$ tall) and a tall grass (Calamagrostis arundinaceae) grew in this area. None of these, except Crataegus, grew in the plots quantitatively surveyed.

\begin{tabular}{|c|c|c|}
\hline $\begin{array}{l}\% \quad \text { Genus \& Species } \\
\text { cover }\end{array}$ & Family & Pollen type \\
\hline
\end{tabular}

\section{On fen - plot a $(2 \times 2 \mathrm{~m}$ plot $)$ \\ CANOPY \\ Monocotyledones: \\ 100\% Phragmites australis (Cav.) Trin. ex Steudel}

\section{UNDERSTOREY}

Sphenopsida:

2\% Equisetum palustre L.

Angiospermae:

Dicotyledones:

$+\quad$ Epilobium sp.

1\% Lycopus europaeus L.

Monocotyledones:

5\% Carex sp.
Gramineae

Phragmites australis

Equisetaceae

Equisetum

Onagraceae

Labiatae

Cyperaceae
Epilobium angustifolium t. or E. hirsutum t. Mentha t.

Carex hallerana t. or C. flacca $\mathrm{t}$. 


\begin{tabular}{|c|c|c|}
\hline $\begin{array}{l}\% \quad \text { Genus \& Species } \\
\text { cover }\end{array}$ & Family & Pollen type \\
\hline
\end{tabular}

Edge of fen - plot b ( $2 \times 2 \mathrm{~m}$ plot $)$

Sphenopsida:

3\% Equisetum palustre L.

Equisetaceae

Angiospermae:

Dicotyledones:

$15 \%$ Cirsium creticum (Lam.) D'Urv.

Compositae

5\% Lycopus sp.

2\% Mentha aquatica L.

$10 \%$ Pulicaria dysenterica (L.) Bernh.

1\% Teucrium scordium L.

Monocotyledones:

2\% Cyperus cf. longus $\mathrm{L}$.

10\% Juncus inflexus L.

$50 \%$ Scirpus holoschoenus L.

Close by:

Angiospermae:

Dicotyledones:

Corylus avellana L.

Potentilla reptans $\mathrm{L}$.

Trifolium pratense $\mathrm{L}$.

Dryland near fen - plot c ( $2 \times 2 \mathrm{~m}$ plot)

Sphenopsida:

$+\quad$ Equisetum palustre L.

25\% Equisetum telmateia Ehrh.

Angiospermae:

Dicotyledones:

$2 \%$
$1 \%$
Buglossoides purpurocaerulea
(L.) I.M. Johnston

$+\quad$ Cirsium eriophorum (L.) Scop.

$2 \% \quad$ Clinopodium vulgare $\mathrm{L}$.

$2 \% \quad$ Corylus avellana $\mathrm{L}$.

$1 \%$ Crataegus cf. monogyna Jacq.

15\% Crepis $\mathrm{sp}$.

$+\quad$ Cuscuta sp.

$+\quad$ Daucus carota L.

15\% Dorycnium graecum (L.) Ser.

10\% Ononis spinosa L.

$1 \%$ Prunus sp.

$2 \%$ Quercus cf. cerris L.

1\% Rubus cf. ulmifolius Schott

$+\quad$ Taraxacum sp.

1\% Teucrium chamaedrys L.

$+\quad$ Teucrium polium $\mathrm{L}$.

Monocotyledones:

7\% Brachypodium silvaticum (Hudson) Beauv. Gramineae

$15 \%$ Carex sp.
Labiatae

Labiatae

Compositae

Labiatae

Cyperaceae

Juncaceae

Cyperaceae

Corylaceae

Rosaceae

Leguminosae

Equisetaceae

Equisetaceae

Rosaceae

Boraginaceae

Compositae

Labiatae

Corylaceae

Rosaceae

Compositae

Convolvulaceae

Umbelliferae

Leguminosae

Leguminosae

Rosaceae

Fagaceae

Rosaceae

Compositae

Labiatae

Labiatae

Cyperaceae
Equisetum

Cirsium t.

Prob. Mentha t.

Mentha t.

Solidago t.

Teucrium

Prob. Cyperus longus t.

Juncaceae

Carex hallerana t.

Corylus

Potentilla

Trifolium repens $\mathrm{t}$.

Equisetum

Equisetum

Agrimonia t.

Buglossoides

purpurocaerulea

Cirsium t.

Mentha t.

Corylus

Crataegus

Cichorium t. or Taraxacum t.

Cuscuta epithymum t.,

C. europaea t. or C. monogyna

Daucus

Dorycnium

Ononis t.

Prunus avium t. or P. spinosa t.

Prob. Quercus trojana t.

Prob. Rubus t.

Taraxacum t.

Teucrium

Teucrium

Dactylis t.

Carex hallerana t. or

C. flacca t. 


\begin{tabular}{|c|c|c|}
\hline $\begin{array}{l}\% \quad \text { Genus \& Species } \\
\text { cover }\end{array}$ & Family & Pollen type \\
\hline
\end{tabular}

Close by:

Angiospermae:

Dicotyledones:

Opopanax hispidus (Friv.) Griseb. Umbelliferae ?Seseli t.

Open dry grassland (steppe), not influenced by moisture from fen - plot d ( $2 \times 2 \mathrm{~m}$ plot)

Sphenopsida:

$1 \%$ Equisetum palustre $\mathrm{L}$.

Equisetaceae

Equisetum

Angiospermae:

Dicotyledones:

\begin{tabular}{|c|c|c|c|}
\hline+ & Alyssum murale Waldst. \& Kit. & Cruciferae & Hornungia t. \\
\hline $7 \%$ & Crataegus sp. & Rosaceae & Crataegus \\
\hline+ & Cuscuta sp. & Convolvulaceae & $\begin{array}{l}\text { Cuscuta epithymum t., } \\
\text { C. europaea t. or C. monogyna }\end{array}$ \\
\hline+ & Dorycnium sp. & Leguminosae & Dorycnium \\
\hline+ & Eryngium sp. ?campestre L. & Umbelliferae & Prob. Eryngium \\
\hline $7 \%$ & Galium verum $\mathrm{L}$. & Rubiaceae & Galium t. \\
\hline $1 \%$ & $\begin{array}{l}\text { P.W. Ball \& Heywood } \\
\text { Prunella sp. }\end{array}$ & $\begin{array}{l}\text { Caryophyllaceae } \\
\text { Labiatae }\end{array}$ & ?Dianthus t. \\
\hline $1 \%$ & Prunus spinosa $\mathrm{L}$. & Rosaceae & $\begin{array}{l}\text { Prunella t. } \\
\text { Prunus spinosa t. }\end{array}$ \\
\hline+ & Sanguisorba minor Scop. & Rosaceae & $\begin{array}{l}\text { Prunus spinosa t. } \\
\text { Sanguisorba minor }\end{array}$ \\
\hline $10 \%$ & Teucrium chamaedrys L. & Labiatae & Teucrium \\
\hline $10 \%$ & Teucrium polium $\mathrm{L}$. & Labiatae & Teucrium \\
\hline $25 \%$ & Thymus sp. & Labiatae & Prob. Mentha t. \\
\hline+ & Trifolium sp. & Leguminosae & $\begin{array}{l}\text { Trifolium dubium t., } \\
T \text {. repens t. or } T \text {. montanum t. }\end{array}$ \\
\hline \multicolumn{4}{|c|}{ Monocotyledones: } \\
\hline $15 \%$ & Carex sp. & Cyperaceae & $\begin{array}{l}\text { Carex hallerana t. or } \\
\text { C. flacca } \mathrm{t} \text {. }\end{array}$ \\
\hline $5 \%$ & Dactylis glomerata agg. & Gramineae & Dactylis $\mathrm{t}$. \\
\hline $15 \%$ & Festuca sp. ?glauca Vill. & Gramineae & Festuca t. \\
\hline+ & Phleum sp. & Gramineae & ??Dactylis t. \\
\hline+ & Triticum aestivum $\mathrm{L}$. & Gramineae & Triticum t. \\
\hline
\end{tabular}

Dry grassland close to plot d; transitional to damper ground of plot c (reconnaisance survey) Angiospermae:

Dicotyledones:

$\begin{array}{ll}\text { Anchusa azurea } \text { Miller } & \text { Boraginaceae } \\ \text { Carpinus } \text { sp. } & \text { Corylaceae } \\ \text { Centaurea } \text { sp. } & \text { Compositae }\end{array}$

Cichorium intybus L.

Clematis sp.

Convolvulus arvensis L.

Crataegus cf. monogyna Jacq.

Dipsacus fullonum L.

Echinops microcephalus Sibth. \& Sm.

Euonymus europaeus L.

Fragaria vesca $\mathrm{L}$.

Fraxinus ornus $\mathrm{L}$.
Compositae

Ranunculaceae

Convolvulaceae

Rosaceae

Dipsacaceae

Compositae

Celastraceae

Rosaceae

Oleaceae
Anchusa arvensis t.

Carpinus t. or Ostrya t.

Centaurea cyanus t.,

C. napulifera t., C. solstitialis t.,

C. graeca t. or C. amplifolia

Cichorium t.

Clematis vitalba gr. or

Ranunculus aquatilis gr.

Convolvulus

Crataegus

Dipsacus t.

Echinops

Euonymus europaeus t.

Fragaria vesca t.

Fraxinus ornus 


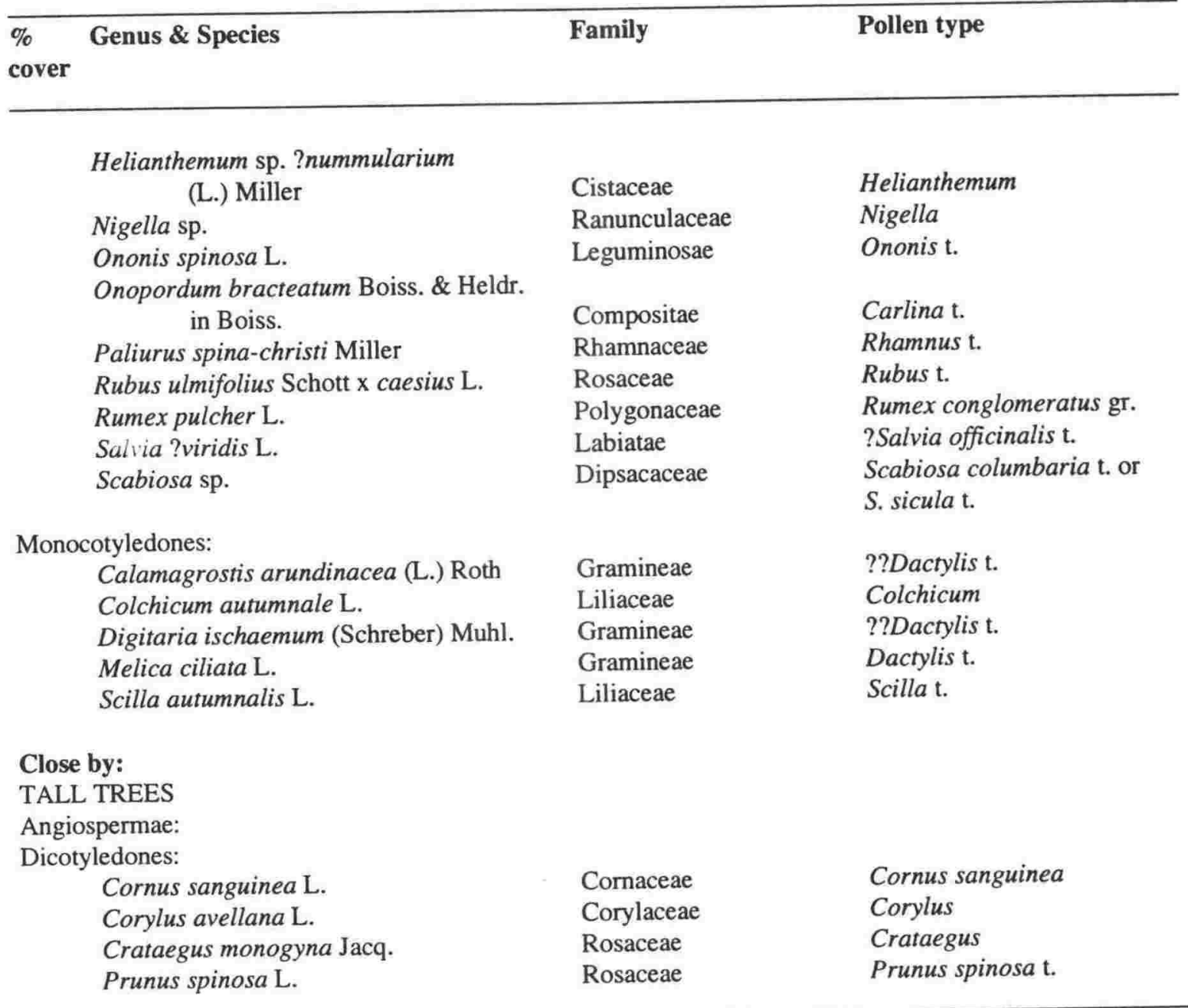




\section{References}

Andersen, S.T. 1970. The relative pollen productivity and pollen representation of North European trees, and correction factors for tree pollen spectra. Determined by surface pollen analyses from forests. Danmarks Geologiske Undersфgelse II Rakke, $\mathrm{Nr} 96$.

Andersen, S. T. 1973. The differential pollen productivity of trees and its significance for the interpretation of a pollen diagram from a forested region. In, H.J. B. Birks and R.G. West (eds), Quaternary plant ecology: 109-115. Blackwell Scientific Publications, London.

Birks, H.J.B. 1973. Past and present vegetation of the Isle of Skye. A palaeoecological study. Cambridge University Press, Cambridge.

Birks, H.J.B. 1977. Modern pollen rain and vegetation of the St. Elias Mountains, Yukon Territory. Canadian journal of botany 55: 2367-2382.

Institute for Geological and Mining Research (I.G.M.R.) 1979. Geological Map of Greece 1:50000, Ayiofillon Sheet.

Institute for Geology and Subsurface Research (I.G.S.R.) 1959. Geological map of Greece 1:50000, Metsovon Sheet.

Institute for Geology and Subsurface Research (I.G.S.R.) 1960. Geological map of Greece 1:50000, Pentalofon Sheet.

Institute for Geology and Subsurface Research (I.G.S.R.) 1972. Geological Map of Greece 1:50000, Grevena Sheet.

Institute of Geology and Mineral Exploration (I.G.M.E.) 1980. Geological map of Greece 1:50000, Panayia Sheet.

Institute of Geology and Mineral Exploration (I.G.M.E.) 1987a. Geological map of Greece 1:50000, Dheskati Sheet.

Institute of Geology and Mineral Exploration (I.G.M.E.) 1987b. Geological map of Greece 1:50000, Konitsa Sheet.

Moody, J. and O. Rackham (in prep.). Observations on the historical ecology of Grevena.

Moore, P.D. and S.B. Chapman (eds) 1986. Methods in plant ecology. Blackwell Scientific Publications, London.

Savina, M. 1990. Geology and geomorphology of the Anelia site. Unpublished Grevena Project report.

Tutin, T.G., V.H. Heywood, N.A. Burges, D.H. Valentine, S.M. Walters and D.A. Webb (eds) 1964-80. Flora Europaea. Cambridge University Press, Cambridge. 5 volumes.

Watson, E.V. 1968 (2nd ed.). British mosses and liverworts. Cambridge University Press, Cambridge. 



\section{Appendix IX}

\section{Surface samples - Pollen and charcoal counts}

Site

Slide numbers

\section{GYMNOSPERMAE}

Abies

Pinus (Diploxylon)

Juniperus

Taxus baccata

Ephedra fragilis

\section{DICOTYLEDONES}

Salix triandra

Salix fragilis type

Populus

Juglans regia

Alnus glutinosa

Carpinus type

Ostrya type

Corylus

Fagus

Castanea sativa

Quercus ilex type

Quercus trojana type

Quercus frainetto type

Ulmus glabra type

Urtica dioica

Urtica urens type

Urtica pilulifera type

Arceuthobium oxycedri

Polygonum bistorta type

Polygonum aviculare type

Rumex type

Oxyria digyna

Rumex acetosella group

Rumex acetosa group

Rumex crispus pp.

Emex spinosa

Chenopodiaceae type

Herniaria type

Paronychia type

Dianthus type

Lychnis flos-cuculi

Scleranthus annuus type

Stellaria holostea

Helleborus

Nigella

Actaea spicata

Adonis

Caltha type

Ranunculus aquatilis group

Anemone nemorosa group

Thalictrum aquilegifolium

Thalictrum lucidum

Chelidonium majus

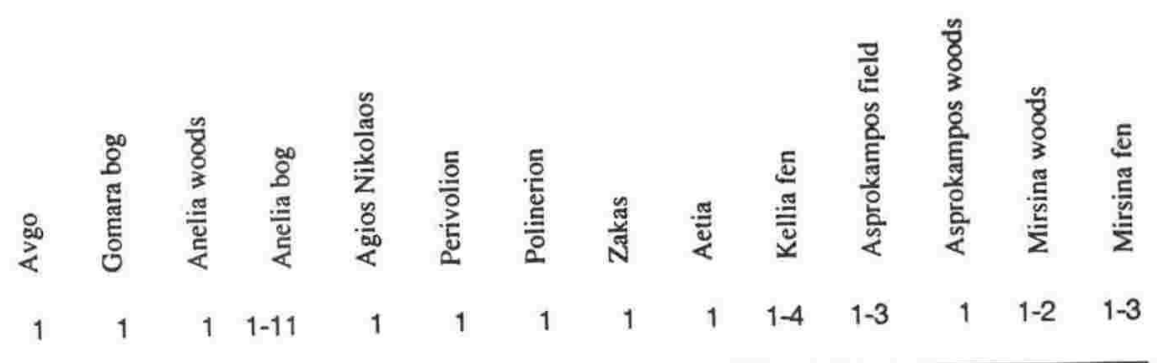


Site

Slide numbers

Papaver rhoeas type Hornungia type

Arabis

Sinapis type

Umbilicus

Chrysosplenium alternifolium

Saxifraga stellaris

Saxifraga tridactylites type

Parnassia palustris

Platanus orientalis

Filipendula

Rubus type

Sorbus type

Rosa

Crataegus

Sanguisorba minor

Potentilla

Fragaria vesca type

Ononis type

Lotus type

Vicia cracca type

Galega officinalis

Medicago sativa

Dorycnium

Lathyrus sativus

Geranium

Linum usitatissimum type

Linum austriacum type

Euphorbia

Pistacia

Acer

Buxus sempervirens

Tilia cordata type

Daphne

Elaeagnus angustifolia

Helianthemum

Myricaria germanica

Tamarix

Lythrum salicaria type

Lythrum portula

Trapa natans

Myrtus communis

Myriophyllum spicatum

Theligonum cynocrambe

Cornus sanguinea

Cornus mas

Hydrocotyle vulgaris

Smyrnium

Bupleurum

Carum

Berula erecta

Trinia

Conium maculatum

Cnidium silaifolium

Seseli type

Oenanthe aquatica group

Oenanthe pimpinelloides group

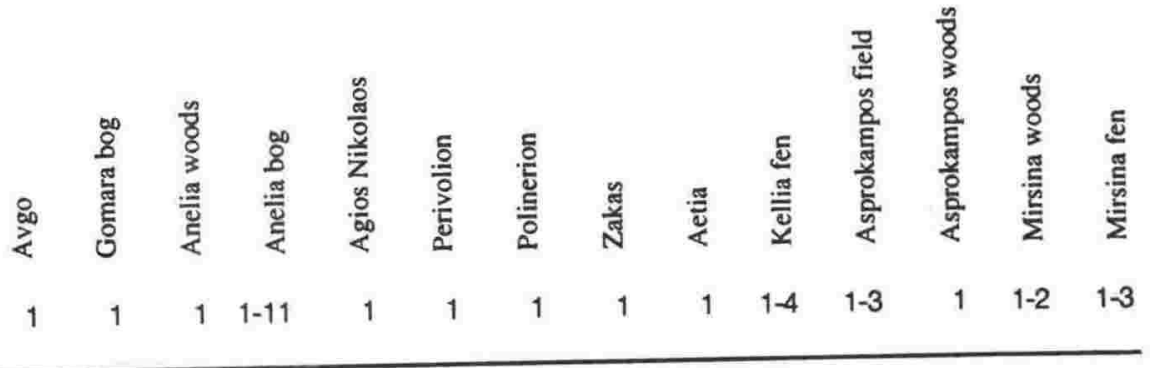

3

2

1

3

4

4

$\begin{array}{lllll}1 & & & 5 & \\ & 4 & & 4 & 2 \\ & 2 & 2 & & \\ & 1 & & & \\ & 1 & & & \\ & & & & \\ & 1 & & \\ & 1 & & & \\ & 3 & & \\ & & & & \\ & 2 & & \\ 1 & 3 & & \\ & 2 & & \\ & 1 & & \\ & 1 & & & \end{array}$

2

2

2

1

2

2

1

\section{2}

3

2

1

2

1

1
1

1 
Site

Slide numbers

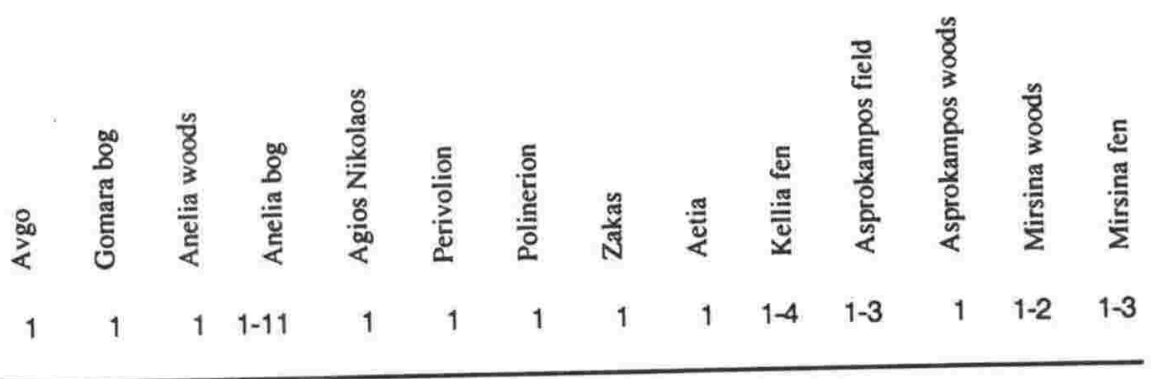

Chaerophyllum temulentum type

Ammi

Pimpinella saxifraga

Angelica sylvestris

Bunium

Eryngium

Pimpinella anisum type

Torilis japonica type

Daucus

Coriandrum type

Pyrola

Vaccinium myrtillus

Soldanella

Lysimachia

Asterolinon linum-stellatum

Samolus valerandi

Fraxinus excelsior type

Fraxinus angustifolia

Fraxinus ornus

Phillyrea

Gentiana lutea type

Cuscuta europaea type

Alkanna type

Callitriche obtusangula

Teucrium

Prunella type

Mentha type

Solanum dulcamara

Solanum nigrum type

Scrophularia type

Gratiola officinalis

Veronica triphyllos type

Pedicularis

Galium type

Plantago major

Plantago afra

Plantago alpina type

Plantago lanceolata type

Plantago coronopus

Plantago bellardii

Plantago media

Plantago argentea

Plantago albicans

Plantago amplexicaulis

Plantago atrata

Valeriana officinalis type

Scabiosa columbaria type

Knautia

Campanula erinus type

Solidago type

Artemisia

Carlina type

Achillea type

Centaurea cyanus type

Cirsium type

Centaurea solstitialis type

2

2

2

21

1

1

5

2

1

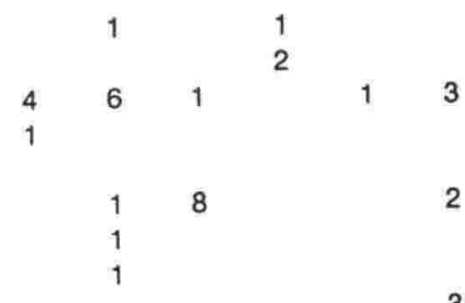

$\begin{array}{llllll}1 & 18 & 1 & & 2 & 9 \\ 4 & & 2 & 1 & & 1 \\ & 1 & 1 & & 2 & 1 \\ & & 1 & 2 & & \\ & & & 2 & & \\ & & & & & \end{array}$

23

$\begin{array}{llll}39 & 1 & 1 & 4\end{array}$

5

9

28

12

3

2

4

6

1

Centaurea graeca type 
Site

Slide numbers

Crupina

Lactuca type

Cichorium type

Hieracium type

Taraxacum type

\section{MONOCOTYLEDONES}

Damasonium alisma

Hydrocharis morsus-ranae

Potamogeton crispus type

Muscari comosum

Muscari neglectum type

Allium sphaerocephalon type

Asparagus

Gladiolus type

Festuca type

Glyceria type

Dactylis type

Agropyron type

Triticum type

Secale cereale

Hordeum type

Avena type

Phragmites australis

Zea mays

Sparganium erectum

Sparganium angustifolium

Typha angustifolia type

Cyperus michelianus

Cyperus longus type

Schoenus nigricans

Carex hallerana type

Carex flacca type

Limodorum abortivum

Cephalanthera

\section{PTERIDOPHYTA}

Equisetum

Pteridium aquilinum

Asplenium type

Gymnocarpium dryopteris

Blechnum spicant

Indeterminate

Unknown

Total trees \& shrubs

Total terrestrial pollen \& spores

Total pollen \& spores

\section{CHARCOAL}

Charcoal $<25$ um $^{2}$

Charcoal 25-50 um ${ }^{2}$

Charcoal 50-100 um ${ }^{2}$

Charcoal 100-200 um ${ }^{2}$

Charcoal 200-400 um ${ }^{2}$

Charcoal $>400 u^{2}$

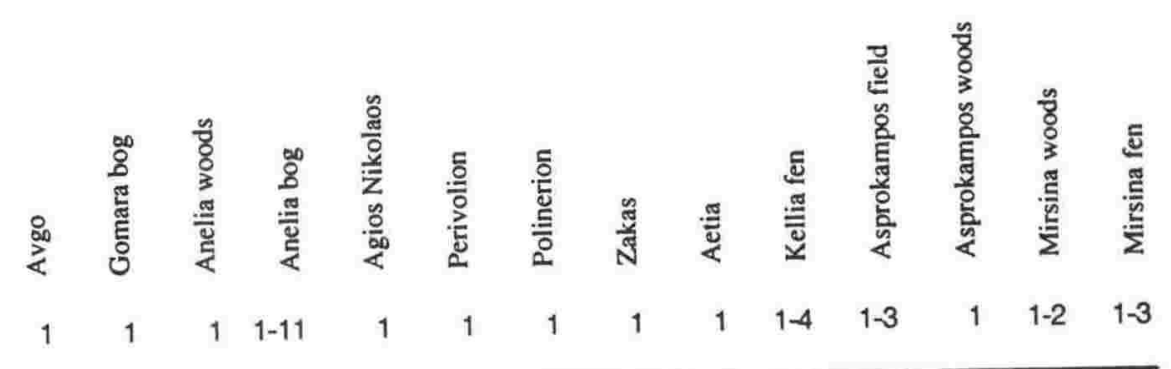

26

$\begin{array}{rrrrrr}1 & & 26 & & 1 & 1 \\ 1 & & & 1 & 6 \\ & 2 & & 9 & & 1\end{array}$

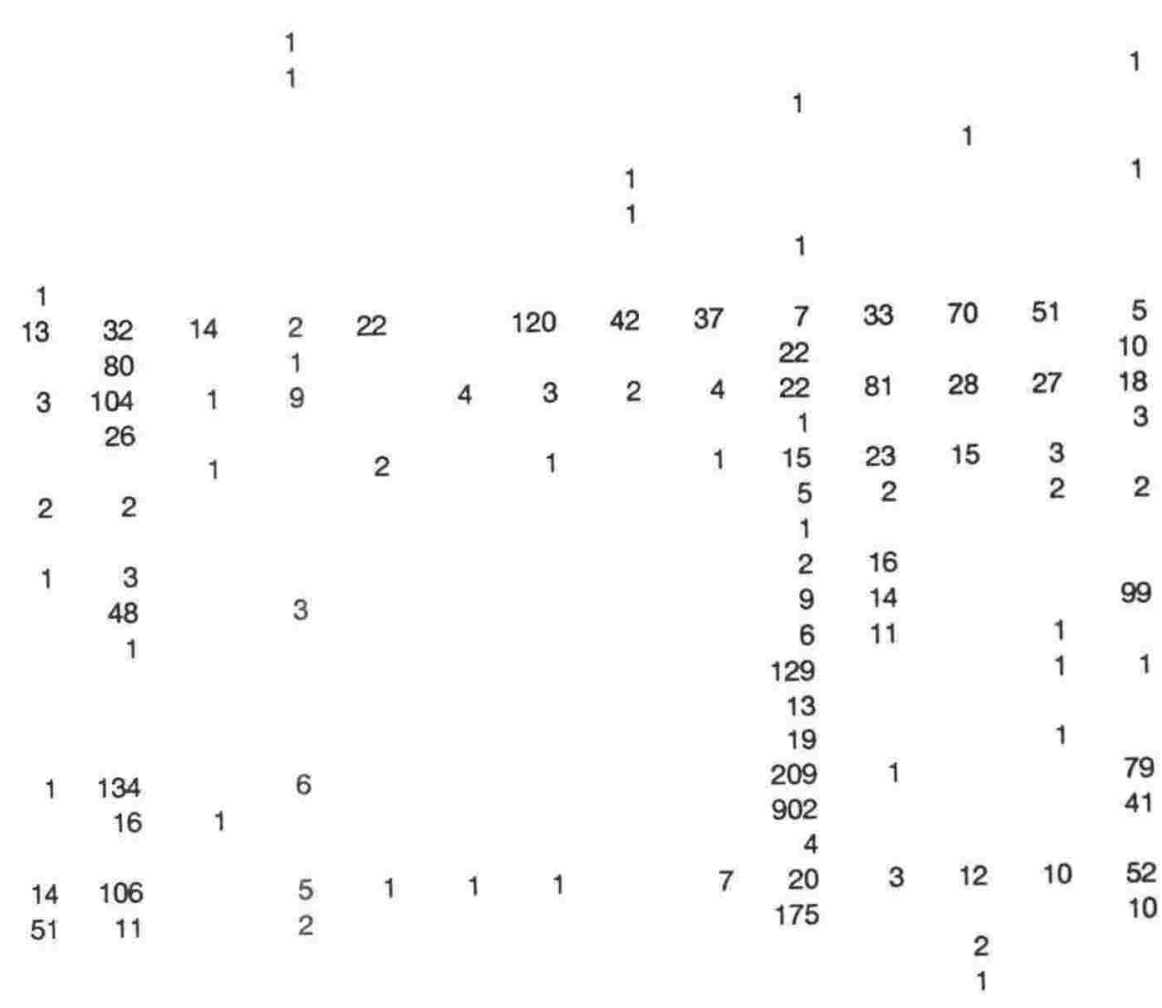





\section{Appendix X}

\section{Ecological data of pollen types}

This table lists all the pollen and spore types represented in all the pollen diagrams. The names which appear in this table are the same as those used in the pollen key and pollen and spore count tables. Taxonomic names of vascular European species follow Tutin et al. (1964-80) and of non-vascular species Watson (1968).

Life-form classification is based on that of Raunkiaer (1937), where plants are classified according to the position of the perennating buds or persistent stem apices in relation to soil level. This classification is a convenient method of indicating how plants pass the unfavourable season, and is useful because of the correlation with climate. The seven primary classes are used mostly; secondary classes are included where data was available. Life-form data was not readily available for many perennial herbs so these have been assigned to the hemicryptophyte class because it is immaterial for this study whether they are herbaceous chamaephytes, hemicryptophytes or geophytes. Symbols and their meanings are as follows:

1) Phanerophytes: Woody plants with perennating buds or stem apices more than $25 \mathrm{~cm}$ above soil level MM Mega- and Mesophanerophytes with perennating buds or stem apices borne on aerial shoots more than $8 \mathrm{~m}$ above the ground (trees)

M Microphanerophytes with perennating buds 2-8 $\mathrm{m}$ above the ground (small trees, tall shrubs)

N Nanophanerophytes with perennating buds between $25 \mathrm{~cm}$ and $2 \mathrm{~m}$ above the ground (shrubs)

2) Chamaephytes: Woody or herbaceous plants with perennating buds or stem apices above soil surface but below $25 \mathrm{~cm}$

Chw Woody Chamaephytes (small shrubs)

Chh Herbaceous Chamaephytes (herbs)

Che Cushion plants

3) Hemicryptophytes: Herbaceous (very rarely woody) plants with perennating buds at ground level or in litter (most biennial and perennial herbs)

Hp Protohemicryptophytes, with uniformly leafy stems, but the basal leaves usually smaller than the rest

Hs Semi-rosette hemicryptophytes, with leafy stems but the lower leaves larger than the upper ones and the basal internodes shortened

$\mathrm{Hr} \quad$ Rosette hemicryptophytes, with leafless flowering stems and a basal rosette of leaves

4) Geophytes: Herbaceous plants with perennating buds below the soil surface (herbs)

$\mathrm{Gb} \quad$ Geophytes with bulbs

Gr Geophytes with buds on roots

Grh Geophytes with rhizomes

Grt Geophytes with root tubers

Gt Geophytes with stem tubers or corms

5) Hel Helophytes: Perennating buds below water level in soil or mud (mire plants)

6) Hyd Hydrophytes: Perennating buds below water level in water (aquatic plants)

7) Th Therophytes: Perennating bud in seed (annual herbs) 
Habitats are given for each pollen type so far as they are known. Ecological data comes from the author's observations, and the following references:

Arber, A. 1920. Water Plants. A study of aquatic angiosperms. Cambridge University Press, Cambridge.

Aschenbrenner, S.E. 1988. Field report - Grevena Project. Unpublished Grevena Project report.

Behre, K.E. 1981. The interpretation of anthropogenic indicators in pollen diagrams. Pollen et spores 13: 225-245.

Behre, K.E. 1990. Some reflections on anthropogenic indicators and the record of prehistoric occupation phases in pollen diagrams from the Near East. In S. Bottema, G. Entjes-Nieborg and W. van Zeist (ed), Man's role in the shaping of the Eastern Mediterranean landscape: 219230. A.A. Balkema, Rotterdam.

Bottema, S. 1979. Pollen analytical investigations in Thessaly (Greece). Palaeohistoria 21:20-40.

Bottema, S. 1980. On the history of the walnut (Juglans regia L.) in southeastern Europe. Acta bot. Neerl. 29 (5/6): 343-349.

Bottema, S. 1982. Palynological investigations in Greece with special reference to pollen as an indicator of human activity. Palaeohistoria 24:257-289.

Bottema, S. 1990. Holocene environment of the Southern Argolid: A pollen core from Kiladha Bay. In T.J. Wilkinson and S.T. Duhon (with contributions by J.A. Gifford and S. Bottema), Franchthi Paralia: 117-138. Indiana University Press, Bloomington.

Bottema, S. and H. Woldring 1986. Late Quaternary vegetation and climate of southwestern Turkey. Palaeohistoria 26: 123-149. (for 1984)

Bottema, S. and H. Woldring 1990. Anthropogenic indicators in the pollen record of the Eastern Mediterranean. In, S. Bottema, G. Entjes-Nieborg and W. Van Zeist (ed), Man's role in the shaping of the Eastern Mediterranean landscape: 231-264. A.A. Balkema, Rotterdam.

Bozilova, E. and S. Tonkov 1990. The impact of man on the natural vegetation in Bulgaria from the Neolithic to the Middle Age. In, S. Bottema, G. Entjes-Nieborg and W. Van Zeist (ed), Man's role in the shaping of the Eastern Mediterranean landscape: 327-332. A.A. Balkema, Rotterdam.

Dennell, R. W. 1972. The interpretation of plant remains: Bulgaria. In E.S Higgs (ed), Papers in Economic prehistory: 149-159. Cambridge University Press, Cambridge.

Dennell, R.W. 1977. On the problem of studying prehistoric climate and crop agriculture. Proceedings of the prehistoric society 43: 361-369.

Faegri, K. and J. Iversen 1975. Textbook of pollen analysis. Hafner Press, New York.

Hansen, J. M. 1988. Agriculture in the prehistoric Aegean: data versus speculation. American journal of archaeology 92: 39-52.

Haslam, S.M., C.A. Sinker and P.A. Wolselet 1975. British water plants. Field studies 4: 243-351.

Hedrick. U.P. 1972. Sturtevant's edible plants of the world. Dover publications, New York. 
Heywood, V.H. (ed). 1979. Flowering plants of the world. Oxford University Press, Oxford.

Humphries, C.J., J.R. Press and D.A. Sutton 1985. The trees of Britain and Europe. Country Life Books, Feltham, Middlesex.

Huxley, A. and W. Taylor 1989. Flowers of Greece and the Aegean. The Hogarth Press, London.

Moody, J. and O. Rackham, in prep. Observations on the historical ecology of Grevena.

Polunin, O. 1988. Flowers of Greece and the Balkans: a field guide. Oxford University Press, Oxford.

Polunin, O. and B. Everard 1976. Trees and bushes of Europe. Oxford University Press, London.

Polunin, O. and A. Huxley 1987. Flowers of the Mediterranean. The Hogarth Press, London.

Rackham, O. 1991. Flora Greveniotica. Unpublished Grevena Project report.

Rackham, O. 1983. Observations on the historical ecology of Boeotia. Annual of the British School at Athens 78: 291-351.

Raunkiaer, C. 1937. Plant life forms. Clarendon Press. Oxford. [Translated from Danish into English by H. Gilbert-Carter]

Renfrew, J. 1973. Palaeoethnobotany. The prehistoric food plants of the Near East and Europe. Methuen \& Co. Ltd, London.

Sculthorpe, C.D. 1967. The biology of aquatic vascular plants. Edward Arnold Ltd, London.

Sfikas, G. 1978. Trees and shrubs of Greece. Efstathiadis Group, Athens.

Strid, A. (ed) 1989. Mountain flora of Greece. Vol. I. Cambridge University Press, Cambridge.

Strid, A. and K. Tan 1991 (eds). Mountain flora of Greece. Vol. II. Edinburgh University Press, Edinburgh.

Turrill, W.B. 1929. The plant-life of the Balkan Peninsula. A phytogeographical study. Clarendon Press, Oxford.

Tutin, T. G. et al (eds) 1964-80. Flora Europaea. Volumes 1-5. Cambridge University Press, Cambridge.

van Zeist, W. and S. Bottema 1971. Plant husbandry in early neolithic Nea Nikomedeia, Greece. Acta bot. Neerl. 20 (5): 524-538.

Watson, E.V. 1968 (2nd ed.), British mosses and liverworts. Cambridge University Press, Cambridge.

Willis, J.C. 1951. A dictionary of the flowering plants and ferns. Cambridge University Press, Cambridge. 


\begin{tabular}{|c|c|c|}
\hline Pollen type & \begin{tabular}{|l|}
$\begin{array}{l}\text { Life } \\
\text { form }\end{array}$ \\
\end{tabular} & $\begin{array}{l}\text { Notes on geographic distribution, habit, ecological amplitude, } \\
\text { plant communities and uses }\end{array}$ \\
\hline \multicolumn{3}{|l|}{ GYMNOSPERMAE } \\
\hline Abies & MM & $\begin{array}{l}\text { N. temp. Evergreen. Montane fir, between } 500 \& 2000 \mathrm{~m} \text {. On } \\
\text { limestone or serpentine. Shade tolerant. In Grevena Abies grows in } \\
\text { the beech vegetation zone of the Pindos Mtns. Grevena is close to } \\
\text { the northern limit of A. cephalonica (endemic to Greece). Fast- } \\
\text { growing, rather short-lived. Fir can survive long periods of browsing } \\
\text { when young \& does not burn. It is killed when felled. Can be } \\
\text { pollarded, but this was rarely seen in Grevena. Abies appears to have } \\
\text { less power of rejuvenation than oaks and beech \& Turrill (1929:143) } \\
\text { thinks that its abundance has been much reduced by lumbering. }\end{array}$ \\
\hline Picea abies & MM & $\begin{array}{l}\text { N. Hemisph. Evergreen. Montane. Forming an extensive forest } \\
\text { between } c .1400 \mathrm{~m} \& 1900 \mathrm{~m} \text { in NE Greece, partly in pure stands \& } \\
\text { partly together with Pinus sylvestris \& Fagus sylvatica. Not known } \\
\text { in Grevena today. }\end{array}$ \\
\hline Cedrus & $\mathrm{MM}$ & $\begin{array}{l}\text { Evergreen. Not native; planted for timber in Greece. Not known in } \\
\text { Grevena. }\end{array}$ \\
\hline Pinus (Diploxylon) & MM & $\begin{array}{l}\text { N. temp. Evergreen. } P \text {. heldreichii \& } P \text {. leucodermis are endemic to } \\
\text { the Balkans. Form highest altitude forests in Pindos Mtns }(600-2300 \\
\mathrm{m}) \text {. Light demanding. Do not burn easily. Pines are often unable to } \\
\text { re-establish themselves in competition with beech with its dense } \\
\text { shade (Turrill 1929). Used for building \& formerly tapped for the } \\
\text { production of resin. }\end{array}$ \\
\hline Pinus (Haploxylon) & $\mathrm{MM}$ & $\begin{array}{l}\text { Evergreen. Forms forests on siliceous mountains of central Balkans. } \\
\text { Not known in Grevena. }\end{array}$ \\
\hline Juniperus & $\mathrm{M}, \mathrm{N}$ & $\begin{array}{l}\text { N. Hemisph. Evergreen. Light demanding. In Grevena it is } \\
\text { ubiquitous from the lowlands to the mtns on dry soils, forming } \\
\text { brushwood in the low alpine zone. Pseudo-maquis. Grows in open } \\
\text { grassland, as under shrub in open pine \& oak woods. Profits from } \\
\text { grazing (Bottema 1982:263). Takes advantage of destruction of } \\
\text { primary forests (Behre 1990:224). Acts as a nurse crop for } \\
\text { regenerating mtn pinewoods. Juniper wood is very flammable. } \\
\text { Juniper is rather unpalatable, but where abundant may be an } \\
\text { important browsewood. Used for charcoal making \& firewood. J. } \\
\text { communis is used for making gin. }\end{array}$ \\
\hline Taxus baccata & $\begin{array}{l}\mathrm{MM} \text { or } \\
\mathrm{M}\end{array}$ & $\begin{array}{l}\text { N. temp. Evergreen. Grows either on mtns or at lower altitudes in } \\
\text { cooler climates. Grows in edaphically wet \& atmospherically moist } \\
\text { conditions. Usually grows on limestone (but also serpentine), as a } \\
\text { scattered tree in beech or mixed woods, } 800-2200 \mathrm{~m} \text {. In Grevena it } \\
\text { is scattered in mixed woods on limestone in the Aetia gorge and } \\
\text { above Zakas at } c \text {. } 1000 \mathrm{~m} \text {. Foliage is poisonous to animals. Long } \\
\text { lived \& slow growing. }\end{array}$ \\
\hline Ephedra fragilis & $\mathrm{M}$ & $\begin{array}{l}\text { Warm temp. Evergreen. Xerophytic, grows in steppe. Not observed } \\
\text { in Grevena }\end{array}$ \\
\hline Ephedra distachya type & $\mathrm{N}$ & $\begin{array}{l}\text { Warm temp. Evergreen. Xerophytic, usually grows in steppe, but } E \text {. } \\
\text { distachya may grow in very dry alpine areas. Lowlands to } 1950 \mathrm{~m} \text {. } \\
\text { Not observed in Grevena. }\end{array}$ \\
\hline \multicolumn{3}{|l|}{ ANGIOSPERMAE } \\
\hline \multicolumn{3}{|l|}{ DICOTYLEDONES } \\
\hline Salix triandra & $\mathrm{M}$ or $\mathrm{N}$ & $\begin{array}{l}\text { Cosmop. Deciduous. Commonly found on river banks, by lakesides } \\
\text { \& growing in damp ravines. Hybridization plays an important role in } \\
\text { Salix. Part of current riverine flood plain vegetation of Grevena. }\end{array}$ \\
\hline
\end{tabular}




\begin{tabular}{|c|c|c|}
\hline Pollen type & $\begin{array}{l}\text { Life } \\
\text { form }\end{array}$ & $\begin{array}{l}\text { Notes on geographic distribution, habit, ecological amplitude, } \\
\text { plant communities and uses }\end{array}$ \\
\hline Salix fragilis type & $\begin{array}{l}\text { MM, M, } \\
\text { N }\end{array}$ & $\begin{array}{l}\text { Cosmop. Deciduous. In Grevena this type grows in damp localities } \\
\text { from lowland to montane altitudes, by streams, rivers, on marshes \& } \\
\text { fens, \& in wet woods, scrub, hedgerows \& meadows including } \\
\text { streams in mtn woods \& highland ravines. Salix have been known to } \\
\text { be planted as an osier \& pollarded. Fast growing. }\end{array}$ \\
\hline Populus & MM & $\begin{array}{l}\text { N. temp. Deciduous. Light demanding. Grows in damp localities in } \\
\text { lowlands \& lower highlands, near rivers, damp \& muddy areas on } \\
\text { hillsides, valleys, hedgerows \& in damp montane woodlands (nr } \\
\text { Spilaion) as well as on roadsides \& fields. Most spp. sucker freely. }\end{array}$ \\
\hline Juglans regia & MM & $\begin{array}{l}\text { N. Hemisph. Deciduous. Prob. native to the Near East. Walnut does } \\
\text { not appear in Greece until the end of the fourth millennium BP, } \\
\text { thought to have either profited from changes in habitats caused by } \\
\text { humans or been planted in prehistoric times (Bottema 1982:275). } \\
\text { Naturally grows scattered among other woodland trees in the } \\
\text { highlands. Today it is the most cultivated tree in Grevena, although } \\
\text { it is somewhat precarious: every walnut in the region was badly } \\
\text { damaged by frost in 1987. Oral tradition includes walnuts as part of } \\
\text { the suite of subsistence agricultural products grown for human } \\
\text { consumption before 1940. Slow growing. }\end{array}$ \\
\hline Alnus glutinosa & $\begin{array}{l}\text { M or } \\
\text { MM }\end{array}$ & $\begin{array}{l}\text { N. temp. Deciduous. Frequently found growing by water - marshes, } \\
\text { banks of rivers \& streams \& in ravines, but also in stable locations in } \\
\text { the mtns, plains \& lowlands where there is a sufficient water supply. } \\
\text { Deep rooted. Medium shade tolerance \& colonizing ability. Sheds } \\
\text { thick shade. Woods of alder grow in the riverine vegetation of the } \\
\text { Venetikos, Aliakmon \& Pramoritza rivers. Pollen emitted before } \\
\text { leafing. }\end{array}$ \\
\hline Betula pendula & MM & $\begin{array}{l}\text { N. temp. Deciduous. Pioneer. Forms woods, particularly on light } \\
\text { soils, often secondary after forest destruction. Highly self- } \\
\text { propagating, but is short lived, does not grow in shade \& is } \\
\text { eventually replaced by other, larger species. Local \& confined to } \\
\text { mountains i.e. cooler climates. Its spread in the sub-Atlantic period } \\
\text { may indicate colder conditions, but it may also have been favoured } \\
\text { by an increase in the practice of burning, after which it is a common } \\
\text { colonizer (Dimbleby 1969:177). Not known in Grevena today, but } \\
\text { Turrill (1929:143) records it in the montane beech \& fir forests } \\
\text { further north on the Balkan Peninsula. }\end{array}$ \\
\hline Carpinus type & $\begin{array}{l}\text { MM or } \\
\text { M }\end{array}$ & $\begin{array}{l}\text { N. temp. Deciduous. Carpinus betulus represents this pollen type } \\
\text { mostly, while Carpinus orientalis and Ostrya are minor } \\
\text { representatives. In Grevena } C \text {. betulus grows in damp woods in the } \\
\text { Fagus zone \& in the mixed woods at Spilaion. }\end{array}$ \\
\hline Ostrya type & $\begin{array}{l}\text { MM or } \\
\text { M }\end{array}$ & $\begin{array}{l}\text { N. temp. Deciduous. The genus Ostrya and Carpinus orientalis } \\
\text { represent this pollen type mostly, while Carpinus betulus is a minor } \\
\text { representative. Ostrya usually grows in bushy places on limestone } \\
\text { hills \& mtns. In Grevena this pollen type grows in mixed woods, at } \\
\text { Aetia \& Spilaion, on limestone at about } 1000 \mathrm{~m} \text { and between Kranea } \\
\& \text { Milea on ophiolite \& gabbro (where it is frequent). C. orientalis } \\
\text { wood is an alternative to oak wood in the lowlands, but grows only } \\
\text { in damp areas. It can withstand grazing reasonably well and } \\
\text { regenerates quickly. Very palatable (grazed very low in Mirsina } \\
\text { wood). Slow growing. Withstands some shade. Used for firewood. }\end{array}$ \\
\hline
\end{tabular}




\begin{tabular}{|c|c|c|}
\hline Pollen type & $\begin{array}{l}\text { Life } \\
\text { form }\end{array}$ & $\begin{array}{l}\text { Notes on geographic distribution, habit, ecological amplitude, } \\
\text { plant communities and uses }\end{array}$ \\
\hline Corylus & $\begin{array}{l}\mathrm{MM}, \mathrm{M} \\
\mathrm{N}\end{array}$ & $\begin{array}{l}\text { N. temp. Deciduous. Light demanding; colonizer. Known to self- } \\
\text { propagate in mixed woods in northern Greece. In Grevena grows in } \\
\text { mixed woods at Aetia at } 1000 \mathrm{~m} \text { on limestone and in a mixed oak } \\
\text { wood at lower altitude } 8 \mathrm{~km} \mathrm{SE} \text { of Grevena. Also grows in the oak } \\
\text { wood zone near Mirsina fen. Remains of } C \text {. avellana (hazel nuts) are } \\
\text { often found in prehistoric archaeological sites. Pollen emitted before } \\
\text { leafing. }\end{array}$ \\
\hline Fagus & MM & $\begin{array}{l}\text { N. temp. Deciduous. Shade tolerant. Beech grows in a colder } \\
\text { environment than oak \& wetter environment than pine. This type is } \\
\text { represented mostly by } F \text {. sylvatica. It forms pure stands on well- } \\
\text { drained soils between } 1000 \& 1800 \mathrm{~m} \text { on the Pindos, usually in } \\
\text { damp areas. Sheds thick shade which usually keeps the forest floor } \\
\text { bereft of undergrowth. Freely regenerates from fallen seed \& after } \\
\text { felling. Grevena is close to the southern limit of beech. In Grevena } \\
\text { beech woods are usually of coppice type, felled every } 40-75 \text { yrs, but } \\
\text { many have not been cut for centuries. It can withstand long periods } \\
\text { of browsing when young \& does not burm. Beech mast occas. occurs } \\
\text { in prehistoric food plant remains. }\end{array}$ \\
\hline Castanea sativa & MM & $\begin{array}{l}\text { N. Hemisph. Deciduous. Native to Greece, but also cultivated. } \\
\text { Sweet chestnut grows naturally on well-drained, usually acidic soils, } \\
\text { often on slopes at moderate altitudes among other deciduous trees; } \\
\text { usually calcifuge. Extensively planted for nuts \& timber. }\end{array}$ \\
\hline Quercus coccifera type & $\begin{array}{l}N(\& \\
M M)\end{array}$ & $\begin{array}{l}\text { Medit. Evergreen. Xerophytic. Suckers. Usually forms thickets on } \\
\text { dry stony slopes at low altitudes. In Grevena Q. coccifera grows in } \\
\text { the SE on a patch of limestone with red soils in pseudo-maquis as a } \\
\text { dense prickly shrub. Pyrophytic (regenerates quickly). Quick } \\
\text { growing. Used for firewood. Takes advantage of destruction of } \\
\text { primary forests (Behre 1990). }\end{array}$ \\
\hline Quercus trojana type & $\begin{array}{l}\text { M or } \\
\text { MM }\end{array}$ & $\begin{array}{l}\text { Eur. Semi-evergreen or deciduous. Mesophytic. See } Q \text {. frainetto } \\
\text { below. }\end{array}$ \\
\hline Quercus frainetto type & $\begin{array}{l}\text { M or } \\
\text { MM }\end{array}$ & $\begin{array}{l}\text { Eur. Deciduous. The } Q \text {. trojana \& } Q \text {. frainetto pollen types } \\
\text { dominate the foothills of the Pindos and the Grevena plains. They } \\
\text { form extensive woods. There is much hybridization. Oaks form a } \\
\text { continuum in which some individuals are more or less typical of one } \\
\text { or other species, but many are intermediate. Thus the distinction } \\
\text { between the two pollen types } Q \text {. trojana, which represents mostly } \\
\text { semi-evergreen oaks, \& } Q \text {. frainetto, which represents mostly } \\
\text { deciduous oaks, is not very clear morphologically. Oaks are resistant } \\
\text { to browsing; they are quite palatable to livestock, but can live } \\
\text { indefinitely even if bitten down to less than a metre. They are not } \\
\text { killed by felling, but sprout from the stump. They do not burn easily, } \\
\text { but the litter can be ignited by adjacent stubble or grass fires. Young } \\
\text { oaks are killed to the ground by such fires, but sprout. Oaks easily } \\
\text { invade abandoned farmland. Used for building structures, firewood, } \\
\text { fodder \& pasture. Evidence of acorns being used for human } \\
\text { consumption has been found in archaeological sites in Thessaly } \\
\text { dating to the Neolithic Period at Sesklo \& Achilleion (Renfrew } \\
\text { 1973:28). }\end{array}$ \\
\hline Ulmus minor & MM & $\begin{array}{l}\text { N. temp. Deciduous. Shade tolerant. Frequent vegetative } \\
\text { propagation (suckering). Grows particularly by rivers, prefers low } \\
\text { altitudes. In Grevena, U. minor occurs in suckering patches along } \\
\text { watercourses smaller than the Aliakmon \& Venetikos rivers. Pollen } \\
\text { emitted before leafing. }\end{array}$ \\
\hline
\end{tabular}




\begin{tabular}{|c|c|c|}
\hline Pollen type & $\begin{array}{l}\text { Life } \\
\text { form }\end{array}$ & $\begin{array}{l}\text { Notes on geographic distribution, habit, ecological amplitude, } \\
\text { plant communities and uses }\end{array}$ \\
\hline Ulmus procera & MM & $\begin{array}{l}\text { N. temp. Deciduous. Bushy places. Shade tolerant. Frequent } \\
\text { vegetative propagation (suckering). Not seen growing in Grevena. }\end{array}$ \\
\hline Ulmus glabra type & MM & $\begin{array}{l}\text { N. temp. Deciduous. Usually grow in cool forest locations among } \\
\text { other trees. Shade tolerant. U. glabra grows on the damp north } \\
\text { facing slopes in the mixed woods at Aetia and Spilaion. Pollen } \\
\text { emitted before leafing. }\end{array}$ \\
\hline Celtis & MM & $\begin{array}{l}\text { S. Eur. Deciduous. Grows in bushy places in the Mediterranean } \\
\text { region. Not seen growing in Grevena. Hackberry stones have been } \\
\text { found on a number of Near Eastern archaeological sites. }\end{array}$ \\
\hline Morus nigra & MM & $\begin{array}{l}\text { N. temp. Deciduous. Not native. Widely cultivated. Not seen } \\
\text { growing in Grevena. }\end{array}$ \\
\hline Morus alba & MM & $\begin{array}{l}\text { N. temp. Deciduous. Not native. Cultivated. Not seen growing in } \\
\text { Grevena. }\end{array}$ \\
\hline Humulus lupulus & $\mathrm{Hp}$ & $\begin{array}{l}\text { N. temp. Liane. Widely cultivated in Europe, but grows naturally in } \\
\text { hedges, bushy places and on river banks. Not seen growing in } \\
\text { Grevena. }\end{array}$ \\
\hline Urtica dioica & $\mathrm{Hp}$ & $\begin{array}{l}\text { Temp. Ruderal. The common stinging nettle, grows in somewhat } \\
\text { damp, nutrient-rich habitats. Often grows near habitation \& where } \\
\text { the ground is covered with litter \& rubble. It regularly ascends to } \\
\text { alpine levels in places where grazing animals congregate, near snow } \\
\text { patches, at the base of rock overhangs \& by shepherds' huts. } \\
\text { Cultivated in prehistory - ?Grevena (Hedrick 1972). Unpalatable. }\end{array}$ \\
\hline Urtica urens type & Th & $\begin{array}{l}\text { Temp. } U \text {. urens is native to Greece while Pilea microphylla has } \\
\text { become naturalized in the Balkan Peninsula. They are widespread, } \\
\text { but grow at lower than montane altitudes. Ruderal \& weed. }\end{array}$ \\
\hline Urtica pilulifera type & Th & $\begin{array}{l}\text { Temp. Ruderal \& weed. Grows in waste places, waysides \& } \\
\text { cultivated ground. }\end{array}$ \\
\hline Parietaria & Th \& Hp & $\begin{array}{l}\text { Temp. \& trop. Often grows on dry rocks \& walls. Observed growing } \\
\text { in } 1 \frac{1}{2} \text { year-coppiced oak wood. }\end{array}$ \\
\hline Viscum album & $\mathrm{N}$ & Evergreen. Hemi-Parasite. In Grevena it grows on Pinus. \\
\hline Arceuthobium oxycedri & $\mathrm{N}$ & $\begin{array}{l}\text { Hemi-Parasite that grows on Juniperus oxycedrus. It is very } \\
\text { abundant near Aetia. }\end{array}$ \\
\hline Polygonum bistorta type & $\mathrm{H}$ & $\begin{array}{l}\text { Cosmop. Montane. P. bistorta grows in damp meadows \& forest } \\
\text { clearings on acid soil. P. alpinum, which may belong to this type, } \\
\text { grows in grassland, rocky places \& screes on serpentine, granite or } \\
\text { other non-calcareous rocks. Both grow in the Pindos Mtns, } 1400 \text { - } \\
2350 \mathrm{~m} \text {. }\end{array}$ \\
\hline $\begin{array}{l}\text { Polygonum aviculare } \\
\text { type }\end{array}$ & $\begin{array}{l}\text { Th } \\
\text { (mostly) } \\
\& \mathrm{H}\end{array}$ & $\begin{array}{l}\text { Cosmop. Ruderal \& weed of cultivation. Occurs naturally, but is } \\
\text { increased by anthropogenic disturbance. Grows in dry, open } \\
\text { disturbed communities from the lowlands to the mtns, up to } 2500 \mathrm{~m} \text {. } \\
\text { A large number of } P \text {. aviculare seeds were found in Neolithic } \\
\text { deposits at Sitagroi, northern Greece. This may indicate that they } \\
\text { were being cultivated for human consumption. Has been cultivated } \\
\text { in historical times (Hedrick 1972) - ?Grevena in prehistory. Leaves } \\
\text { may have been consumed (Dennell 1977). }\end{array}$ \\
\hline
\end{tabular}




\begin{tabular}{|c|c|c|}
\hline Pollen type & $\begin{array}{l}\text { Life } \\
\text { form }\end{array}$ & $\begin{array}{l}\text { Notes on geographic distribution, habit, ecological amplitude, } \\
\text { plant communities and uses }\end{array}$ \\
\hline Bilderdykia & Th & $\begin{array}{l}\text { Two spp. belong to this pollen type. } B \text {. convolvulus (black } \\
\text { bindweed), grows on cultivated ground \& in waste places. } B \text {. } \\
\text { dumetorum (copse bindweed) grows in hedges or among native } \\
\text { vegetation. Seeds of black bindweed have been found in a number of } \\
\text { samples from Neolithic Bulgaria and also from Sitagroi, northern } \\
\text { Greece (Renfrew 1973:181). The seeds are bigger than those of } \\
\text { Polygonum aviculare \& it may have been use as a supplementary } \\
\text { food (Renfrew 1973:182). }\end{array}$ \\
\hline Rumex type & Th \& H & $\begin{array}{l}\text { N. temp. Occurs naturally, but is increased by anthropogenic } \\
\text { disturbance. Weeds. Has been cultivated in historical times (Hedrick } \\
\text { 1972) - ?Grevena in prehistory. }\end{array}$ \\
\hline Oxyria digyna & $\mathrm{H}$ & $\begin{array}{l}\text { Alpine. Grows in shady, moist rock crevices \& screes on a few high } \\
\text { peaks in the Pindos, generally near late-lying snow-patches, } 2000- \\
2400 \mathrm{~m} \text {. }\end{array}$ \\
\hline Rumex acetosella group & $\mathrm{H}$ & $\begin{array}{l}\text { Sheep's sorrel is a weed of arable land \& poor sandy soils; grows } \\
\text { most vigorously on lime-deficient land. Lowland to } 2000 \mathrm{~m} \text {, occas. } \\
\text { higher. Observed growing on the peaks of Vasilitsa \& Gomara, as } \\
\text { well as nr Perivoli \& in the Vourinos Mtns. } R \text {. tenuifolius observed } \\
\text { growing in Grevena on burnt steppe \& other dry areas. }\end{array}$ \\
\hline Rumex acetosa group & $\mathrm{H}$ & $\begin{array}{l}\text { Grows mostly in moist meadows, shady slopes \& forest clearings on } \\
\text { a variety of substrates, } 500-2000 \text { m. Seen growing in the Pindos \& } \\
\text { Vourinos Mtns. The seeds of Rumex acetosa (common sorrel) are } \\
\text { commonly found in prehistoric sites in Europe \& the Near East. The } \\
\text { green parts of this wild plant may have been eaten. }\end{array}$ \\
\hline Rumex crispus pp. & $\mathrm{H}$ & $\begin{array}{l}\text { Common lowland weed. Commonly grows as a ruderal, as well as } \\
\text { on river gravels. Rarely occurs in mtns where it is restricted to } \\
\text { ruderal habitats. }\end{array}$ \\
\hline $\begin{array}{l}\text { Rumex conglomeratus } \\
\text { group }\end{array}$ & $\mathrm{H}$ & $\begin{array}{l}\text { Open places from lowlands to mtns e.g. clearings in woods, along } \\
\text { roads \& tracks, as well as slopes \& screes. } R \text {. conglomeratus grows } \\
\text { in damp places. Some spp. are ruderals. Observed growing in the } \\
\text { Pindos \& Vourinos Mtns. }\end{array}$ \\
\hline Rumex obtusifolius group & $\mathrm{H}$ & Mostly ruderals, from lowlands to mtns. \\
\hline $\begin{array}{l}\text { Rumex bucephalophorus } \\
\text { group }\end{array}$ & Th \& H & Lowland ruderal. \\
\hline Emex spinosa & Th & Medit. Disturbed ground. Weed. \\
\hline Chenopodiaceae & $\begin{array}{l}\text { Th } \\
\text { (mostly) } \\
\& \mathrm{H}\end{array}$ & $\begin{array}{l}\text { Weeds. Many ruderals, weeds of cultivation \& waste places, \& } \\
\text { halophytes occuring in a wide variety of habitats. Found mostly in } \\
\text { open habitats esp. steppe. Occurs naturally, but is increased by } \\
\text { anthropogenic disturbance. Chenopodium bonus-henricus is a } \\
\text { montane ruderal in Grevena. Many sp. belonging to this pollen type } \\
\text { have been seen growing in Grevena as ruderals, weeds of cultivation } \\
\& \text { waste places. Seeds of Chenopodium album are often found on } \\
\text { prehistoric sites in Europe, sometimes in large amounts; these seeds } \\
\text { may have been used as a food supplement (has been cultivated in } \\
\text { historical times (Hendrick 1972)). }\end{array}$ \\
\hline Phytolacca americana & Hel & $\begin{array}{l}\text { Trop. \& subtrop. Introduced. Naturalized in S. Eur. Perennial herb. } \\
\text { Marshes \& ruderal places. }\end{array}$ \\
\hline Glinus type & Th & S. Eur. Xerophytic. Waste places. \\
\hline Montia fontana & $\begin{array}{l}\text { Hyd } \\
(\mathrm{Th})\end{array}$ & $\begin{array}{l}\text { Cosmop. Submerged in water or on mud or seasonally wet open } \\
\text { ground, usually calcifuge. }\end{array}$ \\
\hline
\end{tabular}




\begin{tabular}{|c|c|c|}
\hline Pollen type & $\begin{array}{l}\text { Life } \\
\text { form }\end{array}$ & $\begin{array}{l}\text { Notes on geographic distribution, habit, ecological amplitude, } \\
\text { plant communities and uses }\end{array}$ \\
\hline Spergula & Th & $\begin{array}{l}\text { Temp. Common weed of cereal fields. Seeds may be used for human } \\
\text { consumption (Renfrew 1973:187). }\end{array}$ \\
\hline Spergularia type & Th to $\mathrm{H}$ & Cosmop. Mostly halophytes. Dry, sandy habitats. \\
\hline Herniaria type & Th to $\mathrm{H}$ & $\begin{array}{l}\text { Medit., Eur. Lowland to montane. Usually in dry sandy \& rocky } \\
\text { places. Pindos, Vourinos Mtns. }\end{array}$ \\
\hline Corrigiola litoralis & Hel (Th) & $\begin{array}{l}\text { Medit. Seasonally wet sand or clay, often impermanent, usually at } \\
\text { low altitudes, but has been found at } c .1850 \mathrm{~m} \text { on mainland Greece. }\end{array}$ \\
\hline Paronychia type & Th \& H & $\begin{array}{l}\text { Cosmop. Several spp. included. Usually grow in dry, sandy or stony } \\
\text { places from the lowland to mtns. Includes weeds \& ruderals. Have } \\
\text { been observed in Grevena at a variety of altitudes. }\end{array}$ \\
\hline Dianthus type & $\begin{array}{l}\text { Th, } \mathrm{H} \text { or } \\
\mathrm{Chh}\end{array}$ & $\begin{array}{l}\text { Eur., esp. Medit. Many spp. included in this type, occur at all } \\
\text { altitudes \& in many different habitats; steppe to bog. Mostly in dry } \\
\text { sunny situations. Benefits from forest destruction. Includes coppice } \\
\text { plants \& many weeds e.g. Stellaria media is a cornfield weed. Many } \\
\text { observed in Grevena at all elevations. }\end{array}$ \\
\hline Silene alba type & Th \& H & $\begin{array}{l}\text { Eur. Usually occur as weeds of disturbed areas \& cultivation at low } \\
\text { altitudes. }\end{array}$ \\
\hline Lychnis flos-cuculi & Hel & N. temp. Damp meadows \& marshes. \\
\hline Agrostemma githago & Th & Medit. Corncockle is a weed of cornfields. \\
\hline Scleranthus perennis & $\begin{array}{l}\mathrm{H} \text { or } \\
\mathrm{Chh}\end{array}$ & Mountain rocks (800-)1500-2600 m. \\
\hline Scleranthus annuus type & Th or $\mathrm{H}$ & $\begin{array}{l}\text { Eur. Dominant weeds on markedly acid sandy soils where they may } \\
\text { form a complete carpet; characteristic of non-calcareous sands. }\end{array}$ \\
\hline Stellaria holostea & Chh & \\
\hline Nymphaea alba & Hyd (G) & $\begin{array}{l}\text { Trop. \& temp. climates. Perennial herb. Shallow fresh water, } \\
\text { floating ls and fls. Not seen in Grevena. Still (to slow) flow. Up to } 3 \\
\mathrm{~m} \text { deep. }\end{array}$ \\
\hline Nuphar lutea & Hyd (G) & $\begin{array}{l}\text { N. temp. and cold climates. Perennial herb. Shallow fresh water, } \\
\text { floating ls and fls. }\end{array}$ \\
\hline Helleborus & Chh & $\begin{array}{l}\text { Eur., Medit. Open woodland \& pasture, } 700-1900(-2100) \mathrm{m} \text {. Damp } \\
\text { habitats in semi-shade in the foothills \& mtns. Common \& } \\
\text { gregarious in pastures at } c .900-1600 \mathrm{~m} \text { in the Pindos foothills where } \\
\text { it is common. Poisonous \& avoided by grazing animals. }\end{array}$ \\
\hline Nigella & Th & Eur., Medit. Weeds of cereal fields in Grevena. \\
\hline Trollius europaeus & $\mathrm{H}$ & N. temp. \& Arctic. Damp mtn meadows, 1400-2100 m. \\
\hline Actaea spicata & $\mathrm{H}$ or $\mathrm{G}$ & $\begin{array}{l}\text { N. temp. Shady places on loose, humus-rich, calcareous soils } 1100 \text { - } \\
1900 \mathrm{~m} \text {. Woods. }\end{array}$ \\
\hline Adonis & Th & Grassy places \& weeds of cultivated ground. \\
\hline Caltha type & Hel & $\begin{array}{l}\text { Temp. Perennial herbs (mostly). Seasonally flooded places. Marshy } \\
\text { meadows by streams \& brooks, often in partial shade, } 1100-2000 \mathrm{~m} \text {, } \\
\text { on a variety of substrates. }\end{array}$ \\
\hline Ranunculus acris group & Hel & $\begin{array}{l}\text { N. temp. Perennial herbs. Several spp. in this group. Some marsh } \\
\text { plants. Habitat is mostly wet meadows between } 700 \& 1900 \mathrm{~m} \text {. One } \\
\text { ruderal. }\end{array}$ \\
\hline $\begin{array}{l}\text { Ranunculus aquatilis } \\
\text { group }\end{array}$ & $\begin{array}{l}\text { Hyd, H, } \\
\text { Th }\end{array}$ & $\begin{array}{l}\text { Mostly marsh or aquatic plants with a floating stem bearing leaves. } \\
\text { In water up to } 0.5(-1) \mathrm{m} \text { deep; still to swift flow. }\end{array}$ \\
\hline
\end{tabular}




\begin{tabular}{|c|c|c|}
\hline Pollen type & $\begin{array}{l}\text { Life } \\
\text { form }\end{array}$ & $\begin{array}{l}\text { Notes on geographic distribution, habit, ecological amplitude, } \\
\text { plant communities and uses }\end{array}$ \\
\hline $\begin{array}{l}\text { Anemone nemorosa } \\
\text { group }\end{array}$ & Grh & Cosmop. Woods \& shady places between $c .700 \& 1800 \mathrm{~m}$. \\
\hline Clematis vitalba group & $\mathrm{N}$ & Cosmop. Deciduous. Liane. Woods \& bushy places. Pseudo-maquis. \\
\hline Ranunculus arvensis type & Th & R. arvensis is a weed of cereals in Grevena. \\
\hline $\begin{array}{l}\text { Thalictrum } \\
\text { aquilegifolium }\end{array}$ & $\mathrm{H}$ & $\begin{array}{l}\text { N. temp. Woods in mountains, } 800-1700 \mathrm{~m} \text {, but occas. } 2000 \mathrm{~m} \text {. } \\
\text { Damp, rocky \& shady places. }\end{array}$ \\
\hline Thalictrum minus & Hp & $\begin{array}{l}\text { N. temp. Dry rocky places in open woodland, scrub \& alpine } \\
\text { habitats, (300-)800-2000(-2500) m, esp. on limestone. }\end{array}$ \\
\hline Thalictrum lucidum & $\mathrm{H}$ & N. temp. \\
\hline Chelidonium majus & $\mathrm{H}$ & Bushy \& waste places. \\
\hline Papaver rhoeas type & Th & $\begin{array}{l}\text { Eur. Weed of cultivation (common cornfield poppy). Seeds of } \\
\text { Papaver rhoeas are commonly found in prehistoric sites in Europe } \\
\& \text { the Near East. It is collected for flavouring food. A common } \\
\text { annual in arable land. }\end{array}$ \\
\hline Glaucium & Th to $\mathrm{H}$ & Eur. Waste places \& cultivated ground. \\
\hline Papaver argemone type & Th & Eur. Weed of cultivation. \\
\hline Hornungia type & Th to $\mathrm{H}$ & Weeds \& ruderals from lowlands to $1800 \mathrm{~m}$. \\
\hline Arabis & $\mathrm{H}$ & $\begin{array}{l}\text { N. temp. A. glabra \& A. turrita ascend to nearly } 1800 \mathrm{~m} \text { in some } \\
\text { places, but is a typical forest spp. }\end{array}$ \\
\hline Sinapis type & Th to $\mathrm{H}$ & $\begin{array}{l}\text { Eur., Medit. Many genera \& spp. belong to this type. Many are } \\
\text { weeds, a few are marsh plants, some are steppe plants, from } \\
\text { lowlands to mtn pine wood-pasture in Grevena. Brassica nigra, } \\
\text { Erysimum cheiranthoides, Neslia paniculata \& Sinapis arvensis are } \\
\text { weeds of cornfields. Camelina sativa which prob. belongs to this } \\
\text { pollen type was cultivated as an oil-seed crop (Rackham 1990:496). } \\
\text { A bag of S. alba was found in the Late Bronze Age site of } \\
\text { Marmariani, Thessaly. Today this sp. is cultivated to provide salad } \\
\text { leaves, \& also for the manufacture of condiment mustard. }\end{array}$ \\
\hline Umbilicus & $\mathrm{H}$ & Medit. Shaded rocky places in woods. \\
\hline Sedum & $\begin{array}{l}\text { Th, } \mathrm{H} \text { or } \\
\mathrm{Chh}\end{array}$ & $\begin{array}{l}\text { N. temp. Mostly in dry stony \& rocky places, ranging from lowlands } \\
\text { to } 2000 \mathrm{~m} \text { in Pindos. }\end{array}$ \\
\hline $\begin{array}{l}\text { Chrysoplenium } \\
\text { alternifolium }\end{array}$ & $\mathrm{Hel}$ & $\begin{array}{l}\text { Perennial herb of wet, rocky \& shady places, chiefly by small } \\
\text { streams in the upper montane zone }(900-1600 \mathrm{~m} \text {, occas. up to } 1850 \\
\text { m). }\end{array}$ \\
\hline Saxifraga stellaris & Hel & Perennial of mtn streams \& other damp places, $1800-2300 \mathrm{~m}$. \\
\hline $\begin{array}{l}\text { Saxifraga tridactylites } \\
\text { type }\end{array}$ & Th \& H & $\begin{array}{l}\text { Damp shady places in rocky habitats, generally between } 500 \& 1500 \\
\text { (up to 2900) m. Mtn woods \& grassland. }\end{array}$ \\
\hline $\begin{array}{l}\text { Saxifraga oppositifolia } \\
\text { type }\end{array}$ & $\mathrm{H}$ & Mtns. Rocky crevices, damp screes \& ravines, up to $2500 \mathrm{~m}$. \\
\hline Parnassia palustris & Hel & N. temp. Mtn bogs, damp meadows, $600-2100 \mathrm{~m}$. \\
\hline
\end{tabular}




\begin{tabular}{|c|c|c|}
\hline Pollen type & $\begin{array}{l}\text { Life } \\
\text { form }\end{array}$ & $\begin{array}{l}\text { Notes on geographic distribution, habit, ecological amplitude, } \\
\text { plant communities and uses }\end{array}$ \\
\hline Platanus orientalis & $\mathrm{MM}, \mathrm{M}$ & $\begin{array}{l}\text { Native to Balkan peninsula, southwards from c. } 42^{\circ} \mathrm{N} \text {. Deciduous. } \\
\text { Thought to have either profited from changes in habitats caused by } \\
\text { humans or been planted in prehistoric times (Bottema 1982:275). } \\
\text { Today it is often planted as village shade trees. Naturally it grows in } \\
\text { woods in the hills, by rivers. Can grow up to } 1500 \mathrm{~m} \text { if there is } \\
\text { enough water. Long lived. Grows naturally in the riverine vegetation } \\
\text { along the two main rivers of Grevena, the Venetikos \& Aliakmon, } \\
\text { and reaches } 970 \mathrm{~m} \text { in Kranea. }\end{array}$ \\
\hline Filipendula & Hel & N. temp. Perennial herb. Dry grassland, damp or wet places. \\
\hline Rubus type & $\mathrm{N}, \mathrm{Chw}$ & $\begin{array}{l}\text { Cosmop., esp. N. temp. An under shrub in open woods } \& \text { in flushes } \\
\text { on the sides of gullies \& ravines. Forms natural hedges in Grevena. } \\
\text { Seeds from the fruits of } R \text {. idaeus (wild raspberry) \& } R \text {. caesius } \\
\text { (dewberry) have been found in many prehistoric archaeological sites } \\
\text { esp. in the alpine region. }\end{array}$ \\
\hline Sorbus type & $\begin{array}{l}\mathrm{MM}, \mathrm{M}, \\
\mathrm{N}\end{array}$ & $\begin{array}{l}\text { N. temp. Deciduous. Open woods \& open stoney cliffs, } 700-2000 \mathrm{~m} \text {. } \\
\text { Grevena oak woods. }\end{array}$ \\
\hline Rosa & $\mathrm{N}$ & $\begin{array}{l}\text { N. temp. Deciduous \& evergreen. Pindos Mtns. Dry rocky places, } \\
\text { stony meadows, open deciduous or mixed woodland, usually } \\
\text { between } 1000 \text { \& } 1500 \mathrm{~m} \text {, occas. up to } 2500 \mathrm{~m} \text {. Rose hips belonging } \\
\text { to Rosa canina have been found on a number of archaeological sites } \\
\text { in Europe; e.g. Bronze Age in Britian. }\end{array}$ \\
\hline Cotoneaster type & $\mathrm{N}$ & $\begin{array}{l}\text { N. temp. Deciduous. Dry rocky slopes, usually on limestone in } \\
\text { meadows, scrub \& open woodland (including deciduous woods), } \\
\text { mainly in mountains ( } 800-) 1200 \text { to } 2200(-2426) \text { m. }\end{array}$ \\
\hline Prunus avium type & MM & $\begin{array}{l}\text { N. temp. Deciduous. } P \text {. avium \& P. mahaleb grow in the mixed } \\
\text { woods on limestone at Aetia \& Spilaion at } c .1000 \mathrm{~m} \text {, as moderate } \\
\text { sized trees, or as a tall trees of open habit, on rocks \& dry hillsides, } \\
\text { thickets \& open woods. Coppice when cut. Prunus dulcis (almond) } \\
\text { may belong to this pollen type. Almonds are common in Grevena } \\
\text { and are often planted as hedges. Cultivated cherry occurs first in } \\
\text { Europe in Roman times. Stones of wild cherries are found in } \\
\text { archaeological sites in Europe in Neolithic times. } P \text {. mahaleb is used } \\
\text { for firewood. }\end{array}$ \\
\hline Crataegus & M & $\begin{array}{l}\text { N. temp. Deciduous. Grows on rocky mountain slopes \& in barren } \\
\text { soil, in high pasture lands, dry scrubland \& woodland mixed with } \\
\text { other shrubs or trees from lowland up to } 2050 \mathrm{~m} \text {. Grows in steppe } \\
\text { near Mirsina fen \& in pseudo-maquis in Grevena. Fruit stones of } \\
\text { Crataegus sp. are occas. found in prehistoric contexts. }\end{array}$ \\
\hline Sanguisorba officinalis & $\mathrm{Hel}$ & $\begin{array}{l}\text { N. temp. Perennial herb. Damp fields \& streamsides, } 1400-2000 \mathrm{~m} \text {, } \\
\text { chiefly on calcareous substrates. }\end{array}$ \\
\hline $\begin{array}{l}\text { Sanguisorba minor } \\
\text { (Poterium spinosum) }\end{array}$ & $\mathrm{H}$ & $\begin{array}{l}\text { N. temp. Occurs naturally, but is increased by anthropogenic } \\
\text { disturbance. Dry grassy or stony places. Light demanding. Spreads } \\
\text { after forest clearance or extensive grazing. }\end{array}$ \\
\hline Geum & $\mathrm{H}$ & $\begin{array}{l}\text { N. \& S. temp., arctic. Scattered in open woods, at woodland } \\
\text { margins, in ravines \& various disturbed habitats, generally at } \\
\text { altitudes between } 500 \text { \& } 1700 \mathrm{~m} \text {, occas. up to } 2000 \mathrm{~m} \text {. }\end{array}$ \\
\hline Potentilla & $\mathrm{H}$ & $\begin{array}{l}\text { Nearly cosmop., chiefly N. temp. \& arctic. A variety of habitats } \\
\text { from dry \& rocky to damp \& shady from } 500-2400 \mathrm{~m} \text {. P. argentea is } \\
\text { a weed of cultivation \& a ruderal. }\end{array}$ \\
\hline
\end{tabular}




\begin{tabular}{|c|c|c|}
\hline Pollen type & $\begin{array}{l}\text { Life } \\
\text { form }\end{array}$ & $\begin{array}{l}\text { Notes on geographic distribution, habit, ecological amplitude, } \\
\text { plant communities and uses }\end{array}$ \\
\hline Fragaria vesca type & $\mathrm{H}$ & $\begin{array}{l}\text { N. Hemisph. In Grevena grows under deciduous oak woods. Wild } \\
\text { strawberry seeds have been found at many prehistoric archaeological } \\
\text { sites in Europe. }\end{array}$ \\
\hline Alchemilla type & Th \& H & $\begin{array}{l}\text { Temp. \& trop. Open habitats, cultivated fields, meadows, pastures, } \\
\text { forest margins, } 1500-2350 \mathrm{~m} \text {. }\end{array}$ \\
\hline Hedysarum coronarium & $\mathrm{H}$ & N. temp. Cultivated ground. \\
\hline Ononis type & $\begin{array}{l}\text { Th } \\
\text { (mostly), } \\
\text { H, N }\end{array}$ & $\begin{array}{l}\text { Deciduous. Temp. to sub-trop; Medit., Eur. \& S. Eur. to Himal. } \\
\text { Mostly weeds or ruderals in open disturbed ground, steppe \& open } \\
\text { woods. }\end{array}$ \\
\hline Trifolium dubium type & $\begin{array}{l}\text { Th } \\
\text { (mostly) } \\
\text { to } \mathrm{H}\end{array}$ & $\begin{array}{l}\text { Temp. \& subtrop. In scrub, rocky woodland \& ruderal habitats; } \\
\text { occurring above treeline in dry, rocky, often overgrazed grassland, } \\
\text { on cliffs \& screes \& by tracksides. At lower altitudes in dry or damp } \\
\text { places \& waste places. Sea level to } 2100 \mathrm{~m} \text {. }\end{array}$ \\
\hline Trifolium repens type & Th \& H & $\begin{array}{l}\text { Temp. \& subtrop. Dry grassy places, disturbed places, fields, } \\
\text { cultivated ground. Steppe in oak woods in Grevena. }\end{array}$ \\
\hline Lotus type & Th \& H & $\begin{array}{l}\text { Temp. Eur. Many spp. included in this type. Occurs mostly in } \\
\text { grassland. L. uliginosus is a marsh plant. Tetragonologus purpureus } \\
\text { is a weed of cultivation. Trigonella foenum-graecum ?cultivated in } \\
\text { Greece for fodder. Pindos \& Vourinos Mtns. }\end{array}$ \\
\hline Lathyrus pratensis type & Th \& H & $\begin{array}{l}\text { N. temp. Mostly dry places, grasslands, fields, cultivated ground. } \\
\text { Sea level to } 2100 \mathrm{~m} \text {. L. pratensis is an exception in growing in } \\
\text { marshes. Edge of oak woods in Grevena. }\end{array}$ \\
\hline Vicia sativa type & Th \& H & $\begin{array}{l}\text { N.temp. This pollen type includes several wild spp. as well as one } \\
\text { cultivated sp. ( } \& \text { one that prob. belongs to this type } V \text {. ervilia) and a } \\
\text { weed of cultivation ( } \& V \text {. pannonica which prob. belongs to this } \\
\text { type). V. sativa (vetch) was cultivated in the Pindos foothills \& on } \\
\text { the Grevena plains before } 1940 \text { for fodder on a subsistence basis. It } \\
\text { is no longer cultivated to the same extent. Seeds of domesticated } V \text {. } \\
\text { ervilia (bitter vetch) were found in Macedonia at the archaeological } \\
\text { sites of Nea Nikomedeia \& Sitagroi in contexts dating to the } \\
\text { Neolithic (Hansen 1988). The seeds are thought to have been used } \\
\text { for human consumption (Renfrew 1973:116). }\end{array}$ \\
\hline Vicia cracca type & Th \& H & Grassy places, disturbed ground, cultivated ground. \\
\hline Astragalus & $\begin{array}{l}\text { Th \& H } \\
\text { (mostly) }\end{array}$ & $\begin{array}{l}\text { Cosmop. On a variety of substrates; on mtn rocks, dry rocky slopes, } \\
\text { pasture \& steppe and }+/ \text { - xerophytic, up to } 2400 \mathrm{~m} \text {. Often thorny. } \\
\text { Grows in steppe in oak wood pasture in Grevena. }\end{array}$ \\
\hline Galega officinalis & Hel & $\begin{array}{l}\text { S. Eur. Perennial herb. Sometimes cultivated as a fodder plant. } \\
\text { Grows naturally in damp meadows \& by rivers. }\end{array}$ \\
\hline Medicago sativa & $\mathrm{H}$ & $\begin{array}{l}\text { Eur., Medit. Medicago sativa sativa (alfalfa, lucerne) was cultivated, } \\
\text { for fodder \& forage, in the Pindos foothills and on the Grevena } \\
\text { plains prior to the introduction of mechanized agriculture. It is } \\
\text { cultivated on a small scale today mostly in the plains. Unfortunately, } \\
\text { M. sativa sativa pollen may occur in the Trifolium montanum and } \\
\text { the Medicago sativa pollen types. Also Medicago sativa is native to } \\
\text { almost all of Europe and it is not possible to distinguish the } \\
\text { cultivated subsp. from the native. The origin of } M \text {. sativa sativa is } \\
\text { uncertain. }\end{array}$ \\
\hline Ornithopus compressus & Th & Medit. Rocks, waysides. \\
\hline Dorycnium & $\begin{array}{l}\text { Chw or } \\
\mathrm{H}\end{array}$ & Medit. Woods, damp bushy places. \\
\hline
\end{tabular}




\begin{tabular}{|c|c|c|}
\hline Pollen type & $\begin{array}{l}\text { Life } \\
\text { form }\end{array}$ & $\begin{array}{l}\text { Notes on geographic distribution, habit, ecological amplitude, } \\
\text { plant communities and uses }\end{array}$ \\
\hline Trifolium montanum type & $\mathrm{H}$ & $\begin{array}{l}\text { T. montanum is new to Greece. This pollen type may also include } \\
\text { Medicago sativa (see above). }\end{array}$ \\
\hline Lathyrus sativus & $\mathrm{Th}$ & $\begin{array}{l}\text { N. temp. The origin of this sp. is unknown, it is widely naturalized. } \\
\text { From oral history it is known to have been cultivated in the Pindos } \\
\text { foothills \& the Grevena plains for fodder on a subsistence basis } \\
\text { before } 1940 \text {. Seeds of } L \text {. sativus (grass pea) are found quite widely } \\
\text { in archaeological sites and may have been cultivated for both human } \\
\text { consumption \& fodder. They have been found on Early Neolithic } \\
\text { sites in Europe. }\end{array}$ \\
\hline Oxalis & $\begin{array}{l}\mathrm{G}, \mathrm{H} \text { or } \\
\mathrm{Th}\end{array}$ & $\begin{array}{l}\text { Cosmop. Dry open habitats, esp. cultivated ground, waste places, } \\
\text { disturbed ground. }\end{array}$ \\
\hline Geranium & $\begin{array}{l}\text { Th to H } \\
\text { (mostly) }\end{array}$ & $\begin{array}{l}\text { Cosmop. esp. temp. Ruderal; often in sheep-manured habitats. Dry } \\
\text { rocky places, gravelly slopes, shady places in ravines, woodland, } \\
\text { meadows, cultivated ground \& waste places, } 500-2200 \mathrm{~m} \text {. Mtn pine } \\
\text { wood-pasture \& oak woods in Grevena. }\end{array}$ \\
\hline $\begin{array}{l}\text { Geranium robertianum } \\
\text { group }\end{array}$ & $\mathrm{Th}, \mathrm{H}$ & $\begin{array}{l}\text { Ruderal; often in sheep-manured habitats. Dry rocky places, gravelly } \\
\text { slopes, shady places in ravines, woodland, meadows, cultivated } \\
\text { ground \& waste places, } 500-2200 \mathrm{~m} \text {. }\end{array}$ \\
\hline Tribulus terrestris & Th & $\begin{array}{l}\text { Medit. Weed \& ruderal. Dry habitats, waste places, waysides \& } \\
\text { cultivated ground. }\end{array}$ \\
\hline Linum usitatissimum type & Th to $\mathrm{H}$ & $\begin{array}{l}\text { S. \& W. Eur. Of the } 2 \mathrm{spp} \text {. in this pollen type } L \text {. bienne is a wild } \\
\text { biennial to perennial while } L \text {. usitatissimum, flax, is of uncertain } \\
\text { origin. L. bienne grows in grassy, stony places. Flax was formerly } \\
\text { cultivated throughout most of Eur. for fibre \& oil from the seed } \\
\text { (linseed); now much less commonly grown, but still recorded as a } \\
\text { casual throughout Europe. Macrofossils of } L \text {. usitatissimum are } \\
\text { found in archaeological sites dating to the Neolithic. Alarge number } \\
\text { of flax seeds were found in Early Bronze Age deposits at Lerna, } \\
\text { Argolid. }\end{array}$ \\
\hline Linum austriacum type & $\mathrm{H}$ & $\begin{array}{l}\text { Temp. \& subtrop., esp. Medit. Rocky \& stony slopes, dry meadows } \\
\& \text { pastures from lowland to c. } 2000 \text { m. Pindos Mtns. }\end{array}$ \\
\hline Radiola linoides & Hel (Th) & Medit. Seasonally damp, bare, sandy or peaty ground; calcifuge. \\
\hline Linum catharticum & Hel (Th) & $\begin{array}{l}\text { Medit. Annual herb. Marshy slopes \& moist, rocky places by } \\
\text { streams, } 1200-2200 \mathrm{~m} \text {, rarely at lower altitudes. On various basic } \\
\text { substrates. Pindos Mtns. }\end{array}$ \\
\hline Linum tenuifolium & $\begin{array}{l}\mathrm{H} \text { or } \\
\mathrm{Chh}\end{array}$ & $\begin{array}{l}\text { Temp. \& subtrop., esp. Medit. Rocky places \& dry, stony meadows, } \\
\text { from lowland to c. } 1900 \text { m, on limestone. Pindos \& Vourinos Mtns. }\end{array}$ \\
\hline Mercurialis perennis & $\mathrm{H}$ & $\begin{array}{l}\text { Medit, Eur. Woods, shady places. Usually in Quercus or Fagus } \\
\text { woods. }\end{array}$ \\
\hline Euphorbia & $\begin{array}{l}\text { Th, H, } \\
\text { Chw }\end{array}$ & $\begin{array}{l}\text { Chiefly subtrop. \& warm temp. Many spp. included in this pollen } \\
\text { type, from a variety of habitats of both dry \& damp places. Many are } \\
\text { ruderals \& weeds of cultivation. E. salicifolia is unpalatable. }\end{array}$ \\
\hline Dictamnus albus & $\mathrm{H}$ & $\begin{array}{l}\text { Eur. Volatile and inflammable ethereal oil is secreted, so that on hot } \\
\text { calm days the air round the plant may sometimes be ignited. Grows } \\
\text { at the edge of deciduous oak woods \& in the patches of steppe } \\
\text { grassland in the oak woods in the Grevena plains. }\end{array}$ \\
\hline Rhus coriaria & M & $\begin{array}{l}\text { Subtrop. \& warm temp. Semi-evergreen. Grows amongst brushwood } \\
\& \text { in rocky places at low \& medium altitudes. Rocky places, } \\
\text { waysides. Palatable to stock. }\end{array}$ \\
\hline
\end{tabular}




\begin{tabular}{|c|c|c|}
\hline Pollen type & $\begin{array}{l}\text { Life } \\
\text { form }\end{array}$ & $\begin{array}{l}\text { Notes on geographic distribution, habit, ecological amplitude, } \\
\text { plant communities and uses }\end{array}$ \\
\hline Pistacia & $\mathrm{M}, \mathrm{N}$ & $\begin{array}{l}\text { Medit. Evergreen }(P \text {. lentiscus) \& deciduous ( } P \text {. terebinthus). Dry } \\
\text { open scrub \& woods, usually calcareous substrate. } P \text {. lentiscus is } \\
\text { very sensitive to cold. } P \text {. terebinthus grows in pseudo-maquis in SE } \\
\text { Grevena \& Pindhos foothills in mixed woods. Quick growing. Takes } \\
\text { advantage of destruction of primary forests. }\end{array}$ \\
\hline Cotinus coggygria & $\mathrm{N}$ & $\begin{array}{l}\text { N. temp. Deciduous. Usually grows on dry hills, rocky places \& } \\
\text { open woods. Low altitude scrubland. On calcareous soil. Shibljak. In } \\
\text { Grevena, forms shrubbery on eroding slopes too unstable for trees to } \\
\text { be established \& without enough soil for grassland. }\end{array}$ \\
\hline Acer & $\begin{array}{l}\mathrm{N} \text { to } \\
\mathrm{MM}\end{array}$ & $\begin{array}{l}\text { N. temp. Deciduous. Acer grows in damp woods in Grevena such as } \\
\text { in the hornbeam (Carpinus) woods in the eastern plains \& the mixed } \\
\text { woods at Aetia \& Spilaion at } c \text {. } 1000 \mathrm{~m} \text {. Also in the Abies/Fagus } \\
\text { wood between Kranea \& Milea, as well as dry pseudo-maquis in the } \\
\text { SE corner. Shade tolerant. Used for firewood. }\end{array}$ \\
\hline Ilex aquifolium & $\mathrm{M}$ or $\mathrm{N}$ & $\begin{array}{l}\text { Eur. Evergreen. Montane woods \& pseudo-maquis. An understorey } \\
\text { tree in beech \& oak woods. Very flammable, the waxy leaves will } \\
\text { burn fiercely, even on the living tree, but suckers will rise quickly in } \\
\text { great numbers if the soil is not heated too much. Not subject to cattle } \\
\text { grazing. Susceptible to cold winters. }\end{array}$ \\
\hline Buxus sempervirens & $\mathrm{M}$ or $\mathrm{N}$ & $\begin{array}{l}\text { Evergreen. Open dry woodland, pseudo-maquis \& scrub on } \\
\text { serpentine or limestone, from } 400-2000 \mathrm{~m} \text {. Gregarious \& locally } \\
\text { abundant. Pyrophytic: regenerates quickly after burning by shoots } \\
\text { from roots or stem bases. In Grevena box is common in mtn pine } \\
\text { woods \& beech woods, \& oak woods of the foothills. It replaces } \\
\text { juniper as an understorey, on more calcareous soils. It occurs only as } \\
\text { a shrub in Grevena. }\end{array}$ \\
\hline Frangula & $\mathrm{M}$ or $\mathrm{N}$ & $\begin{array}{l}\text { Cosmop. Deciduous. Damp shady places. Rocks, bushy places in } \\
\mathrm{mtns}, 500-1500 \mathrm{~m} \text {, sometimes alpine habitats up to } 2100 \mathrm{~m} \text {. }\end{array}$ \\
\hline Rhamnus type & $\mathrm{M}$ or $\mathrm{N}$ & $\begin{array}{l}\text { Cosmop. Deciduous. Usually grows on dry slopes, brushwood on } \\
\text { limestone rocks, } 500-2300 \mathrm{~m} \text {. Paliurus spina-christi grows in } \\
\text { pseudo-maquis in SE Grevena. }\end{array}$ \\
\hline Vitis vinifera & $\mathrm{M}$ or $\mathrm{N}$ & $\begin{array}{l}\text { N. Hemisph. Liane. Woods \& brushwood. This pollen type includes } \\
\text { both a wild \& a cultivated subspecies. The wild subsp. grows on } \\
\text { river banks \& in damp woods. Domesticated vine seeds have been } \\
\text { identified at Sitagroi, eastern Macedonia dating to the Early Bronze } \\
\text { Age \& also in archaeological sites in Thessaly (Iolkos) \& central } \\
\text { Macedonia (Kastanas) dated to the Late Bronze Age (Renfrew } \\
\text { 1973:28; Hansen 1988). The cultivated subsp. is currently cultivated } \\
\text { in Grevena for household use only, but it is known to have been } \\
\text { cultivated much more in the past, esp. around Spilaion where it was } \\
\text { produced beyond subsistence needs and thus was exported. }\end{array}$ \\
\hline Tilia platyphyllos & MM & $\begin{array}{l}\text { N. temp. Deciduous. Woods, bushy places. Shade tolerant. Very } \\
\text { palatable to stock. }\end{array}$ \\
\hline Tilia cordata type & MM & N. temp. Deciduous. Woods of mtns. Shade tolerant. Very palatable. \\
\hline Abutilon type & Th \& H & Trop. \& subtrop. Cultivated ground \& waste damp places. \\
\hline Althaea officinalis & Hel & $\begin{array}{l}\text { Temp. Marsh mallow grows in damp places, field verges. } \\
\text { Unpalatable. }\end{array}$ \\
\hline Malva moschata & $\mathrm{H}$ & $\begin{array}{l}\text { N. temp. Cultivated and stony ground. Normally a sp. of low \& } \\
\text { moderate altitudes, has been found up to } 1850 \text { m. Pindos Mtns. }\end{array}$ \\
\hline
\end{tabular}




\begin{tabular}{|c|c|c|}
\hline Pollen type & $\begin{array}{l}\text { Life } \\
\text { form }\end{array}$ & $\begin{array}{l}\text { Notes on geographic distribution, habit, ecological amplitude, } \\
\text { plant communities and uses }\end{array}$ \\
\hline Daphne & $\mathrm{N}$, Chw & $\begin{array}{l}\text { Eur., temp. and subtrop. Evergreen \& deciduous. Mostly high } \\
\text { altitude up to } 2100 \mathrm{~m} \text {, in open woodland or stony slopes or } \\
\text { meadows. D. oleoides grows as an undershrub in grazed Pinus } \\
\text { heldreichii woods in the Pindos Mtns. }\end{array}$ \\
\hline Elaeagnus angustifolia & $\mathrm{M}$ or $\mathrm{N}$ & $\begin{array}{l}\text { Eur. Deciduous. Not native. Naturalized in Greece. Grows nr water; } \\
\text { banks of streams \& waterfalls, in river silt. Planted for its fruit \& } \\
\text { fragrant blossoms. }\end{array}$ \\
\hline Hypericum hircinum type & Chw & Temp. Damp places, often beside rivers. \\
\hline $\begin{array}{l}\text { Hypericum perforatum } \\
\text { type }\end{array}$ & $\mathrm{H}$ & Temp. A variety of habitats, at all elevations. \\
\hline Viola hirta type & $\mathrm{H}$ & $\begin{array}{l}\text { Cosmop., chiefly N. temp. Damp grassland, scrub \& woodland, } \\
\text { generally below } 1000 \mathrm{~m} \text {. }\end{array}$ \\
\hline Viola riviniana type & $\mathrm{H}$ & Cosmop., chiefly N. temp. Woodlands. \\
\hline Viola arvensis type & Th & $\begin{array}{l}\text { Cosmop., chiefly N. temp. Weeds of fields \& open scrubland below } \\
\text { c. } 1100 \mathrm{~m} .\end{array}$ \\
\hline Helianthemum & $\begin{array}{l}\text { Chw \& } \\
\text { Th }\end{array}$ & $\begin{array}{l}\text { Eur., Medit. Dry stony alpine \& grassy pastures, scrub \& woodland } \\
\text { clearings, } 400 \text { to } 2400 \mathrm{~m} \text {, on a variety of substrates, but most } \\
\text { frequently on limestone. }\end{array}$ \\
\hline Cistus monspeliensis type & $\mathrm{N}$ & $\begin{array}{l}\text { Medit. Dry sunny places, scrub, open woods, uncultivated ground, } \\
\text { garrigue \& maquis. Pyrophytic. C. incanus, which prob. belongs to } \\
\text { this type, occurs as an undershrub in the steppe of the oak woods in } \\
\text { the Grevena plains. }\end{array}$ \\
\hline Myricaria germanica & $\mathrm{N}$ & $\begin{array}{l}\text { N. temp. River gravels \& other open habitats. Forms thickets in } \\
\text { riverine vegetation along the Aliakmon \& Venetikos. }\end{array}$ \\
\hline Tamarix & $\mathrm{M}$ or $\mathrm{N}$ & $\begin{array}{l}\text { Eur., Medit. Halophytic. Damp places, river banks, hedges \& } \\
\text { roadsides, from lowlands to mountains. } T \text {. pariflora (Balkan } \\
\text { tamarisk) forms thickets in the riverine vegetation along the } \\
\text { Aliakmon \& Venetikos. }\end{array}$ \\
\hline Elatine alsinastrum & $\begin{array}{l}\mathrm{Hel}(\mathrm{Th} \\
\text { or H) }\end{array}$ & $\begin{array}{l}\text { Temp. Annual or perennial. Shallow, usually still water, on wet mud } \\
\text { or sand, or in seasonally flooded places. Submerged roots. }\end{array}$ \\
\hline Lythrum salicaria type & $\begin{array}{l}\text { Hel }(\mathrm{Th} \\
\& \mathrm{H})\end{array}$ & Cosmop. Fens, marshes, riversides. Unpalatable. \\
\hline Lythrum portula & $\mathrm{Hel}(\mathrm{Th})$ & Cosmop. Wet places \& shallow pools. \\
\hline Trapa natans & Hyd & $\begin{array}{l}\text { C. \& S. Eur. Annual water plant with floating is and submerged } \\
\text { roots. In nutrient rich but not strongly calcareous water. The fruits } \\
\text { were used for food in the Alpine region in prehistoric times. }\end{array}$ \\
\hline Myrtus communis & M & $\begin{array}{l}\text { Medit. \& SW Eur. Evergreen. Usually grows only on limestone. } \\
\text { Brushy places, damp ground. }\end{array}$ \\
\hline Circaea lutetiana & G & N. temp. and arctic. Woody \& shady places. \\
\hline Ludwigia palustris & Hyd & $\begin{array}{l}\text { Cosmop. Perennial water plant; still \& flowing waters, seasonally } \\
\text { flooded places. }\end{array}$ \\
\hline $\begin{array}{l}\text { Epilobium angustifolium } \\
\text { type }\end{array}$ & $\mathrm{H}$ & $\begin{array}{l}\text { Temp. \& arctic. E. angustifolium grows in woods \& shady places, is } \\
\text { common in woodland clearings, esp. if fire is used. Oenothera } \\
\text { biennis grows on disturbed ground, waste places, dunes. }\end{array}$ \\
\hline Epilobium hirsutum type & Hel & $\begin{array}{l}\text { Temp. \& arctic. Perennial herbs, mostly along margins of water } \\
\text { courses, fens \& other wet, \& disturbed places. Usually at low } \\
\text { altitude, but locally up to } c .2200 \text { m. Pindos Mtns. }\end{array}$ \\
\hline
\end{tabular}




\begin{tabular}{|c|c|c|}
\hline Pollen type & $\begin{array}{l}\text { Life } \\
\text { form }\end{array}$ & $\begin{array}{l}\text { Notes on geographic distribution, habit, ecological amplitude, } \\
\text { plant communities and uses }\end{array}$ \\
\hline $\begin{array}{l}\text { Myriophyllum } \\
\text { verticillatum }\end{array}$ & Hyd & $\begin{array}{l}\text { Cosmop. Perennial herb. Aquatic. Submerged freshwater plant. Still } \\
\text { to slow flow. Shallow to } 1 \mathrm{~m} \text {. }\end{array}$ \\
\hline Myriophyllum spicatum & Hyd & $\begin{array}{l}\text { Cosmop. Perennial herb. Aquatic. Submerged freshwater plant. Still } \\
\text { to swift. Up to } 2 \mathrm{~m} \text {. }\end{array}$ \\
\hline Theligonum cynocrambe & Th & $\begin{array}{l}\text { Stony \& dry places, dry rocks \& walls \& their crevices. Arable } \\
\text { fields \& ruderal places. }\end{array}$ \\
\hline Cornus sanguinea & $\mathrm{N}$ & $\begin{array}{l}\text { N. temp. Deciduous. Woods, bushy places. Oak woods near Mirsina } \\
\text { fen. }\end{array}$ \\
\hline Cornus mas & $\mathrm{N}, \mathrm{M}$ & $\begin{array}{l}\text { Eur. Deciduous. Light-demanding. Open woods, bushy places. } \\
\text { Brushwood. Steppe/oak woods of the Grevena plains, nr the } \\
\text { Aliakmon River. Cornelian cherry stones have been found in } \\
\text { Neolithic deposits at Nea Nikomedeia (van Zeist \& Bottema 1971) } \\
\text { \& Sitagroi, northern Greece (Renfrew 1973:142). The fruits may be } \\
\text { eaten fresh or preserved or made into brandy, as they do in northern } \\
\text { Greece (Renfrew 1973:142). }\end{array}$ \\
\hline Hedera helix & Chw & $\begin{array}{l}\text { Temp. Evergreen. Climbing on trees, rocks, \& walls, or covering the } \\
\text { ground in woods. Pseudo-maquis. }\end{array}$ \\
\hline Hydrocotyle vulgaris & Hyd & $\begin{array}{l}\text { Temp. Aquatic; shallow, still (slow moving), fresh water \& damp } \\
\text { places. }\end{array}$ \\
\hline Anthriscus caucalis & Th & Eur. Dry places. \\
\hline Smyrnium & $\mathrm{H}$ & $\begin{array}{l}\text { Medit., Eur. Rocky slopes, meadows, scrub, woodland, roadsides \& } \\
\text { cultivated places from lowlands up to } 2200(-2400) \mathrm{m} \text {, chiefly on } \\
\text { limestone but also on shist; sometimes gregarious in manured places. } \\
\text { Weeds \& ruderals. }\end{array}$ \\
\hline Ligusticum & $\mathrm{H}$ & N. Hemisph. Rocky, gravelly places, $1800-2050 \mathrm{~m}$. \\
\hline Bupleurum & $\begin{array}{l}\mathrm{N}, \mathrm{H}, \mathrm{Th} \\
\text { (mostly) }\end{array}$ & $\begin{array}{l}\text { Eur. Dry open habitats such as cultivated land, waste places, } \\
\text { waysides. Weed. }\end{array}$ \\
\hline Sison amomum & $\mathrm{H}$ & Eur., Medit. \\
\hline Carum & $\mathrm{H}$ & $\begin{array}{l}\text { Temp., subtrop. Rocky slopes, meadows \& pastures, from lowlands } \\
\text { to } 2650 \mathrm{~m} \text {. }\end{array}$ \\
\hline Berula erecta & Hel & Perennial herb of shallow water. Still to fast flow. \\
\hline Trinia & $\mathrm{H}$ & $\begin{array}{l}\text { Eur., Medit. Gravelly places, rocky \& stony meadows \& pastures, } \\
\text { slopes in open woodland, } 500-2650 \mathrm{~m} \text {. Vasilitsa, Pindos Mtns. }\end{array}$ \\
\hline Sanicula europaea & $\mathrm{H}$ & $\begin{array}{l}\text { Cosmop. Woods in mtns. Grows under Abies borisii woods at } 2000 \\
\text { m above Samarina. }\end{array}$ \\
\hline Conium maculatum & $\mathrm{H}$ or Th & N. Temp. Weed. \\
\hline Cnidium silaifolium & $\mathrm{H}$ & $\begin{array}{l}\text { Scree, rocky \& stony meadows, slopes in open woodland \& forest, } \\
400-2200 \mathrm{~m} \text {, on a variety of substrates. }\end{array}$ \\
\hline Seseli type & $\mathrm{H}$ & $\begin{array}{l}\text { Eur. Grassy slopes in open woodland \& meadows \& open rock } \\
\text { ledges, } 800-2100 \mathrm{~m} \text {, on a variety of substrates. Pindos Mtns. }\end{array}$ \\
\hline Oenanthe aquatica group & Hyd & $\begin{array}{l}\text { N. temp. Perennial. } O \text {. aquatica grows in still or slow flowing water } \\
\text { of seasonally wet places \& } O \text {. lachenalii in wet grassland. }\end{array}$ \\
\hline $\begin{array}{l}\text { Oenanthe pimpinelloides } \\
\text { group }\end{array}$ & $\mathrm{Hel}$ & $\begin{array}{l}\text { N. temp. Perennial herbs. The habitat of } O \text {. pimpinelloides is not } \\
\text { known, but } O \text {. tenuifolia, which might belong to this pollen type, } \\
\text { grows in marshes. }\end{array}$ \\
\hline Oenanthe fistulosa & Hel & N. temp. Perennial herb of wet places, shallow water. \\
\hline
\end{tabular}




\begin{tabular}{|c|c|c|}
\hline Pollen type & $\begin{array}{l}\text { Life } \\
\text { form }\end{array}$ & $\begin{array}{l}\text { Notes on geographic distribution, habit, ecological amplitude, } \\
\text { plant communities and uses }\end{array}$ \\
\hline $\begin{array}{l}\text { Chaerophyllum hirsutum } \\
\text { type }\end{array}$ & $\mathrm{H}$ & $\begin{array}{l}\text { N. temp. In clearings \& meadows in woods up to } 1800 \mathrm{~m} \text {, rarely in } \\
\text { alpine pastures up to } 2000 \mathrm{~m} \text {. Pindos Mtns. }\end{array}$ \\
\hline $\begin{array}{l}\text { Chaerophyllum } \\
\text { temulentum type }\end{array}$ & $\mathrm{H}$ & N. temp. Weed. \\
\hline$A m m i$ & Th or $\mathrm{H}$ & Medit. Weeds. \\
\hline Pimpinella saxifraga & $\mathrm{H}$ & Old World. Mtn meadows \& ravines in woodland, up to $1800 \mathrm{~m}$. \\
\hline Torilis arvensis & Th & Medit. \\
\hline Anethum graveolens & $\mathrm{Th}$ & Medit. \\
\hline Falcaria vulgaris & $\mathrm{H}$ & Medit. \\
\hline $\begin{array}{l}\text { Peucedanum austriacum } \\
\text { type }\end{array}$ & $\mathrm{H}$ & Eur. Pastures, rocky meadows, open woodland, $900-2200 \mathrm{~m}$. \\
\hline Angelica sylvestris & Hel & $\begin{array}{l}\text { N. Hemisph. Perennial herb. Damp, shady places by streams, } \\
\text { medium altitude, ascending to } c .1800 \mathrm{~m} \text {. }\end{array}$ \\
\hline Peucedanum schotti & $\mathrm{H}$ & $\begin{array}{l}\text { Eur. Grassy meadows \& rocky, grassy slopes in Pinus woodland, } \\
1200-1700 \mathrm{~m} \text {, on ophiolithic substrates, occas. on schist. }\end{array}$ \\
\hline Bunium & $\mathrm{H}$ & Serpentine screes \& gravel, c. 1700 m. Pindos Mtns. \\
\hline $\begin{array}{l}\text { Foeniculum vulgare ssp. } \\
\text { vulgare }\end{array}$ & $\mathrm{H}$ & Medit., Eur. Dry rocky places. \\
\hline Anthriscus sylvestris type & $\mathrm{H}$ & $\begin{array}{l}\text { Eur. In clearings, meadows \& by streams in montane \& submontane } \\
\text { woodland from } c .400-1850 \mathrm{~m} \text {, predominantly on calcareous } \\
\text { substrates, but also on serpentine. }\end{array}$ \\
\hline Pastinaca sativa & $\mathrm{H}$ & $\begin{array}{l}\text { N. temp. Meadows, woodland clearings up to } 1500 \mathrm{~m} \text { (rarely } 1950 \\
\text { m). }\end{array}$ \\
\hline Eryngium & $\mathrm{H}$ & $\begin{array}{l}\text { Trop. \& temp. Dry places \& woods. Often gregarious in pastures, } c \text {. } \\
500-2050 \mathrm{~m} \text {. E. amethystinum grows in the Pindos foothills in the } \\
\text { oak wood-pasture and oak pasture vegetation zone. Pindos \& } \\
\text { Vourinos Mtns. }\end{array}$ \\
\hline Scandix type & Th & $\begin{array}{l}\text { Eur., Medit. Open habitats, often as weeds. Cultivated \& disturbed } \\
\text { ground. }\end{array}$ \\
\hline Heracleum sphondylium & $\mathrm{H}$ & $\begin{array}{l}\text { N. temp. \& trop. One subsp. grows in open woodland, rocky \& } \\
\text { gravelly slopes, by mtn roads } 700-2200(-2500) \mathrm{m} \text {, on a variety of } \\
\text { substrates. Another subsp. grows in damp woodland, meadows \& } \\
\text { streamsides, } 500-1950 \mathrm{~m} \text {, on limestone \& schist. Pindos Mtns. }\end{array}$ \\
\hline Pimpinella anisum type & Th & $\begin{array}{l}\text { Old World. Widely cultivated for its aromatic fruits \& often } \\
\text { naturalized. Pimpinella anisum (anise) was found in the Late Bronze } \\
\text { Age settlement at Thera-Therasia, Greece. It may have been } \\
\text { collected for flavouring food. Two wild spp. which may belong to } \\
\text { this type grow in dry places. One of these P. tragium grows in the } \\
\text { Pindos (Vasilitsa) \& Vourinos Mtns. }\end{array}$ \\
\hline Anthriscus cerefolium & Th & $\begin{array}{l}\text { Eur. In Europe var. cerefolium cultivated as a herb \& often } \\
\text { naturalized. Not observed by author in Grevena. }\end{array}$ \\
\hline Torilis japonica type & Th to $\mathrm{H}$ & Medit. Weed; may occur in arable fields. \\
\hline Daucus & Th or $\mathrm{H}$ & $\begin{array}{l}\text { Eur. Weed; may occur in arable fields. Seeds of wild carrot have } \\
\text { been found in Neolithic and Bronze Age deposits in Switzerland; the } \\
\text { tuberous root may have been used for human consumption (Renfrew } \\
\text { 1973:171). }\end{array}$ \\
\hline
\end{tabular}




\begin{tabular}{|c|c|c|}
\hline Pollen type & $\begin{array}{l}\text { Life } \\
\text { form }\end{array}$ & $\begin{array}{l}\text { Notes on geographic distribution, habit, ecological amplitude, } \\
\text { plant communities and uses }\end{array}$ \\
\hline Coriandrum type & Th & $\begin{array}{l}\text { Medit. C. sativum is cultivated for its aromatic fruits \& widely } \\
\text { naturalized in Europe. C. sativum seeds have occas. been found in } \\
\text { prehistoric deposits. They were found in large quantity at Sitagroi, } \\
\text { northern Greece, in an Early Bronze Age level. It may have been } \\
\text { collected for flavouring food (Renfrew 1973:171). }\end{array}$ \\
\hline Pyrola & $\begin{array}{l}\mathrm{H} \& \\
\mathrm{Chh}\end{array}$ & $\begin{array}{l}\text { N. temp. Woods \& alpine meadows, c. } 1800 \mathrm{~m} \text {. Pindos Mtns } \\
\text { (Perivolion, Vasilitsa). }\end{array}$ \\
\hline Erica herbacea & Chw & $\begin{array}{l}\text { Eur. esp. Medit. Evergreen. Grows in the Pindos Mtns \& foothills on } \\
\text { rocky slopes, usually in open Pinus nigra \& } P \text {. heldreichii } \\
\text { woodland, } 1000 \text { to } 1900 \mathrm{~m} \text {, on serpentine, and also in beech woods, } \\
600-1600 \mathrm{~m} \text {. }\end{array}$ \\
\hline Vaccinium myrtillus & Chw & $\begin{array}{l}\text { N. Hemisph. Deciduous. Mainly in open, somewhat damp subalpine } \\
\text { grassland or heath, occas. in woodland }(900) 1600-2200 \text { m, chiefly } \\
\text { on shist \& granite where it may be gregarious \& dominant, rarely on } \\
\text { limestone or serpentine. Bilberries have been recovered from a few } \\
\text { archaeological sites in temp. Europe. }\end{array}$ \\
\hline Soldanella & $\mathrm{H}$ & $\begin{array}{l}\text { Alps of Eur. Damp montane places, } 1600-2400 \mathrm{~m} \text {. Pindos Mtns. The } \\
\text { fls expand at very low temperatures, often coming up through the } \\
\text { snow. }\end{array}$ \\
\hline Cyclamen & Gt & $\begin{array}{l}\text { Eur. Usually in woods, thickets \& rocky places. C. repandum grows } \\
\text { in deciduous oak woods in Grevena, but only in shade and is set } \\
\text { back when coppicing occurs. Cyclamen sp. grows in the Grevena } \\
\text { foothills in the oak wood-pasture and oak pasture near Polinerion. }\end{array}$ \\
\hline Androsace villosa & $\mathrm{H}$ & $\begin{array}{l}\text { N. temp. Tufted xerophytes. Limestone rocks \& stabilized screes, } \\
1800-2300 \mathrm{~m} \text {. Pindos Mtns. }\end{array}$ \\
\hline Primula & $\mathrm{H}$ & $\begin{array}{l}\text { N. hemisph. Moist shady places, in meadows, pastures \& woods, } \\
700-2300 \mathrm{~m} \text {, on a variety of substrates. } P \text {. vulgaris is not drought } \\
\text { resistant and grows in the beech-fir woods, } 600-1600 \mathrm{~m} \text {. } P \text {. veris } \\
\text { grows in glades \& } P \text {. elatior is a coppice plant in the beech-fir } \\
\text { woods. }\end{array}$ \\
\hline Lysimachia & $\mathrm{Hel}$ & $\begin{array}{l}\text { Temp. \& subtrop. Wet places; wet grassland, wet woods, fens, lake- } \\
\text { shores, ditches, river banks, wasteland. }\end{array}$ \\
\hline Anagallis arvensis type & Th & Eur. Cultivated ground, waste places. \\
\hline Anagallis tenella type & $\mathrm{Hel}$ & $\begin{array}{l}\text { Eur. Perennial herbs of damp open habitats; bogs, ditches \& lake } \\
\text { shores. }\end{array}$ \\
\hline $\begin{array}{l}\text { Asterolinum linum- } \\
\text { stellatum }\end{array}$ & Th & Medit. Dry, open habitats. \\
\hline Samolus valerandi & $\mathrm{Hel}$ & Cosmop. Perennial herb of wet, usually saline or calcareous habitats. \\
\hline Armeria maritima type & $\begin{array}{l}\mathrm{H}, \mathrm{Chh} \\
\text { or Chc }\end{array}$ & $\begin{array}{l}\text { N. temp., E. Eur., E. Medit. Halophytic. Mtn meadows \& rocks, } \\
800-2300 \text { m. Pindos Mtns. }\end{array}$ \\
\hline Fraxinus excelsior type & MM & $\begin{array}{l}\text { Eur. Deciduous. Damp base-rich soils in woods or scrubland at high } \\
\text { altitude, also on floodplains \& river banks. Burns easily. Rather light } \\
\text { demanding, fast growth but short longevity and provides little shade. } \\
\text { Used for fuel. Very palatable to stock. }\end{array}$ \\
\hline Fraxinus angustifolia & MM & $\begin{array}{l}\text { S. \& E.C. Eur. Deciduous. Damp places; river banks, floodplains \& } \\
\text { deciduous woods. Rather light demanding \& provides little shade. } \\
\text { Used for fuel. Very palatable to stock. }\end{array}$ \\
\hline
\end{tabular}




\begin{tabular}{|c|c|c|}
\hline Pollen type & $\begin{array}{l}\text { Life } \\
\text { form }\end{array}$ & $\begin{array}{l}\text { Notes on geographic distribution, habit, ecological amplitude, } \\
\text { plant communities and uses }\end{array}$ \\
\hline Fraxinus ornus & MM & $\begin{array}{l}\text { Medit. \& S.C. Eur. Deciduous. Grows naturally at medium altitudes. } \\
\text { In Grevena it is frequent in damp hornbeam woods of the eastern } \\
\text { plains \& scattered in the drier deciduous oak woods of the plains \& } \\
\text { foothills. Also grows in the mixed woods of the limestone gorge of } \\
\text { Aetia at } 1000 \text { m. Used in the past for syrup-processing (Bottema \& } \\
\text { Woldring } 1990: 260 \text { ). May have been planted. Firewood. Very } \\
\text { palatable to stock. }\end{array}$ \\
\hline Ligustrum vulgare & $\mathrm{N}$ & $\begin{array}{l}\text { Eur. Deciduous or semi-evergreen. Woods margins \& scrub, at } \\
\text { medium \& low altitudes; somewhat calcicole. }\end{array}$ \\
\hline Phillyrea & MM, M & $\begin{array}{l}\text { Medit. Evergreen. Grows on limestone only. P. media grows in SE } \\
\text { Grevena in pseudo-maquis. Quick growing. Used for firewood. } \\
\text { Takes advantage of destruction of primary forests. }\end{array}$ \\
\hline Centaurium & $\begin{array}{l}\mathrm{H}, \mathrm{Th} \\
\text { (mostly) }\end{array}$ & $\begin{array}{l}\text { Open habitats, damp grassland at low altitudes or dry grassland on } \\
\text { plains \& mtn slopes, open woodland up to } c .1500 \mathrm{~m} \text {, or occas. to } \\
2000 \mathrm{~m} \text {. Pindos Mtns in Pinus woods. }\end{array}$ \\
\hline Gentiana lutea type & Hel & $\begin{array}{l}\text { Cosmop. Perennial herbs of damp shady places, marshes \& pastures } \\
\text { in hills \& mtns. Pindos Mtns. }\end{array}$ \\
\hline Convolvulus & $\begin{array}{l}\text { G, H, } \\
\text { Chc }\end{array}$ & Temp. Weeds from low altitudes to $1900 \mathrm{~m}$. In dry habitats. \\
\hline Cuscuta epithymum type & Th & $\begin{array}{l}\text { Trop. \& temp. Parasite. C. suaveolens is parasitic on cultivated } \\
\text { species of Medicago and Trifolium and other herbs. C. australis is } \\
\text { parasitic on Polygonum \& other herbs. C. planiflora is parasitic on } \\
\text { dwarf shrubs and annuals. C. approximata is mostly on shrubs. }\end{array}$ \\
\hline Cuscuta europaea type & Th & $\begin{array}{l}\text { Trop. \& temp. Parasite. C. europaea is usually on Urtica dioica or } \\
\text { Humulus lupulus but also on many other hosts. C. campestris is } \\
\text { parasitic on cultivated species of Medicago and Trifolium and other } \\
\text { herbs. C. palaestina is parasitic on dwarf shrubs. }\end{array}$ \\
\hline Cuscuta monogyna & Th & Trop. \& temp. Parasite. Mostly on trees and shrubs. \\
\hline Calystegia & $\mathrm{G}, \mathrm{H}$ & $\begin{array}{l}\text { Temp. \& trop. Hedges, verges, damp places \& open ground. } \\
\text { Unpalatable. }\end{array}$ \\
\hline Cynoglossum type & $\mathrm{H}$ & $\begin{array}{l}\text { Temp. \& subtrop. Dry open habitats. Mtn rocks, waysides, hedges, } \\
\text { woods, } 800-2300 \mathrm{~m} \text {. }\end{array}$ \\
\hline Heliotropium & Th & Trop. \& temp. Cultivated ground, wasteplaces \& waysides. \\
\hline Myosotis type & $\begin{array}{l}\text { Th } \\
\text { (mostly) } \\
\text { to H }\end{array}$ & $\begin{array}{l}\text { Old World temp. Several spp. included in this pollen type. Some } \\
\text { spp. grow in dry open habitats \& rocky pastures while a few grow in } \\
\text { wet habitats. Ruderal. Pindos Mtns. M. palustris is unpalatable. }\end{array}$ \\
\hline Lappula & $\begin{array}{l}\text { Th } \\
\text { (mostly) } \\
\text { or H }\end{array}$ & Temp. Dry places; cultivated fields \& other disturbed areas. \\
\hline Echium & $\begin{array}{l}\text { Th or } \mathrm{H} \\
\text { (mostly) }\end{array}$ & $\begin{array}{l}\text { Eur., Medit. Weed of dry stony waysides, cultivated \& uncultivated } \\
\text { soils. }\end{array}$ \\
\hline Alkanna type & $\mathrm{H}$ & Medit., S. Eur. Dry, rocky places. Usually between $400 \& 2300$ m. \\
\hline Borago officinalis & Th & Medit., Eur. Dry, often waste places. \\
\hline $\begin{array}{l}\text { Buglossoides arvensis } \\
\text { type }\end{array}$ & Th & Dry open habitats \& cultivated ground. \\
\hline Anchusa arvensis type & Th \& H & $\begin{array}{l}\text { Eur. Weed of cornfields, wasteplaces \& waysides. A. arvensis grows } \\
\text { on the small spring-fed clayey fens at Kellia. }\end{array}$ \\
\hline
\end{tabular}




\begin{tabular}{|c|c|c|}
\hline Pollen type & $\begin{array}{l}\text { Life } \\
\text { form }\end{array}$ & $\begin{array}{l}\text { Notes on geographic distribution, habit, ecological amplitude, } \\
\text { plant communities and uses }\end{array}$ \\
\hline Nonea & $\begin{array}{l}\text { Th } \\
\text { (mostly) } \\
\text { to } \mathrm{H}\end{array}$ & Medit. Sunny hills, stony ground, fields. \\
\hline Pulmonaria mollis & $\mathrm{H}$ & Eur. Woods. \\
\hline Anchusa officinalis type & Th or $\mathrm{H}$ & $\begin{array}{l}\text { Eur. Cultivated ground, grassy places, waysides. Generally at low \& } \\
\text { moderate altitudes, occas. up to } 2000 \mathrm{~m} \text {. Pindos Mtns. }\end{array}$ \\
\hline Verbena & Th \& H & $\begin{array}{l}\text { Trop. \& temp. Ruderals. Waste ground, waysides. V. officinalis } \\
\text { grows on the road verge on the Grevena plains, in steppe/oak wood } \\
\text { vegetation near Asprokampos. }\end{array}$ \\
\hline Callitricha obtusangula & Hyd & $\begin{array}{l}\text { Cosmop. Perennial herb. Aquatic. Plant with submerged \& floating } \\
\text { Is in fresh or brackish, usually slow-moving water. }\end{array}$ \\
\hline Callitriche stagnalis type & Hyd & $\begin{array}{l}\text { Cosmop. Aquatic. Plant with submerged \& floating ls in fresh, still } \\
\text { or slow moving water. }\end{array}$ \\
\hline Teucrium & $\begin{array}{l}\mathrm{H}, \mathrm{Chw}, \\
\mathrm{N}\end{array}$ & $\begin{array}{l}\text { Cosmop. Several spp. included in this pollen type. Some spp. grow } \\
\text { in dry places, in open woods, on banks. In mixed deciduous \& } \\
\text { evergreen open woodland, dry stony meadows \& rocky places. } \\
\text { Other spp. grow in damp places \& beside water. Usually ( } 500- \\
\text { ) } 1000-2400 \mathrm{~m} \text {. Generally on limestone, occas. on serpentine. } T \text {. } \\
\text { polium, a Mediterranean garrigue plant, is an undershrub in the oak } \\
\text { wood/steppe of the Grevena plains. T. scordium grows on the small } \\
\text { spring-fed clayey fen at Kellia. }\end{array}$ \\
\hline Stachys sylvatica type & $\begin{array}{l}\text { G, Th \& } \\
\text { H } \\
\text { (mostly) }\end{array}$ & $\begin{array}{l}\text { Cosmop. Usually dry, open habitats, waste places, cultivated } \\
\text { ground. Grassy meadows, rocky places \& screes. Sea-level to } 2500 \\
\text { m. Oak woods in Grevena. }\end{array}$ \\
\hline Stachys angustifolia & $\mathrm{H}$ & Dry places. \\
\hline Scutellaria type & $\begin{array}{l}\text { Th to } \mathrm{H} \\
\text { (mostly) }\end{array}$ & $\begin{array}{l}\text { Cosmop. Two genera \& several spp. are included in this pollen type. } \\
\text { Some grow in damp humus rich places, deciduous woods; others } \\
\text { grow on dry hills, fields, stony places. Occas. up to } 2650 \mathrm{~m} \text {. Ballota } \\
\text { nigra is a ruderal. Pindos Mtns. }\end{array}$ \\
\hline Prunella type & $\mathrm{G}, \mathrm{H}$ & $\begin{array}{l}\text { Three genera \& several spp. included in this pollen type. Some spp. } \\
\text { grow in woods, grassland \& waste places, usually in damp soil in } \\
\text { shady places. Others grow on rocks, in bushy places, forest clearings } \\
\& \text { meadows at medium to subalpine altitudes, up to } 2100 \mathrm{~m} \text {. }\end{array}$ \\
\hline Salvia officinalis type & $\begin{array}{l}\text { Th, H } \\
\text { (mostly), } \\
\mathrm{N}\end{array}$ & $\begin{array}{l}\text { Trop. \& temp. Dry places, rocky places, waysides. Stony alpine } \\
\text { meadows, rock ledges or stabilized screes. Scattered in scrub \& } \\
\text { open woodland, usually at } 500-2200 \mathrm{~m} \text {. S. officinalis widely } \\
\text { cultivated as pot herb. Grows on road verge on the plains of } \\
\text { Grevena, near Asprokampos. }\end{array}$ \\
\hline Mentha type & $\mathrm{Hel}$ & $\begin{array}{l}\text { Old World. Seven genera \& many spp. included in this pollen type. } \\
\text { Most grow in marshes, but some grow in dry habitats. Mentha } \\
\text { aquatica is unpalatable. }\end{array}$ \\
\hline Solanum dulcamara & $\mathrm{N}$ & Liane. Grows in damp woods, hedges \& river banks. Shade tolerant. \\
\hline Solanum nigrum type & Th to $\mathrm{H}$ & Weed of arable \& wasteland. \\
\hline Scrophularia type & $\begin{array}{l}\text { Chh, G, } \\
\text { Th, H } \\
\text { (mostly) }\end{array}$ & $\begin{array}{l}\text { N. temp. Many genera \& spp. included in this pollen type. Grow in a } \\
\text { wide variety of habitats from aquatic to dry stony waste land, } \\
\text { woodland clearings \& disturbed areas; from lowlands to mtns. }\end{array}$ \\
\hline Gratiola officinalis & Hel & A perennial herb of ditches, river-banks \& wet meadows. \\
\hline Rhinanthus type & Th & $\begin{array}{l}\text { Hemi-parasitic and parasitic. Damp meadows in forest openings, } \\
\text { gravel cliffs \& ledges, } 1300-2300 \mathrm{~m} \text {. Pindos Mtns. }\end{array}$ \\
\hline
\end{tabular}




\begin{tabular}{|c|c|c|}
\hline Pollen type & $\begin{array}{l}\text { Life } \\
\text { form }\end{array}$ & $\begin{array}{l}\text { Notes on geographic distribution, habit, ecological amplitude, } \\
\text { plant communities and uses }\end{array}$ \\
\hline Odontites type & Th & $\begin{array}{l}\text { Medit., S. Eur. Parasitic. Stony mtn slopes or dry hills, sandy or } \\
\text { grassy places. }\end{array}$ \\
\hline $\begin{array}{l}\text { Veronica anagallis- } \\
\text { aquatica type }\end{array}$ & $\begin{array}{l}\text { Hel } \\
\text { (mostly), } \\
\text { Th, Chw }\end{array}$ & $\begin{array}{l}\text { Annual \& perennial herbs of two genera are included in this type. } \\
\text { Odontites are hemi-parasitic annuals that grow in meadows, } \\
\text { pastures, roadsides, cultivated fields \& other disturbed habitats. } \\
\text { Some Veronica spp. grow in cultivated ground \& others grow in } \\
\text { marshes. }\end{array}$ \\
\hline Veronica triphyllos type & $\begin{array}{l}\text { Th, H \& } \\
\text { Chh }\end{array}$ & $\begin{array}{l}\text { Weeds \& ruderals. Dry open habitats. On cultivated ground, waste } \\
\text { places, in grassland, thickets, hedges, open Pinus woods, lowlands } \\
\text { to } c .2400 \mathrm{~m} \text {. Pindos Mtns. }\end{array}$ \\
\hline Pedicularis & $\mathrm{H}$ & $\begin{array}{l}\text { N. Hemisph. Hemi-parasitic alpines. } P \text {. comosa, } P \text {. brachyodonta, } \\
P . \text { graeca, } P \text {. hoermanniana grow in the Pindos Mtns. }\end{array}$ \\
\hline Putoria calabrica & $\mathrm{N}$ & $\begin{array}{l}\text { Medit. Rocks \& rock crevices, often on loose conglomerates, river } \\
\text { gravels, hills \& road cuttings at low \& moderate altitudes, occas. to } \\
2000 \mathrm{~m} \text {. }\end{array}$ \\
\hline Crucianella & Th & $\begin{array}{l}\text { Eur., Medit. Dry stony meadows \& gravel, } 500-2000 \mathrm{~m} \text {. Pindos } \\
\text { Mtns. }\end{array}$ \\
\hline Sherardia arvensis & Th & $\begin{array}{l}\text { Eur. Blue field madder. Cultivated ground, waste places \& dry } \\
\text { grassland. }\end{array}$ \\
\hline Galium type & $\begin{array}{l}\text { Th, H } \\
\text { (mostly), } \\
\text { Chh }\end{array}$ & $\begin{array}{l}\text { Cosmop. Ecologically heterogeneous, from lowlands to montane } \\
(2000 \mathrm{~m}) \text {. Usually dry open habitats. Dry or moist meadows, } \\
\text { grassland, pastures, rocky heaths, open woods, waste land \& } \\
\text { cultivated ground. A few marsh spp. }\end{array}$ \\
\hline Plantago major & $\mathrm{H}$ & Ruderal \& weed of waysides \& cultivation. \\
\hline Plantago afra & $\mathrm{Th}$ & Dry places. \\
\hline Plantago alpina type & $\mathrm{H}$ & Lowland \& montane. Rocky places, dry pastures. \\
\hline Plantago lanceolata type & $\mathrm{H}$ & $\begin{array}{l}\text { Weed of cultivation. Meadows, waysides, waste ground. Occur } \\
\text { naturally, but increased by human disturbance. Require open } \\
\text { ground. Grow in open disturbed areas and/or sometimes grazed } \\
\text { areas. }\end{array}$ \\
\hline Plantago coronopus & $\mathrm{H}$ & Xerophytic. Waysides. \\
\hline Plantago bellardii & $\mathrm{Th}$ & Dry, sandy ground \& waste places. \\
\hline Plantago media & $\mathrm{H}$ & $\begin{array}{l}\text { Ruderal. Waysides, somewhat damp meadows, particularly in rocky } \\
\text { places, }(800-) 1600-2500 \mathrm{~m} \text {. }\end{array}$ \\
\hline Plantago arenaria & $\mathrm{Th}$ & Poor fields, sandy places, waysides. \\
\hline Plantago argentea & $\mathrm{H}$ & Dry grassy \& stony places, $1400-2000 \mathrm{~m}$. Pindos \& Vourinos Mtns. \\
\hline Plantago albicans & $\mathrm{H}$ & Dry places. \\
\hline Plantago amplexicaulis & Th & Dry places. \\
\hline Plantago atrata & $\mathrm{H}$ & Mountain pastures. \\
\hline Sambucus nigra & $\mathrm{N}$ & $\begin{array}{l}\text { N. temp. Deciduous. In the wild grows near water, in damp woods, } \\
\text { hedges \& waste places. Often cultivated in Eur. Seeds of elder have } \\
\text { been found on a number of prehistoric sites chiefly in the Alpine } \\
\text { region of Europe. Elder berries are used for wine making. }\end{array}$ \\
\hline Viburnum & $\mathrm{N}$ & $\begin{array}{l}\text { Temp. \& subtrop. Deciduous \& evergreen. Scattered in open damp } \\
\text { woods, edges of woods, thickets, hedges, rocky places. Sometimes } \\
\text { forms thickets. Highlands sometimes ascending to } c .1800 \mathrm{~m} . V \text {. } \\
\text { tinus used for firewood. }\end{array}$ \\
\hline
\end{tabular}




\begin{tabular}{|c|c|c|}
\hline Pollen type & $\begin{array}{l}\text { Life } \\
\text { form }\end{array}$ & $\begin{array}{l}\text { Notes on geographic distribution, habit, ecological amplitude, } \\
\text { plant communities and uses }\end{array}$ \\
\hline Valerianella rimosa type & Th & $\begin{array}{l}\text { Temp. Disturbed ground or other dry, open habitats ascends to } 2000 \\
\text { m. Often in heavily grazed areas. Pindos Mtns. }\end{array}$ \\
\hline Valeriana tuberosa & $\mathrm{H}$ & $\begin{array}{l}\text { Eur. Mainly on stony slopes \& meadows up to } 2100 \mathrm{~m} \text {. Grows in } \\
\text { grassland of pine wood-pasture vegetation in Grevena. }\end{array}$ \\
\hline Valeriana officinalis type & $\mathrm{H}$ & $\begin{array}{l}\text { Bushy places, rocks, woods. Chiefly low \& moderate altitudes, but } \\
\text { ascending to } 2450 \mathrm{~m} \text {. }\end{array}$ \\
\hline Valerianella locusta type & Th & $\begin{array}{l}\text { Chiefly low \& moderate altitudes, ascending to } 2000 \mathrm{~m} \text { in subalpine } \\
\text { screes \& grassland, often in heavily grazed areas. }\end{array}$ \\
\hline Succisa pratensis & $\mathrm{Hel}$ & Damp meadows, marshes, fens, damp woods. \\
\hline $\begin{array}{l}\text { Scabiosa columbaria } \\
\text { type }\end{array}$ & $\mathrm{H}$ & $\begin{array}{l}\text { Eur., Medit. Dry rocky slopes, forest openings, alpine meadows; } \\
\text { lowlands to } 1300-2500 \mathrm{~m} \text {. }\end{array}$ \\
\hline Dipsacus type & $\mathrm{Hel}$ & Eur., Medit. Biennial herb of woods, waysides, marshes. \\
\hline Knautia & Th & $\begin{array}{l}\text { Eur., Medit. Scrub \& disturbed ground, mtn meadows \& wood } \\
\text { margins, rocky places in subalpine \& alpine meadows. May occur } \\
\text { up to } 2300 \mathrm{~m} \text {. }\end{array}$ \\
\hline Jasione & $\mathrm{H}$ & $\begin{array}{l}\text { Eur., Medit. Dry rocky alpine meadows \& grassland, stabilized } \\
\text { screes \& gravel or open woodland. Lowland to c. } 1800-2500 \mathrm{~m} \text {. } \\
\text { Pindos Mtns (Vasilitsa). }\end{array}$ \\
\hline Campanula erinus type & Th to $\mathrm{H}$ & $\begin{array}{l}\text { N. temp. \& esp. Medit. Dry grassy, stony places, scrub \& forest } \\
\text { margins or open forest, } 1000-2300 \mathrm{~m} \text {. }\end{array}$ \\
\hline Campanula rotundifolia & $\mathrm{H}$ & $\begin{array}{l}\text { N. temp. \& esp. Medit. Rock crevices \& ledges, rocky meadows, } \\
\text { screes c. } 1300-2600 \text { m, variety of substrates. }\end{array}$ \\
\hline Asyneuma type & $\mathrm{H}$ & $\begin{array}{l}\text { Steppe \& mtn grassland. Fields, stony places, } \\
(400-) 1200-2200 \mathrm{~m} .\end{array}$ \\
\hline Solidago type & $\begin{array}{l}\text { Th \& H } \\
\text { mostly, } \\
\text { Chh, G }\end{array}$ & $\begin{array}{l}\text { Ecologically heterogeneous. Grows in habitats ranging from alpine } \\
\text { rocks, mtn pastures, scrub, woodland pastures, open grassland, } \\
\text { cultivated ground \& waste places to damp places, fens \& marshes. } \\
\text { Lowlands to } 2300 \text { m. Helianthus annuus (sunflower) is currently } \\
\text { cultivated in Grevena. Steppe in oak woods in Grevena. }\end{array}$ \\
\hline Echinops & $\begin{array}{l}\mathrm{H}, \text { rarely } \\
\mathrm{Th}\end{array}$ & $\begin{array}{l}\text { Eur. Dry rocky places at low \& medium altitude, regularly ascending } \\
\text { to subalpine levels. }\end{array}$ \\
\hline Artemisia & $\begin{array}{l}\text { Chh \& } \\
\text { H } \\
\text { (mostly) }\end{array}$ & $\begin{array}{l}\text { N. Hemisph. Common on arid soil (steppe). Occurs naturally, but is } \\
\text { increased by human disturbance (weed). }\end{array}$ \\
\hline Carlina type & Th to $\mathrm{H}$ & $\begin{array}{l}\text { Eur. \& Medit. Dry meadows, open rocky places, waste places, } \\
\text { cultivation ground, } 900-2300 \mathrm{~m} \text {. Grows in the grazed Pinus } \\
\text { heldreichii woods in the Pindos. Onopordon acanthium (Scotch } \\
\text { thistle) seeds were found in Early \& Middle Bronze Age deposits at } \\
\text { Lerna, south Greece. }\end{array}$ \\
\hline Picnomon acarna & Th & Cultivated ground, waste places. \\
\hline Achillea type & Th \& H & $\begin{array}{l}\text { N. temp. Steppic \& alpine weeds. Ecologically heterogeneous. } \\
\text { Grows throughout Grevena. }\end{array}$ \\
\hline Centaurea cyanus type & $\begin{array}{l}\text { Th, } \\
\text { rarely } \mathrm{H}\end{array}$ & $\begin{array}{l}\text { Dry open places; cultivated ground \& waste places. Weed of cereals } \\
\text { in Grevena. }\end{array}$ \\
\hline $\begin{array}{l}\text { Centaurea napulifera } \\
\text { type }\end{array}$ & $\mathrm{H}$ & $\begin{array}{l}\text { Grassy \& stony slopes in mtns \& clearings in forests between } 1000 \\
\& 2100 \mathrm{~m} \text {, occas. up to } 2750 \mathrm{~m} .\end{array}$ \\
\hline
\end{tabular}




\begin{tabular}{|c|c|c|}
\hline Pollen type & $\begin{array}{l}\text { Life } \\
\text { form }\end{array}$ & $\begin{array}{l}\text { Notes on geographic distribution, habit, ecological amplitude, } \\
\text { plant communities and uses }\end{array}$ \\
\hline Cirsium type & $\begin{array}{l}\text { Th, H \& } \\
\text { G }\end{array}$ & $\begin{array}{l}\text { N. temp. Mostly weeds \& ruderals of pastures, disturbed ground \& } \\
\text { wasteland. Also grows in grassland, scrub, stony places, damp shady } \\
\text { places, wet meadows \& marshes (one sp.), 1100-2000(-2200) m. }\end{array}$ \\
\hline Xanthium & Th & $\begin{array}{l}\text { Medit. Occurs naturally, but is increased by human disturbance. } X \text {. } \\
\text { spinosum is native of N. America. Weeds. Unpalatable. }\end{array}$ \\
\hline $\begin{array}{l}\text { Centaurea solstitialis } \\
\text { type }\end{array}$ & Th to $\mathrm{H}$ & $\begin{array}{l}\text { Several genera \& many spp. Occurs naturally, but is increased by } \\
\text { human disturbance. Grows in disturbed situations. }\end{array}$ \\
\hline Centaurea graeca type & $\mathrm{H}$ & $\begin{array}{l}\text { Mostly dry grassland, rocky places \& scrub on hills \& mtns. Also } \\
\text { cultivated \& waste ground. }\end{array}$ \\
\hline Crupina & Th & S. Eur. Dry grassland \& stony slopes. \\
\hline Sonchus type & Th to $\mathrm{H}$ & Almost cosmop.Weed. Cultivated ground, fields \& waste places. \\
\hline Lactuca type & Th to $\mathrm{H}$ & $\begin{array}{l}\text { N. temp. Woods \& scrub, damp woodland openings, dry rocky or } \\
\text { stony places, waste ground, } 1400-2300 \mathrm{~m} \text {. }\end{array}$ \\
\hline Scolymus & Th to $\mathrm{H}$ & Medit. Corn fields, cultivated ground, waysides. \\
\hline Cichorium type & Th to $\mathrm{H}$ & $\begin{array}{l}\text { Eur., Medit. Ruderal. Dry open habitats, fields, waysides, waste } \\
\text { places. Pindos foothills \& plains in Grevena. }\end{array}$ \\
\hline Hieracium type & Th to $\mathrm{H}$ & $\begin{array}{l}\text { N. Hemisph. Ecologically heterogeneous. Lowlands to } 2500 \mathrm{~m} \text {. } \\
\text { Mostly dry open habitats, disturbed ground. Often as weed. }\end{array}$ \\
\hline Taraxacum type & Th to $\mathrm{H}$ & $\begin{array}{l}\text { Temp. Ecologically heterogeneous. Mostly weeds of dry open } \\
\text { habitats, such as meadows, pastures, open woodland, rocky alpine } \\
\text { grassland, cultivated fields \& waste places. Also shady places, moist } \\
\text { \& swampy patches. Lowlands ascending to } 2000 \mathrm{~m} \text {. The seeds of } \\
\text { Lapsana communis are commonly found in prehistoric sites (from } \\
\text { Neolithic times) - the green parts of this plant may have been eaten. }\end{array}$ \\
\hline \multicolumn{3}{|l|}{ MONOCOTYLEDONES } \\
\hline Sagittaria sagittifolia & Hyd & $\begin{array}{l}\text { Temp. Perennial herb. Marsh or shallow water at the edges of lakes, } \\
\text { ponds, canals or slow rivers. Submerged, floating or emergent. } \\
\text { Shallow to moderately deep. }\end{array}$ \\
\hline Alisma & Hyd & $\begin{array}{l}\text { N. temp. Perennial herb. Aquatic. Ls floating, submerged or } \\
\text { emergent. Shallow waters (still to slow), muddy places \& damp } \\
\text { ground. }\end{array}$ \\
\hline Damasonium alisma & Hyd & $\begin{array}{l}\text { Eur., Medit. Perennial herb. Aquatic; shallow, still water. Floating } \\
\text { (submerged). }\end{array}$ \\
\hline $\begin{array}{l}\text { Hydrocharis morsus- } \\
\text { ranae }\end{array}$ & Hyd & Eur. Perennial herb. Aquatic; floating ls and surface fls. \\
\hline Stratiotes aloides & Hyd & Eur. Perennial herb. Aquatic. Floats in summer, sinks in autumn. \\
\hline Vallisneria spiralis & Hyd & $\begin{array}{l}\text { S. Eur. Perennial herb. Aquatic. Submerged, but fls float to the } \\
\text { surface when open; pollination at surface. Still water. }\end{array}$ \\
\hline Triglochin bulbosa & $\mathrm{Hel}$ & Medit. Perennial herb. Marshes; fresh water- or salt-marshes. \\
\hline $\begin{array}{l}\text { Potamogeton crispus } \\
\text { type }\end{array}$ & Hyd (G) & $\begin{array}{l}\text { Cosmop. Perennial herb. Aquatic. Wide variety of fresh-water } \\
\text { habitats - in deep or shallow water, stagnant to fast-flowing. } \\
\text { Submerged or floating. Can grow subterrestrially in dried-up } \\
\text { situations. Fls wind pollinated above surface. }\end{array}$ \\
\hline Potamogeton pectinatus & Hyd (G) & $\begin{array}{l}\text { Eur. Perennial herb. Aquatic. Base-rich or brackish water, up to } 1 \mathrm{~m} \\
\text { deep. Fls pollinated below the water surface. }\end{array}$ \\
\hline
\end{tabular}




\begin{tabular}{|c|c|c|}
\hline Pollen type & $\begin{array}{l}\text { Life } \\
\text { form }\end{array}$ & $\begin{array}{l}\text { Notes on geographic distribution, habit, ecological amplitude, } \\
\text { plant communities and uses }\end{array}$ \\
\hline Colchicum & $\mathrm{Gt}$ & $\begin{array}{l}\text { Eur. Pindos Mtns. Open habitats, rocky or gravelly slopes, } \\
\text { grassland, meadows, pastures, cultivated \& disturbed ground. } \\
\text { Lowlands ascending to } 2000 \mathrm{~m} \text {. }\end{array}$ \\
\hline Narthecium scardicum & Hel & N. temp. Marshy places by springs \& brooks, $1600-3200 \mathrm{~m}$. \\
\hline Veratrum & $\mathrm{H}$ & $\begin{array}{l}\text { N. temp. Scrub, wood margins \& mtn meadows, } 1200-2000 \mathrm{~m} . \mathrm{V} \text {. } \\
\text { nigrum grows on limestone only. }\end{array}$ \\
\hline $\begin{array}{l}\text { Asphodelus fistulosus } \\
\text { type }\end{array}$ & $\begin{array}{l}\text { Th or } \\
\text { short- } \\
\text { lived Grt }\end{array}$ & $\begin{array}{l}\text { Medit. Steppe plant. Roadsides, cultivated ground. Intolerant of } \\
\text { shade. }\end{array}$ \\
\hline Asphodelus albus & $? \mathrm{Grt}$ & $\begin{array}{l}\text { Medit. Steppe plant. Meadows, heaths, open woods \& mtn pastures. } \\
\text { Intolerant of shade. }\end{array}$ \\
\hline Fritillaria & $\mathrm{Gb}$ & N. temp. Open woods, scrub \& grassy places, $400-2500$ m. \\
\hline Gagea pratensis & $\mathrm{Gb}$ & N. temp. Grassland \& disturbed ground. \\
\hline Tulipa sylvestris & $\mathrm{Gb}$ & $\begin{array}{l}\text { N. temp. Meadows, grassy \& rocky places (esp. steppe), occas. in } \\
\text { open woodland, (500-) } 1100-2200 \mathrm{~m} \text {. }\end{array}$ \\
\hline Lilium & $\mathrm{Gb}$ & $\begin{array}{l}\text { N. temp. Mtn meadows, open woods \& scrub, } 650-2200 \mathrm{~m} \text {. Beech } \\
\text { woods in Pindos Mtns. }\end{array}$ \\
\hline Anthericum liliago & $\mathrm{H}$ & Open woods, dry grassland \& stony slopes, $500-2000$ m. \\
\hline Scilla type & $\mathrm{Gb}$ & $\begin{array}{l}\text { Temp. Widespread in dry, open habitats, usually at low \& moderate } \\
\text { altitudes, occas. ascending to } 2400 \mathrm{~m} \text {. Beech \& oak woods in } \\
\text { Grevena. }\end{array}$ \\
\hline Muscari comosum & $\mathrm{Gb}$ & Dry grassland \& cultivated ground. \\
\hline Muscari neglectum type & $\mathrm{Gb}$ & $\begin{array}{l}\text { Dry places in woods, fields, pastures, meadows, ascending to } 2500 \\
\mathrm{~m} \text {. }\end{array}$ \\
\hline $\begin{array}{l}\text { Allium sphaerocephalon } \\
\text { type }\end{array}$ & $\mathrm{Gb}$ & $\begin{array}{l}\text { N. hemisph. Mainly on disturbed ground; prob. spread by human } \\
\text { activity. Dry places, fields \& cultivated ground, (800-) } 1400-2200 \mathrm{~m} \text {. } \\
\text { A. sativum (garlic) is widely cultivated. }\end{array}$ \\
\hline Asparagus & Grh & $\begin{array}{l}\text { Old World. Mostly in dry places. Grows wild in woods \& bushy } \\
\text { places \& pseudo-maquis. }\end{array}$ \\
\hline $\begin{array}{l}\text { Narcissus papyraceus } \\
\text { type }\end{array}$ & $\mathrm{Gb}$ & Eur., Medit. Meadows, pastures \& cultivated fields. \\
\hline Sternbergia & $\mathrm{Gb}$ & E. Medit. Open stony slopes \& dry scrub, (1000-)1200-2300 m. \\
\hline Iris pseudacorus & $\operatorname{Hel}(G)$ & N. temp. Perennial herb of marshes \& ditches. \\
\hline Iris spuria type & $\mathrm{Hel}$ & N. temp. Perennial herb of wet places. \\
\hline Gladiolus type & Gt & $\begin{array}{l}\text { Eur. Common weed of crops. Dry rocky, heath, scrub \& open } \\
\text { woodland. }\end{array}$ \\
\hline Gynandriris sisyrinchium & Gt & Medit. Dry places, stony hillsides. \\
\hline Festuca type & $\begin{array}{l}\text { Th, H, } \\
\text { Grh \& } \\
\text { Chh }\end{array}$ & $\begin{array}{l}\text { Cosmop. Wild grasses of grasslands. Often viviparous in mtns. } \\
\text { Festuca spp. dominate the grassland of pine wood-pasture } \\
\text { vegetation in Grevena. See Dactylis below. }\end{array}$ \\
\hline Glyceria type & $\begin{array}{l}\text { Th to } \\
\text { Grh \& } \\
\text { Hel }\end{array}$ & $\begin{array}{l}\text { Cosmop. Glyceria spp. are perennial growing in marshy ground or } \\
\text { shallow water. Glyceria grows on seasonally dry lakebed at Gomara, } \\
1750 \mathrm{~m} \text {. Glyceria is eaten by stock. Bromus spp. are mostly annual } \\
\text { weeds and ruderals, but grow in grassland also. }\end{array}$ \\
\hline
\end{tabular}




\begin{tabular}{|c|c|c|}
\hline Pollen type & $\begin{array}{l}\text { Life } \\
\text { form }\end{array}$ & $\begin{array}{l}\text { Notes on geographic distribution, habit, ecological amplitude, } \\
\text { plant communities and uses }\end{array}$ \\
\hline Dactvlis type & $\begin{array}{l}\text { Th, H \& } \\
\text { Grh }\end{array}$ & $\begin{array}{l}\text { Eur., Medit. Wild grasses of grasslands mostly (some marsh spp. } \\
\text { such as Phalaris arundinacea). Irrigation agriculture is used in NE } \\
\text { Grevena today to grow grass for fodder. Increased by the } \\
\text { establishment of pasture but grazing may reduce the flowering } \\
\text { capacity of wild grass, while wood cutting may increase grass pollen } \\
\text { values. Two small-grained millets, Echinochloa crus-galli (wild) \& } \\
\text { Setaria italica (cultivated) may belong to this pollen type. } \\
\text { Macrofossils of these have been found in prehistoric contexts in } \\
\text { Europe that suggest they were both eaten by humans. Setaria italica } \\
\text { is known to have been cultivated for fodder and bird seed in S. } \\
\text { Europe where it is often locally naturalized (Tutin } \text { et al 1980:264). }\end{array}$ \\
\hline Agropyron type & $\begin{array}{l}\text { Th \& } \\
\text { Grh }\end{array}$ & $\begin{array}{l}\text { S. Eur. Mostly wild grasses that grow in dry habitats, except one } \\
\text { species which may belong to this pollen type, Panicum miliaceum } \\
\text { (broomcorn millet). This small grained species was formerly widely } \\
\text { cultivated as a cereal, now more locally \& mainly for fodder in C., } \\
\text { S. \& E. Eur. It has been identified in C. \& E. Europe in Neolithic } \\
\text { contexts. }\end{array}$ \\
\hline Triticum type & Th & $\begin{array}{l}\text { Cultivated cereals. Wheat is now the major crop in Grevena. It is } \\
\text { grown mostly on the plains, but before mechanized agriculture was } \\
\text { introduced it was cultivated in the Pindos Mtns \& foothills as well as } \\
\text { on the plains on a subsistence basis for bread \& pasta. Domesticated } \\
\text { wheat of } T \text {. dicoccon (emmer) is found in nearly all Neolithic } \\
\text { archaeological sites in Greece, including Thessaly, from about } 6000 \\
\text { BC (Renfrew 1973:26; Hansen 1988). Domesticated bread wheat } \\
\text { T. aestivum has been found in Macedonia \& Thessaly in sites dating } \\
\text { to the Middle \& Late Neolithic respectively (Hansen 1988). } \\
\text { Domesticated wheat is self-pollinating. }\end{array}$ \\
\hline Secale cereale & Th & $\begin{array}{l}\text { Cultivated cereal. Rye has been found in N. \& C. European } \\
\text { archaeological sites dating to the Neolithic. Introduced into Greece } \\
\text { by the Romans (Renfrew 1973:83). Prior to mechanized agriculture, } \\
\text { rye was grown in the Pindos Mtns \& foothills and on the Grevena } \\
\text { plains on a subsistence basis as food for human consumption. Today } \\
\text { it is a minor crop, confined mostly to the plains. Cross-pollinated } \\
\text { (Hector, in Renfrew 1973:82). }\end{array}$ \\
\hline Hordeum type & $\begin{array}{l}\text { Th } \\
\text { (mostly) } \\
\text { \& Grh }\end{array}$ & $\begin{array}{l}\text { Includes cultivated cereals as well as some wild grass species. } \\
\text { Domesticated barley (H. distichon \& H. vulgare) has been identified } \\
\text { from Neolithic sites throughout Greece, including Thessaly, along } \\
\text { with the domesticated wheat } T \text {. monococcum (einkorn), included in } \\
\text { this pollen type (Renfrew 1973:26; Hansen 1988). Domesticated } \\
\text { barley has been identified in Bulgaria by 5000BC (Dennell 1972). } \\
\text { Prior to mechanized agriculture, barley was grown in the Pindos } \\
\text { foothills and on the Grevena plains on a subsistence basis as food } \\
\text { for human consumption \& for fodder. It is the second most } \\
\text { important crop grown in Grevena today. Domesticated barley is self- } \\
\text { pollinating. The wild species grow on disturbed ground, sometimes } \\
\text { as persistent weeds on cultivated ground, in dry grassland, and on } \\
\text { dry sandy or stony ground. Elymus caninus grows only in the shelter } \\
\text { of woods. Hordelymus europaeus which is new to Greece also } \\
\text { grows in woods, as well as other shady places. }\end{array}$ \\
\hline
\end{tabular}




\begin{tabular}{|c|c|c|}
\hline Pollen type & $\begin{array}{l}\text { Life } \\
\text { form }\end{array}$ & $\begin{array}{l}\text { Notes on geographic distribution, habit, ecological amplitude, } \\
\text { plant communities and uses }\end{array}$ \\
\hline Avena type & Th & $\begin{array}{l}\text { This pollen type includes cultivated cereals as well as some wild } \\
\text { spp. of oats. Evidence of cultivated oats was found at Achilleion, } \\
\text { Thessaly, dating to the Neolithic (Renfrew 1973:88). Prior to } \\
\text { mechanized agriculture, oats (prob. A. sativa) were grown in the } \\
\text { Pindos foothills and on the Grevena plains on a subsistence basis as } \\
\text { food for livestock. Not known to be cultivated today. The wild spp. } \\
\text { grow on dry, rocky and waste ground including roadsides and as } \\
\text { weeds on cultivated ground. }\end{array}$ \\
\hline Phragmites australis & $\begin{array}{l}\mathrm{Hel}(\mathrm{Grh} \\
\text { or } \mathrm{H})\end{array}$ & Cosmop. Wild grass (1-4 m tall). Marsh plant; fresh, shallow water. \\
\hline Zea mays & Th & $\begin{array}{l}\text { Cultivated cereal. Introduced from Am. Some now grown on the } \\
\text { plains of Grevena. }\end{array}$ \\
\hline Arum & Grt & Eur., Medit. Woods, hedges \& disturbed areas. \\
\hline Dracunulus type & Grt & Medit. Woods \& scrub. Shady places. \\
\hline Lemnaceae & Hyd & $\begin{array}{l}\text { Cosmop. Perennial herbs. Aquatic. Free floating. Fronds floating or } \\
\text { submerged, fls at water surface. }\end{array}$ \\
\hline Sparganium erectum & $\mathrm{Hel}$ & $\begin{array}{l}\text { N. temp. Marshes (semi-terrestrial). Creeping rhizome with stems } \\
\text { projecting out of water with ls \& fls higher up. Up to } 1 \mathrm{~m} \text { deep } \\
\text { water. Grows on Kellia fen today. }\end{array}$ \\
\hline $\begin{array}{l}\text { Sparganium } \\
\text { angustifolium }\end{array}$ & Hyd & $\begin{array}{l}\text { N. temp. Aquatic; floating, with creeping rhizome with stems } \\
\text { projecting out of water with ls \& fls higher up. Perennial. } 0.5-2 \mathrm{~m} \\
\text { deep water. }\end{array}$ \\
\hline Typha angustifolia type & $\mathrm{Hel}$ & $\begin{array}{l}\text { Temp. \& trop. Perennial herbs of marshes or shallow water, at } \\
\text { margins of lakes, ponds and rivers. Grows on Kellia fen today. }\end{array}$ \\
\hline Typha domingensis type & Hel & $\begin{array}{l}\text { Temp. \& trop. Perennial herbs of marshes or shallow water, at } \\
\text { margins of lakes, ponds and rivers. }\end{array}$ \\
\hline Scirpus & Hel & $\begin{array}{l}\text { Cosmop. Lakes, ponds \& streams. Still to moderately fast flow. In } \\
\text { still sheltered water } 1-2+\mathrm{m} \text { deep, in flowing usually } 30-80 \mathrm{~cm} \text { deep. } \\
\text { Grows on Kellia fen today. }\end{array}$ \\
\hline Cladium mariscus & $\mathrm{Hel}(\mathrm{G})$ & $\begin{array}{l}\text { Trop. \& temp. Fens \& lake margins, usually in base-rich water. } \\
\text { Stems } 125-250 \mathrm{~cm} . \text { Perennial. }\end{array}$ \\
\hline Cyperus michelianus & ?Hel & Annual. River-banks, marshes or wet grassland. \\
\hline Cyperus longus type & Hel & River-banks, marshes or wet grassland. Grows on Kellia fen today. \\
\hline Schoenus nigricans & Hel & Usually in fens \& other at least seasonally wet, base-rich habitats. \\
\hline Carex hallerana type & Grh & $\begin{array}{l}\text { Temp. Terrestrial. Widespread in damp places \& in dry, rocky } \\
\text { places, scrub \& open woodland, ascends to } 2500 \mathrm{~m} \text {. }\end{array}$ \\
\hline Carex flacca type & $\begin{array}{l}\mathrm{Hel}(\mathrm{H}, \\
\mathrm{G} \& \mathrm{~T})\end{array}$ & $\begin{array}{l}\text { Temp. Mostly marsh plants. Marshes, fens, shallow lakes, margins } \\
\text { of streams, and grasslands. Ascends to } 2200 \mathrm{~m} \text {. }\end{array}$ \\
\hline Epipactis type & G & $\begin{array}{l}\text { N. temp. Mostly in undisturbed shady woodland habitats, sometimes } \\
\text { on rocky slopes or marshes. }\end{array}$ \\
\hline Limodorum abortivum & G & S. Eur. Woods \& shady grassland. \\
\hline Cephalanthera & G & $\begin{array}{l}\text { N. temp. Perennial herb. Woods \& other shady places. Undisturbed } \\
\text { areas. }\end{array}$ \\
\hline \multicolumn{3}{|l|}{ PTERIDOPHYTA } \\
\hline Equisetum & $\begin{array}{l}\mathrm{Hel} \\
(\mathrm{Grh})\end{array}$ & Cosmop. Marshes. E. telmateia grows on Kellia fen today. \\
\hline
\end{tabular}




\begin{tabular}{|l|l|l|}
\hline Pollen type & $\begin{array}{l}\text { Life } \\
\text { form }\end{array}$ & $\begin{array}{l}\text { Notes on geographic distribution, habit, ecological amplitude, } \\
\text { plant communities and uses }\end{array}$ \\
\hline Ophioglossum vulgatum & $\mathrm{Hel}$ (Gr) & $\begin{array}{l}\text { Trop., temp. Damp grassland from low altitudes to c. 2000 m, on } \\
\text { varying substrates. }\end{array}$ \\
\hline Botrychium & $\mathrm{Gr}$ & $\begin{array}{l}\text { Cosmop. A variety of habitats from damp grassland to dry, rocky } \\
\text { slopes, usually at high altitudes (1500-)1700-2600 m. }\end{array}$ \\
\hline $\begin{array}{l}\text { Adiantum capillus- } \\
\text { veneris }\end{array}$ & $\mathrm{Gr}$ & Damp rocks. \\
\hline Pteridium aquilinum & $\mathrm{Gr}$ & $\begin{array}{l}\text { Cosmop. Pioneer. Not affected by cattle grazing. Encouraged by } \\
\text { burning. Common and often gregarious in woodland clearings at } \\
\text { montane altitudes (generally 500-1400 m), occas. ascends to 1800- } \\
\text { 1900 m. }\end{array}$ \\
\hline Thelypteris palustris & $\mathrm{Hel} \mathrm{(Gr)}$ & Fens \& marshes. \\
\hline Thelypteris phegopteris & $\mathrm{Gr}$ & Shady places. \\
\hline Asplenium type & $\mathrm{H}$ & $\begin{array}{l}\text { Cosmop. Mostly small plants of shady rock crevices \& ledges, dry to } \\
\text { damp. On a variety of substrates, in maquis \& woods. Up to 2700 m. }\end{array}$ \\
\hline Athyrium filix-femina & $\mathrm{H}$ & $\begin{array}{l}\text { Damp, shady places by streams, generally in woods, ascending in } \\
\text { seepage meadows \& by brooks to 1800 m, occas. 2100 m. }\end{array}$ \\
\hline Athyrium distentifolium & $\mathrm{H}$ & Screes \& rocky outcrops on exposed mountains. \\
\hline Dryopteris filix-mas type & $\mathrm{H}, \mathrm{G}$ & $\begin{array}{l}\text { Shady \& semishady places in woods \& at high altitudes, also on } \\
\text { screes \& in rock crevices, 600-2900 m. }\end{array}$ \\
\hline $\begin{array}{l}\text { Gymnocarpium } \\
\text { dryopteris }\end{array}$ & $\mathrm{Gr}$ & $\begin{array}{l}\text { Among rocks in woods \& in shaded rock crevices, on non- } \\
\text { calcareous substrate, 1400-2300 m. }\end{array}$ \\
\hline Blechnum spicant & $\mathrm{H}$ & Damp humus-rich rocky places up to c. 1700 m. Usually calcifuge. \\
\hline Polypodium & $\mathrm{H}$ & Cosmop. Grows on shaded, mossy rocks, (600-)1200-2100 m. \\
\hline Salvinia natans & Hyd & Water plant. Free floating \& submerged ls. Floating spores. Annual. \\
\hline
\end{tabular}




\section{Appendix XI}

\section{Relative dispersal and representation}

This table summarizes relative dispersal and representation of pollen and spore types whose source plants were noted in the areas surveyed and/or were present in surface pollen samples. Known pollination mechanisms have been included as an indication of pollen production, that is, anemophilous plants generally produce more pollen than plants with other pollination mechnisms (Knuth 1906:7; Erdtman 1969). Pollination mechanisms have been derived from Knuth (1906, 1908), Willis (1951), Faegri \& van der Pijl (1971), Proctor \& Yeo (1973) and Heywood (1978).

Letters used to symbolize types of pollen dispersal mechanisms (DM) are: $\mathrm{A}=$ anemophilous; $\mathrm{E}=$ entomophilous; $\mathrm{H}=$ hydrophilous; $\mathrm{EpH}=$ ephydrophilous. Where two symbols appear, the first is the more common method of dispersal.

Relative dispersal (RD) (termed 'relative export' by Flenley (1973:137)) has been scored (after Flenley 1973) as:

1) High: pollen types which occur in all or almost all samples, wherever collected.

2) Medium: pollen occurs in samples from adjacent vegetation.

3) Low: pollen occurs only in samples from vegetation in which parent plants are growing.

Representation ( $\mathbf{R})$ of pollen types has been scored (after Dodson 1976) as:

1) Over-represented: where pollen relative percentage is greater than the relative abundance of source species within the vegetation. Those species which have 'high' relative dispersal and therefore appear in areas where the source vegetation is not present are ipso facto over-represented.

2) Well-represented: where pollen relative percentage approximates source species relative abundance in the vegetation.

3) Under-represented: where pollen relative percentage was zero or smaller than source species relative abundance in the vegetation.

4) Insufficient data (i.d.): where there is doubt about the presence of the source plant for the pollen or insufficient pollen observed.

Pollen types are presented in the same order as in the pollen diagram: high altitude shrubs, high altitude herbs, coniferous and deciduous woodland trees, woodland understorey trees \& shrubs, woodland herbs, riverine trees, evergreen woodland trees, plants of open dry rocky habitats, herbs of open-ground, cultivars, bog \& marsh plants, and aquatic plants. 


\begin{tabular}{|c|c|c|c|c|}
\hline Pollen/spore type & DM & RD & $\mathbf{R}$ & Comments \\
\hline \multicolumn{5}{|l|}{ Alpine shrubs } \\
\hline Armeria maritima type & $\mathrm{E}$ & (no pollen) & under & \\
\hline Daphne & E & i.d. & i.d. & \\
\hline Vaccinium myrtillus & $\mathrm{E}$ & medium & well & \\
\hline \multicolumn{5}{|l|}{ Alpine herbs } \\
\hline Oxyria digyna & A & i.d. & i.d. & \\
\hline Pedicularis & $\mathrm{E}$ & i.d. & i.d. & \\
\hline Plantago atrata & A & i.d. & i.d. & \\
\hline Polygonum bistorta type & $\mathrm{E}$ & i.d. & i.d. & \\
\hline Pyrola & $\mathrm{E}$ & i.d. & i.d. & \\
\hline Soldanella & $\mathrm{E}$ & i.d. & i.d. & \\
\hline \multicolumn{5}{|l|}{$\begin{array}{l}\text { Coniferous \& deciduous } \\
\text { canopy trees }\end{array}$} \\
\hline Pinus (Diploxylon) & A & high & over & $\begin{array}{l}\text { Well known over-representation } \\
\text { (Wright 1972; Sheehan 1979; Jahns } \\
\text { 1993). }\end{array}$ \\
\hline Fagus & A & high & under & $\begin{array}{l}\text { Coppiced. Represented in the pollen } \\
\text { spectra at all sites, but in the sample } \\
\text { collected from under the canopy of a } \\
\text { beech wood was under-represented. }\end{array}$ \\
\hline Quercus frainetto type & A & high & ?well & Coppiced. \\
\hline Quercus trojana type & A & high & ?well & $\begin{array}{l}\text { Coppiced. Badly or not represented in } \\
\text { degraded woods (Bottema 1974). }\end{array}$ \\
\hline Abies & A & medium & under & $\begin{array}{l}\text { Found to be strongly under-represented } \\
\text { in stands where fir was growing } \\
\text { (Bottema 1975; Sheehan 1979), but } \\
\text { Jahns (1993) found Abies to be better } \\
\text { represented. }\end{array}$ \\
\hline Acer & $\mathrm{E}$ & low & under & Under-represented (Bottema 1974). \\
\hline Carpinus type & A & medium & well & $\begin{array}{l}\text { Good production \& usually has good } \\
\text { transport, but low shrubs are under- } \\
\text { represented (Bottema 1974). }\end{array}$ \\
\hline Castanea sativa & $\mathrm{E}, \mathrm{A}$ & medium & over & $\begin{array}{l}\text { Pollen dispersal is poor (Bottema 1974; } \\
\text { Bottema \& Woldring 1990:260). }\end{array}$ \\
\hline Cornus mas & E & low & under & Under-represented (Bottema 1974). \\
\hline Cornus sanguinea & E & low & under & Under-represented (Bottema 1974). \\
\hline Corylus & $\mathrm{A}, \mathrm{E}$ & high & over & $\begin{array}{l}\text { It is often noted that Corylus produces } \\
\text { much less pollen as an under-shrub than } \\
\text { as a tree (Iversen 1941; Ritchie \& } \\
\text { Lichti-Federovich 1963; Faegri \& } \\
\text { Iversen 1992:5) }\end{array}$ \\
\hline
\end{tabular}




\begin{tabular}{|c|c|c|c|c|}
\hline Pollen/spore type & DM & RD & $\mathbf{R}$ & Comments \\
\hline Crataegus & $\mathrm{E}$ & low & under & \\
\hline Euonymus europaeus type & $\mathrm{E}$ & (no pollen) & under & \\
\hline Fraxinus ornus & $\mathrm{E}$ & medium & over & Well represented (Bottema 1974). \\
\hline Malus & $\mathrm{E}$ & (no pollen) & under & \\
\hline Ostrya type & A & high & over & $\begin{array}{l}\text { Good production \& good transportation } \\
\text { (Bottema 1974). }\end{array}$ \\
\hline Prunus spinosa type & $\mathrm{E}$ & (no pollen) & under & \\
\hline Pyrus & $\mathrm{E}$ & (no pollen) & under & \\
\hline Rhamnus type & E & (no pollen) & under & \\
\hline Sorbus type & $\mathrm{E}, \mathrm{A}$ & low & under & $\begin{array}{l}\text { No pollen identified from the site, } \\
\text { Aetia, where it was recorded growing, } \\
\text { but one grain recorded at Gomara which } \\
\text { must be a result of long distance } \\
\text { dispersal. }\end{array}$ \\
\hline Taxus baccata & A & medium & over & $\begin{array}{l}\text { Occurs in only small amount in the } \\
\text { mixed woods at Aetia \& Zakas. Is } \\
\text { represented in these two pollen spectra } \\
\text { in small numbers, as well as a few other } \\
\text { spectra in small numbers. }\end{array}$ \\
\hline Tilia cordata type & E, A & low & under & \\
\hline Ulmus glabra type & A & medium & under & $\begin{array}{l}\text { No pollen recorded at the mixed woods } \\
\text { site, Aetia, where it is recorded as } \\
\text { growing, but a few grains were recorded } \\
\text { in the spectra at Gomara \& Anelia } \\
\text { which must be the result of long } \\
\text { distance dispersal. }\end{array}$ \\
\hline \multicolumn{5}{|c|}{$\begin{array}{l}\text { Woodland understorey trees } \\
\& \text { shrubs }\end{array}$} \\
\hline Arceuthobium oxycedri & $\mathrm{E}$ & i.d. & i.d. & \\
\hline Buxus sempervirens & $\begin{array}{l}\text { E, A } \\
\text { (occas.) }\end{array}$ & low & well & $\begin{array}{l}\text { Rather good production, but poor } \\
\text { dispersal (Bottema 1974). }\end{array}$ \\
\hline Clematis vitalba group & $\mathrm{E}$ & (no pollen) & under & \\
\hline Hedera helix & E & (no pollen) & under & \\
\hline Juniperus & A & ?medium & under & $\begin{array}{l}\text { Found to be under-represented } \\
\text { (Sheehan 1979; Jahns 1993). }\end{array}$ \\
\hline Rosa & $\mathrm{E}$ & low & under & \\
\hline Rubus type & $\mathrm{E}$ & low & under & \\
\hline \multicolumn{5}{|l|}{ Woodland herbs } \\
\hline Actaea spicata & $\mathrm{E}$ & i.d. & i.d. & \\
\hline Agrimonia type & E & (no pollen) & under & \\
\hline
\end{tabular}




\begin{tabular}{|c|c|c|c|c|}
\hline Pollen/spore type & DM & RD & $\mathbf{R}$ & Comments \\
\hline Anemone nemorosa group & $\mathrm{E}$ & high & over & $\begin{array}{l}\text { A few grains of this pollen type were } \\
\text { found in the spectra of sites where it had } \\
\text { not been seen growing. }\end{array}$ \\
\hline Arabis & $\mathrm{E}$ & medium & over & \\
\hline Arum & $\mathrm{E}$ & (no pollen) & under & \\
\hline Asparagus & E & i.d. & i.d. & \\
\hline Asplenium type & A & i.d. & i.d. & \\
\hline Blechnum spicant & A & medium & over & \\
\hline Cephalanthera & $\mathrm{E}$ & i.d. & i.d. & \\
\hline Cyclamen & E, A & (no pollen) & under & \\
\hline Dorycnium & $\mathrm{E}$ & low & under & \\
\hline Eryngium & $\mathrm{E}$ & i.d. & i.d. & \\
\hline Fragaria vesca type & $\mathrm{E}$ & low & under & \\
\hline Fritillaria & E & (no pollen) & under & \\
\hline Gymnocarpium dryopteris & A & i.d. & i.d. & \\
\hline Helleborus & E & low & under & \\
\hline Limodorum abortivum & E & i.d. & i.d. & \\
\hline Orchis & E & (no pollen) & under & \\
\hline Pimpinella saxifraga & E & i.d. & i.d. & \\
\hline Primula & $E$ & (no pollen) & under & \\
\hline Saxifraga tridactylites type & $\mathrm{E}$ & i.d. & i.d. & \\
\hline Scilla type & E & (no pollen) & under & \\
\hline Stellaria holostea & E & i.d. & i.d. & \\
\hline Thalictrum aquilegifolium & $\mathrm{A}, \mathrm{E}$ & i.d. & i.d. & \\
\hline Thalictrum lucidum & $\mathrm{A}, \mathrm{E}$ & i.d. & i.d. & \\
\hline Umbilicus & E & i.d. & i.d. & \\
\hline Valeriana officinalis type & E & i.d. & i.d. & \\
\hline Viola hirta type & $\begin{array}{l}\text { E, auto- } \\
\text { gamous }\end{array}$ & (no pollen) & under & \\
\hline Viola riviniana type & $\begin{array}{l}\text { E, auto- } \\
\text { gamous }\end{array}$ & (no pollen) & under & \\
\hline
\end{tabular}




\begin{tabular}{|c|c|c|c|c|}
\hline Pollen/spore type & DM & RD & $\mathbf{R}$ & Comments \\
\hline \multicolumn{5}{|l|}{ Riverine trees } \\
\hline Alnus glutinosa & A & high & over & $\begin{array}{l}\text { A few pollen grains recorded in every } \\
\text { surface sample, although it is not } \\
\text { recorded as growing at any of the } \\
\text { surface sample collection sites. It grows } \\
\text { in the riverine vegetation of Grevena. }\end{array}$ \\
\hline Fraxinus angustifolia & A & medium & over & $\begin{array}{l}\text { Not recorded as growing near the } \\
\text { surface samples sites. }\end{array}$ \\
\hline Fraxinus excelsior type & A & medium & over & $\begin{array}{l}\text { Not recorded as growing near the } \\
\text { surface samples sites. }\end{array}$ \\
\hline Myricaria germanica & $\mathrm{E}$ & i.d. & i.d. & \\
\hline Myrtus communis & $\mathrm{E}$ & i.d. & i.d. & \\
\hline Populus & A & medium & well & $\begin{array}{l}\text { Two pollen grains recorded in Mirsina } \\
\text { fen, these are probably from the riverine } \\
\text { vegetation along the Aliakmon which is } \\
\text { closeby. One grain recorded at the } \\
\text { Gomara site must be from long distance } \\
\text { dispersal. }\end{array}$ \\
\hline Salix triandra & E, A & low & well & \\
\hline Tamarix & $\mathrm{E}, \mathrm{A}$ & i.d. & i.d. & Poor dispersal (Bottema 1974). \\
\hline \multicolumn{5}{|c|}{ Evergreen woodland trees } \\
\hline Ephedra fragilis & A & low & under & $\begin{array}{l}\text { One grain identified in the Mirsina fen. } \\
\text { It may have been growing in the steppe } \\
\text { surrounding this site, although it was } \\
\text { not observed. It may also be the result } \\
\text { of long-distance transport (Ritchie \& } \\
\text { Lichti-Federovich 1963; Maher 1969; } \\
\text { Markgraf 1980:143; Birks 1981; Faegri } \\
\text { \& Iversen 1992:30). }\end{array}$ \\
\hline Phillyrea & $\mathrm{E}$ & i.d. & i.d. & $\begin{array}{l}\text { oderate pollen production \& poor } \\
\text { dispersal (Bottema 1974). }\end{array}$ \\
\hline Pistacia & A & i.d. & i.d. & $\begin{array}{l}\text { Known to be highly under-represented } \\
\text { (Bottema 1974; Sheehan 1979; Jahns } \\
\text { 1993). }\end{array}$ \\
\hline Quercus coccifera type & A & high & over & Over-represented (Bottema 1974). \\
\hline \multicolumn{5}{|l|}{$\begin{array}{l}\text { Plants of open dry rocky } \\
\text { habitats }\end{array}$} \\
\hline Agropyron type & $\mathrm{A}$ & i.d. & i.d. & \\
\hline Alkanna type & $\mathrm{E}$ & i.d. & i.d. & \\
\hline Asyneuma type & $\mathrm{E}$ & (no pollen) & under & \\
\hline Bunium & $\mathrm{E}$ & i.d. & i.d. & \\
\hline Campanula erinus type & $\mathrm{E}$ & i.d. & i.d. & \\
\hline
\end{tabular}




\begin{tabular}{|c|c|c|c|c|}
\hline Pollen/spore type & DM & RD & $\mathbf{R}$ & Comments \\
\hline Campanula rotundifolia & E & (no pollen) & under & \\
\hline Carum & E & i.d. & i.d. & \\
\hline Cnidium silaifolium & $\mathrm{E}$ & i.d. & i.d. & \\
\hline Crupina & $\mathrm{E}$ & i.d. & i.d. & \\
\hline Echinops & $\mathrm{E}, \mathrm{A}$ & (no pollen) & under & \\
\hline Euphrasia & $\mathrm{E}$ & (no pollen) & under & \\
\hline Helianthemum & $\mathrm{E}, \mathrm{A}$ & low & under & \\
\hline Herniaria type & $\begin{array}{l}\text { Auto- } \\
\text { gamous, } \\
\mathrm{E}\end{array}$ & i.d. & i.d. & \\
\hline Linum austriacum type & $\mathrm{E}$ & i.d. & i.d. & Poorly represented (Wright 1972:190). \\
\hline Linum usitatissimum type & $\begin{array}{l}\text { E, auto- } \\
\text { gamous }\end{array}$ & i.d. & i.d. & Poorly represented (Wright 1972:190). \\
\hline Plantago afra & A & i.d. & i.d. & \\
\hline Plantago albicans & A & i.d. & i.d. & \\
\hline Plantago alpina type & A & i.d. & i.d. & \\
\hline Plantago amplexicaulis & A & i.d. & i.d. & \\
\hline Plantago argentea & A & medium & over & \\
\hline Scabiosa columbaria type & $\mathrm{E}$ & i.d. & i.d. & \\
\hline Seseli type & $\mathrm{E}$ & i.d. & i.d. & \\
\hline Stachys angustifolia & $\mathrm{E}$ & (no pollen) & under & \\
\hline Trinia & $\mathrm{E}$ & i.d. & i.d. & \\
\hline \multicolumn{5}{|l|}{ Herbs of open-ground } \\
\hline Achillea type & E & medium & under & \\
\hline Adonis & $\mathrm{E}$ & i.d. & i.d. & \\
\hline Alchemilla type & $\mathrm{E}$ & (no pollen) & under & \\
\hline Allium sphaerocephalon type & $\mathrm{E}$ & i.d. & i.d. & \\
\hline$A m m i$ & $\mathrm{E}$ & medium & well & \\
\hline Anagallis arvensis type & $\mathrm{E}$ & (no pollen) & under & \\
\hline Anchusa arvensis type & $\mathrm{E}$ & (no pollen) & under & \\
\hline Artemisia & $\mathrm{A}, \mathrm{E}$ & medium & well & $\begin{array}{l}\text { Good dispersal (Bottema \& Woldring } \\
\text { 1990). }\end{array}$ \\
\hline Asterolinum linum-stellatum & $\mathrm{E}$ & i.d. & i.d. & \\
\hline Buglossoides purpurocaerulea & $\mathrm{E}$ & (no pollen) & under & \\
\hline
\end{tabular}




\begin{tabular}{|c|c|c|c|c|}
\hline Pollen/spore type & DM & RD & $\mathbf{R}$ & Comments \\
\hline Bupleurum & $\mathrm{E}$ & i.d. & i.d. & \\
\hline Calystegia & $\mathrm{E}$ & (no pollen) & under & \\
\hline Carlina type & $\mathrm{E}$ & low & under & \\
\hline Centaurea cyanus type & $\mathrm{E}$ & i.d. & i.d. & \\
\hline Centaurea graeca type & $\mathrm{E}$ & i.d. & i.d. & \\
\hline Centaurea solstitialis type & $\mathrm{E}$ & i.d. & i.d. & \\
\hline Centaurium & $\mathrm{E}$ & (no pollen) & under & \\
\hline $\begin{array}{l}\text { Chaerophyllum temulentum } \\
\text { type }\end{array}$ & E & i.d. & i.d. & \\
\hline Chelidonium majus & E & i.d. & i.d. & 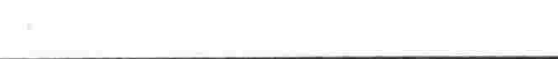 \\
\hline Chenopodiaceae & $\mathrm{A}, \mathrm{E}$ & high & over & $\begin{array}{l}\text { Good production, poor dispersal } \\
\text { (Bottema 1974). }\end{array}$ \\
\hline Cichorium type & E & low & under & \\
\hline Cirsium type & E & low & well & \\
\hline Colchicum & E & (no pollen) & under & \\
\hline Conium maculatum & E & i.d. & i.d. & \\
\hline Consolida type & E & (no pollen) & under & - \\
\hline Convolvulus & E & (no pollen) & under & \\
\hline Coriandrum type & E & i.d. & i.d. & \\
\hline Cuscuta epithymum type & E & (no pollen) & under & $\begin{array}{l}\text { C. epithymum is cleistogamous (Knuth } \\
\text { 1906:59). }\end{array}$ \\
\hline Cuscuta europaea type & E & i.d. & i.d. & \\
\hline Cuscuta monogyna & $\mathrm{E}$ & (no pollen) & under & \\
\hline Dactylis type & A & high & under & $\begin{array}{l}\text { Grazing may prevent grass from } \\
\text { flwring. }\end{array}$ \\
\hline Daucus & E & i.d. & i.d. & \\
\hline Dianthus type & $\mathrm{E}$ & i.d. & i.d. & $\begin{array}{l}\text { Some spp. are pseudo-cleistogamous \& } \\
\text { others cleistogamous (Knuth 1906). }\end{array}$ \\
\hline Emex spinosa & $? \mathrm{E}$ & i.d. & i.d. & \\
\hline Euphorbia & E & low & under & \\
\hline Festuca type & A & high & under & Grazing may prevent flwring. \\
\hline Galium type & E & low & well & \\
\hline Geranium & E & i.d. & i.d. & \\
\hline Gladiolus type & E & i.d. & i.d. & \\
\hline Hibiscus & E & (no pollen) & under & \\
\hline
\end{tabular}




\begin{tabular}{|c|c|c|c|c|}
\hline Pollen/spore type & DM & $\mathbf{R D}$ & $\mathbf{R}$ & Comments \\
\hline Hieracium type & $\mathrm{E}$ & medium & over & $\begin{array}{l}\text { A few grains were identified in several } \\
\text { pollen spectra at sample locations where } \\
\text { plants had not been identified. } \\
\text { Distribution seems erratic. }\end{array}$ \\
\hline Hornungia type & $\mathrm{E}$ & medium & over & \\
\hline Hypericum perforatum type & $\mathrm{E}$ & (no pollen) & under & \\
\hline Kickxia & $\mathrm{E}$ & (no pollen) & under & \\
\hline Knautia & $\mathrm{E}$ & i.d. & i.d. & \\
\hline Lactuca type & $\mathrm{E}$ & i.d. & i.d. & \\
\hline Lathyrus pratensis type & $\mathrm{E}$ & (no pollen) & under & $\begin{array}{l}\text { Lathyrus setifolius \& probably other } \\
\text { spp. are cleistogamous (Knuth 1906:59) }\end{array}$ \\
\hline Lotus type & $\mathrm{E}$ & i.d. & i.d. & \\
\hline Muscari comosum & $\mathrm{E}$ & i.d. & i.d. & \\
\hline Muscari neglectum type & $\mathrm{E}$ & i.d. & i.d. & \\
\hline Myosotis type & $\begin{array}{l}\text { E, auto- } \\
\text { gamous }\end{array}$ & (no pollen) & under & \\
\hline Nigella & $\mathrm{E}$ & low & under & \\
\hline Ononis type & $\mathrm{E}$ & low & under & \\
\hline Oxalis & $\mathrm{E}$ & (no pollen) & under & $\begin{array}{l}\text { Photo-cleistogamous and sometimes } \\
\text { thermo-cleistogamous (Knuth 1906:55) }\end{array}$ \\
\hline Papaver rhoeas type & $\mathrm{E}$ & i.d. & i.d. & \\
\hline Paronychia type & $\mathrm{E}$ & i.d. & i.d. & \\
\hline Pimpinella anisum type & $\mathrm{E}$ & i.d. & i.d. & \\
\hline Plantago bellardii & $\mathrm{A}$ & i.d. & i.d. & \\
\hline Plantago coronopus & $\mathrm{A}$ & medium & over & \\
\hline Plantago lanceolata type & $\mathrm{A}, \mathrm{E}$ & medium & over & $\begin{array}{l}\text { Over-represented, high dispersal } \\
\text { (Bottema 1974). }\end{array}$ \\
\hline Plantago major & $\mathrm{A}$ & i.d. & i.d. & \\
\hline Plantago media & $\mathrm{A}, \mathrm{E}$ & medium & over & \\
\hline Polygonum aviculare type & $\begin{array}{l}\text { Auto- } \\
\text { gamous, } \\
\mathrm{E}\end{array}$ & medium & $?$ & \\
\hline Potentilla & E & low & under & \\
\hline Prunella type & E & i.d. & i.d. & \\
\hline Pteridium aquilinum & $\mathrm{A}$ & medium & well & Representation variable (Bottema 1974) \\
\hline Rumex acetosa group & $\mathrm{A}$ & medium & over & \\
\hline Rumex acetosella group & $\mathrm{A}$ & i.d. & i.d. & \\
\hline
\end{tabular}




\begin{tabular}{|c|c|c|c|c|}
\hline Pollen/spore type & DM & RD & $\mathbf{R}$ & Comments \\
\hline Rumex conglomeratus group & A & (no pollen) & under & \\
\hline Rumex crispus pp. & A & i.d. & i.d. & \\
\hline Rumex type & A & low & under & \\
\hline Salvia officinalis type & E & (no pollen) & under & \\
\hline Salvia verticillata & E & (no pollen) & under & \\
\hline Sanguisorba minor & A & i.d. & i.d. & \\
\hline Scleranthus annuus type & $\begin{array}{l}\text { E, auto- } \\
\text { gamous }\end{array}$ & i.d. & i.d. & $\begin{array}{l}\text { Photo-cleistogamous or sometimes } \\
\text { thermo-cleistogamous (Knuth 1906:56). }\end{array}$ \\
\hline Scrophularia type & E & medium & well & \\
\hline Scutellaria type & E & (no pollen) & under & \\
\hline Sinapis type & $\mathrm{E}$ & medium & over & \\
\hline Smyrnium & $\mathrm{E}$ & i.d. & i.d. & \\
\hline Solanum dulcamara & $\mathrm{E}$ & i.d. & i.d. & \\
\hline Solanum nigrum type & $\mathrm{E}$ & medium & under & \\
\hline Solidago type & E & high & well & \\
\hline Sonchus type & E & (no pollen) & under & \\
\hline Stachys sylvatica type & $\mathrm{E}$ & (no pollen) & under & \\
\hline Taraxacum type & $\mathrm{E}$ & i.d. & i.d. & \\
\hline Teucrium & E & low & under & \\
\hline Theligonum cynocrambe & ?E & i.d. & i.d. & \\
\hline Torilis japonica type & $\mathrm{E}$ & i.d. & i.d. & \\
\hline Trifolium dubium type & $\mathrm{E}$ & (no pollen) & under & \\
\hline Trifolium montanum type & $\mathrm{E}$ & (no pollen) & under & \\
\hline Trifolium repens type & $\mathrm{E}$ & (no pollen) & under & \\
\hline Urtica dioica & A & low & well & \\
\hline Urtica pilulifera type & A & low & under & \\
\hline Urtica urens type & A & low & under & \\
\hline Verbena & $\mathrm{E}$ & (no pollen) & under & \\
\hline Veronica triphyllos type & E & low & under & Some spp. are pseudo-cleistogamous. \\
\hline Vicia cracca type & E & i.d. & i.d. & \\
\hline Viola arvensis type & $\begin{array}{l}\text { E or } \\
\text { auto- } \\
\text { gamous }\end{array}$ & (no pollen) & under & \\
\hline
\end{tabular}




\begin{tabular}{|c|c|c|c|c|}
\hline Pollen/spore type & DM & RD & $\mathbf{R}$ & Comments \\
\hline Xanthium & A & i.d. & i.d. & \\
\hline \multicolumn{5}{|l|}{ Cultivars } \\
\hline Avena type & A & low & under & $\begin{array}{l}\text { Domesticated oats are autogamous \& } \\
\text { pseudo-cleistogamous (Knuth 1906:56). }\end{array}$ \\
\hline Elaeagnus angustifolia & E & i.d. & i.d. & \\
\hline Hordeum type & A & low & under & $\begin{array}{l}\text { Domesticated barley \& emmer wheat } \\
\text { are autogamous. Barley is } \\
\text { cleistogamous \& emmer is pseudo- } \\
\text { cleistogamous. }\end{array}$ \\
\hline Juglans regia & A & high & over & $\begin{array}{l}\text { Production \& dispersal may be good } \\
\text { (Bottema 1974). }\end{array}$ \\
\hline Lathyrus sativus & E & i.d. & i.d. & \\
\hline Medicago sativa & E & i.d. & i.d. & $\begin{array}{l}\text { A few grains were identified from } \\
\text { locations that suggest they were from } \\
\text { the wild subspecies. }\end{array}$ \\
\hline Platanus orientalis & A & medium & over & $\begin{array}{l}\text { Pollen dispersal is not very good } \\
\text { (Bottema \& Woldring 1990:260). } \\
\text { Under-represented (Bottema 1974). }\end{array}$ \\
\hline Secale cereale & A & medium & under & Pseudo-cleistogamous (Knuth 1906:56). \\
\hline Triticum type & $\begin{array}{l}\text { Auto- } \\
\text { gamous }\end{array}$ & medium & over & $\begin{array}{l}\text { These scores are based on the local } \\
\text { vegetation of the sampling sites. On a } \\
\text { regional basis Triticum type is severely } \\
\text { under-represented with low relative } \\
\text { dispersal. }\end{array}$ \\
\hline Zea mays & A & medium & under & $\begin{array}{l}\text { These scores are based on the local } \\
\text { vegetation of the sampling sites. On a } \\
\text { regional basis severely under- } \\
\text { represented with low relative dispersal. }\end{array}$ \\
\hline \multicolumn{5}{|l|}{ Bog \& marsh plants } \\
\hline Anagallis tenella type & E & (no pollen) & under & \\
\hline Angelica sylvestris & E & i.d. & i.d. & \\
\hline Berula erecta & E & low & under & \\
\hline Caltha type & E & i.d. & i.d. & \\
\hline Carex flacca type & A & low & ?well & \\
\hline Carex hallerana type & A & high & over & \\
\hline Chrysosplenium alternifolium & E & i.d. & i.d. & \\
\hline Cyperus longus type & A & low & well & \\
\hline Cyperus michelianus & A & medium & over & \\
\hline Dipsacus type & E & (no pollen) & under & \\
\hline Epilobium hirsutum type & E & (no pollen) & under & \\
\hline
\end{tabular}




\begin{tabular}{|c|c|c|c|c|}
\hline Pollen/spore type & DM & RD & $\mathbf{R}$ & Comments \\
\hline Equisetum & A & low & under & \\
\hline Filipendula & $\mathrm{E}$ & i.d. & i.d. & \\
\hline Galega officinalis & $\mathrm{E}$ & i.d. & i.d. & \\
\hline Gentiana lutea type & $\mathrm{E}$ & i.d. & i.d. & \\
\hline Glyceria type & A & medium & well & \\
\hline Gratiola officinalis & $\mathrm{E}$ & i.d. & i.d. & \\
\hline Juncaceae & A & (no pollen) & under & \\
\hline Linum catharticum & $\mathrm{E}$ & (no pollen) & under & \\
\hline Lychnis flos-cuculi & $\mathrm{E}$ & i.d. & i.d. & \\
\hline Lysimachia & $\mathrm{E}$ & low & well & \\
\hline Lythrum portula & $\mathrm{E}$ & low & under & \\
\hline Lythrum salicaria type & $\mathrm{E}$ & low & under & \\
\hline Mentha type & E & low & under & \\
\hline Oenanthe aquatica group & $\mathrm{E}$ & i.d. & i.d. & \\
\hline Oenanthe pimpinelloides group & $\mathrm{E}$ & i.d. & i.d. & \\
\hline Parnassia palustris & $\mathrm{E}$ & low & well & \\
\hline Phragmites australis & A & medium & well & \\
\hline Pinguicula & $\mathrm{E}$ & (no pollen) & under & \\
\hline Ranunculus acris group & $\mathrm{E}$ & (no pollen) & under & \\
\hline Salix fragilis type & $\mathrm{E}, \mathrm{A}$ & i.d. & i.d. & \\
\hline Samolus valerandi & $\mathrm{E}$ & i.d. & i.d. & \\
\hline Saxifraga stellaris & E & i.d. & i.d. & \\
\hline Schoenus nigricans & A & i.d. & i.d. & \\
\hline Scirpus & A & (no pollen) & under & \\
\hline Sparganium erectum & $\mathrm{A}$ & low & over & \\
\hline Typha angustifolia type & A & low & well & \\
\hline Typha domingensis type & A & (no pollen) & under & \\
\hline $\begin{array}{l}\text { Veronica anagallis-aquatica } \\
\text { type }\end{array}$ & $\mathrm{E}$ & (no pollen) & under & $\begin{array}{l}\text { A few sp. are pseudo-cleistogamous } \\
\text { (Knuth 1906:55). }\end{array}$ \\
\hline \multicolumn{5}{|l|}{ Aquatics } \\
\hline Callitricha obtusangula & ?EpH/A & i.d. & i.d. & \\
\hline Damasonium alisma & $? \mathrm{E}$ & i.d. & i.d. & \\
\hline Hydrocharis morsus-ranae & $\mathrm{E}$ & i.d. & i.d. & \\
\hline
\end{tabular}




\begin{tabular}{|c|c|c|c|c|}
\hline Pollen/spore type & DM & $\mathbf{R D}$ & $\mathbf{R}$ & Comments \\
\hline Hydrocotyle vulgaris & $\begin{array}{l}\mathrm{E} \text {, auto- } \\
\text { gamous }\end{array}$ & i.d. & i.d. & \\
\hline Myriophyllum spicatum & $\mathrm{A}, \mathrm{H}$ & i.d. & i.d. & \\
\hline Potamogeton crispus type & A & i.d. & i.d. & \\
\hline Ranunculus aquatilis group & $\begin{array}{l}\mathrm{E} \text {, auto- } \\
\text { gamous }\end{array}$ & i.d. & i.d. & $\begin{array}{l}\text { R. aquatilis is autogamous } \\
\text { cleistogamously under water where } \\
\text { water is too deep for the flowers to } \\
\text { reach the surface (Knuth 1906:55) }\end{array}$ \\
\hline Sparganium angustifolium & A & low & well & \\
\hline Trapa natans & $\begin{array}{l}\text { Auto- } \\
\text { gamous }\end{array}$ & i.d. & i.d. & $\begin{array}{l}\text { Flowers almost always open in air, } \\
\text { rarely under water, although there is } \\
\text { some hydro-cleistogamy (Knuth } \\
\text { 1908:452) }\end{array}$ \\
\hline Utricularia & $\mathrm{E}$ & (no pollen) & under & \\
\hline
\end{tabular}




\section{References}

Birks, H.J.B. 1981. Long-distance pollen in Late Wisconsin sediments of Minnesota, U.S.A.: A quantitative analysis. New phytologist 87 : 630-661.

Bottema, S. 1974. Late Quaternary vegetation history of northwestem Greece. Doctoral dissertation Rijksuniv. te Gronigen, Gronigen, Netherlands.

Bottema, S. and H. Woldring 1990. Anthropogenic indicators in the pollen record of the Eastern Mediterranean. In S. Bottema, G. Entjes-Nieborg and W. Van Zeist (ed), Man's role in the shaping of the Eastern Mediterranean landscape. A.A. Balkema, Rotterdam.

Dodson, J. 1976. Modem pollen spectra from Chatham Island, New Zealand. New Zealand journal of botany 14: 341-347.

Faegri, K. and Iversen, J. 1992: Textbook of pollen analysis. 4th edition by K. Faegri, P.E. Kaland, and K. Krzywinski. John Wiley \& Sons Ltd, Chichester

Faegri, F. and L. van der Pijl 1979 (3rd revised ed). The principles of pollination ecology. Pergamon Press, Oxford.

Erdtman, G. 1969. Handbook of Palynology. Morphology. Taxonomy. Ecology. Munksgaard, Copenhagen.

Flenley, J. R. 1973. The use of modern pollen rain samples in the study of the vegetation history of tropical regions. In, H.J.B Birks and R.G West, Quaternary plant ecology: 131-142. Blackwell Scientific Publications, Oxford.

Heywood, V.H. (ed) 1978. Flowering plants of the world. Oxford University Press, Oxford.

Iversen, J. 1941. Land occupation in Denmark's Stone Age. Danmarks geologiske undersøgelse 2 rk. (66).

Jahns, S. 1993. On the Holocene vegetation history of the Argive Plain (Peloponnese, southern Greece). Vegetation history and archaeobotany 2: 187-203.

Knuth, P. 1906-9. Handbook of flower pollination. 3 Vols. Clarendon Press, Oxford. [Translated by J.R. Ainsworth Davis]

Maher, L.H. Jr. 1969. Ephedra pollen in the sediments of the Great Lakes region. Ecology 45: 391-395.

Proctor, M. and P. Yeo 1973. The pollination of flowers. Collins, London.

Ritchie, J.C and S. Lichti-Federovich 1963. Contemporary pollen spectra in Central Canada. I. Atmospheric samples at Winnipeg, Manitoba. Pollen et spores 5 (1): 95-114.

Sheehan, M.C. 1979. The Postglacial vegetational history of the Argolid Peninsula, Greece. Unpublished PhD thesis. Indiana University.

Willis, J.C. 1951. A dictionary of flowering plants and ferns. Cambridge University Press, Cambridge.

Wright, H E. Jr. 1972. Vegetation History. In, W.A. McDonald and G.R. Rapp (eds), The Minneapolis Messenia Expedition: reconstructing a Bronze Age regional environment. Minnesota University, Minneapolis: 188-199. 



\section{Appendix XII}

\section{Results of diatom analysis}

Presented here are relative frequency graphs plotted by Dr M. Harper from the results of the diatom analysis undertaken on the sediment core of the Gomara wetland site. Plots are based on the ecological indicator values of van Dam et al (1994) and ecological groups are arranged in order of occurrence.

Figure XII.1 pH tolerance

Figure XII.2 Moisture requirements

Figure XII.3 Habitat

Figure XII.4 Trophic levels

Figure XII.5 Oxygen requirements

Figure XII.6 Organic nitrogen uptake metabolism

\section{Diatom results according to wetland pollen zones}

Samples are combined according to the local wetland pollen assemblage zones defined in Chapter 4 .

$189 \& 172 \mathrm{~cm}$ (zone $G W-6$ ) Diatom concentration is relatively low in the basal sample, but increases twofold in the upper sample. Most species are alkaliphilous (mainly occurring at $\mathrm{pH}>7$ ) recording $60 \%$ in the basal sample and increasing to almost $90 \%$ in the upper sample. Diatoms preferring neutral water conditions reach almost $30 \%$ in the basal sample, but decline to less than $10 \%$ in the upper sample. Alkalibiontic diatoms (exclusively occurring at $\mathrm{pH}>7$ ) record about $5 \%$ in the basal sample, but then drop to less than $1 \%$. Acidophilous diatoms (mainly occurring at $\mathrm{pH}<7$ ) and those indifferent to water $\mathrm{pH}$ record less than $5 \%$.

About $40 \%$ of diatoms live in permanent water, while $40-50 \%$ live in ephemeral water. Only about $5 \%$ live in fairly permanent water. Less than $5 \%$ live in wet places (wet soil and mosses) and about $10 \%$ live in unknown moisture conditions.

Relative frequencies of epipelic diatoms (those that live on silt) are the highest of the sequence (slightly more than $30 \%$ to slightly more than $40 \%$ ). Representation of diatoms that live in shallow water is also high, being slightly more than $30 \%$ to almost $60 \%$, but the lower value (basal sample) is the lowest value of the sequence for this habitat. Epiphytic (those that live on plants) and epilithic diatoms (those that live on rocks) record intermediate values for the sequence (slightly more than $20 \%$ in the basal sample, then slightly more than $10 \%$ ). Less than $1 \%$ of the diatoms live in open water.

Relative frequencies of diatoms indifferent to trophic level (oligo- to hypereutraphentic) are the highest of the sequence in zone GW-6 (slightly more than $35 \%$ in the basal sample, then about 55\%) and those of hypereutraphentic diatoms are the highest of the sequence in the basal sample (about 10\%), but then they decline sharply. Frequencies of eutraphentic diatoms are the highest of the sequence in this zone (about 20-25\%). Meso- to eutraphentic diatoms record the lowest frequencies of the sequence (about $15-20 \%)$. Mesotraphentic, oligo- to mesotraphentic and oligotraphentic diatoms record low relative frequencies (less than 10\%).

Diatoms requiring $100 \%$ oxygen are dominant occurring at about 55 and $70 \%$. Diatoms requiring $>75 \%$ oxygen are next most abundant at $25-30 \%$ and those requiring $>50 \%$ and $>30 \%$ oxygen occur at less than $10 \%$.

Diatoms that are obligately nitrogen-heterotrophic (needing continuously elevated concentrations of organically bound nitrogen) record the highest relative frequencies of the sequence in the basal sample (about 10\%), but then decline to zero. Facultatively nitrogen-heterotrophic diatoms (needing periodically elevated concentrations of organically bound nitrogen) record about $1 \%$. Nitrogen autotrophs tolerant of organically bound nitrogen are dominant in this zone, recording slightly more 
than $60 \%$, then about $85 \%$. Diatoms intolerant of organically bound nitrogen record $25 \%$ in the basal sample, then decline to slightly less than $15 \%$.

$154 \& 139 \mathrm{~cm}$ (zone $G W-5$ ) Diatom concentration continues to increase, reaching the highest value for the sequence in the lower sample (with the exception of those samples in zone GW-1) but decreases in the upper sample to below the value of the top sample in zone GW-6.

Alkaliphilous diatoms continue to be dominant (about $80 \%$ ), and those preferring neutral water conditions are next most abundant (about 15\%). Alkalibiontic diatoms increase from about $1 \%$ in the lower sample to about 5\%. Acidophilous and $\mathrm{pH}$-indifferent diatoms are less than 5\%.

Relative frequencies of diatoms that live in permanent water increase dramatically in this zone to over $80 \%$, but then decline to about $60 \%$. The relative frequency of diatoms that live in fairly permanent water remains at about $5 \%$ in the lower sample, but then increases sharply to about $30 \%$, whereas the relative frequencies of those diatoms that live in ephemeral water are much reduced (about 10\%). Frequencies of diatoms that live in wet places and those whose moisture preference is unknown are very low $(<5 \%)$.

The relative frequency of epipelic diatoms declines in this zone (about 15\% and 20\%), while that of epiphytic/epilithic diatoms remains at about $15 \%$. Diatoms that live in shallow water are very abundant (between $60 \%$ and $75 \%$ ), while those of open water habitats remain extremely rare.

A decline in trophic status results in relative frequencies of meso- to eutraphentic diatoms reaching the maximum for the sequence at slightly above $80 \%$; they then decline to $70 \%$. There is a corresponding decline in the relative frequencies of oligo- to hypereutraphentic diatoms (slightly greater than $5 \%$ ) and eutraphentic diatoms (about 5-10\%). Mesotraphentic, oligo- to mesotraphentic and oligotraphentic diatoms are all represented at less than $10 \%$.

Diatoms requiring $100 \%$ oxygen remain dominant being represented by relative frequencies of about 75 and $85 \%$. Diatoms requiring $>75 \%$ oxygen remain next most abundant, but decline to about 10 and $20 \%$. Diatoms requiring $>50 \%$ and $>30 \%$ oxygen remain at less than $10 \%$.

Diatoms in the nitrogen autrotroph group that are tolerant of organically bound nitrogen remain dominant but have declined to between 55 and $60 \%$. Diatoms intolerant of organically bound nitrogen have increased significantly to about $40 \%$. Facultatively nitrogen-heterotrophic and obligately nitrogen-heterotrophic diatoms represent less than $5 \%$.

$119 \mathrm{~cm}$ (zone $G W-4)$ Diatom concentration continues to decline in this zone. The relative frequency of alkaliphilous diatoms declines $(60 \%)$, while that of neutral and $\mathrm{pH}$-indifferent diatoms increases (about $20 \%$ and $15 \%$, respectively). The relative frequency of acidophilous and alkalibiontic diatoms remains low $(<5 \%)$.

Diatoms that live in permanent water, fairly permanent water and ephemeral water are represented almost equally in this sample, about $30 \%$. This represents a decline in diatoms that live in permanent water, a very slight increase in those that live in fairly permanent water and a sharp increase in those that live in ephemeral water. Those that live in wet places are represented by less than $5 \%$ and those in the unknown category have a similar relative frequency.

Diatoms of shallow water habitats decline (about 45\%), but remain dominant. Epiphytic/epilithic diatoms increase dramatically to almost $40 \%$, while epipelic diatoms remain unchanged (about $20 \%$ ), as do open water diatoms.

Meso- to eutraphentic diatoms remain dominant (50\%). A possible slight decline in trophic level is shown by an increase in oligo- to hypereutraphentic diatoms (to 15\%), although mesotraphentic and eutraphentic diatoms are unchanged $(<10 \%$ and $10 \%$ respectively). Oligotraphentic diatoms remain 
at under $5 \%$.

Diatoms requiring $100 \%$ oxygen remain dominant at almost $80 \%$. Those requiring $>75 \%$ oxygen decline very slightly to about $15 \%$, and those requiring $>50 \%$ and $>30 \%$ are very low at less than $5 \%$.

Nitrogen autotrophic diatoms intolerant of organic nitrogen now become dominant (about 60\%), while diatoms tolerant of organic nitrogen have declined to about $40 \%$.

$106 \mathrm{~cm}$ (zone GW-3) Diatom concentration increases slightly in this zone. Alkaliphilous diatoms increase slightly to about $70 \%$, while those preferring neutral water conditions remain at about the same relative frequency of about $20 \%$ and acidophilous and alkalibiontic remain at less than $5 \%$. Diatoms that are indifferent to water $\mathrm{pH}$ conditions decline to about $5 \%$.

The relative frequency of diatoms that live in permanent water increases again (about 65\%). Diatoms that live in fairly permanent water and ephemeral water both decline (about 10\% and 20\%, respectively). Those of wet places and unknown habitat are unchanged (less than 5\%)

Shallow water diatoms increase again to almost $60 \%$, epiphytic/epilithic diatoms decline slightly and epipelic decline more sharply to almost $10 \%$ (the minimum of this habitat for the sequence).

Meso- to eutraphentic diatoms remain dominant, increasing slightly to almost $65 \%$. Oligo- to mesotraphentic, mesotraphentic and eutraphentic diatoms decline (the first substantially to about 5\%, the others only slightly). Hypereutraphentic diatoms reappear, but at less than $5 \%$ and oligotraphentic diatoms remain at about $5 \%$.

Diatoms requiring $100 \%$ oxygen remain dominant at almost $80 \%$. Those requiring $>75 \%$ oxygen decline to less than $10 \%$ and those requiring $>50 \%$ and $>30 \%$ oxygen increase, but to less than $10 \%$.

Nitrogen autotrophic diatoms tolerant of organic nitrogen again become dominant at about $65 \%$, while diatoms intolerant of organic nitrogen decline to $30 \%$. Facultatively nitrogen-heterotrophic and obligately nitrogen-heterotrophic diatoms increase, but are less than $5 \%$.

$84 \& 56 \mathrm{~cm}$ (zone $G W-2)$ Diatom concentrations in these two samples are minima of the sequence, being extremely low in the upper sample. The relative frequencies of alkaliphilous diatoms increase to almost $80 \%$, then drop to $60 \%$; $\mathrm{pH}$-indifferent diatoms decline to less than $5 \%$, but then increase to almost $15 \%$. Diatoms with a neutral $\mathrm{pH}$ tolerance decline to about $15 \%$ (both samples). Acidophilous and alkalibiontic diatoms increase slightly to about $5 \%$.

Diatoms that live in permanent water continue to be represented by very high relative frequencies $(60-65 \%)$. Diatoms living in fairly permanent water and in ephemeral water fluctuate slightly between 10 and $20 \%$. Diatoms of wet places remain rare $(<5 \%)$, while those of unknown moisture requirements increase $(>10 \%)$.

Diatoms of shallow water remain dominant with relative frequencies about 50 and $70 \%$. Epiphytic/epilithic diatoms are the next most abundant, recording about 15 and $40 \%$. Epipelic diatoms increase slightly to almost $15 \%$, while diatoms that live in open water remain at less than $1 \%$.

Meso- to eutraphentic diatoms remain dominant with relative frequencies of about 55 and $75 \%$. Mesotraphentic diatoms increase to almost $10 \%$ and then decline to about $5 \%$. Oligotraphentic, oligoto mesotraphentic and eutraphentic diatoms are all represented at less than $10 \%$.

Diatoms requiring $100 \%$ oxygen remain dominant at relative frequencies about 65 and $85 \%$. Diatoms requiring $>75 \%$ and $>50 \%$ oxygen both decline slightly in the lower sample, but then increase to about $15 \%$. Those requiring $>30 \%$ oxygen are represented at less than $5 \%$. 
Nitrogen autotrophic diatoms intolerant of organically bound nitrogen are dominant in the lower sample (about $55 \%$ ), but then decline to $30 \%$, while diatoms tolerant of organic nitrogen record only $40 \%$ in the lower sample, but then become dominant at $65 \%$. Diatoms of unknown organic nitrogen tolerance are about $5 \%$.

$35,10 \& 1 \mathrm{~cm}$ (zone $G W-1$ ) Diatom concentration increases dramatically in this zone to highest levels of the sequence. The relative frequencies of alkaliphilous diatoms are maxima of the sequence (with the exception of that recorded at $172 \mathrm{~cm}$ ). Conversely those of indifferent preferences for water $\mathrm{pH}$ condition and alkalibiontic decline to either zero or almost zero. Acidophilous diatoms and those preferring neutral water conditions remain at about the same relative frequencies, the former increasing slightly to almost $10 \%$ and the latter declining slightly (10\%).

The relative frequency of diatoms that live in permanent water increases slightly, reaching greater than $80 \%$ at $1 \mathrm{~cm}$ (sharing the sequence maximum with the sample at $154 \mathrm{~cm}$ ). Diatoms that live in fairly permanent water decline from about $10 \%$ to about $1 \%$ in the top sample. Diatoms that live in ephemeral water decline slightly to maintain an almost constant relative frequency in this zone at $10 \%$. Those that live in wet places, and those of unknown moisture requirements remain at the same levels as those in the previous zone (less than $5 \%$ and about $10 \%$, respectively).

Diatoms that live in shallow water remain dominant and increase to $80 \%$ in the top sample. Epiphytic/epilithic diatoms decline to about 10 and $25 \%$. Epipelic diatoms increase slightly and then decline in the top sample to less than $10 \%$. No diatoms of open water are represented.

Meso- to eutraphentic diatoms remain dominant at about $65-80 \%$. Oligotraphentic, oligo- to mesotraphentic, mesotraphentic, eutraphentic and hypereutraphentic diatoms are all represented at relative frequencies of less than $10 \%$, but may show a slightly higher trophic level for this zone than GW-2.

Diatoms requiring $100 \%$ oxygen remain dominant and increase to the maximum relative frequency of the sequence of about $95 \%$ in the top sample. Diatoms requiring $>75 \%$ and $>50 \%$ oxygen decline to less than $5 \%$ in the top sample, and those requiring $>30 \%$ oxygen increase to about $5 \%$.

Nitrogen autotrophic diatoms tolerant of organic nitrogen are dominant in this zone at $70-85 \%$, while diatoms that are intolerant of organic nitrogen are much less abundant at about $15-25 \%$. Facultatively nitrogen-heterotrophic and obligately nitrogen-heterotrophic diatoms are less than $5 \%$.

\section{Reference}

van Dam, H., A. Mertens and J. Sinkeldam 1994. A coded checklist and ecological indicator values of freshwater diatoms from the Netherlands. Netherlands journal of aquatic ecology $28(1)$ : 117-133. 


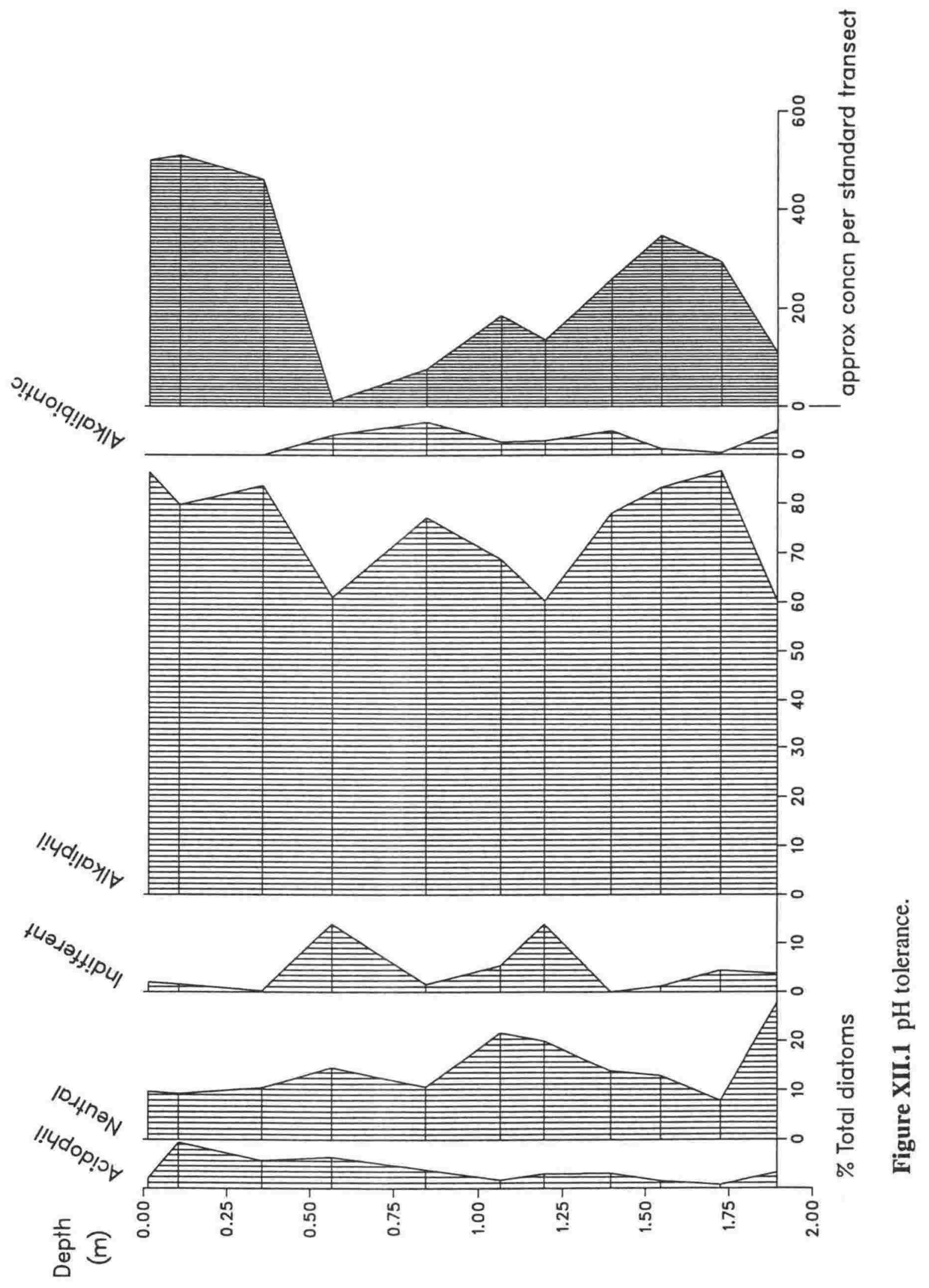




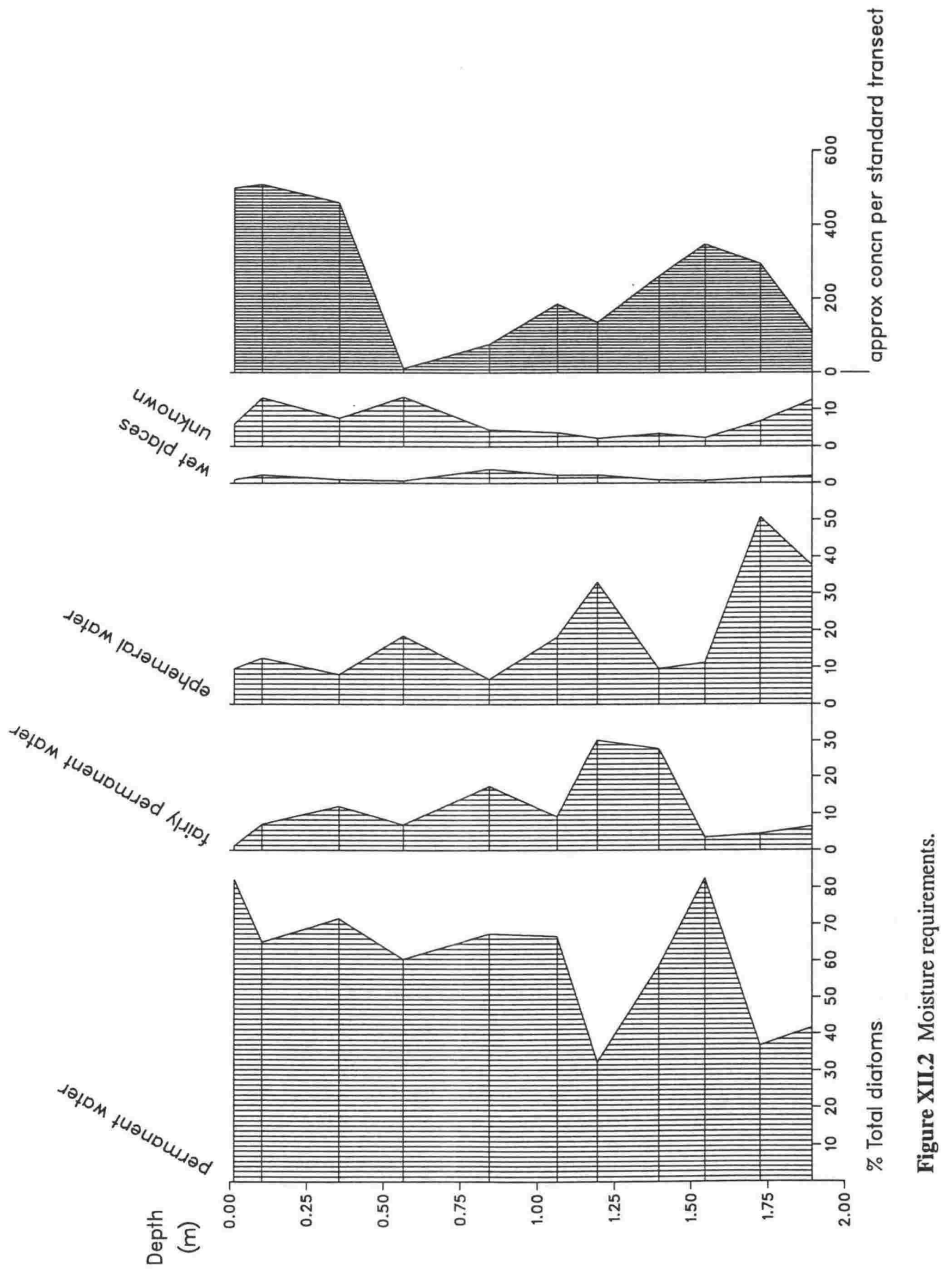




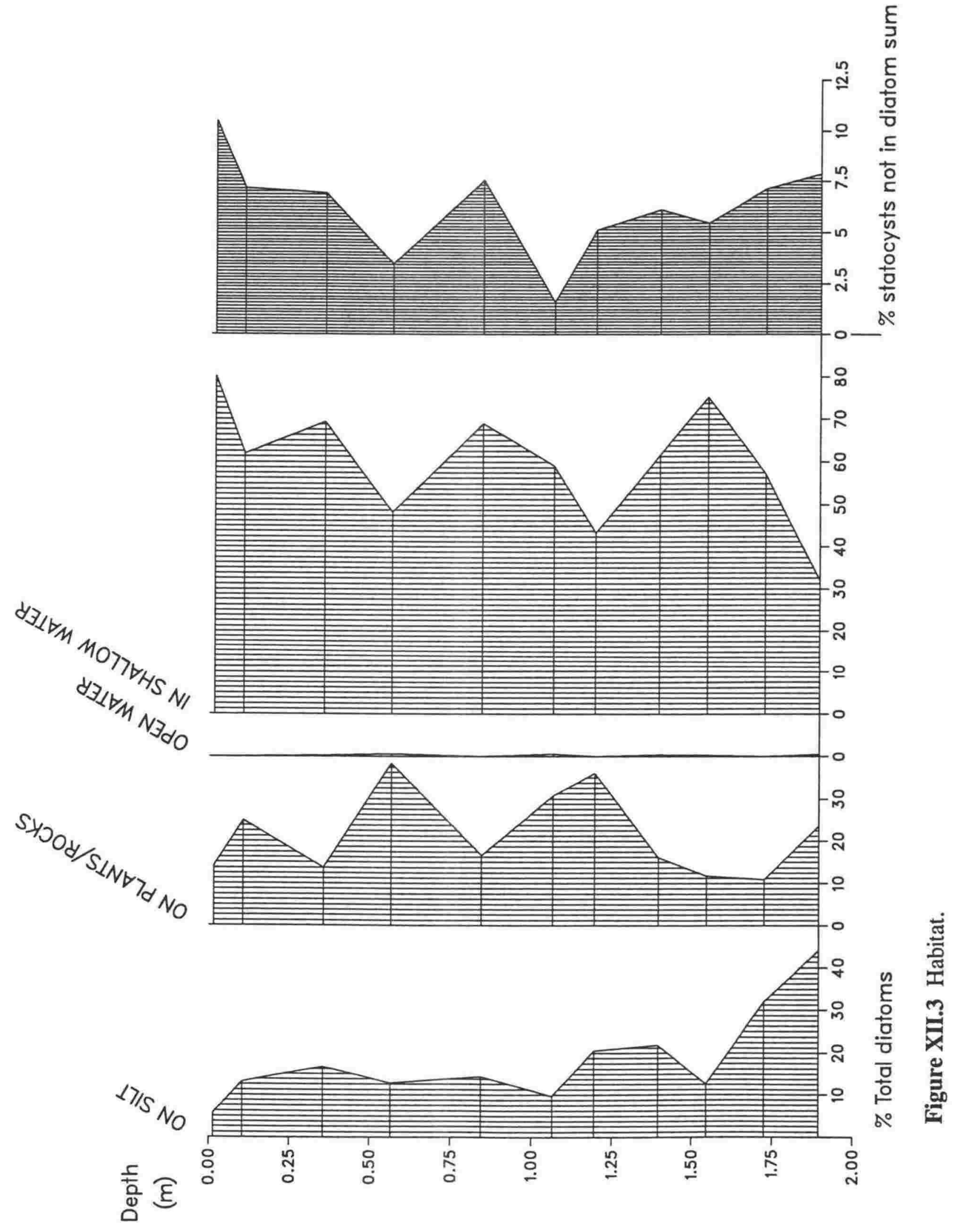




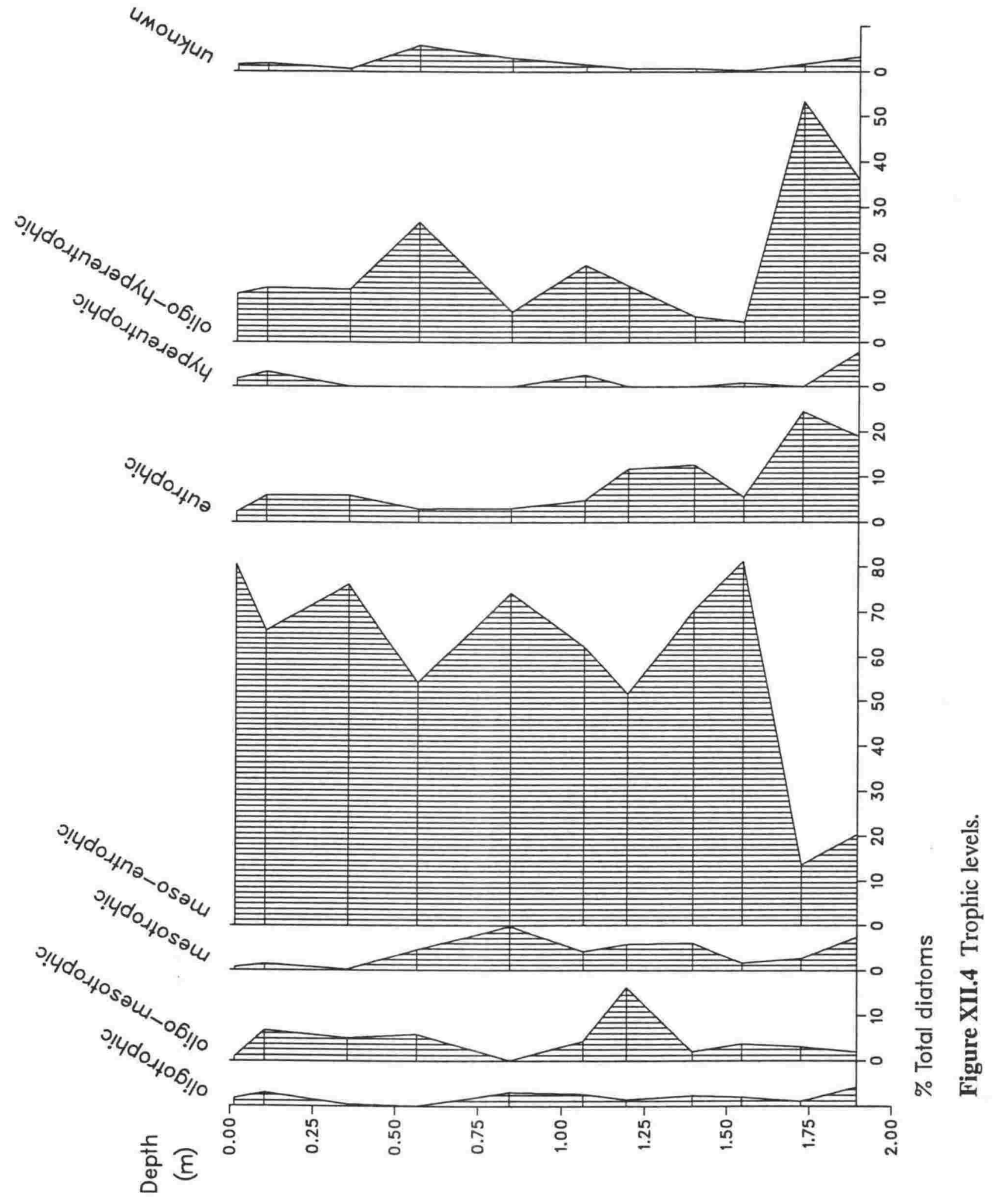




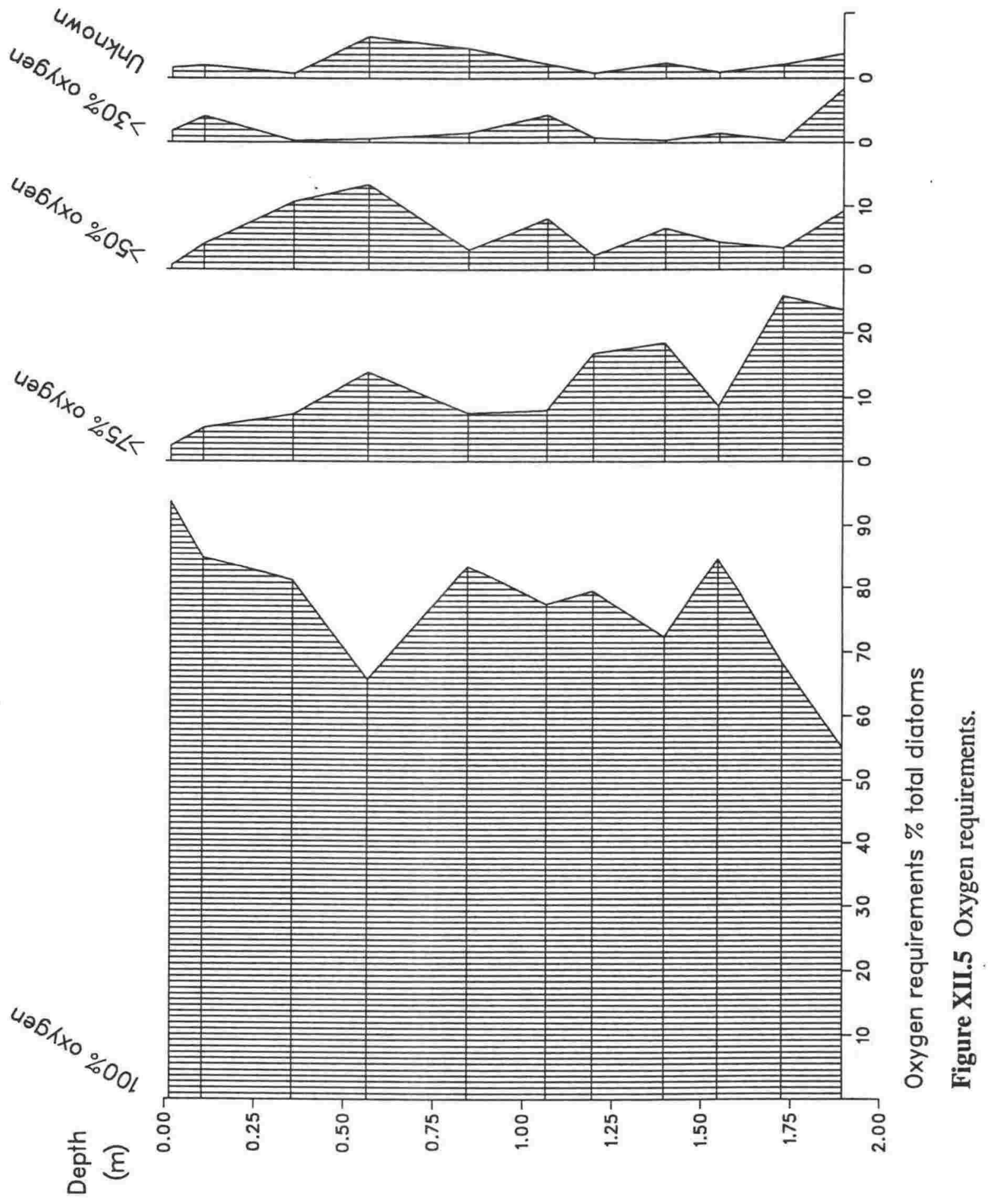




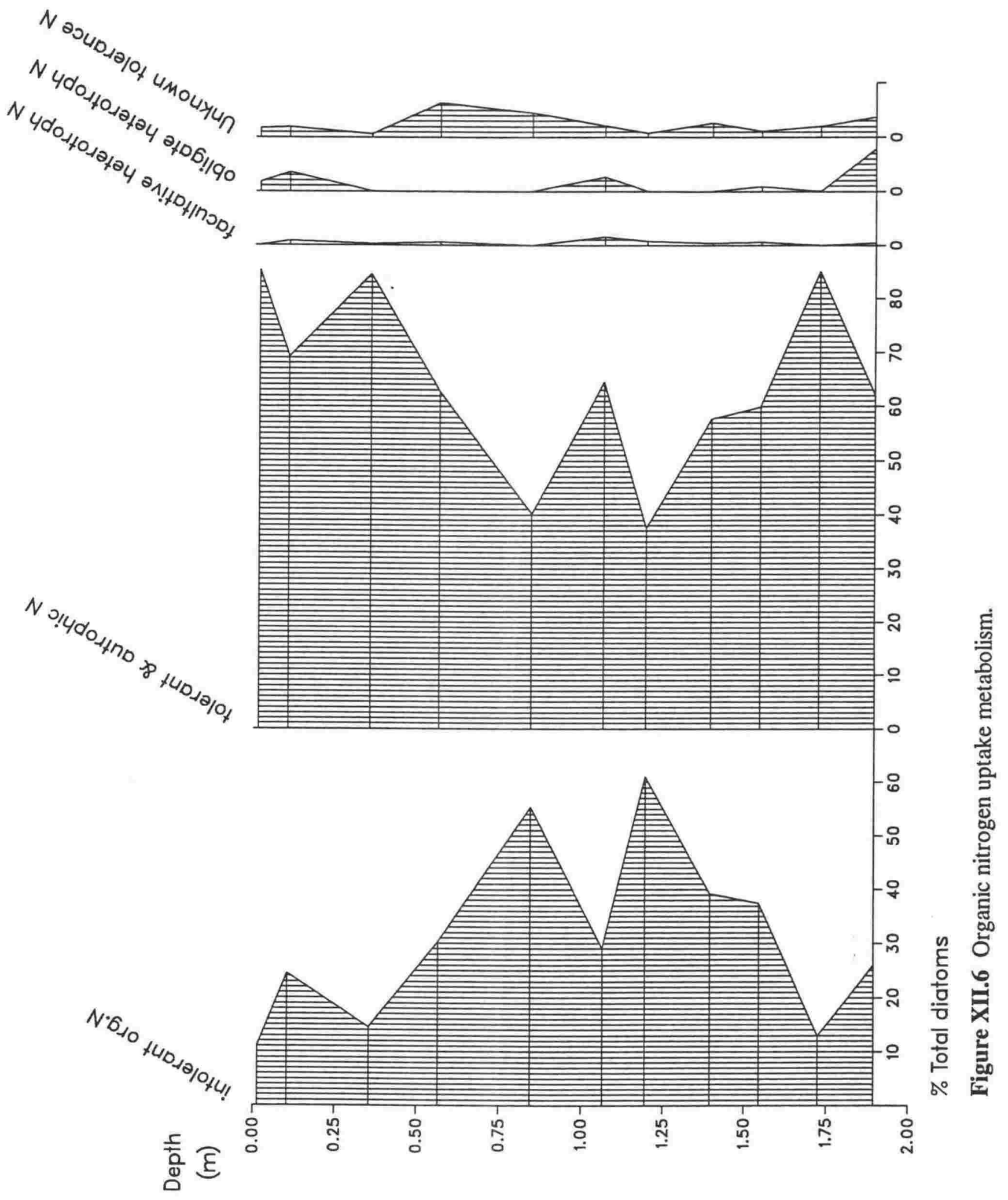



Appendix XIII

Sediment sequences - Pollen and charcoal counts 
Absolute counts data

Slide numbers

Dilution factor

Aliquot size (ul)

Sample weight (g) (wet)

Sample fraction counted

Lycopodium suspension added (ml)

Lycopodium count

$\begin{array}{rrrrrrrrrrrr}1 & 1 & 1 & 1 & 1 & 1 & 1 & 1 & 182 & 1 & 1 & 1 \\ 25 & 25 & 25 & 25 & 25 & 25 & 25 & 25 & 25 & 25 & 25 & 25 \\ 200 & 200 & 200 & 200 & 200 & 200 & 200 & 400 & 600 & 200 & 400 & 400 \\ 1.241 & 1.175 & 1.102 & 1.097 & 1.124 & 1.105 & 1.053 & 1.02 & 1.007 & 1.023 & 1.017 & 1.037 \\ 0.008 & 0.008 & 0.008 & 0.008 & 0.008 & 0.008 & 0.008 & 0.016 & 0.024 & 0.008 & 0.016 & 0.016 \\ 2 & 2 & 1 & 1 & 1 & 1 & 1 & 0.5 & 0.5 & 0.5 & 0.5 & 2 \\ 1213 & 1031 & 723 & 535 & 920 & 754 & 480 & 1049 & 932 & 512 & 993 & 2832\end{array}$

\section{GYMNOSPERMAE}

Abies

Picea abies

Pinus subgenus Diploxylon

Pinus subgenus Haploxylon

Juniperus

Taxus baccata

Ephedra fragilis

\section{DICOTYLEDONES}

Salix triandra

Populus

Juglans regia

Alnus glutinosa

Betula pendula

Carpinus type

Ostrya type

Corylus

Fagus

Castanea sativa

Quercus coccifera type

Quercus trajana type

Quercus frainetto type

Ulmus glabra type

Celtis

Urtica dioica

Urtica urens type

Urtica pilulifera type

Parielaria

Viscum album

Arceuthobium oxycedri

Polygonum bistorta type

Polygonum aviculare type

Bilderdykia

Rumex acetosella group

Rumex acetosa group

Rumex crispus p.p.

Rumex obtusifolius group

Rumex bucephalophorus group

Chenopodiaceae type

Montia fontana

Spergula

Herniaria type

Paronychia type

Dianthus type

Lychnis flos-cuculi

Stellaria holostea

Nymphaea alba

Nigella

$\begin{array}{rrrrrrrrrrrr}9 & 26 & 10 & 29 & 19 & 2 & 16 & 33 & 9 & 10 & 9 & 35 \\ 837 & 412 & 204 & 282 & 581 & 156 & 181 & 284 & 154 & 220 & 170 & 467 \\ 29 & 1 & & 1 & & 4 & 4 & 3 & 2 & & 1 & 2 \\ & 1 & & 10 & 1 & & & & & 1\end{array}$

Trollius europaeus

Actaea spicata

Adonis

Caltha type

Ranunculus acris group

Ranunculus aquatilis group

Anemone nemorosa group

Thalictrum aquilegifolium

Thalictrum minus

Thalictrum lucidum

Papaver rhoeas type

Papaver argemone type

$\begin{array}{rrrrrrrrrrrrr}1 & 3 & 1 & & 2 & 3 & & 3 & 1 & & 3 & \\ & 1 & & & 1 & 4 & 1 & 3 & & 1 & \\ 4 & 4 & 4 & & 6 & 9 & 2 & 2 & 4 & 2 & 1 & 8 \\ & 1 & & 1 & & & & & & & & \\ 4 & 3 & 2 & & 8 & 4 & 2 & 1 & 4 & 2 & 1 & 14 \\ 50 & 59 & 20 & 20 & 80 & 47 & 26 & 16 & 15 & 38 & 5 & 35 \\ 3 & 5 & 5 & 2 & 8 & 11 & 3 & 2 & 4 & 2 & & 3 \\ 63 & 76 & 63 & 48 & 212 & 117 & 233 & 154 & 126 & 154 & 42 & 268 \\ 3 & & & & & & & & & & & \\ 19 & 6 & 3 & 7 & 3 & 3 & 3 & & 1 & & & 32 \\ 24 & 19 & 19 & 6 & 37 & 27 & 10 & 35 & 16 & 18 & 8 & 32 \\ 72 & 99 & 117 & 57 & 97 & 89 & 27 & 66 & 35 & 39 & 18 & 55 \\ 5 & 2 & 1 & & 1 & 1 & & & 1 & & & \\ & & & & & & & & & & & \\ 5 & 8 & 5 & 2 & 4 & 7 & & 2 & 1 & & & 2 \\ & 1 & 11 & & 1 & & & 7 & & & & 7\end{array}$


Gomara

Depth $(\mathrm{cm})$

Hole number

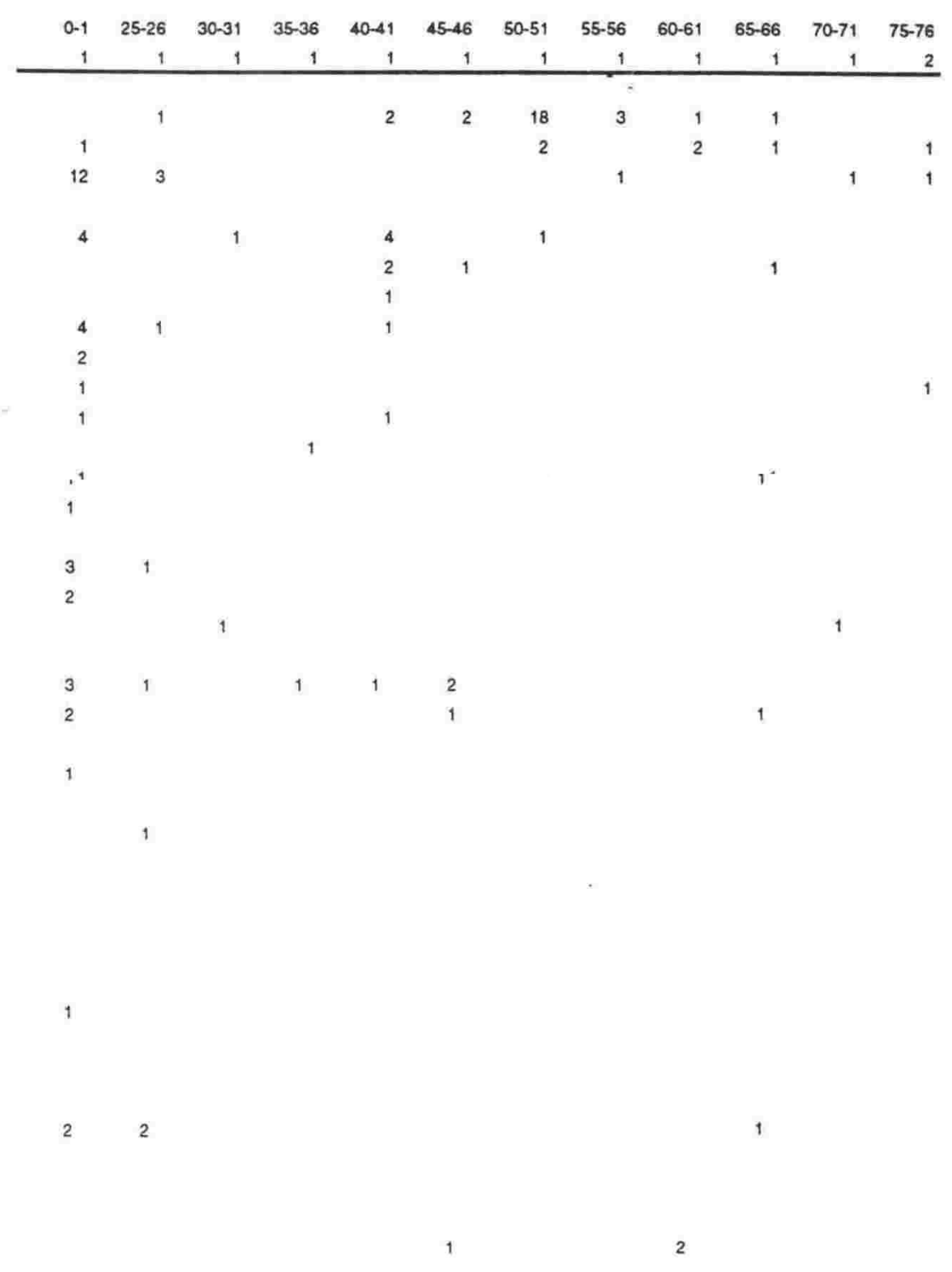

Homungia type

Arabis

Sinapis type

Sedum

Chrysosplenium altemifolium

Saxifraga stellaris

Saxifraga oppositifolia type

Platanus orientalis

Filipendula

Rubus type

Sorbus type

Rosa

Cratageus

Sanguisorba minor

Geum

Potentilla

Ononis type

Trifolium dubium type

Trifolium repens type

Lotus type

Vicia cracca type

Astragalus

Galega officinalis

Medicago sativa

Dorycnium

Trifolium montanum type

Lathyrus sativus

Geranium

Linum austriacum type

Mercurialis perennis

Euphorbia

Dictamrus albus

Rhus coriaria

Pistacia

Acer

Ilex aquifolium

Buxus sempervirens

Rhamnus type

Tilia cordata type

Daphne

Hypericum perforatum type

Viola hirta type

Viola riviniana type

Viola arvensis type

Helianthemum

Tamarix

Elatine alsinastrum

Lythrum salicaria type

Myrtus communis

Circaea lutetiana

Myriophyllum verticillatum

Myriophyllum spicatum

Corrus sanguinea

Contus mas

Hedera helix

Hydrocotyle vulgaris

Ligusticum

Bupleurum

Sison amomum

Berula erecta

Trinia

Seseli type

Oenanthe fistulosa

Chaerophyllum hirsutum type

Chaerophyllum temulertum type

Ammi

Pimpinella saxifraga

Anethum graveolens

Falcaria vulgaris

Peucedanum austriacum type

.

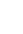


Gomara

Depth (cm)

Hole number

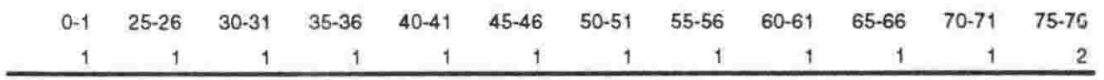

Angelica sylvestris

Peucedanum schotti

Burium

Foeniculum vulgare ssp. vulgare

Anthriscus sylvestris type

Pastinaca sativa

Eryngium

Anthriscus cerefolium

Torilis japonica type

Daucus

Coriandrum type

Vaccinium myrtillus

Soldanella

Primula

Anagallis arvensis type

Anagallis tenella type

Samolus valerandi

Ameria maritima type

Fraxinus excelsior type

Fraxinus angustifolia

Fraxinus omus

Phillyrea

Centaurium

Gentiana lutea type

Calystegia

Cynoglossum type

Heliotropium

Lappula

Echium

Alkanna type

Buglossoides arvensis type

Anchusa arvensis type

Nonea

Teucrium

Stachys angustifolia

Scutellaria type

Prunella type

Mentha type

Solanum dulcamara

Solanum nigrum type

Scrophularia type

Gratiola officinalis

Rhinanthus type

Veronica anagallis-aquatica type

Veronica triphyllos type

Pedicularis

Crucianella

Galium type

Plantago major

Plantago afra

Plantago alpina type

Plantago Lanceolata type

Plantago coronopus

Plantago bellardii

Plantago media

Plantago arenaria

Plantago argented

Plantago albicans

Plantago amplexicaulis

Plantago atrata

Sambucus nigra

Valeriana tuberosa

Valeriana officinalis type

Jasione

Campanula erinus type

Solidago type

Echinops

Artemisia

Carlina type

Achillea type

3

$\begin{array}{lllll}3 & 1 & 3 & 1 & 2 \\ & 1 & 3 & 4\end{array}$


Gomara

Depth $(\mathrm{cm})$

Hole number

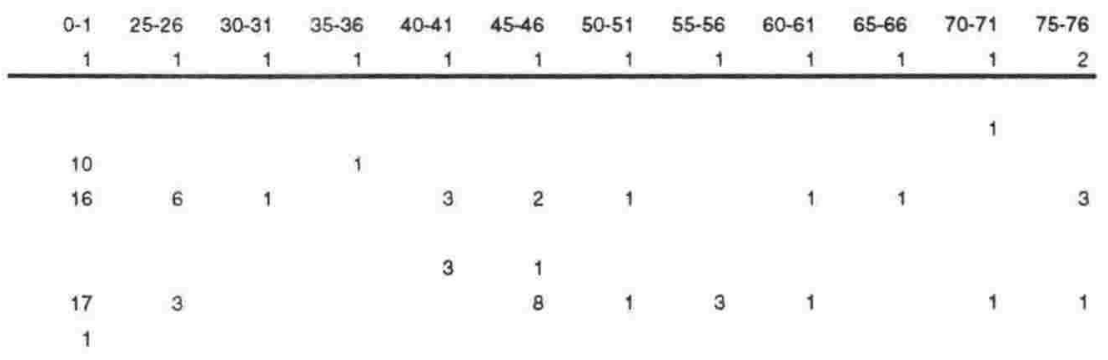

Centaurea napulifera type Cirsium type

Centaurea solstitialis type

Centaurea graeca type

Lactuca type

Hieracium type

Taraxacum type

MONOCOTYLEDONES

Sagittaria sagittifolia

Potamogeton crispus type

Potamogeton pectinatus

Veratrum

Asphodelus albus

Fritillaria

Gagea pratensis

Lilium

Scilla type

Asparagus

Stembergia

Festuca type

Glyceria type

Dactylis type

Agropyron type

Secale cereale

Avena Triticum type

Phragmites australis

Zea mays

Dracunculus type

Lemnaceae

Sparganium erectum

Sparganium angustifolium

Typha angustifolia type

Typha domingensis type

Cladium mariscus

Cyperus michelianus

Cyperus longus type

Carex hallerana type

Carex flacca type

Cephalanthera

\section{PTERIDOPHYTA}

Ophioglossum vulgatum

Botrychium

Adiantum capillus-veneris

Pteridium aquilinum

Asplenium type

Athyrium distentifolium

Blechrum spicant

Polypodium

Salvinia natans

Unidentified monolete spores

Unidentified trilete spores

\section{TOTALS}

Indeterminate

Unknown

Number of terrestrial types

Total wetland pollen \& spores

Total terrestrial trees \& shrubs

Total terrestrial pollen \& spores

Total pollen \& spores

$\begin{array}{rrrrrrrrrrrr}32 & 20 & 16 & & 20 & 17 & 5 & 1 & 5 & 4 & 2 & 15 \\ 80 & 83 & 7 & 10 & 39 & 67 & 16 & 23 & 4 & 6 & 4 & 11 \\ 104 & 51 & 8 & 11 & 17 & 58 & 17 & & 16 & 16 & 8 & 4 \\ 26 & & & & & & & & & & & \\ 2 & 4 & & 2 & 7 & 9 & 3 & 10 & 2 & 2 & & 4 \\ 3 & 1 & & & 1 & 1 & & & 1 & & & \\ 48 & 44 & 11 & & 4 & 11 & 6 & 30 & 2 & 11 & 5 & 19 \\ 1 & & & & & & & & & & & \end{array}$

\section{CHARCOAL}

Charcoal $<25$ um2

Charcoal 25-50 um2

Charcoal 50-100 um2

Charcoal 100-200 um2

Charcoal 200-400 um2

Charcoal $>400$ um2

\begin{tabular}{rrrrrrrrrrrr}
22 & 15 & 24 & 10 & 12 & 12 & 4 & 39 & 9 & 6 & 4 & 18 \\
& 2 & & & 1 & & & & & & & \\
84 & 80 & 36 & 31 & 55 & 64 & 37 & 39 & 41 & 39 & 29 & 42 \\
416 & 481 & 236 & 204 & 435 & 755 & 642 & 544 & 480 & 323 & 203 & 129 \\
1144 & 738 & 461 & 460 & 1079 & 491 & 515 & 608 & 377 & 493 & 262 & 927 \\
1631 & 962 & 529 & 513 & 1221 & 730 & 604 & 680 & 437 & 558 & 292 & 1010 \\
2069 & 1460 & 789 & 727 & 1669 & 1497 & 1250 & 1263 & 926 & 887 & 499 & 1157 \\
& & & & & & & & & & & \\
3161 & 5995 & 2071 & 654 & 4033 & 1090 & 218 & 436 & 109 & 545 & 327 & 545 \\
1417 & 3161 & 3597 & 872 & 3379 & 327 & & 981 & 436 & 327 & 327 & 109 \\
1308 & 2507 & 2180 & 654 & 2834 & 218 & & 327 & 545 & 109 & 109 & 218 \\
436 & 1199 & 1635 & 218 & 1199 & 218 & & 109 & 218 & 218 & 218 & \\
218 & 327 & 218 & 109 & 218 & & & 109 & & 218 & & 109 \\
109 & 436 & 327 & 109 & 109 & & & & 109 & 109 & & \\
\hline
\end{tabular}


Gomara

Depth $(\mathrm{cm})$

Hole number

$\begin{array}{llllllllllllll}80-81 & 85-86 & 90-91 & 95-96 & 100-101 & 105-106 & 107-108 & 110-111 & 115-116 & 120-121 & 125-126 & 131-132\end{array}$

Absolute counts data

Slide numbers

Dilution factor

Aliquot size (ul)

Sample weight (g) (wet)

Sample fraction counted

Lycopodium suspension added (ml)

Lycopodium count

\begin{tabular}{llllllllllll}
2 & 2 & 2 & 2 & 2 & 2 & 2 & 2 & 2 & 2 & 2 & 2 \\
\hline
\end{tabular}

\section{GYMNOSPERMAE}

\section{Abies}

Picea abies

Pinus subgenus Diploxylon

Pinus subgenus Haploxylon

Juniperus

Taxus baccata

Ephedra fragilis

$\begin{array}{rrrrrrrrrrrr}1 & 1 & 1 & 1 & 1 & 1 & 1-3 & 1 & 182 & 182 & 182 & 1 \\ 25 & 25 & 25 & 25 & 25 & 25 & 250 & 250 & 250 & 250 & 250 & 25 \\ 400 & 200 & 100 & 200 & 200 & 200 & 1200 & 400 & 800 & 800 & 800 & 200 \\ 1.043 & 1.041 & 1.077 & 1.068 & 1.078 & 1.115 & 1.397 & 1.1 & 1.08 & 1.086 & 1.085 & 1.12 \\ 0.016 & 0.008 & 0.004 & 0.008 & 0.008 & 0.008 & 0.0048 & 0.0016 & 0.0032 & 0.0032 & 0.0032 & 0.008 \\ 1 & 1 & 2 & 1 & 1 & 1 & 2 & 2 & 2 & 2 & 2 & 1 \\ 1581 & 1036 & 1089 & 904 & 896 & 758 & 794 & 266 & 534 & 584 & 557 & 889\end{array}$

\section{DICOTYLEDONES}

Salix triandra

Populus

Juglans regia

Alnus glutinosa

Betula pendula

Carpinus type

Ostrya type

Corylus

Fagus

Castanea sativa

Quercus coccifera type

Quercus trojana type

Quercus frainetto type

Ulmus glabra type

Celtis

Urtica dioica

Urtica urens type

Urtica pilulifera type

Parietaria

Viscum album

Arceuthobium oxycedri

Polygonum bistorta type

Polygonum aviculare type

$1036 \quad 1089$

$\begin{array}{rrrrrrrrrrrr}43 & 29 & 15 & 36 & 36 & 29 & 8 & 5 & 9 & 15 & 23 & 18 \\ 650 & 322 & 442 & 382 & 359 & 299 & 96 & 122 & 176 & 226 & 218 & 335 \\ 1 & & & 3 & 2 & & & & & & & \\ 1 & 1 & 2 & 5 & 5 & 2 & 1 & & & 1 & 5 & 7 \\ & 3 & 1 & & 2 & & & & & & & \\ 1 & & & & & & & 1 & & \end{array}$

Bilderdykia

Rumex acetosella group

Rumex acetosa group

Rumex crispus p.p.

Rumex obtusifolius group

Rumex bucephalophorus group

Chenopodiaceae type

Montia fontana

Spergula

Hemiaria type

Paronychia type

Dianthus type

Lychnis flos-cuculi

Stellaria holostea

Nymphaea alba

Nigella

Trollius europaeus

Actaea spicata

Adonis

Caltha type

Ranunculus acris group

Ranunculus aquatilis group

Anemone nemorosa group

Thalictrum aquilegifolium

Thalictrum minus

Thalictrum lucidum

Papaver rhoeas type

Papaver argemone type

$\begin{array}{rrrrrrrrrrrr}2 & & & & & & 1 & 1 & & 1 & & 2 \\ 11 & 2 & 2 & 5 & 2 & 5 & 3 & 5 & 3 & 6 & 6 & 6 \\ & & & & & & & & & & & \\ 14 & 5 & 6 & 6 & 7 & 10 & 6 & 5 & 5 & 4 & 4 & 4 \\ 45 & 30 & 58 & 49 & 29 & 40 & 25 & 22 & 37 & 29 & 22 & 48 \\ 8 & 3 & 6 & 2 & 1 & 6 & 5 & 4 & 6 & 7 & 7 & 4 \\ 551 & 249 & 257 & 243 & 241 & 134 & 51 & 65 & 89 & 115 & 93 & 141\end{array}$

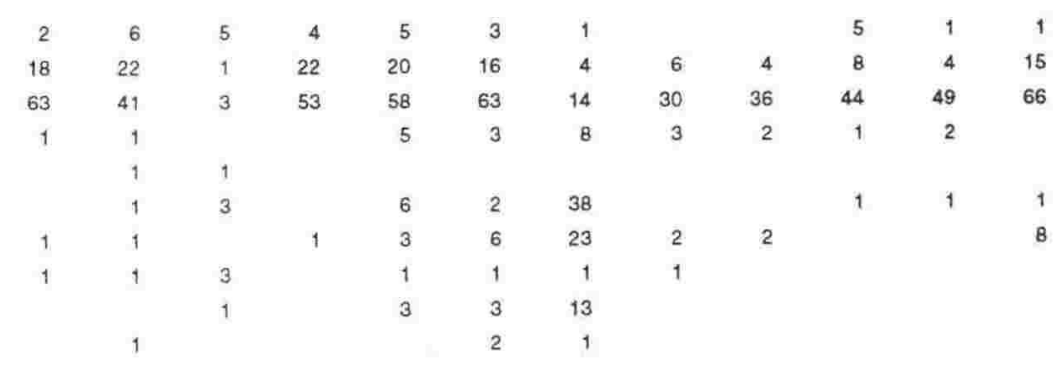




\section{Gomara}

Depth (cm)

Hole number

Homungia type

Arabis

Sinapis type

Sedum

Chrysosplenium alternifolium

Saxifraga stellaris

Saxifraga oppositifolia type

Platanus orientalis

Filipendula

Rubus type

Sorbus type

Rosa

Crataegus

Sanguisorba minor

Geum

Potentilla

Ononis type

Trifolium dubium type

Trifolium repens type

Lotus type

Vicia cracca type

Astragalus

Galega officinalis

Medicago sativa

Dorycnium

Trifolium montanum type

Lathyrus sativus

Geranium

Linum austriacum type

Mercurialis peremis

Euphorbia

Dictamnus albus

Rhus coriaria

Pistacia

Acer

llex aquifolium

Buxus sempervirens:

Rhamnus type

Tilia cordata type

Daphre

Hypericum perforatum type

Viola hirta type

Viola riviniana type

Viola arvensis type

Helianthemum

Tamarix

Elatine alsinastrum

Lythrum salicaria type

Myrtus communis

Circaea lutetiana

Myriophyllum verticillatum

Myriophyllum spicatum

Cornus sanguinea

Cornus mas

Hedera helix

Hydrocotyle vulgaris

Ligusticum

Bupleurum

Sison amomum

Berula erecta

Trinia

Seseli type

Oenanthe fistulosa

Chaerophyllum hirsutum type

Chaerophyllum temulentum type

Ammi

Pimpinella saxifraga

Anethum graveolens

Falcaria vulgaris

Peucedanum austriacum type $\begin{array}{llllllllllllllll}80-81 & 85-86 & 90-91 & 95-96 & 100-101 & 105-106 & 107-108 & 110-111 & 115-116 & 120-121 & 125-126 & 131-132\end{array}$

\begin{tabular}{|c|c|c|c|c|c|c|c|c|c|c|c|}
\hline 2 & 2 & 2 & 2 & 2 & 2 & 2 & 2 & 2 & 2 & 2 & 2 \\
\hline & & 4 & 1 & & 1 & 4 & & 2 & & 2 & 2 \\
\hline \multirow[t]{4}{*}{4} & 2 & 8 & 2 & 6 & & & 3 & & & & 4 \\
\hline & & 2 & & 1 & 1 & 2 & 1 & 1 & 5 & 1 & 2 \\
\hline & 1 & & & & 1 & 1 & & 1 & & 3 & \\
\hline & & & 1 & & & 5 & & & & & \\
\hline
\end{tabular}




\section{Gomara}

Depth $(\mathrm{cm})$

$\begin{array}{lllllllllllllll}80-81 & 85-86 & 90-91 & 95-96 & 100-101 & 105-106 & 107-108 & 110-111 & 115-116 & 120-121 & 125-126 & 131-132\end{array}$

Hole number

Angelica sylvestris

Peucedanum schottii

Bunium

Foeniculum vulgare ssp. vulgare

Anthriscus sylvestris type

Pastinaca sativa

Eryngium

Anthriscus cerefolium

Torilis japonica type

Daucus

Coriandrum type

Vaccinium myrtillus

Soldanella

Primula

Anagallis anensis type

Anagallis tenella type

Samolus valerandi

Ameria maritima type

Fraxinus excelsior type

Fraxinus angustifolia

Fraxinus ormus

Phillyrea

Centaurium

Gentiana lutea type

Calystegia

Cynoglossum type

Heliotropiam

Lappula

Echium

Alkanna type

Buglossoides arvensis type

Anchusa arvensis type

Nonea

Teucrium

Stachys angustifolia

Scutellaria type

Prunella type

Mentha type

Solanum dulcamara

Solanum nigrum type

Scrophularia type

Gratiola officinalis

Rhinamthus type

Veronica anagallis-aquatica type

Veronica triphyllos type

Pedicularis

Cricianella

Galium type

Plantago major

Plantago afra

Plantago alpina type

Plantago lanceolata type

Plantago coronopus

Plantago bellardii

Plantago media

Plantago arenaria

Plantago argentea

Plantago albicans

Plantago amplexicaulis

Plantago atrata

Sambucus nigra

Valeriana tuberosa

Valeriana officinalis type

Jasione

Campanula erinus type

Solidago type

Echinops

Artemisia

Carlina type

Achillea type

\begin{tabular}{|c|c|c|c|c|c|c|}
\hline & & 1 & & & 2 & \\
\hline & 2 & 1 & & 2 & & \\
\hline e & & & 4 & & & \\
\hline \multirow[t]{6}{*}{ type } & 3 & 9 & & 7 & 5 & 4 \\
\hline & & & 1 & & & \\
\hline & & & & 1 & & \\
\hline & 2 & & & & & \\
\hline & 1 & & & & & 1 \\
\hline & 1 & 1 & & & & \\
\hline
\end{tabular}


Gomara

Depth (cm)

Hole number

$\begin{array}{lllllllllllllll}80-81 & 85-86 & 90-91 & 95-96 & 100-101 & 105-106 & 107-108 & 110-111 & 115-116 & 120-121 & 125-126 & 131-132\end{array}$

Centaurea napulifera type

Cirsium type

Centaurea solstitialis type

Centaurea graeca type

Lactuca type

Hieracium type

Taraxacum type

MONOCOTYLEDONES

Sagittaria sagittifolia

Potamogeton crispus type

Potamogeton pectinatus

Veratrum

Asphodelus albus

Fritillaria

Gagea pratensis

Lilium

Scilla type

Asparagus

Stermbergia

Festuca type

Glyceria type

Dactylis type

Agropyron type

Secale cereale

Avena/Triticum type

Phragmites australis

Zea mays

Dracunculus type

Lemnaceae

Sparganium erectum

Sparganium angustifolium

Typha angustifolia type

Typha domingensis type

Cladium mariscus

Cyperus michelianus

Cyperus longus type

Carex hallerana type

Carex flacca type

Cephalanthera

\section{PTERIDOPHYTA}

Ophioglossum vulgatum

Botrychium

Adiantum capillus-veneris

Pteridium aquilinum

Asplenium type

Athyrium distentifolium

Blechrum spicant

Polypodium

Salvinia natans

Unidentified monolete spores

Unidentified trilete spores

TOTALS

Indeterminate

Unknown

Number of terrestrial types

Total wetland pollen \& spores

Total terrestrial trees \& shrubs

Total terrestrial pollen \& spores

Total pollen \& spores

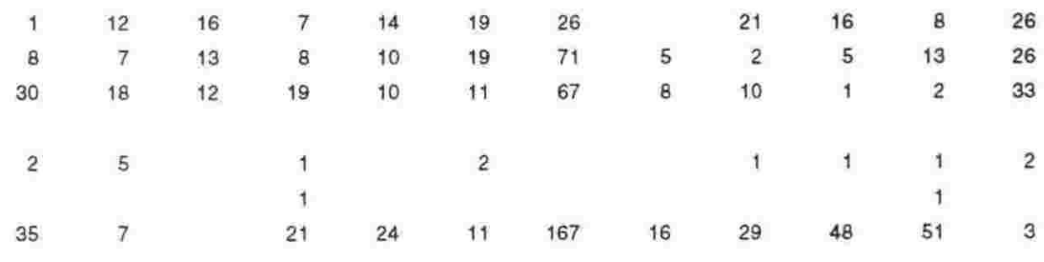

\section{CHARCOAL}

Charcoal $<25$ um2

Charcoal 25-50 um2

Charcoal 50-100 um2

Charcoal 100-200 um2

Charcoal 200-400 um2

Charcoal $>400$ um2

\begin{tabular}{rrrrrrrrrrrr}
4 & 2 & 25 & 8 & 4 & 14 & 11 & 3 & 6 & 2 & 5 & 21 \\
& & 15 & & & 1 & & & & 1 & & 2 \\
46 & 44 & 39 & 43 & 47 & 43 & 100 & 30 & 34 & 43 & 45 & 51 \\
136 & 68 & 100 & 108 & 128 & 133 & 308 & 48 & 73 & 119 & 137 & 120 \\
1423 & 731 & 806 & 817 & 786 & 619 & 248 & 270 & 374 & 476 & 440 & 655 \\
1523 & 810 & 904 & 906 & 880 & 708 & 790 & 311 & 445 & 548 & 498 & 799 \\
1663 & 880 & 1044 & 1022 & 1012 & 856 & 1109 & 362 & 524 & 670 & 640 & 942 \\
& & & & & & & & & & & \\
872 & 1090 & 763 & 1090 & 654 & 1090 & 7303 & 327 & 327 & 654 & 763 & 2180 \\
654 & 327 & 218 & 327 & 218 & 327 & 4469 & 218 & 327 & 109 & 654 & 2071 \\
109 & & & 436 & 109 & 327 & 1635 & 109 & 218 & & 327 & 545 \\
109 & 109 & 109 & 218 & & 109 & 327 & 218 & & & 109 & 545 \\
& & & 218 & & & 218 & & & 109 & & 218 \\
& & & & & & 109 & 109 & & & & 109 \\
\hline
\end{tabular}


Gomara

Depth (cm)

Hole number

Absolute counts data

Slide numbers

Dilution factor

Aliquot size (ul)

Sample weight (g) (wet)

Sample fraction counted

Lycopodium suspension added (ml)

Lycopodium count

\section{GYMNOSPERMAE}

Abies

Picea abies

Pinus subgenus Diploxylon

Pinus subgenus Haploxylon

Juniperus

Taxus baccata

Ephedra fragilis

\section{DICOTYLEDONES}

Salix triandra

Populus

Juglans regia

Alnus glutinosa

Betula pendula

Carpinus type

Ostrya type

Corylus

Fagus

Castanea sativa

Quercus coccifera type

Quercus trojana type

Quercus frainetto type

Ulmus glabra type

Celtis

Urtica dioica

Urtica urens type

Urtica pilulifera type

Parietaria

Viscum album

Arceuthobium oxycedr

Polygonum bistorta type

Polygonum aviculare type

Bilderdykia

Rumex acetosella group

Rumex acetosa group

Rumex crispus p.p.

Rumex obtusifolius group

Rumex bucephalophorus group

Chenopodiaceae type

Montia fontana

Spergula

Hermiaria type

Paronychia type

Dianthus type

Lychnis flos-cuculi

Stellaria holostea

Nymphaea alba

Nigella

Trollius europaeus

Actaea spicata

Adoni.

Caltha type

Ramunculus acris group

Ranunculus aquatilis group

Anemone nemorosa group

Thalictrum aquilegifolium

Thalictrum minus

Thalictrum lucidum

Papaver rhoeas type

Papaver argemone type

$\begin{array}{llllllllllll}135-136 & 140-141 & 145-146 & 150-151 & 155-156 & 160-161 & 165-166 & 170-171 & 175-176 & 180-181 & 185-186 & 189-190\end{array}$

\begin{tabular}{llllllllllll}
2 & 2 & 2 & 2 & 2 & 2 & 2 & 2 & 2 & 2 & 2 & 2 \\
\hline
\end{tabular}

$\begin{array}{rrrrrrrrrrrr}182 & 182 & 1 & 182 & 2 & 1 & 1 & 1 & 1 & 1 & 1 & 182 \\ 250 & 250 & 25 & 250 & 250 & 250 & 25 & 250 & 250 & 250 & 25 & \\ 800 & 800 & 200 & 800 & 400 & 400 & 200 & 400 & 400 & 400 & 200 & \\ 1.108 & 1.171 & 1.188 & 1.132 & 1.146 & 1.28 & 1.625 & 1.526 & 1.536 & 1.461 & 1.309 & 1.126 \\ 0.0032 & 0.0032 & 0.008 & 0.0032 & 0.0016 & 0.0016 & 0.008 & 0.0016 & 0.0016 & 0.0016 & 0.008 & 0.0088 \\ 2 & 2 & 2 & 2 & 2 & 2 & 2 & 6 & 6 & 6 & 4 & 4 \\ 512 & 510 & 1222 & 501 & 258 & 281 & 1202 & 934 & 917 & 903 & 2199 & 2751 \\ & & & & & & & & & & & \\ 11 & 12 & 16 & 5 & 2 & 5 & 60 & 11 & 20 & 17 & 36 & 20 \\ 148 & 159 & 328 & 122 & 109 & 166 & 1232 & 337 & 443 & 355 & 769 & 594 \\ & & & & & 1 & & 2 & & & & \\ 2 & 2 & 5 & & & & 1 & & & & 5 & 10 \\ 1 & & & & & & & 2 & 4 & & 1 & \end{array}$

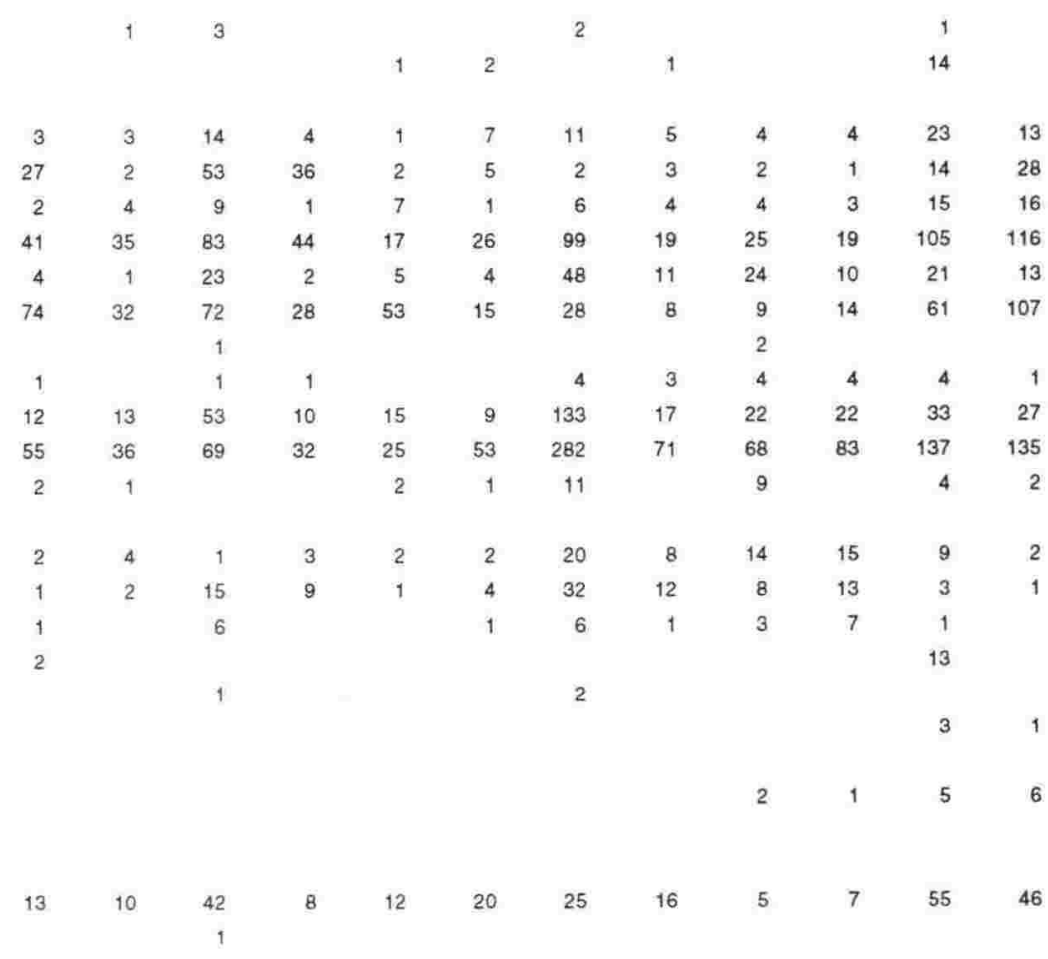


Gomara

Depth (cm)

Hole number

Homungia type

Arabis

Sinapis type

Sedum

Chrysosplenium alternifolium

Saxifraga stellaris

Saxifraga oppositifolia type

Platanus orientalis

Filipendula

Rubus type

Sorbus type

Rosa

Crataegus

Sanguisorba minor

Geum

Potentilla

Ononis type

Trifolium dubium type

Trifolium repens type

Lotus type

Vicia cracca type

Astragalus

Galega officinalis

Medicago sativa

Dorycnium

Trifolium montanum type

Lathyrus sativus

Geranium

Linum austriacum type

Mercurialis perennis

Euphorbia

Dictamrus albus

Rhus coriaria

Pistacia

Acer

Ilex aquifolium

Buxus sempervirens

Rhamnus type

Tilia cordata type

Daphere

Hypericum perforatum type

Viola hirta type

Viola riviniana type

Viola arvensis type

Helianthemum

Tamarix

Elatine alsinastrum

Lythrum salicaria type

Myrtus communis

Circaea lutetiana

Myriophyllum verticillatum

Myriophyllum spicatum

Cornus sanguinea

Cornus mas

Hedera helix

Hydrocotyle vulgaris

Ligusticum

Bupleurum

Sison amomum

Berula erecta

Trinia

Seseli type

Oenanthe fistulosa

Chaerophyllum hirsutum type

Chaerophyllum temulentum type

Ammi

Pimpinella saxifraga

Anethum graveolens

Falcaria vulgaris

Peucedanum austriacum type $\begin{array}{lllllllllllll}135-136 & 140-141 & 145-146 & 150-151 & 155-156 & 160-161 & 165-166 & 170-171 & 175-176 & 180-181 & 185-186 & 189-190\end{array}$

\begin{tabular}{|c|c|c|c|c|c|c|c|c|c|c|c|}
\hline 2 & 2 & 2 & 2 & 2 & 2 & 2 & 2 & 2 & 2 & 2 & 2 \\
\hline & & 1 & & 1 & & 4 & 4 & & 1 & & 4 \\
\hline & & & & & 3 & 2 & & & 1 & 2 & 1 \\
\hline \multirow[t]{6}{*}{3} & 1 & & & & & & & & & & 2 \\
\hline & 2 & & & & & & & & 1 & & \\
\hline & & 1 & & & 1 & 4 & & & 1 & 9 & \\
\hline & 1 & & & & & & & & 1 & & \\
\hline & & 1 & & & & & & & & & 1 \\
\hline & & & & & & & & & 3 & & \\
\hline 1 & 1 & & & & & & & & & & 1 \\
\hline 1 & 1 & 7 & 1 & & & & 1 & & & 1 & 3 \\
\hline & & & & & & & 1 & & & & \\
\hline
\end{tabular}




\section{Gomara}

Depth $(\mathrm{cm})$

Hole number

Angelica sylvestris

Peucedanum schotti

Bunium

Foeniculum vulgare ssp. vulgare

Anthriscus sylvestris type

Pastinaca sative

Eryngium

Anthriscus cerefolium

Torilis japonica type

Daucus

Coriandrum type

Vaccinium myrtillus

Soldanella

Primula

Anagallis arvensis type

Anagallis tenella type

Samolus valerandi

Anmeria maritima type

Fraxinus excelsior type

Fraxinus angustifolia

Fraxinus ormus

Phillyrea

Centaurium

Gentiana lutea type

Calystegia

Cynoglossum type

Heliotropium

Lappula

Echium

Alkanna type

Buglossoides arvensis type

Anchusa arvensis type

Nonea

Teucrium

Stachys angustifolia

Scutellaria type

Prunella type

Mentha type

Solanum dulcamara

Solanum nigrum type

Scrophularia type

Gratiola officinalis

Rhinanthus type

Veronica anagallis-aquatica type

Veronica triphyllos type

Pedicularis

Crucianella

Galium type

Plantago major

Plantago afra

Plantago alpina type

Plantago lanceolata type

Plantago coronopus

Plantago bellardii

Plantago media

Plantago arenaria

Plantago argentea

Plantago albicans

Plantago amplexicaulis

Plantago atrata

Sambucus nigra

Valeriana tuberosa

Valeriana officinalis type

Jasione

Campanula erinus type

Solidago type

Echinops

Artemisia

Carlina type

Achillea type
135-136 140-141 145-146 $150-151$ 155-156 160-161 $165-166$ 170-171 $175-176 \quad 180-181$ 185-186 $189-190$

\begin{tabular}{llllllllllll}
2 & 2 & 2 & 2 & 2 & 2 & 2 & 2 & 2 & 2 & 2 & 2 \\
\hline
\end{tabular}


Gomara

Depth $(\mathrm{cm})$

Hole number

Centaurea napulifera type

Cirsium type

Centaurea solstitialis type

Centaurea graeca type

Lactuca type

Hieracium type

Taraxacum type

MONOCOTYLEDONES

Sagittaria sagittifolia

Potanogeton crispus type

Potamogeton pectinatus

Veratrum

Asphodelus albus

Fritillaria

Gagea pratensis

Lilium

Scilla type

Asparagus

Stembergia

Festuca type

Glyceria type

Dactylis type

Agropyron type

Secale cereale

Avena/Triticum type

Phragmites australis

Zea mavs

Dracunculus type

Lemnaceae

Sparganium erectum

Sparganium angustifolium

Typha angustifolia type

Typha domingensis type

Cladium mariscus

Cyperus michelianus

Cyperus longus type

Carex hallerana type

Carex flacca type

Cephalanthera

\section{PTERIDOPHYTA}

Ophioglossum vulgatum

Botrychium

Adiantum capillus-veneris

Pteridium aquilinum

Asplenium type

Athyrium distentifolium

Blechnum spicant

Polypodium

Salvinia natan.

Unidentified monolete spores

Unidentified trilete spores

TOTALS

Indeterminate

Unknown

Number of terrestrial types

Total wetland pollen \& spores

Total terrestrial trees \& shrubs

Total terrestrial pollen \& spores

Total pollen \& spores

CHARCOAL

Charcoal $<25$ um2

Charcoal 25-50 um2

Charcoal 50-100 um2

Charcoal 100-200 um2

Charcoal 200-400 um2

Charcoal >400 um2 $\begin{array}{llllllllllll}135-136 & 140-141 & 145-146 & 150-151 & 155-156 & 160-161 & 165-166 & 170-171 & 175-176 & 180-181 & 185-186 & 189-190\end{array}$

\begin{tabular}{llllllllllll}
2 & 2 & 2 & 2 & 2 & 2 & 2 & 2 & 2 & 2 & 2 & 2 \\
\hline
\end{tabular}




\title{
Anelia
}

\author{
Depth (cm)
}

Absolute counts data

Slide numbers

Sample weight $(\mathrm{g})$ (wet)

Lycopodium suspension added (ml)

Lycopodium count

$\begin{array}{rrrrrrrrrrrrr}1-11 & 1 & 1 & 1 & 1 & 1 & 1-4 & 1.3 & 1 & 1 & 182 & 1 & 1 \\ 0.980 & 1.001 & 1.002 & 1.003 & 0.973 & 1.008 & 1.014 & 1.042 & 1.004 & 1.025 & 1.730 & 1.604 & 1.675 \\ 2 & 0.5 & 0.5 & 0.5 & 0.5 & 0.5 & 0.5 & 0.5 & 0.5 & 0.5 & 0.5 & 0.5 & 0.5 \\ 5726 & 1167 & 3172 & 2553 & 2273 & 2669 & 3545 & 924 & 1745 & 1253 & 2557 & 2257 & 1005\end{array}$

\section{GYMNOSPERMAE}

Abies

Pinus subgenus Diploxylon

Jumiperus

Taxus baccata

Ephedra fragilis

\section{DICOTYLEDONES}

Salix triandra

Salix fragilis typ.

Populus

Juglans regia

Alnus glutinosa

Betula pendula

Carpinus type

Ostrya type

Corylus

Fagus

Castanea sativa

Quercus coccifera type

Quercus trojana type

Quercus frainetto type

Ulmus glabra type

Humulus lupulus

Untica dioica

Urtica urens type

Urtica pilulifera type

Parietaria

Viscum album

Arceuthobium oxycedri

Polygonum aviculare type Oxyria digyna

Rumex acetosella group

Rumex acetosa group

Chenopodiaceae type

Glinus type

Spergula.

Spergularia type

Herniaria type

Paronychia type

Dianthus type

Lychnis flos-cuculi

$\begin{array}{rrrrrrrrrrrrr}1 & 5 & 5 & 3 & 1 & 1 & 1 & 1 & 3 & 4 & 45 & 4 & 20 \\ 50 & 172 & 274 & 190 & 142 & 258 & 190 & 172 & 216 & 180 & 331 & 204 & 187 \\ 2 & 16 & 62 & 19 & 33 & 38 & 25 & 52 & 4 & 13 & 3 & 17 & 6 \\ & & & & 1 & & & & 2 & & 1 & 2 & \end{array}$

Agrostemma githago

Scleranthus perennis

Scleranthus annuus type

Stellaria holostea

Nuphar lutea

Helleborus

Nigella

Actaea spicata

Caltha type

Ranunculus acris group

Ranunculus aquatilis group

Anemone nemorosa group

Ranunculus arvensis type

Thalictrum aquilegifolium

Thalictrum minus

Thalictrum lucidum

Papaver argemone type

Homungia type

Arabis

Sinapis type

Chrysosplenium alternifolium

Saxifraga stellaris

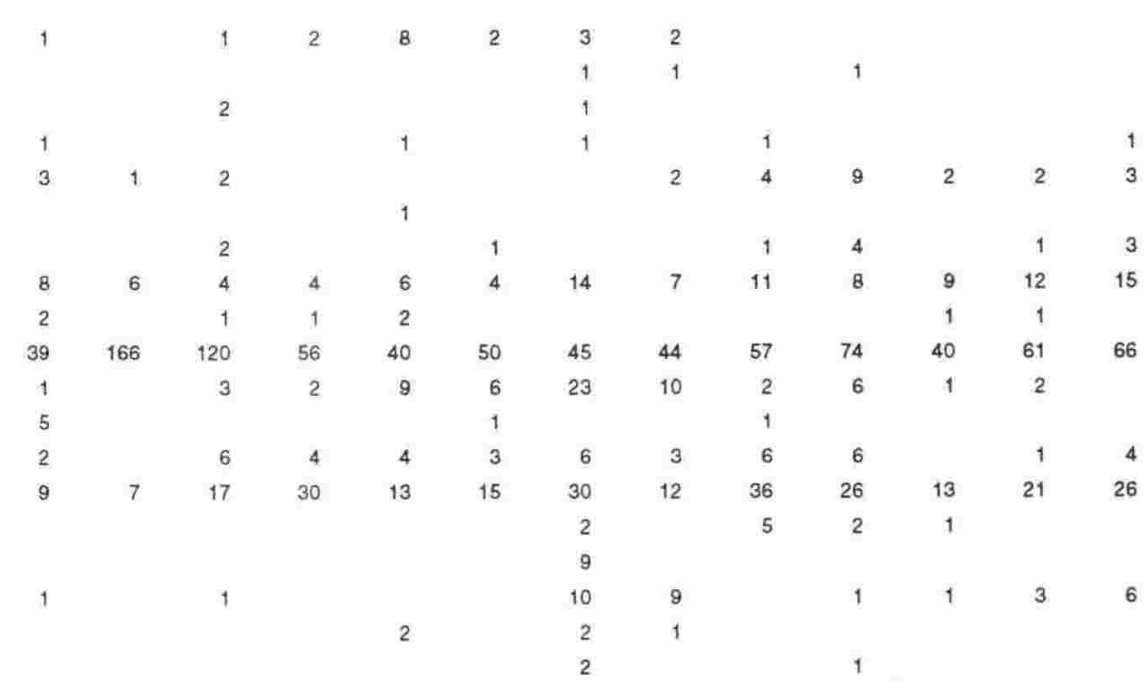




\section{Anelia}

Saxifraga oppositifolia type

Parnassia palustris

Platanu" orientalis

Filipendula

Rubus type

Sorbus type

Rosa

Sanguisorba minor

Geum

Potentilla

Hedysarum coronarium

Ononis type

Trifolium dubium type

Trifolium repens type

Lotus type

Vicia cracca type

Galega officinalis

Ornithopus compressus

Dorycnium

Trifolium montanum type

Linum austriacum type

Linum catharticum

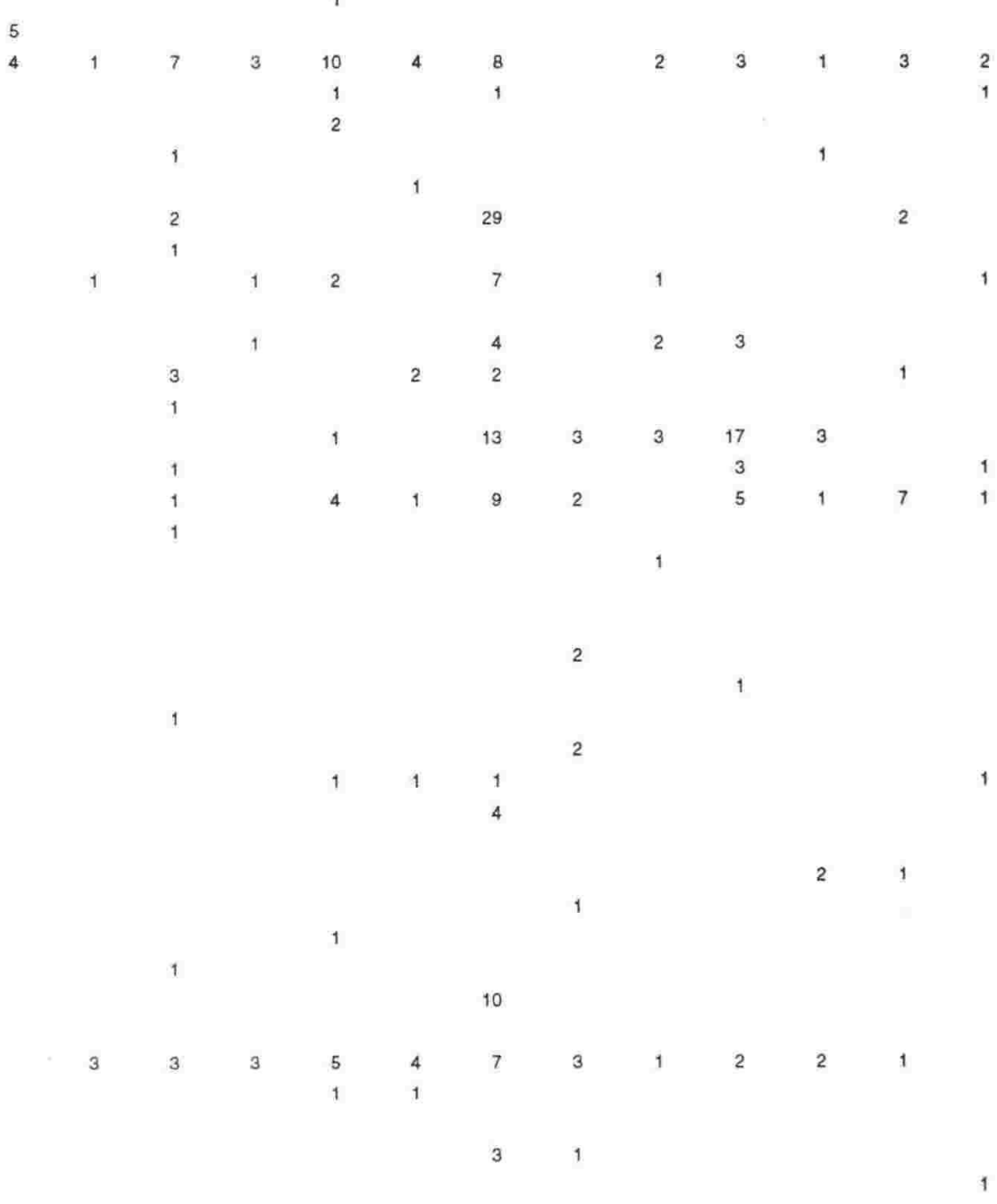

istacia

Acer

Buxus sempervirens

Frangula

Rhamnus type

Vitis vinifera

Tilia cordata type

Daphne

Hypericum perforatum type

Viola hirta type

Helianthemum

Myricaria gemanica

Tamarix

Elatine alsinastrum

Circaea lutetiana

Epilobium angustifolium type

Myriophyllum spicatum

Comus mas

Hedera helix

Hydrocotyle vulgaris

Bupleurum

Berula erecta

Trinia

Oenanthe aquatica group

Oenanthe pimpinelloides group

Chaerophyllum hirsutum type

Chaerophyllum temulentum type

Ammi

Pimpinella saxifraga

Tarilis arvensis

Anethum graveolens

Peucedanum austriacum type

Angelica sylvestris

Anthriscus sylvestris type

Eryngium

Scandix type

Torilis japonica type

Daucus

Erica herbacea

Vaccinium myrtillus

Cyclamen

Androsace villosa

Primula

Lysinachia

Anagallis tenella type

Asterolinon linum-stellatum

Samolus valerandi

Fraxinus excelsior type 


\section{Anelia}

Fraxinus angustifolia

Fraxinus ormus

Cynoglossum type

Heliotropium

Myosotis type

Lappula

Alkanna type

Buglossoides arvensis type

Anchusa arvensis type

Teucrium

Scutellaria type

Mentha type

Solanum dulcamara

Solanum nigrum type

Scrophularia type

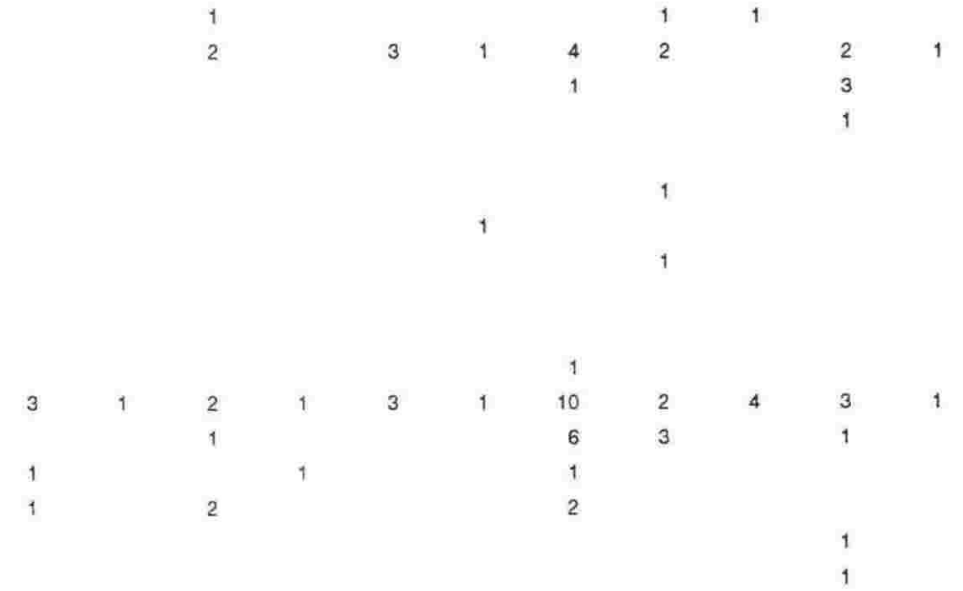

Rhinanthus type

Odontites type

Veronica anagallis-aquatica type

Pedicularis

Galium type

Plantago major

Plantago afra

Plantago lanceolata type

Plantago coronopus

Plantago bellardii

Plantago media

Plantago arenaria

Plantago argentea

Plantago albicans

Plantago atrata

Sambucus nigra

Viburnum

Valeriana officinalis type

Jasione

Campanula erinus type

Campanula rotundifolia

Solidago type

Echinops

Artemisia

Carlina type

Achillea type

Centaurea cyanus type

Centaurea napulifera type

Cirsium type

Centaurea solstitialis type

Centaurea graeca type

Lactuca type

Cichorium type

Hieracium type

Taraxacum type

MONOCOTYLEDONES

Sagittaria sagittifolia

Damasonium alisma

Hydrocharis morsus-ranae

Stratiotes aloides

Vallisneria spiralis

Potamogeton crispus type

Narthecium scardicum

Veratnum

Anthericum liliago

Scilla type

Muscari comosum

Muscari neglectum type

Narcissus papyraceus type

Stembergia

Iris pseudacorus

Iris spuria type

Gynandriris sisyrinchium

Festuca type

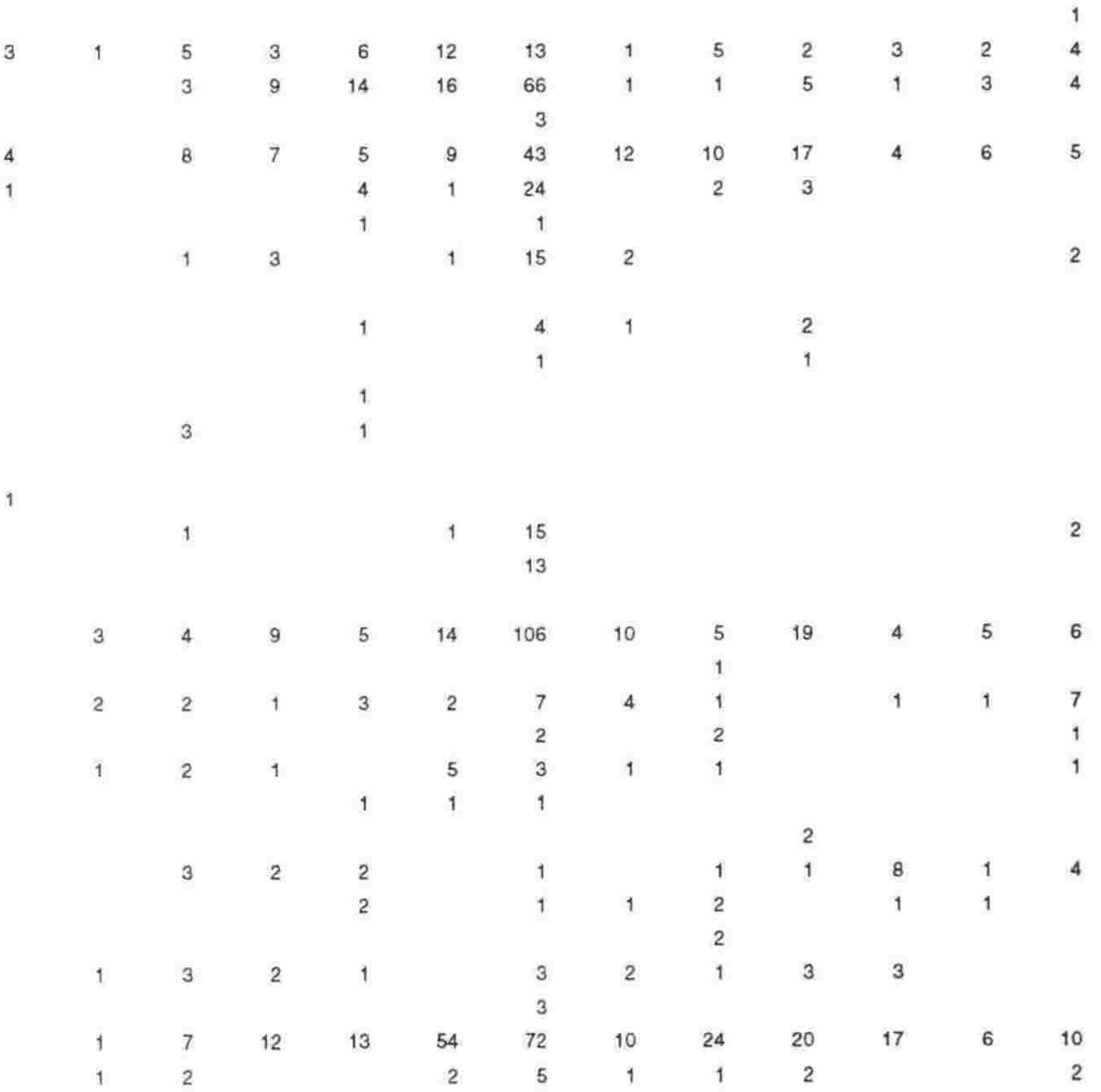




\section{Anelia}

Depth $(\mathrm{cm})$

Glyceria type

Dactylis type

Agropyron type

Secale cereale

Hordeum type

Avena /Triticum type

Phragmites australis

Sparganium erectum

Sparganium angustifolium

Typha angustifolia type

Typha domingensis type

Cyperus michelianus

Cyperus longus type

Carex hallerana type

Carex flacca type

Cephalanthera

\section{PTERIDOPHYTA}

Equisetum

Ophioglossum vulgatum

Botrychium

Adiantum capillus-veneris

Pteridium aquilitum

Asplenium type

Athyrium filix-femina

Athyrium distentifolium

Dryopteris filix-mas type

Blechnum spicant

Polypodium

Salvinia natans

\section{TOTALS}

Indeterminate

Unknown

Number of terrestrial types

Total wetland pollen \& spores

Total trees \& shrubs

\begin{tabular}{|c|c|c|c|c|c|c|c|c|c|c|c|c|}
\hline 1 & 4 & 11 & 4 & 11 & 22 & 54 & 20 & 35 & 27 & 19 & 11 & 29 \\
\hline \multirow[t]{5}{*}{9} & 6 & 4 & 10 & 27 & 20 & 106 & 10 & 12 & 8 & 3 & 2 & 23 \\
\hline & & & & & & & & 1 & 3 & & & 1 \\
\hline & 1 & & 4 & 2 & 2 & & 2 & 3 & 5 & 6 & 1 & 2 \\
\hline & & & & & & & & & 1 & & & \\
\hline & & & 1 & 3 & & & & 1 & 6 & 6 & 1 & 3 \\
\hline \multirow[t]{4}{*}{3} & 3 & 7 & 11 & 27 & 104 & 521 & 4 & 9 & 5 & 2 & 6 & 25 \\
\hline & 1 & 2 & 1 & 2 & & & & & & & 4 & \\
\hline & & & & & & & 1 & 1 & & & & \\
\hline & 1 & & & & & & 1 & & & & & \\
\hline \multirow[t]{2}{*}{6} & & 1 & 2 & 16 & 1 & 5 & 9 & & & & & 1 \\
\hline & & 8 & 7 & 7 & 3 & & 2 & 2 & 3 & & 3 & 2 \\
\hline 5 & 200 & 465 & 244 & 283 & 239 & 240 & 235 & 385 & 333 & 23 & 379 & 178 \\
\hline 2 & & & & & & & 1 & & & & & \\
\hline
\end{tabular}

Total terrestrial pollen \& spores (excluding bog \& marsh plants, aquatics, unknown \& indeterminate) Total pollen \& spores

\begin{tabular}{rrrrrrrrrrrrr}
166 & 414 & 653 & 445 & 497 & 665 & 1240 & 483.3 & 546 & 586 & 722.3 & 470 & 536 \\
200 & 634 & 1180 & 728 & 892 & 1058 & 2207 & 799.3 & 1099 & 988 & 782.3 & 908 & 795 \\
& & & & & & & & & & & & \\
218 & 327 & 763 & 327 & 327 & 1417 & 1417 & 2943 & 327 & 436 & 8066 & 2507 & 5450 \\
0 & 0 & 872 & 327 & 109 & 872 & 763 & 981 & 545 & 327 & 8502 & 981 & 2834 \\
0 & 109 & 218 & 327 & 109 & 327 & 545 & 654 & 327 & 218 & 4687 & 1090 & 2180 \\
0 & 109 & 218 & 109 & 0 & 109 & 218 & 109 & 436 & 327 & 1853 & 981 & 1090 \\
0 & 0 & 0 & 0 & 109 & 0 & 0 & 218 & 0 & 0 & 654 & 545 & 327 \\
0 & 0 & 0 & 0 & 0 & 109 & 109 & 0 & 0 & 109 & 1744 & 218 & 218 \\
\hline
\end{tabular}




\section{Anelia}

Depth $(\mathrm{cm})$

Absolute counts data

Slide numbers

Sample weight $(\mathrm{g})$ (wet)

Lycopodium suspension added (ml)

Lycopodium count

\begin{tabular}{|c|c|c|c|c|c|c|c|c|c|c|c|c|}
\hline 1 & 1 & 1 & 1 & 1 & 1 & $1 \& 2$ & 1 & 1 & 1.7 & 1 & $1-4$ & 1 \\
\hline 1.329 & 1.170 & 1.254 & 1.553 & 1.593 & 1.520 & 1.647 & 1.410 & 1.910 & 1.567 & 1.642 & 2.045 & 7 \\
\hline 0.5 & 0.2 & 0.2 & 0.2 & 0.2 & 0.5 & 0.5 & 0.5 & 0.5 & 0.5 & 0.5 & 2 & \\
\hline 827 & 265 & 587 & 363 & 281 & 958 & 985 & 1096 & 5454 & 5328 & 1106 & 6510 & \\
\hline
\end{tabular}

GYMNOSPERMAE

Abies

Pinus subgenus Diploxylon

Juniperus

Taxus baccata

Ephedra fragilis

$\begin{array}{rrrrrrrrrrrrr}14 & 13 & 26 & 23 & 34 & 41 & 44 & 64 & 28 & 38 & 76 & 52 & 49 \\ 279 & 238 & 177 & 250 & 191 & 200 & 248 & 232 & 92 & 169 & 270 & 175 & 157 \\ 12 & 9 & 4 & 8 & 15 & 12 & 3 & 12 & 3 & 7 & 6 & 6 & 6 \\ & & & & 1 & & & & & & & & \end{array}$

\section{DICOTYLEDONES}

Salix triandra

Salix fragilis type

Populus:

Juglans regia

Alnus glutinosa

Betula pendula

Carpinus type

Ostrya type

Corylus

Fagus

Castanea sativa

Quercus coccifera type

Quercus trojana type

Quercus frainetto type

Ulmus glabra type

Humulus lupulus

Urtica dioica

Urtica urens type

Urica pilulifera type

Parietaria

Viscum album

Arceuthobium oxycedri

Polygonum aviculare type

Oxyria digyna

Rumex acetosella group

Rumex acetosa group

Chenopodiaceae type

Glinus type

Spergula

Spergularia type

Hemiaria type

Paronychia type

Dianthus type

Lychnis flos-cuculi

Agrostemma githago

Scleranthus perennis

Scleranthus annuus type

Stellaria holostea

Nuphar lutea

Helleborus

Nigella

Actaea spicata

Caltha type

Ranunculus acris group

Ranunculus aquatilis group

Anemone nemorosa group

Ranunculus arvensis type

Thalictrum aquilegifolium

Thalictrum minus

Thalictrum lucidum

Papaver argemone type

Homungia type

Arabis

Sinapis type

Chrysosplenium alternifolium

Saxifraga stellaris

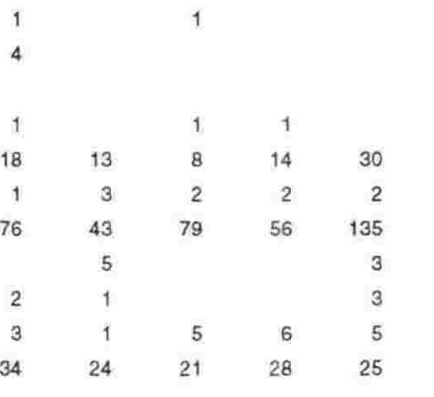

$\begin{array}{rrrrrrrr}2 & 1 & 1 & 2 & & 2 & & 1 \\ 1 & 3 & 3 & 1 & 3 & 4 & 1 & 6 \\ 1 & 4 & 1 & & 1 & 1 & 2 & 1 \\ 9 & 12 & 14 & 20 & 29 & 20 & 18 & 19 \\ 1 & 7 & 2 & & & 3 & 1 & 4 \\ 38 & 30 & 62 & 113 & 46 & 57 & 46 & 55 \\ 1 & & 18 & 1 & & & & 1 \\ 2 & & & & & 1 & & \\ 2 & 2 & 6 & 5 & 4 & & 2 & 4 \\ 35 & 24 & 35 & 30 & 15 & 36 & 20 & 29 \\ & 1 & & 1 & 1 & & 1 & \\ & & & & & & & 5 \\ 2 & & & 1 & 2 & 19 & 5 & 5\end{array}$

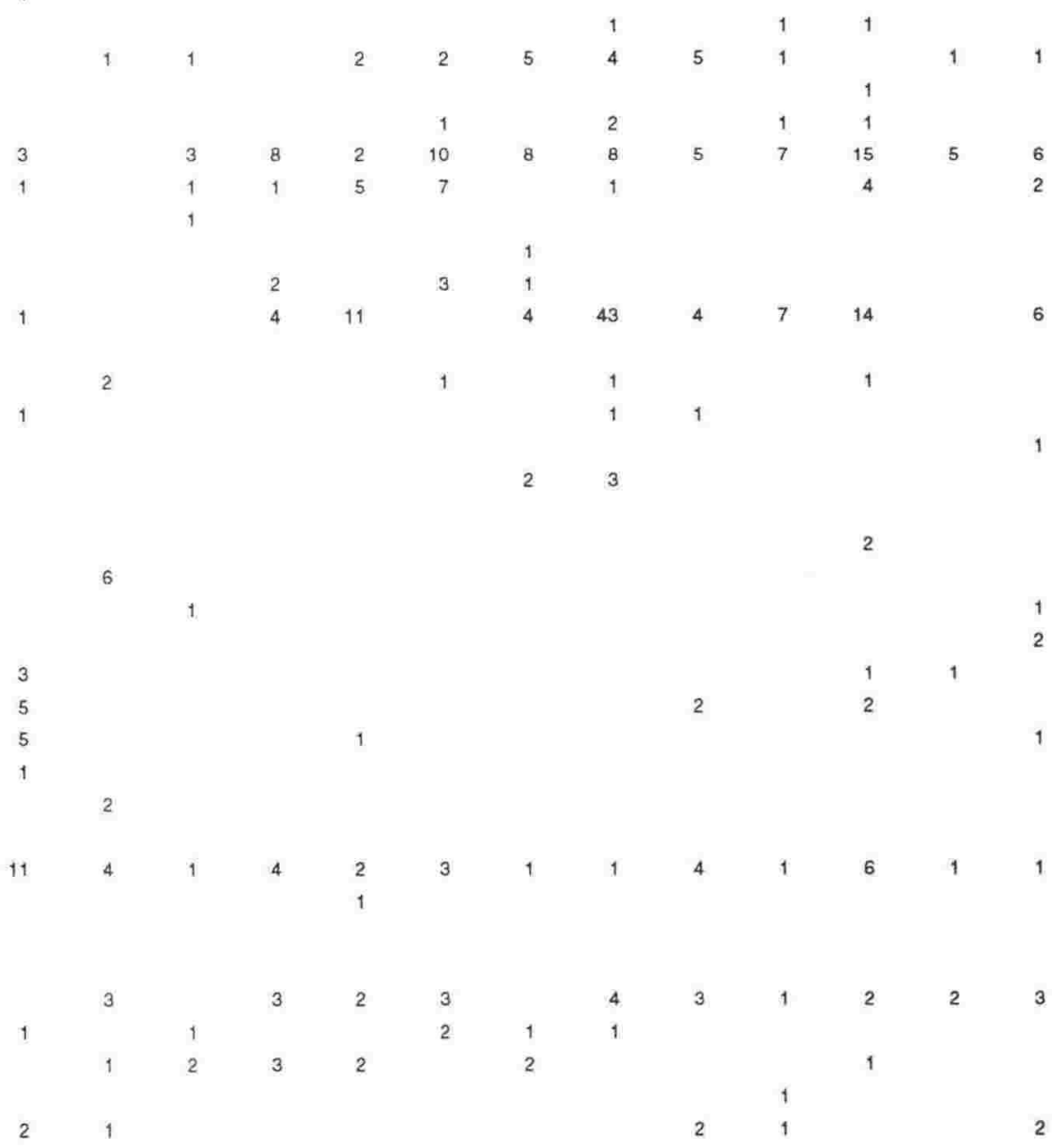




\title{
Anelia
}

\author{
Depth $(\mathrm{cm})$
}

Saxifraga oppositifolia type

Pamassia palustris

Platanus orientalis

Filipendula

Rubus type

Sorbus type

Rosa

Sanguisorba minor

Geum

Potentilla

Hedysarum coronarium

Ononis type

Trifolium dubium type

Trifolium repens type

Lotus type

Vicia cracca type

Galega officinalis

Ornithopus compressus

Dorycnium

Trifolium montanus type

Linum austriacum type

Linum catharticum

Pistacia

Acer

Buxus sempervirens

Frangula

Rhamnus type

Vitis vinifera

Tilia cordata type

Daphne

Hypericum perforatum type

Viola hirta type

Helianthemum

Myricaria gemmanica

Tamarix

Elatine alsinastrum

Circaea lutetiana

Epilobium angustifolium type

Myriophyllum spicatum

Cornus mas

Hedera helix

Hydrocotyle vulgaris

Bupleunum

Berula erecta

Trinia

Oenanthe aquatica group

Oenanthe pimpinelloides group

Chaerophyllum hirsutum type

Chaerophyllum temulentum type

Ammi

Pimpinella saxifraga

Torilis arvensis

Anethum graveolens

Peucedanum austriacum type

Angelica sylvestris

Anthriscus sylvestris type

Eryngium

Scandix type

Torilis japonica type

Daucus:

Erica herbacea

Vaccinium myrtillus

Cyclamen

Androsace villosa

Primula

Lysimachia

Anagallis tenella type

Asterolinon linum-stellatum

Samolus valerandi

Fraxinus excelsior type 


\section{Anelia}

Depth $(\mathrm{cm})$

Fraxinus angustifolia

Fraxinus ormus

Cynoglessum type

Heliotropium

Myosotis type

Lappula

Alkanna type

Buglossoides arvensis type

Anchusa arvensis type

Teucrium

Scutellaria type

Mentha type

Solanum dulcamara

Solanum nigrum type

Scrophularia type

Rhinanthus type

Odontites type

Veronica anagallis-aquatica type

Pedicularis

Galium type

Plantago major

Plantago afra

Plantago lanceolata type

Plantago coronopus

Plantago bellardii

Plantago media

Plantago arenaria

Plantago argentea

Plantago albicans

Plantago atrata

Sambucus nigra

Vibumum

Valeriana officinalis type

Jasione

Campanula erinus type

Camparula rotundifolia

Solidago type

Echinops

Antemisia

Carlina type

Achillea type

Centaurea cyanus type

Centaurea napulifera type

Cirsium type

Centaurea solstitialis type

Centaurea graeca type

Lactuca type

Cichorium type

Hieracium type

Taraxacum type

\section{MONOCOTYLEDONES}

Sagittaria sagittifolia

Damasonium alisma

Hydrocharis morsus-ranae

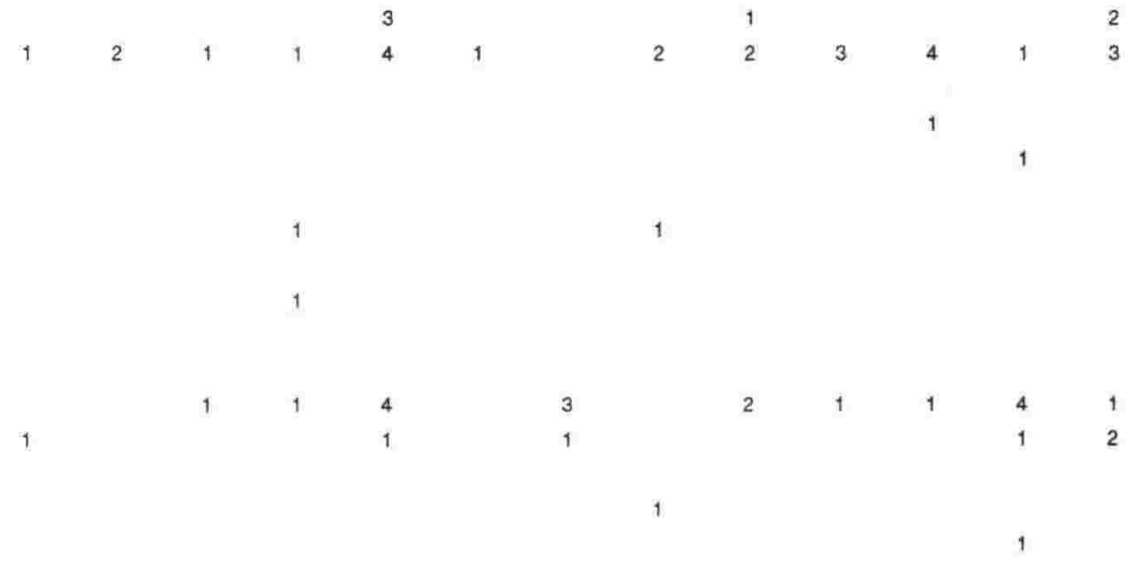

Stratiotes aloides

Vallisneria spiralis

Potamogeton crispus type

Narthecium scardicum

Veratrum

Anthericum liliago

Scilla type

Muscari comosum

Muscari neglectum type

Narcissus papyraceus type

Stembergia

Iris pseudacorus

Iris spuria type

Gynandriris sisyrinchium

Festuca type

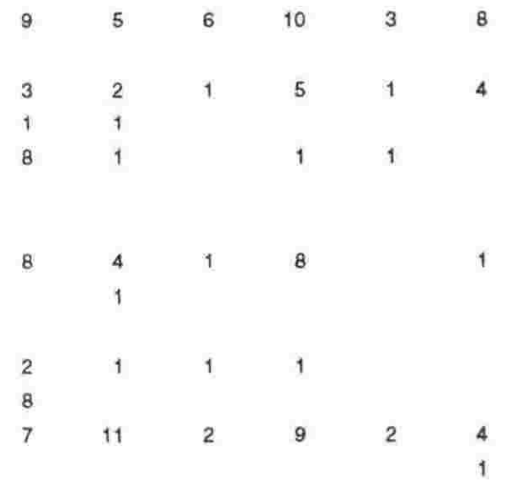




\section{Anelia}

\section{Depth $(\mathrm{cm})$}

Glyceria type

Dactylis type

Agropyron type

Secale cereale

Hordeum type

Avena /Triticum type

Phragmites australis

Sparganium erectum

Sparganium angustifolium

Typha angustifolia type

Typha domingensis type

Cyperus michelianus

Cyperus longus type

Carex hallerana type

Carex flacca type

Cephalanthera

\section{PTERIDOPHYTA}

\section{Equisetum}

Ophioglossum vulgatum

Botrychium

Adiantum capillus-veneris

Pteridium aquilinum

Asplenium type

Athyrium filix-femino

Athyrium distentifolium

Dryopteris filix-mas type

Blechum spicant

Polypodium

Salvinia natans

\section{TOTALS}

Indeterminate

Unknown

Number of terrestrial types

Total wetland pollen \& spores

Total trees \& shrubs

Total terrestrial pollen \& spores

(excluding bog \& marsh plants,

aquatics, unknown \& indeterminate)

Total pollen \& spores$$
99
$$

CHARCOAL

Charcoal $<25$ um2

Charcoal 25-50 um2

Charcoal 50-100 um2

Charcoal 100-200 um2

Charcoal 200-400 um2

Charcoal $>400 \mathrm{um} 2$ $\begin{array}{lllllllllllll}120-121 & 125-126 & 130-131 & 135-136 & 140-141 & 145-146 & 150-151 & 154-155 & 160-161 & 165-166 & 170-171 & 175-176 & 180-181\end{array}$

$\begin{array}{rrrrrrrrrrrrr}13 & 13 & 19 & 15 & 10 & 21 & 14 & 105 & 20 & 19 & 27 & 16 & 42 \\ 6 & 14 & 8 & 5 & 2 & 13 & 13 & 72 & 15 & 8 & 18 & 6 & 17 \\ 1 & 1 & & & & & & & & & & & \\ 1 & 1 & 1 & 4 & & 2 & 4 & 3 & 4 & 2 & 3 & 4 & 6 \\ 4 & 1 & & 1 & & 4 & & 3 & 5 & 3 & & 3 & 6 \\ 4 & 5 & 10 & 9 & 6 & 11 & 11 & 14 & 4 & 8 & 10 & 6 & 1\end{array}$

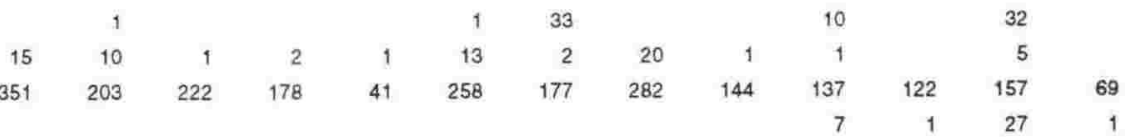

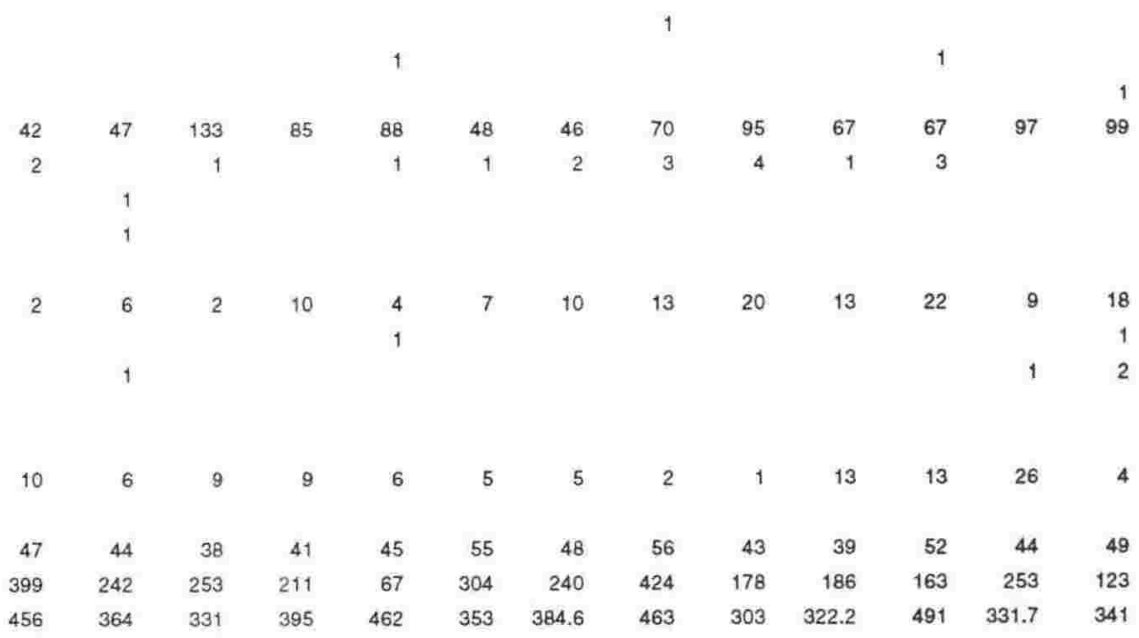

\begin{tabular}{rrrrrrrrrrrrr}
2071 & 2180 & 4687 & 10355 & 8829 & 4905 & 5014 & 7957 & 46761 & 13298 & 19838 & 13843 & 14715 \\
2071 & 1635 & 2398 & 6322 & 9265 & 5777 & 3379 & 4251 & 43709 & 8393 & 12971 & 8611 & 9701 \\
1308 & 872 & 1199 & 3924 & 3924 & 2834 & 3270 & 3270 & 32700 & 6758 & 6976 & 5559 & 5450 \\
763 & 218 & 1417 & 2071 & 2725 & 1526 & 1853 & 3052 & 14715 & 2943 & 4578 & 1962 & 3488 \\
545 & 0 & 436 & 545 & 1199 & 654 & 872 & 1635 & 5886 & 1962 & 1853 & 1090 & 981 \\
218 & 109 & 436 & 436 & 654 & 218 & 327 & 436 & 2834 & 654 & 2507 & 436 & 654 \\
\hline
\end{tabular}




\section{Anelia}

Depth $(\mathrm{cm})$

Absolute counts data

Slide numbers

Sample weight ( $\mathrm{g}$ ) (wet)

Lycopodium suspension added (ml)

$\begin{array}{rrrrrrrrrrrrr}1 & 1 & 1-5 & 1 & 1 & 1-3 & 1 & 182 & 182 & 182 & 1-3 & 1 & 1-3 \\ 1.878 & 1.922 & 1.986 & 1.662 & 1.637 & 1.719 & 1.470 & 1.314 & 1.008 & 1.039 & 1.184 & 0.666 & 1.994 \\ 0.5 & 0.5 & 0.5 & 0.5 & 0.5 & 0.5 & 0.2 & 0.2 & 0.2 & 0.2 & 0.5 & 0.2 & 0.5 \\ 1429 & 3170 & 1193 & 1505 & 953 & 2472 & 351 & 253 & 528 & 439 & 765 & 400 & 1469\end{array}$

GYMNOSPERMAE

Abies

Pinus subgenus Diploxylon

Juniperus

Taxus baccata

Ephedra fragilis

$\begin{array}{rrrrrrrrrrrrr}38 & 47 & 69 & 111 & 105 & 61 & 71 & 51 & 36 & 34 & 48 & 21 & 32 \\ 167 & 166 & 172 & 207 & 160 & 157 & 203 & 271 & 240 & 131 & 186 & 292 & 128 \\ 17 & 8 & 6 & 6 & 6 & 1 & 5 & 6 & & 7 & 3 & 2 & \end{array}$

\section{DICOTYLEDONES}

Salix triandra

Salix fragilis type

Populus

Juglans regia

Alnus glutinosa

Betula pendula

Carpinus type

Ostrya type

Corylus

Fagus

Castanea sativa

Quercus coccifera type

Quercus trojana type

Quercus frainetto type

Ulmus glabra type

Humulus lupulus

Untica dioica

Urtica urens type

Untica pilulifera type

Parietaria

Viscum album

Arceuthobium oxycedri

Polygonum aviculare type

Oxyria digyna

Rumex acetosella group

Rumex acetosa group

Chenopodiaceae type

Glinus type

Spergula

Spergularia type

Hemiaria type

Paronvchia type

Dianthus type

Lychnis flos-cuculi

Agrostemma githago

Scleranthus perennis

Scleranthus annuss type

Stellaria holostea

Nuphar lutea

Helleborus

Nigella

Actaea spicata

Caltha type

Ranunculus acris group

Ranunculus aquatilis group

Anemone nemorosa group

Ranunculus arvensis type

Thalictrum aquilegifolium

Thalictrum minus

Thalictrum lucidum

Papaver argemone type

Homungia type

Arabis

Sinapis type

Chrysosplenium aliernifolium

Saxifraga stellaris

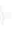

0.5

69

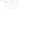




\section{Anelia}

Depth (cm)

Saxifraga oppositifolia type

Pamassia palustris

Platanus orientalis

Filipendula

Rubus type

Sorbus type

Rosa

Sanguisorba minor

Geum

Potentilla

Hedysarum coronarium

Ononis type

Trifolium dubium type

Trifolium repens type

Lotus type

Vicia cracca type

Galega officinalis

Ornithopus compressus

Dorycnium

Trifolium montanum type

Linum austriacum type

Linum catharticum

Pistacia

Acer

Buxus sempervirens

Frangula

Rhammus type

Vitis vinifera

Tilia cordata type

Daphre

Hypericum perforatum type

Viola hirta type

Helianthemum

Myricaria germanica

Tamarix

Elatine alsinastrum

Circaea lutetiana

Epilobium angustifolium type

Myriophyllum spicatum

Cornus mas

Hedera helix

Hydrocotyle vulgaris

Bupleurum

Berula erecta

Trinia

Oenanthe aquatica group

Oenanthe pimpinelloides group

Chaerophyllum hirsutum type

Chaerophyllum temulentum type

Ammi

Pimpinella saxifraga

Torilis arvensis

Anethum graveolens

Peucedanum austriacum type

Angelica sylvestris

Anthriscus sylvestris type

Eryngium

Scandix type

Torilis japonica type

Daucus

Erica herbacea

Vaccinium myrtillus

Cyclamen

Androsace villosa

Primula

Lysimachia

Anagallis tenella type

Asterolinon linum-stellatum

Samolus valerandi

Fraxinus excelsior type 


\section{Anelia}

Depth $(\mathrm{cm})$

Fraxinus angustifolia

Fraxinus ormus

Cynoglossum type

Heliotropium

Myosotis type

Lappula

Alkanna type

Buglossoides arvensis type

Anchusa arvensis type

Teucrium

Scutellaria type

Mentha type

Solanum dulcamara

Solanum nigrum type

Scrophularia type

Rhinanthus type

Odontites type

Veronica anagallis-aquatica type

Pedicularis

Galium type

Plantago major

Plantago afra

Plantago lanceolata type

Plantago coronopus

Plantago bellardii

Plantago media

Plantago arenaria

Plantago argentea

Plantago albicans

Plantago atrata

Sambucus nigra

Vibumum

Valeriana officinalis type

Jasione

Campanula erinus type

Campanula rotundifolia

Solidago type

Echinops

Artemisia

Carlina type

Achillea type

Centaurea cyanus type

Centaurea napulifera type

Cirsium type

Centaurea solstitialis type

Centaurea graeca type

Lactuca type

Cichorium type

Hieracium type

Taraxacum type

\section{MONOCOTYLEDONES}

Sagitnaria sagittifolia

Damasonium alisma

Hydrocharis morsus-ranae

Stratiotes aloides

Vallisneria spiralis

Potamogeton crispus type

Narthecium scardicum

Veratrim

Anthericum liliago

Scilla type

Muscari comosum

Muscari neglectum type

Narcissus papyraceus type

Stembergia

Iris pseudacorus

Iris spuria type

Gynandriris sisyrinchium

Festuca type

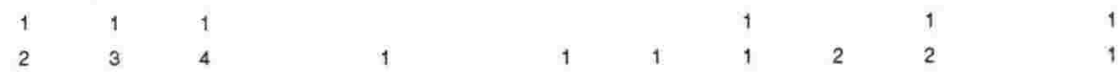
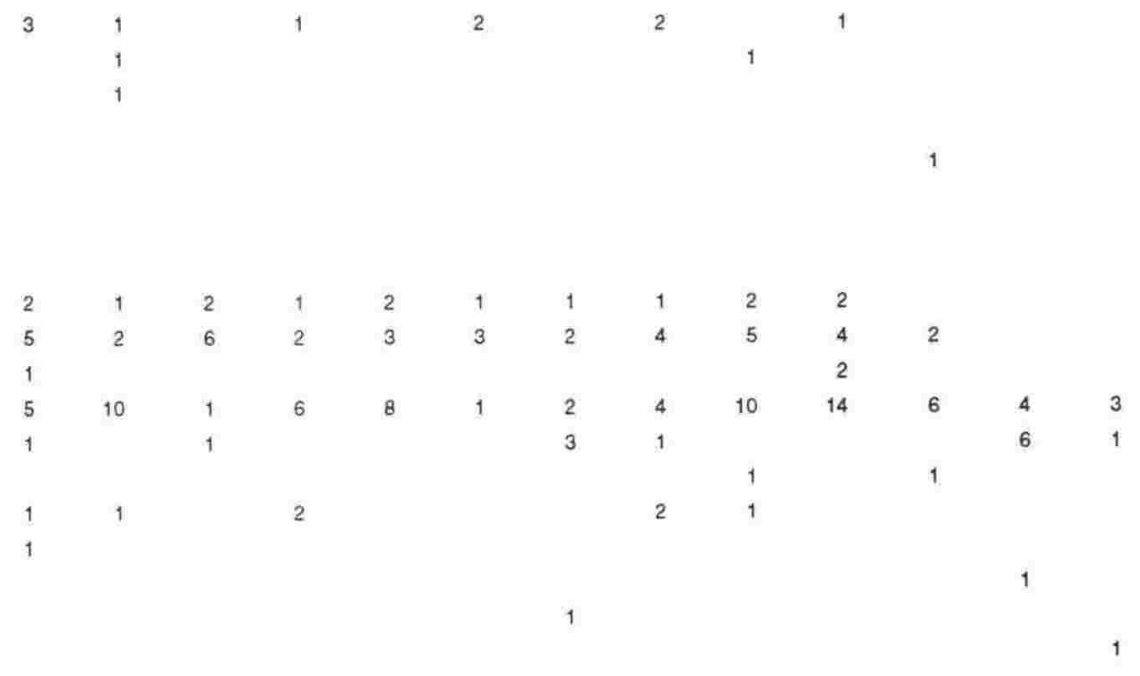


\section{Anelia}

Depth (cm)

Glyceria type

Dactylis type

Agropyron type

Secale cereale

Hordeum type

Avena/Triticum type

Phragmites australis

Sparganium erectum

Sparganium angustifolium

Typha angustifolia type

Typha domingensis type

Cyperus michelianus

Cyperus longus type

Carex hallerana type

Carex flacca type

Cephalanthera

\section{PTERIDOPHYTA}

Equisetum

Ophioglossum vulgatum

Botrychium

Adiantum capillus-veneris

Pteridium aquilinum

Asplenium type

Athyrium filix-femina

Athyrium distentifolium

Dryopteris filix-mas type

Blechnum spicant

Polypodium

Salvinia natans

\section{TOTALS}

Indeterminate

Unknown

Number of terrestrial types

Total wetland pollen \& spores

Total trees \& shrubs

Total terrestrial pollen \& spores (excluding bog \& marsh plants, aquatics, unknown \& indeterminate)

Total pollen \& spores

\begin{tabular}{rrrrrrrrrrrrr}
594 & 647 & 544.7 & 695 & 639 & 444.7 & 728 & 793.4 & 725 & 784.3 & 603.1 & 817 & 382.5 \\
806 & 847 & 677.7 & 933 & 878 & 503.7 & 973 & 1001.4 & 1000 & 959.3 & 686.1 & 953 & 445.5 \\
& & & & & & & & & & & & \\
17549 & 19402 & 17113 & 9810 & 12208 & 14497 & 5995 & 4360 & 1853 & 3270 & 1635 & 2725 & 2725 \\
14715 & 17004 & 11881 & 12971 & 9265 & 10028 & 7848 & 5777 & 1962 & 3052 & 3052 & 2289 & 2289 \\
8611 & 12535 & 8829 & 7630 & 7303 & 9265 & 6758 & 3706 & 981 & 1199 & 4796 & 2071 & 2071 \\
3815 & 5668 & 3488 & 5668 & 4033 & 4578 & 3052 & 2725 & 436 & 654 & 3270 & 1417 & 1417 \\
2180 & 2289 & 1308 & 2943 & 1853 & 1853 & 1417 & 981 & 763 & 545 & 1199 & 872 & 872 \\
2180 & 1526 & 654 & 1744 & 1962 & 1526 & 872 & 763 & 545 & 218 & 1417 & 763 & 763 \\
\hline
\end{tabular}

$\begin{array}{llllllllllllll}185-186 & 190-191 & 195-196 & 200-201 & 205-206 & 210-211 & 215-216 & 220-221 & 225-226 & 230-231 & 235-236 & 240-241 & 245-250\end{array}$

\begin{tabular}{|c|c|c|c|c|c|c|c|c|c|c|c|c|}
\hline 36 & 31 & 36 & 22 & 16 & 24 & 22 & 27 & 34 & 25 & 5 & 12 & 9 \\
\hline \multirow[t]{2}{*}{15} & 15 & 9 & 7 & 18 & 2 & 22 & 16 & 15 & 35 & 10 & 38 & 14 \\
\hline & & & & & & & & & & 1 & 2 & 1 \\
\hline 5 & 10 & 12 & 5 & 9 & 5 & 8 & 20 & 20 & 29 & 5 & 11 & 5 \\
\hline 6 & 3 & 1 & 3 & 1 & 2 & 4 & 1 & 4 & 4 & 5 & 11 & 4 \\
\hline 4 & 3 & 3 & 2 & 11 & 1 & 12 & 7 & 7 & 20 & 13 & 15 & 4 \\
\hline \multirow[t]{4}{*}{1} & 1 & & & & & & & & 4 & 2 & 2 & \\
\hline & & & & & & & & & 1 & & & \\
\hline & & & & & & & 1 & & & 1 & & \\
\hline & & 3 & & & & & & & 4 & 9 & 10 & 4 \\
\hline 1 & & 1 & & & 1 & 1 & 1 & & 2 & 1 & 3 & 1 \\
\hline \multirow[t]{3}{*}{159} & 150 & 74 & 206 & 201 & 16 & 200 & 161 & 221 & 95 & 37 & 76 & 30 \\
\hline & & 2 & & & 2 & & & & & & & \\
\hline & & & & & & 2 & & & & & & \\
\hline
\end{tabular}

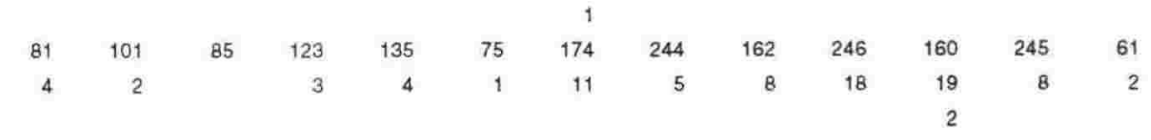

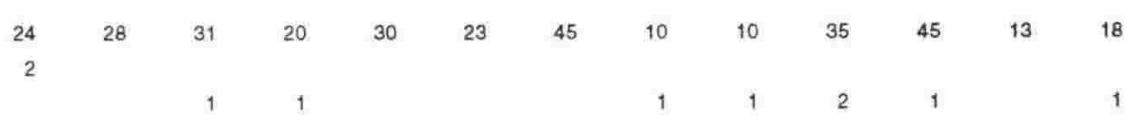

$\begin{array}{rrrrrrrrrrrrr}5 & 12 & 11 & 4 & 7 & 11 & 4 & 5 & 4 & 14 & 8 & 12 & 7 \\ 1 & & & & & & & & 1 & & & & \\ 60 & 54 & 45 & 40 & 46 & 39 & 54 & 48 & 52 & 54 & 42 & 49 & 39 \\ 206 & 188 & 122 & 234 & 232 & 48 & 241 & 203 & 270 & 161 & 75 & 124 & 56 \\ 357 & 374 & 336.7 & 458 & 379 & 300.7 & 374 & 413.4 & 374 & 298.3 & 305.1 & 406 & 236.5\end{array}$

CHARCOAL

Charcoal $<25$ um2

Charcoal 25-50 um2

Charcoal 50-100 um2

Charcoal 100-200 um2

Charcoal 200-400 um2

Charcoal $>400$ um2

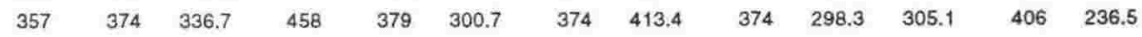


Kellia

Depth (cm)

Absolute counts data

Slide numbers

Sample weight (g) (wet)

Lycopodium suspension added $(\mathrm{ml})$

$\begin{array}{rrrrrrrrrrrr}1.4 & 182 & 1 & 1.5 & 182 & 182 & 1 & 1 & 1 \& 2 & 1.6 & 1 & 182 \\ 0.569 & 1.191 & 1.121 & 1.247 & 1.458 & 1.582 & 1.522 & 1.826 & 1.992 & 1.897 & 1.783 & 1.695 \\ 2.0 & 0.5 & 0.5 & 0.5 & 0.5 & 0.5 & 0.5 & 0.5 & 0.5 & 0.2 & 0.5 & 0.5 \\ 5833 & 3531 & 5762 & 3376 & 3653 & 4624 & 2303 & 2926 & 4214 & 2241 & 2650 & 3668\end{array}$

GYMNOSPERMAE

Abies

Cedrus

Pinus subgenus Diploxylon

Juniperus

Taxus baccata

Ephedra fragilis

Ephedra distachya type

DICOTYLEDONES

Salix triandra

Salix fragilis type

Juglans regia

Alnus glutinosa

Betula pendula

Carpinus type

Ostrya type

Corylus

Fagus

Castanea sativa

Quercus coccifera type

Quercus trojana type

Quercus frainetto type

Ulmus minor

Ulmus procera

Ulmus glabra type

Morus nigra

Morus alba

Humulus lupulus

Urtica dioica

Untica urens type

Urtica pilulifera type

$\begin{array}{rrrrrrrrrrrrr}19 . & 3.6 & 10 . & 12.4 & 6.3 & 5 . & 3.7 & 1 . & 1.4 & 2.2 & 4.3 & 3.3 \\ 136.6 & 111.6 & 202 . & 121,3 & 79.3 & 81.7 & 97.3 & 62 . & 44.7 & 60.4 & 59.7 & 76 . \\ 22 & 4 & 12 & 11 & 8 & 5 & 4 & 23 & 20 & 20 & 23 & 16 \\ 2 & & & & & & & & & & & \end{array}$

Polygonum bistorta type

Polygonum aviculare type

Bilderdykia

Oxyria digyna

Rumex acetosella group

Rumex acelosa group

Rumex crispus p.p.

Rumex conglomeratus group

Rumex obtusifolius group

Chenopodiaceae type

Phytolacca americana

Spergula

Spergularia type

Hemiaria type

Corrigiola litoralis

Paronychia type

Dianthus type

Silene alba type

Lychnis flos-cuculi

Agrostemma githago

Scleranthus annuus type

Nymphaea alba

Nuphar lutea

Helleborus

Nigella

Trollius europaeus

Actaea spicata

Adonis

Caltha type

Ranunculus acris group

Ranunculus aquatilis group

Anemone nemorosa group

$\begin{array}{rrrrrrrrrrrr}92 & 121 & 45 & 27 & 5 & 2 & 9 & 10 & 3 & 7 & 5 & 2 \\ 9 & 3 & 4 & 2 & & & 1 & 1 & & & & \\ & & 1 & & 1 & 2 & & 2 & 1 & & & \\ 3 & 1 & & 3 & 1 & & & & & & & 3 \\ & & & & & & & & & & 1 & \\ 2 & & & & & 2 & & & 1 & & 1 & \\ 12 & 7 & 4 & 9 & 14 & 11 & 4 & 3 & 4 & 4 & 15 & 5 \\ 5 & 1 & & 2 & 3 & & & & 1 & 1 & & 1 \\ 8 & & & 1 & 3 & 1 & 4 & & 1 & & 2 & \\ & 4 & 9 & 2 & 2 & 1 & & 1 & 13 & 1 & 2 & 1 \\ 5 & 3 & 7 & 1 & 6 & 5 & 11 & 7 & 6 & 3 & 7 & 5 \\ 25 & 34 & 22 & 6 & 20 & 12 & 11 & 21 & 25 & 22 & 16 & 19 \\ 90 & 49 & 23 & 44 & 66 & 61 & 60 & 67 & 60 & 77 & 106 & 84 \\ & & & & & & & & & 1 & 2 & 1\end{array}$

(1)

(1)


Kellia

Depth (cm)

Clematis vitalba group

Thalictrum aquilegifolium

Thalictrum minus

Thalictrum hocidum

Chelidonium majus

Glaucium

Homungia type

Arabis

Sinapis type

Chrysosplenium altemifolium

Saxifraga stellaris

Saxifraga opposififolia type

Platanus orientalis

Filipendula

Rubus type

Sorbus type

Cotoneaster type

Prunus avium type

Crataegus

Sanguisorba officinalis

Sanguisorba minor

Geum

Potentilla

Alchemilla type

Ononis type

Trifolium dubium type

Trifolium repens type

Lotus type

Lathyrus pratensis type

Vicia sativa type

Vicia cracca type

Astragalus

Galega officinalis

Dorycnium

Trifolium montanum type

Lathyrus sativus

Oxalis

Geranium robertianum group

Tribulus terrestris

Radiola linoides

Limum catharticum

Linum tenuifolium group

Euphorbia

Pistacia

Cotinus coggygria

Acer

Ilex aquifolium

Buxus sempervirens

Frangula

Rhamnus type

$\begin{array}{lllll}4 & 7 & 4 & 5 & 2 \\ 4 & 2 & 6 & 1 & 1 \\ 8 & 5 & 4 & 1 & 3 \\ 2 & 2 & 1 & & 1\end{array}$

Vitis vinifera

Tilia platyphyllos

Tilia cordata type

Abutilon type

Althaea officinalis

Malva moschata

Daphne

$\begin{array}{lll}4 & 6 & 6\end{array}$

1

Elaeagnus angustifolia

Hypericum hircinum type

Hypericum perforatum type

Viola hirta type

Helianthemum

Cistus monspeliensis type

Myricaria germanica

Tamarix

Elatine alsinastrum

Lythrum salicaria type

Lythrum portula

Trapa natans

Myrtus communis 
Kellia

Depth $(\mathrm{cm})$

Ludwigia palustris

Epilobium angustifolium type

Epilobium hirsutum type

Myriophyllum verticillatum

Myriophyllum spicatum

Connus mas

Hydrocotyle vulgaris

Anthriscus cancalis

Ligusticum

Berula erecta

Trinia

Sanicula europaea

Oenanthe aquatica group

Oenanthe pimpinelloides group

Chaerophyllum hirsutum type

Chaerophyllum temulentum type

Ammi

Pimpinella saxifraga

Torilis arvensis

Peucedanum austriacum type

Angelica sylvestris

Bunium

Anthriscus sylvestris type

Pastinaca sativa

Eryngium

Heracleum sphondylium

Anthriscus cerefolium

Torilis japonica type

Daucus

Coriandrum type

Pyrola

Erica herbacea

Vaccinium myrtillus

Soldanella

Androsace villosa

Primula

Lysimachia

Anagallis arvensis type

Anagallis tenella type

Asterolinon linum-stellatum

Samolus valerandi

Ameria maritima type

Fraxinus excelsior type

Fraxinus angustifolia

Fraxinus onus

Ligustrtum vulgare

Plitlyrea

Centaurium

Convolvulus

Cuscuta epithymum type

Cuscuta europaea type

Cuscuta monogyna

Calystegia

Alkanna type

Borago officinalis

Buglossoides arvensis type

Anchusa arvensis type

Pulmonaria mollis

Anchusa officinalis type

Verbena

Callitriche obtusangula

Callitriche stagnalis type

Teucrium

Stachys sylvatica type

Prunella type

Salvia officinalis type

Mentha type

Solanum dulcamara

Solanum nigrum type

Scrophularia type 
Kellia

Depth (cm)

Odontites type

Veronica anagallis-aquatica type

Veronica triphyllos type

Pedicularis

Putoria calabrica

Sherardia arvensis

Galium type

Plantago major

Plantago afra

Plantago lanceolata type

Plantago coronopus

Plantago bellardii

Plantago media

Plantago argentea

Plantago albicans

Plantago amplexicaulis

Plantago atrata

Viburnum.

Valerianella rimosa type

Valeriana officinalis type

Valerianella locusta type

Succisa pratensis

Scabiosa columbaria type

Dipsacus type

Knautia

Jasione

Campanula erinus type

Campanula rotundifolia

Asyneuma type

Solidago type

Echinops

Artemisia

Carlina type

Picnomon acama

Achillea type

Centaurea cyanus type

Centaurea napulifera type

Cirsium type

Xanthium

Centaurea solstitialis type

Centaurea graeca type

Crupina

Sonchus type

Lactuca type

Scolymus

Cichorium type

Hieracium type

Taraxacum type

\section{MONOCOTYLEDONES}

Alisma

Hydrocharis morsus-ranae

Stratiotes aloides

Vallisneria spiralis

Triglochin bulbosa

Potamogeton crispus type

Potanogeton pectinatus

Colchicum

Narthecium scardicum

Veratrum

Asphodelus fistulosus type

Asphodelus albus

Gagea pratensis

Tulipa sylvestris

Muscari neglectum type

Allium sphaerocephalon type

Asparagus

\begin{tabular}{|c|c|c|c|c|c|c|c|c|c|c|c|}
\hline \multirow{3}{*}{39} & & & & & & & & & 1 & & \\
\hline & 20 & 12 & 9 & 3 & 4 & 4 & 10 & 1 & 9 & 2 & 4 \\
\hline & 2 & 1 & & 1 & 2 & 2 & 9 & 3 & 1 & 10 & 13 \\
\hline & & & & 3 & & & & 1 & 1 & 3 & 2 \\
\hline 18 & 5 & 10 & 11 & 6 & 5 & 7 & 23 & 15 & 12 & 11 & 30 \\
\hline 1 & & 1 & & & & & & & & & \\
\hline & & & & & & & 1 & & & & \\
\hline & 1 & 1 & & 2 & 1 & & & 1 & & & 92 \\
\hline
\end{tabular}

Gynandriris sisyrinchium

Festuca type

Glyceria type

\begin{tabular}{|c|c|c|c|c|c|c|c|c|c|c|c|}
\hline \multirow[t]{4}{*}{23} & 28 & 13 & 11 & 6 & 8 & 4 & 8 & 8 & 9 & 6 & 5 \\
\hline & & & 1 & & & & & & & & \\
\hline & & 1 & 1 & & 1 & & 1 & 1 & & & \\
\hline & 2 & & & & & & & 1 & & & \\
\hline 2 & & & 4 & 1 & & & 2 & & & 1 & \\
\hline \multirow[t]{2}{*}{1} & & & 1 & 1 & & 1 & & 1 & & & \\
\hline & & & & & & & 1 & & & & \\
\hline \multirow[t]{2}{*}{67} & 76 & 62 & 213 & 22 & 6 & 12 & 4 & 1 & 4 & 11 & 4 \\
\hline & & & & & & & & & & 1 & \\
\hline 6 & 3 & & 6 & 2 & 2 & & 1 & 2 & 9 & 2 & 4 \\
\hline 1 & & 1 & 1 & & & & & & & & \\
\hline \multirow[t]{3}{*}{1} & 1 & & & & 1 & 1 & & 1 & 1 & 2 & \\
\hline & & & & & 3 & & & 3 & 10 & & 2 \\
\hline & 13 & 7 & 3 & 2 & 6 & 4 & 5 & & 7 & & 3 \\
\hline 1 & 1 & 7 & 3 & 3 & 1 & 1 & & 6 & 3 & 5 & 5 \\
\hline 11 & 8 & 12 & 13 & 16 & 12 & 9 & 29 & 39 & 32 & 15 & 20 \\
\hline 2 & 3 & 2 & 9 & 5 & 6 & 6 & 15 & 24 & 22 & 21 & 17 \\
\hline
\end{tabular}


Kellia

Depth $(\mathrm{cm})$

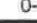

Dactylis type

Agropyron type

Triticum type

Secale cereale

Hordeum type

Averia

Phragmites australis

Zea mays

Anum

Sparganium erectum

Sparganium angustifolium

Typha angustifolia type

Typha domingensis type

Scirpus

Cladium mariscus

Cyperus michelianus

Cyperus longus type

Schoenus nigricans

Carex hallerana type

Carex flacca type

Epipactis type

Cephalanthera

\section{PTERIDOPHYTA}

Equisetum

Ophioglossum vulgatum

Botrychium

Adiantum capillus-veneris

Pteridium aquilinum

Thelypteris palustris

Thelypteris phegopteris

Asplenium type

Athyrium filix-femina

Athyrium distentifolium

Dryopteris filix-mas type

Gymnocarpium dryopteris

Blechnum spicant

Polypodium

Salvinia natam.

Unidentified monolete spores

\section{TOTALS}

Indeterminate

Unknown

Number of terrestrial types

Total wetland pollen \& spores

Total terrestrial trees \& shrubs

$\begin{array}{rrrrrrrrrrrr}28 & 8 & 7 & 4 & 2 & 1 & 2 & 1 & 2 & 7 & 1 & 2 \\ 66 & 55 & 48 & 59 & 55 & 49 & 41 & 49 & 62 & 50 & 48 & 52 \\ 1594 & 790 & 303 & 331 & 131 & 84 & 55 & 220 & 110 & 82 & 83 & 99 \\ 444 & 352 & 344 & 251 & 219 & 196 & 214 & 203 & 191 & 208 & 264 & 235\end{array}$

Total terrestrial pollen \& spores (excluding bog \& marsh types, aquatics, unknown \& indeterminate) Total pollen \& spores

\begin{tabular}{rrrrrrrrrrrr}
778 & 614 & 546 & 640 & 395 & 325 & 323 & 457 & 562 & 460 & 485 & 633 \\
2400 & 1412 & 856 & 975 & 528 & 410 & 380 & 678 & 674 & 549 & 569 & 734 \\
& & & & & & & & & & & \\
26596 & 23217 & 10246 & 10137 & 11772 & 11663 & 7085 & 11336 & 20274 & 29430 & 3924 & 13734 \\
21800 & 25179 & 12317 & 8829 & 13734 & 12317 & 5014 & 8938 & 17331 & 16132 & 2943 & 10355 \\
14933 & 17767 & 9047 & 5886 & 7957 & 11336 & 4251 & 4469 & 7957 & 10464 & 3052 & 7630 \\
5014 & 11445 & 6976 & 3706 & 3161 & 7194 & 2398 & 2398 & 4033 & 4251 & 2507 & 2507 \\
2289 & 3815 & 2180 & 2180 & 1199 & 4469 & 1199 & 1744 & 1962 & 1308 & 763 & 1526 \\
2180 & 4360 & 1090 & 1090 & 1308 & 3815 & 109 & 1744 & 2071 & 1308 & 436 & 545 \\
\hline
\end{tabular}


Kellia

Depth $(\mathrm{cm})$

Absolute counts data

Slide numbers

Sample weight (g) (wet)

Lycopodium suspension added (ml)

Lycopodium count

\begin{tabular}{|c|c|c|c|c|c|c|c|c|c|c|c|}
\hline 1 & 1 & 182 & 1 & 1 & $1-3$ & 1 & 1 & 1 & 1 & 1 & \\
\hline 671 & 1.718 & 1.141 & 1.315 & 1.802 & 1.752 & 1.755 & 1.701 & 1.771 & 1.603 & 1.868 & \\
\hline 0.5 & 0.5 & 0.5 & 0.5 & 0.5 & 0.2 & 0.5 & 0.5 & 0.5 & 0.5 & 0.5 & \\
\hline 190 & 3299 & 4396 & 4381 & 4392 & 1242 & 2373 & 4829 & 4664 & 1779 & 4294 & \\
\hline
\end{tabular}

\section{GYMNOSPERMAE}

Abies

Cedrus

Pinus subgenus Diploxylon

Juniperus

Taxus baccata

Ephedra fragilis

Ephedra distachya type

\section{DICOTYLEDONES}

Salix triandra

Salix fragilis type

Juglans regia

Alnus glutinosa

Betula pendula

Carpinus type

Ostrya type

Corylus

Fagus

Castanea sativa

Quercus coccifera type

Quercus trojana type

Quercus frainetto type

Ulmus minor

Ulmus procera

Ulmus glabra type

Morus nigra

Morus alba

Humulus lupulus

Urtica dioica

Urtica urens type

Urtica pilulifera type

Polygonum bistorta type

Polygonum aviculare type

Bilderdykia

Oxyria digyna

Rumex acetosella group

Rumex acetosa group

Rumex crispus p.p.

Rumex conglomeratus group

Rumex obtusifolius group

Chenopodiaceae type

Phytolacca americana

Spergula

Spergularia type

Hemiaria type

Corrigiola litoralis

Paronychia type

Dianthus type

Silene alba type

Lychnis flos-cuculi

Agrostemma githago

Scleranthus annuus type

$\begin{array}{rrrrrrrrrrrrr}12.3 & 6 . & 10 . & 3.7 & 5.7 & 3.3 & 7.7 & 4.7 & 8 . & 8.3 & 5 . & 5 . \\ & & 1 . & & & & & & & & & \\ 99.7 & 83.3 & 109.7 & 107 . & 95.7 & 63 . & 79 . & 96.7 & 63.7 & 78.3 & 74.7 & 79.7 \\ 15 & 10 & 10 & 5 & 3 & 17 & 9 & 18 & 17 & 19 & 20 & 28 \\ & & & & & & 1 & & & & 1 & \\ 1 & & & & & & & 1 & 1 & 1 & & 1 & 1\end{array}$

Nymphaea alba

Nuphar lutea

Helleborus

Nigella

Trollius europaeus

Actaea spicata

Adonis

Caltha type

Ranunculus acris group

Ranunculus aquatilis group

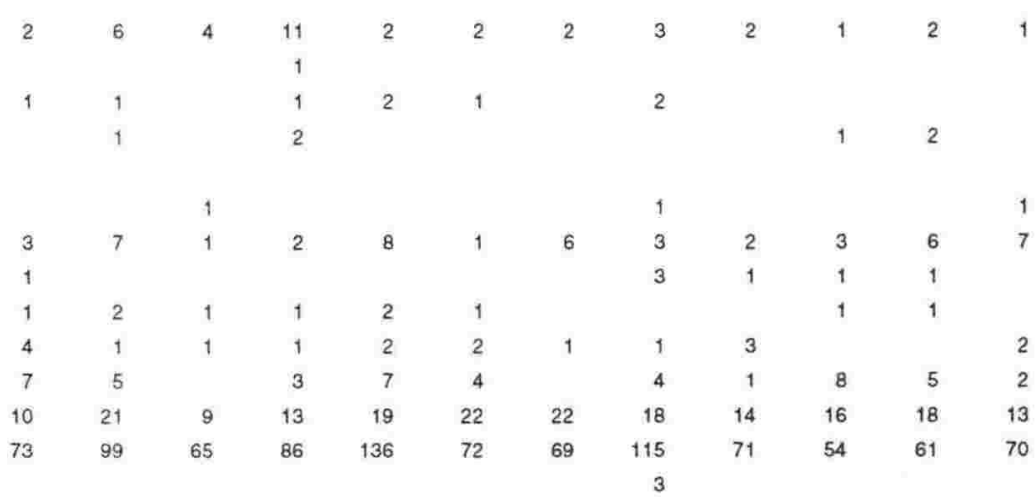

Anemone nemorosa group 
Kellia

Depth $(\mathrm{cm})$

Clematis vitalba group

Thalictrum aquilegifolium

Thalictrum minus

Thalictrum lucidum

Chelidonium majus

Glaucium

Homungia type

A rabis

Sinapis type

Chrysosplenium altenifolium

Saxifraga stellaris

Saxifraga oppositifolia type

Platanus orientalis

Filipendula

Rubus type

Sorbus typ.

Cotoneaster type

Prumus avium type

Crataegus

Sanguisorba officinalis

Sanguisorba minor

Geum

Potentilla

Alchemilla type

Ononis type

Trifolium dubium type

Trifolium repens type

Lotus type

Lathyrus pratensis type

Vicia sativa type

Vicia cracca type

Astragalus

Galega officinalis

Dorycnium

Trifolium montanum type

Lathyrus sativus

Oxalis

Geranium robertianum group

Tribulus terrestris

Radiola linoides

Limum catharticum

Linum tenuifolium group

Euphorbia

Pistacia

Cotinus coggygria

Acer

Ilex aquifolium

Buxus sempervirens

Frangula

Rhamnus type

Vitis vinifera

Tilia platyphyllos

Tilia cordata type

Abutilon type

Althaea officinalis

Malva moschata

Daphne

Elaeagnus angustifolia

Hypericum hircinum type

Hypericum perforatum type

Viola hirta type

Helianthemum

Cistus monspeliensis type

Myricaria germanica

Tamarix

Elatine alsinastrum

Lythrum salicaria type

Lythrum portula

Trapa natans

Myrtus communis

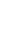

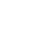


Kellia

Depth $(\mathrm{cm})$

Ludwigia palustris

Epilobium angustifolium type

Epilobium hirsutum type

Myriophyllum verticillatum

Myriophyllum spicatum

Cornus mas

Hydrocotyle vulgaris

Anthriscus caucalis

Ligusticum

Berula erecta

Trinia

Sanicula europaea

Oenanthe aquatica group

Oenanthe pimpinelloides group

Chaerophyllum hirsutum type

Chaerophyllum temulentum type

Ammi

Pimpinella saxifraga

Torilis arvensis

Peucedanum austriacum type

Angelica sylvestris

Bunium

Anthriscus sylvestris type

Pastinaca sativa

Eryngium

Heracleum sphondylium

Anthriscus cerefolium

Torilis japonica type

Daucus

Coriandrum type

Pyrola

Erica herbacea

Vaccinium myrtillus

Soldanella

Androsace villosa

Primula

Lysimachia

Anagallis arvensis type

Anagallis tenella type

Asterolinon linum-stellatum

Samolus valerandi

Anneria maritima type

Fraxinus excelsior type

Fraxinus angustifolia

Fraxinus ormus

Ligustrum vulgare

Phillyrea

Centaurium

Convolvulus

Cuscuta epithymum type

Cuscuta europaea type

Cuscuta monogyna

Calystegia

Alkanna type

Borago officinalis

Buglossoides arvensis type

Anchusa arvensis type

Pulmonaria mollis

Anchusa officinalis type

Verbena

Callitriche obtusangula

Callitriche stagnalis type

Teucrium

Stachys sylvatica type

Prinella type

Salvia officinalis type

Mentha type

Solanum dulcamara

Solanum nigrum type

Scrophularia type 
Kellia

Depth $(\mathrm{cm})$

Odontites type

Veronica anagallis-aquatica type

Veronica triphyllos type

Pedicularis

Putoria calabrica

Sherardia arvensis

Galium type

Plantago major

Plantago afra

Plantago lanceolata type

Plantago coronopus

Plantago bellardii

Plantago media

Plantago argentea

Plantago albicans

Plantago amplexicaulis

Plantago atrata

Vibumum

Valerianella rimosa type

Valeriana officinalis type

Valerianella locusta type

Succisa pratensis

Scabiosa columbaria type

Dipsacus type

Knantia

Jasione

Campanula erinus type

Campanula rotundifolia

Asyneuma type

Solidago type

Echinops

Antemisia

Carlina type

Picnomon acama

Achillea type

Centaurea cyanus type

Centaurea napulifera type

Cirsium type

Xanthium

Centaurea solstitialis type

Centaurea graeca type

Crupina

Sonchus type

Lactuca type

Scolymus

Cichorium type

Hieracium type

Taraxacum type

$\begin{array}{rrrrrrrrrrrr}2 & 10 & 4 & 7 & 4 & 10 & 4 & 8 & 2 & 7 & 7 & 1 \\ 13 & 11 & & 9 & 7 & 1 & 4 & 2 & 3 & 3 & 8 & 4 \\ 1 & & 1 & & 1 & & & & & 1 & & \\ 1 & 10 & 7 & 6 & 15 & 14 & 19 & 28 & 20 & 24 & 8 & 8 \\ & & 1 & 1 & & & & & & 1 & & \end{array}$

\section{MONOCOTYLEDONES}

Alisma

Hydrocharis morsus-ranae

Stratiotes aloides

Vallisneria spiralis

Triglochin bulbosa

Potamogeton crispus type

Potamogeton pectinatus

Colchicum

Narthecium scardicum

Veratrum

Asphodelus fistulosus type

Asphodelus albus

Gagea pratensis

Tulipa sylvestris

Muscari neglectum type

Allium sphaerocephalon type

Asparagus

Gynandriris sisyrinchium

Fesfuca type

Glyceria type

$\begin{array}{rrrrrrrrrrrr}1 & 3 & 4 & 3 & 10 & 1 & 13 & 14 & 9 & 12 & 9 & 17 \\ 10 & 10 & 11 & 11 & 24 & 14 & 21 & 13 & 16 & 24 & 19 & 7\end{array}$


Kellia

Depth $(\mathrm{cm})$

$\begin{array}{llllllllllll}60-61 & 65-66 & 70-71 & 75-76 & 80-81 & 85-86 & 90-91 & 95-96 & 100-101 & 105-106 & 110-111 & 115-116\end{array}$

Dactylis type

Agropyron type

Triticum type

Secale cereale

Hordeum type

Avena

Phragmites australis

Zea mays

Anum

Sparganium erectum

Sparganium angustifolium

Typha angustifolia type

Typha domingensis type

Scirpus

Cladium mariscus

Cyperus michelianus

Cyperus longus type

Schoenus nigricans

Carex hallerana type

Carex flacca type

Epipactis type

Cephalanthera

\section{PTERIDOPHYTA}

Equisetum

Ophioglossum vulgatum

Botrychium

Adiantum capillus-veneris

Pleridium aquilinum

Thelypteris palustris

Thelypteris phegopteris

Asplenium type

Athyrium filix-femina

Athyrium distentifolium

Dryopteris filix-mas type

Gymnocarpium dryopteris

Blechnum spicant

Polypodium

Salvinia natans

Unidentified monolete spores

\section{TOTALS}

Indeterminate

Unknown

Number of terrestrial types

Total wetland pollen \& spores

Total terrestrial trees \& shrubs

\begin{tabular}{|c|c|c|c|c|c|c|c|c|c|c|c|}
\hline \multirow[t]{3}{*}{7} & 4 & 6 & 3 & 22 & 16 & 13 & 19 & 20 & 16 & 10 & 16 \\
\hline & & 1 & 2 & & & & & & 6 & 3 & 7 \\
\hline & & 3 & & 1 & 1 & & 5 & & 2 & 1 & \\
\hline \multirow[t]{2}{*}{8} & 6 & 2 & & 1 & 2 & 4 & 9 & 6 & 3 & 5 & 8 \\
\hline & 1 & 2 & & 6 & 2 & 2 & 3 & 1 & 3 & 1 & 7 \\
\hline 1 & 1 & 2 & 1 & 5 & 1 & 3 & 10 & 6 & 7 & 4 & \\
\hline 2 & & 2 & 4 & 8 & 5 & 1 & 1 & 3 & 10 & 6 & 8 \\
\hline 2 & 2 & 2 & 2 & 3 & 4 & 2 & 3 & & 2 & & \\
\hline \multirow[t]{2}{*}{3} & & 38 & 154 & 6 & 1 & 9 & 43 & 14 & 14 & 8 & 26 \\
\hline & & 5 & 5 & & & 4 & 35 & 10 & 11 & 3 & 8 \\
\hline \multirow[t]{2}{*}{1} & & 1 & 1 & & & 5 & & & 5 & 1 & 7 \\
\hline & 2 & & 10 & & & 8 & 5 & 4 & & 2 & 5 \\
\hline 13 & 4 & 6 & 12 & 10 & 6 & 4 & 11 & 7 & 6 & 7 & 8 \\
\hline 1 & 9 & 3 & 2 & & 1 & & 1 & & & 1 & 2 \\
\hline 17 & 21 & 25 & 53 & 28 & 12 & 20 & 25 & 20 & 15 & 9 & 10 \\
\hline 2 & 5 & 3 & & & 7 & & & 2 & 1 & & \\
\hline 2 & 2 & 5 & 9 & 2 & & 4 & 1 & 1 & & & 1 \\
\hline
\end{tabular}

Total terrestrial pollen \& spores (excluding bog \& marsh types, aquatics, unknown \& indeterminate) Total pollen \& spores

\begin{tabular}{rrrrrrrrrrrr}
343 & 444 & 359 & 412 & 585 & 532 & 465 & 617 & 438 & 440 & 424 & 427 \\
399 & 506 & 470 & 682 & 671 & 613 & 551 & 763 & 518 & 537 & 485 & 521 \\
& & & & & & & & & & & \\
5341 & 16459 & 6976 & 11336 & 10246 & 15260 & 12753 & 9810 & 13080 & 6758 & 9810 & 3597 \\
5014 & 14606 & 4251 & 15260 & 14061 & 9483 & 9701 & 12753 & 14279 & 9047 & 10573 & 6758 \\
3924 & 10573 & 2398 & 9810 & 11445 & 6540 & 8175 & 13516 & 11445 & 6758 & 6540 & 5341 \\
2398 & 6104 & 654 & 5341 & 5559 & 4687 & 4796 & 5450 & 8611 & 2725 & 3815 & 2180 \\
1308 & 2725 & 218 & 1417 & 2507 & 2289 & 1526 & 2507 & 2834 & 436 & 1090 & 763 \\
436 & 327 & 109 & 327 & 2180 & 981 & 872 & 872 & 1090 & 218 & 436 & 218 \\
\hline
\end{tabular}

\section{CHARCOAL}

Charcoal $<25$ um2

Charcoal 25-50 um2

Charcoal 50-100 um2

Charcoal 100-200 um2

Charcoal 200-400 um2

Charcoal $>400$ um2

$\begin{array}{rrrrrrrrrrrr}1 & 5 & 4 & 3 & 4 & 18 & 2 & 6 & 1 & 3 & 1 & 2 \\ 43 & 45 & 48 & 45 & 48 & 55 & 44 & 51 & 43 & 51 & 48 & 40 \\ 55 & 57 & 107 & 267 & 82 & 63 & 84 & 140 & 79 & 93 & 60 & 92 \\ 238 & 259 & 222 & 243 & 289 & 194 & 210 & 280 & 189 & 197 & 203 & 214\end{array}$


Kellia

Depth $(\mathrm{cm})$

$\begin{array}{lllllllllllll}120-121 & 124-125 & 130-131 & 132-133 & 135-136 & 140-141 & 145-146 & 150-151 & 155-156 & 160-161 & 162-163 & 165-166 & \end{array}$

Absolute counts data

Slide numbers

Sample weight ( $\mathrm{g}$ ) (wet)

Lycopodium suspension added (ml)

Lycopodium count

\begin{tabular}{|c|c|c|c|c|c|c|c|c|c|c|c|}
\hline 1 & $1-6$ & 1 & 1 & $1 \& 2$ & 1 & 1 & 1 & 182 & $1-3$ & 1 & \\
\hline 592 & 1.922 & 1.803 & 1.781 & 1.925 & 1.811 & 1.718 & 1.708 & 1.765 & 1.525 & 1.621 & 1.486 \\
\hline 0. & 0.2 & 0.5 & 0.5 & 0.5 & 0.5 & 0.5 & 0.5 & 0.5 & 0.2 & 0.5 & 0. \\
\hline 42 & 2412 & 3652 & 3217 & 8934 & 5633 & 4022 & 2824 & 5933 & 1080 & 2090 & 195 \\
\hline
\end{tabular}

\section{GYMNOSPERMAE}

Abies

Cedrus

Pinus subgenus Diploxylon

Juniperus

Taxus baccata

Ephedra fragilis

Ephedra distachya type

\section{DICOTYLEDONES}

Salix triandra

Salix fragilis type

Juglans regia

Alrus glutinosa

Betula pendula

Carpinus type

Ostrya type

Corylus

Fagus

Castanea sativa

Quercus coccifera type

Quercus trojana type

Quercus frainetto type

Ulmus minor

Ulmus procera

Ulmus glabra type

Morus nigra

$\begin{array}{rrrrrrrrrrrr}4.7 & .9 & 5.7 & 2.7 & 4.3 & 2.7 & 7.3 & 6.3 & 3.3 & 7.3 & 4.7 & 1.7 \\ & & & & & & & & & & & \\ 112 . & 56.6 & 54.7 & 50 . & 64.3 & 54.3 & 92.3 & 118.7 & 53.6 & 93 . & 115.7 & 117.3 \\ 50 & 32 & 17 & 22 & 28 & 31 & 49 & 32 & 32 & 22 & 26 & 29\end{array}$

Morus alba

Humulus lupulus

Urtica dioica

Urtica urens type

Urtica pilulifera type

Polygonum bistorta type

Polygonum aviculare type

Bilderdykia

Oxyria digyna

Rumex acetosella group

Rumex acetosa group

Rumex crispus p.p.

Rumex conglomeratus group

Rumex obtusifolius group

Chenopodiaceae type

Phytolacca americana

Spergula

Spergularia type

Hemiaria type

Corrigiola litoralis

Paronychia type

Dianthus type

Silene alba type

Lychnis flos-cuculi

Agrostemma githago

Scleranthus annuus type

Nymphaea alba

Nuphar lutea

Helleborus

Nigella

Trollius europaeus

Actaea spicata

Adonis

Caltha type

Ranunculus acris group

Ranunculus aquatilis group

Anemone nemorosa group 
Kellia

Depth $(\mathrm{cm})$

Clematis vitalba group

Thalictrum aquilegifolium

2

Thalictrum minus

Thalictrum lucidum

Chelidorium majus

Glaucium

Hornungia type

Arabis

Sinapis type

Chrysosplenium alternifolium

Saxifraga stellaris

Saxifraga oppositifolia type

Platanus orientalis

Filipendula

Rubus type

Sorbus type

Cotoneaster type

Prunus avium type

Crataegus:

Sanguisorba officinalis

Sanguisorba minor

Geum

Potentilla

Alchemilla type

Ononis type

Trifolium dubium type

Trifolium repens type

Lotus type

Lathyrus pratensis type

Vicia sativa type

Vicia cracca type

Astragalus

Galega officinalis

Dorycnium

Trifolium montanum type

Lathyrus sativus

Oxalis

Geranium robertianum group

Tribulus terrestris

Radiola linoides

Linum catharticum

Limum teruifolium group

Eupharbia

Pistacia

Cotinus coggygria

Acer

Ilex aquifolium

Buxus sempervirens

Frangula

Rhamnus type

Vitis vinifera

Tilia platyphyllos

Tilia cordata type

Abutilon type

Althaea officinalis

Malva moschata

Daphne

Elaeagnus angustifolia

Hypericum hircinum type

Hypericum perforatum type

Viola hinta type

Helianthemum

Cistus monspeliensis type

Myricaria gemanica

Tamarix

Elatine alsinastrum

Lythrum salicaria type

Lythrum portula

Trapa natans

Myrtus communis 
Kellia

Depth $(\mathrm{cm})$

Ludwigia palustris

Epilobium angustifolium type

Epilobium hirsutum type

Myriophyllum verticillatum

Myriophyllum spicatum

Comus mas

Hydrocotyle vulgaris

Anthriscus caucalis

Ligusticum

Berula erecta

Trinia

Sanicula europaea

Oenanthe aquatica group

Oenanthe pimpinelloides group

Chaerophyllum hirsutum type

Chaerophyinu temulentum type

Amuni

Pimpinella saxifraga

Torilis arvensis

Peucedanum austriacum type

Angelica sylvestris

Bunium

Anthriscus sylvestris type

Pastinaca sativa

Eryngium

Heracleum sphondylium

Anthriscus cerefolium

Torilis japonica type

Daucus

Coriandrum type

Pyrola

Erica herbacea

Vaccinium myrtillus

Soldanella

Androsace villosa

Primula

Lysimachia

Anagallis arvensis type

Anagallis tenella type

Asterolinon linum-stellatum

Samolus valerandi

Anneria maritima type

Fraxinus excelsior type

Fraxinus angustifolia

Fraximus ormus

Ligustrum vulgare

Phillyrea

Centaurium

Convolvulus

Cuscuta epithymum type

Cuscuta europaea type

Cuscuta monogyna

Calystegia

Alkanna type

Borago officinalis

Buglossoides arvensis type

Anchusa arvensis type

Pulmonaria mollis

Anchusa officinalis type

Verbena

Callitriche obtusangula

Callitriche stagnalis type

Teucrium

Stachys sylvatica type

Prunella type

Salvia officinalis type

Mentha type

Solanum dulcamara

Solanum nigrum type

Scrophularia type 
Kellia

Depth $(\mathrm{cm})$

Odontites type

Veranica anagallis-aquatica type

Veronica triphyllos type

Pedicularis

Putoria calabrica

Sherardia arvensis

Galium type

Plantago major

Plantago afra

Plantago lanceolata type

Plantago coronopus

Plantago bellardii

Plantago media

Plantago argentea

Plantago albicans

Plantago amplexicaulis

Plantago atrata

Vibumum

Valerianella rimosa type

Valeriana officinalis type

Valerianella locusta type

Succisa pratensis

Scabiosa columbaria type

Dipsacus type

Knautia

Jasione

Campanula erinus type

Campanula rotundifolia

Asyneuma type

Solidago type

Echinops

Anemisia

Carlina type

Picnomon acama

Achillea type

Centaurea cyanus type

Centaurea napulifera type

Cinsium type

Xonthium

Centaurea solstitialis type

Centaurea graeca type

Crupina

Sonchus type

Lactuca type

Scolymus

Cichorium type

Hieracium type

Taraxacum type

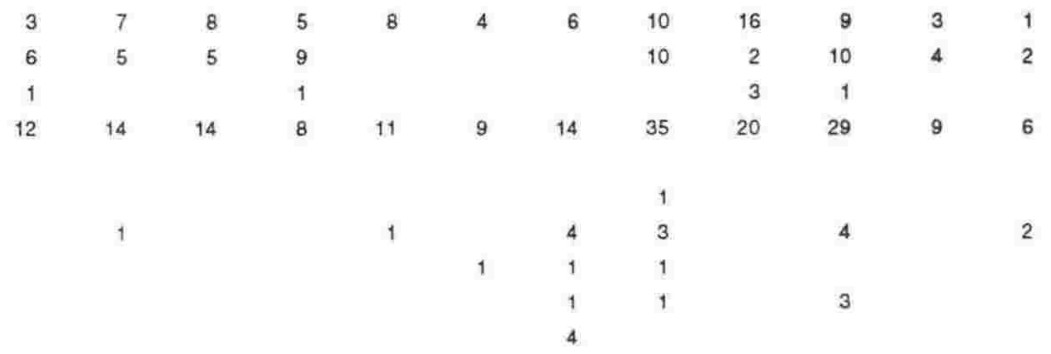

MONOCOTYLEDONES

Alisma

Hydrocharis morsus-ranae

Stratiotes aloides

Vallisneria spiralis

Triglochin bulbosa

Potamogeton crispus type

Potamogeton pectinatus

Colchicum

Narthecium scardicum

Veratnum

Asphodelus fistulosus type

Asphodelus albus

Gagea pratensis

Tulipa sylvestris

Muscari neglectum type

Allium sphaerocephalon type

Asparagus

Gynandriris sisyrinchium

Festuca type

Glyceria type

$\begin{array}{rrrrrrrrrrrr}21 & 1 & 17 & 14 & 17 & 11 & 13 & 11 & 8 & 6 & 10 & 6 \\ 28 & 55 & 33 & 35 & 37 & 38 & 48 & 25 & 35 & 55 & 22 & 34\end{array}$


Kellia

Depth $(\mathrm{cm})$

$\begin{array}{llllllllllllll}120-121 & 124-125 & 130-131 & 132-133 & 135-136 & 140-141 & 145-146 & 150-151 & 155-156 & 160-161 & 162-163 & 165-166\end{array}$

Dactylis type

Agropyron type

Triticum type

Secale cereale

Hordeum type

Avena

Phragmites australis

Zea mays

Anum

Sparganium erectum

Sparganium angustifolium

Typha angustifolia type

Typha domingensis type

Scipus

Cladium mariscus

Cyperus michelianus

Cyperus longus type

Schoenus nigricans

Carex hallerana type

Carex flacca type

Epipactis type

Cephalanther

\section{PTERIDOPHYTA}

Equisetum

Ophioglossum vulgatum

Botrychium

Adiantum capillus-veneris

Pteridium aquilinum

Thelypteris palustris

Thelypteris phegopteris

Asplenium type

Athyrium filix-femina

Athyrium distentifolium

Dryopteris filix-mas type

Gymnocarpium dryopteris

Blechnum spicant

Polypodium

Salvinia natans

Unidentified monolete spores

\section{TOTALS}

Indeterminate

Unknown

Number of terrestrial types

Total wetland pollen \& spores

Total terrestrial trees \& shrubs

$\begin{array}{rrrrrrrrrrrr}22 & 45 & 18 & 18 & 15 & 2 & 33 & 17 & 36 & 49 & 9 & 11 \\ 3 & & 7 & 8 & 5 & 3 & 2 & 8 & 1 & 2 & 4 & 1 \\ & & & & 1 & & & & 1 & & & \\ 11 & 4 & 8 & 5 & 1 & 2 & 5 & 8 & 2 & 1 & 6 & 1 \\ 4 & 1 & 13 & 3 & 3 & 7 & 4 & 6 & 1 & 1 & 6 & 3 \\ 5 & 12 & 5 & & 3 & 1 & 4 & 3 & 4 & 1 & 4 & 4 \\ 5 & 7 & 3 & 22 & 4 & 4 & 9 & 3 & 4 & 8 & 2 & 2 \\ 3 & 1 & 5 & 4 & & 1 & 3 & 5 & & 1 & 1 & 1 \\ & & & & & & & & & & & \\ 8 & 11 & 1 & 2 & 4 & & 15 & 2 & 3 & 17 & & 6 \\ 1 & 1 & & & & & & & & 2 & 1 & \\ & & & & & & 1 & & & 41 & & \\ & & & & & & 2 & 1 & & 1 & & \\ 11 & 12 & 14 & 15 & 15 & 7 & 7 & 7 & 18 & 35 & 34 & 170 \\ & 1 & & & & & & & & & & \\ & 12 & & 1 & & 1 & & 2 & 1 & 6 & & 2 \\ 13 & 20 & 11 & 10 & 4 & 6 & 1 & 1 & 3 & 5 & 4 & 5 \\ 1 & 2 & & & & & 18 & 10 & 6 & 14 & 7 & 60 \\ & 7 & & & 7 & 2 & 1 & 13 & 13 & 37 & 15 & 4 \\ 9 & 22 & 1 & 2 & 1 & & & & 3 & 12 & 3 & 2\end{array}$

Total terrestrial pollen \& spores

(excluding bog \& marsh types,

aquatics, unknown \& indeterminate)

Total pollen \& spores

\begin{tabular}{rrrrrrrrrrrr}
682 & 474 & 527 & 495 & 480 & 410 & 939 & 815 & 630 & 620 & 479 & 455 \\
767 & 655 & 602 & 611 & 565 & 485 & 1061 & 913 & 734 & 892 & 578 & 748 \\
& & & & & & & & & & & \\
8611 & 25179 & 10791 & 7412 & 18421 & 10791 & 23871 & 14606 & 18530 & 13298 & 8502 & 7630 \\
10246 & 15914 & 15478 & 10355 & 22345 & 12099 & 17876 & 11227 & 21909 & 7848 & 9265 & 10028 \\
6104 & 8284 & 8720 & 10028 & 13734 & 9592 & 10355 & 9047 & 15260 & 6976 & 6431 & 5014 \\
3052 & 4251 & 5450 & 7630 & 5450 & 5450 & 6867 & 4142 & 8611 & 2071 & 3270 & 2507 \\
981 & 1308 & 1635 & 3815 & 2180 & 2180 & 3270 & 1744 & 4033 & 981 & 1199 & 545 \\
436 & 981 & 1417 & 1744 & 1962 & 1635 & 872 & 1199 & 1853 & 545 & & 218 \\
\hline
\end{tabular}

\section{CHARCOAL}

Charcoal $<25$ um2

Charcoal 25-50 um2

Charcoal 50-100 um2

Charcoal 100-200 um2

Charcoal 200-400 um2

Charcoal $>400$ um2

$\begin{array}{rrrrrrrrrrrr}1 & 20 & 2 & 7 & 6 & 4 & 8 & 12 & 8 & 22 & 4 & 2 \\ 56 & 47 & 45 & 50 & 45 & 46 & 52 & 61 & 50 & 71 & 48 & 53 \\ 84 & 161 & 73 & 109 & 79 & 71 & 114 & 86 & 96 & 250 & 95 & 291 \\ 374 & 221 & 238 & 219 & 220 & 198 & 276 & 301 & 211 & 259 & 242 & 267\end{array}$


Kellia

Depth (cm)

Absolute counts data

Slide numbers

Sample weight (g) (wet)

Lycopodium suspension added (ml)

Lycopodium count

$\begin{array}{rrrrrrrrrrrr}1 \& 2 & 1 & 1 & 1 & 1 & 1 & 1 & 1 & 1 & 1 & 1 & 1 \\ 1.620 & 1.396 & 1.519 & 1.655 & 1.667 & 1.659 & 1.665 & 1.554 & 1.780 & 1.651 & 1.774 & 1.506 \\ 0.5 & 0.5 & 0.5 & 0.5 & 0.5 & 0.5 & 0.5 & 0.5 & 0.5 & 0.5 & 0.5 & 0.5 \\ 4793 & 2475 & 2623 & 2379 & 1785 & 2080 & 1935 & 2666 & 2862 & 2073 & 2611 & 3316\end{array}$

GYMNOSPERMAE

Abies

Cedrus

Pinus subgenus Diploxylon

Juniperus

Taxus baccata

Ephedra fragilis

Ephedra distachya type

\section{DICOTYLEDONES}

Salix triandra

Salix fragilis type

Juglans regia

Alnus glutinosa

Betula pendula

Carpinus type

Ostrya type

Corylus

Fagus

Castamea sativa

Quercus coccifera type

Quercus trojana type

Quercus frainetto type

Ulmus minor

Ulmus procera

Ulmus glabra type

Morus nigra

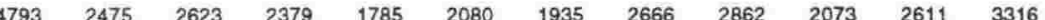

Morus alba

Humulus lupulus

Urtica dioica

Urtica urens type

Urtica pilulifera type

Polygonum bistorta type

Polygonum aviculare type

Bilderdykia

Oxyria digyna

Runex acetosella group

Rumex acetosa group

Rumex crispus p.p.

Rumex conglomeratus group

Rumex obtusifolius group

Chenopodiaceae type

Phytolacca americana

Spergula

Spergularia type

Hemiaria type

Corrigiola litoralis

Paronychia type

Dianthus type

Silene alba type

Lychis flos-cucul

Agrostemma githago

Sclerant/us annus type

Nymphaea alba

Nuphar lutea

Helleborus

Nigella

Trollius europaeus

Actaea spicata

4. $\quad 3.7$

3.7

4.7

$\begin{array}{llllllllllll}93.7 & 128.3 & 123.3 & 132 . & 112 . & 124.3 & 122.3 & 104.7 & 132.3 & 107 . & 79.7 & 126.3\end{array}$

$\begin{array}{llllllllllll}27 & 16 & 20 & 33 & 16 & 15 & 21 & 14 & 7 & 16 & 9 & 6\end{array}$

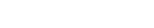

Adonis

Caltha type

Ranunculus acris group

Ranunculus aquatilis group

Anemone nemorosa group

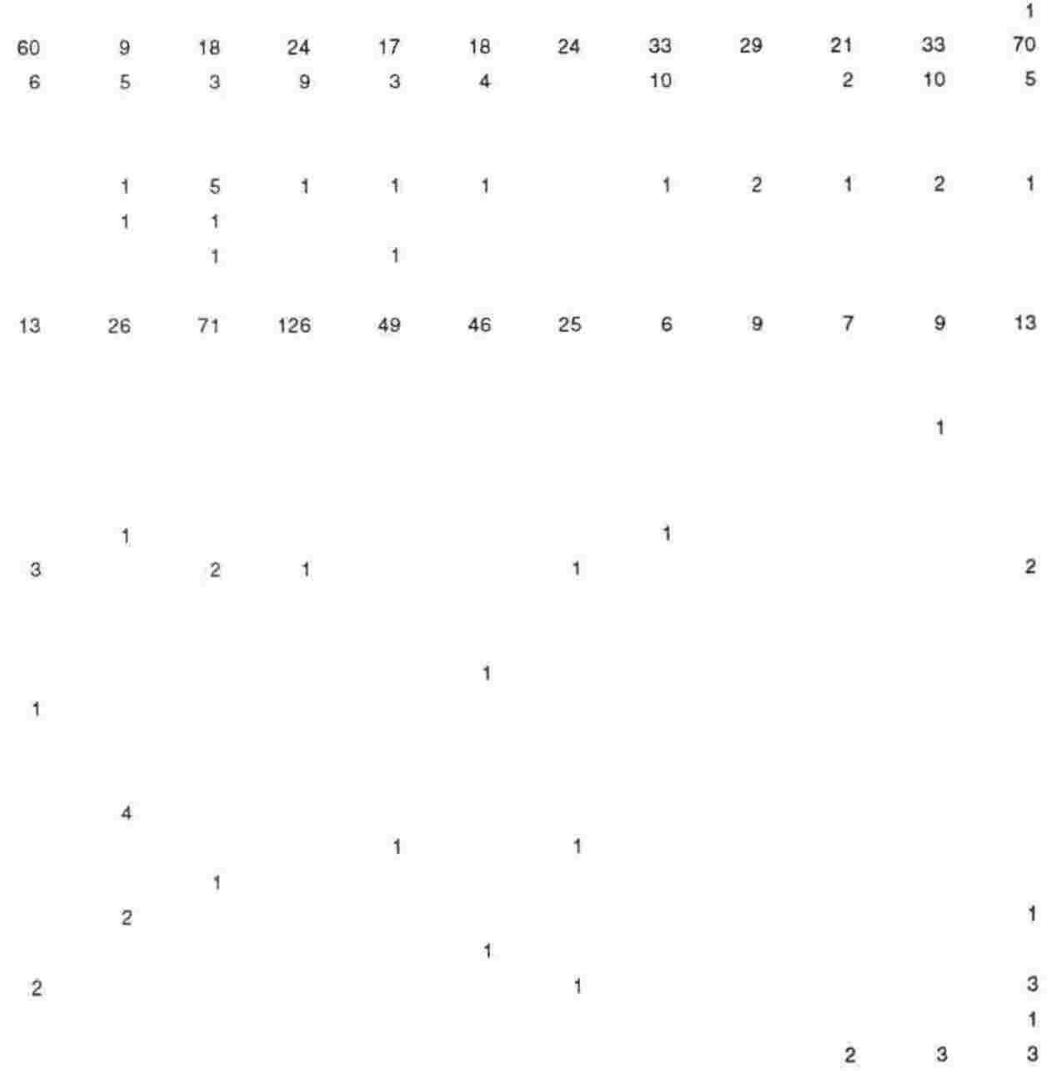


Kellia

Depth $(\mathrm{cm})$

Clematis vitalba group

Thalictrum aquilegifolium

Thalictrum minus

Thalictrum lucidum

Chelidonium majus

Glaucium

Hornungia type

A rabis

Sinapis type

Chrysosplenium alternifolium

Saxifraga stellaris

Saxifraga oppositifolia type

Platanus orientalis

Filipendula

Rubus type

Sorbus type

Cotoneaster type

Prunus avium type

Crataegus

Sanguisorba officinalis

Sanguisorba minor

Geum

Potentilla

Alchemilla type

Ononis type

Trifolium dubium type

Trifolium repens type

Lotus type

Lathyrus pratensis type

Vicia sativa type

Vicia cracca type

Astragalus

Galega officinalis

Dorycnium

Trifolium montanum type

Lathvrus sativus

Oxalis

Geranium robertianum group

Tribulus terrestris

Radiola linoides

Linum catharticum

Linum tenuifolium group

Euphorbia

Pistacia

Cotimus coggygria

Acer

llex aquifolitum

Buxus sempervirens

Frangula

Rhammus type

Vitis vinifera

Tilia platyphyllos

Tilia cordata type

Abutilon type

Althaea officinalis

Malva moschata

Daphne

Elaeagnus angustifolia

Hypericum hircinum type

Hypericum perforatum type

Viola hirta type

Helianthemum

Cistus monspeliensis type

Myricaria germanica

Tamarix

Elatine alsinastrum

Lythrum salicaria type

Lythrum portula

Trapa natans

Myrtus communis 
Kellia

Depth (cm)

Ludwigia palustris

Epilobium angustifolium type

Epilobium hirsutum type

Myriophyllum verticillatum

Myriophyllum spicatum

Cornus mas

Hydrocotyle vulgaris

Anthriscus caucalis

Ligusticum

Berula erecta

Trinia

Sanicula europaea

Oenanthe aquatica group

Denanthe pimpinelloides group

Chaerophyllum hirsutum type

Chaerophyllum temulentum type

Ammi

Pimpinella saxifraga

Torilis anvensis

Peucedanum austriacum type

Angelica sylvestris

Bunium

Anthriscus sylvestris type

Pastinaca sativa

Eryngium

Heracleum sphondylium

Anthriscus cerefolium

Torilis japonica type

Daucus

Coriandrum type

Pyrola

Erica herbacea

Vaccinium myrtillus

Soldanella

Androsace villosa

Primula

Lysimachia

Anagallis arvensis type

Anagallis tenella type

Asterolinon linum-stellatum

Samolus valerandi

Ameria maritima type

Fraxinus excelsior type

Fraxinus angustifolia

Fraxinus omus

Ligustrum vulgare

Phillyrea

Centaurium

Convolvulus

Cuscuta epithymum type

Cuscuta europaea type

Cuscuta monogyna

Calystegia

Alkanna type

Borago officinalis

Buglossoides arvensis type

Anchusa arvensis type

Pulmonaria mollis

Anchusa officinalis type

Verbena

Callitriche obtusangula

Callitriche stagnalis type

Teucrium

Stachys sylvatica type

Pnunella type

Salvia officinalis type

Mentha type

Solanum dulcamara

Solanum nigrum type

Scrophularia type 
Kellia

Depth (cm)

Odontites type

Veronica anagallis-aquatica type

Veronica triphyllos type

Pedicularis

Putoria calabrica

Sherardia arvensis

Galium type

Plantago major

Plantago afra

Plantago Lanceolata type

Plantago coronopus

Plantago bellardii

Plantago media

Plantago argentea

Plantago albicans

Plantago anntexicaulis

Plantago atrata

Viburnum

Valerianella rimosa type

Valeriana officinalis type

Valerianella locusta type

Succisa pratensis

Scabiosa columbaria type

Dipsacus type

Knautia

Jasione

Campanula erinus type

Campanula rotundifolia

Asyneuma type

Solidago type

Echinops

Artemisia

Carlina type

Picnomon acama

Achillea type

Centaurea cyanus type

Centaurea napulifera type

Cirsium type

Xanthium

Centaurea solstitialis type

Centaurea graeca type

Crupina

Sonchus type

Lactuca type

Scolymus

Cichorium type

Hieracium type

Taraxacum type

\section{MONOCOTYLEDONES}

Alisma

Hydrocharis morsus-ranae

Stratiotes aloides

Vallisneria spiralis

Triglochin bulbosa

Potamogeton crispus type

Potamogeton pectinatus

Colchicum

Narthecium scardicum

Veratnum

Asphodelus fistulosus type

Asphodelus albus

Gagea pratensis

Tulipa sylvestris

Muscari neglectum type

Allium sphaerocephalon type

Asparagus

Gynandriris sisyrinchium

Festuca type

Glyceria type 
Kellia

Depth $(\mathrm{cm})$

$\begin{array}{llllllllllll}170-171 & 175-176 & 180-181 & 185-186 & 190-191 & 195-196 & 197-198 & 200-201 & 205-206 & 210-211 & 214-215 & 220-221\end{array}$

Dactylis type

Agropyron type

Triticum type

Secale cereale

Hordeum type

Avena

Phragmites australis

Zea mays

Anum

Sparganium erectum

Sparganium angustifolium

Typha angustifolia type

Typha domingensis type

Scirpus

Cladium mariscus

Cyperus michelianus

Cyperus longus type

Schoenus nigricans

Carex hallerana type

Carex flacca type

Epipactis type

Cephalanthera

\section{PTERIDOPHYTA}

Equisetum

Ophioglossum vulgatum

Botrychium

Adiantum capillus-veneris

Pteridium aquilinum

Thelypteris palustris

Thelypteris phegopteris

Asplenium type

Athyrium filix-femina

Athyrium distentifolium

Dryopteris filix-mas type

Gymnocarpium dryopteris

Blechnum spicant

Polypodium

Salvinia natans

Unidentified monolete spores

\section{TOTALS}

Indeterminate

Unknown

Number of terrestrial types

Total wetland pollen \& spores

Total terrestrial trees \& shrubs

$\begin{array}{rrrrrrrrrrrr}57 & 29 & 36 & 17 & 9 & 11 & 12 & 21 & 29 & 22 & 18 & 21 \\ 9 & 7 & 8 & 9 & 12 & 7 & 13 & 4 & & 1 & 1 & 3 \\ 9 & 10 & 12 & 13 & 17 & 22 & 19 & 8 & 6 & 6 & 4 & 5 \\ 2 & 4 & 4 & 14 & 15 & 17 & 10 & 12 & & 2 & & 2 \\ 5 & 3 & 6 & 12 & 12 & 15 & 12 & 8 & 3 & 6 & 1 & 5 \\ 38 & 5 & 9 & 15 & 12 & 2 & 13 & 4 & 2 & 1 & 3 & 4 \\ 1 & & 3 & 9 & 8 & 4 & 6 & 5 & 1 & & & \\ & & & & & & & & & & & \\ 6 & 4 & 5 & 20 & 14 & 25 & 9 & 20 & 3 & 2 & 1 & 6 \\ & 3 & & 2 & & 1 & 3 & & & & & \\ 1 & & & & & & 4 & & 4 & & & \\ 82 & 168 & 136 & 128 & 88 & 113 & 100 & 129 & 118 & 140 & 58 & 415 \\ & & & & & & & & & & & \\ 6 & 1 & & 8 & 4 & 2 & 4 & & 14 & 18 & 7 & 32 \\ 2 & 4 & 23 & 19 & 17 & 25 & 16 & 32 & & & & \\ 36 & 56 & 34 & 33 & 38 & 13 & 34 & 22 & & & & \\ 8 & & 2 & 1 & 3 & 4 & 7 & 5 & 73 & 74 & 53 & 176 \\ 3 & 5 & 11 & 10 & 14 & 13 & 3 & 9 & & & & \end{array}$

Total terrestrial pollen \& spores

(excluding bog \& marsh types,

aquatics, unknown \& indeterminate)

Total pollen \& spores

\begin{tabular}{rrrrrrrrrrrr}
561 & 496 & 609 & 736 & 536 & 581 & 604 & 489 & 479 & 477 & 524 & 664 \\
847 & 802 & 919 & 1060 & 793 & 864 & 892 & 825 & 790 & 784 & 701 & 1347 \\
& & & & & & & & & & & \\
21691 & 7630 & 15805 & 15805 & 8720 & 12317 & 13625 & 10682 & 9701 & 7194 & 8175 & 12644 \\
21364 & 8502 & 17658 & 17658 & 13843 & 20274 & 18312 & 14061 & 16241 & 17876 & 15914 & 19184 \\
9919 & 5232 & 9483 & 9483 & 7848 & 8175 & 8611 & 7303 & 11445 & 10355 & 10137 & 10246 \\
4033 & 1962 & 2725 & 2725 & 4469 & 4033 & 3488 & 3379 & 4033 & 2289 & 3270 & 3815 \\
654 & 109 & 436 & 436 & 981 & 872 & 981 & 545 & 981 & 1090 & 327 & 654 \\
109 & 327 & 109 & 109 & 218 & 218 & 545 & 218 & 327 & 218 & 109 & 327 \\
\hline
\end{tabular}

\section{CHARCOAL}

Charcoal $<25$ um2

Charcoal 25-50 um2

Charcoal 50-100 um2

Charcoal 100-200 um2

Charcoal 200-400 um2

Charcoal $>400$ um2

$\begin{array}{rrrrrrrrrrrr}7 & 3 & 4 & 4 & 3 & 4 & 7 & 7 & 4 & 3 & 2 & 9 \\ 53 & 52 & 62 & 49 & 44 & 45 & 62 & 57 & 46 & 48 & 58 & 62 \\ 279 & 303 & 306 & 320 & 254 & 279 & 281 & 329 & 307 & 304 & 175 & 674 \\ 225 & 251 & 222 & 220 & 202 & 239 & 226 & 194 & 241 & 225 & 214 & 333\end{array}$


Kellia

Depth (cm)

$\begin{array}{llllllllllllll}225-226 & 230-231 & 235-236 & 240-241 & 245-246 & 250-251 & 255-256 & 260-261 & 265-266 & 270-271 & 275-276 & 280-281\end{array}$

Absolute counts data

Slide numbers

Sample weight $(\mathrm{g})$ (wet)

Lycopodium suspension added (ml)

Lycopodium count

$\begin{array}{rrrrrrrrrrrr}1 & 1 & 1 & 1-3 & 1-3 & 1 \& 2 & 1.3 & 1.5 & 1 \& 2 & 1.5 & 1 & 1 \& 2 \\ 1.601 & 1.536 & 1.672 & 1.758 & 1.774 & 1.806 & 1.789 & 1.834 & 1.666 & 1.918 & 1.787 & 1.762 \\ 0.5 & 0.5 & 0.5 & 0.2 & 0.5 & 0.5 & 0.5 & 0.5 & 0.5 & 0.5 & 0.5 & 0.5 \\ 2422 & 1445 & 1634 & 1266 & 11681 & 7419 & 8113 & 18673 & 6713 & 19845 & 3836 & 3359\end{array}$

GYMNOSPERMAE

Abies

Cedrus

Pinus subgenus Diploxylon

Juniperus

Taxus baccata

Ephedra fragilis

Ephedra distachya type

\section{DICOTYLEDONES}

Salix triandra

Salix fragilis type

Juglans regia

Alnus glutinosa

Betula pendula

Carpinus type

Ostrya type

Corylus

Fagus

Castanea sativa

Quercus coccifera type

Quercus trojana type

Quercus frainetto type

Ulmus minor

Ulmus procera

Ulmus glabra type

Morus nigra

Morus alba

Humulus lupulus

Urtica dioica

Urtica urens type

Urtica pilulifera type

Polyganum bistorta type

Polygonum aviculare type

Bilderdykia

Oxyria digyna

Rumex acetosella group

Rumex acetosa group

Rumex crispus p.p.

Rumex conglomeratus group

Rumex obtusifolius group

Chenopodiaceae type

Phytolacca americana

Spergula

Spergularia type

Hemiaria type

Corrigiola litoralis

Paronychia type

Dianthus type

Silene alba type

Lychnis flos-cuculi

Agrostemma githago

Scleranthus annuus type

Nymphaea alba

Nuphar lutea

Helleborus

Nigella

Trollius europaeus

Actaea spicata

Adonis

Caltha type

$\begin{array}{rrrrrrrrrrrrr}5.3 & & 1.3 & .3 & 2.4 & .3 & 1.3 & 2 . & 2.3 & 3.2 & 3 . & 2.4 \\ 81.7 & 69.7 & 57.7 & 78.4 & 59.6 & 38.3 & 53 . & 56.4 & 77.6 & 51.3 & 49.7 & 78 . \\ 10 & 4 & 5 & 5 & 2 & 2 & 12 & 4 & 2 & 6 & 1 & 8 \\ 1 & 1 & & 1 & & & & & & & & & \\ 1 & & & & & & & & & & & & \end{array}$

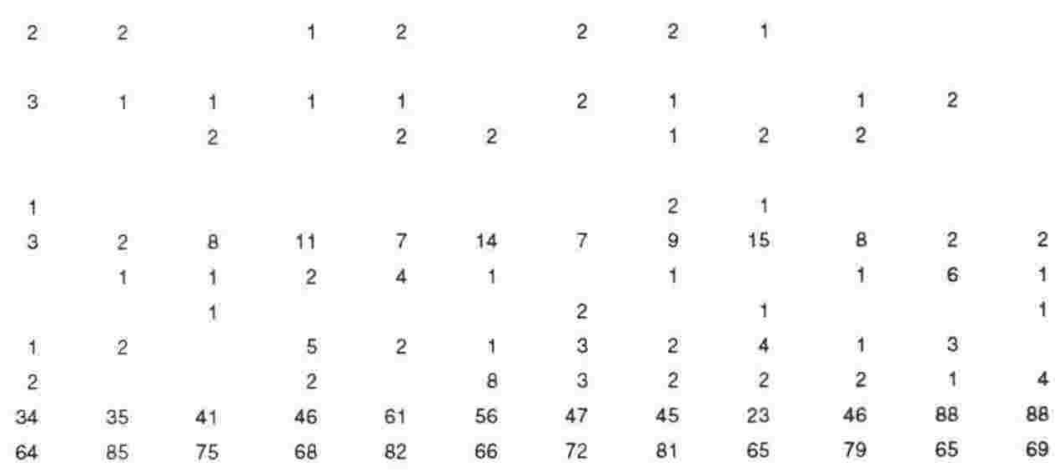

Ramunculus acris group

Ranunculus aquatilis group

Anemone nemorosa group 
Kellia

Depth (cm)

Clematis vitalba group

Thalictrum aquilegifolium

Thalictrum mirus

Thalictrum lucidum

Chelidonium majus

Glaucium

Hormungia type

Arabis

Sinapis type

Chrysosplenium altermifolium

Saxifraga stellaris

Saxifraga oppositifolia type

Platanus orientalis

Filipendula

Rubus type

Sorbus type

Cotoneaster type

Prunus avium type

Crataegus

Sanguisorba officinalis

Sanguisorba minor

Geum

Potentilla

Alchemilla type

Ononis type

Trifolium dubium type

Trifolium repens type

Lotus type

Lathyrus pratensis type

Vicia sativa type

Vicia cracca type

Astragalus

Galega officinalis

Dorycrium

Trifolium montanum type

Lathyrus sativus

Oxalis

Geranium robertianum group

Tribulus terrestris

Radiola linoides

Linum catharticum

Linum tenuifolium group

Euphorbia

Pistacia

Cotinus coggygria

Acer

Ilex aquifolium

Buxus sempervirens

Frangula

Rhamnus type

Vitis vinifera

Tilia platyphyllos

Tilia cordata type

Abutilon type

Althaea officinalis

Malva moschata

Daphne

Elaeagnus angustifolia

Hypericum hircinum type

Hypericum perforatum type

Viola hirta type

Helianthernum

Cistus monspeliensis type

Myricaria germanica

Tamarix

Elatine alsinastrum

Lythrum salicaria type

Lythrum portula

Trapa natans

Myrtus communis

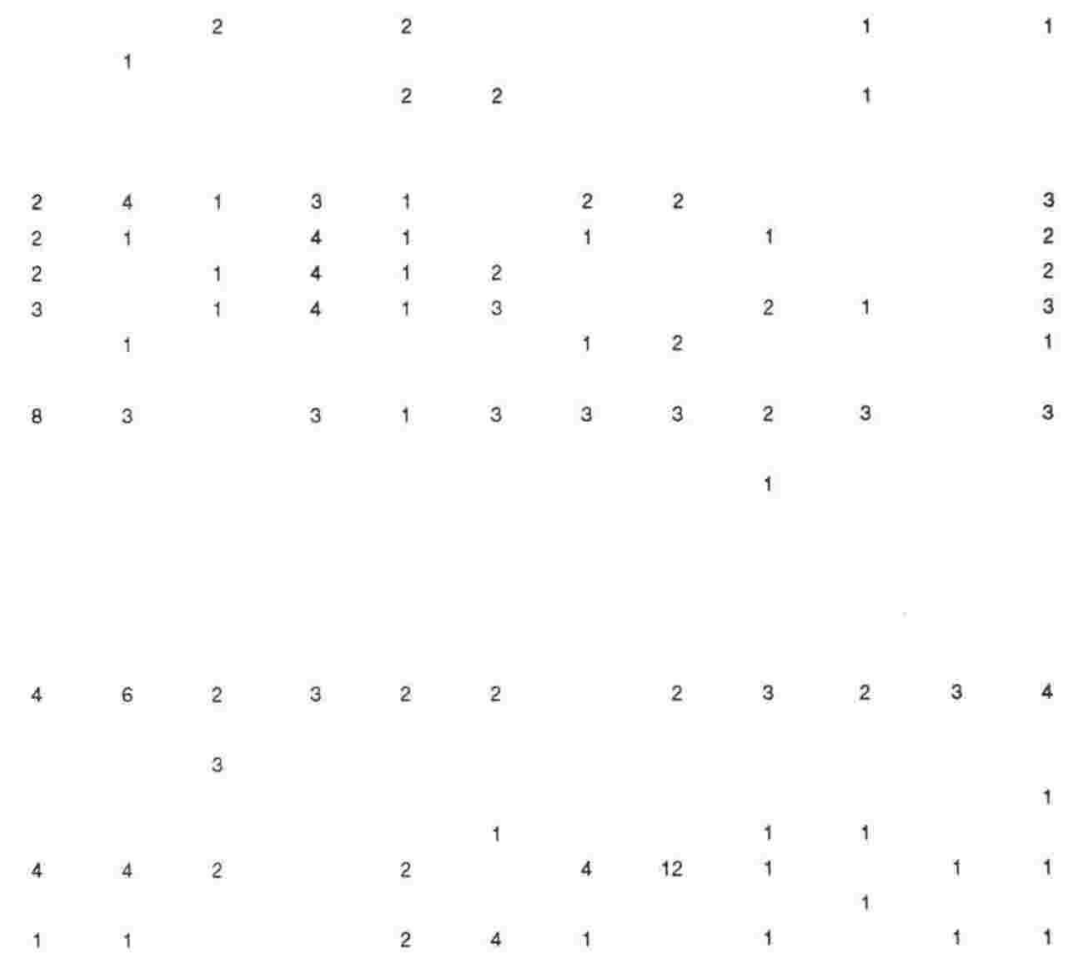

2

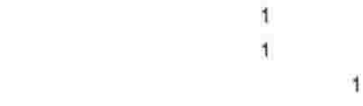

3 
Kellia

Depth $(\mathrm{cm})$

Ludwigia palustris

Epilobium angustifolium type

Epilobium hirsutum type

Myriophyllum verticillatum

Myriophyllum spicatum

Cornus mas

Hydrocotyle vulgaris

Anthriscus caucalis

Ligusticum

Berula erecta

Trinia

Sanicula europaea

Oenanthe aquatica group

Oenanthe pimpinelloides group

Chaerophyllum hirsutum type

Chaerophyllum temulentum type

Ammi

Pimpinella saxifraga

Torilis arvensis

Peucedanum austriacum type

Angelica sylvestris

Bunium

Anthriscus sylvestris type

Pastinaca sativa

Eryngium

Heracleum sphondylium

Anthriscus cerefolium

Torilis japonica type

Daucus

Coriandrum type

Pyrola

Erica herbacea

Vaccinium myrtillus

Soldanella

Androsace villosa

Primula

Lysimachia

Anagallis arvensis type

Anagallis tenella type

Asterolinon limum-stellatum

Samolus valerandi

Ameria maritima type

Fraxinus excelsior type

Fraxinus angustifolia

Fraxinus ormus

Ligustrum vulgare

Phillyrea

Centaurium

Convolvulus

Cuscuta epithymum type

Cuscuta europaea type

Cuscuta monogyna

Calystegia

Alkanna type

Borago officinalis

Buglossoides arvensis type

Anchusa arvensis type

Pulmonaria mollis

Anchusa officinalis type

Verbena

Callitriche obtusangula

Callitriche stagnalis type

Teucrium

Stachys sylvatica type

Prunella type

Salvia officinalis type

Mentha type

Solanum dulcamara

Solanum nigrum type

Scrophularia type

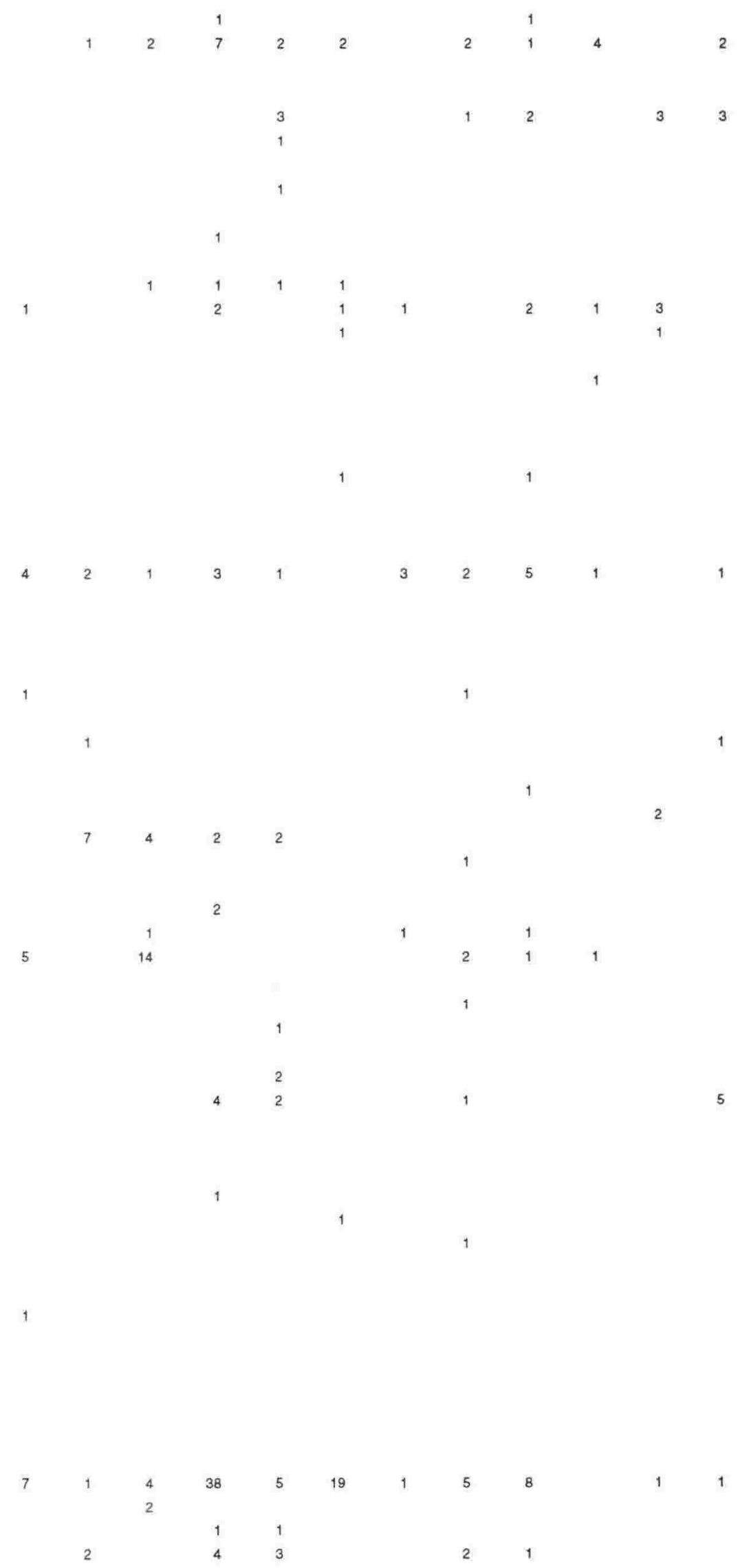


Kellia

Depth (cm)

Odontites type

Veronica anagallis-aquatica type

Veronica triphyllos type

Pedicularis

Putoria calabrica

Sherardia arvensis

Galium type

Plantago major

Plantago afra

Plantago lanceolata type

Plantago coronopus

Plantago bellardii

Plantago media

Plantago argentea

Plantago albicans

Plantago amplexicaulis

Plantago atrata

Vibumum

Valerianella rimosa type

Valeriana officinalis type

Valerianella locusta type

Succisa pratensis

Scabiosa columbaria type

Dipsacus type

Knautia

Jasione

Campanula erinus type

Campanula rotundifolia

Asyneuma type

Solidago type

Echinops

Artemisia

Carlina type

Picnomon acama

Achillea type

Centaurea cyanus type

Centaurea napulifera type

Cirsium type

Xanthium

Centaurea solstitialis type

Centaurea graeca type

Crupina

Sonchus type

Lactuca type

Scolymus

Cichorium type

Hieracium type

Taraxacum type

\section{MONOCOTYLEDONES}

Alisma

Hydrocharis morsus-ranae

Stratiotes aloides

Vallisneria spiralis

Triglochin bulbosa

Potamogeton crispus type

Potamogeton pectinatus

Colchicum

Narthecium scardicum

Veratrum

Asphodelus fistulosus type

Asphodelus albus

Gagea pratensis

Tulipa sylvestris

Muscari neglectum type

Allium sphaerocephalon type

Asparagus

Gynandriris sisyrinchium

Festuca type

Glyceria type

\begin{tabular}{|c|c|c|c|c|c|c|c|c|c|c|c|}
\hline 8 & 14 & 6 & 9 & 5 & 8 & 6 & 9 & 9 & 10 & 20 & 13 \\
\hline & \multirow[t]{3}{*}{1} & & 2 & 3 & & 1 & 1 & 2 & 1 & 2 & \\
\hline & & & 1 & & & & 1 & & 1 & & \\
\hline & & & 2 & 1 & & 1 & 1 & & & & \\
\hline 2 & 1 & 4 & & 2 & 2 & 3 & 5 & 1 & 4 & 5 & 4 \\
\hline 5 & 5 & 7 & B & 3 & 8 & 5 & 7 & 4 & 13 & 3 & 5 \\
\hline 10 & 11 & 24 & 21 & 24 & 15 & 17 & 18 & 15 & 13 & 13 & 16 \\
\hline \multirow[t]{2}{*}{3} & 1 & & 1 & 1 & 2 & & 11 & & & & 2 \\
\hline & 1 & 1 & 1 & 1 & & 1 & 28 & & 4 & & \\
\hline 9 & 2 & 7 & 5 & 13 & 9 & 9 & 22 & 15 & 28 & 12 & 17 \\
\hline 10 & 9 & 15 & 5 & 7 & 5 & 10 & 14 & 15 & 8 & 6 & 1 \\
\hline 1 & & & 2 & 1 & & & 1 & & & & \\
\hline 3 & 2 & 1 & 8 & 6 & 7 & 6 & 10 & 15 & 12 & 9 & 20 \\
\hline 41 & 34 & 18 & 30 & 33 & 20 & 21 & 91 & 30 & 64 & 20 & 38 \\
\hline 11 & 7 & 7 & 5 & 16 & 12 & 13 & 19 & 13 & 28 & 19 & 16 \\
\hline
\end{tabular}


Kellia

Depth $(\mathrm{cm})$

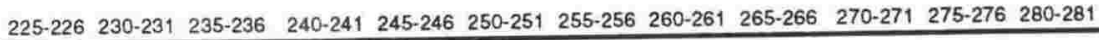

Dactylis type

Agropyron type

Triticum type

Secale cereale

Hordeum type

Avena

Phragmites australis

Zea mays

Anum

Sparganium erectum

Sparganium angustifolium

Typha angustifolia type

Typha domingensis type

Scirpus

Cladium mariscus

Cyperus michelianus

Cyperus longus type

Schoenus nigricans

Carex hallerana type

Carex flacca type

Epipactis type

Cephalanthera

\section{PTERIDOPHYTA}

Equisetum

Ophioglossum vulgatum

Botrychium

Adiantum capillus-veneris

Pteridium aquilinum

Thelypteris palustris

Thelypteris phegopteris

Asplenium type

Athyrium filix-femina

Athyrium distentifolium

Dryopteris filix-mas type

Gymnocarpium dryopteris

Blechnum spicant

Polypodium

Salvinia natans

Unidentified monolete spores

$\begin{array}{rrrrrrrrrrrr}27 & 50 & 30 & 54 & 80 & 34 & 32 & 48 & 41 & 47 & 32 & 29 \\ 6 & 3 & 1 & 4 & 4 & & & 1 & 2 & 4 & 3 & 3 \\ 4 & 19 & 6 & 10 & 13 & 11 & 3 & 1 & 5 & 6 & 13 & 8 \\ 4 & 6 & 7 & & 3 & 1 & 3 & & 1 & 2 & 1 & 4 \\ 4 & 12 & 3 & 5 & 7 & 4 & 3 & 7 & 5 & 5 & 2 & 3 \\ 6 & 2 & 7 & 5 & 17 & 2 & & 4 & 3 & 3 & 8 & 1 \\ & 1 & 1 & & 2 & 1 & 4 & & & & 1 & \end{array}$

TOTALS

Indeterminate

Unknown

Number of terrestrial types

Total wetland pollen \& spores

Total terrestrial trees \& shrubs

$\begin{array}{rrr}2 & & \\ 179 & 930 & 290 \\ 62 & 63 & 20 \\ & 2 & 3 \\ 3 & 2 & 3 \\ 78 & 309 & 37\end{array}$

$$
9
$$
3 
Kellia

Depth $(\mathrm{cm})$

Absolute counts data

Slide numbers

Sample weight $(\mathrm{g})$ (wet)

Lycopodium suspension added (ml)

Lycopodium count

$\begin{array}{rrrrrrrrrrrr}1 & 1 & 1 & 1 & 1 & 182 & 1 \& 2 & 182 & 1 \& 2 & 1 & 1 & 1 \\ 1.696 & 1.440 & 1.466 & 1.550 & 1.547 & 1.828 & 1.862 & 1.685 & 1.760 & 1.884 & 1.927 & 1.857 \\ 0.5 & 0.5 & 0.5 & 0.5 & 0.5 & 0.5 & 0.5 & 0.5 & 0.5 & 0.5 & 0.5 & 0.5 \\ 2265 & 1880 & 2325 & 1898 & 1594 & 3893 & 4514 & 4018 & 6198 & 3469 & 3560 & 3135\end{array}$

\section{GYMNOSPERMAE}

Abies
Cedrus

Pinus subgenus Diploxylon

Juniperus

Taxus baccata

Ephedra fragilis

Ephedra distachya type

\section{DICOTYLEDONES}

Salix triandra

Salix fragilis type

Juglans regia

Alnus glutinosa

Betula pendula

Carpinus type

Ostrya type

Corylus

Fagus

Castanea sativa

Quercus coccifera type

Quercus trojana type

Quercus frainetto type

Ulmus minor

Ulmus procera

Ulmus glabra type

Morus nigra

Morus alba

Humulus lupulus

Urtica dioica

Urtica urens type

Urtica pilulifera type

Polygonum bistorta type

Polygonum aviculare type

Bilderdykia

Oxyria digyna

Rumex acetosella group

Rumex acetosa group

Rumex crispus p.p.

Rumex conglomeratus group

Rumex obtusifolius group

Chenopodiaceae type

Phytolacca americana

Spergula

Spergularia type

Hemiaria type

Corrigiola litoralis

Paronychia type

Dianthus type

Silene alba type

Lychnis flos-cuculi

Agrostemma githago

Scleranthus annuus type

Nymphaea alba

Nuphar lutea

Helleborus

Nigella

Trollius europaeus

Actaea spicata

Adonis

Caltha type

Ranunculus acris group

Ranunculus aquatilis group

$\begin{array}{rrrrrrrrrrrr}2.7 & 2.7 & 3 . & 1.3 & 2 . & & .7 & 2.4 & & 2 . & 2 . & 2.3 \\ 66.3 & 95.3 & 93.7 & 81.7 & 74.7 & 52 . & 54 . & 74 . & 62.4 & 41.3 & 44.7 & 49.3 \\ 3 & 3 & 1 & 4 & 4 & 4 & 1 & & 1 & 5 & 1 & 3 \\ 1 & & & & 2 & & & & & & & \end{array}$


Kellia

Clematis vitalba group

Thalictrum aquilegifolium

Thalictrum minus

Thalictrum lucidum

Chelidonium majus

Glaucium

Hornungia type

Arabis

Sinapis type

Chrysosplenium alternifolium

Saxifraga stellaris

Saxifraga oppositifolia type

Platanus orientalis

Filipendula

Rubus type

Sorbus type

Cotoneaster type

Prumus avium type

Crataegus

Sanguisorba officinalis

Sanguisorba minor

Geum

Potentilla

Alchemilla type

Ononis type

Trifolium dubium type

Trifolium repens type

Lotus type

Lathyrus pratensis type

Vicia sativa type

Vicia cracca type

Astragalus

Galega officinalis

Dorycnium

Trifolium montanum type

Lathyrus sativus

Oxalis

Geranium robertianum group

Tribulus terrestris

Radiola linoides

Limum catharticum

Linum tenuifolium group

Euphorbia

Pistacia

Cotinus coggygria

Acer

Ilex aquifolium

Buxus sempervirens:

Frangula

Rhamnus type

Vitis vinifera

Tilia platyphyllos

Tilia cordata type

Abutilon type

Althaea officinalis

Malva moschata

Daphne

Elaeagnus angustifolia

Hypericum hircinum type

Hypericum perforatum type

Viola hirta type

Helianthemum

Cistus monspeliensis type

Myricaria germanica

Tamarix

Elatine alsinastrum

Lythrum salicaria type

Lythrum portula

Trapa natans

Myrtus communis 
Kellia

Ludwigia palustris

Epilobium angustifolium type

Epilobium hirsutum type

Myriophyllum verticillatum

Myriophyllum spicatum

Cornus mas

Hydrocotyle vulgaris

Anthriscus caucalis

Ligusticum

Berula erecta

Trinia

Sanicula europaea

Oenanthe aquatica group

Oenanthe pimpinelloides group

Chaerophyllum hirsutum type

Chaerophyllum temulentum type

Ammi

Pimpinella saxifraga

Torilis arvensis

Pencedanum austriacum type

Angelica sylvestris

Bunium

Anthriscus sylvestris type

Pastinaca sativa

Eryngium

Heracleum sphondylium

Anthriscus cerefolium

Torilis japonica type

Daucus

Coriandrum type

Pyrola

Erica herbaced

Vaccinium myrtillus

Soldanella

Androsace villosa

Primula

Lysimachia

Anagallis arvensis type

Anagallis tenella type

Asterolinon linum-stellatum

Samolus valerandi

Anneria maritima type

Fraxinus excelsior type

Fraxinus angustifolia

Fraxinus ornus

Ligustrum vulgare

Phillovea

Centaurium

Convolvulus

Cuscuta epithymum type

Cuscuta europaea type

Cuscuta monogyna

Calystegia

Alkanna type

Borago officinalis

Buglossoides arvensis type

Anchusa arvensis type

Pulmonaria mollis

Anchusa officinalis type

Verbena

Callitriche obtusangula

Callitriche stagnalis type

Teucrium

Stachys sylvatica type

Prunella type

Salvia officinalis type

Mentha type

Solanum dulcamara

Solanum nigrum type

Scrophularia type 
Kellia

Depth $(\mathrm{cm})$

Odontites type

Veronica anagallis-aquatica type

Veronica triphyllos type

Pedicularis

Putoria calabrica

Sherardia arvensis

Galium type

Plantago major

Plantago afra

Plantago lanceolata type

Plantago coronopus

Plantago bellardii

Plantago media

Plantago argentea

Plantago albicars

Plantago amplexicaulis

Plantago atrata

Viburrum

Valerianella rimosa type

Valeriana officinalis type

Valerianella locusta type

Succisa pratensis

Scabiosa columbaria type

Dipsacus type

Knautia

Jasione

Campanula erinus type

Campanula rotundifolia

Asyneuma type

Solidago type

Echinops

Artemisia

Carlina type

Picnomon acama

Achillea type

Centaurea cyanus type

Centaurea napulifera type

Cirsium type

Xanthium

Centaurea solstitialis type

Centaurea graeca type

Crupina

Sonchus type

Lactuca type

Scolymus

Cichorium type

Hieracium type

Taraxacum type

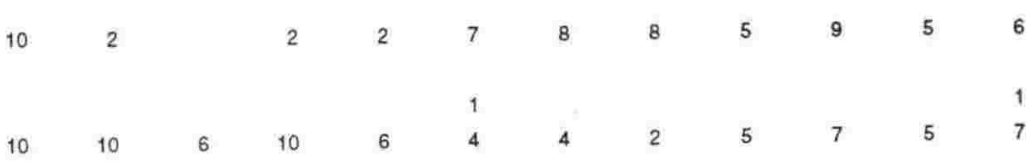

MONOCOTYLEDONES

Alisma

Hydrocharis morsus-ranae

Stratiotes aloides

Vallisneria spiralis

Triglochin bulbosa

Potanogeton crispus type

Potamogeton pectinatus

Colchicum

Narthecium scardicum

Veratrum

Asphodelus fistulosus type

Asphodelus albus

Gagea pratensis

Tulipa sylvestris

Muscari neglectum type

Allium sphaerocephalon type

Asparagus

Gynandriris sisyrinchitum

Festuca type

Glyceria type

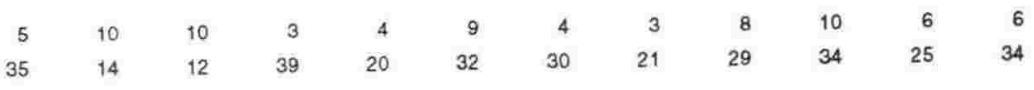


Kellia

Depth (cm)

285-286 $290-291 \quad 295-296 \quad 305-306 \quad 310-311 \quad 315-316 \quad 320-321 \quad 325-326 \quad 330-331 \quad 335-336 \quad 340-341 \quad 345-346$

Dactylis type

Agropyron type

Triticum type

Secale cereale

Hordeum type

Avena

Phragmites australis

Zea mays

Anum

Sparganium erectum

Sparganium angustifolium

Typha angustifolia type

Typha domingensis type

Scirpus

Cladium mariscus

Cyperus michelianus

Cyperus longus type

Schoenus nigricans

Carex hallerana type

Carex flacca type

Epipactis type

Cephalanthera

\section{PTERIDOPHYTA}

Equisetum

Ophioglossum vulgatum

Botrychium

Adiantum capillus-veneris

Pteridium aquilinum

Thelypteris palustris

Thelypteris phegopteris

Asplenium type

Athyrium filix-femina

Athyrium distentifolium

Dryopteris filix-mas type

Gymnocarpium dryopteris

Blechnum spicant

Polypodium

Salvinia natans

Unidentified monolete spores

\section{TOTALS}

Indeterminate

Unknown

Number of terrestrial types

Total wetland pollen \& spores

Total terrestrial trees \& shrubs

$\begin{array}{rrrrrrrrrrrr}22 & 4 & 10 & 12 & 25 & 25 & 35 & 27 & 19 & 38 & 33 & 34 \\ 5 & 1 & 1 & 3 & 2 & 4 & 2 & 5 & 8 & 6 & 6 & 5 \\ 5 & 1 & 3 & 10 & 7 & 2 & 3 & 9 & 3 & 3 & 4 & 2 \\ 2 & & & 2 & 5 & 3 & 1 & 2 & 1 & & 2 & 2 \\ 2 & 1 & 1 & 3 & 3 & 3 & 5 & 12 & 1 & 3 & & 1 \\ & & 1 & 1 & & 2 & 1 & & & & 1 & 1 \\ & & 2 & 3 & 2 & & & & 2 & & & \\ 4 & 1 & 7 & 9 & 20 & 131 & 272 & 10 & 104 & 77 & 69 & 62 \\ & & & & & 1 & & & 1 & 5 & & \\ 4 & & & & & 2 & 2 & & 1 & 7 & 2 & \\ 128 & 430 & 285 & 661 & 845 & 125 & 76 & 177 & 53 & 205 & 59 & 76 \\ & & & & & & & & & & & \\ 65 & 58 & 235 & 268 & 63 & 5 & 1 & 1 & 6 & 2 & & \\ 1 & & & & & 6 & 3 & 21 & 4 & 2 & 2 & \\ 14 & 18 & 15 & 38 & 27 & 2 & & & & & & \\ 214 & 682 & 914 & 877 & 311 & 11 & 14 & 28 & 24 & 28 & 6 & 5 \\ & & & & & 1 & & 3 & 1 & & & \end{array}$

Total terrestrial pollen \& spores (excluding bog \& marsh types, aquatics, unknown \& indeterminate) Total pollen \& spores

\begin{tabular}{rrrrrrrrrrrr}
492 & 387 & 485 & 572 & 533 & 587 & 503 & 660 & 611 & 455 & 504 & 423 \\
973 & 1595 & 1960 & 2491 & 1831 & 926 & 925 & 938 & 846 & 825 & 670 & 608 \\
& & & & & & & & & & & \\
10464 & 1308 & 1417 & 3597 & 1308 & 22890 & 29975 & 31392 & 43491 & 22672 & 22454 & 18203 \\
11772 & 3488 & 2616 & 4251 & 2507 & 28994 & 31392 & 50794 & 81096 & 28122 & 30411 & 21582 \\
7957 & 1526 & 2725 & 3488 & 2071 & 21037 & 23871 & 30302 & 51121 & 12753 & 17222 & 14497 \\
3270 & 1853 & 545 & 1199 & 763 & 10900 & 10791 & 9265 & 17113 & 5995 & 6104 & 4469 \\
1308 & 436 & 436 & 109 & 218 & 4251 & 2834 & 2507 & 4905 & 1308 & 981 & 981 \\
545 & 218 & 436 & & 109 & 2725 & 1744 & 654 & 436 & 436 & 327 & 218 \\
\hline
\end{tabular}


Kellia

\section{Absolute counts data}

Slide numbers

Sample weight (g) (wet)

Lycopodium suspension added $(\mathrm{ml})$

Lycopodium count

$\begin{array}{rrrrrrrrrr}1-3 & 1 & 1 & 1 & 1 & 182 & 1 \& 2 & 1 & 1 & 182 \\ .1 .868 & 1.877 & 1.918 & 1.829 & 1.826 & 1.879 & 1.893 & 1.902 & 1.936 & 1.490 \\ 0.2 & 0.5 & 0.5 & 0.5 & 0.5 & 0.5 & 0.5 & 0.5 & 0.5 & 0.5 \\ 1139 & 1858 & 1610 & 3568 & 2683 & 2354 & 3198 & 4140 & 3643 & 3188\end{array}$

\section{GYMNOSPERMAE}

\section{Abies}

Cedrus

Pinus subgenus Diploxylon

Juniperus

Taxus baccata

Ephedra fragilis

Ephedra distachya type

\section{DICOTYLEDONES}

Salix triandra

Salix fragilis type

Juglans regia

Alnus glutinosa

Betula pendula

Carpinus type

Ostrya type

Corylus

Fagus

Castanea sativa

Quercus coccifera type

Quercus trojana type

Quercus frainetto type

Ulmus minor

Ulmus procera

Ulmus glabra type

Morus nigra

Morus alba

Humulus lupulus

Urtica dioica

Urtica urens type

Urtica pilulifera type

Polygonum bistorta type

Polygonum aviculare type

Bilderdykia

Oxyria digyna

Rumex acetosella group

Rumex acetosa group

Rumex crispus p.p.

Rumex conglomeratus group

Rumex obtusifolius group

Chenopodiaceae type

Phytolacca americana

Spergula

Spergularia type

Hemiaria type

Corrigiola litoralis

Paronychia type

Dianthus type

Silene alba type

Lychnis flos-cuculi

Agrostemma githago

Scleranthus annuus type

Nymphaea alba

Nuphar lutea

Helleborus

Nigella

Trollius europaeus

Actaea spicata

Adonis

Caltha type

Ramunculus acris group

Ranunculus aquatilis group

Anemone nemorosa group 
Kellia

Depth (cm)

Clematis vitalba group

Thalictrum aquilegifolium

Thalictrum minus

Thalictrum lucidum

Chelidonium majus

Glaucium

Homungia type

Arabis

Sinapis type

Chrysosplenium altemifolium

Saxifraga stellaris

Saxifraga oppositifolia type

Platanus orientalis

Filipendula

Rubus type

Sorbus type

Cotoneaster type

Prumus avium type

Crataegus

Sanguisorba officinalis

sanguisorba minor

Geum

Potentilla

Alchemilla type

Ononis type

Trifolium dubium type

Trifolium repens type

Lotus type

Lathyrus pratensis type

Vicia sativa type

Vicia cracca type

Astragalus

Galega officinalis

Dorycnium

Trifolium montanum type

Lathyrus sativus

Oxalis

Geranium robertianum group

Tribulus terrestris

Radiola linoides

Linum catharticum

Lintun tenuifolium group

Euphorbia

Pistacia

Cotinus coggygria

Acer

Ilex aquifolium

Buxus sempervirens

Frangula

Rhamnus type

Vitis vinifera

Tilia platyphyllos

Tilia cordata type

Abutilon type

Althaea officinalis

Malva moschata

Daphne

Elaeagnus angustifolic

Hypericum hircinum type

Hypericum perforatum type

Viola hirta type

Helianthemum

Cistus monspeliensis type

Myricaria germanica

Tamarix

Elatine alsinastrum

Lythrum salicaria type

Lythrum portula

Trapa natans

Myrtus communis 
Kellia

Depth $(\mathrm{cm})$

Ludwigia palustris

Epilobium angustifolium type

Epilobium hirsutum type

Myriophyllum verticillatum

Myriophyllum spicatum

Cornus mas

Hydrocotyle vulgaris

Anthriscus caucalis

Ligusticum

Berula erecta

Trinia

Sanicula europaea

Oenanthe aquatica group

Oenamthe pimpinelloides group

Chaerophyllum hirsutum type

Chaerophyllum temulentum type

Anmi

Pimpinella saxifraga

Torilis arverssis

Peucedanum austriacum type

Angelica sylvestris

Bunium

Anthriscus sylvestris type

Pastinaca sativa

Eryngium

Heracleum sphondylium

Anthriscus cerefolium

Torilis japonica type

Daucus

Coriandrum type

Pyrola

Erica herbacea

Vaccinium myrtillus

Soldanella

Androsace villosa

Primula

Lysimachia

Anagallis arvensis type

Anagallis tenella type

Asterolinon lirum-stellatum

Samolus valerandi

Armeria maritima type

Fraximus excelsior type

Fraxinus angustifolio

Fraximus ormus

Ligustrum vulgare

Phillyrea

Centaurium

Convolvulus

Cuscuta epithymum type

Cuscuta europaea type

Cuscuta monogyna

Calystegia

Alkanna typ

Borago officinalis

Buglossoides arvensis type

Anchusa arversis type

Pulmonaria mollis

Anchusa officinalis type

Verbena

Callitriche obtusangula

Callitriche stagnalis type

Teucrium

Stachys sylvatica type

Prunella type

Salvia officinalis type

Mentha type

Solanum dulcamara

Solanum nigrum type

Scrophularia type

21 
Kellia

Depth (cm)

Odontites type

Veronica anagallis-aquatica type

Veronica triphyllos type

Pedicularis

Putoria calabrica

Sherardia arvensis

Galium type

Plantago major

Plantago afra

Plantago lanceolata type

Plantago coronopus

Plantago bellardii

Plantago media

Plantago argentea

Plantago albicans

Plantago amplexicaulis

Plantago atrata

Viburnum

Valerianella rimosa type

Valeriana officinalis type

Valerianella locusta type

Succisa pratensis

Scabiosa columbaria type

Dipsacus type

Knautia

Jasione

Campanula erinus type

Campanula rotundifolia

Asyneuma type

Solidago type

Echinops

Artemisia

Carlina type

Picnomon acama

Achillea type

Centaurea cyanus type

Centaurea napulifera type

Cirsium type

Xanthium

Centaurea solstitialis type

Centaurea graeca type

Crupina

Sonchus type

Lactuca type

Scolymus

Cichorium type

Hieracium type

Taraxacum type

MONOCOTYLEDONES

Alisma

Hydrocharis morsus-ranae

Stratiotes aloides

Vallisneria spiralis

Triglochin bulbosa

Potamogeton crispus type

Potamogeton pectinatus

Colchicum

Narthecium scardicum

Veratrum

Asphodelus fistulosus type

Asphodelus albus

Gagea pratensis

Tulipa sylvestris

Muscari neglectum type

Allium sphaerocephalon type

Asparagus

Gynandriris sisyrinchium

Festuca type

Glyceria type

3


Kellia

Depth $(\mathrm{cm})$

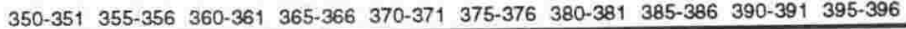

Dactylis type

Agropyron type

Triticum type

Secale cereale

Hordeum type

Avena

Phragmites australis

Zea mays

Anum

Sparganium erectum

Sparganium angustifolium

Typha angustifolia type

Typha domingensis type

Scirpus

Cladium mariscus

Cyperus michelianus

Cyperus longus type

Schoenus nigricans

Carex hallerana type

Carex flacca type

Epipactis type

Cephalanthera

\section{PTERIDOPHYTA}

Equisetum

Ophioglossum vulgatum

Botrychium

Adiantum capillus-veneris

Pteridium aquilinum

Thelypteris palustris

Thelypteris phegopteris

Asplerium type

Athyrium filix-femina

Athyrium distentifolium

Dryopteris filix-mas type

Gymnocarpium dryopteris

Blechrum spicant

Polypodium

Salvinia natans

Unidentified monolete spores

TOTALS

Indeterminate

Unknown

Number of terrestrial types

Total wetland pollen \& spores

Total terrestrial trees \& shrubs

$\begin{array}{rrrrrrrrrr}26 & 21 & 21 & 46 & 42 & 37 & 12 & 46 & 41 & 77 \\ 2 & 7 & 11 & 10 & 4 & 1 & 2 & & & 3 \\ & & & & & 1 & 12 & 9 & 6 & 1 \\ & 4 & 3 & 13 & 1 & 5 & 23 & 15 & 12 & 4 \\ 3 & 5 & 5 & 2 & 3 & 1 & 1 & & & 1 \\ & 1 & & 2 & 1 & 1 & & 1 & 3 & \\ & 4 & & & & 4 & 1 & & 1 & 5\end{array}$

15

$\begin{array}{llllrrr}2 & 98 & 33 & 82 & 73 & 5 & 3\end{array}$

Total terrestrial pollen \& spores

(excluding bog \& marsh types,

aquatics, unknown \& indeterminate)

$\begin{array}{rrrrrrrrrr}411 & 357 & 495 & 600 & 536 & 650 & 651 & 995 & 804 & 679 \\ 532 & 496 & 592 & 800 & 737 & 802 & 793 & 1160 & 911 & 781\end{array}$

CHARCOAL

Charcoal $<25$ um2

Charcoal 25-50 um2

Charcoal 50-100 um2

Charcoal 100-200 um2

Charcoal 200-400 um2

Charcoal $>400$ um 2

$\begin{array}{rrrrrrrrrr}9 & 2 & 7 & 4 & 4 & 13 & 8 & 16 & 25 & 19 \\ & & & & & & & 1 & & 1 \\ 44 & 38 & 42 & 41 & 49 & 53 & 46 & 40 & 33 & 53 \\ 112 & 137 & 90 & 196 & 197 & 139 & 134 & 148 & 82 & 82 \\ 205 & 189 & 183 & 306 & 232 & 219 & 192 & 278 & 196 & 213\end{array}$

\begin{tabular}{rrrrrrrrrr}
46107 & 34444 & 25615 & 20383 & 17331 & 45017 & 82840 & 63874 & 23871 & 42837 \\
29648 & 34771 & 26051 & 28994 & 20274 & 38695 & 78044 & 69978 & 43055 & 65836 \\
18748 & 20383 & 15478 & 18094 & 14170 & 21909 & 39676 & 50249 & 28885 & 34662 \\
7739 & 6649 & 5886 & 7303 & 5123 & 7303 & 12426 & 17113 & 14388 & 13298 \\
3379 & 2180 & 2071 & 1526 & 1417 & 2398 & 3815 & 3924 & 4905 & 4142 \\
1090 & 436 & 545 & 872 & 218 & 763 & 1744 & 981 & 981 & 1090 \\
\hline
\end{tabular}





\title{
Appendix XIV
}

\section{Stratigraphy of exploratory boreholes in Anelia \& Kellia wetlands}

\author{
Anelia \\ Depth below Description of stratigraphy \\ ground surface \\ (cm)
}

\section{Hiller exploratory core a:}

0 - $94 \quad$ wet fibrous peat; medium brown, oxidises very dark brown; paler at base, grading into grey clay

94-102 light-medium grey (slightly yellow) clay

102-110 same clay as above with angular pebbles $>1 \mathrm{~cm}$ size (?colluvium)

\section{Hiller exploratory core $b$ :}

$0-95$ wet fibrous peat; medium brown, oxidises very dark brown; paler at base, grading into

grey clay

95-112 light-medium grey (slightly yellow) clay with some plant stems

$112-125$

$125-128$ medium brownish grey clay, more plant material

$128-138$

$138-150$ ?

$150 ?-161$

161-167

167-175

$175-181$

$181-225$ medium brown? Detritus herbosus medium brownish grey silt, much fibrous plant material medium greyish brown clay with more fibrous plant material than above unit sloppy clay with wood fragments (twigs, ?bark to $2 \mathrm{~cm}$ size) - medium to dark brown medium brown clay with some fibrous plant material grey clay, rare plant fibres laminated medium brown \& dark grey silt pebbly greenish-grey clay; dark grey at top, medium grey at bottom; angular pebbles to $1 \mathrm{~cm}$ (?colluvium)

\section{Hiller exploratory core :}

$0-45$ wet fibrous peat; medium brown; oxidises very dark brown

45- 60 grey-brown clay with abundant plant fibre

60- 66 fibrous peat ?Detritus herbosus

66- 68 brownish grey clay with some plant fibre

68- 71 same as $60-66 \mathrm{~cm}$

71- 84 light-medium grey clay; some fibrous material

84-86 dark brown detritus

86-165 pebbly gritty medium grey clay, darker in top $4 \mathrm{~cm}$

\section{Hiller exploratory core d:}

0- 84 wet fibrous peat (wetter below $50 \mathrm{~cm}$ );

84-92 medium greyish brown clay with abundant plant material

92-107 medium grey clay with common fibrous plant material

107-115 medium brown clayey Detritus herbosus

115-132 grey clay with some plant fragments \& silt, minor sand

132-145 dark greyish brown clayey detritus with some woody material

145-200 gritty medium grey clay

\section{Hiller exploratory core e:}

$0-110$ wet fibrous peat; slightly silty between $95-110 \mathrm{~cm}$

110-116 grey clay

116-119 medium brown clayey detritus

119-120 grey clay 
120-125 medium brown clayey detritus

125-135 clayey grey grit

135-160 grey clay with some plant material

160-165 muddy grey gravel, angular fragments to $1 \mathrm{~cm}$

165-190 slightly laminated medium brownish grey to medium greyish brown clay \& silt with some fibrous material

190-215 medium dark grey clay with roots \& occasional grit

215-240 blue/grey clay with grit

Hiller exploratory core f:

$0-82$ wet fibrous peat; some grey silt below $70 \mathrm{~cm}$

82- 88 fine grey gravel with some fibrous plant material, clay matrix

88-110 grey gritty clay

110-125 brownish grey-greyish brown silt \& clay with detritus

125-137 grey silty fine gravel

137-165 brownish grey mud with some fibrous plant material

165-170 medium brownish grey Detritus herbosus with pebbles

170-185 medium brownish grey muddy fine gravel with some detritus

185-225 brownish grey silt with some detritus

225-280 pebbly blueish grey clay

\section{Hiller exploratory core g:}

0- 57 wet fibrous peat; 7.5YR 3/3 dark brown

57- $80 \quad$ mud \& muddy sand with plant fibres; 5GY 4/1 dark olive grey

80- 95 mud \& fine sand with some plant fibre, some grit; 10YR 5/8 yellowish brown - 10YR $4 / 2$ greyish yellow brown

95-125 muddy grit, average 3-4 mm particle size; also sand; 10YR 4/4 brown; bottomed on pebble

\section{Hiller exploratory core $h$ :}

0- 87 wet fibrous peat as elsewhere

87-105 mud with some fibrous plant material; 5 Y $4 / 2$ greyish olive

105- $\quad$ gravel; unrecovered

\section{Hiller exploratory core $i$ :}

0-89 wet fibrous peat as elsewhere; silty in last $5 \mathrm{~cm}$

89-105 mud with some fibrous material; 10YR 4/2 greyish yellow brown

105-150 muddy sand \& fine gravel (alluvium); 5GY 5/1 olive grey

\section{Hiller exploratory core $j$ :}

0-71 wet fibrous peat as elsewhere

71- 85 sandy fine gravel; admixture of organic muck; same colour as above

85- 94 silt; 10YR $2 / 2$ brownish black

94-126 clay; 2.5 GY $5 / 1$ olive grey

126-170 grit \& clay; same colour as above

\section{Hiller exploratory core $\boldsymbol{k}$ :}

0-22 wet fibrous peat as elsewhere

22- 45 mucky gravel, sandy gravel with organic muck; same colour as peat

45- 65 silt with fine organic material; 7.5YR 3/1 brownish black

65-100 muddy sand \& fine gravel; 2.5 GY $5 / 1$ olive grey 


\section{Hiller exploratory core l:}

0 - 33 wet fibrous peat as elsewhere

33- 61

interbedded detritus, and clay with detritus; colour varies from brown to grey, 7.5YR

4/2 greyish brown

61-66 Detritus herbosus; same colour as 0-33 cm

66- 90 mud with detritus; 5GY 5/1 olive grey

90- 98 muddy fine gravel; same colour as horizon above

98-157 silt \& fine sand with gravel layers; 5GY 5/1 olive grey

157- bottomed on gravel

\section{Hiller exploratory core $m$ :}

0 - 10

$10-25$

25- 36

36- 41

$41-112$

$112-$

wet fibrous peat as elsewhere, with occasional grit

peaty clay with pebbles; 10YR 3/1 brownish black

wet fibrous peat as elsewhere

clayey detritus; 10 YR $3 / 3$ dark brown

mud with detritus; 10 YR $4 / 2$ greyish yellow brown

bottomed on gravel

\section{Hiller exploratory core $n$ :}

0 - 20 wet fibrous peat as elsewhere

20-30 clayey detritus; 10YR 3/3 dark brown

30- 37 clay with some detritus

37- 95 pebbly sandy mud; 2.5 Y $3 / 1$ brownish black, greenish below $80 \mathrm{~cm}$

95- bottomed on gravel

\section{Hiller exploratory core o:}

0-95 wet fibrous peat as elsewhere

95-115 gravel; 5Y 5/1 grey

115-124 clay with plant fibres; 2.5 Y $5 / 2$ dark greyish yellow

124-125.5 clayey peat; 10 YR $3 / 2$ brownish black

125.5-131 same as $115-124 \mathrm{~cm}$

131-132.5 clayey peat with fine gravel

132.5-182 clay \& silt with some plant material

$182-186$

$186-190$

190-220

220-232

232-240

240-245

245-250

clay but more peaty than above horizon; 5 Y $3 / 1$ olive black

sand with silt to very fine sand; $2.5 \mathrm{GY} 4 / 1$ dark olive grey

grey clay

brown peat

grey clay

peaty

gravelly 


\author{
Kellia \\ Depth below Description \\ ground surface \\ (cm)
}

\title{
Hiller exploratory core a:
}

0- 25 stiff mud; 10YR 4/2, greyish yellow brown

25- 60 stiff mud with some plant material; 5 Y 5/1-2, grey to greyish olive

continued with soil auger:

60-100 stiff greyish olive mud as above with calcareous grit \& plant material

100-135 same as above, but more gritty \& more plant material

135-140 same but sandy

140-170 same as $60-100 \mathrm{~cm}$

170-175 peaty material mixed with same mud with calcareous grit

175-205 as 60-170 cm

205-235 same mud, but less calcareous grit, much plant material

235-340 same mud, but much firmer \& less plant material, less calcareous grit

340-415 softer silt-rich mud, with fine calcareous grit and no plant material

415-445 same as $60-100 \mathrm{~cm}$

445-470 same mud with some orange mottling; quartz pebble at $467 \mathrm{~cm}$

470-480 sand; 2.5 Y 5/3, yellowish brown

480-490 gravel

\section{Hiller exploratory core $b$ :}

0 - 18 stiff mud; 10YR $4 / 2$, greyish yellow brown

18- 20 sand; $2.5 \mathrm{Y} 6 / 3$ dull yellow

20-22 same as top horizon

22- 25 sand as at $18-20 \mathrm{~cm}$

25- 97 stiff greyish olive mud with some plant material as in section 'a' from $25-60 \mathrm{~cm}$, sandy below $85 \mathrm{~cm}$

97-100 grey muddy sand

\section{Hiller exploratory core :}

0 - 20 stiff mud; 10YR 4/2, greyish yellow brown

20-25 sand as in exploratory core b

25-133 stiff greyish olive mud with occasional plant material; sandy at 75-82, 108-112,

$119-121,126-131 \mathrm{~cm}$

133-138 greyish olive muddy sand with grit at base

138-273 greyish olive mud as above, slightly sandy in places; noticeable sandy layers at

160-165, 182-185, 186-190, 194-195 cm

273-290 peaty silt; 10YR $2 / 3$ brownish black

290-350 same as $25-133 \mathrm{~cm}$

continued with soil auger:

$350-410$ same as $25-133 \mathrm{~cm}$

410-430 greyish olive silty mud, very little or no plant material; stiffer

430-435 greyish olive mud with brown-orange mottles

435-470 brown clay with orange mottles \& grit to $3 \mathrm{~mm}$

\section{Hiller exploratory core $d$ :}

0-15 mud; 10YR $4 / 2$, greyish yellow brown

15- 17 sand as in 'b'

17-150 stiff greyish olive mud with plant fragments \& sandy layers (sand minor); calcareous grit; sandy layers at $35-48 \mathrm{~cm}$ and $132-140 \mathrm{~cm}$; fruit at $65 \mathrm{~cm}$; concentration of coarse plant material at $90-110 \mathrm{~cm} ; 5 Y 5 / 2$ greyish olive 
continued with soil auger:

$150-180$

same as above

$180-200$

change in colour at 180 to $5 \mathrm{Y} 5 / 1$ grey; mud stiffer from $180 \mathrm{~cm}$

200-225

no sample retrieved

continued with D-section corer:

225-240

browner more organic matter, 10YR $3 / 2$ brownish black

240-260

mud, slightly darker $5 Y$ 4/1 grey

260-280

no sample retrieved

continued with soil auger:

280-300 mud, very stiff with less plant material

300-305 mud, 5Y 5/1 grey; no plant material

305-310

310-360

$350-360$

$360-373$

373-395

$395-408$

intermixed grey and $2.5 \mathrm{Y} 6 / 3$ dull yellow mud, no plant material

mud with occasional mottles of 10 YR $5 / 8$ yellowish brown; minor plant material; carbonate nodules $2 \mathrm{~mm}$ rare; darker from $340 \mathrm{~cm}$ i.e. $2.5 Y$ Y $5 / 3$ yellowish brown;

$408-500$ sandy with carbonate and quartz

no sample retrieved

muddy sand, orange mottles and ?charcoal also manganese mottles
mud with orange mottles and quartz grit at top, sand fines upwards from coarse sand at base

muddy fine sand and silt, orange and black mottles and some carbonate grit, these increasing with depth

\section{Soil auger exploratory core e:}

$0-15 \quad$ stiff clay with some silt; 10 YR $4 / 2$, greyish yellow brown

15-110 stiff greyish olive clay with calcareous grit; sand layers at $25-28,45-47 \mathrm{~cm}$; quite peaty continued with soil auger:

at $100 \mathrm{~cm}$; charcoal and peaty at $110 \mathrm{~cm}$

$110-300$

mud as above; $130-136 \mathrm{~cm}$ - grey clay with much plant material as elsewhere; sample between 160-180 cm not retrieved (prob. too soft); $180-200 \mathrm{~cm}$ - less plant material and with calcareous grit; $220-240 \mathrm{~cm}$ - some brown mottling of peaty material; at $275 \mathrm{~cm}$ less plant material \& firmer; calcareous grit; $280-300 \mathrm{~cm}$ stiff grey clay with some plant material \& calcareous grit

300-360 muddy sand; $300-320 \mathrm{~cm}-5$ Y $5 / 3$ greyish olive; $320-340 \mathrm{~cm}$ - more orange than above; 10YR 5/6, yellowish brown with iron staining; $340-360 \mathrm{~cm}$ - same as above with more iron staining

\section{Soil auger exploratory core $f$ :}

$0-10$

$10-24$

24- 32

32- 64

64- 68

$68-170$

$170-172$

172-183

183-198

198-200

200-260

260-290

290-317

$317-320$

320-355

$355-380$ peaty mud; 7.5YR 4/2 greyish brown; grades into horizon below mud with calcareous grit; $2.5 \mathrm{Y} 6 / 3$ dull yellow sand medium to coarse (up to $4 \mathrm{~mm}$ ); orange mottled especially at base; some plant material same stiff olive greyish clay (5Y 5/1) with plant fragments and calcareous "sand" layers $1-2 \mathrm{~cm}$ medium sand same stiff grey clay with plant material \& calcareous grit iron stained gravel same clay as above sandy layer with abundant calcareous grit same clay as above mud much softer with more abundant plant material; not much material retrieved mud remains soft, some plant material same mud, but firmer from $290 \mathrm{~cm}$ with some calcareous grit grey mud with large orange/yellow mottles firm grey mud with calcareous grit \& little plant material yellow sand with orange mottles 


\section{Soil auger exploratory core g:}

0 - 10 friable brown soil with roots and some peaty mud same as previously but drier 10- 45 stiff greyish olive mud with calcareous grit, some small orange mottles, $2 \mathrm{~mm}$ at $10-20 \mathrm{~cm} ; 2.5 \mathrm{Y} 6 / 3-5 / 3$ dull yellow-yellowish brown; drier than previously; at $30 \mathrm{~cm}$ small (grit size) pottery fragment; more calcareous $30-35 \mathrm{~cm}$; less gritty $35-40 \mathrm{~cm}$

45-73 laminated silt \& clay; 2.5Y 5/2-7/4 dark greyish yellow-light yellow, general appearance $2.5 \mathrm{Y} 6 / 3$ dull yellow; calcareous and with occasion plant material e.g. $3 \mathrm{~cm}$ of Equisetum rhizome at $70 \mathrm{~cm}$

73- 80 medium grained sand with orange mottles and some plant material

80-110 olive grey (2.5GY 5/1, olive grey) calcareous clay and silt with orange mottles some plant material; soft and slightly moist at bottom; Equisetum fragments

\section{Soil auger exploratory core $h$ :}

0 - 15 peaty silt with some orange mottles; $7.5 \mathrm{YR} 3 / 2$ brownish black; more peaty at bottom

$15-25$

25-115 greyish olive clay carbonate rich with plant material; very wet from $40 \mathrm{~cm}$ below ground surface level; peaty layer at $100 \mathrm{~cm}$ as in exploratory core ' $\mathrm{e}$ '

\section{Soil auger exploratory core $i$ :}

$0-15$

15- 20

20-296

silty peat; 7.5 Y $3 / 2$ olive black

296-307

peat with clay and silt; $5 Y$ 7/2 light grey

307-376

stiff olive greyish mud as elsewhere, carbonate rich with coarse plant material; very wet from $80 \mathrm{~cm} ; 75-95 \mathrm{~cm}$ very peaty, $7.5 \mathrm{Y} 3 / 2$ olive black

\section{Detritus}

376-386

soft olive greyish mud with very little plant material; gastropod collected while cleaning hole, ?from $370 \mathrm{~cm}$

386-406 mixed grey and brown clay brown clay

Soil auger exploratory core $j$ :

0- 7 peaty silt; moist at top; 10YR 3/4 dark brown

7- 12 peaty silt and clay $7.5 \mathrm{Y} 3 / 2$ olive black

12- 14 peaty silt and clay 5 Y $7 / 2$ light grey

14-100 stiff olive greyish mud with coarse plant material as elsewhere; rich in plant material $20-30 \mathrm{~cm}$; calcareous from $35-50 \mathrm{~cm}$; less calcareous and more plant material below $50 \mathrm{~cm}$; very moist from $60 \mathrm{~cm}$; fine grained with much plant material as from $50 \mathrm{~cm}$; peaty material from $80-100 \mathrm{~cm}$

\section{Soil auger exploratory core $k$ :}

$0-20 \quad$ peaty silt and clay; 7.5 Y $3 / 2$ olive black

20-160 stiff olive greyish mud with plant fragments and calcareous grit; carbonate rich at $40-50 \mathrm{~cm}$; stiff between $80-115 \mathrm{~cm}$ with less organic material

160-180 same mud, but less plant material; carbonate rich

180-280 same mud, but no plant matter; very carbonate rich

280-300 silty olive greyish mud; no plant material; little carbonate material

\section{Soil auger exploratory core $l$ :}

$0-10$ peaty silt; 10YR 4/2 greyish yellow brown

10-20 peaty silt and clay 10YR $2 / 1$ black

20-35 peaty silt and clay - clayey peat; 10YR $4 / 1$ brownish grey

35-100 stiff calcareous olive greyish clay with plant fragments as before; bivalve at $50 \mathrm{~cm}$

\section{Soil auger exploratory core $m$ :}

0- 20 crumbly silt and clay with fresh roots, carbonate; some peat; slightly moist; worm at $20 \mathrm{~cm}$; 10YR 5/3 dull yellowish brown, paler at base (all material this colour is 
probably disturbed after drainage)

20- 57 clay and silt, with carbonate and some plant material laminated; small orange mottles; 10 YR $6 / 3$ dull yellow orange

57- 67 stiff olive greyish clay with orange mottles

67-100 stiff olive greyish clay with plant fragments and carbonate; gastropod at $90 \mathrm{~cm}$

\section{Soil auger exploratory core $n$ :}

0- 50 crumbly very slightly moist; top same as for ' $\mathrm{m}$ '; remnant lamination in places; paler at base; gastropods at $c .38 \mathrm{~cm}$; carbonate; minor organic fragments

50- 65 carbonate-quartz grit plus ?black nodules of same size; muddy matrix; concentration of soil nodules i.e. concentrated with water washing

65- 97 as 0-50 $\mathrm{cm}$ with rare small orange mottles

97-100 olive grey clay with orange mottles

100-120 firm grey clay with much plant material

120-140 stiff grey clay with plant material \& calcareous grit as elsewhere

140-180 stiff clay with ?charcoal \& calcareous material; 7.5GY 5/1 greenish grey (sample

collected)

180-220 more organic; darker shade, $2.5 \mathrm{GY} 4 / 1$ dark olive grey

220-280 very stiff with plant material \& calcareous material

280-290 small orange mottles

290-307 more calcareous \& less plant material \& more mottles

307-330 much calcareous material with small orange mottles; $2.5 \mathrm{Y} 6 / 3$ dull yellow

\section{Soil auger exploratory core 0 :}

0 - 20 slightly moist, crumbly clay as at ' $n$ '

20-25 carbonate nodule grit, minor quartz grit

25- 60 slightly laminated brown clay and silt with small orange mottles; carbonate

60-100 stiff clay with carbonate and plant fragments; darker than above, 10YR 5/2 greyish yellow brown; Equisetum at $75 \mathrm{~cm}$; much Equisetum from $80-100 \mathrm{~cm}$

\section{Soil auger exploratory core p:}

$0-15 \quad$ silty peat with living roots

15- 30 peaty clay lighter in colour slightly laminated with orange mottles

30- $40 \quad$ medium sand with abundant carbonate

40- 75 stiff olive-grey clay with coarse plant fragments; carbonate and sandy layers

75- 79 olive-grey sand with abundant carbonate and abundant plant material

79-100 as for $40-75 \mathrm{~cm}$

\section{Soil auger exploratory core q:}

$0-10$

10- 20

peaty mud with roots; 10 YR $3 / 2$ brownish black

$20-100$

mud with coarse plant material (oxidised material); $2.5 \mathrm{Y} 5 / 3$ yellowish brown

stiff olive-grey mud with scattered coarse plant material; sandy and peaty and carbonate rich at 32-45 and 65-67 cm; concentration of coarse plant material at: 85-87 and $95-97 \mathrm{~cm}$; small corms or fruit at c. $50 \mathrm{~cm}$ 